He

H.
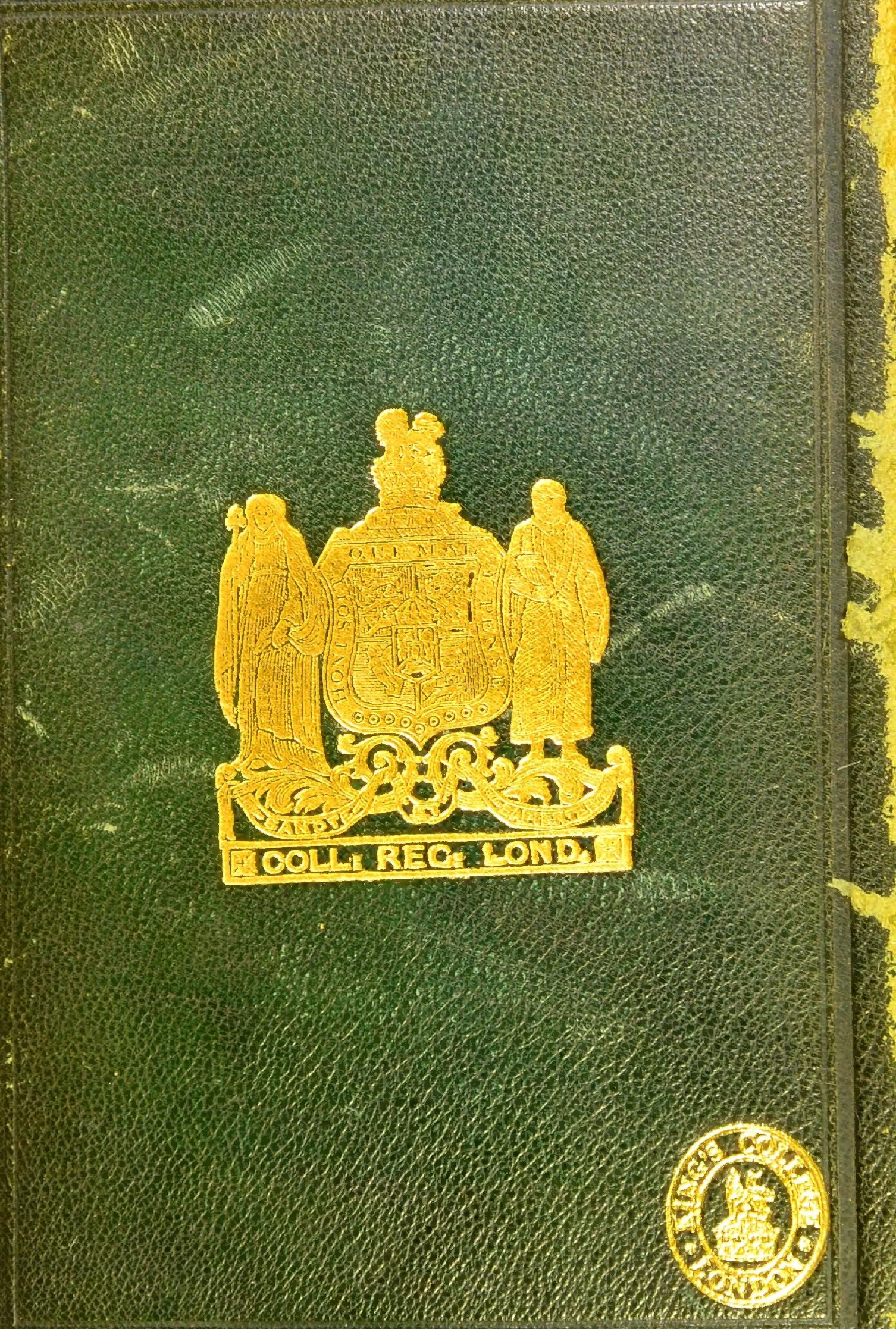


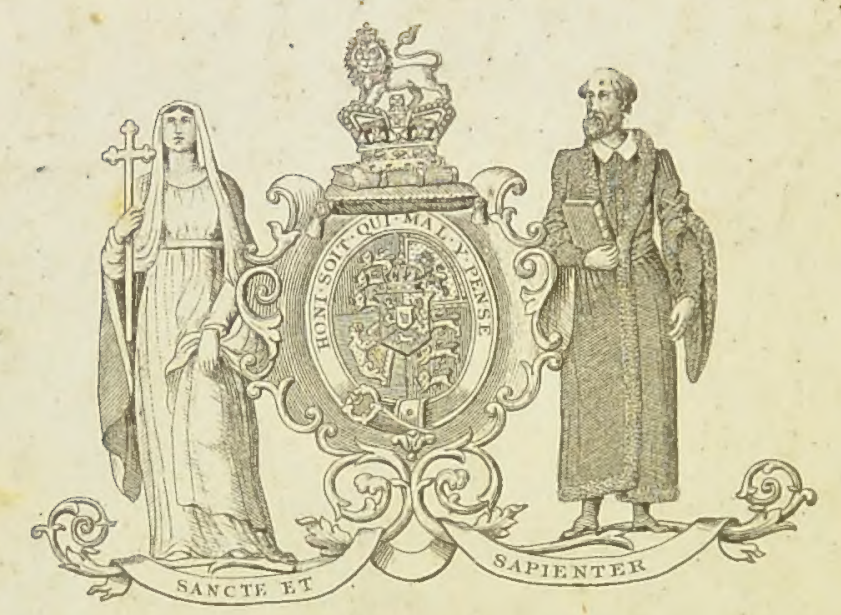

- Presented by the Council

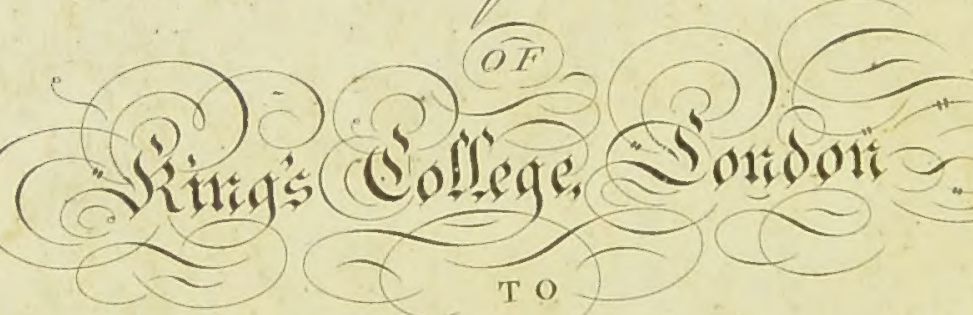

George Oliver Pierce as a lure for mathematics King's College School Christmas, 1890. Treake" Exhibition

STORE 


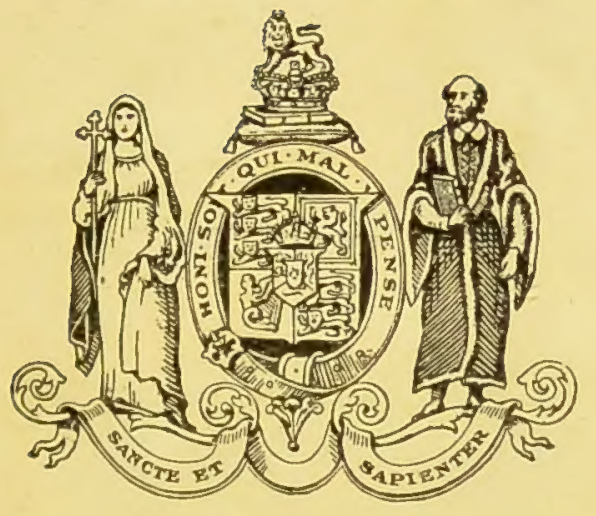

KING'S COLLEGE LONDON 


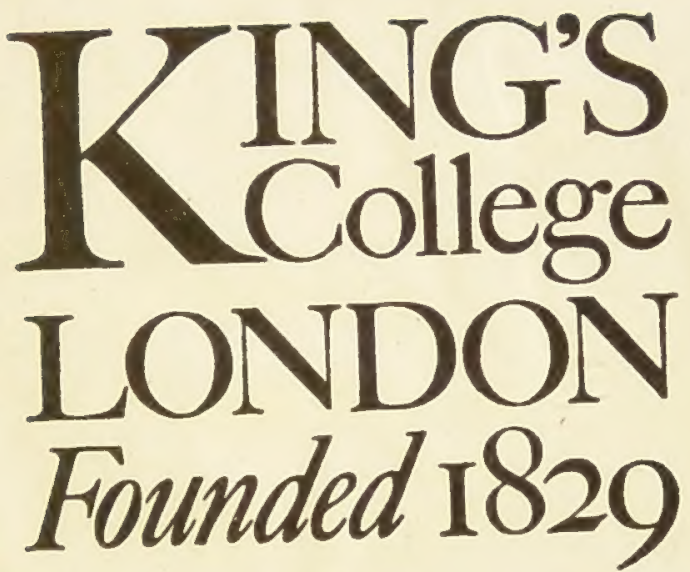

Phillips, Library

materic medica

and therepcutics

1886

$\mathrm{Cm}^{\mathrm{C}} \mathrm{Si}$.

PHI

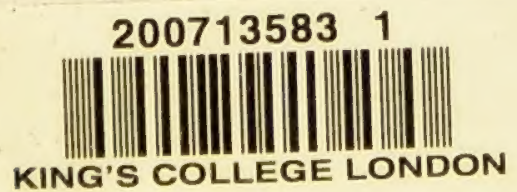





\title{
MATERIA MEDICA
}

\section{THERAPEUTICS}

\author{
VEGETABLE KINGDOM \\ ORGANIC COMPOUNDS \\ ANIMAL KINGDOM
}

BY

CHARLES D. F. PHILLIPS， M.D.

FELLOW OF THE ROYAL SOCIETY OF EDINBURGH, AND MEMBER OF THE ROYAL COLTEGE OF PHYSICIANS OF LONDON, ETC.

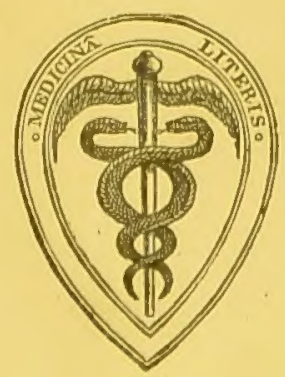

LONDON

J. \& A. C H U R CH I L L

11, NEW BURLINGTON STREET 
472888

G. 15907

Sift of De So. Rues

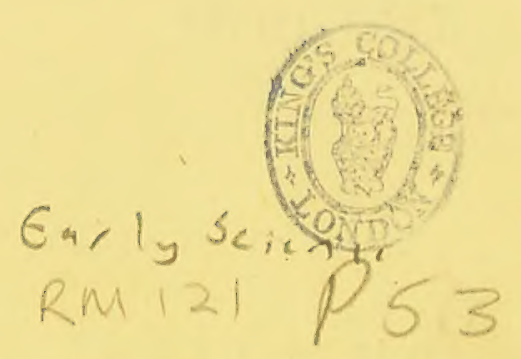

WHEATSTONE LIB. 
TO

\section{SYDNEY RINGER， M.D., F.R.S.,}

THIS VOLUME IS INSCRIBED

IN RECOGNITION OF IIIS DISTINGUISHED WORK ON THE SUBJECTS OF WHICH IT TREATS,

$$
\text { AND OF }
$$

VALUED FRIENISHIP FOR MANY YEARS. 
Digitized by the Internet Archive in 2015

https://archive.org/details/b21297046 


\section{P R E F A C E.}

THE present is i revised, enlarged, and almost entirely rewritten edition of my work on "The Vegetable Kingdom." It completes a Treatise on Materia Medica, of which the volume referring to "Inorganic Substances" was published in 1882, and it includes many substances, such as carbolic acid and ethers, which I had originally intended to treat of in a third volume; hence the somewhat large size of this one, for I have been reluctant to adopt smaller type, or a less open page, helieving that the form chosen will be that most generally appreciated. I have endeavoured to give a sufficiently full account both of the physiological and therapeutical action, without too minute detail, having always in view the practical and of scientific work.

Although many allied treatises have been published since my first edition, I still think there is need for such a one as this.

I desire to noknowlerlge my obligations to the standard works on Pharmacolngy and Therapentios in English, French, and German, ns woll ns to mnny moungraples and journals; amongst the latter, free use has been male of 
the "Lancet," "British Medical Journal," "Medical Times," "Practitioner," "London Medical Record," and of the "Revue des Sciences Médicales." The abbreviations, are, I trust, sufficiently explicit for reference.

The greater part of this volume was printed before the publication of the "British Pharmacopœia of 1885"; but the additions to and changes from the previous edition will be found described in a summary at the end of this work.

I have to thank my friends Dr. Allchin, Dr. Mackey, Dr. S. H. C. Martin, and Mrr. A. Pearce Gould, for valuable help and suggestions while the work was passing through the printer's hands.

10, Henrietra Street, Cavendish Sodare, W. March 1st, 1 sise. 


\section{O N T E N T S.}

\section{VEGETABLE KINGDOM.}

RanUNCULACE :

The Aconite Family

MagNoltaCe :

The Magnolia Family ...

Menisperuace正:

The Calumba Family

PAPateracele:

The Poppy Family

Cruciferde:

The Cress Family...

VIOLACE及:

The Violet Family

PoLyGathaCe AE :

The Rhatany Family .

ERyTHROXYLACT As :

The Coca Family...

IINACF,E:

The Flax Family ... ... 152

MATYACE :

The Nallow Family $\quad \ldots \quad 155$

AURavtracee:

The Orange-tree Family ...

S'T FRCULIACT, I: :

The Cacao Family

1
PAGE

Rimamaceds :

The Buckthorn Family ... 218 ANACATRDIACLE:

The Sumach Fanily ... 222

BUtraracem:

Camelitaceas:

PAGE

SAPINDACre

GUTTIFER刃:

The Gamboge Family . $\quad 183$

Canellaces :

The Canella Family $\quad \ldots \quad 186$

VITACEX:

The Grape-vine Family ... 187

ZYGOPHYLLACE.T:

The Guaiacum Family ... 191

RUTACEA :

The Rue Fanily ... ... 195

StMaRUbacex:

The Quassia Fumily $\quad \ldots \quad 214$

Criastractas:

The Spindle-tree Family... $\quad 216$

The Myrrh Family _.. 230 


\section{LEGUMTTOSE :}

The Pea Family ...

SANTHLACE :

The Sandal-wood Family

ROSACEI :

The Rose Family ...

FAMANELACEX:

The Witch-hazel Family

MTrTACEA :

The Myrtle Family

GrayataCex:

The Pomegranate Family

CUCURBITACE :

The Cucumber Family ... 308

UMRELLIFERA :

The Parsley Family _.. 319

Caprtfoltace a :

The Honeysuckle Family

\section{Cinchon ACE F :}

The Cinchona Family ...

\section{VALERIANACEAE :}

The Valerian Family ...

CompostTx:

The Aster Family

LOBELIACEA :

The Lobelia Family ...

Ertcactis :

The Heath Family

SAPOTACFA:

The Gutta-percha Family

S'ITRACACHE:

The Benzoin Funily

Pyrolaceas:

The Pyrola Tramily _... 437

OLEACEA :

The Olive-tree Family ...
APOCYNACEX:

XGH

The Doghane Family ... 447

SpIfirlaCe

The Nux-Vomica Family

ASCLEPIADACE :

The Wax-Flower Family

Gentranacez:

The Gentian Family ... 494

Convolvolace 2 :

The Convolvulus Family 498

SOLAyaCE无:

The Nightshade Family... 505

SCROPHULARIACE $\mathrm{x}$ :

The Foxglove Family _.. 590

LABIATE:

The Sage Family ... $\quad \ldots \quad 622$

Polygonacex :

The Rhubarb Family .. $\quad .633$

MrRisticaced :

The Nutmeg Family _.. 642

LATRACEX :

The Cinnamon Family ... 645

A RISTOLOCHIACEAE:

The Birthwort Family ... 659

JUGLANDACEX:

The Wahut Family $\quad$.. 662

THYMELACEE :

The Mezereon Family ... 664 EtrHORBIACEA :

The Croton Family ... 666 Pirtichac :

The Peppor Family ... 677

SALICACEA :

The Willow Family ... 684

UT,MACEA :

The Elm Family ... ... 712 


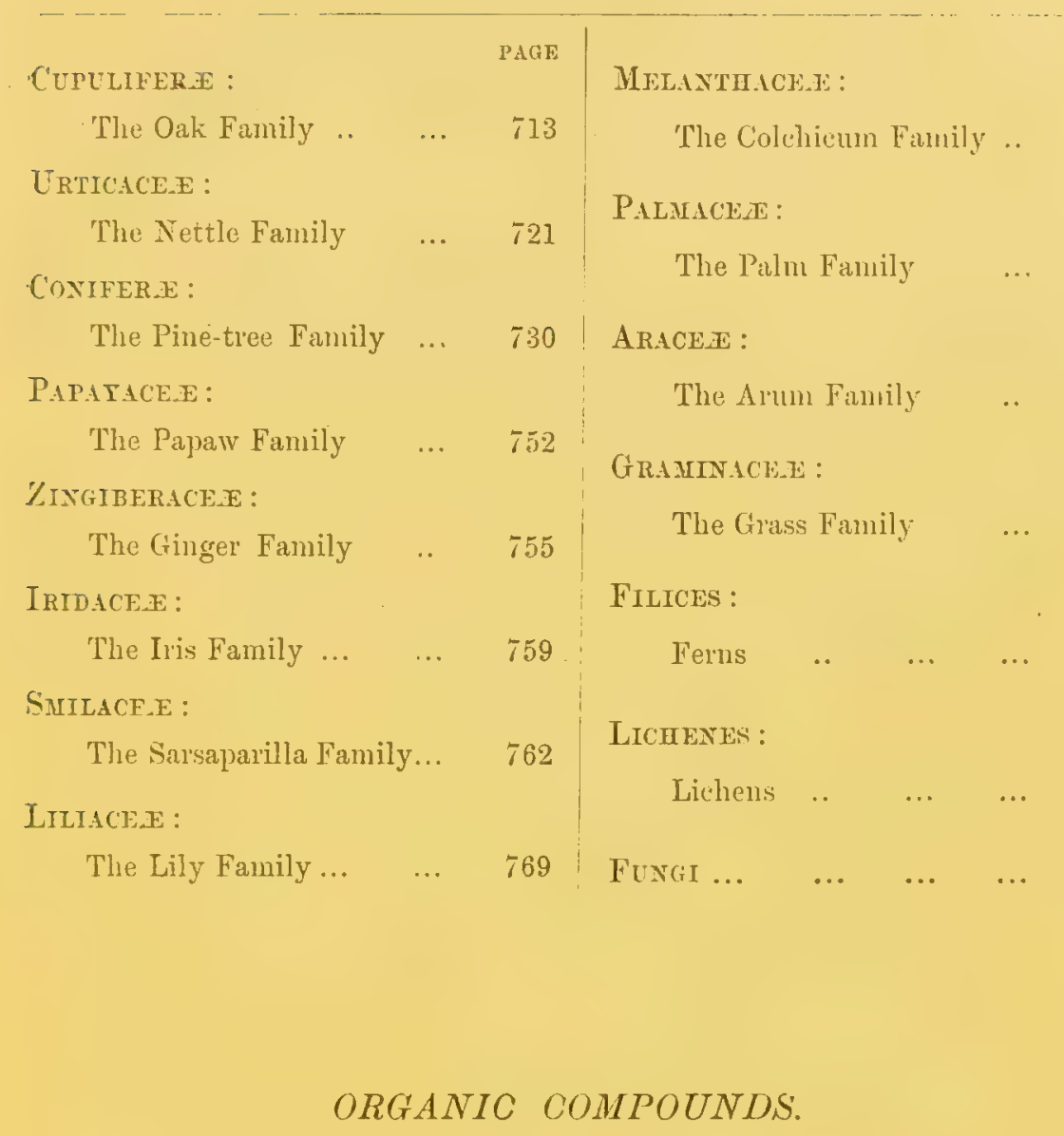

PAGK

\begin{tabular}{|c|c|c|c|c|c|}
\hline ACrdiar C'ArBolicum & $\cdots$ & 830 & A:снут, Iontdu & $\cdots$ & $\cdots$ \\
\hline SODA SULPHO-CARBOLAS & ... & 865 & AтHYL Bromat: & I... & ... \\
\hline KIKEASOTEM ... & $\cdots$ & 868 & Sodm Ethriats & $\cdots$ & .. \\
\hline Ftechein $\quad \ldots$ & $\ldots$ & $8 \div 2$ & Eitier Acrictes & ... & ... \\
\hline RESORCIN $\ldots$ & $\cdots$ & 872 & 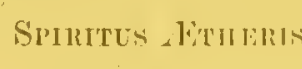 & S NTRO: & si \\
\hline Cinbon-Compounus: & & & Aнта Nitris & & \\
\hline rascous amd Liınul & $\cdots$ & 875 & Tínilin & & $\ldots$ \\
\hline AlCoHIOL $\quad \ldots \quad \ldots$ & $\cdots$ & 877 & & & \\
\hline CEREVISI FeRMENTU & $\ldots$ & 907 & ANTIPTIRIN ... & $\cdots$ & $\cdots$ \\
\hline Jrve (Malt) & & 0() 8 & CHLOHOFOHMUM. & $\cdots$ & $\cdots$ \\
\hline P'ARALEHŸ & $\cdots$ & 9()$!$ & 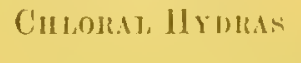 & $\cdots$ & $\cdots$ \\
\hline iETாЕ & $\cdots$ & 912 & 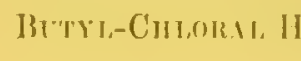 & Irolls & $\cdots$ \\
\hline
\end{tabular}




\section{ANIMAL KINGDOM.}

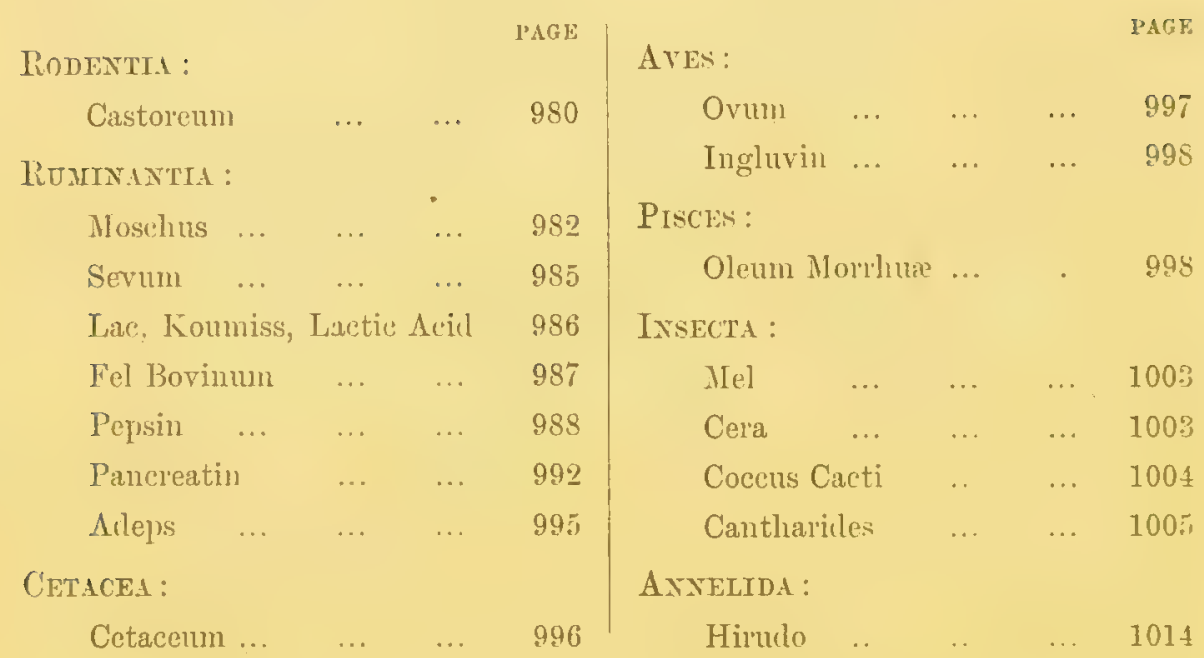




\section{MATERIA MEDICA}

AND

\section{THERAPEUTICS.}

\section{VEGETAB L E KI NGDOM.}

\section{RANUNCULACEA.}

\section{- THE ACONITE FAMILY.}

THis contains about a thousand species of exogenous plants, distributed throughout Europe, Asia, and North America, the medicinal varieties growing largely in mountainous regions. The majority are terrestrial and herbaceous, with simple, deeply-divided leaves, dilated petioles, no stipules; regular flowers, with five sepals and petals, and numerous stamens and ovaries-all free. Many genera are, however, irregular: in some, the petals are absent, and the calyx resembles a corolla; in others, the stamens are only five, and the ovaries two or three.

A narcotic acridity, the most usual property of the order, renders many species very active, often poisonous; some, however, are only bitter and tonic (Aconitum heterophyllum), and the leaves and roots of others are eaten as vegetables in Lapland and India (A. septentrionale, A. multifidum). 
The chief species employed medicinally are-

$\begin{array}{llll}\text { Aconitum Napellus . . } & \ldots & \text { Aconite or monkshood. } \\ \text { Pulsatilla nigricans . . } & \text {. } & \text { Pulsatilla. } \\ \text { Hellebŏrus niger _. } & \ldots & \text { Hellebore. } \\ \text { Podophyllum peltatum } & \ldots & \text { Podophyllum. } \\ \text { Hydrastis Canadensis } & \ldots & \text { Yellow-root. } \\ \text { Delphinium Staphisagria . . } & \text { Stavesacre. } \\ \text { Actæa racemosa _. . . } & \text { Actæa or black snake-root. }\end{array}$

\section{ACONITUM NAPELLUS.}

\section{(ACONITE.)}

DESCRIPTION.-The common or purple aconite, known in gardens by the name of "monkshood" or "wolfsbane," is a native of the alpine forests of Sweden, France, Italy, and other parts of Central and Southern Europe; in our own country it grows apparently wild, but is probably only naturalized; upwards of thirty varieties have been described.

The root is perennial, tapering or spindle-shaped, with many lateral rootlets, and having one or more pyriform tubers attached to its upper portions; it is of dark brownish colour externally, white internally, and has an earthy odour. (It has been fatally mistaken for horse-radish; but that root is of nearly uniform thickness, is destitute of fleshy lateral rootlets, and exhales, on scraping, a characteristic pungent odour.) The flowering-stems rise early in the spring, and attain the height of 3 or 4 feet; they are simple, erect, and glabrous. The leaves are numerous, alternate, petiolate, and deeply divided; their breadth is about 2 inches, but they vary in size, the upper ones being often small, trifid, and sessile, or nearly so ; in colour they are of a dark and shining green, and their surface, like that of the stem, is glabrous. The violet-purple flowers constitute a large, erect terminal raceme, each bloom being about an inch and a half in length, of irregular shape, the modified calyx forming a remarkable hood, which shelters a couple of hammer-shaped abortive petals, and is, with 
these, characteristic of the genus. Numerous blackish stamens cluster round the three or four pistils, which ripen into follicles, containing many angular, dark-coloured seeds. The time of flowering is about midsummer, but, when cultivated in gardens, the raceme often develops lateral branches, which bloom in succession for many weeks.

ACTIVE INGREDIENTS.-The properties of aconite mainly depend upon aconitia, or aconitin, $\mathrm{C}_{33} \mathrm{H}_{43} \mathbf{N O}_{12}$ (or according to Duquesnel $\mathrm{C}_{54} \mathrm{H}_{40} \mathrm{NO}_{2}$ ), which may be obtained from good dry roots in the proportion of about 1 per cent. It varies somewhat in character according to its preparation, but is usually met with as an amorphous, granular, yellowish-white or grey powder. Morson obtained it in crystals (1862), also Groves and Duquesnel (1864), and this crystalline form is now common.

Aconitia (B.P.) is said to be soluble in 150 parts of cold and 50 of hot water, and is still more soluble in alcohol, ether, and chloroform (British aconitin, however, requires 4,000 parts of water for solution-Martindale). It melts with heat, and burns with a smoky flame, leaving no residue if there be free access of air ; it has strongly marked alkaline characters. With sulphuric acid it gives a brown colour, but the perfeetly pure drug is said to give no characteristic colour-reaction with acids or oxidizing agents.

Commercial aconitia, indeed, is not a simple substance, but a mixture probably of several alkaloids; it may vary in power to a dangerous extent, for which reason it has now been erased from the pharmacopoias of Germany and of the United States (1883), but I think this a retrograde step.

Dr. Alder-Wright finds that an inert bitter base, picraconitin, which is itself erystalline, is contained in many specimens of the alkaloid, e.y., in Groves' and Duquesnel's, and its varying proportion is the cause of some of the difference in their effects; also that another (unnamed) alkaloid exists, which further explains their variation in strength (Lancet, ii., 1877, and Pharm. Journ., ii., 1880, and i., 1883). There is still room for definite research on these points.

Dr. Springmuihl endeavoured to show that the true alkaloid was prepared only in Germany; but the contrary seems really to 
be the fact. The English preparation is very much more active some of the French aconitin (Petit, Duquesnel) is stronger still, and fatal results have followed the substitution of these for the German form by an ignorant chemist-for instance, when $\frac{1}{16}$ grain of nitrate of aconitin (Petit) was dispensed instead of Merck's (B. M. J., i., 1882 ; Lancet, ii., 1880).

The best-known aconitins are arranged by Plugge in the order of their strength as follows-1. Duquesnel's; 2. Morson's; 3. Hottot's ; 4. Hopkin and Williams'; 5. Merck's; 6. Schuchart's; 7. Trommsdorff's (Virchow's Archiv, 1882). In such a list Petit's should be placed high, and Friedlander's towards the end. Japaconitin, from A. Japonicum, is said to equal or surpass in activity any of the others (Harnack and Mennicke, Berl. Klin. Woch., Oct., 1883). (There are two kinds of Japanese roots, which are well described in the Pharm. Journ., ii., 1880.)

A second and closely allied alkaloid, pseudaconitin, $\mathrm{C}_{36} \mathrm{H}_{49} \mathrm{NO}_{12}$, has been obtained mainly from the Aconitum ferox (Bish) of India, and seems to have been at one time (1858) used as aconitin, and preferred to that procured from the Napellus; it occurs both in the crystalline and amorphous form. It has been called also fer-aconitin, and the first one nap-aconitin, but A. Napellus yields both.

Another alkaloid, aconella, has been described by T. and H. Smith, who consider it identical with narcotina. Lycoctonin is an alkaloid obtained from $\mathrm{A}$. lycoctonum, which does not yield ordinary aconitia.

Another base, napellin, a white, amorphous, bitter substance, has been described by Hübschmann, but its exact relations are uncertain. These basic substances are combined with an acid, termed aconitic acid, $\mathrm{C}_{6} \mathrm{H}_{6} \mathrm{O}_{(3}$, which may be isolated in colourless laminæe or warty masses, and occurs largely in the leaves: it is found also in the larkspur, equisetum, etc.

ABSORPTION AND ELIMINATION.-Liégeois has shown that the alkaloid is readily absorbed by the skin and subcutaneous tissue; by the mucous membrane of the mouth, rectum, and still more rapidly by the stomach (within four minutes) and by the lungs. It is more quickly taken up than strychnia. 
Its elimination is not easy to trace, but occurs mainly by the skin and kidneys. In the Lamson case, Drs. Stevenson and Dupré found aconitin in the urine (B. M. J., i., 1882). Laborde and Duquesnel have also found the alkaloid pass unchanged in the urine, and conclude that it is eliminated also by the liver and the salivary and gastro-intestinal glands (Des Aconits et de l'Aconitine, Paris, 1883). Laborde experimented as to how long after absorption it remained in the blood, by transfusing this from the veins of a poisoned animal into a healthy one, and found that the greater part of the poisoned blood might be thrown in without toxic effects, although the operation was effected within a few minutes after the beginning of the poisoning.

Physiological Action.-External.-Aconite, in the form of tincture, powdered root, or leaf, simply applied to the unbroken skin, does not produce pain or redness; but its prolonged contact, accompanied by friction, creates a characteristic burning, numbness, and tingling. This is especially marked in mucous membranes, as on the tongue and lips; on tasting, or especially on chewing the drug, the flow of saliva is increased, heat and tingling are felt, followed by numbness, and a sense of swelling of the parts touched, which effect remains for many hours; placed upon the eyelid, aconite causes a flow of tears, but no sensation of heat. A minute fraction of the allicloid brought into contact with the eye will produce severe irritation for several hours, and, if inhaled, will bring on profuse nasal and bronchial catarrh (Wright, Lancet, ii., 1877). When the tincture is painted on the temple the pupil generally becomes contracted, but sometimes dilated; when aconitin ointment is lightly painted on the conjunctiva, contraction quickly occurs (Fleming).

PHysiological Action. - Intemel. - Circulatory System.-Lowering of the force and frequency of the heart's action is the usual first effect of full doses of aconite in all classes of animals. In frogs the depression is followed by increased rapidity and evident weakness of the action, which soon becomes irregular, with long pauses, and is finally arrested in diastole 
(Achscharumow, Reichert's Archiv, 1866). Laborde describes somewhat differently the action of aconitine on a frog's heart, viz., (1) a short period of quickening, with irregular contraction; (2) a period of doubled force and completeness of contraction; (3) slow, progressive weakening and arrest. These effects are produced, he considers, through the spinal nerves, and more immediately through the vagus, the drug not acting as a primary poison to the cardiac muscles;-but that it does exert a direct influence upon the heart-substance or ganglia seems proved by the fact that when placed on the organ after removal from the body it produces effects similar to those above described. The electric contractility of the heart-fibre is not destroyed at the moment of death: but afterwards the muscle does not react to a galvanic eurrent. The description of Böhm substitutes for stage (2), " a short convulsive phase wherein the three eavities contract irregularly and independently of each other."

In mammalia similar results occur-i.e, quickening, irregularity, and slowing-and lessening of arterial pressure has been demonstrated; it may be preceded by a temporary increase.

According to Dr. Fleming, the drug exerts a direct sedative effect on the vascular system, reducing, more or less, with the dose, the strength, volume, and, at first, the frequency of the pulse, which may sink as low as 60 , or even 40 per minute; so long as it is slow it usually remains regular, but becomes intermittent and irregular as it rises again in frequency. If only two or three full doses-10 or 20 minims of the tincture-lave been given, the heart recorers itself in from twelve to twenty-four hours; but if the drug has been taken for a week or more, the cardiac depression persists for several days.

The slowing of the pulse is attributed by Achscharumow and Lewin to "stimulation of the cardiac inhibitory centres" in the medulla oblongata.

In the early stage of aconite-poisoning the pulse may be quickened for a time, and the face flushed, with a feeling of heat and fulness in the head; on several occasions I have seen epistaxis occur. Later, the countenance becomes 
pale, the lips blue, and the tongue cold; the limbs also become cold and pulseless. Fatal cases in man generally terminate by syncope from eardiac paralysis. I have myself experienced violent palpitation of the heart, with much precordial oppression, one hour and a half after taking 20 minims of the tincture; it lasted for about fifteen minutes, and recurred at intervals during the following day.

Irregularity and intermittence of pulse occur in some patients-the subjects of weak heart-after comparatively small doses, such as 1 or 2 minims of the tincture, repeated three times at intervals of two hours. Torsellini explains the different effects of the drug by the presence of varying amounts of the alkaloids, which have different powers; thus, nitrate of aconitin slocked the heart of a frog, napellin rendered the action irregular, whilst lycoctonin quickened (Record, 1884).

Respiratory System.-All observers are agreed as to the depressing effect of the drug upon respiration. Mackenzie found this to be "one of the earliest, most invariable, and most prominent features of aconitism" (Practitioner, vol. xx.).

In the frog, small doses render the respirations irregular, but generally deep and slow,-sometimes quick and shallow, -long pauses occurring at intervals; if the dose has not been excessive, a return to the normal condition takes place. After large doses the respirations are reduced to two or three in a minute, ceasing for some minutes at a time, and finally altogether, the heart continuing to beat for hours afterwards.

In mammals the breathing becomes slow and laboured-with occasional temporary quickening - and with spasmodic attacks of partial asphyxia from inability to inspire; expiration is prolonged, and after it follows a long pause-the whole mode of breathing markedly resembling that induced by section of the vagi. Moreover, according to the last-named observer, if, in an animal already aconitized and breathing in the peculiar manner described, the vagi be divided, no further change occurs, implying that the effect of the drug and of the section is similar in kind; that effect is plausibly held to be a paralysis of the terminals of the sensory branches of the vagi, which paralysis extends along the course of the nerves to their roots, and thus implicates the respiratory centre. Sensation is inı- 
paired in the mucous membrane of the air-passages, and the expired air is cool. The motor branches of the vagi are not affected.

In physiological experiments, death has commonly been traced either to asphyxia, or to a variety of collapse "characterized by markedly lessening respiration from a peculiar influence on its nerve-centre, the heart continuing to beat more or less strongly" (Brown-Séquard). In Laborde's observations, respiration was irregular throughout, "from spasmodic action of respiratory muscles"; death occurred from apnoen rather than from syncope. In cases of poisoning in man, symptoms of asphyxia also are often present, but sometimes there is no marked disturbance of respiration (Lancet, i., 1882); there is, however, frequently a harassing, short, dry cough. Dr. Fraser states that pseuduconitin acts more on the respiratory, and aconitin on the circulatory system (B. M. J., ii., 1881).

Nervous System.-There has been much difference of opinion as to the effects of aconite on this system, and as to the mode of their production; and the conclusions of Achscharumow (quoted in the first edition of this work) have been modified by later researches.

The point of most interest clearly brought out is the paralysis of sensory nerves induced,- -and I believe, with Mackenzie, that this commences at their periphery rather than at the perceptive centre (as maintained, amongst others, by Liégeois and Hottot, 1861). Certainly the posterior columns of the cord are unaffected long after the sensory nerves and nerve-roots have lost their functions.

In some animals there are symptoms as of increased cutaneous sensibility before the paralysis occurs; but these are due probably to altered rather than to heightened power of feeling, and to sensations like the tingling felt by some persons, or the "quasi-electric twitchings beneath the skin" described by Hirtz. Ultimately, sensation is quite abolished, and the same condition spreads along the trunk of the nerve to its root; " to it is probably due the ataxic gait and diminished power of locomotion in aconitized animals." This was noted also by Laborde "as one of the first and essential sigus of aconitepoisoning." 
Abolition of sensation is very marked in some cases in man. Thus, in a case recorded by Dr. Taylor in his work on Poisons, the patient, a quarter of an hour before death, lost all power and sensation in his limbs, the sharpest pinches producing no impression. This is further illustrated in a fatal case of poisoning by 3 drachms of aconite liniment, in which the patient was convulsed, and there was entire loss of sensibility of the skin (B. M. J., ii., 1883). J. Harley, experimenting on animals, found such anæsthesia most marked about the head and neck (St. Thomas's Hosp. Reports, v.).

With regard to the special influence of aconite on motor nerves, the general conclusion of late years has been that it is comparatively slight, and certainly less marked than that exerted on sensory branches. Laborde and Duquesnel, though locating the main effect of aconite in the medulla oblongata and spinal cord, also support this view, stating that sensation is first impaired, whilst "the excitability of the motor nerve is altogether unaffected by physiological doses." It was long ago stated by Pereira, that a dog so far under aconite as to be insensible to the pain of needles run into the skin or paws, will yet recognize and follow its master.

On the other hand, Achscharumow, Gréhaut, and, quite recently, Plugge maintain that paralysis of motor nerves is produced, beginning at their periphery (Rev. de Méd., Dec., 1883). It is singular that direct contradiction should exist on a question of fact, but there are naturally difficulties in interpreting results obtained chiefly on frogs, and in any case the question of motor paralysis must be one only of time and quantity of poison. No one would maintain that such a poison as aconite would invade only one section of the nervous system, and I consider the facts are sufficiently covered by this conclusion of Laborde and Duquesnel, viz., "that whilst the sensibility of 'mixed' nerves, and also of sensory nerves, is early impaired, and the function of a motor nerve relatively unaffected, yet, by a toxic overwhelming dose, motor power may be affected and more or less rapidly abolished" (De l'Aconite, ete., p. 311).

The failure of muscular irritability is said by these observer's to be synchronous with failure of motor-nerve power; this constituting a difference between the action of aconite and of 
curare. Plugge, however, and with him many others, find that the irritability survives much larger doses than are required to destroy the function of the nerve-endings, and this, on the evidence, may be accepted ; still, it does not warrant the conclusion that the two drugs mentioned are alike in their intimate action.

Harnack and Mennicke, writing still more lately, trace again a central action to the drug, " no other organic base so constantly paralysing the motor system from above downwards-the brain being first affected, voluntary movements cease, and power of co-ordination; then the cord loses its functions," etc.; but I cannot agree with their conclusions.

Just as some altered condition of sensibility precedes its abolition, so apparently some stimulation of motor nerves and centres precedes their paralysis. Guillaud formulates his results thus: (1) primary excitation of the said parts, (2) temporary exhaustion, (3) secondary excitation (and then, in fatal cases, paralysis). Mackenzie found such excitation constant in frogs, and traced to it the convulsions of spinal origin that commonly occur in them under aconitin. Japrconitin is specially credited with exciting powers.

Böhm and Wartmann also recognized excitation of spinal motor centres after minute doses, but not after large doses. The convulsions that nay occur in mammals, Wood considers to be cerebral, "since they do not occur in those portions of the body separated by spinal section from cerebral influence." In part they may depend on asphyxial conditions. The motor centres in the brain are, however, not easily paralysed, since the muscles respond to stimulation of such centres long after poisoning is far advanced. It is proved that the power of voluntary movement may remain after reflex activity is lost; this is contrary to the conclusions of Achscharumow; and although such loss of reflex activity is considered spinal, by Liégeois, it is more probably connected with the sensory paralysis already referred to.

The functions of the sensorimm are, as a rule, unimpaired, but after full doses there may be depression of the mental powers, with lessened ability to think or talk, tinnitus, vertigo, beadache, and other symptoms to be explained by a diminished supply of arterial blood to the brain. 
In toxic cases there is generally much restlessness, sometimes fitful sleep, occasionally convulsions, which may be slight or severe. Stupor and delirium have occurred, but the mind often remains unclouded to the last. Muscular tremor, stiffness, cramps, pain in the joints, and rigidity, especially about the jaws and throat, are commonly experienced.

The eyes are described as glaring and continually moving; the state of the pupils varies much in the early stages, contracting and dilating, with a general tendency to the former condition; later, there is extreme dilatation which persists if convulsions occur. The body-temperature may be lowered one or more degrees.

Drs. Ringer and Murrell conclude aconite to be to frogs a "protoplasmic poison," which destroys the functions of neuromuscular tissues, causing general paralysis of the nervecentres, motor and sensory, and of the nerves and muscular tissue of the heart (Journ. Physiol., i., 1879). These observer's also conclude that in the frog the phenomena observed were not due to deficiency of blood-supply.

Digestive System.--Small doses exert no special effect on the stomach, the tongue remaining clean, and the bowels regular. Any marked symptoms produced are of irritative character, such as pungent heat and constriction in the palate and fauces, with thirst, numbness and trembling of the tongue, and a feeling of burning in the stomach. Vomiting is not uncommon after the first few doses, but if these are moderate it soon subsides; after very large doses it may be severe, and often attended with jaundice, abdominal pain and distension, sometimes with diarrhœa.

Urinary System.-Aconite commonly increases the secretion of the kidneys, and not only the water but the solids. Sometimes I have known it cause pain in micturition, with irritability and some spasm of the neck of the bladder, leading to partial retention. Mackenzie concludes, from observations on about twelve cases, that this diuretic action is fairly constant in febrile conditions, but does not occur in certain forms of valvular heart-affection and chronic Bright's disease.

Cutaneous System.--Small doses have no marked effect; full ones usually, but not constantly, cause an increase of per- 
spiration, which is secondary, probably, to a sedative influence on the circulation, and hence more marked in febrile conditions. After toxic doses, cold clammy sweats occur; but if recovery is brought about, the skin becomes at first hot and dry, and then covered with profuse warm perspiration.

The influence on the cutaneous sensory nerves is evidenced by a sense of formication as well as numbness; this I have myself experienced after a dose of 30 minims of tincture B.P.; and Störck recorded fourteen cases of aconite-poisoning where there was general itching, and afterwards an eruption of red spots, followed by desquamation. The sense of formication may remain after the acute toxic symptoms have disappeared, and heat, itching, and redness of the conjunctivæ, and profuse lachrymation are usual. (An analysis of nearly 100 cases of aconite poisoning may be found in the Philadelphia Med. Times, Nov. 19, 1881.)

SYNERGISTS.-Aconite, especially when given in small, frequent doses, stands almost alone in its power of regulating and lessening over-fulness in the arterial system without too much depression of the heart; but more or less allied in action are gelseminum, veratrum, hydrocyanic acid, bromides, depressants generally, and cold.

ANTAGONISTS.-In cases of poisoning by the mouth, the stomach-pump or emetics should be used, and followed by free stimulation with hot brandy-and-water, ammonia, ether, etc.; the horizontal position should be enforced, and the circulation assisted by warmth and friction. I have found strong infusion of coffee serviceable. Dr. Thelwell Pike has recorded an instructive case in which 3 to 4 drachms of aconite liniment were swallowed, and when the patient was apparently dying from collapse, three or four hypodermic injections of 20 minims of ether at short intervals had the best result, and recovery soon followed (Lancet, i., 1880); other instances have since been published (ibid., ii., 1880, and i., 1882) ; and this mode of stimulation should certainly be adopted.

In a recent painful case, a medical man in France ordered for a patient 0.04 gramme ( $\frac{3}{5}$ grain) of nitrate of aconitin every 
hour; the patient took as much as $0 \cdot 17$ gramme (21 grains), divided into five doses, in the twenty-four hours; vomiting, burning in the throat, and, after the last dose (which was retained), collapse were produced. No doubt the earlier develoyment of this last symptom was prevented by the persistent romiting. The doctor, to show the innocence of the remedy, took of the solution 50 to 60 drops, equal 0.08 to 0.12 gramme ( $1 \frac{1}{5}$ to $1 \frac{4}{5}$ grain) of the drug. The toxic effects commenced in a quarter of an hour, and four hours later he was found pale, with small irregular pulse and other symptoms, including those of active cerebral congestion. Ether was injected, and convulsions set in; a second injection was made, but consciousness did not return, and death soon occurred. At the autopsy there was noted great pallor of the skin and muscles, but much congestion of internal organs (Bull. de la Soc. de Thérap., Fév., 1883). The occurrence of convulsion soon after the ether injection was probably only a coincidence, not a sequence, but it suggests caution in the presence of an active congestion, which itself is unusual. There is some evidence that digitalis is directly opposed to aconite in its action on the heart. Fothergill found, in frogs, that when cardiac paralysis had reached an extreme degree, the contractile power might be gradually restored to normal by the administration of digitalis; the converse does not seem to hold good (On Digitalis, p. 6). Dr. Dobie has reported the case of a man who took an ounce of Fleming's tincture, and recovered after having 20 minims of digitalis tincture injected under the skin, and three teaspoonfuls of the same administered (within an hour), together with ammonia and brandy; galvanism was applied to the cardiac region (B. M. J., ii., 1872).

Nux vomica has also been said to be physiologically antagonistic to aconite.

Dr. Ringer has shown that atropic antagonizes much of the action of aconite (Journ. Physiol., vol. ii.); and Dr. Murrell has recommended $\frac{1}{60}$ grain to be injected hypodermically in aconitem poisoning: he has seen benefit also from inhalations of amyl nitrite.

THERAPEutical ACTion.-Baron Störek was the first to draw public attention to the medicinal value of neonite 
(De Stramonio, Hyoscyamo, et Aconito, Vindob., 1762). $\mathrm{He}_{\theta}$ administered it in many different diseases, including intermittent fever, chronic rheumatism, and gout, and relates many instances of the success which attended its exhibition. Being well acquainted with the potency of the drug, he recommended small doses at first, to be very gradually increased if necessary. His observations soon led to the employment of aconite in other diseases. In consequence, however, of its potency, and the uncertainty of its mode of action, it was, and is still, distrusted; but when properly handled, it will be found a most valuable remedy in those diseased states characterized by high temperature, a quick full pulse, and a dry hot skin; in short, in certain pyrexial and inflammatory conditions. Its effects in lowering the temperature, diminishing the burning heat, and allaying febrile excitement, correspond closely to that formerly produced by bleeding as practised by our predecessors in similar morbid states. It is especially of service in the earlier stages of inflammation, and in the fevers of the strong and full-blooded, and those of sanguine temperament and plethoric habit; whilst not without some value in the later subacute stages of inflammation, it is distinctly contra-indicated when the patient's vitality is depressed, and the "typhoid state" has supervened.

Acute Specific Fevers.-The value of aconite in moderating febrile action in these disorders is now largely recognized. In scarlet fever and measles it favours perspiration and the development of the rash, and is specially suitable for cases with a high temperature; it modifies this, and seems to render the course of the disorder milder. Sometimes it will lower the pulse and not the temperature: thus, in four cases of measles, I noted that under aconite the pulse fell to about 72 on the second day of the eruption, and continued so during the eruptive stage; while the temperature ranged between $101.6^{\circ}$ to $103^{\circ} \mathrm{F}$. until defervescence set in : this, however, is unusual.

Bakewell writes of it as "an excellent febrifuge," after trial during a severe epidemic of variola in Jamaica; he gave it with nitre (Med. Times, i., 1872). Varicella seldom requires treat ment beyond a restricted diet, warmth, and attention to the 
bowels; but when there is much pyrexia, and abundant eruption, with burning and itching, aconite, when taken internally, quickly gives relief.

In erysipelas, especially when the pyrexia is high, aconite is one of the most effective remedies, except the disease be running an asthenic course, when the drug is contra-indicated. In my experience of thirteen idiopathic cases, where the temperature varied from $102^{\circ}$ to $105^{\circ} \mathrm{F}$, and in which the only remedy (except an aperient) employed was aconite, all recovered within five days after being attacked.

Liston, Fleming, and Dr. J. Le Cour (Union Médicale, No. 92, 1861) have recorded good illustrations of its value; the doses, however, given by the latter produced physiological effects which I should consider better avoided.

Septic Fevers.-Puerperal Fever.-If this disorder comes under treatment soon after the initial chill, the results to be obtained by aconite are highly satisfactory. In five cases where the fever came on from the second to the fifth day after. confinement, with severe rigors, a pulse of 120 to 140 , and a temperature of from $103^{\circ}$ to $105 \cdot 6^{\circ} \mathrm{F}$., with the usual symptoms of peritonitis, minim doses of tincture were given every one to two hours, with an occasional dose of castor oil; repeated hot poultices and laudanum fomentations; and all patients made good recoveries. In puevperal mania, with much fever, restlessness, scanty secretion of milk, etc., it has also been used with marked success. Dr. Lambart records three cases of puerperal fever, two of which proved fatal,-in experienced hands and with the best recognized treatment of some years ago,- - but in the third case, which occurred recently, aconite apparently had the best results; on the third day after confinement, rigor and sweatings set in, with a pulse of 120 , temperature $102^{\circ}$ to $105^{\circ} \mathrm{F}$., and every symptom of a severe attack, which progressed. from two to three days, until the patient was ordered 4 minims of the tincture every two hours: next day she was better, and shortly made a good recovery (Lancet, i., 1882). Dr. Counsellor writes of similar experience with three cases (ib.), and Dr. W. S. Playfair also advocates the treatment.

Septiccemia.-The analogy between this and puerperal fover 
was first suggested by Sir James Simpson, and is now generally acknowledged; hence its treatment by the same remedy is not unreasonable, and I have cause to think that if this be commenced early, during the initial rigor, or soon afterwards, excellent results may be obtained. Isnard has reported three cases of traumatic pyæmia illustrating this (Union Médicale, 1861). The same remarks apply to surgical fever; and in the "urethral fever" which sometimes follows the use of the catheter, Mr. R. Harrison states that he and others have found 2 minims of Fleming's tincture, given shortly before the operation, and repeated, if necessary, afterwards, "almost unfailing" (Lancet, i., 1878).

In the class of cases so far mentioned, 1 minim of the tincture may be given every fifteen minutes for the first hour, and afterwards less frequently, according to the severity of the symptoms. As the pulse slows, the interval between the doses should be prolonged.

In Rheumatic Fever, if aconite be administered early, the heart is not so frequently affected, the patient suffers less from pain and swelling in the joints, and the duration of the fever is shortened.

The treatment was first prominently brought into notice by Lombard of Geneva, whose very favourable statements were subsequently corroborated by Dr. Fleming, who introduced the tincture which bears his name (1845). In the practice of the majority of English physicians, however, the drug has not established a permanent reputation either for efficacy or manageableness, owing partly to the use of excessive doses, which, by their dangerously depressing effects, render it difficult to carry out the treatment with that regularity which is essential; it is highly important to adopt the system of small doses, frequently repeated.

Nothnagel states that neither its power to shorten the disorder, nor to avert heart complications, has as yet been distinctly established, and, although not agreeing with him, I admit that carly treatment by aconite means also early commencement of medical care, nursing, etc., and to this may be partly credited the freedom from heart affection above mentioned. 
In affections of the heart, following on rheumatic fever, aconite will be found useful during the acute stage, often relieving the palpitation, dyspnœa, sense of suffocation, and irregular cardiac action. The treatment is equally applicable to endocarditis and pericarditis. Pain and cough, with the sense of tightness, are often relieved; but the aconite should not be continued if symptoms of depression supervene. Of this treatment Dr. Walshe says: "I feel satisfied that if pericarditis be caught at its very outset, if the pyrexia be well marked and the general conditions sthenic, tincture of aconite in small frequently repeated doses ( 2 to 4 minims, B.P.) may be given ('fas est et ab hoste doceri'), with a well-nigh certainty of lessening the frequency and force of the pulse, lowering the temperature, and possibly controlling the inflammation. Cautiously used, it can do no harm, but in overdoses it will accelerate, instead of slackening, the rate of the heart's motion, and dangerously lower its power" (Dis. Heart, 4th Ed. 1872, p. 241).

Dr. Bomford has recorded three cases of tropical remittent. ferer, resembling acute rheumatism in symptoms, and in which he traced much benefit to small repeated doses of aconite, finding them lower the pulse and the high temperature, clean the tongue, help sleep, and promote a healthy action of both the skin and kidneys (Practitioner, i., 1881).

Inflammatory Diseases.-In those morbid states, which, for the most part, have no fixed period of duration, and which offer the possibility of arrest if treated sufficiently early, we have in aconite a drug of the greatest value, as seen in the following:-

Periostitis, when not specific, is well treated by aconite; the absorption of recent exudation may be promoted by it. It will also relieve the pain of osteitis, and is especially indicated when the disease is acute; later on, iodide of potassium acts better. Toothache, dependent on local congestion or inflammation of the periosteum, with throbbing dull pain (relieved by pressure), and a feeling of elongation of the tooth may be benefited; the pain of caries is not so amenable. The drug must be given in small frequent doses, or it may be painted over the gum, combined with iorline, or applied on cotton wool.

Ophthulmin.-In acute inflammations of the eye, conjunctivitis, 
sclerotitis, etc., aconite is of more value than is generally known; and I have seen cases of rhenmatic iritis where it has quickly allayed the frontal pain, and by relieving congestion has promoted the absorption of recently-effused lymph. In syphilitic iritis it is less effective than leeching, and in scrofulous ophthalmice with profuse discharge, swelling, and photophobia, it is useful, but uncertain. Blodig has recorded numerous instances of its value (Med. Times, i., 1861).

In membranous laryngitis (croup), although the best remedies are often unavailing, I have seen decided relief from aconite in the early stages; it has lessened the pyrexia, relieved the hard dry cough, and in some cases seemed to abort the inflammation. I believe it can control the exudation, and in later stages, in conjunction with iodine or other remedies, can also help to promote absorption. Its use, however, must not prevent the employment of fomentations, inhalations, etc. A case of laryngitis is reported in the Lancet (i., 1882), in which $\frac{1}{2}$ minim every half-hour for twenty-four hours appeared very satisfactory.

Tonsillitis.-I have often observed this inflammation, even when severe, take a comparatively mild course under the influence of aconite-the pyrexia, pain, and swelling subsiding without suppuration or permanent enlargement of tonsils. This applies as well to follicular as to simple tonsillitis.

Adenitis, etc.-Aconite is often effective in acute inflammation of the lymphatic glands, such as occurs in strumous subjects from cold, etc. In inflammation of the mammary gland it is specially valuable, and, if given early and frequently, will control the symptoms and prevent the development of suppuration. Mr. P. Swain has recorded the case of a puerperal woman with rigors, a temperature of $103.6^{\circ} \mathrm{F}$, and a pulse of 120 , etc.: 2 minims of the tincture were ordered every ten minutes for four hours, then every hour, extract of belladonna being applied locally. At night the temperature was $101^{\circ}$ and the pulse 88; afterwards, quinine was given, and the patient did well (Lancet, ii., 1878). Such frequent administration of 2 minims would not always be prudent.

Catrorh-Bronchitis. - In the first stage of acute catarrh, with 
chilliness, pain and fulness across the forehead, sneezing, and flow from the eyes and nose, aconite often relieves; also in the same stage of bronchitis it reduces fever and alleviates the dry teazing cough, and the sense of rawness and tickling about the larynx and trachea.

Pnewmonia-Pleuro-pneumonia.-Aconite is useful chiefly in the early stage-that of congestion; it has but little influence over consolidation, but it will control and remove the congestion which generally exists at the circumference of the solidified portion of the lung. Nine of my cases, not selected, but taken consecutively as they occurred, showed the value of the drug at the commencement of the disease. The age of the patients ranged from twenty to sixty-two years; in each case the attack was ushered in by rigors, dry cough, dyspnoea, and the usual symptoms accompanying rise of temperature, which varied in these cases from $102^{\circ}$ to $105^{\circ} \mathrm{F}$.; with a pulse of 110 to 140 : examination of the chest showed dulness over the affected part and fine crepitation. In some very favourable cases of pneumonia, treated without medicine, the fever subsides upon the third or fourth day; but it commonly lasts from six to ten days, even though the best ordinary remedies be administered. In these nine cases, however, the fever lasted in no instance longer than six days after the rigor; and in three to six days after the temperature had fallen to $99^{\circ}$, the physical signs became almost normal. In most, both pulse and temperature declined to the healthy standard at the same time; but in some, the former remained frequent when the latter fell to $99^{\circ}$-the pulse remaining quick from debility; the proportional fall of pulse and temperature is more usual. Eleven other cases of pneumonia in the second stage I also treated with aconite, but it had no effect in removing the consolidation.

Dr. Sydney Ringer has drawn attention to the value of the early treatment by aconite (Lancet, i. 1869); and since that date, and the first edition of this volume, much evidence of a similar kind has been recorded.

Dr. Dobie has published three or four cases in which good results were evident on the second or third day (Practitioner, June, 1879). Spark also writes to the same effect (ibid.); 
and Dr. Rabagliati, agreeing with their observations, argues that the drug exerts two distinct actions, according to its dose: an unduly large amount is apt to be followed by a reactive congestion, whereas small frequently-repeated doses induce a spanæmic condition of the capillaries and antagonize the congestions produced by cold (ibid., ii., 1879).

Tuberculosis.-In early progressive stages, I have found the pyrexial access with its symptoms of thirst and heat of skin allayed by aconite, and local pulmonary congestions sometimes subside under its use. In the later stages, when the rise of temperature depends on septic absorption, some, though less marked, benefit may be gained from it. Various observers have found it relieve the sweating of phthisis (Med. Times, ii., 1855).

Peritonitis.-In ordinary cases, as distinguished from septic forms characterized by tympanitic distension and pain in the abdomen, pyrexia, small hard quick pulse, flexed thighs, costal breathing, etc., aconite offers a fair chance of cutting short the disease.

In certain affections where elevation of temperature does not occur, or only to a trifling and temporary extent, this drug has also been used with much advantage.

Neuralgia.-In this disorder the efficacy of aconite has been. variously estimated, owing in part to the vague way in which the word "neuralgia" is employed. The drug is not to be depended upon where the principal cause of the pain is some persistent peripheral irritation; and though I have found it useful in such cases, it is in the neuralgias more properly so called, especially those of the face or brow, that it gives the best results. Dr. Habershon related a good illustration of its power in a severe case, at Guy's Hospital, of facial neuralgia of many years' duration, where 2 minims of the tincture thrice daily gave ease in a few days (Ranking, $i$, 1862). Dr. Fleming also has tabulated upwards of forty cases, including sciatica, angina pectoris, and iutercostal neuralgia, many of them very severe and rebellious to other treatment, yet relieved by this remedy.

Gubler speaks highly of the curative powers of aconitin; he "does not know a neuralgia of the fifth nerve-even 
tic-douloureux-that resisted it"; one patient who came to him after suffering for three months day and night, recovered with this remedy, omitted it, relapsed, and recovered again on resuming it; another patient, for whom Nélaton had resected filaments of the nerve with but temporary relief, and who was on the point of committing suicide on account of the severity of the pain, recovered completely under the alkaloid.

The same writer considers the preparation by Hottot and. Liégeois good, but prefers a solution of the nitrate of aconitin, $\frac{1}{140}$ grain for a dose ( $\frac{1}{2}$ milligramme of nitrate equals $\frac{1}{280}$ grain of aconitin itself); the dose may be gradually increased up to $\frac{1}{12}$ grain of nitrate (equal nearly 6 milligrammes, $v$. p. 25). Of all the patients treated by him, only one had an untoward symptom, viz., loss of consciousness, which occurred after taking $1_{2}^{1}$ milligramme in sixteen hours : it is not desirable, however, to give this drug when heart disease of any kind is present (Paris Médical, Fev., 1877).

A number of cases of neuralgia cured under aconitin and napellin are given by Laborde (op. cit.), and Séguin has recorded seven severe cases of trigeminal neuralgia, two of them of many years' duration, cured by the aconitin of Duquesnel. He notes how much the susceptibility of different patients varies, one getting rather severe effects from $\frac{1}{200}$ grain, whilst another took $\frac{1}{84}$ grain without feeling its physiological action; an average dose was $\frac{1}{100}$ grain. No doubt this uncertainty has limited the general use of so valuable a drug (N.Y. Med. Journ., ii., 1878 ; Med. Times, i., 1879).

Weir Mitchell relates another case of eighteen years' duration, relieved for a time only by resection of the nerves; $\frac{1}{140}$ grain of Duquesnel's aconitin was given, at first three times daily, and the dose increased gradually up to $\frac{1}{96}$ grain four times, then seventeen times, in the day; the pain lessened, and finally ceased, without other than slight physiological effects (Record, 1879).

Oulmont concludes the remedy to be specially suited for" "congestive neuralgias," meaning cases where the pain is not associated with persistent lesions, and is not markedly intermittent (Practitioner, i., 1880); but its value is by no means restricted to such conditions. Gubler found it equally effective 
in the "acrodynic" form, when the pain was felt chiefly at the extremities of the limbs, where Pacinian corpuscles are most numerous. Napellin has been used successfully in facial neuralgia (Revue, Juillet, 1884).

The external use of aconitia ointment is a valuable resource, and the liniment (B.P.) is a good preparation, especially if its absorption be promoted by the addition of chloroform; but even the ordinary tincture of aconite will often give relief. Thus, painting it along the track of the brachial plexus was sufficient to cure a severe traumatic neuralgia of the arm. The most effective method of using it is over a blistered surface.

Mr. Robert Smith gives a graphic account of his severe sufferings every night for seven weeks from neuralgia of the fifth pair, persistent in spite of competent treatment-improved by vesication, but at once and permanently cured by applying to the blistered surface, next day, $1 \frac{1}{2}$ drachm of the tincture on lint; he states that he has found this treatment uniformly successful in many cases, though 10 to 15 or 30 minims are safer doses to employ than the one he used for himself (Med. Times, ii., 1868). In my own experience, this is a painful and not uniformly successful mode of treatment.

In Myalgia, Muscular Rheumatism, and various forms of non-inflammatory aching pain in tendons, ligaments, and other fibrous structures, aconite is often of considerable service. In cases of stiff joints the liniment is valuable, when applied with friction, and combined with hot douches and passive movements.

In the different varieties of Headache I have found aconite very useful, when applied locally and given internally: for instance, in hemicrania, or sick beadache; in the bilious, catarrhal, and rheumatic forms; in that arising from abrupt suppression of an habitual discharge, whether uterine or hæmorrhoidal; also in the "nervous headache" brought on by anxiety, emotion, or fright.

Dr. Symonds recommended it in somewhat similar cases of "nervous headache, probably congestive;" also, in the more chronic condition of " constant soreness of the scalp, with tendency to ache," he records some excellent results, but adrises caution in its use: he generally ordered 1 to 2 minims of Fleming's tincture for a dose, to be repeated in two or three 
hours; but one lady, who found great relief from $\frac{1}{2}$ grair. of Morson's extract, took on one occasion a second, and then a third pill of it, and showed serious symptoms of poisoning (Med. Times, i., 1858).

In Cardiac Disorder, non-inflammatory, but characterized by increased pulsation or pain occurring, e.g., from nerve causes or even from dyspepsia, I have known aconite useful; and Dr. Fleming found it, in conjunction with appropriate general treatment, act well in all forms of "functional palpitation."

In simple hypertrophy it is available, but when there is mitral reflux, some danger attends its use. Large doses should certainly not be given if any serious organic disease be present; aconitin in any dose is inadmissible in such cases.

Hæmorrhage. - In active hæmorrhage, dependent on congestion or inflammation, with much vascular excitement, full bounding pulse, and dry hot skin, aconite exerts some of its best effects; but I have also seen it of service in the passive form, with venous engorgement, small rapid pulse, and cold extremities: in these cases, then, a depressed and embarrassed condition of the circulation is not always to be considered as forbidding the careful use of the drug. In hæmorrhage and hæmoptysis connected with tubercle, aconite is not only beneficial for the acute forms, with profuse flow and general febrile excitement, but also in those cases where a mouthful of blood is brought up occasionally without, at first, other more serious symptoms; and again, where a moderate hromoptysis has continued for some time, or recurred at short intervals, rendering the patient weak and anæmic, I have known 1 to 3 minims every half-hour for 6 or 8 doses arrest the hæmorrhage and relieve the general symptoms, after gallic and sulphuric acids, turpentine, opium, iron, and other apparently more likely remedies had failed.

I have also known it control various other hæmorrhages, such as epistaxis and that from hromorrhoids.

Uterine Congestion.-In cases of sudden suppression of the menses, such as occurs from rheumatism, chill, or shock, aconite will often promptly re-establish the natural flow, especially if the pationt be kept warm so as to favour perspiration. 
In Dysmenorihœea and in Menorrhagia of congestive character, without organic disease, it is a valuable remedy, when commenced early. More benefit is derived in the latter form of disorder when it occurs in plethoric, nervous subjects. It is important to give the drug in frequent minim doses in the manner previously detailed; M. Chéron recommends for dysmenorrhoa minim doses of the tincture every fifteen minutes for six hours on the first day, and again on the second if improvement has commenced; if not, the dose must be doubled (Bull. de Thérap., Jan., 1883). This would be a dangerous amount of the English tincture, but the French one is evidently milder: it is, I believe, made from the leaves.

Apoplexy.-In cases of this kind occurring in plethoric subjects, when the pulse is full and strong, and the face flushed and turgid, aconite is suitable; but if there is a feeble pulse, a pale face, and threatening syncope, it is unsafe.

Irritable Bladder, etc.-The nocturnal enuresis of children, especially when connected with ascarides and accompanied by some febrile reaction, may be controlled by aconite. In cases of retention of urine with spasmodic stricture, particularly if caused by a chill (sub-inflammatory), aconite also acts well. In one case of locomotor ataxy, where retention often occurred after a slight chill, and commonly required the catheter, the use of the instrument was obviated during one severe attack, minim doses being given every quarter of an hour: this treatment was adopted, when called for, during the two or three following years, with the result of rendering catheterism unnecessary.

Diarrhœa, etc.-Aconite is of use in diarnoca caused by a chill, especially in the young: also in non-tropical dysentery and dysenteric diarrhoca, when the patient suffers from high fever, and pain in the abdomen of a griping and cutting character, these latter symptoms preceding a frequent inclination to stool.

PREPARATIONS AND DOSE.-Tinct $m(1$ in 8$)$ : dose, $(\alpha)$ for acute cases, in children, is min. every hour or two, for about six hours, or according to the effect; $(b)$. for adults, 1 min. every quarter or half-hour for about six doses, then less frequently; $(c)$ in 
chronic cases, adult, 3 to 5 min. three or four times daily (the B.P. dose is given at 5 to 15 min.); Fleming's tincture and Turnbull's are about five times stronger. Extractum (from fresh leaves and flowers): dose, $\frac{1}{2}$ to 2 gr. (B.P.). Fleming's extract is made with alcohol; dose $\frac{1}{10}$ to $\frac{1}{2} \mathrm{gr}$. Linimentum (aconite powdered 20, spirit 20, camphor 1). Aconitia or aconitin (Duquesnel), -dose $\frac{1}{400}$ gr., gradually increasing to $\frac{1}{84} \mathrm{gr}$.; Hottot's or Morson's may be given in slightly larger amount ( $v$. active ingredients, p. 4). Aconitice nitras, $\frac{1}{140} \mathrm{gr}$. ( $\frac{1}{2}$ milligramme), cautiously increased to $\frac{1}{12} \mathrm{gr}$. (nearly 6 milligrammes). Oulmont mentions $\frac{1}{4}$ milligramme of nitrate of aconitin as sometimes poisonous, and points out that the activity of all the preparations depends partly on where the root was grown, that from the Vosges being less active than that of Dauphiné (Revue de Thérapeutique, 1883). Unguentum aconitice (8 gr. to the ounce).

ADULTERATIONS. - In the imported roots there are often doubtful and inert specimens. The U.S.P. admits only such as are "whitish internally and enclose a pith having about seven rays;" this excludes roots that are brownish, hollow, and horny.

\section{PULSATILLA PRATENSIS.}

(Pulsatilia.)

DESCRIPTION.-An herbaceous perennial with numerous deciduous leaves, which are mostly radical, hairy, and deeply pinnatifid, with linear lobes. The flower-stalk is 3 to 4 inches high, bears a solitary, pendulous, dark purple, and silky blossom, with six sepals of about an inch in length, numerous stamens, and ovaries, which, when ripe, become feathery-tailed achænia. It is indigenous to Central and Eastern Europe, and extends into Asia, growing upon dry plains and hillsides.

Active IngRedients.-Anemonin, $\mathrm{C}_{15} \mathrm{H}_{12} \mathrm{O}_{6}$ (Husemann), seems to represent nearly all the active properties of the plant; it is contained also in the fresh roots of Anemone pratensis and nemorosa, Ranuneulus flammula, and other species. 
It occurs in colourless, shining, orthorhombic prisms, very friable, scentless, and neutral in reaction; when melted it has a burning taste, and causes numbness of the tongue, lasting for several days.

Anemonin is soluble in chloroform and in olive oil, and moderately so in boiling water, alcohol, and ether, but is almost or quite insoluble in these liquids cold.

Physiological Action.-Extemal and local.-Locally it acts as an irritant, whether applied directly to the skin or inhaled in powder. Bulliard relates the case of a man who used the bruised root to the calf of his leg to relieve rheumatism, and inflammation and gangrene of the whole limb followed in consequence.

Orfila speaks of the fresh plant as producing violent inflammation of any tissue with which it is brought in contact. I have made several experiments with the bruised dry plant moistened, and have seen vesication as the result. The inhalation of the dust has produced itching of the eyes, colic, vomiting, diarrhoea, etc. The local inflammation produced by anemonin applied to the conjunctiva of a rabbit is said to be slight, but that induced by its vapour is intense (Heyer); and if placed on the tongue it causes pricking and burning, with persistent numbness and white patches (Murray).

Physiological Action.-Intemal.-Digestive System. -Ordinary medicinal doses, such as 5 minims of tincture, act as a tonic, improving appetite and digestion; doses of 10 to 30 minims, if continued, often create a bitter taste in the mouth, with white and coated tongue, anorexia, flatrlent eructations, and nausea; làrge doses, 30 minims and upwards, if repeated several times, produce a sense of burning and numbuess in the mouth, and irritate the gastro-intestinal tract, causing vomiting, colic, and diarrhœa, with a tendency to hæmorrhoids.

Anemonin acts as a stimulant or irritant to the digestive tract, according to the quantity taken, although, as is often the case, the mucous membrane of the alimentary canal shows no sign of hyperæmia post mortem.

Circulatory System.-Pulsatilla resembles aconite some- 
what in its action on this system. It lowers the force and frequency of the heart's action, such lowering being sometimes preceded by temporary excitation; it lessens the rate of respiration, and causes cough, with sense of constriction and oppression of the chest, and, in advanced stages of its action, stertor.

"The extract of the herbaceous part of the plant, given to rabbits in 5 to 10 -grain doses, reduces the pulse, the frequency of respiration, and the temperature; it causes dyspnœea and stertor, debility, and afterwards paralysis of the limbs, stupor, dilatation followed by contraction of the pupil, and death without convulsion" (Stillé).

Nine grains of anemonin proved fatal to rabbits in three or four hours, and after death the heart-walls were found relaxed, its cavities and great vessels full of dark clotted blood (which elsewhere was fluid); the vessels of the brain and medulla oblongata were also congested, and the lungs cedematous.

Nervous System.-The extract of pulsatilla causes vertigo as if from alcoholic intoxication, heat, heaviness and tension in the forehead, and also depression of spirits and disturbing dreams.

Dr. Shapter, basing his conclusions apparently on clinical observation, considers "that it exerts a primary sedative influence on the sympathetic or ganglionic nervous system, subduing reflex and vascular sympathetic neuroses" (Practitioner, July, 1882).

From the symptoms of partial paralysis, first of the hind and then of the fore limbs, the mydriasis and, later, myosis, and the final stupor caused by anemonin in animals, as well as from the affection of respiration already described, and the convulsions caused by extract of pulsatilla, Clarus concluded that it had the power of paralysing the medulla oblongata and the spinal cord.

Cutaneous System.-Pulsatilla is said to produce an eruption on the skin resembling measles. I have myself on several occasions known it cause a kind of nettle-rash, with burning, tingling, and itching of the surface. According to Orfila, it produces pain in the eyes as if sand were in them, with profuse lachrymation and itching of the lids; and Bergius has observed cedema of the eyelids from exposure to the hot vapours of the extract (Bull. Gén. Thérap., Jan. 30, 1883). 
Genito-Urinary System.-The extract in 3-grain doses and upwards generally induces a copious flow of urine, but sometimes, probably when the dose is too large or too often repeated, a condition of renal and vesical irritation with frequent passing of small quantities of urine, tenesmus, and even slight strangury. Pain in the testis and spermatic cord has also been noted; and in women, "bearing-down pain," and a discharge of dark coagulated blood from the uterus, also a feeling of distension of the breasts.

SYNERGISTS.-Therapeutically, bromides.

ANTAGONISTS.-Stimulants: caustic alkalies, tannic acid, and metallic salts are said to be chemically incompatible.

THERAPEUTICAL ACTION.-Inflammation.-Pulsatilla may be employed in conjunctivitis, and in most of those acute and subacute inflammations of mucous membranes in which there is discharge of mucus or muco-pus, as in the early stage of rheumatic and gonorrhoal ophthalmia. A novel use of the drug is in acute epididymitis, for which it is commended by Dr. Borchain after observation of twenty-four cases: he gave 2 minims every two hours, and found it relieve within three days, better than other treatment (Record. May, 1884).

It is useful in inflammation of the external auditory canal, so often met with in children-not when the lining membrane is red, swollen, and painful, but later on, when a thin acrid or puriform discharge appears. Both for these cases and for the eye disorders mentioned a lotion may be prepared with 1 drachm of the tincture to 4 ounces of water, and freely applied to the affected parts eight or ten times daily; suitable doses according to age being given internally. The so-called "stye," tending to recur repeatedly as it does in scrofulous children, may often be aborted by this treatment.

Nervous Disorders. - Pulsatilla is said to be a more suitable remedy for females, especially those of a melancholic tendency, than for males. The class of cases in which Dr. Shapter found this drug useful includes-(1) reflex eclampsia, from uterine irri- 
tation; (2) "sympathetic neuroses"- -g., a youth of seventeen, suffering from cardialgia, functional palpitation, throbbing of vessels, heat and general flushings, got more relief from pulsatilla than from bromides, iron, or any ordinary treatment; and a woman of twenty, with anæmia, turgescence of thyroid, and amenorrhoea, found equal benefit. Dr. J. I. Tucker found it good in heuduches caused by excessive mental application; it relieved some cases more than bromides or valerian in 3 to 10 minim doses (Record, 1880). (3) Aisthesiæ, such as facial neuralgia (which was relieved after failure of croton-chloral), feverish conditions attendant on dysmenorrhœa, and nervous exhaustion from overwork. "It controls irritability and excitability of the ganglionic system, but is not a vascular sedative, unless in reflex conditions, such as arise from hay fever" (loc. cit.).

Dyspepsia.-Pulsatilla is useful in many cases of dyspepsia occurring in phlegmatic subjects with headache and nervous depression, white and coated tongue, nausea, flatulence, sometimes with pain in the epigastrium, coldness and clamminess of the extremities, and either constipation or diarrhœe. When the diarrhoea is attended with mucous discharges or active piles, the pulsatilla relieves these also: about 5 minims should be taken every four hours.

Uterine Disorder.-In cases where the catamenia are scanty or delayed, or suddenly arrested by fright or chill, pulsatilla is often of value in re-establishing the flow: it is also of much benefit in dysmenorrhoea, when the discharge is blackish and clotted. If this medicine should fail to restore the menses at the first period, it is still worth while to persevere for some time.

In mental disorder connected with abrupt suppression of the puerperal discharges, or of the monthly periods, I have seen some excellent results from this medicine, which actively contributes to restore the discharge; it is also useful in hysteriu.

Lencomhoeal discharges, attended by pain in the loins, feeling of weariness, and derangement of the nervous system, have been remedied by a course of pulsatilla continued for a few weeks.

In catarhal conditions of the respiratory mucous membrane, such as occur during measles, also sometimes in asthma and pertussis, pulsatilla (or anemonin) affords relief. 
Tapeworm.-An extract of the root employed internally has been found valuable in cases of tapeworm.

PREPARATIONS AND DOSE.-Tinctura pulsatilla (made from the cut-up plant by macerating one part in eight of alcohol and percolating): dose, for adults, 5 to $15 \mathrm{~min}$; for infants, 1 min. Anemonin: dose, $\frac{1}{2}$ to $2 \mathrm{gr}$. and upwards.

\section{HELLEBORUS NIGER.}

(Black Hellebore-"Christmas Rose.")

DESCRIPTION.-An herbaceous perennial with large pedate leaves, on stems 6 to 8 inches high, bearing one or two white or green, pendent, rose-like, five-petalled flowers, which appear in the late winter or early spring. The rhizome is rough and knotty, black outside (whence the specific name), white within, and giving off many long fibres, which are brownish-yellow when fresh, dark when dried: it resembles the rhizome of actæa and of adonis, but may be recognized by chemical tests (Pharm. Journ., ii., 1882).

(The same name, "Hellebore," has been applied to Veratrum, from which this plant must be distinguished.)

Active IngRedients.-(1) Helleborin (Bastick, 1852), $\mathrm{C}_{36} \mathrm{H}_{42} \mathrm{O}_{6}$, a non-volatile, neutral substance, of bitter taste, causing slight tingling on the tongue; soluble in alcohol, less so in ether, and still less in water. Marmé and Husemann obtained it in shining, colourless needles. It is more abundant in II. viridis than in H. niger, but in both is in very small proportion. Boiled with dilute sulphuric acid, it is changed into sugar and helleboresin, $\mathrm{C}_{30} \mathrm{H}_{33} \mathrm{O}_{4}$.

(2) Helleborein, a slightly acid glucoside, $\mathrm{C}_{26} \mathrm{H}_{44} \mathrm{O}_{15}$, which, when boiled with dilute acid, gives sugar and helleboretin, $\mathrm{C}_{14} \mathrm{H}_{20} \mathrm{O}_{3}$ : this has a fine violet colour, but no physiological action. 
(3) Some other crystalline principles have been obtained, and an organic acid, probably aconitic (equisetic).

Physiological Action.-External.-The flowers of the plant, when crushed and applied to the skin, cause redness and vesication, but these effects are not produced by the leaves or root. The root-fibres-the part used in medicine-have, when bruised, a disagreeable smell; when dry, they become less acrid, but more nauseous and bitter to taste. Neither of the glucosides affects the skin, but helleborin is moderately, helleborein intensely, irritant to mucous membranes.

\section{Physiological ACtion.-Internal.-Digestive System.} -Small doses stimulate the abdominal organs, augmenting the hepatic and pancreatic secretions, and quickening peristalsis, etc. (Stillé). In toxic doses the drug acts as a powerful irritant, causing pain and swelling of the tongue and fauces, secretion of viscid mucus, thirst, sense of suffocation and burning, colic, bilious vomiting and purging; post mortem, the stomach and intestines, particularly the rectum, are found much inflamed, suggesting some analogy with the action of colchicum. Helleborin causes in animals licking and chewing movements, teethgrinding, some salivation, pain, vomiting, and straining; the crop or stomach is found congested or inflamed.

Helleborein, given in small doses for a long period, is said to producé cumulative effects, as shown by anorexia, nausea, and vomiting, which, however, cease on suspending the drug ; sometimes increased secretion, pain, and gastro-enteritis have occurred from it.

Nervous System.-The nerve-symptoms caused by the drug seem to depend upon helleborin: when this was given to animals, excitement and restlessness occurred, followed by general tremor and paresis, first of the hind limbs, then of the whole body; anæsthesia was also produced, the pupils being widely dilated during narcosis. Death occurred, apparently, from cerebral paralysis, and followed, in pigeons from $\frac{1}{2}$ grain ; in frogs and rabbits from 1 to $1 \frac{1}{2}$ grain; in dogs from less than 4 grains. (Entozon were not affected by it.) Post mortem, congestion of brain and cord were found; sometimes, softening of 
these organs and extravasations of blood. The action of helleborein was exerted more on the heart and intestinal canal, but semi-paralytic weakness of limbs and severe convulsions occurred after large doses.

Circulatory System.-Helleborein is not quite so poisonous as helleborin; perhaps because less of it is absorbed unchanged, the remainder being split up into products which are inactive, such as helleboretin. Its special action upon the heart resembles that of digitalis, but is quantitatively much weaker: small, repeated doses slow, larger doses quicken, and, finally, arrest the heart pulsation suddenly: this effect is presumed to occur through the vagus nerve. The blood-pressure is heightened both in the early and later stages. The respiration, at first quickened, becomes slow and difficult, but it continues after cessation of the heart's action. The same respiratory change occurred during narcosis from helleborin, but this did not reduce the heart's action unless given in very large doses. Post mortem, the lungs were found hyperæmic and congested.

Glandular System, etc.-Under helleborein, salivation was always produced, and the action of the kidneys increased; those organs were found hyperæmic after death. In female animals the uterine mucous membrane was invariably congested.

Therapeutical Action.-Previously to the introduction of medicines derived from the mineral kingdom, the value attached to hellebore was immense, but it is now scarcely ever used, unless by veterinary surgeons. The early physicians commended it in the treatment of insanity, both maniacal and melancholic, and great benefit sometimes followed its employment. It has also been given with apparent success in dropsy, worms, cutaneous disorders, and as an emmenagogue. I have prescribed the tincture with success in some dropsical effusions, especially in general anasarca following scarlet fever.

Marmé has recommended helleborein in cardiac disease and dropsy, its solubility and non-irritant properties when injected being advantageous ('Zeits. f. rat. Medic., 3, xxvi. 1).

PREPaRations AND Dose.-Pordered root: dose, 2 to $8 \mathrm{gr}$.; as a drastic purgative, 10 to $20 \mathrm{gr}$. Tinctura (L.P., 1851) 
(5 oz. root to 2 pints spirit): dose, 5 to $60 \mathrm{~min}$. Decoctum ( $2 \mathrm{dr}$. root-fibres to 1 pint water): dose, $\frac{1}{2}$ oz.; may be taken every four hours until effects are produced.

The Adonis vernalis is a ranunculaceous plant lately introduced into practice. It has large yellow flowers, divided leaves, and black rhizomes something like hellebore.

According to Dr. Cervello, the active principle is a glucoside, adonidin, which is colourless, odourless, amorphous, and bitter; soluble in alcohol, but precipitated by tannin: he says it is more active than digitalin (Pharm. Journ., 1882-3).

Therapeutically, the tincture of adonis has been used as a cardiac tonic and sedative, being said to act like digitalis, but without so much risk of "accumulative" effect.

It has marked diuretic powers, and has been of service in cardiac dropsy and in the heart-failure of Bright's disease. In other cardiac disorders the results were doubtful or negative (Bubnoff, Revue, i., 1884; Deutsches Archiv, 33).

\section{PODOPHYLLUM PELTATUM.}

(May Apple, or Mandrake.)

DESCRIPTION.-This plant is widely diffused in the United States, and is sometimes grown in English gardens. By some botanists it is referred to the Berberidex. The stem bears two broad palmate, six- to seven-lobed leaves, and a single flower, which is white, pendent, and fragrant; the ovary ripens into an oval, yellowish berry, sometimes called "wild lemon." The rhizome spreads extensively underground, and when dried, occurs in simple or branched cylindrical brownish pieces, smooth or wrinkled, about the size of a large quill or little finger, marked with scars of leaf-scales, and having numerous rootlets attached. The powder resembles that of jalap. 
Active INGREDIENTS. - The rhizome contains 3 to 5 per cent. of a peculiar duplex resin, termed podophyllin, more correctly, podophylli resina; (2) an alkaloid, berberin (contained also in calumba); (3) another colourless alkaloid; (4) an acid, an odoriferous principle, which may be obtained in scales; and (5) saponin.

Podophyllin is best prepared by exhausting the powdered drug with alcohol, by re-percolation, and slowly pouring the strong tincture into a large quantity of water, slightly acidulated with hydrochloric acid, when the resin subsides. After drying, it forms a light, brownish-yellow powder, having a tinge of green; it is soluble in caustic, less freely in carbonated, alkalies, and is precipitated by acids. Ether separates it into two portions - the one soluble in the menstruum, the other not-both equally purgative (Flückiger and Hanbury). Klump has recently stated that the insoluble portion does not purge even in 2-grain doses, whilst $\frac{1}{4}$ grain of the soluble part acts freely (Trans. Pharm. Soc., 1883). An aqueous extract of the plant is not cathartic. Some recent analyses of podophyllin (at Dorpat) assign to it three principal constituents: (1) podophyllotoxin, which is colourless, bitter, soluble in alcohol, highly poisonous to animals; (2) picro-podophyllin, crystalline bitter, physiologically active; (3) podophyllic acid, which is soluble in warm water, and combines with alkalies (Archiv f. exper. Path., xiï., S. 19).

The latest researches of Podvysotzky describe podophyllotoxin as a resinous, amorphous, bitter, active substance, obtained from the root and the resin of podophyllin: it is a mixture of (1) picro-podophyllin and (2) podophyllinic acid. The emetic and drastic properties of the drug depend on picro-podopluyllin, of which large doses cause vomiting, small ones purging; but being costly, he recommends podophyllotoxin for use; it is best given in alcoholic solution, but is precipitated by much water or alkalies (Record, 1883).

PHYSIOLOGICAL ACTION.-External.-Podophyllum acts as a local irritant, and its dust is apt to cause inflamed eyes, and a vesicular or pustular eruption on the skin of men employed 
to grind it. Applied to mucous membranes it has produced escharotic effects.

\section{Physiological Action.-Intemal.-Digestive System.} - The fruit of the podophyllum is subacid, but insipid in flavour, and may be eaten without injury; the leaves are somewhat more active; but the special properties of the drug reside in the rhisome and rootlets : these are bitter, acrid, and nauseous in taste, and have a narcotic, disagreeable odour. The impure resin, podophyllin, is the preparation commonly employed; it has a similar acrid taste, and by whatever channel administered, if in a dose of 2 grains and upwards, acts as an irritant to the gastro-intestinal mucous membrane. Percy injected an alkaline solution subcutaneously in dogs, and observed, after a few hours, colic, tenesmus, and vomiting. Anstie injected an alcoholic solution into the peritoneum of dogs and other animals, with the uniform result of provoking vomiting, bloody stools, and death from exhaustion, with symptoms of a peculiar respiratory paralysis. Ulceration of the duodenum was found in several, and inflammation in all cases; but the absorption had been so prompt and complete that no inflammation of the peritoneum was produced.

The question of the cholagogue action of podophyllin has been much discussed. At one time it was generally acknowledged, and the name "vegetable calomel" was given to the drug; but in this country such an action was denied by the late Dr. Hughes Bennett, on the strength of a number of experiments upon dogs with doses of from 2 to 8 grains, which diminished the secretion of bile. Anstie also, on finding that fatal doses could be injected without causing visible change in the liver, concluded that the drug had no direct action on that organ, and that the increase of bile sometimes induced in man is an accidental result of intestinal irritation. Experimenting also on dog's, Rutherford found the hepatic effect to depend on the dose: when it was very large, and caused much purging, the amount of bile poured out was diminished; when the dose was smaller, but still irritant, the secretion was increased at first, but soon lessened; whilst with quite small quantities increase occurred, and continued for some time (Practitioner, vol. xxiii.). 
In fact, his experiments showed that, given in appropriate doses, podophyllin was one of the most powerful cholagogues known.

The clinical experience also of a number of observers, both in America and in this country, corroborates the conclusions of Rutherford as against Anstie and Bennett. Dr. John King has recorded much evidence of it (Lancet, i., 1862). Dr. Gardner "found no drug so certain in this respect" (Med. Times, ii., 1870). Ramskill speaks of it as "the best cholagogue he knows," and states that one dose will often get rid of a quantity of bile. There seems some confusion between stimulating the liver, and evacuating bile already poured out; the drug, however, is credited with both actions. Dr. Garrod, finding the later stools caused by it to be largely mucous and serous, considers that it acts rather by emptying the gall-bladder.

Squibb concludes that it acts specially on the upper part of the intestinal tract, much as aloes does on the rectum, and hence stimulates indirectly both pancreas and liver (Med. Times, i. 1870). For my own part, judging from clinical results, I am convinced that the drug, given in suitable doses, will, whatever its modus operandi, both correct deficient secretion of bile and promote its excretion.

It produces at first copious, rather consistent, then watery stools, and acts somewhat like, but slower than, jalap; a purgative dose requiring, as a rule, six to ten hours for its effect. Demarquay calculated twelve hours. Braun specially notes that it increases peristalsis, which increase may be both seen and felt, and the first motions produced are commonly firm. With too small doses, straining is caused without evacuation; $\frac{1}{2}$ grain acts in most instances as a cathartic; 2 grains as a drastic purgative; 4 grains and upwards as an emeto-cathartic, causing vomiting, pain, watery stools, and prostration.

The following illustrates the physiological action of the drug as it develops in some persons, even from medicinal doses. A gentleman, aged twenty-five, in good health, took $\frac{1}{6}$ to $\frac{1}{8}$ grain twice daily for a fortnight, and experienced lassitude, nervous depression (especially in the morning), vertigo or a sense of fulness of head, anorexia, coated tongne, nausea, 
and occasionally vomiting of mucus and bile; pain in the bowels, with copious semi-solid or liquid stools, pale greenishyellow or brown, which, when frequent, produced excoriation and burning at the anus. Three months later he took a single dose of $1 \frac{1}{2}$ grain, and besides many of the above symptoms, hæmorrhoids and prolapsus ani occurred. The doses used were probably too large from a therapeutical point of view : I know several patients who always suffer from prolapsus or from hæmorrhoids after taking only $\frac{1}{4}$ to $\frac{1}{2}$ grain.

Other effects ascribed to podophyllin are a "special stimulation of the gustatory nerve" (B. M. J., ii., 1876), profuse sweatings, and symptoms of collapse, such as fainting, vertigo, ete. (which I have occasionally seen). Mr. Forster has observed serious results dependent on over-irritation; severe pain in one case from $\frac{1}{4}$-grain dose, copious hæmorrhoidal flux in another, and miscarriage in two other patients (B. M. J., i., 1879). Swelling of tongue, glands, etc., has been attributed to small doses, $\frac{1}{30}$ grain (Lancet, i., 1880); but such effects are so exceptional as to need further confirmation.

Circulatory System.-Podophyllin in full doses exerts a markedly depressing influence on the circulation, weakening the action of the heart and the force of the pulse, which may become almost imperceptible; collapse and cold elammy sweats may occur in consequence. Whatever influence it exerts on respiration is secondary and reflex, and is usually slight in amount.

Genito-Urinary System.-No definite effect on the male organs has been traced to podophyllin; but, like many other cathartics (aloes, for instance), it determines a flow of blood to the uterus and neighbouring parts, so that if given near the . menstrual period it commonly hastens its appearance and increases its quantity - this I have myself observed several times, and clinical evidence to the same effect may be found in the Lancet (ii., 1863). Some prolapse of the vagina and inlpaired power of the sphincter of the bladder are apt to follow the strong action of the drug.

The urine, at first pale and copious, soon beomes diminished in amount, and deposits a yellow sediment, which is probably coloured by biliary acids. 
SYNERGISTS.-Calomel in 1-grain doses will act slowly enough to effect a suitable combination with podophyllin, but 3 to 5 grains would act before the latter drug. The rhubarb, or colocynth and henbane pill, also acts well with it, and henbane or belladonna lessens its liability to gripe. Ramskill recommended cannabis indica for this purpose. Dried carbonate of soda also combines well with it in pill.

ANTAGONISTS.-Acids are chemically incompatible with a solution. Astringents antagonize the laxative effects. In collapse caused by the drug, ammonia, alcohol and warmth are indicated. Hot infusion of ginger is said to relieve the griping pain.

Therapeutical Action.-Dyspepsia. - When first introduced into practice, podophyllin was largely employed in those conditions of indigestion in which constipation formed a marked symptom, and welcomed partly on account of some prejudice against mercury, it is now in rather less demand, for it will not replace the former as an alterative: and though highly valuable in some cases, it is uncertain, and sometimes, as already mentioned, very unpleasant. Sir Andrew Clark, who was one of the first to publish English experience of the drug, after stating the objections to ordinary laxatives, recommended this one as "acting slowly, surely, and after the manner of nature." He considered 1 grain a maximum dose, and preferred $\frac{1}{2}$ grain given with taraxacum at breakfast to secure an action on the following morning; this he sometimes reduced to $\frac{1}{4}$ grain given with ipecacuanha, and if it did not then auswer well, he considered it unsuitable to the case (Med. Times, i., 1862). Dr. Ramskill, using it about the same time at the London Hospital and elsewhere, wrote still more strongly in its favour, as the best cholagogue, "useful in all conditions of arrested bile-secretion and sluggish liver, whether simple, or complicated with cardiac enlargement, phthisis, etc." (ibid.). Dr. Gardner advised it in similar cases, and as an aperient in gout (especially with belladonna and leptandrin), in the torpid liver of tropical countries, and in jaundice; he has seen the yellow skin, eyes, etc., quite cured by one dose, "with the evacuation of an 
incredible quantity of bile" (Lancet, i., 1862). Squibb describes its aperient action as "slow and certain, not exhausting, and not constipating afterwards, but apt to be harsh, disagreeable, and insufficient, and so peculiar that a considerable number of persons do not bear it well: used in large doses as an active cathartic, it almost always causes great complaint (cf. p. 36). Duckworth, comparing it with calomel, finds it "uncertain, griping, and requiring to be repeated" (Practitioner, vol. xvii.). Demarquay, from observations on forty hospital patients, found a dose of 5 to 6 centigrammes ( $\frac{3}{4}$ to 1 grain nearly) acted well, with little pain, but some straining. There was often free discharge of bile, and not undue purgation unless too large a dose were given. In chronic constipation, satisfactory results were obtained with two to three weeks of treatment (Med. Times, i., 1874).

My own experience is, on the whole, very favourable in dyspepsia with hepatic derangement, characterized by loss of appetite, brown tongue, acid regurgitation, foul taste in the mouth, flatulence, and a tendency either to constipation or to diarrhoea, $\frac{1}{10}$ grain of podophyllin every night and morning will often produce good results. It is useful in chronic vomiting after meals, and in obstinate heartburn connected with liver derangement. As a laxative in general, and especially for the purpose of removing habitual constipation, podophyllin should be given in moderate doses. A single dose of $\frac{1}{4}$ grain, or at most $\frac{1}{2}$ grain, though sometimes slow in acting, will usually produce watery and bilious purging; and often this effect, instead of being followed by a constipative reaction, will be succeeded by increased and long-sustained activity of the bowels. For habitual constipation it is perhaps better, however, to give from $\frac{1}{6}$ to $\frac{1}{4}$ grain, with 4 grains of pil. col. et hyose. at night when necessary; this treatment is very useful when the constipation is accompanied by nervous and bilious headache. The drug has been presumed to be specially serviceable in constipation with any tendency to cerebral congestion (Med. Times, i., 1880).

Rousselet advises for chronic constipation a pill containing $\frac{1}{\hbar}$ grain, to be taken daily, adding, if necessary, one pill daily to the dose till the effect is produced, continuing such dose for a 
fortnight daily, afterwards every two to three days for two to three months; he finds it best taken with soup at the last meal (Record, 1877). Rivière recommends a similar amount in cases of hremorrhoids (ibid.).

When the motions have become white or clay-coloured, a few doses of $\frac{1}{20}$ to $\frac{1}{10}$ grain every twelve hours for a child, or of $\frac{1}{16}$ to $\frac{1}{8}$ grain for an adult, will frequently restore the natural character of the evacuations, and regulate the bowels. These doses are not irritant; on the contrary, by improving the hepatic condition they relieve symptoms of intestinal irritation, such as vomiting and diarrhœe. Administered less frequently, they are also serviceable in cases of prolapsus of the rectum in children, especially when this is connected with constipation and straining at stool. Braun recommends somewhat larger doses in constipation ( $\frac{1}{12}$ to $\frac{1}{6}$ grain for children under one year, $\frac{1}{6}$ to $\frac{1}{3}$ grain up to the age of four), but points out, what I believe to be often true, that the suitable dose depends rather less on age than on duration of constipation; he recommends one dose to be taken daily at bedtime.

Podophyllotoxin, prepared from the chloroform extract of the root, he finds more certain, as well as more active. He gives, in solution, $\frac{1}{60}$ to $\frac{1}{30}$ grain under one year, up to four years $\frac{1}{30}$ to $\frac{1}{15}$, and above that $\frac{1}{10}$ to $\frac{1}{8}$ (Practitioner, Jan., 1882).

In pyrexial conditions in children, due to gastric or hepatic disorder or to worms, I have found podophyllin of service-when, for example, there are such symptoms as headache, delirium, sleeplessness, dry brown tongue, nausen or vomiting, and pain in the stomach. (As a vermifuge, it was much employed by the aborigines of America.)

In the early stage of typhus, when much congestive headache, delirium, or sleeplessness occurs, with constipation or with evidence of hepatic congestion, the remedy is indicated, and doses of $\frac{1}{8}$ to $\frac{1}{4}$ grain (with henbane), repeated about every six or twelve hours, will often give much relief to the general distress, and somewhat lower the temperature. This medication must be stopped as soon as mild laxation occurs, otherwise in such maladies the debility induced might be mischievous. Wilmot has recommended podophyllin even in cholera, on the ground of its promoting bile-excretion better than calomel 
(Med. Times, ii., 1866); and for the same reason Ramskill has advised it in dysentery.

In Rheumatic Fever it has been more than once asserted that this drug relieves by some specific power in addition to its aperient action. Thus, Dyer found the joint-pains quickly subside under its use (B. M. J., ii., 1874). Snow also described it as a valuable remedy given in $\frac{1}{4}$ to $\frac{1}{2}$-grain doses every three hours, believing that more than usual is required for a full effect in this disorder (ibid., i., 1877). I cannot, however, think this amount judicious.

Secondary Syphilis, etc.-Dr. Sisson, on the strength of one or two cases, has recommended the drug in secondary syphilis, and certain of its American advocates maintain "that it can do everything that mercury can do, and more safely." Dr. Sisson's principal case had been suffering several years, and had conjunctivitis, skin-rash, and ulceration, but recovered quickly with $\frac{1}{6}$ grain thrice daily. Dr. Marston, in his cases, also treated by podophyllin, rather attributed recovery to the mercury previously used, and there is no convincing evidence in favour of the former (Lancet, i., 1864). A case of ague, with enlarged spleen and other serious complications, did well under it (ibid., pp. 87, 116). Dr. Ward found it valuable in asthenic dropsy, etc., and Gardner in bronchitic and febrile conditions (Lancet, i., 1862).

PREPARATIONS AND DOSE.-Podophylli radix (the rhizome dried and powdered): dose, 10 to $20 \mathrm{gr}$. (seldom used). Podophylli resinc (podophyllin): dose, $\frac{1}{20}$ to $1 \mathrm{gr}$. $\left(\frac{1}{4}\right.$ to $\left.1 \mathrm{gr} ., \mathrm{B} . \mathrm{P}.\right)$; advantageously combined in small doses with calomel, rhubarb, or pil. colocynth. et hyos. (v. Synergists). A tincture may be made by dissolving $1 \mathrm{gr}$. of podophyllin in $1 \mathrm{dr}$. of spirit (to which essence of ginger may be added) : dose, from 3 min. upwards. Podophyllotoxin: dose, for an adult, $\frac{1}{4}$ to $\frac{1}{3} \mathrm{gr}$. at a time; dose, for a child, from $\frac{1}{100}$ to $\frac{1}{20} \mathrm{gr} .:$ the second dose should not follow sooner than eight or ten hours after the furst (alkalies during treatment to be avoided). 


\section{HYDRASTIS CANADENSIS.}

(YELLOW-ROOT.)

DESCRIPTION.-This is a small herbaceous perennial, indigenous to most parts of the United States and Canada, where it grows in watery and shady places, blossoming in May and June. The rhizome is knotted and tortuous, of deep yellow colour internally; the stem is erect, about 12 inches in height, bearing two broad and palmate leaves, and one terminal small whitish flower, which is followed by a head of crimson drupeolæ much like a raspberry.

ACTIVE INGREDIENTS.-The dried rhizome contains (1) about $1 \frac{1}{2}$ per cent. of an alkaloid called hydrastia or hydrastin, $\mathrm{C}_{22} \mathrm{H}_{24} \mathrm{O}_{6}$; (2) a resinous body called sometimes by the same name, but more properly "resina hydrastis"; (3) another active principle, not much known and not yet named (B. M. J., Nov., 1880). The pure alkaloid crystallizes in white, glistening, four-sided rhombic prisms, opaque when dry, melting at $135^{\circ} \mathrm{F}$., alkaline, tasteless in the solid state, bitter in solution; soluble in spirit, ether, chloroform, and benzol, almost insoluble in water (Mahla, Silliman's Amer. Journ., vol. Lxxxvi., 5\%). Most of the simple salts of hydrastin, including the nitrate, are uncrystallizable. The resin is of yellowish colour, little soluble in spirit; it is said to be identical with berberin-the same substance as that mentioned under podophyllum. It was first discovered in the common barberry, and has since been found in various medicinal substances that have a bitter. taste and yellow colour.

Physiological Action.-The powdered rhizome, when applied to the skin, blisters it; its odour is powerfully narcotic, and its taste bitter. The tincture or infusion made from it is also bitter, and promotes the flow of saliva, and probably of 
gastric juice, and gives rise to a sense of warmth at the epigastrium; it induces a copious flow of urine, in which the resinoid principle can be detected. According to Schatz, it excites contraction of the vessels of the mucous membranes, and stimulates the generative system (Centralb. f. Gynäkol., 1883). The resinous extract used by Rutherford, in his experiments on animals, proved " a moderate hepatic, a feeble intestinal stimulant" (B. M. J., i., 1879). Large doses cause noises and a sensation of rushing in the ears, much like the effect of quinine; they do not induce any marked disturbance in the alimentary canal.

SYNERGISTS.-Most vegetable tonics, especially berberin and calumba; quinine partially.

ANTAGONISTS.-The alkalies, tannin, and muriatic acid are chemically incompatible; the acid precipitates berberin.

\section{Therapeutical Action. - Extemul. - Ulceration,} Mucous Discharges.-The aborigines of America seem to have been well acquainted with the value of yellow-root in such conditions.

In the chronic indolent ulcer, common on the lower part of the leg, a lotion applied in the usual manner will often be found very effective; and I have seen it act well in the still more obstinate ulcerations that occur about the nose and eyelids, and resemble rodent ulcer, having a reddish-yellow, dry, glazed, and slightly granulating base, and little discharge. An average strength of lotion is 1 drachm of tincture to 8 ounces of water, and the remedy should be given internally at the same time.

In chronic cory: w, whether syphilitic or not, with a congested condition of the Schneiderian membrane, accompanied, as it is commonly, with minute ulcerated patches and muco-purulent discharge, a similar lotion, injection, or nasal douche, used several times daily, will often induce a healthy action of the affected part, if there be no polypus or other persistent source of irritation. The remedy is equally good in mucopurulent dischriges from the conjunctiva and from the meatus of the ear, and forms a good mouth-wash or spray in cases of stomatitis, and of unhenlthy mucous secretions from the fauces. 
For abrasions and fissures about the nipple, such especially as occur during lactation, a compress with hydrastis lotion is a good application. The infant should not be put to the breast quite so often as usual, and the nipple should be protected. For erosions and ulcerations of the cervix uteri, and also for vaginal discharges and for vesical catarrh, injections containing hydrastis are often valuable: for the former class of cases, direct application of the strong extract is good. Gonowhoer will commonly be much benefited by injections of the usual strength ( 1 drachm to 8 ounces), though M. Bredin recommends them somewhat stronger, and adds morphia with very good result (Lancet, 1874). I generally order such injections to be used every halfhour for seven or eight hours, afterwards thrice daily for several days. In prostatom rheen they are also useful.

In Hæmorrhoids, whether internal or external, accompanied with hæmorrhage, muco-purulent discharge, pain during: defreation, and other usual symptoms, relief will be obtained from lotions and ointments containing this remedy, which should be at the same time taken internally. It is also useful for Abrasions and Fissures about the Anus, but it may require to be supplemented by stronger measures; for instance, in one case of rectal ulceration and fissure in an elderly subject, though lotions of hydrastis were used for many weeks with temporary advantage, cure did not result till after application of nitric acid, when it followed quickly.

Prolapsus Recti is often amenable to hydrastis, which relieves the congestion and swelling of the mucous nembrane.

Cancerous and Strumous Ulcerations are sometimes favourably influenced by the same remedy, as shown by Dr. Payne in some cases where the breast and the rectum were affected. In strumous conditions and glandular enlargements, I have myself seen advantage from it; since the general health is commonly impaired in such cases, it may also be well given intermally as a tonic and alterative.

Therapeutical Action.-Intemal.-Hepatic Disorder.-Like podophyllin and some other resins of the same nature, hydrastis has been found clinically to stimulate, or at least modify, the secretion and excretion of bile, and the condition 
of the gastric mucous membrane. I have given it with good result in "sluggish states of the liver," accompanied with headache, yellowish complexion, constipation, etc., either in the form of $\frac{1}{2}$ drachm of tincture several times daily, or a 3-grain pill of the resin over-night, followed by a saline in the morning.

It is a very suitable remedy for catarrhal inflammation of the bile-ducts and duodenum, and also for a similar condition arising from calculous irritation: the jaundice subsides under its use; and since it presumably acts by lessening the congestion of, and discharge from, the mucous surface, it may contribute to the easier passage of inspissated bile or " gall stone." When dropsy has followed on hepatic obstruction, hydrastis is credited with being a good diuretic.

In Uterine Hæmorrhage, whether puerperal, from fibroids, or menorrhagic, Schatz prescribed it in many cases with success (loe. cit.).

In Atonic Dyspepsia it is sometimes ordered instead of calumba and the milder bitter infusions. Bartholow credits it with being " one of the best remedies for the gastric catarrh of chronic alcoholism, and probably the best substitute, if given in sufficient doses, for alcoholic stimulants when their habitual use has to be abandoned."

Intermittent Fever, etc.-Hydrastin is largely employed in America as a remedy for intermittents; Bartholow ranks it next to quinine, and recommends its administration in the same manner. It is said to be valuable in any form of chronic malarial poisoning, and exerts some power over enlarged spleen.

The fluid extract of the drug contains both hyclrastin and berberin; the latter has a somewhat similar action and forms the greater part of what is known as the "eclectic" preparation. Hydrastin, the alkaloid, has been given also for typhoid fever with profuse swenting, for excessive dirmrhen, and for septic poisoning, in doses of 2 grains and upwards.

PREPARATIONS AND DOSE.-Tinctura (1 part in 10 of proof spirit) : dose, 5 to $30 \mathrm{~min}$. and upwards. The allactoid mydrastin or hydrustiu: dose, 1 to 5 gr. Resina (also called hydrastin, and said to be identical with muriate of berberin): dose, 2 to $6 \mathrm{gr}$. 
The American officinal preparation is a fluid extract (which has lately been transferred from the secondary to the primary list of the materia medica): dose, 5 min. to $1 \mathrm{dr}$. A lotion may be made with 1 or $2 \mathrm{dr}$. of tincture to $\frac{1}{2}$ pint of water; an injection with $1 \mathrm{dr}$. of allaloid to $8 \mathrm{oz}$. of mucilage $\left(\frac{1}{2} \mathrm{oz}\right.$. or more for each injection).

\section{DELPHINIUM STAPHISAGRIA.}

(Stavesacre.)

DESCRIPTION.-This is a stout, erect, biennial herb, growing 3 to 4 feet high, branching slightly; the lower leaves are like those of the vine, palmate, with seven to nine lobes, the upper ones smaller; the flowers are bluish-grey, borne in a thin terminal raceme; they are followed by three large downy follicles, each containing about twelve seeds, which are about 3 lines long, shaped like an irregular four-sided pyramid, one side being broader than the rest, and curved; they are sharp-angled, rough, wrinkled and pitted, blackishbrown in colour, offensive in odour; the testa encloses a soft whitish albumen; the leaves, flowers, and stem are pubescent.

The plant is native to Greece, Italy, and Asia Minor, and was well known to the ancients.

Active INGREDIENTS.-The latest researches as to the chemical constituents of the seeds give the following:-

(1) Delphinin, $\mathrm{C}_{22} \mathrm{H}_{35} \mathrm{NO}_{6}$ - obtained in rhombic crystals 1 inch long; soluble in 11 parts of ether and 15 of chloroform, also in absolute alcohol.

(2) Staphisngrin, $\mathrm{C}_{22} \mathrm{H}_{33} \mathrm{NO}_{5}$-amorphous; soluble in less than 1 part of ether, and slightly so in water; apparently the anhydride of (1), but quite different from it.

(3) Delphinoidin-amorphous; soluble in 3 parts of ether; resembling delphinin in action; formula not fixed.

(4) Delphisin-obtrined in crystalline tufts; sparingly 
soluble in ether, etc.; it exists in minute proportion only, and the total amount of all the alkaloids does not exceed 1 per cent. (Marquis, 1877).

(5) A fatty, non-drying oil, in a proportion of $2 \gamma$ per cent., may be obtained by exhausting stavesacre seeds with boiling ether ( $\mathrm{F} . \&$ H.).

Physiological Actron. - Extemal. - Delphinin (like veratria), when applied to the skin, produces a sensation of prickling and heat, even burning; in the nostril it causes sneezing; on the conjunctivæ, inflammation; on the tongue, irritation and burning.

Physiological Action.-Intemal.-Turnbull states that $\frac{1}{2} \mathrm{gr}$. of pure delphinin given several times daily sometimes purges, but without much irritation and without vomiting; others report violent vomiting; it generally causes diuresis, and also sensations of heat and tingling in various parts of the body (On the Medical Properties of Ramunculacere). Given internally to cold or warm-blooded animals, it usually causes salivation, etc., lowers the heart-pulsation and the respiration, and ultimately induces general prostration and loss of sensibility, of voluntary and of reflex power, often spasm and convulsion (v. Praag, Falck); even small doses prove fatal to rabbits and cats, generally by asphyxia.

It paralyses especially the heart-muscle and the nerve-centres, and this almost without any preliminary stimulation (Böhm). In a case of undue excitation of the spinal cord, von Albers prescribed the drug, and after a few days it produced salivation, heat and redness of mouth and fauces, nausea, vomiting and tenesmus, weak pulse, and irritation of the skin, so that its use clearly requires caution. According to Böhm, strphrisagrin is a less active poison, not depressing the pulse or causing convulsion. Rigor mortis is very marked, and passive venous congestion is found; sometimes also patches of inflammation in the intestine.

Therapeutical ACtion. - Extemul. - Phtheiriasis, etc.-The best-known use of stavesacre is for the destruction of pediculi, especially about the head-whence the popular 
name "lice-bane." The powdered seeds may be mixed with ordinary hair-powder, and then thoroughly brushed into any affected part, or dusted over the body (for they are very effective for pediculus corporis).

An ointment containing 1 part of the oil to 7 of lard has been found valuable in "prurigo senilis." It has also been advised by Bourguignon for scabies, but, according to Küchenmeister, it is uncertain.

An ointment containing the powdered seeds has also been used for "fungous ulcerations."

Tinea Tarsi.-Like several other ranunculaceous plants, staphisagria is useful in certain affections of the eyes: it allays irritation and itching; checks excessive secretion from the conjunctivæ, Meibomian follicles, and ciliary glands, and renders the discharge less puriform; when ulceration is present, it helps to promote healing.

Neuralgia.-An alcoholic solution (1 part in 60, 15, or 8), painted upon the skin, is of service in neuralgia, and may be dropped into the cavity of an aching tooth; in rheumatic neuralgia, an ointment or liniment of similar strength may be employed.

Therapeutical Action.-Intemal.-It has also been recommended for internal use in the maladies last mentioned, especially in obstinate neuralgia affecting the facial and superficial spinal nerves of the neck. In rheumatic periostitis and shifting pains in the long bones it is to be favourably regarded. Stillé refers to its use in dropsy and in spasmodic asthma.

Van Praag gave delphinin in $\frac{1}{10}$ to $\frac{1}{8}$-grain doses with advantage in acute rheumatism, finding it act as a sedative to the heart and to the muscular and nervous systems; but both in this and in chronic rheumatism my own experience with the drug has been unsatisfactory.

M. Bazin gave the extract in $1 \frac{1}{2}$-grain doses several times daily in eczema.

I have known the tincture sometimes apparently useful in long-standing amenorrhoen, and also in the nausea of pregnancy.

A decoction of the seeds has been occasionally employed as an 
anthelmintic, and with tolerable success, but it is inferior to veratria (Küchenmeister).

PREPARATIONS AND DOSE.-For external use, the seeds may be infused in vinegar, $1 \mathrm{oz}$. to 2 pints; or $3 \mathrm{oz}$. of the powdered seeds may be macerated for twenty-four hours in benzoated lard in a sand-bath at $212^{\circ} \mathrm{F}$., and strained through linen (Bourguignon). Tinctura (of seeds, 1 part in 10): dose, 5 to 20 min. Decoctum (of seeds, $\frac{1}{2}$ oz. to 1 pint) : dose, 1 to 2 oz. Delphinin : dose, $\frac{1}{16}$ to $\frac{1}{8} \mathrm{gr}$.

\section{ACT EA RACEMOSA-RADIX CIMICIFUGAE.}

\section{(Black Shake-root-Black Cohosh.)}

DESCRIPTION.-An herbaceous perennial, the stem growing from 3 to 6 feet high, erect and simple, except at the upper part; leaves few, alternate, doubly and triply ternate or pinnate, the upper ones gradually smaller, the lobes. or leaflets thin, glabrous and shining, and 1 to 3 inches long; flowers numerous, small, cream-coloured, and disposed in an erect and terminal raceme of about 9 inches in length; sepals four ; petals not developed; stamens numerous ; ovary becoming a small, many-seeded follicle. It is common in the woods of Canada and the United States, and is often found, in English gardens, blossoming at midsummer. The part used in medicine is the rhizome, which is short, knotty and branching, about $\frac{1}{2}$ inch thick, and having numerous brittle, wiry rootlets, dark brown in colour, of bitter acrid taste, and narcotic smell. "The larger roots, when broken, display a thick cortical layer with converging wedges of open woody tissue, three to five in number, a characteristic structure easily observed with a lens" (F.\& H.). 
ACTIVE INGREDIENTS.-Besides gum, sugar, starch, resin, and tannic acid, a volatile oil has been described as existing in the fresh root. Conard could not verify this, but found a neutral crystalline substance of acrid taste, slightly soluble in ether and water, and freely in alcohol and chloroform, not in benzol, turpentine, or bisulphide of carbon; formula not certain (Pharm. Journ., i., 1871).

American "eclectics" prepare from the root an impure resin, termed cimicifugin or macrotin, by the same method as podophyllin is obtained, in the form of scales or dark brown powder.

ABSORPTION AND ELIMINATION.-I know of no special observations on the mode of absorption of this drug, but it is eliminated by the skin, the kidneys, and probably by the pulmonary mucous membrane.

Physiological Action.-Internal. - (To obtain this it is essential that preparations from the fresh root be employed.)

Digestive System.-The taste is bitter, somewhat astringent and acrid. Small doses, 10 to 20 minims, improve appetite and digestion, and increase the flow of saliva; large amounts, sometimes even 40 minims (if continued), are apt to cause gastric irritation, evidenced by anorexia, dryness of pharynx, fotor of breath, eructations, flatulence, nausea, vomiting, and tendency to diarrhoea.

Circulatory System.-Dr. Young states that ordinary medicinal doses do not affect the pulse (Amer. Journ., v., ix.) ; but I think they exert a regulating and tonic effect on the vascular system. Full doses act somewhat like digitalis in lowering the pulse-rate and increasing arterial force and tension ; continuance of these doses, however, causes a depression of the pulse and circulation generally, with vertigo, sense of sinking and faintness at the epigastrium.

Nervo-muscular System.-The contractility of unstriped muscular fibre is said to be increased by actæa, as it is, though more markedly, by ergot. 
Young and others describe various nervous. symptoms produced by actæa, viz., " irritability, restlessness, headache, flushing of face, vague uneasy feelings in the limbs, slight tremors, vertigo, and impaired vision, with dilated pupils." Large doses have produced "considerable soporific and anodyne effects."

Genito-Urinary System.-Actæa is said to stimulate the sexual instinct in men, and the uterine functions in women. Among the North American Indians it has long been in popular use as an ecbolic and abortifacient (Wheeler, Boston Med. Journ., Sept., 1839). I formerly made many observations as to its action upon the parturient uterus: I did not find it so reliable as ergot, but when it operated well the contractions produced by it were sufficiently strong, without being painfully continuous, and in some instances more like the natural pains of labour.

The urine is increased in quantity under its influence, and exhales the odour of the drug.

Cutaneous System, etc.-Actra commonly augments the secretions of the skin and of the nasal and bronchial mucous membranes, and its characteristic odour may be perceived in the perspiration, especially if this be profuse.

SYNERGISTS.-Ergot, digitalis, colchicum; to some extent belladonna; also cold.

ANTAGONISTS.-Astringent iron preparations are chemically incompatible. Ammonia and circulatory stimulants counteract some of its effects.

Therapeutical ACtion.-Chorea.-The success of this drug as a popular remedy in chorea first led to its use by the profession, but in this country it has not been generally prescribed. Stillé regards it as valuable, especially in cases where the nerve disorder is independent of other definite disease, noting that for good results it must be given in efficient doses, e.g., to the production of such specific effects as vertigo and dimness of sight. Other practitioners have corroborated this. 
I have had some excellent results from this drug in longstanding chorea occurring in hysterical subjects, and in those with menstrual irregularity and rheumatic pain in various parts. For instance, a delicate somewhat hysterical girl, aged eighteen, had a moderately sharp attack of rheumatic fever, followed by some affection of the aortic valves. Several weeks afterwards severe general chorea supervened, which continued for nearly twelve months under various treatment, including bromides, valerian, iron and arsenic, without benefit. I then prescribed 30 minims of tincture of actrea thrice daily, and improvement commenced in ten days. She ultimately got quite well of the chorea, although it is recognized that such cases are particularly obstinate.

Acute Rheumatism.- In this complaint I have upon several occasions found actra serviceable. It has quickly relieved the pain, and subsequently reduced the frequency of the pulse; the profuse perspiration under its influence often becomes less acid.

McDonald has specially commended it in rheumatism (Ranking, ii., 1861); and more recently, Bartlett has reported twenty-nine subacute or chronic cases, of which twenty-two were cured by actæa. There was no distinctive symptom (such as whether worse during the day or night) which could be decided on to indicate its use; in a few instances it caused giddiness, headache, or disturbance of stomach (Practitioner, vol. x., 1873).

Actæa appears to possess a powerful affinity for the musculer system, and we may daily observe examples of its efficacy in removing localized rheumatic affections, such as wry-neck, lumbago, pleurodynia, intercostal and abdominal rheumatism; uterine pain, when presumably rheumatic, is also amenable to it. In ordinary colds, associated with rhermatism, with aching pain in the face, often with sore throat, it is a good remedy; for the neuralgia also, and the headache of such patients, and especially for headache occurring in connection with the menstrual period, I have often prescribed it with advantage.

Cardiac Disease-Dyspnœa.-There is much resemblance between the action of digitalis and of actra; and though 
the former is more powerful and more dependable, the latter is sometimes a useful alternative, and is comparatively free from risk. In various forms of cardiac disorder, where weakness and intermittence of action were marked symptoms, sometimes when the pulse has been too quick, at other times when abnormally slow and irregular, with much dyspnœa and even orthopncea, cold and odematous extremities, and scanty secretion of urine, I have known these unfavourable symptoms greatly relieved by actæa, the heart's action being rendered stronger and more equable, and a copious flow of urine induced.

In Bronchial Catarrh it acts well, relieving the hacking cough, and aiding expectoration (Med. Times, i., 1868).

Uterine Disorders.-In this class of cases the action of the drug seems more akin to that of ergot, with greater power of relieving pain and reflex neuroses connected with the pelvic viscera. For instance, when spinal irritation is aggravated during or just after a menstrual period; and again in dysmenorrhœa, with severe pain at first and scanty loss, followed later by dark and rather excessive discharge-in such cases I have seen it act extremely well. I have also prescribed it successfully in suppression of the menses, brought on by cold and attended by rheumatic pains, especially in persons of nervous habit.

Dr. King, one of the best American authorities, describes it as useful in amenorrhœa, dysmenorrhœa, leucorrhwa, etc., and asserts that as a pren'tus cucclerctor it may wisely be substituted for ergot, bringing on the expulsive action of the uterus speedily and energetically, whilst after labour it is effectual in allaying general excitement of the nervous system and relieving uterine pains and post-partum hremorrhage; he finds the uterine contractions produced by it less powerful and less continuous than those caused by ergot.

Puerperal Hypochondriasis. - A remarkable instance of the value of actrea in this disease is reported by the late Sir. James Simpson (Med. Times, ii., 1860). A lady, the mother of several children, was twice the subject of the most painful mental despondency, commencing about a month after her confinement. Upon one of these occasions her delivery had taken place in 
London, where she had the advice of several eminent physicians; but her complaint took a long and tiresome course, seeming to defy all remedies, and terminated only gradually and slowly. It was arranged, upon a subsequent occasion, that the confinement should take place in Edinburgh, in order that the patient should be placed under the care of Sir J. Y. Simpson. Her child was born, and some weeks subsequently she returned to England, apparently in perfect health; but in the course of another month the lady again went to Edinburgh, but now in the lowest possible state of depression and mental misery. "I tried many plans," says the eminent authority named, "to raise her out of this dark and gloomy state;-all failed. At last, fancying from some of her symptoms and complainings that there might be a rheumatic element in the affection, I ordered her 50 drops of the tincture of actrea daily. After taking one dose, she refused to continue the medicine, the drug having a taste similar to that of laudanum, and all opiates having a tendency to make her worse. On being assured that there was no opium in the medicine, she recommenced it, without any expectation, however, of good resulting to her, having lost faith in every species of medicine. When I next saw her, some eight or ten days afterwards, she was changed in a marrellous degree, but all for the better. On the third or fourth day after resuming the actra, so she informed me, the cloud of misery which had been darkening her existence suddenly began to dissolve and disappear, and in a day or two more she felt perfectly herself again, in gaiety, energy, and spirits. So pleased was she with the effects of the actra, that nothing could induce her to cease from the use of it for a further period of six or eight weeks; and the last time she passed through Edinburgh she told me she had prescribed her new-found and priceless remedy to more than one melancholic subject, with success nearly as great as she had experienced in her own person."

Nerve Disorders, etc.-In some cases of spermatorrhoea and nocturnal emissions with melancholic hypochondriasis, I have prescribed actrea with benefit. It is an excellent touio to the nervous system, and it can remove the tremors and vertigo of delirium tremens, having the advantage over digitalis of 
increasing the appetite, rather than diminishing it; under its influence the patient often sleeps calmly and awakes refreshed. In painful or convulsive states due to nerve-disease, Bentley found it very efficacious (Lancet, i., 1862).

PREPARATIONS AND DOSE. - Extractum cimicifugce fluidum (U.S.P.): dose, $\frac{1}{2}$ to $2 \mathrm{dr}$. Tinctur'a actrece racemosce (1 part to 4): dose, 10 to 50 min. Cimicifugin: dose, 1 to $4 \mathrm{gr}$. 


\section{MAGNOLIACEA.}

\section{THE MAGNOLIA FAMILY.}

A faMily of about 100 noble arborescent exogens, found chiefly in North America, China, and Japan, but not native in Europe. The leaves are large, alternate, and simple; the flowers, usually handsome and lily-like, are trimerous, the sepals and petals varying from six to thirty; the stamens are numerous, and hypogynous; the ovaries are also numerous, but agglutinated into a coniform body. Exceptions to this structure occur in some plants formerly distinguished as the Winteraceæ, but, though anomalous, and apparently unlike the rest of the order, they possess no substantially different characters.

The flowers of the Magnoliaces have commonly a delightful perfume, and the other parts of the plant are bitter, with an agreeable aroma.

\section{Pharmacentical Species.}

Drimys Winteri .. . . . . . Winter's bark.

Illicium anisatum . . . . . . . . Star-anise.

\section{DRIMYS WINTERI. \\ (Winter's Bark.)}

Description.-A forest tree of South America, where it attrins the height of 30 or 40 feet; it has large elliptical leaves, and pale cream-coloured flowers nearly 2 inches in diameter; the petals lanceolate. It was first brought under the notice of Europeans by Captain Winter, who accompanied Sir Francis Drake in 1578 to the Straits of Magellan, but through 
mistake the plant became confounded with the Canella alba. The bark occurs in curved pieces or quills under $\frac{1}{4}$ inch thick, externally grey, internally brown, marked with longitudinal ridges; it has a peculiar odour and warm, pungent taste, and breaks with a short fracture.

ACTIVE INGREDIENTS.-Winter's bark contains volatile oil, resin, colouring matter, tannin, acetate and sulphate of potash, chloride of potassium, oxalate of lime, and oxide of iron. Of these the really active ingredients are probably the tannin and the volatile oil. The latter is divisible into two substances: one a thin greenish-yellow fluid, the other a fatty body of acrid burning taste.

THERAPEUTICAL ACTION.- The union of the styptic tannin and the stimulating volatile oil renders Winter's bark a tonic, valuable in various disorders. It was employed for much the same purposes as canella, which superseded it, and which has itself, in turn, fallen nearly out of use. It is a fairly active stomachic tonic, well applicable to chronic atonic dyspepsia, and has also been given in scurvy, and other conditions of debility.

PREPaRations and Dose.-Infusum: dose, 1 to 2 oz. Pulvis (of the bark) : dose, 30 to $40 \mathrm{gr}$.

AdULTERATIONS.-Canella of various species.

\section{ILLICIUM ANISATUM.}

(STAR-ANisE.)

DESCRIPTION.-An evergreen shrub, indigenous to Cochin China, Siam, and parts of China, 8 or 9 feet high, with obovate entire leaves, and solitary yellow flowers, which are followed by stellate clusters of woody follicles. These, in particular, and other parts of the plant in a less degree, yield the odour of anise. 
The follicles, about eight in each star, are boat-shaped, beaked, wrinkled, brown, usually open on the upper surface, and containing a single smooth brown seed. The Japanese variety, I. religiosum, a sacred plant, "shikimi," grows higher, and has pointed leaves: some specimens of the fruit can hardly be distinguished from the true star-anise, but the taste is disagreeable, and the smell more resembles laurel (Eykman, Pharm. Journ., i., 1881).

ACTIVE INGREDIENTS.-Mainly a volatile oil, containing (1) anethol or anise-camphor, $\mathrm{C}_{10} \mathrm{H}_{12} \mathrm{O}$, which is commonly liquid, but solidifies at low temperatures; (2) a smaller quantity of an oil isomeric with oil of turpentine.

The oil of star-anise is identical in chemical, but not in all physical, properties with the oil of Pimpinella anisum, Fœniculum dulce, and of species of anethum and artemisia. It is said to be nearly insoluble in proof spirit, whilst the oil of true anise is soluble (hence the common substitution of the former for the latter, e.g., in the tinct. camph. comp., is not quite satisfactory).

Physiological Action.--That of the true drug seems little other than stimulating, but the Japanese kind acts as a strong irritant to the intestinal tract, causing vomiting, purging, convulsion, and even death (loc. cit., Revue, Oct., 1884).

Therapeutical Action.-The Chinese employ the fruit as a carminative, but it is also stimulant, somewhat anodyne, and diaphoretic.

PREPARATIONS AND DOSE.-The fruit made into a confection, or the volatile oil; the dose is much the same as that of true anise, and of anise oil.

Adulterations.-Fruits of Illicium religiosum, introduced before exportation from China and Japan.

It is probable that other members of the Magnoliacere possess more active therapeutical power than the so-called Winteracer. Perhaps the most widely esteemed for tonic and also diaphoretic 
powers (on the continent of America, for it has not been used in Europe) is the Magnolia glauca. This tree is common in the morasses of the middle and southern United States, and bears the names of "beaver tree" and "swamp-sassafras." The bark has an aromatic odour, which lessens on keeping, and a warm pungent and rather bitter flavour. The bark of the root has similar properties, and, for pharmaceutical purposes, is considered preferable.

The U.S.P. likewise includes the Magnolia acuminata, called "cucumber-tree," and the M. tripetala or "umbrella tree." The bark of both these trees is reputed to possess the same properties as that of the M. glauca. The tincture of the fruits has been found equally valuable with that of the bark; and an infusion of the fruits, while green, in whisky or brandy, is extensively employed in the United States against intermittent fevers, and for rheumatism and catarrhs. 


\title{
MENISPERMACEA.
}

\section{THE CALUMBA FAMILY.}

Tre members of this family are climbing exogenous shrubs or undershrubs, natives almost exclusively of the woods of tropical Asia and America. The leaves are simple, broad, and angular. The inconspicuous flowers usually racemose, diocious, pale greenish-yellow, and trimerous. The stamens are six or nine, hypogynous, often monadelphous; the carpels are either solitary or whorled; the fruit is either baccate or drupaceous; when ripe, it is usually shaped like a horse-shoe, whence the name "menispermum," literally moon-seed, or lunate seed. The predominant qualities of the plants of this order are bitter and narcotic.

\section{Pharmacentical Species.}

Cocculus palmatus vel Jateorrhiza , Calumba Calumba. Cissampelos pareira .. . . . Pareira. Anamirta paniculata. . . . . CocculusIndicus-Fishberry.

\section{COCCULUS PALMATUS VEL JATEORRHIZA CALUMBA.}

\author{
(Calumba.)
}

DESCRIPTION. - The name of this drug being similar to that of the chief city of Ceylon, the idea long prevailed that the plant was a native of that island. But the word "calumba," or "columbo," is simply an altered spelling of the native Africon appellation, the plant being indigenous to that portion of the east coast of Africa which includes Mozambique. In the thick forests which cover its shores, the plant grows in the greatest 
profusion, and thence the drug used in England is imported. The root is perennial, and constituted of numerous tubers, which are branched, fleshy, and of varying thickness; externally, brown; internally, deep yellow. The stems are annual, slender, and climbing, those of the male plants simple,of the female somewhat branched; in both, the lower portion is thickly clothed with glandular hairs. The leaves are alternate, petiolate, and more or less circular in outline, but with three, five, or seven deep lobes, according to age and position. The flowers, green-coloured and small, are produced in axillary racemes; the fruit is drupaceous, about the size of a hazel-nut, and densely covered with long, glandular hairs.

For medicinal purposes the roots are collected in the dry season, which, in the eastern part of Africa, is March. The main body of the root is not disturbed, being not only too fibrous for use, but the source of new supplies of the lateral tubers, which are alone collected. Soon after being taken from the soil these are cut into transverse slices, which are received in England in the dried state; they are depressed in the centre, from 1 to 3 inches in diameter, and from $\frac{1}{4}$ to $\frac{1}{2}$ inch thick; the taste is bitter, slightly aromatic, and mucilaginous.

ACTIVE INGREDIENTS.-The two ingredients which probably are alone efficient are berberin and starch, for the bitter, crystalline alkaloid, columbin, $\mathrm{C}_{42} \mathrm{H}_{44} \mathrm{O}_{14}$, is most likely inert (Schroff, Falck). Berberin, $\mathrm{C}_{20} \mathrm{H}_{17} \mathrm{NO}_{4}$, exists in the root in combination with columbic acid; it forms, from an aqueous solution, small glittering yellow crystals, which have a bitter taste and a neutral reaction; at a temperature of $120^{\circ} \mathrm{C}$. they melt into a reddish-brown resinous mass. It is little soluble in cold, more so in boiling water, very soluble in alcohol, insoluble in ether. Charcoal precipitates it from a watery solution, but alcohol will separate it again from the chareoal. Its solutions have no influence on the plane of polarization. The starch is an important element, and exists in the root to the amount of 33 per cent.

Physiological Action.-Berberin has been mentioned as a constituent of several ranuneulaceous plants (podophyllum, 
hydrastis, etc.); it is possessed of poisonous properties, which, however, are weak and variable. It causes in animals symptoms of irritation of the gastro-intestinal tract, with secondary nervedisturbance. According to Falck and Guenste, rabbits died in from eight to forty hours after a subcutaneous injection of $7 \frac{1}{2}$ to 15 grains. Dogs, on the other hand, were not killed even by the largest doses injected into the blood. Given to them by the stomach, it excited some tremor, restlessness, and thirst, and a few watery evacuations. Injected into the veins it caused the same symptoms, with the addition of salivation, dyspnœea, and paralysis (especially of the hind legs); but all these symptoms gradually wore off, the tremor being the last to disappear. In pigeons and fowls repeated injections of berberin solution into the crop caused vomiting, watery evacuations, quick breathing, and loss of appetite; given in pills amounting to a quantum of 4 to 8 grains with each day's food, the drug produced progressive loss of appetite, to the extent of causing marked inanition (Deutsche Klinik, xiv., xv., 1854; De Columbino et Berberino Observationes, Inaug. Diss., Marburg, 1851).

Digestive System.-On man, berberin does not produce actively poisonous effects; but several observers record pain in the belly and watery diarrhœa, as the result of swallowing doses of 7 to 15 grains-effects which at least are remarkable, and different from those either of small doses of berberin itself, or from the ordinary medicinal employment of infusion of calumba.

Therapeutical action.-Calumba is powerfully tonic, and has the recommendation of being free from nauseous flavour, acridity and astringency, while there exists in it so much starch as to render it demulcent. It promotes appetite, and assists digestion without causing nausea, sickness, or headache-symptoms which so frequently follow the exhibition of tonies; hence it can be usefully employed when other remedies of its class would be rejected.

Dyspepsia, etc.-Calumba is employed with benefit in all affections of the stomach and bowels which are accompanied by increased secretion or excretion of bile; also in those conditions 
when nausea, pyrosis, and flatulence are the main symptoms; it may be combined with soda in these cases. Again, in the "bilious attacks" to which delicate females are often subject, the conjoined use of infusion of calumba and of effervescing draughts is in most cases beneficial; while in dysentery, when inflammatory symptoms have subsided, it has proved so serviceable that, in Germany, it is known as "Ruhr-wurzel," or "dysentery-root." The utility of the drug is specially observable when the complaint is chronic and attended by ulceration. In habitual diarrhoea, when tonics are admissible, calumba also possesses value.

Not only does it arrest the vomiting produced by tartaremetic and ipecacuanha, but, when combined with these medicines, their operation is rendered milder. The distressing vomiting of early pregnancy is frequently allayed by the use of calumba; so also are the vomiting and diarrhoa accompanying dentition. Vomiting caused by kidney disease and renal calculi, and even when occurring in cholera, has also been alleviated by the employment of calumba.

After attacks of fever, the infusion is an excellent preparation, and in puerperal fever it is, according to Denman, preferable to cinchona. In hectic, and in the last stages of phthisis pulmonalis, it has also been found excellent for checking colliquative diarrhoea, allaying irritability, and imparting tone to the stomach.

Dr. Ringer speaks of the drug in the following words:"Calumba is used as a tonic to increase both appetite and digestion. It has a slight irritant action upon the stomach, as indeed most bitter substances have, and is considered by this property to be able to set aside slight changes in the mucous coat of the stomach, and, in this indirect way, to assist appetite and digestion. It is easily tolerated by the stomach, and is thus employed when this organ is in a weak state, as during the convalescence from an acute disease, when it is often found that calumba is borne with benefit, while stronger tonics may upset the stomach."

Dr. Wilson Fox says that calumba is very useful as a mild tonic in dyspepsia, but places nux vomica and cinchona before it (Dis. of Stomach: Reynolds' System). 
PREPARATIONS AND DOSE.-Extractum: dose, 2 to $10 \mathrm{gr}^{2}$. Infusum: dose, 1 to $2 \mathrm{fl}$. oz. Critumber raclix: dose, 5 to $20 \mathrm{gr}$. Tincture: dose, $\frac{1}{2}$ to $2 \mathrm{fl}$. $\mathrm{dr}$.

The infusion will not keep more than a few days; it may be usefully combined with aromatics, with orange-peel, alkalies, and neutral salts, or with iron. The root may be chewed like rhubarb.

\section{CISSAMPELOS PAREIRA.}

(Pareira Brava.)

DESCRIPTION.-This drug is derived from the pareira, a native of Africa, the West Indian Islands, and the adjacent parts of America; and is commonly known as "velvet-leaf." The root is woody and branching; the climbing stems glabrous or pubescent, the ieaves nearly circular, peltate, glabrous above. In the male plant, the racemes of flowers are axillary, and nearly solitary; in the female, the flowers are fasciculated into racemes longer than the leaves. The small yellow flowers are simple; those of the male tetramerous and octandrous; of the female dimerous, with a solitary ovary, which ripens into a round or reniform scarlet berry. The pareira brava sold in the shops is probably derived from more than one species of cissampelos, for very different quantities of extract are yielded by various samples: the true pareira is a strong bitter. The root occurs in pieces 3 to 6 inches long, 1 to 3 inches in diameter, twisted, dark brown outside, pale brown, rather waxy, inside.

ACTIVE INGREDIENT.-Buxin, $\mathrm{C}_{1 \mathrm{~S}} \mathrm{H}_{21} \mathrm{NO}_{3}$, an alkaloid which occurs also in plants of the Lauracere and Euphorbiacer, as well as in others of the Menispermacer; discovered by Fauré in box-bark, it is substantially identical with the bibirin found in the "bibiru" tree, and the pelosin discovered in pareira.

Buxin, when pure, is a white amorphous substance, of bitter taste, and alkaline reaction ; insoluble in water, freely soluble in 
absolute alcohol, ether, chloroform, and acetone. It neutralizes acids, and forms with them uncrystallizable salts. Concentrated sulphuric acid changes it to a brownish-yellow resinoid body.

Physiological Action.-This is tonic, a specific influence being exerted over the mucous membranes; it is also diuretic. In 1648 pareira was brought to Paris by M. Amelot (the French ambassador in Portugal), with the reputation of being a lithontriptic; and its efficacy was so highly vaunted that even Helvetius was convinced that calculi about the size of olives disappeared beneath its influence, and that lithotomy would no longer be needed.

The effects upon the general system are not well known, though large doses may prove aperient.

THERAPEUTICAL ACTION.-The use of pareira at the present day is almost limited to the treatment of discharges from the genito-uninary mucous membrane; gonorrhoa, leucorrhoea, and chronic inflammation of the bladder being often successfully treated with it.

In the last-named disease, Sir B. Brodie considers pareira superior to uva-ursi in lessening the secretion of ropy mucus, and at the same time diminishing inflammation and irritability of the organ.

PREPARATIONS AND DOSE.-Decoctum: dose, 1 to $2 \mathrm{oz}$. Extractum: dose, 10 to $20 \mathrm{gr}$. Extractum paieire liquidum: dose, $\frac{1}{2}$ to $2 \mathrm{dr}$. Alkalies, acids, or anodynes, especially hyoscyamus, may be added.

ADULTERATIONS.-The most important adulteration is the stem of the plant itself; the cortex of this is brown-grey: section shows the layers to be eccentric; it is not waxy. 


\section{ANAMIRTA PANICULATA.}

\section{(Cocculus Indicus - Fishberry.)}

DESCRIPTION.-This is a native of Malabar, and other parts of the East Indies, whence the seeds were originally obtained by the Arabians, whose ancient medical writers were the first to mention them. It is a strong climbing plant, with roundish leaves about 6 inches across, leathery, and shining. The racemes of flowers are lateral and compound, the ovaries becoming one-seeded drupes (not unlike those of the common bay-tree-Laurus nobilis), which in the dried state have a thin brown shell, and a crescentiform, yellow, oleaginous seed. This never entirely fills the cavity of the shell, by which character the fruit of the anamirta may be distinguished from the solid one of the bay-tree. Both shell and seed are acrid and bitter.

ACTIVE INGREDIENTS.-The properties of cocculus seem to be fully represented by cocculin, or picrotoxin, $\mathrm{C}_{12} \mathrm{H}_{14} \mathrm{O}_{5}$, which crystallizes out of a clear solution in stellate bundles of colourless, shining needles, free from water of crystallization. It is odourless, neutral in reaction, and bitter in taste ; easily soluble in alcohol; less so in ether, amylic alcohol, chloroform, aqueous solutions of alkalies, and in concentrated acetic acid; slightly soluble in water.

It dissolves in sulphuric acid, with yellow coloration, becoming purplish, brown, and green, with bichromate of potassium (Köhler). Barth and Kretschy reported it to be a mixture of three or four substances, of which a toxic bitter one should retain the name picrotoxin; a second bitter, not toxic, being picrotin; a third, having neither quality, is anamivtin. (Chirone, however, found picrotin toxic in a ratio of about 30 to 1 of picrotoxin.)

Schmidt has since brought evidence to show that the two latter are really decomposition products, and that the stellate colourless crystals have a constant formula, which he gives as $\mathrm{C}_{30} \mathrm{H}_{34} \mathrm{O}_{13}$, and a constant melting point of $199^{\circ} \mathrm{C}$. (Pharm. Journ., i., 1884). 
ABSORPTION AND ELIMINATION.-Picrotoxin is readily absorbed from any tissue. Flies are poisoned by the blood of creatures that have died from the drug. Elimination occurs chiefly by the kidneys, partly by the skin.

Physiological Action.- Nervous System.-The phenomena produced by cocculus and picrotoxin resemble in many respects those of an epileptic paroxysm; in some instances and in certain stages they also resemble the symptoms of tetanus and of strychnia-poisoning.

In frogs, doses of $\frac{1}{30}$ to $\frac{1}{15}$ grain of picrotoxin induce at first restlessness, with weakness of movement, sinking in of the eyes, somnolence, temporary loss of reflex irritability, and in about fifteen minutes rapid seizures of opisthotonos (the belly being much protruded), semicircular swimming movements, and tonic spasm, with violent eructation of air from the mouth. After an interval of exhaustion, emprosthotonos occurs, with irregular movements of hind limbs. Death is usually delayed for many hours or days (Roeber, Archiv f. Physiol., 1869, Reichert).

In mammalia there has been observed a similar alternation of tonic and clonic convulsion, the latter of singular but definite character, such as swimming, thrusting the limbs backwards and forwards, and rolling over and over. The powdered seed given to animals shows equally an action upon the cerebro-spinal system, causing tremor, tetanic convulsions, insensibility, and, in very large doses, death.

Dr. Glover, one of the earliest observers, gave 20 grains to a dog, and noted within twenty minutes vomiting, salivation, and general tremor; within an hour strong convulsion, with opisthotonos; gradual recovery ensued in two hours. Similar but more severe symptoms occurred in another dog after 30 grains, without fatal results (Lancet, i., 1851). Deadly effects are induced in fish by the powdered seeds when thrown into water; the drug stupefies them, and they float on the surface. Wheat poisoned in a similar manner has been used for the destruction of game. Experiments on animals have been repeated with greater attention to detail by Dr. Crichton Browne, who noted drowsiness with some stupor, then restlessness and unsteady 
gait, and later the convulsive paroxysms which he describes as closely resembling those of epilepsy, there being a similar spasm first of one group, then of all of the muscles, with biting of tongue, foaming at the mouth, loss of consciousness, and afterimpairment of muscular power; there were also retraction of head and arching of body, tetanic in character (B. M. J., i., 1875). Chirone and Testa agree with this writer in describing an "artificial epilepsy" as the result of the drug. Vulpian considers the resemblance traced to be somewhat exaggerated, but in a fatal case recently reported, where a man took whisky in which a handful of berries had been steeped, the symptoms of crying, foaming at mouth, loss of consciousness, and recurrent convulsion certainly recall that disorder. Death from paralysis of respiration followed in three hours (Revue des Sc. Méd., Oct., 1884).

A point of interest and importance is involved in the pathological explanation of such attacks, and in the question as to what parts of the brain and cord are chiefly acted upon. Glover, arguing from certain backward movements of his animals, as well as from post-mortem results, and from the observations of Fleurens, which he considered parallel, suggested that the cerebellum and corpora quadrigemina were most affected. Chirone and Testa locate the primary action "in the bulb and commissural fibres," and this especially because the convulsions are as intense, or even more so, after removal of the cerebral hemispheres, and so long as the bulb and fibres are left, but cease when the latter parts are removed; an excitant effect (secondary) is exerted also on the spinal cord proper, especially in batrachia; in higher animals, the latter action is less marked than the former. Dr. C. Browne dissents from the conclusion that because convulsions are severe so long as the bulb. etc., is left, therefore they originate in this (bulb) rather than in the hemispheres, pointing out that it is common for "impressions and movements represented in a lower centre (bulb, etc.) to be re-represented in a higher one" (hemisphere), and he considers that the parts first affected are certain areas in the cortex cerebri, corresponding almost to the "motor areas" of Dr. Ferrier, since electric stimulation of such parts produces symptoms like some of those in picrotoxin-poisoning; even so, 
however, he recognizes that the medulla oblongata is, if not first, yet soon and specially, affected by the drug.

M. Bonnefin maintains rather that it acts at once on the whole extent of the cord, because he observed convulsive movements of the hind limbs after a transverse section (of the cord). Vulpian, however, states that repeating these experiments he could only detect late and partial movements, which should be more correctly attributed to asphyxial conditions, and adds the weight of his authority to the conclusions of those who locate the first and chief action of cocculus in the medulla oblongata, and distinguish it from that of strychnia, which involves the whole cord (Leçons). Dr. C. Browne describes some paresis of sensory nerves; but according to Roeber, the electrical reactions of the nerve-trunks and of the muscles remain normal. The action upon the pupil varies, but this is commonly at first contracted, afterwards dilated; the fundus of the eye has been found hyperæmic. The temperature of the body rises for a longer period than after strychnia; but when spasm has set in, the production of heat-force is lessened, and in later stages temperature is lowered (Lewis, Record, 1877). Post mortem, the meninges are found engorged, but the nervecentres themselves normal or anæmic (Husemann), with sometimes congestion of the fundus oculi.

When cocculus is used in the brewing of beer, it gives the drink more "bottom," and naturally renders it more intoxicating; drowsiness and a stupid condition, with vertigo, staggering gait, and after-headache with nausea, are symptoms ascribed to it; larger doses induce complete (temporary) loss of voluntary power.

Circulatory System.-Both cardiac and respiratory action are greatly quickened until spasms set in, when the former is rendered very feeble, and the latter irregular (Brown). There is some variation in the account of the effects on the circulation by different observers, dependent, probably, on difference in dose. Roeber describes it as returded, and Planat states that by small doses, cardiac pulsation is rendered slover until the occurrence of convulsion, when there is induced rapid action, which subsides again as coma develops. Vulpian reports it slightly retarded and lessenerd in force, but concludes that any effect on the 
heart is but slight, and is largely secondary to action on the medulla.

Post mortem, the venæ cavæ and the flaccid heart (especially the right side) are very full of dark blood; the lungs are also engorged, and present apoplectic spots, and there is large accumulation of mucus in the mouth, throat, trachea, and bronchi.

Digestive System.-The peristaltic action of the alimentary canal is constantly stimulated by picrotoxin, the free and loose action of the bowels which occurs being attributed by Dr. C. Browne to defective absorption of the intestinal contents: he could find no sign of local irritative action; the drug is apt to cause nausea, but not usually vomiting. On the other hand, in a fatal case, when less than 2 scruples of the powder, mixed with other things, were taken by a boy of twelve, the symptoms were mainly those of irritation of the digestive tract, pain, vomiting, and purging; death occurred from the results of gastro-enteritis (Taylor).

Secretory System.-The urine is apparently augmented; the saliva greatly; and probably the secretions of the intestinal glands, and of the pancreas and liver. Dr. Ringer states that the alkaloid acts upon the sweat-centres in the spinal cord, thus inducing freer perspiration.

SYNERGISTS.—Strychnia, brucia, ergot.

ANTAGonists. - Chloral hydrate is a physiological antagonizer to picrotoxin; but the latter scarcely at all antagonizes chloral. Acetic acid relieves some symptoms of picrotoxinpoisoning.

Therapeutical Action.-External.-Skin Diseases.Cocculus indicus has long been considered useful for the destruction of pediculi; also in certain skin diseases, such as porrigo, scabies, and ringworm, for all of which purposes it is best employed in the form of ointment. The last-named disease is advantageously treated also with decoction of cocculus, the slsin being well washed with common brown soap and hot water every night and morning before its application. 
THERAPEUTICAL ACTION.-Internal.-Dyspepsia," Sick Headache." - In forms of vomiting, sometimes even when severe and persistent, accompanied by dull and heavy pain in the head, giddiness, and intolerance of light and sound, also in nausea with violent retching, cocculus is valuable.

Cocculus relieves severe epigastric pain, especially when aggravated by pressure, and accompanied by flatulent distension of the stomach (or intestines), with nausea, some colic, giddiness, headache, and dryness of mouth. Again, when the colon is distended, the bowels constipated, and the motions hard and lumpy, it proves, in many cases, of singular service, and also relieves those mild cases of tympanites met with in peritonitis and in enteric fever; further, in pregnancy it relieves the frequent desire to urinate when referable to pressure on the bladder from the same cause.

Dysmenorrhœa, etc.-Cocculus mitigates this affection, especially in females of nervous temperament and thin delicate frame, when it is preceded by paroxysmal colicky pains in the hypogastric region, with back- and hip-ache, and either scanty or profuse discharge, which is generally pale. In leucorrhœa, cocculus is also useful, especially when the discharge is of sero-purulent character, and accompanied by pain in the lumbar region. In chlorotic cases, where menstruation takes place at irregular intervals, and is pale and scanty, patients receive benefit from the employment of the drug.

Nervous Affections, such as hysterical hemiplegia, choreic hemiplegia, and epileptic hemiplegia, may be usefully treated with cocculus. I have seen well-marked hysterical paralysis -where the sensibility and the muscular power were both impaired-yield quickly to it; and more particularly when they have been accompanied by menstrual irregularities, and by spasms attacking different organs. Some epileptic cases which are attributable to onanism likewise derive benefit from cocculus.

Reil employed tincture of cocculus seeds in chorea, in hemiplegia arising from cold, and in paralysis of the sphincter vesicre; and in every instance with advantage to the patient.

Gubler recommends picrotoxin in chorea, and reports improvement from it in a case of glosso-labio-laryngeal paralysis. 
Ischudi advises its use in paralysis of the lower extremities, and of the sphincters.

Epilepsy.-Picrotoxin has been recommended in this disorder, especially for weak anæmic subjects. Planat concludes that it certainly has "anti-convulsive" powers, and benefits such cases, as it does eclampsia and spasm generally (Record, 1880). Hammond, Hurd, and others have also seen some advantage from it, but its position is by no means well-established. Vulpian saw no benefit from it, and throws doubt upon its value.

Night-Sweating.-Its action in this condition is curious, but certainly rests on better clinical evidence than the above. To twenty cases, many of them advanced in phthisis, Dr. Murrell gave from $\frac{1}{180}$ to $\frac{1}{90}$ grain hypodermically, once or twice at bedtime, and improvement was, as a rule, rapid, and lasted for some days (Practitioner, Oct., 1879).

PREPaRATions and Dose.-Tinctur'a cocculi ind. (1 part of berries to 4 of alcohol-Planat): dose, 1 to 5 min. and upwards. Unguentum (80 gr. of the seeds to 1 oz. of lard). Picrotoxin: dose about $\frac{1}{8}$ to $\frac{1}{6}$ gr.; subcutaneously, $\frac{1}{60}$ to $\frac{1}{20} \mathrm{gr}$. 


\section{PAPAVERACEAE.}

\section{THE POPPY FAMILY.}

Herbaceous exogens, of which there are about 200 species. The leaves are generally alternate, occasionally all radical, and either simple or pinnatifid. The showy flowers are normally tetramerous, but in a few genera trimerous. The stamens are usually numerous, always hypogynous, and with innate anthers. The ovary is superior and free, ripening either into a capsule or slender pod; the fruit is many-seeded and dehiscent.

The juice of the Papaveraceæ is frequently coloured; it resembles milk in the common opium-poppy, while in other species it is red or orange-yellow. The odour of the plants is often offensive. The seeds (except in the Mexican Argemones), do not contain any narcotic principle.

\section{Pharmacentical Species.}

Papaver somniferum .. . . . Opium poppy.

Papaver Rhœeas .. .. . . . . Scarlet corn poppy.

Sanguinaria Canadensis .. .. Blood-root.

Chelidonium majus .. . . .. Celandine.

Argemone Mexicana . . . . . Mexican poppy.

\section{PAPAVER SOMNIFERUM.}

(The Optum Poppy.)

DESCRIPTION.-This plant, familiar to the ancient Greeks, was probably indigenous to the Levant and south-western parts of Asia, but is now met with in most countries, and is naturalized in England. 
It is an annual, with a white tapering root and erect stem, which is 3 to 4 feet high, glabrous, and, like the leaves, of a peculiar glaucous hue: the latter are simple, sinuate, lobed, notched, and amplexicaul. The flowers are produced on long, terminal, somewhat bristly peduncles, and have two large, concave, deciduous sepals, four crumpled, lilac-coloured petals, with a purple spot at the base, numerous blackish-purple stamens, and a nearly globular ovary with eight to ten sessile stigmata forming a star on the summit-the familiar dried "poppy-head" of the shops.

Opium is the inspissated juice of the unripe capsules, collected after incising them, and is met with in various forms and qualities according to the place of its production.

Turkey opium is considered the best, and occurs in roundish flattened lumps of various sizes, covered with capsules of a kind of rumex-dark and hard externally, softer and reddish brown within: exposed to the air it becomes brittle, and is readily powdered; it is soluble in water, alcohol, and dilute acid. A rough test of its purity is to draw a line with it on white paper; if it be adulterated the line will be continuous, and vice versâ. Under the microscope it shows some needles and short imperfect crystals, which are not sufficiently developed to warrant positive conclusions as to their nature: it contains about 8 per cent. of morphia.

Egyptian opium usually occurs in hard flattened cakes about 4 inches in diameter, covered with remnants of poppy leaf (not with rumex fruit). The fractured surface is porous, of dark colour, with shining particles of quartz, etc., imbedded. Starch granules are usually visible under the microscope. The proportion of morphia is estimated at 6 per cent.

Persicm opium is found commonly in short rounded cones, but also in flat circular cakes between $1 \mathrm{lb}$. and $2 \mathrm{lb}$. in weight. It is of firm consistence and charncteristic odour. Good qualities contain 8 to $10 \mathrm{per}$ cent. of morphia. This and Indian opium are highly crystalline under the microscope.

European opizm, with 16 to 20 per cent. of morphia, has been manufactured on the Continent, especially in France, but is seldom met with in commerce.

East Indian opium, as made up for the Chinese market, is in 
globular lumps, termed "cakes," with a diameter of 6 inches, and is rolled in the leaves and broken stem of the plant. For the Indian market it is formed into square cakes.

Matwa opium is made up in bricks: it is not so pure as that of Bengal, but contains about 9 per cent. of morphia.

ACTIVE INGREDIENTS. - These are very numerous; the most important are morphin and codein, which occur combined with meconic acid; next, narceia, somewhat allied; then papaverin, which may be considered intermediate between these and a group comprising nareatin and thebaia. Meconin, or opianyl, landanin, laudanosin, and cryptopin, have lately attracted attention; less important constituents are opianin, porphyroxin (opin), rhœadin, papaverosin, lanthopin, codamin, and thebolactic acid.

Morphia, $\mathrm{C}_{17} \mathrm{H}_{19} \mathrm{NO}_{3}$, forms small, white, silky needles, or (if slowly crystallized out of alcohol) colourless, semi-transparent, six-sided, oblique, rhombic prisms. It has a distinctly alkaline reaction, and a bitter taste, but no smell; melts at about $330^{\circ} \mathrm{F}$., and is destroyed by a higher degree of heat; is insoluble in cold water or ether, soluble in 400 parts of boiling water, much more so in chloroform and in alcohol. It neutralizes acids, forming salts which are, for the most part, crystallizable, odourless, bitter, soluble in water and alcohol, insoluble in ether, chloroform, and amylic alcohol. Nitric acid dissolves morphia with effervescence, producing a rich orange colour; perchloride of iron strikes a deep indigo blue, turning to green when the reagent is added to excess; strong sulphuric acid and bichromate of potash produce a green tint, owing to reduction of oxide of chromium, reactions due to the deoxidizing power of the alkaloid. If a mixture of iodate of potassium and sulphuric acid (i.e., a solution of iodic acid) be added to a solution containing a minute trace of morphia, iodine is set free, and may be recognized by the starch test, or if ether be shaken with the mixture it becomes violet red (from dissolving the iodine).

Morphice acetas, the,acetate, occurs in snow-white foathery crystals or in powder; soluble in 6 parts of water, 100 of spirit. The solution has an acetous odour.

Morphice hydrochloras, the hydrochlorate (muriate), is met 
with either in powder or in white acicular silky prisms (somewhat like quinine), or in large transparent prisms; it is soluble in about 26 parts of water, 10 of glycerine, 80 to 90 of alcohol.

Morphice sulphas.-The sulphate forms bushy groups of needles; soluble in 2 parts of water.

Morphice tartws, the neutral tartrate, also crystalline, is still more soluble, and hence is specially adapted for hypodermic use (B. M. J., i., 1883).

The bi-meconate is resinoid.

Chastaing has found that morphia is not only a base, but has some properties of an acid or a phenol, and Guimaux has obtained from it the following alkaloid, codeia, which he considers stands to it in the relation of a methylic ether (Lancet, ii., 1881).

Codeia, $\mathrm{C}_{18} \mathrm{H}_{21} \mathrm{NO}_{3}$, is a powerful base, capable of precipitating the solutions of salts of iron, lead, nickel, copper, and cobalt. It crystallizes out of a pure ethereal solution in small, anhydrous, colourless crystals, which melt at $150^{\circ} \mathrm{C}$.; from a watery solution in hydrated crystals, which, if the process be slow, form large, regular, transparent octahedra and prisms, and melt on heating into an oily fluid, odourless, and of a weakly bitter taste. Codeia is soluble in $1 \%$ parts of boiling and 80 parts of cold water; easily soluble in alcohol, chloroform, and ether: its salts are mostly crystalline, bitter, and almost insoluble in ether. Two are used in medicine, of which the hydrochlorate crystallizes in star-shaped groups of short needles; is soluble in 20 parts of cold, and in less than one part of boiling water: the nitrate crystallizes out of boiling water in small prisms, which melt under heat, and cool again into a resinoid mass.

Narceia, $\mathrm{C}_{23} \mathrm{H}_{29} \mathrm{NO}_{9}$, crystallizes from solution in water, alcohol, or dilute acetic acid, in long, white, four-sided prisms, or clusters of fine needles, which have a bitter, styptic taste, melt at a high temperature, and solidify amorphously on cooling, and are insoluble in water, slightly soluble in cold, easily in boiling alcohol. Narceia dissolves, but not easily, in weak mineral and vegetable acids, if pure, without discoloration ; if impure, often with a blue tinge: although a weak base, it forms salts with these acids. Concentrated sulphuric acid first colours it brown, and then gives a bright-yellow solution. 
Papaverin, $\mathrm{C}_{21} \mathrm{H}_{21} \mathrm{NO}_{4}$, crystallizes out of alcohol in white masses of needles or scales; it is weakly alkaline in reaction, almost insoluble in water, cold alcohol, or ether, but boiling alcohol takes it up freely, and chloroform extracts it either from an acid or alkaline watery solution. Concentrated sulphuric acid changes it to a deep violet blue, and forms a violet solution, the colour of which fades slowly: its salts are mostly difficult of solution in water.

This alkaloid is not to be confounded with a substance also unwisely named papaverin, which exists in the unripe poppy capsule, and is a resinoid body.

Narcotin, $\mathbf{C}_{22} \mathbf{N}_{23} \mathrm{NO}_{7}$, crystallizes out of an alcoholic or ethereal solution in colourless, transparent, pearly prisms, or grouped needles on the right-rhombic system : precipitated by an alkali from an acid solution, it presents the form of light, white flakes. It is odourless, tasteless, and neutral in reaction. Cold water hardly dissolves it; boiling water only in very small proportion, alcohol and ether moderately, chloroform with great facility. Its neutral solutions rotate the plane of polarization to the left, acid solutions to the right. With acids, narcotin forms salts of acid reaction; usually uncrystallizable; soluble in water, alcohol, and ether; and extremely bitter to the taste. Concentrated nitric acid converts it into a red resinoid body, red vapours being also given off; this resin, on boiling with potash, develops methylamin.

Thebaia, $\mathrm{C}_{19} \mathrm{H}_{21} \mathrm{NO}_{3}$, homologous with sanguinarin, crystallizes in silvery, four-sided scales, or in needles, or in small horny crystals, sharp and styptic in taste. It is strongly alkaline in reaction, and electro-negative on friction; is almost insoluble in water, and in aqueous solutions of ammonia or alkalies; freely soluble in alcohol and ether, less so in benzol. Dilute acids dissolve it easily, and form salts which will crystallize out of an alcoholic or an ethereal solution, but not from a watery one. Concentrated acids, and also chlorine and bromine, change it into a resinoid body. In concentrated sulphuric acid it dissolves with production of a blood-red colour, which later changes to yellow.

Opianyl, or meconin, $\mathrm{C}_{10} \mathrm{H}_{10} \mathrm{O}_{4}$, forms tasteless, white, shining needles, slightly soluble in cold water; soluble in ether, 
alcohol, and acetic acid. It dissolves without colour in pure sulphuric acid, but the solution becomes purple on heating.

ABSORPTION AND ELIMINATION.-Opium and its soluble compounds are absorbed by all the tissues without difficulty -from the subcutaneous tissue with remarkable quickness. It is eliminated partly by the skin, mainly by the kidneys, and has been detected in the urine of a patient who had taken only $\frac{3}{4}$ grain of the extract. In habitual opium-eaters it ceases to be detected in the urine about seven days after omission of the drug.

Landsberg, after administering morphia to dogs, failed to detect it either in the urine or the nerve-centres (Lancet, i., 1881), but Marmé recovered it unchanged from the urinewhen not less than $\frac{1}{7}$ grain had been taken during the day; after larger doses, it was found also in the fæces:- a decomposition product,-_"oxydi morphin" appearing in the tissues when toxic amounts had been taken in small quantities at a time (Deuts. Med. Woch., Ap., 1883). Orfila, Bouchardat, Dragendorff, and others, also report that the drug is eliminated unchanged, but Lassaigne, Christison, Buchner, Landsberg: (Record, 1884), and, later, Burkart maintain,-I think more correctly,--that the greater part of the drug is decomposed in the system, and its absence from the urine of patients does not prove their not taking it (Rev. des Sci. Méd., Oct., 1884.)

Sir H. Thompson, having injected $\frac{1}{2}$ ounce of liq. opii sed. several times without effect into the bladder of a man suffering from chronic cystitis, concludes that opium is not absorbed by that viscus - 20 minims taken by the mouth readily acted in the same patient (Clin. Lect., 7th ed.); the ease, however, does not decide the question for the healthy bladder.

Physiological Action.- Nervous System.- When taken in medicinal doses, just enough to produce characteristic effects without approach to poisoning, a quiet dreamy condition is induced, "during which ideas and images float before the mind, and by their endless and effortless repetition shorten time, which seems to lose itself in rest." The imagination is stimulated rather than the reason, although some persons have maintained that they could study and write better under the 
influence of the drug. Dr. C. R. Francis states that if the drowsiness be resisted the intellect becomes clearer, but general sensibility is diminished (Med. Times, i., 1882). After a varying time, the condition merges into sleep, which may be either "light and dreamful," or heavy, according to the dose: similarly, on awakening, the subject may either feel well, or be depressed, or suffer from headache and nausea; sometimes these symptoms may occur instead of the expected sleep.

The action of toxic doses ( 1 to 4 grains and upwards) presents somewhat different aspects according to circumstances. When the preparation taken is such as to permit rapid absorption, the (adult) patient quickly becomes conscious of a sense of fulness in the head, which seems to commence in, and spread from, the nape of the neck; in a few minutes great drowsiness sets in, and a sensation of general heat, which increases to an almost intolerable degree, and is accompanied by sweating, usually so profuse as to suspend all other secretions. Later, the surface becomes clammy and cold; the drowsiness passes into semicoma; the patient, unless roused, lies unconscious, and is heedless of everything about him, but if roughly spoken to and shaken, can still be induced-though the speech is thick and hesitating-to answer questions, and even to get up and walk about; on leaving him, however, he immediately subsides into his former stupor. The pupils are commonly contracted to the size of a pin's point, and become insensible to light; there is buzzing in the ears. The pulse, which in the first or hot stage was rapid and somewhat full, becomes feeble and irregular, and falls to the normal rate, or even below it; simultaneously with this alteration, the features become pinched and ghastly, and flushing gives place to livid pallor, while the museles of the limbs are affected with spasmodic jerkings.

The mouth and fauces are dry, and there is commonly nausea, and often vomiting. The general depression of the system is marked. The respiration becomes more and more embarrassed; in the later stages the presence of a quantity of mucous secretion in the tubes makes itself known by the rattling sound of the breathing. The respiration comes to a standstill usually some minutes before the entire cessation of the heart's action. 
The symptoms differ considerably in young children, especially in infunts; there is a more rapid passage into profound stupor; but what is remarkable is the much greater frequency with which convulsions occur. The convulsive movements vary from mere twitchings of the facial muscles to rhythmical startings of the limbs; severe clonic convulsions, which may be unilateral or bilateral; and even tetanic spasms.

It is interesting to note that the tendency to convulsive movement, which in man is rare except in the first few years of life, is a constant feature of opium-poisoning in many of the lower animals. By experiments upon these, additional light has been thrown upon the mode of action of the drug: the injection of 1 or 2 grains causes in the frog convulsion, afterwards paralysis and suspension of respiration; the convulsions are mainly reflex, but certain experiments after the division of nerves indicate that they arise in part from the nerve-centres, and also from a direct action on peripheral nerves and on muscles. Birds are remarkably little affected by the drug, and their pupils remain unaltered by it; large doses, such as 10 grains, of morphia given to pigeons by the mouth, or 3 grains by the skin, will, however, induce unsteadiness in movement, laboured breathing, convulsion, and death. In the mouse, a fraction of a grain of morphia produces spasm, not sleepiness; the respiration becomes irregular, and later, either recovery occurs, or coma and apnœa cause death. On dogs and horses the drug acts more as it does on man, causing sleepiness and sweating, or, in toxic dose, convulsion and death. It would seem that the lower a creature is in the scale of organization the more the symptoms, such as convulsion, indicate affection of the cord; and the more highly developed the cerebral hemispheres are, the more they are affected, as shown by sleepiness, stupor, or delirium. Some of the alkaloids of opium-e.g., thebaia-are more fatal to animals than to man, and vice versâ.

According to recent experiments by Zuelzer on animals, opium greatly increases destructive change in nervous tissue, and consequently the elimination of phosphoric acid (Rev. des Sci. Méd., Juillet, 1884).

On the pupil no special effect is produced by local applications 
of opium or morphia, but the well-known evidence of their constitutional effect is contraction, which may be extreme; it depends probably on stimulation of the nerve-centres concerned, and the later dilatation which sometimes precedes death, on paralysis of the same (Wood); in horses the pupil is commonly dilated ( $\mathrm{J}$. Harley); in dogs it dilates before contracting.

Circulatory System.- In man the pulse is at first slightly increased in frequency, afterwards lessened while it becomes stronger and fuller; later, it either recovers its usual condition or becomes rapid and weaker. The slowing seems to be due to stimulation of the cardiac inhibitory nerves, and occurs very quickly and markedly if morphia be injected near the cerebral centres of these nerves; the rapid, feeble pulse of toxic cases is traced to paralysis of the peripheral terminations of the vagi. The drug is said first to stimulate and then depress the motor ganglia within the cardiac substance (Gscheidlen), and it seems to exert a similar action on the vaso-motor system. In the experiments of Ringer and Sainsbury on frogs' hearts, there was evidence of primarily increased force, but ultimately contractile power was abolished; the heart was always arrested in diastole, and they trace the effect rather through the vagi than the ganglia (B. M. J., i., 1883).

Respiratory System. - The gradual paralysis of this system has already been mentioned; it is due to an action of the drug, not on the peripheral nerves, but on the respiratory centre in the medulla, since it occurs equally if the vagi be divided.

Filehne states that in animals after the primary slowing of respiration there is a stage during which it returns to the normal rate, but brings forward experimental proof that the excitability of the respiratory centre is not really restored during this stage, which gradually deepens into the final asphyxia (Arch. f. Exper. Path., Bd. x., xi.).

Digestive System.-Opium readily disorders the gastric functions, since it lessens peristalsis, as well as the natural secretions, the discharge of bile, etc.

Röhrig (Med. Times, ii., 1873) stated that opium increases the biliary secretion; but Rutherford, in two experiments on dogs, found that morphia had no effect in this way, and that the 
liver was still capable of stimulation (e.g., by salicylate of soda) after the injection of morphia into the duodenum (B. M. J., i., 1879). In some patients taking opium the stools became clay-coloured; but this may be explained by the fact that peristalsis is lessened, and thus more of the bile poured into the intestine may be re-absorbed than normal.

The diminution of bile-secretion is given as a reason for the characteristic sallowness of opium-eaters.

Nothnagel, inquiring as to the mode of production of constipation by morphia, first applied a salt of soda to the exposed intestine of an etherized rabbit, and found it cause an ascending contraction, but under the moderate influence of morphia this effect was not produced, but only annular contraction; under let'ge doses, however, it again appeared, whence he concluded that in the former ease fibres of inhibitory nerves were stimulated, leading to a temporary paralysis of action (constipation); whilst with the larger doses the same nerves were paralysed, causing sometimes an increase of movement, and hence purgation (Virchow's Archiv, Bd. lxxxix., 1).

Petrone confirms this, and compares it to the action of digitalis on the heart, small doses stimulating the splanchnic (inhibitory) nerve, and large doses paralysing the same (Record, 1883).

Genito-Urinary System.-The urine is generally diminished in amount under the influence of opium, but irritability of the bladder, with frequent passage of small quantities, sometimes occurs under morphia. On the other hand, retention may be experienced from partial paralysis of the bladder. An aphrodisiac effect has been traced to medicinal doses of the drug, and connected with its influence on the cerebral centres (Med. Times, ii., 1861). It is said to be experienced at the commencement of the opium habit, though in later stages there is marked impairment of sexual desire. Dr. Francis gives some evidence to show that the effect is rather a heightening of sensory perception than a true stimulation (loc. cit.).

Cutaneous System.-Perspiration is commonly increased by small doses of opium, and with toxic amounts, if the skin becomes at first dry and warm, it is soon covered with moisture, and in later stages with a profuse cold sweat. In opium-eaters perspiration is lessened. 
Occasionally this drug, and more frequently morphia, given either by the mouth or skin, will induce a general erythema, which specially affects flexor surfaces, and somewhat resembles a scarlatinal rash; it is accompanied with much itching, sometimes by sudcmina, and followed by slight desquamation. Such effects can only be set down to "idiosyncrasy"; they are perhaps more frequent in elderly persons than in others. Charcot connects them with the rheumatic diathesis. (For some remarkable cases following moderate doses, $x$. Berl. Klin. Woch., No. 46, 1882.)

Physiological Action of Morphia.-This alkaloid represents most nearly the essential element of opium in its action upon man; for him it is the most powerful in blunting sensation and in producing sleep, or death; not so in animals, if we rely upon some experiments of Cl. Bernard, who found that 2 grammes of the hydrochlorate injected into the vein of a clog did not prove fatal, whilst 10 centigrammes of the same salt of thebaia caused death in five minutes; the latter dose given to man produces scarcely any effect, whilst the same quantity of morphia is almost surely poisonous. Grasset and Amblard found that small doses of morphia given to dogs produce sleep, but cause some muscular contractions, and even convulsions; so that they conclude this alkaloid to be not so wholly opposite to thebaia in action as previously thought (Rev. des Sci. Méd., i., 1883).

In animals it is also said to be less soporific than narcein, but more so than codeia. If an equal quantity of the latter and morphia be injected into two dogs, sleep follows in both; but on awakening, the dog under the influence of codeia behaves as usual, whilst the one under the morphia seems stupid and strange, and does not recognize friends.

Morphia, more than the other alkaloids of opium, lessens the secretions of the intestinal tract. Moreau illustrated this effect by injecting into a ligatured portion of intestine in a living animal a solution of sulphate of magnesia; after a certain time, in the normal animal a quantity of fluid is found in the ligatured part, whilst under morphia little or none is poured out. 
Morphia also (more than the other constituents of opium) disorders digestion, impairing appetite, and often causing nausea and vomiting, especially in nervous and irritable subjects; injected under the skin it seems to stimulate more or less the circulation and the frequency of respiration, heightening the colour and causing some perspiration; such effects are not remarked after taking it by the mouth.

Physiological action of Codeia.-- "In man a single dose of 5 centigrammes ( $\frac{5}{7}$ grain) causes, in about half an hour, some heaviness of head and confusion of ideas, with some weakness of the lower extremities" (Rabuteau); but these symptoms are temporary. The pupils are slightly contracted, the pulse unchanged; the appetite and stomach are unaffected; there is neither diarrhœa nor constipation as a rule, but in some persons nausea and slight headache may be felt from grain doses. Two grains caused a sense of fatigue, and some itching of skin, especially of the extremities; also contraction of pupil, lasting for one day; slight somnolence, but not sleep. Persons vary much in their experience as to this latter point. I have known $\frac{1}{4}$ and $\frac{1}{2}$-grain doses, given at bedtime, commonly followed by natural sleep in subjects habitually wakeful; the soporific effect of bromides is markedly increased by it. Bouchut found it distinctly soporific; Gregory not so, even in 3-grain doses; $\frac{3}{4}$ to $1 \frac{1}{2}$ grain makes dogs sleep, but not so soundly as morphia or narceia -it is more a restful state than real sleep. Its power in lessening sensibility is much less than that of morphia, and nearly a grain has been injected in cases of neuralgia and sciatica without relief to the pain.

In cases where unduly large doses have been taken medicinally, semi-coma has been produced, with tinnitus aurium, sense of pressure in the temples, impaired vision, and slowing of pulse (Schroff). In the instructive case published by Dr. Myrtle, 4 grains were taken in pill with $\frac{1}{20}$ grain strychnia. For two or three hours the patient felt stimulated and exhilarated; then his sight suddenly failed; he became giddy and partly unconscious. Four hours after the dose " he had a terrified look, was exceedingly pale, sweat standing in large beads on his forehead, pupils slightly contracted, pulse scarcely percep- 
tible, surface cold and clammy, voice reduced to a whisper." After an emetic, stimulants, and warmth, the symptoms subsided within forty-eight hours, though nausea and retching were severe; pulse 56. The amount of urine passed during the period was much less than usual, and contained no sugar, though this had been abundant the day before (B. M. J., April, 1874). The symptoms were not those of drowsiness or coma, but mainly of collapse and gastric irritation, with some delirium.

In rabbits and dogs, with toxic doses, Wachs observed muscular tremors, stretching out and then weakness of limbs, twitching of lips and of eyeballs, sometimes of the jaw muscles; the animals sometimes ran backwards, or in a circle, became restless, and opisthotonos and inspiratory spasm set in. This last proved quickly fatal if the dose was very large; if not, clonic spasms occurred, ending in convulsion and death. The parts most affected seem to be the cerebellum and medulla oblongata, and the symptoms much resemble those caused by picrotoxin. In cats, Stocker found cerebral congestion (determined by examination of retinæ), with dilatation of the pupil; much reflex excitability; in one case, epileptic convulsion. Salivation and purging occurred in two cases, without vomiting.

Post mortem, there was hyperæmia of the cerebral membranes, and effusion in the ventricles; no congestion or hæmorrhage in the brain substance itself. The heart cavities were distended with dark blood, the lungs and viscera congested, the gall bladder full; the alimentary canal, the pancreas, and also the bladder were anæmic (Das Codeia, Eine Monographie, Marburg, 1868).

In the few fatal cases recorded in man there has been some uncertainty either as to dose or purity of the drug. Thus, a child with broncho-pneumonia got "an over-dose," and passed into a state of collapse (Med.-Chir.-Rev., i., 1876); and a man of forty-five took, in twenty-four hours, a syrup which the chemist asserted contained only about 2 grains, and died comatose fourteen hours afterwards. But, as Rabutean points out, we have no evidence that morphia, which is cheaper, was not contained in the syrup; and the various syrups of codeia in 
use in France vary much in strength, from 5 to 30 centigrammes in 30 grammes of vehicle.

PHYSIOLOGICAL ACTION OF NARCEIA.-The properties of this alkaloid have been much disputed. Claude Bernard pronounced it the most hypnotic of the opium series; whilst Fronmüller, in more than twenty observations, found it inert. Dr. J. Harley also states that any powers he detected in the specimens used by him depended on adulteration with some of the other alkaloids. Schroff takes a similar view; but, on the other hand, the opinion of Bernard has been more or less supported now by many observers, and if narceia be not so active as he thought, it is impossible to deny to it some narcotic power. This is exerted more in animals than in man : thus, 1 grain of the hydrochlorate of narceia injected under the skin of a young dog caused a profound sleep, during which painful operations could be performed; in a healthy man, however, 2 grains scarcely produce any somnolence, but in weakly persons keeping their bed, it will often act well (Rabuteau).

Brown-Séquard many times observed, from doses of 3 to 4 grains, a tranquil, refreshing sleep, which was markedly less deep than that produced by a dose of morphia twenty times smaller. Eulenburg remarked similar effects from narceia (prepared by Merck), and calculated the dose required at trice that of morphia for corresponding results.

Barnay, writing more recently, corroborates these observations, finding the sleep produced by narceia very like natural sleep, and its action not followed by skin-irritation or nausea (Record, 1877).

Rabuteau has relieved the pain of uterine cancer by its injection into the vagina; and Petrini, the pain of neuralgia by its subcutaneous injection.

Bouchardat (fils) describes gastric irritation from the drugdryness of mouth, nausea, vomiting, and sometimes diarrhœa (from $1 \frac{1}{2}$ grain injected), also muscular twitching's, and slowing of pulse and respiration (De ln Narcéine-Thèse, Paris, 1865). Rabuteau found it relieve diarrhœa in man when given in fairly large doses ( 1 to 3 grains), but not so effectively as morphin or opium; it lessened also the secretion of the buccal and other 
mucous membranes, though it did not cause the usual inconveniences of opium. Béhier and Laborde found it act specially well in the diarrhcea and cough of phthisis, but sometimes difficulty in micturition followed its use.

Physiological Action of Papaverin.-This substance has little action upon man, even in comparatively large doses ( 3 grains and upwards); and in rabbits and dogs the same amount may be injected, without evident effects upon the pupils, the heart, or the nervous system. It is not, however, inert; C. Bernard ranked it second as a convulsant, and less than a grain placed under the skin of frogs will cause convulsion either directly or on slight disturbance (Schroff, Rabuteau).

Baxt states that it has the power of paralysing reflex irritability, and also the action of the heart (independently of any action on the vagus), and that it has some hypnotic properties (Virchow's Archiv, lxx., 1869); but these last are very doubtful, and have not been verified by Bernard or other observers. It does not prevent osmosis into the intestine, nor control diarrhœa ; on the contrary, it sometimes has an opposite effect.

Physiological Action of NARCotin.-This substance does not correspond well with the name given to it, for it has little or no narcotic action, and is the least poisonous of the opium-alkaloids. In frogs, indeed, $\frac{1}{3}$ grain injected under the skin may cause convulsion without proving fatal; and in cats, 5 grains induce, after several hours, tremor, tetanic rigidity, contraction of pupil, and clonic spasms, succeeded by coma and death (Kauzmann).

In man, however, upwards of 7 grains may be given without other effect than a bitter taste in the mouth and a slight congestion of the eye with contraction of the pupil-no evidence at all of cerebral influence.

Bailly has given as much as 45 grains in twenty-four hours; and similar large doses have been given in India, by Dr. O'Shaughnessy and others, for anti-periodic effects.

It does not cause constipation.

Physiological Action of TheBaia.-This is a tetanizing poison, with some analogy to strychnia; of nuch power in 
animals, but markedly less active in man. In the experiments of F. W. Müller with dogs and rabbits, restlessness and tetanio spasms set in soon after the poison was given, and were followed by paralysis; recovery was preceded by vomiting; 2 to 3 grains were fatal.

Pigeons were less readily affected, but fatal doses caused general tremor, and afterwards a tetanic attack, followed by isolated spasms and a paralytic condition.

On the hearts of frogs it seemed to act as a direct poison, since they ceased to beat sooner in a solution of it than in plain water. Müller showed also that thebaia and morphia do not antagonize each other, for a mixture of the two injected into a rabbit caused death like the former alone, and with similar rapidity (Monographie, Marburg, 1868).

It has no soporific effect, but seems to aid the action of chloroform, and in man $\frac{1}{7}$ grain of the hydrochlorate, when injected, has relieved neuralgic pain more permanently than an equal amount of morphia (Rabuteau). Half to one and a half grain has been taken by this observer without other effect than some confusion of head, like that of slight intoxication; there was no action on the pupil or the pulse, and no astringent effect.

The three last-named alkaloids-papaverin, narcotin, and thebaia-may be grouped together as excito-motors, or convulsants; whilst the three former-morphia, codeia, and narceia-are sedatives, and act usually by moderating reflex excitement.

The three following have been recently investigated by Falck (Archiv. f. ges. Phys., xxv.):-Landanin, $\mathrm{C}_{20} \mathrm{H}_{25} \mathrm{NO}_{3}$, caused quick respiration, tetanus, and death in rabbits, in about twenty-five minutes,-first lowering, then raising temperature.

Laudanosin, $\mathrm{C}_{21} \mathrm{H}_{27} \mathrm{NO}_{4}$, acted similarly, about 1 grain causing death in rabbits, which became restless, quivering, and salivated, with dilated pupils; the heart-action was increased (from stimulation of the accelerator nerves), temperature was also elevated, convulsions, like those of strychnia, occurred, and the respiration, at first accelerated, was finally arrested: large doses stopped the heart-action at once. 
Cryptopin quickened respiration, then caused spasm, and later adynamia and death.

IDIOSYNCRASY-TOLERANCE.-The preceding description of the action of opium and its constituents must be qualified by allowance for the peculiar condition termed "idiosyncrasy": for, on the one hand, unusual, often severe, symptoms may occur in some persons from very small doses, and on the other, very large ones may fail to produce their ordinary results. An unusual effect of a small dose is seen in the following case. A woman, aged thirty, "neuropathic," after 6 minims of "Sydenham's laudanum" (= about $\frac{3}{4}$ grain opium), had syncope and contraction of pupils, afterwards hallucinations, and general anæsthesia, though consciousness remained; coffee was given; recovery followed in about two hours. Several weeks afterwards, $\frac{1}{2}$ centigramme of morphia hydrochlorate $\left(=\frac{1}{1 \pm}\right.$ grain) hypodermically was also followed by hallucination. Huchard, who relates the ease, terms the condition "ataxie thérapeutique" (Rev. Méd., t. ii., 1883). Some subjects are exceedingly intolerant of hypodermic morphia, getting violent vomiting, or pruritus, or mental excitement from moderate doses, although these effects may often be avoided by combining. a minute dose of atropia. In a case of mitral disease with anasarca, etc., serious coma followed $\frac{1}{3}$ grain (Lancet, ii., 1882); and the subjects of gall-stone, of albuminuria, of alcoholism, and of disorders interfering with aëration of the blood, are specially sensitive to the drug. This would seem to favour Landsberg's hypothesis that the drug in such subjects is not readily decomposed in the blood, whilst in those who take large doses with impunity, the power of decomposing it is greater (v. p. 78). From small doses of opium some persons get erythema and headache, and from full doses excitability and insomnia.

Dr. Kane has published an able report on the subject, containing twenty-five instances of death apparently due to morphia, and sometimes to only small doses. Three were of delirium tremens, and in one of these only $\frac{1}{4}$ grain had been given; in several, the fatal issue followed a moderate amount, e.I., $\frac{1}{12}$ grain injected, after $\frac{1}{4}$ grain had becn taken by the 
mouth; and again, $\frac{1}{6}$ grain by the skin, after 30 drops of laudanum had been swallowed (asthma). Dangerous syncope requiring active treatment has occurred from $\frac{1}{4}$ and even from $\frac{1}{6}$ grain of morphia (Lancet, i., 1880). Infants are commonly very susceptible, yet one, aged four months, is said to have taken habitually 6 to 8 drachms of laudanum in twenty-four hours (to relieve colic, due probably to improper feeding), the mother stating that "less than 50 drops produced no effect" (B. M. J., i., 1880). As with other poisonous drugs, a condition of "tolerance" may be induced in many persons by continued use, and the dose requires to be increased; but there are curious differences in this regard also. Thus, Dr. Hudson (U.S.) records a case of cancer where $\frac{1}{16}$ grain morphia remained a sufficient daily dose throughout the illness (Record, 1881); whilst Dr. P. Boulton and Dr. Cass have reported instances where so much increase was made that 700 grains were used in ten months, and again 20 grains of morphia in twelve hours, without narcotism (Lancet, i., 1882).

Under the influence of pain, or of mental disorder, as a rule, but not invariably, larger doses are tolerated; and in diabetics this is specially observed. I have seen several cases in which considerable amounts of morphia were taken daily without evident inconvenience for many years, and Dr. Murrell states that in the Fen districts upwards of two quarts of laudanum per week are consumed by some working-people (B. M. J., ii., 1881); but, on the other hand, 3 to 5 grains daily will in some persons induce the symptoms of chronic opium-poisoning, anorexia, constipation, torpor, etc. Such curious differences partly explain the contradictions of opinion that exist on the questions of opiumeating, or smoking, and opium traffic: the one side seeing little else but advantage in the drug, whilst the other regards only exaggerated instances of its fatal abuse.

SYNERGISTS.-The cerebral effects of opium are augmented by alcohol, by chloral, camphor, etc. Quinine, which by Gubler is regarded as antagonistic, is so only in toxic doses; as a remedy, e.y., in inflammation and congestion, it is an adjunct to opium. The sudorific action is favoured by ipecacuanha. 
Ringer, comparing opium specially with chloral, finds that both depress the centres at the base of the brain, but the former exerts a much more marked primary stimulation (B. M. J., i., 1883). As to the synergic action of chloral given in disease, some caution is required. In a few cases where this had been taken as a composing draught, and followed shortly afterwards by an ordinary dose of morphia, I have seen the effect of the latter much exaggerated, profound narcosis and even convulsion occurring: the same fact has been noted by others, e.g. by Lamotte (Lancet, March, 1880). There is, however, a difference of opinion on the subject. No doubt some persons can take the two drugs in suitable doses combined, or at moderate intervals, without other than good effect (Lancet, ii., 1873, and ii., 1880) ; but very sensitive patients cannot safely do so, nor, in my experience, those with weak heart, or bronchial disorder.

(As to morphia with chloroform, $\imath . \mathrm{p} .112$.)

ANTAGONISTs.-Alkaline carbonates, metallic salts, and tannates are chemically, but not physiologically, antagonistic to opium. Belladonna and the mydriatics generally neutralize, within limits, some of the actions of opium, especially the paralysis of the respiration and the heart.

This subject has already been referred to under Atropia, and may here be summarized somewhat in the words of Bartholow. (1) Morphia and atropia are antagonistic in their action on the cerebrum, and the result of such antagonism is torpor, which, with large doses, deepens into coma; so that remedial antagonism does not extend to lethal doses.

(2) They are antagonistic in action on the pupil, though not constantly, and the effect of atropia preponderates; (3) also in their action on the henrt, but that of atropia is more powerful and prolonged; (4) also on respiration -morphia retarding the movements and lessening excretion of carbonic acid, atropia increasing . both; (5) also on arterial tension-opium (in full doses) retarding the heart and paralysing the arterioles, atropia the reverse, and the latter preventing largely the depression, coldness, sweating, and nausen caused by the former. (6) On the kidneys-morphia diminishing, atropia increasing, the secretion. (7) $\Lambda$ s to the bladder, the former lessens its sensibility and impairs the contrnetile power, whilst 
the latter increases the action of the sphincters; so that, although different, they are not antagonistic in action.

(8) In therapeutics such opposing actions will secure effects not to be obtained from either alone. In cases of poisoning, he estimates $\frac{1}{20}$ grain of atropia to be used for each grain of morphia (N.Y. Med. Record, 1880 and 1883).

A large number of instances in which atropia has been given with apparent success in severe opium-poisoning have now been placed on record in various countries. Some of the earliest were those reported by Dr. Johnston from China, to the number of sixteen, of whom ten recovered (Med. Times, Sept., 1872). The amounts taken varied from 1 to 4 drachms of opium extract; and the amount of atropia injected, from $\frac{1}{2}$ to 1 grain (altogether), generally given in two doses. In a recent case, 11 grains of morphia were taken, and $\frac{1}{35}$ grain atropia injected twice, and recovery followed (B. M. J., i., 1883). In another, 30 grains of sulphate of morphia were taken; an emetic and coffee were given, and $\frac{1}{24}$ grain of atropia; in fifteen minutes, $\frac{1}{16}$ grain; and this again in another fifteen minutes; and in half an hour, $\frac{1}{8}$ grain: then the pupils began to dilate, but the stupor increased, and $\frac{1}{8}$ grain was injected hourly till distinct improvement began: altogether, $1 \frac{1}{16}$ grain was used (Lewis, Record, 1880). In four cases reported by S. Wallian, $\frac{1}{5}$ grain atropia in three doses $\left(\frac{1}{20}, \frac{1}{20}, \frac{1}{10}\right.$ grain) was sufficient, and its effects were shown in about two hours by dilatation of pupil and return of consciousness: its influence on vision, motor power, etc., remained for about forty-eight hours (N.Y. Med. Record, 1883).

Coffee, bromides, and digitalis oppose the effect of opium on the cerebral circulation. Strong hot tea injected into the rectum has been found useful in acute poisoning (Sivel). An "antiopium pill," much prescribed in hospitals in China, is composed of quinine, henbane, capsicum, eamphor, and bitters (Med. Times, ii., 1871).

There is some evidence of antagonism between opium and strychuic injected in animals (Maon, Med. Times, ii., 1871).

Also two cases of opium-narcosis are recorded in which veretrum viride was used with apparent benefit. One occurred in an infant, to whom 1 drachm was given every hour, of a mixture containing 20 minims of tinct. verat. virid. in 3 ounces 
water, and narcotism disappeared after the third dose ; the other in an adult, in whom, when respirations were reduced to less than four per minute, 6 drops of tinct. verat. virid. injected under the skin modified the symptoms in half an hour (Record, 1880 ; another case, B. M. J., ii., 1871).

Tannic acid is advised by German authorities (ibid.); nicotin by Bonnacorsi, who concludes that with both poisons death occurs by asphyxia; but with morphia this arises by paralysis, with nicotin by a tetanizing action on the circulatory centres (Record, 1883). Certainly there is some reason to believe that habitual smokers, and perhaps also those who are addicted to chewing tobacco, are less susceptible to the influence of opium and its preparations than others who do not make use of tobacco.

THE OPIUM-HABIT.-Judging from clinical experience, there can be no doubt of the evil results in the large majority of persons of the habitual taking of opium and its alkaloids; and since the more general use of the hypodermic syringe, these have become so well recognized as to receive the special name of "Opium-" or "Morphia-habit," Morphinism (Morphiomanie, Morphiumsucht). Pain or sleeplessness is the most usual cause of its first adoption, and it would seem that one month's habitual use is an average time to confirm the habit (Mattison, N.Y.). If $\frac{1}{15}$ or $\frac{1}{4}$ grain represents the early dose, increase is soon made to $\frac{1}{2}$ and 1 grain daily, because "it loses its effect." According to Richardson, most hubitués increase to 3 grains of morphia daily, some to 5 or 6 grains: under the former dose they may go on with their usual work; but above that quantity, symptoms of "exhaustion and mental incompetence" appear. Most persons do not exceed 6 grains daily-an amount that has beeu continued for two years; but 10 and even 18 grains of morphia daily have been taken. Dr. Sharkey quotes one patient who took 24 grains (Lancet, ii., 1883), and for seventeen years he had done his work well. In this matter there is clearly also an "idiosyncrasy," another illustration of which may be quoted from Dr. Yost. A man aged twenty-five-subject to "nervous trouble," e.g., "loss of motion of all parts supplied by spinal nerves," etc., traceable to drink, and partly to heredity, and which commenced at about fourteen years of age-began taking 
opium about ten years later, and soon took from 1 to 2 ounces of laudanum daily (U.S. Pharm., 1 grain in 12 minims), which amount he gradually increased (within nine months) up to $1 \mathrm{lb}$. daily. Slight constipation was the only recorded inconvenience. At the end of the nine months the habit was stopped suddenly, but without other ill effect than moderate insomnia (Med. Surg. Rep., Philad., 1883).

The main symptoms presented by the subject of the morphiahabit may be grouped somewhat as follows:-

Digestive System.-Impaired appetite, nauseous or unusual taste, dry red tongue, at first nausea and vomiting; but not severe dyspepsia, colic, or flatulence, as with alcoholic subjects. There may be difficulty in swallowing solid food or pills, on account of altered mucous secretion. Constipation is obstinate, the light-coloured fæces "as hard as wood," and either requiring mechanical removal, or very strong purgatives, "expulsive power being mil",; the liver is torpid.

Nervous System.-Perturbed sleep; disturbance of special senses-smell, sight, and hearing; " intolerance of bright light and shrill sounds ;" pupils commonly contracted. There is often loss of motor power, with spasm, and partial paralysis; impair. ment of memory, and usually of the mental power, though at times imagination is more lively; tremor, occasional difficulty in articulation, and symptoms resembling those of intoxication. The tremor is peculiar in that it seems to result from a twist of the limb on itself, due to the alteruative contraction of pronator and supinator: it is distinguishable from senile and alcoholic tremor (Jouet, Thèse de Paris, 1883).

As to the moral condition-if it can be said that morphiasubjects are "less untruthful" than alcoholic, it must not be understood that they are truthful: as a rule, no reliance can be placed on their statements, and Richardson remarks that "they do not intentionally tell falsehoods, but they seem to say anything, regardless of truth or falsehood" (Lancet, ii., 1883). They are sometimes so far irresponsible for their actions that I have known-as with alcohol-a few instances of their being certified as insane, and placed.in an asylum, though they recovered perfectly on omission of the drug. There are marked abeyance of will and control-power, alternations of excite- 
ment and great depression, no tendency to humour or sociability, but rather to gloom, selfishness, and shunning of society; occa sionally a semi-hysterical state, especially in women. The expression of the face is careworn and old.

Circulatory System.-The heart-action is slow and regular, often weak, without bruit; but whilst the effect of the narcotic is passing off it may become irregular, with reduplication of the second sound. As a rule, the pulse is small, resistant, and slow, but sometimes it is rapid. Pyrexia is said to occur after hypodermic injection, the temperature rising to $100^{\circ}$ to $101^{\circ} \mathrm{F}$. and upwards ; Dr. Richardson considers this frequent enough to constitute a distinction from alcoholism. Autopsies of morphiomaniacs show general venous stasis-and this especially in the lesser circulation and the kidneys, more than in the nerve-centres (Burkart).

Respiratory System.-This is commonly lowered in function, the breathing becoming slower and shallower; bronchial and sibilant râles may be heard, and there is unusual secretion of tenacious bronchial mucus.

Cutaneous System.-The skin is pale, sometimes cyanotic; it becomes pigmented, hard, and otherwise altered at the site of repeated injections, where also small abscesses occur; there are occasional profuse perspirations.

Genito-Urinary System.-Micturition is less frequent than normal, the bladder being sometimes paralysed for a time; the urine is irregular in quantity, pale, neutral. Sexual perception is for a time increased, and sexual desire sometimes greatly so; but, as a rule, impotence follows.

Treatment of the Opium-Habit.-(a) In patients suffering from diabetes, malignant disease, or a markedly intermittent pulse, it is probably better to regulate the dose of morphia than to omit the drug altogether.

(b) Where morphia has been commenced for relief of pain, independent of organic disease, and has been continued for its own sake, omission of it may prove that the original malady is cured, and the drug is no longer required; if, however, the pain return, endeavour to relieve it by other means.

(c) To break the habit we may either (1) begin by a substitution of other anodynes, such as chloral or alcohol, but unless this be done with great caution it may only replace one 
habit by another; or (2) we may proceed by gradual reduction of the dose, ordering at the same time regular diet, change of scene, and suitable moclicinal adjuvants ; or (3) abruptly cut off the morphia, taking special care of the patient for a week or two whilst the craving lasts. This method is strongly advised by many authorities (Fleming, Thin, Maclagan), and in moderate or comparatively recent cases should certainly be adopted; but, in the opinion of Richardson and some others, it is not "rationally possible" in older cases, since it leads to vomiting, purging, and other severe effects. A case of miscarriage from the practice is reported (Féré, Rev. Méd., ii., 1883), and according to Burkart it may, in some conditions of the circulation, be fatal. That it can, however, often be adopted without more than temporary suffering is evidenced by Dr. Sharkey's case, previously quoted, where more than 20 grains of morphia acetate had been taken daily for years, and yet rapid recovery ensued after about three days of the symptoms of purging and collapse alluded to (loc. cit.). I have myself adopted this plan in many cases, and think it the best when it is practicable; but in certain patients, especially elderly ones, I have found its difficulties too great, and have been obliged to arrange for a gradual weakening of the solution, sometimes without the knowledge of the patient.

The symptoms that are liable to occur when leaving off the habit, besides vomiting and constant watery diarrhœea, are salivation and profuse (cold) perspiration, hyperæsthesia of the whole surface and excitement, insomnia, intense craving for the drug, and prostration in various degrees up to absolute collapse. As the patient gradually improves, a condition of tearful depression or of undue excitability may remain for some time.

The treatment of such symptoms should be conducted on general principles, including careful regulation of diet, rest, and nursing. Ammonia and capsicum, phosphoric acid, and chirata are useful nerve-stimulants; belladonna often relieves the "nervous distress," and, combined with aloes, is suitable for the after-constipation; with quinine it is recommended for the attendant amenorrhœa (Chévon, Rev. Méd., i., 1883). For the sleeplessness, lupulus and cannabis are valuable; and for the 
general condition, different forms of galvanic and hydropathic treatment.

Of cases occurring in my own practice, and illustrating varions effects, I briefly note the following:-

(1.) A lady, aged forty, who first began opium for flooding consequent on a fibroid tumour, increased the dose until she took 20 grains and upwards of solid opium twice daily for twenty-five years, without the occurrence of narcotism. Gradual breaking off the habit was effected by weakening the dose without her knowledge: the main after-symptom was depression.

(2.) A medical man, who received a head-wound in the Crimean war, and began the opium-habit, increased the dose to 40 grains daily (in two doses); this amount he took for over twenty-five years. He carried on his work, but was often irritable, and showed occasional symptoms like those of alcoholism; he had no constipation or bladder troubles, but often sexual excitement. The daily dose was continued till his death at seventy-three, from "gradual break-up."

(3.) A man, aged forty-eight, having taken nearly 16 grains of opium daily for about fourteen years, has developed occasional epileptiform attacks, and frequently misplaces words. $\mathrm{He}$ still continues the habit, and degeneration of vessels is setting in, but whether from the drug or in the natural course of events it is difficult to say.

(4.) A lady, with diabetes, daily took 16 to 18 grains of opium, and applied upwards of 16 ounces of laudanum externally to carbuncles. The dose was gradually reduced to 5 grains with advantage.

(5.) A lady, with advanced phthisis and severe sciatica, for two years injected about 8 grains of morphia acetate daily: she improved in health, but the morphia gradually lost its effect. This patient took no wine, a fact contrary to Dr. Richardson's experience, "that he has not known a case of opium-habit amongst those temperate in alcohol." I have myself met with several instances.

(6.) A lady, who began hypodermic-morphia for neurnlgia, etc., increased the dose to 5 to 8 grains daily, and still contimues its use; she has estranged all friends by the persistent spreading of untruthful reports; there is no narcotism. 
(7.) A lady, suffering from uterine growth, who took 17 to 18 grains daily of solid opium, was several times placed in an asylum as insane, but eventually, on omission of the drug, became, and continued, perfectly rational : tetanic contraction of muscles also occurred in this case.

I have known another patient certified as insane on two occasions, and detained for several weeks, he having kept the opium-eating secret from every one; when the supply was stopped, the mental symptoms disappeared.

Therapeutical Action OF OPIUM.-As remarked by Pécholier, in his monograph on this subject, the action of opium is essentially complex, and is neither limited nor uniform: if sedative to the nervous system, it is often at the same time stimulant to the circulatory, and in some persons to intellectual and muscular activity; whilst the after-depression, which is a secondary effect (like fatigue after exercise), has also to be allowed for. The great contra-indication for its use is hyperrmia, - a hard, full pulse being one guide at least to this condition; whilst if the pulse be small, soft, and compressible, opium, as a rule, can be well borne.

Insomnia.-To decide when the hypnotic power of opium can properly be utilized in disease, requires careful consideration of the carse of the insomnia. If this be dependent, e.g., upon dyspepsia, the liability of the drug to arrest the gastric secretions makes it unsuitable; and even in nervous cases such an effect is often a drawback to its use. Hence, and especially since the introduction of chloral, bromides, etc., it is certainly. less employed than formerly-less, possibly, than it deserves to be. It is most serviceable in the early stages of sleeplessness from mental distress and anxiety; and Dr. George Johnson has recorded many instances in which a grain of opium at night, continued for one or two weeks, induced sound and refreshing sleep after long periods of unrest, and apparently altered the whole course of a threatening disorder (Med. Times, ii., 1853). It acted well, not only in such cases, but also when the sleep (without it) had been heavy and unrefreshing, and full of oppressive dreams and delusions.

In the more serious conditions of similar kind, on the border- 
land of actual mental disease and melancholia, Dr. Maudsley testifies to the advantage of grain doses at night given with aloes (Practitioner, vol. ii.). In the sleeplessness of acute mania, however, although opium was largely employed by our predecessors-Dr. F. Winslow, for instance, spoke of it as " our sheet-anchor in incipient insanity with active vascular excitement" (Ranking, i., 1860), - yet modern practice takes more account of its disadvantages.

Clouston, giving 1-drachm doses of tinct. opii two or three times daily, as required, found it sometimes quiet the patient, though not so well as other drugs, and it often impaired appetite, etc. (Med.-Chir. Rev., ii., 1870); also its sedative effect soon lessened, the body-temperature was lowered, the patients lost weight, and nutrition was interfered with. Dr. Maudsley points out that opium restrains the brain working, and, like physical restraint, may injure as much as it saves (Lancet, ii., 1871); he objects to it specially in "sthenic mania," and in hysterical and epileptic forms of insanity.

Dr. Mickle reports no special curative results in twenty melancholic patients treated by moderate doses of opium, but general mitigation of distress (B. M. J., ii., 1873; Practitioner, v., 26). Some more recent observations are more favourable: thus, of twenty-seven cases of insanity recorded by Aug. Voisin as treated successfully by hypodermic-morphia, twenty were of melancholia, the others of hysterical and religious mania. The average doses were 5 to 8 centigrammes (= $\frac{3}{4}$ to $1 \frac{1}{7}$ grain) daily (Bull. de Thérap., t. 100, 1881).

In delirium tremens the malady characterized par excellence by sleeplessness, there is a similar difference in professional opinion as to the use of opium. Strongly recommended by Blake, especially during the second stage (Practical Essay on Delirium Tremens, 1840), and doubtless often serviceable in his hands, it has proved a double-edged weapon, apparently fatal in its effects in many instances, so that Stillé says he "has observed with intense regret a tendency to revive the practice of giving large doses of opiates in this disense, . . . for the mortality under them was enormous, and is remembered by him as one of the most painful features of his hospital life" (Dispensatory, 1879). Modern writings are full of cautions against the treatment 
(Lancet, i., 1878). Dr. G. Johnson, indeed, says that $\frac{1}{2}$ to 1-drachm doses of laudanum may be tried for a few doses, but should not be continued for fear of inducing cardiac paralysis; but there is some evidence that the dose above stated, and commonly given, errs on the side of timidity. Thus, in Roser's treatment, which is well vouched for, he ordered 1 grain of morphic every hour till narcotism was induced; and Nicholson, after an army experience of fifty cases, states that this has given him far better results than any other form of medication (Med. Times, i., 1876). Leared injected 5 grains in separate doses in the course of four hours with success (ib.), and Hunter $\frac{3}{4}$ to 1 grain several times (Med. Times, i., 1859). Mr. R. Park has injected 3 and 5 grains respectively of morphia acetate during attacks of delirium tremens, and with advantage, but such practice is not free from risk; he notes the tolerance of the drug in acute mania, and also that ten minutes or half an hour may elapse before symptoms of its action appear (Practitioner, i., 1880).

In the Berlin Hospital, it is said that the former morphiatreatment has replaced the more modern chloral, and with the advantage of diminishing by one-half the mortality from delirium tremens which had occurred under the latter (Med. Times, i., 1879).

The presence of organic brain disease does not, as taught by some, always contra-indicate opium : it does so if coma or congestion is a prominent symptom; but if intense pain, vomiting, and restlessness are marked, morphia, especially by the skin, has been found of the greatest advantage (Moxon, Lancet, i., 1875). It should be remembered that a sudden termination by coma may be part of the malady.

For the insomnio of specific fevers better sedatives may now be exhibited, the objection to opium being-as stated by Sir Wm. Jenner with regard, for instance, to typhoid fever-that it disturbs digestion and secretion, and may exert a fatally paralysing influence on the heart and the nerrous system (Med. Times, ii., 1879). The high authority of Dr. Grares may be quoted in favour of the occasional value of the drug in the restlessness and muttering delirium of typhus fever, and especially when given in combination with antimony; but its judicious use requires much experience. Contraction of the 
pupil is a decided contra-indication under all circumstances (Dublin Journ. Med. Sci., July, 1838).

Dr. Ringer recommends the small frequent dose when there is muttering delirium with muscular tremor and great prostration, mixing 60 minims of laudanum or 1 grain of morphia with 4 ounces of water, and giving a teaspoonful every five or ten. minutes for three or four doses; if sleep be not then induced, the medicine should be omitted for half an hour, and after that a few more doses may be given if required. This method is said to give calm sleep, the patient waking up refreshed, free from wandering, with moist skin and improved appetite and digestion.

Pain.-As a general anodyne, opium is without an equal. It will, at least temporarily, relieve pain of any kind; whether it be functional, or dependent on inflammation, or the result of wounds, or of destructive organic processes such as cancer.

The most characteristic example of the anodyne influence of opium is seen in its effect upon simple nerve-pain, such as neuralgia; or upon the pain of a severe wound, in which nerves are cut, torn, or bruised: in such cases it relieves quickly, but one point requires attention. When the pain, however severe, is felt more as a local distress, without seriously affecting the nervous system, small doses of opium will be found to act as well as, if not better than, large ones; but where the secondary general distress is great, and the patient is excited and kept on the rack by pain, the best practice is to place him in a state of tolerably deep stupor. The smaller doses of opium may, however, be effectively used as prophylactics, even in cases where the pain (then only just commencing) would otherwise be of the severest type; as, for example, in acute neuralgia. Hypodermic-morphia is now the most effective method of giving the drug in such cases.

As a local anoclyne opium is useful, though often inferior to aconite, belladonna, and some other drugs. It may be applied to the unbroken skin in the form of liniment, or of lnudauum on cotton-wool or-much better-sprinkled on the surface of a hot linseed poultice. As an application to painful ulcers it is often valuable, but sometimes irritates, as stated below. The 
best ointment for the purpose is made with 1 part of soft extract to 9 or 12 of simple ointment.

Ulcers.-The value of opium in forwarding reparative processes in certain kinds of ulcers and wounds is one of the most interesting facts in therapeutics. First in importance under this head is its action, when boldly and promptly given, in the phagedænic ulcer; doses of 1 or 2 grains every three or four hours frequently exert a surprising influence, not only in easing pain, but in arresting the destructive processes. Mr. G. Pollock, in a recent address, has well illustrated the fact.

In hospital gangrene, Holmes thinks it "of no special use;" but Agnew found it very valuable (Lancet, ii., 1879), and Pollock states that "administered internally, it arrests or modifies any tendency to ulceration or sloughing, soothes pain, and husbands the power of the patient; the irritable surface or gangrenous edge, under its influence, generally and soon becomes changed to a clean, healthy granulating surface, with the characteristic whitish margin of skin. The external application of opium appears occasionally to assist towards such beneficial results, but seldom or never is potent of itself to curb the action of ulceration" (B. M. J., i., 1884). Pollock objects to it only in "traumatic spreading gangrene," and finds it of little use in the carbuncle or gangrene of diabetes or embolism, but of the utmost value in phagedæna, whether syphilitic or not, as well as in severe and ulcerative stometitis, which it will cure independently of nitrio acid or painful caustics.

In the indolent or weak ulcer, with large flabby granulations, the internal administration of opium ( 3 or 4 grains daily), together with its external application in lotion or ointment (either alone or combined with lead, nitric or carbolic acid, etc.), often changes the character of the sore, and induces rapid healing.

In the "irritable" ulcer, the local application of opium is not so good, although, a priori, it might have been considered so. The disease may be aggravated by it, some inflammation being excited; other treatment is usually better.

Cutaneous Disorder.-In various forms opium is beneficial, e.g., in pruritus and prurigo, when it may be combined with borax or alkaline salts; and in acute ccsema, applied in lotion or powder, as well as given internally. 
An opium ointment, or morphia solution, relieves the pain of a blister, and, as remarked by Purdon, that of "gutta rosacea" (Ranking, i., 1860).

Spasm.-In various forms of spasmodic disorder, opium has much power. In localized spasm of muscle-for instance, in a deep-seated part-hypodermic-morphia is our most effective remedy; and when for any reason this is not advisable, we may relieve by fomentations or poultices, with laudanum added to them. In cases of intestinal colic, full doses of the drug may be ordered internally, castor oil or other purgative being first given if required, as it is, e.g., in the colic due to lead or irritant foods.

A specially important modern use of opium is in acute obstruction of the bovels, a very serious condition, which was formerly often treated by purgatives with disastrous results; for, whether dependent on spasm or paralysis of a portion of intestine, or accidental snaring by a band of lymph, the effect of increased peristalsis is to contract the gut from above, and commonly increase the malady, besides possibly inducing adhesive inflammation. Dr. Brinton observing this, essayed a different method of treatment, viz., by doses of opium sufficient to abolish all spasmodic contraction; and he proved, clinically, that about $\frac{1}{2}$ grain given every four hours for two or three days, or longer, will, in suitable cases, not only arrest the most dangerous symptoms, such as stercoraceous vomiting, but result in painless evacuation of quantities of semi-fluid dark-coloured fæces (Treatise on Intestinal Obstruction) ( $v$. Belladonna).

Even cases of obstruction from fiecal impaction, which are often efficiently treated by purgatives, will also do well at times under opiates, the hardened masses being gradually passed along the yielding intestine: this treatment is, however, best adapted where the gut has been injured by inflammation or congestion. Many remarkable recoveries from such conditions are now on record: in one, for instance, the obstruction lasted for thirty-nine days, and yet recovery took place under the daily injection of from $\frac{1}{2}$ to 1 grain of morphia (Lambart, Lancet, i., 1880) ; and Costello refers to a number of similar cases successfully treated by 12 to 20 grains of 
opium daily, which produced, apparently, partial paralysis of the intestine, and resulted in free purgation after thirty and even forty days of treatment (Lancet, ii., 1880). Even in the obstruction of strangulated hernia, hypodermic-morphia has been advocated (Jaubert, Rev. Méd., 1883); but I cannot agree with this teaching, since the narcotism masks serious, probably fatal, symptoms, which can only be remedied by the necessary operation.

With regard to the treatment of the grave form of localized spasm termed angina pectoris, opium stands now in a different position from that which it formerly occupied. The high authority of Dr. Walshe recommended it as the best remedy in the emergencies of this frightful disease, and hypodermicmorphia is often a valuable resource; but recent observation has shown that more speedy relief may be obtained by nitrite of amyl, nitro-glycerine, ether, brandy, and other diffusible stimulants.

Duchard has especially shown the value of morphia in the distress connected with aortic stenosis (Record, 1878), and large doses ( 3 to 5 grains) of morphia have been successfully used in severe "heart disease" (Practitioner, 1880); but in cases of mitral reflux with venous congestion, dyspnoca, ascites, etc., opium is dangerous. Two instructive cases of this kind, in which death followed a dose of opium, are recorded by Trousseau (Med. Times, i., 1859).

In spasmodic asthma, opium will sometimes do good, especially in the form of hypodermic-morphia. In suitable cases, i.e., when the nerve element is the principal one, and catarrh absent or moderate, this treatment proves quickly efficacious, and possible ill effects of the sedative are commonly obviated by combining it with a fractional dose ( $\frac{1}{120}$ grain) of atropia. In one case, described as "exophthalmic goître," with urgent orthopnoa, œdema, palpitation, etc., hypodermic-morphia seems to have been very serviceable. As a rule, in my experience, such subjects are too "nervous" to bear the drug well, though large doses will doubtless calm excited heart-action.

In the spasm of tetamus, intramuscular injections of the drug have proved useful, especially in traumatic cases (Demarquay, Med. Times, i., 1872); and opium is said to be successfully 
prescribed for tetanus in the East (Med. Times, i., 1864),it has certainly been largely given. There seemed the more reason to hope for good results from its use, because in this disease the system shows an extraordinary tolerance of it, $-20 \mathrm{or}^{\circ}$ 30 grains having been taken, with the simple result of relieving: spasm to a greater or less extent, without producing decided stupor. At present, however, I believe that no high authority regards opium as a remedy of considerable curative power in tetanus; it would seem rather that the cases which have recovered under it belonged to the class, not inconsiderable in number, which recover spontaneously. Still it proves of the utmost advantage in cases of severe and lacerated wounds, and, according to the experience of American surgeons during the Civil War, "often seemed to ward off tetanus, and to save amputation of a limb" (Lancet, ii., 1866).

Morphia has been of use in strychnia-poisoning (Med. Times, ii., 1868); and Hunter proved it of some advantage in chorea (Med. Times, i., 1859).

Inflammation.-The value of opium in inflammation was formerly one of the most disputed topics in therapeutical literature; but the observation of Brochet (1828) probably represents what is now the general opinion of experienced practitioners, viz., that the drug is far more useful in inflammations of membranous than of parenchymatous structures. In many cases where the peritoneum or pleura or the gastrointestinal mucous membrane is inflamed it is a valuable remedy ; and diffuse inflammation of the subcutaneous tissue, especially when the periosteum is involved, is well controlled by full and repeated doses.

Stillé compares the good effect of the drug in inflammation to that of rest upon an injured limb. "It removes or holds in check causes of irritation, and thus allows the recuperative powers of the inflamed part to act without hindrance. It is in this manner, probably, that must be explained the unequal, and indeed opposite, effects of opium in the inflammation of different organs." As some guide to its judicious use, we may note the teaching of Sir T. Watson, that special caution is required in prescribing it for such inflammatory disorders as tend to cause death by coma or apnooa, or whenever there is 
duskiness of the lips or face, showing imperfect aëration of blood. "On the other hand, it is, cceteris paribus, in cases where the tendency is towards death by asthenir that opium as a remedy for inflammation is most serviceable; thus, it has a capital effect often after depletory measures in cases of peritonitis and of enteritis." Dr. Stokes also notes asthenia during inflammatory disorders as indicating opium. "In cases of recent inflammation of serous and mucous membranes, where depletion is inadmissible, and the system is in a state of collapse, opium has a powerful effect in controlling the disease. It may be given with benefit and safety; it raises the powers of life, and its poisonous effects are rarely observed."

In puerperal peritonitis it has doubtless proved beneficial, but should not be employed as a matter of routine, since other remedies, e.g., aconite, are decidedly better suited for individual cases.

In inflammation affecting the brain or its membranes this remedy is commonly inadmissible; but Gross and others have recommended it as a sedative, as lessening arterial pulsation, and on the hypothesis that any congestion caused by it would be passive and not injurious.

In rheumatic inflammation affecting the joints (wheumatic fever), special value may be assigned to opium, which is commonly used more or less in every ease to relieve pain and procure sleep; some, however, advocate it as the chief medicinal treatment, giving 1 grain every three or four hours during the acute stage (Cazenave, Corrigan, Fuller). There seems to be remarkable tolerance of the remedy. Dupuytren's method of giving it by rectal injections of about 10 minims of laudanum two or three times daily has the advantage of saving gastric irritation (Ranking, i., 1860).

Dr. Handfield Jones has reported two good illustrations of the value of opium in arthritis when the salicylates and other remedies had been tried without much relief (B. M. J., ii., 1871).

In Acute Gout, hypodermic-morphia is sometimes most valuable, but, as a rule, opium is not advisable, for fear of checking elimination. Its use in any case should be preceded by purgation; and in nervous, weakly subjects it is well combined with camphor (Copland). 
Catarrh.-The effect of opium in allaying irritation and hyper-secretion of mucous membranes is often remarkable, and the commonest example is found in catarrhal irritation of the nares, the larynx, and the bronchial tubes. Dr. Thudichum finds special advantage from smoking the drug (Amer. Journ., July, 1884).

In ordinary febrile catarrh its good effect is greatly heightened by combination with ipecacuanha, e.g., in Dover's powder, 3 to 10 grains of which will act favourably as a sudorific.

It is in the enrly stages of these affections that opium is specially applicable; at more advanced periods it is often hurtful. This is particularly the case in fully developed bronchitis; and when secretion is copious, and expulsory power feeble, it is possible to do even fatal mischief by opium. In general it may be laid down as a maxim, that moderate doses only are useful in the irritative affections of the air-passages.

Dr. Charles Hare has some practical remarks on the value of $\frac{1}{40}$ to $\frac{1}{20}$ grain of morphia (in mucilage) for "needless coughing" (B. M. J., ii., 1883). Rossbach recommends hydrochlorate of morphia and of apomorphia, of each 3 centigrammes ( $\frac{3}{7}$ grain), with dilute hydrochloric acid, and water 150 grammes (nearly 5 fluid ounces), $\frac{1}{2}$ ounce every two or four hours, "for lessening frequency of cough and increasing fluidity of sputa" (Berl. Klin. Woch., No. 19, 1882).

Clinical experience is corroborated by the conclusions from Filehne's experiments already referred to: thus, if the dyspncea depend on deficient blood in the medulla, while no pulmonary obstruction exists, morphia will do good; but if it depend on deficient arterialization of the blood, then its use requires the greatest caution.

In catarrhal irritation of the gastric mucous membrane, opium sometimes proves valuable. In gastritis (acute or chronic) the tongue may, as Dr. Wilson Fox says, to some extent be taken as an indication for opium, i.e., it is beneficial when the tongue is red and irritable, and the papillse pronounced, but should not be given when the tongue is large, pale, and flabby ; its constipating effects may more than undo temporary advantage. However, in chronic gastric cotarrh, attended with pain and pyrosis, small doses (perhaps combined 
with the mineral acids) are sometimes very useful; and constipation may be met by mild aperient waters, such as Pullna or Friedrichshall, if necessary.

In the vomiting of pregnancy, opium is effective, especially in the form of hypodermic-morphia; in one case, where this was of the greatest service, a condition of much excitement supervened on its cessation at four and a half months, but the romiting did not recur (Record, 1878).

In catarrhal affections of the intestines, especially in autumnal diarrhcea, and in English cholera (early stage), the good effects of opium, combined or not, according to circumstances, either with mineral acids or with chalk and aromatic confection, are well known. In the early stages of Asiatic cholera, notwithstanding all that has been said against it, practitioners who have had the largest experience still declare that opium is one of the most valuable agents; it is given on the principle of lessening: shock to the nervous system, as well as of controlling discharges, but it is useless in the stage of collapse and in that of reaction.

Mr. Hardman and others have recorded rapid and permanent benefit, in severe cases of choleraic diarrhœa, from hypodermicmorphia (Lancet, ii., 1880).

In Sea-sickness, Dr. G. Johnson recommends opium; and Mr. Vincent, after much practical experience, finds hypodermic-morphia more satisfactory than any other remedy. A common and useful prescription which affords considerable relief is a rectal injection of 20 to 30 minims of laudanum.

Urinary Disorders.-For inflamed catarhal conditions affecting the kidneys and urinary passages, and especially for nephralgia and vesical pain connected with calculus, and in acute cystitis, opium is valuable, and is often well applied in the form of suppository. Mr. Liston combined it with henbane (opium 2 grains, henbane 10 grains). In renal colic, morphia hypodermically in doses up to $\frac{1}{2}$ grain is best.

In spasmodic urethral stricture and retention a full dose of opium after a hot bath will often relieve. In gonorrhœal and spermatic discharges an opiate injection is good; and opium given night and morning is valuable in the treatment of orchitis (Rouse, St. George's Hosp. Rep., 1870).

The tincture in 1-minim doses, gradually increased if neces- 
sary, acts beneficially in prolapsus ani in children over four years of age.

Uterine Disorder.--In cancer of the uterus, as of other parts, opium in various forms gives more relief than any single remedy. In "irritable uterus" and ovarian pain it is also valuable, but should not be pressed to such an amount as to deaden the pain without taking steps to relieve it by other treatment.

Severe dysmenorrhoa may need hypodermic-morphia, which will always relieve for the time. In acute suppression of the periods from mental shock and excitement, opium is valuable, as it is in threatened abortion from the same cause. During and after childbirth it is often used as a sustaining and quieting remedy.

In Convulsion, especially when dependent on cerebral congestion, or whenever the kidneys are seriously affected, it has been usual to avoid opium ( $v$ p. 89 ) ; but, recently, evidence has been offered of its value in albuminuric cases, in the form of morphia injected under the skin. Thus, Loomis and Austin Flint record cases that did well under it (Lancet, ii., 1875), and its diaphoretic action has been found useful (Record, 1880). A case of chronic albuminuria with convulsion, and one of granular kidney with insomnia and orthopnœa, are reported as relieved by it (Practitioner, vi., 1871).

In Epilepsy, many years ago, opium was commended by high authority, but is now seldom if ever prescribed, and ordinary hypnotic doses are more likely to do harm than good. In some few cases, however, of " nocturnal epilepsy" I have known doses of 2 minims of the tincture, given three times daily, beneficial in warding off the attacks.

Puerperal Convulsions.-The high authority of Scanzoni may be cited in favour of the beneficial influence of morphia in this form of convulsion, although it is probable that in most cases better results may be obtained by the employment of chloroform, bromide of potassium, aconite, or hydrate of chloral; yet in some instances morphia has succeeded when these have failed (Rev. Méd., ii., 1883). Opium may be useful in those restless, nervous, hysterical subjects who suffer occasionally during pregnancy with twitchings, startings, dyspnoea, intolerance of light and noise, and disturbed sleep; 
also in puerperal convulsion dependent on anæmia from profuse hremorrhage, with pallor and coldness of face and of the general surface, small quick or imperceptible pulse, restlessness, or even delirium; it would be likely to do harm to plethoric subjects.

Diabetes. - The utility of opium in diabetes is still under discussion. Striking instances are on record of the power of full doses to reduce the amount of sugar-elimination and of diuresis, and to improve the general bodily condition; but, on the other hand, the drug has often been tried without the least good effect (Thompson, Clin. Soc. Trans., vol. iv.). The doses given have been exceptionally large, McGregor (in 1845) ordering as much as 90 grains daily. Dr. Pavy, in one of his early cases illustrating the value of the drug, did not succeed in arresting the sugar-excretion with less than 10 grains daily, and in another, 7 grains thrice daily were required (Med. Times, i., 1869; ; 10 narcosis was produced. In a recent case under Dr. Bastian the dose of extract was gradually increased to 12 grains daily with benefit, and then gradually diminished (B. M. J., i., 1882). Codeia has, however, been found still more advantageous (v. p. 114).

Therapeutical Action of MORPhiA.-Certain differences between the remedial action of morphia and of opium depend partly on the presence of other ingredients in the latter, and partly on the easier administration of the former. Morphia is more applicable to the relief of pain, and less potent in the production of sleep, than opium; it is less useful, also, than the latter in checking hyper-secretion from mucous membranes. On the other hand, it can be more effectively employed, especially in small doses, for the relief of spasmodic affectionsfor instance, whooping-cough, in which the treatment adrocated by Dr. Edward Smith with doses of $\frac{1}{24}$ grain three or four times daily, is often extremely effective; and spasmodic asthma, in which small doses of morphia (especially if hypodermically injected) afford a relief which would be sought in vain from opium.

Practically, the most important advantages of morphia over opium are evidenced in the modern practice of hypodermic 
injection. First adopted in 1857, by Dr. Alexander Wood, as a local remedy for pain, it has since developed, in the hands of Hunter, Eulenburg, Béhier, Lewin, and others, into a method of unexpected power, and one adapted for the administration of many other substances. No drug, however, so fully illustrates the value of hypodermic injection, or the dangers that attend indiscriminate resort to it as morphia. Its most striking effects are seen in the rapid relief of pain, the severest neuralgias being often relieved in a few minutes by the injection of $\frac{1}{16}$ or $\frac{1}{8}$ to $\frac{1}{4}$ grain: nor is the alleviation always merely temporary; in recent cases it is possible, by injecting once or twice a day (according as the attacks recur), to completely efface the malady. In my experience, the best results are obtained in neuralgias of the trunk and limbs, rather than in facial neuralgias; in the latter, I have more frequently known unpleasant consequences, such as syncope or vomiting. It is of great importance not to use too large doses, both because of exciting such effects, and also for fear of inducing the morphia-habit (v. p. 93). But, as Dr. Allchin has well observed, it is necessary to bear in mind that pain once established for any long period, induces what he terms a "habit of pain," which it is essential to break, and for which purpose a longer use of the sedative may be necessary, even for a short time beyond the actual cessation of the pain itself.

The golden rule is to employ just the amount sufficient to arrest pain without producing either narcotism or stupor, or even contraction of pupil, headache, or constipation. In the severe pain of serous inflammations, especially pleurisy, but even peritonitis, all the relief which formerly was sought from the lancet or leeches may usually be afforded by the hypodermic injection of $\frac{1}{16}$ to $\frac{1}{4}$ grain of acetate of morphia: one or two of these daily during the early stages will save the patient considerable suffering.

It has been a question whether, in using such injections for relief of pain, they should be given near the affected part, or in any convenient indifferent place-Wood, Eulenburg;. Béhier, Lawson, and others, advocating the former; Hunter, Anstie, the Committee of the Med.-Chir. Soc., etc., adopting the latter plan, which is now genernlly recognized as effective in most cases. 
Possibly, when the pain is traumatic, as from a fractured rib, or localized in one nerve, as in sciatica, an injection near the seat of pain may relieve best. In most cases of febrile disease (fevers, acute inflammation, pyæmia, etc.), if it be necessary to use opium at all, this is, as a rule, the best method of giving it, since it produces less disturbing effect on the stomach; and in acute diseases it is of the greatest importance not to interfere with digestive power. The hypnotic effect of such injections is not materially different from that of morphia swallowed (allowing for difference in dose and rapidity of action).

The question of the use of morphia injection as an adjunct to chloroform-inhalation deserves special attention. On the one hand, it is claimed that this proceeding leads to the production of complete anæsthesia with less chloroform, less primary excitement, after-vomiting, or risk of syncope, and more aftersleep and comfort (especially if atropia be combined), (Lancet, i., 1865); and this statement has lately been reinforced by a report of many successful cases by Dr. Crombie (Practitioner, ii., 1880). Cl. Bernard, and also Brown-Séquard, whilst supporting the practice, strongly advise the combination with atropia in this as in other cases, since a greater therapeutical power is developed, and there is less risk of cardiac or respiratory paralysis. (Three minims of the injectio morphiæe et atropiæ hypodermica contain $\frac{1}{2}$ grain of acetate of morphia and $\frac{1}{60}$ grain of sulphate of atropia: dose, 1 to 3 minims.) There are some dangers, however, such as the production of coma (Anstie), and some contra-indications, especially shock (stupeur générale) with low body-temperature (Poncet, Compt. Rend. de la Soc. de Biologie, 1883); and serious results have followed often enough to prevent such injections becoming a matter of routine. Jacob has especially shown their risk when used before ether inhalations, possibly because of their preventing due clearance of secretion poured into the bronchi (B. M. J., i., 1881). That dangerous and even fatal consequences may follow from comparatively small doses of morphia injected has been already stated to $(v . p .89)$. It is important to avoid injecting it directly into a vein; otherwise, besides the general symptoms, local pain, numbness, and swelling are liable to occur. 
THERAPEUTICAL Action OF CoDeia.-Insomnia.As a hypnotic, codeia has received commendation from trustworthy authorities, though Garrod and others depreciate its powers. Berthé and Aran found it procure sleep in cases of tormenting cough, as well as in the pain of gout and of cancer. Krebel praised it, especially in cases of nervous insommia, and that dependent on rheumatic pains, and states that the sleep it causes is light and refreshing, not followed by the disagreeable after-effects of opiates; to sensitive subjects he gave only $\frac{1}{15}$ to $\frac{1}{10}$ grain; 1 grain was the maximum. Reissner employed codeia subcutancously, and found it analogous to morphia both in beneficial and inconvenient effects. For this use a phosphate of codeia is best, since it is soluble in four parts of water and contains 70 per cent. of the alkaloid; this is said to act like morphia, but with less tendency to cause toxic symptoms (Record, October, 1883).

In France, codeia is largely employed as a hypnotic, and Claude Bernard placed it (along with morphia and narceia) in the soporific group of opium-alkaloids.

The nitrute of codeia, which was recommended by Magendie because of its solubility, must on that account be given in somewhat smaller doses than the alkaloid itself.

Pain.-As a remedy for pain, codeia, on the whole, is inferior to morphia. Erlenmeyer employed it subcutaneously for neuralgia without effect; and according to English experience, also, it is inefficient. Yet, in abdominal neuralgias and in rheumatic sciatica it has been strongly recommended by Berthé, Aran, Krebel, and others.

In Irritation and Hyper-secretion of the Bronchial Mucous Membrane it may be valuable when morphia or other opiates are not well borne, and for this purpose it was. strongly recommended by Vigla and Aran; Dr. Anstie emplnyed it with satisfactory results in such cases in the dose of $\frac{1}{6}$ grain every three to six hours. Dr. Saundby has written to recommend the alkaloid in phthisis, and in the cough and catarrh of gouty subjects and others intolerant of opium (B. M. J., i., 1879); he gives 1-grain doses, but this is larger than I find necessary.

In Diabetes it has now become an accepted remedy. 
Dr. Pavy has published a series of cases in which the sugar disappeared from the urine under opium, morphia, or codeia; this last was given in 10-grain doses thrice daily, and had the advantage of not producing narcosis like the others sometimes did (Guy's Hosp. Rep., v. and xv.).

Dr. Cavafy reports a case that did well with 15 grains thrice daily (St. George's Hosp. Rep.), but this amount seems dangerously large. I have already referred to Dr. Myrtle's case of toxic symptoms after 4 grains.

Dr. Ord had good results with 1 grain of the sulphate twice daily, after diet alone had failed; the patient gained $7 \mathrm{lbs}$. weight in one week. Dr. Allchin also relates a favourable experience, and concludes that codeia has a far more beneficial effect in the glycosuria of old and middle-aged persons, than it has in the diabetes of the younger, for whom he prefers opium. Dr. Shingleton Smith has, however, reported three cases under thirty years of age, all of which " exhibited marked improvement whilst taking codeia," which improvement ceased when the drug was withheld, and was renewed on its repetition. Morphia had a good effect in two of the cases, but the improvement was much less marked with it than with the other alkaloid (B. M. J., i., 1882).

Dr. Mackey observed excellent results from it in two cases, quite as much in relieving nervous restlessness and producing sleep, as in reducing the amount of sugar; in one elderly subject it has been continued in $\frac{1}{2}$ to 1 -grain doses almost every night for two years, without losing its sedative effect. Dr. Bradbury reports similar results in a patient aged sixty-nine; the amount of urine passed was lessened one-half, though the proportion of sugar was not much altered (B. M. J., i., 1883).

The drug has so much advanced in professional favour in Germany that at present the supply can scarcely equal the demand.

THERAPEUTICAL ACTION OF NARCEIA.-The various views of authorities range from absolute denial of any medicinal virtues in narceia, to the statement that it is the best promoter of sleep and soother of pain that exists; there can be little doubt that each of these extreme views is incorrect. 
The substantial agreement of the best French authorities (Bernard, Béhier, Delpech, and Bouchardat) with two of the best German experimenters (Erlenmeyer and Eulenburg) negatives the idea that narceia is nearly an inert substance: it is plainly a hypnotic of considerable power (though it fails to affect some individuals), and is comparatively free from the tendency of opiates to produce after-headache, nausea, skin-imitation, or convulsion (Barnay). Sometimes, however, difficulty in micturition has followed its use. It is a good remedy in irritative cough, especially in that of phthisis, for which Béhier has particularly recommended it (see also Bouchut, Bull. de Thérap., i., 1865).

Laborde supports this recommendation, and extends it to pertussis, Barnay to chronic bronchitis (Record, 1877). Béhier, and more recently Petrini, at the Hôtel Dieu, have reported the hydrochlorate valuable in neuralgic pain, sciatica, etc., especially when used in subcutaneous injection. This, however, requires 1 or 2 minims or more of acid for solution, which then is not complete, though it may be made so by placing in warm water. In a case of violent intercostal neuralgia it proved locally very painful, and did not relieve the nerve-pain for longer than an hour, whilst morphia, without giving local pain, relieved for many hours.

Narceia itself excites much local irritation, probably because of its insolubility; and Husemann suggests for injection the more soluble lactate. Rabuteau states that from 1 to 3 grains by the mouth produced sleep in invalids; and a vaginal injection of $1 \frac{1}{2}$ ounce of a 5 per cent. solution of the hydrochlorate relieved the pain of cancer of the cervix. To ne it seems sudorific, as well as hypnotic and anodyne.

Rabuteau, in some recent experiments, finds that 5 to 10 centigrammes of this alkaloid given by the skin distinctly aid the anæsthetic effect of chloroform, which also is rendered less nauseating. Narcotin and codeia did not act in the same manner, but papaverin and thebaia slightly so (Compt. Rend. de la Soc. de Biol., 1883).

Therapeutical action of Papaverin.-This alkaloid has been the subject of conflicting statements; but the 
clinical researches of Baxt and of Leidesdorf have probably convinced impartial observers that genuine papaverin has real powers. Baxt and Sander reckon it equal in strength to morphia, but this seems doubtful; on the other hand, the trials of Hoffmann and of Reissner, which gave negative results, must surely have been made with bad specimens of the alkaloid. It seems probable that for cases of insomnia with nervous excitement, papaverin is an effective hypnotic in doses of about a grain. The phosphate is not adapted for subcutaneous injection, on account of its irritating properties, but the hydrochlorate was successfully employed in this way by Leidesdorf.

Therapeutical Action of Thebaia. - The experiments of Reissner, Ozanam, Schroff, and Eulenburg seem to prove that this, which is a powerful tetanizing poison in animals, possesses neither hypnotic, anodyne, nor astringent qualities, nor, indeed, any medicinal virtues. Rabuteau, however, and also Arzeronny, have more recently published observations which tend to show that it is sometimes more analgesic than morphia itself, $\frac{1}{8}$ grain (by injection) of thebaia having relieved pain more quickly, and for a longer time, than the same quantity of the former allaloid (Gaz. Hebdom., 1872).

\section{Therapeutical ACtion of MECONIN, OR OPI-} ANYL.-John Harley's experiments on animals seemed to indicate that opianyl would prove a valuable tranquillizing hypnotic, without unpleasant after-effects, but I know of no confirmation of his observations.

I must conclude these remarks on opium and its alkaloids with a few cautions. The most important of these refers to the extreme susceptibility of young children (at any rate during the first two or three years of life) to the poisonous influence of these drugs. Instances are not uncommon in which a single drop of laudanum, given to a child three or four months old, has produced profound stupor lasting for many hours; and in one case within my knowledge $\frac{1}{20}$ grain of acetate of morphia produced coma and convulsions in an infant of seven months.

Another eaution must be given as to the possible formation of the opium-habit. It is a familiar fact that the continued 
use of this medicine in any form is apt to induce, first, a tolerance of, and then a violent craving for it. It is easier to point out this danger than to say how it can be avoided, since many patients, suffering from severe and incurable disease, must and will have opium daily; but we may postpone the increase in the dose required to relieve pain, by commencing with the smallest quantities, and never sanctioning a large advance. As a rule, the hypodermic injection secures the greatest economy in dose, but it is often impossible to trust the discretion of the patient's attendants in this matter, whilst it is equally impossible for the medical man always to administer it himself. Unfortunately, this application of the remedy has rapidly passed into the hands of many who persist in being their own doctors, and great mischief, and many serious and even fatal accidents, have followed. These are more fully referred to under "Morphia-Habit;" and it need only be said further, that whenever this medicine is ordered it should be done with the same care and scruple as alcohol, and with an equal consideration of the possible consequences.

PREPARATIONS AND DOSE.-The following preparations are officinal:- $(A)$ Opium.-Pulvis : dose, $\frac{1}{10}$ to $3 \mathrm{gr}$. or more. Confectio (strength, 1 in 40 ): dose, 5 to $20 \mathrm{gr}$. Emplastmon: 1 in 10. Enemu: $\frac{1}{2} \mathrm{dr}$. of laudanum with $2 \mathrm{oz}$. of mucilage of starch. Extractum (about double the strength of good raw opium) : dose, $\frac{1}{2}$ to $2 \mathrm{gr}$. Extractum liquidum (1 gr. of extract in 22 min.): dose, 10 to $40 \mathrm{~min}$. or more. Linimentum (equal parts of laudanum and soap liniment).

Pilula scuponis composita (1 gr. powdered opium in nearly $6 \mathrm{gr.}$ ) : dose, 3 to $5 \mathrm{gr}$. Pulvis opii compositus (1 part powdered opium in 10): dose, 2 to $5 \mathrm{gr}$. Tincture (1 gr. powdered opium in about $14 \frac{2}{3} \mathrm{~min}$.): dosè, 5 to $40 \mathrm{~min}$. or more. Tineture ammoninta (1 gr. powdered opium in $96 \mathrm{~min}$.$) :$ dnse, $\frac{1}{2}$ to $1 \mathrm{dr}$. Trochisci (each lozenge contains $\frac{1}{10} \mathrm{gr}$. of extract of opium): dose, 1 to 6 lozenges. Vinum (22 $\mathrm{min}$. equal $1 \mathrm{gr}$. extrnct of opium) : dose, 10 to $40 \mathrm{~min}$.

Tincture comphorre composite (paregoric) (1 gr. in $\frac{1}{2}$ oz.) :

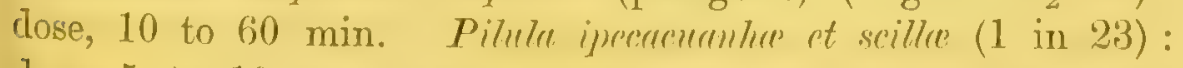
dose, 5 to $10 \mathrm{gr}$. Pilulu phumbi cum opio (a 4-gr. pill con- 
tains $3 \mathrm{gr}$. of lead acetate and $\frac{1}{2} \mathrm{gr}$. pulvis opii): dose, 3 to $5 \mathrm{gr}$. Pulvis cretre aromaticus cum opio (1 in 40): dose, 10 to 40 gr. Pulis ipecucunhice compositus (1 in 10): dose, 5 to $15 \mathrm{gr}$. Pulvis lino compositus (1 in 20): dose, 5 to $20 \mathrm{gr}$. Suppositoria plumbi composita (1 gr. of opium in each suppository).

(B) Preparations of the Alkaloids.-Liquor morphice acetatis ( $\frac{1}{2} \mathrm{gr}$. in $1 \mathrm{fl}$. dr.): dose, 10 to $60 \mathrm{~min}$. Injectio morphice hypodermica (1 gr. of acetate in 12 min.): dose (by the skin), 1 to $6 \mathrm{~min}$. Liquor morphice hydrochloratis (1 gr. in $123 \mathrm{~min}$.$) : dose, 10$ to $60 \mathrm{~min}$. Suppositoric morphice ( $\frac{1}{2} \mathrm{gr}$. of morphia hydrochlorate in each). Suppositoria morphice cum sapone (same strength). Trochisci morphice (each contains $\frac{1}{36}$ gr. of morphia hydrochlorate). Trochisci morphice et ipecucuanhe (each contains $\frac{1}{36} \mathrm{gr}$. of morphia hydrochlorate and $\frac{1}{12} \mathrm{gr}$. of ipecacuanha): dose, of either lozenge, 1 to 6 . Codcia: dose, $\frac{1}{4}$ to $2 \mathrm{gr}$. Codeice Sulphas : dose, $\frac{1}{4}$ to $2 \mathrm{gr}$., in pill, lozenge, or syrup. Codeice Phosphas (for hypodermic use, solubility 1 in 4).

Narceia: dose, $\frac{1}{2}$ to $1 \mathrm{gr}$. or more in pill; of the hydrochlorate, $\frac{1}{4} \mathrm{gr}$. by the skin to commence with.

The above directions for dosage are as full and precise as I can well make them, but they must be taken with qualifications according to circumstances; and especially it is needful to remember the cautions given above respecting the extreme susceptibility to the drug of infants, young children, and those affected with disease of the kidneys.

Besidesthe officinal preparations, there are some which require mention as being commonly ordered. Aqua opli, distilled from opium, is a good deal employed in Germany, though not much in England, as a lotion for the eyes. Unguentum opii (1 part extract of opium, 9 simple ointment). Morphire olects, usually made 5 per cent.-i.e., 5 gr. morphia to 95 of oleic acid: 5 to $10 \mathrm{~min}$. applied to the thighs or abdomen produce sleep in infants: Solution of bi-meconate of morphic (Squire), same strength and dose as laudanum. For hypodermic injection it is concentrated to one-twentieth its volume, when $3 \mathrm{~min}$. are equal in effect to $\frac{1}{2} \mathrm{gr}$. of acetate of morphia. Liquor opii secktivus (Battley) (50 per cent. stronger than laudanum, and generally acting better): dose, 5 to 20 min. Jeremie's 
solution of opium (Savory and Moore) is almost exactly like Battley's solution. Sydenham's landanum (imitated by the tinctura opii crocatc of the Prussian Pharmacopœia) is greatly used abroad, and frequent references to it will be found in French authors : it is very strong-1 gr. of opium in $8 \mathrm{~min}$. Nepenthe (Ferris, Bristol) is about the same strength as laudanum, dose the same; it resembles liquor opii sedativus in the mildness of its action. A glycerole of this is prepared for hypodermic use (1 gr. in 16 min.). "Opium denarcotisatum," recently admitted into the U.S. Pharmacopœia, is prepared by repeatedly washing. powdered opium with ether, drying, and bringing to a definite weight with sugar of milk; this process frees the opium from nar'cotin, fat, resin, etc. A similar result is obtained by Messrs. T. \& H. Smith by frequent washings with distilled water, filtering and neutralizing, and then adding proof spirit in such a manner as to obtain a paler liquid than laudanum, but one free from repulsive taste and smell, and remaining clear when mixed with water.

\section{APOMORPHIA.}

Preparation.-In 1869, Matthieson and Wright discovered that on heating morphia for two or three hours in a closed tube, with excess of hydrochloric acid, water and a new substance, which they termed apomorphia, were formed; thus, $\mathrm{C}_{17} \mathrm{H}_{19} \mathrm{NO}_{3}$ (morphia) became $\mathrm{H}_{2} \mathrm{O}+\mathrm{C}_{17} \mathrm{H}_{17} \mathrm{NO}_{2}$ (apomorphia). When this is precipitated with sodic bicarbonate, dissolved in chloroform or ether, and treated with strong hydrochloric acid, chloride of apomorphia deposits on the sides of the vessel, and by neutralizing with soda the pure drug may be obtained as an amorphous white powder, which changes to green on contact with air and moisture, and forms green-coloured solutions with water and alcohol, but red and violet with ether and chloroform. The chloride occurs in small whitish acicular crystals which also turn green on exposure: soluble in 7 parts of water, insoluble in ether and in chloroform. Auric chloride precipitates it purple, changing to browu when boiled.

Apocodeia has been obtained also from codeia. 
ABSORPTION AND ELIMINATION are rapid, and occur by all tissues and excretions.

Physiological Action.-Digestive System.-The best-known effect of this drug is the production of romiting, which follows its administration either by the mouth or the skin, and usually quickly-i.e., in less than five minutesthough sometimes not for twenty-five minutes.

One-fifth of a grain of the chloride taken by a healthy man caused within twenty minutes dizziness and depression, an uncomfortable sensation in the head, nausea, pallor, and salivation; in twenty-five minutes after the dose he vomited freely twice, then felt relieved, drank some wine, and in half an hour regained his usual condition. Subcutaneous injection of $\frac{1}{10}$ gr. of the chloride in a healthy adult produced similar sensations and free vomiting in ten minutes (Gee, Barth. Hosp. Rep., vol. v., 1869). Subsequently, Dr. Hensley induced vomiting in a drunken man in three minutes by injecting $\frac{1}{5}$ grain, and in a drunken woman also in three minutes by injecting $\frac{1}{10}$ grain, and in another drunken man in six minutes by $\frac{1}{10}$ grain. As a rule, the nausea produced is little, and the emptying of the stomach complete, but the vomiting may recur two or three times: diarrhoea is not unusual.

Circulatory System.-It is important to note that, in some instances, the emetic action has been accompanied or followed by profound depression and dangerous symptoms of cardiac failure, although experimental observations on animals showed no effect upon blood-pressure, and even a rise of pulse during emesis (Siebert and Moerz, Schmidt's Jahrb., Bd. clv. to clvii.). Reichert found it cause first, irregularity and quickening of pulse; and after vomiting, slowness of circulation, and lowering of temperature (Phil. Med. Times, i., 1880). The effects on M. Pécholier illustrate its possible disadvantages: being weakened by illness, he injected, to relieve pain, etc., 13 milligrammes of apomorphia (= about $\frac{1}{6}$ grain) ; two minutes afterwards he had painful retching without vomiting, and was intensely prostrate; breathing stopped, then recurred irregularly, "leaving a state of inexpressible agony." His collengues thought it well to inject a second dose, which induced some 
vomiting, and then collapse, lasting for thirty minutes, with stertorous respiration, livid face, and feeble irregular pulse. Sinapisms and subcutaneous ether revived him in a few hours. M. Pécholier asks, "What is to be thought of a medicine that in such a dose can nearly kill?" and notes that the proximity of the vomitive and respiratory centres in the bulb explain the symptoms of asphyxia (Ann. d'Hygiène, Août, 1882).

As a rule, apomorphia causes, at first, increased rapidity of respiration, then a lowered rate, and finally arrest; it is presumed to "first excite, and afterwards exhaust, the irritability of the respiratory centre," since the same results occur when the vagi are divided (Bartholow). The vomitive action appears to be less in conditions of impeded hrmatosis, and also when narcosis is profound.

Nervous System.-Locally applied, apomorphia interferes with tissue-change; given internally, it first stimulates and then depresses the cerebral functions and motor nerves, and quickly paralyses sensory nerves. The convulsions produced seem to be spinal in origin, the emesis to depend on "stimulation of vomitive centres in the medulla oblongata," which later are lowered in tone.

Quehl asserts that in animals it affects neither the motor, sensory, nor vaso-motor nerves, and that the emetic effect ceases after section of the vagi (Schmidt's Jahrb., Bd. clv.). Pécholier's case, however, certainly leads one to believe that in the human subject the sensory nerves are affected, for sinapisms were applied to nearly the whole surface of his body for half an hour without his feeling them.

As a rather exceptional instance of the influence of the drug on cerebral function may be quoted the case of a man under treatment for chronic morbus Brightii, who had on each of two successive days taken an emetic draught without effect, and was injected with $\frac{1}{10}$ grain of apomorphia; in four minutes he vomited freely, and contimued to do so at intervals for half an hour; he then passed into a mildly delirious state for a short time, and afterwards slept. Other observers have occasionally noted heaviness of head, giddiness, and drowsiness (Riegel and Böhm, Arch. f. Klin. Med., Bd. ix.).

Given in large doses to animals, the chloride produced grave 
nervous symptoms; thus, when 2 grains were injected into a dog, two or three minutes after vomiting it began to run round the room in a curiously persistent and methodical manner, but afterwards recovered. Three grains injected into a cat caused greater excitement: besides the running round there were occasional high leaps and somersaults; the pupils were dilated to the extreme, and became insensible. Two more grains produced epileptiform convulsions; and upon the injection of yet two more, the convulsions were followed by relaxation, the heart beat forcibly, and next morning the creature was found dead. All the organs were natural, and free from hyperæmia. Reichert also found, post mortem, no cerebral hyperæmia, nor congestion of the pons, as described by Quehl.

In general terms it may be concluded that apomorphia first excites the nervous system, causing delirium and convulsion, and may be followed by paralysis. It is more allied in action to thebaia and narcotin than to morphia and codeia.

Therapeutical Action.-Poisoning.-The special value of this drug has been proved in cases of irritant poisoning and of overloaded stomach (v. p. 120). It is better aroided in conditions of extreme debility, of impeded brematosis, and of profound narcotism; also in the very young. The conclusions of Dabadié, that it is the best emetic known, and can be given in full doses without accident, are too favourable (Thèse, Rev. d. Sci. Méd., 1882).

It is commonly held that some change in the drug will account for the occasional untoward effect; but a competent writer asserts that he has frequently injected, without any other than good result, 4 or 5 minims of a 1 in 50 solution of the chloride, that had been made for three months (Lancet, i., 1882).

It has been given as an expectorant, but we have, I consider, better-proved remedies. It is said to be well combined with morphia (v. p. 107).

PREPARATIONS AND DOSE.-Apomorphice chloridum: dose, $\frac{1}{16}$ to $\frac{1}{10}$ gr. by the skin, to be repeated if necessary; double that amount by the mouth, as an emetic; $\frac{1}{32}$ to $\frac{1}{16} \mathrm{gr}$. as an expectorant. 


\section{PAPAVER RHEAS. \\ (Scarlet Corn-Poppy.)}

The common corn-poppy has a therapeutical action similar to that of opium, but very slight in degree, and the active ingredients are uncertain in quantity. The preparation is a syrup from the petals; and the dose from one fluid drachm upwards. The petals are chiefly used for the red colouring matter they yield to water,- - the colour is much deepened by alkalies.

\section{SANGUINARIA CANADENSIS. \\ (BLOOD-ROOT-Puccoon.) (Not Officinal.)}

DESCRIPTION.-A low-growing perennial, native of North America, but common in English gardens. In early spring the rhizome sends up one or two leaves, and a slender scape, 4 to 6 inches high, bearing a single large white flower. The leaves, which are reniform, do not attain their full size till after the petals have been shed in April or May. The rhizome is 2 to 4 inches long, about $\frac{1}{2}$ inch thick, fleshy when fresh, and emitting, on section, a dark red acrid juice; after drying, it is reddish brown, and breaks with a short, waxy-looking fracture (Stillé).

ACTIVE INGREDIENTS.-The powers of sanguinaria chiefly depend on the presence of the alkaloid sanguinarin, or chelerythrin, $\mathrm{C}_{19} \mathrm{H}_{17} \mathrm{NO}_{4}$, which has been obtained also from celandine. It forms colourless crystalline needles, which cling together in lumps, or stellate groups; when dry, they are opaque. It is insoluble in water; soluble in ether, alcohol, and the fixed and volatile oils.

A second alkaloid has been termed porphlyroxin, from its supposed identity with a constituent of opium: it may be obtained in white tasteless crystals (Gibb).

The ingredient termed puccin, formerly considered to be 
another alkaloid, has been resolved into a sulphate of sanguinarin with a trace of resin (Hopp, 1874).

Physiological Action. - Digestive System. - If a dry crystal of sanguinarin be nibbled, it seems tasteless; but the alcoholic solution has an acrid, bitter flavour.

Small doses of the infusion, tincture, or alkaloid, improve both appetite and digestion; larger doses nauseate, and if pushed, cause salivation, vomiting, and purging, with burning pain at the stomach, and afterwards collapse (Dr. Meade Smith, Amer. Journ., 1876).

Wareing describes it as an "acrid emetic," and its marked vomitive properties have led Gubler to trace a resemblance between sanguinaria and apomorphia. Rutherford found it "a feeble intestinal, but a powerful hepatic, stimulant" (Practitioner, ii., 1879).

Circulatory System. - Full doses cause "a progressive lowering of the pulse and arterial pressure, partly by a direct action on the heart, partly by paresis of the vaso-motor centres -this stage is preceded by irritation of these centres, which is more marked after moderate doses." Irregularity and palpitation of the heart are complained of, and a fatal issue followed large doses, in four instances, at the Bellevue Hospital, New York: "racking burning pains and tormenting thirst" are recorded as the chief symptoms.

In frogs, Weyland found that 0.0156 gram. proved fatal in about two hours, the heart-action being arrested; its muscle was found to retain some electric excitability.

The respiratory act is rendered slower and shallower from direct central action, and death commonly occurs from asphyxia. The mucous membrane is liable to be much irritated by the drug, and the powder provokes considerable sneezing.

Nervo-muscular System.-Convulsions occur in various classes of animals, but have not been recorded in man. Smith traces them to an action on the spinal cord, the centre of Setschenow being excited: reflex excitrbility becomes, however, lessened, and the condition of the spinal centres is later depressed - the pupils are dilated and vision impaired. Vertigo, anæsthesia, prostration, unconsciousness, and occasional rigidity 
of limbs have been reported. The muscles retain their electric irritability, so that the drug does not resemble veratria as a muscle-poison.

Synergists.-Varions emetics and alteratives. Digitalis and veratrum, also apomorphia, are similar in their sedative and nauseous effects. Rhubarb, podophyllin, and aloes assist its action on the liver and intestinal tract.

ANTAGonists.-Alkalies, tannic and gallic acids, and most metallic salts are chemically incompatible. Opium relieves the pain and depression produced by it.

Therapeutical Action.-Sanguinaria being stimulant and escharotic, may be applied with advantage to the surface of atonic and ill-conditioned ulcers, and is said to keep down excessive and unhealthy granulations. Dr. Fell asserted its power to cure cancer, and thus probably excited some prejudice against it. A recognized mode of applying the remedy is given in the Medical Times, ii., 1858.

Used as smuff, it relieves nasal polypi, and in ointment it has been found beneficial in gangrene, tinea, and impetigo.

Tully prescribed doses of $\frac{1}{4}$ to 1 grain of the resin as a cholagogue, and Mothershead as "an excitant of the liver;" they have also found it useful in atonic dyspepsia and duodenal catarrh.

In disorders of the respiratory organs, sanguinaria has some repute, but more in America than in this country. It acts as an expectorant and diaphoretic. The late Dr. D. G. Gibb wrote strongly in favour of its use for various stages of bronchitis, phthisis, and pneumonia - generally chronic in character-and many American authors have expressed the same opinion (Ranking, i., 1860, and Naphey's Therapeutics). It is often combined with lobelia or ipecacuanha. For chronic mescel cutery it has been given internally, and applied locally. The acetum is employed as a gargle in scarlatinal and ulcerative anginu. The effects of the drug in diphtherin and croups searcely justify the high recommendation of it by some practitioners.

Sanguinaria, either alone or combined with podophyllin, is 
said to be useful in many cases of hysteric due to disturbance of the system from pain or moral causes; the following is a good formula in such cases:-Podophyllin, 3 grains; sanguinaria, 8 grains; soap, 8 grains; extract of hyoscyamus, 3 grains: make 20 pills: one pill to be taken morning and night (Van der Espt, Journ. de Méd. de Brux., 1868).

Bartholow endorses the opinion of Dr. Coe and others, that sanguinaria is a good emmenagogue for functional disorders of this kind occurring in anæmic or chlorotic subjects, but recommends it with aloes or iron.

As an aphrodisiac, when there are relaxation of the genital organs, diurnal losses, inaptitude from irritability, etc., he recommends it with ergotin or stillingia. It has also been used in chronic syphilitic, rheumatic and strumous affections.

Preparations and DOSE.-Pulvis, dose, $\frac{1}{2}$ to $3 \mathrm{gr}$. when its stimulant effects are required; 8 to $16 \mathrm{gr}$. as an emetic (the powder should be made into pills on account of its irritant properties).

Infusum vel decoctum (an ounce of the powder to $10 \mathrm{oz}$. water): dose, as an emetic, $\frac{1}{2}$ oz. to $1 \mathrm{oz}$.

Acetum (120 parts of the powder, 1,000 parts distilled vinegar, and 30 parts alcohol; macerate in the vinegar for one week; filter, and add the spirit): dose, as an expectorant, 10 to $30 \mathrm{~min}$. three or four times a day.

Tinctura (made with 12 parts of the powder; diluted alcohol, 1,000 parts-macerate and filter): dose, as an emetic, $2 \frac{1}{2}$ to $3 \frac{1}{2} \mathrm{dr}$.; as a stomachic and expectorant, 30 to $60 \mathrm{~min}$.

Extractum alcoholicum: dose, $\frac{1}{8}$ gr. to $\frac{3}{4} \mathrm{gr}$.

The alkaloid sanguinarin is but little used: dose, $1 \mathrm{gr}$. triturated with 20 to $30 \mathrm{gr}$. of sugar of milk, and divided into 10 to 30 doses, according to the effect desired. 


\section{CHELIDONIUM MAJUS. (CelandiNe.)}

\section{(Not Officinal.)}

DESCRIPTION.-This plant forms usually a small succulent bush, with branching stem about 2 feet high, and large alternate leaves, hairy above, smooth below, deeply pinnatifid, the lobes rounded and bluntly notched. The flowers, produced in scanty umbels on long peduncles, have four bright yellow, fugitive petals, and are followed by a long capsule. The stem contains an orange-red acrid juice. The so-called "lesser celandine" belongs to the Ranunculaceæ.

ACTIVE INGREDIENTS.-Chelidonium root contains at least two alkaloids. (1) Chelidonin, $\mathrm{C}_{19} \mathrm{H}_{17} \mathrm{~N}_{3} \mathrm{O}_{3}$, which crystallizes in colourless, glittering tables, is insoluble in water, and nearly so in ether and alcohol, but more soluble in fixed and volatile oils. Solutions of its salts, when tested with alkalies, let fall the chelidonin as a voluminous cheesy deposit, which gradually becomes horny-a characteristic test. Sanguinarin, or chelerythrin (already described under Sanguinaria, p. 123). Probst isolated also chelidoxanthin, which crystallizes in small yellow needles, unaltered by acids or alkalies, soluble in hot water.

The alkaloids are combined with malic and chelidonic acids, which latter seems identical with succinic acid.

Physiological ACTiON.-The fresh plant and the juice irritate the skin, and also, when taken internally, the gastro-intestinal mucous membrane, causing violent diarrhoea,-but exact observations as to the action of this drug are wanting. Some narcotic effect has been noticed in cases of poisoning.

Chelidonin has a bitter taste, and irritates in the mouth; but Probst and Reuling agree that any dose up to 5 grains produces no poisonous effects either on animals or on man; while the sanguinarin found in the same plant proved 
fatal to rabbits in doses of $\frac{1}{3}$ grain. The symptoms were like those seen in Orfila's experiments, and death occurred in ten minutes (Ann. Chem. Pharm., xxix., 13).

Therapeutical ACTION.- Celandine was formerly reputed a good aperient, diuretic, and sudorific, as well as a powerful "deobstruent," and employed in jaundice, acute and chronic hepatitis, gallstones, and other hepatic affections; also in hæmorrhoids, and in pneumonia with hepatic complications. Writers have praised it in paralysis, spasmodic coughs, and neuralgia (Dr. Buchmann); also in eczema and herpes. The fresh juice is still used as an application for warts, but more dependable remedies have practically superseded this one in general practice.

PREPARATIONS AND Dose.-Succus: dose, 20 to $40 \mathrm{~min}$. Infiusum ( $\frac{1}{2}$ oz. of plant to 1 pint of water): dose, 1 to $2 \mathrm{oz}$. or more. Extractum: dose, $10 \mathrm{gr}$.

\section{ARGEMONE MEXICANA. (PRickly Poppr.)}

\section{(Not Officinal.)}

DESCRIPTION.-A beautiful annual, found wild in the East and West Indies, at the Cape, and in most tropical and subtropical countries. It is about 2 feet high, with sessile alternate leaves, having prickly teeth and white spots, yellow flowers, and ovate prickly capsules, containing numerous black seeds; the plant has a bitter, acrid taste, and exudes a yellowish juice when wounded.

ACTIVE INGREDIENTS.-A small proportion of morphia has been found in the leaves and capsules (Charbonnier, 1868); also a bland drying, fixed oil, of light yellow colour, from which Frölich obtained a soda-soap and butyric, valerianic, and other acids.

Physiological Action.-The juice acts as a local irritant, and resembles gamboge in several properties. The 
seeds are anodyne and narcotic, and the oil derived from them is said to cause romiting and purging. Flückiger, however, could not verify this with plants grown in the East Indies. An infusion of the plant is reported diuretic and diaphoretic.

Therapeutical Action.-In Mexico the fresh juice or bruised leaf is applied to warts, indolent ulcers, and syphilitic sores; also to corneal opacities in chronic ophthalmia.

The infusion is prescribed for strangury; the oil for neuralgic headache; and the juice in dropsy, as a cathartic like gamboge.

PREPARATIONS AND DOSE.-Oleum: dose, 15 to $30 \mathrm{~min}$. Infusum ( 3 drachms of the seeds to a pint of water): dose, 1 to 2 oz. 


\section{CRUCIFERAE OR BRASSTCACEAE.}

\section{THE CRESS FAMILY.}

A family of about 2,000 species of herbaceous or occasionally suffruticose exogens, found chiefly in Europe and the temperate parts of Asia. The leaves are alternate, exstipulate, and simple, though often deeply incised. The inflorescence is usually racemose, and destitute of bracts; the flowers are tetramerous; the sepals, petals, tetradynamous stamens and ovary are free; and the fruit, peculiar to the order, is a silique. No deleterious plant exists among the Cruciferæ; many species are used as food, and some are noted for acridity, pungency, and anti-scorbutic qualities.

\section{Pharmacentical Species.}

Sinapis nigra. . . . . Black mustard.

Sinapis alba ...... White mustard.

Armoracia rusticana .. Common horse-radish.

\section{S I N A P I S.}

(MUSTARD.)

DESCRIPTION.-There are two principal species; the black (Sinapis nigra), and the white (Sinapis alba) or salad mustard. The first-named is an annual with spreading, glabrous stem, 3 to 4 feet high : the lower leaves are large, lyrate, variously lobed and toothed, and roughened in places. The flowers are small and pale yellow, and followed by bluntly-quadrangular pods, pressed close to the stem, and containing each a few 
round seeds, covered with shallow depressions, and having a pungent odour when macerated with water; they are free from starch.

The Sinapis alba grows wild in every part of Europe, and is a plant of much the same character as the S. nigra, but not so tall. The flowers are larger, the pods spreading, bulging and hispid, and contain pale-yellow seeds, which are larger than those of the Sinapis nigra. Its properties are very similar.

Active INGREDIENTS.- ( 1 ) The fixed oil, which forins about 25 per cent., can be obtained from either white or black mustard by expression of the ground and sifted seeds. It is almost devoid of acridity, and consists of olein, stearin, and glyceride of erucic acid.

(b) Erucic or brassic acid, $\mathrm{C}_{22} \mathrm{H}_{42} \mathrm{O}_{2}$, crystallizes in long, slender, white needles, which are insoluble in water, but freely soluble in alcohol and ether.

(c) Myronic acid, $\mathrm{C}_{10} \mathrm{H}_{19} \mathrm{NS}_{2} \mathrm{O}_{10}$, exists in black mustardseeds in the form of myronate of potash, $\mathrm{C}_{10} \mathrm{H}_{18} \mathrm{KNS}_{2} \mathrm{O}_{10}$, which salt is soluble in water and dilute alcohol,--insoluble in ether, chloroform, and benzol. It has a cool, bitter taste, and neutral reaction; when heated, it gives out a pungent smell.

(d) Myrosin is an albuminoid substance, somewhat analogous to emulsin, and acts as a ferment to the myronic acid in mustard-seeds, as soon as water is mixed with the flour. By this reaction is produced-

(e) The volatile oil of mustard, which is not found in the dry seeds : it is colourless or light yellow; has a pungent odour and taste, and a neutral reaction. It largely consists of the sulpho-cyanide of allyl, $\mathrm{C}_{4} \mathrm{H}_{5} \mathrm{NS}$, mixed with cyanide of allyl, and is almost, if not quite, identical with an oil obtained from horse-radish.

White mustard-flour also undergoes a fermentation process, when mixed with water; but the oil developed, though of pungent character, is not the volatile oil of black mustard: by Will (1870) it has been termed sincllhin, $\mathrm{C}_{30} \mathrm{H}_{44} \mathrm{~N}_{2} \mathrm{~S}_{2} \mathrm{O}_{10}$, and has been obtained in small glass-like prisms, neutral, soluble in water, sparingly so in alcohol. 
(f) Sinapin, $\mathrm{C}_{16} \mathrm{H}_{23} \mathrm{NO}_{5}$, is a readily decomposed alkaloid which exists in both white and black mustard-seeds, under the form of the sulpho-cyanide. (This substance has also been called sinapia, sulpho-cynapisin [Berzelius], sulpho-cynapic acid, sinapisin, etc.). Sinapin itself is not to be obtained pure in a solid form, but can be procured as a watery solution, indirectly, from its bisulphate. The solution has a marked alkaline reaction, and a light-yellow colour; but, during evaporation, changes, through green and red, to a noncrystalline brown residue.

Physiological Action.-The well-known irritant and acrid qualities, both of white and black mustard-seeds, are dependent on the fermentation products, already described, which are the result of the mixture of mustard with water. The volatile oil of black mustard, ascending in the steam of hot water which is poured on mustard-flour, acts either as an irritant (causing sneezing, watering of the eyes, etc.), or, if the vapours be not too strong, as a soporific. A general mustard-bath, even if warm, $86^{\circ} \mathrm{F}$., induces at first a sensation of chill, with sometimes twitching of the limbs; reaction sets in after leaving the water, with some sense of stinging of the skin. In baths of a higher temperature, $95^{\circ}$ to $98^{\circ} \mathrm{F} ., \mathrm{I}$ have not noticed this effect, but a distinctly soporific action, as well pointed out by $\mathrm{Mr}$. Newington (v. "Inorganic Substances," p. 173).

Mustard-flour mixed with a little water and applied to the skin produces redness, vesication, and even ulceration and gangrene, if pushed to an extreme. The volatile oil acts with still more severity, producing great pain, redness, and vesication even when diluted; one drop placed on the tongue causes intense burning pain in the fauces and stomach.

It is one of the most poisonous of the ethereal oils: rabbits have been killed in two hours by a drachm, and in fifteen minutes by half an ounce; extreme frequency and afterwards feebleness of the heart-action were produced; also loss of sensation and of muscular power, oppression of breathing, convulsion, coldness, and collapse; there was violent gastro-enteritis, and the odour of the oil was perceptible in the blood, urine, and breath. 
Digestive System.-Prepared mustard is used as a condiment, and in due proportion stimulates the gastric mucous membrane, and promotes the digestion of tough foods: like other stimulants, however, if used in excess, it impairs the tone of the digestive organs. The seeds taken whole in teaspoonful doses have a laxative action, and pass through the bowel without perceptible change.

It has been shown that they by no means act merely as mechanical irritants. On the contrary, in their somewhat slow passage along the alimentary canal, they swell up and become mucilaginous (especially white mustard-seeds, of which the external coating is thin), and then give out the true acrid products of the fermentation of myronic acid and myrosin. It is urged that from the slow and equable manner in which this takes place, we have, in uncrushed mustard, a singularly effective and manageable stimulant to all the functions of the alimentary canal; but the objection is that the seeds are apt to accumulate in some one place, such as the appendix vermiformis, and then excite dangerous inflammation; otherwise there can be no doubt that both laxative and diuretic effects can be produced in this way (Journ. de Méd. de Brux., t. I., $1870)$.

ThERAPEUTICAL ACTION.-The distilled water of mustard-seed has been employed in cases of itch.

The principal use of mustard is, however, in the form of poultice, or cataplasm. In various affections of the brain, such as the stupor and delirium of low fever, apoplexy, and in cases of poisoning by opium, or other narcoties, it is most valuable, and should then be applied to the feet and ankles; its speedy operation gives it an advantage over cantharides. So, too, in pulmonary and cardiac affections, the poultice may be applied to the chest with the most beneficial effects; and both this and the liniment are excellent counter-irritants in inflammation, neuralgic pain, and spasm; as well as in pleurodynia, gout, rheumatism, and arrest of the catamenia. The hot mustardbath has been found exceedingly useful in violent manin, and also in the insomnia of nerve-excitement: and even mustard font-baths, and plasters, exert some special soporific effect. The 
bath has proved valuable also in pneumonia, especially in children (Weber).

Dyspepsia, etc.-In atonic dyspepsia with constipation the white mustard-seed may be given as above described: it was at one time held to be almost a panacea for such conditions (Med.Chir. Rev., A pril, 1827), ${ }^{1}$ but it is probable that, as a tonic stimulant, in cases of dyspepsia with torpid liver and loss of appetite, mustard would be best employed in the form of alcoholic solution of the volatile oil. In the Austrian army this has been substituted for dietetic purposes. The solution of 24 drops of the oil to the ounce of spirit, as originally recommended by Meyer and Wolff, is an excellent and powerful medicine both in chronic eatarrhal dyspepsia and chronic bronchial catarrh (in doses of 3 to 5 drops in some emulsion): it has also received much praise as a dinuetic in dropsy, but the special indications for its use in this disorder have not been defined.

A tablespoonful of mustard-flour in warm water is one of the readiest and best emetics, and is especially indicated when it is desirable to stimulate the action of a failing heart, whether in ordinary disease, narcotic poisoning, the collapse of cholera, or in certain forms of paralysis : an excessive dose may, however, cause much irritation and even gastro-enteritis.

PREPaRations ANd Dose.-As stomachic, of the seeds 1 to 2 teaspoonfuls in 3 to 4 oz. of water once or twice daily. As an emetic, from a teaspoonful to a tablespoonful of mustard-flou mixed with water. Cetceplasma sincupis: boiling water, $10 \mathrm{fl}$. oz. ; linseed meal, powdered mustard, of each $2 \frac{1}{2}$ oz. Oleum sinapis. Linimentum sinapis compositum (contains the ethereal oil, with mezereon, camphor, eastor oil, and spirit). Charta sinapis (U.S.): black mustard, in powder, 90 gr.; solution of gutta-percha, as much as will give it a semi-liquid consistence, and let it be spread with a suitable brush on one side of a stiff piece of paper four inches square, and allow it to dry; before applying it to the skin it should be dipped in warm water for fifteen seconds.

1 Mr. C. T. Cooke's pamphlet, On the Efficacy of White Mustard-seed in Affections of the Liver, Internal Organs, and Nervous System (1826), was translated into almost all the Europenn languages. 
Adulterations.-Flour of mustard is commonly adulterated with wheaten flour, coloured yellow with turmeric, and rendered pungent by means of capsicum.

COCHLEARIA ARMORACIA; or, Armoracta Rusticana. (Horse-RAdish.)

DESCRIPTION.-Horse-radish is a perennial, growing wild in many parts of Europe, and now naturalized in English kitchen-gardens. The root is 12 to 18 inches long, 1 or 2 inches in thickness, tapering, white, and tenacious of life. Aconite has been mistaken for it $(v$. p. 2). The radical leaves are 12 to 18 inches long, 2 or 3 inches broad, oblong, crenate, dark green, and with long petioles. The insignificant copious white flowers are produced in large corymbose clusters upon stems about 2 feet high: the pods are roundish.

ACTIVE INGREDIENTS.-The pungent odour and acrid taste depend on a volatile oil, $\mathrm{C}_{4} \mathrm{H}_{5} \mathrm{NS}$, which is produced by the action of myrosin on myronic acid in the presence of water; it is chemically identical with oil of black mustard, but differs in odour; being volatile, it is readily dissipated by heat or exposure to the air: one drop is sufficient to odorize a whole room. It is soluble in alcohol, less so in water; it is heavier than the latter, and of a pale-yellow colour, which becomes darker by age.

Physiological Action.-Eixternul.-The volatile principle of horse-radish is very irritating to the nostrils, and causes the eyes to flow profusely with tears; the scraped root or the oil causes redness and even vesication when applied locally.

Physiological Action.-Intemul.-Taken internally it gives a sweetish, hot, acrid taste, causes a sense of warmth at 
the stomach, and promotes digestion, especially of animal food. It increases perspiration, and the secretion of the kidneys, imparting to the latter a peculiar odour. Tiedemann found the same odour of the drug in the breath of a dog after injecting the juice into its crural vein.

Over-doses of the oil or infusion cause much soreness and relaxation of the throat, burning heat in the stomach, nausea, and vomiting.

Therapeutical Action.-Sydenham highly valued horse-radish in cases of dropsy supervening upon intermittent fever, but in modern practice it is only occasionally employed. Its effects are similar to, though more energetic than, those of mustard-seed, and it may be used as a stimulating vehicle for more active remedies in cardiac, hepatic, or renal dropsy in feeble subjects. It has been given also with good result in atonic dyspepsia, chronic rheumatism, and paralytic affections, especially of the tongue, and in this last it often helps mastication by its powerful sialagogue property. A syrup prepared from a concentrated infusion of the root, and swallowed leisurely (a teaspoonful at a time), or used as a gargle, removes hoarseness arising from relaxation of the throat; and this condition may also be relieved by employing horse-radish as a masticatory.

The infusion taken with draughts of warm water readily produces vomiting, and may be used alone, or to assist the operation of other emetics. As an antiscorbutic it was much praised by Cullen and others.

Preparation and Dose.-Spivitus Amoracire compositus (made with the fresh root, orange-peel, nutmeg, spirit, and water): dose, 1 to $2 \mathrm{fl}$. dr.

An infusion may be made with an ounce each of freshlygrated horse-radish and bruised mustard-seed in a pint of hot water, macerated for two hours. Dose, a wineglassful three or four times daily. 


\section{VIOLACEA.}

\section{THE VIOLET FAMILY.}

Av order of exogens numbering about 300 species, the technical characters of which nearly correspond, though the plants fall into two distinct divisions-one belonging to northern temperate countries, especially to Europe and North America; the other to South America.

The properties are chiefly manifested in certain species of the latter, belonging to the genus Ionidium, all of which have lanceolate or ovate leaves, and small, axillary, solitary flowers. The roots of these plants are emetic, as expressed in the name of the most noted of them, Ionidium Ipecacuanha; they also go by the name of "False Brazilian Ipecacuanha." Pelletier obtained from them 5 per cent. of emetin.

The roots of the Violacer of the northern hemisphere are also reputed to have emetic properties.

\section{Medicinal Species.}

\section{Viola odorata. (The Common Sweet Violet.) (Not Offeinal.)}

DESCRIPTION.-This well-known plant is distinguished by its perennial ront, flagelliform runners, roundish-cordate leaves, and fragrant blue or white flowers, the sepals of which are obtuse.

ACTIVE INGREDIENT.-This is probably the alkalnid violin, discovered in all parts of the plant, but especially in the 
rhizome, by Boullay (1828). It is a pale-yellow, bitter powder, which, on the application of heat, first melts and then burus like a resin. It is still uncertain whether violin is or is not identical with emetin, the active principle of ipecacuanla, but it appears to be more soluble in water, and less soluble in alcohol and ether. Pereira affirmed the operation of the two substances to be similar, and states that the violet-root might be employed as a substitute for ipecacuanha; but the experiments of Orfila and Chomel throw doubt upon this, and show that violin is inconstant in its action; possibly it may be an impure form of emetin, as suggested by Husemann.

Physiological Action. - Violin is irritant to the alimentary canal, though the degree in which it will produce vomiting or purging in any individual man or animal is always uncertain. Its extreme effects are seen in such experiments as those of Orfila, when he placed $5 \frac{1}{2}$ grains in the stomach of a dog, and ligatured the œsophagus: in twelve hours the animal became exceedingly depressed, with rapid pulse, and in forty-eight hours died in convulsions; gangrenous inflammation of the stomach was found on dissection. The same quantity of violin subcutaneously injected, proved fatal to a dog in ten hours; a corresponding dose killed a dog when injected into the jugular vein. Chomel's experiments upon men. proved that the power of violin to produce vomiting or diarnhoa varies much. Garrod says that the root of the Viola odorata is purgative and emetic in doses of 30 to 60 grains.

Therapeutical Action. - In the present state of uncertainty as to whether violin is an independent substance, or merely an impure emetin, it seems impossible to accept as satisfactory any statement respecting the medicinal value of the plant; but it may be mentioned that syrup of riolets has been found a useful remedy in spasmodic nervous cough attended by dyspnoca, and little or no expectoration; in some cases of whooping-cough it lessens the spasms.

For hysteria, attended by depression of spirits and constant weeping, it is in popular repute, especially in places where the plant is largely grown. An equal part of oil of almonds and 
syrup of violets, administered in a dose of one to two teaspoonfuls, serves as a pleasant laxative for new-born infants.

Preparations and Dose.-Mel viole (1 part of fresh violet flowers to 5 parts of honey): dose, $1 \mathrm{dr}$. Symupus vioke: dose, $\frac{1}{2}$ to $2 \mathrm{dr}$.

The Viola canina is said not only to have roots with emetic properties, but to be useful as a depurative, and it has been recommended in some cutaneous disorders. In Italy, the common pansy, Viola tricolor, is employed for similar cases, and especially for tinea capitis. 


\section{POLYGALACEA.}

\section{THE RHATANY FAMILY.}

As extensive family of exogenous shrubs and herbaceous plants, of which the typical genus, Polygala, is the only one represented in Europe; for example, in the wild English milkwort, Polygala vulgaris. The leaves are usually simple, alternate, and without stipules. The flowers are irregular: the stamens are few and hypogynous, and the ovary becomes a many-seeded capsule.

\section{Pharmacentical Species.}

Polygala senega

Krameria triandra
Senega.

Rhatany.

\section{POLYGALA SENEGA.}

(Senega, or SNate-Root.)

DESCRIPTION.-This plant, indigenous to the United States, is an herbaceous perennial, with a branched and woody root. The stems are annual, and rise to the height of 15 inches: they are simple, and clothed with alternate lanceolate leaves, the small pink-white flowers forming a terminal erect spike. The root-crown is about $\frac{1}{2}$ to 1 inch in diameter, very knotty; the root itself is only $\frac{1}{4}$ inch in diameter, $t$ to 6 inches long, and divided into several branches, fleshy and round when fresh, but after drying twisted and showing a ridge which projects; it is brittle, yellowish-grey outside, whitish within. 
ACTIVE INGREDIENTS. - The most important is the glucoside, polygalin or senegin, called originally polygalic acid (Peschiar), and probably identical with saponin, monesin, and other kindred substances found in various plants. It is an amorphous light-coloured powder, neutral, freely soluble in water, little so in cold strong spirit, and not at all in ether. The latest formula given is $\mathrm{C}_{32} \mathrm{H}_{54} \mathrm{O}_{18}$, but it is not fixed. Concentrated sulphuric acid dissolves it with a reddish-yellow colour, that changes to bright red. The watery solution is peculiar, from its lathering like soap.

There is probably a second active ingredient in senega, specially soluble in alcohol, and which does not exist to any large extent in infusions or decoctions.

It contains also pectin compounds which render solutions gelatinous, sugar, a fixed oil, resin, etc. The only part of the plant used in medicine is the root, and the inner part of this is nearly or quite inert, the virtues residing exclusively in the bark. They are brought out by water, more completely by spirit; but it is said that neither the decoction nor the infusion possesses value equal to that of the simple powdered root.

Physiological Action.-External.-The root is nearly inodorous, but has a peculiar bitter, pungent flavour, and when chewed leaves an acrid sensation, which is deepened if the saliva be swallowed.

Senegin is also odourless, but highly irritant to the nostrils, and has a taste, sweet at first, afterwards sharp and pungent: both it and allied substances, especially monesin, if applied to the skin or mucous membrane, cause redness with some anæsthesia, and if there be any breach of surface severe pain and irritation, followed by ulcers which give out a plastic exudation, and become covered with a grey-coloured membrane.

Physiological Action.-Intermel.-Digestive System, etc.-Stillé describes the following symptoms from taking 20 grains of senega three times within six hours: umpleasant irritation of fauces and throat, with copions salivation; then hent at the epigastrium, and violent vomiting of mucus; increased 
warmth and moisture of skin, and colic with diarrhœa: increased urination with scalding: larger doses excite severe burning gastric pain, vomiting, and purging.

Schroff found that in man, doses of $\frac{1}{3}$ grain to 3 grains of senegin caused some nausea, with a bitter taste and pricking in the mouth. From $2 \frac{1}{2}$ to 3 grains also produced irritative cough, and secretion of mucus in the bronchial tubes, lasting for several hours; but no effect was manifested upon either the kidneys or skin.

Circulatory System.-Applied directly to the heart, or reaching it through the circulation, senegin reduces the frequency and force of its contractions, and finally paralyses it.

Nervous System.-A solution of the allied saponin injected into a frog's thigh "impairs the motility and sensibility of the limb, and abolishes the reflex excitability of the muscles"; this occurs even if the limb be severed from the body.

I believe that the drug exerts upon the nervous system a powerful influence, somewhat resembling that of arnica.

Therapeutical ACtion.-Snake-bite.-The earliest use of senega appears to have been by a tribe of North American Indians, called Senegaroos, who esteemed it an antidote to the bite of the rattlesnake: they applied it both externally and internally. Last century, Dr. Tennant, an American physician, published the secret to his countrymen, and was rewarded with a grant of money by the Legislature. Whether superior, as a specific, to many other plants which are similarly employed, is still uncertain.

Chest Disorders.- In the advanced stages of pmemonic, when there is much prostration, and the cough dry, harsh, and painful, with a sense of oppression across the chest, this medicine promotes expectoration, and relieves other distressing symptoms.

In the bronchitis of old people, and especially when complicated with emphysema, senega often acts beneficially.

In bronchitic asthna and empliysema it not only diminishes the secretion, but promotes easy expectoration, and relieves the opfression,- - for which purposes it has been highly commended by many physicians. In whooping-cough it is also sometimes used. 
Dr. Areher (U.S.) introduced it as a remediy of great power in croup, while Dr. Barton and many others regarded it as a valuable auxiliary to other medieines employed in this disease: it is desirable that further inquiries should be instituted in regard to this point.

Dropsy; etc.-Dr. Percival and others have praised senega as a diuretic that may be employed in dropsies, especially renal; but recent writers have denied its action on the kidneys or skin, and this is certainly not powerful enough to constitute it an important remedy in such disorder. 'In chronic rheumatism it has, however, been more generally considered of value, as stimulant and diaphoretic, though Nothnagel allows only its power as an expectorant.

Amenorrhœa, etc.-Senega-root has been employed with adrantage in amenorrhœa, a pint of the saturated decoction being given daily during the fortnight preceding the monthly function. On the other hand, it is interesting to note that senegin (or rather monesin) has been successfully employed by Martin St. Ange, in 2-grain doses, as a remedy for uterine hrmorrhage (Husemann).

PREPARATIONS AND DOSE.-Senegre raclix (in powder): dose, 10 to $20 \mathrm{gr}$. Infusum ( 1 in 20): dose, 1 to $2 \mathrm{fl}$. oz. Tinctura ( 1 in 8$): \frac{1}{2}$ to $2 \mathrm{fl}$. dr.

ADULTERATIONS.-The roots of two different plants are occasionally found mixed with senega. (1) Of the ginseng. (Panax quinquefolium), readily distinguished by the greater size, the more or less fusiform figure, and the absence of a peculiar ridge, which extends the whole length of the true senega-root upon its concave side; (2) of the Gillenia trifoliata, also negatively distinguished by the absence of the projecting line. 


\section{KRAMERIA TRIANDRA.}

\section{(Rhataxy.)}

DESCRIPTION.-A Peruvian under-shrubby, anomalous plant, growing upon dry hills, and producing beautiful rosecoloured flowers throughout the year.

The root is horizontal, long, much branched, with several knotty heads, and provided with a thick, smooth, or, in old roots, scaly, bark; within it is red; externally reddish-brown. The stem is procumbent, and divided into numerous widely-spreading branches, 2 to 3 feet in length. The leaves are alternate, sessile, oblong, acuminate, entire, and hoary upon both surfaces. The flowers are terminal or axillary, solitary, and supported on short peduncles: individually, they consist of four purple sepals, four petals, three fleshy stamens, and an ovoid orary, which, when ripe, becomes a dry hairy drupe, burred with dull red hooks.

ACTIVE INGREDIENTS. - The most important is krameric, or ratanhia-tannic acid, $\mathrm{C}_{14} \mathrm{H}_{22} \mathrm{O}_{11}$, a dark-red, shining, amorphous substance, little soluble in cold water, precipitated dark green by ferric salts, flesh-colour by gelatine, not at all by tartarated antimony. The bark also contains ratanhia-red, which may be obtained from ratanhia-tannic acid by the action of dilute acids (F. and $\mathrm{H}$.). Ratanhia-red seems to have the same composition as the tannin obtained from horse-chesnut and from tormentil, $\mathrm{C}_{26} \mathrm{H}_{22} \mathrm{O}_{11}$. A substance called rhatanin, $\mathrm{C}_{10} \mathrm{H}_{13} \mathrm{NO}_{3}$, is dissolved out in the watery extract, and crystallizes in coherent masses of slender crystals. It is said to be identical with angelin, the resinous alkaloid of the leguminous plant Fereira spectabilis.

PHysiological Action.-Rhatany acts locally and internally like other vegetable astringents, lessening secretion, and producing tonic effects, which from continued or excessive doses merge into those of irritation. 
SYNERGISTS.-Vegetable astringents.

INCOMPATIBLES.-Iron and other metallic salts. Ipecacuanha.

Therapeutical Action.'-External.-A solution or ointment of rhatany is useful in fissure of the nipple; and applied in the following manner it has obtained a special reputation for fissure of the anus:-About 60 to 90 grains of the extract in 5 ounces of water are injected into the rectum night and morning after an emollient enema; the astringent causes some pain at first, and is retained only for a few moments.

In plaster, rhatany has been applied to atonic ulcers and to tumours; in injection, it is of some use in dysentery, leucorrhœa, gleet, ozæna, etc.; and in relaxed throat as a gargle.

THERAPEUTICAL ACTION.-Internal.-In various disorders accompanied by discharge, whether mucous or hæmorrhagic, rhatany is useful when given internally. Sir H. Halford esteemed it in fluor albus, and in passive hæmorrhage, uterine or otherwise; it is, in these conditions, more indicated in anæmic than in plethoric subjects.

It is of value, also, in chronic diarrhoea, and in incontinence of urine from debility.

In the atonic dyspepsin of leucophlegmatic subjects it proves a good substitute for cinchona, if that remedy disagrees; the extract may be given in pill, if a liquid form of the drug cannot be taken. It has been recommended in the advanced stage of typhoid fever, on the ground that it possesses some of the qualities of port wine.

Rhatany is an excellent tonic to associate with diuretics and eathartics.

PREPARATIONS AND DOSE.-Extractum: dose, 5 to $20 \mathrm{gr}$. Infusum: dose, 1 to $2 \mathrm{oz}$. Tinctura : dose, $\frac{1}{2}$ to $2 \mathrm{fl}$. dr. The pulvis cutechu compositus contains 2 parts in 10 , together with kino, cinnamon, and nutmeg: dose, 15 to $30 \mathrm{gr}$. 


\section{ERYTHROXYLACEAE.}

\section{ERYTHROXYLON COCA. (COCA OR Ctca.) \\ (Not Officinal.)}

DESCRIPTION. - A small shrub, indigenous to the mountains of Peru and Bolivia, about 4 feet high, with spreading purplish hranches, small yellowish five-petalled flowers in axillary elusters, an oblong red, smooth fruit, and alternate leaves about 2 inches long, obovate, obtuse, entire, thin, smooth, and reticulate, with prominent midrib, on each side of which is a curved line: they have a tea-like odour, and a bitter aromatic taste.

Active IngRedients.- (1) About 26 per cent. of a crystalline alkaloid, cocaïn, or cucaïn, $\mathrm{C}_{17} \mathrm{H}_{21} \mathrm{NO}_{4}$, has been isolated in colourless prisms, soluble in alcohol and ether, but only in 704 parts of water: heated with hydrochloric acid, it is decomposed into methylic alcohol, benzoic acid, and ecgonin; the hydrochlorate of cocaïn has been prepared soluble in all proportions of water (Merck). A citrate and salicylate have also been prepared.

(2) A volatile alkaloid, termed by Lossen hygrina, and obtained as a thick, pale-yellow oil of burning taste and an odour of trimethylamin.

(3) A peculiar tannin, cocatannic acid, which gives a green colour with iron salts.

Physiological ACtion.-Digestive System.-The action of coca on this system is not marked, but the sensations of thirst and of hunger may be diminished by its use. Espinosa, however, found no diminution of appetite (Edin. Med. Journ., 1876). Von Ausep (Wurzburg) reports that in mammalia 
small doses accelerate the peristaltic movements of the intestines, and lessen the secretions of all mucous membranes.

Circulatory System.-Locally applied to the conjunctiva, fauces, and larynx, hydrochlorate of cocaïn eauses contraction of the blood-vessels, rendering the mucous membrane white; this is further illustrated by the fact that bleeding is found to be lessened if, in operating on these parts, cocain has been used (W. Phillips, Moore, Emerson, etc., N.Y. Med. Journ., Nov. 22, 1884). The increase of pulse-frequency and of blood-pressure caused by the full action of the drug are commonly preceded by a temporary depression. Respiratory power is improved under its use, but, on the other hand, excessive doses are said to cause death in animals, by paralysis of respiration or of heart-action.

Nervous System.-Recent operations with the alkaloid have demonstrated that local anæsthesia of mucous membranes may be produced by hydrochlorates of cocaïn. Schroff was the first to describe this, and Dr. Van Anrep predicted its therapeutical value in 1880. Koller, of Vienna, was, however, the first to demonstrate it clearly by experiments on man and animals with a 4 per cent. solution, applied in small quantity at short intervals (Lancet, ii., 1884.)

The local action on the conjunctiva is accompanied by an increased secretion of tears, a slight burning sensation, and then a feeling of dryness; anæsthesia of the cornea and conjunctiva follows, so that the former may be scratched and the latter pinched by forceps, without any pain or reflex movement. In fifteen to twenty minutes after instillation the pupil begins to dilate, the dilatation never becoming very great, though with a large dose lasting sometimes eight hours. The pupil reacts to light and accommodation even when dilated; but there is some paresis of the ciliary muscle. The anæsthesia varies in extent and duration with the amount and frequency of application. Dr. Bettman says that 2 minims of a 4 per cent. solution caused anæosthesia of the cornea in two minutes; this remained complete for ten, and died away in fifteen minutes (N. Y. Med. Journ., Nov., 1884). The anæsthesia, however, may be kept up by another dose; and by this frequent instillation the iris may be annesthetized. Local anæsthesia has likewise been produced in the 
mucous membranes of the nose, fauces, gums, larynx, urethra, vagina, and rectum, for which regions solutions of 10 to 20 per cent. and upwards may be required. Emerson states that no action is exerted on the skin unless it is abraded (N. Y. Med. Journ., Nov., 1884).

Espinosa reported headache, heat, and sleeplessness from observations on himself; but others have noted more agreeable stimulation of the nervous system with pleasant hallucinations or mental excitement. Ott, after chewing 60 grammes of leaves, noted loquacity, high spirits, brilliancy of eyes, and desire of active movement; afterwards headache, drowsiness, and disturbed sleep (Record, 1876). In the lower animals small doses stimulate sensibility, and may even cause convulsion, whilst toxic amounts paralyse; large doses induce loss of co-ordinating and of motor power, making it probable that the posterior columns of the spinal cord are most affected. The observations of Poppig, attributing to it great narcotic power, have not been generally credited, but Mr. Caudwell, experimenting on himself with valoid of coca, found that 3 drachms caused profound sleep for four hours, while 1 ounce induced insomnia: with hydrochlorate of cocaïn, he found that $2 \frac{1}{2}$ grains caused insomnia, with increased capacity for exertion; 3 to 5 grains produced giddiness in addition and intense frontal headache; the pupils were dilated for six hours (B. M. J., i., 1885)).

Confirmed coca eaters in their own country are described as haggard, gloomy, and of listless, solitary habit.

Nutrition. - The influence of the drug on nutrition is important, being, doubtless, allied to that of coffee and tea, and availing to lessen waste of tissue and assist in supporting strength on less food than would otherwise be needed. The alkaloid has been reckoned about six times as effective as caffein.

The experience of mountaineers in the Andes especially illustrates this fact, and the analyses of Carter and A. Bennet show reduction in the amount of urea excreted (B. M. J., 1875). On the other hand, Espinosa reported increase in excretion of urea and of carbonic acid, contradictions not yet satisfactorily explained; and the careful experiments of 
Dowdeswell gave altogether negative conclusions (Lancet, i., 1876). These contradictory results may depend on the fact, that dried coca leaves are not so active as the fresh; the alkaloid seems to become decomposed in the leaves.

SYNERGISTS.-Tea, coffee, and special nerve-stimulants.

ANTAGONISTS. - Metallic salts and mineral ucids are chemically incompatible with the infusion and fluid extract of coca, the acids causing formation of benzoic acid and another alkaloid (ecgonin). Physiologically, the agents which increase waste oppose its action.

Therapeutical Action. - Extermal. - Pain. - The local anæsthetizing action of cocain has led to its employment in many conditions in which pain is a symptom, or in which painful operations have to be performed.

Thus in eye diseases, Koller recommended it in conjunctivitis, and diseases of the cornea, associated with photophobia, especially in herpes corneæ. Instillation of a few drops of a 2 per cent. solution relieved the pain and photophobia, though he observed no beneficial effect on the course of the malady (op. cit.). These observations have been supported by Howe, Bader, Power, and others (Lancet, ii., 1884), and Emerson remarks that though cocain contracted the vessels of an inflamed and thickened conjunctiva, it had no effect on those of a vascular cornea, perhaps because the latter contained no muscular coat (op. cit.). It is chiefly in ophthalmic surgery that cocain has found its application. After the repeated instillation into the eye, as described above, operations for lachrymal stricture, tumour in the lid, strabismus, cataract, and those for the removal of foreign bodies in the coruea, for pterygium and staphyloma, have been performed without pain in most instances: in some a little pain was felt; its degree depending on the amount of alkaloid instilled. No irritation or subsequent ill effects have been noticed from the use of the drug. Cocain also removes the pain caused by silver nitrate and the cautery. In the nose, the inferior turbinate bones have been eauterized without pain after the local application of the drug (Butlin, Bettman, N. Y. Med. Journ., 1884). 
For the larynx, cocain may be employed either in the form of solution or spray. It permits the examination of a sensitive throat, and allows the easy removal of growths from the vocal cords (P. James, F. Semon, Lancet, ii., 1884; A. Smith, N. Y. Med. Journ., 1884). It relieves also the dysphagia of phthisical laryngitis, so that food may be taken with comfort; and its application in crystal to the gum has rendered painless the extraction of a tooth.

Internal urethrotomy has been done under its local use, and vaginismus and hæmorrhoidal pain have also been relieved.

Though the action of the alkaloid on the unbroken skin is but slight, yet small operations may be performed after a local hypodermic injection ( $\frac{1}{2}$ grain). Superficial abscesses may thus be opened, and warts removed or cauterized.

\section{Therapeutical Action.-Intemal.-Over-fatigue-} Debility.-There is much recent evidence that coca lessens the sense of fatigue under exertion-for instance, the remarkable walks and mountain ascents of Sir R. Christison. After proving that a sixteen-mile walk was sufficient to fatigue him, he chewed sixty, or, on another occasion, ninety, grains of the leaves in the middle of the exercise, and found that his sense of fatigue was removed, and he accomplished the return journey with perfect ease (B. M. J., 1876). Such good results have not been obtained by all observers, but Surgeon-Major Charles reports somewhat similar effects on an ascent of Mont Blanc, and especially noted relief to thirst: also the drug seems to have special power in obviating the effects of a too rarefied atmosphere. A sportsman describes marked improvement in steadiness of nerve and of heart-action under its use: he took 2 ounces of tincture (Lancet, ii., 1876). To weakly subjects, easily fatigued, and to convalescents, I have often given it with advantage. Dr. Squibb has given it to relieve undue drowsiness, and found that 3 drachms of fluid extract did this about as well as 3 grains of caffein.

The hydrochlorate of cocain has been successfully used in exhaustion from sunstroke, loss of blood, and diarrhoea (Pharm. Journ., 1884).

Besides these uses of coca, Hicks has recently reported 
advantage from it in back-ache, when the urine is high-coloured and contains excess of uric acid and urates; in dyspnoea and palpitation from muscular weakness, in mental exhaustion and "low spirits," as well as in sick headache and in alcoholic craving (N. Y. Med. Journ., Feb., 1884).

PREPARATIONS AND DOSE.-The Vin Mariani $i$ la Coca is a popular and an agreeable cordial. Tincture ( 1 in 6$)$ : dose, 10 to $30 \mathrm{~min}$. Extract. coca liquid ( 1 in 6): dose, 10 to $30 \mathrm{~min}$. Extract. (foliorum), 5 to $15 \mathrm{gr}$. Valoid of Coca ( 1 part equal to 1 part of fresh leaves) : dose, 1 to $3 \mathrm{dr}$. Elixir (1 in 6), 1 to $4 \mathrm{dr}$.

Cocrinu hydrochlorcts: dose, 5 to $20 \mathrm{~min}$. of a 1 per cent. solution in water. For ophthalmic purposes cocain may also be dissolved in melted vaseline or castor oil ( 1 in 20): or prescribed in dises (Savory \& Moore).

For local application to mucous membranes, solutions of 2 to 20 per cent. and upwards are employed.

There is reason to think that preparations of the fresh leaf are much more dependable than those of the dry; in Peru they are considered inert if kept for more than three months. Dr. Squibb asserts that a large proportion of the commercial leaf is valueless from want of care in packing, etc.; it requires as much attention as the tea-leaf (Pharm. Journ., 1884). 


\section{LINACE $A$.}

\section{THE FLAX FAMILY.}

A sMaLL order of exogens, almost exclusively herbacoous, and generally of slim delicate habit. The leaves are simple and undivided. The flowers are regular and pentamerous, with all the parts free, excepting the five carpels, which are consolidated into a superior and few-seeded ovary, ripening into a capsule. This order is specially characterized in the hypogynous sub-class by the carpels completely disuniting when ripe, and leaving no central axis.

\section{Pharmaceutical Species.}

Linum usitatissimum . . . . Common flax.

Linum catharticum .. . . . . . Purging flax.

\section{LINUM USITATISSIMUM.}

\section{(Common Flax.)}

Description.-An annual, indigenous to Egypt. The wiry stems rise to the height of 2 to 4 feet; the numerous leaves are alternate and lanceolate. The blue flowers are about an inch across, borne in terminal and corymbose panicles, numerous, and fugitive; the capsules are globular, the size of a pea, and contain ten brown glossy seeds. They are about $\frac{1}{6}$ inch long, ovate, flattened, pointed, and oblique at the apex, inodorous, with mucilaginous, rather bitter taste; the epithelium swells in water.

ACTIVE INGREDIENTS.-The albumen of the seeds yields to boiling water an inodorous and almost tasteless mucilage, $\mathrm{C}_{12} \mathrm{H}_{20} \mathrm{O}_{10}$, convertible by nitric acid into mucic acid. By expression the kernels or cotyledons yield a bland, inodorous, and sweetish oil, sp. gr. 0.939. It is much more soluble in alcohol than olive-oil, and, as it is one of the "drying oils," after proper preparation it loses the original unctuousness: it 
is not congealed except at a temperature below $0^{\circ} \mathrm{F}$., and boils at $600^{\circ} \mathrm{F}$. It yields, on saponification, glycerine and linoleic acid, $\mathrm{C}_{16} \mathrm{H}_{26} \mathrm{O}_{2}$, with a small amount of oleic, palmitic, and myristic acids.

The seeds, before being submitted to any process, contain about $\frac{1}{5}$ of mucilage and $\frac{1}{6}$ of oil.

Physiological Action. - Linseed is emollient and demuloent, and the oil is a mild laxative. It has been thought available for human food; but when used as an article of diet, it relaxes the digestive organs, and produces a viscid, slimy mucus, and a deleterious acid in the primæ viæ. These effects may be obviated by the addition of bitter extractive, but linseed, in any case, affords but little nourishment, and is found to impair digestion, as long ago noticed by Galen. Cattle fatten upon the refuse of the seeds.

THERAPEUTICAL ACTION.-External.-The most ordinary use of linseed is as a soothing emollient poultice, for which purpose the powdered or crushed seeds are mixed with boiling water.

The oil, mixed with lime-water, commonly known as Carron oil, from the large ironworks where it was most used, is a cheap and fairly good application for recent burns. Without the limewater, linseed oil would not be suitable, for it stiffens the skin as it dries, and may become acrid and irritating.

A decoction of the seeds contains much mucilage and some of the oil, and forms a useful lotion for irritable skin conditions, and an injection for abrasions or ulcerations of mucous membranes. The oil is well added to enemata when hrmorrhoids are present.

Therapeutical Action. - Intemel. - The infusion, "linseed tea," though a simple, is often a valuable adjuvant in cases of irritation of the fauces, bronchial catarih, strangury, and congestive disorders of mucous membranes generally. Blackerby writes of the oil as "one of the best remedies in phthisis;" he gives it with honey and whiskey as a substitute for cod-liver oil (Record, 1881). Whon good and pure, obtained by expression of the seeds, not by hent, which renders it dis- 
agreeable, it is sometimes a corrective of habitual constipation; a little tincture of rhubarb added, assists its action.

PREPARATIONS AND DOSE.-Limi semina. Lini farina. Lini oleum. (The dose of the fresh oil as an aperient is from 1 to 2 oz., but it is seldom used.) Cataplasma lini. Infusum lini: dose, ad libitum. Linseed jelly may be made with $\frac{1}{2} \mathrm{lb}$. of the seeds to 3 pints of cold water; simmer for two hours, strain, sweeten, and flavour.

\section{LINUM CATHARTICUM. (Purging Flax.)}

(Not Officinal).

DESCRIPTION.-An annual, native of most parts of Europe, England included. The stems, which rise many together from the same root, are 6 to 8 inches high, much forked at the upper part, the ends of the branches drooping gracefully till the flowers open, when they become erect, and remain so until the capsules are ripe. The lower leaves are opposite; all are oval or oblong. The flowers are minute and pure white.

This plant gives out its virtues to water, which becomes of a greenish-brown colour, and tastes somewhat warm and bitter.

ACTIVE INGREDIENTS.-The active principle is said to be the neutral body linin, discovered by Pagenstecher. It occurs in shining, silky, white crystals, which are changed to violet blue by concentrated sulphuric and phosphoric acids; they are soluble in alcohol, ether, and chloroform, almost insoluble in water; yet they have the same persistently bitter taste which belongs to the watery infusion of the plant.

Physiological Action.-The experiments of Schröder have put it beyond doubt that linin will produce, in exaggeration, the drastic irritant effects on the intestinal canal which are characteristic of purging flax when administered in other forms.

THERAPEUTICAL ACTION. - Two ounces infused in a pint of water may be recommended to delicate subjects as a gentle purgative.

PREPARATION AND DOSE.-Infilsum: dose, 1 oz: or upwards. 


\section{MALVACEAE.}

\section{THE MALLOW FAMILY.}

Ax order of about a thousand species of exogens, most of which are tropical and arborescent. The leaves are alternate, stipulate, simple, and either undivided, or more or less deeply lobed and cut. The flowers are regular, rosaceous, and pentamerous; the stamens are very numerous and monadelphous, and have reniform one-celled anthers. The fruit, though varying, is always dry; capsules and achenia are the usual forms. No instance of a deleterious plant is known in this order, which is remarkable for its mucilaginous qualities, and in several instances for its fibre.

\section{Phomaceutical Species.}

$\begin{array}{lllll}\text { Althæa officinalis } & \ldots & \ldots & \ldots & \text { Marsh-mallow. } \\ \text { Gossypium herbaceum } & \ldots & \ldots & \text { Cotton. }\end{array}$

ALTHAA OFFICINALIS. (Marsh-MaLlow.)

(Not Officinal.)

DESCRIPTION. - A strong ligneous perennial, of which the stout erect stems attain the height of 6 to 9 feet. The leaves are more or less five-lobed, irregularly serrated, and of a peculiar hoary green colour, owing to their being covered, like the stems, with a soft velvet-like pubescence. The flowers, 
produced in short axillary clusters, are about an inch in width, lilac with dark centre, and very abundant: the fruit consists of. a whorl of rather large achænia. This plant grows wild in most parts of Europe, Britain included, and chiefly near the sea.

The root is the part used in medicine, preferably at the age of two to three years; it occurs in nearly cylindrical pieces, 5 to 8 inches long and $\frac{1}{2}$ inch in diameter, white both inside and out, and marked with circular scars and longitudinal folds; it has a slight peculiar odour and sweetish taste. It is apt to change and become mouldy in a damp atmosphere.

ACtive IngRedients. - (1) Asparagin (an alkaloid, which will be described with the liliaceous plant, asparagus); and (2) Mucilage, starch, pectin, phosphate of calcium, sugar, and a trace of fatty oil and tannin found in the outer bark.

Therapeutical action. - Extemal. - The mucilage which is copiously extracted from althæa by aqueous decoction has certain undoubted uses; e.g., as a fomentation for inflamed surfaces; or as an enema in tenesmus; or, according to Montgomery, as a vaginal injection in cases of diffeult labour: a mucilaginous poultice is made from the roots.

Therapeutical Action.-Intemal.-The French prepare with althæa pectoral lozenges, which somewhat relieve bronchial and laryngeal irritation. A decoction of the root and stem has been resorted to as a remedy in flatulent colic; and a lukewarm decoction of the flowers has been used to relieve irritant cough.

In inflammatory irritation of the throat, stomach, and intestines, it exercises a mechanical protective power: when, however, it is assumed that by internal employment it can exert the same kind of action upon the trachea and brouchi, or upon the genito-urinary tract, the assertion stauds on a different basis; for it implies that mucilaginous matter has in similar action after absorption into the blood as upon immediate contact with tissues-which is improbable. 
PREPARATIONS AND DOSE.-Sympus: dose, $1 \mathrm{fl}$. dr. to 1 fl. oz. Decoctum: dose, ad libitum.

The German "Brustthee,"-French "espèces pectorales,".contains marsh-mallow, liquorice-root, orris, coltsfoot, mullein, and star-anise.

"Quatre-fleurs," - contains mallow, marsh-mallow, mouse-ear, coltsfoot, red poppy, violet, and mullein.

The so-called "Pâte de Guimauve" contains no mallow, but acacia (Stillé).

\section{GOSSYPIUM HERBACEUM.}

(CotTon.)

DESCRIPTION.-The cotton or " cottor-wool" of commerce is the produce of several different species of this genus. They are large shrubs, ligneous perennials, or small trees; the leaves differ in size and figure, but are always more or less divided into pointed lobes; the rose-like flowers are axillary, 2 inches across, yellow or pink, with dark centre; and are succeeded by woody many-seeded capsules. The cotton consists of long tubular filaments that spring from a portion of the testa, and are densely entangled around the seeds.

ACTIVE INGREDIENTS.-Cotton is a modification of lignin or cellulose $\left(\mathrm{C}_{6} \mathrm{H}_{10} \mathrm{O}_{5}\right)_{n}$, and in all its ordinary chemical properties corresponds with woody fibre. It is insoluble in water, alcohol, ether, oils, and vegetable acids. It is dissolved by strong alkaline leys, and decomposed by strong mineral acids.

Physiological Action.-This seems to be exerted mainly upon the uterine system, which is stimulated by the drug.

Therapeutical ACTiON.-Exterml.-Cotton-wool itself is employed in the treatment of burns and scalds, for allaying' 
pain, diminishing inflammation, and excluding the air; also as an artificial membrana tympani, and for protecting the meatus against cold, wet, loud noises, etc. It has also been used as a topical application in cases of erysipelas, and for inflamed joints in gout or rheumatism. In these cases it should be overlaid with oiled silk or gutta-percha paper, so that the parts may be kept in a state of perspiration. As a dressing for surgical cases it has been saturated with 5 to 10 per cent. of salicylic or benzoic acid, iodoform, etc. Cotton-wool is used also for extempore pessaries, etc.; it can be made "absorbent" by removing all fat. Dr. Von Brun has introduced it freed from grease as a dressing for wounds; and Prof. Tyndall recommends it as a filter in respirators.

Absorbent gauze and cotton-wool tissue are much used in wound-dressing, and in the form of balls in place of sponges.

The oil expressed from the seeds is very bland, and may be applied to all the same purposes as almond oil.

THERAPEUTICAL ACTION.- Internal.-The root of the cotton-plant in decoction has long been employed in the East Indies as a demulcent in cases of strangury and gravel.

In the Southern United States, according to the reports of Drs. Bouchelle and Shaw, a similar preparation is resorted to for causing or intensifying uterine contraction, for which purpose it was much valued by the female slaves, and used for dysmenorrhoea and suppression of menses from cold, as well as for procuring abortion (Amer. Journ. Med. Sc., 1841 ; Charleston Med. Journ., xi., 118). Some of these statements would indicate its being more energetic even than ergot: it has recently been recommended as a substitute for that drug, being cheaper. Prochovrick finds its expulsive action not so great, but its risk of tetanizing less.

It proved useful in several of my own cases suffering from hremoptysis, and is spoken well of in the hremorrhage of abortion (Record, 1884).

An emulsion prepared from the seeds is, in the West Indies, considered useful in dysentery and in chest disorders; in India it is said to be employed as a gralactrgogruo. 
PREPARATIONS AND DOSE.-The decoction of the root is made by boiling 4 ounces of the inner bark in a quart of water, until reduced to one-half: dose, a wineglassful every twenty or thirty minutes.

Tinctur Gossypii Radicis (Squire), (dried bark of the root of the cotton plant) 1 ; proof spirit, 4 ; digest for seven days: dose, 1 drachm, three times a day, as an emmenagogue and parturient.

Cotton-wool is medicated with iodine, iodoform, carbolic acid, salicylic acid, eucalyptol, corrosive sublimate, arnica, glycerine, and with perchloride of iron; and is used in the preparation of pyroxylin.

\section{PYROXYLIN, OR GUN-COTTON. $\mathrm{C}_{18} \mathrm{H}_{23}\left(\mathrm{NO}_{2}\right)_{7} \mathrm{O}_{15}$.}

When cotton is immersed for three minutes in a mixture of nitric and sulphuric acids, 5 fluid ounces of each, then well washed, and dried upon a water-bath, it is converted into p!rroxylin. The fibre does not alter in appearance, but is found to have acquired highly explosive properties, while its weight has increased 70 per cent. At a temperature of about $300^{\circ}$ pyroxylin inflames and explodes; but by immersing in water, or keeping wet, the explosive power of the cotton is held in abeyance, and in this condition it may be carried any distance. 


\section{COLLODIUM. \\ (Collodion). $\mathrm{C}_{36} \mathrm{H}_{22}\left(\mathrm{NO}_{48}\right) \mathrm{O}_{30}$.}

Collodion is a colourless, inflammable fluid, having an ethereal odour, and rapidly drying upon exposure to the atmosphere. When the drying is complete, pyroxylin is left as a transparent film, insoluble both in water and rectified spirit.

Collodion is a solution of pyroxylin in ether and alcohol. It - is prepared by taking pyroxylin, 1 ounce; ether, 36 fluid ounces; and rectified spirit, 12 fluid ounces; mixing the two latter, and allowing the pyroxylin to dissolve in them.

It is usefully employed in cases of cracked nipple, fissures of the anus, superficial ulcers of various kinds, and superficial burns and wounds, to all of which it forms a protective covering; also in erysipelas; as a stopping it is applied upon cotton to carious teeth; and is likewise an excellent application to slight cuts, such as are made by a razor while shaving. It also forms a good coating for pills.

The collodium Alexile, "flexible collodion," is made by adding to 6 fluid ounces of collodion 2 drachms of Canada balsam and 1 drachm of castor oil : it must be preserved in a well-stoppered bottle.

When carbuncles, boils, inflammatory pimples, erysipelatous surfaces, etc., are painted over with the flexible preparation, its value becomes very evident. 


\section{A URANTIACEAE.}

\section{THE ORANGE-TREE FAMILY.}

Exogenous evergreen shrubs and small trees, natives chiefly of the East Indies, with alternate, often compound, leaves, filled with translucent oil-cysts; long green spines are often developed from the axils. The flowers are tetramerous or pentamerous; the petals free; the hypogynous stamens either few or many; the superior ovary is many-celled, and ripens into a succulent fruit, the carpels of which are consolidated, while the seeds are attached to the axis. The species of this order are not numerous; they have often excellent fruit, and none are deleterious.

Citrus aurantium $\quad \ldots \quad \ldots \quad \ldots \quad \ldots$ Sweet orange.

Citrus vulgaris .

Citrus limonum $\quad \ldots \quad \ldots \quad \ldots \quad \ldots$ Lemon.

Eglë marmelos . . . . . . . Bael.

\section{CITRUS AURANTIUM.}

(The Siwhet Orangir.)

DESCRIPTION.-The stature attained by this tree is $12 \mathrm{nr}$ 15 feet. The leaves have an ovate pointed lamina, articulated to the broad petiole. The flowers consist of an urceolate calyx, four white petals an inch in length, twenty or thirty stamens, the filaments of which are somewhat polyadelphons, and a large columnar pistil ; the ovary ripens into the golden-yellow spherical fruit.

ACTIVE INGREDIENTS. - The flowers contain volntile oil (called oil of Neroli), bitter extractive, and other substances: 
The rudimentary (umipe) fruit contains volatile oil, a bitter extractive (hesperidin), bitter astringent matter, citric and malic acids. The rind of the ripe fruit contains a volatile oil $\left(\mathrm{C}_{10} \mathrm{H}_{16}\right)$, isomeric with oil of turpentine, hesperidin, and a little gallic acid. The juice contains citric and malic acids, mucilage, albumen, sugar, citrate of lime, and water.

Physiological Action.--Like other substances of an aromatic nature, orange-peel produces in the stomach a grateful sense of warmth. M. Imbert-Gourbeyre states that in the South of France, where the orange is largely cultivated for the sake of the rind, the persons who are engaged in removing it are affected in a singular manner. Their hands become inflamed with an erythematous, papular, or vesicular eruption; they suffer from headache, dizziness, timnitus aurium, deafness, neuralgia, oppression in breathing, constriction of the throat, nausea, pyrosis, irritation, and thirst; are disturbed by dreams, and experience cramps and twitchings of the muscles, with, occasionally, convulsions of epileptic character. These symptoms continue only so long as the occupation is pursued (Gaz. Méd. de Paris, 1853).

Therapeutical ACTION.-Orange-peel stimulates the digestive system, and is valuable as qualifying the action of other bitters in the treatment of dyspepsia. It is most usually employed to cover the taste of quinine, and associated with purgative medicines of griping character, or when the bowels are distended with flatus. In febrile inflammatory complaints, orange-juice allays thirst and diminishes the sense of heat. M. Inbert-Gourbeyre states that he has successfully employed the essential oil for hysterical and other nervous affections.

The leaves have been used, in the form of powder and infusion, in certain spasmodic disenses, and have been prescribed for hysterical females in place of ordinary tea.

The unipe seeds, while hard and dry, are rounded in a luthe, and constitute the "issue-pens" of the shops.

PREPARATIONS AND DOSE.-Aur(untii Horis uqun: Ilose, 1 to 2 oz. Symunus anrentii floris: dose, 1 to $2 \mathrm{dr}$. 


\section{CITRUS VULGARIS, OR CITRUS BIGARADIA.}

(The Seville or Bitter Oraxge.)

This is a smaller tree than the preceding; the leaves are more elliptical; the flowers are more decidedly white, and yield a stronger perfume; the rind of the fruit is not yellow, but deep-orange, and the pulp is acid and bitter. A good distinctive character is found also in the remarkable unevenness of the fruit.

The rind (murntii cortex) contains a volatile oil, and is employed as a constituent of the compound infusion, the mixture, and the compound tincture of gentian. This and other parts of the plant are used for the scme purposes as the sweet orange.

PREPaRations and Dose.-Infilisum: dose, 1 to 20 oz. Infusum anurentii compositum: dose, 1 to $2 \mathrm{oz}$. Sylypus : dose, 1 to 2 dr. Tinctura: dose, 1 to $2 \mathrm{dr}$. Vimum anrantii (used for the vinum ferri citratis and vinum quinæ). Tincture murentii recentis: dose, 1 to $2 \mathrm{dr}$. Orange-flower water is also prepared from it (v. p. 162).

\section{CITRUS LIMONUM.}

\section{(The Lrimax.)}

DESCRIPTION.-The appearance and leaves of this tree nearly correspond with those of the orange; but the flowers, instend of being pure white, are externally purplish. The fruit is oblong, pointed at each extremity, pale yellow, and with an uneven surface. The pulp is acid, and the oil-cysts in the rind are concave, whereas in the orange they are convex. The recent and outermost part of the rind of the fruit has an aromatic, bitter taste; the odour is strong and peculinr.

ACTIVE INGREDIENTS.-These qualities depend upon the' presence of a volatile oil, which, when pure, is colourless or 
pale yellow, limpid, fragrant, and of warm, bitter taste. It is soluble in anhydrous alcohol; less so in rectified spirit. The composition is $\mathrm{C}_{10} \mathrm{H}_{10}$ - that is to say, it is isomeric with the oils of turpentine, savin, juniper, and copaiva. The juice of the fruit consists of citric acid $\left(\mathrm{H}_{3} \mathrm{C}_{6} \mathrm{H}_{5} \mathrm{O}_{7}, \mathrm{H}_{2} \mathrm{O}\right)$, sp. gr. 1.039, malic acid, gum, bitter extractive, and water. Of these substances the citric acid, of which there are about 32 grains in every ounce of lemon-juice, is the most important. It may be obtained in colourless crystals, of which the right rhombic prism is the primary form: they are very soluble in water, less so in rectified spirit, and insoluble in pure ether.

Physiological Action.-Oil of lemon-peel is stimulant, carminative, and diaphoretic, and hence acts as a grateful stomachic. Applied externally, it is stimulant and rubefacient. The juice of the ripe fruit is refrigerant and antiscorbutic; and similar, but feebler, properties pertain to citric acid. The juice, mixed in water, is employed as a beverage. It allays thirst, diminishes heat, abates undue perspiration, and quickens the action of the kidneys.

THERAPEUTICAL AC'TION.-The chief employment of oil of lemon-peel is as a carminative, but it is used generally in connection with medicines of more energy.

Rheumatism.-Lemon-juice has been recommended as a remedy in acute rheumatism and in gout, and has been successfully employed in England, France, Italy, and the United States. At present, however, it is not looked upon with much confidence ( $c$. Citric Acid). Its effects are about the same as those of a mild alkaline treatment.

Narcotic Poisoning.-Lemon-juice is valuable in eases of poisoning by narcotic substances, such as opium. After the poison has been removed from the stomach, its effects may be partly counteracted by freely drinking the juice, or citric acid in solution.

Scurvy.-By far the most inportant use of lemon-juice is in scurvy. Both it and the juice of the lime have been adopted, after much experience, as the most convenient prophylactic and 
curative in sea-scurvy; and, as such, lime-juice is ordered to be carried in stock by the ships of the Royal Navy and merchant service.

But it has long been known that neither citric acid nor any substance peculiar to the lemon is needed for the preventiou and cure of this disorder: and given alone, the acid has proved but feebly curative. Many other fruits and regetables ${ }^{1}$ are antiscorbutic, always provided that their juices are in a fresh state. So markedly is this the case that, instead of attributing the cure to the acid, some have ascribed the antiscorbutic effects to the citrates and malates of potash which the juices contain. This theory, however, has proved no more stable than the other; and at present it can only be said that various fruits and vegetables are both preventive and curative in scurvy, and that lemon-juice is a very convenient form of remedy, being easily preserved by the addition of a small proportion of spirit (10 per cent.).

It is of service also in febrile and inflammatory complaints, and in hæmorrhagic conditions, such as purpura, being generally given as lemonade; it has been found efficient in malarial fevers.

Combined with potash, it forms a citrate, which is mildly diaphoretic and diuretic, and often allays restlessness and wakefulness in pyrexia.

It is adapted, also, for lithic acid deposits, but is sometimes objectionable in phosphatic ones.

As a perfume, oil of lemon is a useful adjunct to sulphur ointment and to evaporating lotions.

PREPARATIONS AND DOSE.-Olenm limonis: dose, 1 to 4 min. Symums: dose, 1 to $2 \mathrm{dr}$. Tincture: dose, 1 to $2 \mathrm{dr}$. Sucrens: dose, $\frac{1}{2} \mathrm{dr}$. to $4 \mathrm{oz}$. Acichm citricum: dose, 10 to $30 \mathrm{gx}$. in a wineglassful of water.

ADULTERATIONS.-Much of the so-called lemon-juice of commerce is said to be prepared from tartaric acid flavoured.

1 1)r. Buzzard (Article "Scurvy," Reynolds' System of Merlicine, vol. i.) mentions oranges, lemons, limes, cabbage, lettuec, potutoes, onions, musturd and cress, dandelion, sorrel, scurvy grass, cactus, grapes, apples, sauerkinut, etc. 


\section{ÆGLË MARMELOS.}

(Bael, or Iriman Bael.)

DESCRIPTION.-This tree is a native of all parts of the East Indies. It is thorny, with ternate leaves, the white flowers being followed by large globular fruits, covered with a smooth, hard rind, almost a shell. Internally, the ten to fifteen cells contain, besides the seeds, much transparent viscid pulp, which, when dry, becomes horn-like; while fresh, it may be drawn out into threads, 3 or 4 feet in length, and so attenuated as to be scarcely visible. As found in European commerce, the fruit is mostly broken, dry, and with some pulp adhering to the rind.

ACTIVE INGREDIENTS.-No accurate analysis of the composition of bael has yet been made. The rind contains mucilage and pectin, but no tannin; the ripe fruit, however, contains an astringent principle allied more or less to tannic acid, to which the active properties appear to be due.

Physiological Action.-Little is yet known on this point. Christison describes it as " an incomprehensible remedy, having no aroma or taste, and certainly no astringency." The liquid extract of the fruit is a mild aperient when given in moderate doses, but when in large ones is an active cathartic.

Therapeutical Action.-In India the fruit is considered a valuable remedy in dysentery and diarrhoca, and all atonic affections of the botvels; also in irritation of the mucous membrane of the stomach, and as an injection in leucorrhoea, etc. It relieves diarrhoca without constipating the bowels, and is recommended also for the relief of habitual constipation. "It is not only astringent when diarrhoer exists, but possesses the singular property of being also aperient if 
the bowels are costive" (Waring). I have administered it in some obstinate cases of chronic dysentery and diarrhœa, with the best results.

Six J. Fayrer has published an account of the drug and it's uses, especially drawing attention to the necessity of employing good ripe fruit, and to its value in chronic, not in acute dysentery (Med. Times, i., 1878). Christison relates two illustrations of its value in such cases (ibid, ii., 1878).

PREPARATION AND DOSE.-Extractum belce liquidum: dose, $1 \mathrm{fl}$. dr. to $\frac{1}{2} \mathrm{fl}$. oz. 


\section{STERCULIACEAE.}

\section{THE CACAO FAMILY.}

A ccrious and interesting order of exogenous trees, shrubs, and herbaceous plants, mainly, but not exclusively, tropical. In some respects it resembles the Malvacer, but is distinguished by the two-celled anthers and the non-columnar filaments. The leaves are alternate and simple; the flowers usually small, their elements in fours or fives; the stamens hypogynous; and the superior ovary commonly ripens into a many-seeded capsule. The medicinal species are-

$\begin{array}{lllllll}\text { Theobroma cacao } & \ldots & \ldots & \ldots & \ldots & \ldots & \text { Cocoa. } \\ \text { Sterculia acuminata } & \ldots & \ldots & \ldots & \ldots & \text { Kola nut. }\end{array}$

\section{THEOBROMA CACAO.}

(COCOA.)

DESCRIPTION.-A small tree, indigenous to Demerara, where whole forests are composed of it. The large lanceolate leaves are entire; the minute reddish flowers are axillary; the five petals are vaulted below, ligulate above; the fifteen stamens are monadelphous at the base; the ripe capsule is 7 or 8 inches long, oval, pointed at each end, flesly while recent, ligneous when dry, and filled with the seeds-the well-known "cocoa-beans" of commerce. These are $\frac{1}{2}$ to $\frac{3}{4}$ inch long, oblong, flattened, with a prominent raphé along one edge, and brownish in colour. 
ACTIVE INGREDIENTS.-The oleum theobromæ, B.P., is a concrete oil obtained from the ground seeds by expression and heat, and appears to be composed chiefly of stearin with a. little olein. The consistence is that of tallow; the colour yellowish; the fracture is clean; presenting no indications of foreign matter; exposure to the air does not render it rancid; in water it is insoluble, but in alcohol, ether, and oil of turpentine it dissolves; it melts at a temperature of $95^{\circ} \mathrm{F}$; has a bland, agreeable flavour, and an odour resembling that of chocolate. Good shelled cocoa-beans contain about 52 per cent. of this oil.

The seeds also contain a substance of different character, which must be taken into account in estimating the effects of cocoa and chocolate used as a beverage, viz., theobromin. This alkaloid, $\mathrm{C}_{7} \mathrm{H}_{8} \mathrm{~N}_{4} \mathrm{O}_{2}$, forms microscopic white rhombic needles with a strong bitter taste, which develops slowly on the tongue; it is soluble in water, less so in alcohol, very little in ether. Hot solutions become opaline as they cool. The percentage of theobromin in cacao seeds has been variously stated, but is probably small, between 1 and 2 per cent.; it is allied to caffein.

Physiological Action.-The oil of cacao can only be considered as a bland oleaginous food.

Theobromin is a powerful poison, which may be absorbed either from the stomach or the subcutaneous tissues, and the action of which has been especially investigated by Mitscherlich. In rabbits the early symptoms were grinding of the teeth, slowing of respiration (often as much as to the third or the fourth of the normal rate), and increased rapidity of pulse, with weakened heart-pulsation. The further symptoms varied according to the rapidity of absorption: when this was great, there were convulsions of spinal origin; but when absorption was slow, there was gradual loss of voluntary power. The secretions, appetite, throat, etc., did not seem affected. Retehing and vomiting were occasional symptoms.

In frogs there was great distension of the lung's and of the bladder. Death occurred with phenomena of vagus- and spinalcord-paralysis. Dissection showerd appenrances corresponding 
to the symptoms: when death occurred very rapidly, there were long-continued irritability of the voluntary muscles, and persistence of peristaltic movements; when the poisoning had been slower, the heart and muscles lost their normal irritability, the ventricles were contracted, the auricles, great venous trunks, and small vessels, in all parts of the body (especially in the mucous and serous membranes, the brain, liver, and kidneys), were hyperæmic; there were extravasations of blood almost always in the lungs, and frequently in the mucous membranes and under the peritoneal covering of the bladder; the blood was dark-red. Mitscherlich concluded that theobromin was a poison of the same general kind as caffein, but fatal in much smaller doses.

THERAPEUTICAL ACTION.-The present employment of the oleum theobromæe is in the preparation of suppositories containing tannic acid, mercury, morphia, or lead. Theobromin has not yet been applied to any therapeutical purpose, but could probably be used with advantage in nervous affections of the same kind as those which are relieved by caffein.

In regard to the dietetic use of cocoa and chocolate as substitutes for tea and coffee, it may be said that the chief advantage of the two former is the predominance of nutrient over stimulating ingredients in their composition, in virtue of the sugar and starch which they contain. Cocoa and chocolinte are really foods, with only so small a proportion of alkaloidal stimulant as probably just serves to render digestion more active.

\section{STERCULIA ACUMINATA. (KoLA NiT-Gotrot.)}

\section{(Not Officinal.)}

DESCRIPTION. - The true or female kola nut is yielded by the S. Acuminata, a tree 30 to 60 feet high, resembling the chestnut, growing wild on the West Coast of Africa, and now naturalized in the East Indies. A false or male kola, somewhat like this, is prodnced by a species of Garcinia, and contains 110 
alkaloids. The seed (scarcely to be called a nut) has a kernel about 2 inches long, like a peanut, but with a groove insteacl of a point at the germinal end. The pod may contain as many as five white or red seeds: the taste is sweet, astringent, afterwards bitter. The nut is used on all political and social occasions in West Africa, as a present, a symbol, a charm, a sacred thing, ete.

ACTIVE INGREDIENTS. - According to the exhaustive analyses of E. Heckel and Schlagdenhauffen, the dried seerls contain a larger proportion of caffein-viz., 2.348 per cent.than is found in any coffee, and in a free, uncombined state; also theobromin, 0.023 per cent.; tannin, 0.027 per cent.; fat, 0.58:3 ; with much glucose and starch and a red colouring; matter (Pharm. Journ., i., 1884).

Physiological ACtion.-Litie its congeners tea and coffee kola is said to sustain the system under great fatigne with limited food-supply: it is the ordinary stimulant of most West African tribes, owing its power mainly to the alkaloids above mentioned.

THERAPEUTICAL Action.-Kola has been recently introduced as a remedy for alcoholism, both to lessen the craving, and to relieve sickness, headache, and other aftereffects. It is said in its native country to be valuable in atonic diarrhoca, dysentery, and liver disorder: also to render foul water potable and harmless. This latter effect may be dependent on mucilage precipitating certain impurities.

PREPARATION AND DOSE.--As a sustaining beverage, I tablespoonful of the powder to a pint of boiling milk or water, or $\frac{1}{2}$ teaspoonful of the powder in any liquid after neals; as an anti-intoxicant, $1 \mathrm{dr}$. of powder in milk or water. 


\section{CAMELLIACEAE. \\ COFEEA ARABICA. \\ (Coffee.)}

DESCRIPTION.-The coffee-tree is a handsome erergreen, indigenous to Arabia, but long since diffused over the torrid zone. It is 8 or 10 feet high, with opposite ovate leares, 3 to 4 inches long, acute, and shining; the abundant white, star-like flowers, $\frac{3}{4}$ inch across, are produced in axillary clusters, and are followed by oval red fruits resembling cherries, each fruit containing two plano-convex seeds, the "green coffee" of commerce.

The seeds, when roasted, are used for the infusion or decoction which forms the domestic drink. The leares of the plant contain, however, a large proportion of the same ingredients.

ACTIVE INGREDIENTS.-Those of the seed vary according as it is raw or roasted. In the raw seed, caffein, $\mathrm{C}_{8} \mathrm{H}_{10} \mathrm{~N}_{4} \mathrm{O}_{2}$ (methyl-theobromin), is probably the only important ingredient; it crystallizes in pure white silky needles, which can be sublimed by a heat of about $350^{\circ} \mathrm{F}$.; its rapour has no smell; it has a neutral reaction, and a weak bitter taste; it is moderately soluble in water, alcohol, and ether. It combines with the stronger acids to form acidly reacting salts; dissolves, colourless, in concentrated sulphuric and nitric acids; but if the solution with the latter be evaporated, there is a reddishyellow residue, which gives, with ammonia, a brilliant purple colour.

In the raw seed, caffein exists as a tannate. In the process of roasting, as was first shown by M. Persome, a portion of this tanuate of caffein becomes transformed into methylamins. 
which (in the form of acetate) has been proved by M. Béhier (ibid.) to possess stimulant qualities (Practitioner, Oct., 1868).

The volatile and aromatic ingredients of coffee are developed in the roasting ; they are not all accurately known, but in the process, the grain swells, loses weight by drying, and is found to contain less caffein, but to develop a special aroma due to urféone, which is formed by the action of heat on chloriginate or caffeate of potash. It is oily, heavier than water, in which, when boiled, it is slightly soluble, and to which a minute quantity communicates the characteristic odour (Rabuteau). It is possible that part of the increased physiological power which roasting confers upon the seed is due to a partial change of the caffein into methylamin. M. Aubert has recently remarked that coffee-extractive free from caffein is not inert.

Coffee-tannin, or caffeic acid, is somewhat astringent. It has been obtained from cuprea bark, and conversely quinic acid has been obtained from coffee.

In addition, Payen found 13 per cent. of fat (palmitin and olein), glucose, dextrin, vegetable acid, casein, volatile oil, etc.

Absorption and Elimination.-These are both rapid, since physiological effects are quickly produced by the drug, and pass away with moderate quickness if the dose be not repeated. Whether caffein be altered in the system is not easily ascertained, because of the difficulty of detecting it in the excretions.

PHysiological Action.-Infusion of coffee is celehrated for its stimulating and refreshing effects, exciting muscular and mental activity, promoting cheerfulness, etc. Its action varies, however, according to certain conditions, especially of the stomach, so that if for any reason it be not well digested-for instance, if taken too strong, or on an empty stomach-it conses discomfort instend of the stimulus mentioned.

To some persons it always seems a more or less powerful poison, and probably even less susceptible individuals would suffer from toxic symptoms if they took it in very large doses. After, however, a full and stimulating meal or promlomgerl mental lahour, if the functions and mind are singgish. 
coffee in most people then exerts a favourable action, presumably by contracting the cerebral vessels, quickening digestion, and stimulating bile-secretion; excess, however, quickly leads to mischief in an opposite direction-dyspepsia, constipation, etc.

Coffee undoubtedly exalts the general reflex excitability : one of the consequences of this is its effect in banishing sleep; another is the production of an irritable state of the heart, in which the slightest excitement is sufficient to bring on violent palpitations. Rabuteau asserts that caffein, or long-boiled infusion in which the caféone has been dissipated, does not prevent sleep-this effect being due to the caféone-an opinion not supported by experiments given under Coca.

Nervous System.-Caffein seems to stimulate, in a direct manner, both the brain and spinal cord, the former more in man: two or three grains are apt to cause headache in susceptible subjects.

Eight grains induced tremor, excitement, delivium, confusion of thought, finally sleep; and twelve grains similar, but more exaggerated effects, with active persistent thinking (Lehmann, Pratt). Fatal doses rather diminish or even paralyse reflex functions; and Dr. A. Hughes Bennett argues that they specially paralyse the posterior columns of the cord (B. M. J., i., 1874).

Jacobi, making observations on a case with exposed brain, noted that under coffee "the mount of blood circulating in the brain is smaller, but is brought to the nerre-tissues muder increased pressure."

The action of caffein on the nervous system is specially illustrated by the recent researches of Aubert, Peretti, and others; when injected in sufficient quantities into rabbits, rats, cats, and dogs, it always exalts reflex excitability, and produces convulsion or tetanus. It also causes a rigidity of the extremities, probably dependent upon a direct netion on the muscular tissues.

The citrate of caffein in large doses produces irritant symptoms, as in a case where 1 drachm was taken: burning in the throat, faintness, epigastric pain, and tremors immediately followed; vomiting soon oceurred, with the passage of 
much urine; then collapse: the patient recovered under treatment in nine hours (Routh, Lancet, i., 1883).

Circulatory System.-A few grains of caffein g'enemlly cause increased action of the heart-in some subjects even malied palpitation; the same doses, given in some cardiac disorders, slow the pulse (Lepine), and toxic doses do this after a preliminary quickening. Binz states that, in moderate closes, it quickens the heart's action, both by its direct effect on the organ, and by exciting contraction of the arteries: the blood-pressure and the pulse-frequency are both augmented; there is rapid rise of temperature-all for a comparatively short time. In rabbits it quickens the pulse, but there are periodic intermissions, in which the heart appears to be distended. In dog's the frequency of the pulsations increases, while the blood-pressure is invariably lessened: the former effect Aubert thinks clue to an action on the excitory apparatus of the heart, the latter to paralysis of the cardiac nerves arising from the cardiac ganglia. Upon man he did not find caffein act very powerfully; a dose of 7 to 8 grains produced only confusion in the head, tremor of the hands, and quickening of the pulse.

Genito-Urinary System.-Coffee has the reputation of lowering the sexual appetite, and of lessening the menses. On the kidneys, in most persons, it acts as a decided stimulant, greatly increasing their secretion; and Dr. Brakenritge, in an able paper, has adduced arguments to show that this stimulation is specially exerted on the renal glandular epithelium (Edin. Med. Journ., Aug., 1881). He used the alkaloid and found that, when combined with a vascular cliurctic (such as digitalis), it had marked diuretic power in cases of general dropsy due to hent disense; if the arterial blool-pressure, however, were fairly normal, caffein acted well by itself (1) 178).

Nutrition. - There is much evidence that roisted coffee controls the processes of organio combustion and tissue-waste. Bocker early reported (1849) that the excretion of urea was diminished under its use, and Eustiatiales proved the same fact by comparative experiments on himself ('Thise de Paris, 1870). It is true that Romx and some others give a contrary 
opinion, and find even an increase of urea on first commencing the drug: in explanation of which it is suggested by Brackenridge either that previously retained wrea is at first excreted, or that altered conditions of the coffee elements, or of the analysis, make the difference in the result (Compt. Rend., 1872).

Clinical experience is certainly in favour of the first statement, and amongst other illustrations may be cited that of the Charleroi miners, who, although insufficiently fed, can do a large amount of work and retain health when adding to their diet several pints daily of carefully-made infusion of coffee. It is said also that other miners in France and Belgium, though living on better food (without coffee), were unable to do the same work (Gasparin, Rabuteau). Joucoud asserted that he could fast for several days without injury on rations of coffee only (Thèse, 1860).

SYNERGISTS.-Tea and other agents in this group.

ANTAGONISTS. - There is remarkable antidotal power between coffee and alcohol, probably exerted through the brain circulation: the same remark applies to opium or narcotics. The poisonous action of caffein can be overcome, or much lessened, by the use of artificial respiration.

Therapeutical ACtion.-Coffee in strong infusion is well known as a remedy for various conditions of nervous depression.

Opium-poisoning affords perhaps the most familiar field for its reviving power, and in such case a common and successful practice is to ply the patient with repented cups of the strongest black coffee, very hot; the progressive torpor of the nervous centres is thus counteracted, and the patient's attention is kept sufficiently aroused to enable him to co-operate with the physician in the necessary unremitting muscular exercise. The grent object is to maintain the activity of respiration and of the eliminntive processes during a certain period; and coffee effects this, partly by direct stimulation of the brain-centres, and partly by general nugmentation of reflex excitability. For the purpose of recovering patients from opium-stupor, it does not 
appear that caffein can replace coffee. Dr. Campbell (America) proposed it for this purpose, but it did not succeed in his hands; and in a subsequent case, recorded by Dr. Anstie, it had only a temporary influence. Either the methylamin or the aromatic principles of roasted coffee must exert some powerful influence of a stimulating kind.

In various Adynamic Fevers coffee has often been used with much success; and some physicians employ it instead of alcohol. Dr. Radcliffe did so at the Westminster Hospital in typhus and typhoid, with good results. Its effect is not wholly due, perhaps, to an action on the nervous system, but also to its power of diminishing the febrile waste of nitrogenous tissues. i

As an Antiseptic and antiperiodic, coffee is adopted by many physicians, especially in the Philippine Islands, whence it was introduced into Europe by Dutch physicians; and opinion seems as strong in Holland as in the East, that coffee will cure intermittents as effectually as quinine. Upon this subject there is no recorded English experience.

In Spasmodic Asthma coffee was especially recommended by the late Dr. Salter, who speaks of cufé noir as one of the most generally useful of all remedies for asthma. I have often seen benefit from it, when taken very hot, in small quantities of concentrated infusion, and upon an empty stomach. Asthmatics should not use coffee as a daily beverage, but reserve it for the time of an attack.

In Whooping-cough it has been recommended, and also in Hay Fever (Worthington).

In Headaches dependent on the nervous system, rather than on the digestive organs, coffee is a valuable but rather uncertain remedy.

In typical Migraine caffein has been extensively given in the form of the so-called citrate, but this salt is sometimes not to be depended on, and then caffein hypodermically may be substituted. I believe that Eulenburg was the first to speak of the value of the alkaloid in this complaint; he employed for injection a solution in water with a little spirit, in which some 2.5 or 30 minims contained the dose $(0.06$ gramme, or about 1 grain); and he found great benefit from its use.

In other Neuralgias caffein has also been found of service 
by some authors; for instance, Anstie employed it successfully in intercostal neuralgia accompanying shingles. A solution in glycerine has been recommended by Lorent-1 grain in $2 \frac{1}{2}$ minims-but this gives a deposit, and requires using at the moment of solution.

In Diarrhœa, especially in children, coffee is remedial by virtue of its astringent properties, which depend upon the peculiar coffee-tannin, but this property is unequally exerted upon different persons, and there are some with whom it always acts rather as an aperient.

In Delirium Tremens coffee is often of service, and the hypodermic injection of grain doses of caffein has proved useful in the insomnia of chronic alcoholism. It must be borne in mind that there is a restlessness and insomnia dependent on lowering of general nervous power, quite as marked as the similar condition produced by exalted reflex irritability.

In Cardiac Disorders, caffein citrate has been lately used. Lepine prefers it, in 10 to 15 -grain doses, to digitalis for slowing the contractions and reviving cardiac energy; it acts quicker and is better borne, but on the other hand it is dearer, and causes in some patients insomnia and restlessness: he finds it difficult to hit the precise dose (Record, 1882). Huchard found the drug augment arterial tension and lessen the rate of pulsation, with the special advantage of increasing the urine. He notes that cirrhotic patients are often intolerant of it; also that after a preliminary slight increase of urea its excretion is lessened (ibid.).

The results of Riegel are very similar: he finds it produce the best effects of digitalis, more rapidly, and without cumulative action, so that it is easily taken by weakly subjects (Berl. Klin. Woch., 1884).

It is, however, in cases of crirlinc drops:y that the best results are recorded. Gubler was one of the first to illustrate this with the hydrobromate (Record, 1878); and Dr. Brakenridge has made still more detailed observations, in which he shows that, whilst digitalis almost certainly acts through the vascular system, augmenting the blood in the renal glomeruli, citrate of caffein specially stimulates the glandular epithelium, and gives instances in which the two drugs supplemented each other in a remarkable manner. He states also that with much dropsy 
it is best to give digitalis for one to four days before commencing the caffein, this acting best when the blood-pressure is nearly normal. Moreover, too-large doses of caffein must be avoided, as the kidneys may be injured by over-stimulation: 3 grains, once, twice, or thrice daily, are amply sufficient (loc. cit.).

Many subjects suffering from acute renal or chronic liver disorders are intolerant of the drug, headache and vomiting being produced; in the majority of patients, however, tolerance is soon established, and the drug may cease to produce its ill effects. Dr. Shapter reported corroborative cases with the citrate (Practitioner, i., 1879).

Still more ample confirmation of its therapentical power was afforded by Dr. D. J. Leech, in a careful report of many cases treated by the citrate in the Manchester Infirmary (Practitioner, i., ii., 1880). Out of seven cases of cardiac dropsy the effect was good in four, in several of which digitalis had failed. In the dropsy of sub-acute nephritis it has also acted well : not so in chronic parenchymatous cases; of five such, it was only effective in one.

Leblond has published a good study of caffein, and finds it often a safe substitute for digitalis, and that it has special value in albuminuria (Gaz. Hebdom., 1884).

Francotte reports recently that although, in his own person, caffein, even in gramme doses daily, did not increase the water or the urea excreted, yet in several cases of cardiac dropsy it produced free diuresis, and relieved after digitalis had failed (Centralb. f. Med., 1884).

PREPARATION AND DOSE.-Tanret notes that pure caffein requires ninety-three parts of water for solution, and that many of its salts, such as the acetate, are decomposed in the presence of water; those with mineral acids are also unstable: so that he recommended as suitable for hypodermic use, double salts, with soda and benzoic or salicylic acid. The double benzoate has been used by several French physicians without local irritation (Pharm. Journ., i., 1883).

Cuffeimu vitres: dose, $3 \mathrm{gr}$. and upwards. Lloyd has shown this to be a definite and sufficiently stable compound (Plamm. Joum., i., 1881). The effervescent form is not always to be depended upon. 
TEA, the product of several species of Camellia thea, a genus which belongs to the order Camelliaceæ, is remarkable in a pharmacological point of view, from containing in its leaves an alkaloid, thein, believed to be not merely isomeric, but identical with caffein. If this be the case, the popular idea which attributes many of the qualities and virtues of tea to the subtler aromatic ingredients, is amply confirmed, seeing that there is an unmistakable difference between the general effects of the two beverages. At present, however, we know nothing of the action of these additional ingredients.

Concerning the differences between the effects of tea and coffee, we might say that tea is more refreshing and stimulating in states of fatigue of brain or muscle, and that when taken in excess (more particularly in chronic excess) it more powerfully affects the stability of the motor system, causing a tremulousness of the limbs, which is less frequently produced by excesses in coffee: on the other hand, coffee is much more prone to cause distressing palpitations of the heart; and, in some persons of weak digestive powers, when taken with substantial food will altogether arrest the digestion of the latter.

PARAguAy TEA, OR MATÉ, the national drink of the inhabitants of a large area of South America, is made from the leaves of the Ilex Paraguayensis (Aquifoliaceæ). It contains caffein in considerable proportion, and is supposed to be intermediate in effect between tea and coffee. The infusion is sucked through a tube, without mills or sugar, and extremely hot. 


\section{SAPINDACEA.}

\section{PAULLINIA SORBILIS. (Guarana.)}

(Not Officinal.)

DESCRIPTION.-Guarana occurs in cylindrical hard cakes of uneven surface, reddish-brown in colour; of slight odour and astringent, bitter taste. The Paullinia sorbilis, from the seeds of which it is prepared by powdering, moistening, and drying, is a climbing shrub, with pinnate leaves and small flowers; the beaked capsule contains one to three shining brown seeds.

ACTIVE INGREDIENTS.-Starch, gum, tannin, oil, and a crystalline alkaloid-guaranin-now proved identical with caffein, of which it has as much as 4 or 5 per cent., being more than either tea or coffee contains.

Physiological Action.-In frogs, the alkaloid is said first to excite the system, then to impair co-ordinating power and cause convulsion; and to slow the heart-action. Mantegazza reported also in man symptoms of stimulation, mental and bodily wakefulness, lowered pulse, and some irritation of the bladder. MeDowall, however, found that, in healthy conditions, very large doses caused no definite symptoms, only when taken after a full meal acting somewhat like coffee (West Riding Asylum Reports).

Therapeutical Action.-In Brazil it is used as an astringent in diarrhoea and dysentery; in France, in mucous and sanguineous discharges, as well as in rheumatism; but in this country it is known almost solely as a renerly for migraine or headache. Introduced chiefly by Dr. Wilks, it has been found serviceable in recurrent forms of these affections, and especially when connected with uterine disorder, or when 
induced by alcohol, and associated with throbbing, heat, etc.; in short, in congestive headache, like coffee (B. M. J., i., 1872), overdoses may induce palpitation.

Seguin states that in many of his cases, when taken early, it cut short an attack (Med. Times, i., 1878); but Day reports less favourably of it (ibid., ii., 1881).

Its effects are often only temporary, and it is not now prescribed so much as formerly.

PREPARATIONS AND DOSE.-Dose: 20 to $30 \mathrm{gr}$. of powdered guarana (infused in a teacup of boiling water) every half-hour for two or three doses (for migraine). Extractum furdum: dose, 10 to $30 \mathrm{~min}$. and upwards. Tincture (1 in 5): dose, 20 to $60 \mathrm{~min}$.

ADULTERATIONS.-These are not infroquent. Lecomte points out that there are two kinds of guarana in the market, only one of which is pure; the other is almost inactive, probably from the occurrence of fermentation. 


\section{GUTTIFERA OR CLUSIACEAE.}

\section{THE GAMBOGE FAMILY.}

A smaLl, interesting order of exogenous trees and shrubs, natives exclusively of the tropics, and principally of South America. The leaves are opposite, entire, coriaceous, destitute of pellucid dots, and have the veins disposed in a pinnate manner. The sepals and petals vary from two to eight; the stamens are numerous and hypogynous; the superior ovary is either" one- or many-celled, and either one- or few-seeded; and the fruit is either succulent or dry. The inspissated juice of various species constitutes gamboge.

\section{GAMBOGIA.}

(Gamboge.)

DESCRIPTION.-A gum resin obtained from a Garcinia (probably G. Hanburii) by making incisions into the bark, or breaking off twigs and receiving the milky juice into vesselsgenerally bamboos-where it hardens: it is met with either in cylindrical sticks (or pipes), 1 to 2 inches in diameter, and 4 to 8 inches in length, striated externally-or in irregular masses : it is brittle, and yields a yellow powder, slightly odorous, which forms a yellow emulsion with water and is soluble in ether to the amount of four-fifths. It is almost tasteless, but after being chewed for some time, "causes an acrid sensation in the fruces."

ACTIVE INGREDIENTS.-The active principle is the resin, $\mathrm{C}_{40} \mathrm{H}_{23} \mathrm{O}_{9}$, which is soluble in alcohol and alkalies: and precipitated from its solutions by basic acetate of lead. When fused with potash, the resin yields acetic acid with phloroglucin, pyro- 
tartaric acid, and isuvitinic acid. Gamboge contains 15 to 20 per cent. of grum, and a large percentage of gambogic acid, $\mathrm{C}_{20} \mathrm{H}_{24} \mathrm{O}_{4}$.

Physiological Action. - The general physiological action of gamboge is that of a powerful, but rather uncertain, irritant of the alimentary canal. In animals it does not seem to cause local irritation, pain, or fever, and not constantly vomiting or purging (Stillé), but sometimes death from collapse. In man, when applied to a raw surface, it irritates; taken in small doses, it augments the secretions of the intestine and the frequency of the stools; but it is said by Abeille that if the small doses be gradually increased up to 20 grains daily, all purging ceases, and a copious diuresis takes its place (Bull. de Thérap., xxxvii.-viii.). According to Rutherford, it is not a hepatic stimulant. In single doses of from 3 to 5 grains it causes nausea (sometimes bilious vomiting), colicky griping, tenesmus, and watery evacuations, and sometimes increases the urine. Still, much of what was formerly stated about the amount of water discharged from the bowels and kidneys in consequence of the administration of gamboge was erroneous; and in regard to the stools, it has been proved by Radziejewsky that there is no more water in them after gamboge than after croton oil. Injected hypodermically in dogs, it causes local abscess without purging; but introduced into the alimentary canal, it becomes effective, because the alkaline secretions promote its absorption.

Taken in large doses the drug produces inflammation of the intestines, with violent abdominal pain, vomiting, and purging, which may be followed by collapse and death. Christison quotes a fatal case from a drachm dose, and mentions Morrison's quack pills (which contain, or then contained, gamboge) as a source of dennger; he cites several trials and inquests, in which death was reported from inflammation produced by these pills, and it seemed probable that gamboge was the fatal ingredient (On Poisons).

At the same time he mentions the curious fact that certain constitutional states seem to prevent the more violent effects of gamboge. Rasori and his followers, in Italy, used to give drachm doses in inflammatory diseases, with no other result 
than brisk purging; and Linoli gave it in inflammatory dropsy in increasing doses up to the extraordinary amount of 850 grains in twelve days, and 1,044 grains in a month. Christison might also have quoted Rayer, who gave gamboge to the extent of nearly 40 grains daily, for six weeks together, in cases of dropsy, without injury. It would be rash, however, to presume upon such facts (which are not yet explained) in the general administration of gamboge; and, indeed, very moderate doses have been known to produce unexpectedly violent effects, such as inflammation of the bowels. After death, redness, ulceration, and even gangrene of the mucous membrane have been found.

It is curious to note the observation of Stillé, that "there is no evidence of its having ever produced fatal effects" (Dispensatory, 1879).

THERAPEUtical ACtion.-The position of gamboge in medicine is unsatisfactory. On the one hand, its known irritant properties make it a dangerous and improper medicine in any case where there is likelihood of the existence of intestinal inflammation or congestion, or any tendency to uterine hæmorrhage; and, on the other hand, it has now been proved that although gamboge is more irritant than jalap, scammony, or colocynth, it is by no means so hydragogue as was formerly supposed. On the whole, considering the great superiority of elaterium as a hydragogue cathartic, and of digitalis and bitartrate of potash as diuretics, there hardly seems any place left for gamboge in the treatment of dropsical affections.

Dysentery.-There is one different application of the drug which deserves mention. Malgaigne and Betz found very small doses (about $\frac{3}{4}$ grain in twenty-four hours) valuable for clysentery, especially in young persons, but their mode of treatment has not been generally accepted.

PREPARATIONS AND DOSE.-The pordered resin is given in pills, but seldom alone, often with jalap or cream of tartar: dose from $\frac{1}{4}$ to $5 \mathrm{gr}$., according to the circumstances above detailerl. It may be given in solution, thus: 10 gr. with 60 gr. of potnssium bicarbonate in $20 \mathrm{~g}$. of cinnamon water; dnse, 30 min. thrice daily in water. Pil. gymbogice (omp). (contains 1 part in (f) with aloesand cimnamon): dose, is to $10 \mathrm{gr}$. 


\section{THE CANELLA FAMILY.}

\section{CANELLA ALBA. \\ (Canelia, or Wild Cinnamox.)}

DESCRIPTION.-An order represented by this one plant, which is a common West Indian aromatic shrub, with evergreen, obovate, alternate, coriaceous leaves, and corymbs of small purplish flowers.

The bark, deprived of its epidermis, and then dried in the shade, is officinal, and occurs in flat or quilled pieces, of light buff colour outside, internally paler: they are brittle, with a white granular fracture. The odour is aromatic and spicy; the taste warm, pungent, and bitter.

ACTIVE INGREDIENTS.-By distillation the bark yields a warm aromatic oil, which is a mixture of eugenic acid, $\mathrm{C}_{10} \mathrm{H}_{12} \mathrm{O}_{2}$, an oil like the chief constituent of cajuput oil, with two neutral oxygen-containing oils.

The principle, canellin, is said to be identical with mannite.

ThERAPEutical Action.-In England, canella bark is principally used as an aromatic adjunct to tonic and purgative medicines. It has been employed in scurry, but is not an important remedial agent.

Combined with aloes (one part to four) it forms a popular remedy, well known by the name of " hiera picra."

Preparation AND Dose.-Pulcis: dose, 15 to $20 \mathrm{gr}$.; it is an ingredient of vinum rhei. 


\section{VITACEAE.}

\section{THE GRAPE-VINE FAMILY.}

Ax order of about 300 species of exogenous shrubs, natives chiefly of warm countries, and well marked by the long, slender, climbing stems, and the handsome exuberant foliage. Most of them have powerful axillary tendrils. The flowers are insignificant, and usually pentamerous; the few stamens are hypogynous, and opposite the petals; the superior, two- to sixcelled ovary ripens into a few-seeded, pulpy berry, which is often one-celled by abortion.

\section{VITIS VINIFERA.}

\section{(The Grape.)}

DESCRIPTION.-The grape-vine is prubably indigenous to the shores of the Caspian Sea. The long and scandent stems, the tendrils, the large and beautiful three- to five-lobed leaves, changing in autumn to red, the thyrses of minute green and honey-scented flowers, and the fruit, distinguish it from any other plant. The grape is really a two-celled ovary, which ripens into a one-celled juicy berry, containing four hard seeds.

ACTIVE INGREDIENTS.-These are water, sugars, gum, tannin, azotised matters, bitartate of potash, sulphate of potash, tartrates of lime, magnesia, alum, and iron; chlorides of potassinm and of sodium, tartaric, citric, racemic, and malic acids. 
The relative proportions, especially of the sugar and the acids, vary with different species and seasons; and these differences have important practical bearings; the variations in the proportion of albuminoid matter are also of consequence. The seeds contain a bland oil, which is a mixture of glycerine compounds of erucic, palmitic, and stearic acids.

PHysiological ACTION.-The large amount of water in the juice of the grape renders it highly diuretic when eaten in quantities, and this action is assisted by some of the other ingredients, particularly the vegetable acids (which pass off in the urine as alkaline carbonates), and the salts of potassium, sodium, etc.

With less constancy grapes act in a laxative or decidedly puryutive manner; and if this effect be carried to excess, particularly in children and delicate persons, there may be excoriation of the tongue, chronic diarrhœa, and an aphthous condition of the whole alimentary canal.

Again, in the early stages of a "grape-cure," excitement of the heart and circulation is sometimes caused; but this effect is only temporary.

The amount of albuminoid matter, though small, is far from valueless; and this, with the large amount of sugar, renders the grape highly nutritious.

Therapeutical Action.-The "grape-cure" has no doubt been greatly over-estimated as to its efficacy: in some cases good may be done, but many patients are injured by the treatment, especially women and childreu. At the present day there are numerous grape-countries where, during the vintage, patients are treated by the administration of 3 to 6 pounds, or more, per diem. The principal places are Meran, in the Tyrol; Vevay, on the Lake of Geneva; Durkheim, in Bavaria; Bingen, Rudesheim, Kreuznach, St. Goar, etc., on the Rhine banks; Grünberg, in Silesin; Aigle, in Savoy.

The remarks made on the physiological action of the grape indicate the probability of there being at least two ways in which the treatment may be efficacious ; in fact, according as the grape contains much sugar and little acid, or vice versâ, there 
will be a preponderance either of the so-called "alterative" purgation, or of the alimentative and tonic effects."

The conditions which are especially benefited by the laxative action of the grape-cure are those somewhat vaguely grouped mder the denomination of "abdominal plethora." There are numerous cases in which the biliary secretion is inactive, and the digestion feeble, in consequence of sluggish portal circulation. In these instances it is usual to administer grapes that are not over-ripe, and, as a rule, the comparatively non-saccharine kinds. The same course should be adopted with women suffering from abdominal congestion which has given rise to amenorrhœea, with its accompanying train of symptoms-headache, vertigo, oppression, and palpitation of the heart. In not a few cases it will be found (especially where there has been marked engorgement, and even a tendency to enlargement of the liver or of some other abdominal viseus), that the grape-cure is not a sufficiently energetic remedy to commence with, but that it comes in effectively after a preliminary course of mineral waters and other medicinal agents.

Chronic catarrhs of the mucous membranes (bronchial, intestinal, and urinary) are frequently benefited by the grape-cure.

Dyscrasic maladies, so-called, are sometimes also modified by it, e./., skin disorders of the eczematous, ecthymatous, and impetiginous classes, linked to the rheumatic diathesis. As regards phthisis, the evidence seems much more doubtful; and it may be questioned whether the supposed cures (other than those of mere pulmonary catarrh) were not effected rather by change of climate than by the specific treatment.

As a tonic mode of treatment, adapted to convalescents from acute diseases and the effects of hremorrhages, and from various states of debility, there seems ample evidence that the reconstituent powers of the grape are often efficncious : digestion is strengthened, appetite increased, and flesh is gained; for these purposes the sweeter grapes are preferable.

It is unnecessary to enumerate all the diseases saicl to be curable by means of this fruit; the above are those respecting"

'This is well explained by H. Curchod, in his "Cure de laisins" (Paris: J. B. Baillière et fils, 1860); to whieh the reader may also be referred for lists of nearly all the important works on the grape-cure. 
which there is some evidence of real benefit. At the same time, a few words must be said as to the accompanying diet. Formerly, especially by Germans, it was recommended to keep the patient almost entirely without other food; but except in a few cases, in which something like a starvation-cure might be expected to do good, this plan is not a sound one. It is possible that abdominal engorgements, due to overeating, or to intemperance in drink, might be benefited by a short course of the treatment, but proper nutrition for any extended length of time cannot be maintained in this way, and for delicate subjects it is altogether improper. Moderate meals, with a little Bordeaux wine, may with advintage be allowed. 


\section{ZYGOPHYLLACEA.}

\section{THE GUAIACUM FA M I Y.}

A small order of exogens exhibiting great diversity of aspect, but with technical characters well marked. Whether arborescent, shrubby, or herbaceous, all have opposite and stipulate leaves, devoid of translucent dots, and usually paripinnate. The flowers are complete and regular, or nearly so; the sepals and petals are either four or five; the hypogynous stamens are twice as many; the superior ovary is more or less five-furrowed, and ripens into a five-winged and few-seeded capsule, the pericarp not laminating.

\section{GUAIACUM OFFICINALE.}

(Guaiacunr.)

DESCRIPTION.-A tree indigenous to the West Indies, and the warmer parts of continental America, where it attains the height of 30 to 40 feet. The leaves are paripinnate; the leaflets, produced in two or three pairs, are oval and entire; the slightly clustered and brilliant blue pentamerous flower's are nearly an'inch across.

The heartwood, commercially known as "lignum vitre," is of a dark greenish-brown colour, and one of the lhardest and heaviest yet discovered; it possesses little or no smell, but upon friction or burning it yields a perfume; the taste is bitter and somewhat warm. By means of incision, also by natural exudation and $b_{y}$ ilplilication of heat, the tree yields guaincm resin. This occurs either in greenish-brown or reddish masse:, or in dark brown tears, which are variable in size, roundish, 
oval, and often covered with a greenish dust. Internally it is reddish-brown; the fracture is shining and vitreous; the laminæ, when sufficiently thin, are brittle and nearly translucent. It has a slightly aromatic and balsamic odour; on being chewed, it softens, and communicates a mildly bitter and acrid taste, followed by a peculiar burning and prickling sensation in the back of the throat. The sp. gr. is 1.25. Not more than 9 per cent. of it is soluble in water ; but alcohol dissolves about 91 per cent., acquiring a deep brown colour, and from this the resin is again precipitated by water: it is soluble also in ether and in alkaline solutions. It has acid properties forming soluble salts with alkalies, and being re-precipitated from them by acids.

ACTIVE INGREDIENTS.-About 10 per cent. of guaiaretic acid, $\mathrm{C}_{20} \mathrm{H}_{20} \mathrm{O}_{4}$, which is crystalline; and about 70 per cent. of guaiaconic acid, $\mathrm{C}_{33} \mathrm{H}_{40} \mathrm{O}_{10}$, with other subordinate substances.

Physiological Action.-Guaiacum, given in small doses, stimulates the vascular system, and often produces diaphoresis; in large doses, it produces dryness of the mouth, burning in the throat, a sensation of heat in the stomach, loss of appetite, heartburn, flatulence, nausea, vomiting, and purging.

These symptoms, which vary in degree according to the dose and the length of time that the patient is kept under the influence of the drug, may be accompanied by palpitation of the heart, headache, apparently of congestive type, confusion of mind, giddiness, fainting, and general lassitude. Stiffness, of rheumatic character, is felt at the same time in the nape of the neck and the lumbar region, with pains in the bones of the legs, the limbs feeling as if swelled. These symptoms are often accompanied by profuse perspiration, and are sometimes followed by an exanthematous eruption, and by ptyalism. Then guaiacum fails to act upon the skin, it often operates as a diuretic.

Therapeutical ACtion.-Syphilis.-Our first knowledge of guaiacum was obtained through the Spaniards, who brought it to Europe from St. Domingo, about the year 1508, with the reputation of its being antisyphilitic. The name of 
"lignum vitæ" was given to it almost immediately, and by 1 j19 many thousands of patients are said to have been cured!

The mode of exhibiting the medicine was in decoction, this being frequently made with port wine. It was employed in every form of the disorder, and in every stage of its progress, the physicians prescribing at the same time the use of purgatives, baths, and appropriate diet. Resort to mercury was discontinued, and even censured, and for a period of two centuries the new medicine enjoyed the highest repute. Boerhaave went so far as to assert that guaiacum-wood was competent to expel the venereal poison from the system. Yet those who most strongly believed in its efficacy were constrained to admit occasional failures; and we can now see that many conditions which guaiacum was supposed to cure were probably not venereal, so that the drug acquired a celebrity it did not deserve.

The ancient reputation of guaiacum as an antisyphilitic remedy was in great part due to the celebrated name of one of the earliest patients who benefited by its use. Ulric von Hutten, the satirical and military champion of the early Reformers, has himself related the story of his cure, effected apparently by means of a compound decoction of various woods, of which guaiacum was assumed to be the most important.

Catarrh and Gout.-In chronic pulmonary catarrh, especially when occurring in gouty subjects, guaiacum manifests a beneficial action: even in gout it once had great renown. Cullen speaks of it in terms of commendation. It is suitable for cases of rheumatism when the pains are markedly relieved by warmth: also in chronic rheumatoid arthritis.

Sciatica.-Fuller has recorded cases of sciatica with sympathetic irritation, in which guaiacum and sulphur (40 grains of each), three times daily, relieved pain and constipation (B. M. J., i., 1864).

Hæmaturia.-Upshur has reported a case of paroxysmal hæmaturia which was benefited by guaiacum in 1-drachm doses three times a day; also two other successful cases (New York Med. Record, 1880).

Tonsillitis.-Dr. Hannah and Mr. Bell (London Med. Gaz., 1840) advise the use of guaiacum in tonsillitis, stating that it reduces the pain and inflammation with marked rapidity. 
Many others have made the same observation, but it is a particularly unpleasant remedy, and in my experience has not acted so well as stated: in the form of lozenge it is often prescribed.

Menstrual Disorders.-Guaiacum is useful in amenorrhcea and in dysmenorrhœa of atonic character: 10 to 15 grains in warm milk, each morning for some time, often has the desired effect, acting probably like other vascular stimulants.

PREPARATIONS AND DOSE.-Guaiaci resina: dose, 10 to $30 \mathrm{gr}$. Mistura: dose, $\frac{1}{2}$ to $2 \mathrm{fl}$. oz. Tinctura guaiaci ammoniata: dose, $\frac{1}{2}$ to $1 \mathrm{fl}$. $\mathrm{dr}$.

Squire suggests a mixture of equal parts of glycerine and tincture of guaiacum (prepared with rectified spirit) as a pleasant way of giving the drug (B. M. J., i., 1879): the mixture, however, does not remain clear on the addition of water. Equal parts of the tinct. guaiaci (Ph. U.S.A.) and liquor potassæ give a clear mixture miscible with water (B. M. J., i., 1880).

Guaiacum-resin enters into the composition of pil. hydrarg. subchlor. comp., and is a constituent of the well-known mixture called "The Chelsea Pensioner"-Sulphur, 6 pts.; mustard, 6 pts.; powdered guaiacum, 3 pts.; rhubarb, $1 \frac{1}{2}$ pts.; nitre, $1 \frac{1}{2}$ pts. : mix. Honey or treacle sufficient to make it into an electuary. Dose : a teaspoonful every alternate evening for rheumatism; the same dose may be taken in the morning as an aperient.

Guaiacum-wood forms an ingredient in decoct. sarsæ comp. 


\section{RUTACEAE.}

\section{THE RUE FAMILY.}

Av order of exogenous shrubs, numbering 500 species, and belonging chiefly to warm countries. The leaves are charged with oil-cysts; the flowers vary considerably in position, regularity, and numerical structure, but have hypogynous stamens, and a large disc below the lobed ovary, which ripens into a few-seeded capsule, the pericarp consisting of separable laminæ. The principal members of this order are-

Ruta graveolens .. .. .. Rue.

Galipea cusparia .. . . . Angustura, or cusparia bark.

Barosma crenulata.. . . . Bucku, or buchu.

Pilocarpus pinnatifolius .. Jaborandi.

\section{RUTA GRAVEOLENS.}

(RUE.)

DESCRIPTION.-An evergreen, glaucous bush, native of Southern Europe, 3 or 4 feet high, abundantly clothed with alternate decompound leaves, of nauseous odour, and producing: its tetramerous yellow flowers in terminal corymbs.

ACTIVE INGREDIENTS.-Rue contains a bitter, neutral, crystalline principle, called rutin, $\mathrm{C}_{25} \mathrm{H}_{28} \mathrm{O}_{155}$, not known to possess physiological activity, and an etherenl nil, which appears to be the source of the virtues of the plant: this is of 
a light-yellow colour (turning brown on keeping), and has a bitter acrid taste and peculiar odour: sp. gr. 0.911 . It consists of two substances, of which one is a hydrocarbon of the turpentine-camphor type; and the other an oxygen-containing oil, which until lately was not understood. The researches of Gorup-Besanez and others have proved it to be methyl-caprinol, or methyl-keton, $\mathrm{C}_{10} \mathrm{H}_{19}\left(\mathrm{CH}_{3}\right) \mathrm{O}$. It boils at about $440^{\circ} \mathrm{F}$.; is colourless if looked at from the side, but shows a violet-blue fluorescence when looked at from above; it has a specific gravity of 0.82 , and crystallizes at $42.8 \mathrm{~F}$; ; it congeals at a low temperature in shining scales; by the action of nitric acid, caprinic, pelargonic, and other acids are developed.

ABSORPTION AND ELIMINATION.--Elimination is easily traced by the odour of the drug in the breath, the perspiration, and the urine.

Physiological Action. - External. - Topically, rue exerts a powerful irritant action; if the bruised and moistened leaves be applied to the skin, they speedily cause redness and inflammation; if chewed, they excoriate the mouth.

\section{PHySiological Action.-Internal.--Digestive System.} - Small doses of the oil cause warmth at the epigastrium; large ones may give rise to inflammation and swelling of the tongue, salivation, thirst, epigastric pain, colic, and vomiting, with hiccough, and, in a word, to all the symptoms of a violent irritant;-after death, inflammatory lesions are found in the stomach and duodenum.

Nervous System.-Rue is a powerful irritant narcotic. Orfila injected 15 minims into the renous system of $a$ dog; in two minutes there followed vomiting, vertigo, and paresis of the hind limbs; the animal, however, recovered. With human beings, the effect of an overdose on the general nervous system is to produce great prostration, combined with restlessness and excitement, confusion of ideas, and stupor, vision becoming dim, and the pupils contracted; there is giddiness, with tottering gait, and some convulsive movements; the skin is cold (Hélie, Med.-Chir. Rev., v., 58). 
Genito-Urinary System.-The symptoms just described, when occurring in pregnant women, commonly precede abortion, which may certainly be caused by the drug. Trousseau traces it to congestion of the uterus, and stimulation of its muscular fibres, which contract strongly and expel the foetus;though not till some considerable time after taking the drug.

Rue has also, from ancient times, been reputed to possess the power of exciting the uterus, and was formerly much used as an abortifacient by rustics, and by irregular practitioners. In England, the common people and the "herb-doctors" now more frequently give savin for this purpose; but in Hindostan the women still put faith in me.

Beau considers its power in this direction greater than that of ergot, but less than that of savin, to which drug he thinks it bears the same relation as ipecacuanha does to tartar-emetic (Bull. de Thérap., v., 43).

In smaller doses it stimulates the torpid non-pregnant uterus, and acts as an emmenagogue. The observations of Hélie show that it has a specific effect on the uterine walls; but it is, of course, always difficult to know whether a remedy which has power to act on the uterine muscular system is also a true stimulant of the ovarian function. No doubt in a considerable proportion of cases, where the menstrual flow is restored under the influence of remedies, this is only effected in a much more indirect manner: but among the emmenagoguesthose which tend to produce the menstrual How without reference to improvement of the general health, the quality of the blood, etc.-rue will probably be found to occupy a very genuine position. Moderate doses are said also to promote the sexual appetite and vigour in men; they tend to increase the urinary as well as other secretions, but toxic doses may cause strangury and suppression.

Circulatory System.-Another phenomenon which has been noted as the result of an overdose of rue is weakness and slowing of the pulse (Hélie). Possibly the alleged weakness referred merely to the small size of the pulse, for the true condition is most likely that of high arterial tension, from stimulation of the muscular coat of the small vessels. 
SYNERGISTS.-Essential oils and other stimulant emmenagogues.

AntAgonists.-O Opium and belladonna (Gubler).

Therapeutical Action. - Extemal. - The bruised leaves of rue laid upon the forehead often arrest epistaxis. The oil, mixed with honey and placed on warts, is said to destroy them.

Therapeutical Action.-Intemal.-Rue has somewhat unaccountably fallen into discredit with the majority of the regular profession in this country; and in Germany, Nothnagel speaks of it as a superfluous drug, to be classed with thyme, origanum, and other remedies of the like efficacy. This is, I consider, incorrect: even the exaggerated ancient ideas of the value of rue were probably nearer the truth, for the essential oil deserves the repute which it attained as antispasmodic, stimulant, emmenagogue, and anthelmintic.

In Flatulent Colic, more especially when occurring in children, it is an exceedingly useful remedy; it may be either given by the mouth, or administered as an enema; for infants, Dr. A. T. Thompson recommends the latter.

Infantile Convulsions, dependent on flatulence in some part of the alimentary canal, can be beneficially treated in the same manner.

Hysteria, especially when associated with amenorrhoa, is sometimes much benefited by rue, an experience corroborated by that of M. Alibert. The cases which seem most suited for this remedy are similar to those of amenorrhœa and dysmenorrhoea, in which muriate of ammonia was found to do good service by Anstie and Cholmeley, viz., those connected with functional inactivity or torpor of the uterine system. It is contra-indicated in congestion or inflammation of the pelvic viscera in pregnancy.

It may sometimes be employed in the uterine hæmorrhage of weakly subjects (Beau), though, as a rule, ergot is better.

In Epilepsy, rue has been vaunted as a specific, its reputation having been originally founded by Galen, who directed 
rue-leaves to be sprinkled in the beds of those who suffer from priapism and erotic dreams. Murray and others have claimed for it the power of curing epilepsy; but the utmost that can be allowed is, that in cases where the malady is wholly or partly dependent on seminal weakness, small doses may limit the nocturnal discharge, and thus mitigate the nervous prostration so favourable to a continuance of the fits.

Asthenopia.-In some of these cases rue has been given apparently with benefit; it seems to strengthen the ciliary muscle, to keep up the accommodative effort, and also to enable the patient to read more continuously without the letters and words becoming so confused, or the eye feeling so painful and weary. It should be applied in the form of lotion ( 1 part in 10 infused for one hour), three times daily, and the oil administered in 3-minim doses, morning and night.

PREPaRATIONS AND Dose.-Pulvis foliom : dose, 20 to $40 \mathrm{gr}$. Oleum: dose, 1 to $6 \mathrm{~min}$. It is sometimes employed in the form of enema: dose, 2 to 5 min.

\section{GALIPEA CUSPARIA. \\ (Angutstera, or Cusparia Bark.)}

DESCRIPTION.-A handsome evergreen tree of the tropical American forests, rising to the height of 60 or 80 feet. The very large leaves are alternate and trifoliate; the white or lilac flowers are produced in erect racemes. Dr. Hancock describes a second species, distinguished chiefly by its smaller leaves, which he asserts to be the true source of the bark, and names it Galipea officinalis.

The bark is met with in flat or curved pieces, from 1 to 3 inches long, and from 1 to 2 inches wide. Externally it is reddish, rough, often with a greyish corky layer; internally, smooth and lighter in colour. It breaks with a smooth fracture of resinous appearance, showing white streaks of calcium 
oxalate. Angustura has a strong: peculiar, and slightly offensive odour, with an abiding bitter aromatic taste, which is said to induce salivation.

ACTIVE INGREDIENTS.-Angustura is composed of a bitter, crystallizable principle called cusparin; a hard and bitter, as well as a soft, balsamic resin; volatile oil; gum and woody fibre; also oxalate of lime, as mentioned: no starch or tannin. Cusparin may be obtained in tetrahedral crystals, slightly soluble in water, more so in alcohol, acids, and alkalies, insoluble in volatile oils and ether. Its composition is unknown, and from the experiments of Saladin it would appear to have little activity. Herzog and others have not isolated Saladin's cusparin, but found an alkaloid called angusturin, $\mathrm{C}_{20} \mathrm{H}_{40} \mathrm{NO}_{14}$. It turns red with sulphuric acid, and green with oxidizing agents (such as nitric acid) added to the sulphuric acid (F.\& H.).

The hard resin is brown, soluble in potash, alcohol, and acetic ether; insoluble in oil of turpentine and sulphuric ether. The soft resin is slightly acrid, greemish-yellow, soluble in alcohol, ether, oil of turpentine, and oil of almonds; insoluble in solution of potash.

The volatile oil is obtained by the distillation of the bark with water. It retains the peculiar odour of the bark, and in taste is acrid; it is lighter than water, and is of a yellowishwhite colour.

PHysiological ACTiON.-In its operation, angusturn is said to resemble cascarilla, but the stimulating effects are less decided, and it is nearer allied to the pure bitters.

Digestive System.-Administered in large doses, it induces nausea, flatulence and purging. Observations are still wanting, however, as to its specific properties, and at present we can only regard this drug as an aromatic tonic and stomachic, adapted for substitution when there are objections to cascarilla, cinchona, or calumba, and especially of use in the tropics. Rabuteau, taking the infusion himself, noted increase of appetite, and more frequent need of food, as if digestion were completed more quickly than usual. In one set of observa- 
tions, lasting five days, he found the pulse rather lessened in frequency, and the excretion of urea slightly diminished.

Therapeutical Action.-Fever.-Dr. Hancock, who had excellent opportunities for watching the effects of angustura, declares it adapted to the worst forms of bilious fevers: indeed, all fevers of adynamic type, especially when accompanied by severe stomach disorders, are considered amenable to it.

In Diarrhœa and Dysentery, when chronic, angustura may be employed with benefit; and in subacute cases, when there is a considerable amount of mucous discharge, its efficacy seems unquestionable: it is adapted also for debility connected with anorexia and atonic dyspepsia.

PREPARATIONS AND DOSE. - Cusparice cortex (in powder): dose, 10 to $40 \mathrm{gr}$. Infiusum: dose, 1 to $2 \mathrm{fl}$. oz.

Adulterations. - The bark of the nux vomica tree has, either by accident or design, been substituted for that of the galipea, and has caused serious accidents. Hence the distinguishing names of true and false angustura: the true, or galipea bark, being West Indian; the false (nux vomica), East Indian. The true, on having its inner surface touched with nitric acid, does not become blood-red, which colour is a characteristic test of the false on account of its containing brucia.

Rabuteau has found that true angustura bark also reddens sometimes with nitric acid, and therefore proposes to test the infusion for alkaloids with phospho-molybdic acid, or ioduretted solution of indide of potassium. The true will give no precipitate, but the nux vomica will give a yellow one with the former, and a brown with the latter (in the cold). The taste is another means of distinction, the strychnia bark being extremely bitter and nauseous. Stillé remarks that the two barks "bear no resemblance," the false one being thicker and harder, externally grey, with bright rustcoloured patches and white warts, the inner surface brown, the fracture smooth and without striæ. 


\section{BAROSMA, OR DIOSMA.}

(Bucku, or Buchu.)

DESCRIPTION.-An erect shrub, native of the Cape of Good Hope, where it grows from 2 to 4 feet high. The leaves are ovate-lanceolate, nearly sessile, and minutely crenate: they contain many large oil-cells, which show as transparent dots when held to the light. The pentamerous and purplish flowers, borne upon rather long peduncles, are produced from the upper axils. Bucku leaves generally include those of - (1) Barosma, or Diosma betulina = D. crenata of Lindley; (2) Barosma crenulata $=$ D. crenata of Linnæus, B. crenata of Kunze, D. latifolia, etc.; (3) Barosma serratifolia.

The first constitute most of the commercial "short buchu," and are $\frac{1}{2}$ to $\frac{3}{4}$ inch long, obovate, usually obtuse, with recurved apex; base almost entire; margin sharply serrate, each serrature containing a gland.

The second are also found in "short buchu," but some leaves are longer; they vary from $\frac{3}{4}$ to $1 \frac{1}{4}$ inch in length, are ovate or obovate, obtuse and crenate, each division having a gland.

The third species have leaves thinner and longer-from 1 to $1 \frac{1}{2}$ inch; lanceolate, tapering to both ends, margin serrulate with glands, mid-rib prominent, and four lateral veins running parallel to the margin.

All are smooth, of dull-green colour, paler on the lower surface; they have a strong peculiar odour, and bitterish aromatic taste.

ACTIVE INGREDIENTS.-Bucku leaves contain a rolatile oil (B. betulina, 1.56 per cent.), yellowish-brown in colour, lighter than water, and smelling like peppermint; "barosma camphor" separates from it at a low temperature; they also contain a peculiar bitter extractive, called barosmin or diosmin, which is said to be brownish-yellow, bitter, and slightly pungent, soluble in water, but insoluble in alcohol and ether. (The existence of barosmin is doubtful.-F. \& H.) Resin, 
gum, and lignin are present. Some varieties contain much more than others of the camphor, or stearoptin (also called diosphenol), which may be crystallized in colourless needles having the formula $\mathrm{C}_{14} \mathrm{H}_{22} \mathrm{O}_{3}$ (Pharm. Journ., ii., 1881).

Physiological Action.-Small doses cause a sense of heat in the stomach, which soon diffuses itself over the body; they also quicken the pulse, relieve nausea and flatulence, increase the appetite, and cause slight moisture of the skin; at the same time the flow of urine is augmented, the colour of the excretion becomes darker, and a brown sediment is deposited, accompanied by a strong aromatic smell. In large doses, bucku produces a burning sensation in the stomach, with vomiting, purging, and strangury.

\section{THERAPEUTICAL ACTION. - Urinary Disorders.-} Bucku is eminently useful in chronic maladies of the genitourinary organs, especially in those arising from chronic inflammation of the mucous membrane of either the whole or a portion of this tract, and accompanied by copious secretionsuch as catarrh of the bladder following gonorrhoea or the improper use of injections. When the catarrh implicates the ureters, or the kidneys themselves, producing considerable mucopurulent discharge, associated as it is often with incontinence or retention of urine, bucku renders more help than most other drugs, though it cannot be relied on in every constitution. It is also given in irritable conditions of the urethra, such as spasmodic stricture and gleet, and in prostatic disorders. Lithiasis, with rapidly increasing secretion of uric acid, has been beneficially treated by bucku, administered in combination with an alkali, such as solution of potash. It is recommended, also, in atonic dyspepsia connected with urinary disense, in chronic rheumatism, cutaneous affections, and for dropsy.

The use of bucku appears to be unknown, or quite neglected, in Germany. Buchheim, Husemann, and Nothnagel do not even mention it.

Preparations and Dose.-Pultis: dose, 20 to $40 \mathrm{gr}$. Infusum: dose, 1 to $2 \mathrm{fl}$. oz. and upwards. Tincturll : dose, 1 to 2 fl. dr. 


\title{
PILOCARPUS PINNATIFOLIUS.
}

\author{
(JABORANDI.)
}

DESCRIPTION.-A shrub growing freely in Brazil, and introduced to European practice in Paris by Dr. Coutinho in 1873. The same name, jaborandi, or jamborandi, is commonly given in South America to many plants with similar properties, and especially to certain species of Piperacer.

The leaves of the above plant are unequally pinnate, and have from four to ten leaflets, with a terminal one. The leaflets are about 4 inches long, unequal at the base, ovate, entire, coriaceous, with prominent mid-rib, and marked by pellucid glands ; slightly aromatic in odour, still more so in taste.

ACTIVE INGREDIENTS.-The leaves contain a volatile oil and an alkaloid, pilocarpin, which is soluble in alcohol, ether, chloroform, ammonia, and dilute acids (Byasson, Hardy, 1875). It is amorphous, and when pure is liquid, like syrup, and colourless; many of its salts are crystalline, and the sulphate and acetate are deliquescent. The nitrate is the salt usually employed. According to Kingzett the formula is $\mathrm{C}_{23} \mathrm{H}_{34} \mathrm{~N}_{4} \mathrm{O}_{4}$, and trimethylamin is yielded on distillation with canstic potash. A second alkaloid, jaborin, also liquid, has been described, but is probably derived from pilocarpin. The volatile oil contains several hydrocarbons, of which one is named pilocarpene, $\mathrm{C}_{10} \mathrm{H}_{16}$ "

PHysiological Action.-External.-The powdered leaf excites local irritation after prolonged contact with the skin.

\section{Physiological Action.-Internal.-Digestive System.} -The leaves, when chewed, have an acrid taste, and an excessive flow of saliva, sometimes to the amount of one or two pints in an hour, is generally, though not always, caused by any preparation of the drug. The secretion contains the usual salts and ptyalin, and some urea, but less than normal of the albuminous 
compounds, and but little sulphocyanide of potassium; it is this salivation partly which causes the vomiting and diarrhœa that sometimes occur. As noted in a dog with fistula, the amount of gastric juice and pancreatic secretion was increased, but the bile only slightly (Rutherford).

Cutaneous and Glandular Systems. - It is on these that the drug exerts its characteristic effect. An infusion of 30 to 90 grains of leaf, or $\frac{1}{2}$ grain of a salt of pilocarpin by the mouth, or $\frac{1}{6}$ to $\frac{1}{3}$ grain injected under the skin, causes in a few minutes flushing and sweating of the face, then of the axillæ, trunk, and limbs; in many cases the amount of perspiration is very great, running down in streams, and soaking the clothes, especially if the patient remains warm in bed; but it occurs (though it may be delayed) independently of this condition, and continues four to five hours. It has been estimated at from 9 to 15 ounces, and is at first acid, afterwards alkaline; the chlorides are in some excess, and the urea is markedly so, viz., from 10 to 15 grains in the day (four or five times its ordinary amount); the carbonates and phosphates are in very small proportion.

Straus showed that small doses ( $\frac{1}{7}$ to $\frac{1}{6}$ grain) given by the skin produced first a fine secretion of sweat in the areola round the punctures, and much smaller doses induced this local sweating, only without any general symptoms (Compt. Rend., 1879).

Jaborandi has been called "the only direct and essential diaphoretic in the materia medica," but sometimes (though rarely) it does not act in this manner at all.

The secretion of tears, and that of the mucous membranes, is increased, and that of the bronchi sometimes so greatly as to threaten suffocation.

Under the influence of such excessive secretions the temperature is generally lowered from to 1 degree $\mathrm{F}$.

That the drug can increase also the secretion of the mammary gland is evident from such a case as the following:-A lady, aged twenty-eight, had had three children, but never any secretion of milk; in a, fourth confinement, jaborandi in powder (presumably not good) was given, and ointment of Calabar bean applied, but without result. After a fifth confinement, the 
tincture was given in $\frac{1}{2}$-drachm doses thrice daily in peppermint water; an abundant flow of milk set in, with free perspiration-little salivation; the remedy was continued for six weeks, the lactation lasted ten months, and similar effects followed the medicine after another confinement (B. M. J., ii., 1880).

On the kidney small doses may act as diuretic (Gubler), and in cases of dropsy they often increase the secretion; but under ordinary conditions, when sweating is free, the amount of urine is commonly lessened; it is voided with pain, which is due probably to some spasm or contraction of the bladder.

When injected directly into a ligatured loop of intestine, the glandular secretions are markedly increased by it (Heidenhain).

Circulatory System.-An infusion injected into the jugular vein of rabbits or dogs lowers the heart-action and pulse (Langley); it slows, and finally arrests in diastole the heart of frogs; but in man its ordinary administration causes a rise of pulse-sometimes forty to fifty beats per minute (Stillé, Ringer)_at first it may be fuller and of higher tension, but soon becomes weaker. Palpitation is occasionally noted. The primary flushing of the face is followed by pallor.

Nervous System.-It is now commonly agreed that the special effect of the drug on the sweat-glands is due to an action on the peripheral nerve-supply (Luchsinger, Marmé, Pfliger's Archiv, xv., 482), and not on the "sweat-centres" in the cord, since all the spinal nerves to a limb may be divided, and yet the sweating occurs in that limb. Some days after section, when degeneration of nerve-terminals has taken place, then sweating does not follow the use of the drug. Small doses injected directly into the salivary glands increase-very large doses arrest--the secretion, even when the salivary nerves are divided, so that there is probably some direct action on gland-cells as well as on peripheral nerves.

The slowing of circulation, when it occurs, is traced to a stimulation of cardiac inhibitory nerves. An effect of the drug on the general nervous system is shown by the production, not unfrequently, of frontal headache, giddiness, dulness, and drowsiness.

It has special effects on the eye, and its local application commonly contracts the pupil. It also "increases tension of 
the accommodation-apparatus, with approximation of near and far points of vision, diminished sensibility of the retina with amblyopia, and glistening scotomata" (Tweedy). These effects are temporary only.

SyNERGISTS.-According to Bartholow, aconite, gelseminum, veratrum viride, and paralysers of the vaso-motor system are synergistic.

AnTAGONISTS.-Caustic alkalies and metallic salts are chemically incompatible. Belladonna and its alkaloid are physiological antagonists: thus, whilst profuse sweating is going on, $\frac{1}{100}$ grain of atropia injected will dry the skin in five minutes, and will also remove the headache, the vesical pain and spasm, and the salivation (Ringer, Vulpian). In a case of atropia-poisoning, when nearly 1 grain had been taken by a man aged nineteen, and the symptoms of unconsciousness, etc., were serious, hypodermic injections of pilocarpin had a marked effect on the recovery (B. M. J., i., 1881); and in another case, when more than 2 ounces of belladonna liniment were taken, and complete stupor had set in, $\frac{1}{5}$ grain of pilocarpin injected every fifteen minutes for four doses caused rapid improvement, and probably saved life (ibid.). It did not induce sweating.

To judge from one case recorded by Galabin, pilocarpin acts unfavourably with chloroform, there being a decided tendency to cardiac failure (B. M. J., ii., 1879).

\section{Therapeutical Action.-External and Local.-Eye} Diseases.-In some forms of inflammation with exudation, in rheumatic iritis, in paralysis of the third nerve, in glaucoma, and in many of the cases in which eserin is useful, equal advantage has been derived from pilocarpin (Practitioner, v., 22 ; Snell, B. M. J., i., 1882).

Galezowski prefers it to the former for contracting the pupil, as being less irritant to the conjunctiva.

The local use has been generally combined with the hypodermic.

Alopecia. $-\Lambda$ liniment recommended by Bartholow contains 1 part in 4 of extrnet of jaborandi, but it is combined with cantharides, which renders the special effect of the former 
drug doubtful. There can, however, be little doubt of its value when given internally in promoting growth of hair (Schmitz, of Cologne).

Therapeutical Action.-Intemal.--Skin Diseases. -A case illustrating the last statement occurred in a woman in whom alopecia was general and complete, and had been so for a long time; and after ten hypodermic injections of the alkaloid, the growth of hair began as well on the scalp as on other parts of the body (Lancet, i., 1882).

In another patient, to whom the remedy was given for the results of nephritis, hair naturally light became dark, and grew more after similar injections (ibid.). It is especially in the irritable, neurotic forms of skin disease, such as prurigo and pruritus, that the drug has been found useful by Simon and Pick (Practitioner, v., 25, 26).

The former compares its action to that of the bath, and did not find it effective in other disorders : the latter found some advantage from its use in a few cases of pruritus and urticaria, as well as in alopecia with pityriasis; none in "alopecia areata," or in psoriasis; his results in eczema were doubtful; he usually injected $\frac{1}{6}$ grain twice daily.

In cutaneous syphilide, condyloma, pharyngeal disease, etc., Lewin (Berlin) has noted definite cures from the same remedy (Practitioner, v., 27), but if pressed it may produce serious discomfort, and, generally speaking, it is inferior to corrosive sublimate: also, it renders the skin very sensitive to cold. Mr. Lockwood has obtained good curative results at the Lock Hospital.

In three cases of fotid perspiration of feet, permanent good is said to have followed hypodermic injection of pilocarpin (Practitioner, i., 1881).

Renal Diseases. - From the diaphoretic action of jaborandi, its administration in congestive kidney disorder was suggested, and there is certainly some evidence of its value. One of the earliest observers was Demme, of Berne, who gave it mainly to children, and noted that in those under four years of age sialagogue effects were more marked than diaphoresis-above that age, the latter occurred as in adults : in cases of desquamative 
nephritis after scarlet fever, of cardiac dropsy with deficient excretion, and in other cases of suppression, he records benefit, though occasionally vomiting and faintness were troublesome (Practitioner, v., 21).

Dr. Craig has reported a severe case of post-scarlatinal dropsy, with albuminuria, in which digitalis and other remedies had failed, and pulmonary œdema and convulsion occurred, but the symptoms were relieved by jaborandi in doses of 10 to 30 grains, and recovery followed (Edin. Journ., 1875).

Bruen and others have recorded equally good results in the dropsy of Bright's disease, but they are by no means constant. Ringer found the ill effects, vomiting and depression, often more marked than the good, but recommends its use when urremic symptoms are urgent. It is most applicable to cases with hypertrophy of left ventricle without obstructive lesion.

Dr. Allchin, in his practice at the Westminster Hospital, invariably treats all cases of Bright's disease with the drug in $\frac{1}{3}$-grain doses hypodermically, daily or on alternate days. The occasional unpleasant effects, such as vomiting (which seems to be more liable to occur when the solution used is more than a day or two old) and depression, are almost invariably counterbalanced by the subsequent feeling of relief that the patient experiences.

Careful notice should be taken of the quantity and specific gravity of the urine daily voided, lest a dangerous accumulation of solids in the tissues should result from the loss of fluid by the skin. It would seem, however, from the very distinct relief that often follows the profuse sweating, even in cases where the urinary secretion is scanty and uræmia is imminent, that there must be some considerable excretion of solid waste in the perspiration.

The same remark, as to inconstancy of effect, applies to observations of uræmia and uræmic and puerperal convulsions. In some cases a full dose of pilocarpin has quickly cansed sweating and subsidence of convulsion (Boegehold, Record, 1880 ; Bruen, B. M. J., ii., 1879 ; F. Barnes), whilst in others, depressing effects have been marked, and in one, profuse bronchial secretion threatened asphyxia (B. M. J., i., 1881). Fordyce Barker discourages the use of the drug in 
puerperal cases, since its depressing after-effect prevents sleep (Lancet, i., 1879). Some cases of "diabctes insipidus" have obtained benefit (Laycock, Lancet, ii., 1875), and also cases of mellituria, but in almost an equal number no relief has been experienced. The hydrochlorate or nitrate of pilocarpin given occasionally, in $\frac{1}{2 v}$-grain doses, relieves the dryness of mouth in diabetic patients.

In Septicæmia, Mr. Macnamara has seen some very good results. In one patient with pus in various joints, pnewmonia, muttering delirium, temperature $107^{\circ} \mathrm{F}$, and other serious symptoms, the effects of $\frac{1}{2}$ grain of pilocarpin injected under the skin were truly surprising; in ten minutes he was in profuse perspiration, with moist tongue, asked for food, began to talk, and the temperature fell to $103^{\circ} \mathrm{F}$; a similar condition recurred on the following day, with marked improvement, but he died that night (Diseases of Bones and Joints).

Pulmonary Disorders.-Jaborandi is said to be specially useful in incipient catarrh, whether nasal or bronchial, 1 to 2 drachms of the tincture, or $\frac{1}{4}$ to $\frac{1}{2}$ grain of the alkaloid, being given at bedtime, so as to induce profuse perspiration. In pleuritic effusion benefit has been obtained, and in pleuropneumonia the symptoms have been relieved (B. M. J., i., 1880).

It is noteworthy that by small doses of the same drug$\frac{1}{20}$ grain of the nitrate at bedtime by the mouth-night sweats have been markedly restrained, not very quickly, still effectually in most of thirty cases recorded by Dr. Murrell (Practitioner, v., 23). Later, he gave $\frac{1}{2}$ grain on alternate nights. He found the cough and other symptoms also relieved, and recommends the remedy in "winter" cough." It may be used in spray for chronic bronchitis.

In Asthma, the efficacy of the drug, sometimes at least, is striking. Dr. Berkart, after giving a caution as to its use in cases with cardiac degeneration, reports the case of a stout, flabby man, aged sixty-three, unable to lie down at night from asthma (bronchitic), with scanty expectoration. Shortly after the injection of 10 minims of a 2 per cent. solution of the alkaloid, and when perspiration set in, the breathing was relieved, and the improvement continued long afterwards (B. M. J., i., 1880). In nnother, aged sixty-six, after one injection "the 
patient was able to lie down for the first time for many years." In another, collapse occurred, but was at once removed by atropia. Dr. Berkart finds the remedy useful "in all eases of chronic pneumonia, when the heart-state offers no contra-indication."

Dr. Mackesy (Waterford) has also recorded a good case in a man of fifty, when the dises of Savory and Moore were used$\frac{1}{12}$ grain had no result, but $\frac{1}{4}$, and later $\frac{1}{3}$ grain, continued daily for a week, proved of great advantage (B. M. J., ii., 1880).

Dr. Mackey reports to me that in one neurotic lady, in whom a severe attack of bronchial asthma had lasted for four hours, the injection of $\frac{1}{3}$ grain (discs) determined the cessation of the paroxysm within five minutes, and it did not recur so badly again for many months; but the consequencessalivation, sweating, pain in micturition, and general depression-were somewhat severe.

In another, $\frac{1}{6}$ grain (dises) gave distinct relief in about half an hour, during an attack also mainly bronchial in character; on another occasion, when more purely "nervous," the relief was not marked; whilst on another, when $\frac{1}{12}$ grain was taken by the mouth every hour or two for four doses, the nausea and depression were very trying.

In Diphtheria good hopes of benefit were raised by Guttmann's eighty cases of recovery; he gave about $\frac{1}{50}$ grain to children, $\frac{1}{20}$ to adults for a dose, and wine after (Practitioner, v., 26); but this favourable experience has not been general. The cases of Laschkewitz were all fatal. It is agreed that the profuse exudation may detach the false membrane, but it is, in itself, of some danger; and still more so, the depression from profuse sweating, pulmonary œdema, etc. Some moderately bad cases did well with it, and it is perhaps best adapted for pharyngeal diphtheria in adults; but the balance of opinion is. now against it (Lancet, ii., 1881 ; i., 1882).

Nerve-Disorders. - Besides pruritus, convulsion, asthma, and diabetes, which have been previously discussed, pilocarpin has been given in hiceough, and in various forms of muscular spasm. In a case of hiccough, which had lasted several weeks, one injection of pilocarpin, $\frac{1}{2}$ grain, finally cured, after failure of other remedies (Ortille). 
Mr. McKeown has recommended pilocarpin in the more serious disorders of double optic neuritis, even when meningitis of the base of the brain is present: he states that under this remedy the exudation cleared up, the disc and the calibre of the vessels became normal (B. M. J., i., 1882).

In certain cases of "detachment of retina, with opacities of the vitreous," an injection of $\frac{1}{6}$ grain pilocarpin night and morning was tried for some time, and with apparent improvement in vision; but this was only temporary, and on the whole the inconveniences of the treatment were much greater than its advantages (Lancet, ii., 1882).

Lauderberg has even shown reason for believing that cataract may develop under this treatment, though opacities in the vitreous may clear up (ibid.). More recently, additional evidence in its favour has been furnished by Dr. Caro (Naples), who reports six cases of "exudative choroiditis" and serous retinitis with opacity of vitreous, of which four were cured under injections of the muriate of pilocarpin, $\frac{2}{7}$ grain, repeated about sixteen times (Rec. d'Ophthalm., Sept., 1884).

Hydrophobia. - Several instances of recovery under the free sweating of jaborandi have been recorded, notably one by Dumont. A man got pain in the arm two months after a bite on the hand, and after severe symptoms had set in $\frac{1}{3}$ grain pilocarpin was injected, and repeated six times. It caused severe symptoms of sweating and salivation, but was followed by temporary relief, though, ultimately, by sudden death from cardiac failure. In the other case, which recovered, the diagnosis was somewhat uncertain; sedatives also were used (Lancet, i., 1882). The failure of the drug was unfortunately evident in two cases carefully recorded by Dr. Charteris (Lancet, ii., 1880). In one of these, however, marked, though temporary, relief was given.

In a case of Locomotor Ataxy, pilocarpin injection is said to have given relief to "lightning pains" after failure of morphia (Oscar Jennings, Lancet, ii., 1882).

Specific Fevers.-The drug should be specially useful in cases when the eruptions of the exanthemata do not readily or properly develop, and Gubler recommended it highly in such conditions. In a case of collapse in a child, with romiting, 
purging, and very serious symptoms dependent on "suppressed" scarlatina, about 5 minims of the fluid extract were given (with ammonia) for some hours without effect, but after the injection twice of $\frac{1}{30}$ grain pilocarpin, improvement occurred with copious passage of urine (Park, Lancet, i., 1883). It was for this use of the drug, and in a similar case, but which ended fatally, that so much odium was unjustly incurred by Dr. Macnaughton Jones in Cork (B. M. J., ii., 1880). It has been recommended in mumps.

In Ague, Griswold found, in six cases, that an injection, given in the cold stage, cut short the attack (Practitioner, v., 23), but Ringer failed to corroborate such good results.

In Cholera even, it was found of service in New York (Simmons). It is noteworthy that no relief to acute "rheumatic fever" is claimed from the drug, but it has acted well in sciatica and muscular rheumatism.

In Uterine Inertia it has sometimes proved useful (Practitioner, v., 22), but cannot be depended upon like ergot (Clay, Galabin, B. M. J., i.., 1879). Its value in increasing the secretion of milk has been already mentioned (Physiological Action).

PREPARATIONS AND DOSE. - Jaborandi: Extractum (alcoholic): dose, 2 to $10 \mathrm{gr}$. in pill. Extractum fuidum (liquor): dose, 10 to $60 \mathrm{~min}$. Infusum ( $1 \mathrm{oz}$. to 1 pint boiling water) : dose, 1 to 2 oz. Tincture ( $2 \mathrm{dr}$. percolated from $1 \mathrm{dr}$. leaves) : dose, 10 to $60 \mathrm{~min}$.

Pilocarpiæ: Hydrochloris: dose, $\frac{1}{20}$ to $\frac{1}{2} \mathrm{gr}$. by the mouth; $\frac{1}{10}$ to $\frac{1}{3} \mathrm{gr}$. by the skin. Nitras, idem. Guttre, $2 \mathrm{gr}$. in the oz. for eye diseases. Injectio hypodermica (1 gr. to $20 \mathrm{~min}$. of distilled water): dose, 2 to $6 \mathrm{~min}$.

The pure alkaloid is not used medicinally. 


\section{SIMARUBACEAE.}

\section{THE QUASSIA FAMILY.}

Av order of about forty species of exogenous trees and shrubs, natives chiefly of the tropics, and noted for the intense bitterness of their wood. The leaves are alternate, usually compound, dotless, and exstipulate; the flowers are tetra-pentamerous, the hypogynous stamens equalling the petals in number; the ovary is superior and four- to five-celled, and ripens into four or five one-seeded drupes, which are placed around an axis.

\section{PICRAENA EXCELSA.}

(Quassia Wood.)

DESCRIPTION.-A stately West Indian tree, attaining the height of 100 feet. The leaves are pinnate; the flowers small, yellowish, and paniculate; the black drupes the size of peas. The wood is imported in billets without the bark, is tough and yellowish-white, often marked with irregular black lines (caused by a fungus), and an efflorescence of nitre; it is commonly sold in the form of chips or raspings.

ACTIVE, INGREDIENTS.-The wood of the picræna owes its qualities to a peculiar neutral principle called quassin, or quassite, $\mathrm{C}_{10} \mathrm{H}_{12} \mathrm{O}_{3}$. When separated it appenrs in the form of small, white, prismatic crystals, destitute of odour, but intensely bitter; readily fusible, soluble in alcohol, less so in ether; it is moderately, but slowly, soluble in cold water, more so when acids or alkalies are added; a small quantity of permanganate 
of potash quite removes the bitter taste. There is no tannin or gallic acid in the bark.

Physiological Action. - The bitterness of quassia serves to improve appetite and stimulate digestion, but overdoses excite gastric irritation. It is poisonous to fish and insects, and even, it is said, in concentrated form, to rabbits and dogs. When not fatal, it has caused partial paralysis.

Therapeutical Action.-As a vermicide enema, for injection into the rectum in cases of thread-worms, it is very useful, but occasionally, when too strong, has caused serious irritant symptoms. Alternate injections of the infusion and of lime-water often suffice for the cure.

In dyspepsia, and especially in that due to intemperance, it is of service, and has the advantage of cheapness, but is commonly considered inferior to gentian.

In hysterical affections the repulsiveness of the drug aids its medicinal effects.

Preparations and Dose.-Pulvis: dose, 10 to $20 \mathrm{gr}$. Extractum, 3 to $5 \mathrm{gr}$. Infusum: dose, 1 to $2 \mathrm{fl}$. oz. Tinctura: dose, $\frac{1}{2}$ to $2 \mathrm{fl}$. dr. It is often combined with iron, forming an astringent bitter tonic mixture. The so-called "bittercup" now sold is made from quassia wood.

ADULTERATIONS.-Chips of other woods of similar flavour; but quassia may be distinguished by its intense bitterness.

The so-called sulphate of quassin, which excited so much attention in Italy a few years ago, was proved by De Luca to be a mixture of various bitter substances, but containing no quassin. 


\section{CELASTRACEAE.}

\section{EUONYMUS ATRO-PURPUREUS.}

(Spindle Tree-Wahoo.)

DESCRIPTION.-An ornamental shrub, 6 to 12 feet high, indigenous to the United States. The leaves are petiolate, opposite, ovate, serrate; the flowers cymose, dark-purple, enclosed in a crimson, five-angled pod; the fruit in crimson, pendulous, smooth, four-lobed capsules. The bark and rootbark are used in medicine, and occur in curved or quilled pieces, not more than $\frac{1}{8}$ inch thick; externally, light-grey mottled; internally, brownish-white.

ACTIVE INGREDIENTS.-A neutral principle, termed euonymin, bitter and amorphous, has been extracted; it is soluble in alcohol and water. Yellow acicular crystals hare also been obtained, as well as asparagin, euonic and other vegetable acids, resins, starch, etc. A glucoside, euonymite, has been described.

Ordinary so-called euonymin is an olive-brown extract, generally in powder.

Physiological Action.- Recognized by many observers in America as a cholagogue, tonic, and diuretic, Rutherford specially directed attention to its first-named effect, as shown by his experiments on animals; it acted always as a "powerful hepatic, though feeble intestinal, stimulant;" personally he associated the effect of 2 grains with some depression (B. M. J., i., 1879). Four grains of euonymin produced in myself nausea, vomiting, flatulent eructations, profuse purging with tormina, cold sweats, and prostration, but this is not a usual effect. 
Therapeutical Action. - In "bilious" conditions. euonymin is now an accepted, though not widely-known, remedy. There is some uncertainty either in the preparation, $o r^{2}$ in its action on different subjects; for whilst several observers report a distinct purgative effect from 1 and 2 grains (B. M. J., ii., 1879), others find only some improvement in the symptoms of "biliousness," and advise either a combination with rhubarb, etc., or a saline draught to secure a distinct aperient effect. With such adjuvants, however, a good preparation will often act exceedingly well, and without any drawback. It does not irritate so much as podophyllin.

PREPaRATIONS AND DOSE.-Enomymin: dose, 2 to $3 \mathrm{gr}$. in pill, with extract of hyoscyamus q.s. and "3 gr. pil. rhei c0., or I gr. of euonymin, combined with $4 \mathrm{gr}$. of iridin, is a good purgative dose, and similar synergic drugs. Tincture (1 in 4): dose, 10 to $40 \mathrm{~min}$. 


\section{RHAMNACEA.}

\section{THE BUCKTHORN FAMILY.}

Av order of about 250 exogenous trees and shrubs, with simple, undivided, usually alternate leaves, small and mostly greenish flowers, the parts of which are in fours or fives. The perigynous stamens are opposite the petals, and the superior and two- to four-celled ovary is seated upon a fleshy dise. The fruit is either fleshy or dry. The order abounds with plants possessed of active properties, the most noted of them belonging to the typical genus Rhamnus. The principal species are :-

Rhamnus catharticus .. . . . Purging buckthorn.

Rhamnus frangula ... ... . Black alder.

Rhamnus purshiana .. .. .. Cascara sagrada.

\section{RHAMNUS CATHARTICUS.}

(Purging Buchthorn.)

DESCRIPTION.-A spinous shrub, 6 to 10 feet high, common in English hedges, with ovate, glabrous, and serrated leaves, and abundance of minute tetramerous flowers, followed by small, globular, black berries. The seeds, four in number, are smooth, elliptical, and plano-convex. The fresh berries contain a greenish sarcocarp, and yield a juice of the same colour, changing to red on exposure, of unpleasant odour, and bitter acrid taste.

ACTIVE INGREDIENTS. - The purgative properties of buckthorn are probably due, for the most part, to an uncrystallizable bitter substance called rhamnocathartin, the descriptions of which are still somewhat vague. It has been questioned 
whether this principle may not be identical with rhamnin, another ingredient of buckthorn berries; but the latter is almost or quite tasteless, and can be shown by the microscope to present a regular crystalline structure. Rhamnocathartin must not be confounded with cathartic acid, $\mathrm{C}_{180} \mathrm{H}_{96} \mathrm{~N}_{2} \mathrm{O}_{32}$, the active principle of senna, and found also in the bark of the Rhamnus frangula. Rhamnocathartin seems to be a certain purgative in 2 or 3 -grain doses, but is disagreeable to take, and slow in action.

Physiological Action.- The juice of the berries causes dryness of the mouth and throat, with extreme thirst, nausea, in certain cases vomiting, griping pains that extend throughout the abdomen, and violent purging with watery evacuations.

Therapeutical Action.-The juice was at one time prescribed in dropsy, gout, and rheumatism, but is now seldom employed except in domestic practice, where it is used as a laxative, suitable especially for children, in the form of syrup.

PREPARATIONS AND Dose.-Symumus: dose, $\frac{1}{2}$ to 1 oz.; for young children, $\frac{1}{2}$ to $2 \mathrm{dr}$.

\section{RHAMNUS FRANGULA}

\section{(Black Alioer)}

is allied to the R. catharticus, and grows largely in Holland, whence the bark is imported in quills with rough grey-brown cortex. It contains, besides cathartic acid, mmodin (described under Rhubarb), and is recommended as a "tonic laxative, especially useful in hremorrhoids." It is said not to cause griping or sickness, unless too young a bark be employed. A fluid extract is made (dose, 1 to 4 drachms), and an "aperient fruit lozenge" contajining it is in popular demand. 


\title{
RHAMNUS PURSHIANA.
}

\author{
(Cascara Sagrada.)
}

DESCRIPTION.-A shrub or small tree, found on the west coast of North America: it has elliptic, toothed, pubescent leaves, large flowers arranged in cymes, and three-lobed black drupes. The bark, which is commonly known under the old Spanish term (cascara sagrada, sacred or chittem bark), is in curved pieces, externally smooth and grey, internally yellowish: it has a smooth fracture.

Active InGREDIENTS.-Professor Prescott reports the presence of three resins - a brown one, of strong bitter taste; a red, nearly tasteless one; and a third, yellow, also tastelesstannic and other acids, a fatty and a volatile oil, and a neutral, crystalline body, soluble in absolute alcohol and in benzole, not in ether or chloroform.

ACTION.-Almost all observations on this point are clinical, rather than physiological.

It has been much vaunted, especially in America, as a remedy for chronic constipation, and is said to act specially on the vas()motor system, "stimulating the secretory apparatus of the alimentary canal, and increasing the peristaltic action of the intestine:" also it is said to be without unpleasant effects, being a laxative tonic rather than a cathartic; and its continued use tending to cure the disorder, rather than to make the patient habitually require the drug.

Many have found it uncertain in effect. In a case of constipation accompanying diabetes, doses of 30 minims and upwards of liquid extract, tried on three occasions, caused only griping and straining without good effect. In another case, connected with deficient liver action, no satisfactory result was obtained from three or four trials of the same. In a third case of habitual constipation in an anæmic delicate woman, the extract failed, but the "cordial" in which it 
is combined with berberis answered well; and in a fourth case of obstinate constipation, dependent mainly on profound nervedepression, insufficient feeding, etc., the latter preparation was really satisfactory, as compared with salines, rhubarb, etc. (cf. B. M. J., i., 1883). A report of several hundred observations (Australian) is in the main very farourable, but griping and depression occurred sometimes from 10 to 20 minims of the liquid extract (B. M. J., ii., 1883). In short, although a useful alternative remedy, I believe it will not be found to justify the extravagant reports of its efficacy, and in my opinion it loses its effect after continuous taking for some time.

PREPARATIONS AND DOSE.-Extractum: dose, 2 to $8 \mathrm{gr}$. in pill. Fluidum : dose, 10 to 60 min. "Cordial," with berberis and aromatics: dose, $20 \mathrm{~min}$. twice or thrice daily as stomachic; 1 to $3 \mathrm{dr}$. as laxative. It may be usefully combined with salines. 


\section{ANACARDIACEAE.}

\section{THE SUMACH FAMILY.}

As important, though not extensive, order of exogens, composel chiefly of large trees and shrubs, and belonging principally to the tropics. The leaves vary from ovate to ternate and pinnate: the flower's are inconspicuous, usually pentamerous, commonly unisexual by abortion, and with as many hypogynous stamen: as there are petals. The ovary is generally superior and onecelled, and contains a solitary ovule, singularly and characteristically attached by a cord to the base of the cell; while the cord itself is so lengthened as to make the orule appear pendulous. The fruit, when ripe, is ordinarily drupaceous and indehiscent.

The plants of this order often abound with a resinous juice, which is sometimes acrid and poisonous. When the acrid matter is not developed, the fruits are usually edible, as the spondias and the mango.

\section{Medicinal Species.}

Pistacia lentiscus........ Mastich.

Pistacia terebinthus .. .. .. Chian turpentine.

Anacardium occidentale .. .. Cashew nut.

Rhus toxicodendron ... .. Poison oak.

\section{PISTACIA LENTISCUS.}

(Mastich.)

A shrub indigenous to the shores of the Mediterranean. The leaves are pinnate, composed of eight to twelve leaflets, which are oval, entire, and glabrous; the small green flowers are borne in axillary panicles; the berries are small, pea-like, and brownish-red when ripe. 
The sweet and fragrant resin called mastich is obtained from this tree by incision, and if pure is in small, pale-yellow, friable tears, which, when chewed, become soft and ductile: it is entirely soluble in ether and in chloroform; soluble in alcohol to the extent of 80 per cent.

The composition of this larger (soluble) portion is $\mathrm{C}_{20} \mathrm{H}_{32} \mathrm{O}_{3}$, and of the residue (which has been termed "masticine") $\mathrm{C}_{20} \mathrm{H}_{31} \mathrm{O}_{22}$; the latter is soluble in ether. Mastich also contains a small amount of volatile oil.

Dissolved in ether it is used for "strengthening" the gums, and as a masticatory, on account of the agreeable odour which it communicates to the breath; cotton saturated with it makes a "filling" for carious teeth. Debout recommends mastich in incontinence of urine in children: 1 drachm twice a day for four days is successful in many cases (Gaz. des Hôp., No. 150, 1860 ; Med. Times, i., 1860). It has been employed in chronic diarrhoa and dysentery; also in chronic catarrh; and in old and obstinate gleet.

\section{CHIAN TURPENTINE.}

\section{(Not Officinal.)}

Chian, Scio, or Cyprus turpentine is an oleo-resin procured by incision of the terebinth tree, Pistacia terebinthus, a common inhabitant of Syria and the Greek Archipelago. The leaves of this tree are pinnate; the seven to nine leaflets are. oval, entire, and glabrous; and the small panicled flowers are followed by roundish, purple fruits. When pure, the turpentine is thick, yellowish, and sweet-scented, the odour somewhat resembling that of lemon or fennel. The taste is ngreeable, and though sharp, by no means acrid.

Essentially it consists of a resin and a volatile oil, the latter holding the former in solution.

In physiological action it nuch resembles similar substances derived from trees of the order Coniferæ; but therapentically it has obtained at least a temporary special reputation on account of the statement of Mr. John Clay, that it can cure 
true scirrhus and epithelial cancer of the uterus and breast. In the Lancet of March, 1880, he relates four remarkable cases which presented all the characters of malignant disease-pain, ragged ulceration, offensive discharge, cachexia, etc. ; and after a more or less prolonged use of pills containing 6 grains of this drug with 4 grains of sulphur, or of a mixture containing the same with ether, the pain was relieved, the discharge altered and lessened, and the foul ulceration contracted and almost or quite healed. A few corroborative cases have been reported, and Mr. Clay has reiterated his views, attributing many failures to the adulteration of the drug which has been, no doubt, practised; but a largely preponderating amount of professional experience is quite against the real possession of any such powers by Chian turpentine, - witness the reports of Tait, Hickinbotham, H. Morris, Hulke, and others (Lancet, ii., 1880). A curious instance of how mistakes may arise is given by Dr. Playfair in the account of a case of sloughing uterine fibroid, simulating cancer, and also simulating improvement under turpentine, when really it was due to detachment and extrusion of the growth from the uterus owing to natural causes (Med. Times, ii., 1880). Some desiccative powers may be fairly credited to the drug, and are exerted more markedly in some cases than others-i.e., vascular growths apparently become harder and drier,--but its action in the cure of true cancer has still to be proved.

In Prostatorrhœa and Gleet, Adams found Chian turpentine, in 5-grain doses, very successful (Med.-Chir. Rev., i., 1852).

\section{ANACARDIUM OCCIDENTALE. (CASHEW Nut.)}

\section{(Not Officinal.)}

DESCRIPTION.-The cashew is a common tree both in the East and West Indies. The trunk is short and crooked; the leaves are obovate, 4 to 8 inches in length, glabrous, and of hard texture; the small, pale-yellow flowers grow in terminal panicles, and are succeeded by fruits of remarkable character, the peduncle swelling into the semblance of a pear, 
and acquiring a fine crimson or yellow colour, while the mut, borne on the summit, is brown, reniform, and rather more than an inch in length. The shell is hard and brittle, and encloses a single white nut.

ACTIVE INGREDIENTS.-The pericarp contains much thick acrid oil, which consists of anacardic acid and about 10 per cent. of a vesicating principle, cardol, $\mathrm{C}_{21} \mathrm{H}_{30} \mathrm{O}_{2}$, isolated as a yellowish oil of aromatic odour, and soluble both in alcohol and ether.

Physiological Action.-External.-This is of irritant character-staining, inflammation, swelling, and even vesication being produced by continued local contact of the oil, or of its vapour when the nut is roasted.

Physiological Action.-Internat.-The nut-kernel is edible, and there is no record of serious internal irritation from the oil: three or four drops may be taken without discomfort, either because of insolubility in, or of dilution by, the intestinal secretions.

Therapeutical ACtion.-Oil of cashew, locally applied, has been much lauded as a remedy for leprosy. The discoverer, Dr. Beauperthuy, communicated his secret to Dr. Bakewell, at whose suggestion Dr. Milroy was sent out to Cumana by the College of Physicians, to examine into the value of the alleged remedy and the truth of the reports. The result was, that while admitting that the oil had a certain value in alleviating the disease, Dr. Milroy was of opinion that much of the recorded success was to be attributed to hygienic measures in connection with a liberal allowance of fresh meat, whilst internally perchloride of mercury was also given.

The oil has been used as a caustic for warts, corns, ringworm, and obstinate ulcers. 


\section{RHUS (RHUS TOXICODENDRON). (PoISON OAK.)}

\section{(Not Officinal.)}

DESCRIPTION.-A shrub indigenous to the United States, where it is common in the woods. It is naturalized in Europe, and is better known as "sumach poison." It usually grows erect, about 3 feet high, but if near trees or walls, climbs by rootlets to the height of 30 or 40 feet; it is still, however, considered a variety, not a distinct species of the same plant, and is known as Rhus radicans (Linn.), or "poison ivy." The Rhus glabrum (smooth sumach or strghorn tree) is a distinct species, commonly placed amongst the Terebinths, to which the $R$. toxicodendron is referred by some authors. In both varieties of the latter the leaves are alternate, and composed of three large leaflets, 2 to 4 inches in length, ovate, acute, usually entire, glabrous, and shining. The small pentamerous greenish-white flowers are borne in panicles; the fruit consists of roundish, pale-green berries. When a branch or leaf is broken off there exudes a yellowish, milky juice, which in a little while turns black; even the atmosphere surrounding the shrub is said to be tainted with emanations from it.

ACTIVE INGREDIENTS.-The researches of Maisch have proved that the canse of the acridity of sumach-juice is the presence of a volatile acid-toxicodendric acid-which, when isolated, is found to produce similar characteristic effects upon the skin and mucous membranes. Stillé found in the leaves a notable quantity of ammonia, and Khittel obtained tannin, oil, wax, etc.

Physiological Action.-Extemal.-Contact with the plant or its exhalation, in some persons, induces local irritation, redness, and swelling, especially of exposed parts, and where the skin is delicate, e./., near the eyes, and where there are many hair-follicles. Exposure to high temperature, e.g., during distillation of the plant, almost always increases such symptoms 
(Maisch, Proc. Amer. Pharm. Assoc., 1865). If the eyes are affected there is much burning pain, with inflammation of the lids; papules and vesicles may form on any inflamed partthere is generally much œedema and severe itching, not confined to the seat of eruption, but diffused over the body, and, in short, a condition very similar to erythema, or eczema, of varying degrees of severity.

The same results have followed from contact with fresh "Japanese lacquer," and have been proved to be dependent upon an allied species of the same plant.

\section{Physiological Action.-Internal.-Digestive System.} - Irritation of internal organs may follow from simply external contact with the plant, whilst the effect already described may equally be produced by its internal administration-thus the mucous membranes may become red, swollen, and very irritable, as seen in the mouth and throat; hence thirst, nausea, or vomiting, pain in the abdomen (which is worse after food), diarrhœea, with tenesmus, and often passage of blood. Strangury also occurs, and there may be diuresis, or in some cases retention of urine.

Nervous System.-Evidence of action on this is shown by dulness and confusion of thought, with vertigo, restlessness, and depression. After large toxic doses there is a markedly febrile condition of typhoid character, with parched lips, sordes, etc., and delirium; it is sometimes intermittent, and marked by profuse sweatings. A common characteristic of the dmig is the pain and stiffness caused all over the body, but particularly in the lumbar region and in the joints-fibrous structures seeming to be most affected; in the lower extremities a sense of numbuess may be experienced.

These symptoms resemble those of some varieties of rheumatism, and it is noteworthy that they are made worse by warmth, and even by rest; there is often slight swelling of the affected parts.

The above-described effects of rhus, though so distressingto the sufferer, appear to be seldom fatal, and, as already remarked, a certain constitutional predisposition appears to be a condition of their occurrence. I have myself witnessed 
several instances of its poisonous influence, and can vouch for the manifestation of most of these phenomena.

SYNERGISTS.-Arnica and aconite are somewhat allied, also bryony.

ANTAGONISTS.-Alkalies and salts of lead chemically nentralize toxicodendric acid, and are practically found very useful-acetate of lead especially-in relieving the local pain and inflammation: Dr. White recommended "black-wash," and afterwards zinc oxicle in powder. Ice has also relieved, as well as lotions of lime-water and of linseed oil. Stillé recommends "alum-curd" at all stages of the inflammation; later, perchloride or sulphate of iron carefully painted over the inflamed surface. Internally, in severe cases, opium may be required; coffee relieves some of the symptoms; and laxatives may be necessary, with spare diet, rest, etc.

Therapeutical Action.-The properties of the Rhus toxicodendron were first brought into notice about the year 1798, by Dufresnoy, a physician at Valenciennes. Alderson, in England, likewise made some interesting observations with regard to them. Rhus was included in the London Pharmacopoeia in 1836.

Skin Diseases.-Dufresnoy's attention was attracted to the plant by the circumstance of a young man, who had suffered from a six years" "dartre" upon his wrist, being cured by accidental subjection to its infuence; and shortly afterwards this physician employed it successfully in various cases of obstinate herpetic eruption. Herpes zoster, pemphigus, and eczema, especially when accompanied by burning or itching sensations, represent the class of ermptions which are benefited by the extermal and internal use of rhus; in erythema also, and erysipelas, particularly when vesicles and bulle develop, it is a good remedy.

Rheumatism.- It is valuable again in various subacute and chronic rheumatic nffections of the fibrous tissues; the symovial membranes seem to be less amemable than the fibrous structures, such as tendons, ligaments, and fascir. 
In the after-stage of acute rheumatism, when the temperature has fallen to $100^{\circ} \mathrm{F}$. or lower, and the patient still suffers from wearing stiffness, and aching of subacute character in the joints, rhus is useful. It should be applied externally in the form of liniment or on compresses, and be given internally, in 5 or 10 -minim doses, every two to four hours.

In cases of "scarlatina rheumatica," it is often of great service, and especially if typhoid symptoms are present.

Paralysis.-Dufresnoy administered the dried leaves, in doses uf half a grain or a grain, twice a day, in cases of paralysis. The patients recorered to a certain extent; the first symptom of imprevement consisting in sensations of pricking or twitching in the limbs, analogous, apparently, to those induced by strychnia. Experience had not at that time defined the class of paralytic affections amenable to rhus, but it is now probable that it includes only cases that depend upon a rheumatic condition.

Enuresis, etc.-The Rihus aromatica, a kindred plant, has recently been strongly recommended in incontinence of urine connected with paralysis of the sphincter: thus a case is recorded of a young married woman, suffering from this disorder after a prolonged labour, who recovered under this medicine after the failure of many others (Record, 1880).

It has been found useful in catarrh of the bladder, and even in enlarged prostate (Amer. Journ., Aug., 1880).

Preparations and Dose.-Of the dried and powdered lenves, $\frac{1}{2}$ to $1 \mathrm{gr}$. three times a day. Tinctura Rhois ( $2 \frac{1}{2}$ oz. of leaves and young twigs to 1 pint of proof spirit): dose, 3 to $10 \mathrm{~min}$.

The expressed juice of the fresh leaves preserved by alcohol (a liquid extract) is employed in the United States, and is probably a better preparation: dose, 1 to 5 min. Linimentum ( 2 dr. of the tincture to $4 \mathrm{or}$. of soap liniment). An Emplesstrum Rhiois is also prepared. 


\section{BURSERACEA.}

TREes or shrubs, with alternate or opposite compound leaves, sometimes stipulate and dotted; flowers perfect, or sometimes diclinous by abortion; calyx persistent, with two to five divisions; petals and stamens perigynous; ovary one- to five-celled, superior; fruit dry, one- to five-celled; seeds exalbuminous; cotyledons plicate, rarely flat.

The plants of this order contain a large quantity of balsam and resin.

\section{Pharmaceutical Species.}

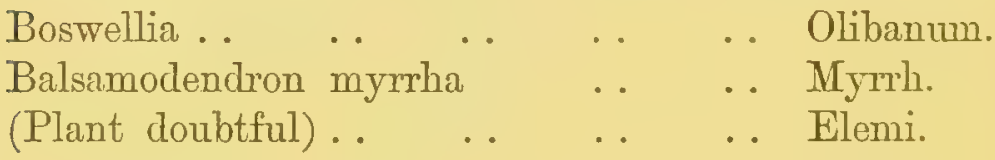

\section{OLIBANUM.}

DESCRIPTION.-Olibanum is the resin produced by two or three species of Boswellia-trees indigenous to Indin, Arabia, and Africa. The leaves are pinnate, and the small pinkishwhite flowers are borne in racemes. From incisions in the bark a milk-white liquid flows and hardens, and is foumd in commerce in the form of roundish oblong tears of yellowish or red colsur, eovered with whitish powder. They are of balsamic odlour and taste, and soften on chewing; they form an emulsion with water, and are partly soluble in alcohol. 
Active INGREDIENTS.-( 1 ) A volatile oil ( 4 to 7 per cent.), which consists of olibene, $\mathrm{C}_{10} \mathrm{H}_{10}$, and of an oxygenated oil, and is of the odour of turpentine; (2) a resin, $\mathrm{C}_{20} \mathrm{H}_{30} \mathrm{O}_{3}$, not soluble in alkalies; (3) gum, 25 to 35 per cent.

Action.-Olibanum is astringent and stimulant, whence it is occasionally employed in chronic catarrhal affections of mucous membranes; also as an ingredient of stimulant absorbent plasters, and of fumigating pastilles. It is the frankincense of the Old Testament, and was used in embalming. Hippocrates prescribed it in pulmonary disease, etc.

\section{BALSAMODENDRON MYRRHA.}

\section{(Мукен.)}

DESCRIPTION.-The spontaneous exudation of a tree indigenous to the neighbourhood of the Red Sea. It is of small size, with spinous branches, a few pinnate leaves, and insignificant green axillary flowers, followed by little oval, purplish drupes. Myrrh itself, in its pure and concrete state, is a gum-resin in reddish-brown tears or masses, faintly trans. lucent, pleasantly odorous, especially when rubbed, and possessing a warm, aromatic, and bitter taste: in cold weather it is readily powdered; in warm weather, not till after drying; and cvaporating the volatile oil. With water it forms a yellowish emulsion; in alcohol it is partly soluble.

ACTIVE INGREDIENTS.-Myrrh contains 2 per cent. of myrrhol, $\mathrm{C}_{10} \mathrm{H}_{14} \mathrm{O}$, a volatile, pale-yellow oil, soluble in alcohol and in ether (the formula given is the same as that of thymol, but it varies somewhat according to oxidation, ete.);-from 30 to 40 per cent. of a resin, called myrrins, $\mathrm{C}_{45} \mathrm{H}_{32} \mathrm{O}_{10}$, soluble in alcohol, ether, and acetic acid, and chnnging under prolonged heat into myrrhic noil, $\mathrm{C}_{14} \mathrm{H}_{32} \mathrm{O}_{8}$; - and 40 to 50 per cent. of gum. 
Physiological ACTION.-Like other resins, myrrh is a stimulant. Mucous surfaces are affected by it, and the secretion therefrom is augmented, in particular that from the bronchial tubes.

Taken in small or medicinal doses, it excites sensations of warmth, and quickens the desire for food, promoting the healthy action of the digestive organs, and giving tone in general to the system. It does not purge or constipate, but was formerly often combined with purgatives, and obtained reputation as a "deobstruent."

Large doses are somewhat hurtful, the sensation of warmth in the stomach being so great as to become unpleasant, and the pulse being slightly increased in fulness and frequency.

In its remote action this drug is moderately tonic, but it exerts none of that influence over the nevvous system which marks the action of gum-resins derived from the Umbelliferæ.

THERAPEUTICAL ACTION.-Extemal.-Ulceration, etc. - Lotions and ointments containing myrrh are found serviceable in cases of foul and indolent ulcers. Spongy and ulcerated gums are likewise well treated with the tincture; and the same, diluted with water, forms an excellent gargle in ulcerated throat. As a dentifice, usually in combination with other substances, myrrh has long enjoyed celebrity.

Therapeutical Action.-Intemal.-Dyspepsia, etc. - When this disorder is connected with an atonic condition, tincture of myrrh is a suitable remedy, but is not usually prescribed alone. Delioux de Savignac states that it acts well in gastrodynia, but he generally combines it with bismuth (Bull. de Thérap.). It is an ingredient of the rhubarb, the aloes, and the galbanum pills, and given with iron in the mistura ferri composita assists a cure in cases of anxmia and chlorosis.

Mucous Discharges.-In similar combinations it is a good remedy in cases of chronic hypersecretion from mucous membranes, whether from the bronchial tubes or vaginal or urethral surfaces. It is hence prescribed in pulmonary catarih, and in phthisis when accompanied with much puriform expectoration. 
Campardon has recommended it in pertussis (Bull. de Thérap., ii., 1878). It seems to have some stimulant action on the torpid uterus, and to aid in the cure of atonic amenorrhoca.

Preparations and Dose.-Milyrha: dose, 10 to $30 \mathrm{gr}$. Tinctura (54 $\frac{1}{2}$ gr. to $1 \mathrm{fl}$. oz.) : dose, $\frac{1}{2}$ to $1 \mathrm{fl}$. dr. Pitula aloes et myrrhe : dose, 5 to 10 gr. Gargarisma: dose, $\frac{1}{2} \mathrm{dr}$. of tincture to $1 \mathrm{oz}$. of water.

\section{ELEMI.}

The concrete resinous substance bearing this name appears to be the product. of three different plants: usually it is yellowish and waxy, and possessed of a pleasant odour, and warm, somewhat bitter, flavour. "Moistened with alcohol it disintegrates, and shows small acicular crystals;" it readily liquefies.

ACtive INGREDIENTs.-(1) A colourless, neutral, fragrant oil of elemi, $\mathrm{C}_{10} \mathrm{H}_{16}$, has been obtained; and (2) soluble and insoluble resins, termed brëin and amyrin: also bryoidin, brëidin, and amyric acid.

The properties of elemi, like those of other substances of similar nature, are stimulant. It is not administered internally; but in ointment it may sometimes be advantageously employed. 


\section{LEGUMINOSAE OR FABACEAE.}

THE PEA FAMILY.

Next to the Compositæ this is the most extensive order of flowering plants. It is distributed all over the world, and presents an endless diversity of habit and properties. In many species the flowers are papilionaceous; in others they are minutely rosaceous; but in either case the fruit is a legume. The leaves are generally compound and stipulate.

\section{Pharmacentical Species.}

Physostigma venenosum .. . . . Calabar bean.

Cassia obovata, C. lanceolata, C. elongata Senna.

Cathartocarpus fistula .. . . . . . Cassia fistula.

Sarothamnus scoparius... . . . . . Broom.

Pterocarpus marsupium ... . . Indian kino.

Myrospermum Peruvianum .. . . . Balsam of Peru.

Myrospermum toluiferum .. . . . Balsam of tolu.

Hæmatoxylon Campechianum .. .. Logwood.

Copaifera multijuga, and other species Copaiva.

Acacia catechu . . . . . . . . Catechu nigrum.

Indigofera $\quad \ldots \quad \ldots \quad \ldots \quad \ldots \quad \ldots \quad \ldots$ Indigo.

Pterocarpus Santalinus.. . . . . Sandal wood.

Tamarindus indica . . . . . . The tamarind.

Astragalus verus . . . . . . . . . . Tragacanth.

Glycymhiza glabra . . . . . . . L Liquorice.

Mucuna prumiens . . . . . . . . Cowitch or cowhage.

Araroba, Po' de Bahia.. . . . . . . Groa powder.

Abrus precatorius. . . . . . . . . Indian liquorice or jequirity. 


\title{
PHYSOSTIGMA VENENOSUM.
}

\author{
(Calabar Bean.)
}

DESCRIPTION.--This plant was raised from seed in the Edinburgh Botanic Gardens in 1860, and named physostigma by Dr. Balfour on account of a singular crescentiform hooded appendage to the stigma. In its native country it is a large, climbing perennial, the woody stem, which is 2 inches in diameter, attaining, when full grown, the length of 50 feet. The leaves are trifoliate; the leaflets 2 or 3 inches long, ovate, and acuminate; the pale pink or purplish, and papilionaceous flowers, about an inch in length, are produced in pendulous racemes, and are succeeded by dehiscent and two- or threeseeded legumes about 7 inches in length. The seeds, when ripe, are oblong, 1 to $1 \frac{3}{8}$ inch in length, and of a deep claret colour.

ACTIVE INGREDIENTS.-The seeds, like others of this order, contain starch and legumin, and about 1.3 per cent. of fixed oil, probably inert. The principal alkaloid, called eserin or physostigmin, $\mathrm{C}_{30} \mathrm{H}_{21} \mathrm{~N}_{3} \mathrm{O}_{4}$, (Hesse) is present chiefly in the cotyledons of the seeds, but also in the testa, and is colourless and varnish-like, drying with heat into thin scales, but at ordinary temperatures soon softening again; when perfectly pure it forms crystalline crusts, or glittering rhombic scales; it is tasteless, decidedly alkaline in reaction; easily soluble in alcohol, ether, benzol, and chloroform, but not very soluble in water. If heated for a long time at $212^{\circ} \mathrm{F}$, it alters to a reddish colour, and its acid solutions soon turn red at the ordinary temperature. The physiological test for the dung is contraction of the pupil on local application.

A second alkaloid-calabarina-has been. recently isolated $(1876)$; it is soluble in aleohol and water, insoluble in etleer; it had previously been a source of confusion, since it differs in action from eserin. Hesse in 1878 discovered a third substance -physosterin, $\left(\mathrm{C}_{26} \mathrm{H}_{44} \mathrm{O}+\mathrm{OH}_{2}\right)$-allied to cholesterin; it is found also in peas. 
ABSORPTION AND ELIMINATION.-Physostigma and its alkaloids are readily absorbed, and are eliminated mainly by the bile and the saliva, and apparently by the gastric secretions, since it is found in them after intravenous injection (Fraser); it has not yet been detected in the urine.

PHysiological ACTION.-This may be illustrated by a statement of the effects of the bean as used in Calabar for trial by ordeal, etc. The accused-suspected generally of sorcery - is led to a temple, and before the assembled people forced to chew as many as twenty or thirty of the beans, or to swallow an infusion of the same. In the latter case death follows in an hour or less, but in the former the very number of the beans gives a chance of safety by the vomiting or purging induced. If a few only be taken, death is almost certain, as in savage duels, when the challenger bites a bean in two, eats one half himself, and gives the other to his opponent, who is bound to eat also at once. The symptoms, which commence in five to ten minutes' time, are- a gradual paralysis of voluntary muscles, a stupid apathetic appearance, rertigo, staggering gait, laboured breathing, feeble pulse, coldness of surface and cold sweats, extreme prostration, and death without much apparent suffering. The pupil is often, but not always, contracted, and sometimes spasmodic contractions of muscles occur. If vomiting or purging set in, there is some chance of recovery (Rabuteau).

Nervous System.-In animals poisoned by the druge.g., by $\frac{1}{5}$ grain of eserin injected under the skin-fibrillary muscular tremors first attract attention ; reflex excitability and the sense of pain are soon lost, but tactile sensibility and the "muscular sense" remain longer. The tremor may be accompanied by spasm so strong as to simulate convulsion, and this has led certain observers to class the drug with strychnia; paralysis, however, soon follows, and the effects much more nearly resemble those of curare. According to Rabutenu, they result, as with that drug, from a more or less complete paralysis of the peripheral onds of motor nerves; certainly the muscular tissue itself is not paralysed, for it contracts readily on direct application of electricity. 
It is said by some that galvanization of the nerve-trunk is not followed by the usual contractions; but on this point there is distinct conflict of opinion, since Laschkewitsch, Vintschgau, and Fraser report that galvanization of the crural nerve in either cold- or warm-blooded animals does induce free contraction in the tributary muscles. If this be so,-and it is difficult to disbelieve the latter experimenters, - then we must conclude that the chief action of the drug is exerted on the spinal cord; and Fraser, Harnack, and others have further corroborated this by preventing circulation in a limb, i.e., preventing the poison from reaching the peripheral nerve, and yet finding that limb paralysed as much as the others; whilst, if the circulation of the poisoned blood be cut off from the lower part of the spine, then the muscles supplied by it are not paralysed. Roeber ${ }^{1}$ takes a similar view, concluding that- " 1 . The chief action of the bean consists in a depression and final annihilation of the excitability of the ganglionic elements of the spinal cord; and its operation especially affects the groups of cells in the anterior horns of the grey matter which conduct impulses from the brain to the periphery, and then also attacks the elements of the grey matter in the posterior horns which transmit sensations of pain to the brain. 2. By this functional lesion of the grey matter a complete loss of the motor and reflex activity of the spinal cord is produced, likewise a loss of sensibility to pain; while the sense of touch, and the so-called muscular sense, are retained till the death of the animal. 3. The motor and sensory nerves are not damaged at the commencement or in the development of the affections of the cord: at a later stage there follows a paralysis of the intra-muscular termini of these nerves."

More recent observations, by Papi, record a stage of cxaltution of reflex sensibility preceding the stage of depression; and also indicate a paralysis of the conducting fibres which pass from the upper brain to the spinal centres as the cause of the loss of this voluntary movement; but H. C. Wood thinks this "higlly" improbable," and attributes the heightened reflex irritability to the presence of calabarina.

1 Ueber die Wirkung des Calabar-extractes auf IIcr\% u. Rückenmark. Inaug. Diss. von Hermann Roeber. Berlin, 1868. In Practitioner, ii., 1869. 
As to its action on the cerebrum, it is stated that "physostigma does not affect the centres of conscious impressions, and, under its influence, consciousness is preserved until asphyxial conditions set in" (Bartholow). Extreme feebleness and general prostration are the most marked symptoms in man (Christison).

Difference of opinion exists as to the effect on the sympathetic system-some tracing to the drug the production of spasm, others obtaining only negative results-but, in all probability, it exerts a paralysing action, as curare does, only more quickly and powerfully. Certainly the characteristic contraction of the pupil is best explained by a paralysis of the sympathetic: to attribute it, with G. Sée and Nothnagel, to a tetanizing action on the motor oculi, lasting several days, would be quite against analogy. If a few drops of solution be placed in the eye, in five to fifteen minutes extreme contraction may be observed: it reaches its maximum in thirty to forty minutes, and is accompanied by pricking, tension, discomfort, altered accommodation and temporary myopia, which symptoms may last for four to five days. Atropin will dilate this contracted pupil; the drugs being mutually antagonistic.

From the fact, however, that the contraction is more active and more complete than when produced only by section of the sympathetic, it is possible that some stimulation of the oculomotor occurs; but under the full influence of the drug the sympathetic eertainly is paralysed, and galvanic stimulation of it in the neck does not alter the pupil (Engelhardt): direct galvanism of the iris will, however, dilate it (Fraser), ( $\imath$. p. 242, note). Legros traces the effect of paresis of the sympathetic still further, attributing to it relaxation of the ciliary muscle, which allows distension of the small arteries in the iris, and so produces a mechanical contraction: many cousider this the most plausible explanation.

Internal administration of the drug will not always induce contraction of the pupil, as was markedly illustrated when fortyfive children were poisoned accidentally by eating the beans: only four of them developed this symptom-an important fact for diagnosis from opium-poisoning.

Contraction does not seem to occur in birds, even from local application. 
Circulatory System.-Calabar bean possesses a special power over the movements of the heart, which in small doses it meroly retards, but in large doses completely arrests. At first the systole is strengthened and the arterial pressure raised; and these effects follow even after the vagi have been divided, or paralysed by atropia: hence they cannot be considered due to stimulus of the centre of the inhibitory nerves, but there is evidence that the periphery of these nerves is (temporarily) excited. Thus it has been shown that, after poisoning by Calabar bean, a weaker stimulation of the vagus can inhibit the heart's action than before-proving an increased excitability of the nerve. Again, after paralysing the peripheral ends of the cardiac vagi by atropia, so that a very strong galvanic current failed to influence the heart's action, functional power could be restored to these nerves by injections of Calabar bean, with the result that moderate currents then caused arrest as usual.

The rise of arterial pressure may follow from the stronger heart-impulse, or from contraction of the peripheral small vessels. It has been found to occur even after paralysing the vaso-motor centres, whether by section of the cord or by chloral, but not so markedly: hence possibly there may be some temporary spasm of vessels, though the main effect is from the heart; the rise of pressure is soon followed by dilatation of vessels and paralysis of the cardiac muscular structure and ganglia. This is the usual cause of death in cold-blooded animals, and after very large doses injected hypodermically in mammals; otherwise, in the latter, asphyxia is the more common cause. The interference with respiration, which is especially produced by small doses, is either the consequence of a sudden interference with the heart's action, or of a destruction of the motor power of the respiratory muscles owing to paralysis of the spinal cord.

Digestive System.-After a dose of 0.15 gramme of eserin sulphate, there occurred nausea and giddiness, and within an hour vomiting : similar symptoms may follow full doses of the bear in any form; thirst and dysphagia have also been observed. Intestinal peristalsis is markedly increased by it, and spasmodic contraction of a tetanic kind is said to occur: 
diarrhoea with profuse fluid discharge is usual. A paralysis of the sympathetic would reasonably account for such symptoms, and would be more consonant with the general action of the drug than the tetanus of the muscular coat of the intestine to which they are sometimes attributed. Other secretions-the bile, the saliva, the tears, and the bronchial mucus-are also increased in amount. In a case of general paralysis the quantity of urea excreted was diminished. Sugar has been found in the urine (Laborde and Leven).

Uterine System. - Sulphate of eserin is said to excite contractions in the pregnant uterus (Van der Meyer, B. M. J., ii., 1879).

SYNERGISTS. - Gelseminum, conium, nitrite of amyl, curare, hydrocyanic acid, opium, and depressants of the motornerve and sympathetic systems.

ANTAGONISTS.-Dr. Fraser, by careful experiments, chiefly upon rabbits, proved that the action of physostigma is powerfully antagonized by atropia - that is to say, the fatal effects of a lethal dose of physostigma may be neutralized by the employment of atropia, which so influences certain structures as to prevent the occurrence in them of the modifications otherwise induced by physostigma: so decided is the antagonism that even one and a half times the minimum lethal dose of the latter is rendered inoperative (Trans. Roy. Soc. Edin., 1870-1871). (Dr. Fraser, however, had in some degree been anticipated as early as 1864, when Kleinwachter narrated a case of belladounapoisoning successfully treated by physostigma. In Paris, also, a case of tetanus had been much ameliorated by the internal administration of the powdered kernel of physostigma-enough, it was believed, to cause death, - - followed up by the subentaneous injection of a small quantity of atropia.)

Dr. Fraser's experiments disclose facts of a singular character : for instance, when the two substances are administered simultaneously, from $\frac{1}{20}$ to $3 \frac{3}{10}$ grains of atropia are found to antagonize one and a half times the lethal dose of physostigma; when atropia is given after such amount, any dose between $\frac{3}{10}$ and $2 \frac{1}{10}$ grains prevents fatal action if the interval be ten minutes, and any dose between $\frac{3}{10}$ and 1 grain, with an interval of 
fifteen minutes. When the atropia is given before the same dose of physostigma, $\frac{1}{20}$ grain antagonizes if the interval is twenty minutes or less; with a larger dose of atropia (up to 3 grains) the interval becomes longer; but with more than 3 grains the ratio is not so constant.

The antagonism may be attended by a greater prominence of the effects either of the atropia or of the physostigma, and no special action belonging to either of these two substances requires to be prominently and obviously produced in order that the antagonism shall be successful ( $v$. p. 246, dose, etc.).

Stillé throws doubt on the value of eserin in belladonnapoisoning, but the following instance seems striking. A man aged thirty-five (suffering from bone disease) injected about $\frac{1}{12}$ grain atropia with morphia, and became unconscious, with muttering delirium, dilated pupils, etc. - the history being at first unknown, an emetic was given, but without improvement. In two or three hours the symptoms had grown worse, and the power of swallowing was lost; the dilatation of the pupils then indicating the poison, a "solution of extract of physostigma was injected" (dose not mentioned); there was at first not the least trace of returning consciousness, but in less than five minutes he looked round in a surprised manner, and in another five minutes gave a clear account of the occurrence: he could scarcely be made to believe that nearly eight hours had elapsed since he sent for a doctor-his last previous act of consciousness (Dr. Hudson, New Zealand, B. M. J., June, 1881).

Chloral also seems partially antagonistic. In one experiment of Professor Bennett's 15 grains were given to a rabbit weighing. 3 pounds, and fifteen minutes later $\frac{2}{3}$ grain of extract of Calabar bean;-the animal recovered: eight days afterwards, the latter dose given alone killed in seven minutes. In other cases rleath was deferred and symptoms mitigated, if life was not saved; when the order of giving the drugs was reversed, the results were also satisfactory. In a case of poisoning by Calabar bean, chloral proved antidotal, though one and a half times the fatal dose of the bean had been taken (Husemann, Record, Jan., 1882).

Digitalis has been found to antagonize physostigmin (ibid.). 
THERAPEUTiCal ACTION.-Extemal.-Eye Diseases.In examining the value of eserin in ophthalmic practice we note the fact, that whilst its full physiological action is that of a paralysant to muscular tissue, yet therapeutically it is constantly prescribed to "stimulate the ciliary muscle" when deficient in tone or paralysed." Argyll-Robertson specially introduced it for this purpose (1863), and cases illustrating its power are given by W. Jones (Practitioner, ii., 1869), Hutchinson, Gubler (Med. Times, i., ii., 1874), Soelberg Wells, and others: and spasmodic twitchings of the orbicularis muscle are constantly induced by the ordinary solutions.

On account of the various inconveniences from strong solutions, Soelberg Wells suggested "periodic applications of a very weak solution to excite the sphincter pupillie and ciliary muscle to gentle contraction, without fatiguing and weakening them by over-stimulation "; and in a recent paper" Dr. J. C. Uhthoff relates a favourable experience of this treatment (B. M. J., July, 1883) : he found that solutions of $\frac{1}{80}$ to $\frac{1}{40}$ grain in the ounce caused, not artificial myopia, but only a "more ready reaction of the ciliary muscle to nervestimulus-the very improvement desired when that muscle is

1 Note as to effect on muscular contraction.-In the text, the observation that it is "a paralysant to muscular tissue" is based on Fraser's essay; on the statement of R. W. Smith, "it causes paralysis of both striped and unstriped muscular fibre"; and on the evidence given in preceding pages.

Now, Nothnagel, Rossbach, and G. Sée consider its action on unstriped fibre, e.g., on the iris and on the intestine, to be tetanizing. H. C. Wood also says, "after toxic doses, spasmodic tetanic contraction of the intestines occurs, probably peripheral and due to contact of poison with muscular fibres or ganglionic nerve-cells;" and again, "the physiological action of the drug indicates its use in atomy of muscular coats."

On the other hand, Rabuteau ridicules the idea of any tetanizing action of so paralysing an agent, and insists that it has no action at all on muscles directly, but only through nerves, and that a paralysing one.

I am not prepared to accept Rabuteau as perfectly correct, but with regard to the iris, it is certainly difficult to realize a tetanizing effect continued for three or four days without change: and although Calabar bean paralyses the sympathetic $(r, p .238)$ it does not act solely on this, as it causes contraction of the pupil after extirpation of the lenticular ganglion (Foster's Phys., 2nd ed.). It thus probably acts on some local nerrous mechanism, like atropin. 
deficient in tone"; no pain was caused, but often twitchings of the palpebral portions of the orbicularis: he found much benefit in cases of hypermetropia with failure of accommodation, headache after working, etc., and in high degrees of myopia, when even suitable glasses could not be used comfortably for near-vision.

Eserin also acts well in cases of impaired adjustment, as after diphtheria or other cause of debility.

Dr. MeNaughton Jones summarizes the uses of the drug, locally applied, as-(1) in various forms of corneal ulcer and inflammation; (2) in perforation with prolapse of iris; (3) in mydriatic states, whether natural or artificial (i.e., from atropia, etc.), in diplopia, asthenopia, and altered accommodation; (4) in glaucoma (B. M. J., ii., 1879).

In acute cases of the last-named most serious malady some very good results have been obtained; thus, in a lady aged seventy, in whom glaucoma developed five days after an accident to the extent of $+\mathrm{T} 2$, "with steamy cornea, dilated pupil, impaired vision, etc.," eserin discs relieved the symptoms within twelve hours, and recovery ensued (R. J. Pye-Smith, Lancet, ii., 1881). Dr. Buzzard, Priestley Smith, S. Snell, and others have reported similar cases (ibid.), but still " experience teaches the importance of not placing too strong reliance on the efficiency of the drug, to the fatal postponement of the operation of iridectomy" (B. M. J., June, 1882).

Weber, advising eserin in conical comea, as well as in acute glaucoma, considers "that it increases tension in the vitreous, whilst diminishing it in the anterior chamber; and since the ciliary margin of the iris is detached from the cornea, it allows filtration of fluid through the canal of Fontana" (Record, 1880). Brailey, agreeing in the use of the drug, explains its action rather by the fact that in acute or intermittent cases there is excessive secretion into the vitreous chamber, and relief is given "by causing contraction of intra-ocular muscular fibres, thus rendering tense, and opening out the meshwork in the neighbourhood of the iris-angle."

Knapp and others hold eserin of doubtful value in subneute cases; of none at all, or even injurious, in chronic conditions, as it may cause iritis (Record, 1879). Mr. Solomon has noterl 
from it severe pain and conjunctivitis; Andrew, posterior synechia. Such effects may be due sometimes to unduly strong solutions or (the irritant effects at least) to contained fungi, which latter may be prevented by adding about 4 per cent. boracic acid, or 0.1 per cent. carbolic acid (Kræmer) : in the shops, spirit is commonly added to preserve the preparation.

Therapeutical Action.- Intemal.-In Tetanus the results attained by some observers have been so remarkable as to excite great hopes for the future treatment of that terrible disease; but there are very conflicting statements. Dr. Fraser has borne energetic testimony to its value, and Dr. Eben Watson has collected a considerable number of cases of its use, and shows that the majority recovered Practitioner, ii., 1868, i., 1870; Lancet, i., 1868). He gave what seems enormous doses of the alcoholic extract-one patient, e.g., who recovered, took in forty-three days 1,026 grains, and as much as 72 grains in one particular twenty-four hours. He believes that the dangers of poisoning are to be avoided if care be taken to adapt each dose to the necessities of the moment, and to support and stimulate the patient freely. Mr. C. Macnamara has also had excellent results in a large number of cases (Practitioner, 1872). On the other hand, many observers have tried the remedy in isolated cases of tetanus, with no good effect. Of strychnia-tetanus a case of recovery under Calabar bean is recorded by Dr. Keyworth (Glasgow Med. Journ., Nov., 1868).

Dr. Cheadle has reported two well-marked cases of infantile convulsions relieved by physostigmin ( $\frac{1}{8}$ to $\frac{1}{6}$ grain for a dose) when chloroform even had failed to suspend the spasms (Med. Times, i., 1880).

In Chorea there is much evidence in favour of the bean, principally from Bouchut, who records 437 trials of eserin in the chorea of children; and he asserts that the drug arrests the movements during the attacks, and modifies then in the interval. The effect of each dose (given daily) lasted for two or three hours, but no trembling or convulsion was noticed, and the pupil was only occasionally contracted (Bull. de Thérap., Av., 1875). But it is more 
difficult to be sure that the remedy was the real cause of recovery in chorea than in tetanus. Harley, Ogle, and McLaurie, all testify favourably (Med. Times, 1864-1868). Gubler, on the contrary, reports its failure in one case, and others have had similar negative results : it has certainly not come into general use.

On the other hand, in some forms of paralytic disorder, more success has been attained; thus, Dr. Crichton Browne benefited cases of general paralysis of the insane, and Drs. Ringer and Murrell have recorded success, more or less complete, in two cases of paraplegia (from myelitis), two of locomotor ataxy, and one of "writer's cramp" : $\frac{1}{30}$ to $\frac{1}{20}$ grain of extract of the bean was given every two to three hours (Lancet, ii., 1877).

Constipation, etc.-In torpor of the muscular layer of the intestine with deficient secretion, it is, according to Bartholow, often serviceable, 15 minims of tineture, or $\frac{1}{2}$ grain of the extract, causing a mucous evacuation. The combination with nux vomica and belladonna, though unscientific, is said to be even more effective. In extreme flatulence from a paretic, relaxed state of the muscular layer, especially in women at the climacteric period, great relief has been given by this drug, and consequent relief also to morbid fancies, headache, etc. This use of the drug may be compared with that already mentioned of stimulating the ciliary muscle, and would seem to indicate that the therapeutical action of small doses is directly opposite to the physiological action of large ones.

Night Sweating.-In the profuse perspiration of phthisis, Dr. Murrell has found relief given by $\frac{1}{10}$ grain of extract taken in pilule once to three times in the night; also by $\frac{1}{60}$ grain of an eserin salt: in successful cases the sweating did not recur for three or four weeks (Practitioner, ii., 1883).

PREPaRATIONS AND DOSE. - Pure eserin or physos. tigmin is seldom employed, being difficult to prepare, but Sée ${ }^{1}$ recommends for local use, a solution of 1 in 2,000 or 1 in 1,000 of the sulphate (which is very soluble in water), one or two drops to be applied to the eye; internally, about $\frac{1}{60}$ to $\frac{1}{20} \mathrm{gr}$.,

'Recherches Chim. et Physiol. sur la Fève de C'alabar. Paris, 1865. 
increased gradually to $\frac{1}{12}$ gr., for a dose; for subcutaneous injection, about one-half of the latter quantities. Ophthalmic surgeons now generally use divided squares or dises of impregnated paper or gelatine, each one of which contains a suitable dose; occasionally solutions of 2 and 4 gr. to the ounce are ordered, but more often $\frac{1}{10}$ or $\frac{1}{12}$ gr., and recently $\frac{1}{40}$ or $\frac{1}{80} \mathrm{gr}$. to the ounce (v. p. 242).

The hydrobromate, which forms stellar groups or fibrous crusts, and almost colourless solutions, is also used in similar doses. Merck prepares a crystalline salicylate in colourless shining needles or rhombic prisms-soluble in 24 parts of absolute alcohol,-130 parts of cold, easily in hot, water: dose, $\frac{1}{60}$ to $\frac{1}{20}$, or in some cases $\frac{1}{12}$ gr. The salicylate of eserin is officinal in the German and American Pharmacopœias. The dose of the bean itself is said to be from 1 to $4 \mathrm{gr}$., and of the pharmacopœial extract $\frac{1}{16}$ to $\frac{1}{4}$ gr. or more; but the gravity of each case must be taken into consideration, and severe traumatic tetanus may require frequent repetition of the dose, so that large quantities are taken in each twentyfour hours. The subcutaneous injection of the extract should always be done in the manner recommended by Dr. Haining, viz., by mixing the dose with 10 to $15 \mathrm{~min}$. of water, and neutralizing its acidity with carbonate of potash. Dr. Eben Watson states that he has not found good results from the subcutaneous injection, and prefers to give the drug by the mouth or rectum.

A tincture may be made, 1 part to 4 : dose, $10 \mathrm{~min}$.

In cases of poisoning by belladonna or atropia, the dose and repetitions must be regulated in great part by the apparent effects upon the pupil; but since physostigma in large doses has a tendency to paralyse the heart, close watch must be kept on the circulation, and the remedy given in fractional quantity as often as is found safe. 


\title{
CASSIA OBOVATA; CASSIA LANCEOLATA; CASSIA ELONGATA.
}

\author{
(Senna.)
}

DESCRIPTION. - Cassia is a genus of herbaceous and under-shrubby perennials, with paripinnate leaves-the six to fourteen leaflets of which are usually oblique at the baseand large rosaceous yellow flowers produced in racemes. The legumes are flat, falcate, and three- to six-seeded. The subgenus Senna includes the species mentioned above, which are small shrubs, 2 or 3 feet high, with persistent stipules and axillary racemes; natives of the East Indies, Arabia, Egypt, etc. The kinds most esteemed are the East Indian and the Alexandrine: the leaflets of which are about $1 \frac{1}{2}$ inch in length, lanceolate, entire, and unsymmetrical at the base, with a peculiar tea-like odour, and a nauseous bitter taste.

ACTIVE INGREDIENTS.-The principal one is cathartic acid, $\mathrm{C}_{180} \mathrm{H}_{192} \mathrm{~N}_{4} \mathrm{O}_{82} \mathrm{~S}$, (Watts' Dict., a peculiar sulphurated glucoside, which, when isolated, is amorphous, brown or black in colour, and nearly insoluble in water, spirit, and ether; but when combined, as it is in the plant, with alkaline and earthy bases such as lime and magnesia, is readily soluble in water: boiled with hydrochloric acid and alcohol, it gives rise to sugar and cathartogenic acid, a yellowish-brown substance insoluble in water and ether,-catharto-mannite, etc.

The cathartin of older authors is a complex substance, destitute of active properties; but chrysophanic acid has been found in senna, and has some purgative power: acrid bitter principles, sennacrol and sennapiorin, have also been isolated.

ABSORPTION AND ELIMINATION:-The active principlo of senna is readily absorbed and eliminated by all the secre. tions. The milk of nursing mothers taking it, is apt to gripe or purge the infant. 
Physiological Action.-Digestive System.-Moderate medicinal doses cause first a sensation of warmth in the stomach and abdomen; followed soon by a purgative action-the stools being liquid, and of yellow colour, which is due probably to altered chrysophanic acid. Large doses produce nausea, vomiting, griping, flatulence, depression, and, after a little excitement of the pulse, drastic purgation, with tenesmus; they may also cause hrmorrhoids, and in women may increase the monthly discharge. The action of moderate quantities is usually mild, and the effects of even large doses, though powerful and disagreeable, are never poisonous. Compared with rhubarb, it is more irritant and energetic, but less tonic: it is more powerful and speedy than aloes in operating upon the small intestine; but upon the large bowel its action is less decided. When acrid and griping effects are produced by moderate doses, they are referable probably not so much to the senna as to certain plants with which it is adulterated.

The infusion injected into the veins produces vomiting and purging ; and even the odour of senna-leaves, or of the infusion, is, in certain susceptible persons, sufficient to cause an evacuation of the bowels.

\section{Therapeutical Action. - Constipation. - Senna} being a safe and generally sure purgative, is employed with advantage whenever there is occasion for prompt liquid evacuation of the bowels, and especially of the small intestine. In all forms of inactivity of the alimentary canal it constitutes a reliable domestic remedy: it is much used as a laxative in cases of hæmorrhoids, congestion of rectal vessels, and slight superficial anal fissure, and is well combined with sulphur in these cases: ordinarily the infusion is combined with Epsom salts or tartrate of potash, or with manna or tamarinds: if a more vigorous action than usual is desired, it is said to be well to combine the senna with guaiacum. In order to cover the nauseous taste; Dr. Cullen recommends the admixture of coriander-seeds. In cases of woms and a feeling of fulness in the hend, senua is likewise resorted to with great benefit: children and elderly persons are especially suitable subjects to derive benefit from it. 
Its administration is inadvisable if inflammation of the mucous membrane of the bowels be present, and it should be employed with caution also when there is febrile disorder, the action of this drug upon the pulse being exeitant: in such cases it is better, perhaps, to use one of the saline purgatives.

If the large amount of senna-tea required for an efficient dose be found objectionable, the electuary, or the compound liquorice" powder, or the syrup, may be recommended as more convenient, and to some persons more palatable.

Senna has the additional recommendation of not constipating after its purgative effects have passed away, rendering it particularly valuable when the bowels are constantly, though not seriously, confined, and when aperient medicine has to be often taken.

PREPaRATIONS AND DOSE.-Powdered leares (seldom used): dose, 30 to $120 \mathrm{gr}$. Infusum: dose, 1 to $2 \mathrm{fl}$ oz. Mistura senuce composita: dose, 1 to $1 \frac{1}{2} \mathrm{H}$. oz. Tinctura: dose, 1 to $4 \mathrm{fl}$. dr. Confectio: dose, 60 to $120 \mathrm{gr}$. Symupus: dose, 1 fl. dr. and upwards. Putvis glycyrrhize compositus (contains senna, liquorice, and sugar): dose, 30 to $60 \mathrm{gr}$. The German form contains also sulphur, and is commonly preferred.

ADULTERATIONS.-These consist of the leaves above adverted to as apt to produce griping effects unjustly attributed to the senna. The worst are those of the Coriaria myrtifolia, a poisonous shrub in no way related to the senna plant, with leaves simple instead of pinnate, and symmetrical at the base.

\section{CATHARTOCARPUS FISTULA.}

(Cassia Fistuia.)

The blackish pulp contained within the legumes of this plant was formerly in favour as a mild cathartic, but is now used only as an ingredient in confectio sennæ. 


\section{SAROTHAMNUS SCOPARIUS.}

(Broon.)

DESCRIPTION. - A bushy shrub, native to many parts of Europe, with straight slender twigs 2 or 3 feet long, erect, angular, glabrous, and dark green. The lower leaves are small and trifoliate, the upper ones simple; the large, numerous, bright-yellow, papilionaceous flowers are followed by thin black legumes, $1 \frac{1}{2}$ inch in length, ciliated along each margin. The tops of the green twigs are flexible, pentangular, winged, not thorny, smooth except at the end, which is downy; of peculiar odour when fresh, and of bitter, nauseous taste. The flowers, which are sometimes used, become brown on drying.

ACTIVE INGREDIENTS.-There are two peculiar principles, one of which is neutral, the other a volatile liquid alkaloid. The neutral principle, called scoparin, $\mathrm{C}_{21} \mathrm{H}_{22} \mathrm{O}_{10}$, forms yellow crystals, soluble in water and alcohol, and is free from bitterness or odour. Dr. Stenhouse believes it to represent the diuretic property of broom-tops. The alkaloid, called spartein, $\mathrm{C}_{15} \mathrm{H}_{26} \mathbf{N}_{2}$, is pale when newly prepared, but on exposure becomes brownish, and has a bitter taste. It forms crystalline salts with bichloride of platinum, terchloride of gold, and corrosive sublimate, and is extremely poisonous, being little inferior in this respect to either conia or nicotia. Extractive matters and salts of potash, etc., are likewise found in broom-tops.

Physiological Action.-The plant has cathartic and diuretic powers, which are exerted both by the infusion and tincture. In small doses the former is mildly laxative; in large ones it becomes emetic and purgative and is reputed cholagogue. The alkaloid spartein is narcotic, and sedative to the spinal centres and to the circulation, but it exists in very small proportion, and seems to vary in its effect. Schroff 
killed a rabbit in six minutes with a single drop of spartein, violent tetanic symptoms being produced: but Mitchell and Stenhouse gave 4 grains to a rabbit that lived for three hours. afterwards, and then died in stupor without convulsions. This. difference of experimental results, and the fact that spartein is little soluble in water, make it doubtful whether it takes. any share in the action of decoction of broom-tops.

Therapeutical Action.-In cordicuc dropsy, broom is serviceable, especially when combined with diluents. Cullen found it more trustworthy than any other diuretic. In other forms of dropsy-inflammatory or renal-its use is objectionable; and especially so, it is said, in pleuritic effusion, if there be pulmonary congestion or inflammation.

PReparations and Dose.-Decoctum: 1 to $3 \mathrm{fl}$. oz. Succus: 1 to $2 \mathrm{fl}$. dr. or more. Cullen recommended that $\frac{1}{2}$ oz. of the fresh tops should be boiled in a pint of water until reduced to one half that quantity; two tablespoonfuls of this decoction to be given every hour, until the whole had been taken, or until the bowels were moved.

\section{PTEROCARPUS MARSUPIUM.}

\section{(East Indian or Malabar Kino.)}

Description.-A tree indigenous to the Circar Mountains. The trunk is erect and lofty, but rarely straight; the pinnate leaves have five or seven long deep-green leaflets; the numerous flowers, white tinged with yellow, are borne in large terminal panicles; the legumes are winged, velvety, and one- to twoseeded. The juice, which exudes through an incision in the bark, is pale in colour, but soon hardens, and becomes of $n$ deep blood-red hue, and so brittle that to collect it is a tedious. undertaking. In commerce it occurs in small, irregular, angular, and glistening fragments, the larger pieces nearly black, the smaller ones reddish; in lumps it is opaque, but in thin laminæ translucent and ruby-red. 
The original gum kino, now known by the name of "African," is the produce of the Pterocarpus erinaceus; it is not met with in commerce. There is an Australian or Botany Bay kino, derived from Eucalyptus; also a Bengal kino from Butea; and Jamaica or West Indian kino from the Coccoloba: all these resemble the officinal variety.

ACTIVE INGREDIENTS.-Kino is inodorous, but has a very astringent taste, which ultimately becomes sweetish. Artificial heat does not affect it, but in the mouth it softens readily, turning the saliva blood-red, and when chewed, clinging to the teeth. In cold water it is partially soluble, in boiling water more so, and in alcohol almost entirely. The constituents are a peculiar kind of tannin, called mimo- or kinostannic acid (or catechu-tannic acid), $\mathrm{C}_{13} \mathrm{H}_{12} \mathrm{O}_{5}$, and another astringent principle, called catechin, probably isomeric with catechu-tannic acid (described as a constituent also of pale catechu), with some red gum, pectin, and other unimportant ingredients. Etti (in 1878) isolated kinoin, $\mathrm{C}_{14} \mathrm{H}_{12} \mathrm{O}_{6}$, a compound of gallic acid which turns red with ferric salts; it exists to the extent of $1 \frac{1}{2}$ per cent.

Physiological ACtion.-Kino operates in the same manner as catechu, but being less soluble, its action is frequently less powerful.

Therapeutical Action. - In relazed conditions of mucous membrane (e.g. in the throat), and again in leucorrhoea, kino possesses some value as a topical astringent; but it is chiefly employed in obstinate chronic diarrhœa, often in combination with chalk or opium. It has likewise been found useful as a tonic in intermittents.

Preparations and Dose.-Pulicis: dose, 10 to 30 gr. and upwards. Pulvis kino compositus: dose, 5 to $20 \mathrm{gr}$. (contains $1 \mathrm{gr}$. of opium in 20 of powder). Tinctura: dose, $\frac{1}{2}$ to $2 \mathrm{fl}$. $\mathrm{dr}$. 


\section{MYROSPERMUM PERUVIANUM.}

(Balsam of Peru.)

DESCRIPTION.-A tree indigenous to the forests of Brazil, agreeing in all conspicuous characters with the M. toluiferum, but differing in the form and texture of the leaflets, which, instead of being thin and membranous, are coriaceous, emarginate, and all of equal size; whereas, in the other species, the terminal one is the largest. The balsam is obtained from the trunk by separating and charring the bark in strips, and when these fall off, tle resin is absorbed by rags, and obtained from them by boiling in water. It is imported in earthy or metal jugs or cases. In consistence it is viscid like treacle, sp. gr. $1 \cdot 15$; in colour it is reddish-brown, or almost black; possesses an agreeable odour, somewhat resembling that of vanilla, and a warm, acrid, slightly bitter taste. It is inflammable, and burns with a smoky flame; is soluble in alcohol, and in about five parts of rectified spirit, but insoluble in water.

Balsam of Peru contains cinnamein (cinnamate of benzyl, $\mathrm{C}_{16} \mathrm{H}_{14} \mathrm{O}_{2}$ ) to the extent of about 60 per cent. This is a neutral, acrid, colourless, strongly refracting oil, inflammable, of sharp taste, and slightly aromatic odour; it is heavier than water, and insoluble in that medium, but soluble in alcohol and in ether.

By the action of caustic potash, cinnamein is converted into cinnamic acid, which constitutes about 6.4 per cent. of the balsam; and a light oily fluid called peruvin (benzylic alcohol). Cinnamic acid may also be formed hy exposing oil of cinnomon to the atmosphere.

About 24 per cent. of the fresh balsam consists of resin of Peru, which is really a hydrate of cinnamoin, formed by the cinnamein uniting with the elements of water: the resin increases in quantity with the age of the balsam, at the expense of the oil.

Physiological Action.-Balsam of Peru is stimulant, tonic, and expectorant, the action olosely resembling that of 
storax and benzoin. Acting most decidedly upon the mucous membranes, it promotes digestion, causes warmth and excitement throughout the system, stimulates the pulse, and increases the secretion of the kidneys and the skin. In large doses it produces pain and oppression in the stomach, with nausea, vomiting, colic, and diarrhœa.

Therapeutical Action.-Extemal.-It has been used in plaster for the cure of headache and toothache, and as an ointment for closing recent wounds and for healing ulcers and similar lesions; also in chronic eczema, and in scabies, for which disorders, after potash baths, about 40 drops may be rubbed into the seats of eruption three times daily for two or three days (Husemann). It is a valuable application for sore nipples in the following form, used five or six times daily: balsam of Peru, 2 drachms; oil of almonds, 90 grains; gumarabic, 2 drachms; rose-water, q.s.

Therapeutical Action.-Intemul.-Balsam of Peru is given in chronic bronchitis, asthma, gonorrhoa, gleet, leucorrhoea, and other similar disorders; it tends to lessen and improve the character of the discharge in these diseases.

Preparations and Dose.-Balsamum: dose, 10 to 30 min. and upwards may be given in an emulsion of almonds, mucilage, or the yolk of eggs, with a little sugar. An ointment may be prepared with 1 part in 8 .

\section{MYROSPERMUM (VEL MYROXYLON) TOLUIFERUM. (BAIsaM OF Tol,}

DESCRIPTION. - An elegant tree, native of the warm parts of Brazil, and remarkable for the abundance of resin secreted in the coarse, compact, and heavy bark. The pinnate leaves are crowded with oil-cysts; the white flowers are produced in axillary racemes; the legumes are flattened, straw-coloured, and about 2 inches in length. Upon making incisions in the bark, during the heat of the day, the 
resin exudes freely: while moderately fresh, it is soft and tenacious, translucent, reddish or yellowish-brown, fragrant, and of a pleasant and somewhat sweetish taste; but with age it becomes hard, brittle, granular, and somewhat crystalline; under the microscope, crystals of cinnamic acid may be seen in it. If heated, it melts readily, and, while burning, gives out an agreeable odour: it is soluble in alcohol, ether, volatile and fatty oils, and in solution of caustic alkalies.

ACTIVE INGREDIENTS. - Similar to those of balsam of Peru, chiefly cinnamein, cinnamic acid, and resin: a thin, colourless volatile oil, tolene, $\mathrm{C}_{10} \mathrm{H}_{16}$, has also been found in the proportion of about 1 per cent. The soft balsam contains a larger proportion of oil, but less acid, than when dry and brittle.

Action.- Similar to that of balsam of Peru.

PREPARATIONS AND DOSE.-Balsamum: dose, 10 to $30 \mathrm{gr}$. and upwards. Symupus tolutames: dose, 1 to $3 \mathrm{fl}$. dr. Tinctura tolutana: dose, 15 to 30 or $60 \mathrm{~min}$. (Balsam of tolu enters into the composition of tinct. benzoini comp.) A tolu lozenge is in common use for bronchial affections.

\section{HAMATOXYLON CAMPECHIANUM.}

\section{(LoGwoon.)}

DESCRIPTION.-A tree, native of Campeachy and the West Inclies, low, crooked, and frequently spinous. The leaves are pinnate; the small and yellowish flowers grow in racemes; the legumes are flat, lanceolate, and two-seeded.

For officinal purposes the stems are cut into pieces about 3 feet long, the bark and white sapwood removed, and the heartwond sliced: it is externally dark red, internally reddishbrown, and so heavy as to sink in wator. It is eut into chips, which have a slight agreeable odour and sweetish taste, and on being chewed, impart to the saliva a dark pink colour. 
ACTIVE INGREDIENTS.-Logwood contains hæmatoxylin, or hæmatin, $\mathrm{C}_{16} \mathrm{H}_{14} \mathrm{O}_{6}$, which is sometimes found crystallized in the crevices; it has a rather bitter astringent taste, and is soluble in alcohol and ether, slightly so in water: with alkalies it gives a red or purplish colour; with acids, yellowish or red.

The colouring and the astringent principles of the wood are dissolved out both by water and alcohol; the solutions are deepened in colour by alkalies, and rendered turbid by acids. Other ingredients are a light volatile oil, tannin, and resin.

Physiological Action.-Logwood, in decoction, is a mild astringent, which, however, constipates less than such remedies generally do: its colour can be detected both in the urine and the stools. It is now greatly used by microscopists as a dye for animal tissues.

THERAPEUTICAL ACTION.-Logwood is usefully administered in dysenteries and diarrhœas of long standing, and is specially adapted for diarrhœe in children. It is also employed in hæmorrhages from the lungs, the uterus, and the bowels, and has been found efficient in leucorrhoa. It is commonly prescribed in combination with other astringents, such as chalk, acids, or opium. Dr. Percival used it to check profuse perspiration in phthisis.

PREPARATIONS AND DOSE.-Decoctum: dose, 1 to $2 \mathrm{fl}$. oz. Extractum: dose, 10 to $30 \mathrm{gr}$.

COPAIFERA MULTIJUGA (AND OTHER SPECTES).

(Copaiva.)

DESCRIPTION.-The oleo-resin, inaccurately called "balsam" of copaiva, is the produce of several species of the Brazilian and West Indian genus Copaifera, especially the C. multijugn. This last is a lofty tree, with large pinnate leaves, and panicles of white flowers, succeeded by woody and one-seeded legumes. The copaiva is obtained by inci- 
sion, and appear's in commerce as a limpid and pale or brownish-yellow liquid, sp. gr. 0.95, of peculiar odour, and nauseous, bitter, somewhat acrid flavour: it becomes darker in colour, and more dense, with age and exposure to the atmosphere. Like other oleo-resins, it is insoluble in water, but soluble in alcohol, ether, and oils; also in an equal volume of benzol: with alkalies it forms a kind of soap, insoluble in water.

ACTIVE INGREDIENTS.-These are a resin, which occurs in the proportion of about 52 per cent., and a volatile oil, 40 per cent.- proportions which vary, however, with age and exposure. The resin (copaivic acid, $\mathrm{C}_{20} \mathrm{H}_{32} \mathrm{O}_{2}$ ) resembles common rosin, and crystallizes in small soft prisms, soluble in alcohol, ether, and oils: it enters into combination with alkalies and alkaline earths, so that if copaiva be triturated with one-sixteenth of its weight of magnesia or of slaked lime, copaivates of these earths are formed with the production of a solid mass.

The volatile oil, oleum copaiva, which is obtained by distilling copaiva with water, is a colourless or yellowish liquid, having the smell and taste of the drug, and is isomeric with oil of turpentine $\left(\mathrm{C}_{10} \mathrm{H}_{16}\right)$.

Besides these two principal constituents, there is about 2 per cent. of a soft brown resinoid matter, the nature of which is unknown: it is more abundant in old than in recent copaiva; and is soluble in anhydrous alcohol, ether, and the vils, both fixed and volatile.

Oxy-copaivic acid, $\mathrm{C}_{20} \mathrm{H}_{29} \mathrm{O}_{3}$, and metacopaivic acid, $\mathrm{C}_{22} \mathrm{H}_{34} \mathrm{O}_{4}$, have also been obtained.

ABSORPTION AND ELIMINATION.-The influence exerterl upon the urinary organs proves that copaiva enters the general circulation: and this is further indicated by its tainting the breath, and by its action on the skin, eruptious sometimes being produced which resemble urticaria, roseola, or rubeola: some authors consider these to be only secondary to gastric irritation, but I have seen them occur without any evidence of 
the latter condition. Elimination is rather slow, continuing for nearly four days after ceasing the medicine (Bernatzik). Weikart states that the volatile oil is decomposed in the system, and only copaivic acid passes out in the urine.

Physiological Action.-The most complete inquiries as to the operation of copaiva, when taken into the system, are those of Ricord, who has shown that the skin, bronchial tubes, digestive organs, and the whole of the mucous surfaces, are affected by it; the nervous centres also, occasionally; and the urinary apparatus most markedly and uniformly.

Digestive System.-The action upon the digestive organs is manifested by an increased flow of saliva, flatulence, and some colic; heat at the pit of the stomach, diminished desire for food, nausea and retching, or eructations that possess the peculiar taste and odour of the medicine: when not tolerated by the stomach, purging often follows, sometimes with blood and mucous discharge, a sense of burning in the region of the sphincter ani, and violent tenesmus.

Urinary System.-The influence upon the urinary organs is shown by diuretic effects, and by the changed quality of the secretion, which acquires a deeper colour, a certain degree of bitterness, and a slightly balsamic odour, or, at times, the smell of violets (this peculiar odour being perceptible, likewise, in the stools). There is also an alterative astringent effect exerted on the mucous membrane of the tract over which the urine passes. After standing a little while, it often presents a filmy covering or iridescent pellicle, and the suspension in it of resinous matter produces a manifest turbidity; it deposits, also, a sediment, which chiefly consists of vesical and urethral mucus. A true albuminous condition may be induced by renal irritation from the drug, but as a rule any precipitate by nitric acid is composed of the resin, or of the oxidized oil, which floats and is soluble in alcohol. Should the dose have been unduly large, micturition becomes frequent, and is preceded and followed by itching, smarting, and burning in the urethral passage, and during its progress is accompanied by heat and tenesmus, and even by hæmaturia and ischuria. While these local results appear the whole system becomes excited; the 
pulse is rendered quicker and fuller, and thirst and headache supervene.

The diuretic action of the drug seems to depend specially on its resin, as shown by Dr. Willss (Lancet, i., 1873). Dr. Carter also, giving $\tau$ grains thrice daily to a healthy man (with erythema only), found the quantity of urine increased steadily to 82 ounces in twenty-four hours; the specific gravity diminished; the excretion of solids was increased. He reported two cases of ascites occurring independently of renal or cardiac disease, and cured by the resin acting as a diuretic.

Dixon recorded three similar instances, and Dr. F. Taylor treated upwards of sixty cases of various forms of dropsy with the same drug, and with, on the whole, satisfactory results (Guy's Hosp. Rep., 1876). It cannot, however, be depended upon in cases of tubular nephritis, whence Stillé concludes that it acts upon the secreting elements of the kidneys.

The effects of full doses of copaiva upon the respiratory tract are shown by dryness and irritation in the larynx and bronchi, huskiness, and dry painful cough, with expectoration of a semi-purulent, greenish, nauseous-smelling mucus.

Nervous system. - Its continued use has induced, it is said, epileptic convulsions, rigidity, or paralysis.

The physiological action of the oil of copaiva is nearly similar to what has been described, but is not so powerful; by full doses, micturition is increased, the stools rendered frequent and watery, and sometimes bloody; the breathing hurried; there is palpitation of the heart, and general restlessness; eventually the medicine is excreted by the customary channels without serious injury. Some persons are much more sensitive than others to the action of the drug.

SYNERGISTS.-Cubebs, sandal-wood oil, gurjun oil, matico and other peppers, turpentine, and the true balsams; also alkalies.

Antagonists.-Purgatives (?), acids, cold (Gubler).

THERAPEUTICAL ACTION.-The most common use of copaiva is in the chronic stages of gonorrhert, gleet, and Huor allms. 
Women who may be suffering from gonorrhœa are less successfully treated with it than men, since in women the disorder is not confined to the mucous lining of the urethra, but extends to that of the vagina, where it is obvious that the disease cannot be benefited to the same extent by the local action of the medicine.

The remedy may be given according to either of two methods. The first is to give it at the commencement of the disorder, to arrest or suppress it; the other is to delay the use of copaiva until the inflammatory symptoms have subsided.

The first method is that employed (at all events, to some extent) in America ; in Europe, though it has been followed by some practitioners for nearly seventy years, it is now discarded for the sake of the second, which directs at first antipyretic and soothing treatment, and, when the inflammation has much abated, the administration of copaiva to reduce or stop the discharge. Hunter, Sir Astley Cooper, and Lawrence followed this latter plan, and there can be no question that it is the safer; for, independently of the usefulness of the preliminary treatment, instances have occurred when copaiva, given in the early stages of gonorrhoea, has aggravated the symptoms. Orchitis has been traced to its excessive or too early use; but, on the other hand, Laennec, Kibes, and others adrise the drug in full doses as an excellent treatment for orchitis without leeches, baths, or local applications.

Copaiva has, likewise, been employed with success in chromic irritability and inflammation of the bladder; also, according to Armstrong (1818) and other writers, in leucorrhœea, and in long-standing cases of chronic pulmonary catarrh in torpid constitutions.

In chronic inflammation of the mucous membrane of the bowels, especially of the colon and rectum, it has sonetimes proved a valuable agent: small doses only should be used, otherwise increase of irritation may be caused. Culleu recommends it in hromorrhoids, and formerly it was used as an application to wounds, ulcers, chilblains, etc.

Dropsy.-As already mentioned, copaiva, and especinlly the resin, has proved efficrcious in cardiac ascites, and in hepatic fropsy dependent on cirrhosis (Taylor, Guy's Hosp. Rep., 1876). 
Other maladies in which the drug has been recommended by some practitioners, but in which its use is not generally adopted, are iritis, sclerotitis, and psoriasis.

PREPARATIONS AND DOSE.-Coprita: dose, 15 min. to $1 \mathrm{fl}$. dr. Oleum: dose, 5 to $20 \mathrm{~min}$. It may be given with liquor potassæ and mucilage, or yolk of egg flavoured, nitrous ether being commonly added.

A well-known French preparation, in addition to copaiva, "Potion de Chopart," contains alcohol, tolu, peppermint, and nitrous ether. Capsules contain from 5 to $25 \mathrm{~min}$. each. The resin may be given in pill in doses from 5 to 15 gr., or in emulsion with compound almond powder, or $3 \mathrm{dr}$. with 5 dr. of alcohol, 1 dr. of spiritus chloroformi and mucilage and water to $12 \mathrm{oz}$; dose, $1 \mathrm{oz}$.

Dr. Chapman recommends that the copaiva be poured upon half a wineglassful of water, to which is then added a small quantity of some bitter tincture: by this means the copaiva is collected into a globule, which may easily be swallowed, the taste, so nauseous to many patients, being entirely masked. Rubbed up with magnesia, it may be taken in wafer paper.

ADULTERATIONS.-Fixed or volatile oils, such as castoroil, or turpentine. The former may be detected by the diffused greasy stain left after warming one or two drops on paper, and the latter by its odour on heating (R. W. Smith).

\section{aCACIA CATEChU. (Catechu Nigrum.)}

(Not Officinal.)

DESCRIPTION. - The substance called catechu nigrum, commonly known also by the name of kutch or cutch, is obtained from several different plants, belonging to distinct natural orders, and of these the leguminous Acacia catechu is one of the chief. It is a native of the East Indies, and when full grown forms a tree 30 feet in height, with numerous strong black thorns, doubly pinnate leaves, and cylindrical spikes of 
innumerable small white flowers, the stamens of which are their most conspicuous feature: the legumes are linear, straight, flat, and four- to six-seeded. To obtain the drug, the heart-wood is cut up and boiled with water, which is afterwards concentrated to a thick consistence, and poured into moulds.

Black catechu is imported from India in large irregular masses, made up of layers, which are composed of smaller blackish-brown or reddish pieces with a shining surface, and enveloped in leaves or the husks of rice. In the mouth it slowly dissolves, with an astringent bitter taste, followed by slight sweetness. The active ingredients and action correspond nearly with those of Catechu pallidum, which only is now officinal.

\section{INDIGOFERA (SPECIES YARIOUS).}

\section{(Indigo.)}

Indigo, $\mathrm{C}_{8} \mathrm{H}_{5} \mathrm{NO}$, in large doses has caused romiting, purging, and debility: it sometimes colours the urine and perspiration bluish-green. It is worth while to note the fact that both indigo and its sulphate have at various times been much vaunted in medicine, particularly for epilepsy, but it is exceedingly doubtful whether it really has any effect on that disorder; and the surmise of Hubert Rodrigues, that any apparent good was really the result of valerianic acid, accidentally found in some of the commercial samples of indigo, is probably correct.

\section{PTEROCARPUS SANTALINUS. \\ (Ren Saunders, or Saxdal, Wood.)}

Used in the compound tincture of lavender, and of value only as a colouring agent. It must not be confounded with Santalum album (Santalaceæ, v. p. 2r2). 


\section{TAMARINDUS INDICA.}

(Tamarind.)

The preserved fruit of the tamarind is used as a sweetening agent for covering the nauseous taste of drugs: or for feverish patients, on account of its pleasant and subacid flavour. One ounce boiled with a pint of milk produces an agreeable whey; a tablespoonful, stirred with warm water, in the early morning is a mild aperient.

\section{ASTRAGALUS VERUS. (Tragacanth.) \\ ACACIA VERA. \\ (Acacia, or Gum Arabic.)}

The dried gummy juice of tragacanth is met with in horny laminated flakes, and that of acacia in small semi-transparent irregular masses.

The active ingredient of the latter is arabic acid, $\mathrm{C}_{6} \mathrm{H}_{10} \mathrm{O}_{5}$, an interesting substance from a chemical point of view, and allied to starch (Pharm. Journ., ii., 1883).

Both drugs are used as "demulcents," i.e., soothing or sheathing applications to irritated mucous membranes, and also to suspend medicines which are insoluble in water. Comparative experiments in preparing emulsions, show acacia to be much the more suitable for that purpose (Gerrard, Pharm. Journ., ii., 1880), but glycerine of tragacanth is in more frequent use as a convenient excipient for pills. 


\section{GLYCYRRHIZA GLABRA.}

(Liquorice.)

The rhizome contains a particular kind of sugar, a glucoside, glycyrhisin, which is non-crystalline and unfermentable. Either from this, or from the presence of small quantities of other ingredients, it possesses a certain soothing power in irri tated conditions of the throat and bronchi. It is of little importance except as a sweetening material, for which purpose it forms part of decoctum aloes comp., decoctum sarsæ comp., confectio terebinthinæ, infusum lini, tinctura aloes, etc. It is also used for mechanical purposes in making several of the officinal pills.

PREPARATIONS AND Dose.--Extractum: dose, $\frac{1}{2}$ to $1 \mathrm{dr}$. Extractum liquidum: dose, $1 \mathrm{fl}$. dr. Pulies glycylhiwe comp. (v. Senna, p. 249): dose, 1 to $2 \mathrm{dr}$.

\section{MUCUNA PRURIENS. (Cowitch, or Cowhage.)}

\section{(Not Officinal.)}

The short, stiff bristles, which clothe the legumes of this plant, were formerly much in request as a vermifuge, acting apparently as mechanical irritants to the parasites. A dessertspoonful thrice daily, followed by a purge on the following morning, is often effective against the lumbricus. 


\section{PO' DE BAHIA.}

\section{(Araroba-Goa Powder).}

Description. - A powder obtained from a leguminous tree indigenous to Bahia, probably either from a species of Centrolobium or of Andira: it is contained "in elefts or cavities, which traverse the rood in the direction of its diameter, and are prolonged through the entire trunk," which is cut down, split, and the powder scraped from the clefts (Stillé).

It was formerly sent almost entirely to Portugal, and thence to the Portuguese colonies, especially to Goa. It occurs in commerce as a rough powder, or in irregular lumps mixed with small pieces of the wood: of light-yellow, brown, or purple colour, and bitter taste: "carefully heated, it yields a moss-like sublimate, which is coloured red by alkalies."

ACTIVE INGREDIENTS. - The chief of these is chrysophanic acid ( 80 per cent.): a glucoside, a bitter principle, and gum are also present.

Chrysophanic acid, sometimes called chrysarobin or rhein, $\mathrm{C}_{15} \mathrm{H}_{10} \mathrm{O}_{4}$ (F. \& H.), may be obtained also from a lichen (Parmelia), from rhubarb, from rumex, and probably from other allied plants: Chrysarobin is first obtained, and by treatment with caustic potash forms a chrysophanate of potassium, from which the pure acid is separated. It is commonly met with as a pale, or orange-yellow, powder, but sublimes in bright-yellow acicular crystals, without taste or odour. It is freely soluble in benzol, chloroform, oils, and hydrocarbons; partly so in boiling water, alcohols, ether, and glacial reetic acid; almost insoluble in cold water.

Physiological Action.-Ertemal.-Much local irritation may be excited by "Goa powder," and occurs oceasionally from the diluted chrysophanic acid; at the same time a red or purplish rash may develop, and is followed by some staining, and afterwards whitening of the skin, and coloration of 
nails and hair. These effects pass away under warm bathing, and emollients, with desquamation of the affected parts: dilute solution of potash or of chlorinated lime hastens the disappearance of the stains.

Physiological Action.-Internal.-Taken in sufficient dose, it seems to have an emeto-purgative action : in some cases even half a grain has caused diarrhoea, and seven grains have acted violently, but there is some uncertainty in such effects.

Therapeutical Action.-Eixternal.-Parasitic Skin Disease - Psoriasis. - In such cases remarkably good results may be obtained with these remedies. Sir J. Fayrer and Dr. da Silva (Lima), only about ten years ago directed professional attention in Europe to the long-established use of the powder as a secret remedy in the East, for obstinate forms of ringworm especially (Med. Times, ii., 1874, i., 1875), and soom afterwards its main ingredient was identified by Prof. Attfield, and has since been chiefly employed. Cases of tinea circinata are readily cured by it, as by many other remedies. Dr. Crocker quotes a number of instanoes (Lancet, i., 187\%), but it has proved by no means so successful in tinea tonsurens, and the drawbacks of irritation, staining, etc., prevent its being a very desirable application. In psoricsis, however, its value is often so much greater than that of other local applications, that it must remain as a standard remedy for obstinate cases. B. Squire was one of the first to report such good result, two serere cases yielding completely after six to eight applications of an ointment containing 2 drachms of the acid to 1 ounce of lard (B. M. J., ii., 1876). Still more remarkable is a case recorded by Dr. A. D. Keith, of nine years' duration, and steadily getting worse under all kinds of treatment (including rubber-clothing), and yet completely cured by a few ounces of ointment coultaining 90 grains of Gon powder to the ounce (B. M. J., i., 1877). Mr. Hutchinson showed another case, in which one half of the body was seen quite sound after the acid ointment, whilst the other half, treated by tar at the same time, remained uncured (B. M. J., i., 1878). Ogilvie Will also reported six cases (ibid.), and Neumann concurs in praising the results of 
similar treatment, though irritation has sometimes proved very unpleasant (cf. W. G. Smith, Dublin Journ., March, 18r9). Dr. Napier has reported two cases of psoriasis treated successfully by the intemal use of the acid: $\frac{1}{8}$ grain rubbed up with sugar of milk was the commencing dose, which was gradually increased (Glasgow Med. Journ., 1882).

It may be concluded from such cases that the remedy acts, in part at least, by absorption from the skin in patients when it has only been used externally.

PREPARATIONS AND DOSE.-Goc poucler is applied in India mixed with lime juice, in Brazil with a little vinegar or lard (20 to $40 \mathrm{gr}$. to the ounce): it stains much more than the acid. Ointment of chrysophanic acid may be made of any strength from 5 to $20 \mathrm{gr}$. (or upwards) in the ounce, but a stronger preparation than this is liable to irritate severely. The acid should be well dissolved in the excipient.,...o.g., benzoated lard: it should be mixed and melted thoroughly, and stirred in till cold. The drug may be made into a watery paste and brushed over the diseased patches, and when dry, covered with collodion, and allowed to remain on forseveral days.

Internally: dose, of the acid, $\frac{1}{2}$ gr. gradually increased; $\frac{1}{2} \mathrm{gr}$. has sometimes caused purging. 


\section{ABRUS PRECATORIUS. \\ (Indiax Liquorict, or Jequirity).}

DESCRIPTION.-A small twining shrub, having alternate pinnate leaves with stipules and petioles, and erect racemes of numerous flowers, rather large, pale pink and papilionaceous in form; the legume is long-rhomboidal, containing four or five spherical, smooth, scarlet or white seeds with a black spot at the hilum-rarely the colours are reversed. These seeds are known as "prayer beads" or jumble beads (goonteh), and are used also as standard weights. The integument is curiously constructed with no less than nine distinct layers of cells (Cunningham, Indian Med. Graz.). The root is long and woody, about $\frac{1}{2}$ inch in diameter, internally yellowish, with thin bark, disagreeable odour, and a bitter-sweet taste.

ACTIVE INGREDIENTS.-The careful analyses of C. J.H. Warden, of Calcutta, obtained snow-white silky crystals of a body which he termed abric acid (probable formula, $\mathrm{C}_{21} \mathrm{H}_{24} \mathrm{~N}_{3} \mathrm{O}_{4}$ ), soluble in alkaline solutions, but not much so in alcohol; also an oil and a volatile irritant principle. Neither the acid nor the oil, however, produced characteristic effects, and the action of the seeds was ascribed by MM. Cornil and Berlioz to the agency of a specific organism (Progrès Médical, 1883), their researches being suggested by those of Sattler (Wiener Med. Wochensch., 1883). Warden and Waddell have, however, come to the conclusion, from an extended series of experiments, that the active principle of abrus is a soluble proteid body, which they term chrin.* This has all the physiological properties of abrus-seed. It is best prepared by percolation with cold distilled water after exhausting the seeds first with chloroform, and then with alcohol: the percolate being precipitated by alcohol several times, abrin is obtained in a fairly pure state as a pale-grey amorphous powder, becoming of a gummy transparent appearance when dried in thin layers. It is a proteid, probably a native albumen, though in some respects

* The Non-bacillar Nature of Abrus-poison, C'alcutta, 1884. 
related to globulin. It forms a slightly yellow or slaty solution in cold water and glycerine, from which it is precipitated by boiling, by carbonic, nitric, and hydrochloric acids (soluble in excess), and gives the characteristic proteid test with Millon's. reagent.

Physiological Action.--In India, abrus-seeds are used for poisoning cattle in the form of "suis," i.e., spikes which are thrust into the skin: the symptoms are rapid local cellulitis, affection of the lymphatic glands, followed by general depression, ending in death in from eight hours to two days: post mortem, besides local suppuration the lungs have been found hyperæmic.

An infusion of abrus-seeds has long been known to produce a form of purulent ophthalmia when applied to the eye, a great number of bacteria being found in the discharge. Sattler ascribed such action to these bacteria, supposing that a bacillus innocuous in the air, became pathogenic. The experiments of MM. Cornil and Berlioz tended to confirm this: and they showed further that the subcutaneous injection of carefullyprepared infusion into guinea pigs, caused a train of symptoms more or less septicæmic in character-viz., local odema and phlegmon of the skin, inflammations of serous membranes (especially peritonitis), and ecchymoses in various parts, or inflammation of Peyer's patches, and peculiar yellowish patches in the liver: death generally occurred within thirty-six hours, but the time varied according to the dose injected. In the different lesions micro-organisms were found-the "bacterial rods of jequirity." When the infusion was injected after being sterilized by filtration, or when abric acid was used, none of these effects followed; hence, the French observers concluded that the process was connected with the development of special bacilli. No mention of cultivation-experiments is made.

Dr. Klein brought forward evidence against this theory: taking special precautions to sterilize the infusion, and "cultivating" a portion of this in a solution of peptone, he injected another portion under the conjunctiva of a rabbit. No orgrmisms developed in the cultivation-material, while intense ophthalmia was procluced in the animal. Moreover, there was. 
no bacillus found in the discharge, nor was the latter infective,hence he concluded that the freshly-prepared infusion acts independently of germs, and that the real result of boiling it (which renders it innocuous) is not the destruction of such germs, but the alteration of the active principle (probably a ferment like pepsin), which thus loses its fermentative power (Centralb. f. Med. Wiss., Feb., 1884).

This result is abundantly confirmed by the experiments of Warden and Waddell, who showed that the bacteria developed in lesions produced by subcutaneous injection of abrus-infusion, were of different kinds; and further, that when the infusion was sterilized, death occurred in the usual manner, without the presence of bacteria in the lesions. They further instance the fact, admitted by Sattler and De Wecker (Monogram, Paris, 1883), that the intensity of abrus-ophthalmia is proportional "to the strength of the infusion and to the number" of applications," a fact opposed to the bacterial origin of the inflammation.

Cultivations were made of the seeds, and colonies of rarious bacteria obtained, and the injection of this cultivationproduct produced in none of the animals (cats and chickens) any result whatever: in some cultivations no bacteria were found. These experiments, confirming as they do Klein's conclusion, disprove the "bacterial origin" of the action of jequirity.

In a large number of examinations of discharges, etc., from eyes affected with jequirity ophthalmia, Mr. Benson could not once find the typical bacillus (B. M. J., i., 1884).

Subcutaneous injection of a solution of pure abrin caused death with the symptoms and post-mortem signs of abruspoisoning, identical with those previously detailed. "Abrinpeptone" obtained by the action of pepsin also produced sinilar symptoms.

Abrin is not entitled (according to these observers) to be called a true ferment, though its activity is destroyed at a temperature of $100^{\circ} \mathrm{C}$, since it has no action on albumen or starch.

Its physiological action may be thus summarized:-

Circulatory System.-The action of the heart is lowered, and with toxic doses is wrestecl in diastole: the blood is rendered unduly fluid, and is frund to contain "enormous num- 
bers of blood-plates; "- the white dises are not affected. The respiratory function and the body-temperature are also lowered.

Digestive System.-It is curious that the abrus-seeds may be eaten. with impunity; but in poisoning by "suis" mucosanguineous diarrhœa is usual: there is anorexia, and in animals that survive for a few days, emaciation is marked.

Therapeutical Action.-For exciting inflammation in the conjunctiva, an infusion of the seeds has long been used (and often abused) in South America; it has, comparatively lately, been introduced into ophthalmic practice in Europe, especially for granular lids (trachoma). Moura advised an infusion of 8 grains of the seeds in 100 minims of cold water (Pharm. Journ., 1883). Dr. Weekes used a solution of 155 grains in 17 fluid ounces of cold water ( 1 in about 52) - with which the ophthalmia produced disappeared in about a fortnight without endangering the cornea: it cured the granulations of trachoma rapidly. The ophthalmia begins almost immediately after the application; is intense, and carnses great pain. Dr. D. Webster, of New York, has published thirteen cases of trachoma (some with pannus), treated by jequirity, and speaks highly of the results obtained, the granulations being cured, the pannus improved, and with this the sight. In some cases the effect of the drug was so severe that atropin and iced cloths had to be employed to subdue the inflammation (Arch. of Ophthal., No. 1, 1884) - a fact which points to the danger of the remedy, as ulceration of the cornen may be produced. The drug seems to be safest when the cornea is very vascular; but great care in all cases is necessary. Dr. Shoemaker has used a strong emulsion in lupoid disorders, epithelioma, ete., with the result of causing severe inflammation, but subsequent cure (Med. Bulletin, May, 1884).

PREPARATION as above stated. Further trial may show that a solution of abrin will be more trustworthy than an infusion of the seeds; it is, however, premature to speak on this point. It is important to note that a fresh infusion of the seeds must be used; it sonn decomposes. 


\section{SANTALACEAE.}

\section{THE SANDAL-WOOD FAMILY.}

$\Lambda_{x}$ order of epigynous exogens, the species of which have few characters in common except minutely technical ones. In Europe and North America they are insignificant weeds; in the East Indies they are shrubs or small trees. The leaves are simple; the flowers small and apetalous, with their parts in fours or fives; the fruit is one-seeded, and usually drupaceous.

\section{SANTALUM ALBUM. (White SANdal Wood.) (Not Officinal.)}

DESCRIPTION.-An evergreen tree of 20 or 30 feet in height; native of India, and especially of the mountains of Malabar. The leaves are opposite, ovate-lanceolate, 2 or 3 inches long, entire, glabrous, and shortly petiolate; the numerous flowers are tetramerous, straw-coloured, changing to purple, and borne in terminal thyrsi; the fruit resembles a small black cherry. The "white sandal" of commerce consists of the heart-wood, in billets.

Active INGREDIENTS.-In the Appendix to the Pharmacopoia of India it is stated that the wood of white sandal yields about 2.5 per cent. of the oil, which is of a paleyellow colour, and has a resinous taste and a sweet peculiar smell, best appreciated by rubbing a few drops on the wam hand: it is soluble in 80 per cent. of alcohol, in ether and 
ohloroform (Pridie). A resinous principle and tannin are also contained in sandal wood. M. Chapoteant says that the oil distilled from it is composed of two "essences" $-\mathrm{C}_{15} \mathrm{H}_{24} \mathrm{O}$, boiling point $300^{\circ} \mathrm{F}$., and $\mathrm{C}_{15} \mathrm{H}_{26} \mathrm{O}$, boiling at $310^{\circ} \mathrm{F}$. From this latter he derived a series of ethers, and concluded it to be an alcohol, of which the former is the aldehyd; and as these bodies are very susceptible of oxidation, he finds in this an explanation of a greater therapeutical power than is possessed by copaiva.

ABSORPTION AND Elimination. - Elimination commences about half an hour after taking, and as with copaiva, is evidenced in the urine by its clouding with nitric acid; this cloudiness is from precipitation of resinoid constituents of the drug, and is cleared up by alcohol.

Physiological Actrion.-According to Mr. Robert Park "its physiological effect is generally to constringe all the mucous membranes of the body. Thus, the immediate effect of a dose of it is to cause dryness of the fauces and thirst. During its digestion and absorption, this thirst is kept up and intensified in some cases; in all, if a large dose be given (i.e., 15 to $20 \mathrm{~min}$.), and in many a moderate dose, produces a feeling of 'drawing together of the bowels,' sometimes verging on colic. Some patients have experienced nausea from its markedly disagreeable smell, but as a rule it is much less unpleasant than copaiva. Its action is somewhat more powerfully felt in the kidneys. At a period varying from two to three hours after it has been taken, a sense of fulness is experienced in both renal regions, which lasts for a period varying from ten to twenty minutes" (Practitioner, ii., 1869).

Therapeutical Action.-There is a good deal of evidence to show that the oil is a powerful remedy for gonorrhoea. The principal authorities are Dr. Henderson, who reported relief generally in forty-eight hours without disagreeable effects on the stomach (Glasgow Med. Journ., 1865), Mr. Berkeley Hill (B. M. J., ii., 1867), Dr. Purden, Dr. H. W. Beach (Boston Med. Surg. Journ., Nov. 1868), and Dr. G. Pridie. Mr. Park thus sums up the result of a 
considerable experience:- "The eases in which it is most efficacious are not those of sickly patients with thin mucoaqueous discharges, but those of full plethoric subjects with thick purulent discharge, and even considerable scalding. Given in a case of this kind as soon as possible after the appearance of the discharge, the effect is simply very gratifying both to patient and doctor. The discharge disappears, and the scalding is no more felt; and if the medicine be continued and collateral treatment attended to, in from two to five days the cure is complete-that is to say, there will have been no discharge seen for twenty-four hours, and there is no pain or abnormal symptom whatever. The patient must not be dismissed now, however, without a caution to abstain.rigidly from all stimulants for at least a week, nor must he, within a similar period, take a purge, else he will assuredly bring back the discharge: then this redeveloped discharge is, for some reason I have never been able to fathom, more difficult to deal with; nor have I been able to find any attempt at explanation in the works of either Cullerier, Ricord, Lancéreaux, or any of our own authorities. I will merely add that complications are to be treated separately, though the exhibition of the oil is by no means contra-indicated by the presence of orchitis, prostatitis, or phimosis. It may be given perfectly well while the appropriate treatment for each of these is being carried out. To this rule there is one exception, viz., in orchitis. If it be requisite, as it very often is, to give a purge, let it be given at once, and before the oil is administered: as purges and stimulants (including coffee and, I strongly suspect, tea also) are contra-indicated during its administration. I have never once, however, seen any of the above-mentioned complications occur during a course of the oil,-I mean idiopathically." Stillé concludes that it has no advantage over copaiva, but according to my experience it is more easily taken, is safer, and on the whole, though not always, more effective. I have not seen the bad effect described from an occasional purge during treatment.

Dr. Parras (1865) found this drug modify the inflammatory symptoms in a striking and rapid manner, the scalding being removed in from one to three days, and the thick discharge being reduced to a few drops of muco-pus. 
Durand (Thesis, 1874) reports equally favourably, finding: blennorrhœe in the acute stage relieved in three days by doses which did not cause nausea or any digestive derangement.

In inflammation of the bladder, and in hæmorrhage from the urethra, the remedy is also good. Lober corroborates these observations, and has given as many as twenty-four capsules per diem without disturbance (Lille, 1876).

PREPARATIONS AND DOSE.-The oil itself should be administered in mucilage or capsules. Authorities differ as to the dose, some recommending $20 \mathrm{~min}$., some 30 or 40 . Mr. Park thinks that larger doses than $5 \mathrm{~min}$. are useless and even injurious, but gives this quantity every four hours. 


\section{ROSACEAE.}

\section{THE ROSE FAMILY.}

A LARGE and diversified order of exogens, comprising trees, shrubs, and herbaoeous plants, of wide distribution in the cooler parts of the northern hemisphere. The leaves are alternate, stipulate, either simple and undivided, or compound; the flowers are regular, usually pentamerous, and have their free and often wrinkled petals seated upon the rim of the calyx, which also bears the numerous stamens. The ovary and the fruit present many different forms, which allow of the order being subdivided into the Rosaceæ proper, the Pomiferæ, and the Drupiferæe or Amygdalaceæ, with one or two other sections of minor importance. The leading property of the order is astringency, but many species are capable of yielding prussic acid.

\section{Pharmacentionl Symeries.}

Rosa gallica; Rosa centifolia; Rosa camina .. Roses.

Tormentilla officinalis . . . . . . Tormentil.

Cydonia vulgaris _. $\quad \ldots \quad \ldots$. . The quince.

Amygdalus communis . . . . . . . . The almond.

Cerasus lauro-cerasus . . . . . . . . Cherry-laurel.

Prunus domestica $\quad \ldots \quad \ldots \quad \ldots \quad \ldots$ The plum.

Brayera anthelmintica .. . . . . Kinusso.

Prunus rel Cerasus Virginiana . . . Virginian or

Wild cherry. 
ROSA GALLICA; ROSA CENTIFOLIA; ROSA CANINA. (Roses.)

DESCRIPTION.-The Rosa gallica is a small and prickly shrub, with pinnate leaves, and large erect red flowers. It is a native of Austria and other parts of central Europe, and occurs also in the Crimea.

The R. centifolia, aboriginal to the woods in the eastern parts of the Caucasus, is at once distinguished from the $\mathrm{R}$. gallica by its drooping flowers.

The R. canina, to be found in every English hedgerow, is known by its long and arching shoots, small leaves, which are destitute of glands, and faintly odorous flowers.

ACTION.-The rose has slight astringent and tonic properties. Of certain species the petals are collected and dried for use in various preparations, but chiefly in infusion, as a vehicle for the exhibition of neutral salts in cases of homorrhage and other disorders. For combination with preparations of lead or zinc, and for collyria, rose-water is eminently serviceable; and the conserve prepared from the pulp of the fruit of Rosa canina is useful to give bulk to pills containing calomel, antimony, and other drugs: it is likewise employed in demulcent electuaries, and was at one time considered a suitable medicine for dysentery.

PREPARATIONS AND DOSE.-Aqua rose (made from the Ros(e centifolice petala): dose, ad libitum. Confectio rosre gallicee: dose, 60 gr. or upwards. Infusum rosce acidum: dose, 1 to $2 \mathrm{fl}$. oz. Syrupus rosce gallice (made from the Rosce gallice petala): dose, $1 \mathrm{fl}$. dr. or more. Confectio rosse canince (made from the Ros(e canince fructus) : dose, $60 \mathrm{gr}$. or more. 


\section{TORMENTILLA OFFICINALIS. (ToRMENTIL.)}

\section{(Not Officinal.)}

DESCRIPTION.-An herbaceous perennial, growing everywhere in England upon dry banks. The long slender stems are crowded with ternate or five-lobed leaves; the flowers are small, regular, square, and golden-yellow; the rhizome is ligneous, large for the size of the plant, and internally red: it is usually met with in pieces 2 to 3 inches long, $\frac{1}{2}$ inch thick, branched and covered with roundish protuberanoes or depressions, from which thin rootlets are given off.

ACTIVE INGREDIENTS.-From 20 to 30 per cent. of tannic, or tormentil-tannic acid, also some kinovic and ellagic acid, oxalate of calcium, and tormentil-red.

ACTION.-The root is astringent in a high degree, and operates without causing excitement, whence it is considered by some writers one of the best medicines of its class : it may be used in all cases where tannin is indicated.

Preparations and Dose.-Pulvis: dose, 20 to $60 \mathrm{gr}$. Decoctum ( 1 in 20): dose, 1 to $2 \mathrm{fl}$. oz.

\section{CYDONIA VULGARIS. (The Quince.)}

\section{(Not Officinell.)}

A tree, originally brought from Candia, in most respects similar to the apple, but differing essentially in the fruit, the five cells of which are many-seeded. The seeds themselves are large, ovate, pointed, and plano-convex, the testa containing much mucilage, which is readily given up to boiling water, and 
makes a good demulcent for external application in cases of cracked or excoriated skin: in France it is in popular use, especially for bed sores (a tablespoonful of seeds to half a pint of water-boil to a jelly). A decoction ( 2 drachms of seeds to 1 pint of water, boiled for ten minutes) forms a soothing, neutral eye-lotion.

\section{AMYGDALUS COMMUNIS.}

\section{(The Ammond.)}

DESCRIPTION.-A tree attaining the height of 20 feet, with leaves 3 to 4 inches long, narrow-lanceolate, and finely serrated. The pink flowers, about an inch across, are produced plentifully in March and April, before the foliage is developed. The fruit resembles a small peach, but is ovoid, hard, leathery, and of an ashy-grey colour, and, when mature, opens down one side. Two varieties are distinguished by the shape and quality of the seeds-the sweet almond and the bitter almond,- the latter being thinner, broader, and shorter than the former.

ACTIVE INGREDIENTS.-Both kinds yield by expression a considerable quantity of oil, which is more pure than olive oil, and less liable to become rancid. An essential oil is likewise procurable by distilling almond-water with baryta, so as to separate the prussic acid; in close vessels it is very volatile; exposed to the air it becomes solid, crystalline, and inodorous.

Amandin and emulsin are two proteid compounds which are soluble in cold water, and by forming an emulsion serve to suspend the oil. Amygdalin, a glucoside, which may be crystallized, is found only in bitter almonds (v. p. 281).

Physiological Action.-Sweet almonds have a bland, agreeable flavour, and are nutritive and emollient, but somewhat difficult of digestion (through containing so much oil); they have been known to cause nausea and uuticaria.

Almond oil (not the essential oil, but usually expressed from 
the bitter variety, on account of its cheapness, and the higher value of the residual cake) possesses the dietetic and medicinal properties of the other fixed oils, and is, in its local action, emollient; in large doses it acts as a mild laxative.

Alarming symptoms have been produced in the human subject through eating bitter almonds, and even death has occurred: thus, Orfila relates the cases of two children, in whom the symptoms were marked and rapidly developed; within a quarter of an hour after eating them, pallor and collapse of the features set in, the pupils became dilated, and the respiration sighing, with somnolence and muscular relaxation. In another case, a man fell dead very soon after partaking freely of the almonds; he frothed at the nose and mouth, and the eyes became fixed and glistening. In a recently published case, death was only averted by the use of the stomach-pump and other vigorous measures. The patient, a man, had eaten two handfuls of bitter almonds when fasting, and after an interval of an hour or two, suddenly fell down unconscious (B. M. J., ii., 1881).

The essential (volatile) oil of bitter almonds is a deadly poison (containing in 100 parts nearly 13 of anhydrous prussic acid), one drop of which has been known to kill a cat (Taylor).

A case is reported by Mertzdorf of a man who swallowed two drachms of this oil; his features became spasmodically contracted; his eyes fixed, upturned, and starting from his head; his breathing jerking and hurried; and death followed in half an hour. In a recent case tried at Maidstone, death occurred in a girl of sixteen about two hours after a teaspoonful of the essential oil had been administered as medicine by a clergyman; the dose was calculated to contain about 2 grains of prussic acid (Pharm. Journ., ii., 1883). (On the other hand, in several cases large quantities have been taken without fatal results, proving only that the amount of contained prussic acid is variable.)

Therapeutical Action.-Dr. Pavy has proposed, as a substitute for bread or starchy food for patients suffering from dinbetes, cakes made of sweet almonds.

The "mistura" is useful as a lotion to allay itching of the 
skin, and has been a favourite vehicle for the administration of tartarized antimony to subdue pulmonary inflammation and to relieve cough. As a demulcent and emollient in such cases it is certainly good, as well as in inflammatory affections of the alimentary canal and of the urinary organs.

Almond oil may be employed for the same purposes as olive oil : combined with an equal volume of syrup of roses, or syrup of violets, it is a suitable laxative for infants; is useful also in the preparation of emulsions, and of certain kinds of linctus.

A few blanched almonds are said to relieve heartburn.

PREPARATIONS AND DOSE.-Mistura amygdalce: dose, 1 to 2 oz. Pulvis compositus: dose, 60 to $120 \mathrm{gr}$. Oleum: dose, 2 to $4 \mathrm{dr}$. (Mistura amygdulce amarce [not officinal] requires caution in its use.)

\section{CERASUS LAURO-CERASUS.}

(Cherry-Laurel.)

DESCRIPTION.-A tree indigenous to Trebizond, bushy in habit, and attaining the stature of 10 to 20 feet. The leaves are oblong, 4 to 6 inches in length, shortly petiolate, coriaceous, yellowish-green, and glabrous. The fragrant white flowers, half an inch across, and produced in early spring, are borne in simple axillary racemes about as long as the leaves, and are followed by black drupes the size of small cherries. The leaves alone are officinal: they have a bitter, somewhat acrid flavour, and the odour evolved when they are bruised is offensive and characteristic; drying dissipates it, but the taste remains, and then exhibits a certain amount of astringency.

ACTIVE INGREDIENTS. - Their most important constituent is amygdalin $\left(\mathrm{C}_{20} \mathrm{H}_{27} \mathrm{NO}_{11}\right)$, a substance identical with the 
glucoside of that name which is found in bitter almonds ( $x . p$. 279), although not crystalline, like the latter, but amorphous: it undergoes many chemical transformations, but the most interesting is, that in the presence of certain ferments (emulsin and others not known) and water, it gives rise to the formation of hydrocyanic acid, bitter-almond oil, sugar, and (probably) formic acid: 17 grains of anhydrous amygdalin yielding about 1 grain of prussic acid. This transformation takes place when laurel leaves are distilled with water, and hence laurel-water contains a small, and unfortunately a varying, proportion of prussic acid, which is its chief ingredient.

PHysiological ACtion.-The leaves and the kernels of the fruit possess poisonous properties. "Laurel-water," prepared from the former, is similar in action to prussic or hydrocyanic acid, being capable of inducing sudden insensibility, and death within a few minutes.

Digestive System, etc.-In large medicinal doses, laurelwater sometimes occasions nausea, accompanied, perhaps, by vertigo, and pain in the stomach, as well as by increased secretion of saliva, irritation in the throat, disordered and laborious respiration, pain in the head, obscured vision, and sleepiness. If the dose be increased, there is more decided vertigo, faintness, with sickness, and a sense of constriction at the præcordia; while a dose that just stops short of being fatal, occasions insensibility and extreme feebleness of the action of the heart.

As the result of a poisonous quantity, other symptoms quickly show themselves: the respiration becomes difficult and spasmodic; the pupils are usually dilated (sometimes contracted); the pulse becomes small or imperceptible; and occasionally tetanic convulsions, insensibility, and death, quickly follow. ${ }^{3}$

It is remarkable that the pulp of the fruit is not only free from the poisonous properties of the leaves and kernels (as happens also with the fruit of the yew-tree), but is wholesome and palatable.

1 The most interesting case of fatal poisoning with laurel-water recorded is that of Sir Theodosius Broughton, who was murdered in this way in 1781 . 


\section{Therapeutical ACtion.-Dyspepsia-Vomiting}

Regarded as a medicine, cherry-laurel water, like prussic acid, is narcotic, sedative, and anti-spasmodic, but it does not possess the power of lessening pain in general; nor has it the property of inducing sleep in a direct manner, in the way, for instance, that is effected by opium. It may be employed in all cases where it is customary to resort to prussic acid, i.e., when a sedative is required, but the results of its administration are rendered uncertain by the varying strength of the medicine: its energy is greatest when prepared quite recently from dried leaves, or when moderately young leaves are employed.

In gastralgia, pyrosis, hiccough, and some other forms of dyspepsia, it is a useful remedy, and palpitation depending upon these conditions may be greatly mitigated by it. Relief may also be obtained in cases of romiting unconnected with inflammation of the stomach, e.g., in the prolonged romiting of pregnancy.

Pain.-Laurel-water, like prussic acid, will not mitigate pain in the intestines ; and its employment for such purposes is proved by experience to be often useless, though the attacks of pain in angina pectoris, which closely resemble gastrodynia, are often more quickly alleviated by these remedies than by any others: also, laurel-water sometimes quickly allays the pain in ticclonlonven:x: but for the procuring of sleep, for the relief of pain in general, or as a remedy for diabetes, laurel-water, like prussic acid, though often recommended, will be found a poor substitute for other drugs.

Whooping-cough, etc.-I have frequently employed laurelwater with advantage in whooping-cough, and in the affection termed "spasmodic cough"; also in cases of inflammation of the chest, after the subsidence of the acute symptoms. In repeated catarrh, and in chronic bronchitis, small doses of the same medicine often prove serviceable: it will also relieve some of the more distressing symptoms of phthisis, and is valuable, therefore, as affording temporary comfort.

Prurigo, etc.-In prurigo, impetigo, inveterate psoriasis, and other cutaneous affections attended by severe itching and tingling, laurel-water locally applied affords great relief : and 
the powdered leaves, mixed with flour or linseed-meal, have been employed as a poultice for ulcers.

PREPARATION AND Dose.-Aqua lauro-cerasi: dose, 5 to $30 \mathrm{~min}$. (The strength of this preparation being uncertain, caution is required in the use of it.)

\section{PRUNUS DOMESTICA.}

(The Pium.)

DESCRIPTION.-A well-known tree, with ovate serrated leaves, rosaceous white flowers, and drupaoeous fruit. One of the many varieties yields the prunes of commerce, the finest of which are preserved for food, while the smaller and more aoid ones are employed in medicine.

ACTIVE INGREDIENTS.-Prunes contain a large amount of water, with about 20 per cent. of solid matter, consisting of malic acid, gum, sugar, pectin, lignin, and other unimportant substances, with a purgative principle, the exact nature of which has not yet been accurately determined.

Physiological Action.-Prunes, like most other kinds of plum, are gently laxative, but if eaten in excess they are apt to occasion flatulence, griping, and diarrhœa.

Therapeutical Action.-Employed as an article of diet by persons of costive habit, prunes often induce beneficial effects. They are also considered useful during convalescenoe after febrile and other disorders.

Preparation and Dose.-Dose, 1 oz. and upwards. Prunes, when added to the infusion of senna, render it more palatable, and increase its purgative action: they are contained in confectio sennæ. 


\section{BRAYERA ANTHELMINTICA.}

(Kousso.)

DESCRIPTION.-The dried flowers and tops of an Abyssinian tree, named "kosso," or "kousso," have been employed in their native country for at least two centuries. This tree is one of the few arborescent Rosacere attaining the height of 40 feet: the leaves are imparipinnate; the small, greenish or purplish, unisexual flowers are borne in panicles: for medicinal purposes they are collected before the seeds of the earlier ones are ripe, or while a number of the blossoms are unchanged; the female flowers, which are more usually collected, are brownishred-known as "red kousso"- the male inflorescence is lighter, and greenish-brown. The taste of the dried flowers offers nothing remarkable until after the lapse of a few minutes, when an acrid and unpleasant flavour, somewhat like that of senna, is perceived. The odour is fragrant and balsamic, resembling a combination of senna, hops, and tea.

ACTIVE INGREDIENTS. - It still remains doubtful whether the crystalline acid substance, koussin, is the only active element in kousso ; or whether a peculiar resin, a volatile oil, and two species of tannin, which it contains, are also poisonous to intestinal worms. So far as I know, the only positive evidence is in favour of koussin, $\mathrm{C}_{31} \mathrm{H}_{38} \mathrm{O}_{10}$, which occurs in minute white or yellow rhombic crystals that have a bitter and irritant taste, and a peculiar odour of Russian leather. It is very insoluble in water, easily soluble in alcohol; concentrated sulphuric acid dissolves it yellow, and, if water be added to this solution, white flocky masses are separated. The alcoholic solution gives a brown precipitate with perchloride of iron, and a greyish-yellow one with acetate of lead.

Physiological Action.-Kousso produces no observable effect on the human body, excepting occasional sickness from the nauseousness of its taste, but it is a direct poison to intestinal worms, especially tape-worms. In Abyssinia, where it 
is commonly employed, it is said to have acted so strongly as to cause abortion and death in pregnant women. Koussin also has been successfully given, in a dose of about 20 grains, against tape-worm; it sometimes induces vomiting and diarrhoea, and is better given in three or four divided portions, in some aromatic vehicle.

Therapeutical Action.-As a vermicide for both varieties of tape-worm, and to a less extent for round worms, kousso has received ample commendation. So long ago as 1850 the Lancet gave details of its successful employment by several London physicians: and in Switzerland, where tapeworm (especially Bothriocephalus latus) is very common, from the habit of eating certain meats raw, the value of kousso has been well established. It has also been freely used both in France and Germany, though in the latter country it is not much employed at present. Its nauseous flavour is certainly an objection, but the same defect belongs to the Filix mas, the value of which no one of experience would dispute on that ground.

PREPARATIONS AND DOSE.-Infusum $\left(\frac{1}{2}\right.$ oz. to 8 oz. of boiling distilled water) : dose, 4 to $8 \mathrm{fl}$. oz.; or $\frac{1}{4}$ to $\frac{1}{2}$ oz. of the flowers may be given with honey in confection. Should the worm not come away in three or four hours, a castor oil or saline purge should be administered. Konssin: dose, $20 \mathrm{gr}$. in two or three portions; its taste may be covered by peppermint.

\section{PRUNUS VEL CERASUS VIRGINIANA.}

(Virginian or Wimin Cherrer.)

\section{(Not Officinal.)}

Description.-A North American tree, small in the open field, but 60 or 80 feet high in the forests: it has oblong, petiolate, serrated leaves, white flowers in racemes 4 to 5 inches long, and small round purplish drupes. The bark, which is used in medicine, occurs in irregular fragments, grey, brown, or 
greenish externally, smooth and reddish internally, brittle on fracture. The odour when dry is slight, but on moistening the bark, becomes distinctively that of the bitter almond: the taste is astringent, bitter, somewhat aromatic.

ACTIVE INGREDIENTS.-Tannic and gallic acids, resin, starch, a bitter principle, and a volatile oil, which is pale-yellow in colour, and resembles the volatile oil of bitter almonds in containing prussic acid; it is formed, as in that substance, by the action of emulsin on amygdalin in the presence of moisture: the amygdalin varies in amount; it has been separated in the crystalline form (Procter).

Physiological Action.-This may be expressed in general terms as a combination of tonic and sedative : 15 grains of the powder have the former effect, with some astringency, and cause also a sensation of warmth at the epigastrium. Any doses up to 30 grains commonly quicken and strengthen the pulse at first, but later on, or with larger doses, this becomes slower, and a sedative effect is produced, with lessening of general sensitiveness, and a tendency to sleep. The larger the dose, naturally the more sedative the effect. Eberle "found copious draughts of the cold infusion reduce the pulse from 75 to $50 "$ (Wood). More than 40 grains are apt to cause gastric irritation with sense of fulness, nausea, and diarrhœa (Van der Espi, Lancet, ii., 1880).

Therapeutical Action.-Externul.-An infusion has been used to inflamed eyes and to ulcers.

Therapeutical Action. - Internal.-Dyspepsia.The drug is often valuable in the dyspepsia of nervous delicate subjects, especially when some febrile excitement is present, or after convalescence from acute disease, or in phthisis : it lessens irritation, and improves appetite and digestive power.

For nerrous cough from reflex irritation, for whoopingcough, and bronchial and laryngeal irritation, it is also good.

Cardiac Disorder.-Palpitation, whether purely "nervous," 
or connected with dyspepsia or anæmia, is often much relieved by prunus, which has the further advantage of not causing any toxic effects in medicinal doses, even if continued for some time. Dr. Clifford Allbutt speaks highly of it in several forms of organic disease, and reports many interesting cases of mitral reflux and of dilatation with urgent dyspnœa and bronchial catarrh, also of cardiac debility with anæmia, etc., benefited directly by prunus. $\mathrm{He}$ compares its action to that of digitalis, but it is less powerful: on the other hand, it has the advantage of not causing vomiting or serious depression (Med. Times, 1867).

Preparations and Dose.-Pulvis: dose, 15 to $40 \mathrm{gr}$.

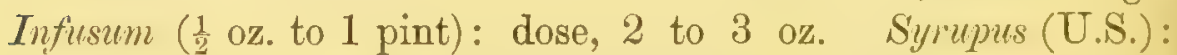
dose, 1 fl. dr. Tinctur ( 1 in 6) : dose, 20 to $60 \mathrm{~min}$. An extract prepared by evaporating the tincture is known as Prunin or Cerasin: dose, 1 to $5 \mathrm{gx}$.

It is difficult to get the sedative elements properly represented in ordinary modes of preparation (Moon, Pharm. Journ., ii., 1881). 


\section{HAMAMELACEA.}

\section{HAMA MELIS VIRGINICA. \\ (Witch-Hazel - Winterbloom.) \\ (Not Officinal.)}

DESCRIPTION.-A shrub, under 10 feet in height, growing in woods in North America : it has crooked branches, with smooth or fissured bark, brown or grey, according to age; alternate leaves, oval-toothed, and about 4 inches long; flowers in axillary clusters of three or four, with a four-cleft calyx and four linear yellowish petals, which bloom late in November; and at the same time, as well as after, comes the nut-like capsule containing two black oily seeds. The leaves only are officinal in the last U.S.P.: they somewhat resemble those of the common hazel, and are fragrant, bitter, and astringent. The plant derives part of its name from its supposed power as a divining-rod in the discovery of water, gold, etc.

Active Ingredients.-According to H. K. Bowman, the bark contains upwards of 8 per cent. of tannin, but it is probable that this is not the only active principle. Bartlett, analysing "Pond's extract," which is commonly taken to represent this drug, finds it contain, besides some gallic acid, a peculiar astringent principle, probably volatile at a low temperature, a diffusive volatile oil, and an oleo-resin, "like terebene"; he finds a general resemblance to Arnica montana (Lancet, ii., 1879). No alkaloid has been found in the leaves or bark (Guy). 
PHysiological Action.-Hector Guy reports, after observing the effects of very large doses in frogs and rabbits, that no signs of poisoning were produced: neither is the plant poisonous to man, nor does it show any special physiological action on the vascular system (Thèse de Paris, 1884). Headache and, in some cases, depression have, however, been attributed to full doses.

SYNERGISTS.-Vegetable astringents. Bartholow classes it with agents increasing waste.

ANTAgonists.-There is little known on this subject. Hamamelis is said to antagonize the uterine action of gossypium.

Therapeutical Action. - Stillé remarks that "the accounts published of the remedial virtues of witch-hazel seem as hard to accept as the popular faith in it as an infallible divining-rod" ; in certain conditions of hcemorrhage, howevernotably passive hæmorrhage - there is a good deal of concurrent testimony to its value. Mr. R. Smith speaks of its value in menorrhagia, phlegmasia alba, and hæmoptysis (Lancet, ii., 1879); in the first, occurring in girls, Mr. Gumning found $\frac{1}{2}$-drachm doses answer well (B. M.J., ii., 1884); Dr. Preston advised it in epistaxis, and Dr. Hall in dysentery; and others have given it in threatened miscarriage.

Dr. Ringer, whilst obtaining no benefit in a case of hæmophilia, has seen it of use in ordinary hremorrhage, and especially in hæmaturia, and has "found it singularly successful and prompt in arresting the chronic hrmorrhage of piles"; others in bleeding wounds. In most cases of hrmorrhoids it is desirable to employ the remedy also locally. Mr. Netherclift speaks in high terms of a much-advertised preparation of the drug, termed hazelin, as a local application to irritable and inflamed piles; bathing with it is practised three or four times daily. and lint steeped in it applied during the interval; all urgent symptoms have been subdued in twelve to twenty-four hours (B. M. J., i., 1881). He has found it good also in chronic varicose and eczematous ulcers, and it is said to have proved 
useful in varix; but $I$ have not been able to verify this. The decoction has been used as a mouth and eye-wash, and as a lotion in various forms of painful growth, in which cases it acts probably like other astringents.

PREPARATIONS AND DOSE.-Decoctum ( 1 oz. bark to 1 pint water): dose, 1 to $2 \mathrm{oz}$. Tinctura ( 1 of the bark in 10 of proof spirit): dose, 5 to $20 \mathrm{~min}$. Injectio: $1 \mathrm{dr}$. tincture to $3 \mathrm{oz}$. cold water. Unguentum: $1 \mathrm{dr}$. tincture in $10 \mathrm{dr}$. ointment. Hamamelin (a powdered extract): dose, $\frac{1}{2}$ to 2 gr. in pill or suppository. Plasters are also prepared with it. 


\section{MYRTACEAE.}

\section{THE MYRTLE FAMILY.}

Av extensive family of ligneous, evergreen, and chiefly arborescent exogens, the species of which are mostly tropical or sub-tropical. The leaves are simple and undivided, almost invariably opposite, exstipulate, entire, and thickly occupied by translucent oil-cysts. Except when the lamina is narrow, they present also a remarkable and characteristic intra-marginal vein. The flowers are regular, usually pentamerous and icosandrous; the ovary is inferior, many-celled and many-seeded; the fruit is either a capsule or a berry. Not one species is deleterious; and, as a whole, the order is noted for its aromatic qualities.

\section{Pharmaceutical Species.}

Caryophyllus aromaticus... . . . The clove.

Pimenta vulgaris . . . . . . . Pimento.

Melalenca cajeputi .. . . . . . . Cajeput oil.

Eucalyptus globulus $\quad \ldots \quad \ldots \quad \ldots$ Blue gum tree.

\section{CARYOPHYLLUS AROMATICUS.}

(The Clove.)

DESCRIPTION.-A tree 20 or 30 feet in height, with a elose pyramidal head. The ovate-lanceolate leaves are about 4 inches in length; the white flowers are borne in small terminal panicles; the ovary, when ripe, becomes a violetcoloured berry-mother-cloves. The cloves of commerce are the unexpanded flower-buds, consisting of the long, inferior, somewhat tetragonal ovary, the four small lobes of the adherent 
calyx, and the incurved corolla upon the summit. The odour of eloves is strongly aromatic; the taste is hot and acrid, but pleasant, and not so fiery as pepper or ginger.

Active IngREDIENTS. - These, according to Trommsdorf, consist chiefly of volatile oil, tannin, a gum-resin, extractive, and lignin. Upon analysis, the volatile oil resolves into a hydrocarbon, which is termed light oil of cloves, $\mathrm{C}_{15} \mathrm{H}_{24}$ (F. \& H.), in which eugenic acid, $\mathrm{C}_{10} \mathrm{H}_{12} \mathrm{O}_{2}$, is dissolved; caryophyllin, $\mathrm{C}_{20} \mathrm{H}_{32} \mathrm{O}$, a substance isomeric with camphor; and, thirdly, a body called eugenin, probably isomeric with eugenic acid; it contains also some salicylic acid, probably in ethereal form (Scheuch). The caryophyllin presents itself in crystals of satiny brilliance, destitute of taste and smell, fusible, volatile, and soluble in alcohol, but insoluble in water. The oil, though clear and colourless when fresh, gradually acquires, by keeping, a dark and reddish-brown hue ; its taste is aromatic and somewhat acrid; the odour is that of the spice, and very strong. It is soluble in alcohol, ether, and the fixed oils, and has a sp. gr. of 1.034 to 1.060 , being one of the few oils that are heavier than water. The infusion and oil of cloves are reddened by nitric acid, and turned blue by ferric chloridecolours similar to those given by morphia (R. W. Smith).

Physiological Action.-Used as a spice, and in moderation, cloves stimulate the digestive organs, and have been credited with emmenagogue and aphrodisiac powers: taken in excess, or too continuously, they exhaust the susceptibility of the stomach, and induce loss of appetite and constipation. The oil, in large doses and undiluted, acts as a powerful irritant; but in smaller quantities, as a diffusible stimulant.

Therapeutical Action.-Extemal.-As a local excitant, cloves have been recommended to be chewer in particular cases of paralysis of the tongue; and the essential oil is at popular remedy for toothache, being applied on cotton-wool to carious cavities in the teeth.

Therapeutical Action.-Interual.-Cloves are generally considered to be the most stimulating of the aromatics. 
They are employed more as a condiment than as a medicine, and often to season food of a somewhat fatty, indigestible character. The infusion, being a warm and grateful stomachic, is advantageously given to relieve the sense of coldness in the stomach which attends certain forms of dyspepsia, especially in cases of the abuse of ardent spirits, of chronic gout, and of flatulent colic; also for the relief of nausea and vomiting. The infusion, or any other preparation of cloves, is seldom prescribed alone unless as a carminative. It is valuable for the purpose of imparting a pleasant flavour to medicines of a distasteful sharacter, and for correcting the irritant properties of drastics. By diffusing the essential oil in water, with some mucilage, an agreeable draught may be prepared.

Cloves are employed, also, in the preparation of an aromatic syrup, which is afterwards coloured with cochineal.

The oil of cloves is largely used in the preparation of specimens for microscopical examination, on account of its power of clarifying them.

PReParations and Dose.-Pulvis: dose, 5 to $10 \mathrm{gr}$. Infusum: dose, 1 to $4 \mathrm{oz}$. Oleum : dose, 1 to 4 min. (Cloves are contained in infusum aurantii co., mistura ferri aromat., and vinum opii; the oil in confectio scammonii, pilula colocynthidis co., and pilula colocynthidis et hyoscyami. Preparations containing cloves are chemically incompatible with lime, iron, strong acids, and gelatine.)

\section{PIMENTA VULGARIS.}

(Pimento.)

DESCRIPTION.-A handsome tree of the West Indies and the warm parts of South America. The oval or oblonglanceolate leaves attain the length of 4 inches; the flowers, which are tetramerous, yellowish-white, and rather small, grow in terminal and branching panicles, and are followed by purplish-black berries the size of peas: these, when dried, are the allspice or pimento of commerce. 
ACTIVE INGREDIENTS. - The virtues of pimento mainly reside in the pericarp and the seeds, from which are obtained two essential oils, one volatile and the other fixed. The former of these (Oleum pimentæ) constitutes about 6 per cent. of the dried berries, and is considered to possess all the active properties, as it has the chemical characters, of oil of cloves; it is separated by distillation, and on it mainly depend the strong fragrance and the warm and aromatic flavour. The fixed oil is greenish, and has an acrid burning taste, and a rancid but somewhat clove-like odour.

Dragendorff (1871) found a small quantity of an alkaloid having the odour of conia (F. \& H.).

Physiological Action.-Pimento is an aromatic stimulant, stomachic, and carminative, and it holds an intermediate place between pepper and eloves: externally employed, it is a rubefacient.

Therapeutical Action.-Pimento is used as a condiment by persons suffering from exhaustion of the digestive system, and especially by those living in tropical regions: it relieves nausea, flatulence, and pain in the bowels; it increases the effects of vegetable tonies, and prevents the griping of purgatives; and is also used to cover the taste of nauseous medicines.

Preparations and Dose.-Aqua: dose, 1 to $2 \mathrm{fl}$. oz. Oleum: dose, 1 to 3 min. on sugar, in pill or emulsion. Pulvis: dose, 5 to $20 \mathrm{gr}$. (It is contained in syrupus rhamni.)

\section{MELALEUCA CAJEPUTI.}

(CAJeput On..)

DESCRIPTION.-A small, slender, erect tree. The leaves, unlike those of most other Myrtaceæ, are alternate; in figure they resemble those of the common willow, being lanceolate, and 3 to 5 inches in length; the flowers are small and whitish, 
and crowded into dense cylindrical spikes at the ends of the branchlets; the stamens are pentadelphous, and much longer than the petals; the fruit is capsular and many-seeded.

ACTIVE INGREDIENTs.-Cajeput oil is fluid and transparent, light green in colour, and of strong but agreeable odour, and warm, aromatic, camphoraceous taste, which is soon followed by a sense of coolness in the mouth. The sp. gr. is 0.914 to 0.927 . It is wholly soluble in alcohol, and boils at $343^{\circ} \mathrm{F}$. Distilled with water, there first passes over a colourless oil, which is the hydrate of eajeputin $\left(\mathrm{C}_{10} \mathrm{H}_{16} \mathrm{H}_{2} \mathrm{O}\right)$, and constitutes about two-thirds of the crude oil. The green tint of the oil is due to copper, which is probably added intentionally. Some species of melaleuca certainly yield a green oil by distillation (Guibourt), but this hue is transient.

Physiological Action.-When swallowed, cajeput oil causes a sense of warmth in the stomach, accelerates the pulse, and increases the perspiration and urine.

Therapeutical Action.-External. - Muscular rheumatism, chilblains, and nervous headaches are often quickly removed by the oil being well rubbed into the parts twice a day; when thus applied externally it is a strong stimulating rubefacient: it is employed on cotton-wool for toothache, and, mired with olive oil, it is applied to the auditory meatus for earache and deafness.

Therapeutical Action. - Internal. - According to Ainslie, "cajeput oil is a highly diffusible stimulant, antispasmodic and diaphoretic, and may be efficaciously given in dropsy, chronic rheumatism, palsy, hysteria, dysmenorrhœea, and flatulent colic" (Mat. Med. Indica, i., 261). In India it is used with much success in choleraic affections; also in nervous vomiting, nervous dysphagia, and for the destruction of ascarides. Five minims of the oil added to a simple soap enema (half a pint), relieves flatulence, and is preferable to giving it by the mouth. 
PREPARATIONS AND Dose.-Oleum cajeputi: dose, 3 to $10 \mathrm{~min}$. on sugar. (It is contained in linumentum crotonis.) Spiritus cajeputi ( 1 in 50): dose, $\frac{1}{2}$ to $1 \mathrm{dr}$.

ADULTERATION.-Turpentine, sometimes.

\section{EUCALYPTUS GLOBULUS.}

(Blue Gum Tree.)

(Not Officinal.)

DESCRIPTION.-A noble tree of rapid growth, attaining a height of 200 or 300 feet, and a diameter of 15 feet; indigenous to Australia and Tasmania, but now flourishing in many parts of the temperate zone. The whitish buds are shaped like a top, the upper part being formed by an operculum of the petals; the flowers, either solitary or in small clusters, are hermaphrodite, with numerous stamens, a short style, and a four- or five-celled ovary. The leaves in young plants are opposite, ovate with prolonged base, pea-green in colour; in older trees they are alternate, lanceolate or sickle-shaped, 6 to 12 inches long, thick and coriaceous, dark-green in colour, and dotted with oil-glands: their peculiar glaucous hue is said to depend upon a film of fatty matter; they have a strong aromatic odour and taste, the latter being followed by a cool sensation. The leaves and flowers are the parts chiefly used in medicine. There are several different species.

ACTIVE INGREDIENTS.-Tannin, cerylic alcohol (?), a fatty acid, salts of potash, three varieties of resin, and, probably the most important, a volatile, oil. According to Cloez (one of the earliest observers-1870), the oil is chiefly composed of eucalyptol; according to Faust and Homeyer, it contains terpene, $\mathrm{C}_{10} \mathrm{H}_{16}$ (boiling point $320^{\circ} \mathrm{F}$.), cymol (cymene), $\mathrm{C}_{10} \mathrm{H}_{14}$, and an oxygenated compound, $\mathrm{C}_{10} \mathrm{H}_{10} \mathrm{O}$, which they name encalyptol; 
they consider that of Cloez to be a mixture of terpene and cymol. Eucalyptol has an agreeable odour, like camphor; its taste is aromatic, bitter, and somewhat acrid.

The original oil of Bosisto is said to be obtained from E. amygdalinus (Pharm. Journ., i., 1884).

AbSORPTION AND Elimination. - Absorption takes place by all the tissues. Elimination occurs mainly through the bronchial mucous membrane and salivary glands; partly also by the skin, and probably by the kidneys.

Physiological Action.-External.-When applied to a raw surface, the oil usually causes burning pain; not so when placed on the healthy skin; Schultze states that it is painless on wounds.

Hypodermic injections of a certain strength ( 1 part in 4 of olive oil) have caused much local irritation, which does not follow, however, if more diluted solutions be used.

The antiseptic powers of eucalyptus are mainly due to the oil. Buchholz states that the latter is three times as potent as carbolic acid in destroying bacteria (Centralbl. f. Chirurg., 1880); this is corroborated by Girou, who estimates its antiseptic power as 1 in 666, that of carbolic acid being 1 in 200 (Rev. des Sci. Méd., t. xxii., 1883).

Like quinine, the oil of eucalyptus restrains the amœboid movement of white blood-corpuscles.

Physiological Action.-Intemal.-Some discrepancy exists as to the exact action of the drug.

On the Digestive System, in man, the oil in 15 to 20 minim doses, continued for a few days, acts as a stimulant, causing a greater flow of saliva and increase of appetite; then as an irritant, producing eructations tasting of the drug, burning sensation in the stomach and rectum, tenesmus, sometimes purging (the stools smelling of the oil) and excessive thirst: vomiting has also been observed.

Circulatory System.-The circulation, especially in the capillaries, is commonly stimulated, and the pulse is quickened. Mosler says that moderate doses lover the action of the heart 
and the blood-pressure, but that large doses cause pyrexia. Other observers state that the temperature (in animals) is lowered by the oil.

Nervous System.-Moderate doses, according to Guimbert, stimulate this system, a sense of lightness, activity, and mental clearness being induced; large doses produce headache, some prostration, and feverishness, and, according to Mosler, sleepiness (Bull. de Thérap., i., 1882).

Cutaneous System. - The perspiration is commonly increased, and herpetic eruptions are sometimes seen under the use of the drug.

Urinary System.-Guimbert states that eucalyptol greatly increases the elimination of urea, but I know of no aecurate confirmation of this. According to Stillé, the odour of the drug is not imparted to the urine except by large doses; but Bartholow says that "after some days' administration the urine becomes most strongly odorous by the presence of eucalyptol." I have myself observed this after giving the tincture for three or four days; and concurrently, a feeling of slight intoxication, with headache, flushed face, vertigo, and dimness of sight, was not uncommon.

SYNERGISTS.-Hydrastis, cinchona, camphor, turpentine, essential oils, and aromatic bitters.

ANTAgonists. - Alkalies, mineral acids, and metallic salts are said to be incompatible chemically.

The depressing effects of toxic doses are relieved by tea or coffee.

Therapeutical ACTion.-Extemal.-As bearing on its influence over lower forms of animal and vegetable life, Garden (U.S.) states that a plant of eucalyptus in a room will keep away mosquitoes, and some French writers assert that its growth in vineyards and the inoculation of the vines with a few drops of essence will prevent phylloxera.

As an external application, preparations of the plant have been found of service in all cases where an antiseptic (and astring'ent) is required.

Thus, in Affections of the Mouth and Throat (stomatitis, 
tonsillitis, even cancer of the tongue and gangrene), a decoction of the leaves is an excellent gargle.

The decoction, or a lotion made with the tincture, or an ointment, are good applications to unhealthy wounds and surfaces, as well as in chronic ulcer; they clean the surface, setting up healthy granulation and cicatrization.

In Affections of Mucous Membranes and of the GenitoUrinary Organs, e.g. in ozæna and in gonorrhoea, eucalyptus is raluable as an injection. Dr. Curries (U.S.) says that vaginal plugs soaked in equal parts of glycerine and eucalyptol greatly relieve the pain and discharge in many forms of uterine catarrh, displacement, and other disorders (Amer. Journ., Oct., 1882). Dr. Sloan (Glasgow) strongly recommends pessaries of a similar kind for footid uterine discharges after parturition (Lancet, ii., 1882); and I am quite satisfied that warm injections containing 2 drachms to $\frac{1}{2}$ ounce of tincture in the pint are very serviceable in such cases: this treatment may also be used for the discharge of epithelioma of the cervix.

In Antiseptic Surgery, Schultz, Siegen, and others have advocated its adoption, and Sir Joseph Lister has utilized it to some extent in this direction. A eucalyptus gauze may be prepared with 3 parts of the oil, 15 of alcohol, and 150 of water; this will absorb discharges for several days without becoming offensive (Lancet, ii., 1880). The oil has been said to preserve blood from decomposition for five months. Air impregnated with the vapour by a special apparatus forms a disinfectant medium for operations, possessing many advantages over the carbolic spray; but it has not been largely tested as yet (B. M. J., ii., 1882) : Mr. Robson's report, founded on about twelve cases, is very favourable.

The steam arising from a fresh hot infusion of the leares seems to have proved effective in some cases of Diphtheria in Australia (J. M. Gibbes, Lancet, i., 1883): Mosler also found inhalations of the oil (10 to 60 minims) mixed with spirits of wine highly useful (Med. Times, ii., 1879).

In Scarlet Fever, with putrid throat and most offensive odour, I have attributed markedly beneficial effects to this eucalyptus treatment. 
Therapeutical ACtion.-Internal.-Malarial Fevers, etc.-It is in these disorders that eucalyptus has been specially praised by many observers, and there are undoubted instances of planting of the trees rendering a malarious neighbourhood much healthier. Prof. Bentley instances tracts of country at the Cape and at Cuba, etc., and adopts a suggestion that the benefit is partly due to the great power possessed by the extensive roots of absorbing water, more than ten times their weight being taken up in twenty-four hours; this acts like subsoil drainage: partly, too, he thinks, the leaves act antiseptically by their odorous emanations (Med. Times, i., 1874). $\mathrm{He}$ refers to a similar result from the draining of the soil by the roots of other rapidly growing plants, such as the sunflower in India. Parts in the neighbourhood of Rome, such as the Tre Fontani, are commonly considered much healthier since the trees were planted.

The drug has also been largely given internally for ague, but there are contradictory statements as to its value. This action is said to have long been recognized by the aborigines of Australia, and to have become known only by accident to Europeans through the cure of a number of severe cases of the disease in a French ship's crew. Physicians in South America reported very favourably of it. Keller (Austria) used it in 432 cases, of which about 70 per cent., including many of obstinate character, yielded to the remedy; he classified his cases according to the type of disease, and found that of tertian ague, 75.5 per cent. were cured; of quartan, 70 per cent.; of quotidian, 67 to 89 per cent.; moreover, the average duration of the disease was nine and a-half days, whereas with quinine it is twelve and a-half days. $\mathrm{He}_{e}$ used a tincture, prepared by macerating the leaves in alcohol for three months -10 pounds of leaves yielding 25 quarts of tincture, of which he gave 2 trachms three times daily.

In 1876, Ur. Curnow reported two striking cases occurring on the Dreadnought: both men had had tertian for several weeks, with regularly recurrent attacks lasting many hours, the temperature rising to $104^{\circ}$ and $105^{\circ} \mathrm{F}$; each was ordered 1 drachm of tincture thrice daily, before the beginning of a paroxysm; in one case there was no further attack 
from that time, in the other the temperature never rose to more than $100^{\circ} \mathrm{F}$, and on doubling the dose recovery ensued (Lancet).

On the other hand it may be stated that Burdel after observing 123 cases, in a malarious province of France, judged the remedy to have "little or no curative power"; only eleven were cured in hospital without relapse (Bull. de Thérap., t. Lxxxiv., 1885). Fichter (Basle) also had negative results, and Dr. H. P. Roberts (Bombay) has related many instances in which no effect followed eucalyptus, and yet the attacks were cut short at once by quinine (Practitioner, v., 20).

It would almost seem, from the varied results obtained, as if different species of the plant were used. Effinger indeed has suggested that Eucalyptus latifolius does not succeed, while E. longifolius does; moreover, the essential oil is said to be absent from the leaves of plants grown in France and Corsica (S. Martin, Bull. de Thérap., 1883), and preparations made from dried leaves are said to be less efficacious than those made from fresh. Sometimes advantage has been obtained by combining quinine with eucalyptus (Practitioner, $\nabla ., 20$ ).

In Facial Neuralgia of periodic character I have frequently prescribed the tincture and extract, and after a few doses the pain disappeared. The local application of the oil on lint to decayed teeth is said to relieve odontalgia.

In Septicæmia the drug is said to be valuable; and one puerperal case reported by Dr. Sloan recovered from an apparently hopeless condition under hypodermic injections ( 1 in 4 of olive oil); much local irritation was produced.

At the climacteric period, Bartholow recommends it as useful for flatulence, palpitation, and flushing.

In Catarrh of Mucous Membranes, after the acute stage is passed, and whether gastric, bronchial, or vesical, benefit has been observed from the tincture given internally: bronchorrhoea and chronic discharges from the bladder are special indications for its use.

In Chronic Ulcer of the Stomach it has frequently proved of service; the tincture, given in drachm doses thrice daily, checks the hromorrhage and vomiting, and improves the general condition of the patient. 
PREPARATIONS AND DOSE.-Eucalyptus gau: (v. p. 300). Tinctura eucalypti (1 part of leaves to 4 of proof spirit): dose, 15 to $120 \mathrm{~min}$. Unguentum (1 part of oil to 2 each of vaselin and paraffin). This is often combined with iodoform, the odour of the latter being lessened. Iodoform and eucalyptus bongies are also made: iodoform $5 \mathrm{gr}$. , eucalyptus oil 10 min., cocoa butter 35 gr. Pessus (v. p. 300).

Eucalyptus rostrata and other species furnish a "red gum," which resembles kino, and is highly astringent. It is used mainly as a local styptic and astringent in lotion, extract, syrup, insufflation, and lozenge. 


\section{GRANATACEA}

\section{THE POMEGRANATE FAMILY.}

THis order includes the single genus Punica, referred by some to the Myrtaceæ, and by others placed near the Lythraceæ, but in certain respects so exceptional as to be better considered by itself. The only species of importance is the following :-

\section{PUNICA GRANATUM.}

\section{(Pomegranate.)}

DESCRIPTION.-A native of Persia and other Eastern countries. The trunk rises to the height of 20 feet; the numerous branches are well clothed with opposite lanceolate leaves, 2 to 3 inches long, entire, dotless, bright green, and deciduous. The flowers are usually solitary, scarlet in every part, and composed of an inferior ovary, surmounted by five large and fleshy lobes, interior to which are five petals, and an indefinite number of perigynous stamens. When ripe the fruit resembles an apple, crowned with the persistent calyx lobes, which usually become erect. Internally, it is divided into several irregular compartments, crowded with small bags of rose-coloured juice, of which each contains a seed the size of a grain of wheat. The rind of the fruit is in curved, irregular pieces, rough externally, brownish-red, lighter within, and showing spaces depressed by the seed. Portions of the calyx commonly adhere to some of the pieces; the fracture is short, taste astringent, odour little or none.

The bark of the root is also in curved pieces, 2 to 4 inches long, thinner than the above ( $\frac{1}{2}$ line), yellowish-grey externally, 
and ridged, internally smooth, with fine striæ, and having fragments of whitish wood attached.

ACTIVE INGREDIENTS.-Not only the root bark, as formerly thought, but also that of the trunk and of the mediumsized branches, contain upwards of 20 per cent. of tannin, also punico-tannic acid, $\mathrm{C}_{20} \mathrm{H}_{16} \mathrm{O}_{13}$-an acrid principle called punicin, resin, and mannite (which includes probably "granatin "), together with extractive and mucilaginous matters. More important constituents in the form of alkaloids were isolated by Tanret (1878); and, as special names appropriate to the plant were already given to other ingredients, he named these "Pelletierines" (after the chemist who discovered quinine, etc.).

He obtained them by mixing the bark with milk of lime, displacing with water, and exhausting with chloroform, and distinguished them by the somewhat different reactions with bicarbonate of soda into-(1) pelletierin $\left(\mathrm{C}_{8} \mathrm{H}_{13} \mathrm{NO}\right)$, which is oily, colourless unless exposed to air, aromatic, dextro-gyrate, and very soluble in water, alcohol, ether, and chloroform; it unites with acids to form crystalline salts, and is contained in the proportion of about four parts to 1,000 of the bark; (2) a crystalline alkaloid, "iso-pelletierin," without action on polarized light; (3) a liquid, lævo-gyrate, "pseudo-pelletierin"; (4) a liquid, "methyl-pelletierin," without rotary action.

\section{PHysiological ACTION.-Digestive System.-A strong} decoction of the bark acts as an irritant, causing nausea, flatulence, vomiting, and purging, the stools being generally of a light-yellow colour.

Nervous System.-The symptoms of its action upon this system are such as giddiness, dimness of vision, numbuess of limbs, and sometimes, it is said, convulsion. DujardinBeanmetz, experimenting both on animals and men with the allinloidls above described, found that they produced similar toxic phenomena, but in different degree-pelletierin, for instance, being much stronger than methyl-pelletierin. He discovered some resemblance to the nction of curare in the effects on the terminals of motor nerves, their function being destroyed, whilst muscular irritability and sensation remained. In frogs, couvul- 
sions commonly precede the loss of voluntary power of movement; in rabbits they occur shortly before death; in man, heaviness of head and vertigo are experienced, with congestion of the eyes and contraction of pupil, -7 grains injected under the skin caused these symptoms quickly and severely for several hours; sometimes a temporary loss of power in the extremities is experienced. The head-symptoms, Dujardin-Beaumetz explained by vaso-motor paresis causing dilatation of ressels at the back of the eye, and probably within the cerebrum; ophthalmoscopic examination confirms this (Bull. de Thérap., t. xeviii., 1880, and Leçons de Clinique, t. i., 1883).

\section{Therapeutical Action. - Intestinal Worms.-As} a remedy for these parasites, pomegranate bark has obtained great repute, dating from the time of Avicenna; and is still highly thought of in France, Portugal, and most European countries. For trenia it is specially preferred, a decoction being made with about 2 ounces of fresh root-bark to $1 \frac{1}{2}$ pint of water, boiled till the fluid is reduced by one-half, and when cold a third part is taken at intervals of thirty minutes, for three doses. The "decoctum granati radicis" (B.P.) is somewhat weaker than this, but Niemeyer, noting that such decoctions commonly gave rise to vomiting, and generally to severe colic, strongly advised the use of a " simple macerntion" or infusion, at all events before employing the decoction. It may be made with the proportions mentioned above, and he has found it succeed as a rule; in one case it brought away three trenire at one time, with the heads. In my experience the decoction has answered well in fourteen cases of many years' duration, that had been rebellious to kousso, male feru, etc., but it is not in common use in this country-possibly having been found uncertain in action, from the bark being too dry, and other accidental causes. According to the observations of Tanret, Dujardin-Beaumetz, and others, the uncertainty should now be greatly lessened by the employment of the allialoids-the pelletierins already described. Bérenger-Feraud has shown that pelletierin and iso-pelletierin are the active tronicides (Bull. de Thérnp., t. xcviii., 1879), and the sulphates of these salts, especially when combined with tannin, have proved very 
efficient; the ordinary dose by the mouth being about 4 grains each of the two latter in solution with 7 or 8 grains of tannin. This remedy acts probably by paralysing the suckers of the worm, and is better given alone and on an empty stomach; a good draught of water should be taken ten minutes afterwards, and a purgative, such as castor oil or senna, administered in about three-quarters of an hour; four hours later the worm is generally voided in a ball or knotted, and "complete in nine cases out of ten." If not entire, then it is better to wait two or three months before repeating the dose. The day and the evening before taking it, spare diet and generally a purgative are to be enjoined.

The author concludes that pelletierin is one of the most useful trenifuges we have (Bull. Gén., Ang., 1882).

Other Uses.-Pomegranate bark is strongly recommended as the basis of a gargle for relaxed gums and throat. It is employed with advantage in chronic diarrhœa; also in menorrhagia, and in prolapsus uteri and ani: it has been used in paralysis and Menière's disease. In phthisis pulmonalis for the profuse perspirations, and in colliquative diarrhœa, pomegranate rind is prescribed with considerable benefit.

In fever, with its attendant thirst, the juice of the pomegranate, especially if mixed with sugar or honey, is particularly refreshing; many consider it preferable to that of the orange.

PREPARATIONS AND DOSE.-Decoctum granati redicis: dose, 1 to $2 \mathrm{fl}$. oz. or more. Pelletierice sulphlats: dose, 4 to $8 \mathrm{gr}$. by the mouth (or 2 to $4 \mathrm{gr}$. hypodermically); for children twelve years old-dose, 2 to 4 gr.; and for infants--dose, $\frac{1}{2} \mathrm{gr}$. Pelletievire tumna: : dose, $8 \mathrm{gr}$. The dose of pelletierin should be followed in about an hour by a full dose of castor oil or senna.

Adulterations. - Sometimes boxwood, which does not contain tannin, and is therefore unaffected by ferric salts; also barberry, which is bright-yellow on fracture. 


\section{CUCURBITACEAE.}

\section{THE CUCUMBER FAMILY.}

'Tre Cucurbits are herbaceous exogens, natives chiefly of tropical countries. The stems, mostly annual, are weak and succulent, through extreme rapidity of growth; ordinarily, they attain a length of many yards, and climb by means of powerful tendrils: the leaves are very large, alternate, usually lobed or palmate, and rough or scabrous: the flowers are monopetalous, regularly pentamerous, and unisexual, the males having sinuous anthers, and the females an inferior three-celled ovary, which ripens into a succulent and many-seeded fruit, often of prodigious size. A few of the species yield edible fruits, but the order, as a whole, has deleterious properties.

\section{Phamaceutical Species.}

Ecbalium agreste .. $\quad \ldots \quad \ldots \quad . \quad$ Elaterium.

Citrullus colocynthis $\quad . . \quad \ldots \quad$. Colocynth.

Bryonia dioica .. . . . . . . . . Bryony.

\section{ECBALIUM AGRESTE.}

(Eiaterium.)

DESCRIPTION.-The plant which furnishes the celebrated drug of this name is indigenous to the south of Europe, and is now cultivated at Mitcham, in Surrey.

It is an axinual, with long, much-branched, trailing stems, and cordate, crenately toothed leaves of grey-green colour; no tendrils: the flowers are yellowish, monocious, and 
axillary, and are succeeded by oval fruits the size of a walnut, greyish-green, and very hispid. When perfectly ripe, the fruit separates violently from the peduncle, the removal of which leaves a hole in the pericarp, through which the pulp and seeds are shot out with great force, owing to the contraction of the pericarpic walls; hence it has been called "squirting cucumber."

ACTIVE INGREDIENTS. - According to some modern writers, every portion of the plant is cathartic; and it is expressly stated that the root may be employed in substance, and that an infusion of it is equally efficacious. It appears, however, from the experiments of Dr. Clutterbuck, that the properties of the herbaceous portions and of the pericarp of the fruit are of little importance, the only part really valuable as a medicine being the juicy pulp which invests the seeds. This juice, as it issues from the natural orifice in the fruit, without pressure, is limpid and colourless. After standing for a short time, it becomes turbid; and in the course of a few hours deposits a greenish sediment, which, being dried, becomes on exposure to light a yellowish-white or grey powder, slightly tinged with green, very light and pulverulent, and constituting the genuine "elaterium" of the materia medica: it is commonly met with in thin flat or curved brittle pieces, marked by the fibres of linen on which it has been dried. Much of the elaterium of commerce is not pure, being prepared by strong pressure of the fruit, and consequently containing the deposit of the ordinary juices, as well as the active principle. This compound elaterium is dark green, approaching black, and is in substance compact and heavy, breaking with a resinous ard shining fracture. The best British elaterium contains 26 pe cent. of elaterin ; the worst about 15 per cent. The French elaterium is considerably inferior, the proportion being only 5 or 6 per cent.

The active principle is elaterin $\left(\mathrm{C}_{20} \mathrm{H}_{23} \mathrm{O}_{5}\right)$, a crystalline substance, which forms colourless, shining, hexagonal tables, has a pungent, bitter taste, and a neutral reaction, and melts at $200^{\circ} \mathrm{C}$. into a yellow fluid, which on cooling forms amorphous yellow masses. According to Buchheim, "it is the anhydride of 
elateric acid, and is changed into the latter by heat; hence the importance of avoiding heat during its preparation." It is not soluble in water or glycerine, but in boiling (not cold) alcohol, and in chloroform. Concentrated sulphuric acid dissolves it dark-red; the addition of water gives a brown precipitate. A few drops of melted carbolic acid followed by sulphuric acid produces a crimson colour changing to orange and scarlet (Lindo).

Physiologigal Action.-The active properties of the drug are fully represented by elaterin, which has been made the subject of numerous experiments; the earliest being those undertaken by Morris (the discoverer) and Golding Bird. Giren even in very small doses, either to animals or man, it produces characteristic violent drastic purging, and 3 grains of extract have proved fatal. Its action on the lower animals is not very certain, and it would seem that the drastic effect only takes place when the drug comes in direct contact with bile; for Köhler experimented on animals by placing powdered elaterin in a portion of intestine which was included between two ligatures (the contents having first been squeezed out of this part of the bowel), and found no effects, whether local or remote. He also tied the ductus choledochus, and then found that the aqueous solution of elaterium, administered by the stomach, produced no purging, but only some remote poisonous effects (Virchow's Archiv, Bd. 1.). Stillé remarks that, "however the drug may be given to dogs and rabbits, it does not vomit or purge them, but destroys with tetanoid phenomena"; on the other hand, he gives evidence to show that its external application will cause purging in man.

Digestive System.-Schroff investigated the effects of large doses upon two of his pupils, giving as much as $\frac{3}{4}$ grain. In one of these young men salivation occurred first, and was followed forty-five minutes after ndministration by nnusen, retching, and vomiting, which recurred four times in two hours, and became bilious in character: the salivation ceased when the vomiting begnn: simultaneously with this, the patient suffered from flatulent belchings, griping, abdominal pains, irritation in the throat, and cerebral torpor: six hours 
after the medicine, there occurred several very copious watery evacuations, anorexia, and depression. In the second case, a similar dose was given, and this quickly produced nausea and retching (which was a good deal relieved by a fit of sncezing): a stool occurred six and a half hours after the administration, and a second two hours later: eleven hours after the medicine, there occurred epistaxis, and a single violent vomiting, which did not recur, although there was constant nausea and a smell like that of rotten eggs: on the second day there were nine fluid stools, and on the third day there were three: the nausea and retching, with feelings of weakness and languor, continued till the fifth day (Lehrb. d. Pharm.).

Nervous System, etc.-Besides the action upon the alimentary canal, elaterin can produce toxic effects upon the nervous system; and according to the way in which it is given, the irritant or the nervous phenomena may predominate. The latter have been produced in cats by direct injection into the veins: and salivation, which was marked, was followed in one instance by restlessness, stertorous breathing, and death in twenty-two minutes. In another, when half the dose was employed, there was salivation, dyspncea, and death from passive hyperæmia and oedema of the lungs in an hour and a half. In rabbits, after subcutaneous injection, Köhler observed salivation, celema of the lungs, coma; tetanus, and death.

THERAPEUTICAL ACTION.-Elaterium, though only occasionally available, is one of the most powerful and valuable remedies in appropriate cases.

In Dropsy it acts by carrying off a large quantity of fluid through the intestines; and hence is suitable in passive dropsical accumulations which are causing mischief by their mechanical pressure. It is used in Bright's disease, when dropsy is marked or coma threatening, and when free intestinal transudation and excretion are needed. It was much employed by Sydenham and his contemporaries, but, owing to its violent effects, fell into disrepute for a time. Dr. Feminr, of Manchester, restored its credit by employing it successfully in cases of hychothorax, and Dr. Hope showed it to possess equal efficney in encline dropsy : since then elaterium has held its gromud, at least in such con- 
ditions, though many practitioners are still afraid of it, and with good reason, since it is apt to produce such severe retching and vomiting as to outweigh any benefit that might follow its aperient effect. The vomiting, however, may be to some extent prevented by combining the drug with about a grain of pulvis capsici.

In obstinate Constipation, elaterium has been proved, by Golding Bird and others, to act effectively; but there are numerous cases in which a good result cannot be looked for, and some in which a drug like elaterium might do great harm.

Gout.-A combination of elaterium and opium has been employed with advantage in this disorder.

Apoplexy.-In those cases of sudden brain-mischief where it is possible that the action of a decided purgative may produce beneficial revulsion, elaterium has been strongly recommended. It is important to remember, however, that if any good is to be produced in this way, the action must be speedy: it is therefore not desirable to give elaterium for this purpose in ordinary doses by the mouth, as its action would probably be slower than that of calomel and croton oil placed on the tongue with a little sugar. Elaterium should be given in suppository, a large dose (2 grains) being rubbed up with hard soap; or, still better, in injection, a solution being thrown up into the rectum.

Dr. Clutterbuck speaks highly of elaterium in mania, especially in the acute form; in one case the attack seemed to be warded off by free purgation with the drug (Lancet, ii., 1884).

In the "endemic bilious fever of the West Indies," Bradburn strongly recommends it in pill, $\frac{1}{4}$ to $\frac{1}{2}$ grain with $\frac{1}{2}$ grain of extract of belladonna; he found no medicine so beneficial (Lancet, ii., 1878).

PREPARATIONS AND DOSE.-Elaterium in pill, with extract of gentian, capsicum, etc.: dose, $\frac{1}{16}$ to $\frac{1}{2} \mathrm{gr}$.

Another combination is with hyoscyamus, said by Ir. G. Harley to be especially necessary when the drug is resorted to in the treatment of renal dropsy: without this safeguard it is apt to set up a persistent diarrhoca, seriously dangerous to the patient, especially if uremic symptoms lave already appeared. 
It is desirable to substitute, when practicable, elaterin for the elaterium which is subject to such serious fluctuations in quality.

As regards dose, it must be reckoned that the former is four to five times as strong as the latter: so that its proper dose, for drastic purposes, will be $\frac{1}{32}$ to $\frac{1}{8}$ gr. (according to the age and strength of the patient), repeated every four hours till decided action is produced, which will usually occur after the second dose. If necessary to maintain the action the drug should be combined with 5-gr. doses of extract of gentian, and repeated every two or three days, an interval then being allowed, as recommended by Dr. Dartwell. Nitrous ether is a convenient solvent.

Smaller quantities ( $\frac{1}{64} \mathrm{gr}$. elaterin, $\frac{1}{16} \mathrm{gr}$. elaterium) are proper to be given in such maladies as gout, unless in the exceptional instances where it is desirable to relieve not only the joint-affection, but a coincident dropsy,-when the usual doses are required.

AdULTERATIONS.-Elaterium is not liable to intentional adulteration; but owing to careless preparation, many specimens are almost worthless, containing as little as 5 per cent. of elaterin. The safest way is to procure it from a first-rate manufacturer whose standard article contains about 20 per cent.

\section{CITRULLUS COLOCYNTHIS.}

(CoLociNTH.)

DESCRIPTION.-A native of South-western Asia, Egypt, etc. The stems are hispid, the leaves many-lobed, and the flowers small, yellow, moncecious, and solitary; the ripe fruit is globular, the size of an orange, bright-yellow, and intensely bitter. The medicinal portion of the plant consists of the soft, white, porous matter which envelopes the small brown seeds.

ACTIVE INGREDIENTS.-The active properties of colocynth depend upon the presence of the glucoside colocynthin, 
$\mathrm{C}_{56} \mathrm{H}_{84} \mathrm{O}_{23}$, discovered by Herberger, and further investigated by Walz and Bastick.

It is usually seen in amorphous yellow masses, but, by careful evaporation of the alcoholic solution, may be obtained in small bundles of yellowish-white crystals. It has an excessively bitter taste, and is very soluble in cold and hot water, and in alcohol, but insoluble in ether. Its character is at once displayed if, besides applying the above tests, the solution be acidified and boiled: colocynthin is then immediately changed into sugar, and a resin, colocynthein $\left(\mathrm{C}_{44} \mathrm{H}_{64} \mathrm{O}_{13}\right)$, which is precipitated.

There is no reason to suppose that another substance, colocynthitin - a tasteless crystalline body, which Walz procured from colocynth by treatment with ether-has any share in the action of the drug.

Physiological Action.-The experiments of Schroff show that colocynthin is a very active irritant: 5 to 7 grains killed rabbits in four hours, with repeated purging, and the development of an extensive gastro-enteritis. In man, small doses quicken peristalsis and increase the intestinal secretions, causing mucous and watery stools with colic; in large doses it proves an emeto-cathartic, and has been known to canse peritonitis and death: one and a half teaspoonful of the powder proved fatal in twenty-four hours. Frictions of the abdomen with the tincture cause purging (Richter). Rutherford describes colocynth as a powerful hepatic stimulant, making the bile more watery, but increasing the amount of solid constituents (Brit. and For. Med.-Chir. Rev., i., 18r 7 ).

Solokowski states that colocynthein is capable of producing colic and diarrhoen, even when given in small doses.

THERAPEUTICAL ACTION.-The medicinal effects of colocynth alone are little referred to, since the dring is commonly given with one or several other agents. It has been found too irritant for convenient use, except with carminative and soothing remedies, or in small doses combined with other aperients: neither colocynthin nor colocynthein has been adopted in practice. 
The ancient Greek physicians, who were well acquainted with its violent effects when injudiciously administered, were accustomed to employ colocynth as a drastic purgative in dropsy, as well as in lethargic and maniacal cases.

In Habitual Constipation, combined as above mentionerl, it often operates mildly and effectively; but care must be taken that it be given at regular, and not too short, intervals. The tincture (Ger. Ph.) is a good form to use.

In obstruction, where the bowels have ceased to act for some days, but where there is no mechanical impediment, colocynth proves valuable, and may be conveniently given in enema, rubbed down with soap and water.

In torpor of the abdominal and pelvic nerves and vessels generally, colocynth often acts as a useful stimulant. Thus, in certain cases of chlorotic amenorrhoa, its rousing influence upon the reotum seems to affect the aterus and ovaries by sympathy.

In Apoplexy and Cerebral Congestion, colocynth acts favourably, upon the principle of revulsion.

In Dropsy, colocynth may prove a useful hydragogue, increasing vermicular action and secretion from the bowels, though it is inferior to elaterium, since the latter drug. evacuates much more fluid in proportion to the degree of intestinal irritation which it causes. (Wood remarks that "it should never be used in dropsy.") As a diuretic, colocynth only acts indirectly, but sometimes does so very beneficially, when employed for this disorder; it is, however, only after some persistence in its use that this effect is likely to be attrined, owing apparently to the cumulative action of colocynthin upon the kidneys when it has circulated for some time in the blood. In its general character as a purgative, colocynth most - nearly resembles aloes, and next gamboge; but it appears to act more uniformly upon the whole intestinal tract than the former, and further differs from it in being devoid of tonic qualities.

PREPARATIONS AND DOSE. - Eirturtum colorynthidis comp.: dose, 2 to $5 \mathrm{gr}$., combined with about un equal quantity of extr. hyoscyami to prevent griping. Pilule colocyuthidis comp. 
(contains also aloes and scammony): dose, 5 to $10 \mathrm{gr}$. Pil. coloc. et hyoscyami (a still milder preparation): dose, 5 to $10 \mathrm{gr}$.

The tinct. colocynthidis of the German Pharmacopœia is an important medicine, but seldom used in this country. It is composed of 8 parts of colocynth, 1 part of tincture of staranise, and 96 parts of rectified spirit: dose, 4 to 7 drops three times a day.

AdUlTERATIOns. - Colocynth pulp is not liable to adulteration by admixture of other substances, but inferior qualities are sometimes sold: these are of greyish or brownish colour, instear of pale yellowish-white.

\section{BRYONIA DIOICA.}

\section{(BRYoNy.)}

DESCRIPTION.-Wild bryony is a climbing perennial; the stems, which are renewed annually, sustaining themselves by means of tendrils, and mantling shrubs and hedges with dense, vine-like, deep-green foliage. The small greenish-white flowers are borne in axillary clusters, the female being upon distinct plants, and succeeded by globular, dull-red fruits, the size of peas. The root is a large and fleshy tuber, of disagreeable odour and nauseous taste.

In continental Europe, especially in the woods and rineyards of the southern parts, grows the Bryonia alba, so called from the decided whiteness of the root. The flowers are monøcious, and the berries black; but in other respects the two plants are much alike. The properties correspond with those of the English B. dinica. The roots are from 1 to 2 feet long, and fleshy: externally, pale-brown and wrinkled; intermally, white, with a thin bark; it is usually dried in transverse slices, which are inodorons and bitter. 
ACTIVE INGREDIENTS. - The active principle of the Bryonia dioica is probably the neutral body bryonin, which appears to be a glucoside, $\mathrm{C}_{48} \mathrm{H}_{80} \mathrm{O}_{19^{\circ}}$ It occurs, when separated, in white amorphous masses, of very bitter taste; is readily soluble in water and in alcohol; insoluble in ether. On boiling the solution with dilute acid, sugar is formed, and a resin (bryoretin) is precipitated.

PHYSiological Action.-The poisonous effects of bryony have long been known, the medicine having once been a favourite with the French physicians, who noticed that in overdoses it often produced sickness, griping, watery evacuations, and fainting, some cases ending fatally. Moderate doses are simply purgative, and may be employed with advantage, provided that care be taken to stop the medicine should irritant effects become developed, in which case opiates and cordials must be resorted to. Orfila's experiments upon animals proved that the root is a violent irritant: introduced into the stomach, it caused intense and fatal gastritis ; into the pleura, it caused pleurisy, with fibrinous effusion. The fresh plant will blister the skin on prolonged contact.

The researches of Collard de Martigny showed that bryomin, when swallowed, possesses a similar but more energetic power of inflaming the stomach, and also any wound or raw surface to which it may be applied. Further researches are needed, however, to discover whether there may not be other physiological properties in bryonin.

To insure uniformity in action, the roots should be dug up in the spring, then cut into thin slices, and dried either in the sun or in a warm room, by adopting which means the acrid principle becomes partly dissipated: the whole of this may be expelled by repeated washing with water; the fecula which remains is not unlike that of the potato, and is said to be a nutritions food.

THERAPEUTICAL ACTION.-In Epilepsy, the juice was administered in the time of Dioscorides; the upper part of the root was laid bare, and a hole scooped in it, which in a few hours filled with juice: one teaspoonful of this was 
taken once or twice daily in water. This method has been followed for the same purpose in our own day, especially when the convulsions are dependent on worms.

In Mania it was formerly much relied upon,-i.e., in days when this malady was habitually treated by free purgation: and among other authorities in its favour is found Sydenham: it has, however, now been superseded by other remedies

In Dropsy it has been recommended as a drastic purgative, and might still be well employed. Dr. Pearson's opinion was that "it would very well supply the place of jalap in our hospitals." The infusion is a good form of administration: half an ounce of the dried root should be placed for an hour in a pint of boiling water, to which should be added an ounce of spirit of juniper when prepared; of this a wineglassful should be taken every four hours until copious watery motions are induced.

This infusion not only purges, but also produces a vigorous action of the kidneys - a circumstance that points to bryony as a valuable drug where it is desirable to quickly get rid of large accumulations of fluid: care, however, is required, on account of its tendency to depress the action of the heart.

In Serous Inflammations, when general pyrexia has diminished or disappeared, but exudation continues, bryony limits the extent of effusion, and helps its absorption.

It is useful also in chronic bronchitis; and in the catarrhal stage of whooping-cough.

In Rheumatism, bryony will sometimes relieve swollen joints, but it is more especially the merely painful and stiff ones that are benefited by it.

In that vague hepatic derangement commonly known as "sluggish liver," and also in the ordinary bilious headache with vomiting, bryony is worthy of commendation.

PREPARATIONS AND DOSE.-Infilsum, as above. Tinctura (dried plant 1 oz., proof spirit 9 oz.): dose, 3 to $10 \mathrm{~min}$. 


\section{UMBELLIFERA OR APIACEA.}

\section{THE PARSLEY FAMILY.}

Av extensive and important order of herbaceous exogens, distributed principally in northern temperate latitudes. The plants are weedy, the stems usually erect, hollow, and more or less branched, with an ordinary stature of 3 to 4 feet; the leaves are alternate, much divided, and broken up into many segments, the petiole expanded at the base. The inflorescence is almost invariably a compound umbel; the flowers numerous, small, pentamerous, irregular, and usually white; the petals and five stamens crowning an inferior and two-celled ovary, which ripens into the distinctive and seed-like fruit called a "cremocarp." Occasionally the habit and complexion of the plants differ widely from the type, especially in the few species of the order which inhabit the southern hemisphere.

Some species produce aromatic and carminative fruits; others furmish a gum-resin; others, with watery juice, are narcotic. There are various poisonous species; many are esculent, while many more are negative or inert.

\section{Pharmaceutical Species.}

Conium maculatum ... . . . . Hemlock.

Narthex asafotida . . . . . . Asafœtida.

Dorema ammoniacum ...... Ammoniacum.

Opoidia galbanifera .. . . . . Galbanum.

... $\ldots \ldots \ldots$... Sagapenum.

Opoponax chironium ... ... ... Opoponax.

Pimpinella anisum ... . . . . Anise.

Foniculum dulce .. . . . . . Fennel.

Cuminum cyminum ... ... .. Cumin.

Coriandrum sativum .. $\quad . \quad \ldots \quad .$. Coriander.

Carum carui. . . . . . . . .. Caraway.

Anethum graveolens .. . . . . . Dill.

Daucus carota . . . . . . . . Cnrrot.

Euryangium sumbul . . . . . . . Sumbul. 


\section{CONIUM MACULATUM.}

(Spotted Hemlock.)

DESCRIPTION.-This celebrated plant is a native of most parts of Europe, and extends into Western Asia, growing ordinarily in damp and shady places. The root is biennial; the stem is 4 to 6 feet high, furrowed, and spotted with brown; the large tripinnatifid leaves, formed of innumerable little segments, are triangular in general outline, and often 20 to 24 inches in length near the base of the plant, all spreading and hanging very gracefully; the compound umbels of minute white flowers appear in June and July; the numerous palegreen fruits are about an eighth of an inch in length, ovate, compressed, separating into two mericarps, each one marked with five ribs: in the furrows are no vittæ.

The leaves have a strong unpleasant taste, and upon being bruised emit a disagreeable odour, resembling that of mice or of cat's urine.

ACTIVE INGREDIENTS. - The special and characteristic medicinal substance contained in hemlock is an alkaloid, called conia, and known also by the names of conin, coniin, and cicutin $\left(\mathrm{C}_{8} \mathrm{H}_{15} \mathrm{~N}\right.$, or, according to Hofman, $\left.\mathrm{C}_{8} \mathrm{H}_{17} \mathrm{~N}_{33}\right)$. It is found, in combination with malic acid, in every portion of the plant, but chiefly in the pericarps of the mature green fruit, and is separable by distillation with water and caustic potash. When the leaves are dried, all the conia volatilizes, so that in this condition they are useless.

The pure alkaloid is, at ordinary temperatures, an oleaginous, colourless, volatile liquid, sp. gr. 0.89, of strong, penetrating, and stupefying odour, and an acrid flavour, somewhat resembling that of tobacco. The volatile character may be shown by dropping a small quantity of the alkaloid upon paper, when the stain will become translucent, and as if greasy, but will disappear upon heating. Conia burns with a bright flame, giving off much smoke; the vapour also is inflammable; the boiling point is between $340^{\circ}$ and $413^{\circ} \mathrm{F}$. At ordinary 
temperatures it is but slightly soluble in water, more so in cold than in hot; in ether and in alcohol it dissolves readily; with a quarter of its weight of water it combines, forming hydrate of conia. It is the only allialoid besides aniline which coagulates albumen. Conia is a strong base, and resembles ammonia, not only in being devoid of oxygen, but in many of its reactions, and has been artificially prepared by the action of ammonia on butyric aldehyde (Schiff). With the vapour of hydrochloric, nitric, and acetic acids it prcduces copious white fumes, completely neutralizing the acid; with nitric acid it becomes bloodred. Exposure to the atmosphere resolves it into ammonia and a bitter non-poisonous extractive matter.

A second ingredient in hemlock, the volatile alkaloid called conydrin or conhydin $\left(\mathrm{C}_{\mathrm{S}} \mathrm{H}_{17} \mathrm{NO}\right)$, discovered by Wertheim, is less poisonous than the former, from which it is distinguished by being solid and crystalline. A third alkaloid contained in hemlock fruits has probably the formula $\mathrm{C}_{7} \mathrm{H}_{13} \mathrm{~N}$. A fourth ingredient, existing only in minute quantity, is a nonpoisonous rolatile oil, which possesses in a high degree the characteristic odour of the plant, but is not the active principle; hence, in judging of the pharmaceutical value of different preparations of hemlock, no stress can be laid upon the presence or absence of special odour. Several animal tissues, when treated with caustic potash, develop a similar odour.

ABSORPTION AND ELIMINATION.-That conia is absorbed is evident from the symptoms, and Orfila has detected it in the spleen, kidneys, and lungs of animals poisoned by it. There has been difficulty in detecting it in the urine,-Harley, for instance, could not find it, but Zaleski and Draggendorff. reporter its presence in abundance during twelve hours after the dose, and in slight quantity for forty-eight hours afterwards. Its volatility makes its elimination by the lungs probable (Wood).

Physiological Action.- We may dismiss from consideration the statements of older authors as to the action of conium upon the glandular system, and especially on the sexual organs. It was formerly said that the continued use of large doses would 
cause the mammæ and testes to waste, and destroy sexual power; but the experience of Dr. J. Harley, and of other recent observers, has shown that while conium has the power of repressing unnaturally high sexual excitement, it has no influence upon the natural function, far less any power to injure the glandular structures; it is said, however, to retard or arrest menstruation. The truth is that all statements based on the employment of the older preparations before the discovery of the alkaloid conia are of little or no value, because the former were probably inert. As to the hemlock used by the ancient Greeks for the execution of criminals (if this plant were really the poison employed), the fatal drink was probably the expressed juice, the succus, which is the only valuable officinal form. All preparations in which heat is employed are probably worthless, because of the great volatility of the alkaloid, and we may disregard all results except such as have been obtained by the use of the alkaloid itself, or the juice, or the (non-officinal) tincture of the nearly ripe fruit, which contains more of the active principle than any other part of the plant. ${ }^{1}$

Since the time of Christison (Trans. Royal Soc. Edin., 1835), the subject has been thoroughly investigated by Kölliker, Albers, Guttmann, Schroff, and many others; and within the last few years by Drs. Fraser and Crum-Brown, who have studied the modifications introduced into the action of conia by sundry chemical combinations.

Nervous System. - The numerous modern researches alluded to clearly indicate conia to be a nerve-poison, with definite and limited affinities, i.e., though one of the most active poisons it operates within a contracted sphere, and mainly as a pure paralyser; upon the motor system it acts not directly on the muscles, but through the nerves alone, without affecting the spinal cord. Verigo credits it with powerful depressant, and Pelvet and Damourette with an excitunt, action on the cord; but the results of the former are presumed to be due to methyl-conia (Arch. Gén., t. vi.). Small doses have the same effect as curare, viz., upon the nerve terminals only, but larger doses affect also the motor trumks. The hind limbs of animals are paralysed first, but the whole

2 Old Vegetable Neurotios, p. 92. Also Practitioner, Oct., 1871. 
system of voluntary motor nerves is ultimately affected, and death ensues by cessation of respiratory movements : in many animals convulsions are seen, with tremor and exaggerated reflex movements. The occurrence, first, of heaviness, then loss of power, and later, loss of sensation in the legrs, gradually extending upwards, and followed by a convulsion and stoppage of respiration, is, in brief, Plato's description of the death of Socrates.

There is considerable variance between Harley and other observers; in part, perhaps, more apparent than real. He finds a full dose of the succus (5 to 6 drachms) act in the following manner:-If the taker keep up a brisk walking exercise, in half or three-quarters of an hour he feels a sense of weariness in the legs, with some giddiness and heaviness over the eyes, but if he persevere in his walk these symptoms soon pass off. If, on the contrary, he remain at rest after the dose, the eyes become first affected; accommodation is interfered with, and there is drowsiness, with dilatation of the pupil; after these come weakness of the legs, pallor, inability to stand steady, a general diminution of muscular power, especially in the hamstring muscles. From the consideration of these facts, and from his results in the treatment of chorea, etc., Harley believes that the central motor tract is affected by conium, and that its action in full and repeated doses is primarily tranquillizing, and subsequently tonic, to the muscular movements. He particularly states that the vertiginous and quasi-paralytic effects are much more pronounced in the quiescent than in the active; so that a delicate child, if lively and running about, will take with impunity a dose that would confuse a sedentary adult.

As regards the production of dizziness, impaired accommodation, dilated pupil, and other brain symptoms, although some observers deny them, a preponderance of evidence is confirmatory ; ${ }^{1}$ but they appear, on the whole, to be trifling in comparison with the effects on the motor system.

Upon the sensory neves conia exerts a direct, but moderate,

1 See especially Schroff's account of the experiments made under his control by Dillnberger and Dworzall. M. Rochefontaine believes that the principle which paralyses nerve-centres may be separated from the one which acts like curare (Compt. Rend., xei.). 
paralysing influence, which is local in action. Applied to a nerve laid bare, it causes no pain, but simply interrupts the conduction of sensory impressions. There is no reason to think that it affects sensation by an action on the nervous centres: it is said, however, that the first momentary effect of applying conia to a wound, or to the mucous membrane of the mouth, is a burning feeling, but this is immediately succeeded by a loss of sensibility, and there is no ground for viewing conia objectively as an irritant. According to Pelvet and Damourette, conia can destroy the epithelium of the mucous membranes and of the skin.

Circulatory System.-Whether conium affects the heart, and, if so, in what manner, is doubtful. The general result of experiments on animals certainly discountenances belief in such action; but in human being's the evidence is more conflicting. J. Harley declares that there is usually little or no effect; the pulse may, if anything, become firmer and fuller, but in any case the known general physiological character of the drug leads us to treat its action on the heart as but of slight consequence: the effect on the blood-corpuscles outside the body is to make the nucleus of the white corpuscles larger, and then to dissolve the corpuscles to a formless mass: within the ressels it induces formation of coagula. Casaubon held it to be a direct poison to the corpuscles, preventing their absorption of oxygen (Thèse, 1863). After death the blood has been found less fluid, so that the French observers already quoted explain that it nourishes the tissues less, and hence lessens the development of neoplasms, cancers, etc.

Secretory System.-Conium was formerly credited with diuretic and diaphoretic action, but on doubtful evidence. Some weiglat may perhaps be given to the affirmation by several American physicians of its diaphoretic effect; but the assertion of Fountain, that this is equally produced by conia and the extract of hemlock, is open to question.

TOXIC ACTION.-Of the fatally poisonous effects either of hemlock or its alkaloid upon man we possess little information; it is desirable, therefore, to take particular notice of any wellobserved case. J. Hughes Bennett recorded one in a man who 
ate hemlock by mistake in a salad; the first symptom observed was weakness of the legs, and as this increased, the man staggered as if drumk, and the arms began to be paralysed; loss of voluntary movement and power to swallow followed; and, lastly, palsy of respiration put an end to his life: consciousness was unimpaired up to death, but sight was destroyed. In another case-a woman-death followed in a few minutes after taking: 10 to 15 minims of the alkaloid; violent palpitation occurred (Husemann). In a third, a man-after previous divided doses, amounting altogether to 180 drops (Squibb's fluid extract), taken to relieve facial spasm-took an additional 50 minims, which caused dizziness and muscular relaxation: in half an hour 50 more, which induced great muscular weakness, inability to stand, and thickness of speech (without relieving. spasm); in another half-hour 50 more, with production of nausea and some tremor; later, all the symptoms became intensified, with loss of the powers of speech and swallowing; pulse 60. He was conscious enough to sign for remedies, but on being raised up died suddenly (quoted by Wood).

The preceding account would be incomplete without reference to the observations of Fraser and Crum-Brown, who ascertained that two varieties of conia are sold, of which one (Morson's) contains not only the drug', but a compound of itmethyl-conic-the properties of which are very different. This influences not only the motor-nerves, but the spinal cord itself; with large doses the former action is developed before the latter, but with small doses the action on the corcl is completea before that on the nerves. The general result is to lessen the tendency to exaggerated reflex and convulsive action. It is evident that the effects differ in proportion to the varying degrees in which the so-called conia may contain methyl-conia; hence the discrepancies of authors.

SYNERGISTS.-To some extent, Calabar bean, curare, aconite, tobacco, opium, and narcotics.

AnTAGONISTS.-Chemically, tannins and iodides; mechanieally, exercise and frictions; alcohol, coffee, stimulants, possibly, to some extent, atropia. 
Therapeutical Action.- Pain. - Almost the only trustworthy fact among the older observations on conium is the local sedative action, which, in pain and irritation, may certainly be very useful: the pain of cancer seems to be particularly amenable to its influence. The best application to a sciwhous breast, for example, in the stage of painful tension, either before or during the process of ulceration, is a mixture of succus conii and glycerine in equal parts, or a weak solution of conia, or a poultice of the fresh leaves. The same applications are useful for enlarged or congested glands or joints. For cancer of the stomach the suceus may be given in doses of 2 to 5 drachms or more; and to allay the pain of cancer of the rectum, 1 ounce may be injected with the same quantity of starch decoction. In painful "scrofulous" photophobia, Dr. Mauthner has employed conia most successfully; using a mixture of $\frac{1}{2}$ grain of it with 1 drachm of almond oil, applied two or three times daily to the conjunctivæ. Irritability and soreness of the air-passages in bronchitis and in the tickling cough of phthisis, can be calmed by the inhalation of the vapour. Harley's formula is the best:-Conia 1 grain, alcohol 1 $\frac{1}{2}$ drachm; dissolve in $\frac{1}{2}$ drachm of the spirit, and add the remainder with 2 drachms of water: 20 minims contain $\frac{1}{12}$ grain of conia, to be placed on a sponge in a suitable apparatus for inhaling.

In Spasmodic and Convulsive Disorders, the remedy, though apparently indicated by its physiological action, has not attained a high repute, notwithstanding the strong statements that have been made in its favour.

In chorea, Dr. Harley found the succus of remarkable value, when given in increasing doses until the characteristic slighter physiological symptoms are produced. One, at least, of his cases is beyond criticism; for the choren was of old date, and the boy (aged six) had also suffered from morbid restlessness from his birth: only after very large doses were given was any decided improvement observed; he took $10+$ fluid ounces in twelve weeks, and under this treatment not merely dicl he lose his chorea, but his congenital restlessness disappenred, and be also gained flesh and strength remarkably.

On the other hand, many practitioners have given patient 
trial to conium in chorea, and have either had no success at all, or else capricious and uncertain results. Ringer speaks of the improvement under conium as only temporary, and adds- "It has yet to be shown that conium will shorten the course of this disease." I have heard many practitioners express a similar view; and many who at first believed that they had found in conium a specific remedy for chorea have afterwards lost all faith in it. We cannot, however, ignore such cases as are recorded by Harley; and on the whole the reasonable conclusion seems to be that there are patients over whom it has a very powerful influence, and others for whom it does nothing: what may be the pathological difference that causes this varying. result, we unfortunately do not know. Dr. Anstie remarked that he had never seen conium do good in the severe choreas of puberty, while it had sometimes appeared strikingly beneficial to young children.

In Tetanus the anticipations of success from conium which once prevailed have not been sustained. Experimentally, Guttmann found that frogs which were tetanized with strychnia were not benefited even by paralysing doses of conia; and the cases, if apparently successful, recorded by some authors, must be received with suspicion. Thus, Mr. Corry's patients were treated with the cxtract in 5-grain doses, and as there is reason to believe this preparation to be inert, recovery was probably spontaneous (Dublin Quarterly Journ. of Med., Nov., 1860). The cases published by Professor Johnson, of Maryland, are, as Ringer points out, most unsatisfactory, for in both of them other powerful remedies were simultaneously employed: nor does it seem probable, from the best knowledge we can get of the physiological action of the drug, that coniun could antagonize either pathological-or strychnia - tetanus, notwithstanding Dr. Harley's arguments to the contrary.

In Epilepsy, also, I regret to say that I have obtained no confirmation of his favourable statements: he limited lis commendation of the drug to those cases in which self-abuse had been the apparent cause, but even in this special relation I have not found it effective. It is probable that self-abuse is never more than the exciting cause acting upon a system predisposed to the disense; consequently, we could hardly expect 
efficient remedial action from any treatment, except one which should either remove the exciting cause, or deeply modify the nutrition of the nervous centres; and there is little hope that conium will prove adequate for either of these purposes. Whatever can be done in the way of lowering the susceptibility to peripheral excitement can probably be better effected by bromide of potassium; while the slower, but more radical, benefit which may be hoped for from improvement in central nervous nutrition, is more likely to be obtained by nutritive tonics such as cod-liver oil. Dr. Wolfenden has, however, recently recorded seven cases of epilepsy treated with hydrobromate of conia, which drug he has found serviceable, except when convulsions are due to a gross lesion. Large dosesi.e., more than 1 grain in a child, or 4 grains in a man-are apt to cause headache, giddiness, and congestion of conjunctivæ (Practitioner, 1884).

In Mental Diseases there seems much more probability that conium will prove efficacious. The practical authority of Dr. Crichton Browne is strongly in favour of its use in acute mania (Lancet, i., 1872); and Dr. J. Wilkie Burman has published some remarkable results of the subcutaneous injection of conia and morphia in the same disease; the latter experimentally confirmed J. Harley's statement that the union of these drugs heightens the effect of each, and that the combined influence is tranquillizing both to the mental and the motor centres (Practitioner, Dec., 1872). Dr. Burman's estimate of this treatment, if correct, is very important: the question ought to be settled by extensive experiments in our large asylums, and this could readily be done. I am bound to state that I have heard of instances where the treatment has been patiently and carefully tried, in apparently suitable cases, without any good effect; but no general decision ought to be arrived at until many more well-conducted trials have been made.

In Delirium Tremens, according to Harley, conium is most useful, especially in combination with opium. It may here be remarked that, should it prove true that conium has a direct influence over some forms of delirium tremens, we might be led to think that it possesses special affinities for the braincortex; but nothing certain can yet be said on this point. 
PREPARATIONS AND DOSE.-Of the powdered lenf: dose, 2 to $8 \mathrm{gr}$. or more. Cutaplasma: 1 oz. of leaf to 3 of linseed and 10 of boiling water. Exticatum: dose, 2 to 6 gr. or more. Pitula conii composite (with ipecac.): dose, 5 to $10 \mathrm{gr}$. Succus: dose, $30 \mathrm{~min}$. to $1 \mathrm{fl}$. dr. or more. Tincture (of the fruit): dose, $20 \mathrm{~min}$. to $2 \mathrm{fl}$. dr. or more. Vapor : extract 1 part, liq. potass. 1 part, aqua $10 ; 20 \mathrm{~min}$. of the mixture to be used in a suitable apparatus ( $v$ p. 327). Conice hyclrobromas: dose, $\frac{1}{2}$ to $\frac{3}{4} \mathrm{gr}$. twice in the day; $4 \frac{1}{2}$ gr. per diem should not be exceeded.

Adulterations.-Various umbelliferous plants (Pharm. Journ., ii., 1883).

\section{NARTHEX ASAFCETIDA.}

(AsAFGetida.)

DESCRIPTION.-A native of Western Thibet, where it attains the height of at least 8 or 9 feet. The stem is 6 to 7 inches in circumference at the base, the leaves larg'e and bipinnate, the flowers yellow, produced in numerous umbels, and the root, which is massive and perennial, blackish externally, but white in the inside, is charged with a dense milky juice, with the odour of the drug. From the living: root is obtained the asafoetida, incisions being made in the upper part, from which the juice exudes and soon coagrulates: in commerce this gum-resin occurs in irregular lumps of a yellowish or pinky-brown colour, breaking with a conchoidal fracture. The freshly fractured surface is whitish and pearly; but exposure to the atmosphere causes it to assume, after a little while, a violet-red or peach-blossom hue, which soon changes to yellowish or pinkish-brown. The taste is acrid aud bitter; the odour is powerful, alliaceous, and peculiar. The resin burns with a white flame and much smoke.

ACTIVE INGREDIENTS.-Besides resin, gum, and other unimprortant substauces, which constitute its chief bulk, asafootida 
contains a variable quantity $(3.5$ to 4.5 per cent.) of volatile oil, upon which its odour and properties essentially depend. The oil, according to Hlasiwetz, is a mixture of sulphurets of ferulyl or laseryl, $2\left(\mathrm{C}_{2} \mathrm{H}_{11}\right) \mathrm{S}$ and $\mathrm{C}_{6} \mathrm{H}_{10} \mathrm{~S}$. When newly distilled, like the essential oils of horse-radish and black mustard, it contains no oxygen. It is lighter than water, and at first is colourless, but exposure to the air causes it to acquire a yellow tinge and also to become acid. The odour is very powerful, and, as evaporation proceeds rapidly, it is soon perceived at a long distance. The taste is at first mild, afterwards bitter and acrid. Phosphorus is probably one of its elements, and sulphur certainly, sulphuretted hydrogen being disengaged during the process of boiling. It dissolves in all proportions of alcohol and ether, but requires more than 2,000 times its weight of water for solution. When the asafoetida itself is rubbed with water, the gummy matters dissolve, the resin and volatile oil are suspended, and an emulsion formed. The resinous matter is soluble in alcohol; and if the alcoholic solution be mixed with water it becomes milky, owing to precipitation of the hydrated resin.

ABSORPTION AND ELIMINATION.-Asafoetida is absorbed by the stomach and rectum, and is eliminated in the respired air and through the skin.

Physiological Ac'Tion.-The first complete investigntions into the action of asafoctida appear to have been those made by Jörg. He ascertained that distinct effects followed the administration of a single grain, but that in different individuals there is very various susceptibility to its influence; in some the characteristic smell of the drug could not be traced in the secretions, implying that more was absorbed than usual.

Digestive System.-Moderate doses, if continued, cause alliaceous uructations, often for twenty-four hours, showing the length of time the medicine is retained in the stomach; the digestion becomes impaired, and the abdomen distended with flatus, which, when discharged, is of foctid, disagreeable character.

In larger doses (10 to 30 grains), asafoctida increases the 
secretions both of the pulmonary and abdominal organs, especially of the liver and bowels, and angments peristaltic action; morbid secretions of the mucous membrane are improved in quality; and if intestinal worms are present, they are expelled.

Genito-Urinary System.-The urine is not augmented in quantity, but becomes acrid and burning. Jörg's statement that the menstrual period is advanced has been confirmed by numerous observations: sexual desire is also excited.

Circulatory System.-The pulse and respiration become quickened, and the secretion of the bronchial mucous membrane is promoted.

Nervous System.-The head is more or less affected with flying pains, often attended by much giddiness. Upon all parts of the system, asafotida in moderate doses appears, in short, to operate as a stimulant, and especially in hysterical subjects, in whom, among other results, it frequently enlivens the spirits.

SYNERGISTS.-Other antispasmodies, galbanum, ammoniacum, valerian.

Therapeutical Actron.-Acting on the nervous system as a stimulant and powerful anti-spasmodic, asafœtida becomes useful in hysterical convulsive affections, globus hystericus, hysterical cough, etc. In hysterical tympanites it may be administered with much benefit in the form of enema, or it may be taken internally; still, in nervous affections nothing more must be expected of this remedy than palliation of certain symptoms: substantial cures can only be looked for from medicines of greater energy.

Dr. Garrod, however, "is inclined, from the result of much observation, to regard asafotida as one of the most valuable remedies of the materia medica; far above all other ordinary anti-spasmodies"; and he thinks "the value of the drug is chiefly due to the sulphur oil contained in it."

According to Dr. Ringer it is servicenble in the flutulune of young children, when unconnected with constipation or diarrhoca, a tensponful being given every half-hour of a 
mixture consisting of a drachm of the tincture to half a pint of water; he states at the same time that when flatulence has constipation or diarrhoea for its accompaniment the asafœtida does little good.

Whooping-cough, asthma, and other nervous disorders have been successfully treated with it. It is useful, again, in certain forms of chronic bronchitis and whooping-cough, by reason of its expectorant power. In chronic catarrhs, especially when accompanied by spasmodic cough and by occasional difficulty of respiration, asafœtida alone, or combined with ammonia, gives decided relief.

Asafœetida has been used for Guinea-worm; it is said to cause its extrusion rapidly (Lancet, i., 1879).

PREPARATIONS AND DOSE.-Of the gum-resin, dose, 5 to $30 \mathrm{gr}$. or more. Tinctura: dose, $\frac{1}{2}$ to $1 \mathrm{fl}$. dr. Pilula aloës et ascifoctidre: dose, 4 to $10 \mathrm{gr}$. Pitula asafoetide composita (with galbanum and myrrh): dose, 5 to $10 \mathrm{gr}$. Spiritus ammonice ficetidus: dose, $\frac{1}{2}$ to $1 \mathrm{dr}$. Enema asafoetidre contains $30 \mathrm{gr}$. of the drug to $4 \mathrm{oz}$. of water.

\section{AMMONIACUM, GALBANUM, SAGAPENUM, OPOPONAX.}

These are gum-resins produced by umbelliferous plants of South-western Asia, the botanical identity of which is not determined. The chemical composition appears to differ little from that of asafotida; the effects also are similar but feebler. The internal exhibition of ammoniacum is almost limited to its employment for chronic pulmonary affections. Externally, it is employed as a discutient plaster, for glandular enlargements and indolent affections of the joints. Empl. hydrargyri cum ammoniaco is useful for "housemaid's knee" and ehronic bursal enlargements, but sometimes excites much local irritation. 
PREPARATIONS AND DOSE.-Of ammoniacum the dose is from 10 to $30 \mathrm{gr}$. or more. Misturr: dose, $\frac{1}{2}$ to $1 \mathrm{fl}$. oz. Galbamm is stronger than ammoniacum: dose, 10 to $30 \mathrm{gr}$. or more; and Sagapenum holds a middle place as to energy: dose, 10 to $30 \mathrm{gr}$. or more. Opoponax seems more nearly allied to ammoniacum.

\section{PIMPINELLA ANISUM.}

(Anise or Aniseed.)

DESCRIPTION.-An annual, indigenous to Egypt, and of very ancient cultivation. The stem, which is 12 to 18 inches high, is round, striated, and branched; the lower leaves are imperfectly three- to five-lobed, the upper ones in many acuminate segments; the small white flowers are produced in terminal and flat-topped umbels, of ten or twelve rays, devoid of bracts; the fruit is ovoid, contracted at the sides, and tipped by the persistent recurving styles. The odour of anise is aromatic, and the flavour warm and grateful, with a degree of sweetness.

ACTIVE INGREDIENT.-From the fruits is obtained an oil, which is a mixture of a fixed oil and the volatile anethol, a substance common not only to several Umbelliferæ, but also to the Illicium anisatum (v. p. 58) and the Artemisia dracunculus. The formula is $\mathrm{C}_{10} \mathrm{H}_{12} \mathrm{O}$ : it is colourless when fresh, but gradually becomes yellowish; it has the characteristic taste and smell of the seeds; sp. gx. 0.98 or 0.99 . It is very soluble in alcohol and ether, but slightly so in water.

PHysiological Action.-Anise oil acts as a rapid poison to lice and to itch-insects; is a powerful narcotic to certain small birds, and in half-ounce doses kills rabbits with symutoms of nareotico-acrid poisnning; it affects cats and dogs but slightly; to the human skin it is somewhat irritant.

Therapeutical Action.-Extemally anise oil has been employed with success in the form of an ointment or sonp 
to destroy lice and itch-insects. Taken internally, in small doses it is a mild stimulant to the stomach and intestines, and to the bronchial mucous membrane; hence it is useful in atonic dyspepsia, colic, flatulence, and chronic bronchitis. It was formerly known as "intestinorum solamen," and by Galen was reckoned among the true anodynes or cordials. It is eliminated both in the urine and the milk, and has therefore been credited with the power of increasing the excretions, but of this there is no good evidence.

PReparations AND Dose.-Oleum: dose, 2 to $5 \mathrm{~min}$. Essentia (1 in 5): dose, 10 to $20 \mathrm{~min}$. An infusion may be made with 2 to $3 \mathrm{dr}$. of the bruised seeds to $\frac{1}{2}$ pint of boiling water: dose for an infant, 1 teaspoonful.

\section{FCENICULUM DULCE.}

(EENNEL.)

DESCRIPTION.-An erect dark-green plant, about 3 feet high. The large leaves are divided into innumerable hair-like segments, many inches in length; the yellow flowers are produced in erect umbels, destitute of bracts, and composed of six to eight rays; the ripe fruit is narrow-oblong, smooth, of a pale-dun colour, about 3 lines in length, and with sharp ridges that have a considerable space between them, the vittæ being distinct in the intervals. (By these characters it is distinguished from the fruit of Fœniculum vulgare, which is ovate, not quite 2 lines in length, pale bright-brown, and with very little space between the ridges; but $\mathrm{F}$. dulce may be only a smaller variety of this.) The herbage is noted for its powerful, not unpleasant odour; the fruit, when required for medicinal purposes, is generally imported from Italy.

ACTIVE INGREDIENTS.-The oil of sweet fennel, constituting the active primciple, is of light straw colour, and retains the odour of the fruit. In composition it corresponds 
with the oil of anise, but it differs from this in being dextrogyrate, probably in consequence of a hydrocarbon it contains.

Physiological Action.-The vapour of fennel oil causes secretion of tears, and occasionally of saliva. Given in large doses to rabbits it proves fatal, in much the same way as anise oil, but, unlike the latter, does not pass out in the urine.

THERAPEutrcal Action.-Fennel is a useful carminative, and is given for flatulence and griping pain. It is said to increase the various secretions, and to have emmenagogue properties: a hot infusion has been prescribed in congestive amenorrhœa and for lessened secretion.

PReparations AND Dose,-Oleum: dose, 2 to $5 \mathrm{~min}$. Aqua: dose, 1 to $2 \mathrm{fl}$. oz. An infusion may be made with $1 \mathrm{dr}$. to $\frac{1}{2}$ pint of boiling water: dose, $1 \mathrm{dr}$. for an infant.

\section{CUMINUM CYMINUM. (Cumin.)}

(Not Officinal.)

DESCRIPTION.-A slender, branching annual, often procumbent, but when erect attaining the height of 15 inches. The leaves are repeatedly cleft into long linear segments; the white or pinkish flowers are produced in numerous small umbels of about four umbellules each, and are without involucrum; the fruits resemble; caraways, but are larger, with the ribs minutely spinous. The supplies of this drug for English use are derived chiefly from Sicily and Malta.

ACtive IngREDIENTS. - The properties of cumin depend on a volatile oil, which is of a pale-yellow colour, limpid, and lighter than water. It is a mixture of cuminol and cymol with a little terpene, $\mathrm{C}_{10} \mathrm{H}_{18}$; Cuminol, $\mathrm{C}_{10} \mathrm{H}_{12} \mathrm{O}$, isomeric with 
anise oil, is colourless, with a sharp burning taste, and a strong smell like caraway: insoluble in water, readily soluble in alcohol and ether. Cymol, $\mathrm{C}_{10} \mathrm{H}_{14}$, also colourless, is strongly refractive of light, and has an unpleasant, camphor-like smell: is insoluble in water, readily soluble in alcohol, ether, and fats.

Physiological Action.-Cumin oil has acquired an unexpected degree of importance from the researches of Grisar, already referred to under chamomile oil: he experimented with it on frogs, in the same manner, and came to the conclusion that cumin oil markedly depresses reflex excitubility, though to a less extent than the other substances experimented with. $\mathrm{He}$ also proved the power of cumin oil to antagonize in a decided manner the tetanic excitement of strychnia.

THERAPEUTICAL ACTION. - Cumin has hitherto only been employed in medicine as a carminative, but the abovementioned experiments of Grisar may lead to its use in reducing reflex excitability in its various forms.

PREPaRATION AND DOSE.-Olenm: dose, 2 to 8 min. with sugar, or in emulsion.

\section{CORIANDRUM SATIVUM.}

(CORIANjerr.)

DESCRIPTION.-An annual of the same general appenrance as anise. The white or pinkish flowers are borne in umbels of four or five rays; the umbellules are many-rayed; the fruit is globular, and obscurely striated, and separates into two hemispherical mericarps. When bruised this plant evolves an intolerable odour, resembling that of bugs, and possessed by no other member of the Umbelliferæ. The fruits while recent partake of the same; when dry, however, the scent becomes grateful. 
ACTIVE INGREDIENTS. - The taste and odour of coriander seeds are referable to a yellowish volatile oil, which renders them moderately warm and pungent. Kawalier believes it to be of the camphine class, and to have the composition of $\mathrm{C}_{10} \mathrm{H}_{18} \mathrm{O}$, being isomeric with borneol.

\section{Physiological action.-Unknown.}

Therapeutical ACtion. - Coriander seeds are added to infusion of senna, and to other purgatives, with a view to covering the flavour, and checking a tendency to gripe.

Preparations AND Dose.-Oleum: dose, 2 to $5 \mathrm{~min}$. Of the powdered fruit: dose, 10 to $30 \mathrm{gr}$. In combination with other medicines, coriander occurs in confection of senna, mixture of gentian, syrup of senna, tincture of senna, syrup of rhubarb, and tincture of rhubarb.

\section{CARUM CARUI.}

(Caraway.)

DESCRIPTION.-A biennial, native originally of continental Europe, but now naturalized in England. The stem is erect, about 2 feet high, glabrous, branched, and furrowed; the leaves are doubly and triply pinnatifid, the segments linear, and those of the lower leaves intercrossing: the numerous small white flowers are borne in terminal, erect, and many-rayed umbels; the fruits, which are elliptic-ovoid and greyish-brown, separate into two narrow mericarps, a line and a half in length, and with a solitary vitta in the channel between the filiform and elevated ridges.

ACTIVE INGREDIENTS. - Caraway owes its properties to a volatile oil, which is thin, colourless, or straw-yellow, with in peculiar penetrating smell, and a hot, bitterish taste: soluble in 
an equal volume of rectified spirit. It consists of two bodies, carvol and carvene: the former $\left(\mathrm{C}_{10} \mathrm{H}_{14} \mathrm{O}\right)$ is a thin, clear fluid, with caraway smell; sp. gr. 0.953 ; it does not boil under $250^{\circ}$ C. Carvene $\left(\mathbf{C}_{10} \mathrm{H}_{16}\right)$ is a camphor-like body; it forms a crystalline salt with hydrochloric acid, and boils at $173^{\circ} \mathrm{C}$.

Physiological Action.-Caraway oil appears to be, in large doses, a fatal nareotic poison to rabbits. One case has been recorded where a man took about a drachm, and was attacked with shiverings and heats, congestion of the head, and delirium.

Therapeutical Action.-Flatulent colic in children is often satisfactorily treated with preparations of caraway, but the chief use is that of a flavouring agent. Caraway-water is a convenient vehicle for saline purgatives; and is good also for covering the taste of nauseous medicines and preventing the griping action of purgatives; for this latter purpose the oil is frequently used in eathartie pills.

Preparations and Dose.-Aqua: dose, 1 to $2 \mathrm{fl}$. oz. Oleum: dose, 2 to 5 min. As an ingredient, caraway is found in the confections of opium and of pepper; also in compound opium powder, compound tincture of cardamoms, and tincture of senna.

\section{ANETHUM GRAVEOLENS.}

\section{(Dil.L.)}

DESCRIPTION.-An annual, probably of Asiatic origin, rising to the height of 2 or 3 feet, and easily distinguished by its yellow flowers and small, flat, shining brown, oral fruits, with paler marginal wing. The leaves are doubly pinnatifid, the segments long and linear. 
ACTIVE INGREDIENTS. - The fruit of this plant owes its properties to the non-volatile and volatile forms of anethol, already described under anise.

Physiological Action.-Similar to that of anise.

Therapeutical Actron.-The medicinal uses of dill are identical with those of anise and caraway; the most extensive employment being as a remedy for the flatulence of infants. In India the infusion is administered to women immediately after parturition, as a stomachic and grateful cordial.

Preparations and Dose.-Aqua: dose, 1 to $2 \mathrm{fl}$. oz.; for infants, 1 to $2 \mathrm{fl}$. dr. Oleum: dose, 2 to $5 \mathrm{~min}$. The oil may be taken upon a lump of sugar or dissolved in spirit.

\section{DAUCUS CAROTA.}

(CARRot.)

A biennial plant, distinguished by the large involucrum of many deeply divided and pinnatifid bracts, and the concavity of the fruiting umbel. The leaves are tripinnatifid; the flowers are white; the fruits ovoid and bristly. The juice of this plant contains a small quantity of volatile oil; a crystallizable substance called carotin, neutral, and of fine red colour; also pectin, albumen, and other less important ingredients: the physiological action has yet to be ascertained.

The root, familiar as a garden vegetable, is employed for poultices for ill-conditioned and sloughing ulcers. The fresh scraped root given in teaspoonful doses once or twice daily is in popular repute as a remedy for ascaricles. Carrot soun is a useful laxative. 


\section{EURYANGIUM SUMBUL.}

(Stmbul.)

DESCRIPTION.-Sumbul, though in Oriental countries a collective term for various aromatics, is in England restricted to the parsnip-shaped root of a plant indigenous to Bucharia, and which, having blossomed in the Botanical Garden at Moscow, and latterly at Kew, is now known to be a genuine member of the Umbelliferæ, though for a long time it was incorrectly referred to the Valerianacer. The plant is a perennial, about 8 feet high, with large, triangular, tripinnate radical leaves, and small upper ones sheathing the stem.

In commerce this drug occurs in horizontal slices of the root, which are light brown in colour, of a worn or spongy appearance, variable in thickness, and from 2 to 5 inches in diameter; the pieces which formed the upper extremity of the root retaining relics of the base of the stem, and portions of the dark-brown papyraceous skin. The odour is musk-like; the taste, after a time, becomes somewhat bitter and balsamic, and by mastication a pleasant aroma is evolved.

Active InGREDIENTS. - The analyses of Reinsch show that when the clear pale-yellow resin extracted by ether from sumbul root is treated with alcohol (of 75 per cent.), and the solution evaporated, a balsam-like body is left, which contains much angelic and valerianic acid. According to Sommer, the dry distillation of this substance brings over a blue volatile oil containing umbelliferon, $\mathrm{C}_{9} \mathrm{H}_{6} \mathrm{O}_{3}$, which crystallizes in transparent, silky needles, destitute of taste or smell: readily soluble in alcohol, ether, and chloroform; subliming at a high temperature. Its solution in boiling water has a blue fluorescence.

Physiological Action.-Upon this subject it is to be regretted that we as yet possess no information beyond the general idea that sumbul exerts on the brain and spinal centres a calming, nntispasmodic influence. 
Therapeutical Action.-Sumbul is a drug of which we probably as yet only partially know the value, and which is not at present much used. In Russia it is given in low ferers of a typhoid type; also in asthenic cases of dysentery and diarrhœa; and it has even been said to have proved successful in cholera.

Dr. Granville, who introduced it into England, advised it in cases of gastric spasm, hysteria, dysmenorrhoea, epilepsy, and nervous disorders.

Dr. Murawieff, a Russian physician, recommends it in chronic bronchitis, moist asthma occurring in old anæmic and scorbutic subjects, in atonic dyspepsia, leucorrhoea, hypochondriasis, and hysteria. I can bear witness to its decided efficacy in chronic bronchitis, and in certain stages of phthisis.

Neuralgia.-It is sometimes surprising to observe the rapidity with which a severe facial, sciatic, or ovarian neuralgia will yield to a few doses of sumbul after resisting powerful remedies. It is difficult to state exactly the character of the attacks thus amenable to sumbul, but they occur most frequently in women of a quick and lively "nervous" constitution. On the other hand, the dull migraine of hysterical women, with phlegmatic constitution and tendency to obstinate constipation, does not yield readily, if at all, to it.

In the restlessness of pregnancy, which is exceedingly distressing to some women, sumbul is often invaluable; a draught of 30 to 40 minims of the tincture, with a little chloric ether, giving quiet nights for a long time together without losing its power.

In the Insomnia of Chronic Alcoholism, sumbul ofter serves a similar purpose very efficiently; and it is considerea by Thielmann, of St. Petersburg, the most trustworthy remedy in delirium tremens.

PREPARATION AND DOSE.-Tincturl : dose, 10 to $60 \mathrm{~min}$. 


\section{CAPRIFOLIACEA.}

\section{THE HONEYSUCKLE FAMILY.}

AN order of about 200 exogenous shrubs, natives chiefly of northern cold and temperate countries, and nearly related to the Cinchonaceæ, differing as to technical characters only in the want of stipules. The leaves, however, are sometimes pinnate, and the flowers irregular.

\section{SAMBUCUS NIGRA.}

(Common Elder.)

It is unnecessary to describe this familiar tree. The principal ingredient of the flowers is a volatile oil, which is yellowish, and either limpid or soft and semi-solid, of strong odour, and "warm, bitterish, afterwards cooling, taste." They contain also some resin, tannin, and viburnic-identical with valerianic-acid.

The flowers, made into an ointment or cataplasm, form a cooling and soothing application to irritable sores. The distilled water, which used to be thought diaphoretic, diuretic, and capable of relieving asthma, dry cough, etc., is frequently inert, but makes a good cooling lotion.

The bark and the root-juice have indeed active cathartic properties, but they are uncertain, often violent, and we possess other more trustworthy agents of this class.

The fermented juice of the berries is in popular use as a cordial wine.

Preparation and Dose.-Aque: dose, 1 to $2 \mathrm{fl}$ oz. 


\section{CINCHONACEA.}

\section{THE CINCHONA FAMILY.}

ONE of the most extensive orders known to botany, and among the exogens unsurpassed in importance. The species, which are almost exclusively tropical, number at least 2,500. They present endless diversities of figure and dimensions, but agree in the possession of opposite and entire leaves, interpetiolar stipules, regular monopetalous flowers, and an inferior ovary. The corolla is either four- or five-lobed, and often provided with a long tube; the stamens are epipetalous and definite; the anthers are straight, and open longitudinally; the fruit is usually two-celled; the seeds are either numerous or only two. No instance of a deleterious plant occurs in the Cinchonacer. Many species are famed for their beauty; others possess great economic value. The pharmaceutical species are:-

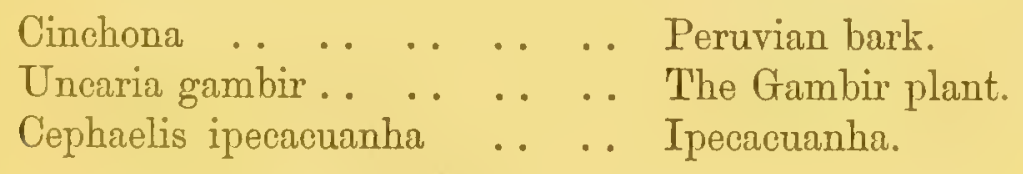

\section{CINCHONA.}

Description.-The plants which furnish Peruvian bark are handsome evergreen trees, indigenous to the mountain forests of Peru, and rising to the height of 30 or 40 feet. The leaves are oval or ovate-lanceolate, 3 to 5 inches in length, and entire; the flowers, which are small, and either pink or white, are borne in terminal panicles or thyrses; the fruits are capsular, 
narrow-oblong, about an inch in length, somewhat woody, and many-seeded.

The medicinal bark is obtained from several nearly allied species: which are the best is not yet satisfactorily settled, partly because of confusion in the nomenclature and of the varying conditions under which the bark has been collected, and partly because of the existence of different "varieties" of the species usually considered best. These are Cinchona condaminea (the officinalis of Linnæus), C. calisaya, and C. cordifolia, to which may be added, as of value, the C. succirubra and C. lancifolia. Now that cinchona cultivation has been established in British India, in Ceylon, and in Java, certainty on the subject will no doubt be secured, but for the present it is not positively known whence are derived the "yellow," "red," and "pale" barks of commerce.

The Cuprea barks, which are now very largely imported from the State of Columbia, are derived from species of Remijia, a genus closely allied to Cinchona and Cascarilla, and growing not only on the Andes, but in comparatively low-lying and colder positions. Chemically they are distinguished from true Cinchonas by the absence of cinchonidin and the presence of a special alkaloid, cinchonamin (besides quinine and quinidin): physically they are remarkable for their great density, and sink in water: the epidermis is thin, longitudinally striated, and the inner surface is very smooth and of wine-red colour: the fracture is not fibrous, but has a horny appearance from the presence of a gum-resin (Triana, Pharm. Journ., i., 1882).

The time and manner of discovery of the virtues of cinchona bark are not distinctly known, but the natives of Peru seem to have been acquainted with it long before the arrival of Europeans in that country. It is said that in 1638 the Countess de Cinchon, wife of the Viceroy, who had been ill for several months with intermittent fever, was advised by a magistrate of Loxa to try the quinquina, of the use of which an Indian had informed him: it proved successful, and the Countess was cured. Two years later she returued. to Europe, bringing it with her, and distributed it powdered 
("poudre de la Contesse"). This story is by some regarded as apocryphal, but the association of the name with the drug. gives a great air of probability to it.

In 1649 the Jesuits received a large quantity, which they distributed throughout Italy: hence the early appellation of "Jesuits' bark." In 1679 an Englishman named Talbor, a contemporary of Sydenham, sold the secret to Louis XIV., and from that time it has been known as "Peruvian bark," and regularly employed in medicine.

ACTIVE INGREDIENTS.-These may be divided into four groups of varying importance, and of these, three are again resolvable into smaller groups. The primary ones are: (1) Alkaloids; (2) Simple Acids; (3) Tannins; (4) the resinoid Kinovin.

1. Alkaloids.-(A) Quinine; (B) Cinchonin; (C) Quinidin ; (D) Cinchonidin; (E) Aricin. (The so-called Quinoidin is an impure residue of manufacture from which is prepared the amorphous quinine of Liebig.)

(A) Quinine, $\mathrm{C}_{20} \mathrm{H}_{24} \mathrm{~N}_{2} \mathrm{O}_{2}$, exists in all the medicinal cinchonas, but is most plentiful in "yellow bark," occurring in natural combination with kinic acid and kino-tannic acid. It is now largely obtained from cuprea bark, which contains up to 2 per cent. The pure alkaloid is a strong base, of characteristic bitter taste; it completely neutralizes acids, and forms both neutral and crystalline salts with them: it is nearly insoluble in water, freely soluble in alcohol, less so in ether. ${ }^{1}$ The solutions are distinguished by a remarkable blue fluorescence; and excess of chlorine-water, with the subsequent addition of ammonia, produces in them an emerald green. Quinine itself is not employed in medicine: the most commonly used salt is the neutral sulphate, $\mathrm{C}_{20} \mathrm{H}_{24} \mathrm{~N}_{2} \mathrm{O}_{2}+\mathrm{SH}_{2} \mathrm{O}_{4}+7 \mathrm{H}_{2} \mathrm{O}$ (F. \& H.), which crystallizes in tufts of fine silky needles, occasionally in scales. These are very light, so that the mass occupies considerable space. In ordinary states of atmosphere and of storage the sulphate contains at least two equivalents of water,

1 The hydrate, with three equivalents of water, is much more soluble in ether than the anhydrous alkaloid. 
which can be driven off at a temperature of $248^{\circ} \mathrm{F}$., but speedily become re-absorbed.

Of late years, especially in Germany, the neutral hydrochlorate of quinine has been preferred by many physicians, particularly by Binz, not only on account of its superior medicinal qualities, but because it is less subject to the fungus which spoils ordinary quinine solutions. ${ }^{1}$

(B) Cinchonin, $\mathrm{C}_{20} \mathrm{H}_{24} \mathrm{~N}_{2} \mathrm{O}$, is most abundant in the paler varieties of bark. It forms clear, colourless, four-sided prisms, which are soluble in 30 parts of water, and are very insoluble in alcohol and in ether: with acids it forms soluble salts, which do not become fluorescent in solution, and are turned lightish brown-yellow by the chlorine and ammonia test.

(c) Quinidin, $\mathrm{C}_{20} \mathrm{H}_{24} \mathrm{~N}_{2} \mathrm{O}_{2}+2 \mathrm{H}_{2} \mathrm{O}$ (isomeric with quinine), is contained in many varieties of bark: it is less intensely bitter, and less soluble in water and in ether ; it gives a similar fluorescence, and the same colour with the chlorine and ammonia test: the sulphate is much more soluble in water than that of quinine.

(D) Cinchonidin, $\mathrm{C}_{20} \mathrm{H}_{24} \mathrm{~N}_{2} \mathrm{O}$ (isomeric with cinchonin), occurs in large, shining, striated, rhombic prisms, which are less bitter than quinine, anhydrous, and scarcely soluble in ether. The solutions are fluorescent, but do not give the chlorine and ammonia test.

(E) Aricin, $\mathrm{C}_{23} \mathrm{H}_{26} \mathrm{~N}_{2} \mathrm{O}_{4}$, is not used in medicine.

2. Simple Acids. - These are kinic and kinovic.

(A) Kinic acid (or quinic acid), $\mathrm{C}_{7} \mathrm{H}_{12} \mathrm{O}_{6}$, forms large, transparent, colourless tablets, with a strong acid taste; they dissolve readily in cold, much less so in boiling water, and are more soluble in weak than in strong alcohol, nearly insoluble in ether.

(B) Kinovic acid, $\mathrm{C}_{24} \mathrm{H}_{38} \mathrm{O}_{4}$, was for a long time supposed to be obtainable only by artificial (chemical) means from the resinoid kinovin, but is now known to be a natural ingredient of raw kinovin, or at all events of the kinovin which is furnished by cinchonas grown in Java. This acid is probably

${ }^{1}$ It is now probable that quinine is derived from a simpler basic body, quinolin or chinolin $\left(\mathrm{C}_{9} \mathrm{H}_{7} \mathrm{~N}\right)$, which, in its free state, is an oily liquid, and which, by combination with methyl and ethyl radicals, etc., has been made to furnish a new series of organic bases termed kairin and kairolin, $q \cdot v$. 
of greater importance than kinic; it occurs as a tasteless, shining crystalline powder: insoluble in water and in chloroform, slightly in ether and in alcohol, readily in alkalies and in solutions of alkaline carbonates.

3. Tannins.-(A) Kino-tannic acid, and (B) Kinovi-tannic acid.

(A) Kino-tannic acid, as prepared by Schwarz' process, is a bright-yellow mass, easily pulverized, very hygroscopic, and having a sour and astringent taste: friction renders it electrical: it dissolves readily in water, alcohol, and ether. Formula, $\mathrm{C}_{42} \mathrm{H}_{30} \mathrm{O}_{35}$ (?).

(B) Kinovi-tannic acid is clear, transparent, yellow, and somewhat bitter : it is soluble in water and in alcohol, but not in ether.

(Whether either of these acids exists in the officinal cinchonas is somewhat doubtful, but when present the amount is 2 to 4 per cent.)

4. Kinovin (or Quinovin), $\mathrm{C}_{30} \mathrm{H}_{48} \mathrm{O}_{8}$, is an amorphous resinoid body, which can be rubbed into a smooth white powder: of manifestly electric properties, and hygroscopic: hardly soluble in water, very soluble in spirit, somewhat less so in ether: when warmed, it evolves a feebly balsamic odour: the taste, though slight, is sharp, and unpleasantly bitter; the reaction is neutral. Dry distillation with lime develops metacetone and resinoid bodies: heating with strong nitric acid causes the evolution of red fumes: concentrated sulphuric acid gradually dissolves it with a dark-red colour: hydrochloric acid vapour conducted into an alcoholic solution causes, as above stated, the development of kinovic acid along with kinova-sugar.

Cinchona-red, $\mathrm{C}_{28} \mathrm{H}_{22} \mathrm{O}_{14}$, is contained in all cinchonas, most in the "red." It is soluble in alcohol and in alkaline solutions; scarcely in water. Proto-catechuic acid has been obtained from it.

The odour of cinchonas is due to a small amount of volatile oil.

There are certain chrmical differences between the various kinds of officinal bark, and especially in the percentage $(\Lambda)$ of alkaloids; (B) of tannin; (C) of quinovin; and (D) of kinovic acid. 
(A) As to the alkaloids, the P.B. requires ycllow bark to contain about 2 per cent. of quinine, but good specimens often have 4 to 5 per cent. (scarcely any cinchonin) : red bark contains about an equal quantity of each, about 4 per cent. altogether; pale bark has only half the above percentage of alkaloids, chiefly cinchonin. The species of cinchona mostly cultivated in India are the "succirubra" and the "lancifolia": quills of the former often have 9 to 11 per cent. of alkaloids, onesixth being quinine; the lancifolia contains a larger proportion of quinidin and cinchonidin. Now that the South American supplies of bark are somewhat inferior, Flückiger considers the "succirubra" the best to adopt as officinal (Pharm. Journ., 1881).

(B) Of tannin, red bark contains about $3 \cdot 2$ per cent.; yellow bark about 2.5 per cent.; pale bark much less. M. Tanret found the proportion of alkaloids and tannin in different extracts to vary considerably (Pharm. Journ., ii., 1883). The process of "shaving" the external bark also alters the proportion in that which grows under it, and is now much imported.

(c) The proportions of kinovic acid and kinovin in the different varieties of bark have not yet been exactly determined, but from the researches of De Vrij we know kinovin is abundant in yellow bark, and it will be interesting to learn whether these two constituents occur largely in the officinal pale bark, and other kinds which are weak in alkaloids, since it may sometimes be desirable to obtain their effects without material admixture of the alkaloids, yet with a small amount of tamin-astringency.

ABsorption AND ELIMINATION. - Quimine is not absorbed by the unbroken skin, but readily from abraded surfaces, the cellular tissue, and mucous membranes. In the stomach the neutral salts of the alkaloid become more soluble under the influence of the hydrochloric and lactic acids of the gastric juice; but in the intestine the alkaline secretions would lessen their solubility and absorption were it not for the carbonic acid present (Kerner).

In any case, the larger part of a modernte dose enters the circulation, since very little is found in the dejections. 
Elimination is rapid, and occurs by every excretory channelthe urine, the perspiration, tears, bronchial mucus, saliva, milk, ete. ; the greater part is effected by the kidney, determining sometimes a stimulation of that organ and of the bladder which may amount to congestion or inflammation. It may be found readily in the urine, twenty to thirty minutes after administration, by testing with ioduretted solution of iodide of potassium (Bouchardat's test). The amount discharged goes on increasing for several hours, then diminishes, continuing slightly for four or five days (Briquet); if a daily dose be given, the same process goes on till the administration is omitted, and for some days after. According to Thau, however, nearly the whole dose taken is discharged as quinine in the course of about twelve hours (Practitioner, ii., 1869). According to Guyochin, the quinine excreted in the urine is in a "modified," though isomeric form; in any case it is agreed that the amount excreted is relatively large, so that the drug seems not to remain in the system, but to pass through it. The more recent researches of M. Personne indicate, however, that only half the amount of quinine taken is eliminated by the kidneys, and this in an unmodified form (abst. Lancet, ii., 1878).

It is interesting to inquire whether more quinine is retained in the body when the drug has been given to a fevered, than when it has been given to a healthy subject; but the only difference shown by Thau's researches is, that in the former case the second period of six hours, and in the latter the first period of six hours, is the time during which the greatest elimination occurs.

Kinic acid is said to change into hippuric in herbivorous animals (Record, 1879). The researches of Bence Jones and Dupré show that quinine (or a substance closely resembling it) is, in minute proportions, a natural constituent of the body.

Physiological Action.-The action of the cinchonas upon the living organism is complex, and we cannot yet exactly distinguish the effects of the bark itself from those of the several ingredients; it will be well, however, to consider them separately.

The action of the former has not been investigated like 
that 'b the alkaloids, and we cannot say whether the different species operate in ways theoretically corresponding with the different proportions of their ingredients; we know, however, that all of them, when given in very large doses, produce the phenomena known as "cinchonism" (described under Quinine), but long before these phenomena commence, certain other disturbances occur.

Upon the mucous membrane of the alimentary canal, cinchona bark produces effects which are probably due to the astringent ingredients. Jörg found that when powdered, and taken in 2-drachm doses, it caused flatulence and eructation, sometimes with nausea, but more frequently with improved appetite; usually with some constipation, though, according to Gubler, more energetic peristalsis, with frequent calls to stool, is noticed: there were no other symptoms, except nocturnal erections in one of the experimenters.

It has been stated that the bark can produce intermittent fever, but this is incorrect. No doubt large doses, taken by very susceptible people, may so upset the nervous system as sometimes to induce a condition somewhat like it; but it is now admitted that the drug does not induce a periodic recurrence of attacks like those of true intermittent fever (v. p. 360).

Physiological Action of Quinine.-It is necessary to consider quinine first in its physiological relations to protoplasm, and afterwards as to its action on the various organs of the body. That it is, in large doses, a protoplasm-poison was first discovered by Binz, and subsequently verified by many other physiologists, notably by Buchanan Baxter (Practitioner, ii., 1873).

In 1849 Buchheim and Engel observed that quinine checked the progress of alcoholic fermentation; and later, the interesting researches of Pasteur directed general attention to the importance of the low organisms present in fermenting liquor. In 1868 Binz published his first researches, showing that quinine restrains the growth and movements of protozoa, and in large amount destroys their life: by the same observers, and by Scharrenbroich and Martin, it was further shown that a similar influence was exerted on the development and the migration of the white corpuscles of the blood; and although there have 
been disputes as to the degree of saturation of the blood with quinine required to arrest the movements of the corpuseles, there is no doubt of the existence of such an action, and it may be carried to an extent fatal to life (B. M. J., ii., 1881). Ceei has shown that 1 part of hydrochlorate of quinine in 800 of water will prevent development of germs (ibid.). The observations of Klebs and Crudeli as to the infective particles of malaria support the same views (Practitioner, ii., 1879). According to Donath, tartrate of quinolin, 0.2 per cent., prevents fermentation of milk and decomposition of urine; 0.4 per cent. prevents putrefaction of blood; 1 per cent. destroys its coagulating power (B. M. J., ii., 1881). In Buchanan Baxter's investigations, hydrochlorate of quinine was dissolved in 0.75 per cent. solution of common salt; a large drop of this mixture having been placed on a slide, the cut surface of a newt's tail was touched with a clean cover-glass; a few seconds having been permitted to elapse, to allow of coagulation beginning, the cover-glass was inverted on the drop. The excess of the fluid was then removed with bibulous paper, and a ring of oil painted round it to prevent evaporation: the following general phenomena were observed:-

"1. Migraton"y movement. The protoplasmic mass is spread out into a thin film, adherent to the glass, and of a most irregular shape. The outline of the film may be well marked in parts, but is never complete; the blurred portions being beset with fine filamentous processes, which are continually changing in size and number, some being retracted, while others are put out. The aggregate result of the changes of form which the corpuscle undergoes is a change in its position; it migrates from one part of the field to another.

"2. Change of form without change of position.

" $a$. The corpuscle is spherical, non-adherent, its surface velvety or shaggy, giving it an indistinct or blurred outline.

" $\beta$. Its outline is sharp; it is more or less spherical, nonacherent, and exhibits a peculiar waxy lustre. Its nucleus or nuclei are usually invisible. If carefully watched it may be seeu to change its shape very gradually; one part of its surface rising slowly into knob-like protuberances, while another part sinks in to a corresponding extent; the protuberances being 
limited by definite contours, and exhibiting the same waxy lustre as the main body of the corpuscle. No filamentous processes are put forth. Such corpuscles, though their movements are undoubtedly vital, do not change their place. But they are capable of resuming the migratory condition as soon as the cause of their temporary quiescence ceases to operate.

" $\gamma$. The corpuscle may be surrounded by projections of another kind. The main body shrinks gradually into an irregular lump, while minute, translucent spherules of various sizes present themselves round its edge. These spherules do not appear to consist of the same substance as the main bulk of the corpuscle; they may even become detached from it, and float away. The appearances are those which might be presented by a coagulum slowly shrinking and expelling a clear fluid from its interstices; the fluid not being miscible with that in which the corpusele is suspended. Whether a leucocyte in this state may, as in the two foregoing conditions, resume its migratory powers, I am unable to state.

"3. Permanent repose or death. The corpuscle is spherical or spheroidal, with a distinct outline; it does not exhibit any sort of movement; its protoplasm is very granular, and its nuclei are well marked. This condition is irremediable, and is the immediate precursor of disintegration.

"The first of these conditions is that of the highest vital activity; the second may pass either into the first or into the third. It is possible so to graduate the dose of a poisonous agent as to obtain the second or the third condition at will. In the following experiments the exact state of the colourless corpuscles is usually indicated; the discordant results of previous observers in the case of quinia being probably due to a neglect of this exactitude. The condition of the red dises is often noted, inasmuch as they furnish the most delicate test of the neutrality of the liquid in which they float-the faintest trace of acid causing the delimitation, a larger proportion the granulation, of their nuclei.'

Digestive System.-Quinine leaves on the tongue a persistent bitter taste, which induces, by reflex action, increase of saliva. Doses of less than $\frac{1}{4}$ grain have no sensible effect, though, according to Rabuteau, they increase the sensation of 
hunger and the gastric secretion; others state that they slow digestion: larger doses, 1 to 3 grains and upwards, cause nausen-rarely vomiting. The action and the secretions of the bowels are generally lessened under effective doses, although diarrhoea sometimes occurs.

Nervous System.-The special action of quinine upon the nervous system is most clearly seen in the symptoms associated with "cinchonism"-viz., loud ringing noises in the ears, headache, vertigo, slight dilatation of pupils, dimness of vision, confusion of ideas, sometimes delirium; later, apathy, sleepiness, and prostration: 15 to 60 grains cause staggering gait, and sometimes deafness, blindness, and aphonia. In animals a toxic dose has often produced eonvulsions and paralysis of the hinder extremities; in frogs, minute doses increase, large ones diminish, reflex excitability. The lowered sensibility of parts, and the lessened muscular action, are probably due not to direct paralysis of nerves or interference with muscular irritability, but to a diminution of reflex action.

It is not easy to say what would be a fatal dose of quinine for man, since very large amounts have been lately given with only temporary bad effects; but if the stomach were to retain a sufficient quantity a fatal result would follow, probably with convulsions, paralysis, and collapse. The extreme cerebral symptoms are traced by many to a congestive or inflamed state of the brain and membrane; but Gubler connects them rather with anæmia (ischæmia), arguing from the antagonism of quinine and opium. Nothnagel assumes a direct action on the cerebral ganglia; and certainly the effects on hearing and vision are best explained by a direct action on their sensory centres (Practitioner, ii., 1882). The soporific effect of quinine is not constant.

Muscular System.-Quinine in moderate doses stimulates, in toxic closes raralyses, involuntary muscular fibre, whether in the vessels, the uterus, or elsewhere. Many observers--especially the French-trace to this even its power over the heart-action, which is paralysed by very large doses; and the contraction of an enlarged spleen is supposed to be produced by stimulation of its muscular fibres; by Binz, however, it is attributed to destruction of germs and of white corpuscles. 
Circulatory System.-Two drachms and upwards of powdered cinchona, 10 to 15 grains and upwards of quinine, distinctly slow the circulation; smaller doses sometimes quicken it in healthy warm-blooded animals. With the larger quantities the heart-action is slowed, the systolic force diminished, and the blood-pressure lowered. Briquet states that these conditions persist often for several days after discontinuing the drug. Cavazzani has recorded additional evidence that quinine slows the heart and contracts capillaries (Abst. Record, 1879). Cerud finds some primary increase, later a decrease, "from action on heart-muscle;" he traces lowered arterial pressure to the same cause (loc. cit.).

Its influence upon the blood is not yet thoroughly known: accorling to Binz and others, it fixes the combination of oxygen with hæmoglobin, so that the oxygen is not so readily disengaged; Monneret concludes that it lessens the blood fibrin and retards coagulation; Briquet, that the fibrin is at first increased, whilst the red globules are diminished, but under continuance of the drug and disorder of respiration, etc., stagnation occurs in the vessels, and coagulating power is impaired.

The white globules of the blood are affected outside the body, as already described. The lowering of temperature follows from the depression of the circulation, etc. : in health it is little or nit, but in pyrexial conditions, especially in intermittents and in typhoid states, the fall may be as much as $1^{\circ}$ to $3^{\circ} \mathrm{C}$, according to the dose and continuance of the drug. Animals killed whilst under its influence show, soon after death, a lower temperature than the average. It has been held coubtful whether this effect is exerted through the nerrous system, by the medium of a (supposed) hent-regulating centre, or whether it is part of a generalized action on the tissues and fluids; but it is now agreed that such lowering of temperature results from an interference with the oxidation processes of the body.

Ranke showed that the elimination of urie acid was lessened under quinine; and Kerner, that about 9 grains of quinine taken daily, lessened the acid one-half, the urea one-eighth : a dose of 38 grains lessened them very much more; such results hare been amply corrobornted. 
Other (local) poisonous actions of the drug are less constant; one of the most singular is that exerted on the skin of some inclividuals, in whom any dose, but especially a large one, produces irritation of the surface, followed by free desquamation; sometimes the cuticle of a hand, or even of a limb, has come off like a glove or stocking. In some recently reported cases of this kind the dose has been only 1 or 2 grains, and this has been taken at other times by the same subjects without the rash appearing (Med. Times, i., 1879); in other cases any dose invariably gives rise to it. The occurrence, though still not frequent, seems more so lately. Thus, Dr. H. Greenhow (Lancet, ii., 1878) states that in forty years' practice he had never seen it; whilst many examples of it may be found in the journals of the last three or four years. Dr. W. G. Smith refers to ten cases (Dublin Journ., and B. M. J., ii., 1878), and Dr. Farquharson, who showed one at the Clinical Society, describes them as either erythematous, like scarlet fever, or papular, more like measles, or " rose-red patches" with marked gastric disturbance (B. M. J., i., 1879).

Another occasional effect is the quickening of the heart's action and the respiration. In some persons the palpitation and hurried breathing are so pronounced as to cause much distress, although many are insusceptible to this influence.

Genital System.-Bark in large doses has occasionally induced sexual excitement; and there is some reason to believe that each of the alkaloids is capable of producing this effect under certain conditions.

That quinine acts as an oxytocic, or stimulant of the uterine morements, is not absolutely proven, but a large amount of evidence has been collected, during the last few years, in favour of the belief that it does possess this action; the latest facts on the subject are those related by Dr. Rancillia (Practitioner, January, 1874; Union Múd., 1873, p. 800). Freuch and Italian physicians have often observed that quinine administered to pregnant women suffering from intermittents caused abortion (nttributed by other authorities to the malaria, and not to the (lrug), but Iinncillin states that in his practice as a veterinary surgeon, at Caen, he had often found the labourpains of bitches brought on actively, even when ergot had failed, 
by the administration of $1 \frac{1}{2}$-grain doses of quinine at short intervals (op. cit.)

Dr. H. C. Wood criticises this evidence, and reports entirely negative results with pregnant healthy cats; and Chiara (Milan) gave 15 grains twice daily for two days to eight healthy women in the eighth month of pregnancy without any effect; and although certainly many cases of abortion during intermittent fever, after taking quinine, are on record (Practitioner, i., 1881 ; ii., 1879), yet also many hundred instances of the contrary may be adduced (v. Wood, and Practitioner, i., 1880; Lancet, i., 1881).

The balance of professional opinion at present-in America, at least-inclines to the view that quinine does not directly originate uterine contraction, but strengthens it when already commenced as in natural labour; it remedies "uterine inertia" (Record, 1878, 1882).

Physiological Action of Cinchonin.--This alkaloid, formerly supposed to be next'in activity to quinine, is now known to be the feeblest of the active ingredients. Bermatzik in 1867 showed that with dogs the fatal dose of cinchonin was one-fourth larger than that of quinine.

More recent observations (Buchanan Baxter, loc. cit.) confirm this, and show that the influence of cinchonin as a protoplasm-poison, though resembling that of quinine, is weaker than it, and also than that of the other alkaloids. This physiological position of cinchonin corresponds to its place in the therapeutic scale.

Stillé states (1879) that sulphate of cinchonin is more poisonous to dogs and frogs than sulphate of quinine. It does not in man so readily produce buzzing in ears and disordered vision, but is more apt to cause pain and oppression in the frontal region, præcordial distress and faintness, also muscular spasm and exhaustion. He agrees, however, that the dose should be twice as large as that of quinine. It is less bitter, more soluble, cheaper, but less reliable.

Bouchardat, Montaro, Martin, and others consider it "more energetic," and describe the symptoms abore mentioned as caused by it (quoted by Paulien, Man. de Thérap., 1882). 
PHYSIOLOGICAL ACTION OF QUINIDIN.-This probably corresponds in every particular with that of quinine. Baxter ranks it equal as a protoplasm-poison. Peacock found it less apt to disagree with the stomach or disturb the nervous system. Strimpell, however, reports it very liable to cause vomiting, at least in typhoid cases (Med. Times, i., 1879). Dougall, also, in his orv person, when in good health, found 10 grains cause nausea and griping.

Physiological ACtion of Cinchonidin.-The great activity of this alkaloid has only of late been appreciated. As a protoplasm-poison, and probably in every other physiological relation, it comes next to quinine and quinidin, and decidedly above cinchonin. ${ }^{I}$

Bockius reports from toxic doses of this alkaloid given to the lower animals, "congestion of the anterior part of the cerebral hemispheres" (which quinine causes). Like quinine, it lowers the quickened pulse of fever.

On man it is said to occasion less disturbance of nervous system, less tinnitus and vertigo; others report romiting (Record, 1876, 1882).

SYNERGISTS.-Stimulants (in stimulant, not toxic, doses) and spices assist the action of quinine.

Capsicum, ginger, etc, make 20 grains act as well as 30 (B. M. J., i., 1879), and use of this fact is made in "Warburg's tincture" (v. p. 362).

Bartholow includes "all agents promoting constructive metamorphosis-bitters, iron, arsenic, aud acids." Gubler adds those that contract the capillaries and act as tonics to the vaso-motor system, including digitalis, bromides, and ergot.

In all malarious cases where there is any tendency to constipation, or where diarrhoen is absent, the action of quinine is increased by the addition of calomel or, perhaps, a little

${ }^{1}$ It is singular that, just as cinchonidin is obtaining its duc recognition as one of the most powerful alkaloids, evidence has been procured which shows that the Countess of Chinchon (whose cure from intermittent fever originated the name "cinchona") was probably treated with a species of bark particularly rich in einchonidin. 
rhubarb. Further, in pyrexia of doubtful origin, where there is a gouty or rheumatic tendency, colchicum added to quinine acts like a "charm": if there be severe diarrhoa, a little laudanum may be added. This quinine and colchicum mixture is said to be much used in America: my personal experience of it is good.

ANTAGONISTS.-On the other hand, daily experience shows that bromides and hydrobromic acid lessen the congestive head-symptoms associated with quinine.

Atropia antagonizes to some extent the effect of large doses on the circulation (Practitioner, ii., 1880).

Gubler specially selects opium as antagonistic, and stimulants in doses "that paralyse the sympathetic"; also iodide of potassium.

THERAPEutical Action. - The curative effects of quinine and the other alkaloids of cinchona may be distributed into four chief divisions: (a) Antimiasmatic, $(\beta)$ Antiseptic, $(\gamma)$ Antiphlogistic, and $(\delta)$ the special actions on morbid conditions of the nervous system.

$a$. The antimiasmatic action of cinchona alkaloids, by means of which they put an end to the morbid processes induced by the so-called paludal poison, is still the most interesting, as it was the most anciently known, therapeutical effect of bark. It is a fact as familiar to the public as to the medical profession, that intermittent and remittent ferers yield promptly to the influence of bark or quinine; but it must be added that the modus operendi can still only be stated conjecturally.

The theory supported chiefly by Binz and his followers implies that the paludal poison consists of, or is conreyed by, low organisms which enter the body and multiply in the blood; and that quinine exerts a restraining power over their development and multiplication.

The fact that quinime has such a porrer orer the lowest organisms has been amply demonstrated, but the dependence of malarial poisoning upon their entrance into the human body is still among the vexed questions of pathology; and while increasing numbers of observers are inclined to affirm it, I 
see no grounds even now for a more positive statement than the following by Nothnagel, in 1871:- "How far these researches with their concordant results - that quinine checks putrefaction and fermentation by destroying infusoria and fungi-will be able to be utilized for pathology, is still uncertain. It is evident that at a time when people are everywhere discovering living organisms and fungi in pathological processes, it is tempting to apply Binz's researches to explain the value of quinine in malarial, puerperal, septicæmic, and (typhous) febrile processes, but this is impossible so long as the fungoid theory of these affections rests upon so unstable a basis as at present" (Arzneimittellehre, p. 347).

Without venturing to reverse this judgment, it may be admitted, however, that there is an increasing probability that the parasitic theory of the above-mentioned disorders will receive a more satisfactory confirmation in the future. Still, there are able pathologists opposed to the germ-theory of "zymotic" diseases, and those who wish to see the opposite view ably presented may be referred to Dr. Bastian's arguments.

Under Sulphurous Acid (Inorganic Substances, p. 317) will be found the instance of the ship Argo, in which ague attacked only those drinking water which had been stored at a malarious place, and other observations on the subject. Klebs and $\mathrm{T}$. Crudeli (v. p. 439), of Rome, state that they can isolate from malarious soils and air definite microphytic forms capable of separate cultivation; and after successive generations of it, rabbits inoculated with this propagated bacillus will become the subjects of intermittent fever (Archiv f. exper. Path., Klebs, July-Oct., 1879). Still later, Drs. Marchiafava and Valenti detected the organisms in human beings. Laveran, and afterwards Richard, stated the existence of certain organisms during the period of accession of fever developed in comnection with the red corpuscles; the pigment granules are formed in these corpuscles during the growth of the organisms (Lancet, $i$., 1882).

In Intermittent Fevers in the acute stages, there are conflicting opinions as to the best mothod of administration. 'Sie especially Appendix $\mathrm{E}$ to the "Beginnings of Life," vol. ii. Macmillan, 1872. 
Probably any one which ensures the daily absorption into the blood of 5 or 6 grains of quinine would cure most cases of moderate severity, but some authors advocate a single large dose of 20 or even 30 grains immediately before an attack is expected; others, a smaller dose either immediately after a paroxysm or while the final (or sweating) stage still lasts. Other's recommend the continuous use of large doses both during the paroxysm and the periods of intermission; while perhaps the majority maintain that quinine should be given only during the intermissions, and then in repeated small doses (2 to 4 grains). Drs. Chasseaud and MoGraith have reported excellent results from the hypodermic use of the drug, which.also proved extremely serviceable in the hands of Dr. W. J. Moore (Med. Times, ii. 1862; Lancet, ii. 1863).

I have found that in treating the ordinary agues of Great Britain there is a decided advantage in employing large doses only in the intermission. In a case of modernte severity and duration - a tertian, for instance, of not more than three weeks' standing-the best plan is to order one 20-grain close to be taken about an hour before the fit is expected. In quoticlian ague which has lasted for some time, or has become quotidian from being previously tertian or quartan, at least 20 grains should be given every day, also about an hour before the expected attrck is due. In quartan ague it is not always sufficient to give one large dose on the critical day, for this type is frequently more obstinate, and I have found it best in such cases to give one 10- or 15-grain dose every day, some time before the usual hour when the fit recurs.

In the severe forms of intermittent fever met with in tropical countries it is almost always well to employ large doses, and it is often necessary to give the medicine during the paroxysm as well as in the periods of intermission. Pernicious remittents still more often require the exhibition of large and continnous doses; 30 to 50 or 60 grains may be given in very severe cases several times a day, and without any special regnrd to the presence or absence of exacerbation : it is right to say that so experienced an authority as Dr. Maclenn deprecates the use of larger doses than 15 or at most 20 grains, but the limit is too strict to suit all cases. At the present time we have no reason to dread 
giving quinine during a paroxysmal period when necessary, for we reckon it among our most active reducers of febrile temperature, and see no reason for withholding it when other considerations make it advisable to speedily impregnate the system with it. Whether the fungoid theory of malaria be true or not, it is certain that something like the saturation necessary to destroy low organisms is the most effective way of dealing with severe malarial fever. Decided, though not severe, cinchonism should be induced; and as soon as the ringing noise in the ears is fairly developed, there will be in almost every case a marked abatement of fever, and a gentle perspiration occurs as the immediate forerunner of convalescence.

It is not only in cutting short the typical symptoms of malarial fever that quinine is valuable; the majority of the complications of these diseases, of whatever kind, yield better to treatment by quinine than to measures addressed immediately to the suffering organ. Among such complications, one of the most remarkable is the tumefaction of the spleen (ague-cake), and in a less degree of the liver. I have rarely seen benefit from measures locally or specially addressed to these organs; nor indeed will quinine always relieve; but, at least in the majority of such cases, its action in reducing enlarged spleen is not less decided than in cutting short the course of the general symptoms. The same may be said of the intense gastric irritability and incessant vomiting almost universal in bad remittents; nothing "quiets the stomach" so effectually as the production of full cinchonism. The distressing pain and hent of head, which drive such patients "almost frautic," are also better treated by quinine than by local or other remedies for the mere symptoms; and the profession owes much to Dr. Maclenn and other Indian writers for the courage with which they have put aside temporizing measures, and have practised and taught the radical treatment by quinine in sufficient doses.

Although, however, we may have full conficlence in the benefits conferred by a bold use of quinine in acute stages of fever, there is no reason to be reckless in the doses we employ, or in continuing them too long; and here I may notice what I believe to be the sole fact which keeps up belief in the assertion that "bark can produce intermittent fever." Many, 
perhaps the majority, of those who have been seriously affected with malarial fever, and have experienced the marvellous benefit of quinine, get into the habit of taking it upon slight indication of illness, and often in extravagant quantities. This cannot be done with impunity, and often throws the nervous system into a state of great commotion. Now, we know well that such disturbance, from whatever cause arising, is sufficient to bring back the phenomena of ague in a person who has once suffered; there are many, indeed, who live comfortably on condition that they pass tranquil and carefully guarded lives, but who immediately suffer from a relapse of aguish symptoms when they are exposed to harassing emotions or any great distress of the nervous system. And thus it may be said with truth that though quinine does not produce intermittent ferer in a healthy person, its untimely and improper use by a person formerly aguish may reproduce the paroxysm with greater or less severity. I have witnessed examples of this occurrence, especially in Americans and Anglo-Indians who come from districts where malaria appears to be a more serious matter than in England.

The combination of quinine with aromatics, etc., known as "Warburg's tincture" contains $9 \frac{1}{2}$ grains in each fluid ounce -half of which quantity is ordered to be taken undiluted after a purgative, and the other half in three hour's time: this has been known to cut short an attack, and we have the authority of Dr. Maclean for stating that it does so better than the same amount of quinine alone: rhubarb and many spices are included in the original formula, which has been published (Lancet, ii., 1875), and the perspiration which follows its use shows eviclence, by its odour, of the aromatics.

In those affections which indirectly result from an original malarial poisoning it is of much consequence to determine the manner in which quinine may be most usefully employed.

In Neuralgias of malarial origin there is generally a more regular periodicity of attack than in the neuralgias which are independent of such cause; and alvantage should be taken of

1 During the whole period of Messrs. Howard and Sons carrying on the manufacture of quinine they have never known amongst their workmen it case of ague or intermittent fever which could by any possibility l referred to the nature of their employment. 
this fact to apply the principles already laid down. Instearl of continuing a number of small daily doses, it is better to reserve the drug for employment when a paroxysm may be shortly expected. About an hour before this time a dose of 5 to 15 or even 20 grains (according to the degree of the patient's previous use of the drug) should be administered, often advantageously in a glass of sherry; this seldom fails to produce decided effects, either aborting the paroxysm or rendering it much weaker than usual. The same plan is to be pursued before the next expected recurrence; and the neuralgia often disappears entirely after a few such doses. Hypodermic injection, especially of the hydrochlorate, is sometimes most effective (Köbner, Practitioner, ii., 1880).

Epilepsy of malarial origin is well combated with quinine. We cannot, however, put in force the principle of single large doses in anticipation of an attack; it is better to give regular moderate doses-about 6 grains a day for several weeks in succession. So iniportant are the benefits secured by quinine treatment when malaria has had any share in production of the disease, that even a small probability of this ought to induce a trial of the remedy; and the mere fact that the patient has resided for some length of time in a malarious country, even though he may not have had distinct malarious symptoms, is sufficient basis for essaying a cure by quinine; indeed, its almost magical operation in such cases is very remarkable, when compared with its uselessness in ordinary epilepsy.

An unusual complication of malarious disorder is torticollis: this has been cured by quinine, after failure of other means (Record, 1879).

$\beta$. In the Septicæmic Fevers-a large and not very accurately determined class-quinine has a part to play only second to that which it fulfils in the malarious fevers; and here again much depends on the mode of administration. In some examples of this group of cliseases, it is true, there are other remedies that frequently prove more directly and specially effective: thrus, in missingeless, at any rate in the early stages, perchloride of iron is the true, and usually successful, remedy; and in Imerperal fever, as alrendy mentioned, aconite is frequently 
serviceable: but in the whole series of this variety of bloodpoisonings, quinine holds a permanent place as a remedy; for whether in erysipelas, in surgical pyæmia, or any other of the infections specially connected with absorption of putrid matters or emanations, when once a certain gravity of organic disturbance is reached,- - when the fever is excessively high, and the nervous system profoundly agitated and depressed,--there is scarcely anything medicinal which offers the same chance of reducing pyrexia, relieving inflammatory complications, and sustaining the vital powers as large and repeated doses of quinine.

It is important to remember that reduction of temperature and of the excessive combustion-processes leading to generation of abnormal heat are greatly aided by the simultaneous administration of alcohol; and Socin has shown that this helps the organism to tolerate doses of quinine which would otherwise produce inconvenient or even dangerous toxic effects (Kriegschirurgin-Erfahrungen).

In Gangrenous conditions, whether the result of aggravated inflammation, or of a specific or septic poisoning, quinine has often proved itself to be of value. It is commonly said that cinchona in infusion acts better than quinine in those conditions; but such is not my experience, eren when employing the stiphate: while it is probable that the use of the hydrochlorate may lessen still further any disturbing influence upon the stomach.

In ordinary Infectious Fevers quinine is a more doubtful remedy than in septicemic fevers. The administration of very large doses (5 to 20 grains or more, frequently repeated) will often produce a notable amelioration of symptoms, and especially of the febrile temperature, but we cannot be sure that any considerable influence is exerted on the general course of the disease; often a striking, immediate antipyretic effect, even many times repeated with unvarying certainty, seems to count for little or nothing in the final event. The instance of typhoid fever serves well to test this matter. In our own country the use of quinine in this disease was introduced but a short time ago by the late Dr. Fuller, and both here and abroas it has been fully tested: that it is at least harmless seems to have been established, though this is contradicted by some observers (Practitioner, ii., 1881); and as much as 3 or 4 drachms have 
been given in divided doses during the twenty-four hours, without injury, and often with benefit. Upon this point it is interesting to quote the recent authority of Dr. Clifford Allbutt, who has used quinine largely in febrile diseases; but in typhoid, neither he nor any other high authority claims to prove either that it is shortened in its course or that its more serious consequences are materially hindered. Neither those who place their main reliance on the removal of heat per se, nor those who think it most important to strike at the combustion-processes which are the origin of abnormal heat, appear now to believe that quinine alone will effect any very material purpose; and the same may be said even more certainly of typhus fever.

There are, however, some of the acute fevers which apparently benefit more by quinine, and especially scarlet ferer, as pointed out by Dr. Peter Hood. In this disease it does appear to be the fact that quinine used in moderate doses from the first has a remarkable effect in averting the graver complications. It is probably not a mere coincidence that quinine thus proves most beneficial to that member of the group of contagious fevers which has some of the closest relations, and even a partial convertibility, with such septic disorders as puerperal ferer.

Besides the direct action which quinine may exercise upon uncomplicated fevers, its use may be specially called for in those septic infections which so frequently occur as complications; as familiar examples I may mention absorption of putrid matters from the throat in scarlet fever, and from the bowel in typhoid fever. In such cases, quinine, with more or less aid from alcohol, offers one of our best resources, and should be given in large and repeated doses.

Whooping-cough is a disease which can as yet only doubtfully be reckoned of septic origin, but after the observations of Biuz it must be considered at least probable that this is the case, and that quinine in full doses is one of the most appropriate remedies.

The drug can be readily given by inhalation of solutions, and this procedure is very useful in many conditions of the larynx, bronchi, and air-cells in which a local antiseptie effect is required; 8 grrains of hydrochlorate, or 16 grains of sulphate, 
may be dissolved in 20 ounces of distilled water, and used in the form of spray. Insufflations of quinine in powder, with salicylic acid, and also with chalk, have been recently much praised for whonping-cough (Record, 1879), but are somewhat disappointing.

In Diphtheria, Zinke has reported well of the same treatment (Record, 1878); and in diphtheritic conjunctivitis, Tweedy certainly obtained excellent results with quinine compresses and local syringing (Lancet, i., 1880).

Chronic Cystitis.-Mr. Nunn has shown the value of quinine injections into the bladder in such cases (Lancet, i., 1878): this treatment is now widely employed, and with good results; 1 ounce of quinine solution containing 2 grains being injected once or twice daily. The same drug has been used locally for gonorihcer.

In Hay Fever, quinine certainly proves beneficial, probably by virtue of its antiseptic powers. The observation was first made by Helmholtz, and subsequently confirmed by Binz, that the injection of a solution of quinine into the nares checked the irritative catarrhal discharge, and also the spasmodic symptoms: I have used 2 grains to the ounce with much advantage.

The Hectic Fever of some chronic diseases is, in a certain number of instances at least, partly due to septic infection. In many examples of phthisis with lumg-softening the rhythmic recurrence of fever is as much due to absorptiou of putrescent matter from cavities, as to exhaustion of the nervous system: also the hectic attending large chronic abscesses connected with carious bone partakes of the character of pyæmic poisoning in minute repeated doses. In both these examples the influence of quinine can ordinarily only be effective when it is given in considerable quantitiesfrom 12 to 20 grains daily. In many examples of septic ferer in chrouic disease, however, there may be good reasons against the administration of quinine, e.g., irritability of the stomach and intestines; but the hydrochlorate will occasionally be better tolerated than the sulphate.

$\gamma$. The employment of quinine in Inflammations is a large subject, which has only been incompletely studied. From time to time some author has strongly asserted its merits in 
regard to a particular inflammatory disease-such, for instance, as peritonitis, in which it was so warmly recommended by Trousseau. Others, again, have expressed in general terms the opinion that quinine is anti-inflammatory; but no scientific basis for this belief existed previously to the observations already mentioned, in which the multiplication and wandering of white blood corpuscles was seen to be checked by the remedy. It may now be considered as established that quinine is indicated in most inflammations, especially of the acuter sort, and its use is only contra-indicated by special peculiarities in the phenomena of the disease:-for instance, the stomach may be unable to bear sufficient doses, when we may try some other form or mode of using it: thus, the hydrochlorate may be borne better than the sulphate,- or injection per rectum, or the subcutaneous method may be advisable. Again, some patients are cinchonized so easily as to make it nearly impossible to give them doses large enough to affect the inflammation, and in a certain proportion of such cases this toxic effect may be mitigated by the simultaneous use of alcohol-a fact equally true of double pneumonia as of traumatic erysipelas (Socin, op. cit.).

In some rare cases that can only be attributed to idiosyncrasy it appears that not merely ordinary cinchonism, but even fatal results have occasionally ensued upon the use of doses apparently not excessive; but such a misfortune is again less likely to occur if the patient be simultaneously treated with alcoholic stimulants.

It would seem that in inflanmatory fever quinine rarely, if ever, tends to aggravate delirium, and if the patient escapes cinchonism, his nervous centres prove usually more tolerant of the drug than they would be in health.

To say, however, that because the difficulties now described do not seriously hinder tho alministration of quinine in many inflammatory cases, therefore it should almost always be administered, would be saying too much, for there nre several other antiphlogistic remedies which, in particular instances, nre suluerior to it.

In Preumonia, at least whilst the skin is dry and pumgently loot, it is much better to commence with aconite, everu if we afterwards employ quinine. 'The action of the former' 
in rapidly dilating cutaneous capillaries and inducing a large transpiration and evaporation from the skin has already been described; and when this action has resulted in lowering the temperature of the body, and thus relieved the distress of the nervous centres, it may perhaps be found that the ead of active inflammation is already reached, and resolution is about to take place. In many cases, however, especially of double pneumonia and such as occur in consequence of general blood-poisoning, of renal disease, etc., no such simple course can be expected; the inflammatory fever will soon regain and maintain its activity, and then quinine with alcohol will probably become our best resource. Still, this is not always the case; and in many instances where a severe pneumonia has complicated an acute or subacute nephritis, digitalis afforded relief superior to any effect of quinine that I have witnessed, even in more favourable cases.

In pneumonia markedly asthenic from the first, the use of quinine in about 5-grain doses every three hours was shown by Sir Dominic Corrigan to be most valuable, especially if preceded by local blood-letting.

Nervous Diseases.-Insolation, or "sun-stroke," or heatapoplexy, is perhaps the principal malady to be considered under this heading; and very remarkable results have been obtained in it with quinine, especially when given by subcutaneous injection. Patients absolutely comatose have been revived within a quarter of an hour by 5 grains thus given; generally 20 are advised (Waller, Hall, and others, Practitioner, June, 1876, and Lancet, ii., 1878). It probably acts by a strong impression on the nervous centres, rather than on the corpuscles, as suggested by Binz.

In Neuralgia, quinine is still considered the sheet-anchor by many practitioners, although the error of such opinion has been pointed out by high authorities. If we exclude the cases partially or wholly due to malarial influence (which form n very small proportion of the neuralgias actually emcountered in practice), it will be found that quinine has not an extensive sphere of curative action. It has some unexplnined preferential influence on the neuralgias of the ophthalmic division of the fifth nerve; but upon neuralgias of other 
merves it frequently fails to produce any decided impression, and not only so, but in some patients much harm has been done by treating an ordinary neturalgia with repeated and increasing doses of it; the nervous system gets seriously upset, there is more or less marked cinchonism, and the pain is aggravated. It is a stereotyped remark in medical works, that the more exactly a disease conforms to a regular type of periodic exacerbations, the more surely will quinine prove useful, but as a general proposition this is untrue. It is only when the neuralgia is due to actual malaria that the rule holds; and in the case of recurrent pyrexias or of hectic, this maxim will fail us unless there be either malaria or else septic poisoning at work.

Among non-malarial neuralgias there are none so typically periodic as migraine; often the attacks occur with the strictest regularity every month or fortnight, yet there is no disease in which quinine is less of a specific: its failures are much more numerous than its successes.

In cases of pain partaling of both rheumatic and neuralgic character, the salicylate of quinine has sometimes acted remarkably well (Lancet, i., 1880). I have given it with much advantage.

Rheumatism.-Dr. H. Greenhow has published an important paper giving results of treatment in forty-three cases of acute rheumatism by a combination of quinine (about 8 grains daily given in pill) and iodide of potassium (20 grains). In about half the number pain ceased and temperature became normal in five days after treatment commenced; but the average stay in hospital was thirty-six days, so that no striking success was obtained (B. M. J., i., 1882). Dr. Garrod speaks well of a combination given in liquid form with potash carbonate, and I have sometimes used it with advantage; but of course one cannot judge of quinime-effect alone, in such combination.

In Epilepsy, when caused by malarin, as already said, quinine is lighly effective, and by various authors it has been considered applicable to the simple non-malarious disense. No such pretensions, however, would at present be supported by our prineipal authorities on nervous diseases. That it "acts 
well with bromide and belladonna" (Practitioner, i., 1881) is no proof of its oum virtues.

Epilepsy can, unhappily, be studied on an immense scale at the Londom hospitals and dispensaries; and no one who has had such experience would now entertain the least hope of doing good with quinine, except in cases of a special nature, which occur but rarely: even of these a considerable proportion are probably obscurely malarial in origin, though the fact escapes the practitioner's observation.

In Tetanus, also, quinine has lost in the estimation of practical physicians and surgeons the high place it once seemed destined to fill. No doubt the variety in which malaria plays a part will be benefited by it; but of the ordinary European eases, traumatic or otherwise, those that recover do so probably from purely natural causes, independent of remedies.

I am reluctant to negative all attempts to benefit tetanuspatients with quinine, but think that in severe cases it would be better to lose no time in applying one of those agents better known to have a direct action in reducing reflex irritability.

In Chorea, quinine has never appeared to me to exert more than a subordinate and almost accidental curative power.

In Chronic Alcoholism, it is much more frequently useful; and, indeed, in all cases of this disorder, where we desire to raise the nervous "tone," quinine has a permanent value: 2 to 6 grains daily are doses large enough.

In "glycosuria from nervous exhaustion" - $a$ condition apparently distinguished from true diabetes-Dr. Worms (Paris) reports much benefit in thirty cases, from quinine, in 3-grain doses twice daily; it markedly diminished the excretion of sugar.

In Laryngismus Stridulus, quinine is a most valuable remedy, although the idea that its action in this disease is simply "nervous" is probably erroneous. Laryngismus is part of a general abnormal state in which the organism is affected in a peculiar manner, and in which we usually observe some of the symptoms of Rickets. In what way quinine so benefits this condition we are not able to tell; but it las appeared to me that we can trace, in many cases, a rapid alteration of that fault of assimilation by which the phosphates, 
instead of being applied to the nutrition of the tissues, are thrown out uselessly in the urine. I do not hold with some authors, that it should be given only in the intervals of the paroxysms, and in very large doses; but I believe it better given in sinall repeated doses, without interruption, using enemata if the child cannot swallow.

In Asthma, any benefit derived from quinine would be traced to an influence on the nervous system; but this does not seem to me the whole explanation.

We have the high authority of Dr. Hyde Salter for its use as the best of all tonies; but he generally gave it with iron and mineral acids, and this agrees with my own belief that the good effect of the drug is exerted not only on the nervous system, but also, and probably more decidedly, on the general nutrition of the body, and on the digestive process. It is one of the greatest troubles of asthmatics that they cannot take more than a very small quantity of food at once without the danger of exciting a paroxysm; under the influence of quinine, or quinine and iron, this disability often much diminishes, to the marked benefit of the patient.

In cases of spasm of the glottis, of pertussis, and infantile convulsions, the hydrobromate of quinine has been specially recommended (Record, 1877).

In Erythema Nodosum, quinine has long been noted for its beneficial action; indeed, it is usually the only remedy needed in addition to a few days' rest; and its exhibition renders the course of the affection shorter than when left to nature. We should probably be justified in reckoning this as one of the examples of the action of quinine upon the nervous system, although we are unable to prove the dependence of erythema nodosum on purely nervous causes.

In Urticaria, quinine is only useful in special cases. The tendency to nettlerash is very personal; in the commoner kind of cases the individual is always liable to an attack after enting freely of some particular food, such as shell-fish, salmon, or' strawberries. To such patients, when they have thus brought on an attack, we need only give an emetic, or an emetic and a purge. But there is a kind of chronic urticnria, in which, though it is more easily induced if indigestion be present, the 
true source of the disease lies evidently in some peculiarity of the nervous system; and medicines directed to the digestive organs cannot be expected to do much good.

In some of these cases arsenic is the best remedy; but in most of them the long-continued use of quinine and mineral acids overcomes the tendency more surely.

In Herpes Zoster quinine often acts well.

In Insanity we can scarcely speak of it as exerting a special effect; but there are many cases in which its tonic power, especially when combined with iron, is very useful (Maudsley).

As a General Tonic quinine produces most decided benefit when the flesh is flabby and the skin too perspiring. In the night-sweats of phthisis it is often well combined with other remedies (Practitioner, ii., 1879).

As a Tonic to Digestion: In atonic dyspepsia, quinine is probably inferior, on the whole, to cinchona in combination with mineral acids; but in some cases the symptoms are accompanied by dilatation of stomach, and fermentation of the retained food, which after a time is passively vomited, often in immense quantity: in this disease there is much reason for thinking that the fermentation is not merely an accidental consequence of the primary affection, but directly aggravates the mischief to. such an extent as to become almost the more important part of the morbid condition. Upon this supposition various antiseptic and antifermentative remedies have been proposed, none of them probably having a more powerful action of the lind required than quinine.

\section{The Therapeutical action of the other} CINCHONA ALKaloids needs but few words after what has been said. The extensive comparative trials made by the Indian Commission brought out the general fact that all the four alkaloids-quinine, cinchonin, quinidin, and cinchonidin -act essentially in the same manner against intermittents; and that cinchonin is weaker than the others. These facts, in conjunction with the observations of Dr. Baxter (showing that the same relations exist in the physiological activity of the respective alkaloids), make us regard the question 
as practically settled; and we have only further to deal with the matter of expense, or such special points as the facility with which one or other alkaloid can be prepared for hypodermic use.

Dr. Dougall, in a prize thesis (Edinburgh), reports upwards of 100 cases of intermittent fever occurring in India, and treated-
39 with quinidin (most active);
35 with cinchonidin (next);
34 with cinchonin (least active).

There was little difference in the duration of the cases, which almost all benefited equally if sufficient doses were given (in proportion to the power of the alkaloid); they were found to act best if given during the paroxysm ( $v . p .360)$, whilst quinine given at that time more often caused headache: it, however, seemed to prevent relapse better than the others. The use of all was commonly followed by "bilious purging," which did good (Edin. Med. Journ., Sept., 1872).

The use of the mixed cinchona alkaloids-a preparation which is much less expensive, since the cost of separating, etc., is saved-has been objected to on the ground of insolubility and of causing more gastric irritation (Lancet, i., 1877). Dr. Hollis has, however, recorded two cases of ague well treated by "quinetum sulphate" - the whole alkaloids of C. succirubra, said to be cheaper and as good as quinine (B. M. J., i., 1879).

Burdel found "quinodin" useful in "telluric fevers" (Record, 1878).

A report from a New York committee accepts cinchonidin as very suitable for medical, but not for surgical cases, especially when the abdomen, bladder, etc, are affected-on account of its tendency to cause vomiting (Record, 1876).

The Therapeutical ACTION OF KINIC ACID is still unsettled : of course its primary interest arises from the fact that, in cinchona bark, quinine exists in combination with it, and that this kinate of quinine is particularly soluble: any special jowers belonging to the acid, as such, we do not know. 
The Therapeutical Action of Kinovic Acid is probably more important. The researches of Kerner (1863) first brought this acid prominently into notice, although it had been thoroughly investigated by Hlasiwitz (1859) from the chemical side (Ann. Chem. Pharm. B. 112). Kerner directed attention to the fact that kinovate of lime is the active principle in Deloudre's Extract, a preparation successfully employed in diarrhœe and dysentery in various European countries and in India. The same salt is said also to be useful in true intermittents, but this requires confirmation. Since the year 1869, when kinovate of lime was introduced into London by Messrs. Hodgkinson, a few physicians have occasionally employed it; but there has been no general adoption of it, and it seems to have been forgotten. One circumstance is worthy of mention, viz., the suggestion of some continental authorities that kinoric acid is especially extracted in making cold infusions of bark, and many good observers have preferred such preparation to any other, particularly in febrile conditions with irritability of the alimentary canal.

\section{The Therapeutical Uses of The TANnIC Acids} OF BARK have not been studied with any care, although they well deserve it; in them evidently resides the powerful and peculiar astringency which the cinchonas possess, and which enables us to administer bark where we could give no other tonic. The separate trial of cincho-tamic and kinori-tamic acids, as remedies for diarrhoa and other fluxes, is much to be desired.

\section{PREPARATIONS AND DOSES OF CINCHONA AND} ITS ALKALOIDS.-Of cinchona itself the only preparations we need notice are the following:-

Pulvis cinch. Alux. vel mb. vel pallid: dose, 5 to $60 \mathrm{gxr}$. Inficsum: dose, 1 to 2 oz. Extructum liquichum (made with yellow. bark): dose, 10 to 40 min. Decoctum: 1 to $2 \mathrm{oz}$. (not dependable). Tincture: dose, 30 min. to 2 dr. (made from yellow bark). Tinct. (inch. romp).: dose, 30 min. to $2 \mathrm{dr}$. (made from pale bark, with aromatics). 
Quinice sutphers: dose, 1 to $20 \mathrm{gr}$. or more. Pilula quinice: dose, 2 to $10 \mathrm{gr}$. [A more soluble pill is made with 3 parts quinine to $\frac{1}{2}$ part tartaric acid: glycerine, q.s. The di-sulphate is said to be still more soluble, and has been adopted in several American pills (B. M. J., i., 1882).] Tinctura quinice: dose, 1 to $2 \mathrm{dr}$. (The tincture commonly sold is said to be much adulterated.) Vimum quinice: dose, $\frac{1}{2}$ to $1 \mathrm{fl}$. oz. Tinctura quinice ammoniate ( $1 \mathrm{gr}$. in $1 \mathrm{dr}$.): dose, 1 to $2 \mathrm{dr}$.

Quinine is now sold in definite doses in wafer, or wafer and chocolate, or in capsules: the chocolate covers the taste. Sugar and gelatine-coated pills are also obtainable. Many practitioners, however, think, and with good reason, that quinine should always be dissolved to aid its absorption. If given in the liquid form, mineral acid, lemon juice, or a little acid out of a seidlitz powder must be added to dissolve it: milk is the best vehicle for administration to children, the bitter taste being in this way well disguised. Quinine solidifies if given with much carbonate of ammonia.

Quinice hydrochtores (neutral): dose, as an ordinary tonic, 1 to $2 \mathrm{gr}$. thrice daily; as an antineuralgic, from 3 to $20 \mathrm{gr}$.; in the acute septic diseases, up to 60 and 120 gr. in the twenty-four hours. (The neutral salt should be crystalline; the acid hydrochlorate forms an undesirable gummy mass.)

The hypodermic injection of quinine, from the pain it causes, is not likely to be used, except in those cases where a certain and rapid action of the drug is required, and where its good effects predominate over the lesser evils of abscess and swelling. A solution of the ordinary sulphate may be employed. Ranking states that the neutral sulphate ( $1 \mathrm{gr}$. in $10 \mathrm{~min}$. of warm water) has answered well, 5 min. injected at a time causing no) ulceration, and rarely any inflammation (Record, 1878). Neither sulphuric, acetic, nor (in spite of Bricheteau's assertim) tartaric acic can be used as a solvent of quinine in the hypodermic solution without running the risk of local inflammation and abscess-though the tartrate has been recently again advocated (Lancet, ii., 1881). The sulpho-tartrate is said to be the best preparation for hypodermic injection, owing to its greater solubility (Ziemssen, Encyclopæclia, vol. ii., p. 664). The kinate is also considered very suitable (Pharm. Journ., 
1878). An aqueous solution of the neutral hydrochlorate is a good form of administration, and though $1 \mathrm{gr}$. required 25 min. of distilled water for solution, yet this amount is equal to 3, if not 4 , grains given by the mouth. Before injecting, the needle should not only be perfectly clean, internally and externally, but should be dipped into carbolic acid lotion, and then driven in to some depth, with its aperture turned away from the under surface of the skin.

Quinine may be given in enema.

Of other salts of quinine, I shall simply mention the following:-

Citrate (rather insoluble). Ferrocyanide.

Acetate.

Kinate.

Hydrocyanate.

Tannate.

Trate.

Tartrate.

Lactate.

Acid lactate.
Valerianate (1 to $3 \mathrm{gr}$.).

Stearate.

Nitrate.

Phosphate.

Bisulphate.

Sulpho-tartrate.

Carbolate (2 gr.).

Salicylate (1 to $2 \mathrm{gr}$.).

Dr. Cameron has specially recommended the bromate and the ioclute as more active than the ordinary compounds, the extra oxygen, it is presumed, contributing to this.

The lactute and citrate, though not so strong and less reliable than quinine, are suitable for children; they are not so bitter (Med. Times, ii., 1880).

There are other salts of quinine which are rather to be considered as preparations of some other substances, such as the arseniute (dose, $\frac{1}{10}$ gr.), antimoniate, hydriodate (gr. j. ter die), ete. In short, the ingenuity of chemists has been somewhat unprofitably exercised (so far as mere pharmaceutical purposes are concerned) in multiplying preparations of this alkaloid without good reason or result.

The so-called citrate of quinine and iron is not always dependable: some wholesale chemists sell two qualities ("A" and "B"), the cheaper of which contains little, if any, quinine. In many important cases, it is better to combine them as required, viz., sulphate of iron and sulphate of quinine, in 
mixture or pill; citrate of iron can be given with quinine in effervescence with citric acid and potash or ammonia; an acetate of iron and quinquidin has been well spoken of (Practitioner, ii., 1880).

Sulphate of quinidin (solubility in water, 1 in 108) acts much like that of quinine in similar doses. The drug of the shops in many cases used to be sulphate of cinchonidin; it should yield the tests above mentioned under quinidin.

Cinchonin sulphate (soluble in 66 parts of cold water) : dose at least twice as large as that of quinine or quinidin salts. The acid sulphate is more soluble, and is convenient for hypodermic injection in doses of 1 to 5 or $6 \mathrm{gr}$. : it has some tendency to irritate.

Cinchonidin sulphate is about one-sixth or one-eighth weaker than quinine or quinidin salts: the dose must be judged accordingly.

Of quinetum and other preparations of "mixed alkaloids," the dose is about the same as that of quinine: the solubility is less.

Quinovic acid-quinovate of lime: dose, in powder, 2 to $8 \mathrm{gr}$. every hour.

\section{CHINOLIN.}

This was first discovered by H. Skraup, in quinine and cinchonin. It is now manufactured synthetically on a large scale by Strauss' method, viz., by heating nitro-benzol, anilin, and glycerine in the presence of sulphuric acid. Bach and Loimann found that in rabbits $\frac{1}{10}$ gramme caused a fall in the temperature of $0.3^{\circ}$ to $1 \cdot 1^{\circ} \mathrm{C}$. Within an hour the fall was succeeded by a rise, in many cases exceeding the original tempernture. Larger doses (3 to 5 grammes) caused a very considerable and permanent fall in temperature, usually ending in death: the respirations were increased in number, irregular, and superficial. Doses of 0.2 to 0.3 gramme renderer the animal helpless and dull, reflex excitability being diminished. Doses of 0.6 to 1 gramme caused complete paralysis, with loss of all reflex action; death in collapse. $\Lambda$ cumulative effect was not observed. 
After death, hyperæmia and odema of the lungs were found. In a healthy man, Donath found that on the first day 1 gramme produced no effect, and on the second day none was observed from 1.5 gramme in three doses. The urine showed no chinolin, so it was supposed to be oxidized in the blood.

As an antiseptic, chinolin is very useful; it preserved blood liquid for twenty-eight days, and kept milk from coagulating for sixteen days: in solution it will not precipitate albumen (B. M. J., ii., 1881).

Chinolin has been given with advantage by independent observers in cases of malaria, whooping-cough, typhoid ferer, and many other complaints. As it is insoluble in water, it is more convenient to use the tartrate or salicylate: dose, 5 to $15 \mathrm{gr}$. , and as the taste is somewhat disagreeable, it is well to add a few drops of spirit of chloroform and syrup of orange; or it may be given in millk.

\section{UNCARIA GAMBIR.}

\section{(Pale Catechu.)}

DESCRIPTION.-A shrub, found in many parts of enstern tropical Asia, climbing, when wild, by means of hooked spines, or modified peduncles; but, under cultivation, compact and not more than 8 feet high. The leaves are ovate; the little green and pink flowers are borne in globular heads.

The catechu (also called gambir) is prepared by boiling the leaves for several hours in a large cauldron, the decoction being eventually poured into moulds, where it acquires the consistence of clay, and is ready for cutting into small cubes, which are then dried in the sun and become quite hard. 
ACTIVE INGREDIENTS.-Pale catechu contains (1) about 40 per cent. of catechu-tannic acid, which is soluble in cold water, but when exposed to a moist atmosphere, tums dark red, and becomes insoluble; (2) catechuic acid-catechin, $\mathrm{C}_{13} \mathrm{H}_{12} \mathrm{O}_{5}$ which is a modification of tannin, but differs in not affecting a solution of gelatine. It changes the persalts of iron to greyish green; crystallizes in snow-white silky needles; is soluble in boiling water, alcohol, and ether, but very sparingly soluble in cold water, which apparently simply softens it, and causes it to swell up; heated it becomes fused; it slightly reddens litmus. The sweet taste of the drug is said to depend upon the presence of catechin.

\section{Physiological Action.-A simple mild astringent.}

Therapeutical Action.-The powder of pale catechu is sometimes sprinkled upon indolent ulcers with good effect: the infusion injected up the nostrils often stops epistaxis. As an internal astringent, it is employed in affections of the mouth and throat, such as relaxed uvula, the troublesome cough induced thereby, and relaxed sore throat, for which purposes it may be sucked or chewed in the form of lozenges: also as a remedy for atonic dyspepsia, especially when attended by pyrosis. It may be given, too, in cases of dirmhere and chronic dysentery, but should be avoided if there be acute inflammatory symptoms, hæmorrhages, mucous discharges, and incontinence of urine. It may be employed in most cases where astringents are indicated, and it is often prescribed with chalk-mixture, opium, ete. The best time for administration is half an hour before meals.

Preparations and Dose.-Pulis: dose, 10 to 30 gx. or more. Pultis cutrechu compositus: dose, 20 to 40 gx. Infuspm: dose, 1 to 1 ! fl. oz. Tinctura: dose, 1 to $2 \mathrm{fl}$. dr. Trochisci: dose, 1 to :3 or more. 


\section{CEPHAELIS IPECACUANHA.}

\section{(Ipecactanha.)}

DESCRIPTION.-An herbaceous perennial, indigenous to moist and shady woods in Brazil, especially in the provinces of Pernambuco, Bahia, and Rio Janeiro. The root is simple, or slightly rounded, 2 to 5 inches in length, 2 or 3 lines in diameter, irregularly bent, and marked for its whole extent with prominent rings, which are unequal and wrinkled. The stem is suffruticose, 2 or 3 feet long, ascending, and thinly clothed with obovate leaves. The small white pentamerous flowers are borne in solitary axillary heads, and are followed by little two-seeded clrupes of a reddish-purple colour.

ACTIVE INGREDIENTS.-The woody portion of the root is nearly inert, the active remedial part being the bark: both portions contain the essential alkaloid, emetin, but in an impure condition, and united with ipecacuanhic acid.

Pure emetin, $\mathrm{C}_{35} \mathrm{H}_{25} \mathrm{O}_{9} \mathrm{~N}$, constitutes about 1 per cent. of the best root; when separated it is a white amorphous powder, inodorous and of bitter taste, soluble in alcohol and in acids; scarcely so in ether or oils; very sparingly in cold, but much more soluble in hot water; precipitated by tamin; fusible at $122^{\circ} \mathrm{F}$. It is difficult to obtain perfectly pure.

The impure form is of greyish-yellow colour, and occurs usually in transparent scales: in taste it is bitter and acrid; is nearly destitute of odour, soluble in water and alcohol, insoluble in ether.

In addition to the peculiar alkaloid emetin, ipecacuanla contains an astringent acid glucoside, $\mathrm{C}_{14} \mathrm{H}_{9} \mathrm{O}_{7}$, somewhat akin to catechin, and called cephaelic or ipecacunuhic acid. It was formerly mistaken for gallic acid; with the persalts of iron it strikes a green colour; it is amorphous, brown, bitter, 
and soluble in alcohol. There is also a trace of volatile oil, with some fatty matter, and a small proportion of starch and g'um.

ABSORPTION AND ELIMINATION.-The former occurs by all surfaces, the latter mainly by the kidneys and by the glands of the gastro-intestinal mucous membrane (Rutherford, Thesis, 1878).

PHysiological Action.-Extemal.-Ipecacuanha is a local irritant both to the skin and the mucous membrane, and when rubbed in, causes an eruption-somewhat like that of antimony-of small separate pristules, with extended areolre, which give rise to severe ulceration, if the application be continued. The inhalation of the powder in ordinary persons produces cough, sneezing, and catarrh, which might be attributed merely to local irritant action, but in certain subjects these symptoms are accompanied by an amount of dyspnoea and feeling of anxiety which is comparable to that caused by spasmodic asthma or the more severe form of hay-fever.

\section{Physiological ACtion.-Intemal.-Digestive System.} Small doses of $\frac{1}{8}$ to $\frac{1}{4}$ grain of the powdered root act in a tonic manner, somewhat increasing the gastric secretions: 2 to 20 grains cause nausea and vomiting, not, as a rule, severe or continuous, nor with much depression: a quarter to half an hour must be allowed for this action. After repeated doses some tolerance is established, and "bilious" purging is more likely to be caused than vomiting. "Sixty grains mixed with a littlo bile and placed in the duodenum powerfully stimulated the liver" (Rutherford and Vignal, loc. cit.). Post mortem, congestion of stomach and intestine is generally, though not always, found, and this whether the drug has been given by the mouth, rectum, veins, or skin: large amounts given by any of these channels are said to have caused ulceration of intestinal glands.

As to whether the characteristic vomiting is primarily due to local irritation or to influence on the nervous system, reference may be made to the remarks under Antimony (Inorganic 
Substances, p. 367), which are almost precisely applicable to ipecacurnha. Emetin produces vomiting more slowly by intravenous injection than by the mouth (Dr. Ornellas, Gaz. Méd., 1873), and the result of Rutherford's latest experiments points wholly to a local irritant action as the primary one.

In animals killed by it, glucose is absent from the liver, according to Pécholier, but this is denied by Rutherford.

Nervous System. - In frogs, emetin enfeebles and ultimately destroys voluntary movements, causing also primary increase and ultimate diminution of reflex activity.

It is presumed to have a special action on the terminal filaments of the vagi in the lungs, because after death the condition of these organs, which is generally hyperæmic and partially hepatized, resembles that found after section of the said nerves (Chouppe). Death usually occurs from paralysis of respiratory muscles. Rutherford reports spinal convulsion, and also deep sleep or coma, from direct action on the brain; but this is not yet corroborated.

Circulatory System.-According to Dr. Duckworth and several other observers, emetin has no definite effect on the circulation, at least until towards the end of fatal cases, and then the heart-action stops suddenly; the organ remains, however, sensitive to galvanic action (Barth. Hosp. Rep., vol. vii.). "There is no proof that the allkaloid causes vaso-motor spasm" (Wood).

By his experiments with emetin on rabbits, Pécholier found that a dose of 0.072 gramme caused immediate excitement, followed by retching and (in two minutes) a remarkable feebleness, depression of circulation and respiration, together with a very curious lowering of temperature in the whole anterior half of the body, while the rectal temperature was raised, from local congestion. If this dose were repeated daily for some time, it was found that the singular temperature-phenomena became more marked, while the excitement and the retching were less so, and death at last took place.

Dissection showed congestion, intense in the stomach, milder in the upper part of the intestines; the lungs were anæmic; the liver contained no glucose. The same results were produced when the remedy was given by the skin. 
Therapeutical Action.-Intemal.-As an Emetic, ipecacuanha is unequalled in its value for a large number of cases. It is not violent, nor very quick in action, but mild and certain, and usually empties the stomach only once; nor is it followed by the collapse which antimony and some other emetics often induce.

Its freedom from nauseous taste (unlike sulphates of zine and copper) renders it advantageous for children: twenty grains of the powder or half an ounce of the wine for an adult, and from one-fourth to one-half these quantities for a child, rarely fail to act, but if necessary may be repeated in fifteen or twenty minutes.

Common Catarrh of the throat and bronchi in the dry stage may be beneficially treated with nearly nauseating doses of the drug; thus from 1 to 2 grains may be given every two or three hours. The smaller dose will induce diaphoresis, and probably also give some relief to the dryness of the mucous membrane; the 2-grain doses will cause a decided increase in the flow of mucus, and in most patients a feeling of nausea, but much relief will be obtained.

There is a different use for ipecacuanha in the later stages of bronchial catarrh, where the secretion is profuse and troublesome. It is then to be given in much smaller doses, $\frac{1}{4}$ to $\frac{1}{2}$ grain of the powder, or 5 to 10 minims of the wine, which possess the power of limiting the mucous secretion, and of improving its quality, diminishing the ropiness which makes it so offensive and irritating. Smaller doses must be given to children, but not in the exact proportion of their age, for they usually bear ipecacuanha very well.

In Catarrh mainly affecting the stomach, ipecacuanha may also prove useful, but needs to be employed more cautiously: the emetic, or even the nauseating doses, would be almost sure to aggravate the inflammatory tendency, and produce unmanageable gastritis; but the smaller doses (5 to 10 minims of the wine) prove highly beneficial. In many cases of chronic catarrhal dyspepsia (and also of atonic dyspepsia independent of catarrhal cause) this remedy is most valuable. In children, acute gastric catarrh is much benefited by it, and so is the moming sickness of chronic alcoholism: it is, however, useless 
in sickness arising from such structural affections of the stomach, as cancer, ulceration, etc.

In several kinds of Vomiting, ipecacuanha is demonstrated to be an excellent medicine, when taken in small doses. One drop of the wine (equal to $\frac{1}{20}$ grain) should be taken in water, and repeated at short intervals till a decided effect is produced. It is now well established that this effect of ipecacuanha is especially, if not solely, produced in cases where the vomiting is sympathetic in character: thus it is seen remarkably in the sickness of pregnancy, for which there is probably no remedy of equal value.

In Hæmorrhage, ipecacuanha acts well. The best doses are those which barely cause slight nausea, -1 grain (or 10 minims) repeated frequently is enough to produce a gentle effect: to cause actual emesis is to run grave risk of heightening the very mischief which we are treating. In the hremoptysis of early phthisis, ipecacuanha may compare with ergot as one of the most valuable styptics we possess. In the passize hamorrhages from engorged bronchial mucous membrane, it is equally effective. In hemutemesis it is of value when the hremorrhage is vicarious of menstruation, but is not so suitable as other remedies.

Dysentery.-In acute tropical dysentery there is now a vast amount of Indian experience to show that the bold use of large doses is most successful. At the earliest opportunity 25 or 30 grains of powdered ipecacuanha are given in a small quantity of fluid, a full dose of laudanum or chloroform being often ordered half an hour previously. The patient is kept still in bed, taking only a little ice or a teaspoonful of water, if thirsty; mustard or turpentine is applied to the belly.

It is surprising how seldom either vomiting or considerable nausea occurs, and in eight or tem hours the remedy can be repeated in diminished dose. Under this trentment the purging of blood and slime, the abdominal pain and tenesmus, soon disappear, the motions become feculent, and there is often a profuse sweat, followed by refreshing sleep; smaller doses are continued for some days, taking care to keep intervals free for the administration of food: even when the motions 
have become healthy, 10 or 12 grains should be given at bedtime for two or three nights.

This treatment is applicable to the great majority of patients, but some are much more sensitive than others; and in such exceptional cases 2- or 3-grain doses, with opium and grey powder, often prove successful.

The general utility of the large doses above described has, however, been conclusively shown by Dr. Maclean, who ably sums up the modern evidence, including his own extended experience, in his article on Dysentery in Reynolds' System of Medicine, vol. i. The credit of reintroducing the plan is due to Mr. Docker (Lancet, ii., 1858), and it has been supported by Dr. Ewart (Lancet, i., 1884).

It is interesting to notice that while ipecacuanha is, par excellence, the treatment for acute dysentery of the ordinary type, which so commonly alternates, epidemically, with outbreaks of intermittent and remittent fever, the other important plant in this family (cinchona) is equally successful in dysentery when complicated with malarial poisoning: in such eases, large doses of quinine perfectly replace the ipecacuanha treatment.

In Diarrhœa, especially when dependent on nervous irritation, and hence particularly in the diarrhœa of young children, and when there is also vomiting or retching, ipecacuanha is a powerful remedy. The wine in 3-minim doses every two or three hours will often check the disorder, even when it has begun to take on pseudo-dysenteric characters with blood and slime. If more convenient, it may be used as an injection in the proportion of 2 drachms of tincture to 8 ounces of water; the drug is added to the water, which should be allowed to boil for one or two minutes.

In Spasmodic Diseases of the Respiratory Organs ipecacuanha is of great value. In the paroxysms of nerrous asthme it was especially praised by the late Dr. Salter, who gave it, however, in one large emetic dose at the outset. This is often very trying, and I prefer repeated 5- or 10 -minim doses of the wine every ten to thirty minutes until substantial relief is obtained, which indeed will often happen, even in severe cases, quickly. In whoojing-cough, 
doses of 1 minim for children under five years, or 2 minims for older patients, may be given every one, two, or three hours with great relief. As a cholagogue, although its action is inferior to others of that class, ipecacuanha is a valuable adjuvant to blue pill or calomel; in this combination it is an excellent alterative.

In Simple Inflammatory Croup and in Simple Pneumonia, ipecacuanha has a comparatively limited value; it does not so quickly produce nauseating effects as tartar emetic, and is also inferior to it as a diaphoretic, while both are inferior to aconite.

In concluding this general estimate of the therapeutical powers of ipecacuanha, it is necessary to give a caution respecting the inconvenience which may be occasioned by administering this drug, even in small doses, to those exceptional patients in whom the drug produces catarrh, asthma, or vomiting whenever they take it. If any sick person declares from past experience that he is liable to this, the practitioner should pause before venturing to give even the minutest dose, for the misery which it causes to such persons is very great.

It is well to bear in mind that some persons suffer severely from the smell alone, and have been known to be affected by such a minute quantity as $\frac{1}{32}$ grain.

PREPARATIONS AND DOSE.-Pulvis: dose, as emetic, 10 to $15 \mathrm{gr}$. for a child, 20 to 30 for an adult; as nauseant, 1 to $2 \mathrm{gr}$. every two or three hours; as tonic in catarrh of the bronchial and alimentary mucous membrane, $\frac{1}{20}$ to $\frac{1}{2} \mathrm{gr}$. Vimum : dose, as emetic, 4 to $8 \mathrm{dr}$. for an adult, 2 to $4 \mathrm{dr}$. for a child; as nauseant, 15 to $60 \mathrm{~min}$; as tonic to mucous membranes, and as stimulant expectorant, 3 to $10 \mathrm{~min}$. Trochisci: dose, $\frac{1}{4}$ gr. in each lozenge.

The compound preparations of ipecacuanha are as follows: -Pil. ipecce cum scillâ : dose, 5 to $10 \mathrm{gr}$. Pule ipecac. comp. (Dover's powder) : dose, 5 to $10 \mathrm{gr}$. Trochisci ipecac et morphice: 1 to 6 lozenges in twenty-four hours. 


\section{VALERIANACEAE.}

\section{THE VALERIAN FAMILY.}

Ax order of herbaceous exogens, natives chiefly of temperate parts of the eastern hemisphere. The leaves are opposite; the small flowers usually cymose, with minute calyx, a monopetalous long-tailed corolla, and definite epipetalous stamens; the ovary is inferior and one- to three-celled. The roots of these plants are in many instances bitter and aromatic, and have tonic, antispasmodic, and anthelmintic properties: some are powerful stimulants, exciting the cerebro-spinal system; others are esteemed as perfumes in Oriental countries.

\section{VALERIANA OFFICINALIS.}

\section{(Common Valerian.)}

DESCRIPTION.-A well-known European perennial, growing chiefly in wet places, and flowering in July. The stem is erect, 4 or $\tilde{5}$ feet high, hollow, furrowed, and nearly simple; the pinnatifid leaves are few ; the delicate lilac flowers are borne in large terminal panicles, and are followed by pappose fruits, resembling those of most Compositx. The dried rhizome is from $\frac{1}{2}$ to 2 inches long and the same broad, with numerous slender roundish rontlets attached and generally curled round : those from dry places are preferred as containing more oil ; they are smaller and lighter in colour than those from damp localities; after drying they develop a peculine cumphoraceoussome say cheesy-olour : the taste is mpleasant to most persons. 
ACTIVE INGREDIENTS.-The ethereal oil of valerian contains the active ingredients of the plant. According to Pierlot the oil consists of valeren, 25 parts; valerianic acid, 5 parts; valerian camphor, 18 parts; resin, 47 parts; water, 5 parts. Valeren, $\mathrm{C}_{10} \mathrm{H}_{16}$, the borneene of Gerhardt, is a colourless oil, smelling like turpentine, and changing under the influence of nitric acid into ordinary eamphor; boiling point $160^{\circ} \mathrm{C}$. Valerianic acid, $\mathrm{C}_{5} \mathrm{H}_{10} \mathrm{O}_{2}$, is a colourless oily fluid, with a smell recalling at once the odour of valerian and that of decayed cheese: it has a strongly acid and burning taste; the specific gravity is about 0.945 ; the boiling point about $170^{\circ} \mathrm{C}$. It greases blotting-paper, but the mark gradually vanishes. It is soluble in all its preparations in alcohol, in ether, and in about 30 parts of water; it is now usually prepared by the oxidation of fusel oil or amylic alcohol. Valerian camphor, $\mathrm{C}_{10} \mathrm{H}_{18} \mathrm{O}$, forms white crystals. Several alcohols and ethers, such as those of borneol (valerianic ether), of acetic and formic acids, are formed by the decomposition of the above ingredients.

ABSORPTION AND ELIMINATION.-The latter probably takes place largely by the kidneys, but in part by the lungs and skin. Rabuteau notes that sixty grains of the valerianate of soda rendered the urine alkaline in five hours, and for nearly two days: hence he concludes that it acts like formiates and acetates of the same base, and is transformed into a carbonate in the system. Valerianic acid is also changed, since it does not pass out as such in the urine.

Physiological Action.-Erternal and Local.-Valerian in several forms acts as a local stimulant or irritant: the powder of the root inhaled by the nostrils excites sneexing; and ralerianic acid applied to the skin causes first a white spot, afterwards irritation and redness, but not to any extreme extent: on the tongue it may give rise to peeling of the epidermis: it coagrulates blood, serum, and mill.

Physiologrcal Action.-Internal.-Digestive System. - Moderate doses may be taken by most persons, not only 
without discomfort, but with the effect of promoting appetite and digestion, and inducing a sense of warmth at the epigastrium. There is, however, some idiosyncrasy with regard to this drug, for I have met with several instances where 2 or 3 ounces of a weak infusion produced vomiting and indigestion. According to Stillé, 2 drachms (of powdered root) may cause heat and weight at the stomach, eructations, vomiting, and diarrhoca; but Trousseau from the same dose experienced only trifling giddiness and other nerve-symptoms, and states that he has taken large doses of infusion and powdered root "without any derangement of the functions of organic life."

Valerianic acid given in large doses to animals is said to cause death, with the usual symptoms of irritant poisoning except diarrhœa (Reissner, De Acido Valerianico, Berlin, 1855).

Circulatory System. - Valerian is commonly stated to stimulate this system, and produce even, like canella, pepper, etc., a temporary heat of skin, but practically it is seldom given without other stimulants, such as ammonia, spirit, or hot water (in fresh infusion).

Baraillier speaks of it as lowering the pulse-rate; and certainly in persons suffering from nervous excitement, with the small frequent pulse of exhaustion, valerian improves the force and volume while lessening its frequency.

Valerianic acid given to animals in from one- to threedrachm doses renders the heart's action feebler, but more rapid: the respiration also is hurried at first, then slowed; there is much prostration, later convulsion, and death in three or four hours.

Nervous System.-Grisar, experimenting with valerian oil on animals, obtained results similar to, but more marked than, those with the oil of chamomile in diminishing reflex excitability and antagonizing the specific effects of strychnir. On the other hand, to cats the drug seems to act as a special stimulant, inducing curious motions, sounds, and even convulsions,-possibly by its odour suggesting certain sexual conditions.

Trousseau, as already mentioned, experiencerl some giddiness and slight stimulus of sight, hearing, and motility, followed by impairment of these functions, and concluded that 
the drug acted on the cerebro-spinal system through the sympathetic.

Stillé describes the first effects of 1- to 2-drachm doses as "rendering the mind tranquil, inclining to good-humour and exertion," but causing also "a lively formication in hands and feet, and a sensation about the head and spine which has been compared to the "aura epileptica." "

Baraillier states that valerian can induce intellectual torpor, somnolence, and sleep; and it has seemed to me, when long continued, to induce a tendency to melancholy and depression of hysterical character : this, in fact, would represent the aftereffect of any nerve-stimulant.

More serious symptoms, such as hallucination and strong mental excitement, have been recorded from very large doses: thus, in a case related by Dr. Abell, the pulse became frequent, tremulous, and irregular, and the pupils were extremely and fixedly dilated; the patient fancied himself beset with dangers; lie staggered, and had a constant desire to urinate. In addition to the above symptoms there may be restlessness and spasmodic movements of the limbs. According to Kummer, there is "a sense of constriction in the pharynx, accompanied by headache," which last he describes as a pain in the right frontal region, extending to the vertex or to the left, and more decidedly to the eyes, causing a sensation of pressure when these organs are used; these head-symptoms appear and disappear periodically, continuing for a few hours at a time. Berbier, in his Materia Medica, gives in account of a patient in the Hôtel Dieu of Amiens, who, after taking 6 drachms of valerian daily for some time, woke up delirious, fancying that the side of the ward opposite to where he lay was in flames. Other patients who had taken the medicine imagined that flashes of fire darted from their eyes.

Genito-Urinary System.-The stimulating influence of the lrug on sexual instinct in some of the lower animals has been already referred to, but it is not recognized in man.

Some diuresis-i.e., increase in frequency of micturition and in the amount of urinary water passed-is commonly attributed to valerian; also, by some German observers, 
"branny, brick-red, and slimy" deposits. Rabuteau experienced no diuretic effect from 30 grans of valerianate of ammonia.

The question of the drug in any form acting as a diuretic is of interest, as bearing on its use in cases of diabetes: it is possible, on the one hand, that the essential principle passing out by the kidney stimulates that organ under certain conditions, whilst in other states of nerve-weakness and relaxation its tonic effect may lessen a secretion already excessive. According to the observations of Bouchard (of which, however, I can find no definite confirmation), valerian should be classed amongst the "médicaments d'épargne," i.e., those which lessen nutritive changes and the consumption of tissue. He found that 2 drachms of extract lessened the daily excretion of urea by 150 grains, and recalls the fact that certain Indian tribes in Mexico train themselves before warfare by a month's regimen on valerian, in order to better support fatigue (Compt. Rend. Soc. Biol., 1873).

SYNERGISTS.-Camphor, the gum resins, ammonia, alcohols, ethers; in some respects, cannabis and opium: strychnia is often combined, and, on the other hand, bromides.

ANTAGONISTS.—Digitalis, and also ergot; in some respects, bromides and quinine.

Therapeutical Action.-Epilepsy.-From the time of Gralen, valerian has had repute in this disorder-a repute much increased for a time by the classical case of Fabius Colonna (sixteenth century). Various cases might be cited from books of the last century, and great names, such as those of Boerhaave, De Hren, etc., might be quoted in its favour, but there remains always some doubt as to the exact diagnosis of a malady which may be simulated by the epileptiform convulsions of various diseases.

In comparatively recent times, however, Dr. Radcliffe has commended valerian in epilepsy, comparing its action with that of turpentine (Lancet, i., 1860$)$; and Stille allows that in large doses, and long continued, it has sometimes eured the 
disorder in females and children, and especially when induced by fright. From my own experience I should rather limit its use to "hystero-epilepsy" than expect benefit in the fully developed malady. I have known it relieve convulsions induced by worms; it acts as a poison to such parasites.

In chorea dependent upon or connected with the same cause I have also seen it useful.

Headache, etc.-It is chiefly serviceable in headaches (hemicrania) of a nervous or hysterical character, and in those which follow profuse or painful menstruation; also to persons of a nervous temperament, especially females subject to attacks of hysterical dyspepsia, and to flushing of the face upon excitement, with temporal or frontal headache, irritability, and flatulent distension which is apt to come on and subside suddenly. In such cases it often acts better when combined with cinchona or ammonia.

It is useful in most nervous affections occurring in excitable temperaments. In hypochondriasis it calms the system, abates excitement of circulation, removes wakefulness and sadness (if present), and induces sensations of quietude.and comfort. In globus, in asthmatical and hysterical cough, and nervous palpitation of the heart, accompanied by dyspnoen, valerian has likewise proved serviceable, also in facial neuralgia of hysteric type. In fevers, too, of a "nervous" character, and in the milder forms of delirium tremens, it has repeatedly done much good. In Germany it is more valued in such eases than with us.

Combined with guaiacum it may prove a useful stimulant in stmmons enlargements of glandular structures.

Diabetes.-In the so-called "diabetes insipidus," or "polyuria," connected as it must be with impaired controlling power of the renal nerves, I have seen advantage from infusion of valerian, though generally wheu combined with phosphoric acid. Some cases practically cured by it are recorded. Trousseau. especially recommended valerian, and S. Mackenzie has fully reported an illustration of its value (Med. Times, ii., 1878). The patient was passing, on an avernge, 7000 c.c. of urine per diem, but four days after commencing $\frac{1}{2}$-drnchm doses of powdered valerian ter die the amount fell to nearly lalf; with 
incrense of dose further improvement resulted and continued. Dr. Prior relates a case in an elderly man in whom treatment by valerian and valerianate of zinc was commenced soon after the malady attracted notice, with good results (Lancet, ii., 1881). Bouchard and others state that it is of value also in true diabetes and in azoturia, diminishing the solids excreted in both cases.

Pruritus.-Many forms of this disorder are connected with nerve-causes, and are more or less amenable to valerian. Thus, in hepatic pruritus not actually or only dependent on bile in the skin, and in senile pruritus independent of atrophy, but possibly connected with spinal disorder, Dr. Wade has specially recommended a valerianate of amyl ("spirit of valerianic ether") as efficacious and better taken than the ordinary infusion (B. M. J., i., 1874). He has related the case of a young married lady suffering severely from urticaria during pregnancy, unrelieved by arsenic and belladonna, but much benefited by a combination of the former with the preparation above mentioned.

In all the disorders named it has been sought to obtain therapeutical results equal to those of valerian itself, by a pleasanter and supposed even more effective combination of valerianic acid with various alkaline or metallic salts or alkaloids: thus, the valerianate of ammonia was introduced as of special efficacy in neuralgias; the valerianate of zinc for vertigo, nervous headache and climacteric symptoms; valerianates of quinine and of iron as antiperiodic and antichlorotic tonics; and similar salts of atropia for epilepsy, and of caffein for pertussis (Record, 1877).

Difference of opinion existing as to the therapeutical activity of the acid itself, there has naturally been equal difference as to its share in the effect of the somewhat powerful remedies combined with it, and it must be held peculiarly difficult to trace the specific effects of valerian in such combinations. Unduly lauded at first, they were soon, and perhaps unduly, neglected, until now it may be said that both the rine and the ammonia compounds find a recognized place in modern practice, apart from the question whether the good results be due more to the acid or to the base. The formor (now officinal) is valuable, 
as already stated, in various kinds of nerve-headache, and especially such as are associated with the climacteric period; the latter in forms of neuralgia in weak, semi-hysterical subjects - such cases, indeed, as are also relieved by the chloride of ammonium.

PREPARATIONS AND DOSE.-Infusum (2 dr. to $10 \mathrm{oz}$.): dose, 1 to $2 \mathrm{oz}$. Tincture : dose, $\frac{1}{2}$ to $2 \mathrm{dr}$. Tinctur ammoniata : dose, 20 to $60 \mathrm{~min}$. Zinci valerianas : dose, 1 to $3 \mathrm{gr}$. Ammonia valerianas: dose, 2 to $10-20 \mathrm{gr}$. Spt. amyl valerianatis comp. (contains valerianate of amyl, 1 part; spirit, 1 part; acetate of amyl, $\frac{1}{24}$ part) : dose, $8 \mathrm{~min}$. 


\section{COMPOSTTAE OR ASTERACEAE.}

THE order Compositæ is the largest known to botany, the number of species approaching 10,000 . It is met with in every part of the world, in every variety of habitat, and presenting every possible diversity of aspect.

The characters consist, first, in the aggregation of (usually) minute flowers called "florets" into heads or "capitula," every head having the appearance of a solitary blossom, and being provided with an involucrum which resembles a calyx; secondly, in the florets, when perfect, being monopetalous and five-lobed, and containing each five syngenesious stamens and a pistil. The ovary is inferior, and uni-ovulate; the style is generally furcate; the calyx, when present, is usually constituted of minute bristles, which in due time become the pappus of the seed-like fruit. In many genera the florets, instead of being regular, are ligulate; in others the dise of the capitulum consists of tubular florets, and a set of ligulate ones renders it radiant, as in the common daisy.

The general character of the order is bitterness, with a certain amount of astringency. Some species contain an acrid resinous matter; others an ethereal oil, which renders them aromatic. In medicine, accordingly, the Composita become valuable as tonics and stimulants; also as purgatives, diaphoretics, and diuretios. None, however, are drugs of the highest energy, nor are any intensely poisonous to man. 


\section{Pharmacentical Species.}

Invila Helenium . . . . Elecampane.

Anacyclus pyrethrum .. . . Pyrethrum, or Spanish chamomile: pellitory.

Artemisia absinthium .. .. Wormwood.

Artemisia santonica .. . . Santonica.

Anthemis nobilis .. . . . . Chamomile.

Taraxacum Dens Leonis. . . Common dandelion.

Lactuca sativa $\quad . . \quad \ldots \quad .$. Garden lettuce.

Lactuca virosa . . . . . Wild lettuce.

Arnica montana . . . . . Arnica.

Tussilago farfara .. . . . . Coltsfoot.

Grindelia robusta . . . . . . Gum plant.

Calendula . . . . . . . Marigold.

\section{INULA HELENIUM.}

(Elecampane.)

DESCRIPTION.-A large perennial, indigenous to most parts of Europe, England included. The erect stems attain the height of 3 to 5 feet; the leaves are ovate, many inches long and broad, serrated and veiny; the handsome yellow capitula, 2 inches across, and produced at the extremities of the branches, are remarkable for the great number of long and slender rays which spread from the dise.

ACTIVE INGREDIENTS. - The thick substantial root, when dried and sliced, has an agreenble camphoraceous odour, and an aromatic, rather bitter, and slightly pungent taste. Crystals may be obtained by distilling, or even carefully heating the roots, and are described chemically as the anhydride, $\mathrm{C}_{15} \mathrm{H}_{20} \mathrm{O}_{2}$, of aluntic acid. This is accompanied by a small quantity of helenin, $\mathrm{C}_{6} \mathrm{H}_{\mathrm{s}} \mathrm{O}$ (also crystalline), and of alent-cumphor (i.e., elecampane-camphor), $\mathrm{C}_{10} \mathrm{H}_{10} \mathrm{O}$, which in taste and smell suggests peppermint. 
The anhydride obtained by distillation of the root with water is impregnated with alantol, $\mathrm{C}_{10} \mathrm{H}_{16} \mathrm{O}$, which may be separated as an aromatic liquid.

The substance most abundant in elecampane root is inulin, which has the same composition as starch, $\mathrm{C}_{6} \mathrm{H}_{10} \mathrm{O}_{5}$, but replaces it, apparently, in most roots of the Compositre, and differs from it in some characters-c./., it forms a clear fluid solution with boiling water, and does not colour blue with iodine, but yellow; it may be obtained in crystals (F. \& H., 2nd Ed.). The root contains also bitter extractive, soluble in water ; an acrid resin, gum, lignin, albumen, and salts of potash, lime, and magnesia.

Physiological Action.-Elecampane acts as a gentle stimulant to the organs of secretion, and is said to be expectorant, diaphoretic, and diuretic. Large doses, cause nausea and vomiting.

THERAPEUTICAL ACTION.- In pulmonary affections, such as catarrh, when accompanied by profuse secretion, but without concomitant febrile disorder, elecampane is considered decidedly efficacious. It is also administered with benefit in dyspepsia, when attended by relaxation and debility; and is found useful in exanthematous cases, on account of its promoting the eruption.

Formerly, elecampane was supposed to possess emmenagogue properties, and with this no doubt is connected the origin of the classical name : it was prescribed by Hippocrates.

PREPARATIONS AND DOSE.-Decoctm ( $\frac{1}{2}$ oz. of the root in a pint of water) : dose, 1 to $2 \mathrm{fl}$. oz. Poudered root: dose, 30 to $60 \mathrm{gr}$. and upwards. 


\section{ANACYCLUS PYRETHRUM. \\ (Pellitory, or Spanish Chamomile.)}

DESCRIPTION.-A perennial, indigenous to the southern borders of the Mediterramean and the south of Spain. The procumbent stems are 12 to 15 inches long, very little branched, and clothed with bipinnatifid leaves, the segments of which are linear; the large, solitary, and terminal capitula are composed of a yellow dise, with numerous rays that are white upon the upper surface and purplish underneath: it resembles ordinary chamomile. Pellitory root is somewhat fusiform, about $\frac{1}{2}$ inch thick, and 2 to 4 inches long, wrinkled, greyish-brown outside, brownish-white within.

ACTIVE INGREDIENTS.-The principal ingredients of pyrethrum are three in number-(1) an acrid fixed resin, pyrethrin, insoluble in caustic potash; (2) a second resin; (3) an acrid yellow oil, both the latter are soluble in the alkali mentioned. The root contains likewise inulin, tannin, and various unimportant substances.

PHysiological Acrion.-The root of this plant, when dry, is scentless: on chewing it, there is soon perceived on the lips and tongue a peculiar pricking sensation, accompanied by heat; acridity and pungency are then detected, and an abumdant flow of saliva and of buccal mucus soon emsues. When applied to the skin, the root operates as a rubefacient; and in all cases the effects appear to be due to the pyrethrin. In the case of a child nged three and a half years, j0 minims of tincture caused soreness of the tongue and, after a few hours, diarrhœn with pain and passage of bloody nucus, twitching of muscles, convulsions, followerl by stupor, and afterwards excitement with quick pulse. Recovery followed in two or three days after giving opium and stimulants. 
Therapeutical Action.-The chief employment of this drug is as a masticatory and sialagogue; it is recommended also in rheumatic and neuralgic affections of the head and face, and in cases of palsy of the tongue. When resorted to for any of these purposes, the patient should chew the root.

Pellitory is again very good as a stimulant in defective secretion of the salivary glands: it may also be of service in cases of partial obstruction of the ducts. A gargle prepared from it was formerly prescribed for relaxed uvula, and partial paralysis of the tongue and lips. The tincture is useful for toothache.

PREPARATIONS AND DOSE.-Tinctura: for toothache, a few drops should be applied upon cotton-wool; as a gargle, 2 or $3 \mathrm{dr}$. of the tincture in a pint of water. As a masticatory, 30 to $50 \mathrm{gr}$. of the dried root. The German Pharmacopoia has "odontalgic pills," made with powdered pellitory, belladonna root, and opium, of each $5 \mathrm{gr}$. with wax, and oils of almonds, cloves, and cajeput, of each 15 min.; divide into 15 pills.

\section{ARTEMISIA ABSINTHIUM.}

(WoRMwOod.)

DESCRIPTION.-A small evergreen bush, with numerous slender branches, 12 to 18 inches in height, and densely clothed with rather broad doubly-pinnatifid leaves, grey with silky pubescence. The small, very abundant, and yellowish capitula are borne in long, leafy, erect, terminal clusters.

ACTIVE INGREDIENTS.-These consist of-(1) a green volatile oil, absinthol, $\mathrm{C}_{10} \mathrm{H}_{10} \mathrm{O}$, having a strong wormwood ordour; (2) a bitter extract, yielding absinthin, $\mathrm{C}_{40} \mathrm{H}_{55} \mathrm{O}_{9}$ (Kromayer), which last is the essential principle of the plint, ancl presents itself in the form of a faintly crystalline powder, 
neutral, of bitter taste and disagreeable odour: soluble in alcohol, ether, and the allialies; slightly so in hot water; the potash solution is brown red. (3) Absinthic acid, now presumed to be identical with succinic acid. The vegetable alkali kept in the shops under the name of "salt of wormwood" is an impure carbonate of potash, obtained by incineration of the plant, and possessing none of the intrinsic qualities of wormwood itself.

ABSORPTION AND ELIMINATION.-Wormwood, taken in any way into the system, impregnates the whole body with the bitter crystalline principle, as is shown by the taste of the flesh of animals poisoned with it. It is said that the milk of nursing mothers becomes bitter if they take wormwood, and according to Borwick, the infant suffers. The alkaline salts, such as santonate of soda, are still more rapidly absorbed.

\section{Physiological Action.-Digestive System, etc.-} In moderate doses wormwood operates in the manner usual with aromatic tonics. It increases the appetite, promotes digestion, slightly accelerates the circulation, and to some small extent augments the secretions.

Large doses are at first excitant, causing a pleasurable degree of warmth to permeate the whole body; subsequently irritation is induced, with considerable pain in the stomach, nausea, giddiness, headache, and confusion of ideas; also faintness, insensibility, and occasionally contraction of the extremities followed by convulsions, with fixedness of the jaws, and foaming at the mouth.

Nervous System.- Small doses, long persisted in, as in the shape of "bitters" - the "absinthe" of the French-seriously injure the nervous system, over which wormwood exercises a specific influence. The infatuation of the Parisians, especially, for absinthe is extraordinary, and the extent to which they indulge in it is frightful.

The idlers of the pare, and even the lower classes, drink petits ver'es of the liqueur, till they absorb an amount of alcohol which in itself is very pernicious, but besides this, the wormwood has special effects, the dangerous character of which has recently 
been investigated by M. Magnan. Absinthe-drinkers are distinguished, for instance, by a particular tendency to epileptiform. symptoms, referable, no doubt, to the action of the oil $;^{1}$ for experiments on animals have shown that the oil causes muscular tremors and shock-like spasms in the neck and fore-limbs, ${ }^{2}$ and, if given in very large doses, trismus and tetanus, alternating with clonic convulsions, foaming at the mouth, involuntary defrecation and discharge of semen, and (apparently) delirious hallucination. After death, the membranes of the brain and cord were always found injected, especially in the region of the medulla oblongata, and there was ecchymosis in the pericardium and endocardium, with hyperæmia of the lungs; the brain was but slightly congested.

Other observers-e.g., Lancereaux and Dujardin-Beaumetzhave denied the occurrence of convulsions (Lancet, ii., 1880). The former stated that in certain cases of convulsions occurring" in obstinate drinkers, the attacks were due to true epilepsy, or to hysteria: but symptoms almost exactly similar to those: described by Magnan occurred in a case reported by Mr. W. Smith (Lancet, ii., 1862), where a man who swallowed about $\frac{1}{2}$ ounce of wormwood oil became completely insensible, and had epileptic fits, with foaming at the mouth, trismus, and retching; he recovered in forty-eight hours.

Marked "degradation of both mental and physical powers" is also traced to absinthe-drinking.

\section{Therapeutical Action.-Dyspepsia, etc.-Worm-} woorl has been much extolled as a stomachic and tonic, and is. a suitable remedy for flatulent dyspepsia, occurring in torpid and debilitated constitutions, and it can certainly promote the assimilation of food.

Fevers.-Before the introduction of the febrifuges now in use, it was celebrated also as a cure for intermittents, but chiefly in domestic medicine.

Worms. - Wormwood is further said to be efficacious as a vermifuge, destroying, if long enough continned, both lumbrici

1 Epilepsy is doubtless an oceasional effect of prolonged excess in mere alcoholic drinks, but it is a much more common result of absinthe-tippling.

${ }^{2}$ Magnan, Comptes Rendus, t. xlvii., p. 14. 
and ascarides. M. Cazin recommends a preparation made by digesting equal parts of wormwood and garlic in a bottle of white wine, of which the dose is from 1 to 3 ounces every morning (Stillé). Haller recommends it as suitable for warding off attacks of gout.

A warm infusion was formerly prescribed in disorders of menstruation, and specially in epilepsy connected with the same.

Preparations and Dose.-Pulvis : dose, 20 to $40 \mathrm{gr}$. Infiusum ( $1 \mathrm{oz}$. to $20 \mathrm{Hl}$ oz. of water) : dose, 1 to $2 \mathrm{oz}$. Essential oil: dose, 4 to 8 drops with a little ether or sweet spirit of nitre.

\section{ARTEMISIA SANTONICA.}

\section{(Santonica or Santonicum-Tartarian Wormwool).)}

DESCRIPTION.-This plant corresponds in general appearance with the other members of its genus, and is technically characterized by its simple branches, pinnatifid and glabrous leaves, and unilateral and deflected flower-spikes. The odour is strong; the taste powerful and camphoraceous. The parts used in medicine are the unexpanded flower-heads mired with short broken stalks, seeds, and very small leaflet. A cluster of the flowers is only $\frac{1}{12}$ inch long by $\frac{1}{2}$ inch broad, so that they are not larger than many seeds, and are commonly known as "wormseed." There are two kinds - "Levant wormseed" (officinal) and "Barbary wormseed :" they are alike in aromatic odour and taste, but the latter is more globular and more downy-it is said not to contain santonin.

ACTIVE INGREDIENTS.-(1) A volatile oil, which is pale yellow, limpir, soluble in ether and alcohol, and contains various oxygen compounds. 
(2) About 2 per cent. of a peculiar crystalline principle, santonin, $\mathrm{C}_{15} \mathrm{H}_{18} \mathrm{O}_{3}$, neutral to test paper, but with acid properties, and called by some santonic acid-by Hesse, the anhydride of santonic acid: it forms soluble salts with lime. The crystals are flat, four-sided, snow-white when fresh, changing to canary yellow on exposure to light: this change is due, not to oxidation, but to the chemical action of sun-light, and does not occur if the crystals be kept exposed in a solution of nitrate of uranium, which prevents the passage of actinic rays (W. G. Smith).

In cold water, santonin is nearly insoluble ( 1 part in 5,000 or more), and 250 parts of hot water are required for its solution. It is insoluble in ammonia, but soluble in potash, soda, or lime, the solution becoming (transiently) red; not soluble in dilute mineral acid, but in strong acetic acid, also in warm olive oil ; the best solvents, however, are chloroform and boiling spirit: strong nitric acid changes it to succinic acid: it is lævogyrate, fusible, and sublimes at a moderate temperature.

ABSORPTION AND ELIMINATION.-Although a certain amount of crystalline santonin may pass undissolved through the bowels, some of it is so far soluble in the gastric fluids that it can be detected in the urine ten minutes after a dose of 4 grains, or within an hour after 1 grain. A part combines probably with soda, and passes into the blood as a santonate of that base.

Elimination is not rapid, about two days being required for ordinary doses (Smith).

Hypodermic injection of a solution in chloroform is the quickest mode of producing the effects of the drug.

Physiological Action.- Nervous System.-This seems to be at first excited, and afterwards, from toxic doses, much depressed: such symptoms as headache, disorder of special senses, muscular twitchings, obscure epileptiform symptoms, prostration, and, in frogs, motor paralysis have been observed. It is curious that toxic symptoms have sometimes followed small dosessuggesting that strychnia or some other more powerful drug 
might have been mistaken for it: thus a child, ten hours after taking $1 \frac{1}{2}$ grain, got strabismus, twitchings, dilatation of pupil, cramp, and convulsion, with slow respiration (Archiv f, exper. Path., vii., 1877). Other cases are reported-in some convulsion, in others coma has been the predominant symptom ; in most recovery takes place. In two or three cases retention of urine has occurred (Lancet, i., 1877 ; B. M. J., i., 1879). Mammalia generally are affected in a similar manner, the drug seeming to act chiefly on the cranial nerves and the medulla oblongata, not directly on the heart (Testa, Record, 1881): much congestion of these parts has been found after death. A child of five and a half years is said to have been killed in fourteen hours by two doses of about 1 grain each, but this must be doubted, for 2 to 4 grains are constantly given to children without any bad effect, although I have known it cause some excitement and restlessness; occasionally sickness or abdominal pain. A peculiar and special effect of santonin is the production of coloured vision, which is very usual, and occurs sometimes within five to ten minutes after the dose. Sir Spencer Wells ${ }^{1}$ noted, in 1848, that objects seemed as if seen through a yellow medium. The urine is commonly coloured yellow if acid, or red if alkaline; in some hours after taking the medicine, the red colour is developed.

ANTAGONISTS.-In cases of poisoning, stimulants (external and internal), frictions, and artificial respiration have proved useful, 一and sometimes chloral.

Therapeutical Action.-Though it will probably be employed, at some future time, for ophthalmic and other medicinal purposes, santonin is at present practically known only as an anthelmintio; being tasteless and inodorous, it can be giveu without inconvenience. To the round-worm it is absolutely fatal, and in somewhat less degree to the smaller ascarides. Its effects are most evident in cases where the presence of worms has given rise to intermittent or remittent febrile symptoms-

$1 \mathrm{Mr}$. Wells's observation was independent; the coloration had, howerer, been noticed as an occasional effect of worm-seed by Hufeland as early as 1806, as an occasional effect of santonin by Callord (1843), and as a constant effect of santonin by Itzstein (1846). 
the so-called "worm-fever." It should be given, like most other anthelmintics, on an empty stomach, and preferably at night and in the morning.

PREPARATIONS AND DOSE.-Suntoninum: dose, for adults, 2 to $6 \mathrm{gr}$. and upwards, given for about six or twelve doses, night and morning, and then followed by a brisk purgative; for children, 1 to $3 \mathrm{gr}$.

Adulterations, eTC.-Boracio acid (which renders an alcohol flame green).

Strychnia has been mistaken for it, and also picric acid.

\section{ANTHEMIS NOBILIS.}

(Chamomile.)

DESCRIPTION.-A perennial, indigenous to Britain and to many parts of continental Europe. The stems and branches are slender, 6 to 12 inches long, more or less procumbent, and clothed with sessile bipinnatifid leaves, the segments of which are linear. The capitula are terminal, less than an inch in diameter, and constituted of a convex yellow dise and numerous white rays. Every part of the plant is bitter, and evolves a strong but pleasant odour. Under cultivation the place of the dise florets is taken by ligulate ones; the capitula are then almost wholly white, and said to be "double."

ACTIVE INGREDIENTS.-Chamomile contains a peculiar dark-blue or dark-green essential oil, the source of its activity, and which has lately assumed an unexpected importance, from the researches of Binz and Grisar. The composition of the oil has been a matter of considerable dispute. Until lately the most generally accepted opinion was that of Gerhardt (Ann. Chim. Phys. (3) xxiv., p. 96), who represented it as a compound of angelic aldehyde, valerianic acid, probably a small quantity of resinous matter, and a peculiar camphor-like body, 
$\mathrm{C}_{10} \mathrm{H}_{16}$, with a boiling point of $175^{\circ} \mathrm{C}$., - to which he gave the name of chamomillin.

More recently, Demarçay (Compt. Rend., t. Ixxvii., p. 360, 1875) has examined the oil, and has come to the conclusion that Gerhardt was wrong in supposing it to contain an aldehyde; on the contrary, he says that it is a mixture of ethers, among which the angelates and valerianates of butyl predominate. The supposed hydrocarbon, chamomillin, appears really to possess the composition of valerianate of butyl.

In 1878 the following was given as the composition of the oil-isobutylic ether, angelicate of isobutyl, angelicate of isamyl, tiglinate of isamyl: thus Demarçay's conclusion as to the oil being a mixture of ethers is confirmed (F. \& H.).

The bitter taste is probably due to anthemic acid.

Physiological Actron.-Nervous System.-By far the most interesting physiological effect of chamomile oil is its power to lower reflex excitability. The important paper of Binz (Med. Centralbl., Feb. 8, 1873) first drew attention to this property of chamomile (among several other essential oils), and Grisar has since worked out the research with special care and very interesting results (Exper. Beit. z. Pharm. Die äther. Oele, Bonn, 1873). His experiments were conducted on the principle introduced into practical physiology by Türek. This consists in suspending frogs with one limb immersed in dilute acid; the time elapsing between this immersion and the withdrawal of the limb from the fluid is carefully marked by a metronome, and forms the test of the degree of reffere cxcitubility.

The element of volition is got rid of by the preliminary adoption of Goltz's process-the division of the cerebral hemispheres by a knife passed through the skull in a line from one posterior orbital canthus to the other. Frogs so prepared are exposed to the acid, their degree of reflex ivritability tested by metronome beats, and then the chamomile, or other ethereal oil, is injected beneath the skin, and successive observations are taken as the system becomes more and more impregnated with the drug. The result of experiments made in this way, and also with decapitated frogs, leaves no doubt that chamomile 
oil, even in doses not fatally poisonous, reduces the reftex. excitability of frogs in a very marked degree, and calms it when artificially excited by strychnia or brucia: or rather, an animal under the influence of chamomile oil is not tetanized by a dose of strychnia which throws an unprotected frog of similar size into characteristic spasms. The infusion acts as an emetic in full doses, especially if taken warm.

Therapeutical Action.-Our knowledge of the medicinal virtues of chamomile has been hitherto empirical, but the experiments just spoken of seem to point the way to a better understanding of the matter.

In Poisoning with Strychnia it will henceforth be a consideration whether we should not employ oil of chamomile, or some of the other ethereal oils, as antidotes;-the experiment is at least well worth trying. At present we are not clear as to the most suitable dose, but it is known that 5 drops of chamomile oil, subcutaneously injected, neutralize the effect of 6 milligrammes ( 0.09 grain of strychnia—a strong dose for a frog).

In Cough which mainly depends on heightened reflex irritability, particularly in hysterical women, chamomile oil may take rank with valerian as a potent remedy if given in sufficient doses ( 4 to 8 minims) : it acts well also in other spasmodic and pseudo-neuralgic affections of hysterical persons, especially in the pseudo angina-pectoris and attacks of colic to which such patients are liable; also in hysterical pain of the fifth nerve.

In Spasmodic Asthma, and in Whooping-Cough, chamomile oil has been found by German physicians very useful.

In Pulmonary Catarrh with excessive secretion and difficulty of expectoration, smaller doses (2 to 4 minimas) are useful, though other substances (which also depend for their effectiveness upon the presence of ethereal oil) are more commonly prescribed.

In Atonic Dyspepsia small doses (2 minims) of the oil are exceedingly useful; also in the diarrhocn of children, especially that arising from worms. 
The infusion of the flowers forms a good aromatic bitter tonic, and acts well in improving appetite, ete., when taken in the early morning.

In deficient liver-action in children, as shown by whitish, somewhat constipated stools, teaspoonful doses of infusion, night and morning, are a simple but effective remedy.

In Egypt and in Rome it was valued in intermittent fever, and is still known abroad as "Roman chamomile," in distinction from "German chamomile" (Matricaria chamomilla).

PReparations and Dose.-For nerve-cases the oil, presenting its original green or blue tint (which fades to yellow after a time), should be employed, in doses ranging from 2 to 8 min. : sugar is the best vehicle, or it may be prescribed in pill with rhubarb; it is a useful addition to sulphur ointment to disguise the smell. Extractum anthemidis: dose, 2 to $10 \mathrm{gr}$. Infusum ( $\frac{1}{2} \mathrm{oz}$. flowers to $\frac{1}{2}$ pint water): dose, as stomachic, 1 to $2 \mathrm{oz}$; as an emetic, 5 to $10 \mathrm{oz}$.

Adulterations.--The flowers of chamomile are sometimes adulterated with those of feverfew, but in the latter the bracts found on the receptacle of every species of anthemis are absent. Good chamomile flowers should yield from $\frac{3}{5}$ to $\frac{4}{5}$ per cent. of oil; it is contained chiefly in the dise or tubular florets.

\section{TARAXACUM DENS LEONIS.}

$$
\text { (DANDELion.) }
$$

DESCRIPTION.-The root is from 6 to 12 or more inches long, $\frac{1}{2}$ to 1 inch thick, roundish, with several short thick heads: when fresh, it is yellowish-brown; when dry, dark brown : it is inodorous, and of bitter taste. It yields a juice which varies a good deal according to the season at which it is gathered; in winter it is thick and albuminous, and in early summer bitter and acrid. 
ACTIVE INGREDIENTS.-Taraxacin and taraxacerin, $\mathrm{C}_{8} \mathrm{H}_{16} \mathrm{O}$; the former is dissolved out by hot water, the latter by alcohol from the insoluble residue: also inulin, mannite, and resin.

Physiological Action.- We have little or no accurate knowledge as to this, but the drug has the properties of a simple bitter. Rutherford reckons it a "feeble hepatic stimulant," whilst popular and also professional opinion credits it with increasing the biliary secretions. Its reputation originally was the vague one of "a deobstruent and blood purifier." It is somewhat diuretic and laxative.

Therapeutical Action.-Hepatic Disorder.-It has been supposed not merely to specifically increase the biliary secretion, but to cure chronic inflammation, indolent enlargements, and even to prove effective in commencing scirrhus of the liver. A great deal of the evidence on which such statements rest has been based on medication in which powerful drugs have been given in conjunction with it. The only distinctly efficacious action of taraxacum appears to be as a mild stomachic, and possibly a duodenal tonic. It certainly does good in simple atonic dyspepsia, and even (temporarily) in failures of digestion which depend upon disease of the liver and other viscera. But there is no proof that it is a specific remedy for biliary disorders. It appears to act solely as a tonic to the digestive functions, and the extent to which it will relieve disorders of these depends on the degree of severity of the original cause; it cannot modify distinct organic changes in any viscus.

PREPaRations AND Dose.-The freshly prepared succus is the best form in which to give taraxacum; but there is difference of opinion as to whether the thick albuminous juice obtained in winter, or the thinner acrid juice of early summer, is the more active. Mr. Squire thinks the winter juice the best. Dose: 2 to $4 \mathrm{dr}$. Extrutum: dose, 5 to $15 \mathrm{~g}$ gr. or more. Decortum: dose, 2 to $4 \mathrm{fl}$. о\%. 


\section{LACTUCA SATIVA.}

(Garden LetTuce.)

An annual, believed to have been brought originally from Asia. The leaves are large, roundish-oblong, very variable in colour, and usually much wrinkled. The flower-stem rises to the height of a yard, branching freely at the upper part, and producing numerous small yellow capitula, all the florets of which are ligulate. When well developed, it contains abundance of bitter and milky sap, which on being inspissated with moderate heat furnishes the substance called lactucarium (a crude form of which is thought to have been the opium of Galen).

\section{LACTUCA VIROSA. \\ (Wild LetTuce.)}

A weed, indigenous to Britain, and to the greater portion of continental Europe. It is distinguished from the garden lettuce chiefly by the leaves being horizontal instead of erect, and by the keel of every leaf being prickly-setose instead of smooth. The odour is rank, and upon the stem there are blood-red spots.

This species yields a good lactucarium; it contains principles similar to those of the L. sativa, and in large quantity.

Lactucarium is procurable also from the L. scariola and the L. altissima, which last is said by Aubergier to yield the best.

It is a complex substance which contains lactucerin, $\mathrm{C}_{19} \mathrm{H}_{30} \mathrm{O}$ (F. \& H.), lactucin, $\mathrm{C}_{11} \mathrm{H}_{12} \mathrm{O}_{3}, \mathrm{H}_{2} \mathrm{O}$, lactucic acid, and lactucopicrin.

Physiological and Therapeutical Action.Lactucarium has been credited by Bouchardat, Frommüller, Fleming, and others with soporific properties; but evidence on the subject is conflicting: Grarrod found the drug of no effect. 


\section{ARNICA MONTANA.}

\section{(Arnici.)}

DESCRIPTION.-A perennial, indigenous to Switzerland and Middle Europe. The rhizome is contorted, rough, and woody, with long thin rootlets; the radical leaves are ovate, obtuse, entire, and ciliated; those of the stem few and lanceolate; the large, radiant dark yellow flowers are borne upon a stalk 6 to 12 inches high, either singly or two or three together. When bruised, the plant evolves an odour which is aromatic and apt to induce sneezing; its taste is bitter and acrid.

ACTIVE INGREDIENTS. - Arnica contains two ethereal oils and a resinous bitter principle-arnicin, $\mathrm{C}_{20} \mathrm{H}_{30} \mathrm{O}_{4}$, which is almost insoluble in water, freely soluble in alcohol and ether; it differs in appearance according to the method of preparation, and is met with as a dark yellow sticky resin, or a golden yellow amorphous acrid mass, which is slightly soluble in water and alkaline liquids as well as in alcohol and ether (Walz, 1861). It is probable that a more important ingredient is trimethylamin, $\mathrm{C}_{3} \mathrm{H}_{0} \mathrm{~N}$, isomeric with, and often termed, incorrectly, propylamin. This may be obtained from the flowers of Arnica montana, as well as from ergot, chenopodium, pyrus, and other plants, and also from the animal kingdom, from guano and from urine, from cod-liver oil, and from herring-pickle, its most usual source. It is a clear, colourless, strongly allialine fluid, which boils at a very low temperature $\left(49^{\circ} \mathrm{F}\right.$.), and then emits a fishy smell; it is freely soluble in alcohol, ether, and water, which last also readily absorbs its vapour. At orclinary temperatures it is a colourless inflammable gas, and the liquid, even when diluted with water, ignites on the application of flame. The hydrochlorate is obtained by neutralizing with $\mathrm{HCl}$, and evaporating : it occurs in white prisms of saline pungent taste, inolorous, deliquescent, soluble in water and alcohol. According to Sigel (1873) the essential oil is a mixture of various bodies, the chief being dimethylic ether of thymohydroquinon, $\mathrm{C}_{12} \mathrm{H}_{18} \mathrm{O}_{2}$. Arnica root also contains about 10 per cent. of inulin (Dragendorff). 
Physiological ACTION.-Local.-Trimethylamin and concentrated aqueous solutions of arnica which contain it with but little arnicin, do not irritate when placed in simple contact with the skin; but if rubbed in for some time, they redden the surface, and, like ammonia, dissolve plugs of secretion at the orifices of sebaceous ducts; they also stimulate and irritate mucous membrane. Pure trimethylamin acts as a decided caustic: a drop of it placed upon the lip produces burning and a flow of saliva; the mucous membrane is reddened, and then the epithelium cast off, leaving a slight sore. Arnica, although an excellent application for bruises and wounds, has been known to produce either actual erysipelas, or a peculiar violet-coloured eruption, attended by great heat and pain ; but these are physiological consequences of the alcoholic, and not of the aqueous solution, which latter contains neither arnicin nor the oil. I have never seen inflammatory consequences follow the application of the purely aqueous lotion to wounds and bruises.

\section{Physiological ACtion OF TRIMETHYlamin.-} Internal.-Nervous System.-Some observers state that doses of about 20 grains, when repeated several times, cause, in rabbits, general distress, tremor, loss of motor power, hyperæsthesia, and increased reflex excitability; whilst 20 to 30 grains injected under the skin produce similar but more aggravated symptoms, followed by depression and death.

Dujardin-Beaumetz, however, says that 75 grains of hydrochlorate of trimethylamin may be given without fatal effect, and when death does occur it is from a local caustic action on the tissues. It is more probable that the drug kills through the spinal cord by depressing the cardiac and respiratory functions.

Circulatory System.-The first effect of moderate doses seems to be increase of frequency of the heart's action, but later an opposite condition occurs, and is indeed developed soon under full doses. In firogs the heart-beats may be reduced from 64 to 20 and 12 : in man 10 grains of the muriate will reduce the pulse 10 bents in a minute, and further doses will lower the temperature $1^{\circ}$ to $2^{\circ} \mathrm{F}$. Congestion of the lung has been found after death, but not invariably: the heart-cavities are full of dark clot. 
Digestive System.-The taste and smell of the pure drug are very offensive to most persons, and 30 to 50 grains cause vomiting (in the dog); the hydrochlorate, however, is less disngreenble. Animals taking the former lose their appetite and become thinner, but neither colic nor diarrhoea is reported to have occurred.

Glandular System.-No definite increase in secretion, either of the skin or kidneys, is constantly observed, but sometimes bloody urine containing bile-constituents has been voided, and congestion of kidneys found post mortem. Husemann could not verify this. Dujardin-Beaumetz states that the excretion of urea is diminished by the drug. Of infusions of arnica flowers the action is by no means the same, stimulant effects being more evident. In horses the arterial and nervous systems are stimulated, and the temperature and amount of urine increased. In man a strong infusion causes irritation of the digestive tract, also headache, giddiness, and restlessness, subsequently depression; at first it increases the frequency of the pulse and respiration, and the amount of most of the secretions. Very large doses cause at once depression amounting to collapse-vomiting, purging, dilated pupils, and a pulse at first quick, afterwards slow. I know of no fatal case from its use.

Therapeutical ACtion.-External.-The physiological action of this drug does not offer a clue to the special power of relieving bruises and lacerations which my own observation satisfies me that it possesses. For reasons already given, I think the infusion the best form to use by way of fomentation and lotion to the affected part; the tincture (containing armicin and volatile oil) is the preparation to be blamed for the irritation sometimes described, but still it is a convenient form if well diluted.

Therapeutical Action. - Intemol. - For Internal Bruises, arnica is an excellent remedy, neutralizing the ill effects of blows, falls, and other mechanical injuries. The absorption of ecchymoses and sanguineous effusions is rapidly accomplished by it, provided the medicine be administered 
shortly after the injury has been sustained. In cases of shake, concussion, and shock, resulting from railway or other accidents, it is also very serviceable.

Under these circumstances I recommend that 5 to 10 minims be taken every two or three hours in a tablespoonful of water. I believe there are few drugs that can so well restore the contused muscular fibre to its healthy condition as arnica, so that its more general use in eases of this description is desirable.

In Hæmorrhages arising from mechanical violence, bleeding from the nose, and hæmoptysis, arnica is also of great service, as well as in pulmonary congestions arising from fractured ribs, etc. In cases of concussion of the brain, induced by a fall, I can speak highly of it. In Germany it is called "Fall-Kraut" ("accident herb").

In Mental Diseases, Schröder Van der Kolk employed arnica largely in the form of infusion of the flowers, and of decoction of the root, using the former in mild cases, and the decoction when a more powerful remedy was required. He found the drug valuable in that condition of idiopathic mania where, the first excitement having diminished, the head nerertheless remains hot, and where a tendency to imbecility or to paralysis is shown. Exhausting diarrhoea and general cachexy are also checked by it. (Van der Kolk's results are the more interesting because obtained with aqueous preparations.) In nerve-prostration with general aching from over-fatigue in weakly subjects, I have used arnica internally with good effect. Possibly also the oil, besides being stimulant to nervous power, may (like chamomile oil, ete.) soothe hyper-excited reflex irritability.

In Paralytic Affections of various kinds, arnica has been found of service by numerous observers, among whom are Alibert and Meyer, who by means of it benefited a case of paralysis of the bladder. Mannior employed it with success in threatened amaurosis, for which disorder it has long been a popular remedy in Germany. It has also been commended in timnitus aurium of nervous origin.

In Typhoid and Typhus Fevers, arnica has been highly extolled, though Nothnagel speaks of it disparagingly. He does not, however, advance any good reasons for this, and as he 
allows that the general "picture" of the physiological actions of arnica gives every indication of its possessing definite powers as a remedy, we may fairly put against his rather vague opinion, and against the prejudice which British physicians have widely felt, the large body of German and French experience which exists as to the favourable action of arnica and trimethylamin in exhausting febrile diseases.

In Chronic Dysentery, when the motions are slimy and purulent, and attended with tormina and tenesmus, an electuary, or weak infusion, or tincture may be used with good effect.

In Rheumatism good evidence has been recorded of the utility of arnica. Dr. Fuller has spoken strongly of its value in rheumatic gout, and investigations concerning trimethylnmin have thrown fresh light on the subject. As long ago as 1854, Arvenarius employed this remedy in acute rheumatism, and in the course of three years treated with it 213 hospital patients, besides many in private practice, and frequently found the joint-pain and the fever arrested by one day's treatment. Guibert and Lagrange also confirmed these results. On the other hand, many observers denied (and still deny) its efficacy, and during several years little was heard of the remedy. The recent researches of M. Beaumetz have, however, again attracted attention, his good results being remarkable. Some confusion has occurred between the real trimethylamin and an isomeric body, "propylamin" (proved by Winckler and Mendius to possess different physical and chemical qualities). Under the former name several compounds of varying composition have been sold, all, however, containing alkalies, ammonia, and trimethylamin. On account of the impurity and variableness of these preparations, Beaumetz employed a definite salt, the hydrochlorate of trimethylamin, and found its effects in rheumatic fever remarkably uniform and striking in lowering of pulse and temperature, relief of articular pain and swelling, and diminution of the excretion of urea : in one case the urea-discharge, which amounted at first to $40 \cdot 74$ grammes per diem, was reduced in six days to 8.55 grammes per diem. The doses employed were from $7 \cdot 8$ to $15 \cdot 6$ grammes in twentyfour hours, in solution, and these amounts do not irritate the throat or stomach: water is a suitable vehicle (Bull. Gén. de 
Thérap., t. Ixxxiv.). Dr. W. H. Spencer, however, found the excretion of urea increased, sometimes more than doubled, whilst the urine was trebled in quantity (Practitioner, Feb., 1875): we cannot, therefore, consider this matter quite settled. In the few cases in which I employed the drug, I found the taste a great drawback to its use, and it has since been largely superseded in practice by the salicylates, which are claimed, probably more correctly, to act in a similar prompt manner.

In serous inflammations, the drug has also been used successfully, but no extended employment of it has been recorded.

PREPARATIONS AND DOSE.-Infusum (of the flowers) (2 dr. to 8 oz.) : dose, $\frac{1}{2}$ to 1 oz. Decoctum (6 dr. of the powdered root to 1 pint of water). Tinctur ( 1 in 20): dose, 5 to $60 \mathrm{~min}$.: the latter dose should rarely be given. As an electury, the dried and pounded flowers, mixed with honey or syrup, are sometimes used.

The only preparation of arnica recognized by the B.P. is the tincture, and although, for reasons already stated, this must be considered a mistake, it has no doubt special virtues of its own: it is more tonic and stimulating than other preparations, since it contains not only trimethylamin, but also arnicin and the ethereal oil.

\section{TUSSILAGO FARFARA. (ColtsFoot.)}

\section{(Not Officinal.)}

DESCRIPTION.-The yellow capitula, borne upon scaly stalks 3 or 4 inches high, make their appearance in waste ground early in the spring, long before the leaves: the latter, when fully developed, are broadly cordate, somewhat angular, as large as the palm of the hand, green above, white and cottony below. 
ACTIVE INGREDIENTS.-All parts of the plant contain nucilage, tamnin, and a bitter principle which is not yet isolatel.

Physiological Action.-Emollient, demulcent, and slightly tonic.

THERAPEUTICAL ACTION.-Coltsfoot is a popular remedy in pulmonary complaints, especially in chronic coughs.

The ancients were accustomed to smoke it for these conditions, whence the expressive name tussilago. The smoke was generally inhaled from the burning root, and the same part, prepared with honey, they used "for ulcerations of the lungs."

Dr. Cullen employed the expressed juice in scrofulous cases.

The leaves, as well as the capitula, may be employed, a handful being boiled in a couple of pints of water till the quantity of fluid is reduced by one-half.

Dose.-Ad libitum.

\section{GRINDELIA ROBUSTA.}

(Gum Plant.)

The genus to which this plant belongs is indigenous to the western parts of North America, and is characterized by having many-flowered heads with yellow ligulate ray-florets and tubular five-toothed disc florets. The leaves of this species are glutinous to the touch, either broad or narrow, more or less serrate; the achenes have one to three teeth at apex, and a two to five lobed pappus. The plant has an aromatic odour and taste, the latter being also pungent and bitter.

ACTIVE INGREDIENTS.-These probably depend upon an alkaloid which has been isolated, but not yot thoroughly investigated : a volatile oil and resin are also active.

Physiological Action.-On the digestive tract this dlug acts first as a stimulant, promoting appetite, but lnter as 
an irritant : it increases the salivary secretion. Upon the circulation and resplintion it acts in a sedative manner, lowering their frequency, and in toxic doses causing death (in frogs) by paralysis of respiration. The nerve-centres, it is said, are at first stimulated, but later certainly their activity is lessened, drowsiness being induced, with lowered sensibility and reflex power: the pupils are dilated. Two drachms of the extract are required to produce such symptoms even in small mammalia: larger doses may cause paralysis. It is said to irritate the kidneys and to increase the urine: it is eliminated in this secretion as well as by the bronchial mucous membrane.

SYNERGISTS.-Conimm and motor depressants.

ANTAGONISTS.-Mineral salts and caustic alkalies: water precipitates the resin. It is antagonized by alcohol, strychnia, cocculus, etc.

Therapeutical Action.-Extemal.-Locally it has been applied in lotion to relieve the irritation caused by Rhus toxicodendron.

Therapeutical ACTION.-Intemal. - Asthma. - In California especially, this plant is recognized as very valuable in relieving spasmodic asthma, and according to Bartholow "few cases fail to be relieved at once" - the prescription suggested by him contains, however, lobelia, belladonna, and iodide of potassium. Prof. Stillé "has been informed of cases (in the aged) in which $\frac{1}{2}$ drachm of fluid extract afforded almost instantaneous relief." In this country we have not at present such satisfactory experience. I have used 15 minims of the fluid extract in a highly neurotic subject, when the malady was for the time complicated with bronchial cataril, with temporary, not striking, relief, which was obtained better by atropin, and also by pilocarpin. Dr. Ringer records his disappointment with grindelia, but it deserves further trial with recent ancl thoroughly genume preparations. It may be given both in the intervals and at the time of attack-then more frequently-also by inhalation. It has been of service in pertussis and other spasmodic coughs, and in hay-asthma. 
In chronic catarrh or suppuration affecting the urinary tract, its resinous properties have proved useful.

PREPARATIONS AND Dose.-Extractum: dose, 2 to $3 \mathrm{gr}$. Extractum fluidum ( $1 \mathrm{fl}$. oz. $=1 \mathrm{oz}$. of plant): dose, 10 to 30 min. every hour or two during the paroxysm (in some mucilaginous liquid). In pertussis : dose, 10 min. every two hours.

\section{CALENDULA.}

\section{(Marigold.)}

DESCRIPTION.-A native annual of Southern Europe, with spreading stem, alternate, sessile, fleshy, entire leaves, and terminal heads of yellow flowers, having a disagreeable odour and bitter taste.

Active IngREDIENTS.-Geiger found an amorphous bitter principle and calendulin, which is yellowish, tasteless, soluble in alcohol, and swells into a jelly when moistenerl with water.

Action.-No definite physiological action has been tracel to this plant, but clinically it has some repute as an external application in lotion, for contusions, wounds, otorrhœe, etc., and ulcerations. I have certainly seen instances of the latter, when suppurating unhealthily, heal better under such treatment than under mineral solutions of lead or zinc, etc. It was at one time esteemed for cancer, and taken internally as well as applieel locally. Internally the extract has been found useful in chronic, vomiting (Stillé), and had formerly some reputation in hepatio congestion and amenorrhoea as "resolvent and stimulant."

Preparation and Dose.-Tincturl (1 in 10): of this a lotion may be made ( 1 part in 10 of water), or an ointment (same strength) with vaselin: an infusion may also be used like that of chamomile. 


\section{LOBBELIACER.}

\section{THE LOBELIA FAMILY.}

As order of several hundred species of herbaceous undershrubby exogens, belonging principally to warm countries, and nearly allied to the Campanulacer. The leaves are simple and alternate; the flowers are pentamerous, irregular, and bilabiate; the five anthers are syngenesious, and the inferior ovary becomes a many-seeded capsule. All the species contain a milky juice, which is often acrid, and sometimes poisonous.

\section{LOBELIA INFLATA.}

\section{(Lobelia.)}

DESCRIPTION.--An annual indigenous to the United States, extending from Canada to Carolina and the Mississippi, and there called "Indian tobacco." The stem varies from 6 inches to 2 or 3 feet in height, and, when the plant is tall, is a good deal branched. The leaves are ovate, sessile, serrate, and hairy; the small bluish two-lipped flowers are disposed in spikes, every blossom lying in the axil of a large and leafy bract: the adherent five-toothed calyx tube becomes "inflated" in the fruit.

The dried plant, as imported into England, is greenish-yellow. The smell is disagreeable and irritating, the taste burning acrid, similar to that of tobacco. The seeds, which are always present in the imported drug (being discharged from the abundant two-celled capsules of the earlier flowers), are very numerous, of a lengthened oval shnpe, reticulated on the surface with projecting hairs, and of light brown colour. 
ACTIVE INGREDIENTS.-The active properties of lobelia are yielded both to alcohol and ether, and appear to ilepend principally upon the presence of lobelin, a peculiar alkaline, pale-yellow liquid, lighter than water, of aromatic odour, and pungent taste. It is soluble in water, more readily in alcohol and ether, and forms crystalline salts with the mineral acids. The plant also contains lobelic acid, which is crystalline, and soluble in water, alcohol, and ether; a volatile oil, to which the odlour of the drug is due; and a resin of acrid taste.

Physiological Action.-Digestive System.-Lobelia readily disorders the stomach. Full medicinal doses-20 grains of the powder-cause speedy and violent vomiting, with persistent nausea, and sometimes purging, copious sweating, and general relaxation; these symptoms are usually preceded by giddiness, headache, and rigors, and are similar to those of tobacco, only requiring larger doses. A single grain incluced these results in a cat in less than two minutes, the creature recovering in about three hours. Administered by the rectum, it produces the same distressing symptoms, viz., sickness, profuse perspiration, and relaxation of the whole body. The patient mostly remains quite still, since to move causes return of the sudden and violent vomiting, with additional prostration: after a time, in favourable cases, he gets short periods of sleep, or sinks into semi-somnolent states, and out of one of these conditions he awalies quite well.

Secretory System.-In small doses, lobelin acts as a cliaphoretic and expectorant, the latter property being manifested without involving the pain of coughing. When it does not cause vomiting, its power is expended in profuse diaploresis or cliuresis, with smarting micturition. The evacuations of the bowels are seldom increased in frequency.

Circulatory System.-The pulse may be irregular, slow, and feeble, or regular and full. The change in blomlpressure is variously described as a proliminary increase, som followed by an opposite condition, or as an immediate fall, followed by a rise (traced to partial asplhyxia), nind then a perpmanent fall ; the heart's action becomes weakenel, the periphorul 
circulation embarrassed, that of the lungs obstructed, oxygenation impaired, and a depression of temperature follows.

Nervous System.-The earliest effects are pain in the back of the head, with a feeling of fulness, tightness, and giddiness; these are followed by general tremor, with a prickling sensation throughout the body, nausea, profuse perspiration, and violent emesis; the pain and giddiness in the head often alternate with the nausea, and on the occurrence of profuse diaphoresis generally subside.

In excessive or full medicinal doses, too frequently repeated, lobelia and its alkaloid act like a powerful acro-narcotic poison.

Before profuse perspiration sets in, there is a feeling of restlessness, with disteusion of the abdomen, and irregular spasmodic respiration. The cheeks are usually suffused, the pupils dilated, and the eyes more brilliant. The senses are rendered more acute; the brain is generally excited; the mind wanders, often lapsing even to wild and furious delirium, although sometimes a calm and placid sensation pervades the system. There is much thirst; the hands and arms are thrown about, and the sufferer rubs or beats his stomach; the secretion of saliva and of mucus is increased; there are dryness, burning, and rawness in the throat, and frequent and difficult deglutition, with irritation of the osophagus, and oppression of the præcordium : spasmodic dyspnœa attends these distressing conditions, with flatulent distension of the abdomen, especially in the neighbourhood of the navel, frequent eructations, and flatulent discharges from the bowels. Prostration of strength and relaxation of the entire muscular system set in, accompanied by heary despondency and fear of death.

In fatal cases, contracted pupils, insensibility, and convulsions precede denth, which occurs usually by paralysis of the respiratory muscles. In the frog, Ott traced to the action of lobelin a progressive paralysis which finally stopped respirntion, and was connected with, if not wholly due to, paralysis of motor nerves, for they were found insensitive to stimuli.

How far, if at all, the spinal cord is directly affected is not clear, but the action is probably limited to the medulla oblongata and respiratory centre. 
Small doses are presumed to excite, and large ones to paralyse the vaso-motor centres and periphery (Ott, Boston Med. and Surg. Journ., 1875).

AnTAgonists. - Caustic alkalies are chemically incompatible with lobelia. Belladonna, ergot, and strychnia are said to be antidotal; but alcoholic and other stimulants, especially coffee, are more reliable.

SYNERGISTS.-Tobacco, emetics and depressants generally, especially antimony; alkalies, iodides, and bromides aid its anti-spasmodic and expectorant action.

Therapeutical Action.-In the United States this plant is considered one of the most active drugs in the materia medica. When Europeans made their first settlement in that part of the world it was found to be the medicine most regularly resorted to by the aborigines: for a long time, however, it was left in the hands of irregular practitioners, and was only introduced to the notice of the profession by the Rev. Dr. Cutler, of Massachusetts, and into English practice in the year 1829, by the late Dr. Reece, who published a treatise upon its "antiasthmatical properties."

The principal value of lobelia appears to be as an anti-spasmodic: in some cases it becomes a useful adjunct to diuretics.

In cases of strangulated hernia it has been employed in the form of enema as a substitute for tobacco. Bartholow states that small doses of the tincture given at bedtime will relieve habitual constipation dependent on atony of the muscular layer and deficient secretion, and that "two drops every hour" have removed impaction of frees, when inflammation was not present. An infusion may be used as an enema, with. the same object, and is safer than tobacco.

Asthma, etc.-Puroxysmal spasmodic asthma, with extreme oppression and ineffectunl striving to cough, is relieved by lobelia in full doses; also dry congh, with continual tickling in the throat, such as hinders the patient from sleeping.

In some instances, it appears to lose power by repetition; and in any case should be administered in closes so small as not to 
canse either nausea or vomiting. If such results follow, the medicine must be immediately suspended.

Other disorders of the organs of respiration, such as catarihal asthma, croup, and whooping-cough have been treated with lobelia, sometimes successfully; but though great expectations were once entertained of its utility, they have not been fully realized.

As an emetic, lobelia is unfit for general use, its operation being very distressing and not free from danger.

I agree with many authors in believing that the administration of lobelia is best confined to nervous temperaments, but in any case it is an uncertain and somewhat dangerous remedy.

PREPARATIONS AND DOSE.-Pulris: dose, as an expectorant, 1 to $5 \mathrm{gr}$; as an emetic, 10 to $20 \mathrm{gr}$. Tinctura: dose, 10 to 30 min., or more (carefully watched). Tinctura lobelice cetherea: dose, 10 to 30 min., also carefully watched. 


\section{ERICACEAE.}

\section{THE HEATH FAMILY.}

Ar extensive order of exogenous shrubs and small trees, mostly evergreen. The stems and branches are tough and wiry; the leaves simple and undivided, of varying size, aciculate, or broadly ovate. The flowers are regular, perfect, dichlamydeous, tetramerous, or pentamerous; the corolla monopetalous, the stamens hypogynous. The anthers open by terminal pores; the ovary is superior; the fruit capsular or baccate, and manyseeded.

Many of the species are astringent, and some narcotic.

\section{ARCTOSTAPHYLOS UVA-URSI.}

(BEARBERTY.)

DESCRIPTION.-A hard, ligneous trailing plant of the moorlands of Northern Enrope and America. The branches spread to a distance of 2 or 3 feet; the abuudant leaves are alteruate, obovate, about an inch in length, entire, leathery, glabrous, and shining; when dried and pulverized they evolve an odour like that of hay or tea; their taste is bitter and astringent: the flowers, which resemble little red berries, are produced in small, crowderl, terminal, and dinoping racemes; the frut is globular, bright red, glossy without and mealy within.

ACTIVE INGREDIENTS. - These are extracted both by water and spirit, and consist of about :35 per rent. of tamuin, 
1.5 per cent. of bitter extractive, a trace of gallic acid, and a peculiar substance called arbutin, $\mathrm{C}_{25} \mathrm{H}_{34} \mathrm{O}_{14}$, a glucoside which forms neutral, colourless, silky needles, of bitter taste, soluble in hot water and alcohol, sparingly in ether. Ericolin, an amorphous, bitter, yellow substance, and ursone, which is crystalline, neutral, and tasteless, are contained in this and other plants of the same order. The infusion of the leaves gives a bluish-black precipitate with perchloride of iron.

Physiological Action.- Taken internally, by the healthy subject, uva-ursi, as a rule, does not act as a diuretic; but when given in disorders of the urinary organs, it would seem both to increase the quantity of the secretion and to affect its quality, lithic deposits becoming lessened by persererance in the use of it as a medicine. The astringent principle passes off in the urine, giving to the latter a dark colour.

In small medicinal doses it increases the appetite, but rather constipates the bowels: in large doses it nauseates, and produces vomiting and purging. It also produces inflammatory irritation of the lining membrane of the bladder and urethra, with tenesmus, and often a bloody discharge which later on becomes purulent.

Therapeutical Action.-The principal value of uraursi is shown in chronic Affections of the Bladder, attended by mucous, bloody, or purulent discharges, with burning in the urethra during urination, and especially when these symptoms are produced by calculus. In nephritic paroxysms, and in irritable states of the bladder dependent on renal disease, it is decidedly effective.

In Leucorrhœa and Gonorrhœa, also in Menorrhagia and Diabetes, uva-ursi has likewise been administered snccessfully: for acute cases it is unsuitable. It should be combined with acids or alkalies, according to the character of the urine.

Contraction of Gravid Uterus.-Dr. Harris of Fayette (Alabama), and Drs. Beauvais and Gauchet in France, assert that uva-ursi possesses the property of causing uterine contraction in the same way that ergot does, but this requires confirmation. 
PREPARATIONS AND DOSE.-Folia (in powder): dose, 10 to $30 \mathrm{gr}$. Infusum: dose, 1 to $2 \mathrm{oz}$.

ADULTERATIONS.-Leaves of the Vaccinium vitis-idra are sometimes found mixed with those of the uva-ursi, probably by accident, as the plants often grow in company. The under surface of the uva-ursi leaves is reticulated; that of the vaccinium leaves is dotted.

\section{SAPOTACEA. \\ G UT TA - PER CHA.}

DESCRIPTION.-This is the dried juice of the Isonandra gutta, a native of Borneo and other islands of the Eastern Archipelago.

ACTIVE INGREDIENTS.-The chief of these is gutta, a hydrocarbon, which forms 80 per cent. of the commercial gutta-percha: a volatile oil, two resins, and colouring matter are also present.

PROPERTIES.- It is elastic when fresh, but becomes brittle after long exposure to air and light. It is soluble in chloroform. and carbon disulphide.

USES.- In hot water, gutta-percha becomes soft, and can be moulder: hence it is much used for making splints, and for tooth fillings, also for surgical dressings, being contained in the "hat-lining" used for antiseptic purposes.

PREPARATION.-Limuor guttr-p) mecher (1 oz. in 8 oz. of chloroform and $1 \mathrm{oz}$. of carbonate of lead): contained in churta sinapis. 


\section{STYRACACER.}

\section{THE BENZOIN FAMILY.}

As order of about 120 species of exogens, arborescent, and chiefly inhabiting warm countries. The leaves are alternate, exstipulate, serrate; the flowers are axillary, usually pentamerous, with a variable number of epipetalous stamens, which are either free or monadelphous; the ovary, generally inferior, is two- to five-celled, and ripens into a drupaceous fruit.

\section{STYRAX OFFICINALE.}

(Storax.)

DESCRIPTION.-A deciduous tree, 15 or 20 feet in height, indigenous to Syria and the Levant. The leaves are ovate and shortly petiolate; the white flowers are about an inch across.

The storax of commerce is the balsamic juice of this tree, and occurs under two forms. "Storax in the tear" is the natural exudation, and is, comparatively speaking, pure: "red storax," imported in lumps, and in England known as Styrax calamita, is procured by incision; it is not so pure, and is not officinal. The former genuine liquid balsam of storax is grey in colour, or, after exposure, brownish yellow, translucent, and of the consistence of honey; soluble, when pure, in alcohol and ether. The odour is peculiar and agreeable; the taste warm and aromatic.

ACTIVE INGREDIENTS. - The constituents are a volatile oil, cinnamic ncid, styracin, and two peculiar resins, one hard, the other soft. The oil, called styrol, $\mathrm{C}_{8} \mathrm{H}_{8}$, is a 
colourless, translucent, volatile liquid, with a burning taste, and the odour of storax; it is soluble in alcohol and ether; boils at about $295^{\circ} \mathrm{F}$. ; burns with a sooty flame; and is of sp. gr. 0.924. Cinnamic acid, $\mathrm{C}_{9} \mathrm{H}_{8} \mathrm{O}_{2}$ (a constituent also of the balsams of Peru and Tolu), is colourless and crystalline; soluble in alcohol, sparingly so in cold water. It is distinguished from benzoic acid by yielding the odour of oil of bitter almonds on being treated with a solution of chromic acid. Styracin, $\mathrm{C}_{15} \mathrm{H}_{16} \mathrm{O}_{2}$, is commonly met with as a crystalline solid, but sometimes in a liquid state: it is soluble in ether, less so in alcohol, and is insoluble in water.

Physiological Action.-Resembles that of the balsams of Peru and tolu.

Therapeutical Action.-Extemnl.-Storax has been used in the form of ointment, as a detergent for foul ulcers, appearing to mitigate the offensive character of the matter secreted; it is specially commended for those of frost-bite. Dr. Mackey has drawn attention to the value of storax ointment in the obstinate affection known as "lichen urticatus," occurring in children (B. M. J., ii., 18\%4), and it is very useful in scabies as an alternative to sulphur, and in various forms of pruritus not traceable to local causes. After extensive frictions with it a deposit resembling albumen has been found in the urine, on the usual tests, but it is probably resinous and soluble in spirit (Record, 1879).

THERAPEUTICAL ACTION.-Interunt.-Stimulant and expectorant, like that of other balsamic substances. The stimulant properties are manifested more particularly in cases of subacute or chronic irritation of the mucous surfaces. It has been used with advantage in affections of the respiratory organs, such as chronic bronchitis, in which it promotes expectration. Chronic catarrhal affections of the genito-urinary membrane are also relieved by storax, and it has been found serviceable in amenorrhoca.

PREPARATIONS AND DOSE.-Styme proprlmatus: dose, 5 to $20 \mathrm{gr}$. (Storax is an ingrediont in the tinct. benzoini 
composita.) An ointment may be prepared with olive oil and spermaceti ointment (equal parts), or with a little spirit and a double proportion of ointment (scented), or with storax 1 oz., oil $1 \mathrm{dr}$, alcohol $2 \mathrm{dr}$.

\section{STYRAX BENZOIN.}

\section{(Benzotn.)}

DESCRIPTION.-A tree indigenous to Borneo and Java, with strong, round, hoary branches, large oblong-ovate leaves, and silky white flowers, in short racemes. The benzoin of commerce is the balsamic juice, obtained by making incisions in the bark, whence it exudes in a fluid condition, but concretes into a solid mass on exposure to air and light. It is imported from Sumatra and Siam; the former, which is darker and inferior, coming by way of India, under the name of "Calcuttr benzoin." The quality differs also according to the period when the juice is collected. The best, which is white, and is called "head benzoin," exudes during the first three years that the tree yields; the "belly benzoin" flows subsequently, and is of a brownish colour; the least valuable, obtained latest, is called "foot benzoin." The best Siam benzoin occurs in white or reddish tears, agglutinated together by a darker coloured and amorphous portion, the fracture presenting an amygdaloid appearance. When heated, it melts, and gives off benzoic acid in the form of white fumes, with a little empyreumatic oil, and then gradually consumes away. It is soluble in alcohol, and in solution of potash. Ether dissolves the resinous element in part. Sp. gr. 1.092. The odour is agreenble, to some persons fragrant: the taste is balsamic and slightly sweet. Sumatra benzoin occurs in large blocks, made up of the "tears" mixed with bark.

ACTIVE INGREDIENTS.-The principal constituents are about 80 per cent. of resin and 10 to 20 per cent. of benzoic 
acid, $\mathrm{C}_{7} \mathrm{H}_{6} \mathrm{O}_{2}$, which was known as long ago as 1608 , and is procurable by sublimation, in soft, white, feathery crystals, with a pearly lustre, and usually with a slight impregnation of the empyreumatic oil, giving it the odour of the original mass. It is now prepared for commerce from the urine of herbivorous animals, i.e., from hippuric acid. It is soluble in alcohol, also in solutions of lime and of caustic alkalies, from which it is precipitated by hydrochloric acid; in water it is sparingly soluble, requiring about 25 parts of boiling, and about 200 parts of cold, for complete solution: it melts at $248^{\circ} \mathrm{F}$., and boils at $462^{\circ}$, entirely subliming when pure. Of the resin, one portion is soluble in ether and in carbonate of potash, the other not: it contains styrol. Some forms of benzoin contain cinnamic acid (F. and H.).

ABSORPTION AND ELIMINATION.-Professor Wöhler, in 1831, expressed his opinion that benzoic acid, after absorption, was probably converted into hippuric acid; and Dr. Ure (1841) states that the administration of benzoic acid causes uric acid to disappear from the urine, hippuric acid replacing it. Keller (1842), on the other hand, maintains that the uric acid undergroes no change, and that the benzoic is directly converted into hippuric acid. Grarrod (1844) likewise takes this view, and states that it renders the urine more acid, as well as somewhat irritating. It is now commonly agreed that benzoic acid takes up glycine in the system, and passes out as hippuric acid, some succinic acid being also formed.

It was supposed by some that this change occurred in the liver, but recent observations point rather to the kidneys is its seat. That it does not take place in the blood or in the intestines appears from analysis, which detects benzoic acid unchangerl in them after administration of large doses; and, indeed, hippuric acid in the blood is known to give rise to severe toxic symptoms (Meisner and Shepard, 1866).

Bunge and Schmierleberg state that when the renal arteries - were tied in a dog, the change did not occur; conversely, if blood containing benzoic acid was passed through a kidney, even removed from the body, the change into hippuric acid occurred; the blood corpuscles seem, however, to have some 
share in the process. Tying the ureters did not interfere with it, i.e., it took place before the urine reaches those chnnnels. It is not clear whence the nitrogen required for this change is obtained, though the urea and uric acid are commonly supposed to be its source. Garrod agrees that the urea is diminished; not so Keller and others-and such effect is clearly not constant. If very large doses of benzoic acid were given, some of it passed unchanged: (the rabbit normally excretes it) (Record, 1880).

In some recent Lumleian Lectures, Dr. Garrod has adduced further evidence to show that the administration of alkaline benzoates will change or remove uric acid in the blood; but the observations of Mr. E. A. Cooke suggest rather a change in its combination than a true destruction, and the question deserves further inquiry (B. M. J., i., ii., 1883).

The acidity of the urine is usually heightened by the drug, but it would seem, when excessive, to be diminished by 10 or 20-grain doses, given, as G. Bird gave it, in solution with salts of soda. Salkowski found that benzoate of soda much increased the elimination of nitrogenous constituents-the amount of urea, for instance, rising under its use from 7 to 11 grammes, and by comparison with the sulphuric acid eliminated, he concluded that the increase was due mainly to destruction of albuminous constituents.

Physiological Action.-If chewed for any length of time, benzoin causes irritation of the fauces: the fumes produced by heat and combustion are also irritating, and the powder, if inhaled, excites sneezing. We possess, however, no exact knowledge of the action of this substance after administration. Schreiber swallowed half an ounce of benzoic acid, divided into forty doses, in the course of two days, with the result of prolonged irritation of the throat, and a sense of warmth, first in the abdomen, and afterwards throughout the body: the pulse at the same time became more frequent, though afterwards it subsided gradually. After the first dny, the perspiration and expectoration were augmented; the quantity of urine unchanged; there was some confusion of head, and digestion was temporarily impaired. In other cases when taken in large doses it is said to 
quicken the circulation, and to increase the flow of urine and the cutaneous exhalations.

Rutherford's experiments on animals showed both the acid and its soda salt in 10 to 20 -grain doses to be powerful stimulants of the biliary secretions; but this effect was not always evident in man, because they are not intestinal stimulants, and do not purge (Practitioner, ii., 18\%9).

Therapeutical Actron. - External.-The compound tincture is an admirable application for sore nipples, and for chapped lips and hands, one part being added to four of glycerine, cold cream or bismuth or zine ointment.

Mixed with water it is used as a cosmetic to remove freckles and other eruptions; e.g., the so-called "virgin's milk" is a spirituous solution in about twenty parts of rose water. Lotions containing benzoic acid or benzoates often relieve pruritus. "Friar's Balsam," "Wade's Drops," and "Jesuit's Drops" are other forms of the compound tincture, and also "Turlington's Balsam," a useful preparation for the healing of wounds and ulcers, and especially valuable to travellers and others who cannot ensily obtain surgical aid.

Its antiseptic powers make it a good addition to ointments, which it prevents from becoming rancid, and in surgical practice it has been employed instead of carbolic acid. Mr. Bryant has shown its value especially as a dressing for severe injuries, such as compound fractures, lint soaked in the tincture being. laid over the wound, and leading to rapid and satisfactory healing (Lancet, ii., 1876-other instances are given in Record, 1880).

Dougall states that benzoic acid is one of the most active antiseptics, 1 part in 533 of water being fatal to infusoria.

\section{Therapeutical Action.-Intrmal.-Diphtheria-} Phthisis.-Much attention has been directed recently to benzoate of soda given by spray and in solution as a remedy for these affections; whatever influence it exerts is tracenble to its antiseptic effects, and these seem to have been exaggerated in the accounts of the earlier cases.

G. Brown reported, from the Prngue Laborntory, that an 
animal "saturated" with it was proof against inoculations of diphtheritic poison; and Klebs, Lezterich, and Hoffmann recorded upwards of forty cases in children and adults treated by it, with only one death (abst. Med. Times, i., 18r9). Later observers have not corroborated these results, and it was given unsuccessfully as a prophylactic in the epidemic at Darmstadt, to the Princess Alice and her family. It is still possible, however, that some value may be justly allowed to the remedy on further investigation.

Its power's in phthisis are at present much discredited. In 1879, Dr. Kroczak, assistant physician to Rokitansky, at Innspruck, reported remarkable curative results in patients with large cavities in the lungs, and advanced septicrmic symptoms, from the use of 5 per cent. solutions of benzoate of sodium inhaled from a Siegle's spray-producer for an hour night and morning (Med. Times, ii., 1879). Yet when Drs. Guttmann, Waldenburg, Fritsche, and others came to adopt the same methods on a larger scale, their good results were mil, and, on the contrary, several patients suffered from romiting and hæmoptysis (Med. Times, i., 1880, p.94). Some advantage may yet be obtained in certain tuberculous cases, for independent observers, such as Schiiller (Greisswald), have demonstrated its power of saving rabbits inoculated with the malady. In man he considers 30 grammes daily to be the minimum amount likely to do good (B. M. J., ii., 1879). Good results have also been obtained by Burney Yeo.

The observations of Salkowski, already quoted, as to the waste-producing effect of the drug; should be borne in mind in using these large doses.

In a case of puerperal fever, Petersen found it apparently of service, and in crysipelcts some observers have reported farourably (Mel. Times, i., 18r9); also in scarlet ferer (Record, $1880)$.

Bronchitis, etc.-Being stimulant and expectorant, benzoin is usefully employed in this disorder for cluronic or subacute cases with profuse semi-purulent expectoration, and especially, though not only, in elderly people.

It may be given internally in combination with other remedies, or by inhalation of heuzoated steam-about 1 ounce 
of the compound tincture being added to a pint of boiling water in a jug or suitable apparatus.

In Follicular and Ulcerative Tonsillitis, I find the same inhalation very useful, and a lozenge of benzoic acid is effective in relaxed tonsillar conditions, in aphonia from. debility, etc.

Cystitis.-In chronic cases with profuse secretion, benzoic acid and its compounds are often good remedies, stimulating the resical mucous membrane, and lessening the foetor and other abnormal conditions of the urine induced by its retention, $e . g$. , in cases of enlarged prostate. Gosselin reported several instances (Med. Times, i., 1874).

Ten grains of the acid with 10 of borax in buchu infusion is a form recommended by Skeene, but the compounds with ammonia and soda are also effective; acid phosphate of soda is usefully combined with ammonia benzoate in these cases.

Cases of Vesical Irritation with phosphatic urine, and of incontinence from various causes, are amenable to the same remedies.

Chyluria also has been cured by benzoates, used sometimes alone, sometimes with tinctura ferri perchloridi (Practitioner, ii., 1882).

Albuminuria. - In several cases, scarlatinal and chronic, Hoffmann reported the excretion of albumen much lessened under benzoate of soda, and the benzoate of calcium has been found useful in 10-grain doses in the albuminuria of pregnancy, etc. (Record, 1881).

As already mentioned, Dr. Ure (1841) and, later, Dr. Golding Bird (1859) recommended benzoic acid as a remedy for uric as well as for phosphatic deposits, Dr. Bird combining it with phosphate and carbonate of soda. This application of it is not so generally recognized, but allied to it is its use in Rheumatism. Senator found it valuable in acute articular cases, and states that 2 to 3 drachms given in twenty-four hours relieved the prin, etc., though withont reducing pulse and temperature; it caused no disagreenble effects, but did not relieve subncute or chronic cases (Practitioner, ii., 1880).

The remedy had been previously used with advantage in gout and rheumatism by Tonjean and others, and Dr. Meliwan 
(Dundee) has recently reported five cases in which it acted well and quickly. He advises its continuance in diminished doses for forty-eight hours after the symptoms have disappeared. otherwise relapse is likely (B. M. J., i., 1881). Dr. Garm? reports clinical experience of the value of alkaline benzoates in gout, gravel, and calculus (B. M. J., i., 1883).

Jaundice.-Dr. G. Harley has specially praised benzoic acic as a remedy for this disorder dependent on " suppression of bilesecretion," giving it generally with liquor potassæ; and Tanner advised the benzoate in "hepatic congestion" (Practice of Medicine).

Dr. Wade notes its power in relieving the pruritus of this disorder, and recommends it for catarrhal cases, etc., generally giving 3-grain doses.

Dysentery.-Guided by the power of the drug to stimulate the biliary secretion, which is often defective in this disorder. Rutherford suggested its use, and Dr. Harris has reported several cases in which 15-grain doses thrice daily of the benzoate of soda proved valuable.

PREPARATIONS AND DOSE.-Beñoimm : dose, 10 to :31) gr. Tinctura benzoini composita: dose, $\frac{1}{2}$ to $1 \mathrm{fl}$. dr., suspendel in mucilage or yolk of egg. Acichm bensoicum: dose, 10 to 1 is gr. (may be given in pill, $5 \mathrm{gr}$. to $1 \mathrm{~min}$. of glycerine; if given in water, phosphate and biborate of soda are useful to aid solution). Ammonire, socke, vel crlcis bensores: dose, 10 to $15 \mathrm{gr}$. Unguentum acidi bensoici ( 1 in 8 or less). Lotio: 1 to. $2 \mathrm{dr}$. to 1 pint.

Benzoic acid is also contained in "paregoric elixir," in courtplaster, and in pastilles. 


\section{PYROLACEAE.}

\section{THE PYROLA FAMILY.}

Ax order of about thirty small perennial exogens, natires of northern temperate latitudes. The leaves are oral or circular, often all radical; the flowering stems, which rise to a height of 6 to 8 inches, bear usually several blossoms, so like those of the Ericacere in general structure, that by some botanists the order is made a section of the last-named.

\section{CHTMAPHILA UMBELLATA. (Chimaphila.)}

(Not Officincl.)

DESCRIPTION-An inhabitant of the United States, and of certain parts of Norther'n Asia. The root is long and creeping; the stems ligmeous and erect; the leaves deep green, lanceolate-cuneate, strongly serrated, irregularly whorled. The cream-coloured flowers, rose-hued in the centre, about. inch across, and somewhat pendulous, usually grow five together in a terminal corymb, and are followed by manyseeded capsules. When bruised, the chimaphila evolves a powerful odlour; the leaves, while fresh, possess consideraible acridity, though in taste warm and sunewhat pungent and nstringent; in the dried state the plant smells like ten.

ACTIVE INGREDIENTS. - The chief ingredients appenr to be a bitter extrnotive matter, in which is sontuined a yellow arystalline principle alled chimaphilin, odourless, tasteless, and very soluble (Fairbank), a resin, tamin, and perhaps a little gallie: acirl; nlso arbutin. 
Physiological Action.-The freshly-gathered leaves, if bruised and applied to the skin, are rubefacient and vesicant. The decoction of the dried plant is tonic, exciting the appetite, and assisting digestion. All the secreting organs, the kidneys in particular, are slightly stimulated by it, and it is said to check the secretion of lithic acid.

ThERAPEUTICAL ACTION.-Chimaphila was found to be in use among the aborigines of North America almost as early as the first settlement there of Europeans; but the notice of the medical profession was not directly drawn to it until 1803. In 1817, Dr. Wolff, of Göttingen, published a treatise, "De Pyrola Umbellata," but in England it has never received the attention it would seem to deserve.

Extemally it has been found useful in ulcers and tumours of various kinds; and internally in albuminuria due to chronic nephritic diserese; also as a diuretic in dropsy, and it is said to act beneficially in hrmaturia.

As a remedy for scrofula, the chimaphila holds a certain amount of reputation: as a tonic, it may be exhibited with advantage, and may prove a useful remedy in gout ancl rheumatism, especially if it really possess the power of lessening the secretion of lithic acid.

PREPARATIONS AND DOSE.-Decoctum. ( 1 part of the leaves to 20 parts of water) : dose, 1 to $2 \mathrm{fl}$. oz. 


\section{OLEACEA.}

\section{THE OLIVE-TREE FAMILY.}

A sMALL order of shrubby and arborescent perigynous exogens, natives principally of northern temperate latitudes. The leaves are usually opposite and simple; the flowers tetramerous and regular, with a definite number of stamens, and a superior ovary, which ripens into a drupe, a samara, or a capsule.

\section{OLEA EUROP AA.}

\section{(The Elropeax Otive.)}

DESCRIPTION.-This tree appears to have been originally brought from the Levant. It attains the stature of about 20 feet; the leaves are lanceolate, 2 or 3 inches in length, shortly petiolate, entire, leathery, dull green above, silverywhitish beneath, and persistent; the little white Hower's are in axillary clusters, and are followed by smooth oval, deep purple drupes, $\frac{3}{4}$ inch long, having a fleshy and large hard stone: clusters of as many as thirty blossoms often produce only three or four perfect olives.

The pericarp abounds in a fixed oil, which is obtained by expression, for which the tree is extensively cultivated in the South of Europe: when pure it is unctuous, pale yellow, or greenish yellow, slightly oflorous, and of bland, sweetish flavour; sp. gr. 0.92 . At a temperature of $38^{\circ} \mathrm{l}$. it begins to 
congeal, and at freezing point a portion becomes solid. Ether clissolves half its volume of the oil, but in alcohol it is only partially soluble, unless the alcohol be in very large proportion.

ACTIVE INGREDIENTS.-The constituents, according to Braconnet, are olein, about 72 per cent.; and palmitin, or margarin, about 28 per cent. According to later analyses, the liquid portion of olive oil is olein, commonly called tri-olein, $\mathrm{C}_{3} \mathrm{H}_{5} \mathrm{O}_{3}, \quad{ }_{3} \mathrm{C}_{8} \mathrm{H}_{33} \mathrm{O}$ : the solid part, known first as margarin, is chiefly palmitin : palmitic, arachic, and stearic acids, with a small quantity of cholesterin, have also been obtained, and nfter saponification, glycerine. Treated with nitrous acid, or with nitrate of mercury, the oil becomes a peculiar fatty solid, called elaïdin. With alkalies and certain other bases, it unites so as to form a soap. Exposed to the atmosphere, it is apt to become rancid, acquiring an unpleasant taste and smell, a deeper colour, and a more dense consistence, which changes are quickened by heat.

Physiological Action.-Externally applied olive oil relaxes the cuticular tissues. Taken into the system, it is mildly laxative, emollient, and in some degree nutritive.

THERAPEutical Action.--In cases where there is much intestinal imitrition, forbidding the use of powerful medicines, olive oil may be given with the best effect. As a very gentle laxative, it is especially valuable, in the form of enema, for infants and young children.

In various forms of Irritant Poisoning, if promptly administered, it mechanically entangles much of the noxious matter, and shields the mucous surface.

As a Vermifuge, it has sometimes been used, in massive doses by the mouth, and also by way of injection.

In cases of Gall-stone, olive oil has prored of service in relieving pain, when taken in 4 to 6-onnce doses; but the biliary concretions supposed to hare been passed have been shown to be masses of a soapy substance, due to the action of the gastric fluids on the oil.

As a mechanical defence from the atmosphere for diseased cutaneous surfaces, olive oil is often rery useful; also 
as a lubricant in such eases, for instance, as hardened wax in the ear; it is said to be specially effective mixed with $\frac{1}{8}$ part of benzole. The value of olive oil in pharmacy, however, is in the making of liniments, plasters, ointments, and other preparations for external application.

The external use of the oil in scarlet fever certainly prevents the diffusion of epidermic scales, and so favours disinfection: some think it protects the kidneys, but this is very doubtful.

Frictions with olive oil have been employed with advantage in wasting diseases, and even in bronchitis, convulsions, and diarrhoea. Several illustrations of their value have been recorded by Dr. Knagg's; he recommends frictions twice daily, and finds them answer the purpose of warm baths without their drawbacks (Lancet, Jan., 1870).

Mr. Marshall has shown the advantage of direct combination of oleic acid with various metals, etc., as oleates, c.g., of mercury, zinc, copper, morphia, atropia, cantharidin, etc., and these are now commonly prescribed for enlarged painful joints, cutaneous disorders, etc. Dr. Shoemaker has brought forward evidence to show that they are not, with the exception of mercurous oleate, so readily absorbed as to produce systemic effects, but their advantages are that a smaller quantity is required than of the older ointments; that they are more cleanly and, if well made, less irritant; and that they penetrate better into glands and follicles: vaseline should not be used as a diluent for oleates (B. M. J., ii., 1884).

PREPARATIONS AND DOSE.-Of the oil as a demulcent or laxative: dose, $1 \mathrm{fl}$. dr. to $1 \mathrm{fl}$. oz.

Olive oil is an ingredient in the linimentum ammoniae, the linimentum calcis, and the linimentum camphorio, and is introduced into varions plasters and ointments.

ADULTERATIONS.-Olive oil is extensively adulternted ivith the chenper kinds of fixed oils, especially with the oils expressed from polyy and cotton-seeds. All other fixed oils congenl much more rendily, so that the almixture of a spurioms oil is detected without much difficulty : a lighter slocifo gravity generally implies almixture. 


\section{SOAP.}

The soaps prepared with olive oil are of two kinds: sapo durus (hard soap), made with soda; and scipo mollis (soft soap), made with potash. Hard soap is of a greyish-white colour, corneous when cold and dry, and then reducible to powder, though if warmed it readily yields to pressure. Water dissolves it, and lime or lead salts precipitate the solution. Rectified spirit dissolves it entirely. Incineration leaves a residue of non-deliquescent ash.

Soft soap, instead of being solid, in consistence resembles honey or jelly; it is yellowish, translucent, and scentless, often speckled with white, owing to the presence of minute crystals, and is soluble in rectified spirit. The residue, after incineration, is an ash which rapidly deliquesces.

These two descriptions of soap, though differing much in physical qualities, are both composed of oleates and palmates. Hard soap consists of oleate and palmate of soda; soft soap of oleate and palmate of potash. Neither of them, when pure, should impart an oily stain to paper.

Soap, whether hard or soft, is in pharmacy useful for little besides external purposes. The supo durus has been given as an antacid, in doses of 5 to 20 grains; it is employed in the preparation of the pilula saponis composita, and of various other pills, not so much, however, for any service it may render as a medicine, as for its suitableness as a vehicle or adjunct; it is valuable, also, in the preparation of certain plasters, such as the emplastrum saponis, and of the linimentum saponis. The sapo mollis has likewise been administered as an antacid, in doses of 5 to 20 grains. It is an ingredient in the pilula saponis composita, and of the linimentum terebinthinæ, and is frequently ordered in chronic eczema, scabies, etc. When dissolved in spirit, it forms a tincture or lotio saponis viridis, for which the following is a good formula (Liveing) :- Potash soap 1 ounce, proof spirit $1 \frac{1}{2}$ ounce; dissolve with water 3 to 6 ounces, filter, and add oil of lavender 20 minims: this is a good applica- 
tion for chronic cases of psoriasis, acne, tinea, and sometimes lupus. It should be well rubbed in at suitable intervals, and allowed to dry on, preferably at night time.

\section{GLYCERINE.}

Glycerine, $\mathrm{C}_{3} \mathrm{H}_{8} \mathrm{O}_{3}$, discovered in 1789 by Seheele, who gave it the name of the "sweet principle of oils," is a thick syrupy liquid produced by the saponification of fats and fixed oils. It is either colourless, or of pale amber tint, unctuous, inodorous when pure, and very sweet to the taste; sp. gr. 1.260; it contains a small percentage of water.

Glycerine does not become rancid, nor does it evaporate, but with heat it decomposes, evolving an irritant vapour called acrolein; at a red-heat it ignites and burns with a blue flame: with water and alcohol it mixes readily, dissolving in any proportions, but in ether it is not soluble. With nitric acid it forms nitro-glycerine ( $v$. p. 444).

External.-It dissolves many different substances, such as iodine, common salt, fixed alkalies, vegetable acids, especially tannic, and many salts of the vegetable alkaloids; hence it is an excellent excipient of many medicinal substances. To lotions it is a useful adjunct, by reason of its soft and unchanging oiliness: it is excellent also as a simple lubricant for dry, chapped, or slightly abraded skin; and in cases of deafness, either alone or in combination with olive oil (in some cases it proves irritant, probably because of its great hygroscopic power).

Internal.-Fincerine gives good results, not only in cases of acidity of the stomach, but in flatulence and pyrosis: Ringer considers that it acts by delaying, or preventing altogether, certain forms of fermentation-just as it prevents the formation of lactic acid in milk.

A good combiuation is made with castor oil, of each 1 or 2 drachms, a few minims of tincture of senega being added to form an cmulsion; the purgative action of the oil is increased, and its nauseous taste lessened. 
One or two dessertspoonfuls of glycerine well whipped up with a small tumbler of milk, and taken in the early morning, nomrishes, helps digestion, and removes biliousness and obstinate constipation.

PREPARATIONS AND DOSE.-Glycerimm! dose, $\frac{1}{2}$ to

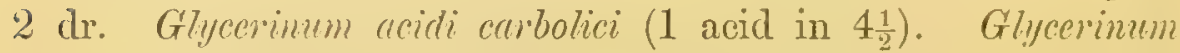
acidl gellici ( 1 acid in $4 \frac{1}{2}$ ). Glycerinum acidi tannici ( 1 acid

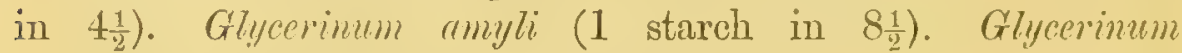
borcacis (1 borax in $4 \frac{1}{2}$ ).

\section{GLONOIN, $\mathrm{C}_{3} \mathrm{H}_{5}\left(\mathrm{NO}_{2}\right)_{3} \mathrm{O}_{3}$. (NITRO-GIJUTRINE.)}

\section{(Not Officinal.)}

PREPARATION.-By acting upon pure glycerine with strong nitric acid.

CHARACTERS.-A bright yellow volatile oily liquid, explosive on percussion.

Physiological Action.-More than twenty years ago the symptoms produced by very small doses of nitro-glycerine were observed by Mr. Field (Med. Times, 1883). Two drops of a 1 per cent. solution in alcohol caused, in three minutes, a sense of fulness and constriction in the neck, nausea, slight mental confusion, and loud rushing noise in the ears; perspiration succeeded, then yawning, headache, some gastric pain, and lassitude.

Dr. G. Harley and Dr. Fuller failed to obtain the same active results as $\mathrm{Mr}$. Field, either from deficient quality of the preparation or from some difference of susceptibility which is known to exist; but Mr. James and Mr. Brady confirmed the previous accounts, and obtrined also evidence of the drug producing partial intoxication and unconscionsness.

Circulatory System.-Dr. Murell, tasting the drug by applying to his tongue the cork of a phial containing a 1 per cent. solution, soon experienced a violent mulsation in the 
head, and "each heart-beat seemed to shake the whole body;" the pulse became fuller, and rose to 100 ; the pen he held was violently jerked with each pulsation, and there was a distressing sense of fulness over the body, as "if he had been runuing violently": walling and stooping much intensified the symptoms, which, however, subsided in the course of ten minutes, leaving" only "a splitting headache" (Lancet, i., 1879).

In thirty to forty subsequent personal experiments, the results were modified by keeping rery quiet; but the pulse always rose, sometimes not more than ten beats. By the sphygmograph he ascertained a close resemblance between the action of glonoin and of nitrite of amyl; both caused marked dicrotism, but the former acted more slowly, often not for six or seven minutes, whilst the latter showed its influence in fifteen to twenty seconds; on the other hand, the former lasted longer, nearly half an hour, whilst the action of amyl was orer in fifteen seconds.

Nervous System.-Nervous and delicate subjects are much more susceptible to its action than the robust. Besides headache, a languid feeling, and in some persons, sleep, or a strong inclination to sleep, is induced; this lasts a varying timefrom one to three hours. Tendency to syncope, to rigors, and sometimes vomiting, followed full doses of from 3 to 10 minims.

Urinary System.-Dr. Murrell utilized a case of epispadias to show conclusively the rapid and marked diuresis produced by the drug. The man was not sensitive to its general action, and could take as much as 25 minims; but after half this quantity, the urine could be seen to spout from the urethra in a jet :3 to 4 inches ligh, instead of dribbling away guttutim as usual.

Therapeutical Action.-Neuralgia.-The remedy was first intrortuced for this disorder, especially for facial neuralgia and toothache, and has proved effective in obstinate cases in doses of 1 to 4 minims of a 1 per cent. solution every three to four hours. Some amount of general distress may loe caused, and the first alministration should he supervised by the prescriber. 
In conditions of Spasm similar doses have been found effectual, and chiefly in spasms due to functional disorder (Field, Med. Times, i., 1858). The records of cases, however, are not sufficiently numerous to enable any one to speak defimitely on the subject. This remark applies to epilepsy, in which disease the drug has been tried, sometimes, it is said, with favourable results, and also to spasmodic asthma, several cases of which seem to have been much benefited (Robson, B. M. J., i., 1880).

Angina Pectoris.-Dr. Murrell has shown how raluable this medicine may prove even in aggravated stages of this disorder, and has recorded the details of four restored by its use to comparative comfort; and other observers have also published cases benefited by it (Jameson, B. M. J., i., 1880; Sawyer, Practitioner, i., 1880), showing that this drug ought to be a great addition to our resources, and deserves further trial.

In Sea Sickness, $\frac{1}{100}$ minim for an adult, and $\frac{1}{300}$ minim for a child, is said to have been successful (B. M. J., ii., 1880), but my experience of it as a remedy is unfavourable.

In Bright's Disease, acute and subacute, nitro-glycerine has beew given with good results, increasing the urine and relieving distressing symptoms of dyspnoea and dizziness, if present (B. M. J., ii., 1880).

PREPARATION AND DOSE.-Of a 1 per cent. solution in alcohol, 1 minim may be given (in water) to commence with, and the dose gradually increased according to circumstances.

\section{ORNUS EUROP压A.}

\section{(MAnna.)}

DESCRIPTION.-A tree indigenous to the South of Europe, and closely resembling the common English ash in habit and foliage, but smaller, and possessed of perfect flowers, which have narrow white petals in conspicuous panicles. Mamna is the 
natural exudation of the stem, of the consistence of cheese, yellowish white, faintly odorous, and of sweetish but sickly flavour.

AC'TIVE INGREDIENTS.-About 60 or 80 per cent. consists of mannite, $\mathrm{C}_{3} \mathrm{H}_{7} \mathrm{O}_{3}$, soluble, when pure, in three to five parts of cold, and in its own weight of boiling water; also in rectified spirit. It melts with heat, and burns with a bluish flame.

Mannite may be extracted from the crude manna by boiling alcohol, from which, on cooling, it separates in shining, whitish, four-sided, acioular crystals: it is odourless, sweet, soluble in boiling, less so in cold, alcohol, and does not ferment with yeast. Other constituents are sugar, of which there are two kinds, one crystallizable, the other amorphous, a small quantity of gum, some resin, and a little nauseous extractive matter.

Physiological Action.-Mildly laxative, sometimes causing flatulence and griping.

Therapeutical Action.-Manna acts well as a gentle aperient for infants and young children, and is suitable for introduction into purgative mixtures, as an adjuvant.

PReparation and Dose.-Manna: dose, for children, 1 to 2 dr. ; for adults, 1 to $2 \mathrm{oz}$.

\section{APOCYNACEA.}

ASPIDOSPERMA QUEBRACHO. (WhTte QUibracho.)

(Not Officinal.)

DESCRIPTION.- $\mathrm{A}$ tall tree, indigenous to the Argentine Republic, having a straight trunk several feet thick, with an oval crown of sparse foliage, the extreme twigs being fine and pendent. The lanceolate leaves are in triple whorls, and end in 
a sharp thorn. The five-cleft yellow flowers are arranged in cymes, and are followed by two-celled ovaries. The wood is remarkably hard.

The bark, which is the part used in medicine, is noted for the great thickness of the corky layer, which is yellow in colour, with numerous white points. The outer surface is grey with lichen, dark red where the epidermis is rubbed off.

Active IngRedients. - From the bark, G. Fraude has prepared an alkaloid termed aspiclospermin, $\mathrm{C}_{22} \mathrm{H}_{23} \mathrm{~N}_{2} \mathrm{O}_{2}$. It occurs in small white prismatic crystals, which are soluble in alcohol and ether, slightly so in water: the chloride and sulphate are, however, freely soluble. Hesse, finding that this alkaloid did not show the therapeutical effects of the drug; examined the bark more closely, and reported the presence of another, which he termed quebrachin, $\mathrm{C}_{21} \mathrm{H}_{22} \mathrm{~N}_{22} \mathrm{O}_{3}$, and which has strongly basic properties. It is slightly soluble in cold alcohol or ether, more so in hot alcohol, from which it crystallizes in delicate colourless needles, which slowly turn yellow in sunlight. It dissolves in eoncentrated sulphuric acid with a bluish tinge, which becomes darker on standing, and intense when a crystal of potassium bichromate is added. From three to six alkaloids have since been found by Hesse in different specimens of quebracho bark-the others being named by him aspidospermatin, aspidosamin, hypoquebrachin, and quebrachamin (Pharm. Journ., i., 1882).

Physiological Action.-The special action of this drug is apparently exerted most on the nervous system and respiratory centre.

In frogs, paralysis of the jatter occurred, with slowing of the heart-beat from sixty pulsations down to ten-a depression "not caused by irritation of the vag'us." Complete motor paralysis of central origin is also described (Penzoldt, Med. Times, ii. 1879, 1880). With rabbits, dyspnoen and paresis of extremities occurred, and larger doses coused death, preceded by paralysis of voluntary movement and by convulsion, which was possibly due to the severe dyspnoca.

In a healthy dog difficulty of brenthing was also induced, but with incrensed firequency of heart-beat; there was also salivation. 
The body-temperature was not affected by full doses, although in other animals it has been lowered.

Penzoldt suggests that the drug acts directly on the blood, and enables it to absorb a larger amount of oxygen than usual. This is unlikely, and the idea is based apparently upon the facts that if quebracho be added to one sample of blood, and distilled water to another, the former on standing becomes more crimson, and that when the supply of oxygen is lessened, as it is under a mercurial vacuum, the colour produced is less red.

The following are the conclusions of G. Guttmann (Areh. $f$. Exper. Path., Bd. xv., Hft. 6, 1881) :

1. Aspidospermin is a poison acting on respiration and circulation in cold- and warm-blooded animals.

2. In the cold-blooded, respiratory paralysis appears first, and death follows from lowering of eardiac activity.

3. In rabbits, the action on the heart is primary-slowing of pulse occurs, with dyspnœa and marked lowering of temperature. ${ }^{1}$ Death is from heart-paresis.

4. In frogs, there is paralysis of voluntary movement. This is doubtful in warm-blooded animals, and in them there is no evident affection of reflex or sensory nerves.

Maragliano concludes that-(1) The alcoholic extracts of quebracho and quebrachin decrease the frequency of respiratory action, as also does aspidospermin, but less markedly. (2) Their influence is exerted only on functional derangements of respiration. (3) The frequency of the pulse may be reduced 20 beats. (4) Hypodermic administration shows its effect in about five minutes; by mouth, in from thirty to forty: of the extract, 40 to 50 centigrammes are a dose (about 7 grains); for hypodermic use, about $\frac{1}{2}$ to $\frac{3}{4}$ grain of sulphate of quebrachin (Cent. f. Med. Wis., Oct., 1883).

Therapeutical Action. - Intemal. - Pulmonary Disorder. - There is some clinical evidence of the power of quebracho to relieve dyspnoea, even when it is consequent on organic alteration: thus Penzoldt states that in emphysema,

It has not proved anti-pyretic in febrile disorders, although chemically it is reluted to quinine. 
phthisis, chronio pnewmonic conditions, and periodic asthma, the above symptom, and also the cyanosis, were relieved. Even in stenosis and in thrombosis of the pulmonary artery, temporary benefit was obtained from it.

Berthold reports the case of a man, aged sixty-five, suffering from asthma. The tincture was given in drachm doses hourly, and after the third hour the respirations were reduced from sixty to thirty. "Shortness of breath" in aged patients with fatty heart was also relieved (Prag. Med. Woch., Jan., 1881).

Laquer, after observing twenty-two cases, reports not so favourably, thus :-(1) In many cases of dyspnœa, emphysema, and chronic bronchitis, quebracho proved a useful palliative in others, especially in the aged, it was of no service. In cases of valvular insufficiency its value is doubtful.

(2) It decreases the frequency of respiration, especialiy when this is extreme; it does not so constantly decrease the pulse. Its continued employment induces unpleasant symptoms, such as headache, partial unconsciousness, and salivation, and the taste is nauseous enough to make its administration sometimes difficult (ibid.).

Krauth gave it in cases of cardiac hypertrophy with general dropsy, in acute nephritis, and in one case of phthisis and one of pleurisy with effusion, and in all, he states, with some benefit to the respiratory symptoms (ibid.).

Dr. Berkart used the liquid extract "in a limited number" of cases of dyspnoea associated with emphysema of the lungs, atheroma of arteries, and degeneration of cardiac muscle." In three minutes after teaspoonful doses the patients felt their breathing easier, the pulse became somewhat fuller, the face was flushed, and gentle perspiration appeared on the forehead. Slight drowsiness followed, but soon passed off, whilst the breathing continued better. (One patient with cardiae dropsy stated that the quantity of urine was increased by three doses of the medicine) (B. M. J., i., 1880).

Dr. A. H. Smith, Chairman of Committee, Therapeutical Society, N.Y., reports on thirty-two patients (Amer. Rev., Jan., 1882). Of eleven cases of spasmodic asthma, with or without emphysema and bronchitis, nine were notably relieved, and two with bronchitis got no benefit; a case of bronchitis and emphysema got better, but one with bronchitis and obesity did not; 
cases of mitral stenosis, insufficiency, hypertrophy with dilatation, were not relieved, but two others (form not stated) were; two cases of Bright's disease, one of aortic aneurism, and one of cancer of lung were relieved, but not those of hysterical dyspnoa, or catarrhal phthisis; one case of intermittent fever with pleurisy was aggravated. Thus, of thirty-two, twentyone were relieved, ten not, and one was aggravated. It caused nausea in some cases, and was found disagreeable to take. It apparently blunts the sense of want of air; and since exaggerated respiratory effort is itself injurious-the muscular effort leading to aspiration of blood to the thoracic viscera, especially when the trouble arises from narrowing of airpassages-any relief to such effort is beneficial.

Dr. Picot reports much advantage to breathing from taking this drug before hill-climbing, and Skoda is said to have used it himself for dyspnoea with benefit.

Dr. F. Röhrer (Zurich) reports "brilliant results in the asthmatic attacks of consumptives," the respirations falling in one case from fifty-four to thirty with the result of calm sleep, previously impossible; in pleuritis, it fell to twentytwo; in emphysema, to eighteen. Cyanosis was lessened or entirely relieved (a dark blue nose became reddened); the subjective sensations of dyspnoea were much quieted for a time (Therap. Gaz., June, 1881 ; Rev. des Sc. Med., xxi., 1883).

Massimi, after observing sixteen cases, including some of Bright's disease, phthisis, and asthma, concludes that it is only desirable to use it "when more rational means fail," and that it is contra-indicated in weakly subjects and in serious heart affections.

PREPARATIONS AND DOSE. - Tincturk (1 part in 5 proof spirit) : dose, 30 to $60 \mathrm{~min}$. or more. Extructuin fluidum: dose, 30 to $60 \mathrm{~min}$. or more. Infusum and Decoctum (made with 1 part to 20 , with a little acetic acid) : dose, 1 to 2 o\%.

Red quebracho is recommended as equally effective. 


\section{SPIGELIACEAE, OR LOGANIACEAE.}

THE NUX-VOMICA FAMILY.

Av order of exogens, constituted of less than 200 species, which occur chiefly in the tropics, and in aspect and stature are much diversified. The leaves are opposite, entire, and stipulate; the monopetalous and four-, five-, or ten-cleft corolla is mostly regular; the epipetalous stamens are few in number, and the free two-celled ovary ripens either into a drupe, berry, or capsule. Many species have very virulent properties.

Strychnos nux-vomica .. Nux-vomica.

Strychnos Ignatia. . . . . . St. Ignatius' bean.

Spigelia marilandica .. .. Carolina pink-root.

Spigelia anthelmia .. .. Demerara pink-root.

Curare (South American arrow poison).

Akazga (West African ordeal poison).

Gelsemium sempervirens .. Yellow jessamine.

\section{STRYCHNOS NUX-VOMICA.}

\section{(Nux-VOMICA.)}

DESCRIPTION.-An East Indian tree, low in growth and irregular in figure, with shining foliage. The small greenishwhite flowers are pentamerous and pentandrous, and produced in cymes; the fruit is a globular berry, not unlike an apple, externally smooth, of a rich orange colour, and containing five thin, grey, circular, flat seeds, about an inch in diameter, covered with short silky hairs, and having an elevated raphe running from centre to circumference on the concave side. The seed is hollow, and its two sides may be readily sepa- 
rated, showing the embryo, with club-shaped radicle. It is hard, horny, and difficult to powder.

The bark was at one time called "false angustura," the source whence it was derived being unknown, but several years afterwards it was found to contain brucin, and then identified as nux-vomica.

ACTIVE INGREDIENTS.-The seeds contain an unusual amount of protein compounds, igasuric acid (which is amorphous, yellowish-white, astringent), and three alkaloids, strychnia, brucia, and igasuria. The most important is strychnia, $\mathrm{C}_{21} \mathrm{H}_{22} \mathrm{~N}_{2} \mathrm{O}_{2}$, which crystallizes out of alcoholic solution in colourless, four-sided, or octahedral right-rhombic prisms, slightly soluble in water (1 in 7000), more so in alcohol of sp. gr. $0 \cdot 863$, especially when heated nearly to boiling-point: the solution is odourless, intensely bitter, and leaves a characteristic metallic after-taste. The proportion of this alkaloid in nux-vomica varies from $\frac{1}{4}$ to $\frac{1}{2}$ per cent. Strychnia is soluble without change of colour in pure sulphuric acid; but if a single crystal be thus dissolved on a white plate, and a drop of solution of some oxidizing substance (e.g., bichromate of potash) be made to mingle at its edge with the dissolved strychnia, there is presented an unique play of colours, including blue, purple, crimson, and red-brown; this change occurs in a few seconds, and then the red-brown gradually fades into a light red, which is persistent for some hours.!

There is a physiological test based on the sensitiveness of frogs: $\frac{1}{1000}$ grain in a drop of water applied to their dried skin will cause spasm in ten minutes: of the acetate of strychnia $\frac{1}{2000}$ injected will do the same, or even, it is said, $\frac{1}{20000}$ applied to the skin when thoroughly dried-i.e., when natural evaporation and elimination are prevented. The physiological is apparently better than the chemical test, (B. M. J., i., 1883), but it applies also to brucia, etc.

Strychnia is a powerful and very stable base, which neutralizes the strongest acids, and precipitates metallic oxides from their solutions, with formation frequently of double salts. The

1 It has been erroneonsly stated that other alkaloids answer to this test, but Dr. Guy has clearly proved that nothing but strychnir produces the particular sequence of colour-changes above described. 
nitrate, hydrochlorate, and sulphate are crystalline, intensely bitter, and soluble in about 50 parts of water.

Brucia, $\mathrm{C}_{23} \mathrm{H}_{26} \mathrm{~N}_{2} \mathrm{O}_{4}$, occurs in transparent four-sided prisms, or if the solution be more quickly evaporated, in pearly scales that resemble boracic acid, or form a cauliflower-like mass; they are soluble in 150 parts of boiling, or 320 of cold, water : the taste is strongly and persistently bitter. Brucia and its salts are turned scarlet or blood-red by strong sulphuric acid.

The proportion of brucia in nux-vomica is variously stated to be 0.12 (Merck), 0.5 (Wittstein), 1.01 (Mayer) per cent.

Igasuria, or igasurin, was discovered by Desnoix in 1853. It is obtained in colourless, silky prisms, and is more soluble than strychnia or brucia-viz., in 200 parts of cold and 100 parts of hot water. Strong sulphuric acid turns it rose-red, the colour changing to yellow and yellowish green. Nitric acid gives a deep-red colour.

Igasuria has the bitter taste and poisonous properties of the other strychnos alkaloids.

All the salts of igasuria are crystallizable. Schiitzenberger states that it is a compound body, yielding no fewer than nine bases, "all colourless, very bitter, and acting like strychnia" (Compt. Rend., xlvi., p. 1274).

ABSORPTION AND ELIMINATION.-Strychnia is absorbed comparatively slowly from the stomach, more quickly from the rectum (Savory), still more so from the bronchi and cellular tissue; it has been detected in the blood, the spinal cord, the pons Varolii, and especially in the medulla oblongata; more recently it has been found in the brain substance, though rery little had been given (B. M. J., i., 1881). The salts are absorbed still more rapidly than the alkaloid itself, especially the hydrochlorate and the nitrate. Fatty or astringent substances taken at the same time delay absorption.

The amount found in the blood is comparatively so small as to suggest the hypothesis that the drug is partly decomposed in the circulation (G. Harley); but the conclusion from recent observations by Kratter is that strychuia is not decomposed in the liver, but excreted unchanged in the urine: that enough circulates to produce all the characteristic symptoms as shown by a striking experiment of Vulpian's, in which the 
blood is conducted from one living dog into the vessels of in other, and soon after strychnia is injected into dog number one, toxic symptoms appear in dog number two, which latter dies before the former, the life of this being prolonged by artificial respiration.

Elimination takes place mainly by the urine and the saliva, and under ordinary medicinal doses is completed in two or three days. Being thus rather slow, it follows that continuous doses may lead to unpleasant symptoms suddenly-after, for instance, a customary dose that has not been previously felt; hence it is important to omit the drug occasionally during a course of treatment. Elimination is markedly quicker in children than in old persons: it begins within one hour and continues forty-eight hours (Philad. Reporter, 1882), and is even more rapid in the case of brucia.

Physiological Action.-External.-Applied locally, strychaia causes contraction of capillaries, pallor, and some loss of temperature by reflex action: on the abraded skin it produces sharp pain and irritation which may go on to suppuration. Similar local irritation, varying in degree, may be induced in the gastric mucous membrane.

It has been proved (by Binz and others) to possess in some degree the influence which quinine so powerfully exerts as a poison to leucocytes, and to various lower animal and vegetable organisms.

Physiological Action.-Internal.-Digestive System. - Very small closes, $\frac{1}{T 0}$ to $\frac{1}{20}$ grain, stimulate the muscular coat of the intestinal tract to more active contraction, increase appetite and regulate peristalsis : larger doses cause diarrhœa.

Nervo-Muscular System.-Full medicinal doses, if continuerl, induce a state of heightened reflex irritability with general discomfort, restlessness, and twitching of the muscles. The special senses are also affected, so that bright light and loud sounds become painful, and in a few exceptional cases a simulation of alcoholic intoxication is produced. Such effects were formerly not uncommon when unwisely large doses were administered in the hope of curing paralysis, ote.

In toxic doses, of half a grain, sometimes less, strychnia 
produces powerful and characteristic tetanic convulsions. The first symptoms are developed in from a few minutes to an hour after the administration, with a sudden sense of suffocation and dyspnœa; the head and limbs begin to jerk in a shuddering manner, and the latter are suddenly stretched out rigidly, the hands clenched, the head bent backwards, and at last the whole body is stiffly arched, so as to rest upon the head and the heels. The soles of the feet are arched; the belly is hard and tense, the chest is fixed, and the breathing nearly arrested. In the height of the spasm, the face looks dusky and congested, and the eyeballs stand out strongly; the jaw-muscles are also affected with spasm, and the throat is dry, with a sense of choling. It is worthy of notice that the jaw-muscles are never so soon or so powerfully affected as in tetanus from ordinary causes, - a fact stated by Brodie at the trial of Palmer (Lancet, i., 1856). The patient often expresses a fear of impending death, but the intellect remains unclouded. After the paroxysm has lasted for a minute or two, there is usually a relaxation, during which the patient suffers only from soreness of the muscles, but before long he experiences sensations which warn him that the fit is returning, and cries out for some one to hold him or rub his limbs.

After one or two or a succession of paroxysms, respiration stops in the middle of a fit, and the heart soon after ceases to beat: death may occur within five minutes, and is seldom delayed more than five hours. The smallest dose which has produced a fatal result is of nux-vomica extract 3 grains, of strychnia $\frac{1}{2}$ grain, but several persons have recovered after swallowing even larger quantities, the paroxysms gradually becoming less violent, and the intervals longer. On the other hand $\frac{1}{3}$ grain of the alkaloid may imperil life, and $\frac{1}{20}$ grain, by hypodermic injection, is certainly dangerous. Chronic poisoning from continued medicinal doses has culminated in a sudden attack like apoplexy (Ziemssen). Tamassia records a rise of temperature in strychniapoisoning as contrasted with a fall under arsenic, corrosive sublimate, and phosphorus (Record, 1878). After death, there are no characteristic lesions generally recognized, but the occurrence of rigor mortis is usually rapid, almost immediate.

The action of strychnia on different animals varies in degree, 
man apparently being one of the most sensitive to it. The monkey is very little so, and birds less than rabbits. Wintzenreid found with brucia that different species of frogs were differently affected; on the Rana esculenta it acted like curare, on the R. temporaria, and on warm-blooded animals, it acted much like strychnia (Lancet, i., 1883). It is worth noticing that in this latter alkaloid, the substitution of a molecule of ethyl for one of hydrogen converts it into a paralyser allied to curare (Fraser).

Mode of Action.-Magendie taught that it acted on the nerve-centres as a direct irritant, like galvanism or other mechanical stimulus, and that ultimately paralysis followed over-stimulation; but the view adrocated by M. Hall, BrownSéquard, Vulpian, and others, supported as it is by many experiments on the lower animals, is the one now generally adopted, viz., that strychnia acts only on the grey substance, especially on that of the spinal cord, exalting its reflex power to such a degree that comparatively slight stimuli, intrinsic or extrinsic, provoke convulsive spasm; if ancesthesia be induced, the spasm is arrested, though on direct touching of the cord it recurs, showing that the anæsthetic only abolishes reflex action.

Cl. Bernard attributed to strychnia "an elective action on the sensory nerves," paralysing them ultimately, just as curare was supposed to do the motor: this is not correct, for according to Vulpian's experiments, sensibility persists even after motor power is destroyed (Leçons, 475); but when Bernard localizes the action upon the central terminal cellule of the sensory nerve, there is no clear difference between his view and that which locates the action in the grey matter. Stannius, again, argued for a primary action on sensory nerve fibres, especially in the posterior columns of the cord and the posterior roots of the nerves-relying mainly on the observation that if these be divided, no convulsion occurs under strychnia; but a more reasonable explanation is, that such division cuts off the path by which impressions commonly travel; and further, convulsions may sometimes be produced, even under the conditions given, by sudden shocks, etc. Valentin found that frogs apparently dead under strychnia, recovered on shaking (Arch. f. Eixper. Path., Bd. xii., 1880). 
Rokitansky describes a special stimulant action on the respiratory centre, and Brunton states that the drug has an action on the respiratory centre in the medulla oblongata similar to that which it exerts upon the vaso-motor centre, and under it respiratory movements become both quicker and deeper.

Rossbach and Nothnagel agree in stating that the drug acts on the whole spinal cord, especially the ganglion cells of the grey substance and of the vaso-motor and respiratory systems, as well as those concerned with reflex action. They find the impressionability of sensory nerves exaggerated, the motor nerves and the muscles not affected: Vulpian, however, found that the functions of the latter were abolished in later stages in batrachians.

Spitza, in a prize essay of the American Neurological Association (1878), thus sums up modern knowledge on this subject: -(1) Strychnia acts on the whole of the grey substance in the same manner, and affects both its sensory and motor elements. (2) The white substance and the peripheral nerves act only as conductors. (3) Strychnia has no local action on nerves or muscles, except on the peripheral extremity of organs of special sense. (4) It causes tonic spasm in all vertebrate animals-in the higher classes clonic spasm also. (5) Its maximum influence is intracranial, and becomes less progressively in the lower portions of the cord. (6) It augments reflex excitability, but all the spasms are not due to this only, some depend on a direct irritation of motor cells. (7) The lower animals it kills by nerve exhaustion, the higher by asphyxia, with venous congestion of the nervous system. (8) After administration it is found in all organs and tissues of the body-more is found in the grey than in the white substance; post mortem, lesions in toxic cases are due to secondary effects of the diug.

M. Conte maintains, in a recent memoir, that sensory nerves are not stimulated in strychnia-poisoning-on the contrny, they are less easily acted upon; also that the activity of both brain and spinal cord is diminished (Bull. de l'Académie des Sciences, 1883).

Death sometimes occurs in animals many hours after strychnia spasms have ceased, and in explanation of this, Jacubowitsch asserted that the polax prolongations of nerve-cells were broken, 
with escape of nuclei; but such change is denied by Vulpian and others, and seems, indeed, incompatible with the recovery, which sometimes occurs after very violent spasm and conditions of apparent death.

Evidence of the stimulant action of strychnia on the sympathetic system is found in the ocular phenomena-the protrusion of the globe and dilatation of pupil, but more especially in the contraction of small arteries by vaso-motor influence, and the consequent increase of arterial pressure.

Circulatory System.-Of such increase in pressure there can be no doubt, and Richter and Mayer have demonstrated it in animals previously "curarized," and lying immovable, i.e.. free from the convulsions of strychnia. The pressure may be seen to rise as the drug begins to act, and is not due to any influence on the heart; for it occurs even after section of the vagi, and it fails to occur after transverse section of the cord in the upper cervical region. This may be taken to prove that the narrowing of vessels depends upon the poison acting on the vaso-motor centres, and especially those in the medulla oblongata: the pressure may rise to twice its normal amount. The pulse is naturally rendered slower at the same time. The primary stimulation and rise in pressure described are followed by opposite conditions, - a fall in pressure and vaso-motor paresis: very large doses produce these latter symptoms directly (Klapp). The systole of the heart is increased in force, and the diastole prolonged (Spitza); the organ may cease to beat and become fixed in a state of spasm at any period of poisoning: the action on it is exerted partly through the cardiac ganglia-partly through the pneumogastries.

In the vertebrata, the heart sometimes does not quite stop when apparent death takes place, and its activity may be restored by artificial respiration if this be quickly commenced. (Vulpian, p. 433).

According to the observations of Dr. G. Harley, ordinary blood shaken with air for a fixed time was found to contain about $11 \frac{1}{2}$ parts oxygen and 6 of carbonic acid, whilst blood containing strychnia gave nearly 18 of the former and 3 of the latter (Lancet i., 1856). Hence he concluded that the presence of the drug lessened oxidation-processes in the body, and so 
accounted for the nervous phenomena-but the latter supposition has not been supported, and would seem difficult to reconcile with the special affection of the spinal cord as distinct from the brain and nerves.

Genito-Urinary System.-The passage of strychnia by the urine doubtless determines resical symptoms (such as frequency of micturition with some spasm) which occur especially in women: the ultimate effect of toxic doses is loss of controlling power of the sphincters, the urine as well as fæces passing involuntarily. The uterus is stimulated to contraction, and menstruation is rather promoted than the reverse: the drug is said to increase the sexual appetite, and there is evidence that it induces erections.

SYNERGISTS.-Electricity favours the effect of strychnia, according to Vulpian's experiment, in which he galvanized the cut end of a sciatic nerve in a frog subjected to strychnia, and found that characteristic spasm occurred in it more violently than in the opposite one, and it lost its proper powers sooner from exhaustion. On the other hand, a case of poisoning is recorded in which the spasm of the chest-muscles ceased under the electro-magnetic current (Record, 1880).

Ignatia, brucia, picrotoxin, thebaia, to some extent ergot, and according to Rabuteau, belladonna, are also synergic.

ANTAgonists.-(1) Chloral hydrate. There is much evidence as to the definite antagonism exerted within certain limits by this drug. Hughes Bemnett early showed that a recognized fatal amount of strychnia was counteracted in its effect by a certain dose of chloral-though of course a very large dose of the former may kill before any antidote has time to act; and, on the other hand, the dose of the latter may be such as to destroy by its own power. The antagonism extends to the soothing or stimulating respectively of the reflex activity of the cord: strychnia will not antagonize the coma of chloral.

The proofs offered by Liebreich of the antidotal power of chloral are weakened by the results of Orr, who showed that the dose considered as fatal by the former did not always prove so (1872). Bennett's experiments, however, are subsequent to 
these (1874). He put the minimum fatal dose of strychnia for rabbits at $\frac{1}{288}$ grain per pound of body-weight,-and out of twenty rabbits poisoned by more than this proportion fifteen recovered under chloral: these died quickly a few days afterwards from a similar dose of the poison given without the antidote. In man, eases in which 4 grains and upwards have been taken have recovered under chloral (Lancet, i., 1875).

Husemann showed that chloral acted better when given alone than with other remedies (Arch. f. Exper. Path., Bd. vi. 9-10, 1882).

Dr. G. Gray quotes a case where 22 grains of strychnia were taken, and after an emetic, ete., one drachm of chloral was given and followed by a second drachm, recovery ensuing (B. M. J., i., 1880).

Vulpian has demonstrated the antidotal power of intravenous injection of chloral in dogs with much exactness (op. cit., p. 568).

(2) Dr. Richardson relieved the spasm by oxygen and amyl (Lancet ii., 1878), and Dr. R. Barnes found good from amyl nitrite (B. M. J., i., 1882), Cockburn from chloroform (ibid.) : these remedies, ether, and also, though more slowly, bromide of potassium, act like chloral. A case of recovery after 3 grains of strychnia, when $\frac{1}{2}$ ounce of the bromide was quickly given, is quoted by H. C. Wood: there was no vomiting, but relaxation of the spasm occurred half an hour after the bromide was given; small doses of it were continued for an hour or two afterwards. On the other hand, McReddie gives cases (in dogs) where chloroform, amyl, eserin, and atropin proved useless (Record, 1883). Delaunay ascertained that stronger animals were more rapidly poisoned than weaker ones (ibid., 1882).

(3) Alcohol pushed to narcosis acts in a similar manner to chloroform, etc., as shown on animals.

(4) Other drugs that might seem indicated from physiological experiment, such as aconitia (Fothergill), prussic acid (Brunton), and nicotin (Haughton), are unsafe, and, with a few exceptions, have not proved useful in practice (St. Barth. Hosp. Rep., xii.).

Cases of animals recovering under prussic acid are given in B. M. J., ii., 1883 ; curiously, Harley found it increase the symptoms. 
In MIr. Burton's case an infusion of tobaceo seemed useful (ibic.). Haynes found that nicotin increased the convulsions of strychnia, and vice versâ, and that animals were killed by injection of a dose of both, when the same dose given singly was not fatal.

O'Shanghnessy recommended cannabis indica as the best antidote, but it has not been largely tried. Dr. G. Harley advised curare, but Vulpian found no good effect from it. Camphor has been given by Pritchard, opium by Puzey, hyoscyamin by Gillespie, Calabar bean by Watson and Keyworth, etc.

Opium has markedly delayed the onset of strychnia symptoms, e.g., when $1 \frac{1}{2}$ and 2 drachms respectively of laudanum were taken at the same time as a packet of "vermin liiller" (Lancet, ii., 1871, and i., 1882). In a case where $1 \frac{1}{\mathrm{~s}}$ grain of strychnia and at the same time 1 or 2 ounces of tinctura opii were taken, symptoms of the former drug did not set in for eight hours,-chloral was given four hours afterwards, and recovery occurred (Edin. Journ., 1883; Lancet, ii., 1882).

(5) Artificial respiration, practised forcibly with a tube in the larynx, certainly prolonged life, and contributed to recovery in poisoned animals (Leube-Rosenthal), but it is doubtful whether any possible method of employing it in man can be efficacious.

(6) Charcoal acts as a mechanical, and tannin and preparations of iodine as chemical antidotes, precipitating and decomposing part of the alkaloid; but if they are first administered in cases of poisoning, they should be followed by emetics or the stomachpump, since the compounds formed are still poisonous (B. M. J., ii., 1882).

(7) A new organic base obtained from the distillation of cinchona and potash, and termed $\beta$ lutidine, is asserted to show marked antidotal properties (Williams and Waters, B. M. J., i., 1882).

THERAPEUTICAL ACTION.-The action of nux-vomica and of its alkaloids is practically the same, so that either may be prescribed according to considerations of convenience and safety.

Paralysis.-The earliest applications of strychnin in medi- 
cine were directed to the cure of paralytic affections, and during some years it was employed indiscriminately, and often in too large doses. It was thought that the drug should be pushed to the production of actual symptoms of poisoning, and doses as high as the $\frac{1}{8}$ and $\frac{1}{4}$ grain twice or three times a day were often reached, with the frequent result of throwing the patient's nervous system into great disorder, manifested by twitchings of the limbs, hyperæsthesia of the retina and the auditory nerve, and a state of perpetual restlessness. It was discovered after a time that such effects were injurious rather than useful: and although it has been stated that tinnitus must be caused before any benefit can be expected (Med. Times, ii., 1878), violent strychnia-action is specially hurtful, and the drug in any dose is valueless in particular forms and stages of paralysis. In paralysis of cerebral origin it is seldom beneficial; on the contrary, its too early use in these affections, especially in such as proceed from hæmorrhage, has often proved mischievous. It would be natural to expect better results from its employment in spinal paralysis, yet even in such cases there is frequent disappointment, and in the early stages of organic lesions it may do much harm if given in large doses. In the so-called "reflex" paralyses it was supposed, by Brown-Séquard, to promise great results, but experience has hardly supported these expectations, a fact not altogether surprising, since the tendency of recent pathology is to render probable in these cases the presence of actual myelitis, produced by irritation transmitted to the cord through the nerves of the kidney, bladder, uterus, or whatever organ it may be in which the original mischief existed. His suggestion that it acter by dilating the vessels of the cord is not supported by later investigation; but I agree with him that in those cases of paraplegia in which there are signs of congestion or inflammation of the cord or membranes, strychnia should be avoided as dangerous: it is only suitable where the symptoms are chronic.

In finctional paralysis, however, such as that connected with hysteria, with sexual excess, or concussion of the cord, or devendent upon opium, lead or alcoholic poisoning, or resulting from diphtheria, strychnia is often of use, decidedly promoting 
recovery of power in the muscles. Some cases of facial palsy and of aphonia have also been improved by it.

There is one form of paralysis-that, namely, which is limited to one or two groups of muscles-in which a remarkable and, effective employment of strychnia has been devised by Mr. Barwell. He adopted it chiefly in cases of chronic infantile paralysis, when the atrophic process had gone so far as to impair the electric sensibility, even to a constant current. He employed a 2 per cent. solution of hydrochlorate of strychnia, and in some instances injected as much as $\frac{1}{6}$ grain. When these cases were first brought forward some mistake was suspected by most persons, since much smaller quantities, subcutaneously injected, had been known to induce alarming symptoms. But the solution, being examined, was found to be exactly of the represented strength; and as Mr. Barwell made a point of injecting quite into the substance of the paralysed muscles, there could be no waste of the fluid. It is now conceded, by all who have studied the matter, that the concentration of his solution localizes its action; it is highly irritant, and, when injected into a muscle, at once sets up inflammation around, and becomes enclosed, instead of mingling with the general circulation; it is less dangerous than a weaker solution.

The local injection of a strength of 1 in 1,000 has been practised in France with success for the treatment of prolapsus ani; of such a solution 10 or 15 drops were used by Dolfear and Foucher, but the operation would probably be safer if performed with the stronger solution of $\mathrm{Mr}$. Barwell. Bianchi has recorded some striking cases of paralysis of special muscles, e.g., of certain fingers used for playing the flute, lasting long, and rebellious to all ordinary treatment, yet cured by intra-muscular injections of strychnia (B. M. J., i., 1878); and Annandale a good cure of "writers' palsy" after nine injections of equal parts of liquor strychniæ and water, beginning with 6 minims every second day, and increasing up to 12 minims (Record, 1878).

When adult spinal paralysis becomes chronic, strychnia (by the mouth) in many cases aids the recovery of the muscles.

In Anæsthesia of functional character, the good effects of the drug are often very marked; a circumstance which illustrates 
a difference between its poisonous and therapeutical actions, since among the former we scarcely find any direct and eviclent effects upon common sensation.

In Atony of the Bladder, with incontinence or retention of urine, such as occurs in old people with enlarged prostate, strychnia is sometimes employed with much benefit. $\mathrm{Mr}$. A. P. Gould has reported a case illustrative of this (Lancet, i., 1881), and the same treatment is applicable to the spermatorrhoea of debility; when, however, the latter clisorcler or incontinence depends on irritability and excitation of the parts, the more usual treatment by bromides or belladonna is indicated; hence this succeeds better if the disorder's are nocturnal only, whilst strychnia is likely to suit if they occur in the day also, i.e., are more persistent, and less spasmodic. In impotence connected with relaxation of the genitals, it is said to be effective.

In Atonic Conditions of the Intestine, strychnia is of great service, especially in that apparently almost hopeless form of constipation in which the large bowel does not contract properly, and becomes passively loaded with more and more freces day after day. In prolapsus ani, arising from such a state of rectum and sphincter, Dr. Schwarz strongly recommends nux-vomica; I agree with him, and can speak with equal emphasis of its benefit in hromorrhoidal tumours of the anus. Five to ten drops of the tincture of nux-vomica, taken in a tumbler of cold water before breakfast and dinner, act as a laxative, and often overcome most obstinate constipation. In some forms of diarrhœa, i.e., when there is debility without irritation, and when it is readily excited by nerve-causes, and the muscular coat of the bowels may be judged wanting in "tone," small doses of the drug are well combinerl with other remedies, such as acids. In subinvolution of the nterus in weakly subjects, strychnia is often of service.

In dysentery also, with much depression and tympanites, it may be useful. Even in cholera it is said to have acted well (but combined with sedatives).

In Dyspepsia of the simple atonic form, strychnia, or tincture of nux-vomica, is often of the highest value; it is generally best given alone, but an excellent combination is the tincture, 
with dilute nitric acid-5-minim doses of the former, and 10 or 15 of the latter. I have already mentioned its good influence upon the gastric irritation of alcoholism; it may be added that heartburn, hiccough, regurgitation, and even pyrosis, when chiefly due to an atonic condition of the muscular walls of the stomach and functional languor, may frequently be cured by a short course of this medicine. It is often very useful in the morning-sickness of pregnant women, also in abdominal cramps and spasms.

On the other hand, cramp and pain in the bowels may be calsed by ordinary (about 5 minims) doses of the tincture of nux-romica in some patients, especially, as I have noticed, in nervous delicate women; it is well, therefore, to begin with 1 to 2 minims.

Brunton, in an interesting paper, describes strychnia as "at once a gastric, vascular, and nervous tonic," aiding appetite and digestion, preventing putrefaction, and exciting the sensibility of the vaso-motor centre (On Tonics, Practitioner, ii., 1878).

In Tremors and Ataxic Movements of many kinds (though not in true locomotor ataxy), strychnia has proved useful, - for instance, in the tremor of chronic alcoholism, part of its beneficial action being probably due to its remoring the usual catarrhal condition of the stomach. In some cases of hysteric tremor, where the hysteria is merely the product of great bodily weakness, induced by illness or exhausting fatigue, it is similarly beneficial. In chorea, particularly in those cases where fright, or the disturbing effect of commencing puberty, rather than rheumatism, is the principal cause, strychnia in minute doses ( $\frac{1}{80}$ grain ter die for a child of ten years, $\frac{1}{B U}$ to $\frac{1}{40}$ grain after puberty) has often been found of much use.

Vulpian and others are sceptical as to the real value of the drug in such cases, but there is certainly some evidence in its favour, e.g., in one boy with chorea, ill for many weeks, and rendered apparently idiotic by the disorder, recovery ensued under doses gradually increased up to $\frac{1}{5}$ grain thrice daily, no physiological effects being evident (B. M. J., ii., 1882). Troussean advised it in such doses as to produce these effects very strongly, but the practice is unsafe. In tetanus, however, 
if used at all, it is pressed with the object of "substituting the strychnic for the tetanic spasm."

In Epilepsy, Mr. Tyrrell strongly adrocated its use, and showed it to be sometimes effective in the idiopathic form occurring in weak anæmic subjects, especially with nocturnal attacks, or when bromides depress too much and do not act well (B. M. J., ii., 1869). For symptomatic or reflex epileptiform attacks it is unsuitable, and in any case its use is rather the erception than the rule.

In cases of Impairment of the Nervous Apparatus of Sight and Hearing, strychnia is believed by some authors to be very useful. As regards vision, no one, of course, expects it to do good in those diseases in which the nutrition of the retina is destroyed by inflammatory or degenerative processes; but there is reason to think that cases of simple atrophy may be really improved by it. Nagel especially brought forward cases of amaurosis, and even of white atrophy, in which improvement occurred (Tiibingen, 1871), and similar results may be verified in functional amaurosis, e.g., from tobacco, and the headache and giddiness secondary to certain eye troubles may be relieved; also amblyopia from debility or remaining after operation for strabismus; but in other cases not much must be expected. Wilcle stated (many years ago) that he had never seen any benefit from the drug in amaurosis from defective nerve-energy (Med. Times, i., 1861). As in some paralyses, it would seem that much better results may be obtained by the hypodermic use of the drug near the eye than in any other way: and it is said that improvement has occurred in the eye near the seat of injection when none was found in the opposite eye. As remarked by Stillé, it is not easy to explain such effects, for experiment does not show any action of the drug on peripheral nerves.

In Neuralgias of various kinds, strychnia has often proved most useful, and especially where the pain is visceral rather than superficial -in hepatalgia, for instance, and in the milder forms of angina pectoris, and in gastralgia. In the latter clisease, with flatulence, eructations, etc., tincture of nux-vomica has long been a favourite and fairly successful remedy, and may be well combined with antacids and cammintives, though there 
are intractable cases, of course, where this, like every other medicine, will fail.

In all forms of neuralgia the dose of nux-vomica or of strychnia should be small; of the latter there is seldom occasion to use more than $\frac{1}{100}$, or at most $\frac{1}{32}$ grain twice or three times a day. Anstie wrote specially to recommend, in angina and in gastralgia, hypodermic injections of $\frac{1}{120}$ to $\frac{1}{60} \mathrm{gr}$. (B. M. J., ii., 1868). In prurigo it is said to be serviceable, and may fairly be tried in purely " nervous" cases.

In Spasmodic Asthma, and in Coryza, nux-vomica and strychnia have often been used with much benefit.

In Weak Heart, strychnia proves an excellent tonic, and is sometimes the first remedy which begins to do good in cases of fatty heart, when many others have been tried. One caution must, however, be added, namely, that any undue pushing of the remedy will produce, even more seriously than in other subjects, a state of nervous worry and restlessness, in which sleep is broken or even destroyed by a tendency to perpetual muscular movement. To the subjects of fatty heart this condition is not merely fatiguing and annoying, but dangerous; and we are therefore bound to inquire for the first symptoms of its appearance when administering strychnia to such persons.

In Dyspncea dependent on the above cause, or on chronic bronchitis, or emphysema, strychnia has been praised, and is sometimes, but not by any means constantly, useful.

Fothergill has written to recommend it as facilitating expectoration, etc., in the cases referred to (B. M. J., ii., 1881).

In Intermittent Fevers it has been proposed to substitute the use of strychnia for that of quinine; but so far as concerns the acute stages, there is no satisfactory evidence of its efficiency. In the stage of convalescence, however, the combination of strychnia with quinine and iron, in the shape of Easton's syrup, is spoken of with much approval by Dr. Maclean and other good authorities.

The compound syrup of Fellows also contains these remedies with hypophosphites, and is much used in such cases, as well as in anæemia and chlorosis: it sometimes disagrees with the stomach. 
PREPARATIONS AND DOSE.-Of Nux-Vomica-Pulvis : dose, 2 to $5 \mathrm{gr}$. Extractum (alcoholic, of the seeds) : dose, $\frac{1}{4}$ to 2 gr. Tinctura (of the seeds) : dose, 1 to 20 min. Bishop has a granular effervescent preparation containing $\frac{1}{12}$ gr. extract, representing about $12 \mathrm{~min}$. of tincture, in each teaspoonful.

Of Strychnia-dose, $\frac{1}{30}$ to $\frac{1}{12}$ gr. ; but much smaller quantities act strongly in certain constitutions, while larger doses than $\frac{1}{12} \mathrm{gr}$. may, in some special cases, though with caution, be administered. Hypodermically the dose is $\frac{1}{90}$ to $\frac{1}{24} \mathrm{gr}$. Liquor strychnice (strength 1 in 120): dose, 5 to $10 \mathrm{~min}$.

Smaller doses are recommended in the therapeutics of this article.

\section{STRYChNos IgNatia. (St. Ignatius' Bean.) (Not Officinal.)}

DESCRIPTION.-A tree indigenous to the Philippine Islands, having smooth branches, ovate, acute leaves, 5 to 6 inches in length, and elongated tubular white flowers in small axillary panicles. The fruit is spherical, ovoid, pearshaped, 4 inches or more in diameter, with a smooth brittle shell, which contains fifteen to twenty olive-shaped seeds, called "St. Ignatius' beans." Linnæus named the plant Ignatia amara.

ACTIVE INGREDIENTS.-Analysis proves most of the active constituents of these seeds to be similar to those of nuxvomica, though in different proportions. Strychnia is found to the extent of about 1.5 per cent., brucia to about 0.5 per cent., and albuminoid matter to nearly 10 per cent.

Physiological Action.-Given in small doses, gradually increased to a poisonous quantity, the symptoms which ignatia produces are as follow:-Increase of the salivary secretion, nausea, heaviness, giddiness, headache, pain in the stomach, flatulence, a feeling in the limbs as if they had gone to sleep, accompanied by great weakness and twitchings throughout the body; constipation at first, and diarrhœe later . on ; constriction about the throat, numbness, torpor, mental 
depression, twitching of muscles, tetanic spasms; a feeling of intense anguish at the pit of the stomach; convulsions; cold sweats; and finally death by dyspnoa or asphyxia.

Therapeutical ACTION.-St. Ignatius' bean contains about four times the amount of strychnia that the strychnos nux-vomica does, yet its effects are not identical with, though somewhat similar to, those of the latter.

Hysteria.-Ignatia is useful in many cases where the patient suffers from a feeling of suffocation, and a sensation as of a ball rising to the throat, whether attended or not by the usual symptoms of an hysterical paroxysm. It not only relieves the convulsive attacks, but frequently prevents their return. Its effects are considerable in controlling, and even permanently removing, the convulsive bursts of crying or laughing, as well as the hiccough, the flatulent distension, and the general hyperæsthesia. Intercostal neuralgia, so common in hysteria, is often removed by the drug; also clavus hystericus, the acute pain in the head, as if a nail were being driven into it. Where there is either great mental excitement or depression, also in hypochondriasis, ignatia has a soothing effect.

The aphonia, as well as the scanty menstruation and profuse leucorrhœa, so liable to occur in hysterical women, I have lnown pass away under the influence of this medicine. I have often used it with good effect in the reflex comoulsions of children arising from intestinal irritation, such as worms or undigested food, and unattended with cerebral congestion.

In dyspepsia with nervous depression, also in conditions of morbid appetite, it is often useful.

PREPARATIONS AND DOSE.-Eitract: dose from $\frac{1}{8}$ to $\frac{1}{4}$ gr. Tincture (1 in 20): dose, 1 to 5 min.; of Corbyn's, 5 to $20 \mathrm{~min}$.

The constituents of the lignum colubrimum of Timor, the produce of the Strychnos ligustrina, correspond with those of ignatia; and this drug is held by the natives in great estimation as a cure for neuralgia, and for "paralysis of the lower extremities." 


\title{
SPIGELIA MARILANDICA.
}

\author{
(Carolina Pink-Root.)
}

\section{(Not Officinal.)}

DESCRIPTION.-This plant is indigenous to the Southern and South-western States of North America. It has a qualrangular, smooth, purplish stem; a thick knotty rhizome, 2 to 6 inches long, bent in different directions, wrinkled longitudinally, and giving off numerous fibres. It is of dark brownish colour; slight aromatic odour; sweetish, afterwards bitter, taste ; opposite, ovate, sessile leaves; and a terminal raceme of flowers, with funnel-shaped corollas, which are crimson externally, and orange within.

ACTIVE INGREDIENTS.-The root and leaves contain a peculiar bitter substance and a volatile oil, discovered by Feneulle, but which is the true active principle is not at present known.

Physiological Action. - The aborigines of America used spigelia as a medicine, and the early settlers adopted it from them as a purgative and narcotic. The experience of Thompson is conclusive as to the fact that some narcotic effects can be produced by it; large doses causing drowsiness, as well as stiffness of the eyelids, flushed face, and quickness of pulse. In the case of a child where about 5 ounces of an infusion (made with 3 drachms of root to 15 ounces of boiling water), were given in divided doses, the skin became hot and dry; pulse 110, and irregular ; face, especially about the lids, much swollen; pupils widely dilated: there was strabismus of the right eye, and at wild, staring expression of countenance. The intellect seemed maffected, but the child was seized with general tremor on trying to assume the erect position; the tongue also was very tremulous. Next morning every symptom had disappeared, except the swelling of the eyelicls. It appears, however, that; such effects are but rarely cansed in practice; possibly the cathartic element prevents the influence of the narcotic. 
TherapeUtical Action.- In the United States, spigelia ranks as probably the best of the anthelmintics, and as such is in general and constant use, especially for the round worm. There are, also, some cases of intestinal derangement in children which simulate in many respects those produced by worms, accompanied by much fever and nervous imitability, and which are often cured by this drug. It is often advisable to unite with it a purgative-for instance, senna.

PREPARATIONS AND DOSE.-Stillé gives the following as the best formula for the medicine:-Spigelia $\frac{1}{2}$ oz., senna and fennel seeds, of each 120 gr., manna 1 oz., boiling water 1 pint -infuse: dose, $\frac{1}{2}$ to $1 \mathrm{oz}$. for a child, 4 to $8 \mathrm{oz}$. for an adult. The American Pharmacopoia contains an extractum spigelice et senme fuidum: dose, $\frac{1}{2}$ to $4 \mathrm{dr}$.; and an infusum spigetice: dose, $\frac{1}{2}$ to $1 \mathrm{oz}$. for a child; 4 to $8 \mathrm{oz}$. for an adult.

AdULTERATIONS. - Sometimes mixed with serpentaria root.

\section{SPIGELIA ANTHELMIA.}

\section{(Demerara Pink-root, or Wormgrass).}

\section{(Not Officinal.)}

DESCRIPTION.-The Demerara pink-root, or wormgrass, is an annual, native of the West Indies and South America. The stem rises to the height of 12 to 18 inches; the leaves are opposite, sessile, ovate, and entire, the upper ones growing in whorls of four. The insignificant greenish-red flowers are borne in short, unilateral, axillary spikes, chiefly near the summit of the plaut, and in structural characters correspond with those of the spigelia Marilandica, though inferior in size and beauty. The root is short, and divided into long thin branches, dark outsicle, white within.

ACTIVE INGREDIENTS.-Unknown. 
Physiological Action.-This plant must be classed with narcotico-acrid poisons, since full doses cause vomiting and diarrhoea, giddiness, stupor, dilated pupils, subsultus tendinum, irregular contractions throughout the body, dyspnoea, convulsions, and death. It is fatal to cattle when they eat it.

THERAPEUTICAL ACTION.-Spigelia anthelmia is useful in rheumatic pericarditis and endocarditis, in rheumatic ophthalmia and facial neuralgia. It is also employed as a vermifuge.

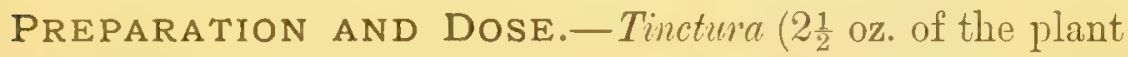
to 1 pint of proof spirit): dose, 5 to $10 \mathrm{~min}$. in water.

\section{CURARE.}

(South American Arrow Poison.)

\section{(Not Officinal.)}

Curare or curara (woorara-woorali) is a poisonous compound prepared by certain savage tribes in South America. It was first brought into Europe from Guiana by Sir W. Raleigh, in 1595. There seem to be two kinds of it, one in calabashes, the other in clay vases, the latter being the stronger. It is not easy to procure, and its exact constituents are not certain; but according to the latest researches of M. Planchon, they are different species of strychnos, mixed sometimes with cocculus, and, according to Claude Bernard, with Paullinia curara (Sapindacere). Preyer identified the last-named in one specimen. In the preparation described by Waterton, poisonous ants and serpent-venom were contained; but in that now imported there is probably no animal poison (Schomburgh).

DESCRIPTION.-Curare, as seen in England, is a blackishbrown substance, of consistency varying from that of an extract to a resin: the taste is very bitter.

It is incompletely soluble in water, i.c., the solution is brown 
and turbid, and when heated gives out an odour resembling that of chocolate. Concentrated sulphuric acid produces a red coloration, gradually becoming deeper, and with shromate of potash the same colour-reactions as strychnia (Koch).

The physiological" action is highly characteristic, and serves at once for the identification of this curious drug. If a drop of the solution be injected beneath the skin of a frog, the animal quickly becomes paralysed in all its voluntary muscles. The extract retains power for an indefinite period, but is weakened by moisture.

ACTIVE INGREDIENTS.-The activity of curare has been proved to depend upon the presence of the alkaloid curarin, $\mathrm{C}_{10} \mathrm{H}_{15} \mathrm{~N}$, discovered by Roulin and Boussingault in 1830, and procured in well-defined crystals by Preyer in 1865. These crystals are four-sided, colourless prisms, of very bitter taste, and weak alkaline reaction. They readily attract moisture, are soluble in water and alcohol, but not in pure ether, sulphide of carbon, benzol, or turpentine.

ABSORPTION AND ELIMINATION.-As with serpentvenom and some other poisons, absorption does not readily occur from the stomach, if the mucous membrane be sound; and hence a comparatively large dose may be given, and but little effect observed, since elimination by the kidneys is rapid, and almost as soon as the drug is taken up by the stomach it passes into the urine, and does not accumulate in the system. Absorption per rectum is also slow, and by the bladder it is almost mil: by the subcutaneous cellular tissue, or by the muscles, as in a wound, it is more rapid; by the lungs still more so, whilst by intravenous injection a smaller quantity develops its effect most quickly. That elimination takes place mainly by the kidneys, and that its rapidity prevents, to a great extent, poisoning by way of the stomach, is shown by the experiments of Bernard and of Hermamn on rabbits and other animals; when they ligatured the renal arteries and then injected the poison into the stomach, toxic effects soon developed.

The same remarks apply to the alkaloid curarin. The fact of its elimination by the urine was distinctly shown by 
Voisin and Liouville, who injected the urine of curarized frogs into healthy frogs, producing the characteristic curara effects; and the matter was carried still further by Bidder, who paralysed a third frog with urine taken from one that had itself been poisoned by the urine of the animal to which the original dose of eurarin had been given. There is no reason, however, to think that the whole of the curarin is eliminated without change: for it is known to be easily acted on by ozone, which destroys its poisonous properties; and probably a certain amount of it always gets oxidized and destroyed in the body.

\section{Physiological Action.-Nervo-muscular System.-} The special action of this drug is to paralyse the voluntary or striped muscular tissue, slight convulsive action sometimes preceding the paralysis. M. Couty has recently reported, from small doses of some native preparations, more marked excitation-jumping, hyperæesthesia, choreic movements and spasms, distinguishable from those of strychnia and of asphyxia,-and concludes that curare is not destitute of convulsant action, and also that it has some effect on the central nervous system (Lancet, i., 1883). The muscles of the limbs are first affected, and then the respiratory muscles, so that death threatens by asphyxia, and may be averted for some time at least by artificial respiration, the paralysis of the muscles continuing.

Hence it has become frequent in physiological experiment, especially abroad, to "curarize" animals, and then keep them alive by artificial respiration, the limbs and trunk remaining: motionless without forcible restraint; $\frac{1}{1000}$ grain is sufficient to place a frog hors-de-combat in about twenty minutes. Animals pierced with arrows poisoned by curare show no symptoms for two or three minutes, and then, if the quantity absorbed be sufficiently large, they soon die, without struggle or apparent pain, in a state of stupor or paralysis. (It is noteworthy that the flesh may be safely used as food.) The paralysis does not depend upon a lesion in the central nervous system whether of the brain or cord, neither is it in the nerve-trunks, for they are found to retain their function; for instance, if a muscle-e.,., a gastrocnemius in a frog-be isolated from its connections and 
left attached to the body only by its nerve (being thus prevented from any direct contact with the poison injected into the body), galvanization of the sciatic nerve will cause the usual contraction of this muscle - not of any other in the body, though, of course, the same nerve supplies many others (Cl. Bernard, Vulpian). Again, if the two gastrocnemii, with their connected nerves, be detached from a frog's body, and the nerves of one specimen, but not the muscle, be immersed in a curare solution, these nerves will be found, on galvanic stimulation, to retain their conducting power; whilst, if the muscle of the other specimen be steeped in a solution (the central ends of the nerves being kept from contact with it), this specimen quickly loses all contractile power, the poison having directly affected the peripheral ends of the motor nerve or else the muscular fibre.

From many experiments it is concluded that neither is this latter directly affected, for it retains its irritability after the action of the poison; there are, in fact, various difficulties in precisely explaining the effect of the drug, but it is most probably exerted on the place of connection between the ultimate nervefibril and the muscular fasciculus, causing a kind of " functional solution of continuity" between the conductor and the tissue in relation with it.

The recent researches of M. Ch. Rouget point to a distinct affection of the "plaque motrice terminale," some modified condition of tissue where the nerve and muscle join each other. It is not that the nerve-fibre becoming then destitute of "myeline," is more exposed to the poison than at other points of its course, because it is equally devoid of myeline at its origin in the brain, and yet is not there affected. (It will be seen that the observations of Onimus [Record, 1880], to the effect that the nervetrunks, and not the terminals, are acted upon, are not accepted as correct.) The intellectual functions of the brain do not seen affected, but there is ground for believing that the stimulus of the will is less actively conducted than normally, though the mechanical stimulus of galvanism works as usual.

Certain parts of the encephalon or of the nervous system are stimulated for a brief period-the pupils are moderately dilated, the secretions somewhat increased, and reflex power persists. 
Sensory nerves retain their function almost unimpaired, and it is suggested by Vulpian, not that they are essentially different in structure from the motor nerves, but that their mode of connection with other organs may be different.

The sympathetic system of nerves, though not paralysed, is impaired in power, for dilatation of small vessels occurs, with lowering of arterial pressure and rise of surface-temperature: still, after section in the neck, the usual symptoms develop, viz., narrowed pupil, retraction of the globe, lessened palpebral aperture, and congestion of conjunctiva; whilst on galvanizing the upper end these conditions are reversed.

In explanation of the comparatively slight action on the sympathetic, it may be said that they do not join on to the smooth muscular fibre in precisely the same way as motor fibres join muscle.

The sympathetic fibres, the inhibitory nerves, the vaso-dilators, and the nerves of secretion preserve their functions, until at least the motor nerves are completely paralysed, i.e., the full influence of the drug is exerted; but the resistance of all the nerves is only a question of degree, for direct injection of the drug into a neighbouring artery will overcome the normal action of any nerves.

Rhythmic movements-those of vegetative life, such as of the heart, the intestines, etc.-also continue under all but the fullest influence of curare, provided that sufficient aeration of the blood be kept up : the intestines, in fact, are more than usually sensitive to stimuli. It has recently been stated that one variety of curare acts only on the smooth muscular fibres, and kills by lowering of arterial tension (Couty and de Superdat). The inhibitory nerves of the heart are notably more quickly paralysed in frogs than in mammalia (Vulpian, op. eit., p. 359).

Circulatory System.-The action on the heart, just referred to, is exerted through the nervous system. It is known, from experiments of $\mathrm{Cl}$. Bernard and of Waller, that the motor fibres of the vagus, both to the heart and to the laryngeal muscles, are derived from the spinal accessory, and we know that galvanizing the vagi will arrest the heart's action, i.e., by stimulating the "inhibitory nerves." Now, under the moderate action 
of curare in the frog, and under its full toxic influence in mammalia, this arrest-action under galvanism does not take place-i.e., the inhibitory nerves are paralysed (though inhibition may still be produced by stimulating the junction of the auricle and sinus in frogs - a point of difference from atropia). Similarly the arrest-action of digitalis does not occur, but the slowing-action of muscarin or jaborandi is not interfered with. Curare itself does not, as a rule, arrest the heart, but somewhat weakens its pulsations; under certain conditions, however, of physiological experiment it can be shown, in full doses, to have a paralysing action upon it (Vulpian).

The weakening is partly dependent on dilatation of peripheral vessels, which is very marked, and is the cause of less blood reaching the heart-cavities for them to contract upon: reddening of the extremities, the face, the eyes, and ears is evident, and is accompanied with marked increase of temperature there. The pulse, though weakened, is quickened, and so also is respiration. Distinct pyrexial phenomena, with chills, were developed in some epileptics under full doses of the drug (Voisin and Liouville); and it generally raised the temperature three or four degrees. According to Vulpian's observations on animals, the rise is temporary and superficial, and the internal temperature is soon markedly lowered, probably, as he suggests, from rapid loss of caloric by the surface.

Glandular System.--It has already been stated that secretion is not arrested by less than fatal doses of curare; but a remarkable change occurs in the composition of the urine, an artificial glycosuria being set up. This occurred both in animals under experiment and in the epileptic patients referred to, i.e., under full medicinal doses. It is presumably connected with alterations of the capillary circulation through the liver-from vaso-motor paralysis. In frogs, when the bladder became enormously distended with urine under curare (due to paralysis), Vulpian noted the occurrence of much oxalate of lime.

SYNERGISTS.-Conium exerts a similar effect in destroying the conduction of efferent impulses to muscles, their physiological functions proper remaining intact. Vulpian finds, in 
the frog at least, the same condition produced in certain stages of poisoning by strychnia, brucia, atropia, hyoscyamin, nicotin, aconitia, Calabar bean, thebaia, even by bromide of potassium, sulphate of magnesia, and other salts, as well as by the ethyl and methyl derivatives of most of the toxic alkaloids: this does not, of course, mean that the totality of symptoms produced is the same as with curare. Schulz, however, finds a close parallelism in the action of hydrobromate of coniin (Zeitsch. f. klin. Med., Bd. iii., 1881).

ANTAGONISTs.-Salt, the halogens, and carbolic acid have been named, and have some influence in lessening or preventing: toxie effects without being truly, antidotal. More value has been ascribed to strychnia; or, rather, curare has been heldby G. Harley especially - to be directly opposed in action to that drug. From one point of view it might seem truly so, since the latter often kills by tetanic spasm of respiratory muscles which the former can paralyse, but practically it is extremely difficult to gauge the suitable dose; if a little too much be given, the curare itself arrests the breathing, especially in the human subject when artificial respiration cannot well be set up: small doses only mask the symptoms of strychnia, which cease for a time,- - to recur when the curare is eliminated. There is no true antagonism, since curare affects the motor nerve-ends, and strychnia the central nuclei. Since chloral was introduced we hear much less of this remedy.

THERAPEUTICAL ACtion.-Although extremely interesting to, and closely studied by, the physiologist, curare has not yet proved itself very serviceable to practical physicians.

In Tetanus much was at first expected, and a certain amount of success was obtained from it : thus, of four Italian cases, three (idiopathic) treated by hypodermic doses of $\frac{1}{6}$ to $\frac{1}{2}$ grain recovered after about 5 to 12 grains had been given, and another (traumatic) after sixty-four injections (Union Méd., 1869). Chassnignne treated a case, which set in severely a fortnight after a wound of the foot, with doses of $1 \frac{1}{2}$ grain by the mouth, and a lotion of $\frac{1}{1000}$ to $\frac{1}{500}$; recovery took place, after one relapse, in sixteen days (Journ. de Méd. et Chir., $2^{\circ}$ série). Busch has recorded his ex- 
perience of twenty-one cases treated in field hospitals during the war in Bohemia : fourteen were fatal; of the whole number eleven were treated by curare, and six recovered, hypodermic-morphia being also used freely in some. Demine, in the Italian war of 1859, saved eight out of twenty-two cases treated by curare: his total average mortality was over 90 per cent., so that the malady is essentially very fatal. Isolated successful cases have been reported by Vella and others, but, according to Vulpian, "success has been extremely rare, and in the majority of cases this remedy has neither prevented nor retarded the fatal issue" (Leçons, p. 395). Still, if it be argued that tetanus does sometimes recover untreated, and that many of the cases just referred to were instances of this, it must also be conceded that in very severe cases every remedy is futile, and to expect this to always succeed is unreasonable. Certain cases of tetanus will, it may be feared, always remain on the list of necessarily fatal maladies; the personal or inherited nervous constitution of the patients rendering them peculiarly easy victims to the kind of influence which sets up this disorder. It need be no serious discouragement to the use of curare that it has failed in such exceptionally severe and rapid cases as those recorded by Vulpian (Union Méd., 1857, No. 7). Some other instances that have been cited against curare in tetanus are equally inconclusive: thus, a case in Professor Schuh's clinic was fatal, but it was one of extreme severity, as attested not only by the rapid course, but by the unusual amount of material lesion found in the cord; the curare, however, produced temporary amendment (Sclumidt's Jahrb., Bd. 118, p. 292). Its effect on strychnia-tetanus has been already referred to. In Epilepsy, Voisin and Liouville thought it exerted sometimes a favourable influence when pushed to physiological effects; they gave from $\frac{1}{7}$ to 2 grains by injection, producing some palsy of the eye-muscles with ptosis and strabismus, pyrexia, and temporary glycosuria.

More general experience is, however, unfavourable to the drug, and Beigel especially has shown it to be ineffective (Berl. Klin. Woch., 1868, No. 9).

In Rabies it perhaps deserves further trial. It had already some reputation in this disorder when Waterton (1812) made a special expedition to Demerara in search of a larger and better 
supply. He watched its preparation, which he describes as from the "woorali vine, a bitter root, two bulbous plants, Indian pepper, two species of ants, and the fangs of two kinds of serpents." Professor Sewell strongly recommended its use, but the cases treated by it are not numerous enough for a definite judgment. In two that occurred at Milan, 58 and 103 days respectively after the bite, death was not prevented. Niemeyer was one of the first to use it, injecting 5 milligrammes ( $\frac{1}{25}$ grain), and then 1 centigramme ( $\frac{1}{6}$ grain), at intervals of three to four hours. Even these small quantities "seemed of temporary service, more so than large injections of morphia," and he strongly urged a trial of large doses. In Dr. Gualla's unsuccessful cases (four) the doses were probably too small (Offenberg).

In Dr. Watson's cases the bad symptoms subsided after the third injection; $\frac{1}{16}, \frac{1}{9}$, and $\frac{1}{6}$ grain having been given. A gir] bitten by the same dog, died of rabies (Amer. Journ. Med. Sci., July, 1876) : but the case recorded by Dr. Offenberg has attracted most attention: it was that of a woman aged twenty-four, cauterized with caustic soda three days after the bite, but developing hydrophobic symptoms eighty days later. Morphia and chloroform failed to benefit, and $\frac{1}{3}$ grain curare was injected three hours after the commencement of the attack. Fifteen minutes after, there being no evident effect, the dose was repeated; then the condition began to improve, the spasms becoming less and with longer intervals. Six injections followed at intervals of one or two hours, according to symptoms, the patient then being better, free from pain in the chest, and having ten minutes' rest between the spasms. After the seventh injection the spasms ceased, and some paralysis of voluntary muscles was evident-the eyelids could scarcely be raised, or the voice made audible; occasional slight arrest of respiration required some artificial movements: the dread of water and sensitiveness to light had now disappeared. Altogether 19 centigrammes (nearly 3 grains) of the drug had been given in four and a half hours. One more injection was required in the next two days, and the patient gradually recovered by the eighth day. This case is quoted by Dr. Dolan, who notes that "with the data we have, we may reasonably hope that, by 
its judicious application, rabies may be shorn of its terrors" (On Hydrophobia, Medical Press Report, 1878).

If the more recent explanation of hydrophobia prove correct - that it depends upon a bacillus which germinates specially in the nerve-centres-it is evident that this drug can only relieve certain of the symptoms, but even so it might be of service (Lancet, ii., 1881).

In Chorea, curare has been tried by many with different results; on the whole, nothing can be said to be definitely established in its favour.

In Facial Spasm, Gualla found curare effective when other remedies had failed.

PREPARATIONS AND DOSE.-Extract: dose, given subcutaneously, about $\frac{1}{4} \mathrm{gr}$., repeated every two or three hours, or oftener, according to the case (the strength of any sample may be ascertained by the physiological test, p. 475).

Of curarin it is impossible at present to state precisely the proper dose. Preyer considered it twenty times stronger than curare, but Beigel's researches with Preyer's own curarin make it doubtful whether the proportion is so high as this: by subcutaneous injection the dose probably ranges from $\frac{1}{60}$ to $\frac{1}{30} \mathrm{gr}$. Marquart, of Bonn, is the best maker.

A good curare may be obtained now from Hopkins \& Williams; Corbyn also supplies guaranteed strength and purity; Savory \& Moore prepare gelatine discs of $\frac{1}{200}$ and $\frac{1}{100} \mathrm{gr}$. cưrare, and various strengths of curarin.

\section{AKAZGA-MCBOUNDOU-BOUNDOU-IKAJA- QUAI.}

\section{(West African Ordeal Poisox.)}

DESCRIPTION. - This is a shrub about 6 feet high, a species of Strychnos at present undetermined. Its principal activity resides in the bark, which has many yellowish tubercles externally, and internally a lightish-brown, hard, contimuous 
layer of cells. The leaves are 3 to 12 inches long, oval, opposite; the seeds are almost globular, downy, about $\frac{1}{2}$ inch in diameter, with a central cavity into which the embryo projects. The specimen sent to Fraser (1867) was in bundles of long, slender, crooked stems of about $\frac{1}{2}$ inch diameter, with either roots or leaf-bearing branches attached. All parts, but especially the bark, have a bitter, faintly aromatic taste.

ACTIVE INGREDIENTS.-From an alcoholic extract of the bark, Fraser obtained by Stas' process an alkaloid which he named akazgia, and which he considers to represent all the physiological powers of the bark. It is a white, amorphous substance, but may be crystallized with some difficulty from an alcoholic solution, in small prisms. It is slightly soluble in water; soluble in alcohol of 85 per cent.; less so in absolute alcohol; soluble in ordinary, less so in pure ether; and has an alkaline reaction. It presents the same remarkable series of colours with sulphuric acid and bichromate of potash, or peroxide of lead, as strychnia. Akazgia and the neutral salts which it readily forms with acids are bitter, but the bitterness is far less intense and lasting than that of strychnia and its salts; they are precipitated by allaline bicarbonates.

Testut (1878) considers there are two active ingredients, one like strychnia, the other narcotic. Rabuteau thought brucia was present; but Ed. Heckel and Schlagdenhauffen, after an exhaustive research, find only strychnia as the convulsive agent (Journ. de l'Anat., 1881, No. 2).

Physiological Action.-There is a strong general resemblance between the action of akazgia and that of strychnia, still more between the former and that of brucia and igasuria. Heckel asserts that the effects are identical. Fraser found that for a rabbit of $3 \mathrm{lb}$. weight the minimum fatal dose was $\frac{3}{6}$ grain, subcutaneously injected. With this quantity the reflex movements were evidently exalted at the end of nine minutes; tetanus nccurred a minute later, and death in eleven minutes after the administration. Smaller doses than this producerl at first serious effects, but the animals slowly recovered. 
Pécholier and Saint-Pierre concluded that akazgia acts in the same manner as strychnia, i.e. on the sensory nervous system, producing, first, exaggerated sensibility, then tetanic convulsions, and at last paralysis and death; also that it only acts secondarily upon the moto-nervous system. Fraser remarks that a certain quantity of the poison is able to produce a condition which as nearly as possible approaches death, and that an extremely small augmentation of this dose is capable of quickly causing violent symptoms and a rapidly fatal termination.

In Vulpian's observations on frogs, the first symptom was general weakness and inertness, and only after several minutes did the evidence of reflex excitability appear in spasm and convulsion; contrary to what is usually seen in strychniapoisoning, the convulsions gradually subside, leaving a state of "resolution" which may terminate in arrest of respiratory movement, or after two or three days the convulsions may recommence, and then end in death or recovery. During this stage, stimulation of motor nerves does not cause contraction of muscles, though direct stimulus of muscles may do sowhich suggests the hypothesis already mentioned under curare, that a "solution of continuity" takes place between the termination of the nerve and the muscle to which it is attached. It seemed to act on the whole cerebro-spinal axis, but more strongly on the medulla oblongata (Leçons, p. 619).

In the native African ordeals, the accused person, after drinking the poison, is required to step over a series of aknzga sticks which are laid upon the ground, or, as some say, held two feet above it; and if he be just able to accomplish the feat, his innocence is supposed to be proved. It is obvious that by giving a quantity just short of the lethal dose the medicineman has it in his power to leave the accused sufficient force to perform this trifling feat; and that if he afterwards falls into a state of coma, or tetanus, it does not matter; while, if he ultimately recovers, as the conjurer may calculate, it will seem an all the more striking vindication of divine justice.

Therapeutical ACtion.-Nothing satisfnctory is known. upon this subject at present; but the above description of the 
physiological action of the plant and its alkaloid gives room to hope that akazga may have special virtues of its own. It appears probable, for instance, that in cases where strychnia in small doses is employed for a considerable time, but with the inconvenience of producing a nervous erethism, the milder akazgia would prove more manageable.

PREPARATIONS AND DOSE.-Wehave not muchexperience on these points, but it would be safer to begin with doses such as are used of nux-vomica and strychnia for the extract of akazga and the alkaloid and salts respectively. The drug cannot yet be easily obtained, nor do we know how far the production of the alkaloid, which seems a difficult process, would allow of its being sold at a reasonable price.

\section{GELSEMINUM OR GELSEMIUM SEMPERVIRENS.}

\section{(Carolina or Yellow Jessamine.)}

DESCRIPTION.-This plant (which is not the same as the yellow jessamine-jasminum of English gardens) is native to the woods of the Southern United States, and has a climbing stem, opposite, ovate, smooth, entire leaves, and axillary clusters of fragrant, yellow, bell-shaped, five-lobed flowers, 1 to $1 \frac{1}{2}$ inch long, which furnish a two-celled flattened fruit. The root and rhizome (the latter about 1 inch in diameter) are the parts used in medicine; they are yellowish-brown externally, marked with longitudinal lines or wrinkles, yellowish on section, with white medullary rays; the odour is "peculiar, heavy, and aromatic."

ACTIVE INGREDIENT.-The alkaloid gelsemia or gelseminia, $\mathrm{C}_{12} \mathrm{H}_{14} \mathrm{NO}_{2}$ (Gerrard), is commonly met with as an amorphous white or yellowish-brown powder, strongly alkaline, bitter, slightly soluble in cold water, highly so in alcohol, ether, and dilute acids: the pure alkaloid is described as a 
brittle transparent solid, crystallizing with difficulty from alcohol, sparingly soluble in boiling water, part of it separating on cooling: on platinum it burns with a yellow flame, leaving no residue. With acids it forms salts of bitter taste, which precipitate white with alkalies; it has no colour-reaction with strong nitric or sulphuric acids, but if to the latter mixture a little manganic oxide be added, with friction, a deep crimsonred is obtained, passing to green (Pharm. Journ., i., 1883): the strychnia coloration is more purple. The hydrochlorate is a whitish granular powder, readily soluble in water. The alkaloid must be distinguished from the alcoholic extract of the drug, which has been called by the same name, or "gelsemin." It exists in the plant in the proportion of about 0.2 per cent., and combined with gelsemic or gelseminic acid, which is fluorescent in solution, and may be crystallized in colourless prisms. Certain chemists reported it identical with æsculin; but although there is some resemblance, and their solutions are fluorescent, Dr. Wormley has amply proved characteristic differences (Pharm. Journ., ii., 1882).

Physiological Action.-Nervous System. - In toxic doses this drug paralyses both sensory and motor nervefunctions, affecting, apparently, the former first in cold-blooded animals, and the second, only in the warm-blooded (Bartholow).

The effect is probably produced directly on the spinal cord, since the nerve-trunks and their peripheral ends retain activity. G. Rouch found that 2 to 5 milligrammes of hydro-bromate of gelsemia injected into frogs caused first an excited condition, afterwards torpor, with loss of voluntary and reflex power.

$\mathrm{He}$ concludes that both in man and the lower animals it paralyses the motor nerves after a temporary excitation like that of strychnia; the heart is affected last, but its action gets gradually slower, and is arrested in diastole: the alcoholio extract prepared in America is specially active as a paralysant (Compt. Rend. Soc. Biol., Déc., 1882).

In some animals tremor precedes paralysis; in others peculiar backward movements, in frogs tetanus, and in various animals convulsions occur. The third nerve is specially affected, causing dilated (sometimes contracted) pupil, double vision, and 
ptosis; occasionally there is protrusion or prominence of the eyeballs, from spasm of the ocular muscles. In man, some idiosyncrasy is found with regard to the effect of the drug, 20 to 40 minims of tincture causing in certain persons more pronounced symptoms than much larger amounts in others; the dose given by Ringer as necessary for full effect, and yet safe, is 1 drachm hourly for three hours.

The eyes and brow are commonly affected first with dull, heavy, or shooting pain, worse on movement; giddiness is complained of, and often diplopia, involving especially the upper part of the field of vision, until ptosis develops; a sense of calm passing into languor is felt, with numbness of the scalp and extremities, then impairment of motor power. Dr. Ringer, specially observing the coudition of the gums, could detect no alteration of sensibility of those parts, though one patient described a "numb pain and tenderness." He remarks, that although the pupil is dilated and accommodation paralysed by the local application of the drug ( 1 grain of gelsemia in 20 minims of water), it is commonly contracted under the internal administration of full doses, unless asphyxial conditions set in, when it dilates fully. The careful observation of animals subjected to its local application only, also showed some primary contraction. Dr. H. C. Wood connects these effects with a peripheral palsy of the oculo-motor nerve, and remarks that the falling of the jaw and loss of speech indicate a parallel paresis of various motor nerves of the head. Fatal, or nearly fatal quantities, such as in a child 21 minims up to 2 drachms of fluid extract, and in adults 2 drachms up to 1 ounce, cause in addition extreme prostration, relaxation of the whole muscular system, inco-ordination of movement; sometimes spasm of larynx and pharynx simulating hydrophobia, drowsiness and gradual loss of consciousness deepening into coma, and death in collapse from paralysis of respirntion, i.e., asphyxia (Practitioner, vol. v. ; Record, 1879 ; Lancet, i., 1882). According to Dr. William Carter, the principal effects produced by large doses are extreme muscular relaxation, without stupor or delirium (B. M. J., i., 1883).

Circulatory System.- Small or moderate doses show no definite effect; toxic amounts depress the force and frequency 
of the heart's action (Ott). This occurred after section of all nerve-supply to the heart, and is therefore concluded to be due to a direct effect upon that organ. In fatal cases its action becomes weak and intermittent, the respirations laboured and irregular. In thirty-three series of observations made by Dr. Ringer in subjects under full doses, the pulse was unaffected in twenty-two, and in the others it was sometimes quickened, sometimes not; hence he judges it to have but little definite power in this direction, and Dr. Burdon Sanderson's experiments tend to the same conclusion. The temperature is unaffected.

Respiratory System.-On this the drug has a markedly depressing effect, which is exerted probably through the respiratory centre (Burdon Sanderson, Lancet, i., 1876). It occurs after division of the vagi, and neither the phrenic nor the intercostal nerves are paralysed.

Secretory System. - Some animals are salivated by gelsemium, and, in advanced stages of poisoning, free perspiration often occurs - as an exceptional symptom, this has been noted even from small doses. A case of inflamed sweat-glands is reported from the use of the drug, but its reliability is not beyond dispute, and at any rate it stands alone (B. M. J., ii., 1881). Temporary vesical paresis with incontinence of urine has been noted (Stillé), and strangury has occasionally happened. The elimination of urea is probably increased, but further observations are required on this point. It is stated that in disease (nephritis with dropsy) there is an increase of the amount of urine, but in diabetes insipidus a decrease, under this remedy.

SYNERGISTS.-In several respects gelsemiun may be allied with aconite and with bromides, and is advantageously combined with the latter especially; some similarity in the head-symptoms to those of quinine may be traced. Bartholow mentions conium, tobacco, and opium as synergic.

ANTAGONISTS. - Caustic allkalies and tannic acid are chemically incompatible; the diffusible stimulants and artificial respiration antagonize its physiological effects. Digitalis has been named, but it seems scarcely prudent to use it. 
In one case of poisoning (but only from 1 to 2 drachms of tincture), morphia is said to have aided recovery (quoted by Wood). An emetic is always of importance. Atropica should antagonize the effect on the heart, and also in small doses should stimulate the respiratory centre; but Bartholow (Practitioner, 1870) speaks of its paralysing that centre, and states that he has found this by experiment. It is, however, only very large toxic doses that do so, and the subject well deserves further inquiry. Physostigma has not proved antidotal ; strychnia has been suggested.

\section{Therapeutical Action.-Local.-Eye Diseases.-} Mr. Tweedy has stated that this drug will dilate the pupil and paralyse accommodation as completely as atropia, whilst its effects will pass off more quickly: a somewhat stronger solution-riz., of 8 grains of alkaloid to the ounce of water -must be used every quarter of an hour, probably four times, and then twice more at intervals of half an hour, for complete paralysis. The maximum dilatation commonly occurs within the hour, and passes off in a few hours (Lancet, i., 1877).

Therapeutical Action.-Internal.-Neuralgia.-In this disorder, especially (but not only) when affecting the face, much relief has been experienced from gelsemium; but either there is more than average uncertainty in its action, or else the preparations of it vary in power, since some observers record much better results than others. (Bartholow states that the alkaloid disappears from the dried root, so that this might explain some failures.)

Dr. Wickham Legg was one of the first in this country to draw attention to its power in "bad toothache," facial pain more or less dependent on carious teeth in many instances; and some cases of "rheumatic pain, worse at night," were relieved by 10 minims every three hours (Lancet, i., 1873).

In other neuralgire the same remedy has proved useful, c.y., in intercostal and myalgic pain, and especially in pelvic forms connected with ovarian or uterine congestion, dysmenorrhen, etc. According to Bartholow, "there is no more generally 
useful medicine in ovarian neuralgia" ; it also suspends "afterpains." In the pruritus of several forms of skin disease it is said to have given relief. It has soothed also the pain of epithelioma in a case where the inferior dental nerve was involved: 20 minims being given three or four times during the attack (Willett, B. M. J., i., 1883).

Spasm.-Certain disorders associated with spasm are also amenable to the drug: for instance, the pain of gall-stone, which has been relieved by 5 -minim doses given every quarter of an hour; also spasmodic, reflex, or "nervous" cough, whooping-cough, and spasmodic asthma.

Insomnia, etc.-When this accompanies mental disorder or over-excitement, or is connected with alcoholism, gelsemium has acted well as a sedative. I have especially observed good effect in the febrile excitement of dentition in children; and when bromide alone had failed, the addition of this medicine has secured rest. A distinction has been drawn between maxillary and frontal neuralgia, the former proving much more amenable than the latter in the experience of some observers (Lancet, ii., 1875 ; B. M. J., i., 1881). Twenty minims, repeated in one or two hours, is the dose recommended; and I have sometimes ordered this three or four times hourly, or 10 to 15 minims every half-hour, with good effect. At other times it has caused giddiness; and in the absence of an officinal preparation it is of importance to state in prescribing whether a tincture made with 2 or with 4 ounces of root to the pint is desired, since both strengths are in use. The liquid extract (U.S.) is much stronger; 5-minim doses, repeated several times, have caused, in my experience, disturbance of vision; 3 minims, repeated six times, induced diplopia and ptosis (B. M. J., ii., 1883). Dr. De Wolfe records that after taking 10 drops for neuralgia, and a second 10 drops in half an hour, he became very drowsy, suffered much frontal pain, got rigors, failure of circulation, and partial collapse, afterwards vomiting: he was relieved by stimulants; the neturalgia did not recur (B. M. J., i., 1881). A case of severe pain lasting many hours, in a child with a decayed molar, was relieved within half an hour by 15 minims; there was no recurrence (ibicl., p. 171). Dr. Ringer speaks also of "decided success" in such cases, but notes the occurrence of 
toxic symptoms, such as giddiness and ptosis, from even 10 minims thrice daily. In "Menière's disease" it proved useful. It is said even to benefit in true inflammatory disease of the cerebro-spinal membranes.

Some few cases of tetanus have recovered under the drug in full doses. Dr. W. Carter refers to three cases (B. M. J., i., 1883) ; and Dr. J. B. Read (U.S.) has published details of one case, a mulatto woman, aged twenty, with well-marked convulsion after injury to the heel: she was given at first 20 and then 40 minims of liquid extract (Tilden) every two hours without any toxic symptoms; improvement began on the third day (B. M. J., ii., 1882). Its use was suggested by its "wellknown power of relaxing all voluntary muscles."

Fever.-The original use of the drug seems to have been in the malarial and other fevers of the Southern States especially; and it has more power of reducing temperature in such conditions than in health: it has also been used with temporary advantage in the hectic of phthisis. In acute inflammations of the lungs and pleurr it is commended by Bartholow, but has not come into general use.

Uterine Disorder.-There is some, though not precise, evidence of its power to dilate a contracted or rigid os uteri, both in the puerperal and non-puerperal state (Practitioner, vol. xviii.).

PREPARATIONS AND DOSE.-Tincture (2 oz. to 1 pint spirit) : dose, 5 to 30 min., according to frequency of repetition. Extractum liquidum (liquid extract U.S.) : dose, 2 to $10 \mathrm{~min}$. every four hours, $\frac{1}{2}$ to $1 \mathrm{~min}$. if given half-hourly. (Both these should be made from the fresh root,- the dried root is said to be often inert.) Gelsemin: dose, $\frac{1}{60}$ to $\frac{1}{20}$ gr. Gelsemire hydrochlorus: dose, $\frac{1}{80}$ to $\frac{1}{20} \mathrm{gx}$. Guttre (for ophthalmia) : 1 gr. to $1 \mathrm{clr}$. of distilled water; dose for hypodermic injoction, 1 to 3 min. Gelsemin (alcoholic extract) : close, $\frac{1}{2}$ to $2 \mathrm{gr}$. 


\section{ASCLEPIADACEAE.}

\section{THE WAX-FLOWER FAMILY.}

A LARGE and curious order of exogens, belonging mostly to the tropics, and including often scandent slender evergreen shrubs and leafless succulents. The leaves, when present, are simple, entire, and opposite. The flowers are pentamerous, regular, usually star-shaped, and remarkable for the singular agglutination of the anthers of the five perigynous stamens to the broad disc-like stigma. The twin ovaries are superior, and ripen into follicles containing numerous seeds, which are generally comose. Many poisonous plants occur in this order, and the milky juice is often acrid; several species are diaphoretic and purgative.

\section{HEMIDESMUS INDICUS.}

\section{(Hemidesmus.)}

DESCRIPTION.-A twining glabrous shrub, native of the East Indies. The smooth and shining leaves vary in form from linear to ovate; the small flowers, green outside, purplish within, are produced in axillary sessile racemes. The root (to the virtues of which part this plant owes its medicinal value) is long and slender, not much branched, invested with a dark-brown bark, and possessed of an agreeable scent, which is not lost by drying. In commerce it is known by the name of "Indian sarsaparilla." 
ACTIVE INGREDIENTS.-Besides starch and some tannin, the root contains a volatile crystalline substance, called hemidesmin or hemidesmic acid, soluble in boiling water.

Physiological ACtion.-Concerning this nothing is definitely known, but the effects of the plant are generally diuretic.

Therapeutical ACtion.-The diuretic virtues of hemidesmus render it useful in certain diseases of the kidneys; and it has been given for some syphilitic skin-diseases, and for indigestion, when it becomes serviceable, like sarsaparilla. In India, where the plant is common, the roots are largely employed as a substitute for the latter drug and act also as tonic and diaphoretic. In England, however, hemidesmus is principally used as a flavouring agent. Lindley stated, in 1838, that much of it was then dispensed in London as a fine kind of American sarsaparilla, and that it was said to be quite as efficient a medicine.

PReParations AND Dose.-Symupus hemidesmi: dose, 1 to $2 \mathrm{fl}$. dr. Decoctum (not officinal) : dose, 1 to $4 \mathrm{fl}$. oz. 


\section{GENTIANACEAE.}

\section{THE GENTIAN FAMILY.}

An order of about 500 species of exogens, almost exclusively herbaceous, with the exception of a few anomalous (chiefly aquatic) forms, of simple and uniform characters. The stems and branches are smooth, straight and erect; the leaves undivided, opposite, sessile, entire, exstipulate, and generally ribbed; the flowers regular, monopetalous, and usually pentamerous; the stamens few and epipetalous; and the superior ovary becomes a two-celled, many-seeded capsule. All the species are bitter to the taste; none are deleterious.

Pharmacentical Species.

Gentiana lutea . . . . . . . . . Gentian.

Ophelia chirata . .

\section{GENTIANA LUTEA.}

(Gentian.)

DESCRIPTION. - An herbaceous perennial, native of the central parts of Europe, and growing chiefly in the subalpine districts. The root is cylindrical, thick, forked, amnulated, brown externally, yellow inside. The stem, renewed year by year, is 3 to 4 feet high, stout and hollow; the ovate leaves are 5 or 6 inches in length; the yellow, pentamerous flowers are nearly an inch across, and are produced in axillary whorls.

Gentian-root has a bitter, non-astringent, somewhat sac- 
charine taste, and a sweet odour; it is very hygroscopic when dried, and becomes corrugated: the best samples are of moderate size, tough, and almost destitute of fibres.

ACTIVE INGREDIENTS.-The active principle of gentian is not (as formerly supposed), the gentianin or gentianic acid, $\mathrm{C}_{14} \mathrm{H}_{10} \mathrm{O}_{5}-\mathrm{a}$ substance which crystallizes in yellow, silky needles, is tasteless, and neutral in reaction, soluble in alkalies, and slightly so in water or alcohol-but gentio-picrin, $\mathrm{C}_{20} \mathrm{H}_{30} \mathrm{O}_{12}$, a bitter glucoside, first obtained by Kromayer, in 1862 , in the form of colourless crystals, which on exposure to the atmosphere become dull white; these crystals are readily dissolved by cold water and by spirit. Potash and soda solutions, and hot solutions of ammonia dissolve them yellow; cold, concentrated sulphuric acid dissolves them without colour, but the solution, when gently heated, changes to a carmine-red; on the addition of water, grey flocculi are deposited.

Gentian contains no starch, but much pectin, and about 12 per cent. of sugar. It does not contain ordinary tannin, and does not blacken salts of iron, though it may turn brown with perchloride; gentianic acid is, however, by some considered a kind of tannin, and called gentio-tannic acid (Ville).

Physiological ACTION.- Gentio-picrin is a bitter closely allied to quinine, both in physiological and therapeutical action. The conflict of evidence which occurred respecting its power as an antiperiodic, arose from the confusion between the true bitter and the tasteless gentianin. The effects of an overdose of gentian itself are dulness, weight in the head, oppression of forehead, and slight giddiness, - symptoms, in fact, much resembling those induced by cinchona: the face becomes flushed, and the conjunctiva injected, the bowels relaxed, and the stools have a bilious character. The drug seems to be eliminated in part by the skin and kidneys, for their secretions acquire a bitter taste. It is probable that besides the gentio-picrin there is some volatile ingredient in gentian which has a slightly inebriant action, since Planché states that water distilled with pure gentian possesses this latter quality. The Tyrolese obtain 
from the root an alcoholic liquid, the sugar being fermented during maceration in water.

ThERAPEUTICAL ACTION.-Gentian-root has long been employed as a valuable tonic, and before the discovery of the cinchonas, occupied the first place in medicine as a febrifuge. It is properly regarded as a pure and simple bitter-that is to say, a bitter without either astringency or aroma. It agrees best with patients of a torpid phlegmatic habit, but should be avoided where the temperament is irritable.

Dyspepsia.-Given in small doses gentian is found beneficial in this disorder (especially when connected with a gouty diathesis), also in hysteria and jaundice, and generally in all those cases of debility for which tonics are exhibited, and which are unaccompanied by symptoms of inflammation. It should be observed, that though "gentian possesses the advantages of a laxative in addition to those of a bitter, its characters as an irritant are more marked, and additional caution is therefore requisite in its use" (Dr. Wilson Fox, On the Diagnosis and Treatment of Dyspepsia). It is likewise valued in scrofula, in intermittents, and as a vermifuge; in the form of infusion it becomes an excellent vehicle for the administration of chalybeates, mineral acids, and neutral salts, with which it is often necessary to combine it.

Intermittents. - Gontio-picrin has been proved efficacious in cases of intermittents by Lange, who published a series of thirty-four cases, in which the attacks were cut short or prevented by $\frac{1}{2}$-drachm doses.

PREPARATIONS AND DOSE.-Pulvis: dose, 10 to $40 \mathrm{gr}$. Extractum: dose, 2 to $10 \mathrm{gr}$. Infusum gentiance compositum: dose, 1 to $2 \mathrm{fl}$. oz. Mistura: dose, $\frac{1}{2}$ to $1 \mathrm{fl}$. oz. Tinctura. gentiance composita: dose, $\frac{1}{2}$ to $2 \mathrm{fl}$. dr. On account of the root containing sugar, its simple infusions will not keep many days. The addition of strong tinctures to such infusions tends to delay the fermentative process, so that the medicine may be kept longer. 


\section{OPHELIA CHIRATA.}

\section{(Chiretia.)}

DESCRIPTION.-This interesting plant, known also by the name of gentiana chirata, is a native of the north of India, and of Nepal, where it is met with as an annual of about 3 feet in height, with a fibrous root and nearly simple stem, which terminates in a leafy panicle of tetramerous yellow flowers. Every portion is scentless, but intensely bitter. For medicinal purposes the entire plant is pulled up by the root, at the time that the flowers are overblown, and then dried and tied in bundles for export.

ACTIVE INGREDIENTS.-These are probably chirettin, $\mathrm{C}_{26} \mathrm{H}_{48} \mathrm{O}_{15}$, a bitter, neutral, resinoid substance, or pale yellow powder, discovered by Höhn, and ophelic acid, $\mathrm{C}_{13} \mathrm{H}_{20} \mathrm{O}_{10}$. The latter occurs in larger proportion than chirettin, and forms a yellowish brown syrup, tasting, at first, sour, and then persistently bitter, having also a peculiar gentianaceous odour.

Physiological Action.-Similar to that of gentian.

Therapeutical Action. - Chiretta is an excellent tonic, and is held in high estimation by the European practitioners in India, who employ it for the same purposes as cinchona when the last-named drug is not procurable. It gives tone to the stomach, obviates flatulency, diminishes the tendency to acidity, and appears to be especially serviceable in the dyspepsia of gouty subjects. Like gentian, it is relaxant rather than constipating.

PREPARATIONS AND DOSE.-Infilsum: dose, 1 to $2 \mathrm{H} . \mathrm{oz}$. Tinctura: dose, $\frac{1}{2}$ to $2 \mathrm{fl}$. dr. 


\section{CONVOLVULACEAE.}

\section{THE CONVOLVULUS FAMILY.}

A FAMILY of herbaceous twining exogens, abundant chiefly in hot countries, and numbering over 700 species. The leaves are alternate, exstipulate, simple, and either undivided, fanlobed, or pinnatifid; the flowers are usually large, regular, pentamerous, monopetalous, and more or less bell-shaped; the five stamens are epipetalous; the ovary is superior, and ripens into a one to four-celled membranous capsule, containing a few large seeds, the cotyledons of which are leafy and doubled up. The roots often abound in a milky acrid juice, which contains a peculiar resin of purgative properties.

\section{Pharmacentical Species.}

Convolvulus scammonia .. . The scammony plant. Exogonium purga . . . . . Jalap.

\section{CONVOLVULUS SCAMMONIA.}

(SCAMmony.)

DESCRIPTION. - Scammony is a native of most of the Levantine countries, and occurs likewise in Persia and CochinChina. The root is perennial, fleshy, 3 to 4 feet in length, and 3 to 4 inches in diameter; the slender and annual stems climb or extend to a distance of several yards; the leaves are arrowshaped; the flowers (rather small for the genus) are yellowish and axillary. On making incisions in the living root, the gumresin scammonium which receives its name from the plant is obtained in abundance. This resin occurs in irregular or 
circular cakes of dark, greenish-grey colour; triturated with water it yields a greenish emulsion; it has a peculiar cheesy odour, and slight acrid taste; the best quality is known as "virgin" scammony.

ACTIVE INGREDIENTS.-The medicinal powers of scammony depend principally upon the presence of scammonin, $\mathrm{C}_{31} \mathrm{H}_{50} \mathrm{O}_{16}$, a glucoside identical with jalapin, and in many respects corresponding with convolvulin, the active principle of jalap; it is sometimes called scammony resin, and is a brownish resinoid body, translucent in thin pieces.

At $302^{\circ} \mathrm{F}$. it melts into a colourless slightly acid fluid, destitute of taste and smell. In water it is nearly insoluble, but in alcohol, hot acetic acid, chloroform, ether, and alkaline solutions, it dissolves with facility. The purest "virgin" scammony contains about 80 per cent.

ABSORPTION AND ELIMINATION.-The absorption of this drug is conditional upon its coming into intimate contact with the alkaline biliary and pancreatic secretions: if these are scanty, or if there be a large amount of mucus coating the bowel, or if the drug be not finely divided, its solubility is slight and its action uncertain; hence the resin sometimes acts even less than scammony itself. A case quoted by Stillé illustrates another peculiarity in the action of the drug: "a healthy woman, nursing a healthy child, two months old, took a dose of scammony for constipation, suffered no purgation herself, but the child was seized with choleraic diarrhœa, which rapidly ended in collapse and death."

\section{Physiological Action.-Digestive System.-Scam-} mony in any form affects the intestinal tract, but, as already explained, its action is uncertain; sometimes it is mild and painless, at other times accompanied with much griping pain, colic, tenesmus, bloody stools, and even enteritis. Its action resembles that of jalap, being commonly, perhaps, somewhat more powerful, whilst its taste is not so nauseous; it is less irritant than gambogre, but is said to have sometimes cnused inflommation and death (in animals) without any purging. 
On the Nervous System some disturbing effects are produced, since convulsions have occurred with contraction (and ultimate dilatation) of pupil, oscillation of eyeballs, and death in half an hour after a hypodermic injection of 7 to 8 grains of jalapinate of soda in animals (Buchheim).

Therapeutical ACTion. - Scammony is one of the oldest known medicines. Hippocrates used it as a drastic purgative, endeavouring to modify the violence of its action by means of sulphur, etc.; and both Greeks and Arabians prescribed it for gout, rheumatism, and other chronic diseases; they were accustomed also to order an acetous decoction to be mixed with meal, when poultices were required for painful affections of the joints. In the materia medica of past empirics, scammony always held an important place: we read that Robert Dudley, Earl of Warwick, strongly recommended a combination of this drug with antimony and cream of tartar, to Marcus Cornachinus, of Pisa, who wrote a book in praise of it. In France, scammony is known to this day by the name of Poudre Cornachine. At present it is chiefly valued as a smart purgative for children, having the advantage of smallness of dose required, and of slight taste: it is generally given in combination with calomel, but if only a mild effect be desired, rhubarb or sulphate of potash may be employed instead of calomel. Scammony may be used as a simple aperient in constipation; also to expel worms, especially in children; it is of service in dropsies and in affections of the head, and acts in the latter cases as a hydragogue purgative, relieving by derivation; hence it is an appropriate purgative in mania and in hypochondriasis: it is well adapted, also, for torpid conditions of the abdominal organs, accompanied by much slimy intestinal mucus.

When aloes produce unpleasant effects upon the hrmorxhoidal vessels, scammony may be substituted with adrantage; while, to modify its own occasionally too severe operation, sugar, gum, or almonds may be combined with it.

PREParations and Dose.-Pulvis scammonii: dose, 5 to $10 \mathrm{gr}$. Scammonin (the pure resin): dose, 3 to $8 \mathrm{gr}$. in powder, or in emulsion, with 3 or 4 oz. of milk. Confectio: 
dose, 10 to 30 gr. or more. Mistura: dose, $\frac{1}{2}$ to 2 丹l. oz. (for child). Pulvis scammonii compositus : dose, 10 to $20 \mathrm{gr}$.

ADULTERATIONS.-The peasants who collect the gumresin are apt to adulterate it with chalky or magnesian earth.

\section{EXOGONIUM PURGA.}

(JALAP.)

Description.-A native of the Mexican Andes. The root is perennial, oblong, fleshy, provided with large tubercles, and full of milky juice; the slender and annual stems twine to the height of 8 or 10 feet; the earlier leaves are hastate, the upper ones cordate; the flowers are axillary, campanulate, 2 or 3 inches across at the rim, purple externally, rosy-purple within.

The portion of the plant employed medicinally consists of the tubercles of the roots, which, when dried, are napiform or pyriform, dark brown, wrinkled, solid, hard, and heavy: they are chiefly imported from Vera Cruz. When powdered, jalap is of a pale yellowish-grey colour; it has a heavy, sickly smell, and a sweetish, sub-acrid taste.

ACTIVE INGREDIENTS.-Jalap contains about 15 per cent. of a resin upon which its cathartic properties depend; also about 20 per cent. of a watery extractive matter, with starch, sugar, and other substances of minor importance.

The pure resin, properly called jalapin, or jalapic acid, $\mathrm{C}_{34} \mathrm{H}_{56} \mathrm{O}_{1 ;}$, is dark brown in colour, brittle, and opaque. It is soluble in alcohol; slightly soluble in ether; readily so in nitric and acetic acid, and in solutions of potash; but in water and in oil of turpentine it is insoluble. Sulphuric acid turns it crimsron.

Jalap also contains a strongly purgative substance, called con- 
volvulin, $\mathrm{C}_{31} \mathrm{H}_{50} \mathrm{O}_{16}$ (rhodeoretin of the German chemists), which is colourless, transparent, and insoluble in ether. It has a slightly acid reaction, and exhibits the chemical characteristies of a glucoside-that is to say, when boiled with dilute acids, it yields sugar and convolvulinol, which is bitter and crystalline, and when treated with alkalies is converted into an acid (convolvulinolic). The resin itself, when treated with caustic alkalies, becomes convolvulic acid, of which convolvulin is the anhydride.

Spurious, or fusiform, jalap, the product of the convolvulus orizabensis, has for its chief ingredient a substance which has been improperly called jalapin, already described under the name of scammonin, as the active ingredient of scammony; this is soluble in ether.

ABSORPTION AND ELIMINATION.-A change occurs in these drugs after their absorption. In some animals they are said to purge after being rubbed into the skin-in man not so; and the milk of nursing mothers is said not to be rendered purgative by them. Neither convolvulin nor its derivatives appear in the urine, nor, except with enormous doses, in the freces: they are supposed to be decomposed and destroyed by combustion within the system.

Physiological Action.-Jalap is a well-known and valuable cathartic, and one upon which great reliance can be placed. In full doses it produces nausea, vomiting, griping in the alimentary canal, often attended by colic and flatulent rumbling, with copious liquid and sour evacuations from the small intestines, and in less degree from the large ones. The action is not accompanied by febrile symptoms, and constipation less frequently follows from it than from most other purgatives. The watery extract is said to purge without griping, and therefore to be well adapted for administration to children. Compared with other medicines, the operation of jalap closely resembles that of scammony: it is more drastic than senna, and less irritant to the mucous membrane than gamboge.

Röhig placed jalap next to croton oil as a hepatic stimulant: Rutherford, however, states that Röhrig's method of 
experimentation was faulty (croton oil having little action on the liver), but he agrees that jalap is a moderate hepatic, and a powerful intestinal stimulant (Practitioner, ii., 1879).

With regard to convolvulin, the researches of Hagentorn show that it is four times more powerful (as a drastic) than the soft resin, and soon after administration produces pain in the belly with liquid purging: $3 \frac{1}{2}$ grains have killed a guineapig in three hours, the purging being following by gastroenteritis ; and in considerable quantity it is, no doubt, an active irritant poison. Bernatzik asserts that when given internally it acts only in a local manner on the alimentary canal, and yet it exerts little or no irritant action upon the skin, conjunctivæ, or nasal mucous membrane. The subcutaneous injection of the convolvulinate of soda, in quantities exceeding 1 gramme (15 to 16 grains), produced, like jalapin, the symptoms of violent and fatal narcotic poisoning in animals.

THERAPEUTICAL ACTION.-Jalap was first known in England as a medicinal substance about the year 1610, though it is only of late that the source of the best kind of the drug has been determined; it is probably now more generally employed than any other vegetable cathartic.

Dropsy.-Jalap certainly has the power of evacuating serous effusions, mainly by free watery purgation, but to some extent also by the kidneys: to increase its action on the latter it is commonly given with acid tartrate of potash, and in cases of liver-disorder with calomel. The permanence of the relief depends, of course, upon the original cause of the malady, e.r., whether it be advanced organic disease or no. It was originally introduced as the Panucea hydropicomm.

As a Vermifuge, jalap acts well in cases of tape and lumbricoid worms. It is said to operate upon them as a poison, and certainly, in many instances, the worms have been found dead when expelled. When used for ascarides, this medicine should be given in combination with calomel.

Habitual Constipation, arising from scanty secretion by the intestines, is overcome by jalap or its resin administered in a moderate dose before rising in the morning, and followed, an hour afterwards, by a tumbler of cold water. 
As a simple purgative for children, it may also be employed with the best results in doses of from 1 to 5 grains of powder.

In inflammatory affections of the brain, or its membranes, when a purge is required, jalap often proves most valuable.

PREPARATIONS AND DOSE.-Pulvis jalapce: dose, 10 to $30 \mathrm{gr}$. Resina: dose, 2 to 5 gr. Extractum: dose, 5 to $15 \mathrm{gr}$. Tinctura: dose, $\frac{1}{2}$ to $2 \mathrm{fl}$. dr. Pulvis jalapce compositus (with potash tartrate and ginger) : dose, 20 to 60 gr. or upwards. 


\section{SOLANACEAE.}

\section{THE NIGHTSHADE FAMILY.}

THis important exogenous family is constituted of probably not less than a thousand species, most of which are herbaceous; but there is a general tendency to the under-shrubby condition, and a few become soft-wooded trees. The leaves are alternate, destitute of stipules, either simple and undivided, or lobed, or irregularly pinnatifid, but never compound. The flowers are regular and pentamerous; generally bell-shaped or stellate. The petals are united from the base upwards, and are plaited in rstivation.

The stamens are epipetalous, correspond in number to the lobes of the corolla, and are alternate with them. The ovary is superior, free, and supports a solitary style, which is tipped with a simple stigma. Internally it is two-celled, or incompletely four-celled; and, when ripe, usually becomes a berry, but, sometimes, a capsule, and in either case is many-seeded.

In one form or another the Solanacese are scattered all over the world, excepting in the extremely cold parts.

The properties of the order are remarkably diversified, but, upon the whole, are to be accounted dangerous and energetic. A few species are bland, or inert; some, like the capsicum, are simply pungent; many, as belladonna, are poisonous.

\section{Phanmecentical Species.}

Dulcamara-Solanum duleamara .. Woody nightshade.

Capsicum fastigiatum .. . . . . Capsicum.

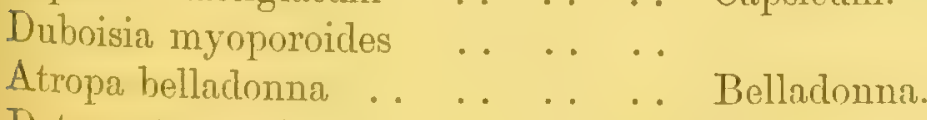

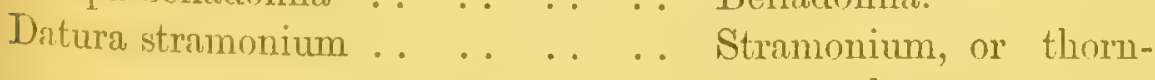
apple.

Hyoscyamus niger .. .. . . . . Henbane-hyoscyamus.

Nicotiana tabasum $\ldots$. 


\section{DULCAMARA-SOLANUM DULCAMARA.}

\section{(Woody Nightshade.)}

DESCRIPTION.-This plant is found wild in Europe, Asia, and North America, especially in moist places. The stem is thin, long, and straggling, the leaves alternate, ovate, with lobes at the base; the flowers, arranged in lateral clusters of six to eight, are star-shaped, violet-blue in colour, with two green spots at the base of each division of the corolla, and prominent yellow anthers forming a cone in the centre of the blossom: the oroid berries are crimson in colour.

The fresh plant has a "heavy" disagreeable odour, which passes away after drying: the taste of the stem, when chewed, is at first sweet, afterwards bitter, as is implied in the specific name. The branches, which are collected for medicinal use in spring and autumn, are about $\frac{1}{4}$ inch in thickness, cylindrical or angular, furrowed or somewhat warty: in the shops, they are generally found cut into sections.

ACTIVE INGREDIENTS.-The alkaloid solamin, $\mathrm{C}_{43} \mathrm{H}_{69} \mathrm{NO}_{16}$, is bitter, rather burning in taste, weakly alkaline in reaction, and crystallizes out of alcoholic solution in minute, four-sided, nacreous crystals; it melts at $235^{\circ} \mathrm{C}$, and solidifies amorphously on cooling; it is slightly soluble in water or ether, moderately so in cold, but completely in 125 parts of boiling alcohol: the amorphous form is more soluble, and according to Winckler is found only in the stem of the plant; it differs in some chemical reactions from the solanin of potatoes. The alkaloid is obtained only in very small proportion from the $\mathrm{S}$. dulcamarn, but is probably its main active ingredient.

A glucoside, dulcamarin, represents, however, more nearly the peculiar taste: it is also found only in minute proportion, $-\frac{1}{10}$ per cent., and chiefly in the stems, and is obtained as a 
yellowish powder, soluble in alcohol and water, not in ether: formula, $\mathrm{C}_{22} \mathrm{H}_{34} \mathrm{O}_{10}$ : when perfectly pure it contains no nitrogen.

Pelletier considered this substance to be really solanin, modified by combination with sugar; whilst $O$. Gmelin and others state a similar opinion with regard to solanin itself, viz., that it is really a compound of sugar with a different alkaloid, which they term solanidin, $\mathrm{C}_{25} \mathrm{H}_{39} \mathrm{NO}$.

Physiological Action.-Both Dr. Garrod and Dr. J. Harley have given large doses (two to three pints of concentrated infusion, or half a pound of the fruit) without definite effects; but other observers have found the plant decidedly toxic. Taylor explains the discrepancy by supposing the amount of solanin formed to vary at different seasons of the year.

In 1877, I saw a child, aged nine and a half years, who had eaten the berries of dulcamara, and was suffering from convulsive spasms and tremor of limbs: there was heat of head, dizziness, dimness of sight with black spots before the eyes, the pupils were dilated and the eyes staring, speech was inarticulate, the tongue was coated, white, and flabby, and eructations, nausea, and retching occurred; the pulse was slow and intermittent, and the skin covered with a cold clammy sweat-no rash; after an emetic, the berries were rejected and the symptoms subsided. The only recorded case in which death followed eating the berries, occurred in a boy aged four, who at first did not suffer, but eleven hours afterwards had vomiting and convulsions, becoming unconscious in the intervals; other children who ate similar berries were not much affected (Taylor).

In one instance, a decoction of the plant is said to have produced in a man dimness of sight, giddiness, and tremor of limbs. Feeble persons especially are said to experience this tremulousness and twitching of eyelids and lips. Vertigo has been observed in most cases when any toxic symptoms have occurred.

Nervous System. - Evidence of an action upon this system is to be found in the cases just mentioned, though the convulsions were possibly reflex.

In rabbits, the extract causes an apathetic state with relaxation of muscles-not true sleep, nor actual paralysis; sensibility 
to pain and touch are blunted; the pupils are not affected; temperature is raised; death occurs in convulsions (Stillé, Dispensatory).

Injection of $4 \frac{1}{2}$ grains of the alkaloid into the veins of dogs caused embarrassment of respiration, spasm, and convulsionthough the possibility of embolism complicates any conclusion from such observation (Fraas and Martin, Virchow's Archiv, iii., p. 225, 1854). The pupils were dilated.

In pigeons, Leydorf observed similar hurried breathing, tremor, and slight convulsion (when the oesophagus was previously tied to prevent vomiting); in these birds also, the pupil was dilated. With regard to such dilatation by solanin statements are discrepant. It seems clear from the experiments of L. van Praag, that even moderate doses will sometimes produce this result (Journ. f. Pharm., i., p. 245). Clarus, on the other hand, applied solanin locally to the eye, and observed slight myosis, with severe irritative effects upon the conjunctiva; while Schroff, in cases where he gave doses (to human patients) sufficient to produce unpleasant toxic symptoms, observed no change in the pupil, and Fronmüller found mydriasis more frequently absent than present (Deuts. Klin., 1865, p. 40).

From his general experiments with animals, Clarus inferred that solanin causes death by its depressing influence on the medulla oblongata, leading to serious congestion of the lungs. Caylus arrived at a somewhat similar conclusion, viz., that it paralysed the nerves of respiration like conia and nicotia (Journ. f. Pharm., i., p. 2 ; Bull. de Thérap, t. lxi.).

Digestive System.- Some irritative effects on the stomach are not unusual. After eating the berries, children have complained of severe pain in the bowels, with much heat in the throat and chest, nausea, thirst, and prostration; sometimes vomiting, purging, and profuse secretion of saliva have been noted; after death (in animals) the stomach has been found much congested.

Circulatory System.-By moderate doses given to animals the pulse and respiration are said to be reduced, but both are commonly quickened in toxic cases, unless a state of collapse sets in: the quickening is possibly dependent on gastric irritation: venous congestion readily occurs, and the 
ears and lips of animals, and the hands and face in man, become cyanosed. After death, the large veins of the thorax and abdomen are found distended with dark blood, and the same appearances have been described in the vessels of the cranial meninges, of the kidneys, and of the liver (Clarus; Malik and Spatzier).

Cutaneous System, etc.-A tendency to sweating rather than to dryness of skin has been described; also an erythematous rash. Carrère states that dulcamara causes violent itching over the whole body, with elevated red papules like flea-bites. Murray found it promote all the secretions.

SYNERGISTS.-Warm diaphoretics.

ANTAGONISTS.- Stimulants and sedatives, according to the toxic symptoms.

THERAPEUTICAL ACTION.-Dulcamara was formerly much used, but has of late years, I think, been undervalued. Dr. Garrod, Husemann (Archiv Klebs., 1875, Bd. iv.), and other authorities, speak of it as almost ineffective, and some therapeutical treatises omit all mention of it; but I consider it by no means a useless medicine.

Catarrh.-Dulcamara has long been used as a diaphoretic, and is to be recommended in nasal, pulmonary, and vesical catarrhs, attended by general dryness of the skin. In the intestinal catarrh of children, whether dependent on chill or on dentition, I have found the infusion serviceable.

Skin Diseases.-Its principal reputation was obtained in skin affections, especially in the dry and scaly forms, such as psoriasis and pityriasis. Linnæous highly commended it, and more recently Crichton and Willan. Neligan prescribed the infusion in large doses, but usually as a vehicle for more active agents, such as iodine and arsenic. Elliotson commonly prescribed a pint to be taken in one day. Bigelow and Bateman confirm the utility of this medicine, the latter declaring it to be one of the most effectual remedies for lepra; he prescribes a decoction of the twigs and leaves. Caylus and Whitehend have recommended dulcamara in syphilis-especially syphilitic 
lepra. The former recommends the alkaloid to be given in the form of 1 to 5 grains of the acetate (Brit. and For. Med. Chir. Rev., ii., 1859, i., 1852). Bretonneau stated that dulcamara, as a decoction, was a good preventive and curative remedy for mercurialism-he began with 2 drachms, and steadily increased the dose (ibid., ii., 1848). I have employed it in humid asthma (especially when the disorder appeared after the suppression of nettle-rash or some other eruption), and always with some success. In delicate constitutions, and in hysterical women, the exhibition of dulcamara has sometimes been followed by syncope and slight palpitation of the heart. Such symptoms are exceptional, but should they occur, the dose should be diminished. It is said to act best in persons of fair complexion and light hair, with a disposition to catarrh. Amongst other maladies in which this drug was formerly much administered are gout, jaundice, and "incipient phthisis;" it was also given to aid the action of mercury, and Dr. G. B. Wood, in his "Dispensatory," recommends it as a fit medicine for subduing excessive sexual desire in maniacs and others.

PREPARATIONS AND DOSE. - Infusum ( 1 oz. of fresh stalks, chopped small, to $10 \mathrm{oz}$. of boiling water): dose, $\frac{1}{2} \mathrm{dr}$. to $2 \mathrm{fl}$. oz. or more. This is much more trustworthy than the decoction, since boiling dissipates much of the active principle.

Adulteration.-The cut stems of humulus lupulus, and some other plants, are occasionally mixed with those of dulcamara.

\section{CAPSICUM FASTIGIATUM.}

\section{(CApstcum.)}

DESCRIPTION.-All the species of this genus are herbaceous, rising to the height of about 18 inches, freely branched, but in a distorted manner, and provided with abundance of ovate leaves. The flowers are insignificant, axillary, starlike in 
figure, usually white, and followed by bright red or yellow pods, which are singularly inflated, coriaceous in texture, smooth and shining, and often remarkably large for the size of the plant. The capsicum fastigiatum is a small shrub which grows wild in India, and tropical Africa and America, and produces, in ench fork, two or three subconical scarlet fruits, from $\frac{1}{2}$ to $\frac{3}{4}$ inch long; on drying they become shrivelled, and brittle when old. This kind is known as African or Guinea or pod pepper, and as "chillies": when powdered it is "cayenne pepper." The taste of the pericarp and of the seeds is extremely fiery. The dried fruit has a peculiar odour, somewhat like that of hay, but pungent.

ACTIVE INGREDIENTS.-The name "capsicin" has been given to various preparations. That of Bucholz (1816) is an impure extract made with alcohol and ether. Buchheim and Fleischer also obtained a dense red, aromatic, fatty liquid termed capsicol $\left(\mathrm{C}_{24} \mathrm{H}_{45} \mathrm{O}_{4}\right)$. Witting's capsicum is basic, and forms crystalline salts with acids. The real active ingredient is apparently that obtained in crystals by Thresh (1876), and termed capsaicin $\left(\mathrm{C}_{9} \mathrm{H}_{14} \mathrm{O}_{2}\right)$; it occurs only in small quantity, and associated with a red fatty matter, composed chiefly of palmitic acid; it is fusible, volatile, intensely hot and pungent to the taste, soluble in alcohol, ether, and alkalies, slowly in turpentine, sparingly in water; it has basic properties, and forms crystalline compounds with barium, calcium, etc. The red colouring matter of capsicum is slightly soluble in boiling. alcohol, readily in oils, ether, and chloroform. Felletár isolated a volatile alkaloid having the odour of conia, but differing from it in various chemical reactions.

ABSORPTION AND ELIMINATION.-The active principle of capsicum is readily absorbed, and is eliminated mainly by the urine; it has no disinfectant power. Applied to the slin, especially when in concentrated solution, it is a powerful and painful rubefacient; and though, unlike cantharides, it does not readily vesicate, it will do so if applied continuously.

Physiological ACtion.--Intenal.-Digestive System. -In the mouth, capsicum causes a burning sensation, also 
in the fauces and throat if swallowed: moderate doses taken into the stomach excite a gentle, not unpleasant, feeling of warmth, which with somewhat larger quantities becomes like "heartburn," and is accompanied with yawning, eructations, and passage of flatus. It has been stated that, under certain conditions, capsicum may produce severe inflammatory swelling of the mucous membrane of the stomach and intestinal tract, and certainly it may sometimes cause pain and thirst and vomiting, occasionally slimy diarrhoea and tenesmus, but, according to the recent observations of Högyes, it rarely produces serious mischief or permanent anatomical change (Archiv, Böhm and Klebs, Bd. ix., 1878). He examined the stomach in living dogs, to which he administered the drug by a tube, and observed nothing more than hyperæmia with increase of salivary and gastric secretion. Fifteen grains caused vomiting twice, but the animal soon got better. $\mathrm{He}$ considered the drug to stimulate the sensory nerves, to promote digestion, and favour peristalsis, but some persons are much more sensitive than others to its irritant action. Taylor observes that there is no recorded instance of its proving fatal, though in one case a "medical botanist" was charged with having caused the death of a delicate boy by its administration. Dr. Taylor found cayenne pepper in the stomach, and "patches of inflammatory redness," but the connection between this and death was not clear enough to convict. The prolonged use of large (not acutely irritant) doses may impair appetite and digestive power, but such effects are not readily produced in the residents of tropical countries where the plant is indigenous.

Circulatory System.-On the frog's heart little or no action is exerted (Högyes). In most subjects, capsicum does not quicken the pulse or raise the body-temperature, but in many it flushes the face, and gives rise to unpleasant sensations of heat and itching (with perspiration), followed by reaction, languor, etc. Some local congestion will account, probably, for such symptoms as itching and tingling in the nose and larynx, hoarseness, aching and tightness across the root of the nose, redness and burning of the eyes, frequent short cough, oppression and tightness across the chest. 
Nervous System.-Throbbing pain in the temples or across the forehead has been complained of. The sweating of the brow and the salivation which immediately follow very large doses are probably due to reflex paralysis of vaso-motor branches running with the fifth nerve. The pupils are dilated by it, musco volitantes are seen, and there is profuse lachrymation. There is little or no evidence of any sedative action of capsicum on the healthy subject, and the contractile power of muscular tissue is not impaired by it. Högyes certainly found dogs become languid and quiet after its injection into the jugular vein, but the operation would account for this. I have known some persons tired and stupid after the stimulant effects have passed off, but otherwise the evidence of its nervemsedative power is derived only from cases of illness, especially the nervous excitement of delirium tremens; of such, two cases are on record. where a full dose of 20 to 30 grains was followed by calm and curative sleep.

Genito-Urinary System.-Burning sensation in the urethra and ineffectual urging to micturate have been described from capsicum, and suggest some analogy with the action of cantharides : possibly the action is sympathetic, due to irritation of the rectum. The urine passed under its influence deposits. a whitish-yellow sediment, in which the alkaloid can be detected.

Therapeutical Action. - Extemal. - Ulcerations, etc.-In unhealthy, languid forms of ulceration, and in slow... healing sinuses or fistulæ, a weak infusion of capsicum is a useful stimulant.

Congestion.- In many cases of local congestion, e.g., chilblain, relaxed congested throat, or neuralgic pain, the application of capsicum as paint, gargle, lozenge, or cataplasm is very serviceable. For chilblains, if the skin be not broken, saturate a piece of flannel with the tincture, and rub until a strong tingling is produced; and continue daily until improvement occurs. For toothache, a small dossil of lint dipped into the tincture and applied to the cavity is an excellent remedy. Mr. Buck has recommended a lotion prepared by boiling a handful of capsicum pods in a pint 
of water, as excellent for sprains, bruises, and congestions of various kinds. A good cayenne spice-plaster is made with equal parts of ground ginger, cloves, cinnamon, and allspice, and one-fourth part of cayenne pepper, all to be thoroughly mixed and put into a previously prepared flannel bag of the desired size; the powder is then to be distributed equally through the bag, and quilted in with needle and thread and applied over the affected part. In the more severe form of inflammatory sore-throat - cynanche tonsillaris - accompanied with pyrexia, pain and swelling of tonsils, furred tongue, much viscid saliva, difficult deglutition, etc., a capsicum gargle ( 1 drachm of tincture to $\frac{1}{2}$ pint of acid infusion of roses or port wine) often relieves if used before suppuration sets in; it requires, however, some caution, that it may not excite undue irritation: it may be prepared with honey.

\section{Therapeutical Action.- Intemal.--Renal Conges-} tion.-I have given tincture of capsicum internally, and also used it externally, for habitual pains in the loins-dependent probably upon sluggish circulation in the renal vessels-and when there was a persistent though slight trace of albumen in the urine. These symptoms had resisted various remedies, but were speedily removed under the above treatment; 5 minims of the tincture being taken thrice daily, and a cataplasm applied for two or three nights in the week. This action is also allied to that of small doses of cantharides.

In the Ague-attacks suffered in Egypt by the French army under Napoleon, Baron Larrey found the same remedy efficacious in removing similar lumbar pain, as well as in checking the severe chills; he gave an infusion shortly before the paroxysm. Further and later observations, however, have proved that this will not replace quinine, though it may assist its action.

Dyspepsia.-In atonic forms of this disorder, such as occur in weakly or overtaxed subjects, with sense of weight and distension, flatulence, eructations, nausea, etc., capsicum may do good. It may be freely used with food, but generally acts better when given in small doses with other medicines, such as vismuth or rhubarb, than in full doses by itself. It should be 
considered more as an adjunct than as a principal remedy, and is an ingredient in many "digestive lozenges" or "cardiac tinctures." It is added to tonic pills to assist their action. In sec-sickness it often does good, and for this disorder I have prescribed 5 grains sprinkled over a small sandwich with excellent results.

Hæmorrhoids are sometimes relieved by its internal use, as they are by black pepper and some other stimulants of the same class. The cases in which I have myself observed benefit from it are of internal hæmorrhoids, occurring in fairly strong subjects from such causes as long riding, when a relaxed condition of the rectum was present.

Cholera.-Capsicum has been strongly recommended in the collapse stage by Parsons: the dose of the powder in alcohol varied from 5 to 30 grains (Ranking, ii., 1873).

Dipsomania - Delirium Tremens. - Moderate doses of 10 to 15 minims of tincture are serviceable in the treatment of drink-craving, by removing some unpleasant gastric sensations and the sense of sinking which impels the patient to have recourse to alcohol. Larger doses of tincture ( $\frac{1}{2}$ drachm) or 20 to 30 grains of the powder have sometimes acted exceedingiy well in relieving the acute symptoms of an attack of delirium tremens. Kinnear was one of the earliest observers of this, and reported several cases in which such doses given "in spirit and water" every four hours produced quiet and sleep after three to seven doses (Lancet, i., 1862).

Fernely infused 40 grains in a pint of boiling water, strained, and flavoured with sugar and citric acid, and found this "capsicum lemonade" well taken and very beneficial in similar cases. Wills reported seven cases in which the patient remained extremely violent in spite of chloral, etc., but went to sleep after the second dose of 30 grains, and woke convalescent (Med. Times, ii., 1873). Dr. Lyous, of Dublin, speaks equally well of this treatment, though he quotes only one case (Med. Press, April $18,1866)$; he presumes it to act in a reflex manner through the terminals of the vagi in the stomach. Dr. Harris records a favourable experience of the same treatment (Lancet, 1878); and Dr. Crowther sends from Australia notes of two aggravated cases in which it was markedly successful: one of them was 
complicated with pneumonia, and after failure of bromides, digitalis, hospital care and feeding, full doses of capsicum soon lessened the tremor and agitation, lowered the pulse, at the same time quickening circulation in the skin, and acted on the kidneys and bowels (Lancet, i., 1879). The treatment has the great advantage over that by opium and digitalis of being safe, and well deserves more general recognition than it has yet received. I have prescribed it for many cases of delirium tremens and drunkenness, and can speak favourably of its power of improving appetite and digestion; lessening tremor and agitation, and inducing sound and refreshing sleep. The dose ordered has generally been from 20 to 30 grains of the powder, or $\frac{1}{2}$ drachm or more of tincture. It is not so serviceable in chronic alcoholic delirium. It is also given by some practitioners in atonic gout, dropsy, and exhausted conditions due to fever.

PREPARATIONS AND DOSE.-Tinctura (nearly 1 in 7): dose, 5 to $20 \mathrm{~min}$. Putvis: dose, $\frac{1}{2}$ to $1 \mathrm{gr}$. in pill; 20 to 30 gr. in delirium tremens.

An infusion may be made as directed in text.

\section{DUBOISIA MYOPOROIDES.}

This plant, a native of Australia and neighbouring islands, is classed by Endlicher with the Scrophulariaceæ, but by Hooker with the Solanacese, and may be said to form a connecting link between the two orders. It is generally now placed with the latter, and in the group Salpiglossidæ.

It is a tree-like shrub, with smooth lanceolate leaves about 11 inches broad and 3 to 4 inches long, alternate, short-stalked, and entire. The flowers are small and in clusters at the end of the branches.

ACTIVE INGREDIENT.-Mainly, if not only, an alkaloid, duboisin, isolated by Gerrard and Petit as a yellow viscous substance. 
In many chemical reactions it resembles atropia-e.g., with tannin, chlorides of gold, platinum, and mercury (v. p. 521), crustic alkalies, etc.,--but it is more soluble in water ( 1 in 120), is a more energetic base, is coloured reddish-brown by strong sulphuric acid, and on heating evolves a "butyric acid" odour, whilst that of atropia is pleasant. It readily dissolves in alcohol, ether, and chloroform. The sulphate and the bromide are orystallizable.

Physiological Action.-According to Dr. Ringer, this is apparently identical with that of atropin. It dilates the pupil, dries the mouth, suspends the secretions of the skin, quickens the pulse, and in full doses causes headache and drowsiness: the hypodermic injection of $\frac{1}{140}$ to $\frac{1}{70}$ grain will cause these symptoms (Lancet, i., 1878).

According to other accounts, it impairs the muscular power, and instead of stimulating the brain, causes rather indifference and inertness, but not usually drowsiness. Marmé states that "if dogs are so far poisoned by morphia that the heart's action has fallen to two or three beats in five seconds, and the respiration become irregular, small doses of duboisin injected beneath the skin immediately strengthen and accelerate the heart, and render the breathing regular." According to Dr. Gibson, $\frac{1}{14}$ grain caused rise of blood-pressure without influence on the pulse-rate; $\frac{1}{7}$ grain and upwards diminished the pressure and retarded the pulse; $\frac{2}{3}$ to $1 \frac{1}{3}$ grain killed rabbits quickly, the heart stopping in diastole: he concluded that the above effects proved an action on the cardiac ganglia. Small doses contracted the arterioles in the frog's web, large ones dilated them; but otherwise, by testing with a rabbit's ear, etc., no effect on the sympathetic was verified (B. M. J., ii., 1881),--by all analogy, however, such effect should be obtainable. Applied to a frog's heart, duboisin, like atropia, does away with the effect of vagusstimulation. The early stimulant effect is not so marked as with atropia.

It resembles atropia in its general action on the eye, but is much more rapid, a smaller dose being required to dilate the pupil and paralyse the accommodation.

It is very readily absorbed from the conjunctiva. In one 
case, shortly after a "minute piece" of the sulphate melted in the eye, the patient became flushed, dizzy, then wildly delirious, restless, picking at objects, and with some convulsive twitching of arms: morphia allayed the symptoms (Norris). It was supposed to cause less local irritation than atropia, but instances to the contrary are on record (B. M. J., ii., 1879); and, according to Risley, "the prejarations now in the market are more liable to irritate than neutral solutions of atropia sulphate (Amer. Journ. Med. Sci., A pril, 1880).

The extract is less liable than the alkaloid to cause irritant symptoms (Tweedy), and a solution of 1 in 20 dilated the pupil fully in fifteen minutes. The effect on accommodation attained its maximum in four hours, and lasted about thirty hours. In four days the natural condition was restored.

Risley concludes that a solution not stronger than 2 grains to the ounce is free from any danger, and acts more strongly on the eye than a 4-grain solution of atropia, whilst its effects last less than half the time.

The "repeatedly well-marked toxic effects, resembling those of belladonna, with delirium," mentioned by Mr. Nettleship, occurred with a 4-grain solution of an "extremely pure crystalline sulphate," sometimes from 2 to 3 drops only (B. M. J., ii., 1879); so that this should be considered too strong for ordinary use.

Therapeutical Action. - Eye Diseases. - Macnaughton Jones found duboisin useful in cases where atropin was indicated, such as keratitis, corneal ulcer, and inflammatory and painful conditions generally. Sometimes it acted well when the latter remedy had to be omitted on account of irritation.

Phthisis.-In moderating especially the night-sweats of this disorder, duboisin has been proved equally as useful as atropia.

Exophthalmic Goître (Graves' Disease). - DujardinBeaumetz recommended $\frac{1}{1+0}$-grain doses of the sulphate by hypodermic injection; and Dr. Hunt has recently reported a case which should encourage a further trial of the remedy. A woman, aged thirty-nine, presented the usual symptoms in a rather severe degree-goittre, protruding eyes, palpitation with 
systolic bruit, cedema, and extreme "nervousness." Ordered $\frac{1}{120}$ grain of the sulphate in water, at first thrice, afterwards twice daily. The palpitation first improved; in three weeks' time the patient was also better as to the emotional state and freedom from oedema. There had been some vomiting, and the bowels were relaxed in the mornings. In two months she "was very much better." There was still relaxation and "much sweating at night." In another two months, whilst her nervecondition was much better, there was still palpitation on excitement; the eyes were but little prominent; the medicine "made her tipsy and sleepy" (B. M. J., May 19, 1883) (v. p. 548).

PREPARATIONS AND DOSE.-Extract of duboisia: dose, 1 in 20 for "eye-drops." Duboisin: dose, $\frac{1}{100}$ to $\frac{1}{60}$ gr. by injection, somewhat more by the mouth. Sulphate of duboisin: dose, $\frac{1}{140}$ to $\frac{1}{70} \mathrm{gr}$. by injection, $\frac{1}{120} \mathrm{gr}$. or less by the mouth. Liquor duboisince sulphatis: $2 \mathrm{gr}$. to $1 \mathrm{oz}$. of water for "eyedrops."

\section{ATROPA BELLADONNA.}

\section{(Beliadonna.)}

DESCRIPTION. - This celebrated plant, the "deadly nightshade," or "dwale" of the vernacular, is indigenous to almost all parts of continental Europe, and occurs not unfrequently in our own country. It grows in waste and stony places, often in lanes and upon hedge-banks, and frequently among ruins. At one period belladonna was so plentiful in the neighbourhood of Furmess Abbey, that the immediate vicinity was known as the "Vale of Nightshade."

The root is perennial, fleshy, much branched, and somewhat creeping. When dried it is found in commerce in rough, irregular pieces of greyish colour, whitish internally, breaking with a short fracture, and having an earthy smell like liquorice 
root. The stems are renewed annually, rising to the height of from 2 to 5 feet, and, owing to the abundance of their branches, constitute an irregular herbaceous bush. The leaves are alternate, ovate, entire, 2 or 3 inches long, acute, and shortly petiolate, having an unpleasant smell if bruised. When dried they are thin, friable, brownish-green above, greyish beneath, of a disagreeable, faintly bitter taste. They lose their odour, but retain active properties if protected from air and moisture.

ACTIVE INGREDIENTS.-The poisonous action of the leaves and root of belladonna depends upon the presence of the alkaloid atropia $\left(\mathrm{C}_{17} \mathrm{H}_{23} \mathrm{NO}_{3}\right)$, which forms silky, glistening crystals, in bundles of rods and needles (four-sided prisms). These are heavier than water, odourless, but of unpleasant bitter taste that lingers on the tongue. The root contains from 0.25 to 0.6 per cent. (the younger roots having the larger proportion), the leaves 0.46 of the alkaloid. This has an alkaline reaction, and requires for solution 300 parts of cold water, 58 parts of boiling water, 3 parts of chloroform, 40 of benzole, or 30 of ether. Rectified spirit dissolves it completely, and amylic alcohol almost with equal facility. Its solution rotates the plane of polarization feebly to the left. Cyanogen gas passed through its alcoholic solution makes it deep red, but the most reliable test is its dilating the pupil of an animal's eye on local application. Atropia is identical with daturia, the active principle of stramonium; only, according to Schroff, daturia is much the more energetic of the two alkaloids. It exists probably as a malate. A second, somewhat resinous alkaloid, uncrystallizable, has been called belladonnin (Hübschmann). The herb also contains asparagin.

By strong acids and alkalies atropia is decomposed into tropia or tropin and tropic acid; it may be re-formed from these bodies (Ladenburg). Then by treating salts of tropin with dilute hydrochloric acid, a class of bodies termed tropeins is produced, and thus oxytoluyltropein or homatropin has been obtained by acting on the amygdalate of tropin by that acid (Pharm. Journ., March, 1880). It is a non-crystalline, oily substance, with mydriatic properties. 
Test For Atropia.-Mr. Gerrard (Pharm. Journ., Mar., 1884) finds that an alcoholic solution of the pure alkaloid with a 5 per cent. alcoholic solution of mercuric chloride gives a yellow precipitate, which becomes red on boiling; the mercuric chloride must be added to the atropia, not vice versî; the precipitate is mercuric oxide ( $\mathrm{HgO})$ : at the same time a compound of mercuric chloride and atropia is formed, which slowly crystallizes from the solution. Duboisia and hyoscyamia give a similar reaction; but no other alkaloid.

The test does not answer well in dilute solutions.

ABSORPTION AND ELIMINATION.-Belladonna and its active principles are quickly absorbed from any part of the body, but, as happens with other medicines, much more quickly from the subcutaneous cellular tissue than from the stomach. It is possible that a difference in its absorption from the latter viscus, or some decomposition of the drug by the secretions, may explain the remarkable tolerance of it by pigeons, rabbits, and herbivorous animals generally, as compared with the carnivorous.

According to Dr. John Harley, "the kidneys are very active in its elimination from the minute it enters, and in two, or at most three hours, all is removed: this occurs at all ages and in all conditions of the kidney " (Gulstonian Lectures, March, 1868). The drug is eliminated also by all the mucous membranes, and sometimes by the skin, in man : the process is rapid, and hence its action is usually of comparatively short duration.

Physiological Action.-External.-An extract or tincture of belladonna applied to or rubbed on a part lessens its sensibility without causing irritation: applied near the eye it induces dilatation of pupil (mydriasis)-an effect which is also and more commonly produced by instillation of an atropia solution,- - the dilatation being limited to the eye touched by it. Occasionally, and especially with strong solutions, local irritative effects are procluced, such as redness, chemosis, and lachrymation (Lawson, Ophth. Hosp. Rep., ii.) ; Meuriot has noticed eczema and boils on the face. Absorption, with consequent 
symptoms of throat-dryness, etc., sometimes occurs. When painted on the skin, belladonna checks sweating, whether this be local or general. It also checks the secretion of milk when applied to the breasts. Its topical action relieves pain in some cases, and reduces cutaneous inflammation.

PHySIOLOGICAL ACtion.-Intemal.-Few subjects in pharmacology have been more discussed than the action of belladonna. Its best-known and most characteristic symptom is the dilatation of the pupil just mentioned, and when this occurs as a consequence of systemic absorption of the drug it marks the commencement of a group of poisonous phenomena which quickly follow any considerable increase of dose: they include dimness of vision or actual amblyopia, dryness and heat of the mouth, fauces, and pharynx, wild expression and flushing of the face, blueness of the lips, sometimes scarlatinoid redness of the general surface, unconsciousness or delirium, which is often noisy with bursts of laughter, at any rate always busy, and usually attended by spectral hallucinations. Other symptoms noted in poisoning by belladonna are thirst, grinding of the teeth, irritation of the nares, hoarseness of the voice, yawning, continual motion of the hands and fingers, raising of the eyelids, vertigo, weakened condition of the muscles and impaired power of will over them, difficulty of swallowing and aversion to liquids, rapid feeble pulse and action of the heart, and quickened, irregular respiration.

The further symptoms noted in fatal cases are swelling of the face, protrusion of the eyeballs, severe conjunctivitis, convulsions or coma; also often colic, nausea, vomiting; and occasionally diarrhœa and incontinence of urine.

Circulatory System. - Examining the action of the drug in more detail, we find that as regards the pulse there is a conflict of evidence. While many authors (especially Schroff, who made some 1,200 experiments) state that the pulse is at first slightly diminished in frequency, and afterwards accelerated, Meuriot found constantly that in from eight to ten minutes after a subcutaneous injection of atropia there was an acceleration of the pulse which lasted for one or two days, after which there followed a retardation. My own 
experience corresponds with the former statement, i.e., that the pulse is at first retarded and soon after accelerated.

According to Bartholow, in some (human) subjects a decided slowing of the heart takes place immediately after a considerable hypodermic dose, but increased rapidity quickly follows. Dr. J. Harley, however, states that a few minutes after doses of $\frac{1}{150}$ to $\frac{1}{36}$ grain there is a sudden acceleration of from twenty beats to double its usual frequency, volume and force being also augmented: he reckons the drug "a powerful cardiac stimulant" (op. cit.). (In rabbits the rate of the pulse is not at all increased.)

At the same time that the heart's action becomes more rapid, arterial pressure is increased. If, however, large doses are given, and especially by intravenous injection, there is an immediate fall in the blood-pressure, though the pulse increases as described.

It has been found that if, after such increase of the pulse, the vagi be divided, no further quickening occurs; and conversely, if these nerves be first divided, and then atropia be given, increase of the quickened action follows: and hence it is reasonably concluded that the drug produces its characteristic effect on the circulation mainly (a) by paralysing the cardiac inhibitory nerves and the terminations of the vagi, but partly (b) by stimulating the cardiac accelerator nerves or nervecentres, and, unless in lethal doses, does not destroy the excitability of these nerves.

If the drug be applied directly to a batrachian heart, it first increases, then diminishes, and finally arrests its action; but to produce such arrest large doses are required. Coincidently with the early stimulant effect, the inhibitory fibres of the vagus are paralysed, the augmentors remaining intact; these, however, soon become affected, and vagus-stimulation has then no result.

The action of the drug upon the capillaries has been disputed, but the balance of opinion is to the effect that they first contract and afterwards dilate under its influence; that an anæmic condition of certain parts is first produced, and afterwards congestion: the secondary result depends somewhat on the dose, and probably does not occur unless this be large, or the subject unduly sensitive. 
Brown-Séquard, however, states that he has seen "diminution in calibre of the blood-vessels of the spinal pia mater in dogs after large doses, and has had success clinically in treating spinal congestion, and even inflammation, by the drug" (Lectures, Lancet, November, 1860); he says nothing about a secondary congestion, and he explains the effect described by its "powerful excitant action on unstriped muscular fibre." We would rather explain it by a stimulant action on the vasomotor centres. All agree that in an advanced stage of atropiapoisoning, after the blood-pressure has commenced to fall, there is an opposite condition of dilatation of capillaries, and this explains the characteristic blush or rash which may occur; some subjects, indeed, seem so peculiarly sensitive, that such rash appears when in no ordinary sense could poisoning be said to be produced: thus, a few minutes after administering $\frac{1}{200}$ grain of atropia (given for whooping-cough to an infant of three months), the child " turned a deep red, like scarlet fever, over its face and the upper part of its body, perspiration was checked, and the skin became hot and dry"--this continued for five hours; similar symptoms, but lasting for a shorter time, were produced by $\frac{1}{800}$ grain; there was no narcotism or dangerous effect (Med. Times, i., 1868). César has reported the case of a pregnant woman in whom 15 minims of belladonna tincture always caused a red rash (Lancet, i., 1879).

Great difference of opinion has existed as to the proportion of cases in which such a rash occurs, and also as to its persistence, and the extent to which it may spread. Dr. John Harley, for example, says that "generally it is nothing more than a mere temporary blush; but in rare cases, and in persons who are liable to vascular irritation of the skin, the redness remains, and its disappearance is attended by slight roughness and desquamation." He mentions two cases, in one of which the patient was "scarlet from head to foot"; and in the other, after the fourth dose of the drug, there was a "scarlatinous tint of skin." Meuriot says that there is a contrast between the cutaneous and the mucous phenomena: the mucous membranes, ten minutes after a subcutaneous injection of atropia, are red, injected, and dry, while the skin is pale and dry like parchment; 
sometimes, however, the skin soon afterwards becomes covered with erythematous redness, and is thus uniform with the mucous membranes. He says that the redness is most frequently seen upon the face and trunk, and does not spread to the limbs.

Many cases are reported in the English, and many more in the American, journals. Dr. Sinclair's case, in particular (Boston Med. Journ.), is very curious, the rash described by him being remarkably like scarlatina, and appearing to correspond with many instances in which I have myself seen a scarlet eruption ensue upon the continued use of the tincture. A similar result followed in a case of accidental poisoning of a child three years and eight months old, by about $\frac{1}{2}$ grain of pure atropia. The child soon became strangely irritable and excited, though unconscious; the face was maniacally distorted; the pupils were widely dilated and immovable; the eyelids were open, and not affected by passing the fingers before them; the pulse was 170 , and somewhat feeble; the skin was pungently hot and dry, and "covered with a scarlatina-like rash" (Holthouse). Dr. Gillespie injected a small portion of extract of belladonna into the urethra of a patient suffering from stricture, and "to his horror, in less than five minutes the patient became as red as a lobster" (Lancet, i., 1877).

Respiratory System.-Breathing becomes quickened when the pulse rises, probably from stimulation of the respiratory centre in the medulla; and in the later stages of toxic action it becomes slow and irregular, and finally ceases, presumably from paralysis of the peripheral ends of the vagi.

Nervous System.-Of the central organs, the brain shows signs of disordered function in a feeling of lightness and giddiness and some confusion of thought; sometimes spectral illusions are induced. Sleep is not directly caused by the drug, though it may follow indirectly the removal of some preventing cause, but the term narcotic cannot properly be applied except to poisonous doses. Sleep, if it occurs, is broken by moaning and startings, and only becomes profound, and passes into coma and convulsion, when a fatal issue is approaching.

The early symptoms of toxic action on the nervous system are, as already mentioned, restlessness, and excited, talkative 
delirium, in which surrounding objects are mistaken for things entirely different; sometimes consciousuess is sufficiently retained for the patient to feel outside, as it were, his own thoughts and delusions, and as if looking on from a different personality.

It is probable, judging from the condition of the retina, etc., that in the early stages at least the state of cerebral circulation is one of ischæmia, i.e., of contraction of vessels, though later on hyperæmia follows. Hammond, indeed, and some others, consider the latter to be the ordinary effect; but no such changes would explain the peculiar effects, which must be the result of a special action on the nerve-cells, causing perhaps "disordered nerve-discharge, which induces adynamia" (Gubler).

On the spinal centres the action is complex, and has been variously interpreted-Brown-Séquard, for instance, describing a diminution of its reflex power; Meuriot, Bloebaum, and others, an increase.

Fraser, experimenting with frogs, ascertained that atropia, within certain limits of dose, caused absolute paralysis of motion and of reflex action for two to four days, and afterwards a tetanic condition, with violent convulsion and intense spinal excitability. It would seem, then, that the drug first abolished and afterwards intensified reflex activity (H. C. Wood) : the hypothesis suggested by Drs. Ringer and Murrell is that both effects are due to a depressant action, - " that the normal cord has a power of resisting impulses received from the periphery, and especially of preventing their wide propagation among the spinal centres. During the first stage of atropia-poisoning it is supposed that the motor portions of the cord are so paralysed as to be unable to form or propagate any motor impulse, and hence the general paralysis. Later on, however, the motor cells have so far recovered themselves as to be able to generate impulses freely, although the resistive power of the cord is still in abeyance; consequently a peripheral impulse plays, as it were, at will, up and down the spinal cord, and instend of giving rise to a simple reflex action, originates a series of reflex movements, involving all the muscles, and constituting a tetanic convulsion." 
A similar action takes place in the higher animals, according to Lemaitre (Arch. Gén., August, 1865), but the convulsant action is earlier developed, and both that and the paralysis are less marked, than in batrachians.

Clinical records of belladonna-poisoning often show a similar combination of symptoms, e.g., spasms, startings, and convulsions of limbs, tremor, or stiffness, epileptiform convulsion, followed by hemiplegia, loss of sensation, excessive general irritability, and exhaustion.

It is evident that the drug does not increase power in the sense of strengthening the cord, but it renders quicker and more unstable the discharge of nerve-force from the spinal cells, seeming to take away their power of conserving energy - an inverse action to that of quinine; thus, after excessive action, exhaustion soon supervenes, and the convulsions are neither so strong nor so continuous as those brought about by strychnia or by tetanus. The drug does not increase motor or reflex power, or the "dynamic tension" of the cord, but lessens the normal check and control over its action, so that symptoms of excitement appear, such as increased peristalsis of the intestines, or repeated erections on slight provocation, a fact which does not prevent the drug from ultimately lessening the contractility of unstriped fibre, and lowering sexual power (Gubler).

On the motor nerves, atropia acts as a direct paralysant, especially in batrachians: thus, if a frog be poisoned by it after ligature of the vessels of one leg, that leg retains motor power when the rest of the limbs and the body have lost it (S. Botkin, Virchow's Archiv, Bd. xxiv.). If the paralysis had been caused through the spinal centre, it would clearly have occurred equally in the ligatured leg, through its nerveconnections; but these were found to react well to galvanism, whilst in the other limb reaction was abolished.

Some contradiction, however, in the results of other observer's with the higher animals are explained by the fact that very large doses are required to paralyse the motor nerves, and death even may be produced before such paralysis is complete. It has been shown that both the nerve-root and the intra-muscular terminals, are affected (Bezold and Bloebaum). Experiments 
also demonstrate that the functional power of the afferent nerves is impaired by the drug, though not nearly to the same extent: thus, Botkin found that in the frog with one set of crural vessels ligatured, irritation of the poisoned and paralysed leg caused reflex spasms in the opposite limb early in the poisoning, though it would not do so later.

The voluntary muscles are not affected by the drug, but nonstriated muscular fibres are markedly so, and apparently in a paradoxical manner, which may be explained as the double action on the spinal cord is explained. Thus Meuriot, finding that, in an animal opened after poisoning, the intestines are strongly contracted, considers atropia "a powerful excitant of non-striated muscle;" whilst Bezold and Bloebaum report that after examining the same parts, as well as the bladder, ureters, and uterus, they observed relaxation and sedation of muscular effort, and this whether the dose were small or large; in the latter case, however, complete muscular palsy occurred. From experiments of Keuchel, we learn that after small doses of atropia-so small as not to affect the motor nerves - galvanization of the peripheral ends of divided splanchnic nerves fails to arrest peristaltic movements of the intestine (as it normally would-Pflüger), and the conclusion is that the drug paralyses the inhibitory nerves of the intestine at their periphery, just as it does the inhibitory nerves of the heart.

"In full doses it paralyses the smooth muscular fibres of the intestines, bladder, etc., and it may be that in any dose it depresses their activity directly ; but it appears certain that by paralysing more quickly the inhibitory apparatus, it sometimes places the intestinal muscular coat in such a position that it will respond more vigorously than normally to external stimuli" (H. C. Wood).

Action on the Eye.-The special action of belladonna upon the eye is to be explained by reference to the nervous system. The mydriasis or dilatation of the pupil is not likely to depend upon a direct action on muscular fibre, since the circular and radiating fibres would be equally amenable to it, and no change would occur; neither is it dependent upon the central nerve-connections of the eye, nor even on the eiliary ganglion, since it 
occur's after section of it and of the oculo-motor nerve as well as of the sympathetic, or the fifth; also even in eyes removed from the body - as of frogs (Hoppe, Valentin), and in man directly after death (Borelli). It results, then, from an action on peripheral nerve-endings in the eye itself, and such action is mainly a paralysis of the branches of the third nerve (the nerve supplying the normal contractor of the pupil), partially a stimulation of similar branches of the sympathetic. The former is evident, from the paralysis of accommodation which is caused, from the strabismus or ptosis which sometimes occurs, and also from the fact that when dilatation has been produced by atropia, if the third nerve be exposed and galvanized no contraction is produced (Grünhagen). The proof of a contrary or stimulating action upon the sympathetic terminals is not so definite, but rests partly on the facts that the dilatation which follows paralysis of the third nerve in man, or its destruction in animals, is not equal to that caused by atropia, and may be increased by the drug; also that the act of dilatation which can break up adhesions is more powerful than can be associated with a simple paralysis (H. C. Wood).

That the sympathetic is concerned in the effect appears further from the observation of Trasbot, who found that when he divided that nerve on one side in dogs, and gave atropia, dilatation occurred only on the uninjured side (Journ. de la Soc. de Thérap., 1867) -an experiment repeated by Ch. Laurent, who records that on removing the left upper cervical ganglion, or dividing the connection between it and the lower one, atropia, whilst dilating to the full the pupil of the right sicle, scarcely affected the left at all; whilst on galvanizing the cut nerve dilatation followed at once. Indeed, these and some other experiments induce Rabuteau to attribute the major part. of the effect to action on the sympathetic-except in the case of very large doses, when he agrees with the general opinion is to paresis of terminals of the third. Gubler draws special attention to the impaived sensitiveness of the retina, since complete blindness and insensibility to light occur sometimes, and partial impairment of vision may persist even after power of accommodation has been restored by eserin: from this impairment, by reflex action, results relaxation of the ciliary muscle, and of the 
circular fibres of the iris, as also contraction of the radiating fibres-contrary phenomena, but associated physiologically to the same end.

The consideration of such retinal anæsthesia may throw some light also on certain clinical results of the administration of belladonna in maladies of the intestines and genito-urinary organs, etc. Thus it may act partly by a sedative and anæsthetic power in relieving constipation when accompanied with spasmodic contraction of the circular muscular fibres, and similarly in hernire and twisting of bowel, etc.; also in the spasm of contracting bronchial fibres in asthma, and in curing nocturnal incontinence it may act by lessening the sensibility of the mucous membrane and the reflex actions connected with this.

Glandular System.-The arrest of secretion of the mucous and salivary and sweat glands, which is so marked an effect of the drug, is dependent upon a paralysis of peripheral nerves, for it follows section of certain branches which supply glands: thus, in Schiff's well-known experiments, the sub-maxillary ceased to secrete as soon as the chorda tympani was divided, though a subsequent so-called "paralytic secretion" may occur; in such case, galvanizing the cut end restored the secretion, but if atropia were previously administered, galvanism failed to act (Keuchel), showing that the nerve-terminals would not convey or react to the stimulus, i.e., were paralysed. Dr. Ringer has made many experiments demonstrating the power of atropia to check secretion from the skin : thus, when perspiring profusely in the hot chamber of a Turkish bath, he found $\frac{1}{200}$ or $\frac{1}{1011}$ grain hypodermically injected completely dry the skin, and keep it dry: notwithstanding the continuance of the bath. Again, a patient, after much sweating in a similar bath, was rubbed on one side of the face thrice daily for a quarter of an hour with belladonna ointment for two or three days; then the bath was repeated, and general sweating over the whole body was found to be greatly lessened. Any local applications of belladonna had more or less effect of the same lind.

Rabuteau says that toxic doses, which induce blood-stasis, slow the pulse, and dilate the capillaries, cause also profuse sweats. The urinary secretion he finds is increased by small doses, which 
heighten, but is lessened by full doses, which diminish arterial pressure. Meuriot had also stated this. Harley speaks of a great increase after small doses; but according to my experience, and that of others, this effect varies. The same observation applies to the intestinal secretion; but certainly diarrhœa often occurs, and is commonly traced to stimulation of the smooth muscular fibres, though it may in some cases be due to a sedative action of the drug, as before suggested.

Post-mortem examination of the bodies of persons who have been poisoned by belladonna shows that putrefaction commences soon after death. The smell is peculiar and intolerable, and the skin is covered with livid spots, while blood escapes from the mouth, nose, and eyes. Should the whole substance of the berries have been swallowed (as usually happens in such cases of poisoning), they are found to be imperfectly digested, in consequence of the poison inducing extreme torpor of the stomach. The heart and lungs are livid; the latter are usually gorged with venous blood, and studded with black spots; and the blood itself is in an abnormal state, the colouring matter of the corpuscles seeming to be dissolved out.

IDIOSYNCRASY, ETC.-It has already been stated that some classes of herbivorous animals are very insensitive to the action of belladonna. Giacomini says that goats eat the leaves with impunity; others assert the same of rabbits. Dogs, however, have died in twenty-four hours from 15 grains of extract (Orfila), which is clearly a much larger dose than man could tolerate. It would almost seem that high development of the brain and nervous system disposes to its more rapid action. Hufeland states that idiots were as little affected as herbivorous animals (1822). Certainly children often bear it remarkably well; women seem to me more sensitive to it than men-it causes headache, etc., more readily in them; whilst men with highly developed brains are more sensitive than any.

Synergists. - Henbane, datura, and the poisonous Solanacere generally, have similar properties-most of their alkaloids also act like atropia. Narcotics and anæosthetics sometimes act well with belladonna, and even opium may be 
combined for therapeutical purposes-e.g., in the hypodermic injection of morphia with atropia, the value of which is in daily evidence.

Prussic acid, and also lobelia, assist the sedative effect on the nervous system : in certain circumstances, arsenic, quinine in full doses, and bromides may be given for the same purpose. Ergot seconds the tonic action of small doses of atropin on the capillaries.

ANTAGONISTS.-1. Against the heart-paralysing action of large doses of belladonna, antidotes are found in digitalis, ergotin, and perhaps strychnia (Gubler). Tea, coffee, alcohol, and diffusible stimulants are valuable in the later stages of poisoning. Quinine in moderate doses, especially in its action on the spinal cord, is in the main antagonistic to belladonna.

2. Muscarin (the active principle of poisonous fungi), jaborandi (and its alkaloid, pilocarpin), physostigma (and its alkaloid, eserin), prussic acid, opium, and chloral, are more active antagonists of the drug, and are to some extent available in cases of poisoning. Muscarin, jaborandi, and pilocarpin have, according to Schmiedeberg and Koppe, the property of stimulating the vagi, and thus slowing the movements of the heart. Dr. Gamgee, in his Classification of Heart Poisons, speaks of these two drugs as stimulating the inhibitory apparatus and stopping the heart in diastole, but concludes that they do not antagonize atropia, although they are opposed by it (Handbook of Physiology). Cases of belladonna-poisoning apparently benefited by these drugs are, however, on record: with regard to the latter especially, one patient that had taken more than 2 ounces of liniment, and, in spite of emetics, remained in a very critical state, began to improve at once after $\frac{1}{5}$ grain of pilocarpin; this dose was repeated three times at intervals of a quarter of an hour, and then the patient was on the fair way to recovery (Grattan, B. M. J., i., 1881).

Physostigma, according to Gamgee, also stimulates the inhibitory cardiac nerves, but affects parts not influenced by muscarin; it does not stop the heart, but removes the paresis of inhibitory mechanism which is induced by atropia. Experiments on the practical application of such antagonism give, 
howerer, varying results; those of Dr. Fraser tend to support it: thus, in rabbits that received three times the minimum fatal dose of physostigma, and five minutes before it a dose of atropia varying from $\frac{3}{50}$ to $\frac{1}{5}$ grain, life was saved-four times the lethal dose could not, however, be successfully antagonized. If atropia was given after the other drug, then much smaller doses were required for a successful result, e.g., $\frac{4}{25}$ grain. But Fraser himself, and also Martin-Damourette, showed (with birds) that non-toxic doses of the two alkaloids injected simultaneously, constantly proved fatal, proving that a certain ratio was necessary between the doses of the two drugs, other proportions seeming to intensify the action of both. Also, as remarked by Wood, any cardiac antagonism between the two must be held doubtful, and whatever antidotal power atropia has, must be looked for in its stimulant action on the respiratory centres. I should therefore be disposed to use atropia in eserin-poisoning, but not vice versâ.

3. The question of the antagonism to prussic acid has been sufficiently discussed under that drug (in the volume on Inorganic Substances), and although of deep physiological interest, it has little practical value.

4. The antagonism of opium is of more importance. It has, indeed, been much disputed, and the experiments of Camus, of Denis on himself, of John Harley, and others, may be quoted as opposed to it;-still there remains a large amount of evidence in its favour. The late Dr. Hughes Bennett conclurles as follows:- "Out of twenty-one experiments in which what was held to be a fatal dose of meconate of morphia was followed by sulphate of atropia, six animals recovered: in one rabbit, a dose of 10 grains of the former was followed in two and a half minutes by $1 \frac{1}{2}$ grain of atropia; the animal recovered in three hours, but afterwards was poisoned in less time by a similar dose given alone."

Dr. Weir Mitchell gave 6 drachms of tincture of belladonna, and $\frac{1}{3}$ grain atropia, in the course of treatment of a patient who hard taken 5 grains of morphia, and with ultimate success; and on the other hand, a patient poisoned by $\frac{1}{4}$ grain of atropia was almost at once restored by $\frac{1}{2}$ grain of morphia injected. Dr. M. Fothergill gave a grain of atropia to relieve 
the poisonous effects of about 15 grains of opium; but this dose is not usually safe.

Gubler points out that some discrepancy of opinion is natural, because the course of poisoning is so variable-many and very bad cases will recover by natural processes alone; but I am myself quite satisfied that the two drugs should be used as mutually antidotal in cases of poisoning, and preferably by hypodermic injection. The main indication for atropia is failure of respiration, such as occurs in opium-narcosis; then about $\frac{1}{40}$ grain of atropin should be given every twenty or forty minutes till the respiration improves. In belladonna-poisoning opium should be administered to the point of quieting restless excitement. The state of the pupils alone is not a dependable guide, as might be inferred from the fact that the dilatation produced by belladonna depends on a peripheral action, as already explained, whilst the contraction of opium is produced through the central organs. Such a markedly different visible effect doubtless led to the original idea of a general antagonism between the two drugs, but the really important difference lies in their effect on the respiratory nerves and nerve-centres.

TREATMENT OF BELLADONNA-POISONING.-If the drug has been taken by the mouth, whether as fruit or as liquid, an emetic should be given, with due regard to the fact that the poison itself may render the stomach insensitive and torpid, and unable to reject its contents. In this case the hypodermic injection of apomorphia should be tried, and failing this, the stomach-pump used. Sometimes it may be advisable to relieve venous congestion in the cerebral vessels by opening an external jugular vein in addition to cold affusion, stimulants to the nostril, and sinapisms to the feet. Friction should be used over the cardiac region. If prostration be extreme, small quantities of liquid food and stimulants are necessary. Ammonia with opium, morphia administered hypodermically, or chloral offer the best alternatives, as already indicated.

Therapeutical Action.-Pain and Spasm.-The fact ascertained by physiological resenrch, that belladonna paralyses the peripheral ends of nerves, indicates its use in several forms 
of pain, and many disorders accompanied by spasm, and in these cases its internal action is greatly aided by local application. Thus in myalgia, lumbago, pleurodynia, sciatica, and some cases of facial neuralgia, a liniment, ointment, or plaster commonly relieves: applied to the epigastrium, I have even known them ease the pain of pyloric cancer, and a lotion containing 2 drachms of extract in a pint of water, applied several times daily, has proved of service in scirrhous and other painful ulcerations. It must be remembered, however, that from such external applications toxic symptoms have sometimes followed (Med. Times, ii., 1856). The effects of a plaster have even resembled mania.

W. Mitchell has pointed out that the spasm best relieved is that of involuntary muscles; in that of voluntary muscles, such as occurs, for instance, after jagged wounds, etc., it has little power, unless, indeed, it be brought into direct contact with the muscular tissue by hypodermic injection.

I have for many years been in the habit of using largely for inflammations equal parts of belladonna extract and glycerine, and it seems to me to relieve materially, e.g., in erysipelas, abscess of mamma, inflamed glands, and lymphangeitis; it is also useful in neuralgia caused by direct injury. Mr. A. Pearce Gould informs me that he has known much benefit derived from the same application in cases of inflammation of the epididymis.

Rectal Fissure, Ulcer, etc.-The same extract will often serve to heal these painful disorders, a small portion being introduced within the sphincter ani night and morning. If not curative, it will at least mitigate the acute burning pain and distressing spasm that occur after defrecation. Often a combination of the same with an equal part of mercurial ointment will act even better, and especially is this the case in chronic syphilitic ulcerations of the rectum. The ointment is more useful in superficial excoriations of the anus than in deep fissures: the tincture should be given internally at the same time.

In painful spasmodic contraction of various parts, similar treatment is often effective: thus, in spasmodic urethral stricture, I order the fresh extract to be applied over the perineum. Dr. Conquest recommended the rubbing of 30 grains 
over the cervix uteri when rigid and unyielding, as in tedious labour. He reported much advantage from this practice, though it is now seldom employed. In dysmenorrhoea, with dark foetid discharge, crampy pains, and cold chills, also in neuralgic uterine pains, a suppository or mild injection, together with internal administration, have been found to relieve; in congestive leucorrhoea, Dr. Ringer advises an injection containing 2 drachms of tincture to the pint of water, with a drachm of bicarbonate of soda, a few ounces to be used at a time, and retained in the vagina as long as possible.

In the case of a healthy married woman suffering from the effects of division of the cervix, with much local congestion and swelling, severe pain and bearing down, the greatest relief was given by minim doses of liquor atropir, B.P., two or three times daily-after failure of many remedies.

In numerous other cases of uterine pain connected with engorgement of the cervix, I have seen similar relief from atropia. The same remedy, either by the mouth or by subcutaneous injection, has also quickly eased " menstrual colic," even when most intense: sometimes it is well to combine it with morphia: its effects must always be carefully watched.

Menorrhagia.-In profuse menstruation, especially when accompanied with congestive headache and severe pelvic pain, I have seen advantage from the hypodermic use of atropia sulphate ( $\frac{1}{100}$ to $\frac{1}{80}$ of a grain). In less severe cases of the same kind I have given drop doses of liquor atropia, B.P., internally, or a few minims of belladonna tincture. In this respect it has some relation to ergotin, and it is interesting to note that both drugs are mydriatic.

In Disorders (chiefly inflammatory) of Secretory Glands, the mammæ, salivary glands, testicles, etc., belladonna is a specially good remedy: its early reputation was, in fact, founded upon its effects "in dispersing tumours," which were formerly in almost all cases supposed to be malignant, and sure to grow worse unless checked by art. In mammary troubles-(1) It arrests secretion when this is desirable from ny cause, such as the death of the baby, bad health of the mother, deformed or sore nipples, etc. (2) It lessens the "milk congestion" which precedes and causes milk abscess, 
and at the same time, by paralysing nerve-terminals, allays reflex irritation and inflammation. It is thus of use, both because it cheoks the inflammation, and also because it stops secretion of milk, and puts the breast at rest, lessens vascularity, etc. (3) In congestion or inflammation of the mammary gland, such as often occurs after confinement, or during lactation when the breasts become red, hard, and swollen, and the milk-flow obstructed, also when the attack is erysipelatous in character, belladonna is one of the best remedies: if there be much febrile reaction, with full, bounding pulse, the tincture should be given in conjunction with aconite-the extract or liniment of belladomna, or, in less severe cases, the plaster, being applied locally. Dr. Miller found that an alcoholic extract, made twice the strength of emplastrum belladonnæ, P.B., kept fluid by collodion, and with camphor added, gave him excellent results if painted over the breasts night and morning (Record, 1878).

When the salivary glands are inflamed, and discharging a profuse amount of their secretion, from any cause, such as ataxy, pregnancy, or the effect of certain drugs, belladonna is useful, though if occasioned by local irritation, this should be removed, or cure cannot be expected. In two obstinate cases of salivary fistula from direct injury, belladonna extract with glycerine was painted over the parotid, with the effect of arresting its secretion, so far that the fistula soon healed without any interference, though in some of these cases belladonna seems to have no beneficial effect.

Orchitis.-When congestion or inflammation of the testicle occurs from any cause, belladonna is of much service; given internally and applied locally, it relieves the induration, enlargement, and lancinating pain; in the acute forms, strapping with the plaster or rubbing in the ointment with mercury acts well.

Tonsillitis.- In this glandular inflammation, belladonna has shown itself specially valuable, when given within the first forty-eight hours of the onset; it removes the pyrexin, pain, and engorgement, and prevents suppuration: 10 to 30 minims many be given every two hours, or 1 minim of liquor atropire every eight to twelve hours, according to the susceptibility of 
the patient to the drug; it acts best when the pulse is somewhat quick and rather hard, but not bounding. In the inflammatory stage of even diphtheritic sore throat, and in "putrid sore throat," I have known it induce a favourable change when other remedies had failed. Handfield Jones used 20 minims of tincture every two hours with advantage (Lancet, Jan., 1871), and Thompson (Philadelphia) asserts that if treatment with $\frac{1}{B U}$ grain of atropia every two hours be commenced within six hours of the onset, it is always successful. I cannot, however, record this frequent repetition of the dose without saying that it seems to me dangerous, since $\frac{1}{120}$ grain twice in the day will, in many persons, cause severe atropinism.

Neuralgia.-The early reputation of the drug as a remedy for this disorder, seems to have been founded on cases of trigeminal neuralgia, published by Dr. Bailey (1819), and cured by doses of 1 grain of extract given every six hours. Trousseau advocated $\frac{1}{5}$ grain every hour.

This treatment is not now as often adopted as at that time, but according to my own experience, belladonna or atropia has often proved effective in the complaint referred to, especially when much vascular excitement is present with flushed, swollen face, and injected eyes; if, however, the attack depends on malaria, other remedies are more suitable. The extract may also be applied to the affected part; but still better results may be obtained from the hypodermic use of atropia. This latter should be commenced in small doses, e.g., 1 minim of the liquor atropire sulphatis, P.B., $=\frac{1}{120}$ grain; this dose may be cautiously increased up to about $\frac{1}{40}$ grain, but, as with morphia, some subjects are extremely susceptible to the drug, and readily develop the unpleasant symptoms of its physiological action, such as dryness of the mucous membranes, disturbed rision, excitement, or even delirium.

In neuralgic pain of the abdominal and pelvic viscera generally, belladonna is an admirable remedy, and in my own experience 10 to 20 minims of the tincture every two hours have remored violent tearing, shooting pain in the neighbourhood of the ileoexcal valve, arising from continued constipation, with, possibly, some stricture, and whilst relieving pain, it has proved useful also in assisting the action of the bowels. An injection con- 
taining $\frac{1}{2}$ ounce of tincture in 2 pints of hot water has also given great relief-more so than tincture of opium.

Constipation-Obstruction.-These conditions are sometimes relieved in a remarkable manner by belladonna. Trousseau taught that doses of $\frac{1}{4}$ to $\frac{3}{4}$ grain of extract given once or twice daily would secure a daily consistent evacuation without purging or looseness; but I find that if such doses be continued long, headache, or other unpleasant symptom intervenes. No rapid laxative effect is to be expected from the drug, but if a $\frac{1}{4}$-grain pill be taken each night for a short time, and any urgent or marked constipation be relieved at intervals by castor oil or colocynth, the ultimate effect of a regular relief of the bowels will be obtained in a more satisfactory manner than by purgatives only. The belladonna may sometimes with advantage be added to the aperient: thus, $\frac{1}{4}$ grain of extract combined with 1 grain of ipecacuanha and 4 grains of compound extract of colocynth acts very well. Spender recommends a combination with aloes, and it is usefully combined with iron, as it neutralizes its constipative tendency.

It is plausibly supposed to act by stimulating and regulating the peristalsis of the involuntary muscular fibres, thus relieving cases dependent upon want of tone or partial imitative spasm of a portion of the intestine. My experience quite corroborates the statement already quoted that the result is a soft consistent evacuation; but it is to be noted that if belladonna, or more especially atropia, be given in too large doses, unpleasant dryness of the mouth and intestinal tract will occur, with resulting constipation.

The use of belladonna in obstruction is comparatively recent, and is of considerable importance, since the disorder is one involving much anxiety in practice. I consider the main indiration for its preference over other remedies in any given case to be the presence of pain and colic, i.e., signs of spasm and congestion. Belladonna corrects irregular and excessive peristalsis, and helps to ward off inflammation. Opium acts best in those cases where the peristaltic action had been sluggish for some time previous to the accumulation of freces. If, in such conditions, a strong purgative be given, it muy act, but, on the other hand, may very likely increase the irritation, 
and all the painful symptoms dependent upon it; but if $\frac{1}{4}$ grain of extract, or 20 minims of tincture, be given every two to four hours, it will often, in my experience, relieve the congestion and pain, and if it do not itself operate upon the bowels (as it often will), at least it prepares the way for the due action of a laxative. I have known more than one case suffering acutely with colic, dulness over the ileo-creal valve, vomiting, pyrexia, and several days' constipation, relieved by such treatment, after failure of purgative doses of calomel, colocynth, senna, mineral waters, etc. (Little or no good can be expected from the belladonna treatment in internal or external strangulation (hernia), or in malignant stricture.)

Many illustrative cases of the value of the drug in obstructive conditions are now on record. Thus, Dr. N. Kerr has reported five treated with 1- to 2-grain doses every hour, and all ending successfully within six to nine hours; but no exact diagnosis is given (Practitioner, v., p. 21).

The late Dr. Murchison reported a striking case in which colic and obstruction had lasted many days, though castor oil, enemata, and opium were used; the patient began to recover, and the bowels to be freely relieved after four pills, each containing $\frac{1}{2}$ grain of belladonna extract given at brief intervals, the same preparation being also freely applied over the abdomen (Lancet, i., 1867). Another case, a man aged seventy-five, presented very serious symptoms dependent on prolonged obstruction, when belladonna was commenced in doses of $1 \frac{1}{2}$ grain of extract every two hours; after four such pills, characteristic delirium set in, but a motion was freely passed in bed, and the patient recovered (B. M. J., ii., 1878).

This treatment proceeds somewhat on the same lines as the treatment by opium, an anti-spasmodic being preferred to a direct aperient; but I consider it, on the whole, more successful and less likely to be injurious if it fail. In a case I saw with the late Dr. Austie, opium had been freely given for several days, but the patient was getting worse ; improvement set in, and the bowels acted the day after prescribing belladonna extract.

Dr. Brinton, who rather favoured the opium treatment, also suggested a combination of the two drugs, and recorded a good case in which $\frac{1}{6}$ to $\frac{1}{3}$ grain of opium, given with 1 to 2 
grains of belladonna extract, acted exceedingly well (Lancet, i., 1863). In another case, $\frac{1}{2}$ grain extract of belladonna with 1 grain of opium was given every three hours till some toxic symptoms occurred, and then the obstruction yielded (B. M. J., ii., 1878). Stewart recommended 3 to 4 grains of belladonna extract dissolved in water, to be given by injection into the bowel (Lancet, i., 1873).

Dysentery.-On the other hand, some observers, e.g., Dr. Smith (U.S.), says that he has used belladonna with advantage in dysentery (Record, February, 1878), and I have myself known it act well in relieving the rectal spasm, so often an attendant of this complaint in the later stages.

Enuresis.-In the ordinary nocturnal form of incontinence of urine, which occurs especially in strumous children, belladonna is of much service. It is important to treat first any local conditions which may excite vesical irritation, such as phimosis, ascarides, etc., and then to give the remedy in full doses, particularly at night-time ; thus 10 or 15 minims or more may be given twice daily, and double that quantity at nighttime, and so soon as physiological effects develop, the amount should be somewhat lessened. Dr. Farquharson has written to recommend still larger doses, e.g., 20 minims to a child of three years, and 1 or 2 drachms to older patients (Practitioner, ii., 1876). Mr. A. P. Gould informs me that he has given as much as 6 drachms a day of the tincture to a boy ten years old, without other effects than dilated pupil and dry mouth and throat. This mode of treatment will very commonly relieve the complaint, but, on the other hand, I have sometimes been disappointed with it, and therefore cannot speak of it so confidently as do some practitioners. It may be presumed to act in the successful cases by lessening congestion and spasm, excited by small quantities of urine, which the normal bladder would tolerate (in the unsuccessful cases it has seemerl to me there was more paralysis than spasm). I think it acts in these cases of enuresis by diminishing the morbid sensitiveness of the neck, and possibly of the bodly, of the bladder also. For the same reason it is useful in cystitis, acute or chronic, and in urethritis, where it lessens pain in micturition. In the incontinence of acute cystitis, produced, c.g., by cold, by an ulcer in or near the bliudder, 
etc., belladonna often gives much relief, especially when applied locally by injection into the bladder, as well as given internally. In the clronic cystitis of elderly people, dependent, as it commonly is, on a paralytic (central) condition, the remedy cannot be depended upon; stimulants, such as strychnia or cantharides, are more suitable. Smeared on the perineum it relieves chordee.

Asthma.-In true spasmodic asthma, I have used belladonna for the last twenty-five years, sometimes with marked success, giving frequent doses of about 5 to 10 minims every half-hour or hour during a paroxysm, and occasionally during the interval. I have known this succeed when other methods of giving the drug had failed; I have also prescribed minim doses of liquor atropiæe every six to twelve hours, giving speedy relief in many of those cases where the belladonna tincture had failed-a few doses are usually sufficient. I prefer this treatment, or the subcutaneous injection of atropia to the method recommended by Dr. Hyde Salter, viz., one full dose at bedtime. He commenced with 10 minims of tinctura belladonnæ, and increased rapidly to 30 to 40 minims or more, if well borne (Lancet, i., 1869). The latter may be the case to some extent; but as to the former, lessening of muscular spasm is a more probable explanation. As in other cases of spasm, a local application is often more quickly useful, and may be secured by smoking a cigarette made with belladonna leaves, which, according to some observers, act better than stramonium (Med. Times, ii., 1867).

Leclere burns the extract in pastille with ipecacuanha and myrrh, and Gallois recommends 1 or $1 \frac{1}{2}$ grain of extract of opium to be dissolved in the water with which the leaves are moistened; a combination of the two alkaloids, morphia and atropia, acts remarkably well when given subcutaneously. Dr. Oliver, of Harrogate, was one of the first to point out this, asserting that relief was always experienced within a few minutes, that the sererest paroxysm could not last after it, and that it was never injurious (Practitioner, i., 1876). I cannot say quite so much as this, for from my experience those suffering from nervous asthma are often very sensitive to the action of atropin, and serere hendache 
and affection of mucous membranes limit its use. Still the combination mentioned is valuable.

In the dyspnoea of emphysema, belladonna often acts well combined with ether and expectorants. In bronchial asthma, Dr. Spender speaks highly of 4 minims of the tincture given every two hours (Med.-Chir. Rev.): and in the capillary bronchitis of children, Dr. H. C. Marsh has seen it of much benefit; to infants he has given 1 minim of the tincture every hour (Med. Times, i., 1881). I can bear testimony to the efficacy of this treatment.

Pertussis.-Trousseau, Bretonneau, and many others have recorded experience in favour of atropia, and certainly I have seen advantage from it or belladonna when the disease first appears. In this stage the good effects of the treatment are very conclusive, as it limits and shortens the catarrh, and other symptoms. In the spasmodic stage, the violent paroxysms of cough interfere more or less with the circulation to and from the brain, often leading to congestion (mainly venous), with pain in the head, protrusion of eyes, redness and lividity of face, vomiting, undue sensitiveness to light and noise, drowsiness, etc. In such conditions I have sometimes observed the dangerous symptoms subside under the action of the drug, although other remedies, such as bromides, asafœtida, etc., usually relieve the spasm much more effectively. The tincture may be well given in 5- to 10-minim doses and upwards, every one to three hours; whilst the linimentum belladonnæ, P.B., diluted with 10 or 15 parts of soap liniment, may be rubbed over the spine night and morning, the effects being carefully watched. One minim of the liquor atropia sulphatis given to children daily, in the early morning, often proves of much service in all stages of the disease; the dose need seldom be repeated through the day, and should never exceed 2 minims in any twenty-four hours.

The disorder is, however, proverbially uncertain in duration and character, yielding much better at some times, and in some patients to different treatments.

Dr. Ringer rather objects to belladonna during the febrile stage, and during dentition, but I have long since come to a different conclusion, having found it relieve the former, and 
especially benefit the congestion of the gums in the latter: it seems to lessen also congestive irritability of the air-passages, and determination of blood to the head. In the later stages when the severity of the convulsive attacks is abating, the value of the medicine is more generally acknowledged, but there is a difference of opinion as to the best dose; some advise full doses to the production of mydriasis and even skin-rash, others the avoidance of such effects. I have commonly given it as already described, but the amount may be increased, stopping short of the severe symptoms.

Dr. Charles Kelly has published some cases of children with whooping-cough, in the wards of King's College Hospital, taking literally enormous doses-e.g., 15 drachms in the course of four days (Practitioner, 1873). The physiological symptoms produced were of only moderate severity, and I cannot but think the tincture was deficient in normal activity, as it would be if made from old plants: certainly I could not myself adrise the giving of such doses. The paroxysms in some of his cases were lessened in severity and duration, but not in number; in others no advantage was derived, and the throat-dryness rather increased the cough; but I do not think these obserrations fair tests of the therapeutical power of the drug, and Dr. Kelly himself concludes that it acted better when given in smaller doses at frequent intervals.

Dr. Fuller reported much advantage from combination with zinc sulphate (Med. Times, i., 1860); others with alum.

Its use by inhalation should not be omitted. I commonly prescribe about $\frac{1}{2}$ to 1 drachm of extract to the pint of boiling water, the steam to be inhaled; but the method recommended by Dr. Caldwell (U.S.), though more complex, is doubtless more effective. He used an atomized spray made with belladonna extract, 6 to 13 grains; ammonium bromide, 20 grains; potassium bromide, 40 grains, in 2 ounces of water (Prnctitioner, vol. xx.).

Either of the above inhalations may be used thrice daily over the face and throat for fifteen minutes, or until the pupils dilate. A long, narrow belladonna plaster from the nape to the third or fourth dorsal vertebra sometimes acts very satisfactorily. 
Convulsions, Centric or Excentric.-In various diseases with increased irritability of nervous centres-as shown by convulsion-I have formed a high opinion of the value of belladonna.

In the convulsions of reflex irritation (of teething, for instance) I have known it act exceedingly well, and this in various types of constitution; in the apparently robust and healthy, and also in scrofulous, feeble children with precocious cerebral activity,so that I have come to look upon evidences of congestion as the main indication for its employment.

In the convulsions of whooping-cough it is valuable, as already mentioned; but not so much so, I think, in convulsions arising from intestinal irritation.

M. Chaussier (1811) and other distinguished practitioners have drawn attention to its efficacy in puerperal convulsions, and to this I can myself testify, though I do not use the remedy in the form he recommended, viz., application of belladonna ointment to the cervix uteri. In his first case thus treated, uncontrollable uterine hæmorrhage and other perilous symptoms occurred, so that the propriety of such treatment was reasonably disputed; but the objections made do not apply to the modern use of hypodermic atropia, or to the internal administration of the tincture (if swallowing be possible).

Epilepsy.-The reputation long and deservedly held by belladonna in the treatment of this malady has been somewhat lessened by the introduction of the bromides, but the method is still of importance. Trousseau valued the drug highly, and though he did not distinguish the kind of cases most suitable for it, he laid down very precise rules for its administration; thus- "during the first month the patient takes a pill composed of extract of belladonna and of the powdered leaves of belladonna, $\frac{1}{5}$ grain of each, every morning if the attacks occur chiefly in the daytime, but in the evening if they be chietly nocturnal. One pill is added to the dose every month, and, whatever be the dose, it is always taken at the same period of the day. By this means the patient may reach the dose of five to twenty pills, or even more." Dr. Wilks (Med. Times, i., 1869) and Dr. John Ogle saw much advantage from 20-minim doses given thrice daily (Lancet, i., 1874), and Osborme from the 
extract combined with zine sulphate; Dr. J. Harley found it useful in attacks arising "from emotional excitement"; and I have myself found it to act best in recent cases in which the stage of congestion was probably not overpassed. Such cases are not as a rule hereditary, nor of very severe type, but several of them have been connected with sexual irritation in men, and disorder of the menstrual function in women, and many with mental excitement, fright, or peripheral nerve-imitation; some have been of the epileptiform-hysterical type rather than true epilepsy, but the relief given by '20- to 30 -minim doses of tincture of belladonna several times daily has been marked. It is now more generally ordered with other nerresedatives, such as bromide; but I still often depend upon it alone, and I have reason to think that when good effects are obtained from it they are more permanent than from the sedative salts.

Schreyer, of Hamburg, has reported sixteen cases of epilepsy treated by 2 grains and upwards of extract of belladomna given twice daily; with this remedy he conjoined spare diet and a course of warm bathing, with cold applications to the head: he states that fourteen of the patients were cured (Lancet, i., 1866).

In Tetanus, though it has often failed, it has proved sometimes successful (Practitioner, vol. xix.), and I have myself on several occasions known it allay long-continued and painful muscular cramps, due, e.g., to cold, damp, or sudden changes of temperature. In one instance the patient when intoxicated (not an uncommon occurrence with him) fell into a ditch, and lay there in the damp for many hours. When I saw him three days afterwards his face was red and swollen, the jaw-muscles were tense, the teeth set, and the niuscles of the body and limbs became rigid and immovable in paroxysms which made him scream with pain; there was profuse sweating; the pulse was quick and feeble: between the attacks the face became denthly pale. I gave him 30 drops of tincture of belladonna at intervals of half an hour, then every hour for four doses, afterwards every four hours, the spine being rubbed also with the extract. Relief was obtained with quiet calm sleep about ten hours after the first dose. 
Waring suggests that belladonna acts in such cases by lessening congestion in the cord and membranes, and this has always been my own view as to its action. In the early stage of meningeal inflammation, for example, it has relieved the severe headache and delirium, also the nausea and vomiting. The suffision of the eyes diminished, light and sound became less distressing, so that the course of the disease was rendered much milder. In congestion from delirium tremens it sometimes gives good results, and in some cases of traumatic spinal meningitis it will relieve, as in two patients suffering severe paroxysms of pain in the lumbar region, hips, thighs, and round the abdomen, aggravated by pressure upon the lumbar vertebre; the pulse being quick and full, temperature $104^{\circ}$. Opium was administered in various ways, but the relief given by it was only temporary, and no permanent benefit ensued until tincture of belladonna was given every half hour or hour, in 10-minim doses, and a belladonna plaster locally applied. In chronic myelitis and meningitis, Dr. Brown-Séquard speaks highly of the same treatment.

Headache.-In various forms of congestive headache, and especially in that dependent on influenza or coryza, when the mucous membrane of the nose and frontal sinuses is dry and congested, I have often seen benefit from the drug. In tinnitus aurium of congestive character I am also accustomed to use it, although aconite and other remedies commonly act better.

Sympathetic Nerve Disorder-Syncope.-Dr. John Harley has strongly recommended atropia as a stimulant to the sympathetic system in cases of syncope or prostration from shock, chloroform, etc., and some, but not much, use has been made of it in such cases. Professor Schäfer has suggested the subcutaneous injection of atropia before the administration of chloroform, to counteract any tendency to syncope, and in cases of slowness and irregularity of the pulse I have myself observed a tonic and stimulating effect. In one man whose pulse averaged forty-four, and in whom slight causes would depress it ten beats or so lower, some faintness occurring at the same time, alcoholic stimulants did not relieve, nor salvolatile nor digitalis, but belladonna frequently did so, and 
he felt this himself so decidedly that he constantly carried the remedy with him and took 5 to 20 minims occasionally.

Exophthalmic Goitre.-Many symptoms of this disorder are clearly traceable to impaired condition of the sympathetic, and the results recorded from belladonna, e.g., by Dr. R. T. Smith, are very remarkable. In two severe cases, one of which had lasted more than twelve months without marked relief, 5 minims given hourly relieved all the symptoms in a few days, lessening the sweating and restlessness, as well as the palpitation and dyspnœa (Lancet, i., 1874). Such results cannot, however, be depended upon,- - digitalis will usually answer better.

Diabetes Insipidus.-For some years I have prescribed belladonna with excellent results, in cases marked by the secretion of an immoderate quantity of pale non-saccharine urine. The malady is commonly traced to sympathetic nerve disorder, often to mental anxiety, grief, ete. One instance may be mentioned: a lady of about thirty for many months was accustomed to pass 130 ounces in twenty-four hours; various remedies had been given without benefit. I ordered 1 minim of liquor atropia twice daily, together with a more generous diet; in about a fortnight improvement set in, and the amount of water was soon lessened to 60 ounces. Dr. Murrell has recorded the case of a boy of fifteen passing about 5 pints daily, who benefited for a time at least by belladonna tincture in doses increasing from 5 to 30 minims; he relapsed, however, on its omission, and afterwards improved equally well with ergot. I have tried the tincture fairly in diabetes mellitus, but without any good result.

Profuse Sweating is a condition allied somewhat to those last mentioned, and its control by belladonna is a therapeutical fact now well known. Bartholow was one of the first to draw attention to this (Prize Essay, 1868), and he recommended $\frac{1}{10}$ grain of atropia at bedtime in the night-sweats of phthisis. He found the use of the remedy also markedly improre the general state; and the same observation has been made by Dr. M. Fothergill (Practitioner, vol. xvii.). He considers "the advantage to be mainly from checking the loss of salts of the body."

For many years I had observed advantage to the general con- 
dition of phthisical patients from the use of belladonna, which I gave in the first place to relieve dry, irritative Iaryngeal cough: whilst it commonly did this, it also lessened sweating. without causing diarrhoa, and soothed nervous irritation.

Dr. Ringer found advantage from it not only in controlling the sweating of phthisis, but also that of debility, of rachitis in young children, and of various exceptional nerve-conditions. Dr. Murrell, with $\frac{1}{100}$ or $\frac{1}{200}$ grain given to sixty successive phthisical patients, found it relieve in about two-thirds of the cases; sometimes it did not produce this effect until the following night, and at other times its influence for good began at once, and extended over several nights; unpleasant dryness of throat may accompany its action. Dr. Fothergill, however, advocates larger doses, stating that they have no evil result. $\mathrm{He}$ attributes the proportion of failures above given to the smallness of the dose, and recommended (by the mouth) $\frac{1}{\frac{T}{5}}$ to $\frac{1}{\bar{\partial} v}$, or, if necessary, $\frac{1}{25}$ grain once daily at bedtime; of course such a dose as this must be reached very gradually, and only exceptionally.

Vulpian speaks well of a pill made with $\frac{1}{2}$ milligramme (about $\frac{1}{140}$ grain) of the sulphate, taken two or three hours before the expected occurrence of the sweat; if more than one pill is required, one or two may be taken at intervals of two hours, beginning six or four hours before the symptom. He advises such treatiment for at least a fortnight-not longer. Homatropin has recently been commended as preferable for night-sweating, and 1 t-grain pill is said to do good without any unpleasant effects on the throat, etc. (Lancet, ii., 1882). The local application of liniment or tincture of belladonna is often useful for the same purpose. Nairne recommends about 2 drachms of the latter with an equal quantity of whiskey as a good and sufficient application for the whole body (B. M. J., i., 1878). For perspiring feet or hands the local application of belladonna is also good.

Urticaria, resulting, as it does, from a partial paresis of superficial nerves and the vessels supplied by them, has been successfully treated by belladonna (Dr. L. C. Smith, Record, 1880). I have also seen relief from it, especially in the case of children, and Bartholow includes cutaneous neuroses, 
prurigo, herpes zoster, erythema, and even eczema, in the list of skin disorders for which the remedy is available. It is, however, more commonly used as an adjunct than alone in such conditions.

Erysipelas.-In this disorder there is much evidence of the value of belladonna; it is specially suitable when the areolar tissue is not much implicated, and the epidermis of a deep-red hue, but not vesicated, and the accompanying heat, pain, swelling, and constitutional disturbance considerable, with a quick, full, but soft and compressible pulse.

In true phlegmonous erysipelas it may also prove of service, but cannot be depended upon. When, however, there seems to be a transference of the disorder to the nerve-centres, or at least when symptoms indicating cerebral congestion arise during the attack, then belladonna or atropia proves very valuable, just as it does in congestion arising from other causes.

I have generally ordered 1 minim of liquor atropiæ two or three times daily, or 5 minims of tincture of belladonna every 2 hours for five or six doses, then less frequently; painting also the extract or glycerine and extract ( 1 in 4 ) once daily over the affected part. In one man with idiopathic erysipelas, apparently from exposure, affecting the head and face very severely, and causing high fever, delirium, with tendency to coma, and prostration so great that life was despaired of, this treatment appeared to relieve the bad symptoms within twenty-four hours, and he made a good recovery. I have seen similar results in a sufficient number of cases to convince me that they may be credited to the remedy. It may be remembered that Liston esteemed it highly, and prescribed it constantly in this disorder.

Lymphangeitis.-Belladonna given internally and applied locally is useful in this affection, as well as in adenitis.

In Severe Burns, Mr. Hutchinson speaks favourably of the constitutional influence of similar treatment (Med. Times, i., 1864).

Nephritis-Pneumonia.-Dr. Harley has spoken highly of the value of belladonna in these inflammations. In the milder form of the latter, and in such cases as scarcely 
pass beyond a congestive condition, I have certainly seen advantage from it, and constantly use it.

Nephritic and Hepatic Colic, caused by the passage of calculi, has several times within my experience been immediately relieved by the hypodermic injection of $\frac{1}{80}$ to $\frac{1}{60}$ grain of atropia: it removes the nauser, vomiting, etc., relaxes spasm, and enables the calculi to pass.

In "chronic albuminous nephritis," Dr. Wood found no advantage from it.

Specific Fevers.-Not only in inflammations, but in some forms of fever, belladonna is of great service. In the early stage, e.g., of typhus, with delirium, sleeplessness, painful sensitiveness to light and sound, etc., I have often seen it relieve and apparently lessen the full development of such symptoms. When the tongue is red or brown, dry or cracked, small doses have the power of causing it to become moist, as remarked by Dr. J. Harley. (I have had many opportunities of observing this, and can quite endorse his statement.) In later stages, when there is partial coma, and other evidence of cerebral congestion of moderate severity, also whenever there is retention of urine, the same treatment is valuable.

Dr. Graves originally advised the drug in cases where the pupil was markedly contracted, and opium contra-indicated, and he reported remarkable benefit, but generally combined it with antimony or musk.

Dr. B. Kelly, also, of Dublin-where, unfortunately, a large experience of typhus and typhoid fevers may always be obtained, - cannot speak highly enough of the good results of belladonna treatment, and seems to consider it directly antidotal. After waiting for the full development of the disease, he commenced with 20 - to 25 -minim doses every four hours, and continued them for about a fortnight. This seemed to do everything one could wish-to quiet the nervous system, moderate the action of the bowels, and diminish unfavourable chest-symptoms. The results are, indeed, described as so invariably good that the observer may reasonably be considered too sanguine in his estimate of the remedy. He has seen no toxic effects from the dose named, only dilatation of prpil and some dryness of throat. During the treatment, stimulants were 
interdicted, and soup, millk, and arrowroot given in regulated quantity (Med. Times, i., 1870).

Without being able to speak so enthusiastically, I can say that I have found it really useful in many of the symptoms; thus, in the excited conditions, in congestive lung-disorder, and in retention and even suppression of urine, and again in paralytic relaxation of the sphincters dependent on central congestion, it is specially indicated. Dr. Harley has reported more than 200 cases of typhus and typhoid well treated with 15-minim doses of tincture every four or six hours.

In Scarlet Fever many practitioners (e.g., Wilson, Gardner, Green) have expressed confidence in belladonna. I have myself used it largely, but more in the milder than in the severe form. In my experience, the majority of cases in which it was used early, and continued through the exanthematous stage, have run a mild course free from complication. I therefore consider that it has exerted a favourable influence, especially in controlling the throat-symptoms. In very severe cases, or where unhealthy conditions of drainage, etc., have existed, then other remedies are much more effective. Whether it has the power not only of relieving, but of actually preventing an attack of this fever, has been much disputed; but although Stillé (ed. 1868) considers it so far established that every one should hate at least the benefit of the doubt, the balance of modern opinion. is against any prophylactic power. In the well-known report of Dr. Balfour, an account is given of 151 boys in a school where an epidemic of the fever occurred. There was fair evidence that this number had not had the disorder, and to seventy-six of them he gave the drug, whilst to seventy-five he gave none, with the result that, of both sets, two only were attacked (Edin. Med. Journ. Sept., 1849).

Some remarkable facts, giving more support to the hypothesis, might be related, and at one time I was almost convinced of its truth, and acted upon it so far as to myself take the tincture when attending many cases. It is true that I have known the malady caught by some who were taking the drug, but I believe that they were not under its physiological influence. Anything short of this, as evidenced by throat-dryness, etc., is quite inefficient; but when such effect is produced, it offer's 
some prospect--though I do not wish to speak positively-of counteracting the fever-poison. Thus, in one family, a mother and child were ill with scarlet fever, and there were five other young children in the house. These latter I kept in distant rooms for two or three days, until their throats became dry and the pupils dilated under the use of the medicine. They were then allowed to communicate freely with the two invalids, but none of them took the infection. The treatment was continued until the patients were able to leave the house for change of air. I have had many similar instances; sometimes a red rash from belladonna would simulate the fever eruption, but it would disappear on omitting the remedy. The continuous treatment is, however, disagreeable, and latterly I have discontinued it in favour of the external use of carbolic acid and other remedies for disinfection of rooms and of the air.

In Small-pox, Hitchman and others have strongly commended belladonna (B. M. J., i., 1879), tracing its good effect to an influence on the sympathetic.

Dengue.-Sir J. Fayrer has recorded that belladonna in 10to 15-minim doses, given hourly for several doses, has afforded much relief in this form of fever (Practitioner, vol. xvii.). External applications of the same with chloroform, rubbed into the back and joints, also relieve the pain and restlessness.

In rheumatic fever, Harley found it very useful.

Iritis and Eye Diseases.-In iritis I am accustomed to order the tincture internally in 5-minim doses every three hours, in addition to the local use of a collyrium containing about 1 drachm of extract to 4 ounces of water. This treatment not only dilates the pupil, and so prevents or breaks down recentlyformed adhesions between the iris and the lens capsule, but also tends to subdue inflammation, and thus to cure iritis in a twofold manner.

The local application of atropia in gelatine dises, or in solution, is now the more usual method, and is of great importance in ophthalmic practice. It is employed not only in iritis, but in corneitis and ulcer of the cornea, in phlyctenular ophthalmia, and many other affections of the eye. By retracting the iris in cases of perforating ulcers' of the cornea, it tends to keep it away from the inflamed parts, and may prevent 
its prolapse ; and in most of the affections referred to it lessens the pain, photophobia, and spasm of lids. It is also constantly employed to dilate the pupil for ophthalmoscopic examinations of the lens and deeper structures of the eye, as well as to paralyse the ciliary muscle in estimating errors of refraction of the eye, such as hypermetropia, myopia, and astigmatism, and to overcome the spasm of the muscles so frequently met with in these affections. With regard to the mode of using solutions, it may be noted that pure atropia being little soluble, the sulphate is commonly preferred, and the P.B. solution of it contains 4 grains in the ounce: if a few drops of this be instilled into the eye, the pupil soon begins to dilate, and is at its widest in about twenty-five minutes; accommodation is then impaired, and near objects cannot be seen distinctly, but about an hour and a half is required for its complete paralysis. When a strong solution of atropia is employed, the power of accommodation begins to return in the course of three or four days, but often is not quite regained, nor is the pupil normal, for a fortnight afterwards. This may be a serious inconvenience, and therefore a weaker solution- $\frac{1}{20}$ grain in the ounce-is preferred when we wish simply to examine the fundus of the eye with the ophthalmoscope: the effects of this solution pass off more quickly.

The hydrobromate of homatropin has recently been recommended for the above purpose by $\mathrm{Mr}$. Tweedy, and has the great advantage that its effects are more transient (Lancet, i., 1880). A solution of 4 grains to the ounce dilates the pupil widely and fixedly in fifteen to twenty minutes, and normal conditions are regained in two or three days; it is also non-irritant.

Atropia in any form should be carefully avoided in all cases where there is increased tension of the globe of the eye; it may unquestionably convert a subacute into an acute gilaucoma, and it is said that this disorder may be induced by its prolonged use in iritis. Toxic symptoms occasionally, though rarely, occur from absorption from the conjunctival surface, or from the nasal passages; thus, Ogilvy, of Leeds, records two such cases (the only ones out of a very large number treated): in one, a man of seventy, after an operation for cataract, solutions of 
2 to 4 , and later of 10 grains to the ounce were used, and after a few applications of the last (the quantity used being equal to about 1 grain) developed dry throat, dilatation of opposite pupil, delirium, and temporary paralysis. Another, aged fifty-nine, after three applications of a 2-grain solution felt giddy and sick, and later had violent vomiting and extreme collapse, requiring free stimulation for recovery. Mr. C. Macnamara informs me that he has seen two cases in which patients threw themselves from windows and sustained serious injuries when under the influence of atropia locally used for ophthalmic purposes.

I have myself seen vomiting, headache, and much prostration follow needle operations when the liquor atropiæ sulphatis B.P. had been freely used every two hours for a day. Mr. Cowell says that it is not uncommon.

(It is important that homatropin should not be confounded with amygdalate of tropin, from which it is prepared, for this latter does not possess mydriatic powers.)

PREPARATIONS AND DOSE.-Emplastrum. Extractum: dose, $\frac{1}{6}$ to $1 \mathrm{gr}$. Tinctura (1 part in 20 of proof spirit): dose, 5 to $20 \mathrm{~min}$. Unguentum (1 part extract, $5 \frac{1}{2}$ lard).

Liquor atropice ( $4 \mathrm{gr}$. to the ounce). If caustic alkali be mixed with it, atropia loses the power of clilating the pupil - a fact first indicated by Dr. Garrod and Professor Liebig.

Atropire sulphas : dose, $\frac{1}{100}$ gr., cautiously increased to $\frac{1}{30}$ gr., and in special cases even to $\frac{1}{10} \mathrm{gr}$.

Liquor atropice sulphatis ( $4 \mathrm{gr}$. to the oz., i.e., $1 \mathrm{~min} .=\frac{1}{120} \mathrm{gr}$.). The tissue-paper and tablets of gelatine now commonly used by oculists are prepared with the sulphate of atropia in such a way as to contain $\frac{1}{250} \mathrm{gr}$., and also $\frac{1}{5000}$ gr. of the salt.

Adulterations.-The leaves of the Solanum nigrum are sometimes substituted for those of belladonna. They are smaller than the latter; obtuse instead of acuminate; and have a bluntly-tonthed instead of an entire, margin. 


\section{DATURA STRAMONIUM. \\ (Stramonium, or Thorn - Apple.)}

DESCRIPTION.-An annual of coarse and weedy habit, rising to the height of 3 or 4 feet, and producing at its upper part many irregular and diffuse branches. The leaves are alternate, ovate, acute, several inches long, sinuated, coarsely toothed, and provided with rather long petioles. The large white flowers come out singly in the axils of the branches and principal branchlets. Individually they are bell-shaped, with five spreading lobes extended into long points; the ovary ripens into a leathery cápsule the size of a walnut, and densely clothed with erect pungent prickles, whence the vernacular name of "thorn-apple." When mature the capsule opens by four concave valves, which separate from the placental portion in the centre, and liberate numerous flat, circular, and blackish seeds.

ACTIVE INGREDIENTS.-The active principle of stramonium is the alkaloid daturin $\left(\mathrm{C}_{17} \mathrm{H}_{23} \mathrm{NO}_{3}\right)$, which, as already mentioned, is identical in formula and in nearly all physical and chemical characters-e.g., in solubility and melting-point -with atropia (F. \& H.); but according to Schroff it is about twice as strong. The seeds yield only about $\frac{1}{10}$ per cent., the leaves and roots a still smaller proportion. It exists in combination with malic acid. Trommsdorff isolated a white tasteless powder, which he termed stramonin; it is insoluble in water, soluble in oils, but has no recoguized physiological effects. The seeds contain also a bland fixed oil, mucilage, resin, etc.

Physiological Action. - Nervous System. - The effects produced on this system are similar in kind to those of belladonna and henbane, with perhaps more tendency to delirium of fatuous and absurd charncter.

Its name, "Jamestown weed," it obtained from the strange 
antics of a party of soldiers there, who ate the herb for salad, "and turned natural fools upon it for several days, blowing up feathers in the air, grinning like monkeys," etc. Both in Malabar and in India, thieves have learnt to drug with datura the food of their victims, so that they lose their senses and sit laughing while they are robbed; and Dr. Chevers records cases where people have been kept "fatuous" for several months, with such an object, for instance, as to prevent their becoming Christians. One missionary, after eating food in which some seeds of it had been cooked, "went off into chattering delirium, and first played strange antics, then became stupid, with dull and heavy eyes, and 'like a different being' for many hours" (Med.-Chir. Rev., i., 1872).

Such effects are not so marked from taking the English herb; thus, a man of sixty-nine drank, when fasting, an infusion made with three of the fruits, and soon afterwards felt giddy. On rising to walk he staggered as if drunk, and "feared he should lose his senses." In the course of half an hour he began to falter in his speech, and then became insensible and restless, muttering to himself; later, whilst kneeling, he stretched out his hands as if in search of something, then seemed partly paralysed, and became quiet; in a few hours afterwards "he raged furiously, requiring two persons to hold him "; but by evening became sensible, and recovered. Mr. Duffin has recorded the case of his daughter, aged two years, who swallowed some of the seeds. After showing irritability of temper and signs of itchiness of skin, she became wildly delirious, striking and biting at imaginary objects of terror; within two hours and a half the voice was lost, she had a croupy barking cough, and was unable to swallow-in fact, the symptoms resembled those of hydrophobia, and only the vomiting of some seeds cleared up the case. She afterwards became insensible, suffered from spasm and convulsion, and finally died collapsed in twenty-four hours. The pharynx and larynx were found greatly congested. A child of two and a half years, who ate a quantity of the seeds, developed "extraordinary mirthfulness_laughed, screamed, and sang alternately." In a girl, attended by Boerhaave after an overdose of the drug (in powder), there occurred delirium, nymphomania, aphonia, tremor, convulsion, tetanic spasm, and 
coma. A woman of thirty-six, who took two teaspoonfuls of an infusion of the leaves, was attacked within ten minutes in a manner very similar to a case already mentioned-with giddiness, dimness of vision, and fainting; in two hours she became insensible, and "all the muscles were convulsed" (Marsh, quoted by Taylor). According to Orfila, datura excites the brain more strongly than belladonna, and causes more violent general action. Post mortem the cerebral vessels have been found turgid with blood, and extravasation has taken place. The pupils are but slightly and temporarily affected by small doses, but commonly fixed and dilated under toxic quantities, the whole eye becoming prominent. Sigmund noted that objects appeared with rainbow tints under the influence of the drug. Hearing is impaired.

Circulatory System.-Stramonium in moderate doses does not specially affect the pulse, but poisonous amounts soon depress the circulation, as evidenced by fainting and prostration. The pulse becomes small and quick, and later on almost imperceptible.

Oulmont and Laurent hold that the alkaloid acts specially on the sympathetic supply to the thoracic viscera, leading to intermittence and ultimate arrest of the heart's action; respiration is at first accelerated, but the temperature usually remains normal.

Digestive System.--Stramonium causes thirst and dryness of the mouth and fauces by arresting the secretion of saliva; also difficulty of deglutition, nausea, and sometimes relaxation of the bowels, not constipation.

Cutaneous System.-The skin may be affected by this drug as it is sometimes by belladonna. In a boy aged six years, under its influence, I have seen vivid redness develop over the whole body, and the same in a girl of twelve, and in four adults. In all instances the redness came on soon after the administration of the stramonium.

In toxic cases the face is commonly described as swollen, flushed or purple, or " of intense scarlet redness" ; in one roung child " the face, neck, and chest were covered with small, shining; star-shaped petechire," which disappeared in about a week.

There is frequently a general itching of the skin, and in several recorded cases where paralysis was present the affected parts were covered with cold sweat (Orfila). 
Therapeutical Action.-Extemal.-Abscess.-The fresh leaves of stramonium made into a cataplasm, and applied to the part, have given relief to the pain of abscess, and are also of service in dispersing indurated collections of milk in the breasts of nursing women.

Hæmorrhoids. - I have many times found an ointment prepared from the fresh leaves, or, as a matter of convenience, made with the extract ( $\frac{1}{2}$ to 1 drachm in $\frac{1}{2}$ ounce of lead or zinc ointment), very serviceable for relieving the pain of external hæmorrhoids.

THERAPEUTICAL ACTION.-Intemal.-Pain.-The internal administration of the drug is useful in several forms of pain.

Gubler speaks highly of it in "congestive neuralgias," and I have known it relieve tic-douloureux and sciatica when given in $\frac{1}{4}$ or $\frac{1}{2}$-grain doses of extract every eight or twelve hours. Its action, however, requires watching, and its exhibition should be suspended if it does not relieve after a few days' trial.

Dr. Marcet (1826) also found it of service in such disorders, and, although it sometimes excited disagreeable nervous sensations alarming to the patient, he found them exceptional and unimportant.

Chronic Rheumatism.-Dr. Sigmund quotes several American physicians who speak very highly of stramonium in this disorder, "considering it less likely to disappoint than any other drug"; he himself prescribed it internally and externally, and found it "an important and valuable remedial agent": but it has not yet had a fair trial in this country.

Asthma.-It is in this disorder, whether connected with bronchial affection or purely spasmodic in character, that the drug is at present most employed, and preferably in the form of smoke.

Its original introduction seems to have been from Indian practice (1802), but Bigelow in his Medical Botany mentions its common domestic use in America, especially for the more purely spasmodic cases, the seeds being smoked like tobreco; it does not answer well, however, for plethoric or intemperate people, nor is it safe when the dyspnoa depends on pleural 
effusion or advanced organic disease. The possible occurrence of serious symptoms from smoking stramonium should be remembered. If incoherent talking, flushed face, or alteration of vision supervene, it should be at once discontinued. The relief given by it to the asthmatic paroxysm in suitable cases is commonly marked and immediate, so that if this be not experienced the smoking need not be long continued.

The dried leaves of this plant, and of its allied species the datura tatula, are of about equal efficacy, and I have known many asthmatic and emphysematous patients derive much benefit from smoking them at the commencement of a paroxysm of "wheezing." It seems, however, that the seeds act with greater promptitude and certainty, and Dr. H. Salter suggested that the leaves should be soaked in an infusion of the latter: they should be fresh, for they lose power after exposure to air and light (Lancet, i., 1857, and ii., 1863).

Datura is a usual ingredient also of asthma pastilles and powders for burning, and a formula containing 2 drachms of the powdered leaves with 1 of nitre, and a scruple of opium is much commended (B. M. J., i., 1879). This is said to be useful in various forms of dyspnoea, notably in that produced by the pressure of a goitre.

The drug is also of some service given internally in such cases. Williams recommended it equally with belladonna; and I have seen speedy relief in several severe cases from $\frac{1}{4}$-grain doses of the extract taken at an interval of three or four hours, and then once or twice daily.

Headland advised 10-minim doses of the tincture in chronic bronchial affections with dyspnoea connected with "dryness of the bronchial tubes" (Lancet, i., 1866); others have combined it with tincture of conium, ether, and alkaline carbonates (B. M. J., ii., 1878).

Epilepsy.-Baron Störck, who was the first to show the value of stramonium, recommended it specially for this and other nerve disorders; Ives and American physicians have also used it; and $I$ have met with some evidence in its farour. One patient, a gentleman aged thirty-nine, who had suffered for many years, and whose mind had become somewhat affected, found himself at one time much relieved by bromides, but later on they 
seened to lose their good effect, though given in largely increased doses. I then prescribed extract of stramonium in $\frac{1}{2}$-grain doses twice daily, increasing gradually to 2 grains, and with the effect of decidedly checking the severity and frequency of the attacks, so that while taking the drug he did not get them oftener than once in six or eight weeks, in place of three to five each week. Against the probability of this being only a coincidence, it should be stated that the fits tend to recur as before whenever the patient is left for eight or ten days without the medicine.

Mania.-Although not so generally used as hyoseyamus, stramonium has been given with advantage in various forms of this disorder. Cullen says, "I believe that there is no drug in the list of the materia medica which warrants a greater degree of confidence than does stramonium in the greater number of maniacal cases." Dr. Davey and many others have demonstrated its power of lessening irritation and producing tranquil sleep. My own experience of it in puerperal mania is favourable; I have used it when the delirium was wild and furious, but intermittent, accompanied with general restlessness and suicidal or destructive tendency, and have found it soothe and promote sleep, whilst the symptoms dependent upon changes in the secretion of milk and of the lochial discharge improved concurrently: from $\frac{1}{4}$ to $\frac{1}{2}$ grain of the extract of stramonium should be administered every six to twelve hours until relief is obtained. It must not be forgotten in these cases to watch the secretions, to use poultices, hot and soothing fomentations, enemata, or gentle laxatives, should occasion require; and, above all, we must not neglect to sustain the patient's sinking powers by means of nutritious and stimulating diet.

Nymphomania. - Professor Wendt (Rust's Magazine) considered stramonium useful in this affection, especially when brought on by undue indulgence; and I think it proves serviceable in cases attended by mental depression, but unconnected with organic disease.

In Rabies it has been given in large doses, so as to secure its full physiological effect, and is said to have been successfully used in India. 
In conclusion, I may remind English readers that, although stramonium is not much used at present, Trousseau and Pidoux held that it was more active than belladonna, and could effect quite as much as that medicine.

PREPaRATIONS AND Dose.-Extractum: dose, $\frac{1}{4}$ to $\frac{1}{2}$ gr. Tincture : dose, 5 to $30 \mathrm{~min}$. (Both from the ripe seeds.) The dried leaves and stems are used for smoking, and in pastilles.

\section{HYOSCYAMUS NIGER.}

\section{(Hexbane-Hyoscyamus.)}

DESCRIPTION.-An herbaceous annual, or more usually a biennial, found wild in most parts of Europe, and not rare in England. It grows principally in waste places, preferring calcareous and sandy soils, especially in the neighbourhood of the sea. The somewhat ligneous stems are branched, about 2 feet high, clothed with large sinuate leaves, usually decurrent. Every portion of the plant, excepting the corolla, is downy, viscid, and possessed of a heavy, offensive odour. The bell-shaped light-buff-coloured flowers appear at first in a dense terminal cluster, but by degrees the stem elongates; so that by the time the blooming is finished there is a long unilateral spike, not unlike that of the digitalis. The oraries ripen into capsules, which are concealed within the accrescent calices, and open, when mature, by transverse dehiscence, allowing the escape of numerous minute seeds, which are wrinkled, reniform, and greyish. The flowers commence to expand in July, and the fruit is ripe by October.

ACTIVE INGREDIENTS.-Of these hyoscyamin or hyoscyamia is the most important; it was first obtained by Geiger and Hesse in 1833, and in 1871 was isolated by Höhn from the seeds, which contain more than the leaves (though only about 0.05 per cent.) : more can be obtained from the fresh than from 
the dried plant. When pure and crystallized slowly, it occurs in stellate groups of silky, odourless needles, generally translucent; from chloroform it crystallizes in tables; from ether it separates in an amorphous condition: it has a sharp, unpleasant taste; is almost insoluble in cold, more soluble in hot water (which, however, is said to render it almost inert, owing to its instability); soluble in spirit, ether, chloroform, and dilute acids. When pure it is permanent in air: the impure form, which is amorphous, or occurs as an oily liquid turning brown on exposure, is hygroscopic and more soluble in water, and has a tobacco-like odour. Purified hyoscyamin is said to be isomeric with atropin, $\mathrm{C}_{17} \mathrm{H}_{23} \mathrm{NO}_{3}$ (I adenburg), but not identical with it, since it forms smaller and less perfect crystals than the latter drug, melts at a lower point $\left(108.5^{\circ} \mathrm{C}\right.$., instead of $\left.113.5^{\circ}\right)$, and the respective derivatives differ in properties: it gives a reaction with corrosive sublimate like that of atropia. Daturin and duboisin are, however, considered identical with hyoscyamin by this observer (Pharm. Journ., March, 1880).

By boiling hyoscyamin with solution of baryta it is split up into hyoscin, a volatile alkaloid, and hyoscinic acid, a crystallizable substance haring an odour of benzoic acid. According to Ladenburg the former is isomeric with tropin, $\mathrm{C}_{8} \mathrm{H}_{15} \mathrm{NO}$, and the acid with atropic acid, $\mathrm{C}_{9} \mathrm{H}_{8} \mathrm{O}_{2}$. They are not, however, precisely alike in properties.

Besides these ingredients, an empyreumatic oil has been obtained from the destructive distillation of the plant: it is said to be identical with a similar oil from digitalis, and is a powerful narcotic. The extract of henbane is rich in nitrate of potash and other inorganic salts. Prideaux speaks highly of the extract generally prepared by Merck, when dark brown or greenish in colour, and of mousy odour: when light, and smelling like gas-liquor, it did not act well, and this was preparerl from the root of the plant, the other mainly from leaves (Lancet, ii., 1878). Merck's extract, when good, seems to be the most manageable preparation for clinical use; he has recently prepared a crystalline form.

Hyroscyamin must not be confounded with an impure resinoid body termed (by the Americans) hyoscyamia, which contains the tme alkalnicl, but is uncertain in strength. 
ABSORPTION AND ELIMINATION.-The active principle of the drug is readily absorbed by the stomach, the skin, the rectum, and other parts. Its effects have been developed after application to open wounds as well as after injection into the bowel. It is eliminated mainly by the kidneys; Harley found it in the urine within twenty-two minutes after hypodermic injection of the alkaloid.

PHysiological ACTion.-External.-When applied to painful parts in lotion or cataplasm, it exerts some soothing anodyne power. Applied to the eye itself, it dilates the pupil, although when only painted round, it often fails to do so. (Pearse).

Physiological Action.-Intemal.-This much resembles what has been already described under Belladonna, and the alkaloids atropia and hyoscyamin have been considered identical. The observations of Ladenburg, above quoted, show that although isomeric they are not really the same in their chemical and physical properties. Laurent found them act alike on the muscular and nervous systems, and Heilman reported that their derivatives showed similar physiological effects; still it is certain that as remedies the two drugs do not act precisely in the same manner.

Nervous System.-According to Dr. J. Harley, sulphate of hyoscyamin injected under the skin in a dose too small to produce dryness of throat, will yet produce giddiness, somnolence, and dilatation of pupil: a somewhat larger amount, quickening the circulation, will intensify these symptoms, and excite flushing of the face and injection of conjunctiva. Compared with belladonna, he considers that it acts more on the cerebrum, and rather less on the sympathetic system; in fact, any stimulant effect on this latter is only evident in man after large doses, and is more transient and less powerful than that of belladonna.

From a subcutaneous dose of $\frac{1}{(i)}$ grain of the crystalline alkaloid, Mr. J. S. Pearse observed giddiness and headache, followed by sleep, unless the aching were too severe. Unstendiness of gait, like that caused by atropia, did not occur, except 
in one patient already ataxic. A solution painted near the eye clid not dilate the pupil, but one containing $\frac{1}{40}$ grain applied to the conjunctiva did so fully in an averag'e time of fifteen minutes. The effect was not so rapidly produced, but lasted longer than that of atropia (Lancet, ii., 1876).

The symptoms recorded by Leared, from an injection of $\frac{1}{40}$ grain, were much more severe. In ten minutes giddiness came on, with a painful sense of compression about the vertex; in half an hour, sickness, and later, delirium, with incoherent talking: the pupils were dilated, and the sense of sight curiously altered, so that objects seemed nearer and of different size than in reality. Thus, the patient grasped wildly at something he thought to be within his reach, but which proved to be a bedpost four feet distant; and a cup being offered him, he always grasped at a nearer point than it really was; he also saw imaginary insects about him. The severe symptoms lasted about four hours, and did not quite pass away for twenty-four (Lancet, i., 1879). Similar delusions as to size and distance of objects occurred in a lady who took $\frac{1}{6}$ grain in pill; the pills in the box seemed to her of different sizes, though really alike (Chubb, B. M. J., ii., 1878). These effects may be ascribed partly to paralysis of accommodation, partly to the action of the drug on the brain.

Coghill, giving $\frac{1}{2}$ grain of hyoseyamin by the mouth at bedtime to a restless patient, observed within an hour involuntary twitching of limbs, general restlessness, and distress, with inability to speak or swallow (Lancet, ii., 1878).

Many observations have been made with Merck's extract, which, when fresh, is very active, and rather more manageable, apparently, than the pure alkaloid. Dr. R. Lawson took 2 grains of it, and amongst other symptoms found the power of voluntary motion impaired, and drowsiness, with interrupted sleep; afterwards, incoherent talking, with delusion and partial aphasia, lasting for some hours (Lancet, ii., 1878). He conclurlexl that the drug could produce "a subdued form of mania, with paresis of voluntary muscular power, ending in quiet sleep" (W. Riding Asylum Reports, vol. v.). Síguin (using the crystalline form) reported similnr results-hallucination, paresis, and sleep-together with dysuria and retention of urime 
(N.Y. Record, March, 1880). Dr. Bacon found the extract " a powerful hypnotic and sedative," and $\frac{1}{2}$ to $\frac{3}{4}$ grain rendered a maniacal patient helpless and comatose in half an hour (B. M. J., ii., 1880). Pitha, injecting $\frac{1}{10}$ grain (crystal) under his own skin, experienced "frightful effects"-meaning, apparently, delirium and "swooning" (Med. Times, i., 1875).

In the action of the ordinary extract and tincture of the drug, in full doses, we may recognize similar effects, differing only in degree. Dr. Cotter took 2 to 3 grains of the former, and experienced a burning glow all over, parching of mouth, dilatation of pupils, protrusion of eyeballs, mental exhilaration, and afterwards somnolence (Med. Times, ii., 1878); and a man, after two pills of colocynth and henbane (B.P.), got dryness of throat and drawing of the mouth, numbness of the arm, giddiness, partial blindness, and much sense of weakness-symptoms which did not disperse until purging occurred four hours afterwards (B. M. J., i., 1879):-in this case some mistake occurred or idiosyncrasy probably existed.

More marked were the symptoms in a man aged sixty, who took by mistake about 6 drachms of tincture in one dose; three hours afterwards he became excited, wandering about the house, muttering to himself, touching everything as if suspicious; he then became drowsy, and, with the idea of relieving him, another dose of the same medicine, containing about $1 \frac{1}{2}$ drachm. of tincture, was administered. Soon afterwards violent delirium developed-he imagined policemen entering the house; his face flushed, the pupils dilated moderately, his hands were in continual motion, and though exceedingly restless he was almost overcome by drowsiness; after recovery all recollection of that time was lost (Lancet, ii., 1874).

In another case, of a woman aged thirty-four, about 1 oumce of tincture was taken by mistake for a "black clraught" at 5 a.m. : soon after, she complained of pricking and heat in the extremities, dryness of mouth, giddiness, and loss of power in the legs; there was a purplish rash and the face wns swollen: four hours afterwards she was almost insensible, the face was scarlet, the pupils very dilated; she was ordered tincture of galls and castor oil, and soon after a copious evacuntion occurred of golden colour and having a strong odour of the 
drug; power was lost in the extremities and sensibility diminished: at 4 p.m. delirium set in, with illusions, later rigors occurred, and finally collapse. It was six days before she regained the use of her legs, and the loss of memory continued for a long time (Lancet, ii., 1873).

In a man under my own observation, who had taken a large quantity of "henbane-tea," there was much drowsiness and prostration, with tremor of the whole body, muscular spasm, and even convulsion, with clenching of the thumbs, rolling of eyeballs, and muttering delirium, obscene in character; he complained of numbness and impaired power in the limbs, and later on as he began to recover, of pain which seemed to be neuralgic, in the hips and lower extremities. (It may be noted that henbanedelirium is commonly obscene in character.)

Symptoms allied to those described have also been reported from hyoscin, which Guauck represents as ten times more active, and approaching atropia in its powers-causing vertigo, sense of oppression, and intoxication or delirium, with unsteady gait, thirst, nausea, itching of skin, redness of face, and dilated pupils, respiration becoming laboured and the pulse retarded; $\frac{1}{60}$ grain by the mouth will cause such effects in greater or less degree, and more markedly when given by the skin. The fall in the pulse-rate he connects with irritation of terminal filaments of the vagus, an effect controlled by the tropic acid present in the complete drug (Centralb. f. Med. Wiss., No. 45, 1881; Record, 1882).

Circulatory System.-Laurent, experimenting with frog's, found the web-capillaries contract after touching with hyoscyamin or injecting it under the skin, and this equally whether the nerve-supply had been cut off or not: small doses he found to increase both pulse-rate and arterial tension; large ones equally raised the pulse, but lowered the tension: direct applicution of the drug to the heart, however, soon slowed its beating (Thèse de Paris, 1870). A small dose of the alkaloid causes slowing of the pulse in man, without loss of its force or volume (J. Harley). Séquin also reports slowing of pulse with increase of tension. A larger amount-enough to cause dryness of throat-iccelerates the pulse ten to twenty beats in the course of ter minutes, and, according to my observations, renclers it harder and fuller: such increase lasts about lialf an 
hour; an hour afterwards it falls rapidly to sixty or even forty per minute; $\frac{1}{60}$ grain causes flushing and increased quickness and fulness of pulse within five minutes, and lasting about one hour with an average increased frequency of eighteen per minute. Temperature is raised $\frac{1}{2}^{\circ}$ to $1^{\circ} \mathrm{F}$. (Pearse). (For effect of hyoscin see previous page.) In the majority of toxic cases flushing is marked and the pulse is quickened, and in later stages becomes small and intermittent, the heart's action being irregular.

After 2 grains of Merek's extract the pulse rose to 120 , and fell again in two hours' time. Lawson, who records this case, gives a caution against administering the drug to very weak persons, on account of such depressing after-effects. Bleeding from the nose $I$ have seen occur in five persons who were taking the extract or tincture for experimental purposes: in each case the face became dusky and appeared swollen. I have also known it induce hæmorrhage from the uterus.

Respiratory System.-When the full physiological action of the drug is developed the breathing is at first hurried and shallow, and the dryness of the air-passages causes a short spasmodic cough, most troublesome on lying down, also a sense of constriction about the throat, with tightness and oppression of the chest: later on the respiration sinks to its usual rate; it may become stertorous.

Digestive System.-Hyoseyamus lessens the salivary and gastric secretions, and causes dryness of mouth and fauces, as a constant symptom of its full action; there is a fœtid odour from the mouth, which, as well as the tongue, becomes coated with a whitish viscid mucus; loss of appetite and much thirst are usually experienced, also nausea or vomiting, burning sensation at the epigastric region, eructations and flatulent distension and rumbling through the bowels.

Some observers describe many of these symptoms as occurring almost constantly from its subcutaneous use (Gill, Practitioner, vol.xx.). Action of the bowels does not follow so usually as with belladonna, but henbane is commonly added to purgatives as an anodyne, and it certainly does not interfere with their action.

So much as 16 grains of extract given to animals does not 
notably affect the bilinry secretion, nor prevent the action of hepatic stimulants (Rutherford, B. M. J., i., 1879).

Cutaneous and Glandular System.-It would seem not so usual for a cutaneous rash to follow the use of henbane as of belladomna, but Dr. Cotter states that one hour after taking 2 grains of extract, a rash, which he could not distinguish from that of scarlatina, came out over his body. In Mr. White's case the rash is described as "purplish" (Lancet, ii., 1873).

I have myself observed a dusky-red eruption in a child that had eaten henbane root, and a similar rash was said to have apperred on the mother and two other children who had also taken it, and suffered from the usual characteristic symptoms of its action : partial desquarnation occurred in all the cases five or six days afterwards. I have in several instances, when giving extract of henbane for experimental purposes, traced to its continuous use the occurrence of small pustules in different parts of the body, and violent itching is not infrequent. The face is commonly, in toxic cases, described as dusky, sometimes the lips red and face dark; the whole skin is hot and dry, as a rule, but I have seen one case with profuse clammy perspiration. Hyoscyamin has the power of checking perspiration, like atropia, but is apparently not so active, since $\frac{1}{60}$ grain by the skin only equals in effect $\frac{1}{120}$ grain of atropia in this respect (J. S. Pearse, loc. cit.). Marked parching and dryuess of mouth, with swelling of tongue, and consequent difficulty of swallowing and of talking, are usual results of full doses.

In many instances there is no marked effect on the remal secretion. Pearse found the quantity, as a rule, not increased,if anything, lessened; and, as a primary effect, I believe this latter is the fact; it is accompanied by some irritability of the bladder. Later on I lave known profuse diuresis of limpid urine oceur-in the case, for instance, of the wonian above mentioned : in two of the children there was also enuresis, in one of them for one night, in another for three nights successively. This symptom dirl not occur in these children except when under the influence of the drug.

Tho SYNERGISTS and ANTAGONISTS are prnctically the same as stated under bolladoma. 
THERAPEUTICAL ACTION. - This is allied to that of belladonna, but of late years has been much more utilized in the treatment of mental disorders. The drug, or its alkaloid when well prepared, has proved an excellent sedative in cases of mental excitement, and when opium is inadmissible. It is well adapted for children.

Pain-Ulceration.-Locally, a cataplasm of the bruised leaves may be applied with advantage to congested mammary glands distended with milk, to painful hæmorrhoids, or to cancerous and strumous ulcerations. For toothache, the vapour of the seeds, inhaled as in smoking, has proved of service, but is a somewhat dangerous remedy.

Whooping-cough, etc.-I have treated many cases of this affection by preparations of henbane with much adrantage, the spasmodic attacks being greatly controlled by it: it has seemed to act better than belladonna when the attacks are markedly worse at night, for I think it soothes and promotes sleep better than that drug.

In many forms of spasmodic throat-cough of tickling irritative character, such as are commonly worse at night or on lying down, and depend partly on relaxed conditions of fauces, henbane will often prove useful.

Constipation.- Hyoscyamus does not seem to possess the same power as belladonna in relieving either this disorder or obstruction, and is used rather as an adjunct to purgatives than for any aperient properties of its own. Certainly it does not confine the bowels like opium usually does, but acts as an anodyne-lessens griping pain, and probably controls the cramping or irregular muscular action which may be induced by irritants such as colocynth.

Febricula.- In cases answering to this term, with dry slin, hard quick pulse, headache, restlessness, etc., henbane will often relieve the symptoms, but aconite is, as a rule, preferable.

Sunstroke, etc.-I have used hyoscyamus with ilecicled success in cases of mild sunstroke or hentstroke, where the patient has suffered in grenter or less degree from faintness, vertigo, headnche, and a sense of tightness across the forehead and chest, a quick and full pulse, sleeplessness, and much nervous imitability. In the convulsions of children, when 
brought about by undue exposure to the sun, I believe hyoscyamus to be a valuable remedy.

In the excitement of delirium tremens, in the delirium of typhus fere', and even when meningitis of subacute character is present, I have seen advantage from the drug or its alkaloid, although belladonna is to be preferred, as a rule, in such cases.

In mercurial tremor, and in that of paralysis agitens, Oulmont found the alkaloid highly useful. It acted best when given hypodermically, $\frac{1}{32}$ grain being employed at first daily, and the dose being gradually increased (Bull. de Thérap., Dec. $15,1872)$.

In a case reported by Dr. Hayes (Dublin)-of tremor in one arm following eighteen months after an injury to the shoulder$\frac{1}{16}$ grain of the alkaloid given in pill night and morning relieved the tremor after the third dose; but when $\frac{1}{8}$ grain was given, delirium set in for several hours, and afterwards paralysis of accommodation. After three days' interval, the drug was resumed in doses of $\frac{1}{32}$ grain three times daily; in two days the tremor was again better, but the eye symptoms produced were so unpleasant that the remedy could not be continued (B. M. J., ii., 1883).

Mental Disorder.-It is, however, by alienists that the therapeutical action of the drug on the nervous system has been most recently tried and verified. Dr. R. Lawson has advanced the hypothesis, that since it produces a form of mania (more or less subdued, according to dose), it may, suitably given, substitute such artificial brain-excitement for the real malady (acute mania), and when the drug-action subsides, the mental condition is found improved. He and others certainly adduce remarkable illustrations of its power: thus, one man aged fifty, in his third attack of recurrent mania, which had lasted for two years, when labouring under much excitement took $1 \frac{1}{2}$ grain of Werck's extract (by the mouth), and experienced for a time its full physiological effect, including profoumd sleep; next day he began to mend, and progressed steadily to recovery. Another man was in a condition of furions mania with delusions, smashing shutters, refusing food, and threatening violence: 1 grain was given after a sleepless night and persistent refusal to eat; " he slept soundly for twelve hours, and on waking took 
a good breakfast, and was free from every trace of excitement." He employed himself usefully for a month, when a visit from his wife produced a fresh outburst. Hyoscyamin was tried with but little effect; it was found that the preparation used had been kept in a warm place and exposed to air. On procuring a fresh supply of Merck's extract, 1 grain produced the same good result as before. Dr. Lawson notes the "potency and permanency of the action of individual doses," also that it is specially suitable for sthenic cases, and for refractory patients liable to outbreaks of excitement; it renders them helpless, impotent, and "silly," and they afterwards, by controlling themselves, endeavour to avoid its being used. With recent cases of acute mania-reduced as they often are to extreme prostration, and requiring to be artificially fed-caution must be exercised, since, although sleep will be induced, danger of serious exhaustion exists, and the passage of a stomach-tube is interfered with by increased throat-dryness.

It is in the chronic monomania of suspicion that some of the best results have been obtained. The malady often results from alcoholism, and its subdued and latent state may be alternated at any time by exacerbations with violent excitement from various causes, turning generally, howerer, on some fresh suspicion of supposed injury or conspiracy. In one case of about six months' duration " a draught of hot air from a flue suggested the delusion that the medical officers were fanning him with pernicious vapour, and he threatened them with extinction. . . . The consequent state of his mind was one of great agitation, leading to ungorernable temper. He had 1 grain of hyoscyamin, slept for about twelre hours, took his food well when awakened, and from that time to this (two years) has never expressed a delusion or shown the least tendency to violence." The effect was equally satisfactory and permanent in another case of the same charncter of ten years' duration (Practitioner, vol. xrii.). For the excitement of senile dementia it is not so suitable.

The formula used was 1 grain of hyoscyamin with s minims of ether and ' 4 minims of alcohol in 1 ounce of water' (Practitioner, vol. xvii., p. 176). In a later paper, Dr. Lawson reconmends a somewhat smaller dose (1) grain), but still remarks 
that the best results are obtained by single large doses: for hypodermic use he advises only one-third of the quantity given by the mouth. Prideaur has corroborated the preceding observations in another asylum, and reports the drug as "most beneficial in many forms of mania," and as having great power in abating mental excitement (Lancet, ii., 1878).

Gill also has recorded several striking illustrations of the same action (Practitioner, vol. xx.). He gave the same extract in smaller doses ( $\frac{3}{8}$ grain), but almost constantly observed vomiting and considerable depression-so that its benefits are by no means without drawback ; and Leared, Coghill, and others have proved this in the cases already noted.

Dr. Gray (N.Y. Lunatic Asylum) considers that in acute mania and melancholia with frenzy there is no remedy that so efficiently calms high nervous and muscular excitement, and brings about sufficient tranquillity for nourishment and rest; it acts, apparently, directly on the cerebral nerve-tissue, and in as marked a manner as opium relieves pain: of the crystalline form he gives by the mouth $\frac{1}{8}$ to $\frac{1}{2}$ grain, of the crude extract up to 1 grain. For simple restlessness and cerebral irritability and hysteria he gives $\frac{1}{60}$ to $\frac{1}{30}$ grain, sometimes with morphia (Med. Times, i., 1880). Dr. Bacon found Merck's extract a powerful sedative and hypnotic in $\frac{1}{2}$ to $\frac{3}{4}$-grain doses by the mouth, and used it with advantage in mania (B. M. J., ii., 1880).

Dr. J. Browne has recently reported several cases in which, certainly, temporary benefit was obtained from Merck's crystalline hyoscyamin, but with some drawbacks. One man, with chronic mania of eight years' duration, when very violent was given $\frac{1}{4}$ grain by the mouth at 8 a.m., he became quieter, but at 6 p.m. lapsed into a state of great prostration, which caused much anxiety : after rallying he slept well, and was not violent again. Other similar cases were given from $\frac{1}{30}$ to $\frac{1}{15}$ grain hypodermically once or twice daily, with. calming and soothing effect, though not cure. He concludes, from a two years' exjerience at the Naval Hospital, Yarmouth, that the hypolermic method of administration is the best; but "claims no curative action for the drug": only it coutrolled violence, 
and "checked the torrent of rushing ideas." He notes that a solution very readily decomposes if prepared by aid of heat (B. M. J., ii., 1882). Dr. Hurd (Michigan) finds amorphous hyoscyamin in doses of $\frac{1}{24}$ to $\frac{1}{2 v}$ grain control convulsive seizures and excitement; and several American physicians, whilst corroborating this, state that larger doses $\left(\frac{1}{12}\right.$ to $\frac{1}{10}$ grain) are required in mania than in melancholia (Amer. Journ. of Insanity, and Canada Med. and Surg. Journ., Sept., 1883). Mr. W. J. Simpson records a case in which $\frac{1}{20}$ grain produced a state of alarming collapse, similar to that seen by other observers, but after rallying from this the patient slept, and had no relapse. He concludes, however, that, as a rule, it did not do more than quiet patients, and required to be given periodically when a fresh outburst occurred; it did not stop the frequency of the attacks, it merely acted for the time being; the dose varied from $\frac{1}{40}$ to $\frac{1}{4}$ grain (B. M. J., ii., 1882).

Chirton also, recording a case of acute mania in a man aged fifty-eight, treated by doses of $\frac{1}{60}$ to $\frac{1}{40}$ grain of hyoscyamin, concluded that though it could induce sleep it led to no permanent benefit (B. M. J., i., 1883).

Puerperal Mania.-I have notes of seven cases that occurred in my practice many years ago, of young married women who shortly after their confinements suffered from "milk fever," or even more serious conditions; two had peritonitis, and one convulsions. The secretions were diminished, there was general pyrexia, with delirium, piercing cries, sleeplessness, and restlessness so extreme as to require constant watching. With our present resources bromide or chloral would probably be used, but at that time I depended upon henbane, giving the extract in 2-grain doses every one or two hours until relief was obtained, and I was well satisfied with its action.

Epilepsy.-There is some evidence of the ralue of the drug in this disorder. Harley specially mentions it, so also does Cotter (loc. cit.). Craig remarks that large doses are required for benefit (Lancet, ii., 1878). Paget, of Cambridge, alludes to a severe case much relieved by it after failure of other remedies (B. M. J., ii., 1880).

Dr. R. Lawson found it very valuable in epileptic excitement: 
one man, epileptic from birth, suicidal and dangerous, unrelieved br a prolonged course of bromide and ergot, was given, on one occrsion when seriously affected, $1 \frac{1}{2}$ grain of hyoscyamin by the mouth. In fifteen minutes he was asleep; "has not since then manifested a trace of excitement, and has been a cheerful ward-helper." In other epileptics good results were obtained, the number of attacks being diminished by it.

Chorea.-I consider henbane a very suitable medicine for the relief of this disorder, and amongst other evidence in support of its power may be quoted the cases of five adults treated by Dr. Oulmont with hyoscyamin in pill, commencing with $\frac{1}{40}$ grain twice daily, increased by degrees to $\frac{1}{3}$ grain for each dose; three of the patients were pregnant. Some throat-dryness and dilatation of pupils were produced, and then the drug was omitted, and the symptoms, which were in course of improvement, became worse again; he considered the drug efficacious in chronic cases (Bull. Gén. de Thérap., Août, 1876).

The same writer had previously related cases of spasm, convulsion, and tetanus relieved by the remedy, which was not, however, beneficial in ataxy (Lancet, ii., 1873).

The night-sweats of phthisis may be controlled by hyoscin, which was used by Fraentzel in the form of iodide; it proved not so generally effective as atropia, but acted well sometimes when that failed (Record, i., 1883).

PREPARATIONS AND Dose.-Extractum: dose, 3 to 10 gr., or more. (This extract is contained in pilula colocynthidis et hyoseyami.) Tincture: dose, 5 min. to $1 \mathrm{fl}$. dr., or upwards. Succus: dose, $\frac{1}{2}$ to $1 \mathrm{dr}$. (Ten drops of liquor potassa added to $1 \mathrm{dr}$. of the tincture of hyoscyamus destroy its active principle.)

Of hyoscyamin the dose must vary somewhat, since it acts differently with different people. In the present state of our knowledge it will be better to prescribe Merck's extract either in the amorphous or, preferably, in the crystalline form: of these, $1 \frac{1}{2}$ and even $1 \mathrm{gr}$. has caused unduly serious symptoms, so that $\frac{1}{2}$ gr. may be stated as a full dose by the mouth, and this may act very strongly (Coghill); it is better to commence with $\frac{1}{8} \mathrm{gr}$. 
Hypodermically, physiological effects are produced by $\frac{1}{60}$ gr. of hyoscyamia, much more markedly by $\frac{1}{40}$ gr. Many insane patients have required from $\frac{1}{20}$ to $\frac{1}{15} \mathrm{gr}$., but the latter dose requires careful watching, since it has sometimes produced symptoms of collapse.

If a large dose be decided upon, it should be given once daily until its full effect has been developed and observed; the smaller doses may be repeated once or twice daily, according to the case.

A formula adopted by several observers is-Hyoscyamin (crystalline), 1 gr.; glycerine and distilled water, āā 100 min.; dissolve without heat, and add carbolic acid (as preservative), 1 min. : each min. $=\frac{1}{200}$ gr. of alkaloid. A similar formula may be prepared with 4 grains to the ounce $=\frac{1}{15}$ gr. in 8 min., but it is not very stable (Dr. T. Browne). Of hyoscin $\frac{1}{60} \mathrm{gr}$. may be given by the mouth.

\section{NICOTIANA TABACUM.}

(TOBAcCo.)

DESCRIPTION.-The Virginian tobacco plant is an herba- ceous annual, 3 to 6 feet in height, with large pale-green, ovate, acuminate leaves, clothed with glandular hairs. The flowers, which grow in terminal panicles, have a five-cleft hairy calyx, and a rose-coloured, funnel-shaped, fire-cleft corolla, and produce capsules which discharge numerous seeds. The dried leaves-the officinal part-are from 6 to 20 inches long, 2 to 6 inches wide, oval or ovate-lanceolate, sometimes oborate, pointed at apex, with entire margin, and prominent midrib; they are thinner than when fresh, mottled, and friable.

ACTIVE INGREDIENTS.-Besides a large proportion of salts, such as the sulphates, nitrates, and phosphates of potash, lime, and ammonia, together with albumen, resin, etc., tobacco contains two alkaloids-nicotianin, and nicotia or nicotin; also, according to Vohl and Le Bon, a third one-collidin.

Nicotianin, or tobacco-camphor, occurs in white foliaceous 
crystals, of tobacco odour, and warm, bitter aromatic taste. It has no action on polarized light. If tobacco leaves be distilled with water, it precipitates after some days from the turbid distillate. The formula is not fixed but it contains much nitrogen.

Nicotia, $\mathrm{C}_{10} \mathrm{H}_{14} \mathrm{~N}_{2}$ (of which the dried leaves contain about 6 per cent.), is a volatile oily liquid, colourless when fresh, but turning brownish-yellow on exposure, of burning taste, unpleasant pungent odour (more leveloped on heating), and strongly alkaline reaction. It boils at $250^{\circ} \mathrm{C}$., and does not soliclify at $10^{\circ} \mathrm{C}$. The specific gravity is 1.027 at $15^{\circ} \mathrm{C}$. It turns the plane of polarization strongly to the left; is very soluble in water (which it also absorbs freely from the atmosphere), ether, rectified spirit, turpentine, and fatty oils; the vapour is exceedingly heavy.

Collodin is described by Le Bon as a liquid of agreeable penetrating odour (Med. Times, i., 1881).

It has been supposed that tobacco smoke contains not only nicotia, but an empyreumatic oil generated in combustion. In 1871, Eulenburg and Vohl published elaborate experiments to prove that pyridin, picolin, and other bases of the same series were the really active constituents of the smoke; they asserted, also, contrary to the general idea, that there was much less nicotin in the tobacco used for chewing (Med. Times). Heubel, however, has since given reason for rather supporting the older view that nicotia is the active agent in both cases, though no doubt prussic and other acids, and various complex organic bodies, carbonic oxide gas, etc., exist in tobacco fumes (Lancet, ii., 1872).

ABSORPTION AND ELIMINATION.-The soluble parts of tobacco are easily absorbed from the alimentary canal, and also from the skin. The latter fact is proved by the following cases related by Dr. Blanchard. A man and wife, to cure themselves of itch, vigorously rubbed into the skin a decoction of tobacco, and in a short time were both affected,- the man (who had scarcely wiped himself) more seriously than the woman. The symptoms were vomiting, violent pain in the stomach, dysuria, jaundice, muscular tremors, painful cramps, and frequent stools, 
resembling those of cholera : both patients recovered under the use of stimulants (Bull. de Thérap., Juin, 1869). I have seen similar deleterious effects follow a cataplasm of tobacco, which I ordered on one occasion to be applied over a strangulated inguinal hernia.

René, in a recent thesis (Nancy, 1878), has given further evidence as to its rapid absorption from all parts of the body, and states that nicotia, when injected into the veins, acts (contrary to what has been thought) very rapidly-" like lightning."

It is probably eliminated mainly by the kidneys and lungs.

Physiological ACTION.-The most familiar of the physiological effects of tobacco are those which are experienced by young smokers, who rarely fail to poison themselves, to a greater or less extent, in their first trials. Nausea, giddiness, vomiting, or a feeling of deadly sickness, with cold sweatings and exceedingly feeble pulse, are the ordinary results of early attempts to smoke tobacco even moderately strong.

The smoke thrown into the rectum is more poisonous than when it is inhaled by the mouth, probably because it is retained. Fatal results have not infrequently followed the administration of the decoction of tobacco in enema. Mr. Eade reports the case of a girl who, in half an hour after receiving such an enema ( 3 drachms to 1 pint), complained of faintness, and of feeling sick, and in another half-hour became quite collapsed, with cold sweats. She vomited, was slightly convulsed, and died in an hour and a half after the first administration of the poison (Taylor). In another case a young man had an enema made with 2 drachms of tobacco to 8 ounces of boiling water, and directly after its injection was seized with convulsions, became speechless, and died in less than two hours (Edin. Med. and Surg. Journ.). An infusion of 30 graius has caused severe symptoms, and eren death.

A lunatic swallowed an ounce or an ounce and a half of crucle tobacco after keeping it some time in his mouth; he became suddenly insensible and motionless, with all the muscles relaxed, very feeble respiration and pulse, strong contraction of the pupils, and (later on) riolent tetanic convulsions and profuse 
purging, with blood and mucus in the stools. After a temporary amendment, consequent on the use of the stomach-pump, the symptoms returned; the patient uttered loud cries; during the convulsions the limbs were rigidly flexed upon the body, and there was grinding of the teeth; death occurred from syncope, seven or eight hours after taking the tobacco (Taylor). Nicotia itself has proved fatal in from three to five minutes. It would appear from a comparison of different reports that in the most swiftly fatal cases the action of the poison has been almost entirely expended upon the nervous system and the heart; while patients who survive longer suffer from severe inflammatory affections of the alimentary canal.

Nervous System.-From the observations of Traube (Allg. Med. Centralzeitung, xxxviii., 1862), Rosenthal (Med. Centralblatt, xlvii., 1863), Krocker (Ueber die Wirkung des Nicotins, Berlin, 1868), Erlenmeyer (Corresp. Blatt. Psych., xvi., 1864), Schroff (Mat. Med. d. reinen Pflanzenstoffe), Van Praag (Arch. Path. Anat., 1855), Kölliker (ibid.), etc., may be derived the following résumé :-Nicotia in toxic doses paralyses the brain, producing loss of consciousness and of voluntary movement, after a more or less brief interval of excitement. The primary action on the spinal cord is exciting, and produces clonic and tonic convulsions. This is followed by paralysis, the cord becoming insensible to direct and to reflex irritation, apparently from affection of the grey matter of the anterior cornun. The motor nerve-trunks are but little affected, but their terminals in the muscular substance are at first strongly irritated and then paralysed.

Small doses affect the heart through the nervous system in a double manner, the vagi being in comparison slightly, the angmentor nerves much more powerfully, affected. The excitement is followed by paralysis. If the vagi be divided, nicotia nevertheless acts on their peripheral twigs, and the ganglionic apparatus in connection with them.

More recent observers conclude that nicotia exerts little or no direct influence on the brain proper, but acts almost wholly on the cord. The convulsions excited-o.g., in frogs-are very peculiar: tetanic cramps laying the front legs alongside the trunk, whilst the hind legs are arched over the back (Krocker). 
This position is traced to the fact of the extensors being more powerfully contracted than the flexors. Motor excitement is commonly followed by muscular relaxation, and, if the dose be a fatal one, by the paralysis already described. Minute doses cause rather muscular tremors (Vulpian), "vibratory muscular wavelets like tetanic shocks" (René).

The power of tobacco to cause tremor in man is often evident, and that the symptom is due to the drug is shown by cases in which it has ceased soon after giving up smoking. Noted marksmen find their hands distinctly less steady after using tobacco, and others notice their writing become "shaky" (cf. Record, 1876). Twitching and cramp of the muscles of the limbs seem to have depended upon smoking in Mr. Allinson's case, and he connected them with "irritation of the solar plexus" (Lancet, ii., 1882). The symptom of tremor is, however, not constant, and allowance must be made for idiosyncrasy as well as for quality and quantity of the tobacco used.

The action upon the pupil has been much disputed, but the most recent and reliable researches seem to prove that nicotin (differing from the mydriatic group of Solanaceæ) produces contraction; certainly this results from moderate doses, or from direct application to the eye, even after its removal from the body. Krocker, who states this, says also that the pupils after toxic doses, though contracted at first, dilate slightly in later stages: others report dilatation as a primary, contraction as a secondary effect (Stillé).

Tscheschichin has observed a constant lowering of superficial temperature, which probably depends on rapid cooling of the body in consequence of vaso-motor paralysis. The dilated earvessels of rabbits poisoned by the drug indicate also such paralysis. The sensory or afferent nerves are said to retain their power.

In doses of $\frac{1}{32}$ to $\frac{1}{16}$ grain nicotia cruses some excitement of the general nervous system in animals, but larger doses give rise to heaviness, giddiness, torpor, sleepiness, indistinct rision, with sensitiveness of the eye to light, imperfect hearing, laborious oppressed breathing, and dryness of the throat. In about forty minutes prostration sets in, the head droops, the limbs seem paralysed, muscular spasms occur, and faintness lapses into unconsciousness. 
Circulatory System.-The direct addition of nicotia to blood turns it dark and disintegrates the corpuscles, but such effects could not be produced by any amount taken during life. It is said, however, that in persons fully under tobacco influence the corpuseles are found less aggregated and more irregular in shape than usual, but they recover their normal condition as the poison is eliminated. Topically applied to a frog's heart, nicotia in a sufficient dose arrests its movements, so that it appears to have a paralysing action on the muscular structure.

According to experiments on animals, the pulse-rate and the blood-pressure at first fall, and afterwards rise-the latter effect appearing due to paralysis of the periphery of inhibitory nerves, for galvanizing the vagi did not affect the heart's action (Rosenthal). In horses the depression of the puise is marked (Stillé). The later rise of the arterial pressure has been attributed to some "vaso-motor action of the drug," but this has not been clearly proved (Wood). Respiration is at first quickened, and there may even be tetanus of the inspiratory muscles; apparently this action is exerted upon the nerve-termini among the muscles, for section of the vagi does not prevent it: the respiratory excitement is followed by paralysis.

Observations upon the effects of smoking tobacco are somewhat discrepant. Whittle found it commonly cause at first quickening of pulse with palpitation; later, slowing of pulse, with palpitation still continuing. He records one case in which, shortly after commenoing the habit, the pulse fell to an average of forty-eight, and after ceasing it, the pulse regained its usual rate of sixty (Med. Times, i., 1878).

"It raised the pulse several beats in intolerant subjects, acting as a heart-stimulant and nerve-sedative" (Lancet, i., 1880).

Dr. Anstie wrote- " The effect of tobacco-smoking in moderation on the majority of persons who are skilled in the use of the pipe is a marked increase of stimulation, the pulse being. slightly increased in frequency, and notably in force, and the sense of fatigue in body and mind being grently relieved. This stimulation is not followed by depression; on the contrary, the smoker seems lighter, more cheerful, and the pulse 
maintains its firmness, in many cases for an hour or two, and then yields no morbid depression. When depression is produced it is produced early, and is a sign that even a small dose will disagree."

Digestive System. - An unduly large dose of tobacco in any form, whether of the vapour, or of the crude drug, by the mouth or rectum, or of nicotia, acts as a gastric imitant, causing nausea and vomiting, with an extreme amount of depression, also distension and diarrhœe, with tenesmus. Intestinal peristalsis is stimulated, and there may even be intestinal tetanic spasms.

If taken by the mouth, there is salivation, which may be excessive, with much sense of burning in the fauces and stomach: if crude tobacco has been swallowed, gastric congestion and inflammation occur, as shown by post-mortem appearances.

Genito-Urinary System.-The action of medicinal doses of tobacco on the genital system is, if anything, sedative, and serious depressant effects have been described by Lallemand, Solly, and others from habitual smoking, which, they say, causes also spermatorrhoea (from relaxation of the ducts). I have not myself seen such results, but can well believe their occurrence in greater or less degree from the excessive use of tobacco by growing youths. I have known many men make use of tobacco for the express purpose of lessening sexual desire. In women it is said that vigorous contractions of the uterus are produced by the drug. The secretion of the kidneys is commonly increased by the drug in any shape.

Tobacco Habit.-The influence of habitual smoking upon the health is a subject apart from its medicinal use, but bears upon its physiological action. The most opposite views have been held concerning it, and the truth probably lies between the extremes. The moderate use, as concluded long ago by Brodie, and more lately by Parkes (B. M. J., ii., 1880), is advantageous to many, as a digestive and laxative, and as a calmative during fatigue or worry; its abuse, as with other potent things, may induce serious disorder.

Although tobncco cannot be called a direct bypnotic, yet by soothing the minor forms of neuralgic pain and general rest- 
lessness it often helps to make sleep possible. To aged persons, provided they have overcome the difficulties of smoking in early life, tobacco often proves useful in quieting nervous restlessness. It is one of the least harmful of those agents by which soldiers and others, who are compelled to undergo strenuous exertion at times, with an insufficient supply of food, are enabled to hold out and do their work effectively. What opium is to a Tartar courier, tobaceo is to the soldier or sportsman, in supporting him under severe and continuous physical efforts, when rest and sufficient food are alike beyond his reach. It should be stated, on the other hand, that many army medical officers are of opinion that the almost universal use of tobacco by soldiers (especially young ones) actually causes or aggravates palpitation of the heart and other diseases of the circulatory system, since the symptoms subside when the habit is left off; in this connection it must be remembered that diseases of the circulatory system rank second in causing invaliding and death in the army.

No doubt there are some persons much more affected by it than others, and some to whom in any dose it is poisonous; from such, however, we cannot argue for the average healthy man. We must recognize that its continued use in more or less excess may cause chronic dyspepsia-partly by direct irritation or by depression of the nerve-supply to the stomach; partly by waste of, or alteration in, the salivary fluids. Depression of the general nervous system may also be traced to tobacco, with restlessness, insomnia, and a tremulous condition of limbs not unlike that of chronic alcoholism. Solly and some few others attribute to it insanity and various frightful results (Lancet, i., 1857); this is sufficiently disproved by Bucknill and specialists of experience (ibid.). There can, however, be no doubt as to the evil effects of smoking in early youth - that is to say, in the years before and immediately succeeding priberty: it hinders the growth and development of the higher nervous centres, and may seriously damage both intellect and character, by its depressing agency continuously exerted during the developmental period; also, by its interference with digestion and assimilation, it may imprir the whole process of consolidation of the bodily frame. Hence the production of 
chloro-anæmia, such as Decaisne found in the majority of thirty-eight lads whom he specially examined with reference to tobacco-effects; and with this disorder were connected palpitation, intermittence of pulse, and a state of general hebetude. Kestral reported similar results (Med. Times, ii., 1868). Impairment of nutrition seems also illustrated by the case of a youth in whom cuts about the hands could not be healed whilst habitual smoking was continued (Lancet, Oct., 1882, p. 648).

Special local changes occur in the throat and in the eyes. The former are classified by De la Sota under three divisions: (1) the erythematous, congested, dry mucous membrane; (2) a greater degree of the same, with vesicles on the palate and lips; (3) a tumid livid-red condition, with granulations and varices about the fauces, which are frequently coated with viscid mucus. Many forms of sore-throat, whether relaxed, congested, or ulcerated, are made worse by persistence in smoking.

With regard to the eyes, more importance was attached some years ago to their presumed serious disorder from this cause than modern research proves to be well founded. The impaired condition of vision was termed-and sometimes is so still-amaurosis, but amblyopia would express it more correctly. "I admit," says the late Mr. Soelberg Wells, "that the excessive use of tobacco (but most frequently with other causes) may produce considerable impairment of vision, and finally .. . even atrophy of the optic nerves. But I cannot, from my own experience, accede to the doctrine that there is anything peculiar in the form of atrophy of the optic nerve. I believe that in the commencement of the amblyopia of smokers and drunkards the disturbance of sight is at first only functional" (1869). Mr. Bruclenell Carter expresses grare doubts as to the existence of the disorder (tobacco-amaurosis); and Mr. J. Hutchinson, though he at first taught its occurrence (1864), was able to publish, in 1876, that a large majority of the subjects of it had recorered, and very few, if any, had become worse-facts inconsistent with amaurosis.

Mr. Hart, after examining 'the eyes of mauy confirmed smokers, denies that white atrophy of the optic nerve occurs in them with unusual frequency : he also points out its occurrence in women and children, and, arguing against Richardson's 
extreme views, shows the disadvantage of "the constant crying of "wolf," " and of the description of "indefinite diseases supposed to arise from ordinary habits of life."

Mr. Nettleship has published fourteen cases of the amblyopia of smokers, in all of which abstinence from tobaceo was followed by recovery or arrest of the disorder (St. Thomas's Hosp. Rep., 1879); and Ely, examining 102 persons occupied in the manufacture, etc., of tobacco, found them fairly healthy, and only two (doubtful) cases of amblyopia amongst them (N.Y. Med. Journ., April, 1880).

Dr. Hirschberg, after a careful examination of the question, and of many cases, concludes that there is no doubt of amblyopia following excessive smoking, and traces it to "a centric scotoma, or limited dimness in the central region of the visual field of each eye." The "scotoma is of more or less oval shape, includes the fixing point, and reaches to the blind spot. If the eye be fixing the middle of the field, a white piece of paper, placed anywhere in the defective area, seems to the patient to be grey; further, as Förster has shown, a red piece is either not seen at all, or appears very dark; and as I have myself observed, a green piece becomes greyish, and a blue one frequently a paler blue or indistinct. The scotoma is. never absolute; the white paper, if well illuminated, will never disappear entirely within it. Corresponding to this impairment of the central part of the field, the acuteness of central vision is reduced to one-third, onetenth, or even in rare cases to one-thirtieth of the normal. Both eyes are equally affected, or one sometimes a very little more than the other. The power of reading common print is abolished; but even in the worst cases the number of fingers can be counted up to a distance of 5 or 6 feet. The fundus at first is normal, but after a time the diso becomes palish. To this definition of tobacco-amblyopia it must be added, that we never meet with the disease in women."

Dr. Hirschberg believes that this disorder never terminates in complete loss of sight_amaurosis, - a malndy dependent on progressive atrophy of the optic nerve, and one which begins and progresses differently. At its commencement (amaurosis), though the patient can still read small print and the dise bo almost normal, the cxtent of the visual field is recluced, 
especially for various colours; a limited central scotoma is not found, nor is the condition stationary for months, nor does a partial recovery occur.

The amblyopic caused by chronic alcoholism is very similar to that caused by tobacco, and, since abuse of the two things often coexists, it is difficult to distinguish accurately their separate effects.

Martin states that the amblyopia from tobacco may be distinguished from the alcoholic disorder, (1) by the pupil being contracted, (2) by the fact that the vision of such patients is not so good at dusk as in daylight, and (3) the absence of delusions, whilst in amblyopia from alcoholism the pupils are said to be dilated, and the malady is also progressive (Thesis, B. M. J., i., 1879) :- these distinctions are not, however, very positive. In Nettleship's cases, vision was better in dull light; and Nelson observed the same thing: the smallest quantity of tobacco he found to produce failure of vision was about $\frac{1}{2}$ ounce daily, and the largest daily amount consumed by his patients was $1 \frac{1}{2}$ ounce; it was from two to six months before the impairment was evident.

A recent paper by $\mathrm{Mr}$. Shears,-based on observations made upon forty men who applied for treatment for impaired vision -mainly corroborates the conclusions already given: the diagnosis of tobacco-amblyopia was arrived at (1) when there was rapid and unusually great failure of sight, not remedied by spectacles, and not due to obvious external changes; (2) where no ophthalmoscopic changes were found, or, at the most, slight pallor or redness of the disc; (3) when the patient was an excessive smoker. Of the forty cases, practically all but three got well or improved greatly; two of these three would not diminish their smoking, and the other was a drunkard. Moderation, without absolutely giving up smoking, was sufficient for recovery (B. M. J., ii., 1884). Inquiries made at large tobacco factories gave to this observer no evidence of injury to the sight from mere contact with the drug.

Dr. Buzzard has published two cases of tobacco-amblyopia, in both of which the patients took alcohol and tobacco to excess, and the symptoms simulated locomotor ataxy in the presence of "lightning pains," and of pallor of the disc (suggesting optic 
nerve atrophy): on diminishing the tobacco and alcohol the acrteness of vision returned to normal (Lancet, ii., 1883).

A myopic medical man reports that a pipe increased the myosis. "A smoke would entirely destroy the effect of my glasses, and when I had them on after smoking I could only see as far as when I had them off on an ordinary occasion" (Lancet, Oct. 14, 1882).

A bad effect on the teeth is commonly traced to tobacco; but Hepburn, on the other hand, says something in its favour: it may discolour them, but the nicotia, and carbonic oxide act as antiseptics and tend to preserve them; they also seem to render the process of decay less painful (B. M. J., ii., 1879).

SYNERGISTS.-Most of the poisonous Solanacer, also lobelia, aconite, and, in fact, all drugs that cause anæmia and depression of the nervous system.

ANTAGONISTS.-Tannin in any form precipitates nicotin, and is therefore chemically incompatible; so also are caustic alkalies and iodides.

Alcoholic stimulants, ammonia, ergot, and in small doses digitalis, and perhaps belladonna, antagonize the depressing cardiac action. Woakes has drawn special attention to the value of camphor as an antidote (Practitioner, vol. i., 1878), and Professor Haughton has demonstrated the antidotal power of strychnia by some interesting experiments (Dublin Hosp. Gaz., Dec., 1856). In a remarkable case of acute tremor of hands, due to excessive smoking, the symptom was almost completely removed within twenty minutes after the hypodermic injection of $\frac{1}{19}$ grain of strychnia (Record, 1876).

On the other hand, Smyley has recorded the case of a boy poisoned by 4 grains of strychnia who recovered under the influence of tobacco infusion (Dublin Quart. Journ., Aug. 1862).

Tobacco-poisoning should be treated by this remedy after the use of emetics, and conjointly with coffee, stimulants, or camphor. There is some clinical evidence that a course of nuxvomica or strychnia will soon lessen or remove the "tobaccoappetite" (B. M. J., ii., 1883).

Mr. Hutchinson has recently published a case in which large 
doses of opium restored vision when much impaired by the use of tobacco; he suggests that the former relaxed a spasm of the vessels set up by the latter (Med. Times, i., 1884).

ThERAPEUTICAL ACTION.-Externally, an infusion or ointment of tobacco has been employed in some skin diseases, especially those of parasitic nature, such as tinea and scabies; also in prurigo; but it is not a desirable remedy.

Constipation-Obstruction.-A few minims (3 to 5) of tobacco wine (U.S, Pharm.), given at bedtime, are said to act favourably in the former condition, and many persons depend upon a morning "pipe" for a regular action of the bowels.

Its mode of action is not very clear, but depends probably on its promoting a more rapid passage of the food along the intestines and increasing the secretions, and possibly stimulating or irritating the muscular fibre.

In obstruction connected with impaction of froces in the cæecum, in plumbism, hernia, and perhaps intussusception, there can be no doubt that a tobacco enema has sometimes proved effectual, although, as already mentioned, its general action has proved not infrequently fatal, and 30 grains given in this manner have destroyed life; not more than about 15 grains (equal to 6 ounces of the enema tabaci, B.P.) should be used at one time. Practically, chloroform and other modes of treatment have superseded this at the present time.

Tetanus, etc.-The reputation of tobacco in this disorder, and in strychnia-poisoning, rests partly on the nimeteen successful cases reported by Mr. Curling (Treatise on Tetanus, London, 1836), partly on the experiments of Professor Haughton. I have not much personal experience on this subject, but should think hypodermic or rectal injection of the alkaloid the best mode of using the remedy; and the following formula has been adopted:-Nicotin, 10 minims; gum mucilage, 40 minims; distilled water, $7 \frac{1}{2}$ drachms : inject 10 minims ( $=\frac{1}{10}$ minim of nicotin) every four hours. When it acts farourably it relaxes trismus, so that nutriment may be taken, and moderates or suspends the tonic convulsion.

Asthma.-Tobacco has often proved useful in this disorder. Dr. Hyde Salter, who thought highly of it, was accustomed to 
push its action to a full extent, often rendering the patient faint and sick, with the design of cutting short a commencing paroxysm. I think, however, that much benefit may be derived with a less degree of physiological effect. It is said that laryngismus stridulus may be quickly arrested by a "snuff plaster" to the neck, but even this is not free from danger.

PREPARATIONS AND DOSE.-Enemu (B.P., $20 \mathrm{gr}$. to $8 \mathrm{fl}$. oz.): dose by enema, $6 \mathrm{fl}$. oz. at a time. Folic (leaf tobacco): dose in substance is usually 3 to $5 \mathrm{gr}$. Infusum (U.S.P., $1 \mathrm{dr}$. to 1 pint): dose, 2 to $4 \mathrm{dr}$. Externally, as a wash, $3 \mathrm{oz}$. of this infusion may be used, provided the skin is not broken. Vinum (U.S.P., 1 oz. to 1 pint): dose, 5 to $20 \mathrm{~min}$; but is rarely used. In strychnia-poisoning and tetanus the dose may be increased, or given at shorter intervals, until physiological symptoms are induced. Tobacco may be used in the form of an ointment, subject to the precautions already stated. Nicotin: dose, $\frac{1}{10}$ min. by the skin (v. previous page), $\frac{1}{5} \mathrm{~min}$. by the mouth.

Adulterations. - Besides the substitution of cabbage and other leaves, Dr. Clark has reported the presence of from 30 to 50 per cent. of water in many samples of tobacco, and of 5 or 10 per cent. and upwards, of sand in snuff (B. M. J., ii., 1884). 


\section{SCROPHULARIACEA. \\ THE FOXGLOVE FAMILY.}

THIs important family numbers over 2,000 species, which are chiefly herbaceous. In the foliage there is nothing uniform or characteristic, except that stipules are never developed; nor does any special feature pertain to the inflorescence, many varieties occurring even in the same genus. The monopetalous flowers are almost always irregular, more or less tubular, and usually tetramerous; the epipetalous stamens are nearly always didynamous; and the superior ovary becomes a capsule, which is many-seeded, the seeds themselves being destitute of wings.

\section{Pharmacentical Species.}

Digitalis purpurea . . . . . . . . . . Foxglove.

Leptandra Virginica . . . . . . . Black-root.

\section{DIGITALIS PURPUREA.}

(FOXGLOVE.)

DESCRIPTION.-A handsome biennial, found in most parts of England and the Continent. It has fibrous roots, and large, ovate, grey-green leaves, which are puckered and obscurely crenate, the blade tapering into a petiole 3 or 4 inches in length. The flower-stem is simple, erect, 2 to 6 feet ligh. and sparingly clothed with leaves, which become gradually smaller, more lanceolate, and sessile from the base upwards. In front, from the middle to the apex, it is hung with tubular crimson blossoms, 
$1 \frac{1}{2}$ inch in length. The filaments of the stamens are curiously bent, the anther-cells are large and divergent, and the ovary ripens into an ovoid capsule, partly concealed in the permanent calyx, and containing innumerable seeds. Every part of the plant, except the corolla, is downy and soft to the touch. The fresh leaves have an herbaceous, unpleasant smell, but when dried this becomes faint and tea-like; the taste is disagreeable. They should be collected in the second year: when two-thirds of the flowers are out, but in September of the first year they are also effective; they should not be kept more than one year; under cultivation the leaves become less. hairy and narrower.

ACTIVE INGREDIENTS.-The chemistry of digitalis is complex, and has been rendered still more so by the application of the same name to different substances by various observers. The main active principle is now generally termed digitalin (by some digitaline, and by Nativelle originally digitalein), and it is only this, and the more recently-prepared digitoxin, that we need practically consider. Of digitalin, $\mathrm{C}_{50} \mathrm{H}_{40} \mathrm{O}_{30}$, there are several forms.

(1) According to the process of Nativelle (1872), it is obtained in small, whitish-yellow, silky needles, or scales, and small lumps, heavier than water, unchanged in air, neutral, of no marked odour, but intensely bitter taste, which develops slowly on the tongue. At $100^{\circ} \mathrm{F}$., it fuses and becomes coloured, and its bitterness is replaced by astringency. It is readily soluble in alcohol of 90 per cent.; also in chloroform, and in chloral solution, which latter assumes a deep greenishblue tint; scarcely at all in ether, beuzol, or even in boiling water ( 1 in 1,000). It does not combine with acids, but strong sulphuric and hydrochloric acids dissolve it with green coloration; with nitric acid it gradually becomes yellow. Although the purest of the preparations, Rouchel states that it contains several ingredients differing in character. It is two or three times more active than the amorphous form (Vulpian-Gubler), and is a substance of extreme energy, requiring in its use the greatest caution.

(2) The amorphous or French digitalin of Homolle and 
Quevenne is neutral, whitish, very bitter, and giving the same chemical reactions as the above, than which, as already stated, it is less active, therefore probably less pure.

(3) The German form, or digitalin of Kosman, is soluble in water, and gives no green coloration with hydrochloric acid; it is less active than the preceding. It is obtained from the seeds, the others from the leaves, and according to Lefort, it results from the oxidation of the amorphous form, like quinine from cinchonin.

It is plausibly supposed by Schmiedeberg that these and many other substances described in digitalis are not true and distinct chemical substances, but mixtures of ingredients preexisting in the plant, or results of chemical decomposition.

(4) The digitalin of the P.B. is described as an "uncrystallizable, light-brown powdery resinoid substance, soluble in spirit and acids, almost insoluble in water and pure ether; leaving no residue when burned with free access of air."

Digitoxin, $\mathrm{C}_{32} \mathrm{H}_{33} \mathrm{O}_{7}$ (which exists only in the proportion of 1 part in 10,000 of dried leaves), was prepared by Schmiedeberg by an elaborate process. It occurs in colourless scales or needles, giving a yellowish green solution with hydrochloric acid, not soluble in water, sparingly in ether, moderately in alcohol: this latter solution is decomposed by dilute acids with formation of toxiresin, an amorphous yellow substance soluble in ether. Both these are too highly poisonous for medicinal use, but some small proportion of digitoxin is contained in the digitalin above described. Besides these two important constituents (of the leaves chiefly), digitalein, an active, bitter, amorphous body, has been obtained from the seeds; and digitalic and other acids, volatile oil, starch, sugar, pectin, etc., from the whole plant.

ABSORPTION AND ELIMINATION.-Absorption occurs from any tissue, but is probably slow, since digitalin is but little soluble, and its effects on the heart do not appear immediately.

Elimination takes place largely by the kidneys, and is also slow, since the drug seems to "accumulate" in the system, i.e., its effects become more marked after some days' use, although 
the dose is not increased, and they sometimes develop more after its omission.

Physiological Action.-Local.--It is commonly said that the powdered leaves or the active principle, when applied to the denuded skin or to mucous membranes produce much pain and irritation, even inflammation, and Gubler states that he suffered a severe laryngo-bronchial inflammation with aphonia, after inspiring amorphous digitalin from a bottle. Rabuteau, on the other hand, asserts that no local irritation is produced by these substances, but they will certainly irritate the conjunctiva.

Physiological Action.-Internal.-Digestive System. - Small doses may be taken for a time without definite effect, but if continued, or if toxic doses be taken, evidence of gastric irritation is shown by pain, flatulence, anorexia, nausea, vomiting, and probably diarrhoa. These symptoms occur in whatever way the remedy is introduced, and are, consequently, not due to a local irritation.

Circulatory System.-After a varying time, according to the dose taken, the pulse becomes slower, falling to forty or less per minute; this depends on a prolongation of the diastole, by which also the heart-action is commonly rendered slower, though sometimes its contractions may be found twice as frequent as the pulse. Of two ystoles one is powerful and another so quick and feeble that the unaided finger does not perceive it in the pulse; in the sphygmographic trace it may be recognized in a slight raising of the long line between two of the slow and distant beats, and this extra and feeble systole may become still more evident under exertion or emotion. "These quick (feeble) beats, as the influence increases, become more numerous, so that the slow beats, which now occur only occasionally, become intermissions, and in a still further stage, the slow bents entirely disappear, and the pulse becomes regular but extremely rapid" (Brunton on Digitalis, p. 21). Hence the pulse may change its character quickly, and this may explain some discrepancy in the accounts of its condition (Rabuteau).

Sanders and others have described the primary condition as 
one always of quickening, but it is now commonly agreed that under therapeutical and physiological doses slowing almost always occurs; with toxic doses the pulse is at first more frequent, and later becomes small, irregular, and intermittent, - a fact well seen in the lower animals.

In poisoning by digitalis the force of the pulse bears no relation to that of the heart's impulse, for while the latter is strong and hammering, the former is small, thready, and nearly imperceptible (Brunton). (Some cardiac fremitus and metallic sound, replaced by a systolic bruit, under digitalis, has been observed, especially by Bouley and Reynal, in horses.)

The rhythm of the exposed frog-heart may be seen to become irregular, the ventricles either contracting only in parts seriatim whilst the rest dilates, or the whole organ contracting very slowly (just as when the vagi are faradized) : the auricles may contract twice for one pulsation of the ventricle, and "palpitating points," like small crimson pouches, may be seen on the latter, which is otherwise white and contracted. Fothergill supposes these to be each under the control of a separate cardiac ganglion : like other observers, he found the hearts (of frogs at least) come to a standstill in firm contraction, much climinished in size, and in shape and colour much resembling an unripe apple-pip (On Digitalis, p.5). Exceptionally there is diastolic arrest-and the arrest in systole is not to be taken, $p e r$ se, as a proof of increased contractile power ; it is a separate effect from ordinary muscular contraction, and akin to the so-called "tomiccontraction" of involuntary muscle. It may be induced even by potash salts and by aconite and other drugs which are known to weaken rhythmic contractility (Ringer). It is very noteworthy that whilst the circulation is rendered slower the arterial tension is raised: this implies an action on the arterioles, because if the heart alone be slowed (as by galvanizing, etc., the vagus), the tension falls.

The primary action exerted on the capillaries is to contract. them, as may be demonstrated in the frog's web, but with more certainty by Brunton's observatious with the kymographion, which he connected with a canula inserted into the crural artery of a dog subjected to the action of the drug. When toric 
effects are developed an opposite condition (of dilatation) occurs, and the tension falls; hence, post mortem, the arterial system is generally found empty, the venous turgid.

Lombard has recorded lowering of arterial tension as the usual effect of the drug. (Thesis, 1877), but his observations were made after injecting infusion into the reins; probably, therefore, the toxic effects were quickly developed.

Theory of Action.-To understand this subject, which has been so much disputed, it is necessary to bear in mind the nervous mechanism of the heart. The heart is supplied by the vagus and sympathetic; it has also an intrinsic nervous mechanism. The vagus contains inhibitory fibres, and fibres which also convey impressions regulating the force of the heart's beat. The sympathetic contains "accelerator" and "augmentor" fibres. These fibres are connected with centres in the medulla ; the vagus with the "cardio-inhibitory" centre, the sympathetic with the "cardiac-motor" centre.

The chief intrinsic ganglia of the heart (in cold-blooded animals) are situated in the sinus venosus. They are supposed to command the automatism of the heart; but it has been shown that the muscular tissue itself (in the tortoise and some invertebrate animals) possesses some automatic power. Other ganglia exist in the frog's heart, in the auricles and auricular septum. The exact functions of this complicated nervous apparatus have not been fully worked out, and for a discussion of the different views we must refer the reader to treatises on physiology.

It is somewhat curious that different observers should think it necessary to restrict the action of digitalis to one of these sets of nerves. Traube, for instance, to the vagus, and Dybkowsky, Pelikan (Zeitschr. Wiss. Zool., xi., p. 278), and others, to the intrinsic ganglia, whilst the primû facie supposition must be that both are affected.

The theory of Traube was generally accepted some time ago, founderl as it was upon such experiments as the following:An infusion of 8 grains of digitalis leaves injected into the veins of dogs raised the pulse from 128 to 132 ; four times the amount injected, lowered the pulse to 32 . Larger quantities quickened it again to 160 and upwards. Then, in one dog, 
after reduction of the pulse from 121 to 48 , the right vagus was divided, and the pulse rose to 66 , and when the left also was divided, to 204; and when both nerves were cut before administering the drug, i.e., when their restraining "inhibitory" influence on the organ was abolished, then the slowing normally produced by digitalis did not occur at all, or if it did so, very slightly. The natural conclusion was that the drug exerted its slowing action by stimulating these nerves. Again, if the conducting power of the nerve be destroyed by the previous administration of atropin (which is known to have the power of paralysing it at the periphery-Bezold and Bloebaum), then also digitalis fails to slow the heart.

Unfortunately for this simple solution of the problem, the above results were not obtained by all experimenters: thus, Boehm, Dybkowsky, and Pelikan, experimenting, it is true, on frogs, found the slowing, the strong contraction, the irregularity and systolic arrest, all produced by the drug after division of the vayi, and even destruction of the cord. Boehm also, and Ackermann, found that atropia did not prevent the above symptoms. On the other hand, they recognized that digitalis exerted some stimulant influence on the inhibitory nerves; but not to the extent stated by Traube.

Although his experiments on mammalia have not been so definitely contradicted, Handfield Jones found that the effects of the drug were not completely prevented even by section of vagi, and another objection to the theory is founded on the conditions of arterial pressure ascertained by the hæmadynamometer. When this instrument was inserted into an artery, and the cardiac action slowed by stimulating the vagi, arterial tension fell; but when the slowing was induced by digitalis, tension did not fall-implying that the drug did not act through the nerve. There is, however, a partial fallacy here, since it is another action of the drug, viz., the contraction of arterioles, which prevents the fall of tension, and, indeed, as we have seen, generally increases it.

Met by such objections, Traube modified his theory so far as to admit a stimulation of the motor as well as of the regulating nerves, to attribute the condition of tension to both these, and to admit a final paralysis. With these qualifications, there 
is certainly partial truth in his view, but in addition we must consider that the drug acts directly on the cardiac ganglia, since the experiments of Ehrenhaus, Legroux, and others, show that the frog's heart, when separated from the body (i.e., from. all nervous supply except its intrinsic one), and placed in a solution of the drug becomes slow in action, intermittent, or quite arrested, according to the strength of the solution. Also if the drug is topically applied to a portion only of the ventricle, it induces persistent contraction of that part, which then dilates less than the rest; whence Ringer concludes that it acts directly on the muscular substance. The special ganglia of the heart on the sympathetic nerve-fibres are now commonly reckoned as three-(1) of Remak, near the lower vena cava ; (2) of Bidder, near the mitral valve; (3) of Ludwig, near the right auricle. The motor action of these is independent of the general nervous system. The third seems to have a moderating rather than an energizing power. It is probably on these, but especially on the two first, that digitalis acts, stimulating or paralysing them according to the dose, and so strengthening the contractions of the heart or arresting it in systole. Such action does not, however, exclude an allied but stronger effect on the terminals of the vagus nerve.

The contraction of small arteries, already alluded to, is effected by a similar stimulus of vaso-motor centres, situated probably in the cord.

It must be remembered, however, that such stimulation is not a permanent increase of power. Digitalis does not act as a food, as a supply of strength to the heart, but only as a caller out, and especially a regulator of what energy is there ; and it is a stimulus which, if unwisely handled, rapiclly develops the paresis of exhaustion.

It is, perhaps, the emphasis placed by certain writers upon its "stimulant or tonic action," without always pointing out what has just been stated, that has given to some clinicians the idea that the "modern theory of the action of digitalis is a dangerous one." Thus, Dr. E. T. Tibbits, writing in 18r9, "did not believe in its action as a cardiac stimulant," nor in the experiments on animals, nor on blood-pressure; he believed it to be strictly a cardiac sedative, and states that "no example is 
recorded of its producing a hard powerful pulse and general vascular excitement" (B.M.J., ii., 1879). It is evident that for a clear apprehending of the modern theory, it is necessary to define as above the limits of "stimulant action," and as Dr. Ringer remarks- "The tonic theory regarding digitalis fails in most instances to explain its usefulness in heart disease: in many cases where it is valuable, the heart beats with excessive force, and the ventricle is found well nourished after death: in general terms, its efficacy is most decided where inegul(vity, rather than weakness, is a symptom "(Handbook)-i.e., it is a regulator rather than a true stimulant of the heart.

Nervous System.-In moderate doses, digitalis acts on the general nervous system as a sedative, calming excitement, and disposing to sleep. It has, however, no true narcotic action. Full doses, and in some susceptible subjects, 10 and even 5 minims of the tincture will cause headache, sense of tension about the forehead, and giddiness. Large doses cause in animals, first some exaltation of sensibility, as shown by spasmodic morements, startings on slight shock, restlessness, headache, vertigo, even hallucinations; afterwards a paresis of the motor and of the general nervous system, and finally profound prostration, and an unconscious, comatose condition, sometimes convulsion (Rabuteau). The sight early becomes dim, a bright halo being sometimes seen; the pupil is dilated. The primary stimulation of the sympathetic system is shown well by an experiment of Gourvats: he divided the right cervical sympathetic of a rabbit with production of the usual phenomena of vascularity, heat, etc., and then injected digitalin: some hours afterwards, the state of the right side was unchanged, but on the left, the central artery of the ear was so small as to be almost imperceptible; the pupil was largely dilated.

Reflex power generally is lessened, according to Weil, by direct excitation of the inhibitory reflex centre (Setchenow's centre), and later by paresis of the spinal cord. The lessening of reflex activity is readily shown in the frog, and the dependence of such lessening upon central influences is demonstrated by the activity returning after section of the upper. part of the cord, i.c., after interrupting communication with the inhibitory centre. 
The temperature of the body is lowered by moderate doses, but not till after two or three days' use, and in consequence, probably, of the changes in circulation and respiration. The depression continues for some time after omission of the drug (Hirtz, Oulmont). Wunderlich and Schneider consider that it occurs independently of the altered circulation, and Ackermann states that the internal temperature only is lowered, not that of the surface. Under toxic doses we have, however, clear evidence that the skin becomes cold and pale, though Demarquay and others have noted (even with such doses) a temporary rise of temperature in dogs. The explanation of these differing results depends partly on varying conditions of the circulation, partly on alterations in the tissue-changes, affecting the temperature in a secondary manner.

Muscular System.- Small doses have little influence on striped muscular fibre; large doses lessen its contractile powers, as demonstrated by galvanism: the unstriped fibre is stimuIated even to the extent of cramp by moderate doses, paralysed by toxic ones.

Genito-Urinary System.-The drug has a sedative and depressing action on the genital system, probably lessening the normal blood-supply to it. Stadion and others found it destroy for a time sexual activity, but Pfaff reports this as sometimes increased, and such effect I have myself noticed. On the uterus, certainly on the enlarged uterus, digitalis acts somewhat like ergot, stimulating the muscular fibres, and probably the coats of the vessels, to energetic contraction, and so arresting hæmorrhagic discharge.

Its influence on the secretion of urine in the healthy subject has been very variously reported, showing, at least, that it is not always the same, and when such increase occurs it is traced by many to an indirect action through the circulation. Thus Traube asserts that " the bulk of the urine is the measure of the blood-pressure on the renal glomeruli," and Legroux and others speak of increased urination as following necessarily on the heightened arterial pressure caused by the drug; but it is difficult to accept this view, since increase is not a constant occurrence: also the pressure on the glomeruli may be lessened on account of the 
renal artery being contracted, and then diuresis would not occur.

Pereira reported diuresis, also Jörg, Hutchinson, and Hammond, and many French observers, noted the same effect in horses. Brunton, in his own case, "found that with small doses the urine varied just as the pulse had done, being generally increased to a slight extent while he took the digitalin, but sometimes not; while with large doses the diuretic effect was marked." When evident symptoms of poisoning appeared there was a sudden fall in the amount (from 50 to 40 ounces), but a rise of greater extent occurred afterwards (to 53 ounces); the original increase was not more than 1 to 2 ounces. In Winogradoff's experiment with $\frac{3}{10}$ to $\frac{2}{7}$ grain of digitalin for five days, there was no increase; in Stadion's for eighteen days there was diminution.

In very large doses, the drug occasionally causes not only diminution but suppression of urine (Christison-Mazel, Edin. Med. Journ., 1884). I have in some healthy persons observed, after taking one dessertspoonful of the infusion three times daily for five to six days, a frequent desire to urinate, but only small quantities of high-coloured water passed at a time; in others the flow was copious, and the water clear. In some instances, where the desire was frequent and the quantity small, there was pain and often emission of blood before and after micturition.

There is much more consensus of opinion as to the diuretic power of the drug in conditions of ill-health, cardiac disorder, and dropsy. It seems probable that the drug lessens the renal engorgement consequent on embarrassed heart-action, at the same time that it lessens the conditions causing dropsy-the fluid already effused is re-absorbed into the circulation, and then rapidly excreted by the kidneys to the amount of four or six pints or upwards daily.

As to the amount of urea and of the chief solids, there was decrease in the case of the two observers above mentioned. Brunton is almost alone in recording an increase of these in his own case. Mégeraud found the excretion of urine augmented under impure digitalin (Homolle and Quevenne), but much more so by very small doses of the crystalline 
form: with powdered digitalis it was, however, lessened, probably because the dose was too large ( 6 grains), and toxic symptoms occurred, e.g., vomiting, and an intermittent pulse of 40 : the urea was diminished notably in all the experiments (Gaz. Hebd., 1870).

Glandular System, Skin, etc.-On other glands, the usual result seems to be increase of secretion: thus, salivation has been recorded, but is not constant; increased secretion from the nasal mucous membrane has been noticed; sweating, after full doses, is generally profuse for a time, though an opposite condition with coldness of surface occurs before a fatal termination; lachrymation is often copious at first, and later on scanty, so that the conjunctiva becomes dry; the Meibomian glands are inflamed, and agglutination of the eyelids during sleep is common. Ferber attributes to digitalis the production of a rash resembling erysipelas (Gaz. Méd. de Strasb., 1862), and two instances of it may be found amongst Traube's observations, but they are not free from doubt; a red rash appeared some days after the drug was omitted; there was no definite febrile reaction connected with it (Med. Times, ii., 1878). Another case is given by Schmidt (Bull. de Thérap., t. xli.).

SUMmaRy OF ACTION.-Pulse.-Small doses slow the pulse, making it also intermittent: large doses make the pulse frequent and feeble.

Arterial Tension.-Moderate doses increase tension, mainly by contracting the arterioles: toxic doses lower tension by dilating them.

Heart.-Moderate doses strengthen and slow the beat, prolonging the systole and diastole, the impulse becoming abrupt (v. p. 593). In frogs the bent is irregular, sometimes slowed, sometimes quickened; final arrest occurs in firm systole.

Respiration.-Accelerated, and then diminished.

Nervous System.-S'mull dloses (continued) cause giddiness, headache, dimness of vision, etc.; sometimes hallucination and delirium. Large doses cause semi-coma, with muscular weakness, and sometimes twitchings: also lowering of temperature. 
Digestive System.-Small doses sometimes cause symptoms of indigestion ; toxic doses, vomiting and purging.

Urinary System.-Moderate doses cause diuresis (slight) with increase of urea (Brunton); the action is commonly more marked in anasarca, but is not constant. Large doses cause suppression in man.

SYNERGISTS.-There are no exact analogues of digitalis, but ergot and perhaps strychnia stimulate muscular contraction somewhat in the same manner. Veratria, colchicin, squill, nitre, and bromides have a similar depressing action on the circulation and sometimes on the kidneys.

Amongst less known synergists are convallaria majalis, adonis vernalis (Botkin), and casca. Dr. C. Allbutt finds tincture of Virginian prume a useful substitute in some cases (Med. Times, i., 1867).

INCOMPATIBLES.-Tannin, quinine, lead, and iron are chemically incompatible, but the last especially is often combined for therapeutical purposes: warmth, opium, iodine, coffee, and diffusible stimulants antagonize its depressing effects. Aconite has some physiological antagonism, but belladonna is safer to use. Saponin, which is allied to senegin, is said to be a still more direct antidote.

In a severe case of poisoning by digitalin the vapour of ammonia to the nostrils proved of much value (Lancet, i., 1880).

ThERAPEUTICAL ACTION.-Cardiac Disease.-Valuable as digitalis is in many disorders of the circulation, it can, when injudiciously prescribed, cause serious danger, and hence needs careful study. It may be well to observe, as a preliminary, that it is particularly adapted to persons of a sanguine or indolent temperament, with soft, lax muscles, and light hair. Dr. Withering long ago expressed his opinion that "digitalis seldom succeeds with men of great natural strength, of tense fibre, warm skin, and florid complexion, nor yet with people of tight and cordy pulse."

It is indicated (1) when compensatory bypertroplay is insufficient, especially in mitral disease, with dilatation of the left ventricle, when the cardiac muscle is unequal to the demands 
upon it, and the contractions are irregular and weak : this drug is then our best "heart-tonic," it increases the force of the ventricular systole, and truly deserves the title "quinine of the heart" (Beau). (2) In cardiac pain and palpitation connected with the same conditions in which its virtues are such that others have named it the "opium of the heart"-(palpitations and cardiac irregularities dependent on anrmia and impaired nutrition are better treated with chalybeates, food, etc.).

Mahomed has published good sphygmographic observations illustrating this (Med. Times, ii., 1872), and other illustrations are in B. M. J., ii., 1868.

Digitalis plays an important part in the treatment of symptoms originally associated by Stokes with "weak heart," and referred to fatty and granular conditions of its museular tissue, also in "temporary cardiac conditions of asthenia" from exhaustion, and in failure from shock-ammonia or nux-vomica may be combined with it. It is curious to recall the fact that formerly this drug was held to be very useful in hypertrophy, but dangerous in dilatation and in muscular weakness of the heart.

To Traube we owe the credit of pointing out that the power of digitalis to correct disturbances in the circulation, caused by organic disease of the heart, is constantly referable to mechanical conditions; and that this is especially true in regard to the wide-spread venous hyperæmia which comnonly accompanies such disease. The venous hyperæmia is dependent on general anamia of the arteries, and originates when hypertrophy causes insufficient compensation, and when the heart is no longer competent to drive a sufficient supply of blood into the aorta. Two physiological effects of digitalis then become remedial - namely, the increase which the drug produces in the force and regularity of the heart's contractions, and the increase which it causes in the contractions of the smaller arteries. Its action is therefore most effective in cases of insufficiency of the mitral valves. In disease of the aortic orifice, compensation by hypertrophy continues to take place for so long a, period that its use may be dispensed with. Niemeyer remarks that "in digitalis we possess a very powerful means of moderating, not only hyperæemia of the lungs, but also the blood-strsis of 
the greater circulation which arises in mitral disease." "If we can succeed," he continues, "in retarding the action of the heart by means of digitalis, we afford time for the auricle to drive its contents into the ventricle through the contracted passage" (Text-Book of Practical Medicine, i., p. 35̃ ).

In simple aortic obstruction with laboured, feeble, or irregular action, or pain and excitement without reflux, and without much hypertrophy, digitalis may be very useful. Dr. Fothergill has pointed out that in some such patients, especially when the obstruction has suddenly arisen, it may even be right to disregard the occurrence, during the administration of the drug, of intermission of the heart's pulsations, except as a reason for increasing the dose, upon which we shall find relief given to the cardiac symptoms. Here the failure of the heart has not been really due to the drug, but to the disease, hypertrophy not having been sufficiently established to do the work of compensation without powerful artificial assistance.

When there is marked aortic insufficiency, attended, as it usually is, with great hypertrophy, the drug is, as a rule, not well borne: theoretically also, at least, there is the danger of exciting a tetanoid contraction, from which the heart might not recover. Even here, however, it is not always the case that hypertrophy is developed in an excessive, or even in a sufficiently compensating, degree; and in instances such as I have occasionally witnessed, in which the inadequate hypertrophy made the overburdened left ventricle liable to sudden failure, with serious or fatal results, digitalis has proved of real service. There is not, in fact, so great a difference between its relations to mitral and aortic disease as was formerly laid down.

Ringer (Handbook) is right in saying that it is better to consider "all the symptoms than to confine the attention simply to the nature of the valvular affection;" and in the following picture represents the class of cases in which digitalis is most strikingly useful. He says-" There is dropsy, which may be extensive. The breathing is much distressed in the earlier stages of this condition only periodically, and especially at night; but when the disease reaches its worst strge, the 
breathing is continuously bad, although it becomes paroxysmally worse. The patient cannot lie down in bed, and is perhaps obliged to sit in a chair, with the head either thrown back, or more rarely leaning forward on the bed or some other support. The jugular veins are distended, and the face is dusky and livid; the pulse is frequent, feeble, fluttering, and irregular. The urine is scanty, high-coloured, and deposits copiously on cooling. The heart is seen and felt to beat over a too extensive area; and the chief impulse is sometimes at one spot of the chest, and sometimes at another. The impulse is undulating, and the beating very irregular and intermittent. The physical examination betrays great dilatation of the left ventricle, with often a not inconsiderable amount of hypertrophy. A murmur is ordinarily heard, having the characters of one produced by mitral regurgitant disease; and there may be also disease of the aortic valves."

As a general rule, it may be laid down that those cases of heart-disease will receive most benefit from digitalis in which there is weakness of heart-pulsation, irregularity (with or without dyspnœa), venous engorgement (with or without dropsy, but especially when accompanied with the latter), and a scanty secretion of urine. In such cases the drug induces very striking effects. The form which is to be preferred is the fresh infusion, made from good leaves; powdered digitalis, which was the form chiefly used by the German physicians in their researches on febrile diseases, is apt to vary greatly in strength. Of the infusion, from 1 to 2 drachms should be given every night and morning, and increased, if necessary, to a dose every three or four hours until a diuretic effect is produced.

Pfaff has laid down rather different rules for its administrntion, arguing that it is better to give large doses at first, and to diminish them gradually for eight or ten days, and then to omit the drug for a time and resume it later in a similar manner. I am not prepared to accept this method at present, but its object is partly to avoid what has been known as the "cumulative" action of the drug; and although there has been some misconception of this term, and it is not by any means specially applicable to digitalis (more than to mercury, strychnia, and 
others), yet it conveys this element of truth, - that by the continuous administration of small doses more is conveyed into the system than is eliminated, and, instead of "toleration" being induced, toxic symptoms steadily develop, until suddenly they culminate in a serious or fatal crisis: this is well exemplified in the following case of slow poisoning by the drug:-A German recruit, aged twenty-two, healthy, but desiring to escape military service, prepared pills containing each $1 \frac{1}{2}$ grain of the powdered leaf, and took two of them three or four times daily for four or five weeks. After the twentieth pill vomiting began, and symptoms of extreme gastric irritation and catarrh developed, such as pain, vomiting, thickly coated tongue, foetid breath, anorexia, etc. The pulse fell to 52; he became very weak and anæmic, ashy grey in colour, and complained of headache, giddiness, tinnitus, dimness of vision, hiccough, faintness, etc., having also some difficulty in swallowing; he stood up (for examination), and then fell in a faint; rallied, but later on attempting to rise became convulsed and died in a few minutes. Post mortem-Blood fluid and dark red; right heart full, left empty; brain anæmic; gastric and duodenal mucous membrane congested and ecchymosed in parts. The total amount taken was calculated at 246 grains (Lancet, i., 18r6).

Dr. H. C. Wood has some excellent remarks on the use of digitalis in cardiac disease. "When the muscle of the heart is for any reason unequal to its task, the systoles become rapid and imperfect, and by this irregular action the ventricles, neither completely filling nor emptying themselves. increase the embarrassment; then, digitalis, by lengthening the diastolic pauses, and increasing the force of the systolic contractions, causes the ventricles to fill themselves in the one act, and to completely empty themselves in the other. By subduing irregular action through inhibitory nerres, by energizing the muscular power of the heart walls, it is of incalculable service; and increasing arterial tension all over the body, it causes the disappearance or the lessening of symptoms due to low pressure in the arteries." Hence in simple dilatation, or simple failure of the cardiac muscle, it acts well; in simple hypertrophy, it does harm. In valvular disease with hypertrophy, however, it may be useful if the latter be not sufficient for the work required 
- "not compensatory enough" -but smaller doses than in dilatation will be required. In mitral stenosis, also in mitral insufficiency, it is commonly beneficial, though by a different mode. In the "irritable heart" of soldiers, connected, as it is, with muscular weakness, and probably dependent on exhaustion of inhibitory nerves, Da Costa found digitalis act better than any other remedy, -in early stages (Amer. Journ., Jan., 1871).

Its use as a cardiac stimulant in syncope or sudden collapse from hrmorrhage "is in its infancy." Dr. Wood states that from 20 to 30 minims of the tincture have been injected into the arm in such cases "with the most astonishing effects." This never caused severe local irritation, but $\frac{1}{50}$ grain of the digitalin has done so. In a later essay, Dr. Wood draws attention to two special points, viz., the cumulative, slowlyproduced effect (which he connects with slow osmosis), and the importance of watching the renal secretion-if this be not free, the former effect is apt to develop suddenly (Philad. Med. Times, 1881). Dr. Gibson corroborates the observations as to its value in collapse, and also as to what has been already stated in regard to cardiac disorder, and the importance of securing free diuresis as a guide to safe administration; but he goes so far as to strongly recommend the drug in fatty heart (Birm. Med. Review, 1881).

The more recent observations of Fernet deserve special attention. He agrees that whatever views be entertained of its physiological effect, practically its use is indicated for slowing and regulating the heart-action, and increasing the urine; as a rule, it is not wanted in aortic, but in mitral disease at the "asystolic" period-i.e., when the systole is imperfect (corresponding to Gubler's "cardiataxic disorder"): for absoIute weakness of heart (the "cardiaplegic state"), stimulants, tonics, and morphia are better: small doses act as well as large, without being dangerous" (Journ. de Thérap., Mai, 1882).

Dropsy.-The diuretic power of digitalis, as well as its influence on arterial tension, renders it of the greatest service in this disorder; and although the diuresis occur's sometimes before the circulation is eviclently altered, it is largely dependent upon such alteration. Thus, in dropsy from a 
dilated heart, "the renal gland-cells cannot act because they are not supplied with the proper kind and quantity of blood; their circulation, like that of other parts of the body, is nearly stagnant. "If, under these circumstances, digitalis be administered, and the circulation becomes comparatively free and active, the result is diuresis wrought out through a double mechanism, partly indirect, and partly produced directly by the drug" (H. C. Wood). In nearly all varieties of dropsy, except those where there is aortic regurgitation and very great eardiac hypertrophy, this drug is of the greatest service; it is wholly free, also, from the tendency to irritate the kidneys, by which many so-called diuretics defeat their own immediate end, and even do harm by congesting a kidney already diseased or mechanically compressed by ascitic fluid. In those distressing cardiac eases, with swollen discoloured face, general anasarca or dropsy, and breathing so difficult and oppressed that suffocation seems imminent, "give digitalis, and you will see a kind of resurrection" (Dujardin-Beaumetz). A tablespoonful of infusion twice daily till definite effects are produced, is often effective; but I have known it quickly cause vomiting, and have succeeded better with 1- or 2-drachm doses. Fernet also prefers the infusion in small doses; it often does not act for two or three days, and then some slowing of the pulse commonly precedes by fifteen to eighteen hours a diuresis, which itself commonly begins during the night: when once set up it continues for several days, and even increases for a time after the suspension of the drug (loc. cit.).

Digitalin has not been found to answer nearly so well in these cases, but $\frac{1}{2}$-drachm doses of the tincture night and morning have proved effective, and local applications of the same in liniment, or of moistened leaves over the kidneys or abdomen, have sometimes produced marked diuretic effects (B. M. J., i., 1868). The pordered leaf, combined with squill and blue pill, has a special reputation. The diuretic action of digitalis is also especially valuable in simple hydrothorax, and in those more passive kinds of pleuritic effusion which most nearly approach the character of dropsy.

In all such cases there is a very good prospect of effecting 
speedy resorption of the fluid by the use of digitalis in the manner just described. In post-scarlatinal dropsy, due to acute and more or less complete suppression of urine, dependent on a catarrhal inflammation of the uriniferous tubules, digitalis is probably superior to other drugs. A potash salt is commonly combined with it, and sometimes iron, but the latter is better reserved for subacute or chromic conditions.

In chronic Bright's disease it is a valuable resource, though the malady is of such a nature as not to admit the same improvement as cardiac dropsy.

In Febrile Diseases, digitalis has been largely employed of late years, especially in Germany. Traube's original paper $(1850)$ asserted, that, in the first place, digitalis as a stimulant to the regulating cardiac nervous system, diminished the lateral pressure in the blood-ressels, and also the rapidity of the blood-current, and simultaneously lowered temperature: and, secondly, that by virtue of these actions, it limited the inflammatory process. As already shown, Traube was probably right in ascribing part of the action of digitalis to its influence on the vagus, but it is impossible to account for the whole of its effects in this manner; and in two important particulars his views were certainly wrong:-(1) The arterial pressure is not lowered, but heightened; (2) although the pulse is slowed by moderate doses of digitalis, there is reason to think that the blood-current is rendered more rapid-a phenomenon which, since the researches of Edward Weber and of Ludwig, is easily intelligible. The statement, however, as to the influence of digitalis in lowering temperature, and diminishing the intensity of inflammatory and febrile processes, at least for a time, proved correct, and since that date the treatment of pyrexial cliseases by digitalis has become very widely spread on the Continent, and has found a few followers in this country.

Typhus and Typhoid Fever.-The reduction of febrile temperatures by means of the cold bath has, it is true, been advocated as better than the use of drug's; but many who have adopted the bath-treatment insist on the great value of digitalis as an auxiliary-as, for instance, Liebermeister, in his trentment of typhoid fever in Basle. The doses given have been very large. Traube commonly employed as much as 2 grammes ( 31 grains) 
of the powdered digitalis-leaves in twenty-four hours, but such doses must be considered excessive, and they probably increased the mortality. E. Hankel (Arch. d. Heilkunde, 1869) reports that in an epidemic of true exanthematic typhus observed in the Leipzig Klinik, eighty cases of the disease were treated, the ordinary dose of digitalis being 47 to 62 grains per diem, but as much as 100 grains being occasionally given. There were thirty-five deaths; and although he attributes this immense mortality to the type of the disease being uniformly severe, twenty-three cases being admitted in a moribund condition, there is nothing remarkably successful in a mortality of 15 per cent.; and we cannot but suspect that some patients may have been fatally affected by the large quantities given. It is hard to draw definite conclusions from Hankel's report; but we may note that as regards the remote influences of digitalis-such as its power to check delirium-only the milder cases seemed to benefit; also, that the patients treated with this drug emaciated greatly, and made slow convalescence.

Wunderlich especially recommends it in the second week of typhoid fever, when the temperature is high, and the pulse rapid; two or three days of the treatment often produces a fall of $2^{\circ}$ or $3^{\circ}$ (Fahr.) of the temperature, and thirty to forty beats of the pulse: he gave only $\frac{1}{2}$ to 1 drachm of the powder in divided doses during three to four days (Med. Times, 1862).

In Erysipelas, digitalis was recommended by Traube; and there is not only much practical evidence in favour of its use, but some antecedent probability that it may do good in this complaint by controlling the amount of blood in the smaller arteries. Many of the objections to digitalis in erysipelas may have been based on the use of improperly large doses; but it has not superseded better known remedies.

In Rheumatic Fever, digitalis has been employed by various authorities-by Hirtz, of Strasburg (Gaz. Méd. de Strasbourg, 1862), by M. Oulmont, and by Traube. The first two used an infusion of $15 \cdot 6$ grains of digitalis to about 4 ounces of eau-sucrée, which, in divided doses, was the allowance for twenty-four hours-a quantity much smaller than that employed by most German authorities. Twenty-four patients were taken indiscriminately, being the whole number 
that presented themselves during a certain period of time. No effect was produced on the pulse or temperature till after about thirty-six hours, when in simple and non-complicated cases, ${ }^{1}$ a sensible fall of the pulse took place, and soon afterwards a decline of the temperature; these continued till the third or fourth day, when nausea and vomiting ensued, and the next day the pulse fell from twenty to forty beats, and the temperature from $1 \frac{3}{4}^{\circ}$ to $21^{\circ}$ (Fahr.). The digitalis was then suspended; but the lowering of pulse and temperature persisted for several days; the symptoms of the disease disappeared, sometimes gradually, and sometimes with surprising rapidity. A few cases were tardy in recovering, and a few had relapses; but, on the whole, those of pure febrile rheumatism did well: several were cured in five or six days, and at the end of ten days left the hospital.

Oulmont, however, states clearly that digitalis was only of use in the primary period of simple joint-fever. When relapses took place, it seemed to effect no real good, although the characteristic stomach symptoms were evoked as before; and in cases complicated with visceral inflammations, it only produced a temporary and unimportant lowering of pulse and temperature. On the other hand, he had reason to think that it tended (used early enough) to avert cardiac complications. When old cardiac disease existed prior to the commencement of the rheumatic attack, its influence seemed to have been favourable in rendering the pulse regular and strong; but in one instance of extensive valvular mischief, where the febrile excitement recurred several times, the patient was seized, at the moment of the fall of the pulse, with an attack of suffocation, palpitation, and precordial anxiety, and seemed in great danger, though the paroxysm soon passed off. Oulmont speaks of this as a reason for caution in administering digitalis in instances of old and advanced heart-disease; but does not distinguish between different valvular affections, nor does he state whether this particular patient was the subject of excessive hypertrophy or not. His general conclusion as to the action of digitalis in febrile rheumatism, is that it benefits solely in so far as it relieves the febrile state; that in first attacks, and in

By this is meant, not complicated with visceral inflammations. 
cases of a simple type, it shortens the febrile period, averts cardiac and cerebral complicatious, and hastens the convalescence; but that it does not touch the cases where the malady is more deeply rooted, and is either complicated with serious internal inflammation, or tends to repeated relapses of the ferer and the joint-affection.

In Acute Inflammations, digitalis is considered by some authorities one of the most powerful direct remedies. We cannot, however, accept as proofs certain observations, where the alcoholic tincture has been used in very large doses; for example, those of $\mathrm{Mr}$. King, of Saxmundham, who held that by single $\frac{1}{2}$ - to 1-ounce doses of tincture, at twenty-four hours' interval, it was possible to cut short acute inflammations, if the remedy were administered before the organs involved became disorganized (quoted by Ringer).

The influence of Traube, Thomas, Hirtz, and other physicians has, on the whole, kept up a high reputation for this drug in such inflammations as the following:-

In Pericarditis, wherein also Niemeyer strongly recommends digitalis when the heart's action is rery rapid and feeble, and there are cyanotic and dropsical symptoms (Practitioner, i., 1868); but I think it rather a dangerous remedy.

In Pleurisy, with high fever, he advises the drug in infusion (10 grains to 1 ounce), whilst in more chronic and less febrile cases, he gives the powder in 1-grain doses, with equal parts of quinine. In the first stage of pericarditis and of pleurisy, my own experience leads me to prefer aconite, as it reduces the pulse and temperature more quickly than digitalis.

In Pneumonia, Lissauer showed its value (Thesis, Berlin, 1856). Bleuler made extensive trials of it, employing about $\frac{1}{2}$ drachm of the powdered leaf (in the form of infusion) daily; this was continued until a decided impression was produced on the pulse; romiting usually set in at the same time. The influence on the mortality seems to have been unfavourable, amounting to 21 per cent., as against 14.5 per cent. with merely expectant treatment; but Bleuler hesitates to draw a positive conclusion from this fact. In the patients who recovered, he was unable to trace any distinct influence of 
digitalis in cutting short the inflammatory process; the defervescence occurred as early as from the fifth to the seventh day, and both Bleuler and Niemeyer, who comments on Bleuler's cases, regard this as being unusually early. But, according to English authorities, this is by no means an uncommon date for defervescence, especially in single pneumonia; everything, therefore, depends on the question whether Bleuler's cases were of a high average severity. He records rather frequent toxic phenomena occurring after the fall of the temperature; but these disappeared as soon as the medicine was discontinued, and convalescence was never delayed.

Niemeyer employed digitalis in pneumonia when the pulse ranged from 100 to 120 , in combination with nitrates of potash and soda; he considered that it reduced the fever, but did not alter the plastic processes.

Wore recent writers express a somewhat similar view. Wood finds it useful in adynamic pneumonia (in fever) for "maintaining the heart's action," not that it can affect the disease itself. Joly, reporting a number of satisfactory cases from a French marine hospital, recommends it "to combat rise in temperature," but for the debility prescribes alcohol. Vulpian, however, maintains that it lessens the phenomena of tissue-combustion.

The approval of so cautious an observer as Ziemssen is of importance: he gives of an infusion (5 grains to 1 ounce water), a teaspoonful every two hours, or about the equivalent of $\tau_{\frac{1}{3}}$ grains daily (for ehildren); and although this occasionally produces intermittence of pulse, he does not find that symptom of itself dangerous.

To quote one more affirmative authority, I may mention the strong testimony of M. Rony-Sancerotte, who, in 35 carefully observed cases of pneumonia, found that cligitalis reduced the fever and kept down the graver symptoms, while intolerance of the drug was very rare (Practitioner, ii., 1869). He remarks that the antiphlogistic operation is less rapirl than that of leeches, but more lasting, and nenrly the same with all patients.

On taking the balance of the statements made by various anthors as to the action of digitalis in puemonin, we may conclude that a good case is made out for continued 
trials of it, and, although it has not come into general use in this country, the statement of Stillé, "that favourable opinions as to digitalis in inflammations are unsupported by direct evidence," is scarcely justified.

It has been applied, in some instances, with remarkably good effect, as a remedy for local inflammations: thus, in orchitis, by Dr. Besnier, who was led to use it through having seen its success in hydrocele (Bull. de Thérap., Fér., 1870). He kept the patients in a recumbent posture, with the scrotum conveniently raised, and enveloped in compresses soaked in a strong infusion, either warm or cold, and covered with oil-silk.

In concluding my remarks on digitalis in inflammation, it may be observed that it is still a question whether the beneficial effects of the drug in febrile disorder can be produced without nauseating and depressing the patient. Alison asserted that the latter result could not be avoided, if the drug were given in sufficient doses to produce an impression with the rapidity required in acute disease (Outlines of Pathology, p. 243). Most recent observers insist, also, on the use of large doses, and, in their writings, nausea and vomiting are treated almost as matters of course: but Alison estimated too highly the danger of this state of things, for the German observations have proved that even marked intermission of the pulse is seldom followed by serious consequences, and that doses ten times larger than any that Alison referred to were given with impunity.

In Hæmorrhage of various kinds, digitalis has, during the last few years, assumed an important place. In metrorrhagia its efficacy was, I believe, first proved by Dr. W. H. Dickinson, who found that doses of 1 to $1 \frac{1}{2}$ fluid ounce of the infusion speedily arrested hæmorrhage of this description, when unconnected with organic disease (Med. Times, ii., 1855). He treated a great number of menorrhagic patients in this way, and never failed to arrest the bleeding by the second or fourth day, according to the quantity of the drug given; thus demonstrating that large doses acted very much better than the small quantities which had previously been held in doubtful repute for the same purpose. His statements were afterwards fully confirmed by Dr. Barclay and Dr. Tilt (Handhook of Uterine Therapentics), and also by Troussean, and it has 
been shown that even in organic disease of the uterus the drug has some power to arrest hæmorrhage, for M. Decaisne treated a case dependent on fungoid growths with complete success by a daily dose of 6 granules of digitalin $(0.0156$ grain in each).

In Hæmoptysis, digitalis has been praised by many writers. Dr. Brunton states that, as soon as the characteristic slowing: of the pulse is produced, the bleeding from the lung is stopped, and speaks of it as the most powerful remedy for hæmorrhage from cavities. Dr. Fuller recommends it (in conjunction with dry-cupping, and ice to the spine) in full doses, e.g., 1 to 2 drachms of tincture, or 6 to 8 grains of the powder, daily. It may be said, perhaps, that these are scarcely what we should call "full doses" at the present day, but, from experience with other remedies, as well as with digitalis, it would appear that smaller doses of hæmostatic medicines are required to check hæmorrhage from the lungs, than to arrest uterine bleeding. Most authors affirm that the bleeding stops when the slowing of the pulse has been produced, but it is not proved that the mere reduction of frequency of pulsation is the active agent; it is more probable that the cessation of hæmorrhage is chiefly due to the contraction of the smaller arteries, and the consequent prevention or diminution of venous stagnation.

In Epistaxis, digitalis, especially in the form of infusion, appears to be as efficacious as in pulmonary or uterine hæmorrhage. This is markedly the case in the bleeding from the nose which sometimes complicates acute rheumatism, and is occasionally so severe, if unchecked, as to greatly confuse the patient and delay his convalescence. Indeed, in all cases of epistaxis, except those which depend upon a general hæmorrhagic tendency, as in scurvy, in purpura, and in the rarer diathetic disease "hæmophilia," digitalis appears to be a prompt and effective remedy. It should be given in one or two large doses, $\frac{1}{2}$ ounce of the infusion, repeated twice, if necessary, at half-hour intervals. If, however, the bleeding has ceased for the time, smaller doses, or 10 minims of tincture every four or six hours, are suitable, especially if the cardiac or general conditions, e.g., dilatation or ocdema, also indicate the drug.

Sugden has recently again advocated it in many forms of hæmorrhage (Record, 1881). 
In Nervous Diseases, digitalis may be made very useful; but the subject has not yet been accurately inquired into. Speaking generally, it would appear that its effects upon the nervous system are strictly dependent on its influence on the circulation, and yet there are certain instances of its acting in a localized manner, not easily explained as a circulation-effect, e.g., in relieving local nerve-pain. Fuller recommends it on this ground for sciatica of pure neuralgic type, and Lehmann speaks of it as an effectual remedy in earache (Amer. Journ. Med. Sci., v., p. 34).

There are many facts, also, which show its sedative action on the "nerves of the sexual apparatus. Brugmans found that this was exerted in both sexes, and in a variety of complaints (Journ. de Méd. de Bruxelles, Nor., 18533). Possibly these effects are partly due to the reduction of the quantity of blood in the organs, an idea to which some probability is given by Dr. E. Mackey's observations on the relief to the congestion and pain of hremorrhoids which is produced by digitalis (B. M. J. i., ii., 1868); for there is much sympathy between the disturbances of circulation in the lower bowel and in the genitalia.

But there is reason to think that digitalis also acts upon the sensation of different parts through the spinal cord, perhaps through the vaso-motor centre in the medulla oblongata.

Its generalized action is seen in the (moderately) successful treatment of intermittents, which I do not include under the section of digitalis in fever, properly so-called, as it stands on a very different footing from the positive effects which have been obtained in typhoid, etc.

Of such localized sedative action, perhaps the most useful instance is in spermatorrhca, in which complaint it was recommended by Corvisart (Bull. de Thérap., xi., 4, p. 18), and has been approved by Ringer (Handbook), who says few remedies are more successful-referring to over-excited and plethoric subjects, not to the anmmic and enfeebled: he recommends 1 or 2 drachms of the infusion twice or thrice daily. In acute gonorrhoea it has been specially advised by BérangerFérand, not as a specific, but for its anti-inflammatory powers and its action on the capillaries. Young and strong subjects, 
seen early in their first attack, were generally cured soon; in bilious nervous subjects, suffering for several days, it did not answer so well; whilst in the wealily, lymphatic, or those who had suffered before, it was not of much use.

The therapeutical effects of digitalis upon cerebral affections are produced, there can be little doubt, through modifications in the cerebral circulation.

In Delirium Tremens, the bold experiments of the late $\mathrm{Mr}$. Jones, of Jersey, first drew attention to this remedy (Med. Times, ii., 1860). He was accustomed to give $\frac{1}{2}$-ounce doses of the tincture at first, and 2-drachm doses subsequently, till calm and sleep were produced; and his facts have been substantially confirmed by the carefil observations of Dr. Peacock (ibid., ii., 1861), who considers the drug chiefly applicable to young and robust persons; obviously, however, this treatment is scarcely a digitalis-treatment, for, as Dr. Anstie observes, the $\frac{1}{2}$-ounce doses of proof spirit were probably of more consequence than the drug (Art. "Alcoholism," Reynolds's Syst., ii.). It is otherwise, perhaps, with the tincture in 20- or 30-minim doses, more frequently repeated, as advised by some; but no evidence can be reckoned satisfactory, except from the use of the infusion of the powder, or of digitalin, and at present it is too scanty for positive conclusion.

Acute Mania.-In various forms of this disease digitalis has been strongly recommended by Homolle and Quevenne, ${ }^{1}$ Lockhart Robertson, ${ }^{2}$ Maudsley, ${ }^{3}$ Blandford, ${ }^{4}$ Van der Kolk, and others; and in a considerable proportion of cases bids fair to supersede the old and dangerous routine administration of opium. It is tolerably certain that the chief proximate cause of excitement and sleeplessness in affections of this kind is the irregular and ill-balanced state of brain-circulation; and for this digitalis can supply a remedial influence which opium cannot. Neither is digitalis a remedy, as formerly thought, too depressing to be employed in acute mania which oncurs in persons of feeble health. On the contrary, some remarkable instances have been observed, in which the maniacal

1 Gaz. des Hôp., 1850, No. 53; Union Méd., 1851, Nos.69, 70.

${ }^{2}$ Journ. Mental Science. ' Practitioner, i., 1869. 'Pructitioner, i., 1869.

${ }^{5}$ Pathol, and Therap. of Mental Diseases. Linglish Trans. 
patients were the subjects of certainly weak and probably fatty heart, and yet the use of digitalis in tolerably full doses was followed by a simultaneous strengthening of the pulse, which became regular, and by a subsidence of the maniacal symptoms. In fact, we see with digitalis, as with opium, that the old theories of action of the two drugs led to conclusions opposed to fact, and practically mischievous; a remark which applies especially to the views as to their employment in acute insanity, which prevailed during the first half of this century.

Neuralgia.-As a direct remedy in this disorder, notwithstanding the evidence of Fuller respecting sciatica, and the more dubious observations of Debout and Serre, respecting migraine, digitalis is not to be relied on (Syd. Soc. YearBook, 1861). According to my own experience, it succeeds only casually and rarely, if at all, and is not worth naming in comparison with the powerful agents which we now use. In those forms of migraine in which there is evidence of great vaso-motor disturbance at the time of the attacks, and especially in those cases where there is an evident relation between the attacks and the commencement of each menstrual flow, it might be worth while, nevertheless, to make careful trial of digitalis, pushing the doses rather fully. Experience has shown that this form of migraine is not without its serious dangers, one or two recorded cases having terminated in fatal cerebral hæmorrhage; and it would, therefore, be desirable, upon the appearance of threatening symptoms, to try the effects of tolerably full doses of digitalis as a means of regulating the brain circulation.

PReParations and Dose. - Pulzis foliomm: dose, $\frac{1}{2}$ to $1 \frac{1}{2} \mathrm{gr}$. Digitalinum: dose, $\frac{1}{60}$ to $\frac{1}{30} \mathrm{gr}$. Infusum (3 $\mathrm{gr}$.

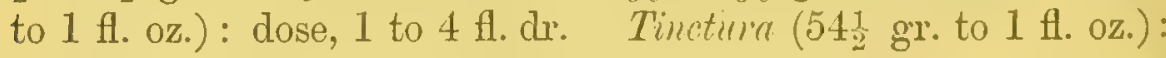
dose, 5 to 30 min.

Adulterations.-Occasionally the leaves of the Inula Conyza, formerly conyza squarrosa, have been employed. This plant is a hard, rough, and erect biennial, 2 to 3 feet high, of the order Composite, and common in the limestone 
districts of England. The leaves, though similar in form to those of the foxglove, and tufted in the same manner, are rougher and much less toothed, and are at once distinguished by their odour, which some call fœetid, others aromatic. The leaves of the verbascum thapsus are also sometimes mixed with those of foxglove.

It may be worth observing that the leaves of the common yew tree are reputed to exert a power similar to that of digitalis; and that in the West Indies the leaves of the Tephrosia toxicaria, a leguminous shrub, appear well adapted for a substitute.

\section{LEPTANDRA VIRGINICA. (BLACK Root.)}

\section{(Not Officinal.)}

DESCRIPTION.-A plant indigenous to Canada and the United States, having an angular stem 3 to 6 feet high, with whorls of four to five lanceolate serrate leaves, and panicled spikes of white flowers. The dried rhizome used in medicine is 4 to 6 inches long, $\frac{1}{4}$ inch thick, blackish, with cup-shaped scars on the upper side, and on the lower numerous rootlets, which are generally broken; it has no characteristic odour, but a bitter slightly acrid taste.

ACTIVE INGREDIENTS.-Besides tannin, resin, and a volatile oil, there is an active, bitter, crystallizable principle of glucoside character, soluble in water, alcohol, and ether: this may be properly termed leptandrin, although that name is commonly given to the precipitate induced by adding water to a concentrated tincture: it is the so-called "eclectic" preparation, and is a dark greenish-brown powder.

Physiological Action.-Digestive System.-The full action of the drug is marked by strongly irritant symptoms -vomiting and purging, with increase of biliary, and probably 
of the other secretions. It is presumed that small doses act in a less degree in the same direction. Dr. Rutherford reported it as a "moderate hepatic, but feeble intestinal stimulant."

THERAPEUTICALly it has been given in 1-grain doses as cholagogue and alterative, alone, or better combined with allied drugs, such as podophyllin, euonymin, etc.; 2 grains are commonly aperient (B. M. J., ii., 1876).

PREPARATIONS AND DOSE.-Leptandrin (the powdered extract is commonly prescribed rather than the true active principle): dose, $\frac{1}{4}$ to $2 \mathrm{gr}$. Extractum fluidum: dose, 20 to 60 min.

\section{DAMIANA.}

A name (Indian) used in commerce for the leaves of certain Mexican plants:-(1) Turnera microphylla v. aphrodisiaca (Turneraceæ) : this has alternate obovate leaves, with three to four teeth on each side, green, rough, and covered with white hairs: the taste is aromatic, somewhat like mint. (2) Aplopappus discoideus (Compositæ) has lanceolate leaves, with usually three short teeth above on each side, coriaceous, with some black dots and resin-like scales, recalling somewhat the leaves of buchu: the taste is "balsamic."

ACtIVE INGREDIENTS.-(1) A volatile oil present in small proportion ( 0.2 per cent.), of turpentine character; (2) a soft brown oleo-resin, acrid, and probably irritant, soluble in alcohol; (3) an amorphous, light brown, bitter substance, soluble in water and alcohol; and (4) a tannin-like acid : there is also a hard, tasteless, insoluble resin, which is probably inactive, and much sugar (Parsons, New Remedies, Sept., 1880).

Physiological Action.-Genito-Urinary System.Certain American writers have recently brought forward 
damiana as having a specific effect in stimulating this system. Thus, Dr. J. T. Caldwell (Baltimore) " is well satisfied, from extended experience, of its powerful influence over the genitourinary organs of both sexes, as in moderate doses it increases the flow of urine as well as the sexual appetite" (Virginia Monthly, N.Y. Record, ete.). He states also that it is a general "nervine" tonic, and that Indian hunters use it to enable them to go through exhausting journeys: its action on the borvels is somewhat aperient, not astringent. Dr. Woodward describes the drug as a "stimulant to the brain, especially to the basilar portion"; also to the sympathetic nerve and the reproductive organs (New Preparations).

Dr. C. G. Polk, whilst calling it a "sexual tonic," states that "it is not a direct stimulant of erotic desire, but acts as a calmative to abnormal sexual appetite, and immediately and directly impresses the nerve-centres which preside over respiration and nutrition" (ibid.). These and other writers report illustrative cases; but, on the other hand, Stillé dismisses the remedy in these words- "Damiana appears to have tonic and laxative qualities. It has been widely and boldly advertised as a remedy for sexual impotence or indifference in either sex; but there is not the slightest reason for confiding in this statement of its virtues. Still less can it be credited with the restoration of an atrophied testis to its nomal size and function, which has been set to its account" (Dispensatory, Ed. 1879). Mr. Parsons refers to three cases of " disordered stomach, with attendant sick headache," apparently cured by the infusion which contrins the oil, the bitter, and the mucilage, without the irritant resin : he prepares it simply by pouring a teacupful of hot water over a teaspoonful of dried leaves.

PREPARATIONS AND DOSE.-Infusim: dose as above. Extructum liquidum: dose, 30 to $60 \mathrm{~min}$., and upwards. Extructum: dose, 7 to $10 \mathrm{gr}$. Elixir: dose, 1 to 2 dr. 


\section{LABIATAE (OR LAMTACEAE).}

\section{THE SAGE FAMILY.}

A LARGE, important family of perigynous exogens, the number of species amounting probably to 2,500. They occupy most of the temperate parts of the globe, and are especially abundant between the parallels of $40^{\circ}$ and $50^{\circ} \mathrm{N}$. L. Almost all are herbaceous or suffruticose; the young stems are generally square; the leaves invariably opposite, exstipulate, and simple; the flowers monopetalous, labiate, and bisexual; the stamens usually didynamous; the superior and four-lobed ovary ripens into four independent achænia, which lie at the bottom of the persistent calyx. No deleterious plant occurs among the Labiates; many are strong-scented, and many others aromatica property depending on a volatile oil, contained in superficial cysts.

\section{Phamacentical Species. \\ Species.}

Lavandula vera

Mentha viridis .

Mentha piperita

Mentha pulegium

Rosmarinus officinalis

Thymus vulgaris
. Lavender.

. Spearmint.

- Peppermint.

. Pennyroyal.

- Rosemary.

Common thyme.

\section{LAVANDULA VERA.}

(LAVENDER.)

DESCRIPTION.-A shrub indigenous to the hills of Southern Europe, growing 1 to 3 feet high, and well-clothed with oblonglinear and persistent leaves of a grey-green colour. The little blue flowers are clustered in spikes 2 inches in length, terminating long and slender stalls. 
ACTIVE INGREDIENTS.-The flower-spikes, which alone are officinal, contain about 1 per cent. of oil, combined with a bitter principle. The former is an oxidized volatile hydrocarbon $\left(\mathrm{C}_{10} \mathrm{H}_{10}\right)$, and contains in solution a camphor-like substance called stearoptene. It is pale yellow; the specific gravity varies, but is usually about 0.95 , the lightest oil being the purest. It is fragrant, of a pungent and aromatic taste, soluble in rectified, and in two parts of proof, spirit.

Physiological Action.-Lavender oil is, in drachm doses, a narcotic poison, causing in rabbits death preceded by convulsions (Fraser, Edin. Med. Journ., 1880). Like other essential oils, it irritates mucous membranes, is contra-tetanic, anti-spasmodic, and diminishes reflex sensibility. It is also a cardiac stimulant, though in large doses a paralyser of the heart's action. It improves digestion, but may cause gastritis. It is antiseptic, and a local anæsthetic, paralysing the terminals of the sensory nerves, and is an effective poison to skinparasites.

THERAPEUTICAE ACTION.-The spirit and tincture are given in cases of hysteria, nervous headache, hypochondriasis, and flatulence. It is well to remark here that the compound tincture of lavender has considerable intoxicating powers, and is frequently used by persons who would hesitate to take spirits or wine for the purpose. In dealing with cases where we have reason to suspect alcoholism, and the use of ordinary stimulants appears to be nil, we should bear this in mind. The leaves and flowers have been employed in sternutatories.

PREPARATIONS AND DOSE.-Oleum: dose, 2 to $5 \mathrm{~min}$. Spivitus: dose, $\frac{1}{2}$ to $1 \mathrm{fl}$. dr. Tinctura lavandulce composita : dose, $\frac{1}{2}$ to $2 \mathrm{fl}$. dr. The oil is employed for scenting evaporating: lotions, ointments, and liniments. It is used in the preparation of the compound liniment of camphor, and the compound tincture is a constituent of the liquor arsenicalis.

AdULTERATIONS.-Oil of spike, prepared from the Lavandula spica, or French lavencler. 


\section{MENTHA VIRIDIS.}

(Spearmint.)

DESCRIPTION.-An herbaceous deciduous perennial, native of all the milder parts of Europe, and of many other regions. Creeping-rooted; the square stems are erect, 12 to 20 inches high; the leaves are ovate-lanceolate, about 2 inches in length, subsessile, serrated, glabrous, and bright green; the small and purplish flowers are produced in terminal and cylindrical spikes. The odour of the herbage is strong and agreeable; the taste pleasant, aromatic, and somewhat bitter.

ACTIVE INGREDIENTS.-Dpearmint owes its general properties to a volatile oil, believed to be a hydrocarbon, and containing a peculiar camphor in solution, as also a substance isomeric with carvol. This oil is lighter than water, sp. gx. being 0.914 , boiling-point $320^{\circ}$ Fahr.; the odour is strong and pungent; the taste is the same as that of the living plant; in colour it is pale yellow, but with age becomes reddish. An oxygenated oil $\left(\mathrm{C}_{10} \mathrm{H}_{14} \mathrm{O}\right)$ has also been described, and is said to be the source of the odour; boiling-point, $473^{\circ}$ Fahr.

Physiological Action.--Stimulant and carminative.

THERAPEUTical ACtion.-Like lavender, spearmint is usefully employed in flatulence and colic, and is a good adjumct to purgative medicines: 1 or 2 drops of the oil added to an ounce of iodoform ointment conceals to some extent the unpleasant smell.

PREPARATIONS AND DOSE.-Menthe viridis oleum: dose, 1 to 5 min. This should be given rubbed up with refined sugar, in a little water. Aque menthe viridis: dose, 1 to $2 \mathrm{floz}$. 


\section{MENTHA PIPERITA.}

(Peppermint.)

DESCRIPTION.-Peppermint resembles spearmint in all its leading characters, but is distinguished by the leaves being broader and more distinctly petiolate, and by the terminal and cylindrical spikes of small purple flowers being interrupted at the base. It is a plant of extremely wide diffusion, and is the most agreeable of its genus in flavour.

ACTIVE INGREDIENTS.-The taste and odour, as in the other species, are due to an oil which, on being distilled from the recent plant while in bloom, possesses a peculiar and penetrating fragrance, with a warm aromatic flavour, followed by a singular sense of coldness in the mouth. The sp. gr. is 0.92 , the boiling-point $365^{\circ} \mathrm{F}$. While new, this oil is pale greenish-yellow, but with keeping the colour deepens. Two isomeric constituents are found in it, one liquid, the other solid. The solid one makes its appearance when the oil is reduced to a temperature of $12^{\circ}$ or thereabouts, and receives the name of "peppermint-camphor" or menthol $\left(\mathrm{C}_{10} \mathrm{H}_{19} \mathrm{HO}\right)$, homologous with thymol. It presents itself in the form of acicular white crystals, which retain the taste and odour of the plant, and are fusible at $92^{\circ} \mathrm{F}$. Menthol is commonly obtained in China from the mentha arvescens. By the action of chloride of zinc, peppermint-camphor is decomposed, with production of menthin $\left(\mathrm{C}_{10} \mathrm{H}_{18}\right)$, a transparent and mobile liquid of sp. gr. 0.85 , or moist in white crystalline masses which liquefy on a slight rise of temperature $\left(9 \%^{\circ} \mathrm{F}\right.$.). Both forms are freely soluble in rectified spirit, ether, chloroform, and oils; sparingly so iu water.

Physiological Action.- Corresponds with that of spearmint. Menthol has antiseptic powers, and is efficient in solution of 1 in 1000 (Edin. Med. Journ., 1880). Applied to 
the skin or tongue, it produces first a cool sensation, afterwards some burning, but it is not caustic. The vapour is highly irritating to the conjunctival membrane, and the after-effect on any part is to lessen sensibility. Interually, menthol acts much like other preparations of peppermint, as a carminative and diffusible stimulant.

Therapeutical Action.-Peppermint is convenient as a cover for the taste of other medicines, and as an adjunct to purgatives: it is administered for the relief of nausea, and of griping pains in the alimentary canal; also to expel flatus, and sometimes as an antispasmodic. Oil of mint is of much use as a palliative in facial neuralgia, sciatica, and gout; for this purpose it is painted over the affected part. Menthol is no doubt the active agent in mint oil, and it produces a temporary paralysis of the sensory terminals, thus explaining its action in neuralgia. Many forms of superficial pain may be quickly relieved, at least for a time, by gentle frictions with menthol-e.g., toothache, tic, myalgia, sciatica, and the spinal pain of delicate subjects; when applied it produces a sense of coldness, objective and subjective. It has long been in use in the East. Mr. Wright was one of the earliest to introduce it here (Lancet, ii., 1870). Mr. M. Morris has recommended a solution of 1 part with 4 of chloroform and 12 of olive oil as an application in chronic ringworm (Lancet, i., 1881).

PREPARATIONS AND DOSE. - Wenthe piperite oleum: ilose, 1 to $5 \mathrm{~min}$. Aqua menthe piperite: dose, 1 to $2 \mathrm{fl}$. oz. - Spiritus menthe piperite: dose, $\frac{1}{2}$ to $1 \mathrm{fl}$. dr. Essentia menthe pizeritce: dose, 10 to $20 \mathrm{~min}$. The solid moist menthol is now . commonly sold as "anti-neuralgic crystal." A linimentum - menthol may be made somewhat stronger than the solution above mentioned (2 to 3 parts), or 1 part to 60 of spiritus -rectificatus with oleum earyophylli. 


\section{MENTHA PULEGIUM. (Pennyroya..)}

\section{(Not Officinal.)}

DESCRIPTION.-Pennyroyal is distinguished from most other species of mint by its procumbent branches. The leaves are ovate, seldom half an inch in length, and entire, or nearly so ; the little purple flower's are produced in axillary and rather distant whorls.

ACtIVE InGREDIENTS.-In composition, oil of pennyroyal resembles oil of spearmint-i.e., is an oxidized hydrocarbon, containing camphor in solution. The colour is pale yellow; the odour corresponds with that of the recent plant, which is peculiar and agreeable; the taste is warm, aromatic and somewhat bitter. The sp. gr. is 0.95 ; the boiling-point $395^{\circ} \mathrm{F}$. Pennyroyal contains tannin in small quantity, with other ingredients of little importance.

Physiological Action.-This corresponds nearly with that of the larger species of mentha. Some consider it specially antispasmodic and emmenagogue.

Therapeutical ACTION.-Chiefly in the directions just named, viz., as an antispasmodic and emmenagogue. It is also esteemed in whooping-cough and hysterical complaints.

PReparations and Dose.-Oleum: dose, 1 to $5 \mathrm{~min}$. Aquel: dose, 1 to $2 \mathrm{fl}$. oz. 


\section{ROSMARINUS OFFICINALIS.}

(ROSEMARY.)

DESCRIPTION.-An evergreen shrub, native of the shores of the Mediterranean, and attaining the height of 3 to 6 feet. The numerous leaves are linear, about an inch in length, entire, and with revolute margins. The blue flowers are produced in short racemes, and appear in February.

Active InGREDIENTS.-The oil, upon which depends the well-known scent of this plant, is colourless; it is of the same chemical composition as the labiate oils already described, and retains both the odour and the taste of the plant; is soluble in alcohol: sp. gr. 0.888 ; boiling-point $365^{\circ} \mathrm{F}$.

PHysiological Action.-Resembles that of the other aromatic labiates; the plant is credited with emmenagogue, galactagogue, and diuretic powers.

Therapeutical Action.-Nervous headache and hysteria are said to be relieved by the smell of rosemary, and the mental powers quickened. The oil has some repute for encouraging the growth of hair, and the green hue of the pomades employed for these purposes is chiefly, if not entirely, orring to the presence of rosemary. The plant is used also as an emmenagogue.

Preparations and Dose.-Olemm: dose, 1 to 5 min. Spiritus : dose, 10 to $50 \mathrm{~min}$. or more. 


\section{THYMUS VULGARIS}

\section{(Common Thyme),}

Including the variety lemon thyme, is indigenous to dry places in the south of Europe, and was introduced to this country about the time of Henry VIII. : it is now widely distributed, and is a small bushy evergreen, with wiry and much-branched stems, about 8 to 10 inches high, clothed with opposite, ovate leaves. The flowers are small and purplish, in a dense whorled spike. The whole plant is aromatic, and the essential oil is fragrant and stimulating.

Active INGREDIENTS.-An essential oil, separated by fractional distillation into two parts-one a liquid, consisting of the hydrocarbons cymen $\left(\mathrm{C}_{10} \mathrm{H}_{14}\right)$ and thymen $\left(\mathrm{C}_{10} \mathrm{H}_{10}\right)$, the other and most important being thymol $\left(\mathrm{C}_{10} \mathrm{H}_{14} \mathrm{O}\right)$, a stearopten, homologous with menthol. Thymol is now prepared by treating essence of thyme first with potash or soda, and then neutralizing with hydrochloric acid, or it may be obtained from Ajowan oil (ptychotis Ajowan). It occurs either liquid or in white rhombic or acicular crystals, neutral in reaction, of characteristic fragrant odour, and pleasant, rather hot taste, sparingly soluble in water ( 1 in 1000 parts), more so in alcohol and glycerine (Pharm. Journ., Jan., 1869). It forms thymolates with alkalies, in which it is soluble. Tincture of perchloride of iron, added to urine containing it, renders the liquid cloudy and greyish-white.

ABSORPTION and Elimination.- - It is probable that thymol may be absorbed from an ulcerated surface; it certainly may be absorbed from the stomach, though slowly. It passes out mainly by the urine, rendering it dark green (brown by transmitted light).

Physiological Action.-Eexternal.-Thymol is astringent and antiseptic, and much resembles salicylio acid in its 
effects. A smaller amount of it than of this or of carbolic acid will suffice to arrest saccharine fermentation; it will retard also coagulation of milk and putrefaction of albumen, and neutralize the foetor of putrid pus (Lewin, B. M. J., i., 1875).

It lessens discharge, and is said only to irritate moist surfaces, such as the lips, not the skin (Husemann). In the dry state it may be non-irritant, but certainly a concentrated solution may irritate so as to produce "minute holes in the skin" (B. M. J., i., 1878).

Subcutaneous injection of thymol is very painful, but a strong solution is said to cause anæsthesia in frogs immersed in it.

Physiological Action.-Intemal.-Digestive System. -Lewin says the drug may be taken in full doses without disturbing digestion, but under the use of continued small quantities, animals emaciate (Kuessner).

Bälz found no definite effects with less than 30 grains (per diem). He describes some pricking and nauseous taste in the mouth on direct contact, and transient heat at the epigastrium; rarely nausea or vomiting, but often diarrhœa (Med. Record, 1878; Arch. der Heilkunde, xiv.). Such symptoms may, however, be obriated, as with salicylic acid, by dividing the dose, or by dilution and flavouring of the mixture. Kuessner took 15 grains in pill daily without other effect than some burning sensation. Fatty degeneration of the liver has been found in animals poisoned by it (Year-Book of Pharmacy, 1876).

Nervous System. - On this system thymol acts like its congeners, causing oppression aud constriction of head, timnitus and deafness, sometimes violent delirium, sometimes collapse, and in one case (of typhoid fever) unconsciousness; 30 to 40 grains reduce the temperature by $3^{\circ}$ to $5^{\circ} \mathrm{F}$. in many pyrexial cases, but with less certainty than salicylic acid. In animals poisoned by it, coma occurred before death.

Circulatory System.-Thymol does not seem to affect the circulation unless it be given in large doses, when its ultimate effect is to lower the blond-pressure (Med. Times, ii., 18\%8); and during illness, at least, it slows the pulse when it depresses temperature (Bälz). Husemann, however, after injecting 30 
grains in rabbits, noted some increase in the pulse-rate, and found the heart continue beating after respiration ceased. The most marked symptom is the slow breathing produced, and, indeed, the toxic action of the drug is mainly shown by a paralysis of the respiratory centre.

Kuessner's observations on dogs and rabbits point to the same conclusion, and he asserts that by artificial respiration he could often save life endangered by thymol-poisoning.

In fatal cases (animals) the blood has been found dark, but fluid, with the corpuscles unaltered.

Glandular System.-Thymol causes a profuse secretion of sweat in the course of half an hour after administration, but not to the same extent as jaborandi or salicylic acid. It stimulates the kidneys, often inducing slight diuresis of a greenish or brownish coloured urine, of peppermint odour: large doses may cause albuminuria, at least in animals, with passage of blood and hyaline casts. Renal inflammation has been found after death.

Therapeutical Action.-Extemal.-Thymol has been employed like carbolic acid in the different operations of antiseptic surgery, but it is costly, and has not proved superior in value; its odour has the disadvantage of attracting flies. It is useful, however, as an astringent aseptic lotion for offensive discharging surfaces, and as a gargle in ulceration of mouth or fauces.

Skin Diseases.-Dr. Crocker has made some observations on the use of thymol in this class of diseases, and finds an ointment containing 10 grains to the ounce of vaseline effective in removing the scales, and curing patches of psoriasis when not in the acute stage (if much congestion be present it is liable to irritate); the same preparation acted well in chronic eczema, and in lichen planus. In general psoriasis a lotion containing 5 grains to 8 ounces of water, with spirit and glycerine, is recommended; and this also cured tinea versicolor, and other parasitic disorders, though not better than sulphurous acid (B. M. J., i., 1878).

Given by inhalation, thymol (1 part in 1000) has relieved the fortic expectoration of phthisis, etc. 
THERAPEUTICAL ACTION.-Internal.-This is but limited In Diabetes, Kuessner reported lessening of sugar in the urine under the influence of daily gramme doses of thymol, but Fürbringer has recorded a contrary result (Med. Times, ii., 1878). The former observer says that he found it useful in Vesical Catarrh and Infantile Diarrhœa, giving 3 to $j$ minims of a 1 per cent. solution.

PREPARATIONS AND DOSE.-Lotio: for wounds' 1 part in 1000 warm water (Spencer Wells); this may be improved by adding glycerine 20 parts, alcohol 10 parts (Volckmann). As a lotion in skin diseases, $5 \mathrm{gr}$. in $8 \mathrm{oz}$. of water with a little liquor potassæe, spirit, and glycerine. Two grains to the ounce of spirit makes a solution miscible with water, and is suitable for internal use. Unguentum: $10 \mathrm{gr}$. to the ounce; this is quite strong enough for safety (Crocker). 


\section{POLYGONACEAE.}

\section{THE RHUBARB FAMILY.}

A LARGE miscellaneous family of exogens, represented at one extreme by the weedy sorrels, docks, and persicarias of our meadows and waysides, and at the other by curious tropical species which become tree-like and produce bunches of juicy fruit. The number of ascertained species is about 500, and of these about twenty-five grow wild in Great Britain.

The leaves are alternate, simple, often large; and usually provided with membranous and ochreate stipules. The perianth consists of five or six pieces, which are more or less petaloid, perfectly free, and in restivation imbricated. The stamens, which vary in number from five to nine, spring from the inner base. The ovary is superior, and surmounted by two or more styles or stigmas; the fruit is usually a triangular or flattened achrnium, and the seed contains a large quantity of farinaceous albumen.

\section{Phannacutical Species.}

Rheum undulatum

R. palmatum.

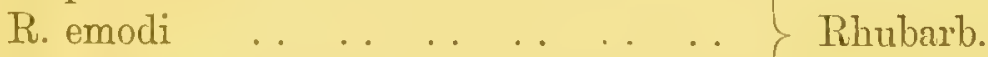

R. compactum

Polygonum bistorta

\section{R H E U M.}

\section{(RHU1BARB.)}

DESCRIPTION.-The above species, which from time to time have been supposed to be the source of the drug in question (designated "Turkey" rhubarb, because received 
by way of Natolia), ${ }^{1}$ are all natives of the temperate parts of Central Asia, perennials with massive root-stocks and immense deciduous leaves, and stout annual flowering-stems, several feet in height, terminating in great panicles of small cream-coloured or purplish flowers, which are composed individually of six sepals, nine stamens, and an ovary that ripens into a large three-winged achænium. The rhubarb of commerce is in segments of the root, which are either roundish and conical, or trapezoidal, flattish, and convex on one side. They are perforated, and the hole contains portions of the cord by which they were suspended. In drying they are trimmed, but patches of the bark are left. The transverse section is marbled reddish-brown or yellow, the white parenchyma being traversed by fine reddish medullary rays. Rhubarb is hard, and breaks with uneven fracture; has an aromatic odour, a bitter astringent taste, and is gritty between the teeth.

ACTIVE INGREDIENTS.-The first ingredient that requires notice is chrysophanic acid, or chrysophan, $\mathrm{C}_{15} \mathrm{H}_{10} \mathrm{O}_{4}$, now known to be the same substance which under rarious impure forms has received the names of Rhabarber-bitter, Rhabarberin, Rheumnin, Rhabarbergelb, Rhabarbergelbsüure, Rhein, Rhaponticin, Lapathin, and Rumicin (Peckolt): it is the principal constituent also of "Goa powder." Chrysophanic acid, as such, does not perhaps exist to any large extent in rhubarb, but is formed by the splitting up of chrysophan. It crystallizes out of alcohol in small orange-yellow prismatic and lustrous needles of a somewhat bitter taste. It is rendered friable by heat, is slightly soluble in water, freely soluble in ether and hot alcohol. From its solution in benzol, on slow evaporation, it is deposited in rather paler rellow crystals, which are oblique rhombic tables. Concentrated sulphuric acid dissolves it red; water added to this solution separates the acid in yellow masses unchanged. Aqueons solutions of fixed alkalies and ammonia change it to a beantiful red; the ammonia solution being evaporated, leaves unchanged

${ }^{\prime}$ Within the last few years there has been introduced, first to Paris, and subsequently to Kew, another species, prorisionally called Rheum officinale, which upon fair grounds is supposed to be the genuine plant. 
chrysophanic acid; but the potash solution, if evaporated, lenves red chrysophanate of potash.

The other vegetable acid present in rhubarb is a peculiar tannic acid, $\mathrm{C}_{26} \mathrm{H}_{29} \mathrm{O}_{14}$, a yellowish-brown powder which attracts water readily, and is soluble in it and spirit, but not in ether. The brown-coloured aqueous solution has an acid reaction, precipitates perchloride of iron blackish-green, reduces gold and silver salts, precipitates lime and albumen, but does not give a precipitate in solution of tartar emetic.

The neutral body chrysophan is a glucoside, which, when boiled with dilute sulphuric or hydrochloric acid, splits up into sugar and chrysophanic acid. Chrysophan is obtained in microscopic orange-yellow prisms; it is present in the proportion of about 9 or 10 grains to the pound of rhubarb-root; is highly soluble in water, insoluble in ether. Concentrated sulphuric acid gives it a brownish colour; from water it subsides in green flocky masses.

Emodin, $\mathrm{C}_{15} \mathrm{H}_{10} \mathrm{O}_{5}$, is an extra product of the (De La Rue) process for the extraction of chrysophanic acid; it forms deep orange-red crystals varying in size up to oblique rhombic prisms of 2 inches in length; these do not melt and decompose below a temperature of $482^{\circ} \mathrm{F}$. Allialine watery solutions turn it red; ammonia gives a violet-red.

Phaoretin, $\mathrm{C}_{16} \mathrm{H}_{16} \mathrm{O}_{7}$, is an extra product of the process for extracting the rhubarb-tannin; it is a dull brown shining resinous body which pulverizes to yellow-brown; is tasteless, melts on heating, and evolves yellow fumes that smell like rhubarb. It is insoluble in water, ether, and chloroform ; very soluble in spirit, also in warm acetic acid.

Aporetin and erythroretin are resinoid bodies which are possibly mere mixtures of other rhubarb constituents; at any rate, nothing accurate is known about them. The grittiness of the root is due to tufts of microscopic crystals of oxalate of calcium.

ABSORPTION AND Elimination.-The colouring and purgative principles of rhubarb are reaclily absorbed, as evidenced by the secretions, especially the milk, the urine, and sometimes the perspiration. Purgation has followerl 
its application to the skin. (The urine coloured by rhubarb may be distinguished from that of jaundice by its becoming purplish-red on the addition of an alkali.)

Physiological Action.-Rhubarb furnishes a striking instance of the wide, though not universal, law that changes in dosage not only alter the degree in which a medicament will act upon the body, but, when carried beyond a certain point, change the mode of action.

Digestive System.-Taken in small doses, such as 4 to 8 grains of the powder, it exerts no purgative action, but, on the contrary, is efficient in checking chronic diarrhœe; it exercises a remarkable tonic influence upon primary digestion, increasing appetite, and enabling the food to be disposed of without discomfort in cases where previously there had been sluggish digestion and flatulence. If, on the contrary, it be taken in large doses, such as a scruple to a drachm, rhubarb induces none of these tonic effects upon the stomach, and acts as a direct laxative, which operation commences in from four to eight hours after the medicine has been taken. According to the magnitude of the dose, and conditionally upon its haring been repeated or otherwise, there may be more or fewer evacuations; but on the whole the character of the stools is fairly constant, they being of loose, but not watery, consistence, and usually of a yellow-brown colour. When the number of eracuations has been considerable, some constipation generally follows, the tamnin then exerting its effect; a small amount, however (12 to 15 grains), may be slowly chewed daily, with the effect of keeping up a free action of the bowels without resulting constipation.

Several hypotheses have been put forward to explain the intimate working of the drug. Is the purgative action produced by a direct stimulation of the muscular fibres of the stomach and intestines, pushing their contents more steadily and rapidly onwards? Most observers are inclined to adopt this idea; and if it were correct, the loose character of the stools might be accounted for without the supposition of any unusual outpour of secretion, by the theory that the frecs simply passed onwards too rapidly to allow of the normal 
mucous secretions being re-absorbed-a process which certainly contributes to the dryness of normal, and still more of constipated, dejections. And it is remarkable that, even in the largest doses, rhubarb does not appear, like jalap, scammony, and other resinous purgatives, to cause inflammation of the mucous membrane; the usual results of an unnecessarily large purgative dose are limited to the production of more or less violent colicky paims, such as do not attend the milder laxative operation.

It has been asserted that rhubarb specially affects the mucous secretions of the duodenum as well as its peristaltic action, and also that it increases the flow of bile. The latter assertion has been corroborated, to some extent, by the recent observations of Rutherford and Vignal, who found that the drug distinctly increased the secretion of bile in dog's.

Another question relates to the respective shares in the physiological action taken by the different ingredients of rhubarb. Nothnagel (Arzneimittellehre), a high authority, considered that the purgative action was proved, by the experiments of Schroff, to depend chiefly upon the chrysophanic acid. Husemann (Die Pflanzenstoffe) takes the opposite view, considering that the chrysophanic acid has little or nothing to do with the purgative effect.

Schroff administered chrysophanic acid (obtained from the lichen, parmelia parietina) in a dose of 8 grains; this was followed by eructation of wind and repeated semi-liquid stools, which commenced twenty-four hours after the dose was swallowed, and continued to recur for five days, during which period there were also observed loss of appetite, fulness of the head, giddiness, and dull depression (Wiener ärtzl. Woch., 1856). On the other hand, Schlossberger, Buchheim, Meykow, and $v$. Auer state that chrysophanic acid produces no definite result on the intestinal tract, even in the same dose ( 8 grains) that was employed by Schroff. There is also a contradiction as to the cause of the colour-change in the urine; Schlossberger ascribing it to the elimination of phaoretin and erythroretin, while Schroff and Buchheim always found the same colouring of the urine in their experiments with chrysophanic acid. Meykow al:io belieres this acid always to be the cause of the 
colour, and says that in experiments where the resinoid ingredients have seemed to produce it they contained an admixture of chrysophanic acid.

The difficulty in supposing chrysophanic acid to couse the laxative action in rhubarb is the small proportion ( 2 per cent.?) in which it exists in the root. It is impossible to understand how there can be sufficient acid from any source in (say) a 30-grain dose of rhubarb to produce decided action on the bowels, seeing that not less than 2 or 3 grains can be supposed necessary for the purpose. Nothnagel thinks it likely that the action of chrysophanic acid is increased by some of the salts present in rhubarb root: which brings to mind the old opinion that a test of good rhubarb was its containing a large number of oxalate of lime crystals; but although the best Russian rhubarb is undoubtedly richer in lime than are the English sorts, the idea of purging by the salt is not now generally entertained. According to Michaelis, the cathartic effect is produced by the resinoid matters, together with the oxalate of lime, while the chrysophanic ("rhabarberic") acid is purely tonic (Journ. Mens. de la Soc. Chim. de Paris, 1868). Kubly believes that phaoretin causes a part of the purgative action (Neues Rep. f. Pharm., xvii., p. 214). I may cite, moreover, the experience of the London Hospital in Pereira's time: English rhubarb was there habitually used, and found to answer well, although it contains less oxalate of lime than the so-called Turkey sort.

Of the various ingredients of rhubarb, very few are freely soluble in water. The rhubarb-tamnin (to which it is inpossible to attribute the purgative action) is almost the only one so soluble, except chrysophan; and this latter, as already said, is trifling in amount. On the whole, therefore, it would appear that the purgative action of rhubarb must be a compound result of several stimuli, any one of which singly would be inadequate. This must especially be the case where such a preparation as the infusion is employed; and in which 24 grains (sliced, not powdered) are infused with 2 ounces of boiling water, and virtue enough extracted to form a draught mildly but decidedly laxative. Where, on the other hand, powdered rhubarb is introduced (either in pill, or partly dissolved and 
partly suspended in water) into the stomach, we may more readily imagine that one or two of the ingredients, being thoroughly dissolved by the gastric juice, might suffice for the production of the effect.

Therapeutical Action. - Although the mode of action of rhubarb is somewhat obscure, there can be no doubt as to the great value of the drug for several purposes.

Ulcers. - Sir Everard Home advised the application of pulverized rhubarb as a local stimulant to indolent ulcers; and though this plan has not been carried out to any considerable extent, the neglect arises, probably, not from its intrinsic inefficiency, but from the circumstance of there being more convenient methods of treatment.

Constipation.-As a laxative, in doses of 20 to 30 grains, it acts with certainty and ease; but such doses as these should not be frequently repeated, since an increasingly constipating: after-effect is apt to follow; and if a mild aperient be required rather frequently, rhubarb should only be given in small doses, combined with other purgatives, as in pilula rhei composita. As a general rule, it is better suited for the aged or the delicate, than for sthenic conditions. It is especially suitable for cases with chronic biliary catarrh or congestion, when hard clay-coloured stools are passed. For a single purgative dose for children, the best plan is to combine it with one-third its weight of carbonate of soda or magnesia, and a little ginger or other carminatives, as in Gregory's powder; but the gritty sensation of powdered rhubarb is not easily obviated. For adults, 6 grains of the extract with 2 grains of ginger, followed, if needs be, by a so called "Seidlitz" draught, may answer the purpose. Rhubarb, with magnesia or potash, has specially good effects in gouty subjects, according to Sir H. IIalford; a modern practitioner has written a special treatise to advocate his own treatment of gout, which is mainly the administration of rhubarb, followed by castor oil. As a laxative in hremorrhoids, it is often very useful.

Dyspepsia.-Combined with gentian or chamomile, it forms a valuable pill in atonic dyspepsia; an excellent stomachic may also be procured by the combination of drachm doses 
of the tincture with some bitter infusion, three times a day. When a tonic and antacid are required, rhubarb is often effectively combined with soda; and 3 or 4 grains of it, with an equal quantity of the exsiccated carbonate of soda, forms a useful pill for meal-times: for flatulent dyspepsia it is very good.

Dr. Budd relates several instances in which 3 or 4 grains of rhubarb, sometimes alone, sometimes with ipecacuanha, before meals, proved of much service in relieving severe urticaria connected with indigestion (Med. Times, i., 18.54), and I have verified this several times.

Diarrhœa.-It has already been mentioned that rhubarb (like various other Polygonaceæ) contains a large proportion of tannin, and hence it has the property of restraining excessive diarrhoea; more especially when this is due to undigested food or other irritant matters, rhubarb acts in a twofold manner, first removing the offending substances, and afterwards constringing the intestinal vessels, and restraining the flow of mucous secretion. It is not, however, suitable to those cases in which diarrhoea or other troublesome symptoms depend upon an inflammatory condition of the mucous membrane, and the popular idea that rhubarb is useful in relieving pain in the bowels sometimes leads to mischief, for the drug may, under these circumstances, induce inflammation by aggravating gastro-intestinal catarrh.

A general review of the therapeutical use of rhubarb shows that it holds a peculiar position. As a purgative it perhaps more resembles aloes than anything else, but its effect is milder, and it has not that specific tendency to act upon the large intestines which characterizes aloes. Its tonic effect is apparently limited to the stomach and intestines, which are affected in two different modes, each of which usefully supplements the other. A great part of its action is undoubtedly exerted after its ingredients have entered the circulation, but a local stimulant effect upon the stomach and intestines occurs before absorption.

PREPARATIONS AND DOSE.-Pulis: dose, as a stomachic, 1 to $5 \mathrm{gr}$; as a purgative, 10 to $30 \mathrm{gr}$. Extractum: dose, 5 to 1i) gr. Infiesum: dose, 1 to $2 \mathrm{fl}$. oz. Pilnle wei composita: 
dose, 5 to $10 \mathrm{gr}$. Pulvis rhei compositus (Gregory's powder): dose, for children, 5 to $10 \mathrm{gr}$; for adults, 20 to $60 \mathrm{gr}$. Syrupus: dose, 1 to $4 \mathrm{fl}$. dr. Tincture: dose, as a stomachic, 1 to $2 \mathrm{fl}$. dr.; as a purgative, $\frac{1}{2}$ to $1 \mathrm{fl}$. oz. Vimm: dose, 1 to

2 fl. dr.

\section{POLYGONUM BISTORTA.}

(Bistort-SNakeweed.)

A common and beautiful English wild-flower, the rhizome of which is of old repute as an astringent. It is met with in pieces about 2 inches long, each piece being bent on itself, transversely furrowed, flattish on one side, rounded on the other. It contains some starch and a considerable quantity of tannic acid, and, when powdered and administered in doses of a drachm, is useful in hæmorrhages, diarrhœa, and chronic dysentery.

Dr. Cullen recommended it in combination with gentian, or other bitter, for intermittent fever.

A decoction may be usefully employed as an astringent injection in gleet and gonorrhoe; also as a gargle for spongy gums, and for relaxed sore throat; and as a lotion for ulcers accompanied by profuse discharge. 


\section{MYRISTICACEAE.}

\section{THE NUTMEG FAMILY.}

Av order of evergreen exogenous trees, containing about fifty species, natives exclusively of the tropics, and chiefly of India. The leaves are alternate, simple, and entire. The insignificant apetalous flowers are unisexual, and are followed by baccate or drupaceous fruits, the seeds contained in which are remarkable for their ruminated albumen.

\section{MYRISTICA MOSCHATA.}

\section{(Nutmea.)}

DESCRIPTION.-A tree attaining the stature of 40 to 50 feet, with oblong, pointed leaves, 3 to 6 inches in length; the yellow flowers are produced in axillary clusters; the fruit is a globular yellow drupe, the size of a small orange, and fleshy in substance. When ripe, it divides into two nearly equal portions, liberating the nut or endocarp, which is about an inch in length, brown, and enclosed by the branches of a large arillus : the latter, scarlet while fresh, turns orange-yellow when dried, and is the "mace" of commerce. The large ovoid kernel well illustrates the ordinal characteristic of ruminated albumen.

ACTIVE INGREDIENTS.-(1) About 2 to 3 per cent. of volatile oil, which is colourless or pale yellow, and has a peculiar smell and taste of the nutmeg itself; sp. gr. 0.95 ; soluble in alcohol; boils at $320^{\circ} \mathrm{F}$., and forms a fulminate with iodine. (2) About 30 per cent. of a concrete oil, which, when extracted 
by means of heat and expression, receives the name "butter of nutmegs," expressed oil of nutmeg, or oil of mace. In commerce it occurs in solid, oblong, brick-shaped cakes, of an orange colour, and possessed of the agreeable and aromatic odour of the nut. Ordinarily, these cakes are wrapped in the leaves of some endogenous plant. This primary or concrete oil is soluble in four times its weight of boiling alcohol, and in twice its weight of ether; upon analysis it yields a fixed oil, or fat, which is solid and crystallizable, melts at $118^{\circ} \mathrm{F}$., and contains myristic acid $\left(\mathrm{C}_{14} \mathrm{H}_{27} \mathrm{OHO}\right)$, in combination with glycerine: this may be crystallized in silky needles.

Physiologrcal Action.-Nutmeg, in moderate quantity, is cordial, carminative, and agreeably stimulating. In excess, however, it proves narcotic, and causes giddiness, oppression of the chest, intense thirst, headache, delirium, and stupor-in particular constitutions, even narcotic poisoning. Fatal apoplexy is said to have resulted from its excessive use, and Cullen is emphatic in recommending persons with a tendency towards that disorder to abstain from nutmeg, even as a condiment.

The volatile oil of nutmeg has also been shown to be a powerful drug. Applied to the human skin it acts as a rubefacient: given to rabbits in doses of 2 to 6 drachms, it proved fatal, with symptoms of narcotic irritant poisoning extending over a few hours or days; the urine had a peculiar smell, which was not that of the oil itself. When the dose was not large enough to kill, prolonged constipation followed recovery from the acute symptoms.

Therapeutical Action.-External.-As a Stimulant the oil is occasionally applied to the skin, especially in paralysis and chronic rheumatism.

THERAPEUTICAL ACTION.-Intermal.-On account of its cordial, carminative, anodyne, and astringent properties, nutmeg has been employed in cliarrhen and in dysentery, as well as in nausea and vomiting: it is best administered in wine or brandy-and-water, or in simple powder. In mild cases it proves an efficient substitute for opium. 
Mace is in India a favourite medicine in low stages of fever, in consumptive complaints, and in humoral asthma; also, combined with other aromatics, in chronic diarrhœa.

PReparations AND Dose.-Pulvis: dose, 5 to $15 \mathrm{gr}$. Nutmeg is contained in pulvis cretro aromaticus, pulvis catechu compositus, spiritus armoraciæ compositus, and tinctura lavandulæ composita.

The concrete oil, or "butter of nutmeg," is an ingredient in the emplastrum calefaciens and the emplastrum picis.

Spiritus myristicre (contains 1 of oil in 50 spirit): dose, 30 to 60 min. Oleum: dose, 2 to 6 min.- contained in pilula aloes socotrinæ, spiritus ammoniæ aromaticus, and mistura ferri composita. 


\section{LAURACEAE.}

\section{THE CINNAMON FAMILY.}

Av order of about 500 species of trees and shrubs, mostly evergeen, and belonging to hot countries. The leaves are simple, almost invariably alternate, undivided, and entire. The inconspicuous flowers are usually clustered, and consist of a single perianth, a small and definite number of perigynous stamens, the anthers of which open by recurving valves, and a superior one-celled ovary, which ripens into a baccate or drupaceous fruit, with a pendulous seed. The plants of this order are innocuous, and are noted generally for being aromatic.

\section{Pharmacentical Species.}

Cinnamomum Zeylanicum. . . . Cinnamon.

Camphora officinarum . . . Camphor.

Sassafras officinale .. . . . . Sassafras.

Nectandra Rodiæi . . . . . Bebeeru or bibiru.

Laurus nobilis .. . . . . . . Sweet bay.

Coto cortex _. . . . . . C Coto bark.

\section{CINNAMOMUM ZEYLANICUM.}

(Cinnamon.)

Description.-A native of Ceylon and Java. The branches are glabrous; the leaves ovate, 3 to 6 inches long, atrongly three-nerved, entire, and of a peculiar yellowish green; 
the insignificant white flowers are six-cleft, silky outside, enneandrous, and produced in thin panicles. The dried inner bark of the cinnamon tree possesses a warm, sweet, aromatic taste, and evolves a fragrant odour.

ACTIVE INGREDIENTS.-These qualities are referable to a volatile oil, $\mathrm{C}_{9} \mathrm{H}_{7} \mathrm{OH}$, which varies in colour, being, when recent, a bright yellow, but after keeping, red. The odour and taste are pleasant, like those of the bark; it is heavier than water, and on exposure to the atmosphere absorbs oxygen, and forms cinnamic acid, two resins, and water. Cinnamon bark also contains tannic acid in considerable quantity, a resin, and cinnamic acid.

Physiological Action. - Cinnamon is warm and cordial to the stomach, astringent, and carminative. The essential oil is devoid of the astringency found in the bark, and in moderate doses is an agreeable stimulant, producing a sensation of warmth in the epigastrium, and promoting assimilation. The too constant use of cinnamon tends, however, to produce costiveness.

In full doses it acts as a general stimulant to the nervous and vascular systems. Some practitioners believe that it exerts a specific influence over the uterus.

THerapeutical Action.-Cinnamon is useful in many cases characterized by feebleness and atony : as an astringent it checks diarrheea, for which purpose it is best combined with chalk, opium, or some vegetable infusion. In the later stages of low fever it is also given with advantage, as well as in ordinary flatulent and spasmodic affections of the alimentary canal: nausea and vomiting are checked by its administration. The oil is sometimes employed as a powerful stimulant in cases of paralysis of the tongue, in syncope, and in cramp of the stomach. The principal value of cinnamon is, however, to modify the flavour of bitter infusions, and when combined with purgatives, to check their griping action: it is an ingredient in many powders, infusions, and tinctures. It has been administered with excellent effect in uterine hæmorrhage. 
PREPARATIONS AND DOSE.-Pulis: dose, 10 to $30 \mathrm{gr}$. Aqua: dose, 1 to $2 \mathrm{oz}$. Oleum: dose, 1 to 5 min. in pill, emulsion, or on sugar. Tinctura: dose, $\frac{1}{2}$ to $2 \mathrm{fl}$. dr. Pulvis cinnamomi compositus: dose, 3 to $10 \mathrm{gr}$.

\section{CAMPHORA OFFICINARUM.}

(CAMphoR.)

DESCRIPTION.-A tree of moderate dimensions, native of China and Japan. The leaves are ovate, acuminate, three-ribbed, and shining; the small yellowish-white flowers are borne in corymbose panicles, and are followed by globular dark red berries, the size of a large pea. Every part of the plant evinces by its odour and flavour the presence of the well-known substance which it secretes.

Active INGREDIENTS.-Camphor, $\mathrm{C}_{10} \mathrm{H}_{10} \mathrm{O}$, is prepared by watery distillation of chips of camphor-wood, gouged from the living tree, and being volatile it is deposited at the top of vessels inverted over the receptacle in which distiliation is conducted. This crude product, of a greyish or pinkish hue, is refined in Europe by re-distillation with charcoal, iron filings, or quicklime: it is thus obtained in the white, concrete, crystalline masses of commerce, being granular, semi-translucent, tough, and difficult to pulverize. The odour is strong, penetrating, and characteristic; the taste is pungent, bitter, and aromatic. It is lighter than water (sp. gr. 0.98); at ordinary temperatures it is solid, but owing to its volatility, it slowly evaporates. Under the influence of heat it rapidly and entirely sublimes; it melts at $175^{\circ} \mathrm{C}$, and boils at $205^{\circ} \mathrm{C}$., and when ignited burns with a bright flame and copious smoke. In water it is very sparingly soluble, so that if water be arlded to the alcoholic solution (which is readily made), the camplor immediately precipitates: it is soluble in ether, and in the volatile and fixed oils. By continuous heating with nitric acid, it becomes oxidized and converted into camphoric acid, $\mathrm{C}_{10} \mathrm{H}_{10} \mathrm{O}_{4}$. 
ABSoRPtion AND ELIMINATION.- Harley states that camphor is so completely and quickly absorbed that he has not been able to detect it in the secretions or exhalations from the lungs or skin. The odour of these, however, gives evidence of elimination by them. Gubler could not find it in the urine, but Cullen detected it in the perspiration, and Tiedemann and Gmelin in the blood.

Physiological Action.-Extemal.-Upon the skin, camphor is well known to act as an irritant. A concentrated solution rubbed in, soon causes heat and bright redness, and applied to a raw surface a sense of burning and even inflammation. Camphor is an excellent antiseptic, and is fatal to the lower organisms: it is this property which is the basis of its use in "Raspail's system ;" he adopted it as an universal panacea.

Physiological Action. - Intemal. - Circulatory System.-Moderate doses (up to 10 grains) cause general stimulation with quickening of pulse, rise of temperature, and profuse sweating.

One of the phenomena which has been often, though not always, noted in severe camphor-poisoning, is dilatation of the superficial vessels, especially of the head and face; this is usually accompanied by delirium.

Nervous System.-Small doses stimulate, large ones narcotize this system, causing disorder of mind and will, depression, collapse, and temporary paralysis, with pallor of face and dilatation of pupils, spasm, or convulsion and delirium. It is not clear whether these severe effects are due to alteration in the blood, or to a direct effect on nerve-centres. Harley maintains that the drug acts mainly on the. cerebral lobes. Very large doses cause death, preceded by the symptoms mentioned.

There are certain concentrated spirituous preparations of camphor, originally intended to be taken in very small. quantities, but which patients not unfrequently prescribe for themselves in large quantities: in this way many serious consequences have occurred. Iu man I am not aware of more 
than one fatal case, but there are many upon record of imminent danger; and one of the earliest still remains instructive, that of Mr. Alexander: he took 40 grains of camphor (mixed with syrup of roses) for experimental purposes, and in twenty minutes became languid and listless; in an hour giddy, confused, and forgetful: all objects quivered before his eyes, and a tumult of ideas floated through his mind: at length he passed into total unconsciousness, and in that state was attacked with strong convulsions and maniacal frenzy: an emetic was then given, and its action expelled almost the whole quantity swallowed: the graver symptoms then disappeared, but singular mental disturbance continued for some time (Christison on Poisons). Considering how small a proportion of the camphor had been dissolved when the emetic was administered, one cannot doubt that less than the 40 grains (if fully taken into the blood) would have proved fatal to this particular person; still, much larger doses have been taken with only temporary and not very severe effects. How much of this variability of effect is due to idiosyncrasy, and how much to difference in the rate of absorption must, however, remain at present undecided. Upon animals many observers have experimented with large and fatal doses, and the postmortem appearances have proved that, besides its action upon the nervous centres, camphor is a direct irritant to the alimentary canal, and also to the mucous tract of the genitourinary organs.

The recent researches of Grisar (upon valerian and other ethereal oils) also included experiments with camphor, which was found in certain doses to exhibit a power of reducing exalted reflex irvitability. It is not improbable that to this we must attribute its capacity of subduing certain forms of diarrhœe.

Genital System.-This is stimulated by small doses, depressed by large ones. On the whole it is probable that this drug possesses the apparently conflicting attributes of acting as a sedative and as a stimulant, the predominance of either effect depending upon dose and occasion.

SY NERG I S T S.- Various other "camphors" (stearoptins), "essential" and volatile oils, diffusiblo stimulants. 
ANTAGONISTS. - Cantharides and other irritants of the urinary tract are markedly antagonized by camphor: oxygen, coffee, galvanism, etc., are antidotal to the narcotic effects.

Therapeutical Action.-Extemal. - Camphor is much employed in the form of liniment for local pains, and is generally credited with good effects, but its action is uncertain.

Some persons recommend its use as a Diaphoretic. It has been already mentioned that in many cases of camphorpoisoning an immense dilatation of the superficial blood-vessels has occurred, and such dilatation, together with copious sweating, can be produced by the use of the vapour, from heating camphor on a plate over which the patient sits with a blanket pinned round his neck (so that the fumes do not enter the throat).

Therapeutical Action.-Internal.-There are at least two ways in which camphor often proves distinctly beneficial.

(1) As a remedy for functional nervous disorders not of the severest type, it is often efficacious. In Nervous Headache, not exactly well-marked recument migraine, but closely bordering upon it as regards the character of the pain, and most common in hysterical females, camphor is very useful. From 3 to 5 grains rubbed down with a little spirit, and suspended in water by the aid of tragacanth, with 20 or 30 grains of carbonate of magnesia, is an excellent if not an elegant form. The magnesia is not superfluous, for it often assists the cure by correcting attendant gastric acidity.

In Hysterical Excitement, and also in Chorea, when not of the graver types, camphor is also of considerable use; and has even been found of service by Van der Kolk in a case of acute mania in which other powerful remedies had failed. In Delirium Tremens and in Spasmodic Asthma it has also been strongly recommended by various authors. But with regard to all these maladies the same remark must be made about camphor as was made by Van der Kolk respecting its use in insanity, viz., that its effects vary widely in different individuals, and that we cannot tell beforehand whether it will act well or not.

(2) In many forms of Diarrhœa camphor proves useful, 
and it is important to define accurately the cases suitable for it. They are evidently those in the production of which nervous irritation has a large share. Among the cases of diarrhoa that occur in high summer heat, there are many distinguished by clear red tongue, in which the mingled exhaustion and irritation of the nervous system are for the most part the true sources of the flux, and here camphor will do much good, a few doses of 3 or 4 grains often completely checking the disorder. It is less useful in proportion as the diarrhoea is more dependent on local irritation from unwholesome food, etc ; and as to the assertion of its power to arrest Asiatic Cholera, I have never witnessed such effects, except in the first (or doubtful) stage, and cannot but suppose that some error in diagnosis has been made in most instances of reported cure.

If we consider the best ascertained effects of camphor, it appears probable that its beneficial action under varying conditions depends upon the power to subdue reflex excitability, indicated in the experiments of Grisar. It is difficult otherwise to explain its ancient high reputation as an anaphrodisiac, and the strong modern recommendations of it in all kinds of irritable conditions of the external and internal sexual organs; thus, for chordee, 5 grains may be given in pill with 1 grain of opium, and this often acts well.

No one who tries the drug in an unprejudiced manner can help observing that it fails as often as it succeeds in any one of these disorders, and it is impossible to discern any proof of a direct action either upon the sexual organs or upon the nervous centres that govern them, except so far as a general state of exalted reflex irritability may exist, and may be calmed by the remedy. It is even probable that a large portion of the so-called stimulunt effects, for which it has been so warmly praised by Copland and others in the treatment of adynamic fevers, etc, is really only a calming of the reflex apparatus, which can be obtained with less of general depression than would be likely to follow the use of other narcotics.

PREPaRations AND Dose.-Camphora: dose, 1 to 10 gr. Aqua (1 gr. in $1 \mathrm{oz}$.) : dose, 1 to $2 \mathrm{fl}$. oz. Spivitus (1 dr. in 9 f. oz.) : dose, 5 to $40 \mathrm{~min}$. Tinctura camphore compositu 
(with opium, benzoic acid, etc.): dose, 10 to $60 \mathrm{~min}$. Linimentum (with olive oil). Linimentum camphorce compositum (with oil of lavender, rectified spirit, and liquor ammoniæ fortior.).

\section{BROMIDE OF CAMPHOR $\left(\mathrm{C}_{10} \mathrm{H}_{15} \mathrm{BrO}\right)$,}

Discovered by Schwartz, is obtained by heating, at $100^{\circ} \mathrm{C}$, one part of camphor with two of bromine. It is solid, crystalline, of camphor-like odour, very soluble in alcohol and ether: it melts at $76^{\circ} \mathrm{C}$, and boils at $274^{\circ} \mathrm{C}$.

Physiological Action.-According to Bourneville, it lowers the pulse-beats, respiration, and temperature in animals, and also induces sleep by an action on the cerebral tissues. Its prolonged or excessive use has caused emaciation (without diarrhœe or vomiting), sometimes excitement with convulsion, sometimes paralysis.

THERAPEUTICALLY it has been used in priapism, delirium tremens, dentition, chorea, ete., and has certainly given good results in some cases of insomnia from nerve-exhaustion: it deserves further investigation.

Dose, 2 to $4 \mathrm{gr}$., best given in pill.

\section{SASSAFRAS OFFICINALE.}

(Sassafras.)

DESCRIPTION.-A deciduous tree of inconsiderable size, native of North America. The leaves are remarkable for the diversity of their figure, some being obovate, others deeply three-lobed, while others again have only a single lateral lobe. The small yellow flowers appear in racemes before the leares 
expand; the fruit is bright blue, and about the size of a pea, while the clavate peduncles are red, the contrast of colours presenting an appearance very remarkable. Sassafras-wood occurs in commerce in large pieces of a greyish-red colour, soft, spongy, light, and possessed of an aromatic odour; more usually it is met with in the form of " chips." The bark of the root, also imported for use in pharmacy, occurs in small pieces of similar character. Sassafras-wood has a warm aromatic taste, and a peculiar fragrance.

ACTIVE INGREDIENTS. - It contains a volatile oil, fatty matter, resin, a principle called sassafrin, tannin in small quantity, and some other less important ingredients. The volatile oil is light yellow; it has a pungent taste like the odour of the wood, a sp. gr. of 1.094, and is itself composed of two other oils, one of which sinks in water, the other floats. The properties are yielded to hot water and to alcohol. The wood contains a less amount of the active principles than the bark or roots.

Physiological Action.-Taken in infusion, both the wood and the bark of the root are reputed stimulant and sudorific; but little is accurately known on this subject: the sudorific action is much assisted by the use of warm clothing. and tepid drinks. Employed as an adjuvant to other medicines, sassafras mitigates disagreeable flavours, and renders them more tolerable to the stomach.

Therapeutical ACTION.-On account of its sudorific and alterative properties, sassafras is advantageously employed in cutaneous disorders, also in rheumatic and syphilitic comIlaints : it is seldom used alone; more usually in combination with sarsaparilla and guaiacum. It should not be resorted to in febrile and inflammatory conditions.

Preparations and Dose.-Oleum: dose, 2 to 10 min. Sassafras is a constituent of the decoctum sarze compositum, but the volatile oil, to which the drug owes its activity, is in great measure dissipated by the boiling. 


\section{NECTANDRA RODIAI.}

\section{(Bereeru or Bibiru.)}

DESCRIPTION.-A tree indigenous to British Guiana, and attaining the stature of 80 to 90 feet. The bark is whitish, the foliage dense and glossy; the leaves are opposite, oblong, about 5 inches in length, acute, and shortiy petiolate. The small yellowish-white flowers come out in thin axillary racemes, which are shorter than the leaves, and are followed by large fruits, the seed being the size of a walnut. Commercially the timber is known by the name of "greenheart." The bark itself is hard, heavy, and brittle, cinnamon-coloured within, and very bitter and astringent.

ACTIVE INGREDIENTS.-Bibiru bark contains an alkaloid called beberia or biberin $\mathrm{C}_{18} \mathrm{H}_{21} \mathrm{NO}_{3}$, a yellow resinous-looking body, which does not crystallize, is soluble in alcohol, slightly so in ether, and sparingly in water. In acids it dissolves, neutralizing them, and forming amorphous yellow salts which are uncrystallizable. There is also a certain quantity of tannic acid, which gives a green colour with a salt of iron. The beberix sulphas of the B.P. $\left(\mathrm{C}_{35} \mathrm{H}_{20} \mathrm{NO}_{6} \mathrm{HOSO}_{3}\right)$, presents itself in the form of thin brown scales, which are translucent, becoming yellow when pulverized, very bitter in taste, and soluble in alcohol and in water.

Physiological Action.-The properties of bibiru bark are tonic, anti-periodic, and febrifugal. The physiological action of biberin has already been described under Buxin (Calumba).

THERAPEUTICAL ACTION.-Intermittent Fevers.-Dr. Rodie discovered that the alkaloid contained in the bark and also in the fruit could be used as a substitute for cinchona, and employed it successfully in intermittents (Edin. Med. Surg. Jour., Oct., 1835). 
In practice, the stomachic and tonic effects of biberin appear to be produced without causing at the same time the headache, giddiness, and ringing in the ears, which often accompany the exhibition of quinia-hence, for patients with whom the latter drug disagrees, it becomes an exceedingly useful substitute: it appears to be generally inferior, but still, when properly administered, has cured intermittents after quinine had failed.

Both the bark and the sulphate of biberin have been exhibited for promoting digestion in anorexia and dyspepsia; also as a general tonic where the constitution is debilitated, e.g., in protracted phthisis, and strumous affections. As an anti-periodic it has also been resorted to in nervous headaches and intermittent neuralgias.

PREPaRations AND DOSE.-Beberice sulphicls: dose, 1 to $3 \mathrm{gr}$. as a tonic; 5 to $20 \mathrm{gr}$. as a febrifuge.

\section{LAURUS NOBILIS. (SweEt Bay.)}

\section{(Not Officinal.)}

DESCRIPTION.-A large, irregular shrub, rather than a tree, though often 30 feet high. The leares are broadly elliptical; the greenish-yellow, tetramerous flowers, produced in considerable abundance in early spring, are unisexual, and often diocious; the hard black fruit resembles a small plum. This is the only species of the order indigenous to Europe.

Active IngREDIENTS. - In England the bay-tree is distinguished from all other out-door evergreens by the cinnamon odour of the bruised leaves; this proceeds from a volatile oil, contained also in the fruit. The latter holds in addition a fixed fatty oil, and other ingredients. The " oil of bays" of commerce (oleum lauri expressum), which is a com- 
pound of the volatile oil and of the fixed fatty oil, is obtained from the drupes, fresh or dried, by means of heat and pressure.

Physiological ACTION.-The volatile oil obtained by distillation of the berries with water is aromatic, stimulant, and narcotic.

In large doses the leaves are emetic.

'Therapeutical Action. - Both the leaves and fruit have been given to strengthen the digestive organs, also to prevent flatus; they are said to act as an emmenagogue.

The oil from the fruit is used as a stimulating liniment in cases of sprain and bruises. It has likewise been given in paralysis, and for the relief of colic, and to mitigate deafness; but these uses are now obsolete.

PREPARATION.-The fruits enter into the composition of the emplastrum cumini, which also contains caraway, Burgundy pitch, and olive oil.

\section{COTO CORTEX. (CoTo Bark.)}

\section{(Not Officinal.)}

DESCRIPTION.-This bark was only imported from Bolivia in 1875, and its exact origin is not yet determined, but is probably from a tree of the natural order Lauraceæ: some have said rather Cinchonacer; others Rubiacer, or the Terebinths. It is in pieces, flat or curved, $\frac{1}{4}$ inch thick, light-brown in colour, smooth exterually, breaking with short, irregular fracture: it has an aromatic odour and taste. Another kind, known as paracoto bark, differs in unimportant particulars. 
Active INGRedients. - Cotoin $\left(\mathrm{C}_{22} \mathrm{H}_{18} \mathrm{O}_{6}\right)$ has been obtained in small, white, curved prisms, which are soluble in alcohol, ether, chloroform, and alkalies; very little so in cold water: solutions are precipitated blackish-brown by ferric salts. It is more frequently met with as a light-yellow amorphous powder with pungent taste (Jobst and Hesse).

Paracotoin $\left(\mathrm{C}_{19} \mathrm{H}_{12} \mathrm{O}_{6}\right)$ is in yellowish-white scales, which are tasteless. Besides these have been found, hydro-cotoin, leucotin, and cotonetin: also a pale yellow volatile oil of pungent taste, and an alkaloid probably identical with propylamin.

Physiological Action.-Not much known. It is said to be antiseptio, but not specially astringent.

\section{Therapeutical ACtion.-Internal.-Diarrhœa, etc.} -If the drug prove at all equal to some recent reports of its value in this disorder, it is a useful addition to our resources. According to Albertoni, there is no medicine so effective in the intestinal catarrh, e.g., of mental alienation and of chronic phthisis and of infancy : he did not find it cause constipation in bealthy subjects, and hence considers it not a direct astringent, but traces its effect to an antiseptic and special action on the intestinal mucous membrane (Record, 1883). Others corroborate its value in the diarrhœa of children especially, in typhoid fever, and in profuse sweating. Fronmuiller speaks well of it in the last-named, and in salivation. Of profuse sweating he reports ninety-one cases, of which twenty-one only were not relieved by one dose; but the effects of this lasted for only a night. Grette (Munich) and Parsons (New York) particularly note its value in infantile diarrhoea ("summer cholera") as not being narcotic. Burney $\mathrm{Yeo}$, in an interesting paper, shows what may be one cause of its failure in some cases, viz., combination with alkaline earths, etc.; for instnnce, when he gave it with mistura cretro it seemed inert, but when given in fluid extract with mucilage and cardamoms, it acted admirably in the colliquative diarrhoca of phthisis (Prnctitioner, vol. xxiii.). 
Preparations and Dose.-Puluis: dose, 1 to $8 \mathrm{gr}$. four times daily. Extractum fluidum: dose, $10 \mathrm{~min}$. (suspended in syrup, mucilage, etc.). Tinctura : dose, $10 \mathrm{~min}$. (suspended, v. Pharm. Journ., 1875). Cotoin : dose, $\frac{1}{2}$ to 2 gr. every two or three hours. A solution of 1 part in 4 of acetic ether is recommended for hypodermic injection: dose, 5 to $10 \mathrm{~min}$. and upwards.

Paracotoin is prescribed in similar doses with analogous results.

The powdered bark, being acrid, is not a good form for internal use. 


\section{ARISTOLOCHIACEAE.}

\section{THE BIRTHWORT FAMILY.}

Ax order of about 150 species of herbaceous, undershrubby, and often climbing plants, widely distributed, and especially abundant in tropical South America. The structure is anomalous, the wood being destitute of concentric zones, though the leaves have reticulated venation: the flowers are monochlamydeous; sometimes regularly trimerous, sometimes irregular; the stamens are either six or twelve, epipetalous, and sometimes gynandrous; the ovary is inferior, six-celled, and many-seeded.

\section{ARISTOLOCHIA SERPENTARIA.}

\section{(SERPENTARIA.)}

DESCRIPTION.-An herbaceous and almost stemless peremnial, indigenous to the woods of the United States. The root consists of a contorted and horizontal head, or rhizomatous portion, and a great number of yellowish fibres. The leaves are alternate, oblong-cordate, shortly petiolate, entire, and acuminate. The insignificant flowers, produced close to the ground, are brownish-purple.

ACTIVE INGREDIENTS. - The root of this plant contains a volatile oil, a resin, and a bitter acrid extractive matter, which is soluble in water. and spirit. The volatile oil is yellowish 
when newly evaporated, but with age becomes brownish; the odour is aromatic, the taste warm, bitter, and camphoraceous. All the properties of serpentary are yielded to water, as well as to proof spirit and to alcohol: by distillation it furnishes a considerable amount of a camphor.

Physiological Action.-Digestive System.-Taken internally, in small doses, serpentaria promotes appetite. In larger doses it causes nausea, flatulence, uneasy sensations in the stomach, and frequent, though not watery stools. According to Jörg, after many experiments, "serpentaria occasions nausea, eructation, vomiting, constriction and pain in the stomach, borborygmi, colic in the small intestine, discharge of flatus, and a disposition to go to stool, but without evacuation, or the evacuation of consistent frecs only." The appetite is sometimes impaired and sometimes increased; the stomach and bowels often become distended with flatus; itching about the anus, and even hrmorrhoids are produced occasionally. Hence it would appear that the medicine acts upon the alimentary canal as an irritant, producing a secretion, not of liquid, but of gas.

Circulatory System.-When absorption is completed, the pulse increases both in frequency and fulness; the surfaceheat of the body is heightened, and secretion and exhalation generally become augmented.

Nervous System.-Serpentaria is stimulant and tonic, and presents some analogy with camphor in its effects on the cerebral functions, being apt to induce headache and a feeling of oppression, and at night to disturb the sleep.

Therapeutical Action.-As implied in the name, the virtues of this plant were originally believed to render it an antidote to serpent-bites. The condition of the system in which the value of serpentaria is best declared are those of atony and torpor. In the low stages of Typhus, when the tongue is dry, and brown or black, and the pulse feeble, it is by some considered a valuable remedy, in combination with sesquicarbonate of ammonia.

In other Adynamic Fevers, the low delirium, watehfulness, 
and other irregular actions of the nervous system which often occur, appear to be amenable to serpentary.

In Remittent Fever, especially when the remission is obscure, this drug is by some thought preferable to cinchona, being seldom irritating to the stomach, and having no injurious effects. Sydenham remarks that in all cases where it is expedient to combine wine with cinchona, the effects are much improved by the addition of serpentaria: it also enables the stomach to retain the cinchona better. He recommended a scruple of it taken in 3 ounces of wine as a remedy for tertians.

In Bilious Vomiting, American physicians report serpentaria to be efficacious in checking nausea and tranquillizing the stomach. For this purpose it is given in decoction, in doses of a table-spoonful frequently repeated.

In cynanche maligna it is employed externally, and as a gargle.

In Dyspepsia, Chronic and Gouty Rheumatism, serpentary is given, and in the last-named is recommended by Garrod as a remedy of some power.

Preparations and Dose.-Puluis: dose, 10 to $20 \mathrm{gr}$. or more. Infusum: dose, 1 to $2 \mathrm{oz}$. Tinctura: dose, $\frac{1}{2}$ to $2 \mathrm{dr}$. It is contained in the compound tincture of cinchona known formerly as Huxham's. 


\section{JUGLANDACEA.}

\section{JUGLANS REGIA. (WaLnut.)}

(Not Officinal.)

DESCRIPTION.-A large elegant tree, growing in Europe, with exstipulate pinnate leaves, and two kinds of catkins, one bearing sterile, the other fertile flowers. In the latter, the perianth is five- to six-lobed, the ovary two- to four-lobed, and ripening into a drupe: the kernel is commonly wrinkled; both leaves and fruit are officinal in the German Pharmacopœia.

ACTIVE INGREDIENTS. - These are probably similar to those of the juglans cinerea, officinal in the U.S. Pharma., and the bark of which contains tannin, a volatile and fixed oil, and an acid, also volatile-juglundic acid, identical with the nucin found in the fruit and leaves of J. regia by Reischauer and Vogel.

This body, known also as juglone, is acrid in taste, of acid reaction, soluble in alcohol and ether, less so in water; may be crystallized in orange-coloured needles, which turn purple with alkalies; the accepted formula is $\mathrm{C}_{36} \mathrm{H}_{12} \mathrm{O}_{10}$. A crystalline sugar, nucit, $\mathrm{C}_{6} \mathrm{H}_{12} \mathrm{O}_{6} 2 \mathrm{H}_{2} \mathrm{O}$, and also a crystalline alkaloid, have been obtained by Tanret from walnut leaves (European).

Action.-This plant seems to illustrate the fact that sometimes no definite physiological action can be made out, and yet a therapeutical effect may be obtained.

In some old French works it is recommended as an astringent, whilst the green fruit has a repute in constipation, and the leaves, locally applied, in scrofulous and even malignant ulcern- 
tion, but it is especially for the symptom of vomiting that the remedy, in form of tincture, deserves notice. Dr. Cooke asserts that it never failed him, even when cerium, prussic acid, and other remedies had done so: he does not, however, distinguish the class of cases (Lancet, i., 1866). To a limited circle it has been known for some time as valuable in the sickness of pregnancy and of uterine disorder; and Dr. Mackey has recorded marked benefit from it in hysterical and other forms of apparently neurotic vomiting (Practitioner, vol. ii., 1878). Dr. Clifford Allbut recommends it in similar cases (Gulstonian Lectures, 1884).

PREPARATION AND DOSE. - Spiritus meis juglandis (fresh walnut 30 oz., rectified spirit 12 oz.: water, q.s., distil, 16 oz.-Southall). This should be clear, light-brown, and aromatic. Dose, 1 to $2 \mathrm{dr}$. (in water); may be repeated every half-hour for some time. The dose of Messrs. Corbyn's preparation is $\frac{1}{2}$ to $1 \mathrm{oz}$.

The juglans nigra $v$. cinerea is used in diphtheria in the form of decoction, gargle, spray, and fomentation (Bost. Med. and Surg. Journ., March, 1881). 


\section{THYMELACEA.}

\section{THE MEZEREON FAMILY.}

As order of about 300 species of exogenous shrubs, with a few trees and herbaceous plants, natives chiefly of the temperate parts of the southern hemisphere. The leaves are simple, undivided, entire, often evergreen. The flowers are monochlamydeous; the perianth is tubular, regular, generally fourlobed; the stamens usually very few ; the ovary is superior; the ripe fruit either dry or drupaceous and juicy.

The bark of the Thymelacer often has caustic properties; if chewed, it causes pain in the mouth, and applied to the skin it acts as a vesicant.

\section{DAPHNE MEZEREUM.}

(The Mezereon.)

DESCRIPTION.-A bushy deciduous shrub, native of Europe, and growing 3 to 5 feet high. The branches are numerous and nearly erect; their upper portions are densely clothed in early spring with sessile, fragrant, tetramerous pink flowers; the lanceolate and glabrous leaves come out subsequently ; the ripe fruits resemble red currants, but are solitary and sessile.

ACTIVE INGREDIENTS.-Besides a resin and a volatile oil, mezereon bark contains daphnin, which is a neutral crystalline glucoside $\left(\mathrm{C}_{15} \mathrm{H}_{10} \mathrm{O}_{9}+2 \mathrm{H}_{2} \mathrm{O}\right)$, bitter, and slightly astringent, but inert; the resin is dark green, acrid, and soluble in alcohol and in ether; the volatile oil is acrid and highly irritant: upon this and the resin depend the active properties. When mezereon-root is boiled in water an acrid vapour is given off. 
Physiological Action.-The berries have long been known to be highly dangerous, and, in a case recorded by Linnæous, are said to have caused death. The bark of the root, in a less degree that of the stem, is excessively caustic. Chewed, it gives at first an impression of sweetness, but soon an acrid, burning sensation in the mouth and fauces, lasting for several hours, and if the impregnated saliva be swallowed, or any portion of the bark itself, the burning extends to the throat and stomach. Pereira says that "all parts" of the plant "swallowed in large quantities" . . . "prove poisonous." The topical action of the bark is that of an irritant, tending to vesication. A decoction, taken internally, appears to promote the action of the skin and of the kidneys (though Dr. Alex. Russell disputes these effects). In large doses it causes irritation both of the kidneys and of the alimentary canal, and often proves larative: it increases the flow of saliva, and causes pain in the stomach and bowels; sometimes vomiting as well as purging.

Therapeutical AĆtion. - Mezereon bark has been resorted to with good effect as a eure for toothache; and as " masticatory, in cases of paralysis of the tongue.

Soaked in vinegar and water, the bark may be applied as a compress, in order to vesicate: and issues and blisters may be kept open with ointment prepared from it.

It has been recommended in rheumatic and scrofulous diseases; also in chronic cutaneous disorders, and in syphilis.

There would, however, seem to be some uncertainty in its action, or perhaps some constitutions only are open to its effects. Mr. Pearson denies its utility in syphilitic disease, but Cazenave and other French authorities prescribe it in these cases.

PREParations. - In England, mezereon is rarely emIlloyed alone: there is but one efficient preparation, viz., the extruetum. meserei retherem (contained in linimentum sinapis compositum). It occurs also as an adjunct to the decoctum sarzac compositum. 


\section{EUPHORBIACEAE.}

\section{THE CROTON FAMILY.}

A LARGE and important class of exogens, diffused extensively over the world, abounding especially in the tropics, and very diverse in appearance. Many are trees, or substantial shrubs; some are milky-juiced herbaceous plants; while some resemble the cactus. The leaves are generally simple and undivided; the flowers usually of simple structure, often minute, and surrounded by large brilliantly-coloured bracts; they are unisexual, and the fruit is a three-celled capsule, the cells containing each a solitary seed, and opening, when ripe, by a vertical suture. The perianth, when present, is hypogynous: the petals, if any, usually free, the stamens either free or monadelphous, the ovules "suspended," and the embryo enclosed in a large quantity of fleshy albumen. The properties of the order are acrid, energetic, and poisonous: the officinal species are the following:-

Ricinus communis ... $\quad \ldots \quad \ldots \quad \ldots \quad$... Castor-oil plant.

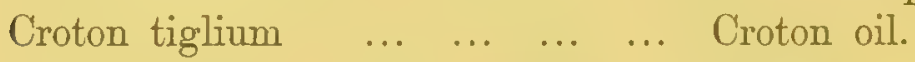

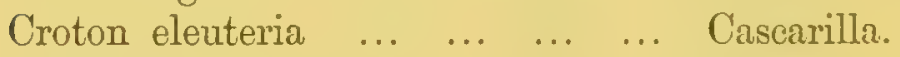

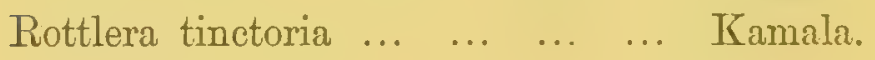

\section{RICINUS COMMUNIS.}

(Castor Oit..)

DESCRIPTION.-The Ricinus communis is a native of the Indies, of South America, and China. In temperate countries it is known only as an annual of moderate size, but under favourable conditions it attains the height of 30 feet, and endures for several years. The large and handsome leaves 
are alternate, palmately lobed, glabrous, and shining. The insignificant flowers are borne in long spikes; the ovaries become trilocular and prickly capsules, the size of a hazelnut, each of the three cells containing a large oval seed, variegated with black and grey. The oil employed in pharmacy is obtained from these seeds by expression. It is "fixed," translucent and viscid, usually of a pale yellow tint; sp. gr. 0.969 ; of little taste or smell, though what flavour there may be is considered nauseous: it is soluble in absolute alcohol, ether, glacial acetic acid, and oil of turpentine.

ACTIVE INGREDIENTS.-Castor oil contains a peculiar glyceride, called ricinic or ricinoleic acid, $\mathrm{C}_{18} \mathrm{H}_{34} \mathrm{O}_{3}$, which may be obtained as a yellowish inodorous liquid of acrid taste, becoming a granular solid below $32^{\circ} \mathrm{F}$. The oil contains also a solid fatty acid related to palmitic acid. Its true acrid principle has not been isolated. In the seeds, besides the oil, is a proteid (to about 20 per cent.) allied to emulsin, and according to Tuson, an alkaloid, ricinin, which crystallizes in prisms and scales. Ricinic acid was formerly thought to be the purgative principle, but more accurate researches do not support this view. Buchheim showed the probability, that (as with croton oil) a saponification process, by mixture with the alkaline intestinal fluids, produces the substance which really operates as a purgative; its effect can be imitated by ricinate of soda. The active drastic principle of the seeds has not been isolated; some trace of it contained in the oil may be the source of its purgative power.

Physiological ACTion.- $\mathrm{A}$ moderate dose of good castor oil acts as a mild purgative,-in some susceptible subjects as an emetic: the discharges produced by it are fluid and frecal and contain some of the oil more or less changed; they are usually passed without griping. The same effects may be produced by frictions of oil upon the skin, and conversely a case (of hydramia) is on record where the oil taken internally was in part excreted by the skin (London Med. Gaz, vol. x.). Half an ounce injected into the ecins did not purge, but depressed the circulation, causing faintness, nausea, and prostration (Hale). In the ordinary way its purgative action is generally succeeded 
by lassitude and tendency to sleep: some constipation is apt to follow it, but when used habitually the dose may be gradually diminished instead of requiring continuous increase.

The beans and seeds of the castor oil plant, when taken internally, have caused violent colic, purging, vomiting, collapse, and death, with evidence of inflammation of the gastro-intestinal mucous membrane. In a case where ten to fifteen seeds were taken by a child, but recovery took place under emetics, etc., constipation was a marked after-symptom (Record, 1882).

THERAPEUTICAL ACTION.-Extemal.-Castor oil is with much advantage dropped into the conjunctival sac after the removal of a foreign body, and soon allays the painful irritation. It is also useful, similarly applied, in scrofulous ophthalmia. As a galactagogue a decoction of the leaves applied to the breasts of nursing women, or administered internally, is said to promote, or even to occasion, the secretion of milk.

Therapeutical ACTion.-Internal.- Constipation, etc.-Castor oil is particularly valuable when this arises from indurated fæces, or after swallowing acrid substances, or from the accumulation of unhealthy secretions.

It is likewise employed with great advantage in disenses attended by irritation or inflammation of the bowels, diarrhœa, dysentery, and enteritis, as a preliminary purgative before commencing sedatives and astringents; it is also sometimes adopted as part of the continuous treatment of such disorders. Thus Dr. Young (Florence) has reported many cases of acute diarrhœa, a complaint often very severe in Italy, successfully treated by the following:-Castor oil, 24 minims; spirits of chloroform, $1 \frac{1}{2}$ drachm; liquor morphire hydrochloratis,

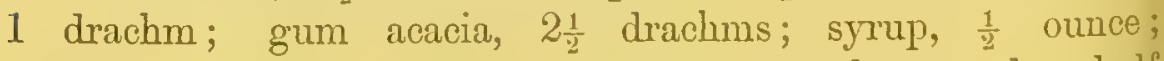
water to 4 oumces: dose, 2 drachms every hour and a half for an adult. Six drops of the morphia solution is the maximum dose: in chronic cases with mucus in the stools, six drops of the oil are ordered for each dose-the mixture does not keep well (Practitioner, i., 1875). I have had good results with the above formuln. Blache gives the oil with mucilage and symu only (Med. Times, ii., 18\%7). 
As a laxative, castor oil operates so speedily and mildly, that it is constantly resorted to where similar operation by medicines of a more powerful nature would cause injury: hence its value in hæmorrhoids, in inflammatory or spasmodic diseases of the genito-urinary organs, in inflammation of the kidneys or bladder, in calculous affections, gonorrhœea, and stricture, and also in affections of the rectum, especially stricture.

Pregnant women employ" it with considerable advantage. It is a valuable evacuant also for children, and is the safest for infants. A larger relative dose may be given to them than to adults, probably from their digesting more of what is swallowed.

PREPARATIONS AND DOSE.-Emulsion.-Castor oil $\frac{1}{2}$ oz., mucilage $\frac{1}{2} \mathrm{Oz}$., syrup of ginger $\frac{1}{4} \mathrm{oz}$, cinnamon water $1 \mathrm{oz}$. Mix thoroughly. The oil may be administered floating upon some aromatic water; or mixed in a cup of hot and sweetened coffee. The combination of an equal part of glycerine increases its efficacy, so that 1 or 2 teaspoonfuls act as well as 1 tablespoonful alone. The combination is best effected by shaling together in a bottle; a few minims of tincture of senega emulsifies the mixture. Patients who dislike the flavour are apt to suck a peppermint lozenge immediately after swallowing the dose; but to cover the taste of this, or any similar medicine, the mouth should be saturated with peppermint before, instead of after taking the oil. The purgative dose is, for adults, from 1 to 4 dr. and upwards; for infants, 1 to $2 \mathrm{dr}$.

An enema may likewise be prepared by the combination of 2 or 3 oz. of castor oil with any mucilaginous fluid.

It is an ingredient of the collodium flexile.

ADULTERATIONS.-Two kinds of adulterntion are practised with castor oil ; the admixture of a small quantity (1) of croton vil; (2) of some cheaper oil of bland and viscid character. 


\section{CROTON TIGLIUM.}

\section{(Croton Oil.)}

DEScription.-The Croton tiglium is a moderate-sized tree, indigenous to India and Ceylon. The leaves are oral and serrated; the small flowers come out in thin terminal racemes, which are usually erect; the ovaries ripen into obtusely triangular capsules, which severally contain three pale-brown, somewhat mottled seeds, which are about $\frac{1}{2}$ inch long, oblong, flattened on one surface, and marked by a raised longitudinal raphe.

ACTIVE INGREDIENTS.-Croton oil, as expressed from the seeds and employed in medicine, is a clear, darkish-brown, rather thick fluid, and contains, according to Schlippe, 4 per cent. of a substance which he calls crotonol $\left(\mathrm{C}_{13} \mathrm{H}_{23} \mathrm{O}_{4}\right)$, and upon which the irritant action of the drug on the skin appears to depend. It is a colourless or pale-yellow turpentine-like substance, with a faint but characteristic odour; it is destroyed by heat, and the action of caustic potash changes it to a brownish resinoid mass. This substance, however, has not been obtained by other chemists, and is not the purgative principle of croton oil, for Schlippe could not produce either diarrhœe or intestinal inflammation when he administered as much as $1 \frac{1}{2}$ grain to rabbits. The oil also contains fixed fatty acids, and two rolatile ones-tiglinic, which is isomeric with angelic acid, $\mathrm{C}_{5} \mathrm{H}_{5} \mathrm{O}_{3}$, and crotonic acid, $\mathrm{C}_{4} \mathrm{H}_{6} \mathrm{O}_{2}$. Neither of these acids appears to. be purgative, and the latest researches render it probable that the drastic principle is only secondarily developed from a saponification process which produces a resinoid body within the alimentary canal.

Physiological Action. - External. - When locally applied, croton oil causes a characteristic eruption, rarying in 
extent and severity with the tenderness of the skin and the strength of the liniment. After some redness and sense of heat, small red pimples appear, which later become pustules, some of which are rounded, some flattened and umbilicated with a red areola; many are confluent: they may continue to develop for three or four days, and then abort or crust over; in about a week they peel off, leaving, as a rule, no cicatrix.

Physiological Action.-Internal.-A small fragment of a croton bean chewred produces smarting, burning, and tingling of the mouth and throat. One minim of the oil is apt to cause burning sensations in the throat and stomachpossibly nausea and sickness. After an hour or two, colic and watery stools occur, with straining and burning about the anus. Upwards of ten motions may follow in the twentyfour hours, but sometimes it does not act well, causing rather general discomfort and fererishness. Three or four drops by enema act very powerfully. After much larger doses the symptoms may be extremely violent,_-vomiting, purging, collapse, and often, though not always, death resulting, the symptoms much resembling those of Asiatic cholera. If the patient survives a large dose, he suffers from gastro-enteritis.

These poisonous effects have been produced, not merely by the oil, but also by the accidental eating of one seed. The inhalation of the dust by those who handle large quantities of the seeds has likewise caused epigastric pain, soreness of the eyes, and swelling of the face; and in one recorded case has even proved fatal.

Therapeutical ACTION.-External.-Inflammation. - In cases of deep seated inflammation the irritant substitutive action of croton liniment externally has proved highly beneficial. Some practitioners have even credited the pustulation of the shaven scalp with the cure of meningitis, and I have myself seen much benefit in such cases; but the remedy is a very severe one, and the tendency of modern opinion is rather against its use, in childron especially : it is more permanent in its effect than blistering. 
In Sciatica and other obstinate Neuralgias and in chronic articular Rheumatism it has given relief.

In Chronic Phthisis and Laryngitis it may be used on the surface over the affected part, and Dr. Park has recently drawn attention to its value in Acute Bronchitis; he says that 2 or 3 drachms of the liniment may be rubbed over the chest even in children without causing more than redness and irritation, and if quickly covered with cotton wool no ill effects are produced, but marked relief; an adult patient can go on with his work during the treatment (Practitioner, i., 1882).

Ringworm.-In chronic recurrent patches of this disorder, pustulation by croton oil is sometimes a valuable, though painful, resource. It has been specially advocated by Mr. Alder Smith, and also by Dr. Liveing and others, with some difference in the mode of its application (Lancet, i., 1880).

The pure oil is perhaps the best to apply in small quantity, and over a limited area; and after it, continuous poulticing should be used for some days to soften crusts and lessen irritation.

Constipation, etc.-Croton oil is valuable in cases where a rapid and drastic effect is desirable; thus it is much used by surgeons in cases of head injury, being easily given, even if the patient be unconscious, in a little butter or with a few drops of castor oil. In other cases, too, such as "head congestions," apoplexy, and uromic coma, croton oil is used; as well as in obstinate fæcal obstructions without inflammation or organic disease, such as occur in plumbism. Small quantities of the drug are usually effective, but as much as 8 or 10 minims have been known to fail. Some vascular depression follows the action of croton oil, but as a rule no seriously disagreeable effects.

PREPARATIONS AND DOSE.-OTeum erotomis: dose, $\frac{1}{4}$ to 1 min., administered in pill (with conserve of roses, or gum and sugar), or better dissolved before mixing, with a little alcohol, or mixed with powdered sugar, and placed upon the tongue, or it may be added to castor oil. Limimentum. contains one part of croton to seven parts of a mixture of equal parts of spirit and cajeput oil. 
ADULTERATIONS.-Croton oil is adulterated with castor oil. The Pharmacopocial test (solubility in alcohol) is inefficacious to detect this, unless more than 33 per cent. of castor oil be present.

\section{CROTON ELEUTERIA.}

\section{(Cascarilla.)}

DESCRIPTION.-Cascarilla bark, so named from reserer, the Spanish word for the rind or bark of a tree, presents a remarkable exception to the acridity of euphorbiaceous plants in general. It is yielded by a small tree, the leaves of which are ovate and entire, about 2 inches in length, and covered on the under surface with silvery pubescence. The flowers come out in axillary and terminal racemes, which are somewhat branched, and thickly studded with sub-sessile, sweet-smelling blossoms, followed in due course by tricoccous capsules the size of peas. The bark is met with in quills or pieces, 1 to 3 inches long, or in fragments. The thin, grey, outer layer is more or less covered with white patches of lichen; it breaks with a short resinous fracture; it is aromatic, much more so on burning; the inner surface is chocolate colour.

ACTIVE INGREDIENTS.-The active elements of cascarilla bark are probably the neutral crystalline principle cascarillin, and, in less degree, two resinoid extracts. Cascarillin forms white, six-sided needles, odourless, of bitter taste, very insoluble in water, but soluble in alcohol and ether. It gives a blood-red colour with concentrated sulphuric acid, and a violet-red with concentrated nitric acid. The formula assigned to it is $\mathrm{C}_{12} \mathrm{H}_{18} \mathrm{O}_{4}$. The bark contains also a volatile oil, of which one element is a hydrocarbon, $\mathrm{C}_{10} \mathrm{H}_{16^{\circ}}$

Physiological ACtion.-No exact experiments have yet been made upon the physiological action of cascurilla or its crystalline principle. It is known, however, that a strong infusion of the bark acts as a stimulant, and in considerable 
dose as an irritant, causing nausea, vomiting, and diarrhoca. It has even been said to produce nareotic effects in very susceptible subjects; but this statement appears open to doubt. The fumes of the bark are intoxicating (Stillé).

THERAPEUTical ACtion.-The bitter-tonic qualities of this drug have recommended it as a substitute for cinchona; and although inferior to that medicine in tonic and febrifugal operation, it has the advantage of agreeing often better with the stomach, being not merely bitter, but aromatic. In irritable conditions of the alimentary canal, cinchona is apt to produce vomiting or purging, but neither of these results ensues upon the exhibition of cascarilla in moderate dose.

In England, cascarilla is chiefly employed in such forms of Dyspepsia as require an aromatic stimulant and tonic.

It is also used in cases of Debility generally; and in chronic Bronchial Affections with a view to cheeking excessire secretion of mucus.

In Germany, where it is a favourite medicine, cascarilla has been used in many other affections, such as low nerrous ferers, intermittents, dysentery, and the later stages of diarrhœa; but of late, it would seem to have fallen into disrepute.

Preparations and Dose.-Putili: dose, 10 to $30 \mathrm{gr}$. Infiusun : dose, 1 to $2 \mathrm{fl}$. oz. Tinctura: dose, $\frac{1}{3}$ to $2 \mathrm{fl}$. dr. 


\section{ROTTLERA TINCTORIA.}

(KAMALA.)

DESCRIPTION.-An evergreen shrub or small tree, which grows wild in Australia, India, Abyssinia, etc., attaining the stature of 1.5 feet; the leaves are alternate and entire; the insignificant flowers grow in axillary spikes, and are followed by capsules the size of a cherry, coated with a deep-red granular powder, which constitutes "kamala:" it is inflammable; does not mix readily with water, but imparts its powers to alcohol.

ACTIVE INGREDIENTS. - Of the three characteristic bodies detecterl by Anderson in kamala,-_rottlerin, rottlera-red, ${ }^{1}$ and a peculiar flocky substance,-it is probable that the first named is the true medicinal principle (Edin. New Phil. Journ., new series, i.). This one-rottlerin, $\mathrm{C}_{22} \mathrm{H}_{20} \mathrm{O}_{6}$-crystallizes out of ether in yellow silky crystals, which melt with heat, and are decomposed at a higher temperature. They are soluble in water, not readily so in alcohol, but easily in ether. Aqueous solutions of alkalies dissolve rottlerin with a deep-rerl colour.

Physiological Action.-In full doses kamala acts as a rather drastic purgative, and may cause violent pain, tenesmus, and sanguiner-mucous strols, sometimes nausea and vomiting.

Therapeutical Action.-The chief medicinal use of kamala is as a vermifuge, especially in tapeworm. Its value in this disorder was first brought prominently into notice by Dr. Mackinnon (Indian Ann. Merl. Sciences, i.), and other observers have spoken strongly in its favour. It may, perhaps, fairly be considered the best remedy for tronia ufter filix mas. An incidental advantage that it possesses over the latter is, that the patient does not need to take any other medicine,

A beatiful dye for silk. 
either before or after the dose of kamala. The worm is commonly voided dead.

It has acted equally well in cases of round and thread worms, though not usually prescribed for them, probably because its effect is often very severe. It appears, from the statements of Mackinnon, that the Hindoos employ kamala successfully as a local application for the destruction of external parasites in itch and in herpes circinatus.

PREPARATIONS AND DOSE.-Pulis : dose, as a vermifuge, 1 to $2 \mathrm{dr}$., which may be given in syrup in the early morning; and repeated in eight or ten hours if necessary. In delicate subjects it will be safer to begin with a smaller dose. Tinctura (not officinal) is made of two strengths - either 1 part of kamala to $3 \frac{1}{2}$ of rectified spirit, or 1 part of kamala to 5 parts of proof spirit. Of the stronger tincture, 1 to $2 \mathrm{dr}$., either in one or two doses, with some aromatic water: of the weaker tincture, 2 to $4 \mathrm{dr}$. 


\section{PIPERACEA.}

\section{THE PEPPER FAMILY.}

Ax order composed of about 600 species of herbaceous and undershrubby exogens, natives exclusively of the hottest parts of the world, where they constitute a large portion of the weedy vegetation. The leaves are usually ovate or lanceolate; the minute apetalous greeī flowers are collected into slender spikes; the stamens are extremely few; the ovary is superior, one-celled and one-seeded, and ripens into a berry.

Orclinarily, these plants are possessed of an aromatic pungency, referable to the presence of a peculiar acrid resin, an ethereal oil, and the crystallizable body called piperin: they are also astringent and narcotic.

\section{Pharmacentical Species.}

Piper nigrum . . . . . . . . . Black pepper.

Chavica Roxburghii .. .. .. .. Long pepper.

Cubeba officinalis ... . . . . Cubebs.

Artanthe elongata ... . . . . Matico.

\section{PIPER NIGRUM.}

(Br.Ack Pepper.)

DESCRIPTION.-A perennial, native of the East Indies. The slender stems climb to the hoight of 12 or 15 feet, or trail upon the ground and root at the joints. The leaves are alternate, broadly ovate, petiolate, and shining; the sessile rerl 
berries, the size of small peas, are borne in racemes, and when dried constitute the common black pepper-corns of commerce; when decorticated, white pepper.

Active IngREDIENTS. - Pepper is hot, pungent, and aromatic, and along with other ordinary constituents of small berries, contains the above-named piperin, a resin, and a rolatile oil. Piperin, $\mathrm{C}_{17} \mathrm{H}_{19} \mathrm{NO}_{3}$, occurs, when pure, in white, crystalline, rhomboidal prisms, which are odourless, and almost tasteless. In cold water it is insoluble, in boiling water it is scarcely soluble; but in alcohol and acetic acid it dissolves readily and, in a somewhat less degree, also in ether. It is volatile, and fuses at $212^{\circ} \mathrm{F}$.

The resin, which exists in large quantities, is soft and acrid, solid at $32^{\circ} \mathrm{F}$, extremely pungent, soluble in alcohol and ether; with fatty bodies it unites readily.

The volatile oil, $\mathrm{C}_{10} \mathrm{H}_{16}$, when pure, is colourless, and has the taste and odour of pepper: sp. gr. 0.9932.

Physiological Action.-Pulverized pepper, allowed to remain in contact with the skin, induces redness and pain. Taken into the mouth, it excites sensations of intense buming; and, if absorbed by the nostrils, burning and severe sueezing. Swallowed in moderate quantities as a condiment, it stimulates the stomach, exciting a sense of warmth, and slightly accelerating the pulse. Digestion is assisted, diaphoresis promoted, and the mucous surfaces excited. It is a warm carminative stimulant, capable of producing systemic excitement, and of lirectly acting upon the mucous menbrane of the alimentary canal and genito-urinary tract. The action is not diffusible but local, and hence, in assisting feeble digestion, it checks a tendency to flatulence, or, if flatus be present, the escape of it is promoted, owing to the local action of pepper upon the rectum.

Taken in excess, pepper induces intestinal inflammation, with violent burning pain in the epigastric region, accompanied by great thirst; in one instance vomiting ensued. In severnl cases that have been recorded, the immoderate use of pepper has been followed even by rigors, convulsions, and delirium. 
THERAPEUTICAL ACTION.--In the form of ointment, pepper is a good application for Tinea capitis: it is well combined also with mustard in the preparation of sinapisms.

In cases of Relaxed Uvula, it may be advantageously used in the form of gargle, or as a masticatory, and thus employed it may be of service in Paralysis of the Tongue, and affections of the mouth and throat, which demand the employment of a powerful local stimulant.

The ancient Greek physicians recommended it with warm water, for the relief of "cold fit." In Dyspepsia, it is effective as a gastric stimulant, and is given also to check romiting, to abate nausea, and to stop singultus.

In the form of confection, pepper is well adapted to those of weak and leucophlegmatic habit, for diseases connected with the rectum, such as fistula, ulcers, catarrh, or hæemorrhoids, the affected parts being gently stimulated. The use of it in this way requires perseverance, and there is the objection that it is liable to accumulate in the colon, so that laxatives are required. Sir Benjamin Brodie says that such accumulation has caused the disappearance of severe hæmorrhoids, the local effect being energetic in the extreme.

As a febrifuge, pepper has been found serviceable by the French and German physicians ; and in our own country it has long been a popular medicine in intermittents, being administered in spirit-and-water when the paroxysms are about to commence.

Piperin is said to possess febrifugal properties that allow of its comparison with the cinchona alkaloids, but while in no way superior, its cost alone would hinder its employment.

PREPARATIONS AND DOSE.-Pullis: dose, 5 to $20 \mathrm{gr}$. Confectio: dose, 1 to $2 \mathrm{dr}$. and upwards. Piperin: dose, $5 \mathrm{gr}$. and upwards. Black pepper enters into the composition of the "Asiatic" pills.

ADULTERATIONS.-The powder is liable to admixture with flour, sago, and other farinaceous matters. 


\section{CHAVICA ROXBURGHil. (Long Peprer.)}

\section{(Not Officinal.)}

"Long pepper" is the name applied to the fruit-spikes of this plant, gathered while immature, and dried. They are 2 or 3 inches long, cylindrical, greyish, and minutely beaded with the rudiments of the berries. The ingredients, action, and doses, are much the same as those of piper nigrum. Whether it be more or less powerful than the latter appears to be undetermined.

\section{CUBEBA OFFICINALIS.}

(Curebs.)

DESCRIPTION.-The plant producing this drug closely resembles the piper nigrum, the only material distinction consisting in the ripe fruits being pedunculate instead of sessile. For medicinal purposes, the berries are gathered while immature: when dried, they resemble black pepper, but are lighter in colour, and readily distinguishable by the long stalks. The importation takes place chiefly from Batavia and Singapore. A portion of the cubebs of commerce is derived, probably, from the Cubeba canina, the fruit of which is less pungent than that of the C. officinalis, and possesses a flavour of anise.

ACTIVE INGREDIENTS.-The odour of cubebs is peculiar, camphoraceous, and aromatic; the taste hot and peppery. They contain a volatile oil, a resin, and a substance callerl cubebin.

The volatile oil, oleum cubebr, $\mathrm{C}_{15} \mathrm{H}_{24}$, is colourless, or pale greenish yellow, and, like that of black pepper, lighter than water, the sp gr. being 0.929 . It yields the odour and possesses the flavour of cubebs, and evaporates on exposure to the atmor- 
sphere. Upon this volatile oil the active properties mainly depend; cubebs, therefore, should not be pulverized for medicinal use until actually wanted. The powder is dark in colour, and of oily appearance.

The resin is soft and acrid; the cubebin, $\mathrm{C}_{10} \mathrm{H}_{10} \mathrm{O}_{3}(\mathrm{~F}$. \& H.), is neutral, and analogous to piperin; it crystallizes in small needles.

ABSORPTION AND ELIMINATION. - Readily absorbed, the active principle is eliminated by the respiratory mucous membrane, skin, and kidneys.

Physiological Action.-Intennal.-Digestive System. -In small doses, cubebs, like other peppers, stimulate the gastric mucous membrane, and so augment appetite and improve feeble digestion. The oil, even in moderate doses, is apt to cause thirst, heat in the throat, nausea, general feverishness, and headache. Larger doses, or conditions of gastric irritation, determine the production of vomiting, colic, and diarrhœea.

On the whole, however, cubebs are less irritant to the digestive tract than copaiva.

Cutaneous System.-A red papular rash, somewhat like that induced by copaiva, has been occasioned, though seldom by cubebs.

Genito-Urinary System.-It is to this generally in man that the special action of the drug is directed, its effects varying according to dose, etc., from simple stimulation with increase of urine, to congestion or inflammation of the kidneys, pain, hæmaturia, and extensive irritation of the urinary tract.

Under full doses, the urine commonly deposits a resinous precipitate on adding nitric acid: this simulates the reaction with albumen, but as in the case of copaiva-deposit, the liquid elears on boiling.

Nervous System.-In rabbits toxic symptoms like those of copaiva may be induced. Gubler records increase of temperature, pains in the head, convulsion, and even paralysis.

Therapeutical Action. - Chronic Gonorrhoea. Practically, at the present day, the principal use mado of cubebs is in the treatment of this disorder. Though efficacious, 
they have sometimes induced conditions very distressing to the patient, such as hæmorthoids, hæmaturia, and deep-seated headaches, sometimes concomitant diarrhœea and nausea so severe as to oblige discontinuance of the medicine. The stimulant operation of cubebs upon the bladder is well illustrated in a case described by Sir Benjamin Brodie (Lond. Med. Graz., i., 300). They are good for catarrh of this organ, and for incontinence from atony, and for leucorrhoa.

Catarrh, etc.-The utility of cubebs is not limited to their action upon the mucous membrane of the genito-urinary organs, but has been proved also in catarrhal affections of the airpassages, especially when accompanied with copious secretion, as well as in chronic inflammation of the mucous membrane of the bronchial tubes and intestinal canal: in such cases oxide of bismuth in combination has been recommended. Originally, they seem to have been resorted to as a gastric stimulant and carminative in atonic dyspepsia, and in India are still accounted useful as stomachic.

Rheumatism has likewise been treated by cubebs, but with questionable success.

PREPARATIONS AND DOSE.-Pulic: dose, 30 to $120 \mathrm{gr}$. Oleum: dose, 5 to 20 min. Tincturu: dose, $\frac{1}{2}$ to $2 \mathrm{dr}$.

The saccharate of cubebs (Delpech) is popular in France: it is tasteless and more readily taken.

\section{ARTANTHE ELONGATA.}

(Matico.)

DESCRIPTION.-A shrub, indigenous to Peru, where it attains the stature of 10 or 12 feet. The leaves are several inches in length, oblong-lanceolate or ovate, pointed, petiolate, serrated, and reticulated in every part with depressed veins.

ACTIVE INGREDIENTS. - Matico leaves contain a light green volatile oil, which becomes thick and crystalline on 
exposure, a dark green resin, a bitter principle called maticin (soluble in alcohol and water, insoluble in ether), traces of tannic acid, and another acid readily crystallizable termed artanthic.

Physiological Action.-Little is yet known beyond the general fact that matico is aromatic, stimulant, and laxative: it has properties in common with turpentine, cubebs, and copaiva.

THERAPEUTICAL ACTION.-Matico was introduced to this country in 1839 as a good internal or chemical styptic: it is useful as a substitute for cubebs, in cases of discharge from mucous surfaces. The tamnin makes the infusion of matico astringent and slightly hrmostatic. The leaf or powder at present is more employed for stanching the flow of blood from trivial cuts and wounds, such as leech-bites, its action being mechanical, like that of other fine webs and powders.

PREParations and Dose.-Pulis: dose, 30 to $40 \mathrm{gr}$. Infirsum: dose, 1 to 4 fl. or. 


\section{SALTCACEAE.}

\section{THE WILLOW FAMILY.}

THIs family is constituted exclusively of the willows and the poplars, all of which are ligneous, though some of the former are diminutive shrubs. They are diøcious; the male and female plants both produce their flowers in catkins, and the seeds are provided with a wing-like, cottony tuft.

\section{SALIX FRAGILIS. \\ (The Crack Willow.)}

DESCRIPTION.-A large and handsome deciduous tree, indigenous to Europe. The leaves are lanceolate, 4 to 5 inches in length, acuminate and serrate; the male catkins are yellorish, the females green and very slender; and in each case they accompany the young foliage. A variety called Salix Russelliana, or the Bedford willow, is by some considered superior to the $\mathrm{S}$. fragilis. In pharmacy it is valued for its bark.

ACTIVE INGREDIENTS. - Willow bark is bitter and astringent. These qualities are referable partly to a certain proportion of tannin, and more particularly to the presence of a crystalline nentral principle called salicin $\left(\mathrm{C}_{7} \mathrm{H}_{6} \mathrm{O}_{3}\right)$. Besides these, there are gum, extractive, and other constituents ordinarily occurring in the bark of trees. 


\section{ACIDUM SAliCYLICUM. (Salicyitc Acid, $\mathrm{C}_{7} \mathrm{H}_{6} \mathrm{O}_{3}$.)}

Formerly obtained by a complex process from salicin, the bitter glucoside contained in willow and poplar bark.

It occurs combined with methyl in the essential oils of wintergreen (gaultheria procumbens, U.S. Ph.), of spiræa ulmaria, of monotropa hypopitys, and as recently reported by Dr. Waring in that of andromeda leschenaultii (B. M. J., ii., 1885).

PREPARATION.-By heating carbolic acid with caustic soda in a suitable vessel, and passing through the mixture a stream of carbonic acid gas, salicylate of soda is formed, and from it salicylic acid may be set free by any strong mineral acid. This simple and ingenious process, devised by Professor Kolbe, has much enlarged and improved our supply of the drug, but a still purer form may be obtained from the essential oils above mentioned, by treating with caustic potash, distilling, etc.

CHARACTERS AND TESTS.-The pure acid occurs in shining white acicular crystals, free from odour, and of slight taste: also as a dull white powder of marked pungent odour. It is soluble in alcohol and ether ( 1 part in 15), in olive oil ( 1 in 120), and in melted lard (1 in 8): warm glycerine dissolves 4 grains in the drachm; warm water readily dissolves it, but cold water takes up only 1 part in 300 . Its solubility in hot water is increased by neutral salts, as follows: the addition of 3 parts of sodium phosphate will raise it to 1 part in 50, whilst 8 parts of borax will make it 1 in 10 (Bose).

Heaterd slowly, it sublimes unchanged, but heated rapidly, it is resolved into carbonic and earbolic acids: at $318^{\circ} \mathrm{F}$. it melts.

An aqueors solution gives a deep violet coloration with persaits of iron. Sulphate of copper is also a good test, a smill quantity giving intense emerald-green coloux to a solution containing 1 part of salicylate in 20 of water, or 1.0 the urine three days after taking even a small dose (Schul\%, Chem. Keitung, 1879). 
A test of purity is readily applied by dissolving 1 part of acid in 10 of rectified spirit, and evaporating slowly in a watchglass: efflorescent crystals form round the edge, quite white in colour if the acid be pure,-yellowish if not so.

Salicylate of Soda is prepared by carefully neutralizing salicylic acid by bicarbonate of soda. It is a neutral salt, which occurs as a whitish powder of unpleasant sweetish taste, but free from odour. Solubility in water, 1 part in 1 ; in spirit, 1 part in $4 \frac{1}{2}$.

Salicylate of Lithium has been used by Professor Sée (Bull. de l'Acad. de Méd., 187\%).

Salicylate of Quinine is prepared by the action of salicylate of soda on sulphate of quinine, or by direct combination with salicylic acid (Dearden, Lancet, i., 1881): it is insoluble in water, but dissolves readily in certain acid solutions, and in hot alcohol (Brown, Edin. Journ., 1876).

Salicylate of Iron is formed by adding a solution of salicylate of soda to sulphate of iron-this gives a claret-red solution, astringent but not irritant (Edin. Journ., Feb., 18ri).

ABSORPTION AND ELIMINATION.-Although salicylic acid is but slightly soluble in organic fluids, both it and its compounds are quickly absorbed. Thiersch states that after application of the acid to wounds, it appears immediately in the urine.

Drasche detected it in this secretion soon after the application of an alcoholic solution to the sound skin, and Byasson found it in the same twenty-five minutes after administration to a healthy man (Cent. f. Chir., 187\%). To a patient with ectropia vesicæ, Bälz gave $\%$ grains of salicylate of soda, and tested the urine collected every thirty seconds from the exposen ureters: violet coloration with perchloride of iron occurred in eight and a half minutes; after salicylic acid itself, the renction did not occur till twenty minutes (Archiv der Heilk., xriii).

Livon and Bernard having injected 2 centigrammes of salicylate of soda under the skin of a guinea-pig, found traces in the milk one hour afterwards; having injected $t$ grammes into the stomach of a dog (after lignture of the osophagus), foumd it 
in the bile in the course of an hour, and in the saliva in two hours; and in other experiments, in the pancreas, cerebro-spinal fluic, frees, etc., successively (Lancet, i., 1878).

Blanchier and Bochefontaine, after injecting it into the blood-current in dogs, detected it in the saliva in five minutes, in the urine in ten minutes, in the bile in twenty. Taken into the stomach, it passed into the saliva in twenty minutes, into the urine in three-quarters of an hour.

In man, the drug does not pass away by the salivary glands, but by the kidneys, as already mentioned; it is eliminated rather more rapidly than from the system of the dog (Graz. Méd. de Paris, Jan., 1879). It has been detected in the serum of a blister by Oulmont, by Buss in the perspiration, and by Bälz in the bronehial secretion.

Although elimination by the urine commences early, it is not soon completed, for the drug has been found in one case fifty hours, and in another eight days, after the last dose. The actual form in which the acid and its compounds circulate is not clearly made out, but it is probably a neutral or alkaline salicylate, as first suggested by Salkowski (Berl. Klin. Woch., 1875). Certainly, outside the body, the acid forms a salicylate in the presence of an alkaline carbonate. Binz, however, suggests that the acid is liberated from this combination (if formed) by the carbonic acid present in the tissues, and records some chemical experiments in proof of this possibility.

Feser and Friedeberger, opposing this view, argue that the acid circulates as an albuminate; Farsky, indeed, produced such a compound, but Fleischer concluded that it was not il permanent one, and in the blood of poisoned animals he found salicylic acid in the serum only, not in the albuminous congrtlum (Cent. f. Med., 1876).

According to Ranke, Lehmann, Senator, and other eminent chemists, salicin clanges, in part at least, into salicylic. acid in the system (Lancet, ii., 1879), though Maclagan contrariicts this, reasoning from the difference of their effects: the gilueoside commonly passes in the urine as a salicylhydricle. It is curious that Spencer, whilst also criticising the views of Senator, and maintaining that only a very small 
amount of the acid is formed in the system after taking much salicin, comes to a conclusion, from clinical experience, directly opposed to that of Maclagan; for he states that after giving salicin to patients with acute rheumatism without any other effect than reduction of temperature, "he saw immediate and brilliant results" with salicylic acid given to the same patients in proper dose: he concludes that the remedies cannot be equivalent, but the advantage is with the acid (Record, 1879).

The acid itself passes in the urine as salicyluric acid (= salicylic acid and glycocoll), and also in part as salicin, and even as oxalic acid (Byasson). Again, salicylate of soda passes in part as free salicylic acid, for Binz detected this two hours after taking 15 grains of the soda salt. A clinical illustration of this may be found in the fact that although salicylate of soda added to urine will not preserve it for more than three days, 15 grains of the same salt taken by the mouth will act so that the urine passed under its influence keeps perfectly clear for ten days (Stuart, Practitioner). Altogether it would seem that salicin, salicylic acid and its salts, form different compounds under varying systemic conditions, and we cannot yet formulate their precise chemistry.

Physiological Action.- Extemal. - The antiseptic power of salicylic is rather greater than that of carbolic acid ; 0.4 per cent. of the former as against $t$ per cent. of the latter prevents development of bacteria in organic solutions (Bucholz). This statement is not, however, admitted by all observers. One part in 20,000 preserves saccharine fluids from fermentation (W. Squire, Lancet, i., 1879), and I part in 2,000 delays putrefactive changes in urine for fourteen days (Meyer and Kolbe).

Köhler, Binz, and others have found the soda salt inefficient as an antiseptic (Practitioner, vol. xx.), but the careful observations of Dragendorff and of Bucholz show that both the soda and ammonia salt have a power only one-third less than that of the free acid in destroying some forms of bacteria (Archiv. f. exper. Pathol., Klebs, 13d. iv.).

Outside the body, 1 per cent. of acid checks the action of ptyalin on stareh, and 2 per cent. that of pepsin on albumen. 
Applied extemally to denuded surfaces, the pure acid produces pain and irritation, though in a less degree than carbolic acid.

Applied to mucous surfaces, $\because . y$., of the nose, in some cases it is tolerated, in others it has given the greatest distress (B. M. J., ii., 1878).

Robin states that its administration in fever has caused, by local action, ulceration of throat and oedema of larynx (B. M. J., i., 1877), but such results must be very exceptional. A minor degree of irritation of the throat, however, often occurs; and from the external use of only a 2 per cent. solution to wounds, Mr. Callender observed redness and vesicular eruption, with some constitutional disturbance (B. M. J., i., 1879; Clin. Soc. Trans., ix.). An objection to the surgical use of "salicylic acid wool" is that the odour and dust are irritating and induce lachrymation, cough, and sneezing; but only usually in the attendants, as the dressing is always covered by ordinary carded wool.

\section{Physiological Action.-Intermul.-Nervous System.} -From 5 to 10 grains of salicylic acid, or 10 to 15 grains of the salicylate of soda, may be taken every four hours for several days by most persons without marked effect; and although some are by idiosyncrasy very susceptible to its action, as a rule a single dose of 60 grains, or several of 20 grains, of salicylate of soda, or 30 of salicin, will be needed to produce characteristic symptoms: these resemble somewhat those caused by full doses of quinine or alcohol; thus, the subject looks dull and flushed, is drowsy, and complains of fulness in the head, headache (which may be severe), giddiness, or "feeling of intoxication," loud tinnitus, and other affections of the special senses, such as partial deafness or blindness, mydriasis, or diplopia ; sensibility is altered-there is tingling of the extremities, ancl muscular irritability is increased, so that sudden startings and twitchings occur, sometimes tremor. All these symptoms may be producerl by salicin, by the soda salt, or by the free acid, but by much smaller doses of the last than of the first (Ringer). The more serious symptoms of delirium, etc., have not, however, occurred from salicin: but if the acid or the soda salt be mon- 
timued, delrium commonly occurs, and this may be of gay and busy, or of depressed frightened type, with delusions, general restlessness, and temporary attacks of unconsciousness: in many cases a regular alternation of delirium and partial consciousness has been recorded: a violent condition is specially apt to occur in drunkards. Moderate doses do not affect sensibility or reflex action in the lower animals (Sée), but under toxic doses, convulsions occur, also anæsthesia of the surface and paralysis of the limbs (Laborde, Bull. de Thérap., xciii.). Injected under the skin of the frog, "it rapidly enfeebled the central nervous system"; the motor nerves and muscular contraction were unaffected (Gaz. Méd. de Paris, 1879). Convulsions are not usual in man, but evidences of a partial paralysis are found in the profuse sweating, erythematous skin-eruptions, bedsores, uncontrolled sphincters, and ptosis.

Most illustrations of advanced nervous disorder have occurred during severe illness: some have been due to it (Philpot, Lancet, ii., 1877), and some have been connected with the accidental taking of carbolic as an adulteration of salicylic acid (Tuckwell, Lancet, ii., 187\%); still, the majority have occurred under such conditions as to prove the power of the pure drug to produce them: 20 grains of salicylic acid, given every three hours, caused, after the third dose, attacks of unconsciousness, which recurred about every quarter of an hour for three minutes at a time; delirium alternated with these attacks later on: when the drug was omitted the symptoms subsided (B. M. J., ii., 1879). Schumacher records actire delirium with painful hallucinations of sight after five 15-grain doses of the acid (Deuts. Med. Woch., 1876, No. 18). Sée describes the delirium as calm, and rarely violent.

Professor Petersen gave to a girl in good general health 60 to 90 grains of salicylate of soda daily for six days, without much effect; then, by mistake, she got in one day nearly 400 grains (part was vomited): she became flushed and restless, with dilated pupils and divergent strabismus; the voice was hoarse, and speech difficult; hallucinations came on, alternating with partial consciousness; the urine contained salicylic acid and albumen; an erythematous rash and a bedsore dereloped: the peculiar mental state lasted about eight days, lucid interrals 
becoming gradually longer; the delusions were painful (causing. tears), but she could not remember them on recovery (Deuts. Med. Woch., Jan., 1877, and Record).

After twelve doses of 20 grains of salicylate of soda, Dr. F. H. Daly says a patient became "restless, incoherent, with all kinds of absurd delusions;" she got better after sleep induced by ebloral, and afterwards resumed 10-grain doses of the salicylate, without ill effect.

The dose that will induce delirium, etc., varies in different cases, but is not likely to be less than 60 grains of acid, or thrice that amount of salicylate of soda: by gradual dosage, toleration of a large quantity may be established.

Salicin has not caused similar serious results; on the contrary, in some instances, when salicylate of soda has induced mental distress and great depression, salicin substituted for it has rather "restored tone to the nervous system" (Maclagan, Lancet, i., 1879).

Circulatory System.-Experiments as to the effect of salicylic acid on the heart and the blood-pressure give varying results. In frogs the beats of the heart were reduced in number, especially by the soda salt; in mammalia they were only sometimes reduced, "independently of dose": with the free acid they were mostly lessened (Chirone and Petrucci, Record, 1878). According to Blanchier, "large quantities affected the heart, modifying first the rhythm and number of its beats, and then arresting its movement" (loc. cit.).

Kinhler having injected large doses into the jugular veins of rabbits, found that both the acid and the salt induced sudden fall of blood-pressure; and as this occurred equally after section of the vagi and the cord, he traced it to a direct depressant action on the ganglia of the heart (Cent. f. Med., 1876).

Danewsky found, with dogs, that salicylate of soda at first increased the contractile force of the heart and caused a rise of blond-pressure, but as poisoning developed, the heart grew weaker and the pressure fell, till the former failed entirely: the frequency of the pulse was sometimes increased, sometimes not.

If the spinal cord were divided, rise in blood-pressure did not occur, implying that it was dependent on vaso-motor spasm, 
and the ultimate effect upon the heart Danewsky judged to be of a directly depressant character.

Feser and Friedeberger record acceleration of pulse in dng's, with "irregularity of circulation"; Bartels and Lürman ohserved the same in man; some connect a fall of the pulse with a fall in the temperature (Record, 1877). Sée, in his experiments, could trace no action on the pressure or the pulserate (Bull. de l'Acad. de Méd., 1877) ; and Balz remarks that it is notorious that the action on the circulation in animals is really but slight. Oltramare, experimenting more recently upon animals, ound that the soda salt injected directly into veins constantly increased the pressure, the number of pulsations, and the systolic force of the heart ; but this effect was transitory, and due to the direct stimulation of the heart, and probably of its motor centres. Under repeated injections the cardiac irritability diminished, and with a toxic dose the pulse became intermittent, the blood-pressure feeble, and the heart ceased to beat-from cardiac palsy, not asphyxia. Post mortem, intense congestion of the abdominal viscera was observed; but if previously the medulla were divided, anæmia was found-whence this physician also concludes that the drug acts on a vaso-motor centre in the medulla (Progrès Méd., Juin, 1879).

Results in illness are not a safe guide to physiological action, but we may note that differences often occur in the circulatory conditions under the influence of these drugs: in my own experience (of acute rheumatism, mainly) the pulse has been commonly and markedly reduced after four to six scruple doses of salicylate of soda-sometimes to sixty in the minute; but in Petersen's case, already referred to, "the pulse was very irregular, sometimes very quick, sometimes as slow as nommal, and then again small and weak"; and in a case of Dr. Whipham's, complicated with pnemonir and pericarditis, and in a state of most extreme exhaustion, salicylate of soda, iustead of depressing, rapidly produced an excellent effect (Lancet, ii., 1877).

Granted that the ultimate toxic effect of the salicylate is to rlepress the circulation, we shall find in practice that no ordinary rlose will depress so much as to cause unensiness; nerertheless, the affects should be carefully wntched. Goodhart describes 
a case of acute rheumatism in which death occurred suddenly after four 15-grain doses of salicylic acid, "from sudden collapse and failure of the heart" (Clin. Soc. Trans., xiii.): it is doubtful whether this result was due to the drug.

Effect on Temperature.--There is a general consensus of opinion that doses, as large as are safe, of salicin, salicylic acid, or its salts, lower the temperature very little, if at all, in healthy animals or men. Sée gave 150 grains daily of the soda salt without effect on the body-heat. Firbringer experimented on rabbits, dogs, and men with the same result. Out of twelve separate observations, Gedl found a slight fall in four (Centralb. f. Med., 1876). Danewsky found any effect produced only "slight and inconstant." Ringer and Bury, if at first they noted a slight depression from large doses of salicin, found later a mild feverish condition which they explain by gastric catarrh (Journ. Anat., xi.). Sée noticed a similar slight rise in chorea and other apyrexial states. Ringer and Morshead gave 160 grains of salicylic acid in one day, without any depression of temperature. Riess, however, in twentythree experiments with salicylate of soda, obtained in four to six hours a constant reduction of about $1^{\circ} \mathrm{F}$; ; and there can be no question that full toxic doses may lower temperature sometimes even with the production of collapse.

In pyrexial conditions, therapeutical doses of these drugs have, like quinine, a much more marked effect (S. Butt, Cent. f. d. Med. Wiss., 1875). It is not uncommon in rheumatic cases to find a temperature of $101^{\mathrm{C}} \mathrm{F}$., which has been persistent for several days, reduced to normal within twenty-four to forty-eight hours after commencing salicylic treatment. Salicin will effect a similar result, but not so markedly or constantly as salicylates (Ringer). Ewald, judging from cases of enteric fever mainly, found the temperature begin to fall about fifteen minutes after a full dose; and generally a profuse perspiration preceded this fall, without being in a definite or evident relation to it (Practitioner, vol. xvi.).

This physician, and also Juste, calculated that the maximum of depression was reached in five to six hours from the dose; and several other observers accept this as an average, though occrsionally the time has been extended to eighteen hours. 
No marked critical evacuation, umless an inconstant one from the skin, occurs to explain the lowering of temperature, and the circulation is often unaffected at the time, so that the explanation of the fall is not clear,-it is commonly traced to nerve-influence ; the hypothesis which connects it with anti-fermentative action within the tissues need not at present be argued at length.

Depression of temperature, more or less permanent, has been observed in all forms of pyrexial disease-in phthisis, pneumonia, infantile remittents, ague, enteric and other fevers, but unfortunately it is not a constant phenomenon, and there are now on record cases of even fatal hyperpyrexia occurring in spite of its free use. After the first fall and rise, a subsequent fall to a less extent often occurs without a fresh dose.

Respiratory System.-Köhler and Danewsky have both made observations as to the respiratory changes which follow on intravenous injection of the acid or its salts, and have found the rate of breathing at first quickened, afterwards reduced. From the results of experiment after section of the vagi, it would seem that irritation of these nerves is caused, together with, possibly, some stimulation of the respiratory centre. Toxic doses fatally paralyse the same structures, so that the breathing becomes slow and laboured, and death commonly occurs from asphyxia, generally by degrees, sometimes suddenly, and often with convulsions (Sée, Buss).

In some recorded cases of poisoning in man the respiration. has been both quicker and deeper than normal, and often stertorous; Petersen records it as "forty per minute, deep and noisy": sighing is not infrequent.

Digestive System.- Small doses of salicin have a stomachic tonic effect: full doses of either salicin, the acid, or the salts are apt to produce gastric irritation and vomiting-the first less so than the others; but nausea has resulted from 10 grains (Lancet, i., 1877). Much may be done to obviate these symptoms by judicious combinations.

Kolbe states that he could himself take 15 to 20 grains of the acid daily without digestive disturbance. On the other hand, Wolffsberg speaks of erosion and hæmorrhage in the gastric mucous membrane from large repeated doses of the acid, and Bälz has heard of an instance of perforation of the stomach 
from the same (loc. cit.). A case of severe gastric pain, followed by sudden death after taling the acid, may be thus explained, but precise details are not given (Bull. de Thérnp., t. xciii.).

Dr. Rutherford found the soda salt a powerful hepatic, but not an intestinal, stimulant, and seeing the chemical relation between salicylic and benzoic acids, suggests that, like the latter, it stimulates the hepatic cells (B. M. J., i., 1879). Bälz speaks of an urgent sensation of ravenous appetite in several cases.

Urinary System.-The urine passed under the influence of these medicines is generally dark green or brown in colour, and contains an excess of indican and of pyro-catechin (Sée, Record, 1877), derived probably from changes in the drugs themselves. Pye-Smith has made the important observation that it will commonly reduce copper-salts, like diabetic urine, probably through containing some glucose derived also from the medicines (B. M. J., i., 1878).

According to Sée and Marot, the excretion of urea and of uric acid is increased. Byasson and most, though not all, observers report the same result; and it came out markedly in the analyses of Barclay in two cases treated by salicylate of ammonia (St. George's Hosp. Rep., viii.). He contrasts them with cases under alkaline treatment, and notes the absence of the diuresis which is produced by the latter. The effect on the quantity of urine, however, seems to vary. Gubler generally found it increased by salicylates when the kidneys were sound; diminished, even suppressed, when they were diseased (Bull. de Thérap., t. xciii.). Bälz found it increased in cases of hepatic disorder.

These medicines may certainly cause much renal irritation, and even acute nephritis, with hæmaturia, albuminuria, and suppression; and there is no more important practical point in their use than to recognize this risk in advanced renal disorder of inflammatory or congestive kind (H. Weber, Clin. Soc. Trans., x.).

Under salicin the quantity of urine was unaltered in Dr. Ringer's observations, and the reaction became less acid or neutral. T. Acland reports it diminished, and hence the 
total amount of urea excreted also diminished in proportion (Record, 1879).

Cutaneous System.-Attention has already been drawn to the profuse ("colossal") secretion of sweat generally induced by these remedies. It is sometimes comparable to that produced by jaborandi, and occurs usually twenty to thirty minutes, more rarely a few hours, after a dose: sometimes it subsides in two hours, in other cases not for several days. There is some evidence that the sweat may be rendered alkaline by them (Ringer). Both this symptom and the occurrence of an irregular dusky rash and a tendency to bedsores are probably dependent on a paralysis of the vasomotor nerves.

Cavafy has recorded a vesicular rash from the salicylate, and Althaus saw an eruption of pemphigus (Lancet, i., 1877). Freundenberg reports the occurrence upon the skin of numerous large petechiæe and vibices after using salicylic acid for six days : they continued to develop for one day after omission of the drug, then became paler, and ended with desquamation; the same effects followed a second administration later (Allg. Med. Cent. Zeit., Oct., 1878). Heinlein relates a case of articular rheumatism in which, after a 60-grain dose of salicylate of soda, intense itching in patches about the face and extremities was followed by an eruption like urticaria: this disappeared by next morning, but recurred over most of the body within half an hour after the second dose (Record, 1878).

\section{Therapeutical Action.-Extemal.-Antiseptic Sur-} gery.-Salicylic acid has been used in spray, lotion, ointment, etc., in the antiseptic treatment of wounds, and although its properties, and comparative freedom from unpleasant odour and toxic effects, promised some advantage over carbolic acid, practically it has not answered so well, and has not come into general use. Callender found a lotion of 2 per cent. caused irritation (Clin. Soc. Trans., ix.), though Sir William MacCormac's experience was the reverse (op. cit, p. 128).

Almost the only preparation of it commonly utilized is a salicylic cotton-wool, wadding, or jute, first suggested by 
Thiersch : it is prepared of 4 per cent. and 10 per cent. strength, and used for absorbing discharge and protecting from the air, and "most rusefully as a supplement to carbolie gauze." Salicylic silk has also been recommended in conjunction with carbolic ganze in a dressing (MeGill, Lancet, i., 1881; Brown, ibid.).

Salicylic lotion answers well for washing out large cavities (MacCormac), and for disinfecting the hands, sponges, etc., but not for instruments, as it oxidizes steel.

Burns - Ulcerations.-An ointment ( 1 part in 10 of vaseline) is a good antiseptic slightly stimulant dressing for these and other granulating surfaces (Wintle and others). Mr. Prowse strongly commends a lotion (1 grain in the ounce of water) for the cure of pustules and ulcerations connected with the internal use of bromides (B. M. J., ii, 1880) ; it should be applied like water-dressing.

In ulceration of the throat, a paint containing 30 to 40 grains to the ounce of glycerine has been found useful (B. M. J., i., 1879).

Wagner treats superficial gangrenous sores by applying a thin layer of the powdered acid, and then wadding : it is valuable also in eczema of the head and face. A case of lupoid ulceration of the face of four years' standing was much benefited by the local use of the acid in lotion and powder (Med. Times, i., 1879).

Thickened Epidermis-Corns.-Dr. Traill Green has published results obtained after employment of salicylic acid as a cure for corns, both hard and soft. The formula he adopts is one recommended by M. Gezow, and is as follows:-Salicylic acid, 30 parts; extract of cannabis indica, 5 parts; collodion, 240 parts. The collodion fixes the acid to the part, and protects it from friction, the cannabis indica acts as an anodyne, and the acid reduces and loosens the corn: the remedy is applied with a camel-hair pencil. In four or five days the patient should use a warm foot-bath and rub off the collodion. If any portion of the corn remains, the acid should be applied again and the treatment continued until the whole of the corn has disappeared (Amer. Med. and Surg. Reporter, 1883).

Psoriasis.-A solution in spirits of wine, 1 part in 16 , 
is said to have a favourable effect on patches of psoriasis, removing the scales and relieving itching, etc. (B. M. J., ii., 1879).

Eczema-Impetigo.-In both these disorders, but especially in eczematous conditions with semi-purulent discharge, I have found lotions of salicylic acid, 1 grain to the ounce, very usseful.

Ringworm.-Mr. W. Cottle speaks well of an alcoholic solution of the acid, $\frac{1}{2}$ drachm in the ounce, rubbed well in thrice daily, for all cases of recent disease ; it is painless, easy to apply, and free from risk of poisoning (Lancet, i., 1880).

Otorrhœa.-In this disorder, especially when resulting from scarlatina, it is recommended, after thorough cleansing of the meatus, to fill it by means of an insufflation with salicylic acid in powder (Record, 1879). An injection containing 3 to 5 grains of salicylate of soda in the ounce of water is often valuable.

Hay Fever.-The caution necessary in judging of a new remedy, and concluding from one or two cases, is illustrated by results in this disorder, for whilst one writer speaks of curing it quickly and pleasantly by using as a snuff the pure acid finely powdered (about 15 grains daily), another describes very severe irritation and pain from following the advice (B. M. J., ii., 1878).

Pertussis.-Neubert states that much benefit may be derived in this disorder from hourly or bi-hourly inhalations of a 1 per cent. solution of salicylate of soda (Jahrb. f. Kinderheillk., xiii.).

As a local application it has also been found of great benefit in acute articular rheumatism by Dr. Orton, who applied it in solution on lint covered with oiled silk (B. M. J., i., 1882).

\section{Therapeutical Action.-Internal--Acute Rheu-} matism.-The main therapentical interest of this group of remedies centres in their influence on rheumatic disorders.

Dr. Maclagan, if not the first to prescribe salicin, was the first to demonstrate its value in rheumatism, and according to his theory the remedy was directed to the "miasmatic origin" of the disease; pain and fever were rapidly relieved in the 
cases recorded by him (Lancet, i., 1876). ${ }^{1}$ The experience of others has not been quite so favourable, but he gives reasons for presuming the drug to have been adulterated, and three years afterwards reiterates his favourable estimate of it as compared with salicylic acid (Lancet, i., 1879).

Giving 30 grains in powder every hour till relief is obtained, then every two to three hours, Maclagan calculates that 1 ounce suffices to cure any ordinary attack; it relieves pain by some specific power independent of controlling the temperature, etc., and even if it has in some cases caused vomiting (Lancet, i., 1877), and in others not acted so well as salicylic acid (Buss, Berl. Klin. Woch., xiii.), and in others failed (Foster, B. M. J., ii., 1876), it has the advantage of never having caused poisonous symptoms. Charteris more recently presses the greater safety of salicin as compared with salicylate of soda (B. M. J., i., 1881); on the other hand, P. W. Latham much prefers salicylic acid (B. M. J., ii., 1880). The more acute the case, the more efficient is the remedy.

Senator published an experience almost equally favourable, but traced the activity of the medicine to salicylic acid said to be formed from it in the blood (Cent. f. Med. Wiss., 241, 1876).

Stricker having shortly afterwards administered the acid itself, reported results still more striking in the rapid relief of rheumatism, hourly doses of 7 to 15 grains relieving severe symptoms within forty-eight hours (Berl. Klin. Woch., xiii.).

Many observers corroborated this statement, but, on account of the throat and gastric irritation often caused by the acid, its compound with socla or ammonia came to be generally preferred.

Dr. C. W. Brown (Boston) of 160 cases, of all ages, treated nine by salicin, the rest by salicylic acid (10 grains every hour while awake, for twelve to thirty-six hours), and noted partial relief in 1.46 day, complete in two to eight days: the average duration of treatment was six days, and stay in hospital eighteen days; two died, one of pericarditis, one of cerebral disorder; eighteen relapsed; nausea or vomiting occurred in 18 per cent.; and some forms of the nerve-troubles already referred to were not infrequent (Boston Med. and Surg. Journ., Feb., 1877).

Mr. Ensor reports from South Africa that a decoction of "willow tops" is commonly and effectually used there for acute rheumatism (Lancet, i., 1876). 
Of seventeen cases treated by the acid at the Massachusetts Hospital, the majority were satisfactory (Boston Journ., 1879).

These data refer to the acid. treatment; the average time till relief by salicin was longer, but the general condition as to appetite and strength better. After the acid, patients were left commonly "in poor physical condition."

Dr. Cavafy, giving 30 grains or more of salicylate of soda every two hours in twenty-one cases, reported that almost all were free from pain and fever in twenty-four to thirty-six hours.

Drs. H. Weber, Broadbent, J. Pollock, and others have recorded favourable experience of the drug (Clin. Soc. Trans.) 1877). Dr. Jacobs (Leeds), referring to seventy eases, found thirty-five so rapidly improved as to leave no doubt of the effect of the remedy (Lancet, ii., 187\%).

Messrs. Finlay and Lucas have reported an interesting series of cases, comparing (1) sixty treated with salicylate of soda, 15 grains every three hours, (2) sixty treated with alkalies, 15 grains every four hours, and (3) thirty-eight treated with quinine-in all 158 cases. Of the first series, pyrexia was relieved in $5 \cdot 7$ days; of the second series, in $3 \cdot 10$ days; of the third, in 11.6 days. Heart-complications occurred in the proportion of 11.6 per cent. in the first series, 6.6 in the second (alkaline) series, $13 \cdot 1$ in the third (quinine) series. Relapses occurred in the proportion of $26^{\circ} 6$ per cent. in the first series, $8 \cdot 3$ in the second, $7 \cdot 8$ in the third (Lancet, ii., 1879). Diesterweg gives still more favourable details of 100 cases; there were only eleven relapses and five cardiac complications (Cent. f. Med., Jan., 1880). Dr. W. Squire speaks also well of the salicylates (Lancet, Dec., 1879). These results represent a general conclusion as to the greater frequency of relapse under salicylic treatment, but such an occurrence is readily explained by the too early omission of treatment and the too early exposure of a convalescent patient: it may be prevented by care, and Dr. Whipham specially notes that patients treated in this manner at St. George's Hospital were sent more safely than before to the Convalescent Home (St. George's Hosp). Rep., viii.). So far as the new treatment shortens the duration of the malady, it ought to lessen the risk of serious complica- 
tions, but the undue proportion of cardiac cases is rather borne out by larger statisties. Pericarditis and endocarditis have in some few instances developed in a patient whilst taking the medicines (Curnow, Lancet, 1876).

Dr. Gilbart Smith has published tables dealing with large numbers of eases treated in the principal London hospitals, from which it is seen-(1) that whereas, before the era of salicylic treatment, the percentage of cases with cardiac complication was 56 , since its introduction the percentage is 63.4 (Lancet, i., 1882); (2) Dr. Fuller's statistics of first cuses under ordinary treatment showed 41.8 per cent. of cardiac affections, whilst more recent statistics of first cases under salicylic treatment gave 60.5 per cent.; (3) of 533 cases with the last-named treatment only, the percentage of cardiac affections was 68.4 (Lancet, i., 1882).

Of Dr. Hood's 350 cases thus treated, 241 had cardiac disease (i.e., 109 only escaped), whilst of a parallel series of 350 treated otherwise 247 escaped. This physician admits that the treatment in question shortens the period of pain, but considers the total duration of treatment is prolonged and exhausting (Lancet, ii., 1881). (This is opposed to the observation of Germain Sée, who says that the ordinary anæmia of rheumatism is absent after the use of this drug.)

Neither will the salicylates always control fever, and a sufficient number of cases of even fatal hyperpyrexia is now on record to show how far these drugs fall short of the complete efficacy at one time credited to them. Sharkey and Ord have failed with salicylie acid (St. Thomas's Hosp. Rep., viii.), Sturges and Lockie with salicin (Lancet, ii., 1876), Roe with salicylate of soda (Lancet, ii., 1877), to say nothing of cases in which only 15-grain doses every four hours were given (B. M. J., i., 18r7), or in which the record is incomplete (Dr. Russell, B. M. J., i., 1877, also Lancet, i., 1880). There is, however, no reason to credit them with such exceptional results as stiff joint (Bradbury, I ancet, i., 1877), or bone necrosis (Lilly, Lancet, ii., 187\%), which may fairly be set down to the disease itself. Ir. W. Carter, summarizing a large number of cases, calculates the failures as 8 per cent. (B. M. J., ii., 18\% 7 ); and I)r. Hermann, jurtging from 160 reports, concludes that 
salicylic acid and salicylate of soda show by far the best results in all points except relapse (London Hosp. Rep., 1878).

Archambault finds the soda salt of the greatest service in the rheumatism of children; they take it readily, and suffer less than adults from giddiness or nerve-symptoms under its use; he gives $1 \frac{1}{2}$ drachm daily for three days, and then omits for a time (Record, 1879).

On the other hand, several observers lay stress on the disadvantages possibly connected with the use of these remedies. Gatti out of 250 cases records alarming symptoms in four -e.y., dimness of sight (for ten hours), delirium, dyspnce, gastralgia. Gubler mentions diarrhœa, cyanosis, and collapse; and Dr. Mahomed refers to serious depression (Lancet, i., 1880).

The general conclusions of Dr. Greenhow are not encouraging. Of ten cases treated by salicin, several relapsed; one got epistaxis; two, vomiting; four, much cardiac depression: the stay in hospital was not shortened. Of fifty cases treated by salicylate of soda, the temperature, it is true, speedily fell, but many got severe nerve-symptoms-vertigo, deafness, headache, delirium,-and others serious depression of the circulation; anæmia was commonly marked (Clin. Soc. Trans., xiii.).

Dr. S. Coupland's conclusions are-(1) that in the majority of cases pyrexia and joint-pains are speedily reduced; (2) that relapses are likely to occur unless the treatment is long continued; (3) that no definite influence upon the heart, or, other complications, can be observed; (4) that the average total duration of the cases is about forty days (Lancet, $i$., 1882).

Vulpian (op. cit.) says that there is now no question as to its utility, and praises it for its rapidity and certainty in reducing temperature and alleviating pain. He quotes the opinions of several eminent French authorities as to its being equally serviceable in the acute rheumatism of children, and also in the rheumatisn of scarlatina. He considers also that the duration of the disease is shortened, and that when proper doses have been given, only exceptional, and relatively very rare, cases last beyond five days.

Whilst we admit this remarkable statement of such an eninent authority, we must bear in mind the difference of 
climate, race, and diet when we contrast the French and English experience on the subject.

Complications, affecting, c.g., the brain, the peritoneum, or pleura, are not amenable to the drug.

My own experience has been very satisfactory. The method I find best is to give 20 grains of salicylate of soda every hour until a drachm has been taken, then to wait for about six hours and repeat similar doses every two hours until another drachm has been taken, and then pause. I have thus often given 3 drachms in 24 hours with great benefit; as a rule, 2 drachms, if continned, are quite sufficient in each twenty-four hours; the physiological symptoms produced will be the best guide. Salicylate treatment ought to be continued for about ten days after the reduction of the temperature to normal.

It will be well also to keep some watch upon the elimination of the acid, e.g., by testing the urine: a few drops of perchloride of iron added to some in a test-tube should give a bright violet colour two or three hours after a dose.

Theories of Action in Acute Rhermatism.-(1) The anti-malarial theory of Dr. Maclagan has been already mentioned; it has not been' generally accepted. (2) Dr. Latham (Cambridge) adduces evidence in favour of the drug combining "with the antecedents of lactic acid and glucose" (to the presence of which in the circulation he attributes the malady): their formation being prevented, the dilatation of minute arteries (which they cause), and the hyperoxidation of muscular tissues, which produces pyrexia and increased formation of urates, do not occur (Lancet, i., 1881). (3) According to Vulpian, some consider it acts as an rncesthetic; but observations on animals disprove any special effect on sensory nerves. (4) Others credit it with a special effect on the vaso-motor system; but it is not likely that any drug can act on the vaso-motor nerves of one region exclusively, and clinical observation is against attributing vascular changes to the drug. (5) Its antipyretic effect scarcely explains everything; it does not act primarily on the fever, but only lessens it by its power over the joint-affection. The pain and swelling generally disappear before the temperature falls. (6) Neitber will a diuretic effect suffice to explain its therapeutiral power: the solids of the urine may be augmented, but 
commonly the amount of water passed is not so. In rheumatic cases the amount of uric acid excreted has even been lessened, although in gouty patients it has been increased (from 80 centigrammes to 3 grammes for three days consecutively). (7) It has been said to act on "ferments," but we have no proof that rheumatism or gout are zymotic diseases. "living protoplasm."

Vulpian applies to this drug his explanation of "the elective action" of drugs generally, viz., that although they probably reach all the tissues, they modify the action only of some; with these they form such combinations (more or less transient) as to induce changes, either chemical or physical, incompatible with the normal exercise of the functions of such tissues or parts; whilst in other tissues, even though a larger amount be present, the modifications induced do not derange the ordinary regular processes of the part. Hence he suggests that salicylate of soda acts in a special manner on the anatomical elements of the articulations, the inflammation of which is the first and principal factor in acute rheumatism-the special action tending to neutralize, check, or terminate such inflammation.

Chronic Rheumatism-Gout.-Professor Sée, who was one of the first French physicians to use this group of medicines, reported advantage from the acid and the soda salt not only in acute and subacute cases, but also in chronic rheumatism and gout, chronic rheumatic arthritis, and lumbago (B. M. J., ii., 1877). Jaccoud and Guéneau de Mussy recorded a similar experience; and Lépine found them good even in saturnine gout (Bull. Gén. de Thérap., t. xciii.) Hewan speaks well of the salicylate of quinine (Lancet, i., 1880).

M. Boulonné (Progrès Méd., Nov., 1879) and the majority of English observers have not seen much advantage from these remedies in such conditions. Dr. Jacob, however, says that of 87 "well-marked cases of chronic articular rheumatism," 61 derived some benefit from sodium salicylate, 15 to 30 grnins thrice daily; relief was not so speedy as in the acute disease (B. M. J., ii., 1879).

Lecorche and Talamon state that. although not efficient in chronic cases of gout, it will prevent acute attacks (Bouchardat, Anmuire, 1882). It angments the discharge of urea by the 
kidneys, and removes tophi. These authors, however, deny its efficacy in acute gout, whilst M. Germain Sée speaks highly of its utility in many cases. A difference of opinion exists also as to its value in gonoriheeal rheumatism-Weber speaking well of it, Guéneau de Mussy the reverse.

Ophthalmia. - There are several recorded cases of much benefit from full doses of salicylate of soda in acute inflammation of the iris and sclerotic, and I have myself seen marked benefit from it in these cases, which are presumably rheumatic in origin.

Neuralgia.-There is reason to think the drug available in those forms of this disorder which depend upon rheumatic, gouty, or arthritic taint.

In the symmetrical neuralgia (sciatica) of diabetes, Dr. Buzzard has proved their efficacy; and there is also evidence to show that sometimes at least salicylic treatment reduces the amount of sugar in the urine (Lancet, i., 1882).

Dr. Maclagan has illustrated the power of salicin in several aggravated cases (Practitioner, vol. xix.) ; and Dr. Boggs has reported instances of sciatica, lumbago, and persistent headache yielding to salicylate of soda (B. M. J., ii., 1878); but, according to Weiss, the treatment is only of service when commenced shortly before the expected attack, and the paroxysms return when the remedy is omitted.

Dr. Abbott has reported three cases of sciatica, and three of acute facial neuralgia, in which the acid or the soda salt was employed with success; two of the eases had had rheumatism: relief was prompt (Boston Med. and Surg. Journ., 1880). Dr. Hewan has written recently to support his former experience of the value of the quinine salt, and says that 6-grain doses give excellent results in neuralgia. According to my experience this salt is not free from the disadvantage of causing tinnitus, etc., as originally reported by Brown in his fever cases (Edin. Med. Journ., 1856).

Intermittent Fever.-According to Dr. Maclagnn's theory, these remedies ought to be exceptionally useful in ague, but experience has not, up to the present time, proved them so. Blom is said to have given salicin with good effect in 1844 (B. M. J., i., 1877), and Dr. Thomson has written of its value 
in the Eastern fen districts (Lancet, i., 1877). Senator, judging from eight cases, thought favourably of salicylic acid, and Thomas (U.S.) speaks well of the antiperiodic action of a solution in nitrous ether, 2 drachms to 6 ounces, $\frac{1}{2}$ ounce being given every half-hour or hour, until six doses are taken (B. M. J., ii., 1877). Zieliwicz reports 207 cases of intermittent fever, mostly in children, treated successfully with full doses of salicylate of soda during the febrile period: he finds the remedy cheaper than quinine, but not so certain or effective (Deutsche Med. Woch., 1879). Rosenstein also found the acid effective, but less so than quinine (Lancet, ii., 1877); Drs. Weber, Ewald, and others could not trace benefit to the acid or the soda salt: they deserve, however, more investigation.

Enteric Fever.-There can be no doubt of the power of salicylates to reduce temperature in this fever, and Riess concluded, from nearly 400 cases, that they acted. favourably and shortened its duration; his mortality, however, was about 24 per cent. (Berl. Klin. Woch., xii.). Drs. Ewald and Goltdammer, who have the next largest experience on this subject, do not agree in Riess' favourable estimate (Practitioner, vol. xvi.); both express a doubtful opinion, Goltdammer saying the duration is not lessened.

Jahn, in thirty-five cases treated by salicylic acid (about 3 drachms daily), reports a mortality of 8.5 per cent., and a mean duration of thirty-seven days, as against a mortality of 30 per cent. under mild hydropathy, and 9 per cent. under energetic cold-water treatment (Deutsche Arch. Klin. Med., xviii., and Lancet, ii., 1877).

Schroeder, on the other hand, found his mortality greater (19 per cent.) in 160 cases treated by the acid than in those without it; the temperature was lowered without definite improvement, and large doses caused symptoms of collapse with acceleration of the pulse (loc. cit.).

Oulmont in ten cases reports also a fall of temperature, but for a time only, and without effect on the symptoms. Dr. De Mussy gave "small doses" of salicylate of soda in twentyseven cases: all recovered; and he concludes that at least the medicine did no harm,- -but there is reason to think that even this can scarcely be said. Murchison recorded serious results, 
delirium and albuminuria, in a case apparently favourable for its use ; it is possible, however, that the large dose of 6 drachms daily was to blame for the symptoms (Lancet, ii., 1877).

Dr. Weber objects to the drug in typhoid; and the general opinion is, that its chances of increasing depression, and also gastric and cerebral irritation, are such as to make it an unsuitable medicine.

Filatow also reports the remedy as not modifying the disease, and as inducing much depression. Weiss has recently published his results of this treatment, helping us to estimate its true value (Allg. Cent.-Zeit., April, 1880): they are based on ninety-six cases of typhoid fever in children, and lead to the conclusions-(1) that salicylate of soda is a powerful antipyretic remedy, which renders the disease milder, though it does not shorten its course; (2) it gives better results than quinine, acids, or baths; (3) large doses must be given at short intervals. No ill effect was observed.

Dr. Moore's estimate of its value is, I think, too high (N.Y. Journ., August, 1879); but more recently, Dr. Tomkins, judging from forty-six cases of marked severity, shows that it is a remedy worth further trial; he gives 20 grains every two hours when the temperature is continuously higher than $102^{\circ} \mathrm{F}$.; if its full action is not obtained in twelve hours the prognosis is unfavourable (Lancet, i., 1881).

In Cholera, Boustead asserts it is very effective (Practitioner, vol. xviii.).

Variola-Scarlatina.-The experience of salicylic treatment in these disorders is not extensive, but Prideaux records the remarkable result of eighty-eight cases of confluent small-pox treated by salicylates without a single death, and seventy-eight of scarlatina with one death (Record, 1878).

The results obtained by Dr. Brakenridge in nine cases of the latter malady were not so good as with sulpho-carbolates (Med. 'Times, ii., 1876).

Infantile Remittent, etc.-Hagenbach is accustomed to use salicylate of soda as an antipyretic in most of the febrile disorders of childhood, including scarlatina and typhoid, giving a single nightly dose of about 15 grains to children a year olr, 30 to 40 grains to those of five: he finds from it a decided 
remission of temperature, and generally lowering of the pulse and respiratory action, and much less necessity for bathing or other treatment; sometimes, however, diarrhœa, vomiting, or restlessness occurred (Record, 1878).

Juste used the remedy in the same class of cases, giving 60 to 70 grains to children, 100 to adults.

Yellow Fever.-In an epidemic of this disease, of intermittent and remittent type, Binz found $1 \frac{1}{2}$-drachm doses of the acid relieve pain, while reducing the temperature and pulse (Practitioner, vol. ii., 1878). Dr. Frèire found hypodermic injections of the soda salt marvellous in eighty-eight cases of the fever in Rio: the dose was 3 to 8 grains, but even 16 to 20 grains were given; the mortality was only 7 per cent.

Erysipelas-Diphtheria.-The same treatment has been advocated for these disorders, but although the acid may be useful locally in the form of a salicylic glycerine (30 grains in the ounce), iron and other medicines are to be preferred internally.

Bartels mentions a noteworthy case of a patient who, while taking full doses of salicylate (for diabetes), died from an attack of erysipelas (Record, 1878). Bälz records an analogous instance.

Pownall, however, strongly recommends the acid internally for the throat symptoms of scarlet fever, and also for diphtherin, ordering from 8 to 16 grains every four hours, but alternately with iron (B. M. J., ii., 1878). Curtis also speaks well of it, but he gave it with chlorate of potash and dialyzed iron (Boston Med. Journ., xcix.). Dr. Walls White recommends the salicylate of iron internally in 4- to 10-grain doses (Glasgorv Med. Journ., Aug., 1879).

Although much praised by some foreign practitioners, careful trials in the Paris hospitals have shown that it is not a trustworthy remedy for diphtheria.

Dyspepsia.-Salicylic acid may be used in cases of this disorder, marked either by deficient gastric secretion or tendency to fermentation of food. It is unsuited for cases of nerrous hypochondriacal character, also for such as are marked by great irritability of, or excessive acid secretion from, the mucous membrane (Federici, Abst. Record, 1879). 
Urticaria.-Several cases unrelieved by quinine have got quickly well after three or four doses of salicylate of soda. The disorder is often of rheumatic ar dyspeptic origin, and is probably then most amenable to the remedy (Record, 1879).

Phthisis.-In the later febrile septicæmic stages of phthisis, benefit has been naturally expected from antiseptic remedies, and salicylates have been found to reduce the pyrexia, but they have not exerted a favourable influence on the course of the malady (De Mussy).

Pneumonia-Gangrene of Lung.-Advocates are not wanting for its use in pneumonia; but other remedies are to be preferred. In gangrene of the lung the very important effect of relieving fœtor may apparently be attained by the internal administration of salicylic acid. In a case recorded by Dr. Draper, the fotor returned when the medicine was omitted, but under its continued employment cure resulted (Practitioner, rol. xviii.).

Tonsillitis.-The relation of this disorder to rheumatic fever is a close one, and an acute attack of it is not unfrequently followed by one of the latter. I think the hypothesis which explains this circumstance by some pyæmic absorption from the tonsils is reasonable; in any case there is good evidence that the salicylates are extremely effective (Dr. Fowler, Dr. J. Hunt). Hormazdji, endeavouring to distinguish the class of cases suitable for it, excluded those connected with-chronic tonsillar inflammation, and also those due to drainage-defect, restricting its value to cases caused by cold or by infectious fever (Lancet, i., 1882); but Dr. Mackey has shown that this restriction is incorrect, and that the salicylates can give quick relief in severe tonsillitis with ulceration, clearly due to defective drainage; that the course of the disorder was shortened (as compared with similar cases in the same family, but differently treated), and relapse prevented : 5 to 10 grains of the soda salt were given every two hours in liquor ammonixo citratis (B. M. J., ii., 1882).

Cystitis.-In chronic cystitis with ammoniacal urine, and in chronic pyelitis, salicylic acid or salicylate of soda is often useful, obviating, for some time, decomposition and footor of the secretions. 
Diabetes.-Cases of success and of failure with salicylic acid have been recorded, and it is difficult yet to say what may be done with it in this disease. Schaetzke has recently recorded the following:-A lady, aged fifty, diabetic probably for more than twelve months, was ordered 45 grains thrice daily; on the first day she had giddiness, on the second day romiting, and the dose was reduced; later on, when sent to Carlsbad, the urine was found free from sugar, and it remained so: a second patient, aged fifty-eight, also evinced intolerance of the same dose, but under a smaller one sugar disappeared in a fortnight: a third, aged twenty-six, gave very similar results (B. M. J., ii., 1879);-but the number is too small for any definite conclusion. Bartels thought that benefit might generally be derived from the drug, but with the risk of delirium or severe nerve-symptoms (Record, 1878). Dr. P. W. Latham relates six cases illustrating variable success; in one, sugar entirely disappeared: he gives interesting analyses, throwing some light on its chemistry (Lancet, i., 1881).

In Syphilis the acid has been employed topically with good result in soft chancres and buboes, being sprinkled over them in fine powder twice daily after they have been washed. In addition, a 2 per cent. solution in warm glycerine is applied about every two hours. After some days the solution is diluted (B. M. J., i., 1882).

Tænia.-In a case where ordinary remedies had proved useless for nine years, salicylic acid seems to have been promptly effectual: 5 decigrammes ( $7 \frac{1}{2}$ grains) were given every hour for four hours, and afterwards castor oil; half an hour later a large tænia solium was passed entire (Allg. Med. Cent.-Zeit., 1879, No. 102). Dr. Ilgin has seen a similar result in six cases: he gives the remedy in somewhat the same manner- $\&$ grains every hour five times successively, - the treatment being preceded and followed by a dose of oil (Record, 1880).

PREPARATIONS AND DOSE.-Wadding containing 4 to 10 per cent. of the acid is used in the dressing of wounds (Thiersch). An ointment may be made with $1 \frac{1}{2}$ part acid dissolved in 2 parts alcohol, and then mixed with lard. Lotions are made with acid 1 part, phosphate of sodium 3 , water 50 ; or with bicarbonate 
of soda $\frac{1}{2}$ part, water 100 ; or with borax 18 , acid 10 , water 100 . A glycerole, with $2 \mathrm{dr}$. of acid and 1 of borax, glycerine q.s. to make a 25 per cent. solution miscible with water or alcohol. An oil, with 1 part of acid to 49 of olive oil.

Internally.-Acidum salicylicum: dose, 10 to $20 \mathrm{gr}$., or more. Sodce salicylas: dose, 10 to $30 \mathrm{gr}$. Salicin: dose, 5 to $30 \mathrm{gr}$. Much larger doses are used by some practitioners than by others, but about $1 \mathrm{dr}$. of the acid, or $2 \mathrm{dr}$. of the soda salt, in divided doses of 15 to $20 \mathrm{gr}$. in the course of twenty-four hours, represents an average amount.

The acid is well given dissolved in liquor ammoniæ citratis (Tuckwell), or with $5 \mathrm{gr}$. each of ammonia and soda carbonate in an ounce of water (Prideaux), or in spiritus ætheris nitrosi (Thomas), or effervescing Vichy or Seltzer-water; mixed with powdered liquorice it is well taken in wafer capsules. Dr. Kemp suggests beef-tea as a vehicle (B. M. J., i., 1881). A granular effervescent form containing $5 \mathrm{gr}$. in each drachm, either of salicin, the acid, or the salt, is made by Savory \& Moore.

Quinice salicylas: dose, 3 to 6 gr., and upwards. Ferri salicylas : dose, 3 to $10 \mathrm{gr}$. in pill. Pulvis saliciylicus cum talco, P.G. (salicylic acid 3 parts, wheaten starch 10 parts, talc 87 parts, mixed to a fine powder): used to correct excessive foetid perspiration of feet. Collodium salicylicum (salicylic acid 60 gr., extract of cannabis indica 8 gr., flexible collodion I oz., dissolve): this forms a solvent for corns and warts. 


\section{ULMACEA.}

\section{THE ELM FAMILY.}

An order of deciduous trees, indigenous to northern temperate latitudes; distinguished by their alternate, simple, ovate, serrate, and oblique leaves, and by their dense and globular clusters of little apetalous flowers, usually with five stamens, produced before the foliage appears, and followed by samaroid seedvessels.

\section{ULMUS CAMPESTRIS.}

\section{(The Common Elm.)}

DESCRIPTION.-Distinguished by the smallness of the leaves, the tetramerous flowers, and the deep sinus in the samara. The bark, which is the part valued in pharmacy, should be stripped from the trunk in spring: it is met with in flat pieces, consisting of the "liber" only, several feet long, and 4 to 6 inches broad; externally of light cinnamon colour, internally grayish-white.

ACTIVE INGREDIENTS.-Elm-bark contains about 3 per cent. of tannin, and a peculiar mucilaginous gummy principle, not met with elsewhere, called ulmin, which is dark brown, insoluble in cold water, slightly soluble in boiling water, very soluble in alkaline solutions.

Physiological Action.-Elm-bark is demulcent and diuretic. Being slightly astringent, it is also in some degree tonic.

Therapeutical Action.-Decoction of elm-bark has been recommended in cases of cutaneous eruption, such as lepra and psoriasis, perhaps as a cheap substitute for sarsaparilla.

PReparation and DOSE.-Decoctum: dose, 2 to 4 fl. oz. When removed from the tree in spring, the bark, in order to preserve its qualities, should be quickly dried. 


\section{CUPULIFERA.}

\section{THE OAK FAMILY.}

A FAMTLY of usually massive and majestic trees, chiefly indigenous to temperate latitudes, and well marked by their penninerved alternate leaves, unisexual monœcious flowers (the males borne in eatkins), and by the fruit, which is nut-like, and contained in a cupule. No species is deleterious; many are noted for their astringency. The pharmaceutical species are-
Quercus robur .. $\quad \ldots \quad \ldots \quad$ The British oak.
Quercus infectoria. . . . . Gall or dyer's oak.

\section{QUERCUS ROBUR.}

\section{(The British OAk.)}

DESCRIPTION.-The oak of our own country is distinguished from nearly allied exotic species by its deciduous, irregularly sinuate leaves (the lobes of which are rounded), and rather small embossed acorn-cups. The portion valued in pharmacy is the iuner bark.

ACTIVE INGREDIENTS.-These are tannic and gallic acid, and pectin, all of which are yielded both to water and spirit. The amount of tannic acid varies with the age of the branch from which the bark is taken, with the season of the year at which it is removed from the tree, and with the time it is kept. For pharmaceutical purposes both acids are prepared from the galls of the Quercus infectoria, but an inferior description of galls may be gathered abundantly in our own country from young plants of the British oak. 
Physiological ACTION.-Oak-bark is powerfully astringent, and resembles in its operation other vegetable astringents containing tannin.

Therapeutical ACTion.-Powdered oak-bark is valuable in cases of passive hamorhage, and in diarrhoe. The infusion or decoction may be employed with advantage as an astringent gargle, also for injections and fomentations of a powerfully astringent character.

PREPARATION AND DOSE.-Decoctum: 1 to $2 \mathrm{fl}$. oz.

\section{QUERCUS INFECTORIA.}

(GAll or Dyer's OAK.)

DESCRIPTION.-The gall-oak or dyer's oak is not a tree, but a mere bush, with small, oblong, shining, prickly-margined leaves, and long, obtuse, solitary acorns. It occurs abundantly in Asia Minor, whence the galls of commerce are imported. These arise from the puncturing of the bark by an insect (the Diplolepis gallæ tinctoriæ), and the deposit of its ova, around which the altered juices of the tree concrete into a kind of nidus. In its perfect state a gall is dark green or brown, nearly spherical, and about $\frac{1}{2}$ inch in diameter; it is hard, often perforated by a small canal through which the insect has escaped, and when broken, shows a central cavity.

ACTIVE INGREDIENTS.-Galls contain about 35 to 40 per cent. of tannic, and about 6 per cent. of gallic, acid; also a substance called ellagic acid, with sugar, starch, gum, extractive saline matter, and other subordinate ingredients. The soluble principles are taken up by forty times their weight of boiling water; the residue is tasteless. The light-brown colour of the infusion is deepened by ammonia, and changed to orange by nitric acid; bichloride of mercury renders it milky, but no precipitate is produced by any of these reagents: the infusion reddens litmus. 
Tannic acid, $\mathrm{C}_{27} \mathrm{H}_{22} \mathrm{O}_{17}$, occurs (when separated) in paleyellow, vesicular masses, or thin and glistening scales, with a strongly astringent taste, and an acid reaction; readily soluble in water and rectified spirit, sparingly so in ether. "The aqueous solution precipitates solution of gelatine yellowish-white, and the persalts of iron of a bluish-black colour. When burned with free access of air it leaves no residue" (B.P., 1867). Treated with dilute acids, it yields gallic acid and glucose.

Gallic acid, $\mathrm{C}_{7} \mathrm{H}_{8} \mathrm{O}_{5} \cdot \mathrm{H}_{2} \mathrm{O}$, presents itself, when prepared, in the shape of crystalline, acicular prisms, or silky needles, sometimes nearly white, but generally of a pale fawn colour. It requires for solution about 100 parts of cold and 3 parts of boiling water. A solution of 20 grains of citrate of potash will take up 15 grains of gallic acid without precipitate in the cold (Long, B. M. J., ii., 1881); it is soluble also in rectified spirit. The aqueous solution gives no precipitate with solution of isinglass, but a black one with persalts of iron. When dried at $212^{\circ}$ the crystalline acid loses 9.5 per cent. of its weight. Burned with free access of air it leaves no residue.

Ellagic acid, $\mathrm{C}_{14} \mathrm{H}_{6} \mathrm{O}_{8}$, appears in the separated state as a white crystalline powder, and differs from tannin and gallic acid in being almost insoluble in alcohol, ether, or water. According to M. Pelouze, it does not exist ready formed in galls, but originates in the action of atmospheric oxygen upon the tannin; and the same, this author informs us, is the origin also of gallic acid (U.S. Dis.). The quantity of ellagic acid contained in galls is trifling, but the intestinal concretions called bezoct's are said to be composed of it almost exclusively.

Physiological Action.-1. Tannic Acid, when taken into the mouth, acts as a powerful astringent upon the mucous membrane of the fauces and of all the parts with which it comes in contact.

Upon the mucous lining of the stomach and intestinal canal large doses produce a peculiar effect by combining with albuminoid matters in a manner like that which comstitutes the essence of the tannin-process in curing skins for leather: hence may follow obstinate constipation and vomiting, thirst, and cutting pains in the abdomen. This tendency 
to combine with proteinous substances gives to tannic acid a decidedly superior power as a local astringent over gallio acid. When absorbed into the blood, it is decomposed, with production of gallic and pyrogallic acids and a humin-like substance. The principal fact in confirmation of this belief is that ordinary tannic acid is not found in the urine, ${ }^{1}$ but only the decomposition-products already named, or some of them. Gallic acid is nearly always present, and can be recognized by its characteristic effect on persalts of iron; but Bartels has shown that occasionally only the humin-like body passes orer, and sometimes even this trace of the tannin is lacking. Neither the liver, the pancreas, the skin, nor the pulmonary mucous membrane appears to excrete tannin unchanged; but the sputa, like the urine, are often coloured almost black.

The powerful coagulating action of tannin upon blood outside the body might give rise to a belief that similar changes occur within the vessels; but of this there is no proof, and there is reason to think that very little tannin as such is absorbed into the blood: in the form of albuminate it has been found largely in the frces, but it is not likely that much passes through the gastro-intestinal mucous membrane without previous decomposition. The remote effects of tannin are therefore with great probability ascribed to its secondary products.

2. Of Gallic Acid the local action, as already said, is far less powerful, but this drug passes rapidly through the body, acting as an astringent on various "organs and functions, and appearing unchanged in the urine.

Therapeutical Action. - Externat. - 1. Of Tannic Acid.-This is mainly local, but is highly useful in a variety of cases, e.g., in quinsy, catarrhal affections, chronic bronchitis, laryngeal phthisis, hamoptysis, etc. Applied in the form of a fine powder, or in gargle or spray, to the mucous membrane of the fances and air-passages, it checks congestion and inflammatory swelling, reduces excessive secretion, and arrests the tendency to cnpillary hæmorrhage. Applied to a bleeding surface anywhere, if no large vessel be engaged, tannic acid is prompt in its styptio

${ }^{1}$ Catechu-tannin and several other varieties do pass off in the urine unchanged. 
action, and will modify the fungating and easily bleeding surfaces of ulcers. But perhaps the most decided instances of its local utility are to be found in its application in the shape of the glycorole of tannin to subacute eczema, blennorrhoeal and leucorrhool states of the urethra and ragina, relaxed throat, intertrigo, rectal fissures, and prolapsus ani.

THERAPEUTICAL AC'TION.-Internal.-Stillé recommends tannic acid in the chronic stages of phthisis as likely to diminish dyspnoea, cough, and expectoration, and to palliate all the symptoms, "converting moist rhonchi, or gurgling, into signs of solidification merely, or of a dry cavity." In the diarrhcen. of children especially, 1- to 2-grain doses of tannin are safe and often valuable.

As an Antidote to Poisonous Alkaloids, tannin holds a high place. The observation was first made in regard to emetin- or ipecacuanha-poisoning, with excessive vomiting; but holds good with opium and its salts, digitalin, nicotin, etc. In Metallic Poisoning, particularly with antimony, tannin has gained some repute, but it is not so effective as albumen (white of eggs).

2. Of Gallic Acid, while the local effects are of comparatively little importance, the remote astringent action is remarkable.

In Hæmorrhage of various kinds, but especially in hæmoptysis, and in hrematuria dependent upon morbid conditions of the mucous membrane of the bladder, it is often exceedingly effective. Other remedies, and particularly ergot of rye, may perhaps supersede it to a considerable extent, but gallic acid remains one of the most useful agents of this class: "Ruspini's styptic" is said to be mainly composed of it.

Albuminuria and Dropsy.-I have formed a somewhat favourable opinion of its action in cansing contraction of the renal vessels, and consequent diminution of the waste of valuable albuminoid matters.

In dropsy and albuminuria, however, the astringent power of gallic acid is probably even more useful than in hæmorrhagic conditions, and there is a general agreement that it can often check the waste of albuminoid matters: it is remarkable that while this is effected there is usually an increase in the flow 
of urine. The action of gallic acid upon the pulmonary and urinary tracts, through which it is eliminated, is used as a strong argument that we may look to the curative effects of drugs on organs by which they are cast out of the body for one of the most promising modes of therapeutical influence.

In these conditions, however, Dr. Parkes gave large doses, without causing any diminution of albumen, and Dr. W. Roberts " has used gallic acid in a number of cases for many weeks, without, in a single instance, any favourable influence on the excretion of albumen, but with, sometimes, serious gastric disturbance" (On Renal Diseases).

In night-sweating and in gastric catarrh it has also been giren.

PREPARATIONS AND DOSE.-Of Galls.-Tinctura galloe (seldom used except as a chemical test): dose, $\frac{1}{2}$ to $2 \mathrm{dr}$.

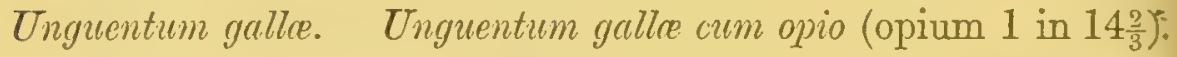

Of Tannic Acid.-Acidum tannicum: dose, 3 to $20 \mathrm{gr}$. Glycerinum acidi tannici ( 1 part in $4 \frac{1}{2}$ ). Suppositoria acidi tannici. Trochisciacidi tamici (each lozenge contains $1 \frac{1}{2}$ gr. of tannic acid).

Of Gallic Acid.-Acidum gallicum : dose, 3 to $20 \mathrm{gr}$. (an ounce of water dissolves about 4 gr. of gallic acid). Glycerinum acidi gallici (1 part in $\left.4 \frac{1}{2}\right)$ : dose, 1 dr., repeated at hourly or halfhourly intervals while hæmorrhage continues.

\section{ACIDUM PYROGALLICUM.}

\section{(Pyrogallic Acrd, or Pyrogaltol $-\mathrm{C}_{6} \mathrm{H}_{6} \mathrm{O}_{3}$.)}

DESCRIPTION. - It may be formed from gallic acid by sublimation, or from extract of galls in a special apparatus, and occurs in thin, light, pearly crystals, freely soluble in water or alcohol, and in 10 parts of melted lard. It readily absorbs oxygen, becoming dark brown on exposure or on contact with alkalies and most metallic salts. It is used for dyeing purposes and in photography. It is odourless, but has a somewhat bitter taste, with a sensation of coolness. 
Physiological Action.-Applied externally in strong solution, it acts as an irritant and browns the skin, but the stains may be removed by chloride of lime or oxalic acid, and even by exposure. A proportion of 1 to 2 per cent. is antiseptic, preventing alcoholic and other fermentations (Bovet, Lyon Méd., 1879).

If applied extensively to the body, absorption may take place, with serious and even fatal result: general erethism, partial suppression of urine, and prostration were noted by Hebra in several cases; and Neisser reports a case of general psoriasis in a robust adult, one half of whose body was rubbed with a pyrogallic acid ointment (10 per cent.), the other half with "rhubarb" ointment, i.e., chrysophanic acid, and who developed in two hours rigors, then vertigo, collapse, and coma, with a temperature of $104^{\circ} \mathrm{F}$; the urine became dark, and in a few days loaded with hremoglobin, death ensuing (Zeits. f. Klin. Med., i., 1880).

It is presumed that the drug is decomposed by the alkaline blood and absorbs free oxygen, so that the corpuscles are destroyed, and there is a true "dissolution of the blood." The renal tubules are blocked with altered epithelium and corpuscles. By other observations it is proved that chrysophanic acid does not cause these symptoms.

\section{Therapeutical Action. - Psoriasis-Lupus.-An} interesting report by Dr. G. Thin would be of itself sufficient to draw attention to this remedy in psoriasis, for it refers to a case of several years' duration, in which careful comparison was made between this and other methods of treatment-e.g., some patches were rubbed with soft soap and ammoniated mercurial ointment, others with oil of cade, and at the end even of several weeks were only slightly improved; whilst patches treated with pyrogallic acid ointment (10 or even 5 per cent.) were "speedily cured." The stronger ointment had to be omitted for a time after a few days' use on account of great irritation, but the 5 per cent. caused no bad effects, except those Dr. Thin thinks essential to its action, such as moderate inflammation about the hair-follicles (Lancet, i., 1881). Arlt was one of the earlier observers of this curative result (Practitioner, 
vol. xxiii.), but Jarisch was perhaps the first to demonstrate it on an extensive scale, and especially showed the value of the remedy in lupus. Of thirty cases, mostly severe, improvement occurred in two-thirds: a 10 per cent. ointment was kept constantly applied for several days, or until it became too painful; it seemed to attack the infiltrated cells (not the healthy), and left cicatrices which became white and soft; recurrence of the growth in other places, required similar treatment for some time (Schmidt, Jahrb., 1880, No. 3).

There is some analogy between this treatment and that by chrysophanic acid; and Hebra, comparing them, gives the preference to the former: he found the latter often caused dermatitis, and that patches of psoriasis would form again on the very site of severe inflammation; neither is it applicable to the face or head, though doubtless it is suitable and effective for circumscribed patches. Pyrogallic acid produced better resultŝ, he found, in three to four weeks' time: he treated lupus also with it successfully (Practitioner, vol. xxv.).

Hæmorrhage.-Dr. Vesey has advocated the use of this acid in hæmoptysis and other forms of hæmorrhage, as having advantages over gallic acid in the requisite dose being smaller (1 grain every half-hour or hour), in its not disordering the stomach or causing either vomiting or constipation, and in its not being disagreeable to take (Dublin Journ. Med. Sci., Dec., 1878). From the case already cited, it must evidently be given with caution.

PREPARATIONS AND DOSE.-Acidum pyrogallicum: dose, $\frac{1}{2}$ to $1 \frac{1}{2}$ gr. The freshly prepared acid may be given in solution, or in pill. Unguentum may be made with melted lard: 5 per cent. is safe, and 10 per cent. should not, as a rule, be exceeded. 


\section{URTTCACEA.}

\section{THE NETTLE FAMILY.}

A LARGE, diversified order of exogens, comprising trees, shrubs, and herbaceous plants, met with in almost every part of the world. Scarcely any single character is universal, except that the flowers are unisexual and apetalous, with one to five stamens opposite the lobes of the small calyx, and the ovary is superior and one-celled, containing a solitary ovule; the style or stigma is simple. (In the limited application preferred by some, the order contains only nettle-like plants; in the comprehensive and more convenient one adopted by others, it covers the Moracer, the Artocarpaceæ, and the Cannabinaceæ.)

\section{Pharmacentical Species.}

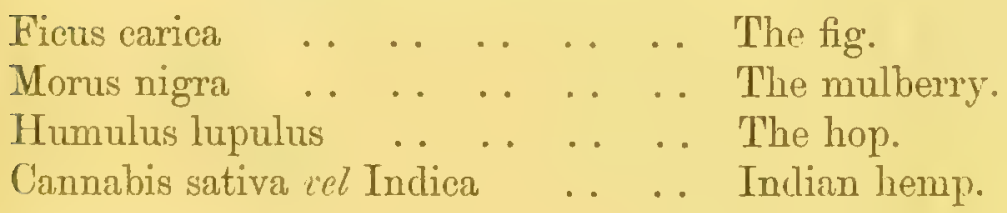

()f the fig it is needless to do more than mention its wellknown character as a mild laxative.

The juice of the mulber'y fruit, mixed with water, may be used as a refreshing drink in febrile conditions. The sym rupus: mori makes a purple colouring as well as sweetening addition to gargles and mixtures. 


\section{HUMULUS LUPULUS.}

(The Hor.)

DESCRIPTION.-A twining, vine-like, deciduous perennial, indigenous to the northern parts of Europe and Asia, and naturalized, if not wild, in Great Britain. The wiry stems mount to a height of several yards; the leaves are opposite, petiolate, 2 to 5 inches across, either three- to five-lobed or cordate, serrated, and rough; the flowers are diocious, those of the male plants forming large light and feathery panicles; those of the females constituting chaffy cones, 1 to 3 inches in length-every scale, when the cone is ripe, having a couple of seed-like fruits at the inner base, and being powdered over its inner surface with minute golden-yellow resinous glands or grains, which easily shake off, and constitute "lupulin." These grains are aromatic and inflammable.

ACTIVE INGREDIENTS.-The active principles of the hop reside chiefly, though not exclusively, in the lupulin, which possesses the peculiar flavour of the plant, and determines, by its abundance or otherwise, the value of any particular sample; it contains 2 per cent. of volatile oil, about 11 per cent. of bitter principle, and 50 to 55 per cent. of resin (Payen).

The volatile oil is obtained by submitting Iupulin to distillation with water; it is said to consist of an oxygenated oil and a hydrocarbon, $\mathrm{C}_{10} \mathrm{H}_{10}$, isomeric with oil of turpentine. In colour it is pale greenish-yellow, though, when re-distilled, it becomes colourless; it is very soluble in alcohol and ether, less so in water, and has a sp. gr. of 0.910 ; the taste is acrid; the ndour resembles that of hops: by keeping and exposure to the atmosphere it becomes a resinous mass. Hops, when long kept, develop some valerianic acid.

The bitter principle, called lupulite or humulin, is yellowishwhite, uncrystallizable, and neutral; it is soluble in alonol, 
sparingly so in water, and insoluble in ether; it contains no nitrogen, and is destitute of narcotic properties.

The resin is bright-yellow, changing to orange colour on exposure to the atmosphere; it is soluble in alcohol and in ether.

Hops contain tannin to the extent of about 5 per cent., also a nitrogenous substance, and a gummy one.

PHySiological ACTiON.-Digestive System.-Infusion of hop acts as an aromatic, somewhat astringent stomachic and tonic, promoting appetite and digestion. The Circulation is somewhat quickened, and the Nervous System, at first stimulated, is later calmed. The latter effect is specially exerted by lupulin, which also seems to have a peculiar sedative power over the genital system.

THERAPEUTICAL ACTION.-EIot hops locally applied, in poultice or fomentation, relieve internal pain and inflammation. With patients who suffer from insomnia, nervousness, or overexcitement, the hop-pillow is deserving of trial ; it relieves pain, and allays nervous irritation, partly, perhaps, by influence of the volatile oil, partly by association of ideas. In the restlessness of early stages of delixium tremens, in drink-craving, and specially in morphia-craving, and in the conditions of prostration and unrest that oscur when leaving off the morphiahabit, I have seen distinct advantage from a tincture of Iupulin, which is naturally much more active than tincture of hop, and deserves a place in our Pharmacopoeia. In alcoholic cases, tincture of capsicum is well combined with it.

In chordee and gleety discharges, and for checking involuntary seminal emissions, lupulin is of value, also in nocturnal incontinence of urice, and in cases of irritable bladder : it has been resorted to as an anodyme in rheumatism.

Infusion of hop is a useful tonic in dyspepsia, and has been found serviceable in gouty spasm of the stomach.

PREPARATIONS AND DOSE.-Lupulin: Close, 2 to $5 \mathrm{gr}$. Infusum bupuli: dose, 1 to $2 \mathrm{fl}$ oz. Extructum: dose, 5 to $15 \mathrm{gr}$. Tincture: dose, $\frac{1}{2}$ to $2 \mathrm{fl}$. dr. Tincture lupuline ( 1 in 8 parts -U.S. Dis.) ; $\frac{1}{2}$ to $2 \mathrm{dr}^{2}$. 


\section{CANNABIS SATIVA VEL INDICA.}

\section{(Hemp-Iniman Hemp.)}

DESCRIPTION.-An annual, indigenous to the cooler parts of India, and found also in Persia. The stem rises to the height of 3 to 6 and 12 feet, and is nearly simple, except towards the summit. The leaves, produced chiefly at the upper part, are quinate or digitate, and supported upon long petioles, the leaflets lanceolate, 3 to 4 inches in length, and sharply serrated. The flowers are diocious, green, and insignificant; those of the male plant are paniculate, those of the females are sessile in the leafaxils. The fruit is a grey achænium.

An Oriental variety of this plant is distinguished by the name of Cannabis Indica; the produce of this one alone is enployed in pharmacy: it is known as gunjah or ganja, and as usually seen in the market, consists of the branch-tops, 2 to 3 inches long, pressed together, brownish-green, having few leaves, but flowers and fruits with adherent resin. In India it is met with more in leaves and small stalks broken up, and is known as bhang, or hashish; the resin, collected by friction with the hands or on leathern clothes, is called churrus-its amount varies with the season, the altitude, etc.

Active IngREdients. - The hemp-plant exhales a powerful and rather repelling odour when fresh, but the scent is much diminished or lost altogether by drying; the taste is feebly bitter. These qualities are referable to the actire principle of the plant, called cannabin, a resinous body possessed of a peculiar fragrance, and a warm, bitter, and acrid taste: it is soluble in alcohol and in ether, but not in water, which throws it down as a white precipitate from an alcoholic solution. Hemp likewise contains a small proportion of volatile oil, with some lignin, albumen, gum, and other less important ingreclients. The seeds do not share in the composition or proprerties of the stems and lenves. 
PHYSIOLOGICAL ACTION.-Observations upon animals appenr to indicate that carnivorous creatures suffer a kind of intoxication from hemp, whilst herbivorous ones seem unaffected even by large doses.

Circulatory System.- In man, when subjected to the operation of this drug, the pulse in some instances becomes quickened, though not remarkably so, and the respiration somewhat slower; in other cases no change is observable. At the same time there is a sensation of warmth in every part of the body excepting the feet, which, ordinarily, seem cold by contrast.

Digestive System.-It is said to increase the appetite for food: the secretions do not appear to be materially affected; it does not cause dryness of the tongue, nor constipation.

Nervous System.--Should the individual taking cannabis be of a temperament favourable to its peculiar action, there is generally an agreeable exhilaration of the spirits, followed by more or less stupor and sleep,-at times there is a delightful state of reverie; having myself experimented with this drug, I can state that this (when it does come on) is agreeable beyond description, but the next day there are sensations of dulness and heaviness, which are anything but enviable. The natives of some parts of India are addicted to the use of it as a pleasant inebriant, but it is certain that in other countries the peculiar energy of the plant is not manifested in the same degree, nor always in the same manner; whence it is evident that climatic and ethnological consiclerations have both to be taken into nccount in estimating the powers of cannabis. Instead of agreeable sensations it would seem that with certain individuals the effects are either pugnacity or foolish antions, with sometimes giddiness, nausea, vomiting, and oppressive thirst. So small a dose as $7 \frac{1}{3}$ minims of tincture has exceptionally caused such symptoms (Lancet, ii., 1871) : full doses have induced a kind of catalepsy.

Thirty Irops, taken in good health by 1r. Clouston, caused in an hour confusion and dulness of head, unusual acuteness of hearing, apparent prolongation of time; and in two hours great drowsiness, which lasted for several hours, and was followed by confusion of thought and incapacity for exertion (Med.-Chir. Riev., Oct., 1880). 
Dr. H. C. Wood has given a graphic account of the effects on himself of "a very large dose," including hallucinations as to time, distance, and hearing - a few minutes seeming to be hours (on account of the great number of ideas presented to the mind), a short distance immense, and a whisper very loud talking; a sense of despair and dread of death were marked (Treatise on Therapeutics). The drug is said sometimes to act as an aphrodisiac, but this is not usual.

The medicinal properties of cannabis are thus summed up by Dr. Glendinning, who employed this drug extensively :- "It acts as a soporific or hypnotic in causing sleep; as an anodyne in lulling irritation; as an antispasmodic in checking cough and cramp; as a nervine stimulant in removing languor and anxiety, also in raising the pulse and enlivening the spirits, without any drawback or deduction of indirect or incidental inconvenience; conciliating tranquil repose without causing nausea, constipation, or other signs or effect of indigestion, without headache or stupor." Notwithstanding these praises, it must be acknowledged that much has yet to be learned in regard to the physiological action of cannabis; as an anodyne it is decidedly inferior to opium.

Acute Poisoning by this drug presents some variety in symptoms. There may be either unconsciousness, with collapse and pale clammy skin, or a cataleptic state with spasm and convulsion; there is always some anæsthesia, so that the Chinese use it for surgical operations. This was very marked in a case recorded by Dr. Strange--when about 20 grains of extract were taken, "the patient declared he could touch nothing" (B. M. J., ii., 1883).

Chronic Poisoning.-Its habitual use induces cedema of face, injection of eyes, weakness and tremor of limbs, with mental feebleness, and uttimately marasnuus.

SYNERGISTS.-Tea, coffee, and tobacco especially, increase the effects of cannabis; the latter is commonly smoked with it in the East. Sedative effects on the nervous system are markedly increased by conjunction with bromides, so that half the usual dose suffices (Clouston). 
Antagonists.-According to Polli, lemon-juice, vinegar, and the vegetable acids arrest the action of cannabis, and serve as real antidotes.

\section{Therapeutical Action. - Neuralgia-Migraine.-} Cannabis has a deserved repute, especially in the latter. Dr. Greene found it act well in patients temporarily relieved by nitrite of amyl, and in six cases, some of twenty years' duration, the frequency of attacks was greatly lessened by perseverance in the use of $\frac{1}{3}$ to $\frac{2}{3}$ grain of extract night and morning (Practitioner, vol. ii., 1872). Dr. Seguin corroborated these observations, and reported relief in half of a much larger number of cases from $\frac{1}{3}$ to $\frac{1}{2}$ grain thrice daily before meals. The treatment should be continued for at least three months, and combined with attention to diet and removal of obvious causes of acidity (Record, 1878).

Dr. Stewart (Canada) notes that it is specially useful in cases with vascular spasm; and Lothrop and others have also recorded good results (Record, 1881).

Tetanus. - Sufficient success in this disorder has been traced to cannabis to render it always well worthy of consideration. Dr. O'Shaughnessy and the late Professor Miller (Edinburgh) have recorded several severe traumatic cases "cured by 2- to 3-grain doses given two or three times in twenty-four hours." Of thirty-five cases tabulated by Dr. Roemer, in 1873, there were twenty-one recoveries, in a successful case 130 minims every hour were given for some time (Lancet, ii., 1877): in another series of cases, good results attended its combination with shloral (ibid., i., 1878).

A writer in the Practitioner (1877) scarcely admits any value in the ordinary administration of the drug; but Dr. John Lucas has more recently recommended smoking the leaves, either with or without tobacco, and to such an extent as to keep the patient always slightly nareotized (Med. Times, i., 1880). Other spasmodic disorder's have sometimes been rolieved by cannabis; thus, Oxley found it useful in chorert, Dr. Handfield Jones in asthma.

In Delirium Tremens it has sometimes acted well, and it is at least freer from risk than ordinary nareotics. 'T'he conı- 
bination of this drug and bromide has been found valuable in mental depression, and even in melancholia with sleeplessness, if it is not very effective in acute mania. Dr. Strange reports that a patient took this mixture regularly every night for six months, "with the effect of removing acute bodily torture and mental anguish completely for the night" (B. M. J., ii., 1883). Dr. Russell Reynolds has found the drug act well in insomnia and other nerve-derangements, "without interfering with any of the functions of organic life"; and Fronmüller reports many instances of sleep produced without any drawback, by 5 to 10 grains of tannate of cannabin; and he has pointed out that in distilling hemp a volatile "oil of cannabin," and a hydrate of the same are obtained, and are both of irritant poisnnous nature, and that in the tannate, which is a brown jowder, of tannin taste, and not unpleasant smell, soluble in allialine solutions, it is presumed that these irritants are got rid of (Record, 1882). He further finds it to be, in doses of 2 to 10 grains, a "reliable, effective, and easily administered hypnotic." In support of this he quotes the result of its administration to more than sixty patients, men and women, in hospital and in private-forty of them were phthisical, the rest chronic invalids, - all suffering from insomnia; thirtyseven of these obtained quiet refreshing sleep, without toxic effects (Record, 1882). In mental disorders Dr. Clouston has often seen more advantage from the combination of cannabis with bromides, than from either separately.

Uterine Hæmorrhage, such as occurs at the period of the cessation of the menses, is often moderately arrested or abater by the use of cannabis. Dr. Churchill, of Dublin, gave for this purpose 5 to 15 or 20 drops of the tincture three times in the day, and with excellent results.

The late Dr. Silver also reported several good illustrations of its value in various forms of menorrhagia with pain and profuse clotted discharge, which other remedies failed to relieve; it was temporarily effective even in fibroid and malignant disease (Med. Times, ii., 1870).

Recently, objection has been taken to the remedy as being uncertain and apt to produce disagreeable nerve-symptoms of the nature already described (Oliver, B. M. J., i., 1883); but, nn 
the other hand, many have corroborated the above statements of its value (ibid., ii., 1883).

The reports show the necessity of securing a good preparation-for which Squire has a special repute.

Uterine Contraction during Labour.-Dr. Alexander Christison claims for cannabis indica a power of promoting contraction equal to that which is possessed by ergot. He states that the effects are perceived more quickly than those of the latter, and are more energetic and of shorter duration, but are not constant. Several observers have stated the same, notably Michel, who traces its action in all cases to contracting the uterine fibres (Record, 1881). It is commonly said to be able to strengthen not to originate such contraction.

Gonorrhœa.-Cannabis indica has been recommended in gonorrhœa, and there can be no doubt that its employment causes diminution of discharge, and relieves the violent burning pain in the urethra which comes on during and after micturition.

PREPARATIONS AND DOSE.-Extractum: dose, $\frac{1}{4}$ to $1 \mathrm{gr}$. or more, cautiously increased; a good pill may be made with sugar of milk and acacia. Tincture: dose, 5 to 20 min. or upwards. Cannabince tannas: dose, 5 to $10 \mathrm{gr}$. (Frommüller). 


\section{CONIFERAE, OR PINACEAE.}

\section{THE PINE-TREE FAMILY.}

ThE order Coniferæ is constituted of about 300 species of ligneous plants, the greater portion of which are evergreen trees, noted for their longevity and altitude. The leaves are usually acicular, the flowers unisexual ; the fruit is well represented in the familiar fir-cone which gives name to the order. In temperate and cold countries the Coniferæ grow plentifully, and become of immense economic value. Two well-distinguished sections have been established-the Abietineæ, or true conifers; and the Cupressineæ, represented in the juniper and the savin.

\begin{tabular}{|c|c|c|}
\hline Terebinthina. . . & & Turpentine. \\
\hline Pix liquida .. & & Tar. \\
\hline Pix Burgundica & & Burgundy pitch \\
\hline Terebinthina Canadensis & & Canada balsam. \\
\hline Juniperus communis . & . & Juniper. \\
\hline Juniperus sabina . . & . & Savin. \\
\hline Thuja occidentalis & & Arbor vitæ. \\
\hline
\end{tabular}

\section{TURPENTINE.}

DESCRIPTION.-Turpentine is a liquid oleo-resin yielded by various species of Pinus, and especially by the Pinus palustris, tecele, and pincester. 
The P.pulustris attains the height of 60 to $\tau 0$ feet: the leaves are a foot in length, brilliant green, and produced in threes; the cones are very long, sub-cylindrical, and muricated. The $P$. teede is somewhat taller; the rigid leaves are also prodnced in threes, but the cones, which are often in pairs, are oblong, pyramidal, and somewhat truncated at the apex. The Pincstrir, on the other hand, produces its leaves in couples, and the cones are short and obtuse. The two first are North American; the third is indigenous to the South of Europe.

Turpentine is obtained by cutting holes ("boxes") in the trees during autumn and winter, removing the bark above, and scraping the wood: in the spring and summer, when the liquid runs best, these " boxes," which hold from four to eight pints, are emptied as they fill, and the contents packed in barrels. "Canada turpentine" is secreted in vesicles in the bark, and these are pierced by a sharp iron tube attached to a can. Turpentine is a yellowish, viscid liquid, of characteristic odour and pungent taste, which separates on standing into a transparent upper layer and a whitish granular lower one: the latter is identical with what concretes on the trees in yellowish-white masses, known as white turpentine (Thus Americanum).

\title{
TEREBINTHIN无 OLEUM.
}

\author{
(Oil, of Turpentine.)
}

PREPARATION.-By distilling the oleo-resin already describerl, with the addition of water; on condensing the distillate the oil separates.

DESCRIPTION.-A colourless, thin, volatile oil; sp. gr. 0.86. It differs somewhat in physical characters according to its source-American turpentine, for instance, turning polarized light to the right; German, to the left. The odour when fresh is mild, but becomes more pungent after exposure, when the nil contains ozone, formic acid, etc., and becomes 
thicker and yellower. It dissolves in 12 parts of alcohol, and in much less of absolute alcohol; also in benzol, sulphide of carbon, chloroform, and ether,-not in water. It combines, lowever, with water to form several compounds, and with gaseous hydrochloric acid to form "artificial camphor." It is oxidized by nitric acid, and is violently acted upon by bromine and iodine.

ACTIVE INGREDIENTS.-The pure oil, $\mathrm{C}_{10} \mathrm{H}_{16}$, is free from oxygen. Distilled with sulphuric acid, it changes into several isomeric oils, e.g., terebene and colophene-the former is a colourless volatile oil of thyme-like gdour, sp. gx. $0 \cdot 86 t$.

ABSORPTION AND ELIMINATION.-The oil is readily absorbed by all surfaces of the body-perhaps most quickly in the form of vapour by the pulmonary mucous membrane. It is excreted by the same membrane, and also by the skin, the bowels, and the kidneys: the urine passed under its influence has the odour of violets, from a chemical change in part of the volatile odoriferous principle. Dr. W. Begbie has noted that, if the kidneys be unsound, more of the drug is excreted by the skin and bronchial membrane.

Physiological Action.-Ertemel.-Locally applied to the unbroken skin, turpentine produces a sense of burning, followed by inflammatory redness and stinging pain; if the application be prolonged, there is vesication. It has marked antiseptic properties: Bond and Kingsett have specially utilized them (Lancet, i., 1881).

Physiological Action.-Intemal.-Digestive System. -Oil of turpentine acts, in very small doses, as a stimulant, in larger ones as an irritant, to the whole of the gastro-intestinal tract: 1 or 2 drachms and upwards cause buruing sensations in the mouth and stomach, with much thirst; larger amounts, nausea and vomiting, and sometimes purging and tenesmus with much abdominal pain: these effects, however, may be modified by judicious combination with demulcents and anodyries. 
Circulatory System.-By moderate doses the pulse is increased in force and frequency; under excessive quantities it becomes feeble, and rapid collapse may occur. According to some researches of Fleischmann and Rossbach, the injection of turpentine into the blood-current lowers the arterial pressure, whilst it increases the pulse-beat in frequency, "denoting paralysis of the central end of the vagus": the general symptoms, such as dyspnoa and cramp, they attribute to minute pulmonary emboli (Abst. Record, 1879). Crusis also concluded that the drug, given to rabbits, "increased coagulability of blood, and cansed minute hepatic and pulmonic emboli" (Thèse de Paris, 1874). Kobert describes its action as "powerfully stimulant on the inhibitory reflex centre, and also upon the vaso-motor centre, thus raising the blood-pressure"; but very large doses paralysed both centres (Centralb. f. Med., 187\%) - the blood became dark and the heart paralysed.

In small doses it is said to cause vascular contraction.

Nervous System.-It is upon and through this system that the main effects of the drug are produced. Hoppe has argued that vaso-motor nerves are first affected by it, but large doses quickly act upon the nerve-centres; and from the fact of the brains of animals poisoned by it smelling strongly of the drug, it has been considered to exert a direct action on the cerebral cells (Bartholow).

Exhilaration, giddiness, and some degree of mental confusion, like that of early alcoholic intoxication, oceur after moderate or continued doses; 1 drachm has an hypnotic effect (Begbie) ; toxic amounts cause delirium, then torpor of intellect, impaired muscular and co-ordinating power, and ultimately paralysis of nerve-function, with convulsion and coma. The pupils are commonly dilated, and vision impaired. Toxic doses in man have cansed "complete muscular relaxation and profound insensibility, with abolition of all reflex movements"; 6. ounces have cansed death with opisthotonos in an adult, and the "brain, lungs, heart, and viscera were found gorged with bloorl"; in the few other fatal cases comparatively slight traces of tissue-change have been verified.

In Fleischmann's experiments with animals, symptoms of peremlysis were much more marked than of stimulution: 2 minims, 
mixed with water and injected under the skin of frogs, caused loss of power of voluntary movement; and the same occurred in rabbits and kittens after injecting an emulsion of turpentine into the stomach.

Sleeping in rooms recently painted is said to have caused death either from collapse or from asphyxia (B. M. J., ii., 1879), the oxygen of the air being absorbed by the oil of turpentine.

Genito-Urinary System.-By small and moderate doses the urine is commonly increased, but beyond a certain limit of dosage the stimulation of the kidney passes into irritation and congestion, with much pain in the loins, strangury, and more or less suppression of the secretion; and often these symptoms may follow inhalation of the vapour. Begbie suggests that the drug circulating in the renal capillaries causes irritation and rupture of the Malpighian vessels, and hence hæmorrhage, whilst when taken in small doses as a remedy for such conditions it acts through the nervous system, contracting the vessels. Priapism has been caused by it, and in women dysmenorrhoa and menorrhagia.

Cutaneous System.-The skin sometimes is found hot and dry under the influence of the drug, and I have known a red rash produced by it: some degree of pruritus is not uncommon, and an increase of perspiration, having the odour of the drug, is usual: desquamation has followed (Lancet, ii.. 1880).

SYNERGISTS.-Stimulants and irritants.

ANTAGONISTS.-Depressants generally: the local irritant action is lessened by demulcents and anodynes, which should be given in cases of poisoning after emptying the stomach. It is a direct antidote to phosphorus ( $r$. Inorganic Srbstances, p. 58), and, according to Binz, large doses of it control the spasms excited by brucia.

THERAPEUTICAL ACTION.-Eirternal-As a liniment, applied either cold or hot, it is valuable in Chronic Rheumatism, sprains, sore throat, and various neuralgic affections; 
also as a fomentation in puerperal peritonitis, pleuro-pneumonia, and all inflammations of serous membranes.

Dr. Copeman, of Norwich, published instanees of the special value of such applications in puerperal cases (Med. Times, ii., $18.2)$, and it is well known in typhoid fever. Vidal has drawn attention to the efficacy of "turpentine compresses" in Peritonitis and broncho-pneumonia (Record, 1881), and I have frequently seen benefit from its application in acute throat inflemmations; it is best applied according to the method advised by Dr. Roberts (U.S.); a piece of flannel, the size of a halfcrown, being soaked in the oil, and placed on the skin over the affected tonsil, and left for three to five or ten minutes, or until considerable redness and smarting are produced.

In Erysipelas it has been used locally with advantage. Liicke had it rubbed in, but Hastreiter records equally good results from simply painting it on the affected part (Record, 1882). Mr. Nunnely prescribed it internally in this affection.

In Diphtheria, the local application of the drug by brush or spray has been found very useful: the vapour also in this disease and in bronchorrhœa, and even in pulmonary gangrene, has acted well (Record, 1878; Med. Times, i., 187\%, i., 1881).

Burns, Scalds, etc.-Severe and dangerous burns and scalds, especially when the local injury is accompanied by great constitutional depression, are successfully treated with turpentine, and occasionally it is found useful in those dry and chronic forms of gangrene which are not preceded by inflammation.

Ringworm.-As a local application in ringworm, it has been advised, and is often of service as an adjuvant to other remedies.

Psoriasis.-In obstinate and chronic cases of psoriasis, Dr. Crocker advocates the application of turpentine, beginning with "l liniment containing ' 2 drachms of oleum terebinthinæ, and 1 ounce of oleum olive, and gradually increasing the strength until turpentine alone is used. Essence or oil of lemons disguises the smell. The eruption is dispersed by this trentment; and strangury is not produced (Lancet, i., 1881). 
THERAPEUTICAL ACTION.-Interual.-In variousdisorders with unhealthy discharge from mucous membranes-in bron(horrhœa, chronic blenorrhœe, and chronic cystitis-turpentine, in doses of 10 to 15 minims, conjoined with local application of the vapour when possible, often acts well.

In Hæmorrhage it has a special and well-deserved reputation, although its nauseous taste sometimes hinders its employment: 25 to 30 minims should be given at a time in hrmoptysis, and the vapour inhaled. It is in passive forms of hæmorrhage and in weak anæmic subjects that it acts best-presumably through the nervous system stimulating the capillaries to contract: cases are recorded in which it produced this effect after failure of acids, lead, and other hæmostatics. Dr. H. C. Wood remarks that the oil of erigeron is much more pleasant to the patient, and has seemed even more efficacious.

In purpura it is a useful remedy (Richardson, Med. Times, ii., 1874; Neligan).

A combination known as "Warren's styptic," is made by slowly mixing 2 drachms of oil of turpentine with 5 of dilute sulphuric acid, then adding 2 of alcohol; it should be kept in a stoppered bottle and given in 40-minim doses with sugar in a teacupful of water : this may be repeated in an hour and again in two hours, and is said to be effective in all varieties of hremorrhage (Med. Times, ii., 1859).

In post-partum hemornhge turpentine has proved extremely valuable, and has the advantage of acting quickly. In six or eight cases after ergot had failed, "a large dose" made the patient at once feel better, the pulse became perceptible, the uterus contracted, and the bleeding ceased (Pollard, B. M. J., ii., 1879).

Dr. Copeland long ago drew special attention to its power in this condition, and J. Hunter esteemed it in hematemesis. Begbie notes that it may be used in small doses even in humuturin (Edin. Journ., July, 1871). In the hrmorrhnge of enteric fever it has special advantages, and often proves invaluable in cases where there is a disposition to this and to extreme tympanites. It is then best given by enema, 30 to 60 minims $1 n$ starch-mucilage, either alone or (if there be much discharge and pain) with 10 minims of liquor opir. The general treatment 
typhoid by turpentine, though fairly successful, is not equal to that by other remedies (Med. Times, ii., 1882). It has, however, proved extremely useful in India, $\frac{1}{2}$ drachm four times daily in mucilage being given (B. M. J., i., 1878):-Dr. White gave 10 minims only (ib., ii., 1877).

Graves praised turpentine much in typhus, finding it both relieve the intestinal symptoms and quiet nerve-excitement. In asthenic bronchitis and pneumonia it may be very valuable.

Tympanitic Conditions of the large intestines, occurring under many other circumstances besides typhoid fever, in gout, for instance, are often greatly benefited by turpentine. It may be given in enema (which relieves attendant constipation) or by the mouth.

In cases of Ulceration of the Bowels, when the tongue parts with its fur in flakes, and when the surface appears smooth and varnished, turpentine is to be recommended; also, in atonic constipution, with gaseous distension of the large bowel.

As a Simple Purgative for any one particular occasion, turpentine is best given in enema. Half an ounce, with an equal quantity of castor oil, mixed with a pint of gruel, usually acts promptly and conveniently.

As a Vermifuge, turpentine once obtained much repute; its chief efficacy is in the treatment of tapeworm, and though, as a rule, inferior to filix mas and kousso for this purpose, I have found it occasionally succeed better.

In Hydatid Cysts, especially of the lung, its internal use is said to have been of service (Begbie, Lancet, ii., 1875).

For Gall Stones, 15 to 20 minims, with 10 of ether, is a "solvent" remedy of some value, known under the name of "Durande": he ordered it two to three times daily for 12.5 days - a method not easy to carry out, but the drug has seemed to me of some service in such cases.

Renal and Vesical Diseases.-In certain chronic cases of albuminuria -in some of typical character, with casts in the urine, in others where the passing of albumen continued for many years without pronounced symptoms of ordinary Bright's disense-I have known 1- to 2-minim doses of turpentine, taken night and morning (not fasting), markedly lessen the amount of albumen, and in the latter class of cases much improve the 
general condition. In chronic cystitis, and in the nocturnal enuresis of children, it is also beneficial.

Skin Affections.-Turpentine may be given internally, with much advantage, for chronic scaly skin affections: the dose may be gradually increased from 2 minims, even to as much as 1 drachm, three times daily, but two precautions are necessary: (1) to give plenty of demulcents-barley water, etc.; (2) to take the drug after food, and not within four hours of going to bed.

Neuralgia.-Like other stimulants, turpentine will often markedly benefit the neuralgia of weak anæmic subjects.

It has a special reputation in sciatica, which it will at times relieve rapidly, after failure of other remedies, though it is not easy to indicate the cases in which it is most suitable. Nothnagel, indeed, says that "scientific indications for its use are entirely wanting." I think it acts best when a vermatic taint is particularly distinet. Sir Thomas Watson mentions, as some guide, the "renal irritation" present in some cases. Dr. King Chambers, noting the proximity of the coecum and rectum to the sciatic nerve, inclines to attribute the benefit to a local action on the intestinal membrane, and Dr. Begbie traces it to the free purgation induced. Dr. Jameson, in an interesting essay, points out that it acts best in cases when age and other circumstances would indicate some degeneration of the nerre-tissue (Edin. Med. Journ., March, 1877); in patients of fifty, for instance, or upwards, when the pain has begun first about the lumbar and sacral regions, and creeps downwards, accompanied by some anæsthesia and loss of power. Of eleven such cases, ten were quickly relieved by 2-drachm doses given with $\frac{1}{2}$ ounce of castor oil, which acted freely: the 10-minim doses, recommended by Anstie, failed sometimes when the above succeeded.

It has been long remarked that the remedy generally agrees with old people, whilst in cases of syphilitic or purely rhcumatic pain, and again in young, hysterical, and nervously irritable subjects, it is not nearly so successful. In my own experience three or four doses of 10 to 15 minims, given in capsules, to a lady of the latter type, suffering from severe acute sciatica, induced extreme renal irritation without relief to the sciatic pain; and 
the possibility of such a result has always to be remembered in administering this drug.

In the "nervous" headache following" excessive mental strain in delicate subjects, 20 minims of turpentine are said to relieve, somewhat like strong tea or coffee may do, and Graves advised it in the "hysterical" nervous headaches of young women.

In Chorea, Dr. Hughlings-Jackson gave 5 to 10 minims to children, with advantage. Epilepsy and various other nervedisorders, e.g., tetanus, have also been at different times treated by it.

Chronic Rheumatism.-Even when this has already produced considerable deformity in the joints, turpentine is one of the few remedies which will frequently give relief to the pains, and occasionally arrest the downward progress of the case.

PReParations AND Dose.-Confectio: dose, 60 to 120 gr. Oleum, as a stimulant, anti-spasmodic, or diuretic, dose, 10 to $30 \mathrm{~min}$.; as an anthelmintic purgative, dose, 2 to $4 \mathrm{fl}$. dr. It may be given in capsules, or in emulsion with alkali and mucilage, or yolk of egg: liquorice disguises the flavour. Enema (contains 1 fl. oz. and $15 \mathrm{oz}$. of mucilage of starch). Linimentum (with camphor). Linimentum terebinthince aceticum. Unguentum:

Resina : dose, 10 to $30 \mathrm{gr}$. if used internally. Emplastrum resince. Unguentum resince. This resin is an ingredient in several other plasters, in turpentine ointment, and the epispastic or blistering paper. 


\section{PIX LIQUIDA.}

(TAR.)

An impure turpentine obtained by the destructive distillation of the wood and roots of the Pinus sylvestris-"Scotch fir."

PREPARATION.-Billets of the wood are packed together in stacks, or inside clay furnaces, covered with a layer of earth, and fired from above: the tar falls, and collects in a ditch below.

DESCRIPTION.-A blackish, semi-fluid substance, heavier than water, of characteristic odour, acrid bitter taste, and acid reaction; soluble in alcohol, ether, chloroform, oils, and caustic alkalies; it communicates to water its taste and acidity without being to any extent soluble in it.

ACTIVE INGREDIENTS.-These are numerous and complex, and vary somewhat according to the wood used, the mode of preparation, etc.; they include acetic acid, acetone, methylic alcohol, xylol $\left(\mathrm{C}_{\S} \mathrm{H}_{10}\right)$, creasote, carbolic acid, etc,

On distillation, acetic acid and water come over, together with a yellow, light, volatile oil (oleum pini sylestris-" oil of tar"), which darkens on exposure: the solid black residue is known as "pitch."

Physiological Action.-External.-Applied to the skin, tar is apt to cause redness, with heat and irritation, sometimes blistering; it may be absorbed, and if extensively applied may induce constitutional symptoms.

It has decided antiseptic powers, and is fatal to the lower forms of regetable and animal life (to insects, for example).

Physiological ACTion.- Internal.- In general terms the action of small doses may be called stimulant, of large ones irritant, and even toxic, for death has occurred from the effects of the volatile oil. 
Digestive System.-The continuous internal use of tar is apt to disorder the stomach and impair appetite; large doses, or an extensive outward application, cause oppression at the stomach, vomiting, purging, and pain in the bowels, the discharges having the odour of the drug.

Circulatory System.-Tar water, i.e., tar in small quantities, quidkens the pulse; large doses depress the circulation.

Nervous System.-Toxie effects on this system are shown by headache, a feeling as of intoxication, and later by a sense of extreme exhanstion.

Secretory System.-All the secretions, especially those of the bronchial mucous membrane, the skin, and the lkidneys, are said to be promoted by the administration of tar, and the inhalation of its vapour: by the latter, copious or unhealthy bronchial secretion is lessened. Irritation of the kidneys may be cansed, with secretion of dark-coloured urine, which smells of the drug, and yields a deposit on addition of nitric acid, distinguishable from albumen by its solubility in alcohol. Reclam has noted that the urine passed under the influence of the drug does not decompose so readily as ordinary urine, keeping for five or six days (Record, 1880).

Cutaneous System.-A red rash occasionally follows the administration of tar, and its too copious external application may cause an acneiform eruption; the same occurs in men who are much exposed to its vapour (Hebra); there is evidence that such skin irritation may develop epithelial cancer (Record, 1881).

\section{Therapeutical Action.-Exterull.-Skin Diseases.} - Tar and its preparations, when judiciously used, are of the greatest service in some forms of these diseases, generally in those of chronic character, dry and pruriginous. When acute symptoms of heat, redness, or discharge are present, then tar may aggravate the malady, and cause much discomfort. In chronic psoriasis it is especially suitable, - an application of the ointment, night and morning for a few times, removing the scales, and lessening the local congestion, with some temporary inconvenience as to colour, odour, ete. It is not easily washed off, but it is better if made with glycerine of starch as a basis. 
Brady published a special formula, viz. : "Glycerine (warmed) 6 ounces, powdered starch 2 drachms, stir in, and add tar 6 ounces, and raise quickly to boiling-point; stir while cooling, and strain if necessary." Tar may be combined with potash or soft soap and spirit, but more elegant forms of it, now generally used, are the oil obtained from the Juniperus oxycedrus, "oleum cadini," oil of cade, and "oleum rusci," from the bark of the white birch; equal parts of either of these, with soft soap and rectified spirit, scented with $\frac{1}{2}$ drachm of oil of lavender in each ounce, make a good stimulating paint to be thoroughly applied once or twice daily; a weaker ointment may be prepared with 10 minims of the oil to an ounce of lard: a decolorized solution of tar may be made with spirit and ammonia. "Liquor carbonis detergens" is an alcoholic solution of its active principles. Similar preparations, but more diluted, are suitable for the chronic, scaly stages of eczema, especially if there be much itching, and McCall Anderson has seen decided advantage from the internal administration of the drug in such cases, beginning with '2 minims of the pix liquida in milk, or in pilular form with liquorice, and increasing to 30 or 40 (capsules are better than pills). He notes that the remedy generally suits, but sometimes causes a red rash, feverishness, vomiting, or diarrhœa; these symptoms, however, soon subside, and may be aroided by management. The same remedy has proved serviceable in chronic psoriasis, especially when a catarrhal tendency coexisted (Lancet, i., 1870).

For prurigo, tar was specially advised by Trousseau, and in severe chronic cases of pruritus ani comnected with an eczematous condition, I have lnown zime ointment with $\frac{1}{4}$ part of unguentum picis liquidum more efficient than any other single application. It is commended highly by Dr. Bulliley in his work on eczema. It is useful in scabies, and is sometimes ordered for ringworm, but is a particularly umpleasant application for the hairy scalp; carbolic acid has practically superseded it. For unhealthy, indolent ulceration tar ointment, or its combination with chalk, is good, but the solutions, tar-water or the liquor carbonis, are more cleanly and pleasant; for chronic hæmorrhoids such applications are often valuable. 
THERAPEUTICAL ACTION.

Internal. - Pulmonary Disorders.-It is in chronic catarrhal affections, especially bronchitis and bronchorrhoea, that the value of tar is best seen. It may be given internally in the form of tar water or pill. A noted treatise by Bishop Berkeley refers especially to the virtues of the former, which may be made according to the formula of Dunglison ( 1 ounce of tar digested for a week with 2 pints of water, and strained) or of Squire ("stir a pint of tar with half a gallon of water for fifteen minutes, and decant"). Two ounces may be taken for a close with mill, and the inhalation of the steam of tar-water should be practised, so as to bring the remedy into direct contact with the affected mucous membrane. Upon this it exerts an alterative effect, improving secretion when deficient, whilst it diminishes a discharge which is unhealthy and profuse. It is also valuable in tubercular phthisis, acting as an expectorant, soothing the cough and relieving the oppression; at the same time it helps the digestive system, increasing the appetite; and if colliquative diarrhoea be troublesome it tends to remove it. When acute inflammation, or hectic fever, exists, tar is better avoided.

Constipation-Hæmorrhoids. - What has been stated as to its relieving phthisical diarrhoea does not contradict the fact that the steady use of a few grains of tar, or of "a pitch pill" at bedtime, acts well in regulating the bowels in the subjects of habitual constipation, and I have known the same remedy relieve hæmorrhoids, especially in puerperal cases.

PREPARATIONS AND DOSE.-Pix liquida: dose, 20 to 60 min.; may be given in capsules. Aqua picis liquidre: dose, 1 to $4 \mathrm{fl}$. o\%. Thunentum picis liquide (better made with glycerine of starch). Pilula picis: made either of pitch, or of tar and liquorice powder, equal parts. Olcum pini syluestris (Firwool oil). A "vapor" of this oil has been recently made officinal. Oteum crdini. Oleum meci. Liquor carbonis detergens. 


\section{PIX BURGUNDICA.}

\section{(Burgundy Pitch.)}

DESCRIPTION.-This product is yielded by the Norway spruce, Abies excelsa, a tree found wherever conifers are cultivated. The stature, when full grown, is 150 feet; the leaves are short, and stand singly; the cones are 3 to $\sigma$ inches in length. The pitch itself is the resinous exudation of the trunk, melted and strained; the best comes from Switzerland, and is hard, opaque, reddish-brown, of a peculiar odour and aromatic taste. It contains a volatile oil like turpentine, and a resin.

Physiological Action.-The local action of Burgundy pitch is that of a mild irritant. Certain constitutions are affected by it in a remarkable manner, vesicular and pustular eruptions occurring from its extensive employment.

Therapeutical Action.-Extemal.-Burgundy pitchplaster is usefully applied to the chest in chronic pulmonary affections; also to the loins in cases of lumbago, to the joints in chronic articular affections, to the buttock in sciatica, or to any external part of the body where there is local pain of a rheumatic character, and also where (as in chronic pleurisy) it is desirable to afford firm mechanical support and pressure.

PREPARATION.-Emplestrum picis.-Burgundy pitch is a constituent of emplastrum ferri. It is well to share off the hairs of the skin to which it is applied, and the plaster should be softened by warmth before removal.

Adulteratrons. - The Burgundy pitch of the shops is rarely quite genuine, the substance sold under this name being mostly prepared from concrete American turpentine, or, if derived from the Abies excelsa, simply melted without being strained. It is usually coloured with palm oil. 


\section{TEREBINTHINA CANADENSIS.}

\section{(Canada Balsam-Canada Turpentine.)}

DESCRIPTION.-Canada balsam exudes from the trunk of the Abies balsamea, a North American conifer attaining the height of 40 feet, with short, solitary leaves, silvery underneath, and erect cylindrical cones, 4 to 5 inches in length. As met with in commerce, the balsam, so called, is a pale yellow, translucent oleo-resin, of the consistence of thin honey, very ductile when warmed, and of a peculiar agreeable odour, with a slightly bitter, acrid taste. It occurs also in small transparent masses, having a vitreous fracture.

ACTIVE INGREDIENTS.-These appear to be much the same as those of turpentine. Transparent turpentines, such as this one, are described as "solutions of abietinic anhydride in the volatile oil, $\mathrm{C}_{10} \mathrm{H}_{16}$," and on exposure to a moist atmosphere they gradually become turbid and opaque from crystallization of abietinic acid. It is not a true "balsam," since it does not contain benzoic acid (Maly).

Physiological Action.-This is little known; probably it is similar to that of other turpentines.

THERAPEUTICAL ACTION.-Canada balsam is not muchemployed medicinally, but may be resorted to in mucous discharges from the genito-urinary organs, such as gleet, gonorrhœa, leucorrhoea, and chronic cystorrhœea. It is useful also in certain forms of chronic rheumatism, especially lumbago and seiatica; and in chronic catarrh, whether pituitous or mucous, occurring in old persons of lax fibre and lymphatic temperament.

PREPARATIONS AND DOSE.-Canala balsam is best exhibiturl in the form of a pill, $5 \mathrm{gr}$. being mixed with half its weight of magnesia: 20 to $30 \mathrm{gr}$. may be given. It is much rsed in microscopical work, and enters into the preparation of charta epispastica and collodium flexile. 


\title{
JUNIPERUS COMMUNIS.
}

\author{
(JUNIPER.)
}

DESCRIPTION.-The juniper and its dark purple fruits are well known. The latter contain three hard ovate seeds which have oil-sacs on their surface, and are imbedded in a brownish pulp containing oil-cells.

ACTIVE INGREDIENTS.-Analysis shows the presence of sugar, resin, organic acids, and juniperin, which is a yellow powder, soluble in alcohol, ether, and hot water, but uncrystallizable; slightly soluble in cold water. Oil of juniper, which is the active principle, is prepared by distillation of the umripe berries mixed with salt and water: that distilled in Britain is better than that imported. It is a colourless or yellowish oil, thickening and darkening on exposure; sp. gx. 0.85 to 0.90 ; boiling-point, $311^{\circ} \mathrm{F}$. to $401^{\circ} \mathrm{F}$.; of peculiar odour and warm aromatic taste; soluble in 10 parts of spirit; neutral in reaction; sulphuric acid colours it red.

The oleum juniperi empyreumaticum, or oleum cadinum-oil of cale-is a brown tar-like liquid, obtained from the wood of Juniperus oxycedrus. The oil of the common juniper rood (not berries) has been substituted for it.

Physiological Action. - Extemally, oil of jumper acts on the skin as a stimulant and irritant; internally, as a carminative on the digestive system, and, like other essential oils, on the nervous and the circulatory system, with a special determination to the kidneys: it causes diuresis in medicinal doses, but in excess it excites much irritation of the bladder and urethra.

THERAPEUTICAL ACTION.-The crushed berries are sometimes used as an application to bruises, swellings, and rheumatic painful parts. Olcum calimum is a more elegant form of tarry stimulant, and is employed in combination with potash or zinc 
ointments for psoriasis, chronic eczema, etc. Oil of juniper is now used mainly as a diuretic, and generally in conjunction with other remedies of the same class, - most commonly, perhaps, in the form of "Hollands." It is given in various kinds of dropsy, mostly of atomic character; often in scarlatinal dropsy, though it is not free from risk in such cases. It is better avoided when any symptoms of acute nephritis are present. Half an ounce with an equal quantity of tartrate of potash to 1 pint of boiling water is an effective diuretic, and with the substitution of magnesian sulphate a good aperient medicine is made, which is in popular and domestic use for renal and uterime congestions. I have seen benefit from it in such cases, but its nauseousness is an objection.

PREPARATIONS AND DOSE.-Oleum: dose, 1 to $3 \mathrm{~min}$. Spiritus juniperi ( 1 in 50): dose, 30 to $60 \mathrm{~min}$.; well diluted. Oleum cadinum, used externally ( $\iota$. p. $\tau 42)$.

AdULterations.- Oil of turpentine; oil of juniper wood.

\section{JUNIPERUS SABINA.}

(SATIN.)

DESCRIPTION.-A low, spreading, evergreen shrub, indigenous to the South of Europe: sometimes it becomes erect, and rises to the height of 10 or 12 feet: while young, the much sub-divided branches are completely covered by minute imbriraterl leaves. The fruit ("galbulus") is small, globular, succulent, and deep purple in colour.

ACTIVE INGREDIENTS.-The leaves, the tops (which are officinal), and the berries have a foetid odour, and a bitter, hot, and acrid taste. Distilled with water they yield a considerable quantity of an essential oil, $\mathrm{C}_{10} \mathrm{H}_{16}$, which is the active element of the plant; the fresh tops afford 2 to 3 per cent., and the berries about 10 per cent. This oil is a yellowish fluid, beoming colourless when rectified: it las a penetrating odour of the fresh plant, and a burning taste. Sp. gr. $0 \cdot 89$ to $0 \cdot 94$. It 
dissolves completely in absolute alcohol, and in twice its weight of alcohol, sp. gr. 0.85 ; it yields another oil boiling at $160^{\circ}$, and having the formula $\mathrm{C}_{10} \mathrm{H}_{10} \mathrm{O}$ (Tilden, 1877).

Physiological Action.-External.-Applied extemally the oil causes redness and a sense of burning.

Physiological Action. - Internal. - Moderate doses stimulate the circulation, causing flushing and heat of the head, and some increase of the pulse-rate: the effect on the rigestive system amounts to a sensation of warmth at the stomach, with perhaps some nausea or malaise: the secretion of the kidneys is commonly increased, and the odour of the drug is detected in it, as also in the breath and perspiration.

Toxic closes cause gastro-intestinal inflammation with violent vomiting, purging (which may be sanguineous), severe colic, renal congestion with discharge of bloody urine and partial suppression, and later evidence of profound nervous disturbance, unconsciousness, stertor, and convulsion,- - death follows in collapse: in pregnant women uterine hæmorrhage and abortion occur, but this results from the violent abdominal and pelvic irritation rather than from any specific action of the drug on the uterus. Fodéré, for instance, gave 100 drops daily for three weeks, without inducing any symptoms of miscamiage. Many instances of fatal poisoning in the human subject have been caused by the practice of administering savin (the oil or decoction of the tops) with a view to produce abortion. Christison records two such cases, and Taylor (On Poisons) expresses the opinion that death from savin, given for this purpose, is far more common than is generally supposed. It is somerhat curious that Orfila is silent respecting the operation of savin, except as regards dogs; which seems to show that, at his date, it had attrncted comparatively little attention on the Continent.

The importance of a true estimate of the action of savin and similar drugs appears on criminal trials. $1 \mathrm{Mr}$. Pascoe was sentenced to transportation for prescribing 14 minims of the oil daily, divided into three doses, to a preguant single woman, mainly on the evidence of a practitioner who 
affirmed this to be a dangerous dose that would only be given for purposes of abortion (Med. Times, i., 1852). No evil effects followed whilst taking the drug, but a week or two afterwards miscarriage occurred.

According to Mitscherlich and Hillefeld, about 2 drachms sufficed to kill middle-sized rabbits in a few hours. After death, the bladder, kidneys, and intestines were found highly congested, and the epithelium of the latter exfoliated.

Therapeutical Action.-Extemal.-Savin is chiefly employed (in the form of ointment) to keep blistered surfaces open and suppurating. The concreted discharges require to be removed from time to time, so that the action of the ointment may be continuous. It is less acrid than ointment of cantharides.

The powder of savin is useful as a caustic for ulcers and for the destruction of warts and other excrescences; in combination with verdigris it may be employed for the removal of condylomata and venereal growths.

Therapeutical Action. - Intemal. - The value of savin given internally has been the subject of much dispute.

In Amenorrhœa, Van der Kolk, and some other writer's, declare it to be useless, but there is a large body of evidence to establish its worth in this respect; ${ }^{1}$ and failure may have been due either to adulteration of the oil or insufficiency of the dose. Cullen expressly states that he had often been deterred from giving it in sufficient doses as an entmenagngue on account of its acrid and heating qualities. I consider that savin, in small doses, is a powerful emmenagogue, and that it can be given, in suitable cases, without risk

In Menorrhagia, Uterine Hæmorrhage, ete., savin has also proved useful. I havo given the tincture, with the best effects, in doses of 5 to 10 minims every one to three hours; and Arnn considered it a most powerful remedy for the

1 Pereira, vol. ii., p. 332; Home, Clinical Fxperiments, J. 337; Nir Charles Clarke; Sir C. Locock (who gave it in eombination with iron and aloes). 
atonic conditions of the uterus which give rise to the abovementioned disorders: he gave the powdered leaves.

In Chronic Gout, and in rigidity of the joints from chronic rheumatism, savin was formerly much employed. Dr. Chapman (U.S.) thought highly of it in the latter class of affections (Elements of Therapeutics).

Nothnagel not only denies these effects of savin, but speaks even of its action in atonic amenorrhœea with a scepticism apparently based on theory and untested by practical experience.

PREPARATIONS AND DOSE.-Sabince cacumina (dried): dose, 4 to $10 \mathrm{gr}$. and upwards. Oleum: dose, 1 to $5 \mathrm{~min}$. (suspended). The dose of oil mentioned by Pereira is 2 to 8 min. two or three times daily; by Ashwell a maximum of 20 , with the same frequency. Tinctura : dose, 5 min. to $1 \mathrm{fl}$. dr. Unguentum.

\section{THUJA OCCIDENTALIS. (ARbor Vtræ.)}

\section{(Not Officinal.)}

DESCRIPTION.-This tree grows in America to the height of 50 feet, and is known as "white cedar." In Europe it is met with as an ornamental shrub.

ACTIVE INGREDIENTS. - The volatile oil is yellowish, of sp. gr. 0.925 , readily soluble in alcohol, and is a "mixture of two oxygenated oils" (Stillé).

The plant contains also sugar, resins, tannin, pinipicrin $\left(\mathrm{C}_{22} \mathrm{H}_{18} \mathrm{O}_{11}\right)$, and thujin $\left(\mathrm{C}_{20} \mathrm{H}_{22} \mathrm{O}_{12}\right)$. Pinipicrin has been found also in the pinus sylvestris, and isolated as a yellow bitter powder, soluble in water and alcohol, fusing when hented. Thujin occurs in small yellow crystals of astringent taste, soluble in water and alcohol. 
PHYSIOLOGicAL ACTION.-In this, thuja much resembles savin, and like it, irritates the skin externally, and the mucous membranes internally, but its systemic effects have not been so much studied.

Therapeutical Action.-Thuja is useful for the cure of warts, especially for those with a narrow base and pendulous body. They may occur upon any part of the surface, and often have an hereditary history. Many of them are removed by cleanliness and the external application of nitrate of silver, glacial acetic acid, or the acid solution of nitrate of mercury, or by cutting off the growth, and dressing the part with an astringent lotion. But these remedies are often inferior to the pure tincture of thuja, applied to the part three times daily for about a fortnight; sometimes the warts fall off in two or three days, leaving the base perfectly healed.

Condylomata about the anus or pudenda, whether of a syphilitic character or otherwise, are often rapidly cured by the external application of this tincture.

The fungous granulations of ulcers may be destroyed by it, and I have found it specially useful in ulceration about the corona glandis. Hoppe has recommended the pure tincture as a lotion for destroying offensive smells from the nostrils, as also from the prepuce, axillæ, feet, etc. (Med. Times, i., 1860). Leaming has found it beneficial in malignant disease when applied locally and given internally, and it has special power in arresting hrmorrhage from such growths: it has been given also for pulmonary hromorrhage and in amenorrhoea, and has been compared with ergot in such cases: it does not affect the gravid uterus (N.Y. Journ. Med., vol. xiv.).

PREPaRATION AND Dose.-Tincture (1 in 10): dose, 3 to 5 min., three times daily, in water. Leaming ordered $1 \mathrm{dr}$. of tincture, or fluid extract. 


\section{PAPAYACEAE. \\ CARICA PAPAYA. (Papaw Tree.)}

(Not Officinal.)

DESCRIPTION.-The papaw tree is one of the few exogens which in figure resembles a palm : it is of rapid growth, soon acquiring its full stature, and is both in flower and fruit all the year round. It has an erect cylindrical stem about 20 feet high, at the summit of which are palmately lobed leaves, from 1 to 2 feet broad, on petioles of equal length. The yellowish white tubular flowers are unisexual and diøcious. The fruit resembles a long melon, containing a soft pulp and numerous seeds. The pulp is cooling, and something like an apricot in flavour.

ACtive IngREDIENTS. - The milky juice which is abtained from excoriations in the green fruit and the stem of the tree, coagulates spontaneously, separating into two parts, a clear liquid and a solid white precipitate-papayotin, sometimes called papain. The latter name is better reserved for the precipitate produced from the juice by alcohol: this is a whitish, amorphous, somewhat granular light powder, tasteless, but with a faint smell : it is soluble in water and glycerine, leaving, as a rule, a small residue. The ferment action is closely associated with a proteid (Wurtz), which Martin has shown to be hemi-albumose (B. M. J., ii., 1885).

Physiological Action.-In the last century Hughes had noted the remarkable property of digesting flesh possessed by the juice of this plant, but it is only recently that more aecurate observations have been made concerning it. Rossbach has ascertained that pieces of fresh rabbit-muscle placed in a 5 per 
cent. solution were softened and partially digested in half an hour. The addition of hydrochloric acid lessened the activity of the process, as also did carbolic acid: the latter did not arrest it. It has been calculated to peptonize 200 times its own weight of blood fibrin (Record, 1882).

Wurtz, who isolated papain, was the first accurately to investigate its action: he found it readily peptonize moist fibrin at $40^{\circ} \mathrm{C}$, leucin being formed as an end-product (Comptes Rendus, 1879-80). Dr. S. H. C. Martin, by extending' Wurtz's experiments, found that papain acted in a similar manner to trypsin, but not so energetically (Journ. of Phys., vol. v., No. 4). The false membrane of croup was dissolved by it in two hours (Zeitschrift f. Klin. Med., Bd. vi., 1883).

A great number of bacteria develop in substances acted upon by the drug, and the bodies of animals in which it has been injected decompose more rapidly than usual. Hypodermically it causes local disintegration of tissue, and commonly abscess, but does not seem to act upon living mucous membrane; for instance, on the stomach, when administered, it exerts no bad effect, but rather, it is said, increases appetite.

On the circulation, the only evidence of special action is a cardiac paralysis induced in rabbits by its intravenous injection.

Therapeutical Action.--Skin Diseases, etc.-In hypertrophy of cuticular elements, such as verrucie, general cuticular thickening, or chronic scaly eczema, a paint containing about 50 grains to the ounce of water with half the amount of borax has been found useful (B. M. J., i., 1882). The skin is apt to become tender, and though the part should be washed once daily, not much friction should be employed.

Péan has employed it by direct injection for solution of cancerous nodules in the breast and elsewhere (Record, 1882).

Diphtheria.-Rossbach has tried it extensively for solution of the membrane in diphtheritis, applying a strong paint directly to it every five minutes for some time (loc. cit.). The treatment is also recommended by Herz as the result of trial in ten cases, nine of which were rapidly cured, and one got rid of the membrane, but died of after-paralysis (Schmidt's Jalırb., vol. cei., 1884). 
Kohts and Asch used a 5 per cent. solution in fifty-three cases, the membrane being rapidly dissolved; in some in which tracheotomy was performed, the papain solution was injected into the trachea. The drug does not act as a specific, but simply as a solvent of the membrane, which re-forms as soon as the treatment is stopped (Zeitschrift f. Klin. Med., Bd. iv., 1883).

Berckholtz does not speak so favourably; but the majority of observers, Fraentzel, Ewald, Schaeffer, agree as to its solvent action on diphtheritic membrane. On account of the high price of papain, Ewald suggests pancreatin as a substitute.

In Dyspepsia it has been employed like ordinary pepsin, and on the same principle it has been added to nutrient enemata, but no large amount of experience with it is on record.

Preparation and Dose.-Papain: dose, 1 to 8 gr., best given simply in water.

Martin has recommended the preparation of food peptonized by papain (loc. cit.). 


\section{ZINGIBERACEA.}

\section{THE GINGER FAMILY.}

Av order of about 250 species of herbaceous endogens, natives of the East Indies. The underground portion of the plant consists mainly of a substantial rhizome; from this arise annual erect shoots, often several feet in height, usually simple, and with alternate, elliptical, and feather-veined leaves. The flowers are spicate or racemose, trimerous, very irregular, monandrous, and bracteate: the ovary is inferior and threecelled; the fruit capsular and many-seeded. None of the species are deleterious; many are distinguished either for pungency or aroma.

\section{ZINGIBER OFEICINALE.}

(Givger.)

DESCRIPTION. - The rbizome is fleshy, with short, irregular branches, and covered with an ash-coloured skin: when dried it is the ginger of commerce. The wand-like foliage-stems rise to the height of 2 feet or more; the leaves are linear-laneeolate; the pale yellowish flowers are borne in coniform spikes 2 or 3 inches in length, and elevated upon short and leafless radical stalks. Every part of the plant is glabrous: the taste of the rhizomo is hot and biting, with a littlo anridity. The aromatic odour of the fresh rhizome is brought out by drying. 
ACTIVE INGREDIENTS.-Chiefly a volatile oil (about $\frac{1}{4}$ per cent.), and a resinous matter ; the sp. gr. of the former is 0.893 : there is also much starch.

Physiological Action.-If a piece of the rhizome be chewed, it causes a flow of saliva, and the powder, if passed into the nostrils, excites sneezing: mixed with water, its application to the skin produces intense heat and tingling. On being swallowed, its stimulating effects are perceived in the alimentary canal, and in the organs of respiration.

Therapeutical Action. - Ginger is useful locally applied in cases of relaxed uvula, and in slight paralytic affections of the tongue, and in toothache; it may be resorted to for the relief of headache, a paste formed of it, and spread upon paper, being applied to the forehead.

Ginger is advantageously given in colic and tympanites. Taken in moderation, it is a valuable stomachic, and, if the body is relaxed and enfeebled, especially in old age, or if gout be present, it promotes digestion. In India, Europeans of delicate constitution are accustomed to use an infusion of ginger instead of tea. It also constitutes an excellent adjuvant, especially to quinine and tonic medicines, to which it communicates cordial and carminative qualities, and in combination with drastic purgatives it is useful to check or prevent nausea and griping.

Preparations AND Dose.-Pulvis: dose, 10 to $20 \mathrm{gr}$. and upwards. Sympup: dose, to $1 \mathrm{dr}$. Tinctura: dose, 15 to $60 \mathrm{~min}$. Tinctura aingiberis fortior: dose, 5 to $20 \mathrm{~min}$.

\section{ELETTARIA CARDAMOMUM.}

(CARDAMOMS.)

DESCRIPTION.-A perennial, growing chiefly in the mountainous parts of Malabar, with stems which rise to the height of 6 or 8 feot, and bear numerous lanceolate leaves, 15 or 20 inches 
long. The flowering scapes are about a third of the stature; the blossoms, produced in thin racemes, are insignificant and greenish white; the numerous seeds contained in the three-celled and somewhat ovoid capsules are the cardamoms of commerce. Other species of the genus probably yield a portion of the drug as received in Europe. The seeds-properly, fruits-are fragrant; the taste is warm, pungent, and highly aromatic.

ACTIVE INGREDIENTS.-These are extracted by water, more easily by alcohol. The most important is a volatile oil of sp. gr. 0.945 , which is present in the proportion of about 4.5 per cent., and rises with the water on distillation. It is colourless, possessed of an agreeable penetrating odour, and strong, burning, camphoraceous, slightly bitter taste, which, however, as well as the scent, is lost by exposure to the atmosphere. Cardamom seeds contain also a fixed oil, some colouring matter, salts, and other (unimportant) ingredients.

Physiological Action.-Cardamoms are gratefully fragrant and pungent, carminative and stomachic, and less heating and stimulating than many other substances of their class.

Therapeutical Action.-Combined with bitters, cardamoms are serviceable in dyspepsia, and, with purgatives, are particularly useful for the prevention of flatulence and griping. The tincture is advantageously added to stomachic infusions; and in cases of flatulent colic, and in gouty and spasmodic affections of the stomach, to mixtures containing other, opium, ete. For rendering mineral waters and saline solutions agreeable to the stomach, there is probably no moro suitable drug.

Preparations and Dose.-Pulvis semimum: dose, 5 to 20 gr. Tinctura cardemomi composita: dose, $\frac{1}{2}$ to $2 \mathrm{fl}$. dr. The drug is introduced into the compound decoction of aloes, aromatic iron mixture, compound semma mixture, and compound tincture of chloroform. 


\section{CURCUMA LONGA.}

\section{(Turmeric.)}

Turmeric is the dried rhizome of the Curcuma longa, a native of India, where, as in China, it is extensively cultivated. It was known to the ancients, and formerly had considerable repute in medicine, being esteemed a powerful remedy in cases of dropsy and intermittent fever. The native Indian doctors assert that the powder is an excellent material for cleansing foul ulcers. It is now employed in England only as a colouring matter, and in the preparation of tests for alkalies, which change its colour from yellow to brown.

\section{MARANTA ARUNDINACEA.}

\section{(ARRowROот.)}

Nearly allied to the Zingiberaceæ, is the beautiful order Marantaceæ, distinguished for the large amount of nutritive fecula contained in the rhizomes, and by the absence of the pungency and aroma which belong to the first named. The most esteemed of these feculas is furnished by the rhizomes of the Maranta arundinacea, a native of the West Iudies, and which, under the name of arrowroot, constitutes, like sago, ans excellent nutritive and demulcent article of diet for invalids and children. 


\section{IRIDACEAE.}

\section{THE IRIS FAMILY.}

Ar order of about 500 species of herbaceous, perennial, and petaloid endogens, noted for little besides the beauty of the flowers, and of slight importance in medicine.

Pharmacentical Species.

Crocus sativus . .

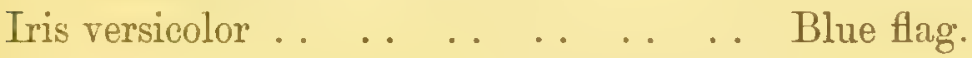

\section{CROCUS SATIVUS.}

\section{(SAFFRON.)}

DESCRIPTION.-The saffron-crocus closely resembles the common garden crocus, but the purple flowers are not produced till autumn, and the stigmata are remarkably developed : picked out carefully and dried, these and the styles form the saffron of commerce. In this condition they have a deep orange colour, a powerful, penetrating, and diffusive odour, a warm, pungent, and rather bitter taste.

Saffron readily imparts its crocus-yellow colour to the saliva, to water, and to substances (such as ginger) triturated with it: the colouring matter is due to a glucoside called polychroit; it changes to blue on the addition of sulphuric acid.

Physiological Action, etc.-Taken internally, as a medicine, saffron is a slightly exhilarating stimulant; the properties which give it this power depend upon a volatile oil, but, unless employed in large doses, the service it may render is little, whilst taken in excess, it is said to have proved fatal. It is sometimes given as an emmenagogue, and in small doses hils 
some reputation as a carminative, antispasmodic, and antihysteric remedy.

Preparations AND Dose.-Pulvis croci: dose, $20 \mathrm{gr}$. and upwards. Tincture: dose, $\frac{1}{2}$ to $2 \mathrm{dr}$.

ADULTERATIONS.-The dried florets of the Carthamus tinctorius, calendula, etc.: also chalk and other powders.

\section{IRIS VERSICOLOR. (Blee FlaG.)}

\section{(Not Officinal.)}

DESCRIPTION.-This plant grows in moist meadow-lands over North America, and has blue flowers with a yellow base, and long sword-shaped leaves. The rhizome, which is the part used in medicine, is in joints, which are 2 to 4 inches long, with projecting leaf-scars, and below, thin rootlets: it has little odour, but an acrid taste.

ACTIVE INGREDIENTS.-These probably reside in a resin, or oleo-resin, named iridin or irisin, not yet fully analysed. It is dark brown in colour, and of bitter nauseous taste. Starch, tannin, etc., are also contained in the rhizome. (Vogel, Pharm. Journ., ii., 1881).

Physiological Action.-Digestive System.-According to the observations of Rutherford on animals, iridin is a powerful hepatic, though but a moderate intestinal, stimulant; it causes purgation (B. M. J., i., 1879). Large doses prove emetic and cathartic, and induce much prostration (Stillé).

Therapeutical Action.-Hepatic Disorder.-Dr. Rutherford, from his personal experience, describes iridin in 4-grain doses as " a certain remedy for biliousuess" ; it produces no disagreeable sensations, and on awaking", the "yellow tongue is clean, and the malaise and headache gone" (loc. cit.). "As iridin, though a powerful hepatic, is not an intestinal stimulant, it is well to give in the morning an ordinary mild saline 
aperient, such as Pullna water. But iridin, though an agreeable remedy at the time, leaves a somewhat depressing effect; and it probably should not be taken oftener than once a week or so." The same treatment has proved very effective in the vomiting of pregnancy (Bury Hart, Med. Times, i., 1884).

Mr. F. Barner, quoting upwards of 30 cases, observed similar favourable results with usually two to four stools yellow in colour. Dr. P. A. Young quotes three cases in which iridin (1 grain, with pilula rhei composita 4 grains) seemed effective in preventing a return of the symptoms of gallstone (B. M. J., ii., 1881). Another writer describes the drug as a mild aperient cholagogue, which induces bilious stools without irritation of the rectum or after constipation (Lancet, ii., 1882).

In my own experience I have found it useful in such conditions as are above deseribed, and when blue pill was either not advisable or did not act well: I have commonly followed it with a saline purge.

It was highly valued as a purgative by the aborigines of America.

PREPARATION AND Dose.-Iridin: dose, 2 to $4 \mathrm{gr}$, alone or with henbane; 1 or 2 gr. combined with rhubarb pill. 


\section{SMILACEA.}

\section{THE SARSAPARILLA FAMILY.}

A small order of herbaceous under-shrubby plants, intermediate in structure between exogens and endogens, and occurring chiefly in the warmer parts of Asia and America. The stems are slender and wiry; the leaves alternate, netveined; and usually ovate; the flowers small, greenish, trimerous, and borne in clusters; the superior three-celled ovary becomes a roundish berry.

\section{SMILAX OFFICINALIS.}

(Sarsaparilla.)

DESCRIPTION.-The true medicinal sarsaparilla is a native of New Granada. The stems are twining, glabrous, quadrangular, and prickly; the leaves are ovate, nearly a foot in length, and the petioles have each a pair of tendrils.

The sarsaparilla of commeree consists partly of a rhizome, to which are attached relics of the stems, partly of the proper roots, which are several feet in length, thin, shrivelled, of the average thickness of a goose-quill, wrinkled, and furrowed longitudinally: according to their bulk and substance these roots are distinguished by the dealers as "lean" or "gouty": the latter usually abound in starch, and are further said to be "mealy." Frequently the roots are more or less clothed with fibrils, and are then called "bearded."

In the South of Europe, preference is given to the thickest 
and most mealy roots, from whatever country obtainea : these are less acrid to the taste than the non-mealy. In England the latter are preferred, since the acrid and nauseous flavour is a criterion of the energy of the drug. Colour can be little relied upon in judging of sarsaparilla, but when the tint is deep orange-red it is usually considered the best; the essential criterion of superior quality is the taste.

ACTIVE INGREDIENTS.-Sarsaparilla contains, besides a varying amount of starch, a volatile oil, and a white crystalline principle, smilacin, (parillin or sassaparin), of which the chemical formula is not yet settled. It is not an alkaloid, as dilute mineral acids split it up into parigenin and sugar (F. \& H.). It is nearly insoluble in cold water; boiling water dissolves it, and the solution, when shaken, lathers like soap. The reaction is neutral; the taste bitter and acrid.

Physiological Action.--I am not aware of any experiments with the volatile oil; and the results of researches upon smilacin are conflicting, but, on the whole, negative. Boecker (Journ. f. Pharm. u. Tox., ii., 1), whose authörity as an experimenter is very high, concludes, from researches which he made with the assistance of Groos, that smilacin is at least entirely devoid of diuretic and diaphoretic power, and that it does not cure syphilitic maladies. On the other hand, the experiments of Pallotta seem to show that, in some persons at any rate, large doses (7 or 8 grains) of smilacin produce gastric uneasiness, slowing of the pulse, vomiting, and perspiration, with faintness. Rabuteau thinks these results probably due to a bitter substance accompanying the smilacin, for Schroff found this bitter cause vomiting and salivation, whereas Heinrich and Devorzak, taking from 3 grains up to 60 grains of pure smilacin, experienced very slight nausea or ptyalism, or action on the pulse: none upon the secretions of the skin or kidneys. The drug passed off by the latter organ, as shown by the urine frothing on agitation, and reddening with strong sulphuric acid. Sarsaparilla itself, in small or moderate doses, does not disorder the stomach, but often improves appetite and digestion, and 
consequently nutrition. Gubler points out that such results often follow from small doses of medicines that in larger quantities are emetic and nausecus like sarsaparilla: to these latter qualities he attributes any diuretic or diaphoretic effect. As to these effects there are contradictory opinions, many believing that only large quantities of a warm decoction cause any sweating or diuresis, and that these results are in reality due to the warmth of the fluid, and to the quantity of water: I am convinced, however, that diaphoresis is one of the most common and genuine results of sarsaparilla.

Therapeutical ACtion.-Syphilis.-Sarsaparilla was introduced into medicine as early as 1560 , when it was employed in Venice as a cure for syphilis. It long enjoyed high repute, but gradually became neglected, till we find Cullen declaring: that he cannot give it a place in the materia medica, never having found it useful in any disease; at the present day there are many who agree with him. Two or three explanations nay be given of this change of view, e.g., the difficulty of explaining its action, the recent proved possibility of removing certain forms of venereal disease without resorting either to mercury or to sarsaparilla, and the frequent adulteration or substitution of a spurious article, before it comes into the hands of the practitioner. The general properties of sarsaparilla are alterative and tonic. Should there be eruptions, ulcerations, or pains of a rheumatic character in any part of the body, these are. often mitigated, and in some cases entirely removed. The best effects are seen in those depraved conditions of the system which are popularly attributed to the presence of some morbid poison, or to a deranged condition of the fluids, whence its familiar repute as a "purifier of the blood." It is of special service in secondary syphilis, either alone or in combination with other remedies, and notably when the disease resists the action, or is aggrarated by the use of, mercury: in such cases it still holds its ground as an important remedy. With respect to this point, it will be interesting to cite first the older British authors, and then to quote one of the latest summaries of the action of the drug by a competent German authority.

Sir William Fordyce recommended sarsaparilla more parti- 
cularly as an auxiliary to mercury, and as well adapted to purify the system after a long course of mercurial treatment. Pearson supports this view, remarking that the contagious matter of syphilis and the mercury together may, in certain habits of body, co-operate to produce a new set of symptoms which, properly speaking, are not venereal. These secondary symptoms (which are sometimes more to be dreaded than the simple and natural effects of the venereal virus) cannot be cured by mercury. Some of the most formidable of them may, however, be removed by sarsaparilla,-the virus still partially remaining; and when by the renewed use of mercury it has been completely subdued, sarsaparilla frees the patient from the sequelce of the mercurial course.

Dr. Good found sarsaparilla succeed chiefly in chronic cases, when the constitution has been broken down, whether from a long persistence of the disease, or from protracted and inefficient mercurial treatment. In conjunction with a milk diet and country air, he considered it of essential importance. The majority of medical men probably coincide with the above opinions, and Dr. Clifford Allbutt (Practitioner, vol. iv.) has given strong testimony of the good effects of the drug, as observed at the Leeds Infirmary, when administered in large quantitiesnot less than a pint of the compound decoction in twenty-four hours. It especially exerted its restorative effects in old and broken-down cases of constitutional syphilis. These results are confirmed by Dr. Carter, who has published two cases of old syphilis with nodes and gummata, which iodide of potash failed to benefit; one pint daily of the compound decoction of sarsaparilla relieved all the symptoms, leading to rapid convalescence (Practitioner, vol. xxiv.).

Modern German opinion is summed up by Nothnagel (Arzneimittellehre). After mentioning that sarsaparilla is always given in conjunction with other vegetable substances which are supposed to act in an analogous and auxiliary manner, he says:- "That this method of treatment often produces good results is positively certain; the particular cases will be presently indicaterl. In what way sarsaparilla and similar remedies arrest syphilis, has not been explained. The old view, that sarsaparilla exerts a specific operation against the syphilis-poison, appears to 
be much more incorrect even than the corresponding phrase as applied to mercury; there is no proof of such specificity. It is now generally assumed that the vegetable remedies (sarsaparilla, guaiacum, etc.) produce a cure by increasing those natural evacuations (diuresis, diaphoresis, purgation) which hasten the metamorphosis of tissues, and thereby the natural elimination of the morbid matter which is the origin of the syphilitic manifestations. This view has much in its favour. It is supported, for instance, by the fact that in many cases a patient suffering from syphilis rapidly gets well by the use of simple warm baths, and the drinking of a warm tea which promotes sweating and urination. These latter cases also favour the opinion so frequently put forward, that sarsaparilla per se is superfluous in the cure, and that the menstruum is the only active agent. This opinion cannot be directly disproved, since it is not possible distinctly to prove the great activity of sarsaparilla.

"Experience teaches us the following facts respecting the use of the vegetable remedies for syphilis:-The "wood-drinks, (Holz-tränke) neither can nor ought to be exclusively employed against syphilis, any more than mercury should be so used. History shows that physicians have repeatedly abandoned the exclusive use of each of these. We have already spoken of the adrantages and applicability of the mercurial treatment, but it is also known that syphilis, under favourable circumstances, may disappear spontaneously. This natural cessation may be assisted by a methodic use of the regetable cure; this mode of procedure is also indicated in the simple, ordinary second phenomena, either in robust, or more especially in scrofulous, tuberculous, or scorbutic individuals: in the former, mercury is usually superfluous; in the latter it is, as a rule, hurtful. Sarsaparilla is also appropriate in the inveterate syphilis of persons who have already undergone various mercurial courses without success : here the vegetable cure is often strikingly effective; also in obstinate and serere secondaries and tertiaries (in the last commonly best in conjunotion with iodine). Sarsaparilla is superfluous in primary indurated chancre, for it hardly at all hinders the occurrence of secondary symptoms; it is also almost always useless in bone 
affections; and, finally, it is inapplicable, from its slow operation, in cases where rapidity of effect is essential (iritis, brain symptoms).

"We cannot enter into detailed discussion of the muchdisputed question of the advantages and drawbacks of the non-mercurial treatment. One point, however, may be brought forward: it does seem certain that the average time required for the vegetable, is longer than that required for the mercurial cure ; it is not true that relapses are rarer after the former than after the latter; on the other hand, it seems certain that they appear earlier and oftener after sarsaparilla, but in a milder form, though indeed cases have been seen in which a thorough vegetable cure has been followed by no relapses whatever. The vegetable cure really does appear to have the advantage of not being followed by the fearful tertiary symptoms to any extent so frequently as happens with the early and forced treatment by mercury; but it must be admitted that in some cases, even of the vegetable cure, tertiary phenomena have been observed, and in others there have been repeated recurrences of secondary symptoms."

In Chronic Rheumatism, sarsaparilla is well combined with sudorifics and anodynes, such as opium and hyoscyamus, especially when there is reason to suspect a venereal taint.

Cutaneous Disorders, dependent on scrofula and conditions approaching elephantiasis, are likewise beneficially treated with sarsaparilla, which acts in these cases as a tonic and alterative : its diaphoretic influence must be assisted by diluents and warm clothing.

In Chronic Abscesses, attended by profuse discharge; in obstinate ulcer; in diseases of the bones; also in Chronic Pulmonary Affections, where there is great wasting; and in many other complaints which indicate a vitiated state of the system, sarsaparilla is useful.

Again, in the irritable condition of the system which often ensues upon severe operations, or from long-continued suppuration, it may be employed with much success, according to the experience of Sir Astley Cooper at Guy's Hospital.

$\mathrm{Mr}$. Lawrence often remarked that physicians liad no confirlence in sarsaparilla, but that surgeons had a great deal; the 
fact being that physicians were less frequently called upon than surgeons to deal with the class of cases in which sarsaparilla is specially beneficial.

PREPARATIONS AND Dose.-The most frequently used preparation of sarsaparilla is the decoctum sarsce compositum, which contains also sassafras, mezereon, guaiacum wood, and liquorice. The simple decoction is not much resorted to: the dose of either should be not less than a pint daily. The extractum liquiclum is the best simple preparation of sarsaparilla; dose about $1 \mathrm{oz}$. daily, in divided portions. Whether or not the solid extract is, as stated, twice as strong as this, is open to doubt. Zittmann's decoction, still used in Germany, contains this drug, with alum, kino, antimony, calomel, senna, and aromatics.

ADULTERATIONS. - Inferior kinds of sarsaparilla are substituted for the true or so-called "Jamaica"; they may be known by the greater amount of mealy matter which they contain, and their less acrid taste. The genuine drug spoils by keeping. 


\section{LILIACEA.}

\section{THE LILY FAMILY.}

Ax order of endogens consisting of about 2,000 species, mostly herbaceous. It is generally diffused in temperate countries, and is wholly terrestrial. The root is frequently bulbous; the flower-stems annual, simple, and scapiform; the leaves almost always curvi-nerved, alternate, and entire; the inflorescence variable; the flowers trimerous and hexandrous, and the superior ovary usually ripens into a three-celled many-seeded capsule.

\section{Pharmacentical Species.}

Urginea scilla . . . . . . . . Squill.

Aloë vulgaris _. $\quad \ldots \quad \ldots \quad \ldots \quad$ Barbadoes aloes.

Aloë socotrina . . . . . . . . . Socotrine aloes.

Convallaria majalis .. . . . Lily of the valley.

\section{URGINEA SCILLA.}

(Squilt.)

DESCRIPTION.-A native of the shores of the Mediterranean. The bulb is very large, weighing sometimes 3 to 4 pounds, and consists of white or reddish fleshy scales; its leaves are broadly lanceolate, 12 or 18 inches long, bright green, channelled and recurved; the flowers, borne on a scape about 2 feet in height, and developed long before the leaves come out, form a large, ovoid, and erect raceme upon the summit; the white of yellowish-green perianth, when fully expanded, is $\frac{3}{4}$ inch across.

When prepared for employment in medicine, the bulb of the 
squill is cut into slices. These, clammy while recent, become when dried, brittle and slightly translucent, and are easily pulverized; but if exposed to the air, they recover moisture, and become flexible. The scent is feeble; the taste disagreeable, mucilaginous, strongly bitter, and somewhat acrid.

Squill yields its active principles to water, acetic acid, and alcohol.

ACTIVE INGREDIENTS.-These are not yet thoroughly understood. The chief part of squill consists of mucilaginous and saccharine substances; Schmiedeberg isolated a peculiar mucilage, sinistrin, homologous with dextrose, $\mathrm{C}_{6} \mathrm{H}_{10} \mathrm{O}_{5}$, but differing from it in being lævogyrate. The bitter principle is called scillitin or skulein, but it has not yet been isolated; it is said to be crystalline, and soluble in hot water. A resinous acrid body soluble in alcohol also probably exists. Besides these, Merck has isolated bodies which he calls scillipicrin, scillitoxin, and scillin. Further, the name scillain has been given to a body, the properties of which are not definitely known (F.\& H.). Under the microscope, crystals of oxalate and carbonate of lime are seen in powdered squill.

Physiological Action.-Locally applied in man, on the denuded skin the fresh juice or powder causes irritation and, it is said, diuresis.

Taken internally, the principal action of squill is exerted upon the lining membrane of the excretory organs, particularly the bronchial, the gastro-intestinal, and the urinary-especially on the last. As a rule it produces, at first, some strangury, aud, as a secondary effect, a copious secretion of water; should it fail to act upon the kidneys, it often causes perspiration.

It is still doubtful whether scillitin is the diuretic principle, and whether (as Schroff thought) it is narcotic in large doses, but it seems evident that the resin induces the phenomena of irritant poisoning, as shown by inflammation of the alimentary canal, etc.

Medicinal doses often cause some relaxation of the bowels; full doses excite nausea and vomiting, and not infrequently purging; excessive doses are poisonous. 
In the lower animals, toxic doses of squill cause pain, vomiting, purging, and loss of power; later, convulsions.

The pulse is rendered slower, and finally the heart is arrested in diastole, before respiration is stopped: the blood-pressure is increased, and with this action on the circulation, diuresis is more or less connected.

Jarmersted, using an extract he called "scillaïn," found that the circulation in dogs and cats was affected in two stages: (1) arterial pressure was raised, and the pulse rendered less frequent; (2) arterial pressure was lowered and the pulse quickened (Record, 1879). Drouot described a stage before these, in which moderate doses of squill caused acceleration of the pulse, and fall of blood-pressure, which afterwards rose, with slowing of the pulse (ibid.)

Husemann and Moeller (B. M. J., ii., 1879), and Merek (Apothek. Zeitung, No. 26, 1879), found that scillipicrin slows the heart and finally arrests it in clicstole; scillitoxin acts more powerfully, and slows, with final arrest in systole, thus resembling Schmiedeberg's digitoxin; scillin acts more feebly, and produces malaise and vomiting, but no direct cardiac effect. The injurious effects of squill (diarrhœa, etc.) are ascribed to scillin (Deutsche Med. Woch., 1876).

Jarmersted and Drouot ascribe the diuretic effect of the drug to the action on the heart; Husemann further stating that he has noticed no kidney lesion in his experiments.

SYNERGISTS.-As regards its diuretic power, squill is allied to digitalis; but in its primary action it is more stimulating to the kidneys, while in general tonic and sedative effect it is less energetic.

Therapeutical Action.-In Dropsy, squill is a valuable medicine, administered either in the recent or the dry state; a small quantity ( 1 to 3 grains) being used at first, morning and evening; and gradually increased until diuretic effects are produced. By some practitioners an amount sufficient to induce nausea has been recommended; but this is hurtful as well as distressing to the patient, and may compel discontinuance of the medicine, since, if once the stomach rebels, 
it is seldom that squill can be again tolerated. The diuretio effects of the drug are sometimes materially increased by combination with mercury, and this is particularly adapted to cases of hepatic dropsy. When the mercurial preparations induce purging, the diuretic action of the squill will be suspendeda result to be obviated either by substituting friction with mercurial ointment, or by counteracting medicines. The same combination is recommended by many in serous effusions into the pleura and pericardium from inflammation (Wood, Chapman); whilst in eardiac dropsy the addition of digitalis is often valuable.

In cases of increased secretion of pulmonary mucus, squill is employed beneficially as an expectorant, and is supposed to operate by promoting absorption, diminishing the quantity of fluid effused, and thus facilitating the expectoration of the remainder. Sir Wm. Jenner insists that squill should never be given in acute bronchitis, for it is likely to act as an irritant and aggravate the malady.

In the Chronic Bronchitis of debilitated patients, attended by profuse loose expectoration of mucous or muco-purulent character, squill is prescribed with great success: it is often combined with ipecacuanha, ammonia or ammoniacum.

In Whooping-cough and Spasmodic Croup, particularly when attended by troublesome sickness, we are still called upon to employ it, endeavouring to secure full exercise of its tonic, and avoid its irritant power, although the emetic effects of the drug are sometimes distressing. In coughs with tickling in the throat, either syrup or vinegar of squill is the best preparation.

Preparations and Dose.-Puliris: dose, 1 to $2 \mathrm{gr}$. Acetum: dose, 15 to $40 \mathrm{~min}$. Oxymel: dose, $\frac{1}{2}$ to 1 dr. Pilula. scillse composita (with ammoniacum) : dose, 5 to $10 \mathrm{gr}$. Symripus: dose, $\frac{1}{2}$ to $1 \mathrm{dr}$. Tinctura: dose, 10 to $20 \mathrm{~min}$.

Contained in the pilula ipecrouanhre cum scillâ. 


\section{ALOË VULGARIS AND ALOت̈ SOCOTRINA.}

(Aloes.)

DESCRIPTION.-The Aloë vulgaris, though now extensively cultivated elsewhere, belonged originally to Barbary and the East Indies. The stem is short, cylindrical, woody, and simple; the leaves are lanceolate, fleshy, flat above, convex below, spinous along the margins, and glaucous. The flowers, borne in a long and erect raceme upon the summit of a slightly branched seape, are tubular, yellow, and pendulous, the stamens projecting beyond the orifice. This species yields the black or dark-brown resin known in commerce by the name of "Barbadoes aloes," which, when pulverized, is of a dirty-yellow colour, always presenting a dull appearance; even thin laminæ are opaque. It is almost wholly soluble in proof spirit. The odour is disagreeable, and is intensified by breathing upon the lump, which in fracture is imperfectly conchoidal.

The Aloë Socotrina, indigenous chiefly to the Cape of Good Hope, is also widely cultivated. Like the $\mathbf{A}$. vulgaris, it is a stout herbaceous perennial, the great mass of the plant consisting of a tuft of large ensiform leaves, from the centre of which arises a scape bearing a raceme of pendulous, tubular, scarlet blossoms. The resin is less opaque and less tough than that of the $A$. vulgaris, and the surface is more shining. It is collected principally in Socotra, and received rî̀ Bombay. Socotrine aloes has a resinous or vitreous fracture; thin layers are sometimes translucent; the odour, instead of being repulsive, is aromatic; when powdered, the colour is bright yellow; and in proof spirit the solution is complete. No difference is perceptible in the bitterness of the two varieties.

ACtive INGREDIENTS. - The most important constituent of either kind of aloes is aloïn, a neutral bitter substance, which crystallizes in needles, is insoluble in cold alcohol, and only sparingly soluble in cold water: in either fluid, however, 
when warmed, it is readily soluble; and if heated to $212^{\circ}$, it rapidly oxidizes and decomposes. Aloïn is likewise soluble in alkaline fluids, forming with them a yellow solution which gradually darkens in colour. More recent researches describe some difference in the aloin of the respective varieties, and name them barbaloïn, socaloin, and nataloïn. The first, $\mathrm{C}_{34} \mathrm{H}_{36} \mathrm{O}_{14}+\mathrm{H}_{2} \mathrm{O}$, occurs in small, yellow, prismatic needles; the second $\mathrm{C}_{34} \mathrm{H}_{38} \mathrm{O}_{15}+{ }_{5} \mathrm{H}_{2} \mathrm{O}$, has the same form; and the third, $\mathrm{C}_{25} \mathrm{H}_{23} \mathrm{O}_{11}$, crystallizes from methylic alcohol in thin, paleyellow, rectangular scales. There is some variation in the formulæ given by different observers.

Aloes also contains a resinoid substance, which differs from all ordinary resins in being soluble in boiling water, and which is formed probably by atmospheric action upon the aloïn. Aloetic acid likewise occurs; it strikes, with the persalts of iron, an olive-brown. The action of nitric acid upon aloes gives rise to the acids named polychromic, chrysammic, picric, and chrysolepic, all of which are crystalline substances, forming solutions of different colours-yellow, red, and purple.

ABSORPTION AND ELIMINATION.-By whatever means introduced into the system, aloes exerts a laxative action: whether swallowed, or injected by the rectum or subcutaneously, or rubbed into the skin, the operation is the same. Nursing mothers who take aloes communicate the laxative effect to their infants.

Physiological Action.-Cullen believed that the specific action of aloes was exercised solely upon the colon and rectum, the drug being simply an evacuant of the frces. Wedekind, on the other hand, held the primary effect to be an increase of the secretion of bile, the purgative or laxative operation being secondary. I lean to the same opinion; and further believe that it is a secondary action which excites the muscular coat of the colon and rectum, leading to expulsion of the freces, rather than the increased secretion thrown out by the mucous surfaces of the large intestines. Aloes, no doubt, does cause such increase. but its good effects are probably owing not to 
this, but to the prior increase in the flow of the bile, ${ }^{1}$ and the excitation of the muscular coat.

When resorted to as an habitual laxative-i.e., when taken continuously-aloes is apt to cause dryness of the throat, an unpleasant warmth throughout the abdomen, uneasiness in the hepatic region, with tightness and throbbing in the right hypochondrium. The pelvic viscera are brought into a more or less engorged condition, and in the portal system a tendency to congestion is developed. These symptoms are accompanied by heat, irritation, and tenesmus in the rectum, often with subsequent hæmorrhoids; the bladder becomes irritated; the urine hot and scanty; the pulse, as a rule, is slightly quickened. In women the menstrual secretion is, under ordinary circumstances, greatly augmented; and when absent, or nearly so, the natural How is restored. Aloes also has the property, when taken in small and repeated doses, of increasing sexual desire (Stillé.)

Administered as a medicine, a dose of 1 to 5 grains of aloes acts upon the bowels in from eight to twelve hours, without nausea or general disturbance, although liable to cause some irritation, griping pains and a sense of warmth throughout the abdomen. The stools are copious, but not too abundant; usually only one or two, fæculent, of dark brownish-yellow colour, and characteristic odour. The number of motions depends more upon the condition of the bowels-i.e., to what degree they are loaded-than upon the strength of the pill; for a single grain of aloes often acts as powerfully as 5 grains, and the latter dose, often not so much as the former. Aloin is two or three times stronger, and also quicker in action. The babitual use of these drugs does not sensibly lessen their activity.

Therapeutical Action.-Constipation.-Aloes, in small doses, operates as a warm and stimulating purgative, particularly adapted to the melancholic temperament. It also

I It is right to mention that several recent observers, of good repute, deny this action on the liver; and Nothnagel says thero are "no convincing: proofs of it." I nevertheless hold to the above opinion, from my own observation of the character of the stools. Experiments on dogs show that 60) grains will augment the quantity of bile secreted in a given time, though it becomes more watery in character. 
exerts a tonic power, and hence has a beneficial influence in chronic dyspeptic affections of the stomach and bowels, with loss of appetite, flatulence, constipation, etc.; the operation being slow, but generally effectual. Chronic cases may be effectively dealt with by a particular plan introduced by Dr. J.K. Spender, of Bath, viz., by giving a pill containing 1 grain of watery extract of aloes and 2 grains of sulphate of iron; at first three times, then twice, and then once daily: it may take some days, or even two or three weeks, to produce a decided effect, but the desired result is usually attained in the end. Acting more particularly upon the colon and the rectum, aloes is efficacious also in expelling ascarides; but for the same reason it is said to occasionally produce hæmorrhoids: this idea has, however, been rejected by many good observers, who maintain that the aloes never caused the piles, which existed already; on the contrary, it often cures piles by removing constipation.

Aloes is well adapted for use in jaundice, especially when there is hypochondriasis.

In Dysenteric Diarrhœa, attended by tenesmus, and becoming chronic, small doses of the drug have an excellent effect; they probably do good by substituting regular and rhythmical peristaltic action for the spasmodic condition which produces tenesmus and prevents the bowel being effectually evacuated.

In Suppression of the Menses, aloes has had a great reputation, but has often been indiscriminately employed in a routine way, not justified by what we know of the physiological action. Given indiscriminately, aloes (as Graves pointed out) is no more likely to restore defective menstruation than any other purgative; indeed, it is likely to do mischief, inasmuch as it tends more than other aperients to engorge the pelvic viscera, which is undesirable except at the regular periods. In nearly all cases of amenorrhoea there are abortive efforts at the performance of the function, which may be traced in the periodical recurrence of pains in the loins, thighs, and hypogastric regions, flushing, etc.: it is at these times, only, that we should administer such substances as cause a direct flow of blood to the uterus; at any other times the congesting influence will only do harm, because the ovarian coudition attending 
menstruation does not exist. Pregnant women should be careful in using aloes as an aperient, though it is doubtless true that aloes has sometimes cured piles in pregnancy, by relieving constipation.

The medicinal effects of the two kinds of aloes, the Barbadoes and the Socotrine, differ but little: the former is said to be more energetic, for which reason it is used by veterinary surgeons, who find the action to take place upon horses in from twelve to twenty-four hours.

Preparations and Dose.-Pulvis: dose, 2 to $6 \mathrm{gr}$. Extractum: dose, 2 to $6 \mathrm{gr}$. Decoctum aloes compositum: dose, 1 to $2 \mathrm{fl}$. oz. Pilula aloes Barbadensis (vel Socotrince): dose, 4 to $15 \mathrm{gr}$. Pilula aloes et asafoetida: dose, 5 to $15 \mathrm{gr}$. Pilula aloes et ferri: dose, 5 to $10 \mathrm{gr}$. Pilula aloes et myrrhce: dose, 5 to $15 \mathrm{gr}$. Tinctura: dose, 1 to $3 \mathrm{fl} . \mathrm{dr}$. Vimum: dose, 1 to $2 \mathrm{fl}$. dr. Much smaller doses than those specified are often very useful.

Adulterations. - Several other species of aloes supply a gum-resin of properties similar to those of the officinal kinds, and advantage would seem to be taken of this in the collection of the drug: such, for example, is the Aloe spicata, which appears to yield "Cape aloes," which is even stronger and has a more disagreeable odour than the Barbadoes variety.

\title{
CONVALLARIA MAJALIS.
}

\author{
(Lilly of the Vatimey.)
}

(Not Offecinal.)

DESCRIPTION.-A small plant, indigenous to temperate climates, with a perennial root-stock, which creeps widely below the surface. Leaves annual, elliptical, 4 to 6 inches in length, tapering to each extremity, usually in couples, arising from the root-stock, their long petioles enclosed in a scaly sheath which resembles a stem. Flowers in a loose, erect 
raceme, upon a scape about 6 inches long; individually campanulate, hexamerous, half-pendulous, pure white, and very fragrant. Fruit, a globular red berry, the size of a pea.

ACTIVE INGREDIENTS. - Convallamarin, $\mathrm{C}_{23} \mathrm{H}_{44} \mathrm{O}_{12}$, is a glucoside of bitter-sweet taste, which may be isolated as a white crystalline powder (Walz, 1858). Tanret has prepared it in a form very like digitalin (Bull. de Thérap., ii., 1882). An acid principle, convallarin, has also been separated; it is a glucoside, since it gives rise, on boiling with acids, to convallaretin and sugar. The odorous principle has been obtained separately in volatile crystals. Martin describes an alkaloid (majalin), an acid (majalic), an essential oil, and a yellow colouring principle (Bull. de Thérap., 1865).

Physiological Action.-External.-The dried powder of the flowers is irritant to the mucous membranes, and when inhaled causes sneezing and lachrymation.

\section{Physiological ACTion.-Internal.-Digestive System.} -All parts of the plant have purgative properties somewhat resembling those of aloes and scammony. Convallarin in 5 -grain doses acts strongly in this way, and a smaller dose of convallamarin has emetic effects (Marmé).

Circulatory System.-This plant slows the action of the heart,-it is said, without lessening its strength: at the same time, respiration is quickened.

Convallamarin also reduces the pulse-rate. Experiments with it have been made on pigeons, a fatal result following doses of $\frac{1}{60}$ to $\frac{1}{30}$ grain : when injected under the skin the heart was arrested in systole.

Troitzki has shown in frogs that an extract of the drug lessens the frequency of cardiac contractions and raises arterial pressure (Centralzeitung., i., 1881).

D’Ary has corroborated this (Journ. de Thérap., Déc., 1881) ; and G. Sée states, in addition, that one drop of extract placed on a frog's heart arrested it in systole, and four drops injected into the veins of a dog produced the same effect in ten 
minutes: in man it restored the proper cardiac rhythm when disordered: he also found it notably increase arterial tension and the contractile power of the cardiac muscle: it lessened exaggerated respiration (Bull. de Thérap., Juillet, 1882). Dr. Ringer classes it in the same group with digitalis as acting directly on the heart-muscle, causing tonic spasm; and he argues that it affects in a similar manner the muscular tissue of the arterioles (B. M. J., i., 1883).

Urinary System.-Troitzki reported a marked diuretic effect; and G. Sée states that this is greater than that of any other drug of the same class, including digitalis.

SYNERGISTS.-Digitalis, caffein, casca.

AnTAgonists.-Amyl nitrite, the ethers, and alcoholic stimulants.

Therapeutical Action.-The plant has been long used in Russia as a popular remedy for dropsy of all kinds, but it is especially in Cardiac Dropsy that it has been lately recommended. G. Sée found it valuable in all forms of heart disease in which there was odema of tissues; he considers it less nauseating and less depressing than digitalis. Dr.,A. E. Sansom, quoting its good effects specially in cases of mitral reflux "with severe symptoms," is "convinced of its power to promote stronger ventricular contraction," but is not satisfied of its superiority to the last-named drug.

By Dujardin-Beaumetz and some other observers it has been found more uncertain; and Dr. Trudeau (U.S.) suggests, in explanation, that it acts best in cases where stimulation of the vight heart is required in order to re-establish the balance of the circulation, and is much more effective in mitral than in aortic disease: its power over oodema and dropsy, however, he reckons "trifling and uncertain" (N.Y. Record, March, 1883).

Functional Palpitation-Dyspnœa.-The same observer finds the drug specially useful in relieving the dyspnoea of emphysema and of ehronie phthisis; and in the orthopnoer of mitral disease it often acts better than digitalis: he also speaks 
highly of it in paroxysmal palpitation and dyspnœa due to nervecauses, as well as in rapid and irregular heart-action dueto debility. This leads him to confirm Dr. Beverley Robinson's statement "that it acts through the nervous system." D'Ary and Borkin have employed it in neuralgias.

PREPARATIONS AND Dose.--Several English chemists now prepare a good extract of the plant (dose, 5 to $8 \mathrm{gr}$.), also a liquid extract (dose, 5 to $20 \mathrm{~min}$.) Tinctura: dose, 10 to 30 min. (Savory \& Moore). Langlebert prepares a symu from the whole plant. Convallamarin: dose, $\frac{1}{8}$ to 2 gr. hypodermically (Bull. de Thérap., ii., 1882). 


\section{MELANTHACEAE.}

\section{THE COLCHICUM FAMILY.}

Av order of about 150 species of herbaceous endogens, widely diffused, and various in physiognomy, but agreeing in the lilylike structure of the flowers, which have extrorse anthers, and the three carpels of the ovary united only at the base, or a little above it, so that the order, as a whole, is trigynous. Except in these particulars it corresponds technically with the Liliaceæ; and were there not so striking a character in the properties, it might be well, perhaps, to regard the Melanthaceæ as a section of the last named. Medicinally, the plants of this order are sedative; but several are acro-narcotic poisons.

\section{Pharmacentical Species.}

Colchicum autumnale

Veratrum album

Veratrum viride

Asagræa officinalis . .
Colchicum.

White hellebore.

Green hellebore.

Sabadilla.

\section{COLCHICUM AUTUMNALE.}

\section{(Colchicum.)}

DESCRIPTION.-The underground perennial portion of this plant consists of a corm, about the size of a tulip bulb, and covered with a brown membranous coat, tho roots descending from the under-surface. In September there rises from the new corm of the current year a flower resembling a 
purple crocus; the leaves do not make their appearance until the following May, when they come up many together, each 12 or 14 inches long, about an inch in breadth, tolerably flat, pointed, erect, and dark green. The capsule is brought into view at the same time, half concealed among the foliage. Internally the corm is white and feculent, containing a milky juice, and having a bitter acrid taste; it is plentiful in the meadows in many parts of England. In commerce it is usually met with in transverse, somewhat kidney-shaped slices, about $\frac{1}{8}$ inch thick, and of white, mealy appearance. The seeds are about one line in diameter, brown, nearly round, with a point or crest, difficult to powder.

ACTIVE INGREDIENTS. - The corm and seeds both contain a crystalline principle, which may be separated from a solution in dilute spirit in needles and prisms, and is called colchicin, $\mathrm{C}_{17} \mathrm{H}_{19} \mathrm{NO}_{5}$. Pelletier and Caventou believed it to be veratria in combination with gallic acid, but Geiger and Hesse proved this opinion to be erroneous. Colchicin is a powerful poison; the taste is bitter, but not so burning as that of veratria; it is destitute of odour, does not excite sneezing, and is soluble in water and in alcohol: with strong nitric acid it exhibits a play of colours commencing with violet.

In the corm there are also contained fatty matter, yellow colouring matter, starch, gum, lignin, and a peculiar acid called cevadic acid. The active properties are partially taken up by water, readily so by alcohol, by dilute spirit, and by vinegar.

Physiological Action on Animals.-Colchicum is acrid and sedative, and to graminivorous animals generally poisonous. Hence they never eat the herbage unless it be inadvertently mingled with their fodder and unperceived by them, in which case it is apt to cause inflammation of the intestines and bloody evacuations, sometimes ending fatally. Störck, who in 1763 published a pamphlet upon colchicum, administered it to dogs, with causation of vomiting, tremor of the limbs, and convulsive movements of the belly. The animals howled, passed urine in large quantity, had bloody eracuations 
and prolapsus of the rectum, and finally died. In some cases they lay prostrate, languid, and perfectly still, with feeble pulse, slow and often irregular respiration, refusing food, and having their eyes glazed and swollen. So peculiarly severe are the effects of colchicum upon dogs, that in France the medicine bears the name of mort au chien, and from my own experiments I can testify to its appropriateness. Cows, when they have eaten colchicum, lose all desire for food and water, cease to muminate, suffer from running at the eyes and nostrils, and from a peculiar kind of diarrhoa; instead of diuresis, as in dogs, there is a tendency to diminution of the urine; the belly becomes distended, and the animal sinks into a half stupefied state.

Upon certain other creatures colchicum appears to produce but little effect. Experiments made with rabbits had scarcely any result beyond exciting some diuresis; upon frogs the effects were trifling and temporary. In all observations made upon the action of colchicum, whether in regard to animals or to the human subject, it must be remembered that the drug varies in energy according to the period of the year at which the corms are collected.

The effects of colchicin have also been tried on a variety of animals. As with colchicum itself, the principal and most characteristic phenomena are those of its drastic operation on the alimentary canal; whether given by the mouth or applied to a wound, colchicin constantly produces these effects, while convulsions are only occasional. Geiger destroyed kittens in 12 hours with doses of $\frac{3}{5}$ grain, the symptoms being those of gastroenteritis; and Bley found one-fourth of this quantity fatal to a kitten of three months; vomiting, purging, and convulsions preceded death. Various experimenters have obtained substantially similar results in operating upon rabbits, pigeons, and dogs, but the researches of Schroff and of his pupil Heinrich showed that the fatality in animals was by no means constantly proportioned to the dose.

Physiological Action on MAN.-The general results of inquiries into the physiological action of colchicum are summed up in the words of Gubler: "Colchioum is a drastic 
purgative, accidentally an emetic, and indirectly a sedative of the circulation; a depressant diaphoretic, sialagogue, and diuretic."

Digestive System. - Heinrich found that of colchicin so small a dose as 0.312 of a grain, taken by the mouth, caused an acrid burning taste, increased flow of saliva, nausea, and retching for several hours. Double the amount induced also symptoms of gastro-enteritis for several days. One fourteenth of a grain produced in a woman severe pain, vomiting of green matter and blood, diarrhœea, and collapse. Colchicin is said to be about 100 times more active than the fresh corm, which will itself produce, in full doses, symptoms like those above described. Small medicinal doses do not perceptibly act on the intestinal tract otherwise than by increasing its secretions, and so inducing a tendency to liquid diarrhcea. Some persons are much more easily affected in this way than others. Intestinal symptoms occur even after hypodermic use of the drug, proving that they are not due simply to a local irritation.

Nervous System.-Headache and vertigo are stated to have sometimes occurred, and even results such as ensue from the use of narcotics have been ascribed to colchicum. But such symptoms, when manifested, are probably referable not so much to the action of the drug per se as indirectly to the exhaustion which follows the employment of excessive doses. Spasms, and sometimes convulsions occur, and according to the observations of Rossbach, are frequent in frogs, and connected. with heightened reflex activity, which is, however, soon lessened, and finally abolished: in warm-blooded animals the primary increase occurs but rarely.

The same observer states that the higher nerve-centres, the cord, and the sensory nerves at their periphery, are paralysed - the motor nerves and voluntary muscles not so.

Circulatory System. - Full doses markedly slow the pulse and depress the heart-action. The slowing effect on the pulse seems always to be produced where the dose is sufficient to produce gastro-enteritis; the latter condition is attended by increased rapidity of the pulse.

In fatal cases, collapse devclops, "the circulation frils more 
and more, the pulse, which has been frequent and feeble becomes rapid and thready, the skin cold, pale, or livid, bedewed with sweat, and death from exhaustion results" (Wood).

Secretory System.-In man, colchicum, administered in small and repeated doses, promotes the action of the secreting organs, and quickens especially that of the intestioal mucous membrane, as already stated. The skin, liver, and kidneys are affected by it, but less obviously. When free perspiration occurs, the action on the bowels is commonly diminished. Moderate doses, regularly repeated, generally increase the amount of urine, but observations are still discrepant as to their effect on the solid constituents. Christison, Maclagan, and others have found uric acid and urea increased after the use of the drug. Graves and Gardner have found the urates diminished. Dr. Garrod (1858) reported that the former substances were sometimes increased, sometimes lessened in amount. The conclusion of Schroff and several physicians of Vienna, after more than sixty observations, was that no increase was produced. Again, Hammond, experimenting on healthy subjects, with care as to diet, etc., found the solids increased by colchicum (1858), whilst Golding Bird reported no such increase (1859).

SYNERGISTS. - Alkalies and purgatives, such as potash and magnesia, assist the eliminant action, "and render it milder."

Veratria and aconitia are allied in their action on the circulation, and in power of relieving pain. Paris states that acids increase the purgative effect.

ANTAGONISTS.-Tannic acid, to some extent, by retarding' absorption; opium and alcohol.

ThERAPEUTICAL ACTION.-Gout.-As a specific for this disease, colchicum first obtained wide celebrity, and principally under the form of the ear médicinale, which became fashionable in France during the reign of Louis XV. In subsequent periods the reputation of colchicum has fluctuated, at times reaching an extraordinary height, at others sinking very low. This periodical discredit of the drug has perhaps been 
due to its varying strength, attributable to the collection of the corms at improper seasons.

At present, and especially since the publication of Dr. Garrod's masterly treatise on gout, scarcely any one disputes the fact that, in the acute stages of the disease, colchicum has the power to produce a remarkable amendment of the symptoms.

Upon the inflammation and pain of acute gout it acts very directly, and Garrod observes that this action is independent of any evident purgation, sweating, or diuresis, though large doses will produce those effects. It cannot be called a lasting or final remedy for gout; it does not prevent relapses, and its power becomes weaker on successive occasions, till finally it will not check pain and inflammation unless given in dangerously large doses.

Sir Everard Home introduced colchicum wine into the London Pharmacopocia, and Sir H. Halford (1831), Sir H. Holland, and Sir T. Watson largely adopted it. Scudamore, whilst prescribing it at times, especially in the mixture, still much used, with magnesian salts and aromaties, recognized some evil results from it, and "could relate almost an infinity of cases in which, after a time, it disappointed the patient"; he further states that "the eau médicinale has been the fruitful source of many cases of chronic gout by enfeebling the nervous system, and occasioning, together with irregular pain and obscure inflammation, a degree of despondency and languor nerer before experienced"; and, moreover, what we should certainly not expect from any moderate use of the drug, that it tends "to render the bowels inactive, to diminish the alimentary secretions, and to weaken the functions of the liver." If this, were always so, colchicum would be more hurtful than remedial, but such effects will not be experienced from a judicious use of it, i.e., from small or moderate doses continued for a short time and supplemented by alkalies and careful diet. Rutherford found it, "in very large doses," a porverful stimulant of the liver and intestines it rendered the bile more fluid. It may be given, after preliminary aperient treatment, in acute or subacute cases, to relieve pain and inflammation, but should not be continuously taken on the principle of warding off impending 
attacks by its use. The same remarks apply probably to Laville's tincture as a colchicum preparation, though colocynth also is a constituent. It more often causes vomiting than the tincture of colchicum.

In Acute Rheumatism there are surprisingly different opinions as to the efficacy of colchicum. Dr. Garrod thinks that in this disease colchicum does good, if at all, by controlling the heart's action. Many authorities describe it as not less's remedial in rheumatism than in gout; Gubler speaks of it in the same terms for both diseases. It is possible that part of its reputation arose from inaccurate diagnosis between them.

On the other hand, colchicum has been losing its prestige as a remedy for acute rheumatism of late years in London hospital practice, and is considered by many physicians useless or altogether noxious, being apt to seriously embarrass the circulation. Certainly the long-continued use of colchicum, or its employment in large doses, is not to be thought of in rheumatism. The peculiar anæmia which attends this disease produces an irritable weakness of the heart, which renders it liable to suffer alarmingly from so depressing a drug.

Badia and Heyfelder have reported very favourably of the subcutaneous injection of $\frac{1}{6}$ to $\frac{1}{3}$ grain of colchicin in chronic articular rheumatism and rheumatic neuralgia, stating that it quickly gives freedom from pain, and more power of movement. On the other hand, it may give rise to local burning pain and even inflammation, sometimes to diuresis and strangury (Practitioner, vols. x.-xxiii.). This treatment has not been extensively employed.

In certain Acute Inflammations experience shows that colchicum often exercises a happy influence, though it may be difficult to explain its modus operendi. In Bronchitis, for example, the occasional fortunate results of colchicum treatment may be really due to the fact that the chest affection is but a part of the gouty diathesis. The same remark applies to its use in Ophthalmia.

A Dr. Waterman represents colchicum as a "true anti-pyretic through the influence over the organic nervous system," suitable for all sthenic pyrexia, iritis, pneumonia, and malarial fever: he states that he has given 1 minim every ten minutes to children, 
and $\frac{1}{2}$ ounce of the wine every thirty minutes to adults, for several doses, but no one would endorse such practice (B. M.J., ii., 1881).

Among the miscellaneous uses for which it may be employed, colchicum is deservedly in favour as an addition to other aperients, when a slight increase of their activity is desirable. Half a grain to 1 grain of the acetic extract is thus often added to a small dose of colocynth or aloes: indeed, we may' sometimes get a very effective combination by adding a dose of colchicum to such a medicine as nux vomica, which otherwise might not act at all as an aperient.

Preparations and Dose.-Pulvis: dose, 2 to $8 \mathrm{gr}$. Extractum: dose, $\frac{1}{2}$ to $2 \mathrm{gr}$. Extractum aceticum: dose, $\frac{1}{2}$ to $2 \mathrm{gr}$. Vinum (prepared from the corms): dose, 10 to $30 \mathrm{~min}$. Tinctura colchici seminum: dose, 10 to $30 \mathrm{~min}$.

Tinctura colchici florum is said to most resemble the eau médicinale, and is by some preferred to other preparations (Squire).

\section{VERATRUM.}

There are two medicinal species of veratrum; the veratrum album owes its virtues to the same alkaloid, veratria (or veratroidia), which is present in veratrum viride, and since it contains also a dangerous ingredient - the resin - which renders its medicinal use undesirable, it need not be considered in therapeutical detail.

\section{Veratrum Album (White Hellebore).}

(Not Officinal.)

DESCRIPTION.-A perennial, with a tuberous, fleshy root, a stout, erect, simple flower-stem, 4 or 5 feet in height, and large ovate, entire, strongly plaited leaves, nearly all radical. The few and smaller stem-leaves are amplexicaulent; the innumerable greenish-white star-like flowers, each about 
$\frac{1}{2}$ inch across, are borne in a large, erect, terminal, and manybranched panicle. It is indigenous to many parts of continental Europe, but not to Britain.

ACTIVE INGREDIENTS.-The root, while fresh, exhales a disagreeable odour; is acrid, bitter, and nauseous; and if chewed excoriates the mouth and fauces. The active properties delend upon the presence of two alkaloids, veratria, $\mathrm{C}_{37} \mathrm{H}_{53} \mathrm{NO}_{11}$, and jervia, $\mathrm{C}_{27} \mathrm{H}_{47} \mathrm{~N}_{2} \mathrm{O}_{8}$ (E. Simon); an acid, formerly supposed to be gallic, but which is most probably veratric; and with this occur some unimportant ingredients, similar, it would appear, to those contained in the corm of colchicum. There is also a highly poisonous resinous body.

\section{Veratrum Viride (Green Hellebore.)}

DESCRIPTION.-A native of North America, closely resembling veratrum album, but distinguished from it by the narrowness and great length of the bracts, which considerably exceeds that of the peduncles.

ACTIVE INGREDIENTS.-Veratrum viride contains two alkaloids, of which the precise characters, and even the names, have been the subject of much controversy.

Some years ago the researches both of C. Bullock and of E. Peugnet were taken to prove that the principal alkaloid was distinct from the one in cevadilla, already known as veratrin, and was therefore better named veratroidia, but the more recent observations of Wormley (Amer. Journ. of Pharm., 1876) tend to show that this is really identical with the veratria of cevadilla, as announced by Pelletior in 1820 (v. p. 798).

The second alkaloid of veratrum viride, called viridia by Bullock and by Dr. G. B. Wood, has been found identical with jervin, an ingredient of veratrum album: I shall therefore describe the alkaloids under the names of veratria and jervia.

Veratria (or veratroidia) is a whitish amorphous powder, without odour, but very irritant to the nostrils, and acrid to 
the taste; soluble in 1,000 parts of water, in three parts of alcohol, in fifty-six of oil, and in ninety-six of glycerine. Concentrated sulphuric acid dissolves it with red coloration. Nitric acid colours it yellow. It forms soluble salts with acids.

Jervia is not easily separated from the resin, but when pure is a white, tasteless, non-irritant powder, insoluble in water, soluble in chloroform, and in alcohol, from which it may be crystallized; it forms salts with acids. Sulphuric acid colours it yellowish green (Stillé).

Veratrum viride does not contain the irritant poisonous resin mentioned under veratrum album.

Physiological Action.-External.-Veratria, applied to the skin in moderate strength of ointment or tincture, causes first some prickling or slight pain, afterwards a sense of coolness, numbness, and more or less anæsthesia; if concentrated it may prove highly irritant, and produce erythema or vesicular eruption with muscular twitchings. On the denuded skin or mucous membrane it causes much burning. The slightest scent of it in some persons, or a minute quantity brought into contact with the nasal mucous membrane, is apt to excite violent irritation and sneezing.

\section{Physiological Action.-Internal.-Digestive System.} -Taken into the stomach, or injected subcutaneously, it is absorbed with great rapidity, and produces salivation, spasmodie action of the intestines, and mucous outpour from their glands. At the same time the patient experiences curious sensations of heat and cold in various part of the body.

Purging, however, which is sometimes a marked symptom, is absent in the majority of instances. It is not possible to say why vomiting or diarrhooa should occur, as it does in a considerable number of cases; it is not the result of inflammatory irritation, for no signs of this are found in the alimentary canal after death, as has been conclusively proved. This vomiting has often prevented even very large doses from proving fatal.

Veratria causes more severe symptoms of gastric irritation than jervia. $\Lambda$ woman who had taken 3 grains (with opium) 
suffered from nausea, thirst, diarrhœa, and tenesmus, with swelling and soreness of tongue and mouth, besicles nerve-symptoms. Dr. Lynch restricts the irritant action wholly to veratria, and the arterial depression to jervia (Record, 1878), but it is not likely that so exact a line can be drawn.

Nervo-muscular System.-A characteristic symptom of the full action of the drug is muscular tremor, twitching, or thrill, and Dr. H. C. Wood reports that this is more strongly marked under jervia than under veratroidia (veratria). After a time the affected animals become unable to stand from weakness, and convulsions set in; sensation is benumbed, but consciousness preserved. The spasmodic condition of the muscles has been the subject of much discussion. Gubler, without sufficient reason (as it appears to me), regards this phenomenon as the reflex effect of the irritation of the intestinal canal; but the researches of Prévost demonstrate that the muscular spasm is due to direct irritation of the muscles by the alkaloid, for it may occur after the nerve-connection with the cord is destroyed, and conversely does not occur if the poison is only pernaitted access through these nerve-connections, not directly to the muscle; neither is it excited by direct mechanical excitation of the spinal nerves, but by that of muscles or by the voluntary muscular efforts of the animal (Stillé). It seems unlikely that the central nervous system is affected at all. The convulsions, and also the alterations in sensibility, can at present be only imperfectly explained.

H. C. Wood considers that the former are cerebral in origin because they do not occur below a point where communication with the brain is cut, though he also states that the drug "has little or no direct influence on cerebral centres." On account of reflex activity being diminished or abolished, he holds it to be "an intensely powerful spinal depressint" : other experiments lead him to consider it also a depressant to vasomostor centres. Pécholier and Redier think that it hos no direct action on the cord (Gaz. Hebdom., Avril, 1883).

Circulatory System.-One of the most constant plienomena is the steady decrease of the pulse, which often renches a very low level; the arterial pressure also is greatly lowered, and these 
effects are probably independent of the conducting nerves, and due to a direct paralysing action on the cardiac muscle (or its ganglia).

Dr. Ringer concludes that the effect observed on a frog's heart (viz., a prolonged and more energetic contraction, but without proper co-ordination) is due to direct action on the muscle, and is independent of the ganglia, because it occurs in the lower two-thirds of a ventricle where there are no ganglia (Practitioner, vol. xxix.). He notes that it is much more effective at high temperatures, suggesting a greater power in febrile conditions. Dr. Lynch (U.S.) attributes the lowering of the pulse to "arterial and capillary contractions," but without sufficient evidence, and probably incorrectly (Record, 1878).

The slowing is dependent partly on quiet. "When there are convulsions, or even when the tremors are marked, the pulse becomes rapid;" also, after a time (following hypodermic injection), the pulse remaining slow, its beats become rery forcible, and later it may become again rapid, and the bloodpressure be much increased, these symptoms being really due to commencing asphyxia, for they cease under artificial respiration. When this is kept up, veratroidia "steadily lessens pulse and respiration." If, however, the vagi be divided, a great increase in the number of pulsations at once occurs, proving, according to Wood, that the drug, in small doses, is a powerful stimulant to the inhibitory cardiac nerves, whilst large ones paralyse them. "Enormous doses thrown directly on the heart by venous injection, at once kill the cardiac muscle." If artificial respiration be not practised, the toxic effect of the drug on respiratory centres is so powerful as to induce death by asphyxia before seriously affecting the heart. The action of jervia does not occur through the vagi, and seems to be exerted directly on the heart-muscle.

Many of the occasional effects are uncertain and irregular in their occurrence; thus, at one time there may be much diuresis, at another much sweating, or much sneezing and lachrymation.

Oulmont further observed a remarkable reduction of temperature, amounting to two, three, or five degrees in the course 
of two hours, and lasting during twenty hours (Gaz. Hebdom., iii., 7,1868$)$.

This latter effect was not observed by Dr. Squarey in some experiments made on patients in University College Hospital with a tincture of veratrum viride (Practitioner, vol. iv.). The patients, four in number, were suffering from slight maladies: in no case was there. any notable depression of temperature, although the pulse was decidedly reduced. Nausea and vomiting were produced whenever more than 20 minims were given at once: in one case there was diarrhoea. The respirations were also reduced in correspondence with the pulse, and there was a feeling of extreme weakness when under the influence of the drug.

The action of veratrum viride may rightly be inferred from the preceding accounts of its alkaloids. Dr. Hadlock says that large doses cause nausea and vomiting, complete relaxation of the system, copious sweat, and a pale, cool skin (Med. and Surg. Reporter, 1870). The pallor may be extreme, and accompanied by syncope, especially if the patient rises suddenly from the recumbent position.

SYNERGists. - Tobacco, aconite, bleeding, and vascular depressants.

Squarey, Lynch (U.S.), and others, would include digitalis, but this is not generally accepted, and is, I believe, incorrect. It should rather be classed amongst antagonists (within limits of dose), and a case cited later on illustrates such action.

ANTAGONISTS.-Opium and morphia markedly lessen the irritant effects. According to Pécholier, stryehnia is a direct antidote to the action on muscles. The more usual ones are alcoholic and other stimulants.

In two cases-one a child-tincture of veratrum is said to have quickly removed opium narcosis (Record, 1880).

Therapeutical Action. - External. - Both veratria and veratrum viride have attracted much attention as remedins during the last fifteen years; the former has been largely 
used in France and Germany: to a much less extent in this country.

In Neuralgias, and other severe localized pains, au ointment made with it is frequently applied with much benefit. In average cases the unguentum, P.B. (8 grains to the ounce), answers well; it produces a slight pricking numb sensation, and speedily relieves pain. In high concentration, however, veratria is liable to prove intensely irritant, and on susceptible skins even the officinal ointment will produce inflammation, and occasionally pustulation. The stronger ointments, which are sometimes recommended, are still more likely to cause such effects in a severe and unpleasant degree: although in many instances in which I ordered one containing 8 grains to 2 drachms, especially in cranial neuralgia, the cases were relieved without any other evident symptom than a moderate stinging, - not more than that of mustard, and less than that of capsicum.

Therapeutical ACtion. - Intenal.-In Pneumonia, veratric has been considered by many a valuable resource. One of the earliest authorities on this subject is Vogt, of Berne, who gave it in doses of $\frac{1}{13}$ grain $(5$ milligrammes) every two or three hours, till it produced vomiting, or retardation of the pulse, and found it very effective (Bull. Gén. de Thérap., 1860). If the stomach proved too irritable the dose was reduced, and the veratria was given either with opium or in an effervescing draught. The mortality was 8 per cent. Aran (Bull. Gérn. de Thérap., xiv., p. 385) highly recommended the same remedy in pneumonia, and this recommendation was afterwards eudorsed by Trousseau. Aran gave it in pills, with small doses of opium, which possibly mitigated the local effects.

Biermer (quoted by Niemeyer) tried it extensively, and equally recommends it: doses of $\frac{1}{20}$ grain, in pill, were employed, and produced decided effects in reducing pulse and temperature; but he remarks that its effects are very variable, and that it is apt to cause nausea or vomiting, and sometimes diarrhœe. The collective results of foreign experience seen to agree with my own, to the effect that the alkaloid may produce striling alleviation in pneumonia, 
but is, on the whole, so unmanageable a remedy as to be of doubtful applicability.

Veratrum viride no doubt stands in a somewhat more favourable position than veratria, yet it is necessary to be cautious in estimating the worth of the various statements that have been made in its favour. Stillé remarks-"The only conclusion to be drawn from a critical study of the mass of conflicting evidence respecting its use in the diseases referred to, is, that the patients would have been better off without it;" and again, "Cardiac sedatives, of which the medicine under consideration is the type, tend to retain in the blood all that is injurious in it, and green veratrum at the same time reduces the patient to a state of such wretchedness that he is unable to take food, or to digest it if he ate it." It seems to me that this, on the other hand, is an extreme view, which expresses only the effect of toxic doses, and benefit may be derived from a few minims of the tincture without any such serious effects. Stillé himself allows that in "some cases of commencing congestion or inflammation its utility is presumable," e.g., after wounds of the head, pericardium, or peritoneum.

H. C. Wood says,- "In the early stages of sthenic pneumonia, it offers, I believe, the best known method of reducing the pulserate and temperature, and ameliorating the disease; also it is a very safe remedy." He quotes a remarkable instance of its value in threatening peritonitis after a punctured wound of the abdomen with protrusion of intestine.

In pneumonia a strong case seems to have been made out for its use. The experience of Dr. Kiemann forms perhaps one of the most favourable testimonies; it is based upon forty cases, and, although five of these proved fatal (a mortality of 12. from the first, and that the results were otherwise decidedly good. The reduction of fever was marked and constant, and although Kiemann acknowledges that veratrum viride is a powerful remedy, never to bo intrustod to any one but a skilled physician, he states that he has not observed it produce collapse, as some authors have reported (Prag. Vtljschr., Bd. iii., 1868).

By Drasche also the antifebrile power of veratrum viride in paeumonia is fully acknowledged: he observes, however, that 
if the medicine be discontinued before defervescence, the pulse at once rises again: and states that, so far from forwarding the process of subsequent resolution, this drug rather delays it (quoted by Ringer).

The testimony of Oulmont is perhaps the most important that we possess to the fact that in pneumonia, and indeed in pyrexial diseases generally, veratrum viride is more effective than the alkaloid.

Stewart has given some instances of its value in the pneumonia of children, when antimony would be dangerous; he found it succeed admirably when ordinary "antiphlogistic" treatment had failed (Practitioner, vol. xxi.). Trousseau compared its action to that of antimony.

In Acute Rheumatism there is much evidence in favour of veratria. It was recommended for this purpose by Trousseau, Alies, Bouchut, Léon, and others, and probably with more justice than in some other acute diseases, yet, after all that has been said, it is not proved to act in any other way than in reducing the febrile phenomena. It should be given in small doses, and may often be advantageously combined with opium.

It is as an Antipyretic, in fact, as well as a special anodyne, that veratria effects some good in the treatment, not only of acute rheumatism, but of a number of pyrexial diseases for which it has been recommended. Some, indeed, of these are unfit subjects for its operation: thus it was at one time praised in the treatment of typhoid fever, but careful investigation of the recorded results of its use are unfavourable; and, on the other hand, its depressant action on the heart has led to disastrous consequerices.

Veratrum viride also has been much commended by Dr. Osgood in acute rheumatism, and possesses considerable power as an antipyretic. I may say of it, as of veratria, that, however doubtful its power of lowering the temperature in the healthy body may be, there can be no doubt of its potency in reducing fcbrile heat, with any attendant increased rapidity of pulse and breathing. Nor are these results to be despised, even though the course of the disease be not shortened, if we can satisfy ourselves that the remedy is safe, since the mere reduction of 
fever is in itself a source of great comfort to the patient. There is much evidence to show that veratrum viride is more manageable than veratria.

Cardiac Disease.-In cardiac irritability connected with slight hypertrophy or even of reflex causation, tincture of veratrum viride is often a suitable remedy (Betot, Practitioner, vol. vii.). It must be considered a direct sedative and depressant, therefore distinct in therapeutical value from digitalis. Mr. E. Wood Forster relates a case illustrating well the contrast in the clinical use of veratrum and digitalis:-A lad of fifteen complained of much pain in the cardiac region, and on exertion suffered from rapid breathing and early sense of fatigue: digestion was good, but temperament was "nervous"; there was no valvular lesion, but slight hypertrophy with strong impulse and "irritability of heart"; the action was not intermittent, but some beats were more feeble than others. Digitalis in 5-minim doses thrice daily gave no marked relief after several weeks, but 3 minims of tincture of veratrum viride distinctly relieved within three days. Mr. Forster credits it with a direct action on the heart (B. M. J., i., 1882).

In the acute stage of Pericarditis it deserves trial; and according to several French observers, it has relieved the palpitation and general distress of exophthalmic goitre, but my experience leads me to think that it does not act nearly so well as digitalis.

Convulsion.- Veratrum viride has proved serviceable in puerperal convulsions occurring after delivery, i.e., of reflex character, but chloral was also given. Even in true epilepsy it has been given by some, and is said to abort the attacks. Mordough used it, combined with morphia, in hypodermic injection (Boyd, Practitioner, vols. xx.-xxii.).

It is a possible resource in the acute stage of delivium tremens, when prostration is not threatening, and it is worth while to remember that it seemed to relieve $n$ case of persistent priapism after failure of antimony, belladonna, and bromides.

\section{PREPARATIONS AND DOSE.-Tinctura veratri viridis:} dose, 2 to $8 \mathrm{~min}$. (in a mucilaginous vehicle). Veratrin (the alkaloid itself is to be preferred to its salts) : dose, $\frac{1}{60}$ to $\frac{1}{16} \mathrm{gr}$., 
twice or at most three times daily. Unguentum (8 gr. to $1 \mathrm{oz}$ : : or sometimes $8 \mathrm{gr}$. to $\frac{1}{4} \mathrm{oz}$.) Both veratria and veratrum viride cause too much local irritation for use in hypodermic injection.

\section{ASAGR无A OFEICINALIS.}

(Sabadilla or Cevadilla.)

A bulbous plant indigenous to Mexico, having linear leares, a slender stem, and spike-like raceme. The fruit has three brownish thin follicles, containing two to six seeds, which are $\frac{1}{6}$ to $\frac{1}{4}$ inch long, narrow, oblong, with one side flattened, one end rounded, the other pointed and peaked; they are of acrid taste and inodorous, but the powder causes sneezing. They contain veratria, and a similar but weaker alkaloid, sabadillin.

The powder has been used locally as a parasiticide, and given internally in doses of 4 or 5 grains as a drastic cathartic in tænia. 


\section{PALMACEA.}

\section{THE PALM FAMILY.}

\section{ARECA CATECHU-ARECA NUT-BETEL NUT.}

\section{(Not Officinal.)}

DESCRIPTION.-The seed of areca catechu, imported from the East Indies, where it is cultivated. The seeds are of a rounded conical shape, about 1 inch long; there is a wellmarked hilum, with a tuft of fibres attached at one part, where the pericarp was connected: the albumen is ruminated.

ACTIVE INGREDIENTS.-Areca nut contains $14 \cdot \gamma$ per cent. of a red amorphous "tannic matter" (areca red), little soluble in water, and yielding, by destructive distillation, pyroeatechin. In the presence of an alkali, ferrous sulphate precipitates areca-red, dark purple; a ferric salt (without the alkali) gives a green tint to a solution.

Fatty matter and gum are also contained in the seeds; but the presence of catechin is denied by Flückiger and Hanbury (op. cit.).

Therapeutical ACTION.-As a dentifrice the charcoal from the nut is used. Areca is the principal constituent of the "betel" of the East, many properties being ascribed to the mixture.

The chief therapeutical employment of areca is as an anthelmintie for both tamia and lumbicus, chiefly for the former. Areca is astringent from the amount of "tannic matter" it contains.

Preparation AND Dose.-Pulvis: dose (as a vermifuge), 1 to $6 \mathrm{dr}$.: for children, dose, $\frac{1}{2} d r$, to be taken fasting. 


\section{$A R A C E A E$.}

\section{TONGA.}

DESCRIPTION. - What is known under this name is a liquid, dark brown in colour, probably a spirituous extract from several trees or creeping plants indigenous to the Fiji Islands. They are sent over in bundles, "the size of a Florence flask," containing bark, leaf, and woody fibre in varying proportions, but all chopped up together and mixed with the object of preventing recognition (Lancet, ii., 1881).

Epipremnum mirabile, Rhaphidophora vitiensis, and Premna taitensis have been named as probable sources (Pharm. Journ., 1880), and two of these have been verified by Mr. R. L. Holmes, who says that the name Tonga is derived from one of the Friendly Islands, whence the drug was first introduced.

The Raphidophora (Arace») is a creeper growing freely in sheltered places, and when climbing it develops a thick stem and large compound leaves; the scraped stem is the part used. The Premna (Verbenaceæ) is a small shrub on dry ground, a tall tree near water-courses; of this the inner bark is used (B. M. J., ii., 1881).

ACTIVE INGREDIENTS.-Mr. Gerrard obtained from the fibrous portions (probably root) a volatile alkaloid, for which be proposes the name tongin, and from other portions, leaf, etc., pectin, glucose, and an essential oil.

Physiological Action.-No definite physiological action or toxic effects were observed by Drs. Ringer and Murrell even after large doses. They did not find the pupil affected, but Mr. Bader reports that the internal use of the drug sometimes caused dilatation (Lancet, i., 1880); its local application increased the power of accommodation, and markedly lessened tension. 
Therapeutical Action.--Neuralgia.-Tonga has the power of relieving nerve-pain without narcosis. Of eight eases reported briefly by Dr. Ringer (several of them from "bad teeth," which were not extracted), "cure" was effected in six. Of four noted by Dr. Vaudrey Lush there was only one failure, and this probably from an inefficient preparation. The early reputation of the drug was founded on its cure of a very inveterate case in Fiji ; and, within my own experience, in facial and other neuralgias it has given quicker relief with less unpleasant effect than many other remedies previously tried. $\mathrm{Mr}$. C. Bader found it valuable when instilled into the eye in asthenopia and in rheumatic iritis.

PREPARATIONS AND DOSE.-An infusion may be made by soaking a muslin bag containing. crude Tonga in warm water for some minutes; the "Tonga bag" is said to retain its power for twelve months. The ordinary preparation (Allen and Hanbury) is liquid; dose, $1 \mathrm{dr}$., repeated as required.

Its importation being almost a monopoly, the medicine is at present too expensive for general use. 


\section{GRAMINACEA:}

\section{THE GRASS FAMILY.}

Ax extensive order of endogens, represented in every part of the world, and familiar in the pasture-grasses and the cereals. In the smaller species the stems constitute straws or reeds; in the larger ones indigenous to the tropics, canes and bamboos. The leaves are alternate, narrow, entire, parallel-veined, and provided with long sheathing petioles; the flowers, small but numerous, and generally aggregated, are composed of chaffy bracteas, disposed alternately upon the rachis, with normally three stamens; the superior ovary has two styles, and ripens into a seed-like fruit of the character illustrated in wheat. This family is almost entirely devoid of deleterious plants, and various species are employed as auxiliaries to medicine.

\section{Phatmacentical Species.}

Claviceps purpurea. . . . . . . . . E Ergot.

Hordeum vulgare . . . . . . . . . Barley.

\section{ERGOT.}

DESCRIPTION.-The origin of this medicinal agent is referable to a minute fungus, the claviceps purpurea, which, attacking the young pistils of various grasses, and especially those of rye (secale cereale), induces a morbid condition of the whole of the part affected. The development of the grain or seed is arrested, and in place of it there is produced a slender, rounded, and solid body, nearly an inch in length, slightly curved, and resembling a little horn, whence the name 
"cornutum" applied to rye when thus affected. These bornlike bodies are the ergot of the Pharmacopœia. They are very conspicuous upon the ears of rye which support them, projecting on every side, sometimes to the number of six or eight: for medicinal use they should be collected while the plant is still uncut, and should not be kept long, especially in powder, for they lose their activity by exposure. Externally they are violet-black; internally firm, pinkish, and mealy, with a short fracture: the odour is faint: if a thin slice be placed in water, and viewed with a high magnifying power, it is found to consist of flocci and spores, compacted into a homogeneous mass.

\section{ACTIVE INGREDIENTS. - Ergot contains about 35 per} cent. of a fixed oil, and about 15 per cent. of a peculiar reddishbrown substance called ergotin, $\mathrm{C}_{50} \mathrm{H}_{52} \mathrm{~N}_{2} \mathrm{O}_{3}$. The fixed oil is lighter than water, and soluble in alcohol, and (at all events when obtained by expression) is inactive as a medicine. Ergotin, presumably the active ingredient, has an acrid, bitter, and disagreeable taste, is soluble in spirit, but insoluble in ether, and in water, which it simply colours red. Hence a doubt as to its being the principle which renders ergot capable of contracting the uterus, since aqueous solutions are prescribed with the greatest advantage when uterine contraction is required.

Rabuteau describes two substances under the name of ergotin, and they certainly require to be distinguished-(1) that of Wiggers, answering the above description, is a resinous reddish substance, very poisonous, and ten times more active than ergot itself; (2) the ergotin of Bonjean, which is much less toxic, and is essentially a watery extract of the drug. Köhler, examining both substances, finds them to be mixtures, the former of all the constituents insoluble in water, the latter of the soluble oncs, neither representing all the properties of ergot.

Ergotinin, an alkaloid deseribed by Tanret as the active principle, is white and crystalline, insoluble in water, soluble in other and alcohol, alkaline in reaction, and of basic properties: it is unstable, and decomposes readily in the air; it is colonred violet-blue by sulphuric acid. The formula given is $\mathrm{C}_{335} \mathrm{H}_{40} \mathrm{~N}_{4} \mathrm{O}_{6}$. The proportion contained in fresh ergot is only 0.12 per cent.

Wenzell described as alkaloids two brown, amorphons, 
bitter, alkaline, soluble substances, which he called ecbolina and ergotina, and presumed to be in combination with ergotic acid; but Dragendorff reported them identical, and of little or no physiological power (1876). The latter, whose observations have been largely accepted, describes other principles, viz., sclerotic or sclerotinic acid (doubtful formula, $\left.\mathrm{C}_{12} \mathrm{H}_{19} \mathrm{NO}_{9}\right), 4$ per cent, and scleromucin, and as less important ones, sclererythrin, the red colouring matter soluble in, and deepened by, weak alkalies; scleroiodin (giving a violet colour with potash or sulphuric acid); sclerocrystallin $\left(\mathrm{C}_{7} \mathrm{H}_{7} \mathrm{O}_{3}\right)$; and soleroxanthin $\left(\mathrm{C}_{7} \mathrm{H}_{7} \mathrm{O}_{3}+\mathrm{OH}_{2}\right)$.

Kobert of Strasburg more recently describes the active constituents under the names of ergotinic and sphacelinic acids, and cormutin, an alkaloid. The first named is a glucoside which corresponds to the sclerotic acid above mentioned; it is soluble in water, and is presumed to form the chief part of Bonjean's "ergotin." The alkaloid occurs in extremely small amount, and is contained in ecbolin (Archiv. f. Exper. Path., ₹. xriii.).

From this enumeration, we can see ground for the remark of Buchheim, "that the low organization of ergot prevents the formation of well-defined principles, and those which are formed are easily altered," also why "preparations possessing some activity may be obtained by very different processes" (Stillé).

Winckler (1826), having separated the fixed oil by means of ether, found in the residue another principle, secalia, afterwards proved identical with propylamin. Trimethylamin has also been obtained by distillation with potash, but is probably formed by decomposition of protein compounds, and does not pre-exist in the drug. A peculiar sugar, mycose, exists in ergot: the presence of lactic, acetic and formic acids has also been prored.

Physiological ACtion.-Extemal.-Ergot and ergotin seem specially irritant to the subcutaneous tissues, when given by hypodermic injection. I have known 5 grains of the latter cause a large livid swelling, with a black centre, rery alarming to the patient, though it does not usually end in suppuration. Latterly such accidents seem less frequent, the preparation probably being purer; still an induration, "more or less sore," may remain for a week or two: less of this is 
experienced if the injection is made deeply into muscular tissue. Five to ten minims of a solution of ergotinin, containing $\frac{1}{50}$ grain in 20 minims, is said not to cause either local abscess or induration (B. M. J., ii., 1882).

\section{Physiological Action.-Internal.-Digestive System.}

-In animals, salivation, thirst, vomiting, and often diarrhoea occur: in fatal cases, inflammatory lesions, and even gangrene of intestines have been found. In the healthy human subject, the symptoms most commonly induced by ergot are colic, pain and spasms in the stomach, salivation, nausea, romiting, a sensation of fulness in the head, painful diarrhoea, occasionally with great prostration, and attended by mucous discharge; of course, doses of 10 to 30 minims and upwards may be given for some days without evident gastric disturbance, but I have seen it occur several times after a few pills containing 3 grains of ergotin, and vomiting, or at least nausea, is certainly not infrequent after such doses as are given during parturition, but Dr. Wood remarks that he has "never seen the fluid extract, even in ounce doses, cause distinct symptoms." The condition of intestinal catarrh above described, and appearances even like those of typhoid fever, have been traced to sphacelinic acid.

Nervous System.-The nerve-symptoms that may be induced by full doses, are such as headache, tinnitus, vertigo, "stupefaction," weight and uneasiness of the limbs, with some staggering of gait; the pupils are commonly dilated. Thxic amounts cause g'eneral spasm, sometimes convulsion, and paralysis, which symptoms are developed to their fullest extent in what is called the convulsive form of "ergotism," epiclemics of which in continental Europe have been traced to eating bread made with ergotized rye. This commences with malaise, irritation of the whole surface of the body, formication, numbness and eoldness of the extrenities, often accompaniod by cramps and by pains in the head and loins. Some time later the digestive organs become affected. There is a sense of tightness and oppression about the epigastrium, heartburn, a feeling of lightness in the head, difficulty in henring, with faintness, twitchings of the muscles of the face, often attender by strabismus, and irregular contractions of the 
joints. These symptoms are generally accompanied by delirium bordering upon mania, with cold sweats; vesicular eruption on the skin, often accompanied with petechix, is also a frequent addition. A ravenous desire for food is often associated with convulsive ergotism.

Kobert, by experiments on animals, distinguished the effects of separate ingredients: thus he found ergotinic acid - given by the skin - narcotize and also depress markedly the reflex and other functions of the spinal cord, lower the blood-pressure, and slow and finally arrest respiration by paralysing the vaso-motor centre in the medulla oblongata. Sphacelinic acid, by contracting the arterioles in the cord, as elsewhere, induced ataxic symptoms, whilst cornutin caused tonic and clonic spasm and convulsions resembling epilepsy; also contractions of involuntary muscular fibre, probably through the central nervous system (op. cit.).

The explanation of the convulsive and paralytic symptoms is not yet clear, but lies, I believe, in the anæmic condition, induced in the nerve-centres; there is much evidence, both from physiological experiment and clinical observation, that such anæmia can be caused by the drug ( $v$. p. 808).

Nothnagel and Rossbach have observed anæsthesin and motor inco-ordination from toxic doses, and general paralysis from still larger doses. The observations of Tuczek are very important, having been carefully made upon 18 out of 500 ergotized patients admitted into Marburg Asylum, and who exhibited a loss of patellar tendon-reflex, paræsthesia, lightning pains, annlgesia, inco-ordination, and apparently all the symptoms of locomotor ataxy; and at the post mortem of several who died selerosis of the posterior columns (columns of Burdach) was found. Exactly similar phenomena could not be produced by experiments on rabbits, but sclerotic acid cansed ataxy in them. (Archiv f. Psychiatrie, Bd. xiii., 1882).

Voluntary muscle is not directly affected by ergot, and Köhler has shown that the function of motor nerves is not impaired, at least by a watery extract. Other ingredients in the drug somewhat stimulated peripheral motor branches, but acted in an opposite manner on sensory nerves; in no seuse sufficieutly to account for paralytic symptoms. 
Muscular System (uterus, etc.).-Upon the smooth or involuntary museular tissue, ergot has a characteristic and peculiar action, stimulating it to contraction, apparently in any part of the body, e.g., in the blood-vessels and the intestines, but chiefly in the uterus. The action on this organ is much more marked at the times of its greatest development, when there is more smooth fibre to be acted upon; it has often caused abortion, both in animals and in women, but does not constantly do so.

Even in the unimpregnated uterus it will often cause contraction and pain, and, on the one hand, according to the case, induce a hremorrhagic flow by its stimulation, or, on the other hand, arrest an excessive loss by closing relaxed vessels. That such action is direct, rather than through the spinal cord, is rendered still more probable by experiments, which show that strong uterine contractions may be caused by the drug after all the nerve-connections of the uterus have been severed (Borrischa). According to Holmes, J. Simon, Rabuteau, and others, the action on smooth muscular fibre, both of the uterus and other parts, is also independent of the sympathetic system. When administered during actual parturition it induces pains, but with the difference that whilst natural pains are intermittent the patient experiencing intervals of rest, those induced by ergot are continuous, and attended by a persistent sense of bearingdown. Ergotinic acid is said to have no action on the uterus, while sphacelinic acid and cornutin cause contraction of smooth muscular fibre, the former producing gangrene by plugging of the vessels following contraction. The two last mentioned cause a rise in blood-pressure (Kobert).

Secretory System.-Most of the secretions, e.g., those of the mammary and sudoriferous glands, are lessened, but the urine is commonly augmented (Rabuteau). Toxic doses, however, induce difficulty of micturition, and a tendency to suppression of urine.

Circulatory System.-In frogs, toxic amounts cause diastolic arrest of the heart, after which it does not react to any stimulus, implying a direct poisoning of the muscular structure; in mammals the action has sometimes been iuduced again by letting blood flow from incisions (relieving congestion-Wood). Under the full physiological action of the drug, the pulse is wealiened 
and lessened in frequency to a moderate extent, but seldom to lower than 60 per minute. According to some observers, toxic doses at first quicken the heart's pulsations.

By an action probably on the muscular coat of the bloodvessels, ergot contracts their calibre; this has been noted, in the frog's web, by Holmes (Schmidt, Jahrb., v. 158); in the limbs and brain-membranes of rabbits by Wernich and others (Virchow's Arch., 1872). On the other hand, vessels in a rabbit's ear, dilated after section of sympathetic, did not contract under hypodermic injection (Vogt). That such vascular contraction is due, not merely to reaction on muscular fibre, but to a "spasm" induced through vaso-motor centres, is plausibly argued by Eberly (Schmidt's Jahrb., Bd. 158), who found the pressure in all the vessels rise very soon after the injection of ergotin, in both cold and warm-blooded animals; in the former, at least, it did not occur after section of the medulla oblongata.

If ergotin, however, be injected direct into the jugular vein, there is at first a great fall of pressure, due probably to the direct depressing effect on the cardiac muscle, overpotvering any increase from contraction of vessels. A similar depressing result occurs after very large doses, and it would seem that "there are generally two periods in poisoning by ergot, one of vaso-motor spasm, and a second of vaso-motor paralysis (Brown-Séquard, Arch. de Physiol., 1870, t. iii.). Coldness of surface is a marked. symptom, at least in early stages.

It is of considerable practical interest to determine whether both arteries and veins really contract under ergotic influence, for Wernich (loc. cit.) asserts a view differing from the one above given, viz., that the veins really are dilated, and contain more blood than normal, and that by consequence, the arteries, receiving less blood, became smaller by a passive collapse; further, that such arterial anæmia determines the contractile movements of the muscular tissue. It seems to me that the chinical evidence we possess of the contractile power of ergot in cases of varicose vein, is against the latter view, and that altogether there is more to be said in favour of the former; still, the question cannot be considered quite decided.

The form of ergotism distinguished from the "mervous" form as "gangrenous," is dependent largely on a lessened bluod- 
supply to the extremities. This commences with a sense of pain and weariness in the limbs, the countenance becomes heavylooking and stupid, and the skin acquires a leaden or cadaverous hue. Afterwards there is malaise, with exhaustion, coldness of the whole body, and numbness of the hands and arms, while formication is perceived in every part of the surface. The lower extremities become affected at the same time as the upper ones; later on, the abdominal muscles are spasmodically contracted, and about the sixth day from the commencement, nausea, vomiting, and diarrhoea make their appearance, with severe colicky pains in the bowels and bladder. In the case upon which these details are founded, upon the fourteenth day two of the children of the family lay as if stupefied: if disturbed, they raved wildly, and complained of pains in the head and limbs; a pruriginous eruption appeared upon the skin, and death occurred on the twenty-first day in violent convulsions.

Bonjean relates a fatal case in which there was pain in the left groin, coldness and pain in the legs, a dark spot upon each calf, formed by an eruption of vesicles, the itching of which was violent, and gangrene of the lower third of each leg. The feet were black and dry; the upper portions of the legs became affected with humid gangrene; in three weeks the sphacelated parts began to separate, and soon afterwards it became necessary to amputate each limb below the knee. Very little blood flowed while the operation was being performed. Symptoms of this kind have been specially associated with sphacelinic acid, which may be shown to induce gangrene in some animals, notably in cocks and pigs: small doses soon affected the combs of the former, making them dry and dark, and after a time gangrenous, so that they fell off: it seemed that in some animals the spasmodic contraction of arterioles was more marked and permanent than in others.

Some further light upon the action of the drug, on the circulatory system, is thrown by an unusual case of poisoning, recently recorded. A nurse, aged twenty-eight, after taking the liquid extract "for several months," to procure abortion, took "two handfuls" of the powder, and was found collapsed and vomiting blood and "red pultaceous fluid ;" she was in a maudlin or stupid condition, with some discoloration over the 
upper part of the body. The skin was pale and cold, temperature $96^{\circ} \mathrm{F}$; the pulse was peculiar, seeming just to touch the fingers and pass so quickly as to be uncountable. Artificial delivery was attempted, but the patient died during the operation. Post mortem, "innumerable small hæmorrhages" were found in the skin, lungs, and mucous membranes : the liver was anrmic (Davidson, Lancet, i., 1882).

SYNERGISTS. - Digitalis, cold, galranism, bromides, possibly arsenic,-are allied in influence on the capillaries: savin, rue, and uterine excitants, in stimulant action on the uterus, which is further aided by iron, whilst the styptic effects are increased by acids, etc.

ANTAGONISTS.-Caustic alkalies, tannins, and metallic salts are chemically incompatible. Tobacco and lobelia to some extent antagonize its action,-and stimulants, especially ethers and amyl nitrite.

\section{Therapeutical Action.-Extemal.-Local Conges-} tions.-The power of ergot to contract small vessels las lately been more utilized than formerly: thus a collyrium containing ergotin with glycerine and water has reliered severe conjunctivitis; Dr. Dobney applies it also in cervicitis and pharyngitis. Hrmorrhoids have been quickly benefited by hypodermic injections into the neighbouring tissue, as well as by suppositories containing 4 grains of ergotin (Dr. Lansing, Record, 1877). Dr. Eldridge applies a solution or plaster of ergotin in gonorrhoea and acne rosacea (Record, 1880), and in congested nose from frost-bite an injection, 1 in 6 , has reliered (Record, 1876).

Erysipelas, etc.-Mr. Millican has dram attention to the value of a solution of ergotin ( 1 in 50) locally in erysipelas of the face, stating that it reduces heat, pain, and swelling. Eczema is also benefited by it, and boils are snid to be aborted. Simple scrofulous, scorbutic, and varicose ulcers have been improved or cured by it.

Varicocele, even when "very extensive," may be cured, it is said, by a single injection of about 2 grains, the needle being 
inserted "so that its point rests amongst the dilated veins, without puncturing them." Very severe pain follows, and there may be faintness; but the effects subside in a few hours, unless great swelling ensues (Bartholow). The same treatment has cured varicose veins in other parts, possibly by inflammatory action.

Hydrocele.-Mr. Walker has recorded cases of radical cure by injection of ergot after failure of iodine, etc. In his first case, which was of long duration, in a man aged sixty-five, 2 drachms of liquor ergotie (Battley) were injected by mistake for tinctura iodi; no pain or inflammation followed, but a complete cure. Dr. Morton, in another case, successfully injected 2 drachms of liquid extract (P.B.), and with but slight pain and inflammation (B. M. J., i., 1883).

THERAPEUTICAL ACTION.-Internal.-In a recent paper Evetzkey summarizes the application of ergot in the following divisions (N. Y. Med. Journ., March, 1882), which may usefully be adopted:-

(1) Disorders of Circulation.-In all forms of hemorhage from any organ, ergot has been found serviceable. I have myself proved its efficacy in purpura, as well as in hæomorrhages from mucous surfaces, in dysentery and enteric fever, in epistaxis, hæmoptysis, and hæmatemesis, even when other means had failed. Very large doses are ordered by some practitioners-1 or 2 drachms or more of liquid extract every two or four hours for many days-and certainly bad results are seldom recorded; but I have seen one case in which gangrene of the hand was plausibly connected with such doses, Igiven for the hæmorrhage of cancer of the uterus. It was the only remedy which had much power in this case, and the bleeding recurred when it was omitted.

Injections of ergotin often give even better results than the internal administration, though with the drawback alrealy mentioned. A very interesting paper by Dr. Curre Ritchie relates a number of cases of hremoptysis, in which the results were striking. Ergotin, from T. and 11. Smith, of Edinburgh (5 grains in 10 minims of distilled water), was subcutaneously injected in seven cases; in an eighth this was found too irritant, and Iangenbeck's formula was successfully followerl, 
viz., 3 grains of ergotin in equal parts of glycerine and rectified spirit. Either one or two injections of 5 grains of ergotin seemed always sufficient to completely arrest the bleeding.

Of the solution of ergotinin (Tanret) I have several times known 5 minims injected under the skin stop severe hæmorrhage from piles.

In menorrhagia, even when connected with fibroid tumours, and other formidable organic affections of the uterus, I have had large personal experience of its efficacy. Dr. Meadows deserves the credit of having pointed out, as long ago as 1868, that sub-involution, chronic subacute metritis, and hypertrophy of the uterus "are alike in this respect, that they are invariably associated with increased vascularity of the organ, though this is mostly of the passive or congestive kind; they are consequently liable to excessive discharges either of mucus or of blood; and they are further characterized by increased bulk of tissue." $\mathrm{He}$ brings forward much valuable evidence to show that these maladies may be successfully dealt with by means of ergot of rye. Leucorrhoea, occurring in the intervals of menorrhagia, is frequently arrested by a decoction of ergot, given by raginal injection. This also conduces to the arrest of the tendency to menorrhagia at the following monthly period.

Aneurism, however, was the disease the treatment of which with ergotin fully aroused attention to the importance of this remedy. In 1869, Langenbeck published a remarkable account of two cases (one probably aortic) successfully treated by the injection of ergotin for many successive days, under the skin covering the tumour. Similar results were afterwards obtained by Dutoit of Berne, and others, and the treatment has since been repeated in various countries, and, on the whole, with an encouraging amount of success. Latterly, however, it has not retained the confidence of the profession-Holmes speaks slightingly of it, and Greenhow calls it "absurd." In a case (popliteal) reported by Mr. Bellamy, it was tried for six successive days without evideut advantage, and then omitted because of sickness and other untoward symptoms (Lancet, 1881); pressure was conjoined. Bartholow, however, speaks of ergot as undoubtedly valuable in aneurisms, especially in such as are beyond the reach of surgery, considering that 
it both slows heart-action and contracts vessels, so promoting coagulation.

In cardiac disease it is sometimes of great service, but the exact indication for it is not yet quite defined by clinical experience. It is said to be unsuited for any cases arrived at the period of asystole, and Meadows reports a case in which attacks of "angina pectoris" followed its administration: we should expect it, à priori, to slow and equalize and regulate the heart-action. In a striking case where cortic obstruction was present, and iron and digitalis had lost power to relieve, the patient being apparently moribund, with dyspnœa, tumultuous heart-action, ascites, œedema of lung, etc.-20 minims of liquid extract every three hours (with 5 of digitalis) quickly effected a change for the better, so that a complete rally took place (Lancet, i., 1881). It is more commonly recommended in dilatation of the heart without valvular disease.

(2) Paresis of any organs containing muscular fibre.-Under this heading may be included deficient uterine action.

Amenorrhea.- Some practitioners frequently prescribe ergot for the restoration of the monthly flow after a long suppression, and where preparations of iron have failed. Neligan states that he has used it in chlorotic amenorrhœea, with the most beneficial results: on the other hand, in cases of threatened miscarriage, when slight hrmorrhage has commenced, accompanied by bearing-down pains, I have often employed this medicine in small doses, or such as are sufficient to partially brace the uterus without inducing uterine pains, with excellent results.

In the use of ergot for inducing premature labour or hastening the process of delivery after it has commenced, the main point to remember is, that the natural passages should be free, or at least readily dilatable. If there be any mechanical obstruction to delivery, the continuous character of the pains is apt to prove highly dangerous, through causing rupture of the uterus, or of other structures. Errgot, moreover, not only induces uterine pains to liberate the foctus, but subsequently promotes the expulsion of the placenta, putting an end to or checking uterine hæmorrhage. In certain cases in parturient women, the death of the child is said to have been caused by the administration of 
ergot; but experience proves that the fault has not lain with the medicine, but with unwise administration; the usual causes of failure and injurious results being either malformation of the pelvic structures, or premature resort to the drug.

- Lead-Palsy.-Another illustration of the stimulant action of ergot on muscle may be drawn from this disorder : a number of cases recovering under its use have been published (Record, 1882), though as it was combined with iodide of potassium no precise deduction as to the value of the drug can be drawn.

Incontinence of uvine from paresis of the sphincter, and, on the other hand, retention from paresis of the muscular coat of the bladder, are both amenable to ergot (Record, 1877). Prolapsus of the rectum from atony and loss of contractile power is beneficially treated by local injections: in some cases these have caused a temporary "spasm of the bladder."

In strangulated hernic it seems also to have been effective, though it is not a treatment to be recommended (Record, 1879). Flatulent dyspepsia, dilatation of the stomach, and constipation depending upon atony of the intestinal muscular fibre, yield sometimes to ergotin.

(3) Tumours. - The uterine growths in which the best results have been obtained from ergot and its preparations are intra-mural fibroids: according to Dr. Percy Boulton, extrauterine growths are little affected by it, and the intra-uterine are better removed by operation (B. M. J., i., 1883).

In the first-named variety it is evident that the remedy can lessen growth both by limiting blood-supply and by stimulating the surrounding fibres to stronger contraction. It is desirable not to give very large doses unless there is free exit (Record, 1879). Of 100 cases recorded by Dr. Byford, twenty-two are said to have been cured (Record, 18\%6). Bell has quoted two or three crucial cases cured in a remarliable manner by vaginal suppositories of 2 to 3 grnins of ergotin, and this certainly has advantages over administration either by the mouth or skin (Lancet, i., 1879). Hildebrandt treated nine cases of fibroid tumours of the uterus by the subcutaneous injection of ergotin. "In four," he says, "the diminution of the tumour was free from doubt; in the others, troublesome symptoms subsided." Delon, in relating a large experience, notes that injections 
into the cervix not infrequently caused faintness, tremor, vomiting, or diarrhœa (Record, 1877).

I have used both the ethereal extract (which Meadows employed) and, by preference, the subcutaneous injection of ergotin. A caution must be given against the employment of the large doses of the latter that have been recommended by several authors. Instead of 4 or 5 grains, which seems to be the quantity ordinarily used, I inject either 1 or 2 grains in glycerine and water, and never inject more than 2 grains in the same place, much preferring to make a second puncture if necessary. I find the smaller doses sufficient for the production of the characteristic action of the drug upon blood-vessels, and upon unstriped muscular fibre generally, and the local effects of the injection are much less severe than with larger doses. A good plan is to inject deeply into the muscles of the shoulder or buttock.

Goitre.-A case of this kind seems to have been vastly benefited by ergotin, in the hands of Dr. Sinclaix Coghill. It was very severe, and an operation was proposed but refused, and from one to three of Savory and Moore's discs ( $\frac{1}{3}$ grain) were injected under the skin sixteen times. From the first, tension and distress were lessened, and, ultimately, considerable reduction of the growth was effected (Med. Times, ii., 1877).

Splenic enlargement.-A case where this was very great, and successfully treated as above described, is recorded by Dr. Miller (N.Y. Record, April, 1876). Vulpian states that the same condition, when occurring in miasmatic and typhoid cases, is also relieved by ergot, but not when due to leucocythæmia.

Prostatic hypertrophy is benefited by it, as shown by Langenbeck and others. The difficulty of micturition is relieved, while the hypertrophy is also reduced.

(4) Abnormal Secretions.-There is a fair amount of evidence that ergot can lessen perspiration when excessive, as in phthisis. Da Costa found 2 grains of ergotin thrice daily very efficient for the purpose, and, comparing it with atropia, notes that it is free from the disagreeable results of the latter, though not so rapid and marked in its effect on the skin (Record, 1882). I have found it useful in the night sweats of phthisis. 
Diabetes insipidus has been cured by large doses given for a considerable time. Dr. Murrell has recorded a marked case, permanently relieved by $\frac{1}{2}$ drachm of the liquid extract given every three hours for some time (B. M. J., ii., 1875). Macaulay has published another (Lancet, $i ., 1882$ ), and these results have been corroborated by several observers. Of saccharine diabetes, Dr. Hunt has reported one satisfactory case under the same treatment (Practitioner, vol. ii., 1880). Acute dysentery has also yielded to it (Record, 1880); but Bartholow remarks that it is best when the chronic succeeds to the acute form, and not in the slowly-developing chronic diarrhœa of hot climates, without acute stage; he gives it with opium or nux vomica, or both. Diarrhœa depending on "nervousness" is benefited by it.

Galactorrhoe is quickly influenced by ergotin, but if pushed too far the medicine will arrest the secretion of milk.

(5) Nerve-Symptoms dependent on Altered Circulation. -Epilepsy, chorea, and some forms of lunacy with congestive attacks, have been successfully treated with it, and in heatstroke it has given good results.

In epilepsy of certain kinds the value of ergot may be considerable, but in practice it has been commonly combined with bromides.

Siemens argues that the induced cerebral anæmia implies hyperæmia of the lower centres in the brain and medulla (a condition which probably exists in epileptics) and that ergot leads to more frequent slight excitement of this part, rendering it less sensitive, and so less liable to epileptic shock (Record, 1882); but the more ordinary explanation would be simply its lessening the amount of blood in the nerve-centres. The same explanation would apply in cases of chronic recurrent mania, in which the drug has been commended by Crichton Browne. In congestive headache and in migraine it has certainly proved useful (Kitchen, Amer. Journ. Insan., July, 1873); and its local injection is said to have been valuable in tic (Record, 187\%).

In certain angeio-meuroses, such as hemicrania due to vasomotor paralysis, and herpes zoster, as well as neuralgias due to inflammation of the sheath of Schwann, although pain is not much relieved, benefit has been obtained. 
In spinal congestions it is perhaps one of our most dependable remedies, and hence it has repute for some forms of paralysis. In epidemic cerebro-spinal meningitis even, and in myelitis, and progressive muscular atrophy, it is relied upon by some practitioners.

Charcot and others commonly give ergot in locomotor atux!, but only for two or three days at a time, and in 2.5-centigramme (nearly 4 grains) doses daily. Grasset notes that if this amount be much exceeded, harm may be done, and quotes the case of a man who was improving, when the dose was increased to 1 gramme daily, and on the second day general paralysis of the arm and of sensation occurred; this passed off on omitting the drug, though the condition of the leg's remained stationary (Lancet, i., 1883).

Sniders and Duboni recommend the drug in ataxic adynamic cases of typhoid fever (Record, 1881). Hayem considers that ergotin has antipyretic properties, superior to quinine and digitalis. The period of defervescence is hastened, and the temperature generally lowered. If a hypodermic injection be given in the afternoon, the evening temperature is lower than that of the morning.

Pertussis.-A similar action on nerve-centres may explain the benefit attributed to ergot in this disorder (Practitioner, vol. xxviii.), but it is not yet generally recognized. It would seem specially appropriate for cases in which epistaxis occurs, and I have so found it.

Acute rheumatism is said to have been cured in many cases by its use. It is asserted that it lowers the temperature and pulse, shortens the length of the disease, and diminishes the probrbility of cardiac complications.

PREParations AND Dose.-Pulvis: dose, 20 to $30 \mathrm{gr}$. infused in boiling water, of which the dregs must also be taken. Extractum ergotre liquidum ( $1 \mathrm{oz}$. to $1 \mathrm{fl}$. oz.) : dose, 10 to 30 min., or more. Infiesum (11 gr. to $1 \mathrm{fl}$. oz.) : dose, 1 to $2 \mathrm{fl}$. oz., or more. Tincture (109 gr. to $1 \mathrm{fl}$. oz.): (lose, 10 to $60 \mathrm{~min}$.

Ergotin (Bonjean's) is a purified arueous extract, soluble in water. Of a solution, $12 \mathrm{gr}$. to $1 \mathrm{dr}$. water, 5 min. are suboutaneously injected for too profuse menstruntion, or $10 \mathrm{~min}$. 
for severer forms of hæmorrhage, the dose being repeated if required in four hours. Ergotin obtained by Wigger's process is a resinoid substance, not crystallized; insoluble in water, alcohol, and acids; soluble in caustic potash.

Ergotin is now purified in Germany by dialysis, but, according to Kobert, this preparation contains ergotinic acid, a principle which does not act on the uterus; moreover, from his researches, which have been detailed previously, it would appear that an alcoholic extract of ergot-after the ergotinic acid has been removed by water-would be the most active preparation, since it would contain both sphacelinic acid and cornutin. This should, however, be tried further before coming to any conclusion.

Misture ergote compositu.-(Liquid extract of ergot, $40 \mathrm{~min}$.; gallic acid, $10 \mathrm{gr.}$; cassia water to 1 oz.-London Hospital): dose, 15 to 30 min., or more.

Ergotinin solution (Tanret): dose, 3 to $10 \mathrm{~min}$. by hypodermic injection.

\section{HORDEUM VULGARE.}

(BARLEXY-MaLt.)

(cf. Malt, p. 908.)

Besides the ordinary use of this as a nutrient (pearl barley) and demulcent (barley-water), it is more largely employed in the preparation of so-called "malt extracts." There are several forms of these- (1) a viscid yellow or brown substance, containing 20 to 30 per cent. of water; (2) a thin solution, containing 60 or 80 per cent. of water; and (3) the same, containing 3 to 4 per cent. of alcohol. Water dissolves, out of the malted grain, albuminoids, phosphates, maltose, dextrin, and diastase, the last an amylolytic ferment, like the ptyalin of saliva, conyerting starch into dextrin and malt-sugar (maltose). The starch must be well cooked to insure a rapid action upon it. The digestive power of diastase is, however, impaired or destroyed by any temperature above $155^{\circ} \mathrm{F}$, and even a lower point than this is 
desirable in the preparation of the extract: hence the expense of the process, which has to be conducted in vacuo with many precartions ; any made at a higher temperature (as in a German process at $212^{\circ} \mathrm{F}$.) contains no active diastase, whatever other nutrient power it may have (Dunstan and Dimmock, Pharm. Journ., March, 1879).

Of the many "malt extracts," Trommer's has a special repute in Germany, Bjorhborn's in Paris, whilst Kepler's (which is made from barley, wheat, and oats), and Maltine (which is also thus made), are largely used in England and America. Our own chemists have now special and good preparations, but of some of them Dr. W. Roberts remarks that "their value as a food has been much exaggerated, and is little more than so much syrup, "- - still they are active as regards the diastase. $\mathrm{He}$ has found, however, equally good results from a cold-water infusion of malt made with 3 ounces of the crushed grain (3 tablespoonfuls) thoroughly mixed with half a pint of water, and allowed to stand for twelve hotrs, then filtered till it passes through bright and sherry-coloured: it has a faint sweetish taste, the odour of beer-wort, is nearly neutral in reaction, of sp. gr. 1025, and is rendered cloudy by nitric acid; its chief. solid constituent is maltose, and it is as rich in diastase as the more expensive "extracts"; it is prone to ferment, and must either be prepared fresh daily, or preserved by adding a little chloroform and keeping from the air. One tablespoonful of this will digest the starch contained in half a pint of gruel, arrowroot, etc., if added to it at a low temperature and stirred in, or a dose may be taken during the progress of a meal (Practitioner, vol. xxiii.).

This preparation is used with the greatest advantage to dilute millk given to infants and young children, and some writers have reported excellent results in malnutrition, etc., from a similar use of a preparation made with hot water; but, for the reasons already given, Dr. W. Roberts's "coldwater infusion" is more likely to contain all the active principlos of the grain. 


\section{FILICES.}

\section{ASPIDIUM FILIX-Mas. (Male Shield-Ferx.)}

\section{LASTR ÆA FILIX-MAS. (Common Shield-Ferx.)}

DESCRIPTION.-A deciduous perennial, abundant in the temperate parts of the northern hemisphere, also in Canada, Mexico, and South America. The subterraneous or radical portion consists of a stout rhizome, 6 to 12 inches long, and more or less clothed with the bases of former fronds, and with brown and chaffy scales, the rootlets descending from between them. The fronds rise to the height of 3 feet-they are nearly erect, with an outward curve, broadly lanceolate, and doubly pinnate; the rachis is very scaly, especially towards the base; the sori, which are abundant, are circular, slate-coloured while young, and disposed in two parallel rows upon the segments of the pinnæ. The rhizome, when dried, is internally of green or yellowish-white colour, slightly odorous, and of a sweetish taste which presently becomes bitterish, astringent, and somewhat nauseous: only the part remaining green should be used in pharmacy.

ACTIVE INGREDIENTS. - A volatile oil, a fatty fixed oil, a resin, tannin, filicic acid $\left(\mathrm{C}_{14} \mathrm{H}_{18} \mathrm{O}_{5}\right)$, crystalline colouring matter, also starch, gum, uncrystallizable sugar, etc. Bock represents the proportion of the fixed oil to be that of 60.0 in 1000 of the dried rhizome, while the volatile oil exists to the extent only of 0.4 . The active principles of the plant, which are probably identified with the oils, or with one of them, are soluble 
in ether. The ethereal extract, termed "oil of male-fern," contains also the resin; it is darkened by the presence of colouring matter, and retains the peculiar odour of the rhizome.

PHysiological Action.-Filix-mas is of very ancient repute as a vermicide, and appenr's to act as a direct poison to the worms, though their expulsion has generally to be accomplished by an aperient: there is, however, a purgative action in the drug, and it often nauseates.

Therapeutical Action.-The chief success of the oil of male-fern has been in connection with tape-worm, and especially Bothriocephalus lutus. Its value in such cases can hardly be over-estimated, and the smallness of the dose required is an advantage. The medicine should be taken upon an empty stomach, and after an interval (say from five to six hours) it should be followed by a gentle laxative. If the alimentary canal be occupied by food, the medicine is too much diluted to be operative, concentration being always very important in regard to vermifuges. A dose of castor oil the night before taking the medicine is often preferable to a laxative subsequently.

PReparations and Dose.-Pultis: dose, 60 to $180 \mathrm{gr}$. Extractum: dose, 15 to 30 min., or $1 \mathrm{fl}$. dr., or sometimes, with caution, more: it may be given with mucilage or peppermint in millk. Of filicic acid, dose, about $30 \mathrm{gr}$. 


\section{LICHENES.}

\section{THE LICHEN FAMILY.}

THE lichens constitute a large order of the section Thallogens of the Cryptogamia. They are either membranous, or chartaceous, or filamentous, always of dry texture, and often pulverulent. The main body of the plant consists of a thallus, and the fructification is produced, ordinarily, in apothecia. Lichens occupy every kind of habitat except water; they are universally distributed, and have no deleterious qualities.

\section{CETRARIA ISLANDICA.}

\section{(Icelann Moss.)}

DESCRIPTION.-This plant consists of an erect, foliaceous and tufted thallus, in colour olive-brown, and having the segments laciniated. The height is 3 or 4 inches; the apothecia are dark brown, flat, and appressed. It grows very abundantly upon the surface of the ground, in exposed situations in high northern latitudes, and received its vernacular name through confusion with the genuine mosses. It is devoid of odour, but possessed of a bitter taste, and in substance is mucilaginous.

ACTIVE INGREDIENTS.-It contains about 80 per cent. of farinaceous matter, which is resolvable into lichenin, or lichen-starch, $\mathrm{C}_{12} \mathrm{H}_{20} \mathrm{O}_{10}$, and the starch-like body called inulin. 
The former is insoluble in alcohol, ether, and cold water, in which last it swells up; but is soluble in boiling water, gelatinizing on cooling. Iodine turns it blue, whereas inulin remains unaffected.

The bitter principle resides in the cortical portion, and is called cetraric acid, $\mathrm{C}_{18} \mathrm{H}_{16} \mathrm{O}_{s}$. When pure it forms acicular white crystals, which are intensely bitter, and almost insoluble in water, though when boiled in water they communicate to it a bitter taste. It is sparingly soluble in alcohol and ether, readily in alkaline solutions, forming soluble compounds. The proportion of acid present is about 3 per cent.; there are found also a little gum, extractive, lichenostearic acid, $\mathrm{C}_{14} \mathrm{H}_{34} \mathrm{O}_{3}$, uncrystallizable sugar, etc.

Alcohol has been obtained from the gilucose of lichenin.

Physiological Action.-Iceland moss, when deprived of its bitterness by prolonged maceration in water, becomes a nutritious food, and is employed as such by the Icelanders. It is demulcent and tonic,-not stimulating or astringent. The bitter principle is said to affect the milk of nursing women (Stillé).

Therapeutical Action. - I reland moss, having no properties that can be strictly called medicinal, takes its place simply with the light and farinaceous foods that often become useful for invalids, the bitterness being first removed by, steeping the plant in some alkaline solution.

It has been recommended for disorders of the stomach, such as dyspepsia and chronic dysentery; also in affections of the respiratory organs, as phthisis and chronic catarrh.

PREPaRATION.-Dcroctum: dose, ad libitum. 


\section{ROCCELLA TINCTORIA-and other Species.}

\section{(Litmus.)}

The Roccellas, of which there are several different species, grow chiefly upon maritime rocks, in warm and sub-tropical countries. In commerce, they are collectively called orchellaweeds. The thallus consists of many round, simple, glaucous, and often nochulose segments, which spring from a common base, and are either erect or pendulous. The length of the segments varies from less than an inch to several inches. The apothecia are scutellate, and normally lateral.

The fine indigo-blue preparation, familiar under the name of litmus, occurs in the form of small rectangular cakes, friable, and is granular in substance.

\section{FUNGI.}

\section{AGARICUS ALBUS.}

(White Agaric-Larrch Agartc.)

(Not Officinul.)

DESCRIPTION.-A fungus without stalk, conical, of grey or yellowish colour externally; "the hymenium on the lower side is in the form of small yellowish spores": it is met with in peeled irregular pieces as large as a closed hand, white, light, 
friable but not easily powdered: it has a faint odour, and a taste at first sweetish, afterwards acrid and bitter. (This drug must be distinguished from the fly agaric, Amanita muscaria.)

\section{ACTIVE INGREDIENTS.- (1) Boletic or fumaric acid (which} has also been found in other fungi); (2) laricin, $\mathrm{C}_{14} \mathrm{H}_{48} \mathrm{O}_{2}$, a white, bitter powder, which forms a jelly with hot water; (3) agaric acid, which crystallizes in needles, soluble in alcohol, not in water; (4) two resins; and (5) agaricin, which may be obtained as a white, crystalline powder, sparingly soluble in water, and of sweet, afterwards bitter, taste. These results of various chemists are open to further inquiry.

Physiological Action.-Large doses cause irritation of the gastro-intestinal tract, with watery diarrhœa, colic, and prostration, sometimes nausea and vomiting. Small doses, on the contrary, have an astringent effect.

Therapeutical Action.-Hyperidrosis.-For a long time agaric has had some repute in the treatment of this symptom, especially when occurring, as it commonly does, in the later stages of phthisis. More recently, Andral, and also Rayer, prescribed it with advantage, generally in 2- to 3-grain doses (the former gave even 40 or 60 ) ; but any dose above 2 grains was liable to cause diarrhoea (Bull. Gén. de Thérap., 1879). Wolfenden speaks well of the remedy (Med. Times, ii., 1881); also Young, who used the tincture in the Glasgow Infirmary under Dr. Gairdner (Glasgow Med. Journ., March, 1882). But the most complete study on the subject is that of Dr. Murrell, who analysed upwards of sixty cases, mainly of phthisis (Practitioner, vol. xxix.). Pills containing 3 grains of the powdered agario did some good, but slowly-in two or three days' time; larger doses ( $\frac{1}{2}$ drachm) of the powder proved disagreeable, and not easily administered- $\Omega$ pill of the extract answered better: advantage was also obtrined from the tincture, and the liquid extrnct, which is stronger,--but on the whole the author concludes that the drug is not equal to atropin, or even to Dover's powder. In nine cases in which I have given agaric in 20 - to 30 -grain doses for night-sweat- 
ing, no special effect has been obtained. Seifert (Wiirzburg) after many trials, concludes that pills containing 5 milligrammes of the alkaloid, administered about five hours before the intended time of the operation, give the best result in checking night-sweats. As the remedy requires increase in dose, it is well to begin with half the amount mentioned: cough is quieted by it, and sleep promoterl.

Preparations and Dose.-Pullis: dose, 2 to $30 \mathrm{gr}$-average, $10 \mathrm{gr}$. Extractum: dose, 3 to $6 \mathrm{gr}$. in pill. Tinctura (1 part in 10 of proof spirit): dose, 20 to 60 min. Agaricin: dose $\frac{1}{12}$ to $\frac{1}{6}$ gr. in pill.

\section{AMANITA MUSCARIA-AGARICUS TEL FUNGUS MUSCARIUS. (Fly Agaric.)}

\section{(Not Officinal.)}

DESCRIPTION.-A fungus indigenous to Europe, growing mostly under pine trees; some species of the same genus are edible, some poisonous. This one has a white stalk, several inches high, with a convex scarlet-coloured pileus, having white outgrowths above, and white "gills" beneath: of mplensant odour and acrid taste. It is found in Europe, Asia, and America; and grows very abundantly in Kamschatka, and is there used in the same manuer as wine, brandy, arrack, opium, etc.

ACTIVE INGREDIENTS. - It contains-(1) a crystallizable acid, soluble in ether; (2) a brown amorphous substance, amannitin, insoluble in ether, soluble in water, and tasteless; (3) a poisonous alkaloid, musearin, separated by Schmiedeberg and Koppe (1869), and presumed to be the chief ingredient. 
ABSORPTION AND ELIMINATION.-The active principle is readily absorbed, and is largely eliminated by the kidneys, so that the characteristic symptoms of its physiological action may be produced by the urine of persons who have taken it.

\section{Physiological Action.-Digestive System.-Even} moderate doses cause burning in the fauces, csophagus, and stomach; increased secretion of saliva; dry, sore, and coated tongue; nausea and a tendency to vomiting, with saltish taste; flatulent eructations, and hiccough; more or less pain in the bowels, increased peristalsis and intestinal secretion, with frequent and liquid evacuations and burning at the anus.

Circulatory System.-Muscarin has a pronounced and characteristic action on the circulation; even a minute quantity, if applied to the exposed heart of a frog, arrests it in diastole, but without destroying its muscular irritability, for contractions may be re-excited by stimuli: section of the vagi does not alter the effects, which are commonly attributed to stimulation of the intracardiac inhibitory apparatus (Prévost, Bull. Gén. de Thérap., xciii.). The heart-action of larger animals is reduced sometimes from 120 to 40 or even 9 beats per minute (Ringer). The smaller blood-vessels, which are said by some observers to contract at first, soon dilate, retain more blood than normal, and simultaneously the arterial pressure is lowered. In man it produces palpitation of the heart, with a feeling of anxiety, and under its continued influence the pulse becomes irregular and intermittent. Doses of $\frac{1}{3}$ graiu were found by Ringer to affect the healthy pulse but slightly, though they commonly lowered the frequency of an extra quick one. Stille's statement is that "in manmals, large doses increase the frequency of the heart's pulsations, whilst limiting their power."

Respiratory System. - On this system the action of muscarin is remarkable, and has been well explained by $D_{x}$. Brunton: others before him had noted intense dyspnoea as it symptom of its action, but he demonstrated on the opener thorax of a nareotized rabbit that so strong a contraction of pulmonary blood-vessels was produced by it, that the lungs became blanched in colour, and then the right side of the heart became largely distended, whilst scarcely any blood reacherl 
the left side; on administering a suitable dose of atropia the normal condition was seen to be quickly restored, and the dyspnoea ceased (B. M. J., ii., 1874). Cough occasionally accompanies its physiological effects, and oppression of the chest is a common symptom.

Nervous System.-According to the observations of Högyes on the lower animals, muscarin depresses the functional power of the central nervous system, and in advanced stages of its action causes paralysis; it also slowly lowers the excitability of the peripheral nervous system, and of smooth muscular fibre: the dilatation of vessels he traces to a paresis of the vaso-motor centre (Lancet, i., 1883). The pupils are contracted under the internal administration of the drug, but dilated by its local application; the sight is not specially affected. A state of rather lively and cheerful intoxication may be produced by the agaricus; and a case is on record of a Calcutta barrister exhibiting such a condition in court, with bursts of laughter, etc., after partaking of a dish of the mushrooms, - " every object appeared to him beautiful" (Chevers, B. M. J., ii., 18rt): headache is not complained of. The symptoms deseribed in fatal cases are, however, much more serious, and are such as anxietas, passing into wild delirium; thirst, colic, purging; dyspnoea, small irregular pulse, and cyanosis; twitching of muscles; and death.

Secretory System.-It has already been mentioned that free salivation is commonly produced by this drug; ; but perspiration is still more usual. Both effects may occur together, or one to a much greater degree than the other. The sweating was free in eleven out of thirteen cases observed by Drs. Ringer and Murrell with a $\frac{1}{2}$-grain dose; profuse with $\frac{3}{4}$-grain to 1-grain,as much as with $\frac{1}{3}$ grain of pilocarpin: Inchrymation occurred iu eight of the cases.

It is remarkable that the urine is lessened in amount, and even sometimes suppressed by it (Prévost): some temporary irritation of bladder has been noticed, as with pilocarpin.

SYNERGIST.-Pilocarpin. 
ANTAGonisTs.-Atropia, digitalis, eserin. Atropia antagonizes all the effects of muscarin; starting a stopped heart, relieving dyspnœa, etc.

Therapeutical ACtion.-Hyperidrosis.-It is in this disorder, and especially in the night-sweats of phthisis, that this, like the last-mentioned agaric, has been most used in medicine : Trousseau, Andral, and more recently Professor Peters, have reported good results; but the observations of Murrell do not give it so high a place as many other drugs. Five minims of 1 per cent. solution of extract of muscarin generally, but not always, relieved the sweating, if given several times (Practitioner, vol. xxv.).

Other uses of the drug are not well established, but Bartholow suggests that, according to its physiological effects, it ought to be valuable in constipation with impaired muscular power of intestine and lessened secretion; in catarrhal jaundice, hayasthma, pulmonary hæmorrhage, and in diabetes. It is contraindicated in pulmonary disorder with excessive secretion.

Preparation and Dose.-Muscarice nitras: dose, $\frac{1}{3}$ to $\frac{3}{4}$ gr. may be given hypodermically. Extructum muscarice: dose, as above. 


\title{
ACIDUM CARBOLICUM.
}

\author{
(CARbolic Acid.)
}

\section{Phentc Acid, $\mathrm{HC}_{6} \mathrm{H}_{5} \mathrm{O}=94$ - Phenic Arcohol-Phenol- Hydrate of Phenyl.}

Discovered by Runge forty years ago. In 1861 it was still made in fractions of an ounce, met with only in chemical laboratories, and priced at $£ 5$ the kilogramme (about $2 \mathrm{lbs}$.).

PREPARATION.-It is commonly prepared from coal tar by fractional distillation: it requires to be carefully purified. In the vegetable kingdom it has been found not only in coal trr, but also in certain plants (e.g., the Andromeda Leschenaultii, in the Neilgherry hills), the essential oil of which consists mainly of methyl-salicylic acid (Broughton, Pharm. Journ., October, 1871); also in benzoin and quinic acid. Hoppe-Seyler reports its extraction from the Isatis tinctoria, which contains indican (Pflüger's Archiv, Bd. v., 1872). Of still greater interest is its alleged detection in the normal urine of cows and horses, and in small proportion in that of dogs and men (Städeler).

Buliginsky pointed out that it could not be present in wine in a free state, and thought it was probably in combination with alkalies, for it was not detected except after acidulating. Hoppe-Seyler concluded that it is not normally present, but is produced by the action of sulphuric acid on urinary constituents (chiefly indican) and subsequent distillation. More recent observations by Brieger and Salkowski go to prove, howerer, that phenol (carbolic acid) is really a normal constituent of the urine, varying in amount proportionally to the decomposition of albuminous substances in the bowel (Centralbl. f. Med. Wiss., 30, 1878). Brieger distilled the urine with sulphuric acid and precipitated with bromine water, and calculated 
the average excretion of healthy persons at 0.0158 gramme: in gastric cancer and in typhoid fever it was somewhat increased, in peritonitis markedly so; in phthisis it remained about normal. In septic, conditions the greatest increase took place, as instanced by a case of gangrenous empyema.

Indican is formed from indol, which again ariginates from the decomposition of albuminous substances in the bowel; but the relation between this and phenol is not constant. In peritonitis both are increased; but in some other disorders, as anæmia, indican is increased, whilst phenol is lessened in amount. Brieger obtained phenol also by aating on putrid pus, distilling it with sulphuric acid. Whether the excess occasionally noted as above be due to increased production in the body, or, as Salkowski suggests, to failure of the tissues to destroy it in its passage from the place of its origin to the kiclneys, it is impossible to say. Phenol has also been obtained by distillation of eastoreum.

CHARACTERS.-Absolute phenol, i.e., perfectly pure carholic acid, occurs as a fine crystalline powder, readily soluble in 20 parts of water at the ordinary temperature. It is less irritant, and has a less disagreeable and persistent odour than the common commercial acid. The latter, when fairly pure, forms colourless needle-shaped crystals, which tend to deliquesce and become fused together, and after keeping or exposure change to pink or brown in colour. A still more impure form of the acid occurs as a dark brown liquid. A trace of moisture suffices to liquefy carbolic acid crystals, but they do not actually dissolve in less than 20 parts of water, a solution which is saturated (Sansom): in alcohol, ether, glycerine, and strong acetic acid, they are soluble in all proportions: solubility in orlinary glycerine distinguishes the drug from kreasote (v. p. 868). Carbolic acid melts at $95^{\circ}$ to $106^{\circ} \mathrm{F}$. (according to purity), forming an oily liquid of tarry odour and corrosive smoky tasto, which leaves on paper a transient greasy stain, has a sp. gr. of 1.065 , and a boilingpoint of $369^{\circ} \mathrm{F}$.

Though commonly called an acid, and cajable of forming carbolates by combination with alkalies, etc., phenol is not 
itself acid in reaction, nor does it decompose carbonates. The ordinary form often contains an homologous substance termed cresol, which does not crystallize, and is more irritating in its properties than the pure acid, but contact for some days with chloride of calcium changes some of the cresylic products into pure carbolic acid, which may then be crystallized out at a low temperature (Gladstone).

TESTS.-(1) A chip of deal dipped into the concentrated acid, and then into hydrochloric acid, becomes of a greenishblue colour on exposure to light. (2) Solution of perchloricle of iron added to a mixture containing carbolic acid changes it to a violet-blue colour. (3) If to the solution of the acid about one-fourth its bulk of liquor ammonire and a small lump of chloride of lime be added, a pale blue colour is produced on heating: this is a very delicate test. (4) On the addition of excess of bromine water to a solution of carbolic acid a flocculent yellowish precipitate of terbromo-phenol is thrown down. This test will detect 1 part of acid in 40,000.

It must be observed, however, that tests for carbolic acid in animal fluids have sometimes failed, even when its presence is evident to the smell.

ABSORPTION AND ELIMINATION.-The power of carbolic acid to coagulate albumen does not prevent its absorption from any tissue of the body, for proof of which we need only refer to the serious and even fatal examples recorded. At a time when carbolic dressings were used in full strength and applied directly to wounds, general toxic symptoms not unfrequently followed, on account of absorption from the wound, or, as some maintain, more through the sweat-ducts of the neighbouring skin (Langenbeck, Zeits. f. Chir., ix., 356). Even from a lotion of 1 in 50, applied after excision of the elbow, and covered with oiled silk, vomiting and partial collapse have occurred more than once (B. M. J., i., 1870).

Sudden collapse and death have followed from frictions with 1 or 2 ounces of commercial acid (Machin, Hoppe-Seyler); and a child that upset over itself about 1 litre of a 30 to 40 per cent. mixture died collapsed in twenty-four hours (Med. Times, ii., 
1879). A fatal result has even followed from painting with carbolic aoid a scalp affected with favus (Deutsche Klinik, 1869, xix., xx.). Absorption from the pleural cavity may be illustrated by a case of empyema washed out with a watery solution, of which some was on one occasion retained, and gave rise to a semi-comatose, collapsed condition (Broadbent, B. M. J., ii., 1872). Husemann considers that absorption by the pulmonary mucous membrane is not very pronounced, but Ringer has known the inhalation of 10 minims cause giddiness and a sense of intoxication. Machin records that all present in a room where carbolic frictions took place were affected with vertigo, headache, etc.; and persons engaged in disinfecting cattle have shown the effect of the fumes by giddiness, stupor, and convulsion (B. M. J., ii., 1872).

Absorption from the rectum is remarkably rapid and complete: an enema (containing only $\frac{1}{4}$ litre of a 1 per cent. solution diluted with warm water) injected in a case of diarrhoea caused tinnitus, giddiness, and partial collapse (B. M. J., i., 1879). This is exceptional, for the same quantity of a solution of 1 in 60, and even 1 in 40 , has been often used as an injection for ascarides; but similar symptoms, less in degree, have occurred from such injections. In a child of three years, an enema containing 5 per cent. was almost fatal; and death with carbolic symptoms after one of 3 per cent. was the subject of a criminal trial in Berlin,- - the patieut suffering from diarrhoea and lung-inflammation at the time (B. M. J., ii., 1879). Absorption from the uterus and even from the vagina (to judge by recorded cases of its use to these parts) does not seem quite so active; and the two cases of renal suppression and coma recorded by I)r. Edwards do not prove the contrary (Practitioner, vols. i.-iii.).

The exact condition in which absorbed carbolic acid circulates is not yet verified: part at least of ordinary doses seems to be oxidized in the system, with formation of a greenish or darkcolourer compound, which often appears in the urine; this is noticed more frequently after external use (which involves exposure to, and oxidation by, the atmosphere), and develops sometimes after the urine has been passed and set to stand (Joseph; Ferrier). Oxalic acid is another product of the 
oxidation of phenol, and has been detected in the urine of animals taking the latter. Certainly some phenol circulates unchanged, and Tortora asserts that the greater part of what is taken passes as such in the urine (Record, 1877).

Patrouillard has indeed recovered some of the pure acid from the urine in a case of poisoning, though the observations of Salkowski and others are opposed to this, since they could not obtain it by distillation without previous acidulation with sulphuric acid; its passage as a carbolate united with the alkalies of the blood has been suggested, though not proved. Küster traces a chronic form of carbolism accompanied with anæmia and dyspepsia to the drug impoverishing the tissues by absorbing sulphuric acid from them. In experiments on dogs, not more than half the amount given could be detected in the urine; none in the freces.

Elimination is moderately rapid. Of small doses given every quarter of an hour, Tortora found traces in the urine two hours after the first administration. Salkowski, giving $1 \frac{1}{2}$ grain thrice daily, obtained the reactions of carbolic acid in the urine on the following day; he remarks that the amount contained is not proportionate to the intensity of the coloursometimes when this was well marked there was no chemical proof of the presence of the acid. This observer, and also Hoffmann, failed to detect it in the breath, but there can be no doubt that it is sometimes excreted by the pulmonary mucous membrane. Lemaire detected it, and considered that the greater part of what was taken up by the stomach was eliminated by the lungs; in many toxic cases the odour of the breath has been distinctive. Hoppe-Seyler found it in the saliva of dogs, the abdomen of which had been painted with the acid; also in the different organs-brain, liver, and kidneys - of animals poisoned by it (Pflïger's Archiv, Bd. v.). E. Labbé, relying on some therapeutical results in cutaneous diseases, and on changes in the slin of frogs, believes that elimination occurs to some extent by the skin.

In many experiments the acid could not be detected in secretions on the day after its last administration, so that the elimination, of medicinal quantities at least, seems to be completed within twenty-four hours. This negatives any idea 
of a "cumulative action," and implies also that if life can be prolonged for that period, the system may clear itself of the poison. Binnendijk has noticed that improvement has often occurred suddenly (B. M. J., ii., 1879).

Physiological Action.--External.-The strong acid acts as a caustic, and if applied to the sound skin causes burning pain, and white coloration of the part, which becomes surrounded with a red areola of congestion. The pain passes away in a moderate time, but irritation may persist for days; the white colour changes to dark or red, and desquamation or slight exudation follows: prolonged application does not blister, but mummifies and leaves a sear. The part subjected to it quickly loses ordinary sensibility, and feels numbed-so much so, that Dr. Bill (who observed this fact about the same time as Professor Wilson) was able, under its influence, to lay bare his own radial artery without feeling any pain (Amer. Journ., Oct., 1870). The anæsthetic, and at the same time destructive, properties of the acid are such, that painless local gangrene has followed the continued application of strong solutions (B. M. J., i., 1873). Mucous membranes are more or less protected from the caustic effect of the acid by its coagulating albuminous secretions on their surface. It causes some burning in the mouth and in the vagina, but little or none when applied to the uterus.

Egg-albumen is not coagulated by solutions of any strength under 5 per cent.: the compound, if formed in the cold, is not a chemical one, for the acid can be removed by washing, and then decomposition occurs as usual, but under strong heat there is formed a congulum which cannot be thus mechanically separated. With globulin similar results are obtained.

\section{Action on Lower Organisms, Putrefaction, etc.-} Lemaire demonstrated (1865) that carbolic acid, even when much diluted, was fatal to lower organisms, both animal and vegetable, and that it prevented yeast fermentation and putrefaction. Sansom, whose work on Antiseptics (1870) has never been sufficiently acknowledged, made more detailed experiments, with similar results. 
With reference to the destructive action of the drug on the lower forms of animal life, Dr. John Dougall concluded it to be less destructive than strychnia, corrosive sublimate, and some other agents (Lancet, ii., 1870); whilst Dr. Crace Calvert, judging from the development of fungi and vibriones in solutions of albumen, held that carbolic and cresylic acids were the only agents that completely prevented it: his observations are, however, somewhat deceptive. Dr. P. C. Plugge placed drops of complex infusions-i.e., of hay, of stagnant water, and of intestinal secretion-in contact with drops of carbolic solution under the microscope, and found that 1 per cent. sufficed to stay the movements of the larger infusoria (paramœcium and colpoda), immediately, of vibriones in a few seconds, of monads in a few minutes: 1 part in 200 acted in the same way in a longer time; 1 in 500 required nearly an hour to destroy the lower organisms; 1 in 1,000 was fatal to the higher forms in fifteen minutes, but not to the lower ones within the hour. ${ }^{1}$ As it is with the development of these latter - vibrio, bacteria, monas-that decomposition is more definitely connected, we conclude that a strength of 1 per cent. is the weakest that can be depended upon for practical use: this sterilizes Cohn's solution when swarming with microzymes. According to some observers, decomposition continues sometimes after destruction of organisms, for this may be effected by $\frac{1}{2}$ per cent., whilst 2 per cent. is needed to prevent completely the putrefaction of albuminous substances (Hoppe-Seyler).

If the carbolic solution be not strong enough to clestroy infusoria already formed, it will at least prevent fresh formation. With regard to the action of the acid on fungi, Manassein found $\frac{1}{16}$ per cent. prevent their germination, and Plugge, making comparative experiments with paste, ascertained that 1 per cent. entirely prevented the derelopment of mould. Dr. Parkes, however, observed that on passing air impregnated with carbolic acid over fungi formed upon organic matter, they "became discoloured, brownish, and

${ }^{1}$ Bacterium termo and Monas crepusculum are held by Pasteur to cause putrefaction; Hallier considers them of plant-nature, and names them Leptothrix; their movements are distinguished from the "molccular morements" of Brown, which are not influenced by acids. 
apparently dead; but revived on readmitting washed air" (free from carbolic acid).

Von Aukum also found that air, when mixed with carbolic vapour, did not hinder the development of lower organisms in complex infusions; and vaccine lymph exposed to carbolized air has preserved its vitality. Hence a probable conclusion, that the agent, unless in great strength, acts rather by preventing changes in germs, "by freezing as it were the living cells, which may be again thawed into life on withdrawal of the acid," and that, as experience has corroborated, it is not very suitable for aërial disinfection. Pettenkofer has stated that the acid preserves inert ferment-cells, but they resume their activity on withdrawal of its influence (Allgem. Zeitung, 1866), but certainly in a solution of sugar mixed with yeast, fermentation is prevented by a strength of 4 per cent.; and in a mixture of sugar and milk the precipitation of easein, and formation of carbonic acid gas, etc., is prevented by half that strength. Husemann states that simple lactic acid fermentation is stayed by 1 part in 265 .

It has been said that the chemical changes induced by myrosin, sinapin, ptyalin, pepsin, etc., are not prevented by carbolic acid, but recent observations show that most of them at least are controlled by a sufficient strength, or a special manner of using it (Bucholtz): thus, if starch be mixed with saliva it becomes changed into sugar, and the addition of carbolic acid in moderate strength will not interfere with the process; but if the acid be mixed with the saliva some time before starch is adder, then no change occurs (Hoppe-Seyler) : the reactions of myrosin and sinapin are prevented only by the strong acid: the action of pepsin does not seem hindered by any medicinal dose, but in the laboratory artificial digestion of albumen is hindered by a sufficiently strong solution ( 1 in 200-Plugge).

Of practical surgical interest are, the statements that healthy pus is preventerl from putrefying by a streugth of 1 per cent. of carbolic aoid. The virus of charlom and of infoctive inflammation generally has been negatived by the sime strength, though Dr. Baxter concludes that no virulent liquid ean be considered disinfected by carbolic acid unless it contains at least 2 per cent. (by weight). Vaccine virus with 1 per cent. of acid 
was uninfluenced in its inoculability; with between 1 and 2 per cent. the results of inoculation were irregular; but with 2 per cent. its infective power was destroyed with certainty. The same proportion (2 per cent.) destroyed the infective power of glanders virus, but 0.5 per cent. did not impair its virulence in the least. Pus, even when in such condition as to cause septicremia if injected, is rendered aseptic by 5 per cent. solutions, so that it will no longer infect the system when injected, though producing perhaps a local abscess. Pus actually putrid is not disinfected by a strength of 5 per cent. (Rosenbach, Centralblatt, 1873).

\section{Physiological Action.-Intemal.-Nervous System.} -The most pronounced effects of carbolic acid are exerted on this system. If 3 to 4 grains of a watery solution ( 1 in 20) be injected under the skin or into the stomach of a frog, it shows signs of irritation, and in three or four minutes becomes quiet and drowsy, remaining with the limbs drawn up; slight twitchings soon commence, reflex excitability is increased, volurtary movements become difficult, convulsions set in strongly and, like those caused by strychnia, without any evident external cause: this stage may last several hours, but sooner or later reflex action is lessened, and paralysis and death follow within twenty-four hours. Such is Salkowski's description of the phenomena; and after death he finds the nerves and muscles still excitable by the induced current, though less so than normal. In rabbits and in dogs the tremors and the convulsions are still more marked, and death occurs sooner (the doses being proportionate). The mode of production of the convulsions has been much discussed; that they are not peripheral in origin. appears from their occurring in a limb after ligature of its main artery, or division of all its tissues except the main nervetrunk, whilst they cease on section of this; that they do not arise in the cerebrum seems evident from their continuance in decapitated frogs; and that they are spinal is concluded by Salkowski from finding that they continued in the hind legs after division of the cord at the junction of the fifth and sixth dorsal vertebro. Bert and Jolyet, Plugge, and most other' observers agree with him on this point; but Professor H. C. Wood (relying, apparently, on some experiments by Labbé and 
by Haynes) inclines to trace the source of convulsion to the base of the brain. In a recent investigation, Dr. Sumner Stone corroborates the former observers: larger doses (15 grains to 1 cubic centimetre) injected into the dorsal lymph-sac of a frog caused immediate paralysis of the posterior, increased excitability of the anterior, extremities; with smaller doses after ligature of the vessels, convulsions equally followed, sensory nerves not being palsied, but rather heightened in function. Dividing the cord on a line with the anterior extremities, he found convulsions occur equally in the posterior, and states, "as a clearly proven fact, that the convulsions are spinal in their origin," and that "it is probable that the spinal action of carbolic acid is confined to the motor column." He further tested the effect on reflex action according to 'Türck's method, i.e., by removing the cerebrum in a frog, and when the shock had passed off, suspending the creature by the nose, and noting the time it would keep the foot immersed in a dilute solution of sulphuric acid before withdrawing it - such time representing the interval before the development of reflex action. In one frog, for instance, ten minutes after the operation, reflex action showed itself in fifteen seconds; twenty-five minutes after, in twenty seconds; - then 0.5 cubic centimetre of a 5 per cent. solution of carbolic acid were injected into the abdominal wall: after thirty-five minutes, no reflex action in sixty seconds; forty-five minutes, action in sixty seconds; forty-eight minutes, action in thirty-eight seconds; fifty-five minutes, reflex action in two seconds, convulsions; sixty minutes, reflex action in one second. From this and similar experiments, Dr. Stone concludes that carbolic acid, when the medulla is intact, first inhibits reflex action; that afterwards this becomes highly exalted, and ultimately quite abolished. When, however, the medulla is inchuded in the section, instead of the primary fall there is a sudden and extreme rise, and he attributes the inhibition to stimulus of a "break-centre" (Setschenow's), believed to preside over reflex action; the abuormal rise he traces to palsy of this centre (Thesis, Philad. Med. Times, Sept., 1879). In man, excitation of the spinal system is by no means so evident; the symptoms are more distinctly cerebral, and afford noore support to Professor. Wood's view, that carbolic acid acts 
upon centres at the base of the brain, than do any experiments on animals. This appears even from moderate doses, and becomes marked in cases of poisoning. Dr. Bill, taking 6 to 8 grains in water, besides local effects of numbness in the mouth and uneasiness of stomach, noticed vertigo, tinnitus, and deafness, especially if the stomach was empty (Amer. Journ. Med. Sci., July, 1872). Similar effects have followed from enemata containing 1 part in 40, and even 1 in 100 (B. M. J., i., 1879). Confusion and heaviness of head, with weakness of limbs and excessive sweating (paralysis of sympathetic), have been noticed from doses somewhat larger than 8 grains, and Danion, with 15 grains, found marked deafness : after 30 grains this deafness lasted half an hour, accompanied by tinnitus and by general formication; 60 grains taken in three doses caused, in addition, quivering of muscles in the calf of the leg. Some degree of tolerance may be established by the continued use of the acid, so that 15 grains may be taken without inconvenience, at least by men ; 6 or 7 grains by women (Husemann). Tolerance is said to be more easily established in habitual drinkers (Fuller). One or two ounces caused symptoms like "those of apoplexy"complete uneonsciousness, stertorous breathing with puffed-out cheeks, and absence of all voluntary movement; the pupils at first contracted, afterwards dilated moderately,-but consciousness never wholly returned, and death occurred in thirteen hours: the blood was clotted in the longitudinal sinus, and the pons and medulla were intensely congested (Ogston, B. M. J., i., 1871). In Mr. Machin's cases, and again in those of HoppeSeyler, the two who recovered described-immediately after the smarting caused by rubbing-in the acid-a sense of tightness, headache, and giddiness, "as if drunk," before falling unconscious. In Dr. Taylor's case, a man swallowed about an ounce of (impure) acid, and directly fell; in two minutes was totally unconscious, and in another mimute dead (Philad. Med. Times, vol. ii.).

In none of these cases were there any convulsions, but they have occurred in a clonic form, and accompanied by spasn of the diaphragm in children, who are more sensitive to the drug than adults: women and weakly subjects are also more readily affected than men; and besides the headache, giddiness, and 
tinnitus, there may be excitement ("like that produced by alcohol"-Lemaire) preceding anæsthesia, paralysis, and unconsciousness. Hoppe-Seyler compares the effects to those of chloroform and other anæsthetics. The collapse, which is pronounced in toxic cases, may be explained as secondary to intense gastric irritation, rather than as a primary nerve-phenomenon.

The Blood and Circulatory System.-When treated with carbolic acid, outside the vessels, or by direct injection, the blood coagulates quickly, becoming brick-red, then brownish-black in colour; the corpuscles and also the nuclei become more distinct, and ultimately granular: the former contract slightly and refract light less (Labbé, Archiv. Gén., 6, xviii., 1871); but both Labbé and Salkowski agree that, within the vessels, after absorption from the stomach, skin, etc., no change of the kind, and indeed no marked change at all, occurs. Hoppe-Seyler says that the form and colour of the red corpuseles and the fluidity of the blood are unchanged, though at some period the venous blood is redder than usual, and in later stages of prolonged poisoning all the blood becomes darker, as in asphyxia generally. After death the condition found is not always alike: e.y., in one case the blood was red and fluid (Brabant, Lancet, i., 1873); in another (a child that survived twenty-four hours) it was dark and much clotted (Med. Times, i., 1878).

Generally, the brain-sinuses and membranes, and often the brain-substance, have been found congested (Neumann, Swain, and others), but the latter has sometimes been described as anæmic (Barlow).

In one instance the corpuscles "seemed softened," adherent by the margins, and not forming into roulecur. ; large white bodies were seen with them (Merd. Times, ii., 1879). Possibly the duration of the poisoning, or some variation in the mode of death, may account for different appearancos, but they do not indicate any marked or certain influence on the circulating fluid. Wallace thought it was disintegrated by the acid, because his patient became anomic, and homoglobin appeared in the urine (B. M. J., i., 1870); Binnendijk also speaks of blood-coustituents in the same secretion (B. M. J., ii., 1879) : but both observers are probably mistaken. Dr. Stevenson concluded thiat the urine in cases of prisoning entained neither blood nor iron, 
as supposed (B. M. J., i., 1870); and Hauxman showed the coloration was not dive to altered hæmatin, by its clearing up on acidifying and applying heat (Zeitschr. des Apothek-Vereins, Jan., 1872). Further, as Dr. Joseph (Archiv d. Heilk., 1868-69) first noted, and Ferrier (B.M. J., i., 1873) and many others have since confirmed, such urine may be passed clear, and only become coloured from above downwards in the course of some hours or days-implying the presence of some oxidizable compound of carbolic acid itself, not any bloodconstituent. The real influence of the acid on the blood is a point of much practical import in relation both to surgical practice and to the treatment of toxic cases. At present we have certainly no evidence that it destroys life, as some other poisons do, by its destructive influence on the blood itself, and hence we are not called upon to admit transfusion as a suitable remedy for carbolic poisoning (B. M. J., ii., 1879); but we have evidence that it can impregnate and circulate with the blood, for it has been obtained in a crystalline form on distillation of that fluid taken from fatal cases (Hoppe-Seyler and others), and hence there is a possibility of its contributing to a thrombosis, as suggested by Mr. Pearce Gould (Clin. Soc. Trans., 1879); and as regards treatment, abstraction of blood from a patient rendered unconscious by the poison seems rational, and has been successful (Mosler, B. M. J., ii., 1872). The blood drawn in this case had an odour of the acid.

Upon the heart itself-in batrachinns-the first effect of a slowly acting toxic dose ( 4 milligrammes) is to lessen the frequency of the bent at the same time that it increases its force; the ventricles contructing to the size of a pin's head, "with a spasmodic rigidity as if galvanized" (Labbć): the auricles, the aortic bulb, and the capillaries also contrnct. This spasm, however, seems to relax later, for the heart is arrested in diastole. Lemaire, Salkowski, and many others are agreed in this observation; but the Iatter argues that the action on the heart is not a primary one, for in acute cases of poisoning (in frogs) he has seen it continue to beat for some time after death. In other cases, according to the dose, the action becomes gradually weaker and slower till the diastolic arrest (Plugge). Hoppe-Seyler investignted the state of vascular tension in dog's 
who were painted over with carbolic acid, and found that when convulsions began there was a slight rise in arterial pressure, afterwards a fall, which continued: in the veins, pressure was higher than normal - the jugular especially was much swollen; this might be from lessened heart-force, or lessened resistance in capillaries, probably from both, but certainly from the latter, since the small vessels might be seen distended, and the surface ( $\circ . g .$, the ear) redder: the arterial spasm described by Labbé either did not occur, or passed away quickly. TVe cannot say that these results with animals illustrate a definite action of the drug on the circulation, but clinical experience indicates its generally depressant character. With 6-to 8-grain doses, Dr. Bill found a lowering of the pulse-frequency four to eight beats in the minute, and when the drug was long continued the heart-action became very feeble. In toxic cases the prise soon becomes small, irregular, and intermittent, the extremities cold, and the general condition one of collapse. If any evidence of stimulation appear, as in some of the experiments referred to, it is only temporary, and is dependent on convulsive disturbance. Cardiac arrest, apparently from shock, is an occasional, but exceptional, cause of death in calbolic poisoning.

Respiratory System.-By medium doses, respiration is quickened for a time, "as if to accelerate elimination." Iu Salkowski's observations on rabbits, toxic doses increased the frequency very much (200 per minute), but the breathing was extremely shallow, and the animal showed signs of great dyspacea. If, after the frequency set in, the vagi in the neck were divided, the breathing became slower and deeper, and the diaphragm acted more completely and regularly. After a time the frequency rose again, but not to more than 60 or 70. If the experiment were reversed--the anid being given after the occurrence of slowing from section of the nervesthen some increase occurred. From these facts he argues that the accelerated respiration depends partly on excitution of peripheral branches of the vagi, and partly on dircet excitation of the respiratory centres in the medulla, resembling that prodnced in the spinal cord as a whole. Ultimately the animal shows signs of defective oxygenation and accumulation of 
carbonic acid, with subsequent paralysis of respiratory centres, and death from asphyxia. Labbé describes convulsive opening and closing of the glottis. Moderate doses in men produce no marked effect on the breathing, and Salkowski gave it to many patients without observing special effects, unless that in some, with lung-gangrene, cough was excited by local irritation. In toxic cases the breathing is generally described as quick and shallow at first, later becoming irregular, interrupted, laboured, and finally stertorous.

Temperature.-A slight lowering of temperature from the continued employment of the acid is usual, but not invariable. In rabbits, a fall of some tenths of a degree occurred after a moderate close; of four-degrees after a large dose-30 to 40 centigrammes (Danion and Feltz, 1869). Küster reports more definite results in dogs, small doses causing a gradual rise $\left(0.9\right.$ to $1.8^{\circ} \mathrm{F}$.) for twenty-four hours; large doses given gradually, a sudden rise and then a fall, but when given all at once, sudden depression and subsequent rise. In certain stages of poisoning in men increased temperature has been recorded, due possibly to secondary causes, such as gastritis (Anderson, Lancet, i., 1869). Rigors, chilliness, and subnormal temperature are more usual (Langenbeck). In the slighter forms of septicemia, carbolic acid is said to lessen pyrexia, but in severe cases to exert no such effect (E. Erb, Schmidt's Jahrb., Bd. 164).

Digestive System.--The taste of carbolic acid is first warm and acrid, then cooling; a sense of warmth along the wosoplagus and in the stomach is also felt, and some increase of saliva occurs. Moderate doses do not interfere with digestion, but rather stimulate it (Labbú); their continued use, however, induces anorexin, gastric pain, catarrh, and diarrhoea. Vomiting is one of the earliest symptoms of a toxic effect from external application, but it rarely occurs even from a poisonous dose when taken by the mouth; there may be nausea, but netual vomiting is induced with difficulty. Dysphagia has been noticed, and some spasm of the esophagus, leading to much trouble in the introduction of a tube. Extreme congestion of the stomach and actunl gastritis have been found post mortem, with hard corrugntions, and white or dark hard 
patches, which are characteristic. In most cases the pharynx, gullet, stomach, and intestines are more or less covered with a white coating, "like white lead"; in one instance, the mucous membrane for fifty inches was thickened, and of bluish-white colour (Dr. Way); and Dr. Moxon has seen a kind of tanning of the stomach (Path. Trans.). Fatty degeneration of the liver has been reported (Neumann), but is not a constant result in either men or animals (Husemann).

Urinary System.- It is not unusual for urine passed during carbolic acid treatment to be coloured dark-olive or blackishgreen, sometimes grass-green; it may be passed in this state, or become so only on standing. Treated with nitric acid, afterwards with potash, and concentrated, it becomes red, changing through green to violet. Carbolic acid, if only added to the urine outside the body, does not answer this test.

This greenish coloration, as already mentioned, is not traceable to an altered blood-condition, but to phenylie compornds which, according to J. H. Bill, are identical with chinon (Amer. Journ. Med. Sci., Oct., 18r0); it occurs more commonly after external than internal use of the drug, and, although one of the earliest indications of toxic effect, is not in itself an index of its degree. Carbolic-poisoning may occur without apparent change in the urine. According to Baumann and Hueter, a more reliable symptom of carbolism is the disappearnnce of sulphates from the urine pori possur with the progress of poisoning, so that at length none can be detected. In testing for this purpose, any albumen present should be precipitated by boiling and acetic acid, and a solution of barium chloride added. In ordinary conditions this precipitates a milky cloud of harium sulphate, but in the presence of carbolic acid oatses either no change or a slight haze only (Berl. Ḱlinische TWoch., No. 28, 1878).

Carbolic acid sometimes causes direct renal irritation, and exceptionally albuminuria; its tendency is to lessen rather than increase the quantity of the secretion.

SYNERGISTS.-Other causties, astringents, and antiseptios are allied in action, but need seldom be combined with carbolio acid for therapeutical purposes; iodine, howover, forms with 
it a carbolate, which is colourless, and seems to have more active antiseptic powers, and to be more readily absorbed than either substance separately.

Sulpho-carbolates, cresylic acid, kreasote, and tar are closely synergic. To a less extent are borax and salicylate of soda, quinine, alcohols, and essential oils (such as those of thyme and eucalyptus), camphor, tannin, etc.

ANTIDOTES AND INCOMPATIBLES.-Alkalies in solution and in excess have some antidotal power (Husemann), and albumen or glycerine should be combined with them. Sansom has shown that gilycerine and olive oil markedly lessen both the caustic and anti-fermentative properties of the drug (op: cit., p. 17). Syrup has not the same effect, but a saccharate of lime is commended by Kunde, and is now on its trial. According to Muter, the permanganate of potash has also some antidotal power (B. M. J., i., 1870). More recently the sulphate of soda (any soluble sulphate would answer equally well) has been brought forward as an antidote, on the supposition that it can form within the system a "phenol-sulphuric" acid, which is harmless (Baumann, loc. cit.). Toxic symptoms in men are said to pass away more quickly than usual under its influence; and if the urine have become dark under carbolic dressings, they may yet be continued without danger, if sulphate of soda be given (Sonnenburg, Deutsche Zeitschr. f. Chirurg., ix., 356). These observations need further confirmation. Iüster says that the sulphate has not answered in severe cases of poisoning (B. M. J., i., 1879). Calvert says that drying oils should not be used as a vehicle for carbolic acid, on account of their tendency to absorb oxygen. Nitric acid should not be employed as a caustic either with, or directly after it, since an explosive compound may be formed.

Therapeutical Action. - Disinfection. - Carbolic acid was, on its first introduction, highly valued as a general disinfectant suitable for all pupposes, but we have already shown reason for doubting its power as an aërial disinfectant, i.e., as a germicide; and since most infectious diseases are propagated through the atmosphere, this remedy is not so 
effective for their arrest as some others, such as sulphurous acid (Med. Times, ii., 1878). In the presence of much moisture also it is inferior to the metallic salts (Sansom; Angus Smith).

Dr. Letheby and $\mathrm{Mr}$. Simon recommended it during an epidemic of cholera, but at New Orleans it failed to control severe incursions of small-pox, scarlet and yellow fever (Official Report, Record, 1876). Mr. Crookes, indeed, found its free local application to be of service in cattle plague; and when a centre of infection can be localized, as in a shed or dairy, then no doubt the drug, in full strength, proves highly valuable: thus, the Hon. W. Hope, "employing a gallon of acid where others had used a pint," scouring the sheds and bathing the animals, and using also lime freely, saved all of more than 100 cows so treated (Dict. of Hygiene, Blyth). For such a purpose the 2 per cent. solution of $\mathrm{Mr}$. Crookes is scarcely strong enough, and 5 to 10 per cent. and upwards should be used; but there arises some danger of toxic effects, since animals are prone to lick the washed surfaces.

Devergie successfully purified the Morgue by a continuous stream of carbolized water containing only 1 part in 4,000 , and such solution is common in public latrines, ete, but there can be little doubt, that the best application of the acid is for disinfection of solid or semi-solid excreta, and then it should be used in good strength: 1 ounce of acid to 2 quarts or rather more of water in which 1 pound of sulphate of iron is dissolved, makes an excellent compound disinfectant for vessels and elosets during a fever case. Its value also for purifying the atmosphere of sick-rooms is not to be igmored, although it may not wholly and always prevent the spread of infectious disease. Much confidence is felt by many practitioners in a sheet wrung from carbolic solution ( 2 to 4 per cent.), and hung in the don'way, and the occasional use of a spray of 10 to 20 per cent. solution; whilst the vapour of the acid obtained by placing it over hot water is of value in whooping-cough and diphtheria, ete. If carbolic acid be chosen for disinfecting a vacant room, the vapour should then he disengaged by heat freely for several hours. There aie, moreover, several good forms of cribolizod powvier: that of M. Bouchardat contains 1 part of acid in 1,000 of plaster of 
Paris; of Parisel, 1 in 100 of coarse meal and 4 of fat; of McDougall, 33 per cent. carbolate of lime, 59 sulphite of magnesia ; of Calvert, 20 to 30 per cent. carbolic acid with powdered refuse from alum works: about $\frac{1}{2}$ ounce of the last should be allowed to every 4 ounces requiring disinfection (Parkes).

\section{TherapeUtical ACTION. - External. - Cutaneous} Disease.-In mild cases of ringworm, the glycerole of carbolic acid painted on twice daily, after thorough cleansing, may prove effective (B. M. J., ii., 1877). Fresh patches may be destroyed at once by a touch with the strong acid, which acts as a caustic, giving pain, and leaving a red stain for a time. It has been already stated that this application to extensive farus has caused poisoning. I have never seen such result in ringworm, but its possibility should be remembered if a large surface be affected. Dr. Alder Smith has recently advocated the use of carbolic acid and glycerine in equal parts, or 1 to 3 , for ringworm, but states that he has once seen toxic effects from applying it to the whole of the scalp of a child (Lancet, $i$., 1880). As a rule, frequent washings are not desirable in tinea tonsurans, and the stronger solutions of the acid painted on the affected spot have given me better results than the lotion containing 2 to 3 drachms in 8 ounces, which is often ordered. In tinea versicolor a mercurial or sulphurous acid lotion acts better than the carbolic.

In diseases dependent on mimal parasites, such as scabies and phtheiriasis, carbolic acid in liniment or ointment is doubtless effective; but since death has followed from frictions with the strong commercial brown acid, and also from a solution in spirit-and-water containing some undissolved crystalline acid, much care must be exercised in prescribing the remedy, and, on the whole, others seem preferable to this one in scabies. Carbolic lotion (5 per cent.) is useful for preliculi capitis, ete. ; when the hair is well washed with this it kills the lice and the nits, and renders shaving the part unnecessary: I have never known it fail. It is said to be effective when applied locally in the treatment of Guinca-rorm (B. M. J., ii., 1869); and certainly carbolic lotion gives the greatest relief in mosquito and ant bites. 
For lichen, prurigo, and irrituble slim-conditions associated with nerve-disorders-also for subacute ecsemu, a lotion containing about 2 drachms in 8 ounces with spirit and glycerine is often efficient, and is one of the best applications of the acid. Mr. Brown, however, finds it " uncertain, like all other" tarry preparations," and not superior to borax or sublimate (Practitioner, vol. iii.).

For chilblains, the following ointment is valuable:-Carbolic acid, 15 grains; tincture of iodine and tannin, of each 30 grains; wax, 1 ounce (Bartholow).

In eczemu, with profuse secretion, and especially when occurring at the flexures of the limbs, neck, etc., carbolized starch powder is very good: it may be prepared with 5 parts of alcohol and 5 of acid, mixed and added to 100 parts of finelypowdered starch (Sansom). It should be applied after carefully drying the part.

In psoriasis, Lemaire, Bazin, and others recommend carbolic acid (1 per cent.), with vinegar and water or glycerine; MacNab, in ointment with lard, 1 part in 4 (Lancet, i., 1870). Sometimes it is effective, though not so much so, in my experience, as the usual tarry preparations. More remarkable is the benefit claimed by Kohn and Hebra from the internal use of 1 to 9 grains (in pill) daily in the disease; of twenty-seven cases all are said to have improved (B. M. J., i., 1869). I have not been able to verify this.

Boils-Small-pox.-According to Dr. Eade (and I can confirm his experience) the application of a few minims of glycerole of carbolic acid to the apex or within the apex of boils, either causes them to abort or arrests their spreading (Lancet, i., 1874-8). Carbolic lotions are very beneficial for the same purpose in rerbuncle, and there is much evidence of their advantage when applied to the voriolons smption in its suppurative stage. Sansom employed a liniment, 1 part in 3 of oil (B. M. J., ii., 18r1). Löffler, Chnuffard, and, more recently, Dr. Alexander Watson have concurred in his estimation of it (Lancet, ii. 1872).

Ear Disease.-In ehronic inflammation of the meatus externus or of the middle ear, with ulceration of the membrana tympani, injections containing carbolic acid are useful. Hagen 
says they do not act as well if the mucous membrane only is affected (Practitioner, rol. ii.).

Strumous Ophthalmia.-A solution containing about 1 minim of acid with glycerine in an ounce of rose water, is effective when applied as lotion or in drops (Practitioner, vol. i.).

Burns-Ulcerations.-In indolent ulcerations, burns, or wounds, with unhealthy discharge, carbolic lotion, or olive oil containing about 10 minims in the ounce, are good stimulant, antiseptic, and slightly anæsthetic dressings. Dr. Boydt concludes that they modify inflammation, prevent septicæmia, lessen suppuration, and induce a smooth extensible cicatrix (Bull. de Thérap., Oct., 18r8).

For aphthous stomatitis and ulceration about the throat, the glycerole of the acid is useful, though somewhat burning and painful to inflamed parts.

In Diphtheria the ordinary glycerine preparation has not been found of much value, but Rothe speaks highly of the following as a local application:-Carbolic acid, spirits of wine, of each 100 parts, tincture of iodine 50 parts, water $500:$ to be brushed on every three hours (Die Carbolsäure, Berlin, 1875).

In Excoriation and Simple Ulceration of the cervix uteri, in chronic inflammatory conditions, endo-cervicitis, and metritis, the glycerole of the acid, or in obstinate cases and those with much discharge, the pure acid itself, applied locally has an excellent effect. It does not give much, if any, pain if it be carefully kept from the vaginal and cutaneous surfaces. Dr. Lloyd Roberts, who has recommended this, remarks "that its action seems intermediate between that of nitrate of silver and of the stronger acids" (Practitioner, vol. i.).

Dr. W. Playfair, who has also written in its favour, employed, at the suggestion of Sir Spencer Wells, equal parts of glycerine, taunin, and of carbolic acid, for uterine catarrh, and even for uterine cancer, with good results (Lancet, ii., 1870, etc.). I can, from my own experience, corroborate these observations.

Cystitis. - In cases of chronic cystitis, with much mucopurulent discharge, painful frequent micturition, etc., carbolized injections, though at first they may give rise to pain for an hour or tro, afterwards relieve markedly (B. M. J., i., 1873).

Hæmorrhoids. - The late Mr. Turner, of Manchester, recom- 
mended the application of carbolic acid to external hæmorrhoids, since he found it coagulate their contents, and, by constriction, empty the venous sac and lead to its gradual obliteration. In internal hæmorrhoids and rectal fissure it has also proved useful. Dr. Pooley (U.S.) injected five or six drops of the crystallized acid, liquefied by heat, deep into the substance of inflamed painful hæmorrhoids; the pain was slight, and when a slough was formed and separated, the parts healed (Record, 1878); but this treatment would not be suitable for internal bleeding piles.

Condyloma-Lupus, etc.-It is as a caustic, i.e., when the pure acid is applied, that this remedy acts best, not only in the cases of uterine disease mentioned, but in mucous tubercles, and, according to Déclat and many others, also in lupus of the face, etc. My own experience as to its value in lupus was disappointing; it proved not so effective as the acid nitrate of mercury, nor as might have been hoped, much less painful. In "Cancroid of the Tongue," Déclat records some remarkable results.

Nævus.-The subcutaneous injection of carbolic acid is a good mode of treating nævi, by coagulating the contained blood, and gradually obliterating the tumour without scar: this I have frequently verified. By it the clot is said to be rendered firmer, therefore not so likely to produce embolism as that following the injection of perchloride of iron; but for greater security a ligature should be placed round the nævus during, and for a short time after, the injection. In a case of extensive nævus the late Mr. Bradley injected 5 minims of the pure acid several times in different places : it acted slowly, but at length successfully; later he modified his process by tattooing with the acid in other cases, and obtained good results more quickly (B. M. J., i., 1876):

Diseases of the Air-Passages.-Carbolic acid is frequently employed in the form. of spray or vapour in disorder of the throat and nose, in subacute and chronic bronchitis, pertussis, gangrene, and phthisis.

In ceterimenl rellexed throut-conditions, in ulcerated throat, and in scarlatinal, and even diphtheritic cases, a spray containing 1 to 2 per cent. often acts better than a brush application. In severe nasal catarrh, and in hay-asthma, the rupour of the acicl often relieves, and it forms the basis of a patent nostrim ("Alkaram"). A solution with ether and ammonia 
in the same form as ordinary "smelling salt" answers rather better (Martindale). Taube (Leipzig), in diphtheria, injects several minims of a 3 per cent. solution into the tonsils twice or thrice daily (Record, Oct., 1878), but in many cases it would be impossible to carry out this method.

For whooping-cough, the vapour of the ordinary brown acid, or of a strong solution of the crystalline, should be generated from a suitable apparatus, and the patients exposed to it for half an hour or an hour twice daily, according to the case. Though I have sometimes seen immediate and marked relief from the first form of inhalation, I think it is not always equally effective. R.J. Lee and others have offered further evidence in its favour (B. M. J., ii., 1875), and Mr. Cole (New Zealand) has recently reported several good cases; he gives it internally as well as by inhalation (Lancet, i., 1878). The alkaline carbolates have a similar efficacy. Thus, Pernot finds the carbolate of soda, vaporized in the sick chamber (over a spirit lamp), diminish paroxysms of cough in two to ten days, and render respiration easier; he never saw the malady increase after this treatment was commenced (Record, Mareh, 1878).

In cases of empyema rupturing into the lung, and again in cases of pulmonary gangrene, the spray or vapour gives much relief by its disinfecting properties.

In chronic bronchitis and bronchorrheen, as well as in cases of simple catarrh, benefit may be obtained by the use of a carbolic spray, expectoration also becoming lessened in amount and improved in character, and cough rendered easier and lighter (Finlay, Med. Times, 1876). As to its value in phthisis, opinion is not so agreed. In the earlier stages, Dr. Marcet used a strength of from $\frac{1}{2}$ grain to $1 \frac{1}{2}$ grain in the ounce, and has recorded instances of its power to relieve difficulty of breathing, cough, excess of secretion (Practitioner, vol. ii.); but in advanced cases, with softening and pyrexia, he finds it likely to depress the heart-action unduly: benefit was sumetimes obtained, but not permanently. Sir Andrew Clark has objected to its use in phthisis (B. M. J., i., 1869), and Moritz reports it not well borne in his cases (Record, 187\%). Considering the susceptibility of delicate subjects, and the ready absorption by lung-tissue, as well as the above clinical results, I think it 
well always to begin cautiously with such inhalations; but when expectoration is profuse and purulent, the patient need not be wholly deprived of a valuable resource. Dr. Eade speaks highly of it, using about 10 grains in half a pint of hot water (Lancet, i., 1878). Dr. Sansom found the strong acid, painted externally over a limited area on the chest, useful as a counter-irritant in the second stage of phthisis, and in emphysema with congestion.

Rheumatism.-The pain of acute rheumatism may often be relieved by wrapping the patient in blankets wrung from hot water: it is said that the efficacy of this treatment is much increased by the addition of an ounce of carbolic acid to the pailful of water (New York Journ., Feb., 1876).

Deep Injections in Erysipelas, etc.-Hueter was the furst to systematize the local treatment of erysipelas by parenchymatous injections of carbolic acid, and in a recent summary records remarkably good results (Berlin. Klin. Toch., xxiv., 1878; Med. Times, i., 1878). Of a solution containing carbolic acid and spirit of wine, of each $1 \frac{1}{2}$ grain in 50 minims of water, he injects 20 to 30 minims into the subcutaneous tissue affected: this suffices to arrest the inflammation in a surrounding area of about "half-a-card," so that six and even twelve injections may be required either at once or after intervals. The earlier this treatment is commencerl, the better it succeeds: in acute cases it may be used twice daily; in chronic, every day or every second day. A strength of from 2 to 3 per cent. is sufficient to do good, and yet not enough to be dangerous, although precautions should be taken not to inject directly into a vein. If the needle be passer in first, unconnected with the syringe, and no blond comes through it, the injection may be safely practised. The pain given is commonly slight and the relief rapid; of seventeen cases, the average duration of treatment was two and a quarter days, and out of 1,000 injections inflammation followed in ten cases only. In a previous year, with ordinary treatment, out of thirty cases four died. In traumatic cases the wound is dressed with wet carbolized wool, or carbolic oil 1 in 20. Aufrecht shortly afterwards adopted a similar method, and with equally good results; Senator has corroborated these 
statements, and the treatment has been applied also in many forms of local inflammation, in glandular swellings, bursitis, synovitis, rheumatism, poisoned wounds, hydrocele, etc. Hagen has used it in the neighbourhood of the larynx in severe angina, threatening diphtheria, and apparently with good success (Schmidt's Jahrb., Bd. clxiv.). Carbolized lotions are also valuable in erysipelas, and a liniment containing 1 part in 10 of oil of turpentine has been recommended (B. M. J., i., 1873). I have often seen benefit from the latter remedy.

Deep Abscess. - A modified form of the same was earlier applied by Dr. Eames to the treatment of deep abscess of the subcutaneous tissue after free incision: he found that injections of carbolized oil lessened the spread of inflammation, and controlled purulent secretion (B. M. J., i., 1873).

Mr. Callender reported good results from over distending: large abscess-cavities after evacuation with carbolic acid solution, 1 part in 30 of water (B. M. J., ii., 18;6).

Neuralgia.-Mertem injected, hypodermically, a 2 per cent. solution in four cases of different forms of neuralgic pains, sciatica, uterine, etc., with relief (Practitioner, vol. xviii.).

For Local Anæsthesia, Sir Erasmus Wilson found that a previous painting with carbolic acid enabled a patient to berr cauterizing of a sensitive growth upon the penis without much pain (Journ. Cutan. Med., 1870), and about the same time, Dr. Bell (U.S.) made careful experiments with a view to utilizing this anæsthetic power for surgical purposes: he found that by bathing the skin for a quarter of an hour in carbolic solution (3 per cent.), and then painting the strong acid along the line of a proposed incision, many minor operations could be performed without pain. Thus he exposed his own radial artery sufficiently for deligation, and opened painlessly a whitlow, abscess, etc. (Amer. Jourm. Med. Science, October, 18r0). Lewis has applicd the actual cautery under the same method without pain (Record, 1876), and it evidently an produce local anrsthesia, but vesication, or even slight sloughing, is apt to follow its use.

Carbolized Dressings. - The application of carbolic acid to the dressing of wounds, with the mimute precantions of Sir Joseph Lister, is of such grent importance as to need special 
attention. It is based upon the fact that not air, but something contained in it, induces decomposition, and upon the principle, that if this something, this "organism or germ," be destroyed or prevented from reproduction, then unhealthy action in wounds is prevented. "This theory suits and explains most of the facts, and until a better be forthcoming we may accept it with a scientific reserve, but without hesitation" (Sir W. MacCormac, Antiseptic Surgery, 1880).

It is clear that the "antiseptic method" does not depend wholly on carbolic acid; but up to the present, that agent has given better results than others, and the details of its use now adopted by Sir Joseph Lister seem calculated to secure the greatest amount of good with the minimum of harm. He employs solutions in vater, 1 in 20 and 1 in 40 parts (5 per cent. and $2 \frac{1}{2}$ per cent.), solutions in olive oil, 1 in 20 and 1 in 10 parts (õ per cent. and 10 per cent.), and crrbolized ymiz', recommending that the latter should now be prepared by saturating it with a mixture consisting of carbolic acid 1 part, resin 4 parts, paraffin 4 parts. This contains half as much more acid as formerly, but is not more irritating; the amount of paraffin is less, and the ganze somewhat more adhesive--resin is used to lessen volatilisation of the acid. To be quite trustworthy, the gauze must be freshly prepared. Küster and others have made apparatus for the purpose, and introduced a variety of formulæ (see MacCormac's Antiseptic Surgery). Abroad, jute is largely substituted for gauze, and by some surgeons oakum.

"Protectiv" is oiled silk coated on both sides with a thin layer of copal varnish, and thus rendered impermeable to carbolic acid vapour. Its surface is covered with a thin layer of dextrine, starch, and weak acid, which enables it to take up uniformly the 1 in 40 solution, in which it is dipped just before application. It contains a trace of lead, and thus becomes stained by contact with septic discharge, sulphuretted hydrogen giving rise to a lead sulphide.

Mackintosh, to prevent the soaking through of clischarge at one spot, etc.; bencluyes of the carbolic gauze; aseptic sutures and bigatures of carbolized catgut, silk, horsehair, or silver wire, and drainage tubrs of rubber, motal, skein of catgut, or decalcified bone or ivory (Neuber of Kiel). Crliss tubes are also used in 
the peritoneum; special care is given also to the disinfection of sponges.

To secure a carbolized antiseptic atmosphere for operation, the 5 per cent. watery solution, carefully filtered, is pulverized by a "spray-producer," the jet being directed upon the part. The handball instrument may be used for small wounds and dressings, but the spray given by it is coarse, and wets the surface too much: in this instrument, the $2 \frac{1}{2}$ per cent. solution should be employed. An instrument worked by the foot (Richardson, Dublin) is more powerful, sending the spray a distance of 6 to 10 feet, but the steam spray producer, at high pressure, is preferable to any other; that of Matthews Brothers, Carey Street, is a good form. It will keep either one or two spray points at work at a pressure of 90 lbs. or 60 lbs. to the square inch respectively, and projects a fine cloud to a distance of 20 feet for two to four hours at a time. In using any of these instruments, it is important to verify at intervals the presence of acid in the cloud, which may be done by the sound and by the taste or smell; also by the colour, for if steam only be issuing, the jet is bluish.

For the purposes of an operation, the hands, the instruments, the sponges, and the surface of the body at the place of operation must be thoroughly cleansed, and then rendered aseptic by a free use of the 5 per cent. watery solution. During the time of producing narcosis it is well to apply a layer of moist aseptic gauze, or a towel wrung out of carbolic acid solution. If an amputation is in question, Esmarch's bandage is applied, and a steam spray directed on the part from the time of the first incision till after the application of the dressing. When the bone is being divided, a weak solution (21 per cent.) poured on the saw suffices to lessen the heat which, in some degree, disposes to necrosis. When the operation is completed, all bleeding points are secured, some for a time with hanging forceps, whilst the larger ones are first tied, with aseptic ligatures, preferably catgut, the ends being cut short when the knot is tied. Sir Spencer WVells commonly employs his strong clamp-forceps for quick temporary arrest of hamorrhage (B. M. J., i., 18\%9): they are especially valuable in ovaristomy. General ooring should be arrested either by 
irrigation with carbolic solution and elevation of the stump, or by spirit of wine, or by hot water $\left(110^{\circ}\right.$ to $120^{\circ} \mathrm{F}$.), or, what is better, by a mass of folded gauze pressed on after being dipped in hot water. When all bleeding is stopped, sutures are introduced, so as to bring the cut surfaces into as close contact as possible; wire with lead buttons or plates may be used for the deeper parts, carbolized silk for superficial stitches. The wound may require washing again with $2 \frac{1}{2}$ per cent. solution to remove clots, and then quite closed, except at one or two points where drainage tubes pass out: these should touch the deepest dependent parts-not the cut bone-and any recess, and be brought to the surface, generally at the angles of the wound, and on a level with the skin. A skein of silk attached to the tube serves for its subsequent withdrawal. Any remaining blood in the wound should be got rid of by gentle pressure of the part with sponges, and then the final dressing may be commenced. First, one strip of "protective," dipped in 1 in 40 solution for a moment before use, should be applied well beyond the ends of the wound, and over this a good quantity of loosely-folded ganze, wrung tightly from the same solution, is placed, so as to press the cut surfaces together. As many layers should be applied as may be required to absorb the expected discharge; over this a layer of salicylic wool is sometimes added, and firm equable compression may be made by a carbolized bandage. An eight-fold layer of dry gauze, well overlapping the wound area, comes next in order, with mackintosh interposed between its outer layers. At the margin of the dressing, salicylic wool is often packed for greater security; and over all, the dry ganze bandage should be firmly applied. In operations near the shoulder-or hip-joints elastic bandage is commonly used at the margins and passed round the trunk; and when the breast or groin is concerned the corresponding limb should be included. Usually a second dressing will be required in twenty-four hours, according to the degree of saturntion with discharge; a change is indicated also if clischarge has penetrated to the margins or external layer's of dressing, if the dressing becomes loosened, if there bo severe continuous pain, or septio odnur, or prolonged rise of templerature over $100^{\circ} \mathrm{F}$.

A second dressing may safely remain two or three days, or 
even a week, and then the drainage tubes may be removed, if empty and dry. When all goes well but slight pain is felt after the first day; the margins of the wound usually show no swelling or redness, and any secretion is scanty, thin and serous in character; there is no true suppuration, no inflammatory fever.

Dressing should be done as quickly as possible; therefore everything necessary must be prepared beforehand. The external surface is gently cleansed by a stream of carbolic solution from a suitable syringe, and to save the bedolothes, it is desirable to move the patient, if possible, to a seat or table prepared with oil eloth : dressing is then reapplied as before under a cloud of spray. As a recompense for all this time and care, the progress of the case is usually good, without drawback; ordinary food is snon taken; little nursing is required; convalescence is rapid: the stump is better, the bone more covered, and there being less cicatricial tissue, there is less retraction.

Abscess.-Before opening a large abscess, acute or chronic, the skin over it should be eleansed as already described, and the steam spray set to work. After incision, the abscess-cavity may be left alone, or it may be washed out with solution ( 1 in 40 ). The latter plan is not free from risk of poisoning if the surface be extensive, and the patient very young and anæmic; and although I have known the method answer well, yet good results are commonly obtained without it. Drainage tubes are needful if the cavity be at all deep or sinuous, and great care must be taken with after dressing. Wonderfully good results have been obtained by the antiseptic method in psoas and pelvic abscess and empyema, and they contrast favourably with the serious and often fatal results of older operations. Dead bone, strumous deposits in suppurating glands, unhenlthy granulation-tissue in sinuses, should be removed at the time of operation.

Compound Fracture.-In these cases the antiseptic method has given the best results, obviating, as it does, pymmia and most of the other preventible causes of death. The wounded limb is first thoroughly washed with soap and water and strong carbolic solution: if the wound be recent, 1 in 40 , and if long exposed, 1 in 20 solution, should be used for its irrigation; this 
must be complete, and openings and counter-openings must be enlarged or made as required, and drainage tubes introduced ; the fracture must then be set in the usual manner, bone being removed if necessary. The dressing already described should then be applied, and sometimes requires changing next day, at other times not for four or five days, and in the most favourable cases only one dressing has been necessary. Sir William MacCormac recommends the plaster of Paris splint in combination with this method.

Osteotomy, etc.-Operations for bone-deformities from rickets, knock-knee, faulty union, ete., have been rendered much safer by antiseptic precautions. Boeckel has published 18:2 successful cases (Paris, 1880). In deficient union of a fracturm. patella, the knee-joint has been opened and the bone-surfaces brought together by wire sutures; but the patients have not, on the whole, made good recoveries.

Operations on Joints. - If a large joint be distended by blond or chronic effusion, serous or pumlent, it may be punctured by a large trocar and washed out with strong carbolic injection without injury and practically without risk. Sometimes the wound is closed, at others a drainage tube is desirable to prevent re-accumulation.

After washing, and whilst using the spray, the trocar is passed into the outer side of, say, the knee-joint, and a stream of 3 to 5 per cent. solution injected and allowed to escape, the joint being manipulated so as to ensure general contact. The process should be continued, it may be, five to ten times, or until the fluid returns quite clear. When the canula is withdrawn the dressing is applied, and then a splint, and in five or six days all will usually be healed, and in a week or two passive movements can be commenced.

Under the spray also freer incisions are made into joints, and recovery takes place without loss of motion. Burse and compound ganglia have been similarly treated with good result.

Abdominal Section.-Formerly wounds of the abdominal cavity involving the peritonewn were considered amongst the most serious in surgery, and were often followed by fatal results; but now operations for tumours, concretions, intussuscention, etc., are undertaken accorcling to the method of Lister, 
with a degree of safety previously unknown. The risk of shock following exposure of so large and sensitive a surface, and the risks of blood-poisoning were increased by its highly absorbent lower, and the difficulty of cleansing its recesses. The former risks were lessened by great attention to warmth, and the latter by perfect cleanliness and the free use of carbolic acid, which seldom irritates the peritoneum. Operations for artificial anus, for radical cure of hernia, for hydatids, and, above all, for ovarian tumour, have been performed under it with a rare degree of suceess. Olshauser reports that of forty ovariotomies performed by him before the introduction of antiseptics, nineteen died; whilst of nineteen cases since, one only died. Of eighty-four cases under antiseptic treatment by Sir Spencer Wells, six only died, and thirty-eight in succession recorered; whilst of a previous eighty-four, "with all the care he could give them," twenty-one were lost. Many similar illustrations might be cited, so that the results are clearly above suspicion of mere chance or accident, and not only is more safety obtained, but much more easy and quick recovery. (It is right to say that since this was written several experienced surgeons have obtained equally good results without carbolic spray.) A small ward should be chosen, and should be disinfected by chlorine. Few persons should be present, and these must be free from recent contact with any septic influence. The hands of the surgeon and assistants must be carefully cleansed with carbolic solution ( 1 in 40 ) before and during the operation, and especially before introducing them into the cavity. The instruments and sponges need careful disinfection, the surface must be washed, and the pubic hair removed. A large piece of oil-silk covers the whole of the abdomen and legs; it has an oval slit in the place where the incision is to be made, the margin of the slit being covered with carbolized plaster below, so that it sticks to the skin. A double spray should be used with $2:$ to 5 per cent. solution. The pedicle is secured within the abdomen in one or more portions by aseptic silk ligature, and the cavity cleared of clot and fluid, deep sutures introduced to include the whole thickness of the wall, and the wound dressed in the usual manner. Drainage tubes are seldom required. Symptoms of 
carbolic-poisoning occur very seldom,-much less often than might be expected.

Head Injuries.-Many severe cases of this lind have been successfully treated in the manner already described. The braiu-substance tolerates the acid well, a 5 per cent. watery solution, and even a 20 per cent. spirituous solution, producing no ill effect, but seeming to avert inflammatory complications. Hence trephining for injury may be performed without the same scruples and risks as formerly; depressed compound fractures may commonly be saved, and even the middle meningeal artery safely found and tied for hæmorrhage (Hueter, Centralblat. f. Chirurgie, August, 1879; Yeo and Femier, B. M. J., ii., 1880).

In the ligature of other vessels, especially veins, the antiseptic method is very serviceable.

Empyema.--Unless great care be taken it is difficult to treat this thoroughly on antiseptic principles, because of the mobility of the parts concerned, and the drawing of air into the chest. Special attention must be given to the working of the spray; the chest-wall must be well washed with 1 in 20 solution, and the incision made as usual. The question of washing out the abscess-cavity will arise, but such a process is full of risk, both of carbolic-poisoning and of convulsive or syncopal seizure, and it should be avoided if possible; certainly, if the pus be healthy, no syringing need be used. A drainage tube must be introduced and securely fastened to the chest-wall by transfixed pins, or threads fixed to the chest by adhesive strapping dipped in hot carbolized lotion, with gauze attached, ete. The next and every subsequent dressing must be made with the strict precautions described.

THERAPEUTICAL ACTION.- Intemal.-In many of the diseases already mentioned as benefited by external applications, or by the spray or vapour of carbolic acid, it has also been prescribed intemally. Thus, Hebra gave it in psoriasis, and Lemaire reported good results from its administration in chronic bronchitis, pertussis, and phthisis. Certainly there is clinical evidence of its occasional value in the two first-mentioned lung disorders, but not much in the last. Mr. Greenway 
has reported good effects from a combination with opium both in bronchitis and pneumonia (B. M. J., ii., 1874), Schutzler from hypodermic injections of $\frac{1}{7}$ to $\frac{1}{2}$ grain in phthisis, as modifying both temperature and general symptoms (Record, 1877).

Vomiting-Dyspepsia.-In these conditions, carbolic acid sometimes proves a very useful remedy. Dr. Wilks speaks well of it for romiting, and Sir Andrew Clark finds it rather preferable to kreasote. I have found 1-minim doses excellent in the nausea and vomiting of pregnancy, and Greenway found it good in the sickness of hysteria as well as in that of pregnancy (B. M. J., i., 1869). In cases of simple hyperæsthesia of the stomach, it may act by virtue of its local anæsthetic effect, allaying irritability: this effect may be further increased by conjoining morphia or bismuth with it. In cases dependent on fermentation of food with pyrosis it acts antiseptically. Dr. Fuller, using 6- to 8-minim doses, found it a grateful stimulant to the stomach, causing evacuation of flatus and lessening its subsequent formation (B. M. J., i., 1869). He compares it to charcoal in action, and finds it sometimes better. It is good for diłated stomach and sarcinæ, and for washing out the riscus whenever dilated with fermenting contents. In the dyspepsin of phthisis I have not seen much benefit. In atonic dyspepsia, with chronic irritation of the mucous membrane, it exerts a beneficial action, being moderately caustic and astringent. Ir. Habershon recommends it in various gastric disorders, functional and organic (Guy's Hosp. Reports, 1870). Dr. Allan found it effective in fermentative dyspepsia, and even in ulcer (B. M. J., i., 1872).

Choleraic Attacks-Dysentery.-In true cholera, little is to be expected from carbolic acid, although isolated cases of a successful result from it are reported. In diarrhoa, when depondent on unhealthy food, it has acted well both by the mouth and by enema. The risks of the latter mode of administration have been already mentioned, and are such as almost to negative its use. In chronic dysenteric cases, enemata have proved successful (Lemaire). Déclat states that, given internally, it proved very useful amougst the soldiers during the siege of Paris; but he generally gave it with opium.

Enteric Fever.-The balnnce of experience up to the present 
is adverse to the use of the acid in enteric fever-Murchison, Buchanan, Labbé, and many others agree on this point. On the other hand, Dr. A. Fergus reports several cases apparently benefited by it (B. M. J., 1869), and Déelat speaks of it in extravagant terms, saying that neither cerebral nor pulmonary complications need prevent its use - that it will relieve both these as well as the diarrhœa and septic condition. He orders from $1 \frac{1}{2}$ to 3 grains for a dose, well diluted, so that 30 grains, or even more, are taken in twenty-four hours; in addition, if grave symptoms supervene, he uses hypodermic injections of $\frac{1}{2}$ to 1 grain. M. Déclat relates several striking illustrations of success traceable to his method, but the style of his work is not calculated to carry conviction, though his statements suggest further research before condemning the remedy as unsuitable (De l'acide phénique, Paris, 1873). Dr. C. Tempesti has recorded two cases recently. One, a girl of ten, was exceedingly ill, in the second week of fever, with delirium, stupor, diarrhœe, hremorrhage, etc.; $\frac{1}{2}$ drachm of the acid was given in the course of a few hours, and next day all threatening symptoms had disappeared. In the other ease, which was somewhat similar, the administration of the same quantity was spread over five days, and had no good result (Record, April, 1873).

Intermittent Fever. - "For a moment," writes Labbé, "one might have believed in the therapeutic virtues of carbolic acid in intermittents." Barraud and Jessier reported twenty illustrative cases; they gave it in grain doses and by injection. On the other hand, Decaisne, Paluel, Markey, and others give upwards of 150 observations which tell against its value. It has unt come into general use.

Zymotic Disease generally.-Though there are not wanting some clinical records and many opinions in favour of this remerly, not only in the forms of disease just mentioned, but also in scarlatina, measles, and small-pox, as well as in cattle plague, the balance of eridence at present is against its exorting a specific influence on their course. Granting even that they are propagated by material prisons akin to "germs," it remains to be proved that these can he neutralized by such doses as ean be safely introduced into the organism: we know, however, as a 
fact, that it can be so far impregnated with the drug as that the secretions during life, and the tissues after death, are rendered much less prone to decompose than usual, and it cannot yet be said that antidotal effects are impossible, and not to be expected; on the contrary, research should be extended still further in this direction.

Mr. Crookes injected carbolic acid directly into the veins of cows suffering from cattle plague, and concluded that it modified symptoms, and in some cases saved life. One bad case, in which 105 grains were injected, recovered.

Diabetes.-Dr. Habershon traced improvement to carbolic acid in some cases of diabetes "of hepatic origin" (Guy's lieports, 1870). Fischer asserts that it enabled certain cases to undergo operation much more safely (Record, 1876); and Ebstein and Mïller reported it of service in the obese rariety of the disorder in its slighter forms. Practically it has not proved itself equal to other remedies.

Worms.-Besides its use in enema for ascarides, carbolic acid has been given internally to destroy and expel tænia: $2 \frac{1}{2}$ grains thrice daily are said to have proved effective when other remedies failed (Practitioner, vol. xvii.).

Preparations AND Dose.-Acidum carbolicum: dose, 1 to $3 \mathrm{gr} .$, may be given in pill, or with glycerine and water. Glycerimum acidi carbolici (carbolic acid 1 oz., glycerine $4 \mathrm{fl}$. oz. ; rub them together in a mortar until the acid is dissolved). Suppositoria acidi carbolici cum sapone (carbolic acid 12 gr., curd soap, in powder, 180 gr., starch, in powder, a sufficiency to form a paste; divide into twelve suppositories, each of which contains $1 \mathrm{gr}$. of the acid).

For "gargle, 1 to " grr. in the oz. : for an injection, 1 to $4 \mathrm{gr}$. in the 0\%.: for a lotion, 10 to $15 \mathrm{gr}$. in the oz. : for an ointment, 10 to $15 \mathrm{gr}$. in the oz.: for limiment, 1 part to 10 to 20 of oil: as a disinfertent, 1 pound to 6 gals., or 1 part (fluid) to 80 . Oleum origumi disguises the odour (Sansom). 


\section{SODA SULPHO-CARBOLAS.}

\section{(Sulpho-Carbolate of Soda.)}

PREPARATION.-By neutralizing sulpho-carbolic acid dissolved in six times its bulk of water, with carbonate of soda; afterwards evaporating and crystallizing.

CHARACTERS AND TESTS.-Occur's in brilliant, colourless, rhombic prisms, cohering in rosettes. It has a slightly saline and bitter taste, no odour: is freely soluble in six times its bulk of cold, two-thircls of boiling, water; slightly soluble in alcohol, not in ether. The watery solution is clear, and does not precipitate with a salt of barium, since the sulpho-carbolate is stable: strong heat will, however, expel the carbolic acid, and then the solution will react with barium, like other sulphates. Perchloride of iron gives a violet colour.

If the crystals be dissolved by boiling in nitric acid, and double the amount of water be added, picric acid is precipitated in yellow scales, containing a quarter of their weight of carbolic acid.

The sulpho-rarbolates of ammonia, potash, magnesia, and calcium crystallize in acicular tufts, more or less white; the analogous salt of copper, in transparent light-blue prisms; that of iron, in small brown micaceous crystals; of ainc, in colourless rectangular plates.

ABSORPTION AND ELIMINATION.--Sulpho-carbolate of soda is readily absorbed, and is, apparently, decomposed in the blood, since the odour of carbolic acid is soon communicated to the breath, whilst sulphate of soda passes in the urine (Sansom). On examining the tissues of two gumen-pigs that harl taken nearly 300 grains in four days, the sulphate was detected in the liver, muscles, etc. but neither carbolic nor sulpho-carbolic acids were evident to tests. It is probable, however, that some free acid passes in the urine, as well as in the breath. 
Physiological ACtion.-Any special powers of the alkaline sulpho-carbolates over the nervous and circulatory systems have not been ascertained: '20 grains taken in water, and repeated several times at short intervals, produce no definite symptoms, and 60 grains given every four hours for several doses cause only slight dizziness or vertigo. The administration of these salts, however, offers an indirect method of giving carbolic acid, and doses much larger than those mentioned would, probably, develop some of its effects; these alkaline compounds, however, have less local irritant action, and up to the present time have not caused poisonous symptoms.

As divect antiseptics, the sulpho-carbolates do not rank high; the soda-salt, however, arrests fermentation more actively than the others.

\section{Therapeutical Átion.-Extemal.-Leucorrhœa-} Gonorrhœa.-The sulpho-carbolates of zine and of copper Lave been employed in lotion containing 3 to 5 grains in the ounce, for discharging wounds and mucous surfaces : they exert an astringent, moderately antiseptic power (Mr. John Wood, Practitioner, 1868). In gleet and balanitis, the stronger solution may be used.

ThERAPEUTICAL ACTION.-Intemal.-Ulcerative Tonsillitis.-In this malady the sulpho-carbolate of soda often acts exceedingly well. Dr. Sansom refers to twenty-one cases, some of them patients subject to prolonged attacks, ending usually in suppuration; the majority recovered under the remedy (10 to 60 grains every three hours) in about four days; the tonsillar congestion subsiding rapidly without any formation of pus. In another series of cases, when sloughing and ulceration had set in, recovery commenced as soon as this trentment was begun; it may be compared with that by salicylate of soda.

Scarlatina.-Of twenty-nine cases, some extremely severe, with suppression of rash and convulsion, all recovered except one (a syphilitic baby). Convalescence was mostly complete in seven to fourteen days, no topical or other treatment being practised, and Dr. Sansom fairly concludes that by the sulpho- 
carbolates the duration of the attack was shortened, the pyrexia lessened, and the throat-symptoms especially benefited. His results certainly deserve more attention than they have yet received.

Variola - Erysipelas. - In these maladies, although the number of cases was not large enough for general conclusions, the results of similar treatment were equally satisfactory.

Skin Diseases-Impetigo, etc.-The soda or lime salt given to the children of the poor suffering from disordered nutrition and digestion (generally accompanied with pustular skineruption, sallow earthy complexion, large abdomen, and other consequences of unhealthy food or atmosphere), assists in their cure; and the iron salt is also said to be especially useful in such cases. The value of these remedies may be well seen in cases of eruption connected with blood-poisoning, or, for instance, where the vaccine virus continues to affect the skin long after the ordinary period. Dr. Mackey has reported cases of children in whom a general vaccinal vesicular eruption has recurred at short intervals during periods of from five to eighteen months from the date of vaccination; in one of these, calf-lymph had been used: at the time of commencing sulpho-carbolate of socla in 2- to 3-grain closes thrice daily, the eruption was as marked and general as at first, but within a few days the resicles were less developed, and in a few weeks they eutirely disappeared (B. M. J., ii., 1884). Analogous cases of "lichen urticatus" are published (B. M. J., ii., 1885).

Enteric Fever.-Sulpho-carbolate of soda has been given in this fever with reported advantage, but the evidence of $\mathrm{Dr}_{\mathrm{r}}$. Ligertwood in its favour is scarcely conclusive. He gave rather small doses-10 grains every three hours in combination with quinine ( 2 grains). The epidemic he refers to is said to have been a severe one, and of twenty-four cases three died: the diarhoon was "not so exhausting" as it commonily is.

Tuberculosis. - The general effects of the remedy in pulmnnary tuberculosis seem to have been satisfactory, for out of 121 cases classified aceording to the first, second, and third strges of the disorder, the majority improved whilst they were taking it; the cough, pain, expectoration, and night-sweating were lessened, and the genernl nutrition improved. Cod-liver oil 
and dietetic treatment were conjoined. In pre-tubercular dyspepsia, sulpho-carbolate of soda was also beneficial.

Rachitis.-The lime salt is said to be useful for this disorder, and has the advantage of being very soluble; it does not, by itself, control diarrhoea, but is a good adjunct to mild astringents.

PREPARATIONS AND DOSE.-Of the alkaline sulphocarbolates, dose, 20 to $60 \mathrm{gr}$. every four hours (adults); $10 \mathrm{gr}$. (children aged seven). Of the iron salt, dose, 10 to $15 \mathrm{gr}$. (adults) ; 2 to $3 \mathrm{gr}$. (children aged one). Of the zine or copper salt, 2 to 5 gr. in the ounce of water for injection or lotion.

\section{KREASOTUM. \\ (Kreasote, $\mathrm{C}_{7} \mathrm{H}_{8} \mathrm{O}_{2}+\mathrm{C}_{8} \mathrm{H}_{10} \mathrm{O}_{2}$.)}

PREPARATION.-By destructive distillation of wood, also of wood-tar oil; its preparation is tedious and difficult, involving repeated distillations and various processes for removal of impurities, such as paraffin, eupion, etc.

Characters And Tests. - Pure kreasote is an oleaginous, colourless liquid, of strong penetrating smoky odour, and caustic burning taste, which is so powerful that it can be detected in a solution of 1 part in 10,000. It is neutral, slightly soluble in water ( 1 in 80 ), soluble in acetic acid, alcohol, and ether. It remains fluid at $1 \tau^{\circ}$ below zero, and boils at $400^{\circ} \mathrm{F}$.

As to its exact nature there has been much doubt, but it is probably a mixture of two homologous bodies-guaincol and kreasol. These are contained also in the fluid obtained by distillation of guaiacum resin. Much of the commercial lueasote is but an impure carbolic acid: the colour-test (B.P.) to distinguish the former (viz, blue-green coloration of a slip of deal acidified) is fallncious, as also is the insolubility in glycerine, for true krensote is soluble in anhydrous glycerine, 
though not in ordinary glycerine containing water. The following distinctions between carbolic acid and kreasote are tabulated by Dr. W. G. Smith :-

\section{Kiecrote.}

Derived from wood-tar.

Liquid even at intense cold. Sp. gr. $1 \cdot 0 \pi 1$.

Sparingly soluble in water. Boiling-point about $400^{\circ} \mathrm{F}$. Dextro-gyrate.

With nitric acid yields oxalic acid and a resinous mass.

With ammonia does not yield anilin; becomes turbid.

With collodion does not gelatinize.

With ferric salts in aqueous solutions turns brown gradually.

Odour more smoky.
Carbolic Acid.

Derived from coal-tar.

In colourless acicular crystals. Sp. grr. 1.065.

Soluble in 20 parts water. Boiling-point $370^{\circ} \mathrm{F}$.

No effect on polarized light.

With nitric acid yields pure picric acid, which forms a yellow crystalline precipitate with potash.

Heated with ammonia, yields anilin, which strikes blue with chloride of lime.

With collodion forms a clear jelly.

With ferric salts in aqueous solutions gives a blue colour.

ABSORPTION AND ELIMINATION.-That lireasote readily passes into the blood and circulates with it is shown by its odour in all the tissues soon after its administration. It is eliminated in the breath, and also in the urine, which it sometimes discolours; but the exact changes it undergoes before reaching the bladder are not known.

Physiological Action.-Extemal.-Applied undiluted to the skin, kreasote produces the same effect as a burn of the first or second degree, with much smarting. On mucous membranes it forms a white coagulum of albumen and muens; in the mouth it excites much irritation, heat, and salivation.

Dilute solutions are astringont and antiseptic; they arrest fermentation, and preserve animal tissues. 
Physiological ACTion.-Intemal.- It is not easy, nor is it necessary, to distinguish minutely between the effects of kreasote and of carbolic acid, which has for the most part supplanted it in general use. The former is, perhaps, less energetic than the latter, and whilst causing, in large doses, similar effects on the Nervous System, such as restlessness, dyspnoea, prostration, and paralysis, does not produce the same acute spasms. The blood in animals after death from kreasote-poisoning is more coagulable than it is after carbolic-acid-poisoning.

In the Stomach, much irritation, sense of heat and constriction, or even inflammation, may be caused if the dose be large. According to Pereira, it increases the amount of urine in health, though it lessens it in disease.

SYNERGISTS.-Astringents, both mineral and organic, but especially carbolic and cresylic acids.

ANTAGONISTS AND INCOMPATIBLES.-Alkalies diminish the activity of kreasote, and saccharate of lime is recommended as an antidote. Oxides, such as that of silver, should not be associated with kreasote, for reduction may take place with heat aud explosion.

Albuminous and mucilaginous liquids antagonize its local caustic effect.

\section{Therapeutical ACTION. - Extemal. - Ulceration,} Hæmorrhage, etc.-In throat-ulceration I have found kreasote gargles often useful. In ulceration affecting the vagina or uterus, and in consequent leucorrhœa, as also in fistulø and simuses, injections and lotions containing it have a good effect: cracks about the nipple may be healed by washing these two or three times daily with lireasote-water. In hwmorrhage it is an effective styptic, and is the basis of some advertised "specifics."

Toothache.-For tonthache dependent on dental caries a minim of lirensote npplied on cotton-wool is a very effective, though somewhat umpleasnit, remedy. It is said to have the drawback of injuring the other teeth, and it may do so by its caustic powers, or by the salivation induced. 
Catarrh, Phthisis, etc.-Inhalations of steam containing kreasote prove a valuable antiseptic stimulant in cases of profuse and purulent secretion from the bronchial membrane: they render it healthier in character, and indirectly relieve cough.

In chronic relaxed conditions of the vocal cords, with more or less aphonia, they have also a good effect.

Skin Diseases.-In several, especially scaly, forms of chronic skin disease, such as psoriasis, kreasote ointment has been recommended, and certainly checks the formation of scales, and has the advantage in colour over tar; but if used of effective strength it is, in my experience, more painful than necessary.

Therapeutical Action. - Internal. - Vomiting. Kreasote in minim doses is often very efficacious in different forms of vomiting, both in that connected with fermentation of food and pyrosis, and in the sympathetic form dependent upon pregnancy, uterine disorder, etc, also in the vomiting of cancer and organic disease generally; and I have had excellent results from it in the vomiting and diarrhoea of typhoid fever.

Diarrhœa-Dysentery.-In some obstinate cases of these maladies, kreasote has succeeded well when other remedies failed.

Diabetes.-Pereira states that kreasote lessens the abnormal amount of urine in saccharine diabetes.

PREPARATIONS AND DOSE. - Trechotum: dose, 1 to 3 min. Misturn kreasoti (contains 1 min. to $1 \mathrm{oz}$, with acetic acid and juniper): dose, 1 to $2 \mathrm{oz}$. Unguentum lireasoti (1 dr. in 1 o\%.). Verpor kirecesoti (inhalation of kreasote): 12 min. to 8 o\% of boiling water.

Adulteratrons.-Oily hydrocarbous, fixed oils, carbolio acill. 


\section{FUCHSIN, MAGENTA, or ROSEIN.}

DESCRIPTION.-One of the anilin series of coal-tar products, technically named mono-hydrochlorate of rosanilin: it occurs in iridescent crystals, which form a deep red solution (utilized for staining bacilli and other microscopic objects).

ACTION.-The physiological action has not been well made out, but the drug is eliminated by the kidneys, and therapeutically seems to have remarkable power in some forms-mainly chronic-of albuminuria (B. M. J., ii., 1879). Drs. Bouchut, Sawyer and Saundby have observed distinct advantage from it in such cases (Practitioner, vol. xxvi.). De Renzi records lessening of urinary mucus. Normal urine is generally reddened by the drug, and if this coloration do not occur, the existence of organic, probably serious, renal disorder is indicated; the lips and mucous membranes are also coloured by contact with its solutions, as well as the froces.

Dose, $\frac{1}{2}$ to 3 or $4 \mathrm{gr}$. in pill form ; $1 \mathrm{gr}$. thrice daily is an average quantity.

\section{RESORCIN.}

DESCRIPTION.-This substance was discovered about twenty years ago, by Barth and ITlasiwetz, of Vienna, in galbanum resiu, when fused with potash. Being found isomeric with orcin, a substance previously recognized in the litmus-lichen, they termed it res-orem; by others it has been called resorcenal, and in technical chemical language meta-di-oxylbenzene: it is isomeric also with hydrochinon, $\mathrm{C}_{6} \mathrm{H}_{4}(\mathrm{HO})_{2}$. It is commonly obtained frum brazilin (from Brazil wood), or by passing benzol vapour through sulphuric acid, and is largely used in the 
manufacture of coal-tar dyes. It is a neutral crystalline body, soluble in water, alcohol, ether and oil, but not in chloroform or carbon bisulphide. Very concentrated solutions deposit feathery crystals, which may also be obtained in white plates resembling benzoic acid; on exposure it becomes pinkish; it melts at $210^{\circ} \mathrm{F}$; on the fire it burms with bright flame. It has a strong, peculiar sweet, afterwards bitter, unpleasant taste, and an odour like that of carbolic acid. A few grains give with strong sulphuric acid an orange-red solution which gradually darkens and finally becomes blue, and on warming, purple-red again.

Physiological Action. - Extemal. - Resorcin has marked antiseptic powers, 1 per cent. retarding fermentation and also decomposition of blood and urine, 2 per' cent. arresting it: the latter strength does not irritate the skin, but more than that has some caustic effect, and forms a white eschar on mucous membranes; it coagulates albumen.

Physiological Action.-Intemal.-Moderate doses of 5 to 10 grains may be taken with little, if any, gastric inritation, which, however, is readily caused by larger amounts, except in septicæmic cases, where 30 -grain doses are tolerated: it is apt to induce tinnitus, giddiness, flushing, and acceleration of pulse, followed, after about ten minutes, by profuse perspiration and subsequent depression of temperature, the last being more marked in febrile malarlies than in nornal conditions: occasionally there is transient delirium (Lichtheim, Lancet, ii., 1880).

Righi reports that the drug is nsullly well borne, but sometirnes induces transient vertigo, timnitus, and congestion of face: rarely pain in the stomach, almost always abunclant sweating (dose not mentioned-Reord, 1884).

Later toxic symptoms are convulsions with rigidity, which may be cansed equally by hypodermic injection. I)ujardinBenumetz describes giddiness, insensibility, incronsed frequency of pulse and respiration, epileptiform and tetanie envulsions, and concludes that the drug affects mainly the central nervous 
system, and not the blood directly: rigor mortis set in fifteen minutes after death (Practitioner, vol. xxix.).

Dr. Murrell has reported the effects of various doses given to a patient suffering from asthma: after $\frac{1}{2}$ drachm the spasm passed away and she slept comfortably; after 1 drachm, and also after $1 \frac{1}{3}$ drachm, giddiness and drowsiness occurred; but after 2 drachms, in addition to these symptoms, she got "pins and needles" all over, then became insensible, and was found lying on her side moaning, with closed eyes and clenched hands, without voluntary or reflex power, pulse weak, temperature $94^{\circ}$ only-recovery occurred gradually. The last specimen of the drug used is said to have been purer than the other (B. M. J., ii., 1881).

Urinary System.-The urine is rendered dark by the drug, and precipitates with nitric acid; the sulphates and nitrogenous elements are said to be increased.

SYNERGISTS.-Carbolic acid and other disinfectants, especially derivatires of coal tar.

Antagonists. - Emollients, emetics, atropia (hypodermically).

Therapeutical ACTION. - Ertemal. - Wounds, U1cers, etc.-Resorcin in solution, 1 to 2 per cent. and upwards, has been largely used in antiseptic surgery, especially on the Continent. Wounds of the cormea and mucous membranes, even when inoculated with septic material, healed kindly under such lotions, which proved useful also in erysipelas and even in cancerous sores. Andeer specially praises its powers in diphtheritic inframmation, and in renereal discharges, and injected 5 per cent. solution in cases of resical catarrh and inflammation, with much advantage (Record, 1884); Righi corroborates this experience.

In offensive Otorrhœa, a 1 per cent. solution is effective: over carbolic acid it has the advantage of being more soluble, less toxic and irritating, and less disagreeable in odour. The application of a similar solution to the glottis in cases of whooping-congh is said to be serviceable (Moncorvo). 
Therapeutical ACTION.-Intemal.-Fever, etc.-In France and Germany resorcin has been extensively given in typhus, typhoid, acute rheumatism, pneumonia, erysipelas, and phthisis, and certainly it may depress the febrile temperature; such depression is, however, of shorter duration than it is after quinine or salicylic acid.

Gastric Ulcer.-For this and other affections of the mucous membrane, such as intestinal catarrh, Andeer has strongly recommended resorcin as having a peculiarly beneficial, and almost specific effect, and I can to some extent corroborate his favourable experience. Carreras found it uscful in the choleriform diarrhoea of young children.

DosE, 5 to 10 or $20 \mathrm{gr}$. well diluted three or four times daily; for young children, $\frac{1}{2}$ to $1 \mathrm{gr}$.

\section{GASEOUS AND LIQUID COMPOUNDS OF CARBON.}

\section{(Not Officinal.)}

\section{CArbonic: Oxide, $\mathrm{CO}=28$.}

Like many of the compounds of carbon, this gas possesses anosthetic powers, as ascertained by Oranam (Brit. and For. Rev., ii., 1856) : it has no odour, and acts rapidly, but is probably too dangerous for ordinary use: it is the active agent in the fumes of lycoperdon (puff-ball), which have been used for nareotic and anesthetic purposes (Richardson, Med. Times, ii., 186\%). Venous blood under carbonic oxide becomes red owing to the combination with hemoglobin, and the wine saceharine (Joum. of Anat., May, 1867).

The Indians have a custom of placing a parturient woman in a hut, filled with the fumes of burning woor, which contrin earbonic oxicle and carbonic acid; and, as I)r. Tyler Smith has prointed rut, the effects are of anzesthetic and oxytocic charnoter (Med. 'Times, i., 1860). 


\section{Bisulphime of Carbon, $\mathrm{CS}_{2}=76$.}

This is a colourless volatile liquid, of strong disagreeable odour; it is largely used in commerce as a solvent for fat, for gutta-percha, etc. When burnt, it is said to act as a good disinfectant by the setting free of sulphur or sulphurous acid (Lancet, ii., 18r6).

It has anæsthetic and intoxicant properties, and perverts the digestion, "causing abnormal appetite" (Lancet, i., 1876). Workers with it are said to become impotent, and Dr. R. Williams states that its internal use controlled masturbation, after bromides had failed (Med. Times, i., 1864).

It was recommended by Dr. Kenyon as a local application in neuralgic headache, used on cotton-wool, protected from the air (Med. Times, ii., 1868). I have frequently tried this, and it seems to act mainly as a counter-irritant: the odour is unpleasant, and the effect not superior to that of chloroform.

\section{Bichloride of Carbon, $\mathrm{CCl}_{2}$,}

Was tried by Simpson as an anæsthetic, and answered perfectly well, but he considered it dangerously depressing to the heart.

\section{Tetrachloride of Cariox, $\mathrm{CCl}_{4}$,}

Was used as an anæsthetic in midwifery by Dr. Protheroe Smith and others (Lancet, i., 1867), and Dr. Sansom male further experiments with it (B. M. J., ii., 186 $)$ ). It is not very volatile, but the first stage of anæsthesia may be produced by it pleasantly, because it is free from offensive odour: its prolonged administration is injurious. 


\section{ALCOHOL (ETHYLIC).}

\section{(Hyprate of Ethyl, $\mathrm{C}_{2} \mathrm{H}_{6} \mathrm{O}=46$.)}

Alcohol is a product of the vinous fermentation of saccharine solutions, which fermentation occurs under the influence of a ferment, - yeast (Torula cerevisiæ), aided by warmth. During the process, carbonic acid is freely given off, insoluble constituents precipitate, the liquid becomes clear, and, after a time, the sweetness of the solution is replaced by a strong spirituous taste and vinous odour. By distillation, alcohol is obtained in association with a variable quantity of water.

All saccharine substances may be made to produce alcohol. Thus it is obtained not only from grape-juice, sugar-cane, beet-root, milk, etc., but-through a decomposition of starch and dextrin - from potatoes, barley, rice, etc. M. Berthelot reports the production of alcohol in the cold by the electrolysis of a solution of sugar (B. M. J., i., 1879).

The different strengths and varieties of alcohol in the Pharmacopocia are:-(1) Absolute alcohol; (2) Amylic alcohol; (3) Rectified spirit; (4) Proof spirit (spiritus tenuior); (5) Brandy (spiritus vini Gallici); (6) Sherry (vinum Xericum); (7) Orange wine (vinum aurantii).

(1) Alsolute Alcohol (Appeudix B.P.). PREPARAtion.Tr) obtain the pure drug unmixed with water, ordinary distillation will not suffice, and the following process is adopted:- Rectified spirit is first digested for two days with carbonate of potash, which absorlos most of the water; the solution is then decanted from the henvier aquenus potash solution, mixed with an excess of freshly-burnt quicklime, ant, after contact with this for twenty-four lours, is distilled from 
it at a gentle heat: the first ounce and a half of distillate being rejected, pure or absolute alcohol then comes over.

CHARACTERS AND TESTS.-Absolute alcohol is a limpid, colourless liquid, with characteristic pungent, spirituous, but not disagreeable, odour and taste; it is light (sp. gr. 0.795) and very volatile; it has a boiling-point of $173^{\circ} \mathrm{F}$, and has never been frozen, even at $-166^{\circ} \mathrm{F}$. It mixes in all proportions with ether and with water, forming transparent solutions; its union with the latter is attended by contraction of bulk and rise of temperature. It dissolves alkaloids, volatile oils and resins, iodine, pure alkalies, etc., but not common salt; so that if any salt dissolves when digested with this alcohol, it proves admixture with water. Such admixture may also be detected by adding a little anhydrous (uthite) sulphate of copper, which would become blue by hydration (Cassovia). Admixture with fusel oil, etc., is detected by the odour, taste, turbidity with water, specific gravity, etc.

The tests for the presence of alcohol itself in other substances are-(1) its odour and other characters when separated from the substance or solution by fractional distillation; (2) when heated with sulphuric acid and red chromate of potassium, an emerald-green colour is produced by reduction of the chromium salt; (3) if a little iodine and soda be added to the warmed liquid, a yellow crystalline deposit of iodoform may be recognized under the microscope in hexagonal plates (Lieben's test). (Other substances closely allied to common alcohol give a similar reaction.)

(2) Alcohol Amylicum (Amylic Alcohol-Fusel Oil, $\mathrm{C}_{5} \mathrm{H}_{12} \mathrm{O}=88$, Potuto Oil) is formed in small proportion (at the same time as ethylic and other alcohols) when saccharime solutions are fermented and distilled. It is most abundant in the spirit obtained. from fermented potatoes, and is separated by continuing its distillation at a temperature of $270^{\circ} \mathrm{F}$, the boiling-point of amylic alcohol which then distils over. It is a colourless or pale-yellow liquid of characteristic heavy offensive odour, and burning taste, sp. gr. 0.818 ; soluble in ordinary alcolool, ether, and oils, sparingly so in water: it dissolves iodine, sulphur, phosphorus, resins, etc. It burns, though not readily, giving a bluish flame; the vapour is irritating to the air-passages. Like 
other alcohols, fusel oil is readily oxidized, changing first into valeric aldehyd, and finally into valerianic acid, which bears the same relation to it that acetic acid does to ethylic alcohol. This oxidation is effected for pharmaceutical purposes by sulphuric acid and bichromate of potash in the preparation of valerianate of soda, and of nitrite of amyl. Amylic alcohol is not used medicinally in any other manner.

(3) Spiritus Rectificatus (Rectified Spivit) is alcohol with 16 per cent. of water. This "spirit of wine" is the form which results from the ordinary distillation of brandy or other "ardent spirits." The odour and taste should be purely alcoholic; sp. gr. $0.838 ;^{1}$ it should burn with a blue smokeless flame, and should remain clear when diluted with water; if not, the presence of fusel oil (amylic alcohol), aldehyd, or similar compound is indicated, and may be further detected by nitrate of silver, which is reduced by them, and falls as a black powder (oxide). "Any quantity beyond a mere trace of these compounds renders the spirit unfit for use in medicine" (R. W. Smith). Rectified spirit dissolves iodine, bromine, alkaloids, oils, gum-resins, ete., and most deliquescent salts (except potash carbonate), but. not ifflorescent salts (soda carbonate), nor salts which are sparingly soluble in water. Digested with acids, it forms ethers, and also compound ethers, such as the spiritus rtheris nitrosi. "Methylated spirit" is rectified spirit with 10 per cent. of methylic alcohol (wood spirit) added.

(4) Spivitus Tenuior (Weak Alcohol, or Proof Spirit) contains 49 per cent. of alcohol, and is prepared by adding to every 5 pints of rectified spirit 3 pints of distilled water at $60^{\circ} \mathrm{F}$. The sp. gr. is 0.920 .

The term "proof spirit" remains from the old method of testing by damping gunpowcler with alcohol: if it fired the powder it was said to be "over proof"; if it burnt off, and left the powder damp, it was " under proof." The weakest spirit with which powder takes fire has a sp. gr". of about 0.920 , and retains the old name. Every additional $0 \cdot \tilde{y}$ per cent. ol' absolute alcohol is said to be one degree over proof. water.

A higher specific gravity signifies a proportionately greater amount of 
(5) Spiritus Vini Gallici (Spirit distilled from French WineBrandy, i.e., Branntwein, burnt or distilled wine) contains about 50 per cent. of absolute alcohol with some volatile oil, œnanthic ether, etc.; it is almost colourless naturally, but becomes yellowish-brown from absorption of colouring matter from the cask, etc.; for medicinal use it should be free from unpleasant odour and taste, and not less than four years old.

(6) Vinum Xericum (Shery-n Spanish. Wine) contains from 15 to 20 per cent. of alcohol, with colouring matter, œnanthic and other ethers, also saline compounds, such as acid tartrate of potash, malates, sugar, etc.; hence it is more liable to become acid in the stomach than a purer alcohol.

(7) Vimum Aurcutii (Orange Wine) is prepared by fermenting a saccharine solution with fresh peel of the bitter orange. It has some taste and odour of the fruit, and a yellowish colour. It contains about 12 per cent. of alcohol, and is slightly acid to test-paper.

ABSORPTION AND ELIMINATION.-Alcohol is readily absorbed from the stomach, the rectum, and from serous. membranes, provided that it be not too concentrated, and the surface be healthy. If it be too strong, it may coagulate albumen, destroy epithelium, and so interfere with osmosis. Rayer proved that alcoholic injections into the pleura and peritoneum could induce systemic effects (Dict. de Médecine, 1829). Symptoms of alcoholism have been traced to the use of compresses soaked in spirit, but they resulted probably from inhalation of the vapour rather than from absorption through the skin (Racle, De l'Alcoolisme, Thèse, 1860). Delmas and Sentex have shown how readily most substances are absorbed by the lung-tissue (Récherches, Paris, 1869); and Ségalas, having injected alcohol into the bronchi, demonstrated its absorption with symptoms of intoxication. Such symptoms occur sometimes in the workmen in wine-cellars, from absorption of the vapour; and Orfila poisoned dogs by making them breathe aix charged with it.

In the stomach, under the influence of warmth and contact with mucus, ete., some portion of the alcohol becomes changed 
into acetic acid (Leuret). According to Bouchardat, Longet, Lallemand, and some other observers, absorption is mainly completed in the stomach; but Bouley, Küss, and others have shown that liquids pass quickly through the pylorus, and their absorption occurs largely in the small intestine. From the intestinal tract (with the exception of what little is converted into acetic acid), alcohol passes unchanged into the blood, where it has been detected by many observers. The absorption is generally rapid, but may be retarded by acids, tannin, mucilaginous, saccharine, and especially fatty matters (Marvaud).

The mode of elimination of alcohol has been much discussed. According to Liebig, Bouchardat, Duchek, and others, it is mainly oxidized, becoming aldehyd, acetic, oxalic, or carbonic acid, and water. This view was commonly accepted until Lallemand, Perrin, and Duroy maintained that the greater part of any alcohol taken passed out unchanged, and that no products of its oxidation could be detected (Du Rôle de l'Alcoöl, etc., Paris, 1860). Despite previous observations and clinical facts, this new view was widely and favourably received; but fresh observers soon threw doubt upon the chromic acid test employed to detect alcohol in the excretions, and proved that ouly a small proportion of what was taken could really be traced (Hall, Smith, Anstie).

In one instance, where 120 grammes were taken, none at all was recovered; in another, 2 grammes only out of 185 ; and in twenty-two observations upon persons drinking a measured quantity of wine, an appreciable amount of alcolool in the urine was only found twice (Baudot). The experiments of Schulinus upon this point were very precise, and practically decided it against Lallemand's views. Measured doses of spirits of wine (45 per cent. strength) were given to different animals by an cesophageal bougie, and so soon as the full effects of alcohol were developed, they were killed by bleeding from the external jugular and insufflating air: an analysis by fractional distillintion of the blood and viscera separately was then made, and repeated observations clearly showerl-(1) that alcolool does not localize itself in certain organs (sueh as the brain or liver), but diffuses itself equally in the system; (2) that only the blood contains proportionately more than other tissues; (3) of the 
alcohol taken, the greater part is decomposed within the organism, and the amount eliminated by the lungs, skin, or kidneys is insignificant (Archiv der Heilkunde, 1866). Lieben corroborated these results (Annalen der Chimie, vii., 1870); and English observers in the same direction were not wanting. Thus, Thudichum and Dupré distilled the collected urine of thirty-three men, passed after drinking between them forty-four bottles of wine (=4000 grammes of alcohol), but recovered only 0.25 per cent.; and in another instance 0.82 per cent. (Privy Council Medical Report, 1868).

Anstie gave large doses of alcohol to a dog for several days, and then distilled its whole body, but could find only a small amount (Practitioner, vol. xiii.). It is true that of toxic doses, such as were employed by Lallemand, a greater proportionate amount is eliminated unchanged; Subbotin, for instance, giving them to rabbits, found that 16 per cent. passed out in the first twenty-four hours, though but little afterwards; still this is not inconsistent with the views as to oxidation above mentioned, but implies only that the process is limited in amount.

With regard to the channels of elimination, observers differSubbotin stating that twice as much escapes by the skin and lung's as by the kidneys; Edes says that the latter organs excrete more of large doses, the lungs more of small ones (Boston Journ., 1872) ; whilst Anstie found the lung-elimination was but little, and Binz reduced it to nil (he says that the odour of the breath and the chemical analysis show only ethers and volatile principles, not really alcohol).

Elimination may take place from any of the excretory organs, but the amount from each probably varies with circumstances.

There is some evidence of the presence of alcohol, or of a substance which gives similar renctions, in the normal organism. (independently of its being taken). Lieben and Dupré found it in the urine of teetotalers; Ford detected it in the blood and tissues of animals, and traced it to a change in hepatic sugar (N.Y. Med. Joun'n., Jan. 18テ2); Béchamp obtained a quantity sufficient to burn, from the urine of persons who had taken no alcohol for a long time previously; and Radjieski from the urine of healthy rabbits (Archiv f. Phys., 1875). 
These results deserve attention, as telling also against the views of Lallemand, who argued that alcohol was absolutely foreign to the organism, and unassimilable by it.

\section{Physiological Action.-Internal.-Circulatory Sys-} tem. - If clibute alcohol be added to freshly-drawn blood exposed to the air, it mixes readily with the serum without much change in its characters, but strong alcohol induces coagulation and darkening in tint, by dissolving out the colouring matter of the corpuscle. Similarly, dilute alcohol may be injected into the blood-current without much change, whilst strong alcohol causes a coagulum which may lead to sudden death.

The blood of persons dying in a state of intoxication is dark and unusually liquid, but this seems rather the result of a partial asphysia than peculiar to alcolool.

Much interest centres upon the effect produced by alcolnol on the gaseous contents of the blood. Dr. G. Harley, adding it in small quantity to blood external to the body, found it lessen both absorption of oxygen and elimination of carbonic acid; and a similar conclusion has been reached, mainly $e x$ hypothesi, by Boeker, Perrin, Bouchardat, and others; but it is not yet possible to give scientific proof of it.

Probably a simple physical effect may explain much of the action of the drug on blood, for we know that the corpusele absorbs what is needed for its healthy nutrition, and gets rid of injurions or effete material, by a process of osmosis. Now, alcohol in the serum induces an osmotic current inwards, and not outwards (Graham and Dutrochet), and thus may interfere with the normal reactions so much as to lower the nutrition of corpuscles. Certainly the physical condition of the corpuscles has much influence upon their relation to, and absorption of, alcohol, as shown by Schulinus and Sulzynoki, who mixed it, in one observation with blood freshly drawn, and in another with blood that had been standing for some hours. On distillation, they obtained much less aleohol from the former (fresh) blow than from the latter, thongh the amount first alded to each was exactly the same. Ir. Parkes suggested certain chemical changes, viz., that snme acetic acid developed from one part of the alcohol taken combined with soda in the blood to form an 
acetate, which, like other acetates, would become carbonate in the process of elimination by the urine. In support of his view he pointed out that the acidity of this secretion is (slightly) increased under alcoholic influence, and the excretion of carbonic acid is diminished. Such results depend upon that portion of alcohol which is oxidized in the system, whilst the physical effect on the corpuscles would depend upon the contact of unchanged alcohol.

The careful observations of Drs. Parkes and Wollowicz on a healthy man, taking graduated doses of alcohol and brandy, showed a decided increase in the force and frequency of the pulse under their use. Thus the average number of beats per minute during the first period, when water was taken, was 73.57; during the alcoholic days, with 1 ounce it went up to 80 , with 8 ounces to $94 \cdot 7$-the average of six days observations giving 88.5 . During the next six days of waterdrinking it fell to 78; but during the following three days, when 12 ounces of brandy were taken daily, the mean was 91.

The effect on the skin-capillaries was very marked. "The face, ears, and neck were flushed, and on the days of large doses the face was slightly swollen. The skin of the trunk also seemed hot to the man himself." The turgescence of the small vessels continued for some time, and was accompanied by a sense of fulness and heaviness in the head, as if intracranial vessels were also enlarged.

Sphygmographic tracings showed, in degree proportionate to the dose, a higher and more vertical up-stroke, which indicated sudden, rapid ventricular contraction and opening of anrtio valves, and quick emptying of the arteries; no definite change in arterial pressure: after the omission of alcolonl, the tracings showed its influence even to the sixth day (Proc. Roy. Soc., No. 120, 1870). Anstie had previously pointed out that in many febrile cases, when alcohol was acting farourably, arterial tension was increased; in other's, when narcotism occurred, tension was lowered. In the healthy, such changes are not marked, and the accounts given by observers are diverse.

The sphygmograms of Marvaud, taken five to fifteen minutes after ounce doses of brandy (diluted), showed signs similar in 
character to, but less marked than, those of Parkes, and in three out of four instances a rise of four to eight beats occurred in the pulse-frequency. The French observer also noted dilatation of peripheral vessels, which he connects with lowered blondpressure, but states that this is soon followed by slowing of the heart-action, diminished volume of the pulse, and increased arterial pressure. He refers the initial excitement of the circulation to lowered arterial pressure from a direct action of the drug on the heart-muscle, independently of nerve-influence, for its injection into the jugular vein of an animal in which both vagi were divided sufficed to cause immediate, though temporary, lessening of pressure.

The subsequent slowing and weakening of heart-contraction with increased tension is traced to the influence of alcohol on the vagus-roots in the medulla oblongata, for by section of these both symptoms may be stayed, and cardiac pulsation brought back to a normal state for a time (Zimmerberg, Arch. Gén. de Méd., 18r0). Or again, the slowing may be traced to an influence on the sympathetic nerve and stimulation of the vaso-motors, accorling to Marey's dictum that the frequency of the heart-beats is in inverse ratio to the difficulty of the organ emptying itself.

Dr. H. C. Wood throws doubt on the conclusions of Zimmerberg, because of the large doses used; and certainly effects vary much according to the dose. Dogiel found the henrt-beats first increased (from stimulation of the accelerator nerves), then diminished (from stimulation of vagi), and finally increased again (from paralysis of vagi), and the arterial pressure first increased, afterwards diminished, and in the latter condition the vaso-motor centres insensible to stimulation (Pflüger's Archiv, 18rt). Dr. Parkes, as we have seen, observed no diminution in the pulse during six days of alcohol-treatment; but Rabuteau records it in a woman to whom he gave 6 ounces of brandy daily (Union Méd., Juillet, 1870). If, however, "physiology still fails," as Ir. Ringer says, "to guide our steps quite plaisly" in studying the action of alcohol on the circulation, we may recognize the clinical fact that in moderate amount it powerfully stimulates (in the ordinary sense of the word), whilst in toxic quantity it strikingly depresses. 
Dr. Wilks, indeed, reckons its stimulating effects as nil compared with its sedative or paralysing ones, but we may accept Dr. Lauder Brunton's definition of it as " something which will enable the body, or one of its parts, to do more work in a given time than can be accomplished without it."

Dr. Richardson reports:- "The first signs caused by a dose of $2 \frac{1}{2}$ ounces consist in a fulness all over the bodyespecially in the head-a flush on the face, rise in temperature, and increase in the heart-action; 5 ounces induced the second stage, with fall of temperature, flagging pulse, and impaired comnection between nervous power and muscular action; in the third stage, when 7 ounces had been taken, these phenomena were increased, and the brain lost all power, except over respiratory functions; 15 ounces is a fatal dose to a man unaccustomed to alcohol and weighing 120 pounds" (B. M. J., ii., 1877).

It must be remembered that the action of alcohol in health is not always a guide to its effect in disense; thus, in fever and in cardiac disease, when the pulse is rapid and weak, alcohol in suitable cases will render it firmer and slower.

Nervous System.-There is much resemblance between the nerve-effects of alcohol and of substances more commonly called "anæsthetic." Both produce stimulation and excitement more or less marked and prolonged, and although after small or moderate doses this is not necessarily followed by depression, yet if the doses are large, sensation, movement, and thought may be completely paralysed by them.

Such results are not secondary to gastric irritation (as suggested by Brodie, Marcet, etc.), but depend upon the direct contact or " molecular impression" of alcohol upon nerre-cells; at the same time, its effects upon the circulation within nervous tissue must be taken into account (Cl. Bernard).

As to these latter, at least, we have direct knowledge from observing the brains of animals, and we find that at the commencement of the action of ether, alcohol, etc., hyperæmia occurs, and later, anæmia; but such changes are not the essential cause of alcoholic symptoms, for they appear equally under other conditions, as from simple pain or excitement.

We do not know the precise alteration produced in nerve-cells 
--there is nothing evident to the microscope in acute cases, and the brain-substance of intoxicated persons has been found apparently healthy, though the membranes are commonly gorged with blood.

Different divisions of the central nerve-system become affected by alcohol in a definite order. In man, (1) the brain proper (the seat of intelligence and will); (2) the cerebellum (which presides over the power of equilibrium and co-ordinate motion); (3) the pons (the centre of general and tactile sensibility); (4) the spinal cord; and lastly, the roots of the nerves of respiration and circulation in the medulla oblongata. This was experimentally proved on animals under ether by Fleurens and Longet, and under alcohol by Lallemand and Perrin. In the spinal cord itself, the sensory tracts seem to be the first affected, then the motor, then the reflex; and although paralysis of sensory nerves is first evidenced at their periphery, the paralysing agent really first reaches their central extremity or root. Dogiel has recently confirmed, on frogs, the paralysis of all these nerve-tracts under large doses of alcohol (loc. cit.).

Methylic, amylic, and other less pure alcohols produce more violent excitement and heavier stupor than ethylic. The various ethers developed in wines and liqueurs, and the organio principles in beer and malt liquors, complicate the results, but to attempt to distinguish between the latter and those of absolutely pure alcohol seems unnecessary. lieferring to the older and purer forms of alcoholic liquid in common use, moderate doses often impart a sense of improved tone and wellbeing, and stimulate the intellectual and imaginative faculty. Such effect, however, is not constant; it is not produced at all in some subjects, and in any case is transitory. Shoner or later, impairment or blunting of nerve-function and sensibility may be traced.

Amongst other observations, those of Dr. Eirlward Smith on the effects of moderate doses of brandy showed lessened conscionsness and lessened sensibility to light, sound, and tnuch (Trans. Roy. Soc., 1859, p. 732).

The higher the ordinary sensibility of a part, the more marked is anæsthesia under the influence of burandy; thus, the cheek and lips are very sensitive, and a feeling of stiffness or 
puffiness in the upper lip, according to Dr. Smith's observations, resembles the first symptom of lowered sensation in eases of paralysis. He found also the dartos, the sphincter of the bladder, and other muscles connected with the genitourinary system become relaxed. Still more marked is the power of alcohol to deaden acute sensation, and to lessen wear and tear of the nervous system. "Every one recognizes," writes Dr. Chambers, "its power of blunting sorrow and pain, of checking the sensation of weariness, mental and bodily, of rendering carnal love coarser, less keen, and less discriminating, taking the points off the stings and buffets, discomforts and nastiness of daily life; but also of corrupting the delicate appreciation of its high delights-in short, of diminishing the sensibility to impressions in mind and body, of lowering the receptive functions of the nervous system."

The special senses are readily impaired by alcohol. Thus, a sportsman who takes it at his lunch cannot shoot so accurately after the meal as before it. A musician, nervous about a public appearance, may gain courage from wine, but his touch and hearing lose some delicacy.

In some conditions, especially of defective elimination and deficient supply of oxygen, or of unusual sensitiveness of the circulatory or nervous systems, alcohol, even in small doses, seems to have a toxic effect, and produces nothing but discomfort, of which headache, dulness, and depression are the most marked symptoms. I have met with several men-in whom indulgence in port wine was found to diminish, or even for a time destroy, the sexual power.

It is possible that such results follow from adulterated specimens, for the ordinary nerve-effect of the distetic use of alcoholic drinks is a beneficial one. More work is done with more comfort and with less impairment of nerve-power, and more nourishment is assimilnted, than without their stimulus. On the other hand, an excess of alcohol perverts or destroys general motor power, sensation, and intellect, leading up through all the stages and phenomenn of drunkenness to congestion, narcosis, coma, or apoplexy.

Habitual excess culminates at intervals (and more often in men than in women) in the "nerve convulsion," delirium 
tremens; whilst the prolonged use of doses somewhat less. than intoxicating induces a loss of nerve-control, with tremor and hysterical symptoms, causeless laughing or sobbing, aphonia, vomiting, and mental symptoms of excitement, depression, restlessness, altered manners and morals, deceitfulness, selfishness, etc. A modified degree of amblyopia-allied to tobacco-amblyopia-has been traced to alcohol by Hirschberg. (B. M. J., ii., 1879).

The question of the direct eausation of insanity by alcoholic excess has been much discussed, and is difficult to solve: 14 per cent. of the total insanity of the lingrlom has been ascribed to it, but, according to Dr. Bacon, 4 per cent. would be nearer the truth (B. M. J., ii., 1880). Dr. Sutherland, in an analysis of 200 cases of insanity, has ably dealt with the difficulty of judging whether the intemperance is a cause, or really a premonitory symptom, of the malady, and, amongst other diagnosties, finds that in the former case the mental symptoms were mostly of homicidal mania or suicidal melancholia; in the latter the melancholia was of more subdued form, and delirium tremens more often occurred (ibid.). Dr. Crichton Browne has stated that perfectly healthy persons, if saturated with alcohol for a sufficient length of time, might be made insane, and that a continnous series of mental disenses might be traced to its toxic action, viz.:-(1) Delirium tremens; (2) mania a potu; (3) monomania of suspicion; (4) alcoholic dementia. Mr. Mould (Cheadle) traces general paralysis often to alcoholic excess. The influence of alcohol in hereditring disease is another extensive field for inquiry, and there can be no doubt that the intemperance of parents may be expressed generally in a stunted growth of children, and in various abnormal comditions of brain, including epilepsy, idiocy, and tendency to crime and insanity of various types. Dr. Fletcher Beach (Dareuth) traced a large proportion of idiotic cases to this canse; Dr. Langdon Down, only 2 per cent.

Digestive System.-Althongh it be true that most liealthy men in favourable onditions will digest food as well, if not better, without any alcoholic liquid, it must yet be allowed that in some persons a moderate quantity taken with meals increases. 
appetite and digestive power, quiets nerve-irritation, stimulates circulation, and hence induces increased secretion of gastric juice. This is especially marked under conditions of exhaustion aud debility, when a little liqueur, champagne, or sherry will secure the digestion of food that would otherwise only fatigue the stomach.

If, however, the alcohol be taken too concentrated, or in too great a quantity, albumen and mucus are coagulated by it, local congestion is produced, and appetite impaired. The large secretion of gastric juice having no food to act upon, irritates the mucous membrane, causing flatulence and secondary palpitations; and when meal-time arrives, and the gastric secretion is deficient, food is only partially digested, and the foundation of anæmia and malnutrition is laid (Dr. T. Williams).

In the experiments of Dr. Parkes, " 1 and 2 fluid ounces of absolute alcohol, given in divided doses (diluted), in twenty-four hours, to a perfectly healthy man, seemed to increase the appetite; 4 fluid ounces lessened it cousiderably, and large quantities almost entirely destroyed it. In most cases of disease, when digestion is weakened, it seems probable that a much smaller amount of alcohol would destroy appetite." Dr. Leren has demonstrated that 25 grammes of a pure brandy improve the digestive power for 200 grammes of meat, whilst 75 grammes completely arrest it (B. M. J., i., 1880).

Claret in the amount of 20 ounces $(=2 \cdot 2$ ounces of absolute alcohol), given to the same man in another series of observations, also impaired appetite; but on neither occasion was primary digestion impeded.

Dr. W. Roberts has recently reported precise experiments as to the effect of certain spirits and wines on the process of digestion, distinguishing between the action on the salivary glands, that on the peptic glands, and that on the muscular contractions of the stomach. Distilled spixits, in the proportions commonly used dietetically, did not retard, but, on the whole, promoted digestion. Wines and malt liquors showed a different result, the former especially inhibiting the action of saliva on starch to a great extent. This was found to be on account of their acidity, and was obviated by a little added alkali. The rapidity of peptic digestion was also 
markedly checked by wine, although the secretion of gastric juice and the contractions of the muscular coat were somewhat stimulated. Effervescent wines retarded less than claret or hock.

Any habitual excess, or habitual use of alcohol between meals-i.e., on an empty stomach-induces more or less gastric congestion with dyspeptic symptoms, anorexia, red, glazed, or coated, patchy tongue, nausea, and frequent vomiting, especially in the early morning, eructations, biliousmess, and constipation alternating with diarrhoea. Gastritis may result, and ultimately fatty degeneration and atrophy of the gastric glands, and of the liver-cells, with increased development of interstitinl connective tissue of that organ-cirrhosis.

Effect on Body-Temperature.-From the sensation of warmth produced in the stomach, and increased circulation in the face and extremities, alcohol has been generally and naturally supposed to increase body-heat. It is true that minute doses may produce (at least in birds and mammals) a rise of

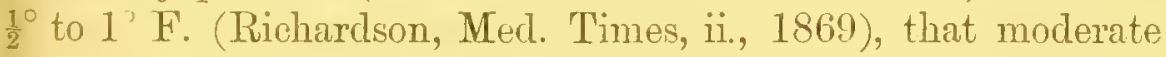
doses often fail to cause any change either way, and that, according to Dr. Parkes, even 8 ounces of alcohol, or 12 of brandy, given in divided doses in one day to a healthy man, caused no reduction, or even a slight rise. Still, a now decisive majority of observers concur in stating that usually a full proportionate to the amount talien follows the absorption of alcohol. Thus, Marvaud, experimenting on himself with precautions as to diet, rest, etc., found a fall of $\frac{2}{10}$ to $\frac{4}{10}$ of a degree $F$., within fifteen to thirty minutes of the slow taking of from $1 \frac{1}{2}$ to 5 ounces of brandy.

Mainzer gave to two men undressed in bed, regulnted doses of from $\frac{1}{2}$ ounce to :3 ounces of hrandy, and took the rectal temperature every five minutes, with all precantions against error; similar observations were male on the same men when not taking any alcohol, and a comparison of temperature curves showed a distinct lowering during the aleoholie periods (Virchow's Archiv., 18r1, Bd. liii.). In animals, unless absoluto drunkenness be produced, any fall of temperuture is but slight, viz., from $2^{\circ}$ to $1^{\circ}$ or $2 \mathrm{~F}$. (Ruge, Virchow's Arehiv., 13.l. xlix.); but full narcotic doses depress as much as $5^{\circ} \mathrm{F}$, and lothin 
ones $9^{\circ} \mathrm{F}$. This was demonstrated in dogs by Dumeril and Demarquay (1848), and more lately Magnan has shown that puppies lost $3^{\circ}$ to $7^{\circ} \mathrm{F}$. when alcohol was added to their food (Etude sur l'Alcoolisme, 1871).

The experiments of Binz and Bouvier on septicæmic fever in animals gave singular results. In a rabbit, for instance, after injecting septic fluid and inducing a febrile reaction of $102^{\circ}$ to $105^{\circ} \mathrm{F}$., about 3 drachms of alcohol diluted with water were introduced into the stomach by means of a sound: fifteen minutes afterwards the temperature was $104 \cdot 1^{\circ}$; in forty-five minutes, $102 \cdot 4^{\circ}$; in ninety minutes, $100.9^{\circ}$; in two hours and a half, $100 \cdot 6$. $^{\circ}$ The following day the pyrexia returned, and increased till the death of the animal. Very large doses lowered temperature as much as $8.5^{\circ} \mathrm{C}$; and if narcosis was induced before fever began, quite prevented its development.

Similarly the rise of temperature which is known to follow section of the spinal cord at the seventh cervical vertebra could be prevented by giving beforehand a certain quantity of alcohol (Wirkung des Alcohols auf die Körpertemperatur, Bomn, 1869 ; Practitioner, 1869-r0).

Drs. Ringer and Rickards showed that analogous results may be produced in man. During complete intoxication a fall of $3^{\circ}$ Fahr. may occur; and in ferer decided reduction may be obtained, though only by toxic doses (Lancet, 1866).

The later experiments of $F$. Riegel, nenrly ninety in number, confirm and complete previous observations, and may be thus summarized :-

Alcohol, even in moderate doses, causes genernlly a lowering of the body-temperature, though, as a rule, this amounts only to some tenths of a degree. Not unfrequently, at least after minute doses, there is no noticeable change; exceptionally, there is an elevation. In convale cents, the diminution of temperature is less than in healthy subjects. In persons who habituully take alcoholic stimulants, the diminution is almost wholly wanting. Its amount is directly proportional to the dose given, but the frequent repetition of the dose diminishes the lowering effect. The fall caused by alcohol is, for the most part, of short duration (Deutsches Archiv für Klin. Med., 1873). 
It has been variously explained as due (1) to a slowing and partial arrest of oxidation, and consequent lessened development of animal heat (Perrin, Mainzer); (2) to increased circulation, respiration causing more rapid evolution of heat (G. Sée); (3) to increased excitement of the nervous system especially (Marvaud), or (4) to vaso-motor palsy increasing heat-evolution (H. C. Wood). In any case, we must connect the fall with the circulation of free alcohol, and not with the changes undergone by a portion of it, for these would rather tend to augment the vital heat.

To such conclusions and reasonings of physiology, we may subjoin the experience of Dr. Rae, "that alcoholic stimulants give no satisfaction to Arctic voyagers," and of Dr. Hayes, that they lessen the power of resisting cold (American Journal of Medical Science, 1859).

Large doses of alcohol lower temperature-it has been presumed by checking metamorphosis of tissue; but Dr. Bevan Lewis, of the West Riding Asylum, from a number of experiments upon animals, concludes this is not the case; after closes of alcohol, the production of heat is at first increased, only it is very quickly dispersed and given out, in consequence of the peripheral vaso-motor paresis produced. As a practical point, the combination of alcohol with chloral causes an extreme degree of such paresis.

Excretion.-The effect of alcohol upon the amount of carbonic acid excreted is difficult to estimate exactly; it seems to vary with different conditions of circulation and digestion. Dr. E. Smith found it vary even with the kind of spirit used, noting a decrease with brindy, whisky, or gin, but some increase with rum. At present we oan only say that according. to several authorities-Boeker, Perrin, Hammond, Davis,-the elimination of earbonic acid is decidedly lessened under alcoholic liquids generally (Journ. de Pharm., 1849; Areh. Gér., tom. iv., sixth series; Phys. Mem. Philad., 1864; Trans. Amer. Med. Assoc., 1855).

As to the excretion of uren results also vary somewhat. Parkes and Wollowicz report "no result of importance when the ingress of nitrngen was constant;" if any change, it was on the side of increase, which might be accounted for by more 
active circulation and urination. Dr. Chambers, giving 4 to 6 ounces of brandy in divided doses with meals, found an increase of six to ten grammes as compared with a non-alcoholic period, but the increase was probably dependent on "a temporary rise of digestive power;" and an opposite condition occurred on the sixth day of the experiment, when the appetite failed, and the urea was diminished. Perrin also recorded a slight increase; but his observations were made with claret, hocks, and beer, which induced much increase in the total quantity of urine.

Except by these observers it is commonly agreed that the direct effect of alcohol is to diminish the excretion of urea, uric acid, and urinary solids generally, with the exception of the sodium chloride, and the clinical results of alcohol-taking with regard to the causation of gout, certainly point in the same direction.

The amount of urinary water is distinctly more during an alcoholic period. Rabuteau investigated this point by hourly measurement of the urine passed. (1) after taking a fixed quantity of water, (2) after the same quantity of brandy, and found that with 3 ounces of brandy, the amount of urine was five or six times more (Union Méd., 18\%0). There can be little doubt that this represents a usual result, due to direct stimulation of the kidneys by free alcohol, passing on to a partial paralysis of vaso-motor nerves.

Upon the amount of intestinal excretion no direct influence is exerted by alcohol, - the quantity of nitrogen in the freces is not increased (Parkes).

Dr. Parkes tested the elimination by the skin, by enclosing the arm in a sealed glass jar, and collecting the perspiration before and during an alcoholic period. During the latter, it gave a pronounced reaction with the bichromate test. It is doubtful whether perspiration is increased or not; Dr. E. Smith asserted that it was lessened, contrary to Weyrich's observations (Parkes' Pract. Hygiene).

Nutrition.-The effect on nutrition is indirect and direct,that portion of absorbed alcohol which circulates and is excreted unchanged by various channels, stimulates, or, in toxic doses, paralyses the nervous, circulatory, and other systems, and thus affects untrition indirectly, improving it, or the reverse, accord- 
ing to dose, etc. Its effects may be compared to those of a galvanic current applied over the sympathetic ganglia, or spinal cord, and indirectly influencing the vascular and nerve supply, and vital functions of connected organs (Marvaud).

That portion of alcohol which undergoes oxidation in the body is clearly a fuel becoming a source of heat production, and possibly, also, indirectly of other forms of vital energy. Dupré has calculated that 1 gramme oxidized, evolves 7,184 heat units; whilst the same weight of lean beef equals only 1,482 units (Practitioner, vol. viii.).

The thermometer cannot gauge latent heat, or that which becomes changed within the economy into force and movement, and to judge by the depression of temperature already described, it would seem that the increase of force produced from alcoholoxidation, is often more than consumed by the increased activity of the cerebro-spinal system. Nevertheless, in moderate doses, and especially when combined with other forms of nutriment, it answer's every purpose of a food, and Dr. Hammond found an increase in weight when he added alcohol to a diet which, without alcohol, was insufficient even to maintain his average weight.

The ultimate result of a continued, even if slight, excess of alcohol is to lead to increased deposit of fat, and to fatty and fibrous degeneration within the viscera, especially the liver and kidneys. It is probable, as shown by Boussingault and Dumas, that some alcohol may be converted into fatty tissue, as sugar and starch are; but the main source of the degeneration is the lessening or arrest of organic oxidation, and the interference with normal vital function.

SYNERGISTS.-Ethers, food, and warmth assist the alimentary effect of alcohol. Capsicum aids its stimulating power, ancl is to some extent a substitute for it.

An'TAGONISTS.-Tea and coffee lessen some of tho oppressive effects of alcohol on the nervous system. Red cinchonit bark is said to be effective in the same way, and in some? instances its froe ndministration lus suceessfully antagonized drink-craving; U'Unger's nostrum contrining it cannot, however, be depended upon (N. Kerr, B. M. J., ii., 1880). Valerian 
and asafœtida are of some use in this condition, and light nourishment, such as beef juice.

Strychnia is a much more active antidote, and Luton has recently urged a more general employment of it in alcoholism. When an acute attack is imminent, small doses should be given several times daily, and in delirium tremens hypodermic injections should be given (Bull. de Thérap., Sept. 30, 1880). On the other hand, Hameau gave to rabbits apparently dead from this alkaloid, 15 minims of alcohol by hypodermic injection, and recovery followed (Record, 1879).

It has recently been affirmed by a French society, that whilst strychnia can control some manifestations of alcoholism, it has no influence in preventing organic alterations, e.g., cirrhosis (Lancet, i., 1884).

\section{Therapeutical Action. - Extermal. - Ulceration,} Wounds.-Alcohol, diluted more or less, according to the amount of irritation present, forms a good antiseptic and stimulant dressing for atonic ulceration and suppurating wounds. Ambroise Paré recommended its use long ago, but it was almost forgotten untī revived by Batailhé. With Syme it was a favourite application. Dr. Blair has shown that compresses moistened with whisky often act well (Glasgow Med. Journ., Feb., 1870). A strong alcohol lotion coagulates albumen, diminishes pus-formation, and arrests hæmorrhage. Horvalle found it exert a local anrsthetic effect, although the first sensation caused on raw surfaces, especially mucous surfaces, is smarting and painful.

Ear Diseases. - Politzer strongly recommends rectified spirit for chronic otorrhoca, and for the destruction of remains of polypi that are beyond the reach of instruments, and in cases of suppuration of the middle ear attended with granulation and polypous growth. The purulent secretion must first be removed from the tympanum by insufflation of air and the use of the syringe, and careful drying. About a drnchm of warm spirit is poured into the meatus, and allowed to remain ten minutes, the head being kept inclined to the opposite side. A sense of warmth is felt; if there be pain the alcohol should be diluted. If possible, it should be applied three times a day, till improve- 
ment occur; the red granulations and growths become pale grey, and the mucus and albumen on the surface are coagulated.

The same treatment is useful for vegetable parasitic growths in the ear; also for nasal polypi (Wiener Med. Woch., Juli, 1880).

Erythema, Bedsores, etc.-A lotion containing spirit of wine is cooling, and exerts an astringent tonic effect on the capillaries in irritable congestive inflammatory conditions of the skin, such as erythema and acne. When there are signs of commencing bedsore brandy is a good wash ; also in tenderness of the nipples, before actual fissure. Nélaton used it to abort boils.

Vaginitis.-At the Loureine Hospital (for venereal disease) Dolbeau found much advantage from strong camphorated alcohol applied for twenty-four hours at a time, on tampons of cotton wool made to fill the vagina.

Hydrocele-Serous Effusions.-In these cases, alcoholic liquids-brandy, or especially port wine-have been injected after withdrawal of the fluid, but they are practically superseded by preparations of iodine. Schwalbe has employed with success injections of 15 to 80 per cent. in varix and vascular growths; he introduces first a canula close to the affected part, and does not inject until satisfied that blood is not flowing from it (Virchow's Archiv, Bd. lxxvi.).

Similar injections have also been used to obliterate hernia (Practitioner, vol. xxiv.).

\section{Therapeutical Action-Intermul.-Acute Disease.-} From the time that Arnauld of Villeneuve recommended "spirit of wine" as an heroic remedy against the most various maladies-cancer, gravel, dropsy, etc.-alcohol has held a more or less prominent place amongst medicinal agents.

When Brown taught that all illness resolved itself into debility, and required stimulation as well as support, alcoholic medication was proportionately in favour; but when the system of Broussais rerluced all disease to conditions of irritation or inflammation requiring to be met by a sedative treatment, alcohol was almost abandonert.

Laennec, Chomel, Pinel, and a few others, continued to pre- 
scribe it in pnemonia and adynamic fevers; but Carmichael Smith is credited with the renewed demonstration of the value of wine and bark in typhus (Description of Jail Distemper at Winchester, 1795). Alison referred the success obtained by Smith with this treatment to a "change of type" in disease, but independently of theory, Graves and Stokes soon afterwards corroborated the results in typhus, typhoid, and other exanthemata, delirium tremens, pneumonia, etc. (On the state of the heart and the use of Wine in Typhus, Dub. Journ., 1839).

Dr. R. B. Todd was the most thorough of modern adrocates for the alcoholic treatment of inflammatory and febrile diseases, and pressed it to the fullest extent. He argued that acute disease could not be cured by reducing measures, nor indeed by any medicine (unless it be a direct antidote of a given poisonous material); and yet, as it tends to cure by a natural process, for the development of which vital porver must be kept up, remedies could only be useful so far as they do this. The strong point of Todd's teaching was his directing attention to the cital strite of the patient rather than to the mere name, or even to the nature of the malady: he insisted also on the great waste of nerve-power during a severe illness, and on the necessity for easily-assimilated food capable of sustaining it. Alcohol, he argued, answered these requirements, acting specially on the nerrous system, improving its power: it did not cause secondary depression, unless in excessive doses, and then only through gastric derangement: it did not cause inflammation, but sustained the animal heat, the heart-action, etc. : to suspend its use might be dangerous, and should be attempted only when much digestive disorder, or a strong alcoholic odour of breath was noticed-on these principles he gave very large doses. On the other hand, his critics maintained that Todd gave alcohol when not requirerl, and too soon in the commencing acute stage, and in too great a quantity; also, that his advice to increase the dose when smaller ones failed to cure was dangerous. F'or these and other rensons, several of Todd's pupils, Anstie, Brinton, and Beale, whilst accepting his general theory and acknowlodging gool results, modified his practice, and especially his doses. 
Tweedie restricted the medicinal use of alcohol to conditions of prostration during fever; Lyons to the typhoid state of pneumonia, etc., and to typhus after the second week-avoiding it in enteric fever. E. Smith was more exact in his indications, ordering it as a direct stimulant of the functions of the stomach, heart, or skin, and in debility, for preventing congestion, improving vital resistance, ete.; yet he recognized also its secoudary, and possibly bad, effects, such as retention of urea, diminution of urine, and of other secretions.

Mrurchison condemned Todd's doses as excessive, and found that his own cases of typhus and typhoid showed better results with moderate quantities. Hughes Bemnett asserted the same with regard to pneumonia. Béhier followed the practice of Todd in the latter disorder, and found under alcohol, "the delirium cease, the pulse fall, the respiration become slower, and abundant perspiration come out, with all which the strength improved." In severe cases of typhoid fever he had, however, no success. Dr. Ferrier pointed out specially the difference in the action of alcohol upon weakened and pyrexial as compared with healthy subjects, and how comparatively seldom alcololism was induced in the former even by large doses.

Gubler remarks that in severe and acute inflammatory ferer, with not only heightened pulse and temperature, but also excess of respiratory combustion, rapid emaciation, and abuudant excretion of urea, alcohol can only do harm. But in that form of pyrexia in which, though the temperature is raised, there is little systemic combustion, when the urine contains a small amount of urea but much uric acid, when there is perversion of function, and much tendency to adynamia, alcohol may render great service as a respirntory food, as lessening the impairment of nutrition and stimulating the vital forces (Traité, p. 8.\%3).

Finally, Jaceoud, one of the most eninent of modern French teachers, considering Tochl's practice as extreme, though sometimes valuable, recognizes in pucumonia, for instance, but ono true indication for alcohol, and that is adynamin: he reommenrls it also in typhoid, variola, scarlatina, erysipelas, and in all continued fevers, finding "that it often lowers temperatum, stimulates the nerve-power, presents to the felorile combustion 
an element easily consumed, and thus restrains consumption of animal tissue, and becomes an 'aliment d'épargne.'

During an interesting discussion upon "alcohol in fever," at the British Medical Association meeting in 1879, Dr. Little showed there were three objects for which wine and brandy were prescribed in medicine - (1) as cordials to enable patients to take and digest a larger quantity of food; (2) to soothe the nervous system ; and (3) because they were supposed to "keep up the vital powers." ... Practically, speaking of severe cases of typhus and typhoid, he thought that while alcohol sustained the circulation, it seemed injurious to the nervous system, increasing restlessness and "muscular weakness"; the diseases in which he found it specially useful were catarrhal typhus and pneumonia.

Dr. MeNaughton Jones summarized nearly 1,000 cases of fever of different kinds, and concluded alcohol to be sometimes of the greatest value in typhus and typhoid; but many cases did not require it at all, and in others it proved injurious, especially if given too early in the case: it did not prevent adynamia, and it was not essential for habitual drinkers; an average time for expecting its requirement was from the eighth to the twelfth day. It had little effect on the temperature: he took as a guide the age, and the condition of heart, pulse, tongue, and head symptoms: a feeble, irregularly-acting heart, a compressible rapid pulse, a fairly moist tongue, and absence of violent head symptoms encouraged him in its use; he considered it "a supporting food" when assimilation was difficult. The young, as a rule, did well without it.

Dr. Wade found, as conditions indicating the use of alcolinl, "increasing rapidity of pulse and respiration, a dry tongue, failing muscular power, increasing delirium, and indications of congestion;" but even when such symptoms were present alcohol might fail to do the good expected. High temperature was not necessarily an indication for it. Alcolol acted rather as a soporific and sedative than a stimulant, and the most certain indication of its doing good was reduction of pulse-rate; this ought to be eviclent in a few minutes after a. "sufficient dose," and so might be ascertained at one risit. There was no single form or type of fever in which it was 
necessarily required, but, on the other hand, when indicated, there was no drug that would supply its place. (F. Vacher has reported on the alternative use of ammonia, ether, etc., in 243 cases of typhus-Lancet, i., 1881).

Dr. Foster argued for the use of alcohol when high temperature was developed, indicating a high state of combustion; it was a substitute for, and spared the oxidation of the tissues. Dr. Hayclen, of Dublin, attached importance to a low temperature as an indication, and Dr. W. Squire to lowering of the surfice-temperature, as compared with that of the interior.

Dr. John Tibbits (of Warwick) regarded alcohol as a "fat-food," providing in fever a "supply of fuel which might otherwise be taken from the muscles;" it lessened tremor, depression, irritability, delirium, and acts probably as an anti-zymotic. In spasmodic stages it was not advisable, in congestion it did well, with venesection. Joly adopts alcohol alternately with digitalis as the treatment of pneumonia(Record, 1880). Dr. N. Kerr, allowing that alcohol could (1) lessen excessive waste, and (2) "repair lesions of circulation" feared "reactionary fever," and advocated rather quinine and cold water, or as stimulants, injections of hot water, ether, etc." "When alcohol did good it acted as a sedative to the nervous system, but the ice-cap, cold affusion, and camphor were equally efficient and less dangerous." Dr. Spedding "measured the amount of his stimulants by the amount of pulmonary complicntions;" he spoke also of their value for children in pyrexial disorder (B. M. J., ii., 1879).

I have summarized these various and somewhat conflicting statements as representing fairly well the average opinions amongst the profession. I may add the words of Dr. Moxon:"In a fever the sense of individual strength is failing, and pluck gives way. Muttering fear becomes horror and violence, then alcohol will bring back the man to hisown help. You make him again come to himself and believe in linnsolf by its aid. The delirium so violent was as that of a shying, timid horse. Alcohol gives the patient courage, and he is fenrless and quiet again. In short, it is a medicine of the mind with some power over the body."

Sir Risclon Bennett recognizes that "alcohol can advanta- 
geously be dispensed with in many cases where it was formerly given as a matter of course," and in simple continued fever commonly omits it during the regular course of the disease, though finding it an important aid, and often indispensable during convalescence. "But in the severer forms of fever, and in many of its more dangerous complications, I believe its use to be imperative, and know nothing that will take its place. In the crisis of many a severe case, when the action of the heart and the pulse are so feeble as scarcely to be felt, it is by alcohol alone that the life of a patient can be saved, and whether it act as a stimulant, or as Dr. Willis would maintain, as a sedative, is immaterial to me, if I find the fluttering pulse become steady, and can again feel the impulse of the heart, and thus tide my patient over the few hours that are to decide between life and death" (Contemporary Review, 1881).

The following statements represent my own views on the subject:--

Specific Fevers-Typhoid.-No rule can be laid down as to the day or the week of a fever on which to commence stimulants: the state of the circulation, digestion, excretion, and cerebral condition must guide our prescriptions. The indications for it are symptoms of cardiac failure with concomitant general depression, a repid, small pulse, dry tongue and shin. The dose must be regulated by the effect produced. When there is early depression in the disease, the prompt exhibition of alcohol is absolutely necessary, the dose being gradually increased. When cardiac failure is feared it must also be given early, but in small doses gradually increased if mecessary. If the quality of the pulse is improved, and its rate diminished, if the tongue and skin become more moist, alcohol is acting beneficially; but care must be taken not to push it too far, or progress will be hindered by an over-dose. In my opinion, though doubtless cases of typhoid recover without alcolol, the majority require it at some period or other of the attack.

Typhus.- Owing to the early and great depression common in this disense, alcohol will generally have to be given soon, and in large doses. In mild cases the same rules apply to its alministration as in typhoid fever, and the remark of sir William Junner should be borne in mind, viz., that if in doulut 
as to giving alcohol in typhus he always gives it, whereas in typhoid, in the same circumstances, he usually withloolds it.

In some cases of high, ferer in chiteren alcohol is of much service; but it must be remembered, not only that they bear fever better than adults, but that the administration of a diffusible stimulant, as alcohol, has a greater effect than in adults, even allowing for the difference in dose. The indications for the drug are practically the same.

Delirium Tremens.-Much judgment is required as to ordering stimulants in this disorder. My own practice is, as a rule, to omit them at once and entirely, supplying their place by different forms of nutriment-strong soups, eggs, coffee, etc., or by medicinal stimulants, such as capsicum or ammonia; still it must be recognized that in some patients the tendency to heart-faihure is so marked that it is safer to add small quantities of alcohol to their nourishment at intervals of three or four hours, at least during the time of great prostration.

The best stimulant to employ is good brandy when one is sure of obtaining it, but should there be any doubt of its quality a good whisky may be substituted with advantage. There are cases where the system requires repict stimulation, and I have never known anything effect this better than small quantities of brandy with champagne: it is superior to, and more permanent in effect than, a much larger anount of brandy and aerated water; it is also more rapid in its action and less likely to cause tympanitis afterwards.

I do not pretend to lay down any rule for quuntity; the physician will do well to order it liberally if the patient gasps or yawns frequently, and he will estimate the quantity required by the smallness and frequency of the prulse. In realing over published cases, I am strongly of opinion that the doses given are too small as a rule. and not frequent enough.

When convalescence begins, the purely alcolonlic trentment should be modified, and good rerl wine (Burgundy) suldstituted gradually for the hrandy or whisky, provided always that the patient has no gouty, rheumatic, or anthritio taint: should this be the case, a good, somnd Bordenux is prefernble.

Nerve Disorder.-It is within my experience that patients suffering from chronic "brain-exhaustion" are often alvised, 
and by very competent physicians, to refrain rigorously from alcohol; much depends, of course, upon the person, the locality, etc., but I think that a moderate quantity of alcohol is indicated in these cases; and in many of them, when sleeplessness is a prominent symptom, an allowance of whisky and water at bedtime will be found of service; also when excessive irritability from over-study or work is present the same remedy often acts well. The danger of the treatment is that the patient may like the stimulant too much, and again, finding so easy a cure for his distress, may be tempted to continue to overwork himself.

The lupulin and alcohol combination found in ale is often of service where other remedies fail to secure sleep; possibly malt may have to do with it also, but I am inclined to credit the sleep-giving properties of ale to lupulin: some people are extremely sensitive to it.

Disorders of Digestion.-Alcohol is rarely required for its direct effect on the digestive organs, but it may in some cases be prescribed with benefit. It is especially in the convalesence from acute illness, when the appetite is poor and the digestive power enfeebled, that its value is most apparent. It acts as a tonic to the stomach, stimulating peristalsis, and thereby aroiding the undue retention of the products of digestion in the gastric cavity, whilst its effect on peptic secretion has already been referred to. It should be given with meals, or occasionally -in small quantity-immediately before.

It is sometimes required for a general stimulant effect in preserving the strength and warding off the collapse of profuse diarrhoca and vomiting: As a stimulant alcohol is. best exhibited as brandy in varying degrees of dilution, and hot or cold, as circumstances may dictate, remembering that its cliffusibility, and hence rapidity of notion, is increased when given warm. In an effervescing form, as represented by champagne, it exerts a sedative effect upon the gistric mucous membrane, and hence may be best borne by the stomach in severe vomiting when amministered thus, especially if iced. The addition of a little brandy to the champagne will increase its stimulant effects whilst retaining all the advantages afforded by effervescence. As a tonic and to improve the appetite, it is advisable to employ beer, burgundy, or port wine. 
Dr. Roberts concludes that persons of weak digestion should use wines only sparingly, so as to get their stimulating, without their retarding effect (B. M. J., ii., 1885).

In children it is a sound rule not to prescribe stimulants for dietetic purposes, yet there are a few cases where they may be ordered in small quantity with great advantage.

Denme has recently written in support of the value of alcohol in the diarrhoea of infants artificially fed. He gives 20 to 30 minims generally with kreasote or benzoate of soda, "to stimulate nutrition and check the too abundant formation of micrococci which load the intestinal glands" (Record, 1880). "White wine whey" has also acted well (B. M. J., ii., 1880), and "iron wine" is a good combination for the same purpose.

Blood-Poisoning.-In cases of severe septicemin, formerly called "surgical fever," alcohol is often of great service. The symptoms, as a rule, manifest themselves after an injury or operation, especially if the genito-urinary or osseous system be implicated. There is a rapid and persistent rise of temperature (varying from $102^{\circ}$ to $106^{\circ} \mathrm{F}$.), the breathing is rapid, there is sudden and marked failure in strength of the heart-action (due to the effect of the poison), the pulse is small and weak, in not a few cases clots form in the right side of the heart, and the patient dies suddenly. In these instances of septicæemia, with marked failure of the circulation, brandy and champagne are often invaluable, keeping up the heart-action until the poison has passed out of the system (cf. Macnamara, On Diseases of Bones and Joints). The quantity to be administered must be regulated by the amount which the patient's stomach can absorb without irritation; if nansea be felt, I have found brandy, in doses of 30 drops, injected with a hypodermie syringe into the subcutaneous tissues, very useful; it must, in fact, be administered in the same way as in instances of threatened death from chloroform. A well-known experiment, conducted by Professor Binz, illustrates this use of alcohol: he produced septic fever by injection of pus in two young healthy dogs of equal weight; one was treated with alcohol (three iloses of 2 drachms each boing introduced, diluterl, into the stomand), and recovered; the other was untreaterl, and died on the second day.

()f pyremin, Inr. Clemens has recorded eight sovere cases which 
recovered under the free use of "red wine," as much being given as the patients would drink (Deutsche Klinik, 1874-5̃); and Dr. Whittaker, amongst others, has shown the value of alcohol in puerperal fever (Obstetric Gazette, Oct., 1880). II severe cases of snake-bite, as well as in poisoning by aconite, veratria, or digitalis, alcohol given even to intoxication has been advised as "the only resource;" certainly three to four ounces of brandy or whisky may be given (and not much diluted) every quarter or half hour, till a definite effect is produced.

In Syncope, or temporary failure of circulation, such as occurs from various causes of exhaustion, hrmorrhage, etc., alcoholic stimulants are often necessary.

In Chronic Illness of any kind, the medicinal prescription of alcohol in any form requires the gravest consideration, since its frequent use might induce drink-craving and a habit of drunkenness; for the mere relief of minor pains and symptoms, it is not justifiable to order a remedy of such seductive, and, possibly, fatal character: at the same time it must be recognized as powerful to relieve, and with due caution its administration may prove most valuable. For instance, in chronic forms of heart-disease, with degeneration, etc., three or four teaspoonfuls of brandy taken with each meal will postpone attacks of syncope and dyspnoen, and enable much more work to be done; as a rule, the stimulant should be rigorously interdicted betreen meals.

In many other forms of chronic disease, on the other hand, alcohol is not only unnecessary but injurious, (./\%, in gouty and rheumatic affections and in cystitis; I have seen, in such cases, a glass or two of wine daily contribute markedly to aggravation of the disorder and destructive alteration of the affected tissues.

In Gout, at any stage, the prescription of alcohol must be the exception. In many, though not in all cases, total abstiuence seems to have prevented attacks in subjects strongly predisposed by inheritance (Lancet, ii., 1876; B. M. J., ii., 1878).

Hypochondriasis - Mental Depression.-In these conditions, consequent either on dyspeptic and other bodily ailments, or on mental anxiety, there is much temptation to "drown care" with alcohol, but the effect is temporary, and can 
seldom be safely obtained. Small doses of 2 to 4 drachms, given always with some form of nourishment, are the utmost admissible. In.markedly hysterical cases, the prescription of alcohol is, in my opinion, very rarely to be justified.

Neuralgia.-In certain cases of chronic recurrent neuralgic main in weakly subjects, and especially if the fifth nerve be nffected, an ounce of whisky taken with hot water will relieve, but as a rule, small quantities of wine at meal times are better. Dr. H. C. Wood suggests its combination with cod-liver oil.

Phthisis - Scrofula.-A special form, "alcoholic" phthisis, has been traced to the immoderate use of "drink," but apart from this, a moderate amount is often of the greatest service during the course of ordinary phthisis, and in later stages, full doses are, I think, justifiable to the extent of some narcosis and lowering of temperature. One case within my knowledge took more than half a pint of whisky during the twenty-four hours for many weeks without any intoxicant effect, but with the production of much comfort. Scrofulous children often derive advantage from wine or porter.

\section{CEREVISIA FERMENTUM.}

\section{(Beer Ytast.)}

PREPARATION.-Fresh yeast is formed whenever an infission of the albuminous principles of malt is made to ferment by the action of old yeast, as in the brewing of beer.

CHARACTERS. - A yellowish-white, viscid, frothy liquid of characteristic odour, and bitter taste. It is formed mainly by a fungus, the Torula cerevisire, which appears under the microsenpe in round or oval cells and spores. It is in an active proliferating state, and gives rise to vinous fermentation in saccharine solutions. It is not destroyed by water or aleohol.

Physiological Action.-This has not been clearly marle out, but topically at least, yeast exerts an antiseptic anil stimulant effect, and it has been supposed to act in the sume manner when taken internally. 
THERAPEUTICAL ACTION.-Extemal.-Suppuration.A poultice of yeast is a cleansing and antiseptic dressing for indolent foul-smelling ulcerations of any kind.

Therapeutical Action.-Intemal.-Debility-Boils - Carbuncles - Suppuration. - Yeast has been given internally in these conditions, and there is some clinical evidence of its value. In cases of general debility, with weak, imperfect digestion, coated tongue, and unhealthy, fœtid stools, it is said to act well as a tonic and aseptic. In continued formation of boils and carbuncles, whether primary or dependent upon diabetes, yeast is often of service, though Dr. Garrod has not been able to verify this from his own experience.

In flatulent distension of the lower bowel, enemata containing yeast have been given.

PREPARATIONS AND DOSE.-Dose: from $z$ to 4 teaspoonfuls. The yeast used should always be fresh, and taken on an empty stomach. when practicable.

Cataplasma fermenti-Mix 6 fluid ounces of fresh beer-yeast with an equal quantity of water at $100^{\circ} \mathrm{F}$., then stir in 14 ounces of flour to the proper consistence. Place near the fire till it "rises" like dough. It is then ready for use.

\section{BYNE (MALT).}

(Extractum Brnes-Extractim Matit-Extract of Malt.)

Under this name a concentrated infusion of malt has been recently much used as a mutrient tonic. It may be adrantrgeously combined with iron or cod-liver oil.

At Guy's Hosprital it has long been prepared in the following manner:-Bruised malt 10 parts, cold water 10 parts; macerate three hours, then add to parts of water at $150^{\circ} \mathrm{F}$.; digest at that temperature for one hour, strnin, boil, and pass through flannel, evaporate to $\frac{1}{11}$ part, again pass through flannel; lastly, evaporate to $\frac{1}{5}$ part ( $\iota$. Hordeum Vulgare, p. 818). 


\section{PARALDEHYD.}

DESCRIPTION AND PREPARATION.-This substance may be described as a "polymeric modification" of aldehyd, which is itself an oxidation product of alcohol forming before acetic acid, and readily passing into it. Paraldehyd represents a combination of three molecules of aldehyd having the formula $\mathrm{C}_{6} \mathrm{H}_{12} \mathrm{O}_{3}$, and may be obtained by treating the latter with dilute sulphuric or nitric acids; it is, at ordinary temperatures, a colourless liquid of ethereal odour (somewhat like that of nitrous ether, which is said to contain it), sp. gr. 0.998. Cooled below $50^{\circ} \mathrm{F}$. it crystallizes like glacial acetic acid. It dissolves in eight parts of cold water, but is less soluble in warm, so that a saturated solution becomes turbid and precipitates on heating.

ABSORPTION AND ELIMINATION.-The former is very rapid, soporific effects being often produced in animals within three to five minutes (Cervello, Archiv f. exper. Path., 1882). The latter occurs almost wholly by the lungs, the odour remaining long in the breath (Gugl): some have found the odour also in the urine, but not the substance itself nor its products.

Physiological Action.- Nervous System.-Two or three grains have been found to cause in rabbits a narcosis of six or seven hours' duration, without other symptoms than some slowing of respiration. Fifteen to sixty grains produce usually, not constantly, in man a drowsy condition or tranquil sleep of several hours, preceded by slight feeling of intoxication, but not followed by headache or unpleasant depression. During the sleep, there is not the ansesthesia or analgesia induced by chloral, but there is diminished sensitiveness to the electro-magnetic current (Hénocque).

The drug seems to act directly on the cerebrum; full doses affect the spinal cord and abolish reflex actions, whilst toxic amounts paralyse the medulla oblongata, and consequently arrest respiration. Tolerance of the drug is quickly established.

Circulatory System. - Whilst certainly less depressing to this system than chloral and some other hypnotics, and indeed 
often quickening at first the circulation, its full effect is of sedative character, the heart-action and the pulse being somewhat slowed, and the arterial tension diminished. Respiration is always more or less slowed, the oxyhæmoglobin of the blood is diminished, and the body temperature lowered (Hénocque; Coudray, Thèse de Paris, 1884).

Digestive System.-The taste of paraldehyd is peculiarly nauseous, to some persons at least, and I have known the drug; when given only in water, produce nausea, anorexia, coated tongue, etc.; this is not, however, the usual experience of continental observers, who speak of it as being well borne. It does not induce salivation.

Urinary System. - The quantity of the urine is commonly increased (Quinlan), but according to Hénocque the solids are not so, since tissue-change is rendered markedly less active (Soc. de Biologie, Mars, 1884). Dr. D.J. Leech could not verify any special effect on this secretion, but noted sometimes free perspiration (Med. Chronicle, Feb., 1885).

SYNERGISTS.-According to Dr. Gugl, the alkaline bromides act specially well with paraldehyd-better than chloral.

AnTAGonists.-Strychnia, and probably caffein. Dujardin Beaumetz gave to one rabbit enough strychnia to kill twentyfive; but having administered previously a large dose of paraldehyd, no other effect followed than weak convulsive attacks (Bull. de Thérap., Jan., 1884).

Therapeutical Action.-Intemal.-Insomnia.-As with all new remedies, varying reports have been received as to the value of this drug in disease, but the general tendency of continental experience, at least, is in farour of its valuable hypnotic power. Of more than 300 administrations by Gugl, the failures were only about 8 per cent. He found it act rather better with the hysterical and nemasthenic than with the hypochoudriacal, and better in mania than in melancholia. It was perfectly well borue in cases of fatty heart and of atheromatous ressels (Wiener Zietschr., Aug., 1883).

(If twenty-eight cases of different forms of mental disorder recorded by $G$. Riggi, it failed only once, when the dose was 
too small; and Morselli speaks equally well of its action, not only in epilepsy, general paralysis, and other nerve maladies, but also in bronchitis and other chest affections (Rev. des Sc. Méd., 1884).

Coudray, on the other hand, found it of no value in the latter class of cases when expectoration was profuse, but good in almost all other varieties of insomnia, in mental disorder, delirium tremens, morphinism, and convulsive neuroses, as well as in tetanus and strychnia-poisoning.

My own experience of the drug is not quite so favourable as the above; in ordinary cases of insomnia, it has not proved a good substitute for chloral, the dyspepsia induced being often considerable, and the taste and persistent odour in the breath render it a disagreeable remedy; but on the other hand, in the insomnia of mental disorder it has acted extremely well, notably in hysteria, with cerebral symptoms, and in general paralysis of the insane. A great advantage is the rapidity of its action : several times when patients were wildly unreasonable and violent, I have known a teaspoonful administered in a glass of claret cause them to doze within ten minutes, so that they could be put to bed and left to sleep off the attack: in such patients the disagreeable taste is little noticed. Mr. G. F. Hodgson notes the "calm sleep produced by it in most diseases," and its advantages over chloral in gouty cases; he finds it objectionable only in conditions of inflamed and irritable throat and stomach (B. M. J., ii., 1885).

Dr. Leech aptly remarks that " chloral, opium, and bromides have their separate areas of usefulness, but there are gapss between them, and paraldehyd fills up one of these; when, for instance, chloral is contra-indicated by a flabby dilated heart, or opium by cyanosis, in cases of delirium tremens or pulmonary congestion" (loc. cit.). In some other cases it acts well combined with chloral or bromides.

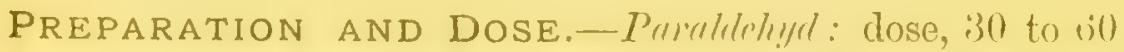
min. and upwards repeated ncoording to effect: it is best given with muilage, syrup, tincture of ornnge, and peppermint water; $2 \frac{1}{2}$ dr. (and probally more) may be safoly taken in the twenty-four hours. 


\section{AETHER (ETHER).}

\section{ÆTHER SULPHURICUS, $\mathrm{C}_{4} \mathrm{H}_{10} \mathrm{O}=74$.}

DESCRIPTION.-A volatile liquid prepared from alcohol. It should contain not less than 92 per cent. of pure (absolute) ether. The old and common name, "Sulphuric ether," is properly restricted to a true sulphate of ethyl, $\mathrm{C}_{4} \mathrm{H}_{10} \mathrm{SO}_{4}$.

Ather purus (pure ether) is so termed when free from alcohol and water.

PREPARATION. - By henting together and distilling a small proportion of sulphuric acid, and a large proportion of alcohol added by degrees. An acid ether (sulphovinic acid) is first formed, and then, in the presence of excess of alcohol, decomposed into free ether and sulphuric acid: the ether distils over, and is condensed, whilst, the liberated acid acts again on a fresh supply of alcohol; this must be added gradually, otherwise a large amount of it would distil orer undecomposed. A small quantity of acid is enough to etherify much alcohol, but the operation is ultimately limited by the occurrence of secondary reactions and by the acid becoming too much diluted; the method is called the "continuous etherification process."

Many other compounds besides sulphuric acid are capable of etherifying alcohol. The crude ether obtained by the above process usually contains sulphurous acid, water, and unchanged alcohol; it is purified by agitation with slaked lime and chloride of calcium and redistillation, and when thus "rectified" contains 92 per cent. of pure ether (by volume) and 8 of alcohol.

Pure ether is prepared by agitating ordinary ether with water, when any contained alcohol will combine with the latter and subside, leaving the ether floating on the top: this is decanted off, and set to stand for twenty-four hours over chloride of calcium and quicklime, in order to free it from water, and then redistilled at a gentle heat. 
CHARACTER AND TESTS.-Ether is a colourless liquid, extremely volatile, of peculiar sweet fragrant odour, and hot pungent taste; at a very low temperature it crystallizes in shining white plates; it boils at or near $105^{\circ} \mathrm{F}$., if perfec'ly pure at $95^{\circ} \mathrm{F}$.; it readily evaporates if exposed to ordinary air, and if applied to any part of the body this evaporation canses a sensation of cold. It is inflammable, burning with a white flame; sp. gr. 0.735 , if above this, an unusual quantity of water or of alcohol is present. Ether and water are to some extent soluble in each other-thirty-six parts of the former taking up about one of the latter-whilst a pint of water dissolves about two ounces of ether; shaken with an equal bulk of water it loses about one-fifth of its volume. When freshly prepared, it does not redden litmus, but develops some acetic acid, if it be kept long. If it contain any oily liquid (such as heavy oil of wine) it becomes milky when diluted with water. It mixes with alcohol in all proportions. It is much employed in pharmacy for its power of dissolving resins, fats, oils, and active organic principles, such as cantharides, the active principle of the male-fern, also iodine, phosphorus, corrosive sublimate, etc.

Pure ether should be of not higher sp. gr. than 0.720 , and should boil at $95^{\circ}$ to $98^{\circ} \mathrm{F}$.; vapour density, 0.37 . It does not congeal at $146^{\circ}$ below zero. It does not coagruate serumalbumen. It dissolves many organic principles, such as aconitia, digitalin, quinine, etc.; but, unlike alcohol, does not dissolve caustic potash or soda.

ABSORPTION AND ELIMINATION.-Wther is very readily absorbed by all tissues, but especially by the lungs. It is also quickly eliminated by these organs, and may be detected in the urine (Pitha, Snow) and in the milk (Seifert). Children are apt to refuse the milk of mothers taking it (Buisson).

Physiological Action. - Extermol. - Ether applier externally causes first a sensation of cold from its rapicl evaporation, also pallor, sometimes "goose-skin;" afterwarls increased redness of the part with dilatation of small vessels anl a sense of heat; to some extent it lessens local sensibility and pain. If evaporation be prevented, the sensation of cold 
will not be felt, and the local irritation will be more, and may amount to blistering; on mucous membranes or surfaces denuded of skin the warm prickling sensation is followed by more marked diminution of sensibility. By increasing evaporation, which may be done by currents of air, fanning, ete, or by finely dividing the ether as in a spray apparatus (Richardson), coldness and anresthesia may be increased so much, that the tissues may be frozen and absolutely deprived of sensation in a few minutes; when circulation returus, however, much tingling and discomfort commonly occur. Peripheral nerves laid bare and treated with ether lose their sensibility in the part touched and above (Flourens, etc.).

Physiological Action.-Internal.-Digestive System. -When taken by the mouth, ether in moderate doses causes a feeling of warmth at the stomach, and has been found to stimulate the gastro-intestinal secretions, especially that of the pancreas (Claude Bernard).

Large quantities, or habitual use of ether, may induce, like alcohol, morning romiting, heartburn, etc. (G. Martin), and sometimes from becoming quickly vaporized, as it does at the normal temperature of the stomach, it may cause much swelling of the abdomen. Nothnagel states that death has occurred from tympanitis thus produced pressing the diaphragm upwards.

Circulatory System.-Ether resembles alcohol in its stimulating effect upon this system, quickening the pulse and capillary circulation for a short time. During ether-narcosis the heart's action has seemed even stronger than normal, according to the sphygmographic tracings of De Morgan, although, if the administration be unduly pressed or prolonged, the heart becomes ultimately exhausted. The pulse, though variable, is commonly quick at first and slow later.

The blood is altered, having been found sometimes all darkcoloured; at other times, all, even the renous blood, of red arterial colour; the former condition being traceable to asphyxia and lessened supply of oxygen, the latter to absence of carbonic acid, as, when respiration remains unaltered, ether may displace this gas almost entirely from the blood (Gubler). When shaken with ether, in small proportion, the blood becomes cherry-red 
and transparent, and the corpuscles, at first altered in shape, and "horned," finally disappear, being dissolved in the plasma. Ether forms a stable combination with hæmoglobin, and so prevents the interchange of oxygen. The blood of etherized subjects exhales a strong odour of the drug.

According to Dr. Richardson, it is too soluble in blood to be a very good narcotic agent, for it is absorbed in the proportion of 1 part to $5 \% 2$ before the blood is sufficiently saturated for complete narcotism, and we are obliged to keep up this proportion by persistent administration and exclusion of air; hence often symptoms of asphyxia and restlessness occur under it.

In the early stages of etherization the amount of carbonic acid exhaled is two or three times more than usual, whilst, after ten to twelve minutes of the process, no trace of the gas can be detected in the expired air; under the full influence of ether the face is deeply cyanosed. Knoll concluded, from a series of rather complex experiments, that both chloroform and ether first stimulated and then exhausted the excitability of the respiratory centre, and in full doses were intense poisons to it. (Ville and Blandin).

Nervous System.-In its action on this system, ether partakes of the properties of inebriants and anæsthetics, and in some respects seems intermediate between alcohol and chloroform: before it was used to deaden sensibility, it was classed with stimulants, such as camphor, and given internally for similar conditions. By drinking it, intoxication, like that of alcohol, may follow, but it comes on and passes off more quickly, because ether is more rapidly absorbed and eliminated. A chronic form of intoxication, accompanied with tremor, vomiting, etc., may also be induced by taking it habitually (Martin), and, on the other hand, stimulation and ultimate narcosis follow from inhaling it like chloroform. The first symptons under' inhalation are usually those of laryngeal irritation, local heat, sense of suffocation and cough: rarely a fatal spasm of the glottis is said to have occurved, but anosthesia of the part som comes on, and tolerance is established; the body beenmes flusher and warm, slight tremors are noticed, and sensibility is somewhat exalted; a calm deep sleepy condition follows, or else lively excitement with incolorent speech and violont movement; 
inhalation being pressed further, thought and its manifestations are abolished; the lines of the face become smoothed, giving an appearance of drunkenness or stupidity, sight is obscured, the pupils dilate, the eyes convulsively drawn upwards are concealed by the falling lids, and the voluntary muscles become completely relaxed. Ultimately, the muscles of organic life are affected, respiration becomes slow, deep, and stertorous, and tactile sensibility is so absolutely deadened that the skin and parts beneath may be pinched, cut, or burnt whilst the patient remains buried in a comatose sleep.

Longet connects this progressive development of symptoms with the gradual invasion of the nerve-centres in the following order-the cerebral lobes and cerebellum, the pons, the cord, and the medulla oblongata.

The time necessary to produce the above phenomena varies according to the amount of atmospheric air allowed to dilute the ether. If the drug be given in large quantity on a saturated towel or sponge, and without special precaution to exclude air, ten to twelve minutes may be needed; if about 40 per cent. of air be admitted, as with Norton's apparatus, three to four minutes suffice; whilst with Morgan's inhaler, shutting out the air entirely, half to one minute is sometimes long enough. The stage of excitement also varies divectly with the amount of air allowed, and the time taken in securing the full influence of the drug, but, as a rule, it is more marked than with chloroform: this is especially so during the early stage of recovery; rigors also are more frequent during this stage under ether than under chloroform; recovery takes place more quickly from the effects of the former than of the latter. The volatility of ether makes it very undesirable to use it without apparatus, as the bystanders would receive almost as much of the drug as the patient.

Temperature is lowered 3 to 4 degrees after an ether-imhalation lasting for thirty to forty-five minutes; this fall is not immediate, but reaches its lowest point about an hour afterwards (A. Dumeril and Demarquay).

SYNERGISTS.-The excitant and antispasmodic action of ether is aided by alcohol and diffusible stimulants generally, 
also by musk; castor, etc.; its refrigerant powers, by all volatile substances; its effect in abolishing motor power, by curare, eserin, cicutin, and their congeners. As an anæsthetic, it belongs to a large group of substances containing carbon either with hydrogen, chlorine, iodine, nitrogen, or oxygen, such as chloroform, amylene, hydrochloric, hydriodic, acetic and nitric ethers, ethylene, aldehyd, carbonic oxide, ete. The combination of alcohol 1 part, chloroform 2 parts, and ether 3 parts (A. C. E. mixture) is useful for many cases.

ANTAGONISTS AND INCOMPATIBLES.-The action of ether is more or less antagonized by acids, astringents, refrigerants, quinine and vaso-motor tonics, but especially by the strychnia alkaloids, which stimulate both the sensory and the motor apparatus; by picrotoxin, by ammonia and its acid compounds, the acetate, carbonate, and chloride, and by currents of pure air or oxygen. The antagonism between ether and chloroform, mentioned by M. Falin, is but partial, and lies only in the more pronounced and prolonged degree of stimulation produced by the former.

Therapeutical ACTion. - Extemel. - Wounds, Hæmorrhage, Ulceration.-By Lordat and others, dilute ether has been found a good dressing for wounds and localized suppuration, and for burus of the first and second degree. Black specially recommends it for ulcerative and inflammatory conditions of the mouth and throat. It has been employed to stay the bleeding of leech-bites and epistaxis. Mr. Berkeley Hill applies it to venereal sores, stating that it is usually painless, but I have known it cause much discomfort.

Hernia.-By directing an ether spray over a hemial sac, contraction of the muscular layer may be induced, and has sometimes proved effectual in reduction, when ordinary taxis has failed.

Operations.--The ether spray is useful in minor operatirns, such as opening abscesses, removing foreign bodies, etc., but the subsequent smarting is great. It has been utilized for the first incisions in Crosarian section and ovariotomy. 
Neuralgia.-For the relief of headache, when cooling: applications are indicated, a lotion containing half an ounce of ether in half a pint of eamphor-water is effective; and acute neuralgia may be sometimes relieved by ether spray or hypodermic injection. Dr. Comegys cured a severe and obstinate brachial neuralgia by the subcutaneous injection of 30 minims of ether twice, at intervals of twelve hours, and relates other similar illustrations of its value (Record, 18i9).

Tetanic convulsive attacks have been sometimes arrested by directing a spray on to the wounded part, and choreic spasms have ceased under the same remedy, applied to the spinal column (Perroud). Several observers have verified this latter observation (B. M. J., i., 187\%), but in six cases of severe chorea in which I tried the remedy, I could find no advantage from it. Lubelski employed it not only in chorea, but also in priapism, onanism, and uterine irritation. It has been applied to the gum for allaying the pain of tooth-extraction, but does not act well, because of the dilution of the ether, and in some cases the freezing has led to local gangrene. Dr. Lawson has recorded a similar result from application to the skin for sciatica The vapour of ether has also been introduced into the auditory canal for several affections of the ear, and for toothache, and in some nerve-cases it may succeed by its stimulant antispasmodic action. In a case of violent hiccough, continuous for" eight days, and traced to "spasm of the phrenic nerve," Pigoni applied ether spray for ten minutes to the epigastrium, and then for five minutes on each side of the throat: the attack was relieved at the time, and was cured after a few applications (Record, 18r9).

Therapeutical Action. - Intermal. - Syncope.- In fainting, or a tendency thereto, ether is a valuable, quickly-acting stimulant. It has been used not only in all ordinary cases, but also in the prostration of cerebral disorder and of cholera. Troussean praises it in gouty metastasis, for preventing. syncope or apoplexy, and relieving delirium and severe gastric pain. Its subcutaneous injection in extreme cases of syncope is now not unusual-Chautrenil advises a drachm to be given in eases of placenta previa and abundant hromorrhage (Union 
Méd., Juillet, 1879). It has been utilized on the field of battle, and advised after any excessive hæmorrhage (B. M. J., ii., 1877).

Angina Pectoris.-In cases of cardiac pain, whether from functional or organic disease, full doses of ether are often efficient.

Lumbago.-A medical man, Mr. David Smith, exemplifies a use of ether in this malady in his own case. Being unable to move on account of pain, he inhaled it,-not to loss of consciousness. In less than an hour he was able to dress and go his rounds with comfort, and states that he has employed it in other cases with equally good results (B. M. J., i., 1879).

Hysteria-Spasm.-In sudden hysterical seizures, in hysterical flatulence, "globus hystericus," and even "hysteroepilepsy," full doses, 30 minims or more of ether, in water alone (or, if necessary, with valerian), and repented at frequent intervals, give much relief. In spasmodic asthma and dyspnoea dependent on mechanical obstruction to the respiration, ether acts well by controlling spasm.

Gastralgia.-Gastric pain, especially when dependent on flatus or irregular muscular contractions, is amenable to ether; it is often given mixed with spirit as "Hoffman's Anodyne."

Biliary Colic.-Ether is a constituent of the "remedy of Durande," which contains also turpentine, and has achieved some repute for relieving the severe symptoms of gallstones. Since these are soluble in ether, it has been thought that they could be dissolved by its internal administration. This is highly improbable, but the remedy acts as an anodyne and antispasmodic, and so gives much relief. I have seen a general improvement in several cases under its enntinued use.

For Anæsthesia.-The anæsthetic properties of ethervapour, discovered by Orfila, and recognized by Brodie and others, were first utilized by the American dentist, Morton, at the suggestion of another dentist, Jackson. Chloroform almost superseded it in Europe, but in America and at a few places, as at Lyons, its special value, and above all, its greater safety, were early recognized. It is this latter fact which, in view of many deaths from chloroform, has led to the more general use of ether in late years (since 18\%0). 
It is more unpleasant than the former to taste and smell : it is apt to cause more laryngeal spasm and lividity of skin; a larger quantity is needed, and its vapour so readily inflames as to make its use by artificial light dangerous; but in many cases, all these disadvantages are compensated for by its power of stimulating, rather than depressing, the heart's action and the circulation.

Mr. Cawtley Dawson, in an able paper summarizing the recorded deaths from ether up to date (B. M. J., i., 1878), concludes that of eighteen, nine only can fairly be connected with the drug, and those not with any directly poisonous action of it, but with asphyxia. He points out that the intense cold may contract the smaller vessels of the lungs, and so greatly embarrass the circulation, from which may ultimately result serous effusion and œedema. In one case, indeed, œedema of lung is assigned as the only cause of death: the patient - apparently recovered from the etherizing-had been carriecl direct into the open air from the operating theatre (Saundby, B. M. J., ii., 187\%). In most cases the lungs are found much congested, containing mucus or even blood (B. M. J., ii., 187\%).

That cardiac failure mu! occur under ether is shown by several recorded cases (Jacob, Lancet, ii., 1879); but in almost all, this was gradual and subsequent to failure of respiration, and it is very noteworthy that such failure frequently happened (if at all) after the operation and inhalation were finished (cf. Lancet, i., 1881). In several of the deaths some abdominal operation was in progress-colotomy or reduction of hernire-cases in which collapse is induced with special readiness (B. M. J., i., 18\% , i., 18r8).

Still, for all cases with feeble heart, and whenever an operation is much prolonged, ether is to be preferred, for though its use is not wholly free from danger to life, such danger is reduced to a minimum: it lies mainly in an obstruction to respiration. De Morgnn puts the comparative mortality as 1 in 23,504 for ether, 1 in 2,573 for chloroform. Ether has, howerer, certain risks peculiar to itself: thus, excitement produced by it may be great, not only at the commencement of inhalation, but also when recovering from it-so much so, that at Boston 
a special room has been set apart for this latter stage: hence, in cases with a tendency to much excitement, delirium, cerebral congestion, or apoplexy, also where quiet is very essential, as after cataract, ether is not suitable. Of 1,200 cases summarized by Dr. Jacob, struggling occurred once in 17; vomiting once in 5; epileptiform convulsions six times (Lancet, ii., 1879). The occurrence of vomiting seems about equally frequent with both drugs: some writers have maintained that it is rather more violent with ether, but does not last so long: (Norton).

Again, bronchitis, and even pneumonia, have followed a prolonged etherization on account of the coldness of the air taken in from the inhaler; unless the sponge be sometimes warmed, it will become covered with thin ice; this danger, however, may be obviated by due care.

For young children, in whom the risk from chloroform is very slight, the pleasanter anæsthetic is to be preferred, also for parturient women; but with such exceptions, ether should as a rule, be used.

PREPaRations, Dose and Mode of AdministraTION.- $E$ ther, dose 20 to 60 min., diluted; if mixed with spirit of wine ( 1 in 3$)$, as in the spiritus $x$ theris, P.B., it dissolves readily in water. Ather purus, not given internally. Spiritus retheris ( 1 in 3), dose: 30 to 90 min. Spiritus atheris compositus ( 1 in 3), dose : 30 min. to $2 \mathrm{dr}$. Tincture shloroformi et morphince (1 in 32), dose: 5 to $10 \mathrm{~min}$. Ather is likewise an ingredient in collodium ( $6 \mathrm{vol}$. in 8 ), and collodium flexile $(6$ vol. in 8).

The amount required for anæsthetic purposes varies with the manner in which it is given. If with fiee admixture of air on a folded towel, 1 or 2 pints may be needed; if with limited access of air, as in Norton's apparatus, or on a sponge inside a felt cone covered with oiled silk (Warrington Haward), from 3 to 10 oz. are often used, whilst with Morgan's inhnler and bag, where the vapour is rebreathed more than once, I liave sometimes seen anæsthesia kept up for twenty to thirty minutes, using only 1 to 2 ounces. Ormsby's inhaler is also excellent and not expensive, but frobaljly the best is Mr. Clover's small 
apparatus: this provides for warming the vapour slightly. The safest method is to give the pure vapour with a breath of air occasionally. If a sponge be used, it should be occasionally changed for one freshly warmed.

\section{ATHYL IODIDUM.}

(Iodide of Ethyi-Hyidionic Ether, $\mathrm{C}_{2} \mathrm{H}_{5} \mathrm{I}$.)

PREPARATION.-By ristilling a mixture of alcohol, iodine, and phosphorus.

CHARACTERS. - A volatile ethereal fluid, with a vapoudensity of 0.78 , sp. gr. 1.920 , boiling-point $148^{\circ} \mathrm{F}$.; soluble in alcohol and ether, little so in water. Dropped on red-hot charcoal it gives off a purple vapour.

ABSORPTION AND ELIMINATION.-This ether is quickly absorbed by the respiratory mucous membrane, and has been detected in the urine ten minutes afterwards, and at various intervals up to thirty hours from administration.

PHysiological ACTION.- It is said to favour oxygenation, and also to stimulate in a reflex manner the respiratory muscles. Symptoms of nerve-exhilaration may derelop in proportion to the dose; nareosis and the ordinary effects of ethereal intoxication may be produced by it. Dr. Richardson reported, besides narcosis, much excitement of the circulation and free glandular secretion (Med. Times, ii., 1870).

\section{Therapeutical Action. - Asthma-Dyspnca. -} Dr. Lawrence, who has probably used this remedy more than other physicians, reports considerable relief from it in both pure spasmodic and bronchitic asthma, and in almost all forms of dyspnoea (N. Y. Med. Record, June, 1880). It not only relieves spasms, but promotes expectoration. He has recently relater 
three additional such cases, and says that extended experience has confirmed the opinion above expressed (N. Y. Med. Journ., June, 1881).

Severe dyspnœa and suffocative feeling from ocdematous laryngitis occurring in the course of chronic pulmunary disease are also said to have been greatly relieved by this remedy (Centralbl. f. Med., May 3, 1879). M. Sée especially corroborates these statements.

Dose, And Mode of Administration. - Eight or ten drops should be first inhaled from a folded handkerehief; afterwards, when confidence is secured, the inhalation may be made, from a phial containing about $\frac{1}{2} \mathrm{dr}$., for ten minutes thrice daily, waiting for a few seconds if nervous symptoms arise.

It may be obtained in small glass capsules incased in silk, containing $5 \mathrm{~min}$. each. Locally applied for a short time, covered, or in the form of a spray, it deadens sensation.

\section{ATHYL BROMIDUM.}

\section{(Bromide of Ethys--Нydrobromic Eтhis, $\mathrm{C}_{2} \mathrm{H}_{5} \mathrm{Br}$.)}

Preparation.-By distilling together alcohol, bromine, and phosphorus.

Characters AND TEST.-A colourless, very rolatile liquid, with a strong ethereal odour, and sweetish, hot taste; sparingly soluble in water, but miscible with it, also with alcohol and ether in all proportions (hence it is ensily adulternterl). Its boiling-point, $104^{\prime \prime} \mathrm{F}$., is intermediate betwern that of chloroform and of ordinnry ether. Sp. gr., 1.400; vapour density, 54. The vapour, which is not inftammable, passed through a glass tube at a rod heat, is resolved into ethylene and hydrobromic acid gas. With ammonia, it yields hydrobromate of ethylamine. 
Physiological Action.-Dr. Richardson, in 1870, reported this to be a good volatile nareotic, acting quickly and with but slight museular excitement, but apt to cause vomiting and a special dryness and irritation of mucous surfaces.

According to Dr. Turnbull, it produces anæsthesia more rapidly than chloroform, and is also more quickly eliminated by the lungs and kidneys (Record, 1880). The heart-action and the respiration are but slightly affected by moderate doses, according to Dr. Levis; the former, and the arterial blood-pressure, are slightly increased (Philad. Med. Times, Jan., 1880).

The odour is not agreeable, and remains long perceptible in the breath of the patient, though not so long in the air of the room; the vapour is not irritating, but rather anæsthetic to the fances and air-passages, and general excitement or struggling is not frequent. In 100 cases reported by Turnbull, there was no asphyxia or fainting, and nausea only occurred twelre, vomiting eight, times, and then after solid food.

Bourneville and Ollier report the drug anæsthetic in rarying degree, according to the subjects taking it: complete muscular relaxation was exceptional (Gaz. Méd. de Paris, Mars, 1881). The observations of Dr. Wood on animals were not so farourable, indicating, as they did, a distinctly depressant action on the heart. "When the cut-out heart of a frog is touched with a drop of the bromide, or even when hung in the concentrated vapour, all movements cease, indicating that the drug acts directly on the heart-muscle or its contained ganglia" (Philad. Med. Times, April, 1880). Its action, however, though allied to that of chloroform on the heart, is less marked, since twenty drops of it were required to produce as much depressing effect as two of chloroform (B. M. J., ii., 1882).

Therapeutical Action.-This ether has been used for all the ordinary purposes of an anæsthetic, and rather largely in America by the physicians above quoted, and others. Mr. Nunnely had previously employed and recommended it (Trans. Prov. Med. Assoc, 1849). In France, M. Terrillon has also given it with good effect. The advantages mainly claimed for it are 
greater safety and greater rapidity and uniformity of action; but opinions are not yet agreed on these points.

In a prolonged operation reported by Marion Sims, the patient was under its influence for an hour and a half, and required $4_{2}^{1}$ ounces. The pulse and general condition remained good, the breathing was rapid and peculiar, the conjunctiva sensible, but the operation was not felt; twitching and opisthotonos occurred three times, and afterwards, though she recovered well, there was much vomiting and headache: three hours after, severe diarrhoea set in, and she died in twentyone hours, with convulsion. It must be said, however, that she was subject to epilepsy, and was found to have acute nephritis (N.Y. Record, April, 1880). Mr. Clover reported that he had made a fair trial of good samples, but found the drug less satisfactory than ethidene dichloride: it produced anæsthesia quickly, and consciousness rapidly returned, but headache, giddiness, and nausea were frequently complained of; the patient had a "drunken look." It may be useful for slight cases, though unsuited for long ones.

More recent observations are those of Dr. Chisholm, who has pointed out that, under certain conditions, the drug is very suitable for short operations, such as can be done in one or two minutes, strabismus, cysts, probing, etc. : it is too evanescent in action to take the place of ether or chloroform. He gives it, on a towel with paper between the folds, and made up into a small, nearly air-tight, cone: into this, about one drachm of bromide is poured, and it is inverted immediately over the moist mouth. A saturated vapour is thus used, and the inhaler must not be removed until the commencement of the operation. Of 300 administrations, the time required for deep narcosis in no instance exceeded sixty seconds. The anæsthetic sleep thus induced lasts about two minutes, and waking occurs suddenly, without beadache, nausea, or vomiting. Such symptoms occur, however, if an operation be protracted, requiring a second or third inhalation, and for such cases the drug presents no advantages (Plililad. Med. News, Janı, 1883).

Dr. Prince corroborates these observations by 500 others; he has used the drug largely as a preliminary to etherization, and, given in the manner described, believes it free from the danger 
previously ascribed to its more prolonged administration (W. R. Williams, B. M. J., i., 1884). Further observations are, however, still desirable before endorsing all the above conclusions.

Hysteria-Epilepsy.-Hysterical attacks have been quickly arrested by this bromide, and epileptic paroxysms much diminished in frequency and violence by its daily inhalation (Bourneville).

Dose, AND MODE OF Administration.-Dr. Levis commences with $2 \mathrm{dr}$. poured on a napkin folded small and enclosed in a large one covering the face. Dr. Turnbull used the same method, recommending especially to "crowd the first drachm on the patient." "With 3 drachms a patient was etherized in three minutes, a painful tooth extracted, and the antrum perforated : recovery was prompt."

Adulteration.-Free bromine, bromoform, and eтen phosphorus, have been found; also ether, which made it explosive. A pure article may, however, now be obtained.

\section{SODII ETHYLAS.}

\section{(Ethylate of Sodium, $\mathrm{C}_{2} \mathrm{H}_{5} \mathrm{NaO}$.)}

PREPARATION.-By treating absolute alcohol with metallic sodium.

CHARACTER.-The ethylates of potassium and sodium are crystalline substances in which one atom of the hydrogen of the hydroxyl radical of absolute alcohol is substituted by one of the metal. The solution is of syrupy consistence and light-brown colour.

Physiological Action.- When brought into contact with organic tissues, ethylates produce at first no action, but as they take up water become decomposed: the contained metal is oxiclized, so that caustic potash or soda is formed in the fresh state, whilst alcohol is re-formed by recombina- 
tion of the hydrogen derived from water (Richardson, Med. Times, ii., 1870).

Therapeutical ACTION.-Extemal.-Cancer-Nævi. -These substances, the sodium compound especially, were introduced by Dr. B. W. Richardson, as caustics with the special advantage of being manageable, effective, and antiseptic. Acting on abnormal growth in the manner above describer, the alcohol that is formed tends to congulate and at the same time prevent decomposition of blood-clot (Pharm. Journ., Dec. 14, 1878). He has given practical illustration of the therapeutical results in such cases (Lancet, ii., 1878, and i., 1881). My own experience is limited to half a dozen cases of the smaller kind of nævi, in which the result, after several applications, was satisfactory.

MODE OF USE.-A solution in absolute alcohol of suitable strength is dispensed by Messrs. Robbins. Not every degree of dilution is safe, since, if too strong, the caustic effect would be too severe. A little is lightly applied with a pointed glass stopper and allowed to dry on the surface: after two or three such applications on successive days, a thick scab or scale forms, and must be left till it is loose; it may be then raised and removed, and fresh application be made, if necessary. No water should touch the part during the cure.

\section{ATHER ACETIOUS.}

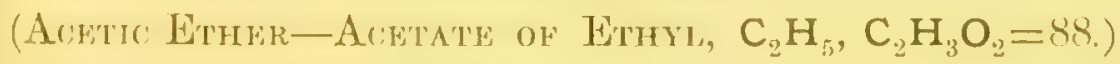

PREPARATION. - By distilling together, dry (i.e., anhyrlrous) acetate of soda, rectified spirit, and sulphuric acil. As with orlinary ether, there are two stages in the reaction:-

(1) $\mathrm{C}_{2} \mathrm{H}_{5} \mathrm{HO}+\mathrm{H}_{2} \mathrm{SO}_{4}=\mathrm{C}_{2} \mathrm{H}_{5} \mathrm{HSO}_{4}+\mathrm{H}_{2} \mathrm{O}$.

(2) $\mathrm{C}_{2} \mathrm{H}_{5} \mathrm{HSO}_{4}+\mathrm{NaC}_{2} \mathrm{H}_{3} \mathrm{O}_{2}=\left(\mathrm{C}_{2} \mathrm{H}_{5} \mathrm{C}_{2} \mathrm{H}_{3} \mathrm{O}_{2}\right)+\mathrm{NaHSO}_{4}$.

The product is freed from water by digestion with half its weight of chloride of caloium, and from free acetic acid ly restifiention with potassic carbonate. 
CHARACTER AND TESTS.-Acetic ether is a colourless, limpid, volatile fluid, with a fruity odour like that of ripe apples, and a burning taste : sp. gr. 0.91. Boiling-point $166^{\circ} \mathrm{F}$. It is soluble, in all proportions, in rectified spirit and ether, and in 11 to 12 parts of water at $60^{\circ} \mathrm{F}$. If soluble in a less proportion of water, it probably contains alcohol. It dissolves essential oils, resins, pyroxylin, cantharidin, etc. By caustic alkalies, it is decomposed into alcohol, and an acetate of the alkali used: with perchloride of iron it gives a Burgundy-red coloration, which disappears on adding strong acids or on boiling.

Physiological Action.-Acetic ether acts as a stimulant and antispasmodic, like ordinary ether, but it is not so powerful; it is, however, more pleasant to the taste and smell.

The presence of acetic ether or of some closely-allied body in the blood of diabetics (acetonrmia), has been considered to explain the coma and urgent dyspnœa with which the malady sometimes terminates.

Therapeutical Action.--In spasmodic contraction with flatulence and colic affecting the stomach and intestine, and in cardialgia, this ether is sometimes preferred.

In the Adynamia of Fevers, it has been given as a stimulant. Dr. H. Wood has proposed its use by inhalation for anresthesia, but it has not been generally adopted.

PREPARATION AND DOSE.-Dose: 20 to 60 min., diluted. In pharmacy, it occurs in small quantity in the tinctura feri acetutis. To ordinary brandy it imparts the flavour of cognao (Smith). 


\section{SPIRITUS ATHERIS NITROSI.}

\section{(Splrit of Nitrous Ether-Swett Sptrit of Nitre.)}

An alcoholic solution of nitrous ether, or nitrite of ethyl, $\mathrm{C}_{2} \mathrm{H}_{5} \mathrm{NO}_{2}$. (It was formerly called "nitric ether," the formula for which is $\mathrm{C}_{2} \mathrm{H}_{5} \mathrm{NO}_{3}$.) This preparation is one of the two organic nitrites in the Pharmacopeia (the other being nitrite of amyl).

PREPARATION.-By cautiously heating together and distilling a few ounces of sulphuric and nitric acids, copper wire, and sufficient rectified spirit. This last is decomposed by the sulphuric acid, with formation (amongst other products) of ether, which is changed into nitrous ether by the nitrous acid formed by the reactions between eopper and nitric acid. $\mathrm{C}_{2} \mathrm{H}_{5} \mathrm{HO}+\mathrm{HNO}_{3}+\mathrm{H}_{2} \mathrm{SO}_{4}+\mathrm{Cu}=\mathrm{C}_{2} \mathrm{H}_{5} \mathrm{NO}_{2}+2 \mathrm{H}_{2} \mathrm{O}+\mathrm{CuSO} \mathrm{S}_{4}$.

Characters and Tests.-Pure nitrous ether is a paleyellow, volatile, inflammable, neutral liquid of agreeable applelike odour, and slightly acid cooling taste: sp. gr. $0 \cdot 840$ to 0.845 . The ordinary diluted spirit of nitrous ether is nearly colourless, and is usually acid to test paper. If ferrous sulphate and a few drops of sulphuric acid be adderl with agitation, a brown compound is produced from absorption of nitric oxide by the iron salt (Smith). If the spirit of nitrous ether be agitated with twice its bulk of a saturated solution of chloride of calcium, alcohol and water are absorbed by this, and a certain proportion, which hould he at least 2 per cent., of pure nitrous ether separates, and rises to the surface; about \& per cent. of the ether should still remain in solution. Dr. Inupré has shown that this test may indicate only :3 per cent. nitrite of ethyl, and from 3 to 4 per cent. is an average amount.

Physiological Action.-When taken internally in ordinary doses, a certain amount of stimulant action on the 
nervous system may be observed in impressionable subjects, especially children, but the usual effect is simply to increase the quantity of the urine and of the perspiration. As to the former secretion, Dr. Grarrod noted increase of its watery part, but diminution of urea and solids; he remarks, however, that the preparation has been so much adulterated that its true value is not yet. determined. Some observer trace serious kidney irritation to the use of full doses in children.

Dr. D.J. Leech has recently drawn attention to the fact, that spirit of nitrous ether is a distinct depressor of arterial tension, also that such depression continues for several hours. After 100 minims taken by a healthy subject, the sphygmogram invariably showed a marked fall in tension, with increased frequency of beat: 50 and even 25 minims caused similar effects, though less in degree: they began within three minutes, and were marked within eight; in thirty a slight risa occurred; in an hour a fall again, and after four hours the tension was barely normal: a full dose in some subjects induces slight faintness and depression (Practitioner, vol. xxxi.). Dr. Leech further argues that the diuretic effects are dependent on the reduction of tension and the dilatation of arterioles. It would seem that any agent which augments the blood-flow through the Malpighian tufts, increases the urine; nitro-glycerine does so through the general circulation (Murrell). Nitrous ether resembles it in such an influence, and hence, "in the absence of proof that it specially stimulates the kidney structure," it may be concluded to have a similar action. This is further suggested by the consideration of its not infrequent inability to act as a diuretic, such failure occurring probably when no reason for decreasing teusion existed, -i.r., when the tension previonsly was not excessive; thus, in ardiac dropsy, it has often failed, whilst in the deficient renal excretion of elderly people it more commonly succeeds. Its diuretic power in any rase is not so persistent as that of digitalis, since its effect on the circulation is not of long duration.

Dilatation of the smaller vessels of the skin will explain in part both the perspiration which follows, in farourable conditions, and the cooling or fehrifuge effect on the general 
system. As to the latter, there may be also some direct influence on tissue-change, tending to lessen its activity.

When given by inlulation, the first symptoms are a dark, livid discoloration of lips, face, and fingers; then coldness of extremities, muscular weakness, and quick feeble pulse; the breathing remains regular, unless exertion is made, when it becomes hurried, with much oppression of chest, and cardiac distress. Giddiness and confusion of mind commonly, and headache always, occurred (D. R. Brown, Pharm. Journ., Mareh, 185i). Richardson recorded similar symptoms with rapid heart-action, and suffusion of skin from taking nitrite of ethyl. Death has been attributed to the inhalation of the vapour from a large quantity which had been spilt in a room, the occupant of which was found dead in bed (Christison).

SYNERGISTS. - The other ethers; also diuretics and dinphoretics, such as acetate of potash or ammonia, with which it is often combined.

INCOMPATIBLES. - If containing free acid, it is incompatible with iodide of potassium, proto-salts of iron, etc., also with guaiacum; for administration with alkalies, it should be rendered neutral.

Therapeutical Action.-Pyrexia-Catarrh. - For these conditions nitrous ether is in frequent use, and, although not a very powerful drug, nor so much relied upon as formerly, it often contributes to relieve by promoting secretion from the skin and kidneys. The former effect is best obtained by giving small doses frequently whilst the body is kept covered and warm, but diuresis is better secured by full doses and moderate exercise with cool air and elothing. It is a more suitable remedy for weakly than for sthenic cases, and is especially indicated in the febrile disorders of children when nerve irritation is indicated hy twitching, starting, etc. Dr. Iteech suggests that it might be useful in angina pectoris, asthma, headache, and some other conditions in which amyl is good.

PREPARATION AND DOSE. - Spivitus wheris nitreni: dose for a young child, 20 to 30 min.; for in indult, : itr. to $\frac{1}{2} n \%$. 
ADULTERATIONS.-Spirit of nitrous ether is liable to change on keeping, and is often also adulterated. The specific gravity does not indicate its quality. It is never quite free from aldehyd, and often contains free acids, acetic, nitrous, or nitric -more rarely oxalic, formic, glycolic, and other acids. Alcohol and water are often added.

\section{AMYL NTTRIS. \\ (Nitrite of Amil, $\mathrm{C}_{5} \mathrm{H}_{11} \mathrm{NO}_{2}=11 \%$.)}

This compound ether is the homologue of nitrite of ethyl (nitrous ether), and bears to amylic alcohol the same relation as that drug does to ethylic alcohol. It was discovered by Balard in 1844, and its chief properties were described by Guthrie in 1859 (Journal of Chem. Soc.), and later by Dr. B. W. Richardson (1865).

PREPARATION.-By acting upon amylic alcohol ( with nitrous acid, - a process analogous to that used for preparing spirit of nitrous ether. Sulphuric and nitric acids, water, and copper wire, are heated with purified fusel oil in a retort to distillation. More nitric acid is supplied gradually, till the alcohol is nearly exhausted, and the product is re-distilled, washed, and rectified over fused carbonate of potash to get rid of moisture. The portion that goes over at $205^{\circ} \mathrm{F}$. (the boiling-point of amyl nitrite) is collected separately.

$\mathrm{C}_{3} \mathrm{H}_{12} \mathrm{O}+\mathrm{Cu}+\mathrm{H}_{2} \mathrm{SO}_{4}+\mathrm{HNO}_{3}=\mathrm{C}_{5} \mathrm{H}_{11} \mathrm{NO}_{2}+\mathrm{CuSO}+2 \mathrm{H}_{2} \mathrm{O}$.

CHARACTERS AND TESTS.-A limpid, faintly yellow very volatile liquid, with an odour not mlike that of orer-ripe pears; sp. gr. $0.8 \% \%$. It is nearly insoluble in water, but freely soluble in ethylic alcohol, chloroform, ether, and benzine, and itself dissolves oils and fats. Its influence in diminishing oxiclation is such that phosphorus burning in a coufined space is extinguished by the varour of a fow drops. 
ABSORPTION AND Elimination.-Nitrite of amyl is readily absorbed by any tissue, except, perhaps, the skin, from which it evaporates before much absorption can take place. Through the lung's its vapour enters the circulation in a few seconds. It is also very rapidly eliminated, mainly by the lungs.

Physiological Action. - Extenal. - Anyl nitrite applied to any tissue impairs or destroys its functional power. This has been demonstrated both as to nerve tissue, central and peripheral, and as to muscular tissue, voluntary and involuntary ; but the paralysing influence is not absolutely destructive, like that, for instance, of the stronger acids, for the part acted upon gradually recovers its power.

\section{Physiological Action.-Intemal.-Circulatory Sys-} tem.-If the vapour of 1 to 5 minims be inhaled, the pulse is almost at once increased in frequency, and the capillaries lilate, as evidenced especially by a rapid flushing of the face; in susceptible subjects, or with somewhat larger doses, a distressing sense of fulness and pain in the head is soon felt, the heart beats violently, and the pulsation of the carotid and temporal arteries becomes visible and throbbing; this condition of the larger vessels continues for about half a minute longer than the dilatation of the capillaries: the same change in the retinn, vessels has been affirmed by $\mathrm{Dr}$. C. Browne and Mr. Alriridge (West Riding Asylum Reports, vol. i.), but denied by R. Pick (Centralblatt f. Med., Dec. 6, 1873). The last observer' states that the degree of dilatation of other vessels diminishes in jroportion to their distance from the head; that the capillaries of the trunk are, however, fully acted upon is shown by a case in wnich the flow of blood into cupping glasses placed over the loins was markedly and immediately promoted by amyl inhalation (Talfourd Jones, Practitioner, vol. vii.).

In frogs, according to Gaspey, dilatation of both arteries and veins occurs at once on inlualing the drug, increases during the first two minutes, and continues for a time proportionate to that of the inhalation: c.g., if this be kept ul for two minutes the enlargement remains for ten or fifteen, and to the 
extent of at least one-third of the original diameter (Virchow's Archiv, 1879). (Filehne had previously stated that such vascular changes did not occur in frogs, and Amez-Droz that the arterioles in the web dilated, not the veins.)

Whilst the pulse-frequency is thus increased, as abore described, the arterial tension becomes quickly and greatly lowered: this we might naturally expect to follow dilatation of the capillaries, and we do not trace it to diminished heartpower (for the strength of the pulsation is not markedly lessened at first), nor to altered innervation, for it occurs whether the vagi be divided or not. From other experiments we know also that cardiac depression does not occur till late, and after toxic doses: thus, if the blood-supply be cut off from a large vascular area by a ligature placed on the descending aorta, fall in arterial pressure and failure of cardiac power do not occur until amyl-poisoning is far advanced (Brunton, Journ. Anat., 2nd series, vol. v., 1871).

According to the recent observations of Dugan, venous pressure becomes augmented as arterial pressure diminishes, which would be explained by lessening the intermediate pressure of canillaries. The ultimate cause of the dilatation of these ressels has been variously traced, by some to a paralysing influence on vaso-motor centres, by others to a direct action of similar character on the muscular walls of the arterioles themselves.

Bernheim and Filehne support the former view by such experiments as (1) galvanizing the cervical sympathetic, and finding this stimulation still cause contraction of vessels dilated under amyl, and (2) cutting off by ligature of the carotid the supply of blood (i.e., access of poison) to a rabbit's ear, and yet finding the capillaries dilate under the influence of the drug (Pflïger's Archiv, Bd. iv.), and doubtless some sedative effect on vaso-motor centres is produced; yet the arterial dilatation and lowering of pressure must really depend on some other cause, for both Drs. H. Wood and Brunton have verified their occurrence after complete "separation of the arterioles from vasomotor centres by a division of the cord." Pick also found after division of the great sympathetic, and even ligature of the carotid, that the vessels of a rabbit's ear still dilated under amyl, and Schüller, after destroying the same nerve, found the 
same result of further dilatation (Berl. Klin. Woch., 18\%t, No. 25). Dugan more recently, repeating and confirming previous experiments, reports that section of all nerves supplying the heart, whether vagus or sympathetic, made no difference in the lowering of pressure, and the dilatation of capillaries (Thèse de Paris, 1879, abst. in Rev. des Sci. Méd., Jan., 1880).

Hence a probable conclusion that the characteristic effect of the drug on the capillaries is produced by a direct paresis of their muscular coat; and this is not disproved by Bernheim's experiment, nor by the analogous one of Dugan, viz., that by stimulating the central end of a cut sciatic nerve, a narrowing of the dilated vessels supplied by it is produced. T'hese only show that the paresis is not complete, and may be counteracted by strong stimuli. Amez-Droz is, I believe, alone in attributing the dilatation to the contact of blood surcharged with carbonic acid with peripheral vaso-motor nerves.

There is more reason to attribute the increased pulse-rute to a depressing effect on inhibitory centres: thus, if communication with these centres be interrupted by division of the vagi, and the cut ends be then galvanized to restore a normal pulse-rate, amyl-inhalation will not quicken the heart-beats (Filehne). Again, the slowing of pulse which occurs in sudden asphyxia and which is recognized as dependent on excitution of the inhibitory centre may be prevented by the use of amyl (Mayer and Friedrich, Archiv f. exper. Pathol., Bd. v., S. 63). Also, if by compression of vessels supplying the brain the drug be prevented from reaching the same centre, increase of pulse does not follow its use, and comparative physiology informs us that "the increase of pulse-rate under amyl is greater in dogs, in which animals the cardiac inhibitory system is more powerful than in domestic rabbits, in which it is feeble" (H. Wood).

The blond of animals, both arterial and venous, when exposed to the action of amyl becomes of a dark chocolate tint, and does not rodden again when shaken with air (unless ammonia be stdled).

In the spectrum of the dark-coloured blood, the two absorptiom-bands of oxyhamoglobin are faint, and those of acid hamatin appour; but in the blood reddened again by ammonia 
the normal lines reappear, whilst when treated by ammonium sulphide, lines of reduced hæmoglobin present themselves, and show that reducing (de-oxidizing) agents can separate the amyl from the compound it has formed with the blood-constituents; in other words, the drug combines with oxy-hremoglobin, but in so unstable a manner as to be readily separable by chemical agents: the compound has been obtained in a crystalline state by Dr. Arthur Gamgee, who names it "nitrite-oxy-hæmoglobin (Philos. Trans., 1868).

Blood to which nitrite of amyl has been added, loses its power of absorbing oxygen, and also of giving up oxygen already contained in it. The air-pump, or contact with carbonic oxide gas, can withdraw or expel oxygen from normal blood, but not from "amyl-blood" (though the presence of oxygen in the latter be proved by spectrum analysis); hence Dr. Gamgee concludes that under such influence the loose oxygen of blood is "locked up," not expelled or remored.

It is true that laboratory experiments do not prove a precisely similar action in man, but taken in conjunction with clinical results they point to diminished oxidation through blood-corpuscles, as a main element in such action. (Franck remarks that mucous membranes are not reddened by capillaries dilated under amyl, on account of the non-oxygenated condition of the blood, "sang asphyxique," (Progrès Méd., Mai 10, 1879). Such diminished oxidation, however, does not account for all the special symptoms of the drug; moreover, the nitrite salts of potash and soda produce very similar effects on blood. Large doses of alcohol equally lessen oxidation, and nitrous oxide arrests it, yet none of these cause the same symptoms as amyl. There must, then, be other elements in its action, and these are probably to be found in commection with the nerrous system, c.g., in some paralysis of inhibitory and motor centres as already described. Certainly the drug, although increasing, for a time, the rapidity of circulation, is not to be reckoned a true cardiac stimulant. Dr. Wood traces, however, to this impaired oxidation, "the production of a thuill of impending suffocation, which leads to increased cardiac and respiratory action," at least for a time, and remarks that this is very different from exerting a direct cardiac stimulation. Filehne's explanation (paralysis of 
inhibitory centres) is, however, more widely confirmed than that of Dr. Wood.

Respiratory System.-During the first stage of the action of nitrite of amyl, the breathing is deep and laboured; afterwards, if the drug be pressed, it becomes greatly hurried and panting, and finally death follows upon its failure. "Mayer" and Friedrich assert that at first nitrite of amyl increases the rapidity and depth of the respiration by stimulating the respiratory centres. Whether this be or be not correct, it is certain that later the respiratory centres are greatly depressed, the breathing becoming both slow and shallow, and death finally occurring from paralytic asphyxia."

The change in the absorptive power of the blood-corpuscle, already described as leading to diminished oxidation, involves lessened excretion of carbonic acid, which also has been experimentally determined.

The post-mortem appearances of the thoracic orgaus seem to vary with the mode of administration of the drug : if it be given quickly, the lungs, etc., are found blanched, the right heart engorged, the left empty ; whilst, if it has been slowly inhaled, the lungs and brain are found congested (Richardson).

Nervo-Muscular System.-Nitrite of amyl has a general influence in relaxing spasm of involuntary muscle, whether arterial, intestinal, or uterine. That moderate doses lave a depressant effect on vaso-motor centres has already been stated; their use is apt to be accompanied by giddiness and headache. Some persons, especially delicate women, and those with highlydeveloped nervous system, are much more sensitive to its netion than others-a minim dose or a distant whiff from a bottle of it will canse in them giddliness, mental confusion, and sense of weakneas or of excitement, whilst stronger persons will easily inhale 5 to 10 minims or more. Freqnent nse lessens the effect in all subjects. Dr. Ringer states that one patient-a woman"after a drop dose, turned doadly pale, felt very giddly, and became partially unconscious, remaining so for ten minutes." In another, nansea and irritation in the throat followed $\frac{1}{3}$ minim; in nother, a tranco-like state occurred after $\frac{1}{30}$ : but these are oxceptions, and ordinary subjects ean take a few minims without haul effect. Dr. Wood has twice seen "alarming 
prostration," but no absolutely dangerous or fatal results have occurred from its use.

Toxic doses diminish reflex activity and power of voluntary motion without causing previous excitement: if any convulsions occur it is at a late period, and they are connected probably with asphyxial conditions affecting the brain circulation.

In cold-blooded animals Dr. Richardson could lower circulation and respiration to such an extent as to induce a trance-like state of suspended animation, from which, however, they could be revived.

There is on record the case of a young lady who took a dessertspoonful of amyl nitrite, and although an emetic was given at once and acted freely, she was found, half-an-hour afterwards, with grayish-white face, dilated pupils, glazed rolling eyes, open mouth, spasmodic and irregular breathing, at one time rapid then slowly drawn and almost ceasing; the pulse, at first irregular and jerking, soon became slow and feeble: the skin was cold, clammy, and bathed in perspiration (which was saturated with amyl), and the patient was the "most limp, limber, relaxed body imaginable." She recovered with the help of warmth, movement, coffee, and opium (B. M. J., ii., 1880).

The main special effect of the drug is exerted upon the spinal cord and motor centres, of which it is a direct powerful depressant; on the motor nerves and the muscles it acts in a similar but less pronounced manner, lessening but not wholly destroying their functional power, for even after death by this agent this is retained for some time. Dr. C. Browne, noting yawning as a common occurrence under amyl inhalation, concludes it to have "a specific action on the motor centre of the mouth." On the other hand, the sensory nerves and nerve-centres are but little affected by nitrite of amyl, for the power of feeling remains almost to the last. It is evident, therefore, that this drug cannot be classed with ordinary anæsthetics, although so valuable for the relief of some forms of pain and spasm.

R. Pick has also observed that if the eye be fixed on a white screen during full inhalation of amyl, a yellow centre with violet and undulating lines surrounding it will be seen; this 
is probably projection of the retinal yellow spot surrounded by its complementary colour (violet), and blood-ressels (Med. Times, i., 1874).

Temperature.-When the first flush is developed there is much sense of warmth affecting sometimes the whole surface, sometimes only the head, face, and neck, while the hands and feet remain cold. Ladendorf found in man during inhalation of small doses, an average rise of half a degree C. (temperature taken in mouth). Dr. Mastin recognized also a momentary rise (mouth and axilla), but there can be no doubt that, in full doses, this drug markedly reduces temperature in healthy and pyrexial subjects. This fall is independent of the nerve-centres, for it occurs after division of the spinal cord, and even after death, in cases where body-heat usuclly rises or continues high for a time,-hence, Wood connects it with arrest of tissue-change, and of oxidation.

Secretion.--Perspiration is increased over the whole surface of which the capillaries are dilated. The urine is also increased in amount, and sugar has been found in it. Dr. F. A. Hoffmann detected this in the secretion of a rabbit after only 0.113 gramme (about 2 grains) was injected under the skin (Reichert's Archiv, 1872). With double this dose the amount of sugar was large, and it continued present for twelve to thirty hours. Its occurrence has been connected with "dilatation of hepatic vessels" (Garrod). Ordinary medicinal doses, however, in man do not cause glycosuria.

SYNERGISTS.-Ether, alcohol, chloroform, chloral, in that stage or degree of their action when they paralyse, or at least impair the power of the sympathetic system of nerves, accord with amy. Bartholow states that "all motor depressants increase its effects."

Antagonists. - Medicines such as nux romicn, cocculus, digitalis, ergot, and belladonnn, which stimulate or increase the functional activity of the spinal cord and sympathetie, in so far antagonize amyl. This, however, being: given by inhalation, acts much more quickly than the former, and has proved effective in strychnia-poisoning 
in animals. On the other hand, in poisoning by amyl itself, the antagonists mentioned would searcely act quickly enough, unless, perhaps, by subcutaneons injection.

Therapeutical Action. - Angina Pectoris.-Dr. Lauder Brunton was led to use nitrite of amyl during an anginal paroxysm, by observing a relation between the pain and the degree of arterial tension. After describing two classes of the affection, he "found from sphygmographic observations during the attack and the intervals, that when it comes on gradually, the pulse becomes smaller, and arterial tension greater as pain increases, owing probably to contraction of systemic capillaries," - the curve becomes lower, the ascent and descent more gradual, and dicrotism disappears. As the nitrite is inhaled, the pulse becomes slower and fuller, the tension diminished, and the breathing less hurried. On those occasions when pain retumed after brief intervals, the pulse remained small in volume, and not till this, as well as tension, became normal, was relief secured. A few minims given by inhalation afforded much more marked and permanent relief than 10 minims given with brandy by the mouth (Lancet, ii., 1867).

Since that time we have had much evidence of the same kind. Dr. Anstie early recorded a case where immediately on the appearance of characteristic flushing, "the heart gave one strong beat and the invalid passed from the state of agony to one of perfect repose and peace" (Clin. Soc. Trans., 1870). Dr. Talfourd Jones and Dr. Ringer have also recorded striking cases, but, as the latter remarks, relief is not invariable. The pain of cardiac aneurism and organic valvular disease is only sometimes relieved by the drug: increased vascular tension and arterial spasm are the conditions really amenable to it. These are sometimes determined by extra exertion, walking, etc., and in such cases the occasional use of the remedy during the exertion suffices to control the attacks; many persons subject to cardiac angina now carry with them glass capsules containing the drug, and by immediate recourse to inhalation of it at the commencement of pain are able to travel and to conduct their ordinary affairs with comparative comfort. Anstie cautions against its use when there is suspicion of advanced atheroma 
of cerebral vessels; and when the heart is fatty, it mist at least be administered cantiously.

Dr. Moxon, in his able Croonian Lectures, has recently thrown more light upon the mode of action of the drug, and has distinguished more clearly the cases which it will, or will not, relieve. He says: "It would appear clear that there are two distinct kinds of angina pectoris, one which is a result of tenderness under over-strain of the great vessels, another, which is a kind of neuralgic heart-ache from inclusion of a cardiac nerve in the inflammatory decay of an artery ; in this latter no signs of high tension are present. In the former kind of pain, high tension in the arteries is a factor, although a morbidly seusitive state of the nerves must at the same time be present. Such pain corresponds to pain and tenderness in the teeth during mastication, and it is lessened by whatever will lessen the strain upon the tender parts, so that amyl nitrite, or, less certainly, nitroglycerine, will bring welcome relief, even if it does not bring entire comfort. But the relief thus afforded is apt to be temporary, and often only partial, so that, even in those cases, we have to bring in other means, and supplement the service which the amyl renders in relieving the strain upon the vessel, by such further help as morphia can give, by reducing general sensitiveness. The conclusion is, that high arterial tension is not to be regarded as the determining cause of angina pectoris, but as one factor of pain in some cases, and that while it is true that amyl gives relief when high tension is present, there is reason to be cautious in pressing the amyl when it fails, for by lowering tension, when already low, the drug may dangerously increase the tendency to stagnation of the blood" (B. M. J., i., 1881).

Spasm-Spasmodic Asthma.- Muscular spasm is a linrge element of the pain felt in many different disorders, and may be often controlled by the nitrite. Thus, in spasm of the stomach, Anstie used it with success, and in gonty subjects suffering from violent chest-pain (connected apparently with gastric eramp, rather than with ardiar anguish), the (ontinued inhalation of nitrite will often give more pelief tham any other remerly of the kinit.

In spasmodic and eardins asthma, it has prover scrvice- 
able, and always deserves trial: but I have been sometimes disappointed with its action. In one lighly neurotic lady, in whom the attack was purely spasmodic, and not complicated with bronchitis, etc., the temporary and slight relief given was more than counterbalanced by tensive headache, faintmess, and nausea; still it may be said that in most simple cases, free from structural lesion, relief is marked and immediate. Talfourd Jones early recorded two good illustrations of this: one in a young woman where the face was dusky, the surface cold, the pulse imperceptible, and dyspnca intense; and another in a man suffering also with bronchial catarrh (loc. cit.). In the case of a delicate lad suffering from acute bronchitic asthma (after wetting his feet in the sea), rapid relief was very striking; and many similar cases are now on record (B. M. J., ii., 1873, etc.).

Syncope, etc.-In cases of syncope with marked pallor of face, it has seemed useful by its instant quickening of capillary circulation, and 1-minim doses have been used to resuscitate newly-born infants (B. M. J., ii., 1880). It has also been given for dang'erous symptoms arising in the first or tetanic stage of chloroform inhalation, and some cases, apparently dying, have been revived by it, though only for a time (Practitioner, vol. xii.). In some anomalous "nerve-cases," with irregular flushing and heat, throbbing dilated veins, nervedepression, etc., it has also been serviceable.

Sea-sickness.-Dr. Clapham first, I believe, reported excellent results in this malady, judging from an experience of 120 cases (1 dancet, ii., 1875), and he has recently recorded further experience of the same kind: he thinks the drug acts by lessening congestion of the spinal cord. Mr. Dingle confirmed these results, observing much benefit from inhalation of 3 drops, repented in two or three hours if necessary: by comparison, he found it act better than morphin, chloral, etc. in commeneing sickness, however, with violent headache, it brought on immediate vomiting before giving ease (Lancet, i., 1879). Mr. Alford and others have also reported favourably: on the other hand, instances of failure are not wanting, and we cannot yet quite estimate the real power of this remedy.

Neuralgia.-In migraine, when there is evidence of vaso- 
motor spasm, e.g., marked pallor of face, inhalations of nitrite have good effect (Berger). Severe facial neuralgia seems also to have been relieved by it after failure of quinine and arsenic; one drop inhaled, relieved the most violent paroxysms (Record, 18\%9).

Dysmenorrhcea. - In some forms of dysmenorrhoca, described as "nervous," 2 to 6 drops of the nitrite, given whenever the pain is severe enough to need it, have acted extremely well (Frickel, M. P. Jacobi, N.Y. Record, Jan., 1875). I have not, however, seen much advantage from it.

Convulsion. - In a case where convulsions occurred after delivery, they were arrested by inhalation of 1 minim of the nitrite: at the same time, however, the contracted uterus relared, and hrmorrhage set in (Jenks, Philad. Med. Journ., ii., 18\% $)$. This scarcely tallies with a case of severe postpertum hamorrhage said to be cured by 5 minims rapidly dilating the cerebral vessels (Kerr, B. M. J., ii., 1879).

Tinnitus Aurium.- Michael refers to twenty-seren cases, of which nineteen were relieved by the inhalation of amyl; 2 to 5 drops were used for a dose, and the remedy was continued so long as the face remained flushed, and omitted when giddiness came on: the tinnitus was sometimes increased during. the inhalation, but improved as the flush began to leave the face. It is said to be suitable for subjective cases dependent on disorder of sympathetic and vaso-motor nerves, not for cases of acute catarth or dependent on mechanical cause (Record, 1880).

Epilepsy.-Or. Crichton Browne showed that artificiallyproduced convulsive attacks in rabbits could be prevented by making them inhale nitrite of amyl, and also that in man an epileptic attack could be arrested sometimes by taking amyl on perception of the aura, and that the "status epilepticus," the continued succession of fits and unconsciousness could be very favourably influencer by the same drug. It has the best chance when there is a markend interval between aura and attack. Ir. T. Jones also fomd it valuable in the state of mental ronfusion and causeless dread inchured by repeated attacks of epilepsy. [)r. Tingor has given 2 to is minims intermally erery three lonurs in cases where the attacks were frequent, and sometimes with better result than was obtained from the bromido of 
potassium; he recommends the patient to take the medicine lying down.

There are some obscure cases in which it is, difficult to distinguish between "petit-mal " or slight epilepsy with transient giddiness, and somewhat similar symptoms dependerit on partial and temporary cerebral congestion from debility, nverwork, ete. As a means of diagnosis, Dr. Weir Mitehell has pointed out, that while nitrite of amyl commonly relieves the fcrmer, it is likely to aggravate the latter condition.

Tetanus - Strychnia-poisoning. - Since, in these conditions, the reflex functions of the spinal cord and the muscular contractile power are highly excited, and temperature and tissue-change increased, this, amongst other sedative remedies, may usefully be employed. Two cases of tetanus recovering under it are already on record (Lancet, i., 1871; Phil. Med. Times, vol. v.), as well as a third in which chloral was giren in addition.

Frogs convulsed by stryehnia were quieted and sared from dying by injections of amyl (Richardson), and rabbits that received subertaneously $\frac{1}{2}$ grain of strychnia at the same time with 10 minims of the nitrite, did not suffer, whilst similar animals died quickly from $\frac{1}{4}$ grain of the alkaloid alone. This antidote has not yet, I believe, been used in man.

Chloral-poisoning.-In several cases amyl has been given with at least temporary advantage (Coghill, B. M. J., i., 1879), and Dr. MoCullough has reported one of an old lady whom he found pale, insensible, and pulseless, after a large dose of chloral; 5 drops of amyl were given by inhalation, and in a few minutes the extreme pallor gave place to a healthy glow, respiration became deep and the pulse fuller (Record, 1880).

PReparation and Dose.-Amyl nitris: dose, 1 to 3 min. on sugar or mixed with mucilage or rectified spirit.

By inhalation, 1 to 5 or 10 min. and upwards on the hand, or on blotting paper or lint held near the mouth or nose, to be removed if unplensant sensations arise. The maximum dose cannot at present be fixed; but much larger doses have been given subcutaneously in a case of cholern without serious result. I.t ought to be used freshly prepared, as it quickly deteriorates 
by keeping. Capsules containing 1,2, 3, or 5 minims may be obtained.

A preparation containing valerianate of amyl has been recommended by Dr. W. F. Wade as an antispasmodic and stimulant It is a compound spirit, containing 1 part of the valerianate in 19 of alcohol, with $\frac{1}{2}$ minim of amyl acetate to each ounce. Dose: 8 min. in water (B. M. J., i., 1874).

(Nitrite of sodium is given for the same cases as nitrite of amyl, in doses of from 1 to 3 grains and upwards.)

AdULTERATIONS.-Much of the commercial nitrite of amyl is very impure, containing appreciable quantities of prussic acid, amylic aldehyd, and ethyl-amylic ether (W. G. Smith).

\section{NITRO-GLYCERINE.}

(Vide p. 444).

\section{KAIRIN, $\mathrm{C}_{10} \mathrm{H}_{13} \mathrm{NO}$.}

(Hydrochloratte of Oxy-CHINOLIN-ETHyl or Hydrochiloride of OXethyt-quinolin Hydride.)

\section{(Not Officinal.)}

PREPARATION.-This basic substance is one of several obtained synthetically by Dr. O. Fischer, by introducing hydrogen into chinolin in the form of hydroxyl or other oxygen-containing groups.

DESCRIPTION.-The hydrochlorate oceurs as a whitishyellow crystalline powder soluble in water, and moderately so in alcohol, not in ether; the aqueous solution is precipitated by ammonia : the taste is saline, bitter, and aromatic, described by some as "persistently nauseous."

Physiological Action.-According to the observations of Filehne (to whom the compounds prepared by Fischer were submitted for experimental trial) the power of reducing febrile 
temperature is possessed by compounds in which the nitrogen atom, besides its combination with two atoms of carbon, as in chinolin, $\mathrm{C}_{9} \mathrm{H}_{7} \mathrm{~N}$, is joined to the carbou of a methyl or other alcohol group-as in the present drug, in kairolin and a few others -all of which are put forward as possible substitutes for quinine.

The full dose of 15 to 20 grains usually induces, in stromg healthy adults, no marked or unpleasant effect, not even lowering the temperature, but occasionally causes gastric disturbance, abdominal pain, and burning sensations in the nose and throat, with lachrymation; in the weakly invalid such doses repeated are liable to induce also profuse sweatings and also cyanosis with depression of circulation (Hoffer).

De Giaxa reports a lessening of the pulse-frequency by five to twenty beats, no change in the blood-pressure; but according to De Renzi, the contractile force of the heart is increased (for a time); respiration is unaffected or slightly lowered in its rate; usually there seemed an increase of energy and activity, and often some muscular spasm, especially in the face; rarely there occurred great depression (Record, 1884). The urine is coloured dark green, and rendered strongly acid; the salts, urea, and water have been found unchanged, but, on the other hand, an increase in the phosphorus-compounds has been reported, and Goluboff traced irritation of the kidney, in an increase of quantity, as well as of mucus, epithelial cells, and even casts.

Girat concludes, from a large number of observations, that the drug acts directly on cellular elements, slowing the processes of oxidation (Thèse de Paris, 1883).

SyNERGISTS.-Quinine, kairolin, antipyrin, and kindred bodies.

ANTAGONISTS.-Btimulants. Atropia, agaricus, etc., control the profuse perspirations.

Therapeutical Action.- It has been used, especially in Germany, in various pyrexial diseases, including specific and puerperal fevers, pneumonia, pericarditis, etc.; its action seems to be "rapid but fugacious." Filehne has reported very favourably of it, and gives minute directious for dosage 
calculated to reduce the temperature to $100^{\circ} \mathrm{F}$., and keep it so; this is effected by hourly doses of 4 to 8 or 12 grains. Dr. P. Guttmann also speaks well of its effects, and found the pulse fall with the temperature. Each dose produced the usual result, implying that the effect is not weakened by repetition: he observed no unpleasant results, except profuse sweating, in phthisical cases. Compared with quinine, it acted more rapidly, but for a shorter time (Berl. Klin. Woch., xxxi.). A severe case of phlebitis after parturition, with a temperature of $106^{\circ} \mathrm{F}$., seems to have been controlled by the drug, the action of which was marked by profuse sweating (Ibid., Sept. 10).

Merkel (Nurnberg), after treating nineteen febrile cases of the kinds referred to, concludes that the drug is one of the most powerful antipyretics, acting in every pyrexial case without collapse or other disagreeable effects (Deutsches Archiv).

The more recent reports of Hoffer are not, however, equally favourable, since the drug proved very uncertain. In one case of intermittent fever it prevented the attack, in another it only postponed it a few hours. In phthisis the temporary lowering was followed by a greater rise, with profuse sweating, rigors, etc.

After giving kairin in six cases of typhoid Shathick reports it antipyretic, but eliminated so soon that the effects pass off too quickly for much practical advantage to ensue, and although of some value, they are not uniform and have certain drawbacks (Bost. Med. Journ., Nov. 1883).

Dr. S. Smith reported rather favourably of the drug, but Dr. Archer's case is not encouraging (B. M. J., i., 1884). The effect in pneumonia is also inconstant, and caution is required in using the drug with delicate subjects (Centralb. f. Med. Wiss., 1883, No. 50). On the whole, it has rather lost than gained recently in professional estimation, on account partly of the transient character of its effects, and partly of the profuse perspirations and serious prostration that may be caused by it.

Dose.-An initial dose is from 8 to $15 \mathrm{gr}$; afterwarls about 4 to 6 gr. hourly, according to the effect on temperature. It is best given in pill or wafer praper, followed by copions draughts of diluents. 


\section{ANTIPYRIN, $\mathrm{C}_{11} \mathrm{H}_{12} \mathrm{~N}_{2} \mathrm{O}$ (Dimethyloxyquinicin).}

(Not Officinal.)

This drug, which also belongs to the group of quinolin derivatives, has alkaloidal properties, and has been synthetically prepared by Dr. Knorr, of Erlangen.

Characters and Tests. - A whitish powder, readily soluble in water, and giving with perchloride of iron and iodine dissolved in potassic iodide, a red-with nitrous acid, a blue colour. The taste is sweetish-bitter-less bitter than that of quinine, and less nauseous than that of kairin.

ABSORPTION AND ELIMination.-The former occurs readily by the skin and by the rectum, as well as by the stomach: the latter chiefly by the kidney. As Maragliano found the drug in the urine thirty-six hours after the last dose given, elimination cannot be considered very rapid, though it commences early, for the iodine reaction is obtained three hours after the first dose.

Physiological Action.-In frogs, antipyrin causes, in doses of 50 to 80 milligrammes ( $\frac{4}{5}$ to $1 \frac{1}{4}$ grains), convulsions, due to an action on the spinal cord; in larger doses it produces paralysis, without spasms, weakening of the heart's action, but no other effect on the circulation (Coppola). According to Demme, however, the drug kills by heart-paralysis. In rabbits, the intravenous injection of 0.01 to 0.2 gramme ( $\frac{1}{8}$ to 3 grains) causes a primary fall of blood-pressure, followed by a permanent rise above the normal, uninfluenced by section of the vagi, and with no change in pulse-frequency (Bettelheim). The primary fall of blood-pressure is denied by Demme (Fortschr. der Medizin, ii., 1884). In heritthy man, most observers deny the action of 
antipyrin in reducing the temperature; but, according to $\mathrm{F}$. Müller, large doses cause a fall of $\frac{1}{10}$ of a degree C. Sulphates are increased in the urine, and the drug has been supposed to be united with a sulphate in elimination. In fever, the temperature is rapidly reduced to the normal, or even sub-normal, with the accompaniment of profuse sweating. Antipyrin acts, according to Maragliano and Bettelheim, by dilating the cutaneous vessels. Untoward symptoms are vomiting and loss of appetite; sometimes a red rash appears, variously described as being like measles or scarlatina.

\section{SYNERGISTS AND ANTAGONISTS.-As under kairin.}

Therapeutical Action. - Pyrexia. - Antipyrin is given solely for a symptom, fever, whether it is part of $n$ specific fever or not. It has thus been given, with excellent effects, in acute rheumatism, enteric and other specific fevers, phthisis, pneumonia, and erysipelas. The fever is almost invariably reduced, and in this respect the drug is a reliable antipyretic. The apyrexia, according to Demme, lasts longer than with kairin; but it is variable, and usually, on omission of the treatment, the temperature begins to rise again. Penzoldt, Sartorius, and Demme have used the drug extensively, and with good results, in the pyrexia of children. Vomiting has occurred in many instances; and this is undoubtedly the great drawback to antipyrin, and may be of serious consequence in cases of typhoid fever and phthisis. Jaccoud says the effects of the drug are illusory, since the internal temperature of the body is not affected, and the distress of the patient is not relieved (Lancet, ii., 1885). The majority of authors, however, speak favourably of the remedy. In cases of typhoid fever, occurring in the Hertford Hospital, Paris, upwards of 100 administrations of antipyrin were made, and with good results. The higher the temperature, the more marked was the fall produced, often in half an hour : the apyrexia rarely lasted longer than six hours, but was maintained by a second full dose (30 gr.), followed by smaller doses, which were regulated by the indications of the thermometer (McKen, Lancet, ii., 1885). Duremberg states that by its judicious use the charts of tuber- 
culous patients may be made to register normal temperatures continuously; he did not find it interfere with appetite, or provoke hrmoptysis (B.M. J., i., 1885).

Dose.-Antipyrin, $30 \mathrm{gr}$., repeated once or twice at intervals of one or two hours, then 10 to $15 \mathrm{gr}$. as required. In weakly subjects $15 \mathrm{gr}$. is a suitable first dose. For children $1 \frac{1}{2}$ gr. for each year of age: it may be given in sweetened water. By the skin, the dose is one-half or one-third the above; by the rectum it is the same as by the mouth, or larger.

\section{CHLOROFORMUM.}

\section{(Chloroform, $\mathrm{CHCl}_{3}=119 \cdot 5$.)}

PREPARATION.-By gently heating together and distilling dilute rectified spirit with chlorinated lime (Soubeiran's process). Slaked lime is added to prevent some secondary decompositions, but these occur more or less, the exact chemical reactions being complex and not exactly verified. Chloroform may also be prepared from methylic and other forms of alcohol, or from chloral by treating with alkalies.

It is purified by several processes: (1) washing with water, which combines with and separates alcohol or chlorine; (2) by agitation with an equal volume of pure sulphuric acid, which chars and removes any oily impurities; (3) by distillation from off a mixture of dry chloride of calcium and slaked lime, which retains any water or sulphurous acid (W. Smith).

Characters AND Tests.-Chloroform is a colourless, heavy, volatile liquid of characteristic ethereal odour and hot sweet taste, sp. gr. 1.48 to 1.496 ; boiling-point $140^{\circ} \mathrm{F}$., slightly soluble in water, completely miscible with alcohol or ether, soluble in oils. It should be neutral in reaction, but often 
decomposes on keeping and exposure, forming chlorine and hydrochloric acid.

It dissolves gutta-percha, iodine, bromine, alkaloids, resins, etc. It burns (not easily) with a green flame, producing hydrochloric acid. It should not become coloured on agitation with sulphuric acid, nor evolve gas on addition of potassium. Dropped into distilled water, it should fall in transparent, round globules.

ABSoRPtion AND Elimination.--Chloroform, whether in liquid or vapour, is readily absorbed by all the tissues, especially by the lung. It passes directly into the blood when inhaled, and is soon eliminated by the lungs, and, in less degree, by all secreting and excreting organs. It passes in the milk in sufficient quantity to affect the infant, and in the urine it quickly gives the reactions of glucose, reducing the potassio-tartrate of copper, etc. The liver and the cerebral substance have been found on analysis to retain more than other parts.

Physiological Action.-External.-Chloroform not only is absorbed itself with facility, but promotes the absorption of other substances through the skin; thus, a solution in it of alkaloids, such as strychnia or morphia, applied to the limb of a frog caused toxic symptoms much more quickly than an alcoholic solution of the same (A. Waller), and if a piece of cotton-wool, moistened with a solution of atropia in chloroform ( 1 in 400), be tied over the forehead and eyes of a healthy man, the pupils visibly dilate in three minutes, completely in five minutes (Parisot).

Much local irritation, redness, pain, and even blistering are caused by the application of undiluted chloroform, but if it be diluted with alcohol, oil, lard, etc., a limited amount of stimulation and a marked lessening of local sensibility will be produced by it.

Physiological Action.-Internal.-Circulatory System. - Small and moderate doses of chloroform given by the mouth stimulate, whilst large doses depress, the heart-action and 
capillary circulation. During inhalation of the drug the pulse mostly becomes quicker and apparently stronger in the first stage, normal in frequency or slow but weakened during the second stage, rapid or irregular and weak during the third. Most observers describe similar changes in the pulse and respiration of the lower animals and of man, i.e., at first acceleration, later retardation; but Dogiel noticed in rabbits a primary decrease in the pulse-frequency during the stage of excitation, and a quickening in the stage of narcosis.

The action of the heart is arrested immediately by injection of chloroform into the jugular vein, and its irritability is destroyed. Chloroform vapour applied directly to the exposed heart has a similar effect (Edin. Med. Journ., 1842).

Whether the sudden cardiac arrest which sometimes occurs under inhalation of chloroform be due to direct action on the heart-substance or is reflex through the pulmonary nerves, cannot be positively asserted; but since the drug is readily absorbed, and so quickly carried from the lungs, its direct action is both possible and probable. In the frog's heart, Dr. Ringer has shown that it stops the beat in diastole, and that the action is restored by ammonia.

During the later stages of narcosis, pulsation in the external jugular and other cervical veins occurs as a symptom of cardiac embarrassment (Noel, Record, 18\%7).

According to Sansom and Harley chloroform inhalation induces first spasmodic contraction, and afterwards dilatation of capillary vessels. These effects may be verified in the frog's weh, and have been traced to a primary stimulating and subsequent depressing effect upon vaso-motor nerves. Similarly in man the early slowing of heart-action has also been traced to stimulation of the inhibitory centre, and the after-increase to paralysis of the same (Dogiel). The decrense does not occur if the vagi be divided. The amount of blood in the brain is lessened under chloroform influence, according to C. Bernard and others, but this is a disputed point. Nothnagel says, regarding it:- " $\mathrm{Cl}$. Bernard recently assumes that the soporific sffect of ehloroform is partly owing to its producing anæmia of the brain, without, however, proving this improbable supposition." Husemann says:- "The statement of Claude Bernard, 
that the effect of chloroform is simply founded on anæmia of the brain, can scarcely be reconciled with the fact that there is very often hyperæmia of the brain in animals killed with chloroform (as also in man)."

A little chloroform added to blood outside the vessels renders it brighter red and more fluid, the corpuseles become contracted, and after a time crystals of hromatin form. If blood containing chloroform be freely exposed to air, the corpuscles become dissolved in the serum, being, it is supposed, oxidized. With regard to the gaseous contents of the blood external to the body, analysis of a mixture of it with ehloroform shows the amount of contained oxygen to be increased, whilst the carbonic acid is diminished (Harley). Bert states that the blood during anæsthesia contains more oxygen than normal, and the explanation would seem to be that after complete oxidation of hæmoglobin under influence of the drug, any more oxygen taken from the air cannot be consumed, and instead of forming carbonic acid, accumulates in the blood (H. Wood) : such changes cannot, however, occur to any extent under the ordinary use of chloroform for anæsthesia.

Respiratory System.-At first, under chloroform, respiration is commonly slowed, afterwards quickened, and then rendered shallow and irregular. There is not the same degree of laryngeal spasm at the commencement as there is with ether. In later stages of narcosis the breathing becomes stertorous, and so far interfered with that blueness of the lips and lividity or partial cyanosis of the face are caused, and sometimes the functional power of the medulla oblongata being paralysed, death occurs from asphyxia. This mode of chloroform-death is, however, not so frequent as that by cardiac syncope.

Nervous System.-The first symptoms of influence on the nervous system are such as rxhiluretion and slight excitement. of the mind and emotions, with diminution of the sensibility to pain and discomfort, and an after-tendency to drowsiness. If the administration be now stopped, the effects subside in a short time with slight after-result, or possibly some headache and languor. If it be pressed, symptoms like those of alcoholic intoxication appear, and constitute what is commonly known as 
the first stage of chloroform-narcosis. This varies in duration, not only according to the dose and mode of administration, but also according to the temperament and habits of the patient. It is marked by discoloured swollen face, incoherence and indistinctness of speech, irregular purposeless movements, and sometimes wild excitement. This stage is not usually so pronounced with chloroform as with ether, but sometimes it is so in strong adults, especially if they are intemperate. Sensibility to pain is lessened, but not wholly abolished: the indications of feeling may be exaggerated, and yet the patient may remember little or nothing of it shortly afterwards.

When consciousness and sensation are abolished, and the muscles relaxed, whilst the patient lies quiet, the second stage, or that of ancesthesia, is developed, and the effects mentioned commonly all occur together. Sometimes, however, muscular rigidity and tendency to excitement persist more or less during unconsciousness. For most surgical or medical purposes it is not necessary to go beyond this condition, but a continuance of inhalation induces a third stage of profound narcosis, accompanied with livid or purplish complexion, stertorous breathing with occasioual complete arrest, general relaxation of museles (often inclusive of the sphincters), and abolition of reflex action: in this stage, danger to life is imminent.

To the condition of the pupil of the eye much attention has been given, but with contradictory result: thus Baudin and Schlöger assert that it is at first dilated, but during complete anæsthesia always contracted. The latter observer says further, that if during the latter condition the pupil recovers its usual state, the unconsciousness is passing off, whilst its sudden and extreme dilatation indicates urgent danger. Dogiel and others found, on the contrary, in animals at least, exactly opposite conditions to the above, whilst W. H. Winslow observed the pupils vary duxing complete anæsthesia, and even when death occurred they were found sometimes dilated, sometimes contracted-he connected the former condition with syncope, the latter with asphyxia (Philadelph. Med. Times, vol. vi.)

It is probable that all degrees of chloroform-effect are pro- 
duced by a direct action of the drug upon the nerve-cells in the brain and cord, and more on the former than the latter; if communication with the muscles be interrupted by dividing the cord, no rigidity or convulsive action takes place below the point of section (Bert, Compt. Rend., 1867).

On peripheral nerve-tissue, however, its effect seems but slight, judging by the fact that if in a frog the iliac artery of one limb be tied and then chloroform administered, conducting power remains equal in the sciatic nerves (Bernstein). Nothnagel observes: "The experiments of Bernstein, which were lately confirmed by Lewisson, prove that a completely bloodless frog (which, as is well known, keeps quite lively for several hours) shows the same symptoms of the chloroform-narcosis as a normal frog, only with the difference that narcosis takes place more slowly; the important fact results with certainty, that chloroform acts directly on the nervous substance, and not indirectly through a change produced in the blood;" and again, "the (chloroform) anæsthesia is chiefly caused by paralysis of the nerve-centres, not by an affection of the peripheral sensitive nerves. That these latter, however, equally as the motor nerves, are affected by chloroform, and in an analogous manner as the central ganglia-cells-viz., that a stage of raised sensibility is at first present and is followed by one of paralysis - Bernstein has proved. But he has equally shown that the ganglia-cells are much more sensitive to the poison than the nerve-fibres, and that at a time when the central organs are completely paralysed, the peripheral nerves are still in no way affected." Clinically, however, we know that chloroform, directly applied, relieves local nerve pain.

Genito-Urinary System.-The bladder sometimes empties itself during narensis, but what is of major importance is, that "subjective erotic sensations" may occur during a comparatively short chloroform sleep-hence charges of assault and rapo against medical men; and although, as Dr. H. Wood remarks, "The valuelessness of testimony of persons as to occurrences during the time of their intoxication with anæosthesia should be recognized by law," every administrator should protect himself by the presence of witnesses. 
SYNERGISTS.-The same as ether. The exhibition of morphia and chloroform together is often useful, as in tinctura chloroformi et morphinæ.

ANTAGONISTS.-In addition to those mentioned under ether, Schäfer argues that atropia acts as a dynamic antidote to the curdic faiture induced by chloroform, which "stimulates the inhibitory cardiac nerves," atropia paralysing the same nerves. In such cases he recommends its injection, not in cases of threatened chlorofom-death from respiratory paralysis (B. M. J., ii., 1880).

Therapeutical Action. - External. - Neuralgia, Lumbago, etc.-In many forms of local pain, liniments containing chloroform are highly useful. If a counter-irritant action is desired, the remedy is often combined with turpentine or camphor liniment, whilst if relief of pain only is sought, its combination with opium, aconite, or belladonna enhances their efficacy. In obstinate cases, especially of facial neuralgia, Bartholow has strongly recommended the "deep injection" of chloroform, putting 5 to 15 minims by syringe as near to the affected nerve as possible. Pain and swelling, and sometimes local induration, are apt to follow, but the after-relief is said to be very great; rarely, a small abscess has formed.

Dysmenorrhcea has been relieved by the local use of chloroform-vapour (through a vaginal tube), and headache by inverting on the part a glass containing cotton-wool with a little of the drug sprinkled upon it.

Spasmodic Asthma may be benefited by prolonged external frictions on the chest and spine with a strong solution in oil (equal parts), and the diluted vapour is useful in pertussis and other reflex forms of cough. As a safe and convenient mode of using such inhalations, Bartholow recommends a teaspoonful of "chloric ether" to be added to a cupful of warm water, the vapour to be directed by a cone into the air-passages: this may be repeated every five minutes for four or five times. Chloroform or chloric ether may also be given internally, as an expectorant and nerve-sedative, to relieve cough.

In violent chorer, and other forms of convulsive disorder, chloroform inhalation is sometimes necessary. 
In the severe colic produced by renal or biliary calculus or by lead, etc., inhalations of pure chloroform pushed to the first or even second stage are sometimes advisable, and, given internally, 3-minim doses thrice daily are said to aid in the passage of gall-stones.

In cases of rectal ulcer and pruritus pudendi the vapour may be locally applied, or an ointment used (1 drachm in 1 ounce of zinc ointment).

Chloroform rubbed on the gums, so as to produce numbness, renders incisions or extractions less painful. Sufficient anæsthesia to permit of almost painless tooth extraction, or other brief operations, without loss of conseiousness, may sometimes be secured by the rapid inhalation of one teaspoonful of the drug: when tingling of the extremities is noticed is the time for operation.

\section{Therapeutical Action. - Internal. - Gastralgia,} Nausea.-In these conditions 2 or 3 minims of chloroform, which may be given on sugar or in mucilaginous liquid, often give rapid relief. The vapour is disengaged within the stomach and bowel, and acts in a direct manner. For fatulent colic the compound tincture is a good remedy. In the nausea of pregnancy, in sick headache, and even in sea-sickness, a few minims of the pure drug are better: for the nausen and cramps of cholera it has proved exceedingly efficacious.

Insomnia. - A full dose of chloric ether ofteu aids sleep in depressecl and exhausted subjects. It has been thus used in the later stages of delirium tremens, but generally combined with tincture of capsicum.

As an Anæsthetic chloroform has been, and is still given for all varieties of surgical and medical procedure, examinntions, operations, dislocations, convulsion, spasm, pains of parturition, colic, etc. Its advantages are the comparative quickness and pleasantness of action of small or moderate doses, the complete muscular relaxation produced, the simplicity of its administration, and its suitability for use by artificial light; but against these must be weighed its essential dangerousness and its large percentage of deaths-about 1 in 3,000 inbalations (H. Wood). 
The danger is difficult, if not impossible, to calculate, for death has happened from small doses and from large, at the commencement and at the end of inhalation, to young and old, to the apparently healthy and the delicate, to those with heart and lung disease, and to those free from it; but, speaking generally, we may say that infants and children under seven or eight years of age are the safest subjects; women, especially during childbirth, also bear it well, and weakly persons already suffering from illness succumb to it less frequently than the strong who need it for operation after injury.

A previous safe inhalation does not guarantee subsequent ones. During parturition the risk seems to be rather of giving rise to hrmorrhage, by lessening uterine contraction, than of causing death: it need never be pressed to the third stage.

Danger is increased by the presence of disease in the nervecentres, e.g., by abscess or tumour of brain, also by delirium tremens; but especially by any obstruction to the free entrance or exit of air to and from the lungs, or to the free play of respiration, e.g., by large tonsils, swollen glottis, tight cinctures, the presence of thoracic effusions, tumours, etc. Advanced emphysema, with venous congestion, induces also an element of danger, and fatty degeneration of heart has been found more frequently than any other morbid change in fatal cases. Valvular lesion does not contra-indicate chloroform if the muscular substance be healthy, and phthisical patients often take it well: a febrile condition is not a contra-indication.

A large proportion of chloroform-deaths has occurred during minor operations, such as teeth-extraction, evulsion of nail, amputation of finger, etc., before anrsthesia was complete. Some of these have been connected with the upright, or nearlyupright, position, but still they confirm a general conclusion, viz., that to commence an operation when anzsthesia is incomplete is more dangerous than to make the patient quite insensible. Syme was accustomed to give the drug freely and largely from the first, and his practice was exceptionally free from accidents which happened in more timid or more cautious hands. Dr. Brunton has specially drawn attention to the risk incurred by giving too little, and it would seem that a reflex irritation proceeding from 
the seat of injury is enough to arrest heart-action when already weakened by the drug. Dental operations are peculiarly hazardous, from the intimate comnection between the root of the fifth nerve and that of the pneumogastric (Bartholow). Too long fasting and any cause of extreme exhaustion also increase the danger of chloroform.

MODE OF ADMINISTRATION.-In view of preventing unnecessary pressure on the diaphragm, and also of lessening: the tendency to sickness, the bowels ought to be relieved a few hours before, and the stomach should not contain food.

A light meal should be taken about three to four hours before the operation, for, as already stated, too prolonged fasting itself leads to danger. I could only attribute to this cause a fatal result in the case of a girl kept waiting all the morning in an out-patient department for a slight eye operation, performed about two o'clock, and also in the case of a man submitted to a minor operation on the foot. In both cases the drug was given by a competent house-surgeon at a large hospital. Vomiting, though not usually a serious danger, has led sometimes to death, from a piece of food blocking the larynx. As to giving a dose of brandy or whisky shortly before the inhalation, opinions are divided; but since one risk arises from heartfaihure and blood-stasis, some stimulant seems advisable, except for children; provided that the amount be not such as to exert an action on nerve-centres antagonistic to that of chloroform.

The subeutrneous injection of morphia is, perhajs, a better preparation for anæsthesia in most operative cases, for if it does not, like alcohol, directly stimulate the circulation, it lessens the sudden shock sometimes inflicted on the heart, and it so far assists the action of chloroform that less of that drug is required: it may be combined with atropia.

It is scarcely necessary to note that the patient's mind should be tranquillized by a quiet mmmer, and that instruments, oto., should be kept from sight. If the first stage los clisturbed by loud talking, or especially by remarks on the case, the "going off " is rendered more difficult. Plenty of fresh air should bo available, and suitable forceps for drawing forward the tougue; 
means of applying artificial warmth, and for the rectal injection of stimulants should be at hand. For the rare cases where death threatens from paralysis of respiratory muscles, a galvanic battery may be required, whilst for the more frequent condition of cardiac syncope and cerebral anæmia there should be means for quickly inverting the position of the patient. The actual mode of administration need only be simple. An ordinary tumbler containing lint enough to absorb one to two drachms, and inverted over nose and mouth, is convenient and efficient. The lips and nose should be slightly oiled to prevent excoriation, and the margin of the tumbler raised at brief intervals to admit air. In Simpson's method a thin fold of lint is held in the same position and the chloroform dropped upon it. This answers well for keeping up anæesthesia, but is often needlessly slow in producing it. It is an ascertained fact that much danger is involved in the breathing of air charged with more than 5 per cent. of chloroform vapour, and Mr. Clover's wellknown bellows apparatus is calculated to supply a definite strength-he recommended commencing with 2 per cent. and increasing steadily to 4 per cent., which should not be exceeded: $3 \frac{1}{2}$ per cent. may be stated as a safer average.

By the lint method the proportion is not estimated, but it may be judged by the observed conditions of the patient, and need not, for clinical purposes, distract the mind of the administrator, who must, however, remember the necessity of diluting the vapour with air at intervals. In view of the danger of insufficient anrsthesia, some recommend commencing with a large quantity - $\frac{1}{2}$ ounce or more of chloroform-applied as closely as may be to the face on folded lint or towel, so as to secure rapid effect and shorten the first stage as much as possible. I have seen this method succeed many times, but I do not think it safe nor easy to defend if an accident should occur. It is more prudent to allow four or five minutes for the first stage. Mere laryngeal spasm or suffocative feeling or struggling is not a reason for suspending but rather for pressing the drug, but for urgent cough or vomiting, intermission should be made. With due care, the second stage may be kept up for an indefinite period.

Chloroform vapour is four times heavier than air, so that it is 
apt to displace the latter from under lint or other covering held over the mouth, and be respired in greater strength than intended.

Lister, however, has shown that the percentage of chloroform vapour in the air inhaled in this, the ordinary way of its administration, is commonly less than 4.5 per cent., and never more than that amount. Snow stated that 5 per cent. of chloroform vapour in the air inhaled induced paralysis of respiration before the circulation failed. A condition suitable for operating is indicated by quietude, muscular relaxation and insensibility of conjunctiva; if touch or pressure on this part does not excite movement of the eyelid, sensation is probably abolished in other parts. The countenance and breathing should be carefully watched. It is not necessary to be constantly feeling the pulse, though its condition should be noted as often as circumstances permit. Indication of cardiac failure will be given by it and also by a sudden pallor of face, though the same symptoms may be only precursors of vomiting; if this latter be not their explanation, immediate attempts must be made to restore the heart's action. The patient should be turned on to the left side, and strong firction applied over it, and also over the extremities, which should be kept warm, and 1 to 2 ounces of brandy should be injected per rectum; but perhaps still more effective is the inversion of the body as proposed and carried out by Nélaton, and after him by Marion Sims and other's. The legs are held uppermost by assistants, and the body kept vertical or slightly slanting with the head low down; the backs of chairs or any convenient object may be used as support. Some very serious cases, apparently even dead, have recovered under this treatment.

If respiration fail during the first (tetanic) stage, the countenance becomes dusky; the pulse may possibly continue beating for a few moments. In this coudition frictions of the chest, and above all, artificial respiration are indicated, the tongue being well drawn forwards. Simpson, Syme, and many others have directed special attention to this point, which, in spite of some recent observations to the contrary, I still considor very important. It not only opens the glottis, but stimulates respiration in a reflex manner; traction forwards of the chin is of some assistance in the same direction. 
If breathing stops during the third stage from relaxation and paralysis of muscles of respiration, then frictions and sudden slappings of the chest, artificial respiration, and faradization of the affected muscles, or of the phrenic nerve should be practised.

The vomiting which may occur after the inhalation, if only moderate, is useful for eliminating some of the drug and for stimulating the circulation, but if it threatens to become excessive, a little ice or iced brandy and soda will relieve, or a hypodermic injection of morphia with atropia. Application of almost boiling water over the heart has been found successful.

PREPARATIONS AND DOSE.-Chloroformum: dose, 3 to 10 min. on sugar, or with syrup, mucilage, or beaten egg. Aqua chloroformi: dose, $\frac{1}{2} \mathrm{fl}$. oz. to $2 \mathrm{fl}$. oz. (1 drachm of chloroform in $25 \mathrm{oz}$. water). Spiritus chloroformi (chloric ether): 1 part in 20 of rectified spirit, sp. gr. 0.871: dose, 10 to 40 min. or more. Tincture chloroformi compositce (2 parts to 8 of spirit and 10 of tincture of cardamoms): dose, 20 to 60 minims. Tinctura chloroformi et morphince ( $\frac{1}{48}$ grain of morphia in 10 minims): dose, 5 to $10 \mathrm{~min}$. Limimentum chloroformi (chloroform and camphor liniment, of each equal parts). An ointment may be made 1 part in 7 . For inhalation, 1 dr. to 4 oz. or upwards, according to duration of operation, etc.

ADULTERATION.-This is a very serious matter, and, in my opinion, has sometimes contributed to fatal results. Of many thousind operations witnessed in the practice of Syme, Simpson, Miller, or concucted by myself, no fatality has occurred, and the preparation used in the cases referred to was invariably pure. The dangerous impurities are (ill-defined) secondary products, arising from careless preparation,-an oily matter of umpleasant odour, hydrochloric acid, and free chlorine. 


\section{CHLORAL HYDRAS.}

(Hymtate of Chuorat, $\mathrm{C}_{2} \mathrm{HCl}_{3} \mathrm{O}, \mathrm{H}_{2} \mathrm{O}=165.5$.)

Discovered by Liebig in 1831, chloral was practically unknown beyond the laboratory until the observations of Dr. Osear Liebreich in 1869.

PREPARATION.-By passing a stream of dry chlorine gas for several days through mhydrous alcohol, there is obtained a dense oily, pungent, volatile liquid, which is anhydrous crude chloral. This is purified by treatment, first, with sulphuric acid (which retains water and any unchanged alcohol), and afterwards with quicklime, which fixes the free hydrochloric acid produced. Liquid chloral, when hydrated by the addition of a small quantity of water, is, with evolution of much heat, converted into a solid-the officinal compound known commonly as chloral (W. G. Smith).

Aldehyd $\left(\mathrm{C}_{2} \mathrm{H}_{4} \mathrm{O}\right)$ is first formed by the action of chlorine on alcohol: (1) $\mathrm{C}_{2} \mathrm{H}_{6} \mathrm{O}+\mathrm{Cl}_{2}=\mathrm{C}_{2} \mathrm{H}_{4} \mathrm{O}+2 \mathrm{HCl}$; (2) $\mathrm{C}_{2} \mathrm{H}_{4} \mathrm{O}+\mathrm{Cl}_{6}=\mathrm{C}_{2} \mathrm{HCl}_{3} \mathrm{O}+{ }_{3} \mathrm{HCl}$. Thus chloral corresponds to aldehyd with $\mathrm{H}_{3}$ replaced by $\mathrm{Cl}_{3}$, or it is tri-chlor-aldehyd. Practically the process is not a simple one, and complex changes occur, which call for the greatest care in the manufacture.

Characters and Tests.-Hydrate of chloral occurs in crystalline masses, opaque on the surface, not unlike lump, sugar, and also in smaller separate crystals, transparent, nondeliquescent, and somewhat resembling sulphate of magnesia: the crystalline forms vary with the medium from which they are obtained: the smaller variety is usually the more pure. The taste is lot, rather acid; the odour pungent, somewhat like that of ripe melon; the crystals are hygroseopic, and very soluble in water and ether, in four times their weight of chloroform, and in alcohol: with this last, chloral combines to form an alcoholate, which has been sometimes substituted for the 
officinal hydrate. At a gentle heat the latter becomes a transparent liquid, which boils at $205^{\circ} \mathrm{F}$. (with broken glass), and volatilizes at a higher temperature on platinum; if cooled, it solidifies again at $120^{\circ} \mathrm{F}$. The solution should be neutral, or nearly so, to test-paper, and should not give a precipitate with nitrate of silver.

Chloral is not easily acted upon by acids: nitric acid, unless fuming, has no effect on it, and sulphuric only dehydrates it, liquid chloral separating and forming an insoluble isomermetachloral - which is an opaque solid. Caustic alkalies decompose the hydrate readily into chloroform and an alkaline formiate $-\mathrm{C}_{2} \mathrm{HCl}_{3} \mathrm{O}_{4}, \mathrm{H}_{2} \mathrm{O}+\mathrm{KHO}=\mathrm{KCHO}+\mathrm{H}_{2} \mathrm{O}+\mathrm{CHCl}_{3}$. The pure drug yields $\tau_{2}$ per cent. of chloroform. The B.P. allows 70 per cent., as tested by mixture with slaked lime and distillation.

ABSORPTION AND ELIMINATION.-Dilute solutions of chloral are readily taken up by the stomach and alimentary tract, also by the mucous membrane of the nose and other parts, and by the subcutaneous cellular tissue, though by this not always with equal quickness or completeness: when given by the rectum it is said to act more powerfully than by the stomach. The absorption of strong solutions is hindered by the coagulation of albumen which they cause. From serous membranes-e.g., the tunica vaginalis-absorption is slow, and large doses have been injected into cysts without any general symptoms (Husemann).

Chloral is eliminated mainly by the lungs and the skin, partially by the kidneys (Gubler). It has not been found as such in the secretions of these organs, and the changes it undergoes after absorption are still under discussion. Recognizing its decomposition on contact with alkalies into chloroform and a formiate, Dr. Liebreich suggested that the same change occurred in the blood. Fminent experimentalists (Richardson, Personne, Byasson and Follet, Horand and Peuch, etc.) have agreed with him, whilst eminent clinicians (Demarquay, Dieulafoy, Krishaber, Gixnldès, Gubler, and many others eited by him; also H. Wood, Amory, W. Mitchell, and indeed most recent writers) take an opposite view, and point to the different 
effects of the two drugrs. The chemical evidence furnished by Personne and others is to the effect, that on distilling at $40^{\circ} \mathrm{C}$. $\left(=104^{\circ} \mathrm{F}\right.$.) the blood of animals poisoned by chloral, chloroform was obtained; but it will be seen that the temperature is higher than the normal temperature of the blood, and the chloroform may be produced during the distillation. Chloral mixed with fresh blood, made to resemble its ordinary condition by the passage into it of carbonic acid gas, gives rise to no chloroform unless the mixture be heated (Hammarsten); and, according to this observer, neither the blood nor breath of dogs poisoned by chloral gives any evidence of chloroform, although the last-mentioned when injected quickly showed itself in both. Rajewsky and Amory confirm this, and prove that when chloroform has been given there is no difficulty in detecting it. Gubler points out how slow is any development of chloroform from chloral, even outside the body; he mixed the latter drug with serum, and again separately with blood obtained by venesection, hæmoptysis, ete., with saliva and mucus, and each of these with alkali in the form of strong Tichy water; but at the end of an hour there was no odour of chloroform, unless heat to $100^{\circ}$ to $104^{\circ} \mathrm{F}$. and upwards was applied. Chloral itself has not yet been detected in the breath after administration, or in any of the secretions; but Demarquay notes an odour of the drug in the air expired by chloralized animals; and A. Tomasciewicz detected it in the urine of lunatics under chloral treatment (Pflüger's Archiv, ix., May, 1874). Amory states that he has found crystals of it in the blood (N. Y. Med. Journ., 1870). Altogether wo must conclude that the chemical change suggested by Liebreich does not occur to any appreciable extent in the ordinary conditions of the body: it must be, in any case, slow, and does not account for its action.

Physiological Action.-Erternul.-Applied in substance or strong solution, chloral hydrate exerts an irritant, eren caustic, effect, and coagulates albumen: on mucous membranes it may cause vesication with severe pain. Injected under the skin it excites much irritation, and in animals hns induced inflammation and gangrene(Lionville). A 10 per cent. 
solution produces only slight burning, and 1 per cent. is scarcely felt. On wounds a chloral lotion forms a thin, semi-transparent, slightly-adherent film.

Chloral has marked antiseptic and preservative powers, which may be utilized without injury to fine tissues. Twenty to 40 grains in the ounce suffice to preserve them indefinitely, and Personne showed to the Academy the body of a dog so preserved for fifty-five days, but apparently just dead. It stays decomposition in organic solutions, and 1 per cent. will prevent lactic acid fermentation (Dujardin-Beaumetz and Hirne) and destroy bacteria in a putrefying infusion of flesh (Dimitrieff); it does not, however, arrest yeast-fermentation.

Brown-Séquard has specially observed the effect of anhydrous chloral applied to the skin of animals, and states that it resembles that of chloroform with some differences; it acts more slowly on the system, but kills more often; induces hæmorrhage and diarrhœa, also diabetes: rabbits pass into a syncopal or trancelike state, and it is noteworthy that when death has followed an application of the drug over 1 to 2 centimetres of the skin of the back, putrefaction is quite absent; all the fluid secretions are increased, so that the muscles are rendered drier: he supposes that a certain amount of hydrated chloral forms in the system under such applications (Med. Times, i., 1881).

\section{Physiological Action. - Intemal. - Circulatory} System.-The special effects of chloral may be produced in frogs in which the blood has been displaced by neutral salt solution (Reichert's Archiv, 1870). This is a further argument against such effects being due to formation of chloroform under influence of the blood-alkalies; and outside the body, corpuscles treated with chloral are not destroyed by it as they are by chloroform. According to some observations, the pulse-rate and arterial tension may be slightly raised under small doses; but usually, even if sufficient to induce a light sleep be giren, the pulse is either not affected or is rendered slower. Capillary vessels in the eye, ear, and face of animals under chloral have been variously described as "enlarged and engorged" (Demarquay) or "contracted" (Guyot, Nicol); the congested condition doubtless represents the full effect of the drug. 
Sedgwick found the inner structures of the eye at first hyperæmic, later anæmic (B. M. J., ii., 1879). Under large doses the pulse becomes very weak and slow, ultimately rapid, thready, and irregular, whilst arterial tension is much lowered (Andrews, Da Costa, Amer. Journ., April, 1870). In fatal cases the heart has been found arrested in diastole with its right cavities distended (Richardson, Med. Times, ii., 1869-70).

The effect on the pulse and on vascular tension is not produced through inhibitory nerves, for it is independent of their section; it may be due in part to a vaso-motor paresis, but must be traced chiefly to a depressant action on cardiac nerves; the cardiac arrest is not direct, for it does not follow the local application of the drug, and in poisoned animals the heart may be stimulated to contract after death; it is effected, probably, through nerve-centres at the base of the brain; after death, congestion of these parts, as well as of the meninges and of the lungs, is found.

Respiratory System.--Under full doses of chloral, respiration becomes slow and deep; but as poisonous effects are developed, it becomes irregular, quick, and shallow, until it ceases. Paralysis of respiration is the usual mode of chloral-death, though cardiac arrest may be simultaneous. The former, like the latter, is effected probably through nerve-centres at the base of the brain : section of the vagi makes no difference in its occurrence.

Nervous System.-The first effect produced by a medicinal dose-10 to 20 grains-of chloral may be more or less excitement, but usually this is slight in amount, and quiet, dreamless sleep supervenes: in favourable cases it resembles natural sleep more nearly than the effect of any other drug, and the patient may be roused from it for food, etc., and then quickly sleep again under the same influence. With such a dose, there is not only no anæsthesia, but often a limited degree of hyperæsthesia (Demarquay, Bouchut, and others), and evidence of irritation or stimulation of spinal, cardiac, and vaso-motor centres. Exceptionally, there have occurred delirium and convulsion, but these were possibly due to impurities in the preparation.

Full doses, which vary in different subjects, but may be stated at from 30 to 60 grains, produce a deep sleep, which may pass into profound coma, with relaxation of muscles and impairment or loss of sensibility and reflex power. 
In the majority of cases the pupil, if at first dilated, becomes minutely contracted when sleep sets in, and remains so for two to three hours, but on sudden rousing it may dilate, and in exceptional cases dilatation seems to be the condition all through the time of chloral influence.

The glycosuria induced by puncture of the floor of the fourth ventricle may be prevented by previous administration of chloral (Echhard).

There is every reason to think that the sleep is caused by a direct action of the drug on the cerebral cells, and the sensory and reflex palsy by direct depressing influence on the spinal cord; for if this be irritated in the later stages of chloralpoisoning, the spasms caused are much less than in the unpoisoned animal (Rajewsky, Schmidt's Jahrb., Bd. cli.). A large dose, doubtless, causes some degree of anæsthesia, but it cannot safely be used for surgical purposes (Giraldés and others). Nussbaum tried it in twenty cases, most of whom certainly slept, but only one, a delicate woman, was anæsthetized. Professor Oré has injected solution of chloral into the blood, and finds the anæsthesia more marked than if given by the mouth: he recommends its subcutaneous use in preference to inhalation of ether, etc., and speaks highly of it for the spasm of tetanus; but the method is very dangerous.

As exceptional symptoms, which point, perhaps, to some paralysing effect on the sympathetic, or are reflex from local gastric irritation, may be mentioned romiting, vertigo, pallor, cold sweating, prostration, an erythematous rash in patches, urticaria, and alteration in vision.

Temperature.--Chloral lowers the body-temperature rapidly, and sometimes in animals as much as $10^{\circ} \mathrm{F}$. in an hour; this occurs, though to a less extent, even if they lie well clothed in a warm place (Ricliardson, Hammarsten, Brunton).

Levinstein records the case of a man who took 6 drachms with suicidal purpose; one hour afterwards his temperature was $102 \cdot 1^{\circ} \mathrm{F}$, and an hour after that it had fallen to $91 \cdot 2^{\circ}$; it raried afterwards up to the time of recovery, which occurred in the course of three days, under galvanism, strychnia, etc. (Lancet, ii., 1874). A smaller reduction than this after medicinal doses has been recorded by Bouchut, Da Costa, and other observers. 
Digestive System.-The hot pungent taste of chloral hydrate excites a flow of saliva, and if the mouth be sore, temporary pain. In the stomach, if the first sensation be "cooling," it is soon followed by warmth, and if the drug be in large dose, or insufficiently diluted, or impure, much irritation, nausea, or vomiting may be produced. Given in moderate dose, it rather stimulates than impairs appetite, and does not cause indigestion (Bartholow).

Chronic Chloralism.-The habitual use of the drug (which is not very uncommon) produces special effects. Dyspnca is one of these; it may be slight, and only felt after exertion, or when the stomach is full, or it may be constant and alarming (H. Wood). Slin-eruptions also occur, in the form of transient red patches (or a disposition to such patches on slight provocation such as taking stimulants), erythema in the course of nerves, or pruriginous papules. I have seen erythema develop in the course of the sciatic nerve after only the second dose of the drug, and Mr. Bryant reported a "rubeoloid rash" after a few days' treatment with chloral for tetanus (Med. Times, ii., 1872). Petechir, ecchymoses, and ulceration, about the nails especially, together with pyæmic symptoms, have been attributed, though not with certainty, to the "chloral-habit." Dr. W. R. Smith has specially reported cases in which the nails were affected, and compares some of the effects produced to those of ergot (Boston Med. Surg. Journ., 1871). A few instances of heat, redness, and swelling of the eyelids have also been traced to the drug (Med. Times, i., 1870). Bartholow describes an "excited hurried manner, voluble speech, vertigo, wakefulness, depression, weakness, and dyspepsia as the ultimate condition of chloral-takerscertainly various signs of impaired circulation and nerve-power are apt to develop in them. According to the report of the Chloral Committee (Clin. Soc.) such pronounced evil effects are not very common; many observers of large experience never having seen them. Fourteen out of seventy practitioners who reported to the committee had seen some ncrve-debility and mental enfeeblement; nine, cutaneons disorders; six, more or less dyspepsia; and two, some urinary irritation. The evidence was not considered sufficient for decisivo judgment (Med. Times, i., 1880). 
SYNERGISTS.-Bromides, chloroform, ether, opium, and its alkaloids assist the action of chloral. Serious collapse has been attributed to opium when given after chloral and bromide (Lancet, i., 1880), and I have seen such effect follow after taking a mixture of chloral and opium.

Antagonists.-Strychnia, eserin, and in a less degree picrotoxin, are clistinctly antagonistic to chloral in several points, though not surely antidotal to poisonous quantities.

Hughes Bennett ascertained the minimum fatal dose of strychnia for a $3-1 b$. rabbit to be $\frac{1}{96}$ grain, and of chloral 21 grains, and then proved the antagonism of the drugs by many experiments: thus, to one rabbit (weighing 2 lbs. 13 ozs.) 14 grains of chloral were given with $\frac{1}{70}$ grain of strychnia, and the animal recovered: seven days afterwards, it succumbed in eighteen minutes to the same dose of strychnia alone. Another animal (weighing 2 lbs. 12 ozs.) got 18 grains of chloral and $\frac{1}{40}$ grain of strychnia, and recovered; seven days after, the same dose of the latter proved fatal in twelve minutes. If chloral were given, not simultaneously, but within eight minutes after strychnia, it proved effective, but if more than ten minutes after, it did not prevent death. Professor Hughes Bennett demonstrated more than once on two rabbits of about 3 lbs. weight, injecting under the skin of each $\frac{1}{96}$ grain of strychnia, and then of one, 15 grains of chloral; in ten minutes the first animal would leap up, and then fall tetanized and dead, the other would go to sleep, and in about two hours wake as if nothing were the matter (B. M. J., i., 1875). With regard to Calabar bean (eserin) the same observer proved that chloral modified largely the nction of a fatal dose, mitigating symptoms and prolonging life, and in some cases saving it.

Sir Crichton Browne found that the minimum lethal dose of picrotoxin for a rabbit weighing 3 lbs. was $\frac{1}{214}$ grain, but 10 grains of chloral administered with this, prevented its causing death. Even 1 grain of the alkaloid could be bome or recovered from if at the same time a full dose (21 grains) of chloral were given: the animal passed into a deep sleep which was broken by clonic spasms (from the picrotoxin) at intervals 
of about three minutes, showing the action of both drugs simultaneously (B. M. J., ii., 1875).

Alkalies are chemically incompatible with chloral.

The failure of the heart and respiratory organs caused by it calls for alcohol, ammonia, coffee, possibly galvanism, and certainly artificial warmth: other external stimulants, flagellation and strong-smelling drugs, artificial respiration, and inhalation of oxygen are also indicated. Atropia is suggested by Wood (cf. Hulke, Lancet, ii., 18r4). The constant excessive use of alcohol induces a condition of system in which it is less susceptible to the action of chloral, and large doses (180 to 240 grains) have been taken by inebriates without fatal results (B. M. J., ii., 1877).

Therapeutical Action.-Extemal.-Ulceration, etc. -A chloral lotion ( 1 to 4 grains in the ounce of water) is a good application to suppurating unhealthy wounds, buboes, bedsore, cancerous ulceration, ete.; it disinfects, deodorizes, and stimulates. In chronic discharging eczema, eczema mammae, and pruritus vulvæ, I have used it also with good result, and in gonorrhoea an injection of the same has proved effective.

M. Dimitrieff has lately made comparative experiments by excising a portion of skin in dogs and infecting the exposed surface so as to induce ulceration: some of these wounds he dressed with a 1 to 2 per cent. solution of chloral, others with simply wet lint: the former soon became healthy, and cicatrized before the latter, which became covered with discharge containing micrococci: these organisms disappeared after using the chloral lotion.

Nævus. - The coagulating power of the drug has been utilized in the treatment of novus, and Dr. Pupi, for instance, cured a rapidly extending erectile growth in the face by injecting 1 part in 10 of water into its base: the cure was complete after three injections, given at intervals of fifteen days (Record, 1880).

Toothache-Neuralgia.- Ilalf to one grain of chloral, wrapped in cottorl-wool, and placed in a painful tooth-cavity, often gives much relief (Spender, Merl. Times, ii., 1880): it dissolves, and the accumulated saliva should be ejected. Tritu- 
rated with camphor, it forms a liquid which is said, by local application, to control neuralgic pain (Lennox Browne) and spasmodic cough (applied over the larynx-Bartholow); but I have not myself had good results with it. In vinter cough, an atomized spray, containing 10 grains of chloral hydrate in the ounce, has proved much more effectual (Phil. Med. Times, 1879).

\section{Therapeutical ACTION. - Internal. - Insomnia. -} When this symptom results from over-work, or anxiety, or from the debility of convalescence after acute disease, chloral is probably the best remedy. In cases when it acts well the sleep given is of natural character, refreshing, and not followed by headache, but in some persons unpleasant excitement, and in others subsequent headache does occur, especially if sleep be not produced or be interrupted; combination with an equal amount of a bromide salt will often act better in such cases.

Dr. Kane, in a recent special study of the subject, calls chloral a "typical hypnotic," and notes its suitability for, and toleration by, children; he gives instances also of its being used to quiet restless horses (N. Y. Record, 1881).

When wakefulness is caused by physical pain, chloral is not so likely to relieve unless in dangerously large doses; it is better then to combine with it a little morphia or atropia, or both, and subsequent depression is thus alleviated. In ferers and febrile affections, chloral has proved exceptionally useful as a sedative, and its power of reducing temperature would, ì priori, be some indication for it. Husemann refers to much experience of its value in Typhoid Fever, particularly when collapse is imminent, and Russell narrates cases when it proved "the best and most reliable hypnotic" (Glasgow Med. Journ., Feb., 18r0). Fraser and Muirhead note that full doses are required for this effect, whilst Dr. Colley Marsh has pointed out the good results to be obtained from 2 to 5 grains given erery four hours ; this treatment, he states, proves most satisfactory if commenced during the second week,-- " restlessness is soothed, the belly becomes less tender, the stools less frequent" (Med. Times, i., 1881).

In Diphtheria, especially when affecting children, the action of the drug is equally beneficial; 1 -grain every hour may be 
given to a child of three years. Dr. Marsh suggests not only an intiseptic action but a "tonic effect on the heart" from small doses. Donovan, on the other hand, has cautioned against its use in pneumonia, pleurisy, and other conditions that impair respiratory power, stating that he has "repeatedly" seen collapse and even death from 24-grain doses given in such conditions: it should certainly be given, if at all, with extra care and in smaller doses in such cases, and especially in chronic bronchitis with weak heart.

In Rheumatic Fever and acute gouty attacks, I have often given this remedy with good effect in relieving pain and procuring sleep. Brudenell Carter, H. Greenhow, Ogle, and Coghill have recorded similar experience, so that the early unfavourable opinion of $\mathrm{O}$. Liebreich as to this point may now be qualified.

Clemens has shown the value of chloral in Metritis and Peritonitis, and in the acute Gastro-enteritis of children, accompanied with vomiting and diarrhœa, 3 to 4 grains given by enema have been found efficacious (Record, 1880).

Mania-Melancholia.-The early reports of the action of chloral in these disorders were mostly favourable. Dr. Tuke found it "more uniform and more lasting in its influence than other hypnotics, and not depressing or constipating." Mercer recorded its advantage in noisy delirium (Med. Times, i., 1871), and Dr. Maury Deas found it "most valuable as a reliable and safe hypnotic" (Cheshire County Asylum Report, 1873). The average dose given was 30 grains at bedtime. Of 2,442 administrations to women, it is only recorded to have failed seven times: of 898 doses to men there were 111 failmes, the greater number being in cases of general paralysis. In many, beyond the securing of sleep, no direct benefit resulted; in about twelve cases it was thought to contribute materially towards recovery: the best results were obtained in forms of alcoholic insanity and atonic melancholia: no injurious effects were traced. Further experience has shown, however, that so favourable an opinion must be qualified. Dr. Deas informs me (1881) that he has found it necessary to use the drug very sparingly and cautiously in general poralysis and other kinds of active brin-disease, for in such cases it not orly often fails to induce sleep, but even a small quantity may cause alarming symptoms. He does not 
advise the drug in any case as a continuous sedative; and, further, he finds 15 grains with an equal amount of potassium bromide better than the larger dose already mentioned. Other specialists have made similar observations, especially as regards general paralysis; and paraplegia and excessive cardiac weakness have been traced to its use. Dr. Rhys Williams reported unfavourably (18 $\mathbf{r}$ ), finding that even 20 grains every four hours for several doses failed to procure sleep in acute cases; and Maudsley, Clouston, and Lindsay express strong objections to its use (Report of Chloral Committee of Clin. Soc., 1880). There is now a general opinion that acute attacks of mania treated by it are longer in recovering, and even, it is said, more likely to end in dementia than those not so treated. (Paraldehyd is often much more suitable.)

Imbeciles and demented persons, however, in whom no active cerebral disease is progressing, generally bear chloral well, a fact which may be compared with Brunton's observation as to the tolerance of the drug by the lower classes of animals with "smooth brains," also with Redier's as to its tolerance by children.

In Puerperal Mania and Convulsion, chloral in 20- to 30grain doses often relieves; it may be given by injection if the patient be unable to swallow. McIntosh reported a good illustration in an anremic woman (Med. Times, ii., 1870), and since then many others have confirmed his experience. Mr. Hugh Thomas obtained immediate quiet after several hours of convulsion, and apparent failure of bromides (B. M. J., ii., 1879); and Guyot, at the Paris Maternity, had good success in thirteen out of fourteen cases (ibid.).

During parturition, Dr. W. S. Playfair has advocated the use of chloral to relieve the pains of labour. He gives sufficient doses to produce a drowsy state-about 15 grains three or four times in the first few hours. It favours dilatation of the cervix uteri, and $\mathrm{D}_{1}$. C. O. Wright advises it in any tedious labour, to shorten the first stage, in the albuminuria of parturient women as well as in eclampsia, and in the vomiting of pregnancy (Amer. Journ. Obstet, 18\%9).

In Convulsion and Spasm of various kinds, chloral is often a good remedy. In uræmic and infantile convulsion, in cramp, hiccough, spasmodic enuresis, laryngismus, and pertussis; also 
in asthma and the dyspnoea of emphysema its value has been often illustrated; also in the early stages of acute catarrh. In cases where bronchitis is present, or there is obstruction to the pulmonary circulation, extra caution is required, though the drug is not wholly contra-indicated. In migraine, which also may be connected with vascular spasm, rectal injections of 15 to 60 grains are said to have proved very beneficial.

Chorea-Epilepsy.-Dr. Althaus has reported trials of it in the former, and $\mathrm{N}$. Allcock traced partial benefit to its use in the latter malady (Med. Times, i., 1872). He records a case where attacks of ague seemed to replace the epileptic convulsion, and judged chloral to be of service in both cases by its relieving spasm; only one other case is, however, given. Dr. Deas "has found it extremely useful in arresting a storm of fits in epilepsy, and thereby checking development of that dangerous condition, the 'status epilepticus.'" In such cases a full dose of say 40 grains, or even more, should be given, and often it is most usefully administered per anmm.

Tetanus - Trismus. - There is much evidence of benefit to be derived in these disorders from chloral, given either alone or with bromides. Dr.J. R. Beck has published thirty-six cases (traumatic), of which twenty-one recovered (St. Louis Med. and Surg. Journ., Jan., 187\%). Wood has tabulated fifty-four others, with a total result of forty-six recoveries against forty-four deaths. Mr. C. Macnamara reports seventeen cures out of twenty cases occurring in India, and he gave the drug as a hypnotic40 grains at bedtime, and only occasionally 30 grains in the morning; noturishment, in the form of milk, brandy, and egg, constituted the rest of the treatment (Practitioner, vol. ix.). More recently, Roberts has summarized n number of cases, on the whole favourable (Amer. Journ. Med. Science, Oct., 187\%).

In a case of traumatic tetanus from an injury to the thumb at the Manchester Infirmary, when the symptoms were fully developed, Mr. Whitcher ordered 20 grains each of chloral and bromide of ammonium every hour till sleep was produced: in six days the man took 110 doses, mostly per rectum, and gradually recovered (B. M. J., i., 1881).

In trismus nascentium, a usually fatal form of the disorder, chloral is credited with saving six cases out of twelve (Widon- 
hofer), and in a case of tetanus occurring in a child twelve days old, Dr. Welch attributes recovery to its being kept under the influence of the drug: 3 to 4 grains in twenty-four hours were sufficient (B. M. J., i., 1881).

Strychnia-Poisoning.-I have already quoted the physiological experiments proving antagonism between chloral and strychnia, and there are on record several instances of this being successfully applied in practice (Ogilvie Will, Edin. Med. Journ., 1875). Dr. Gray gives one in which $22(?)$ grains of strychnia had been swallowed: a drachm of chloral was given slowly "between the clenched teeth," and in ten minutes the convulsions were lessened: later, two half-drachms were administered with completely successful result (B. M. J., i., 1880).

Mansell relates the recovery of a fox-terrier from strychniapoisoning after the administration of 1 drachm of chloral (Lancet, i., 1881).

Nothnagel says, "In tetanus toxicus (strychnia-poisoning) chloral hydrate is a very good remedy, which may be well applied by injection into the rectum. My experiments on animals confirm the statements of Liebreich and Rajewsky, that strychnia-convulsions may be prevented, or may be cut short after their appearance, by comparatively small doses of chloral hydrate. The quick hypnotic effect of the remedy makes it more suitable than opium preparations; still, after large doses death may occur, either from a sudden tetanic access, or without this, from the chloral itself. Very large ('colossal') doses of both substances cannot neutralize their respective actions."

Hydrophobia.-Though chloral cannot be considered a remedy for this disorder, it has certainly mitigated its worst symptoms in the practice of Ellis (B. M. J., i., 18 1 ), Nicholson and Sainter (Lancet, i., 1872), Liouville and Smith. This has also been my experience in four cases, which, however, proved fatal. Mr. Dolan calls it a "valuable auxiliary," and it may be given either as enema or under the skin (Nature of Rabies, etc., 1878).

On the other hand, neither Wolfert (Berl. Klin. Woch., 1871, viii.) nor Verri (Amnali di Med., Nov., 1871) could see any result from it; the paroxysms were not lessened, nor was 
sleep produced. Hence Köhler suggests that cases recovering under its use were really of "sympathetic hydrophobia" (neurosis with hydrophobic symptoms).

Mr. F. A. Southam, after recording seven eases treated in various ways at the Manchester Infirmary, remarks that chloral, by its sedative action on the nervous system, appears to give the most beneficial results, by prolonging life and also by temporarily allaying the spasms; so that even if its curative action be nil, valuable time is gained for the trial of other remedies: he found subcutaneous injections better than giving the drug per rectum (B. M. J., ii., 1881).

Cholera.-Dr. McReddie has recently reported remarkable recoveries even from choleraic collapse under the hypodermic use of from 1 to 6 grains of chloral dissolved in water (B. M. J., i., 1881).

In Sea-Sickness chloral often proves a valuable remedy.

CONTRA-INDICATIONS. - The most important contraindications, to large doses at least, are-(1) weak, irregular heart-action; (2) any serious obstruction to respiration, whether from lung-congestion, bronchitis, or pleuritic effusion : in chronic bronchitis, with weak heart, caution is especially necessary; (3) much irritability of the stomach or intestines also to some extent forbids the use of the drug; (4) given in Bright's disease it is said to favour uræmia; others, however, advise it in the albuminuria of pregnancy.

DosE.-As a sedative, 3 to 8 gr. every two hours; as a hypnotic, 15 to $20 \mathrm{gr}$. in one dose : this amount should not be exceeded unless in cases of urgency. If given per rectum, the dose should not be larger than by the mouth: by the skin $15 \mathrm{gr}$. is a suitable quantity. Redier and also Bonchut assert that the drug is well borne by children, and advocate for them doses of 30 to 60 gr. per diem to secure an anæosthetic effect before operation (Union Mćd., Juillet, 1880). Thirty grains have, however, several times caused grave anxiety (Fuller, Lancet, i., 1871); 45 gr. have induced alarming symptoms (Reynolds, Practitioner, vol. iv.) ; and 80 gr., given in eight separate closes, nearly proved fatal (Watson, quoted by Wood). 


\section{BUTYL-CHLORAL HYDRAS.}

\section{(Hydrate of Butyl-Chloral, $\mathrm{C}_{4} \mathrm{H}_{5} \mathrm{Cl}_{3} \mathrm{O}, \mathrm{H}_{2} \mathrm{O}$.)} (Croton-Chloral-Hydrate, wrongly so-called.)

PREPARATION.--In making chloral by the treatment of a mixture of alcohol and aldehyd with chlorine, the hydrochloric acid produced dehydrates the aldehyd (acetic), with formation of crotonic aldehyd; and this is converted by the free chlorine present into ter-chlorocrotonic aldehyd, introduced into practice by Dr. O. Liebreich as "croton-chloral." It was discovered by Krämer and Pinner in 1870.

Characters and Tests.-Butyl-chloral hydrate occurs in small, colourless, shining tables, not unlike the crystals of chloral hydrate. It is sparingly soluble in cold water. In contact with alkaline solutions it changes into chloride and formiate of sodium and bi-chlorallylene.

ABsorption AND Elimination.-This substance is apparently absorbed and eliminated with the same readiness as chloral hydrate. An analogous decomposition has been supposed to occur between it and the alkalies of the blood ( $v$. p. 964).

Physiological Actron.-The specific effect of small or moderate doses of butyl-chloral is the production of anæsthesia, especially in parts supplied by the fifth nerve, whilst full doses (60 grains) cause deep sleep, without important change in the pulse or respiration (O. Liebreich). Even in comatose conditions produced by still larger doses, circulation goes on, and artificial respiration will restore a moribund animal (B. M. J., ii., 1873). Only by "immense doses" is cardiac paralysis induced. Such results are apparently not constant, for Mering found respiration markedly slowed before the cornea was rendered insensible, and intravenous injections immediately paralysed the heart: he concluded that in animals it acted much like chloral (Archiv Exper. Pathol., Feb., 1875). 
W. Schmidt found that narcosis occurred before anæsthesia (Centralbl. f. Chirurg., 1877); but further observations are required before considering these points settled.

Therapeutical Action.-Neuralgia. - In this disorder, especially when affecting branches of the fifth nerve, great relief may often be obtained by butyl-chloral (B. M.J. and Lancet, 1873-4). When first introduced into this country only small doses of 1 to 2 grains were given, and hence, perhaps, some failure to relieve in the earlier eases in which I made trial of it. Doses of from 3 to 5 grains may, however, be given safely, and repeated every hour if necessary, till about 30 grains have been taken, and then very satisfactory results are obtained. Other forms of nerve-pain-e.g., neuralgic dysmenorrhœe, headache, and sciatica-have also been relieved by this means.

Insomnia.-The hypnotic effects of chloral hydrate may be increased safely by the addition of a small quantity of butyl-chloral. The combination is especially indicated if much nerve-pain be present.

DOSE.-2 to $10 \mathrm{gr}$. every hour, up to a maximum of $30 \mathrm{gr}$. It may be given in pill, or with mucilage, syrup of tolu, or glycerine. 


\title{
A N I M A L KINGDOM.
}

\author{
$M A M M A L I A$.
}

RODENTIA.

\section{CASTOREUM. (CASTOR.)}

\section{(Not Officinal.)}

DESCRIPTION.-The dried preputial follicles with their contained secretion, from the beaver, Castor fiber. There are two varieties-(1) Castoreum Sibiricum seu Europoum, (2) C. Canadense seu Americanum seu Anglicum. It occurs in elongated, flattened, more or less pyriform sacs, united at the narrow end in pairs, varying from 1 to 4 ounces in weight; when fresh they are whitish-pink in colour,-after drying, brown and wrinkled. Four distinct layers may be made out in their covering, viz., cellular, fibrous, vascular, and " an iridescent glandular" coat, with folds and small scales on its inner surface: these contain a thin, yellowish, odorous liquid, which becomes solid and brown on drying.

ACTIVE INGREDIENTS.-Castor contains 1 to 2 per cent. of a pale yellow volatile oil, in which salicin and benzoio acid have been found; also cholesterin, and other bile-derivatives, and a proteid compound : any carbolic acid present is presumed to be due to the process of drying over a smoky fire.

Castorin is the name given to colourless acicular crystals, having a slight odour and taste of the drug, obtained by boiling 
it with lime and water, and treating the residue with hot alcohol; it is somewhat volatile, and soluble in strong alkalies, ether, and oils. There is also a dark brown resinous substance, of acrid taste, soluble in alcohol, insoluble in water: the inorganic constituents of castor include carbonates, phosphates, and sulphates of the alkalies and the alkaline earths.

Physiological Action.-The taste of this drug is bitter and rather nauseous; the odour is aromatic. Its action may be described as nerve-stimulant and antispasmodic; but Stillé doubts whether such effects are seen on the healthy nervous system. Large doses quicken the pulse.

SYNERGISTS.-Musk, valerian, camphor, ether, etc.

ANTAGONISTS.-Similar to those of musk.

THERAPEUTICAL ACTION.-In cases of impaired nervepower from exhaustion, grief, etc., with such symptoms as spasm and tremor, tympanites, etc., whether associated with ordinary hysteria, or uterine colic, or specific fever (in the "typhoid state"), this remedy is serviceable, though now seldom used.

PREPaRations and Dose.-Pulvis: dose, 5 to $10 \mathrm{gr}$. Tinctura: dose, $\frac{1}{2}$ to $1 \mathrm{fl}$. dr.

ADULTERA'TIONS.-Blood and other organic matters, as well as resinous and earthy material. 


\section{RUMINANTIA.}

\section{MOSCHUS.}

(Musk.)

DESCRIPTION.-The dried secretion of the preputial follicles of the Moschus moschiferus, or musk-deer, which inhabits chiefly the mountainous regions of Central Asia. The drug occurs in dark brownish granules, unctuous when fresh, of bitter taste and characteristic odour which lessens on drying, but returns on moistening, especially with alkaline solutions. It is contained in the musk bag (pod, sac, or pouch), which is oval, 2 inches by $1 \frac{1}{2}$, and about $\frac{1}{2}$ inch thick, smooth and flat above, convex and hairy below. The hairs are grey or brown, short, stiff, and arranged in a circle towards an aperture in the centre of the sac, which itself has an external fibrous coat, lined inside with membrane arranged in numerous folds and depressions, containing glands, which form the secretion. The musk brought from Thibet and China is better than that known as Siberian, Russian, or Cabardine.

Active INGREDIENTS.-Besides cholesterin, fat, resin, salts, etc., there is a special odoriferous principle, which has not yet been isolated: it is supposed to be formed by slow decomposition of other constituents in the presence of moisture.

Physiological Action.-This is at first stimulating, especially to the central nervous system, but afterwards more or less soporific or narcotic, and hence has been compared to that of alcohol. There is some reason to consider it specially stimulating to the respiratory centre (Brunton). Jörg reported symptoms of gastric disturbance from the drug, but Trousseau, repeating his observations on himself, found, on the contrary, increased appetite and warmth at the stomach; later on he 
experienced some headache, giddiness, and stimulation of genital organs: the odour of the drug was discernible in all the secretions. In some delicate subjects, headache and even convulsive attacks are induced by the mere odour of musk, which is extremely diffusive, one part sufficing to scent 3,000 parts of an inert powder. When given to animals by intravenous injection, musk has caused some uarcotism, with muscular spasm-also bloody stools, followed by prostration and death.

SYNERGISTS.-Castor, asafœtida, sumbul, valerian. Opium is sometimes well combined with it.

ANTAGONISTS.-Camphor and bitter almonds lessen the odour.

Therapeutical Action.-Nerve Exhaustion.-This is the main underlying condition likely to be benefited by musk, although the symptoms may vary. It may be associated with the severer formos of the specific fevers, with pneumonia, with cholera, brain disorder, etc., and is recognized by muttering delirium or coma-vigil, subsultus tendinum, hiccough, small irregular pulse, nervous palpitation, etc., without absolute coma or collapse. It tends to lessen ataxic phenomena, to steady the circulation, and promote sleep, and by the same action benefits in the restlessness and insomnia of over-fatigue. Its good effects are seen quickly in cases suitable for it.

Récamier was one of the first to record cases of its value in Ineumonia, when the symptoms are unaffected by bleeding, and pass into an adynamic state with nerve-incoherence and ataxic delirium.

'Trousseau saw advantage from it in similar couditions occurring in a drunkard, "when the gravity of the nervephenomena was not sufficiently explained by the intensity of local lesions." He remarks that it is not so much the severe delirium of manifestly fatal tendency to which ho alludes, as a "purely nervous form, when the brain is excited, the patients toss about, try to get up, and talk wildly, very much as in delirium tremens." 
It is worth while to allude also to the extremely favourable reports of the remedy by Sarcone, during a fatal epidemic of fever in Naples. When symptoms developed of extreme nerve-sensibility, emotional distress, and insomnia, he found some benefit from opium, but much more from musk.

Spasm.-There are other more purely spasmodic disorders, independent of fever, in which the same remedy is serviceable, such as laryngismus stridulus, whooping and other spasmodic coughs, spasm of pharynx, neurotic vomiting, etc. Trousseau further quotes authors who state that chorea, hysterical convulsion, and even tetanus have sometimes yielded to it. Whilst referring to some of the wonderful results recorded by older authors in hysteria, he explains them rather by strong: mental influences than by the action of the drug, which he values comparatively little in such cases. I have myself seen good results in hysterical patients suffering from laughing and crying, globus, abdominal distension, ete.

Irregular Gout.-In this disorder, when any internal organs are suddenly affected with pain or colic, Cullen, who had much experience in such cases, expressed a high opinion of musk.

Preparations and Dose.-The solid drug may be given in pill, or suspended in mucilage; dose, 3 to 5 gr. every hour or two, to the amount of 15 or $20 \mathrm{gr}$. per dien. The U.S.P. has a tincture: dose, 25 to $50 \mathrm{~min}$. The drug is costly, and liable to adulteration. The distilled oil of Mimulus moschatus and musk-smelling plants has been said to act similarly: dose, 2 to 4 min. in twenty-four hours (Hanon; Journ. de Pharm., xxv.). 


\section{SEVUM PRAPARATUM.}

\section{(Prepared Suet of Ovis Aries-the Shemp.)}

DESCRIPTION.-The internal fat obtained from the region of the kidney, purified by melting at a moderate heat, and straining. Mutton suet is firmer in consistence, and requires a higher temperature to melt it than other animal fats. It is white, soft, smooth, inodorous, and of a bland taste; fusible at $103^{\circ} \mathrm{F}$.; soluble in ether and boiling alcohol; insoluble in cold water, and nearly so in cold alcohol.

ACTIVE INGREDIENTS.- Suet consists chiefly of stearin, olein, and margarin. Tristearin, $\mathrm{C}_{57} \mathrm{H}_{120} \mathrm{O}_{6}$, obtained crystallized from an ethereal solution, is the chief ingredient. It is concrete, white, opaque in mass, but of a pearly appearance as crystallized from ether; pulverizable, fusible at varying temperatures, though usually at about $143^{\circ} \mathrm{F}$; soluble in boiling alcohol and ether, but nearly insoluble in those liquids cold, and quite insoluble in water.

Otein or triolein, $\mathrm{C}_{57} \mathrm{H}_{104} \mathrm{O}_{6}$, is the more liquid principle of oils, and is difficult to obtain pure: as usually seen it contains more or less of margarin or stearin, or both. It is of oily consistence, varying in quantity in different fats; becoming concrete at $20^{\circ} \mathrm{F}$. ; colourless when pure ; with little odour and of a sweetish taste; soluble in boiling alcohol and ether, insoluble in water.

Margarin is the more solid part of oils, and is supposed to be a mixture of palmitin and other compounds of glycerine and fatty acids; it forms about 28 per cent., and appears in pearly crystals, soluble in cold ether. It resembles stearin, differing mainly in its lower melting-point.

USES.-Mutton suet is nutritious but sometimes difficult of digestion. It is emollient and demulcent. Bniled in milk it is very useful in chronic diarrhoon and wasting diseases. It is contained in the emplastrum cantharides and unguentum hydrargyri. 


\section{LAC.}

Fresh Milk from the Cow-Bos taurus; used in preparing: Mistura Scammonii.

(Saccharum Lactis-Sugar of Milik-Lactose.)

Crystallized sugar obtained from the whey of milk by evaporation.

It occurs in cylindrical masses, 2 inches in diameter, with a cord or stick in the axis, or in fragments of cakes. These masses are formed of rhombic, greyish-white crystals, translucent, hard, gritty, odourless, faintly sweet, soluble in 6 parts of cold and in about 2.5 parts of boiling water, slightly soluble in alcohol, insoluble in ether. It is not subject to alcoholic fermentation, but a large amount of yeast turns it into mannite; dilute acids change it into galactose, a glucose which can undergo alcoholic fermentation.

It is used as a substitute for cane sugar in the diet of infants, as it is supposed to be less irritating to the mucous membrane of the stomach. Cow's milk, diluted with one-third of water, to which a little sugar of milk is added, is considered a good substitute for human milk.

KoUmISs, in its original form, is prepared by fermentation of mares' milk (in Tartary), but is now made in London by fermenting cows' milk with grape sugar, etc. It contains some alcohol, as well as lactic acid, casein, fat, and carbonic acid gas, and has been recommended in cases of mal-nutrition, vomiting, phthisis, etc. It sometimes answers well, but my own experience of it has not been satisfactory.

\section{ACIDUM LACTICUM \\ (Lactio $\mathrm{A}$ (ID), $\mathrm{HC}_{3} \mathrm{H}_{5} \mathrm{O}_{3}$ )}

Is a coloutless, inodorous, syrupy liquid, containing 25 per cent. of water, with 75 of absolute acid; sp. gr. 1.212: it mixes freely with water, alcohol, and ether, not with chloroform: 
vaporizes when heated to $320^{\circ} \mathrm{F}$, and gives off an inflammable gas.

ACTION.-Locally it may be used as a solvent in a spray, or as paint to the false membranes of croup or diphtheria.

Internally, it has been given, like hydrochloric acid, in dyspepsia (for which malady it is often combined with pepsin), and to lessen alkalinity of urine and phosphatic deposits. Some good results have been reported from it in diabetes (Cantani), and my own experience has been favourable. (It is an interesting fact that acute rheumatism has developed under its use-Foster.)

PREPARATION AND DOSE.-Acidum lacticum dilutum (by adding to 3 ounces of the acid, P.B., enough distilled water to make one pint: sp. gr. 1.040) may be used as paint, and, slightly diluted, as spray (protecting the eyes): dose, $\frac{1}{2}$ to 2 fl. dr. (It is important to secure a good preparation.)

\section{FEL BOVINUM PURIFICATUM.}

(Purified Ox-Bile.)

PREPARATION AND CHARACTERs.-Ox-bile is treated, after concentration, with rectified spirit, and the extract evaporated to a syrupy consistence. So prepared, it is a yellowishgreen substance with sweet-bitter taste, soluble in water and in spirit. It consists of two bitter soaps, colouring matter (bilirubin and biliverdin), mucus, and cholesterin. The soaps are formed by the union of glyco-cholic acid, $\mathrm{C}_{26} \mathrm{H}_{43} \mathrm{NO}_{6}$, and tauro-cholic acid, $\mathrm{C}_{26} \mathrm{H}_{45} \mathrm{NSO}_{7}$, with soda, their presence being: indicated by the play of colours, from red to violet, which the solution gives on arling a trace of cane-sugar and an excess of sulphuric acid (Pettenkofer's test).

Physiological and Therapeutical Action.Bile has several uses in the economy ; it emulsifies frts without splitting them up into glycerine and fatty acids; it acts as a 
natural purgative, and is antiseptic. It is given internally when it is judged that the secretion of bile is deficient, and seems to have some power of rendering the frees softer; but little is accurately known of its therapeutical power.

DosE. -5 to 10 gr.; best given in pills or small gelatin capsules.

\section{P E P S N.}

\section{(Pepsin.)}

DESCRIPTION.-One of the active principles of the gastric juice, of the nature of a ferment, which has the property, in an acid medium, of changing albumen, fibrin, and other proteids, into peptones. It may be prepared in several ways. In the ordinary process, known as Beale's, the mucous membrane of the stomach of a recently-killed calf, pig, or sheep, is carefully cleaned and scraped, and the viscid material obtained is dried quickly in thin layers at a temperature not exceeding $100^{\circ} \mathrm{F}$. In the process of Boudault (French Codex), the extract obtained from the stomach is precipitated with lead acetate, the precipitate washed, suspended in water, the lead thrown down by hydric sulphide, and the liquid filtered, evaporated at a low temperature to syrupy consistence, and mixed with starch. The process is complicated, but serves to remove mucus and epithelium, and so leaves a purer active principle. By the method of Schmidt, the gastric juice is first neutralized by chalk, filtered, and evaporated to syrupy consistence, and then precipitated by absolute alcohol. Payen obtained pepsin by treating the filtered gastric juice with about ten times its volume of rectified spirit, the resulting flocculent precipitate being dried and redissolved.

Von Wittich adopts glycerine as the solvent, first pounding the mucous membrane into a pulp with glass, leaving it to macerate for eight days, and then precipitating with alcohol.

Dr. W. Roberts found a boracie acid, and also a chloroform solution act well. Brïcke used phosphoric acid, 5 per cent., precipitating with lime water; this precipitate, after washing, 
he dissolved in dilute hydric chloride, and re-precipitated with a solution of cholesterin, which was removed by ether. According to the method of Scheffer (U.S.), the acid gastric juice is precipitated by chloride of sodium, and the cream-like substance resulting contains syntonin, or acid-albumin, as well as pepsin; the latter is prepared by washing and re-precipitating, and is then generally mixed with sugar of milk (pepsinum saccharatum, U.S.P.). The pepsin essences, elixirs, and wines are prepared by direct extraction of the finely-divided stomach or its mucous membrane.

Dr. A. Tsheppe reports (1883) that the latest and the most active preparation is that of $\mathrm{C}$. Jensen (Philadelphia), but his method is not, I believe, yet published; it is directed to preparing a strong pure pepsin without admixture of foreign substances, but in contra-distinction to those pepsins obtained by precipitation with salt, it contains also the ferment which, like rennet, coagulates milk (Pharm. Journ., ii., 1883).

\section{Characters and Tests.-Pepsin (B.P.) is a light-} brown amorphous powder of faint, not unpleasant, odour, and slightly saline taste, but little soluble in water or spirit. The pure ferment is a colloid substance and does not react like a proteid with the following tests: it does not give the xanthoprotein reaction with nitric acid, is not precipitated by acetic acid and potassium ferrocyanide, nor by tannic acid, mercuric chloride, silver nitrate, or iodine; in other reactions it agrees with the group of proteids. It is rendered inactive in an acid fluid by heating to $130^{\circ}-140^{\circ}$ F. (Mayer, Landois' Physiology, 1885). Two grains of pepsin B.P. in an ounce of water, with 5 minims of hydrochloric acid, should dissolve 100 grains of hard-boiled white of egg (in fine shavings) in four hours, at a temperature of $130^{\circ} \mathrm{F}$.

Scheffer's test is 1 part pepsin to 500 water, with $\frac{1}{2}$ per cent. hydrochloric acid, to digest 50 parts of hard-boiled egg-albumen in five to six hours. The German Pharmacopoeia requires double this strength, and directs the albumen to be cut into small pieces. It is to be noted that the digestive effect varies much with temperature, percentage of acid, concontration of solution, and accumulation of products and division of the albumen. 
Pepsin without acid does not dissolve albumen at all, and the most favourable proportion is found to be 0.2 per cent. Other acids, and especially lactic or organic acids, do not act so well. Of Jensen's pepsin it is said that $\frac{1}{4}$ grain in 18 drachms of water, with 9 minims of hydrochloric acid, digested 125 grains of hard-boiled egg-albumen in two hours at $100^{\circ}$ to $110^{\circ} \mathrm{F}$. This pepsin occurs in small clear scales termed "crystal," is almost tasteless and odourless, and is soluble in water.

Physiological Action.-Pepsin in a dilute acid solution (preferably hydrochloric acid) transforms proteids, at the temperature of the body, into a soluble form, termed by Lehmann "peptone." During this change they are, at first, transformed into a substance having the characters of syntonin, which is an acid albuminate; an intermediate body is formed between this and peptone, and termed propeptone (hemialbumose), which, under the continued action of the gastric juice, passes into a true soluble peptone, formed probably by a hydrolytic action. "The greater the amount of this ferment (within certain limits), the more rapidly does the solution take place" (Landois). Hence a reasonable basis for its administration when such solution is difficult or painfu (dyspepsia). The pepsin itself seems to suffer little change in the process, and in experiments on artificial digestion, if the acid is uniformly renewed and the mixture diluted, the original pepsin can dissolve fresh quantities of albumen. with but little loss. Starch is not affected by it. Peptones can be prepared by other hydrating agents independently of pepsin, e.g., by the prolonged action of dilute acids and alkalies at $40^{\circ} \mathrm{C}$., and various proteolytic ferments (trypsin, ete.).

Therapeutical Action.-External.-Morbid Growth, etc.-The solvent action of pepsin on animal tissue has been utilized in cases of cancer, diphtheritic membrane, and bloodclot in the bladder. Mr. John Clay treated cancerous ulceration and growth from the cervix uteri by its local application, with the result of dissolving much of the morbid tissue, and sometimes leaving a healthy surface. Rosenthal, using a concentrated acid solution ( 1 drachm to 1 ounce of water, with 
20 minims and upwards of dilute hydrochloric acid) applied on a "throat-mop" every hour to the fauces, reported its acting "like a charm" in dissolving the false membrane of diphtheria.

Hollman reports the case of a man, aged 80 , suffering from retention, unrelieved by the passage of a catheter, the bladder containing coagulated albuminous masses mixed with blood. About 16 grains of pepsin (Jensen's) in solution were injected, and some hours afterwards a quantity of dark, viscid, fœtid fluid was drawn off (Record, 1883).

Therapeutical Action.-Internal.-Dyspepsia.-In cases of gastric dyspepsia dependent upon deficient or altered conditions of secretion of the peptic glands, a few grains of a good preparation of the drug taken with the food (sometimes preferably in acid solution) will promote its solution and absorption, and relieve feelings of weight, pain, and distress that would otherwise follow the meal.

We see this especially in cases of anæmia, of phthisis, of convalescence from bronchitis, and other serious maladies, as well as in the dyspepsia of emotional and mental causation.

The remedy relieves also the vomiting of pregnancy, and even of ulceration and of cancer.

In the apepsia of infants and children it has special value, relieving pain, flatulence, and diarrhoea, and greatly promoting their nutrition.

PREPARATION AND DOSE.--There is only one officinal preparation prepared from the pig by Beale's method: dose, 2 to $5 \mathrm{gr}$. It is uncertain in its action.

Of other forms of the drug, some are solid and some liquid. Pepsina ammlacen ("poudre nutritive"): dose, 5 to $15 \mathrm{gr}$. Pepsine surcherreta (U.S.P.) : dose, 5 to 15 gr. Lactopeptin and maltopepsin (containing also pancreatin): dose, 10 to $15 \mathrm{gr}$. Of the fluid forms, Benger's liquor pepticus is one of the best; it is a solution in weak alcohol: dose, 1 to $2 \mathrm{dr}$. Bullock's ghycerinum pepsince ucidum, and the "saline essernee" of Savory and Moore, are both effective: dose, 1 to 2 dr. Liebreich's "pepsin essenz" is good, also Morson's vimum pepsine": dose, 1 to 2 dr. Fairchild's peptonizing powders are a monvenient solid form, sent out in separate glass tubes containing small quantities. 


\section{PANCREATIN-TRYPSIN.}

\section{(Not Officinal.)}

DESCRIPTION.-The proteolytic ferment formed in the pancreas.

PREPARATION.-This may be carried out by several processes. One of the best is that of extracting "perfectly fresh finely-chopped pancreas with four times the weight of 'dilute' spirit" (Benger), which gives a nearly colourless solution with little taste or smell except that of the spirit.

Character and Tests.-Pure pancreatin has not been separated with the same care as pepsin, the preparations in medical use being rather cumplex, and containing several of the other active principles of the pancreatic juice. They have been tested by their powers to emulsify a definite amount of fat: thus, washed fresh butter, half an ounce, shaken for from one to three hours at $100^{\circ} \mathrm{F}$. with half an ounce of water, was completely emulsified by about 1 fluid drachm of "saline essence of pancreatin" (Savory), and also by about the same quantity of the "liquor pancreaticus" (Benger); of pancreatin (Savory) 45 grains were required to produce a similar result,-of another kind, three times that quantity (F. A. Lees, On Digestive Ferments, B. M. J., i., 1880).

Physiological Action.-In the pancreatic secretion there are recognized four "hydrolytio" ferments:-

1. A diastatic ferment, amylopsin, apparently identical with ptyalin, but acting more energetically on raw as well as boiled starch, which it changes into dextrins and grape-sugar. It has a similar action on glycogen.

2. Pancreatin (Corvisart) or trypsin (Kühne). This acts on proteids in alkaline solutions, changing them first into propeptones, and then into true peptones, sometimes called tryptones. If the action is continued beyond this, leucin, then tyrosin and 
other bodies are formed, and at a still later stage, when decomposition occurs, as it is very apt to do, indol is formed, giving an offensive frecal odour, as well as skatol, phenol, and volatile fatty acids.

3. A third ferment, called sterpsin, aids the digestion of fats, chiefly by producing a fine emulsion, but also by causing them to take up a molecule of water, and divide into glycerine and fatty acids, which are saponified by the alkali of the intestinal secretions. This is much more active when artificially prepared four to ten hours after a meal than it is when prepared from a fasting animal.

4. A milk-curdling ferment may also be obtained by extraction with a strong solution of salt (Roberts).

According to the success of chemical processes in obtaining an extract containing these different constituents will of course be the digestive action obtained; but as a rule, what is expected from such extracts as we possess is assistance in the digestion of fat and casein. Milk, for instance, is better digested by pancreatin (or trypsin), whilst egg-albumen is more thoroughly acted on by pepsin. Dr. W. Roberts has shown this by carefully conducted experiments (Lumleian Lectures, B. M. J., i., 1880). Milk subjected, at $120^{\circ} \mathrm{F}$., to the action of pancreatic extract will be seen to become softly curdled; part of the curd quickly dissolves, while part is resistant. The milk soon after loses its glossy white appearance, becoming yellowish grey, and develops a bitter flavour, which is not unpleasant unless the process is allowed to go on to decomposition. At the end of from two to three hours all the casein is converted into peptone, as may be proved by testing with acetic acid. The curtling phase is hindered or altngether prevented by diluting the milk; one quarter of its bulk of water is quite sufficient (Roberts, op. cit.). Pancreatic digestion is much hampered, but not quite prevented, by a slight degree of acidity.

Therapeutical Action.- What has been said under the heading of "Pepsin" applies to pancreatin, and to food prepared with it. These are of much value to delicate or convalescent invalids, securing the assimilation of more nourishment than they could otherwise take. 
Wasting Disease.-In mesenteric and other forms of wasting disease connected with mal-assimilation of fat, Mr. Lees strongly commends an improvised "koumiss," made with milk boiled for a minute or two, and then vigorously shaken with a drachm of saline essences, pepsin and pancreatin, and drunk whilst still frothy.

In the vomiting of gastric irritability, such as occurs in the course of serious illness and in delicate hysterical subjects, also in the vomiting of pregnancy, pancreatin, or pepsin, given with Brand's or other meat extracts, or with koumiss, is often useful. In the vomiting and diarrhoea of infants from mal-digestion of milk, and even when due to organic disease, similar treatment may be of the utmost value.

Dyspepsia.-In judging which of the many forms of this disease are best treated by peptonized food, we should endeavour to distinguish whether the digestion of starch, or fat, or of proteids is most at fault, and choose our remedy accordingly, viz., diastase (maltine), pancreatin, or pepsin.

The administration of pancreatin in any form as a drug is of little use, since the proteolytic and amylolytic ferments are destroyed by the average acidity $(0 \cdot 17$ to $0 \cdot 2$ per cent. hydric chloride) of the stomach. Hence it is best, in cases of dyspepsia and atony of the stomach occurring in fevers and wasting diseases, to use foods peptonized by an extract of paucreas.

If this extract does not contain an active amylolytic ferment, maltine or a watery extract of malt may be added to it to digest the starches more completely. If these remarks be borne in mind, the uses of extract of pancreas as a dietetic remedy are obvious and simple. A word may be said as to the value of peptones themselves. The object of giving digested food is to relieve the stomach of work in some cases, and in others to supply the products which that organ in its enfeebled state is unable to produce. These products are peptones, which, when given as food, have been shown by the experiments of Plósz and Maly to support life and increase the body weight. Peptones may, therefore, in some cases be more valuable than peptonized food. The latter is more palatable, and is suitable for cases in which the stomach can retain food: but when there is constant vomiting on the introduction of food, 
peptones are with advantage added to an enema of peptonized milk or beef-tea and brandy.

PRePaRations AND Dose.-Pancreatin: dose, 2 to 4 gr. Liquor pancreaticus: dose, 1 to $2 \mathrm{dr}$. in water with meals. It contains trypsin, amylopsin, and the curdling ferment. It is beneficially given with bicarbonate of soda.

In the preparation of peptonized food, the directions given by Dr. Roberts are very useful (B. M. J., i., 1880).

The liquor pancreaticus may be poured over a meal half an hour before it is eaten; even in so short a time it renders meat soft, and begins the digestion of amyloids; 1 or 2 drachms is a sufficient quantity: gruel and puddings may also be made in a palatable form (Roberts, Lumleian Lectures).

Pancreatized Mill.-One pint of mills is diluted with $\frac{1}{4}$ pint of water, and heated to about $120^{\circ} \mathrm{F}$; 1 to $3 \mathrm{dr}$. of liquor pancreaticus is then added, with 20 gr. of bicarbonate of soda; the mixture being covered with a "cosey," is kept in a warm place for some time, till, in fact, the curds have nearly all dissolved, or till the bitter taste is well developed. The mixture is then boiled, and may be given by the stomach or rectum.

\section{AJEPS PRAEPARATUS. (Prepared Lard.)}

MODE OF PREPARATION.-The internal fat of the abdomen of the hog, Sus scrofa, is purified by washing with water, and melting at $130^{\circ} \mathrm{F}$, and finally straining through flannel.

DESCRIPTION.-A soft white substance, melting at $100^{\circ} \mathrm{F}$., entircly soluble in ether. It consists of olein, with palnitin and stearin.

USES.-It is of value as an inunction in various forms of mal-nutrition, and also during and after contagious disorders, especially scarlatina. It may be used for dressing blisters, bourns, ete. 
PREPARATIONS.-Lard is an ingredient of adeps benzoatus, emplastrum cantharidis ; unguenta iodi, terebinthinæ, hydrargyri nitratis.

\section{ADEPS BENZOATUS.}

\section{(Benzoated Lard.)}

Contains 1 part of benzoin in 50 of prepared lard.

PREPARATIONS. - The following unguenta:-Aconitina, atropinæ, belladonnæ, calaminæ, chrysarobini, gallæ, hydrargyri subchloridi, iodoformi, plumbi acetatis, potassii iodidi, sabinæe, staphisagriæ, sulphuris, zinei.

\section{$C E T A C E A$.}

\section{CETACEUM. (Spermaceti.)}

DESCRIPTION.-A white spongy substance obtained from cavities in the head of the Physeter macrocephalus, or sperm whale, in which cavities it exists as an oil during the life of the animal, concreting after its death.

CHARACTERS AND TESTS. - When purified by washing, straining, etc., spermaceti occurs in white, pearly, semi-transparent masses, of crystalline foliaceous texture, friable, soft, somewhat unctuous, slightly odorous, insipid; fusible at $112^{\circ}$ to $120^{\circ} \mathrm{F}$., according to purity, volatilized at a bigher temperature, inflammable; insoluble in water, soluble in chloroform and fixed oils, only slightly so in boiling alcohol, ether, 
or turpentine. Pure spermaceti is a palmitate of cetyl, $\mathrm{C}_{16} \mathrm{H}_{33}, \mathrm{C}_{16} \mathrm{H}_{31} \mathrm{O}_{2}$ : when saponified, a monatomic compound, cetylic alcohol, $\left.{ }_{16}^{\mathrm{C}_{16}} \mathrm{H}_{33}\right\} \mathrm{O}$ is formed.

USES.-As a local emollient and demulcent for irritated surfaces and mucous membranes. It was formerly given internally, especially in pulmonary and intestinal disorders, for the same purpose as other oils or mucilage, but it is now seldom used except for external application.

PREPARATION.- Unguentum charta epispastica.-It may be powdered by addition of a little alcohol or almond oil, or suspended in water by means of mucilage.

\section{A VES.}

\section{OVI ALBUMEN-OVI VITELLUS.}

The white and the yolk respectively of the egg of Gallus domesticus.

COMPOSITION.-White of egg consists chiefly of albumen, with some globulin. Yolk of egg contains a globulin called vitellin, and fatty bodies.

THERapeutical Action.-White of egg is a useful antidote in poisoning by corrosive sublimate and copper sulphate, with both of which it forms insoluble compounds. The yolk, when beaten up in "egg flip," is a good nutriment for invalids.

PREPARATION.-Yolk of egg is contnined in spiritus vini Gallici. 


\section{N G L U V I N}

\section{(Not Officinal)}

Is the name given to a preparation from the gizzard of fowls. It is curious that although this contains no true peptic glands, and theory has consequently considered the preparation inert as a peptic remedy, yet practically it has always had some repute, and the modern preparation, which is in the form of a yellowishgrey powder, is unquestionably efficient in the treatment of many kinds of vomiting. My experience of it has been partly in the obstinate vomiting of hand-fed infants, which has often yielded to 1-grain doses after many other drugs had failed, and partly in ordinary conditions of nausea and vomiting attendant on dyspepsia in adults; and the recorded experience of others is much in favour of its value in the vomiting of pregnancy. The dose for adults is given at from 3 to $10 \mathrm{gr}$.

\section{PISCES.}

\section{OLEUM MORRHU $V$ EEL JECORIS ASELLI.}

\section{(Cod-Liver Orl.)}

DESCRIPTION.-A fixed oil obtained from the fresh liver of the cod, Gadus morrhua (formerly called Asellus major), met with chiefly upon the North Atlantic coasts in Europe and America.

PREPARATION.-The cleansed livers, mixed often with those of other fish, are either exposed to sun-heat, and the exuding oil filtered, or they are heated in a steam or water 
bath, or sometimes boiled with water and the oil skimmed from the surface: $28 \mathrm{lbs}$. of the livers yield $12 \mathrm{lbs}$. oil, sp. gr. 0.917 to 0.920 .

CHARACTERS AND TESTS. -- According to the process used, but especially according to the length of time during which the livers are left before being treated, the resulting oil is pale yellow or brown, and either of slightly fishy taste and smell, or of very strong, and even rancid, odour. The oil now preferred is carefully kept free from such products, and is of pale yellow colour, and faint acid reaction. Sulphuric acid added on a white slab gives a violet colour, changing to a dull, reddish-brown, showing the presence of biliary compounds, though not distinguishing from other fish-liver oils.

ACTIVE INGREDIENTS. - A special principle, termed gaduin, may be obtained by a chemical process as a dark brown, brittle, tasteless, inodorous body, soluble in sulphuric acid, and giving a red-blood colour to the solution re-precipitated by water; it is probably a derivative of bile (Stillé). Cod-liver oil resembles other oils in containing triolein (about 7 per cent.), palmitin, and stearin in varying proportions; it has traces of other biliary matters besides gaduin, as well as of iodine $(0.05$ per cent.), bromine, phosphorus, and various acids and alkalies; distilled with ammonia it yields propylamin.

ABSORPTION AND ELIMINATION.-One reason why this oil is more serviceable than others for mutrition is the greater facility with which it is absorbed, a fact which is plausibly connected with the presence of biliary principles, for it has been demonstrated that capillary attraction, and also osmosis through membranes, is much more active for oil containing bile than without it, and if, for instance, two loops of intestine be tied in a living animal, and one filled with cod-liver oil, the other with olive oil, and both returned into the abdomen, from the former, after a given time, much more will be absorbed than from the latter (Nammann). Cod-liver oil has been found also to be more readily oxidized than other oils as tested by permanganate of potnsh. Absorption oecurs, 
as with other fats, chiefly from the small intestine after formation of an emulsion with the pancreatic and hepatic secretions, and such excess of oil as cannot be taken up, passes out unchanged by the bowel.

Physiological ACtion.-This is the same in kind as that of other fats, for cod-liver oil is rather a food than a medicine in the ordinary sense. It improves nutrition and blood-formation, and in suitable dose, the powers of digestion, whilst it provides combustible material for muscular effort, thereby lessening ordinary wear and tear, and increasing the deposition of fat and the body weight. Excessive amounts of it are said to have induced a condition of plethora, with tendency to congestion and hrmorrhage. The improvement produced depends upon its satisfactory absorption, for in some persons it disturbs rather than benefits digestion, and readily causes nausea and diarrhœa. The effect on the blood in cases where it agrees has been definitely determined to be an increase in the amount of contained solids, of the red corpuscles, and of albumen, with lessening of fibrin (Simon).

Its results in disease being, however, not wholly explained by such action on 'nutrition, cod-liver oil is further credited with some undefined "alterative" power.

THERAPEUTICAL ACTION.-Extemal.-Wasting Diseases (Marasmus).-The exhibition of cod-liver oil by friction is often very effective in this extensive class of disorder, where the existence of digestive trouble, such as sickness or diarrhoo, precludes its internal use. Especially in the mal-nutrition of puny children, one or two drachms rubbed well into the abdomen, or under the arms, twice daily, will help to nourish and strengthen; the umpleasant smell may be obviated by scented oils and daily washing:

\section{ThERAPEUTICAL ACTION.-Intemal.-Chronic Rheu-} matism, etc.-The use of cod-liver oil in this disorder preceded its introduction as a remedy for phthisis, and its value is especially seen, according to Stillé, in cachectic subjects of scrofulous constitution who suffer with stiffness of joints and 
muscles, anæmia, and other consequences of damp and crowded dwellings, as well as from rheumatic pains. It is of service also in the cachexia of later stages of syphilis.

Chest Disorder.-It is especially in tuberculosis that the oil proves of such great value, not by specifie action on the morbid growth, but by sustaining the general nutrition whilst climatic and other remedies are used to act more directly on the malady. It is well suited for early, so-called "pre-tubercular" stages with impaired general health; also when limited local disease is present; and again later when eavities have formed and emaciation is rapidly progressing.

We are largely indebted to Dr. Hughes Bennett for demonstrating this. Amongst other cases he has carefully recorded the progress of one from the age of fifteen to that of nineteen. Admitted into Edinburgh Infirmary with all the symptoms of the last stage of phthisis, he began with half-ounce doses of the oil thrice daily. With the addition of sedatives he soon improved; relapsed again on omission of the oil; improved again on resuming it, and finally recovered good health with evidence of cicatrization of cavities. "The pulmonary signs varied according to his ability of digesting the oil." Its effects in phthisis are to nourish the body, to check fresh exudations of tubercular inatter, and to diminish the cough, expectoration, and perspiration (Principles of Medicine, 3rd edit.).

Dr. Bennett, however, reports many advanced cases of a different type, with more marked dyspepsia, in which the oil, as well as other remedies, failed to give more than temporary relief; and in highly pyrexial states, or when much gastrointestinal disorder is present, it is of course unsuitable.

In Chronic Bronchitis with profuse secretion, debility, and anæmia, much advantage may be gained from it, especially in promoting expectoration, or indusing the formation of healthier cells and secretions from mucous membrane by supplying a readily assimilable nutritive material (Brunton). In the later stages of protuswis it is of great service, and ngain in the convalesume from an acute bronchial marth.

Scrofulosis.- In all manifestations of this state, the oil is particularly valuable-in caries and chronic arthritis, in glandular enlargements, especially when internal or when suppurating. In 
strumous ulceration, even in lupus, it has sometimes given excellent results, and should always be tried.

In Rachitis, alone or combined with iron or iodide, it is also of the greatest service.

In Chronic Skin Disorders, associated with mal-nutrition, such as eczema and psoriasis, it is a marked adjuvant to cure, and is of some use in inveterate maladies like ichthyosis.

In cases of intestinal worms it may be advantageously given to relieve irritability, ravenous appetite, etc., as well as for the emaciation.

Neurasthenia.-In cases of this class, whether associated with neuralgic pain, ataxia, hysteria, or even cerebral softening, I have often seen much benefit from cod-liver oil.

To sum up, cod-liver oil is of benefit in all cases of defective nutrition, in convalescence after acute specific or other fevers, and in chronic wasting due to whatever cause; it acts not only as a food, but as a tonic.

DosE.-For children under five years, $\frac{1}{2} \mathrm{dr}$. night and morning is sufficient to commence with; under ten years, $1 \mathrm{dr}$. For adults, $1 \mathrm{dr}$. three times a day, the dose being gradually increased. It is not, as a rule, beneficial to give a larger dose than $1 \frac{1}{2} \mathrm{oz}$. in the day. The oil may be given by itself or in emulsion, many forms of which are now prepared, as with hypophosphates of lime, ete. For children the combination with

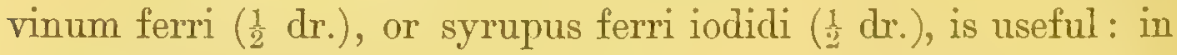
some cases also glycerine $(20 \mathrm{~min}$. to $1 \mathrm{dr}$.) may be added. The best emulsion for adults is made with almond cake. If the oil disagrees at first, $1 \mathrm{dr}$. taken just before going to bed will often establish tolerance. 


\title{
I N V E R T E B R A T A.
}

\author{
INSECTA. \\ HYMENOPTERA.
}

\section{MEL. (HoNEY.)}

DESCRIPTION.-A saccharine secretion deposited in the honeycomb by the hive bee, 'Apis mellifica. It is a viscid, translucent, aromatic liquid, of light yellow colour, which on exposure gradually becomes dark and opaque; it has a peculiar heavy odour. It consists of three different sugars, all derived from the decomposition of cane-sugar, viz., crystallizable canesugar (sucrose) ; grape-sugar (dextrose or glucose), also crystallizable; and inverted sugar, uncrystallizable (a combination of glucose and levulose).

Honey is used chiefly as a vehicle.

PREPARATIONs.-Mel depuratum, which is contained in confectiones piperis, scammonii, and terebinthinæ; mel boracis, oxymel, oxymel scillæ.

Adulterations.-Starch, grape sugar, common salt, etc.

\section{CERA ALBA. (White Wax.) \\ CERA FLAVA. (Yrilow WAX.)}

DESCRIPTION.- Yellow wax is the arule melted honeycomb, after separation of the honey; when hleached it forms white wax. Yellow wax melts at about $140^{\circ} \mathrm{F}$., and white at about $150^{\circ} \mathrm{F}$. Sp. gr. $0 \cdot 960$ to $0 \cdot 965$. It consists of myricine $\left(\mathrm{C}_{45} \mathrm{H}_{32} \mathrm{O}_{2}\right)$, cerotic acid $\left(\mathrm{C}_{27} \mathrm{H}_{54} \mathrm{O}_{22}\right)$, and cerolein, which usually constitutes about 5 per cent. of it; but all three exist in varying proportions. 
PREPARATIONS. - White wax is contained in unguenta cetacei, simplex, and charta epispastica. Yellow wax (bleached) is contained in cera alba; emplastra calefaciens, cantharidis, galbani, picis, saponis fuscum; pilula phosphori; unguenta cantharidis, hydrargyri compositum, picis liquidæ, resinæ, sabinæ, terebinthinæ.

\section{HEMIPTERA.}

\section{COCCUS CACTI. (Cochineal.)}

DESCRIPTION.--This insect is found wild in Mexico and Central America, where also it is cultivated largely for commerce, and from whence it has been imported into the Canary Islands, Spain, Algiers, etc. After fecundation the females attach themselves to the leaves of the Opuntia cochinillifera, on which they feed, and are brushed off and killed by immersing them in hot water, and afterwards drying them in the sun or by the heat of a stove; they are collected in successive crops at stated times.

Characters AND TESTS. - Two principal varieties are found in commerce, known as "silver" and "black grains," the former being reddish grey, almost covered with a white powder: the difference in colour is due either to a different mode of drying, or to the fact that the egg's have been laid by the black variety, and not by the grey. Both kinds may be described as about $\frac{1}{5}$ inch long, oval, conenve, and flat below, convex above, transversely wrinkled and easily reduced to a dark-red powder. The odour is faint, but heavy, and the taste bitter, slightly acid.

ACTIVE INGREDIENTS.-The colour is due to carminic acid, $\mathrm{C}_{14} \mathrm{H}_{14} \mathrm{O}_{3}$, and is intensified by acids, darkened by alkalies. When precipitated by various reagents it is known as carmine or lake. It contains also tyrosin, stearin, and various animal compounds and salts. 
USES.-Mainly as a colouring agent, 5 minims of the tincture reddening an ounce of liquid. It has been supposed to be anodyne and serviceable in neuralgia and in pertussis, for which disorder it has been generally given with potash.

PREPARATION AND Dose.-Tinctura ( 1 in 8$)$ : dose, 30 to $90 \mathrm{~min}$. It is contained in tinct. cardamomi co. and tinct. cinchonæ co.

Adulterations.- Varied and numerons.

\section{COLEOPTERA.}

\section{A N TH A R I E S.}

\section{(Spanish Fimies.)}

DESCRIPTION.-The Cantharis, or Lytta vesicatoria, is a beetle indigenous to Southern and Central Europe, and is commonly collected in Hungary and the warmer parts of Russia. It is $\frac{1}{2}$ to 1 inch long, $\frac{1}{8}$ to $\frac{1}{4}$ inch broad, of a shining coppergreen colour, with filiform antennæ, and a wing-case which has two longitudinal ridges, covering the body: the tarsi of the hinder legs have four, the others five joints: the odour is strong and disagreeable.

The "flies" are collected in the early morning (when more torpid) by shaking them from the trees and shrubs they frequent (lilac, elder, ash, and honeysuckle); they are received in eloths, and killed by boiling water or vinegar, or by the vapour of ether or turpentine. Several other species have similar properties, and have been used especially in America. Varieties of Mylabris are also imported as "Chinese blistering" flies:" they have two orange-coloured bands, and spots on the wing-cases.

The finely powdered cantharis is of 'a greyish-brown colour, with minute shining green particles in it, which are the 
fragments of the feet, head, and wing-cases. If kept from air and perfectly dry, they preserve their vesicating properties for years; but if exposed to damp air, they quickly putrefy.

ACTIVE INGREDIENTS. - Besides acetic and uric acids, fatty and other animal matters, a crystalline substance, cantharidin- $\mathrm{C}_{10} \mathrm{H}_{12} \mathrm{O}_{4}$ or $2\left(\mathrm{C}_{10} \mathrm{H}_{12} \mathrm{O}_{4}\right)$, - is obtained in colourless prisms and scales, by exhausting the powdered beetles witis acetic acid or chloroform. It is soluble in these agents and in warm alcohol, ether, and fixed and volatile oils; partially in cold alcohol; not in pure water, but to some extent when combined with a yellow material in the insects : it may be obtained in colourless prisms and scales. In combination with the elements of water it acts as an acid, cantharidic acid- $-\mathrm{C}_{10} \mathrm{H}_{14} \mathrm{O}_{5}$ or $2\left(\mathrm{C}_{10} \mathrm{H}_{14} \mathrm{O}_{5}\right)$, - and with bases, forms salts which are, however, readily decomposed by other acids. Cantharidin begins to sublime at $185^{\circ} \mathrm{F}$., fuses and sublimes more freely at $410^{\circ} \mathrm{F}$.: it is about fifty times stronger than cantharides.

Cantharides contain also a green oil, non-vesicating, and a volatile, oily, vesicating principle which has the characteristic odour of the insect, sharp, foetid, and nauseating.

ABSORPTION AND ELIMINATION.-That the active principle is readily absorbed by the different tissues is sufficiently proved by the effects, though chemistry has failed to trace the exact process, or the changes, if any, undergone. If applied to the skin, absorption is probably delayed during the stage of acute inflammation, but afterwards the effused serum, charged with the drug, is readily absorbed and produces remote effects. After being introduced into the blood of animals, it has been found in almost all the organs, but especially in the liver, kidneys, salivary glands, and the grey matter of the brain (Liakhnitzky, Dissert. St. Petersburg, 1884). It is chiefly eliminated by the salivary glands, the kidney, the intestine, and other mucous membranes. Cornil found evidence of commencing renal inflammation in rabbits, thirty minutes after the injection of a minute quantity of cantharidin into the areolar tissue (Union Méd., 1881). Cantharidate of soda acts quite in the same manner. 
PHysiological Action.-Extemal.-The main characteristic is that of stimulating or irritating more or less severely; not, like certain caustics, by combining with the water or the albumen of the tissue, but by a direct irritant effect which induces dilatation of blood-vessels, ending in inflammation. A minute portion of cantharidin applied to a delicate part, such as the lip, will cause at once redness, burning pain, and, in about a quarter of an hour, vesication; a small quantity of a good solution of the active principle painted on the ordinary skin will produce, in a few seconds, pain, temporary pallor, followed by vivid redness, and in about half an hour a moderate effusion of serum under the cuticle; the powdered insect, or an ointment containing the same, or the ordinary "emplastrum lyttæ," will produce similar effects in from six to eight or more hours: so that the result differs only in degree and in time, whatever preparation be used, or whatever the place of application.

Blood-ressels, not only of the vesicated part, but also of its neighbourhood, dilate, so that, in proportion to the size of a blister and the amount of effusion, the quantity of blood in other parts may be lessened: the size being, however, of necessity, limited by the pain and the risk of general effects, the change in the circulation does not account for all the effects produced, and we connect them rather with a stimulating action on peripheral nerves : this, as ascertained by experiment, may produce reflex contraction of small vessels at a distance (e.g., in the kidneys, after the application of cantharides to the lumbar region), and is also a probable explanation of the increased heart-action, body-temperature, and general nerve-imitability which follow blistering in some individuals, though part of these effects may be due to the absorbed drug.

It is to be further remarked that, after excessive external use of cantharides, the affected part may become gangrenous, and the contraction of distant capillaries is apt to be followed by a paretic distension, and consequent impaired nutrition and lowered trophic change; we have, indeed, a clinical illustration of this in the occurrence of duodenal ulcer after a severe burn of the surface. The effusion within a blister contrins serum and fibrin, and recurs more or less between each dressing for the first few 
days; a coagulum forms in it, and coats the surface with an adherent film, under which it heals.

\section{PHysiological ACTiON.-Intemal.-Digestive System.} - Small doses ( 1 to 2 minims) of tincture may be taken without evident gastric symptoms, but any excess causes burning at the epigastrium, followed by acute pain, nausea, salivation, a sense of constriction in the throat, vomiting, and dysenteric diarrhœa. Death may ensue from gastrointestinal inflammation, which occurs also after intravenous injection, cantharidin being excreted by the intestinal membrane (Liakhnitzky).

Nervous System.-Contrary to what might be thought, the drug is not a stimulant to the general nervous system; indeed, some writers (Italians chiefly) describe it as a powerful sedative. Certainly, whatever temporary condition of excitement may occur is quickly followed by prostration, which, under toxic doses, soon passes into collapse and coma. Tardieu has recorded cases with severe hydrophobic delirium and convulsion.

Observations in warm-blooded animals show, as a rule, no change in excitability of muscles, or of motor or sensory nerves ; but in the cold-blooded there is a loss of reflexes, probably from a lesion of the reflex centres in the cord. The temperature oscillates, with a tendency to depression. The pupil is dilated under the full action of the drug.

Circulatory System.-The full, and especially the toxic action of the drug is accompanied by a marked increase of pulse-rate, which has been traced to paralysis of the vagus. The arterial tension, if not much affected at first, is decidedly lowered in later stages, and the pulsations become feebler from weakness of the cardiac muscle. Cornil formd evidence, after toxic doses, of congestion of the lining membrane of the bloodvessels, especially in the liver. Respiration is first quickened, and afterwards slowed, probably from weakening of the respiratory centre: in cold-blooded animals it is slowed from the commencement.

Genito-Urinary System.-Minute doses (1 to 1 or 2 drops) of tincture stimulate this system: larger quantities are npt to set up irritation, as shown by burning pains in micturition, 
which becomes more frequent than usual; there may also be early pain in the back. If the doses have been large or continued, the irritation increases to an intense degree, with constant straining to micturate, without being able to pass more than a few drops of urine, which is commonly albuminous or bloody. Swelling, severe pain, and inflammation, even gangrene, of the genital organs have sometimes resulted. There is evidence that by small or moderate doses, r.g., up to 10 minims of tincture, especially of such preparations as contain the essential oil, sexual desire may be stimulated, and this seems a natural consequence of the afflux of blood to the affected parts: it is, however, far from constant, and although in some toxic cases it has shown itself in a most violent manner, with erection, ete., in others it has been wholly wanting, and is indeed incompatible with severe inflammation. In women, abortion has followed-in one case from 24 grains in two doses (Stillé). In animals the gastrointestinal tract is more affected than the genital system, of which there is little, if any, evident irritation. Post mortem, in all cases there are signs of inflammation of this tract, and also of the kidneys and urinary passages. Shakhora stated that the convoluted tubes were most affected, and the glomeruli but slightly; but Cornil, after careful examination, came to an opposite conclusion (op. cit.), which has since been confirmed by Eliasheva (1883). It is curious that the urine may be rendered neutral or alkaline by free blistering of the skin-a. rondition which has been traced to some reflex alteration in the tissue-changes.

SYNERGISTS. - Vesicants and rubefacients. Iron assists a stimulant action on the mucous membranes, especially of the genito-urinary tract.

ANTAGONISTS. - The alkalies sometimes recommender as antidotes are not really so, sinee their compounds with the active principle are as irritant as the latter itself. Camphor, though undervalued in ordinary text-bonks, seems to have a definite antidotal power in relieving the urinary irritation (as pointed out by (troenvelt in 170.5); and in 
toxic cases, after evacuation of the stomach, it should be freely given with albuminous and mucilaginous drinks and opium.

Camphor powdered over a blister will not prevent its irritant effects.

THERAPEUTICAL ACTION.-External.-Counter-irritation by means of cantharides more or less thoroughly applied, though not in such frequent use as formerly, is still often valuable. The effect desired may be either stimulant (locally or generally) or anodyne.

Collapse-Coma.-In such conditions as occur in severe specific fevers, prolonged shock, post-epileptic coma, poisoning by opium, snake-venom, etc., free blistering of the nape, the epigastrium, or the extremities may be valuable as a general stimulant; care is required not to blister so deeply as to cause ulceration.

Epilepsy. - There is evidence that interruption of the path in which the "aura" is travelling may prevent the full development of a paroxysm, and also that such interruption may be effected by a blister applied between the periphery and the centre. This mode of treatment has therefore been recommended.

Neuralgia. - A small-sized superficial blister is a potent means of relief in most forms of neuralgic pain in any part of the body. If deep-seated, the application may be made at the nearest available point; if superficial-.$g$. , at any point of an anterior spinal nerve-it is better made over its posterior root (Anstie).

A "flying blister" - i.e., one allowed to quickly heal, and then followed by another one close by-is, perhaps, the preferable form of application. In intercostal neuralgia and in sciatica this plan of treatment often relieves. The blisters may, of course, be dressed with morphia or other sedative ointments. In facial paralysis, after the first few days, vesication in front of the mastoid is serviceable; and in various forms of hysterical paralysis-of which the most common, perhaps, is that affecting the vocal cords and causing aphonia-resication near the affected parts often acts well. 
Inflammation.-As a rule, blistering is inadmissible during an acute pyrexial state, and though some have advised it early both in pneumonia and pleurisy, I think it better reserved for later stages, e.g., in the former malady when resolution is progressing but slowly, and dulness is persistent.

In later stages of bronchitis, when there is difficulty in expectorating, a copious secretion, tendency to basic congestion, with blueness of lips, dyspnœa, etc., I have seen a blister between the scapulæ of the greatest service.

In hepatitis, local vesication is admissible and effective; but in meningitis it is a more doubtful practice, and is now scarcely ever adopted on an extensive scale, such as over the scalp or nape. In local inflammations affecting the eye or ear, small blisters in the neighbourhood are, however, in daily use. In chronic inflammatory disorder and its results, as seen, for instance, in ovaritis, glandular enlargements, and periostitis, blisters are very satisfactory. Gleet, and similar chronie discharges, resulting from old inflammation of mucous membranes, are also successfully treated by external blistering.

In peritonitis it is scarcely a safe method, for extension of the inflammation of the membrane has been traced to it, and the kidneys are more liable to be injuriously affected.

Pulmonary Disorders.-Besides the inflammations of the lung mentioned, phthisis should be named as a malndy in which occasional blistering, over small portions at a time, of the affected area often greatly relieves pain, discomfort, and dyspnoca; in cmphysema and asthma, the last named symptom is sometimes amenable to the same treatment: plouritic and other serous effusions, especially in the pericardium and the larger joints, may be sometimes got rid of by a few blisters, but it is a somewhat painful mode of treatment, and I have often known it fail in these disorders.

Rheumatism.- The treatment of an acute attack by blisters - preferably circular ones, applied as near as possible to each of the affected joints - has been formulated into a system by $\mathrm{Dr}_{\mathrm{r}}$. H. Davies, who has reported a number of cases showing excellent results in the relief of pain and shortening of the disease. The theory is, that the drug acts partly as an elimiuant, partly as 
a stimulus to the absorbents, through the nerve-endings; but the practice is painful, sometimes extremely so; and on review of a lirge number of cases, it is evident that equally good results lave been obtained by other remedies with less pain to the patient, and consequently this treatment is now comparatively seldom used.

The same remark applies to the treatment of gout, though the occasional blistering of a large or painful joint is quite admissible.

Skin Disease.-In crythematous lupus, when the true skin is much infiltrated, I have seen benefit from vesication induced by cantharides. In chronic hypertrophic eczematous conditions of the hands, with painful fissures (ecrema rimosum), the effect of painting with liquor vesicatorius is often extremely good in relieving itching, etc.: chronic scaly patches of psoriasis may be stimulated in the same way. But perhaps the most frequent use of cantharides to the skin is in the form of lotion or ointment for stimulating the growth of hair; it is thus of use in alopecia areata.

\section{THERAPEUTICAL ACTION.--Intern. - Renal Disorders.} - The marked determination of cantharides to the kidney may be safely utilized in several of these disorders by giving small doses, which act either by stimulating an engorged circulation, or by "substitutive irritation." Ringer and a few other modern writers adrocate such treatment, even on the fifth or eighth day of an acute attack of Bright's disease, when the wine still contains blood.

It is, however, in more chronic congestive conditions of the kidney, and especially in pyelitis caused by calculus or local irritation, that the best results may be obtained. This was well shown by Groenvelt in his book "On the Safe Internal Use of Cantharides," published in the early part of last century, at a time when the drug was held so dangerous a poison that he was imprisoned for advocating its use. Some of his cases, howerer, are still worth perusal; and since that time his treatment has been occasionally, though by no means generally, adopted.

Dr. Mackey has recorded two cases, both in adult women, 
and presenting urgent symptoms of pain, very frequent micturition, discharge of quantities of pus and epithelium continuing for several years, and with occasional paroxysms of febrile exacerbation and partial suppression, yet markedly relieved by the use of from 2 to 20 minims of tincture of cantharides. Both patients ultimately died; and post mortem, in one, the kidneys were found large and full of cavities secreting pus; in the other they contained also calculi (Med. Times, i., 1869). It was remarkable that, with such conditions, so much relief could be given by anything.

In diabetes insipidus the drug has been well spoken of, but I have no personal experience of its effects.

Vesical Disorder.-The class of cases in which it is serviceable come under the general description of "irritability of bladder," probably from weakness of the sphincter. The main symptom is undue frequency of micturition, often either some incontinence at night, or dribbling during the day, especially on slight exertion, such as coughing: these conditions occur more frequently in children or women (not including. cases dependent on uterine disorder), but also in elderly men from enlargement of the prostate or some vesical catarrl. In any case, 2 to 5 minims of the tincture, several times repented, often relieve the symptoms. The urethrul cases amenable to the same treatment are those associated with longstanding mucous or muco-purulent discharge-such as gleet and prostatorrhoca, -in which conditions it is commonly associated with iron: the same combination has been given in impotence. Ringer recommends small doses for chordee.

Uterine Disorders. - Cantharides is sometimes given to stimulate a uterine flow when deficient, in weakly relaxed subjects; and, on the other hand, it has been recommenderl in the excessive losses that often necur in similn lax-filned patients.

There are certain forms of skin disertse, especially those that are dry and scaly, in which the tincture has been sometimes advantageously given, and it has had a less-deserved repute

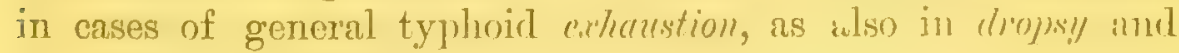
in chronic brouchitis. 
CONTRA-INDICATIONS, besides those of inflammation, or high arterial excitement, are conditions of great debility such as occur in old age or after exhausting illness; of extreme sensitiveness, such as in childhood and in highly neurotic subjects, and in pregnancy; and of superficial hrmorrhage, such as purpura: also in kidney disease, especially if chronic, the llug must be used with due care.

PREPARATIONS AND DOSE.-Acetum cantharidis (1 in 10). Charta epispastica. Emplastrum cantharidis (1 in 3). Emplastmum

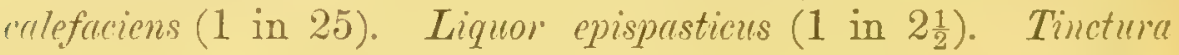
cuntharidis ( 1 in 80); dose, 1 to $20 \mathrm{~min}$. Unguentum ( 1 in 7 ).

The liquor vesicatorius (Bullen and Burt) is a reliable form.

\author{
ANIELIDA.
}

\title{
HIRUDO-SANGUISUGA. (The: Leech.)
}

DESCRIPTION.-An aquatic, hermaphrodite worm with roundish flattened wrinkled body, ' 2 to 4 inches long, tapering towards each end, which is furnished with a flat circular disc, the hinder one being the larger. When out of water, these lises serve as organs of progression. The anterior sucker has " triangular month with three curved teeth edged with upwards of sixty-fire serrations.

The slin of leeches is delicate, the epidermis being slimy; the latter requires cleaning, or they suffer from illness and die.

There are only two officinal species: $\mathbf{S}$. medicinalis, the speckled leech, and S. officinalis, the green leech. The former las a greenish yellow ventral surface spotted with black; the latter is uniformly olive green. They are caught in various ways, either by the hand or by the collector's going into ponds with naked feet, to which the leeches soon 'cling, or by (liver) laits or nets. 
TheraPeUtical USE.- Inflammations. - The special value of leeching is shown in the early stage of local congestion and inflammations, such as arise from injuries, and in orchitis, laryngitis, hæmorrhoids, and inflammations of the ear and eye, cerebral congestions, and congestive fixed headache. Leeches are also of service in a manner less easy to understand in inflammations of deep-seated parts without direct vascular ('onnection with the surface-e.g., in hepatitis, pleuritis, and pericarditis, as well as in pneumonia, peritonitis, and according to some observers in meningitis. In all these disorders, however, they are very much less used than formerly-in the larger hospitals, for instance, when at one time they cost many hundred pounds annually, a few dozens in the year would represent the total employed.

In my julgment, leeching might be with advantage employed much more frequently than is at present fashionable, and $I$ have seen some excellent results not only in relieving pain, but also in cutting short inflammatory attacks.

If we inquire how it is that such good results may be obtained, we can scarcely be satisfied that it is merely the amount of blood withdrewn that will explain them. The local abstraction of blood may, and no doubt does, lessen local inflammation by relieving tension, and by preventing, for a time, the capillary stasis of surrounding parts. In inflammation affecting large surfaces or deep-seated parts we may recognize another effect, known as "remote" or derivative. How this results it is difficult to say, unless it be reflex-local bleeding affecting sensory nerves and producing a reflex vaso-motor effect in the deep-lying trunk underneath.

Each good leech will draw between 1 and 2 drachms of blood, and as much more may be estimated to flow from each bite under the influence of warm fomentation; sometines indeed the flow is much greater, and requires artificial means for its arrest.

MODE OF APPLication.-The skin should be well deaned with warm water, dried, and if necessary shaved, before the application of a leech. These also should be dried with a warm cloth, placed over the site and covered with a 
wine-glass, pill-box, or a leech-glass. If they will not bite, the part may be moistened with sweetened milk, or by a drop of blood from the finger. When full they fall off, but if they should not do so, a little salt, sugar, or snuff sprinkled over the back will detach them. Warm fomentations, poultices, or dry-cupping encourage the bleeding. Great caution is necessary in the case of children. The bleeding is often more than is explicable by the size of the bite: it is probably due to the fact that leech saliva prevents the coagulation of human blood (Haycraft); an indication therefore would seem to be to wash the part well after application. As a rule the bleeding may be stopped by absorbent cotton wool or by a dossil of lint and a bandage. Cobwebs are an ancient resource. If more energetic measures are necessary, cotton-wool soaked in liquor ferri perchloridi or tinctura benzoini composita may be used (it is best to dry the wool before use); a stick of nitrate of silver pushed into the bite, or ligature of the wound may be used in some cases.

Leeches should not be applied at night, owing to the possibility of hromorrhage, nor over loose cellular tissue, where pressure is of not much avail in stopping the bleeding. In inflammation of the eyes they should be applied to the temples, and in that of the scrotum to the penis. If leeches should get into the stomach, nose, or rectum, they may be dislodged by swallowing or injecting a strong salt solution, which will kill them.

One of the best modes of disgorging leeches is to place them in a solution of salt and water at $110^{\circ} \mathrm{F}$. (16 parts in 100); then press them and place in fresh water. After some months they are again fit for use. 
The following substances have been added to the 188.5 edlition of the British Plarmacopoia. Many of them are fully described in preceding pages, though noted as non-officinal at the time of printing :-

Acinum Boricum (syn., Boracicum). Preparation, Unguentum. Carbolicum Liquefactum (10 per cent. water).

Prep., Unguentum Acidi Carbolici.

Chromicum. Prep., Liquor ( 1 in 4 of water).

", Hrdrobromicum Dilutum (Inorganic Substances,

p. 154). Dose, 15 to 50 minims and upwards.

$"$ Lacticum (v. p. 986). Prep., Acidum lacticum dilutum

(15 per cent.): dose, $\frac{1}{3}$ to $2 \mathrm{fl}$. dr.

" Mecoxictu (for making Liquor morphinæ bimeconatis, which is about the same strength as tinctura opii). Oleicum: (for making oleates of mercury and zinc). Phosphoricum Concentratum: (for making Syrupus ferri phosphatis).

Salicylicum (p. 685). Prep., Unguentum.

Alcohol Eтницicum (p. 87\%): (for making Liquor sodii ethylatis). Aloin (p. 773). Dose, $\frac{1}{2}$ to 2 gr.

Apomorphine (previously known as Apomorphia) Hysno(hiloratis (p. 119). Prep., Injectio hypodermica (2 gr. in 100 min. of camplior water): close, 2 to 8 min. (by the skin).

Alumen Potassit.

Argenti et Potassir Nitras ("mitigated" carstic).

Arsenil Iominum (Inorganic Substances, p. 418). Dose, $\frac{7}{30}$ gr. Prop., Liquor arsenii et hydrargyri indicli: close, 10 to 30 min.

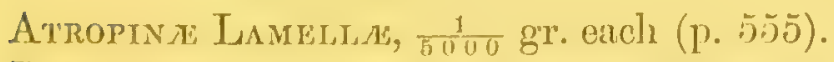

Belladonna: Extractum Aicoholicum. Prep., Emplastrum. Bismuthi Citras.--Bismutut jat Amannil Citras. Dose, 2 to 5 gr. Prop., Iir. bis. and amm. cit. ( $\frac{1}{2}$ to 1 (li.) Butyi,chuorat, Hyoras (1.978).

Caffetina-Cafiein $x$ Cetras (pp. 172, 179).

Calamina Preparata. J'mp., Unguentum. 
Calcil Chloridi Liquor ( 1 in 5 aq.). Dose, 15 to $50 \mathrm{~min}$.

„ Sulphas (Inorganic Substances, p. 514): (for making calx sulphurata).

Cascara Sagrada (p. 220). Prep., Extractum: dose, 2 to 8 gr. Extractum liquidum: dose, $\frac{1}{2}$ to 2 fluid dr.

Chloroformi et Morphine Tinctura (p. 962).

Chrysarobinum (p. 265). Prep., Unguentum (1 in 24)

Cimicifuge Rhizoma (better known as Actra, p. 49).

Prep., Extractum liquidum: dose, 3 to $30 \mathrm{~min}$.

Tinctura : dose, 18 to $60 \mathrm{~min}$.

Cinchonidine Sulphas (p. 346).

Cinchomine Sulphas (p. 346).

Coca or Cuca (p. 146).

Coce Extractum Liquinum. Dose, $\frac{1}{2}$ to $2 \mathrm{fl}$. dr.

Cocaine Hydrochioras (p. 151).

" LaMelit. $\mathbb{E}^{1}\left(\frac{1}{200} \mathrm{gr}\right.$. in each).

Comerva (Codeia, p. 76 ).

Coldodicu Vesicans.

Ctrpr Nitras (deliquescent, corrosive.)

Euaterix (p. 309). Prep., Pulvis compositus (with sugar of milk, 1 in 40); dose, $\frac{1}{2}$ to 5 gr.

Ergotixum (p.803). Dose, 2 to 5 gr. Prep., Injectio hypodermica

( 1 to 2 of aqua camphoræ - to be freshly made).

Gelsemicx (p. 485). Prep., Extractum alcoholicum: dose, $\frac{1}{2}$ to $2 \mathrm{gr}$. Tinctura ( 1 in 8$)$ : dose, 5 to $20 \mathrm{~min}$.

Glycerinum Aluminis ( 1 in $7 \frac{1}{2}$ ).

Plumbi Subacetatis (Inorganic Substances, p. 721). Prep., Unguentum.

Tragacantile (p. 263).

Ionoforsum (Inorganic Substances, p. 77). Prep., Ungruentum -Suppositorium.

Jaborand (p. 204). Dose, 2 to $10 \mathrm{gr}$. Prep., Tinctura: dose, $\frac{1}{2}$ to $1 \mathrm{dr}$. Infusum: dose, 1 to $2 \mathrm{o \%}$.

1 MFem.--In the account given of cocain, its use in eye operations is said to be without drawback, but Mr. Nettleship and others have since recorded cases of panophthalmitis after the use of dises and of solutions not quite fresh; the occurrence is presumably due to "germs," nnd is to be prevented by using borie or sublimate solutions as the rehicle for cocain (B. M. J., Nov. 21st and Dec. 19th, 1885). General toxie symptoms have also followed from instillation of cocain (Lancet, Nov. 7th, 1885). 
Liquor Ammoniz Acexatis Fortiok. Dose, 25 to $75 \mathrm{~min}$.

" Ferri Acetatis. Dose, 5 to $30 \mathrm{~min}$.

30 to $90 \mathrm{~min}$.

" , " $\quad$ Fortior. Dose, 1 to 8 min.

", Dralrsates. Dose, 10 to $30 \mathrm{~min}$. and upwards.

" Morphinze (formerly Morphiæ) Brmeconatis.

Dose, 5 to $40 \mathrm{~min}$.

Sodir Ethrlatis (p. 926).

Lipulinum (p. 722).

Menthut (p. 625).

Morphine Sulphas. Dose, $\frac{1}{8}$ to $\frac{1}{2}$ gre.

Nitroglycerini Tabella, $\frac{1}{100}$ grain in each (p. 444).

Oteatum Hrdrargiri (p. 441).

" Zinci (p. 441). Piep., Unguentum.

Oreo-resina Cubebe (p. 680). Dose, 5 to $30 \mathrm{~min}$.

Oleum Eucalyti (p. 297). Prep., Unguentum.

" Pini Sylvestris (p. 740). Prep., Vapor.

"Santali (p. 272). Dose, 10 to $30 \mathrm{~min}$.

Parafeinum durum, P. molle.

(These with Paraffin, Petroleum, Vaseline, Cosmaline,

Geoline, are various forms of mineral or earth oil.)

Podophrli. Thactura, 1 gr. in $1 \mathrm{dr}$. (p. 41). Dose, B. P.

15 to 60 (5 often used).

Physostigminde Lameit.

Pitocarpin Nitiras. Dose, $\frac{1}{20}$ to $\frac{1}{2}$ gr. (by the mouth, B.P.).

Potassir Craninum (Inorganic Substances, p. 260).

Quintas (formerly Quinim) IIronocinuoras (p. 375).

Rifamint Franguta Cortex (p. 219). I'pep., Extractum: dose, 15 to $60 \mathrm{gr}$. Extractum liquidum : dose', 1 to 4 drachms.

Saltcingm (p. 684).

Santonint Trochisct, 1 gr. in each.

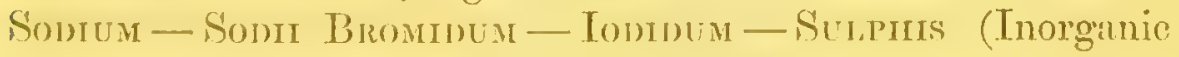
Substances, 1 \%. $77,126,757)$.

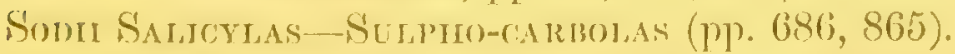

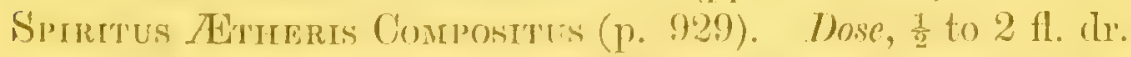

" Cinnamom (made witl oil amel spirit, 1 in 40). 1)ose, to to $1 \mathrm{fl}$. dre

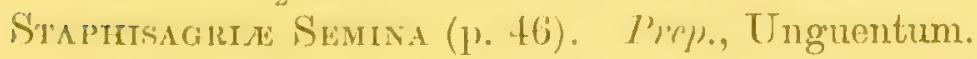


Thrymol (p. 629).

Unguentum Hynrargyri Nitratis Difeturi (1 part to 2 of Paraffin).

Zinci Sulpho-carbolas (p. 868).

Substances omitted from previous Pharmacopoeia.

Areca.

Cammit Iodidum (et UnguenTUM).

Castoreum (et Tinctura). Decoctum Ulmi (et Cortex).

Digitalinum.

Dulcamara (et Infusum).

Enema Tabaci.

Ferri Iodidum (in substance).
Ferri Oxidum Magineticum. " Peroxidum Humidum. Hydrargyri Ionidum Viride. Liquor Atroptж.

Mistura Gentian.e. Pilula Quiniz.

Ritamni Succus ex Syrupus. Strayonit Folia.

\section{Preparations Altered.}

Acetum Scind.2.-Spirit omitted.

Acidum Sulphurosum.-Lowered in strength from $9 \cdot 2$ per cent. to $6 \cdot 4$, sp. gr. 1025 . Dose left at 30 to $60 \mathrm{~min}$.

$"$ Sulphuricum Aromaticum,-Made with cimnamonspirit and ginger-tincture: rather stronger.

Antmoxim Sulphuratum.-Made rather differently, from "Antimonium nigrum purificatum."

Coxfactr. Sulphuris. - Tragacanth added.

Decocrum Alom Comp.- Slightly stronger.

Extractum Cinchones Liquidun.-Standardised to contain 5 per cent. of alkaloids. Dose, 5 to $10 \mathrm{~min}$.

" Sarsa Lirum) (Liq. Sars.j).-Differently made.

Infrisum Cinchone Acinum.-Instead of simple infusion, is made with red bark and $1 \mathrm{dr}$. acid. sulph. arom. to $\frac{1}{2}$ pint.

Iximetro Morpininds Hypodersica. - Increased in strength from $1 \mathrm{gx}$. in 12 to $1 \mathrm{gr}$. in 10 . Dose, put at 1 to 5 min. (by skin), requiring cation.

Taniminsts.-Altered in mode of preparation. 
IAirtor Epispasticus, altered to Collodium vesicans.

" Ionr.-Made with 5 per cent. iodine, $7 \frac{1}{2}$ iodide potassium.

Liztion Morphine (formerly Morphiæ) and the other solutions of alkaloids, and also of arsenic and of permanganate of potash, are made uniformly of 1 per cent. strength, being slightly increased - viz., from 1 in 109 to 1 in 100.

Onemm Phosphoratum.-Made with almond oil and 1 per cent. of phosphorus instead of 0.75 per cent. Dose, 5 to $10 \mathrm{~min}$.

Pilula Phosphori.-Contains $\frac{1}{30}$ gr. in 3 gr. of pill.

Puriss Glixcrrahize Comp.-Sulphur added.

Scammonid Resina.-Substituted in general for scammony.

Suppositoria.-Made with ol. theobromæ.

Tinctura Ferri Acetatis.-Altered in mode of making only. " Ferri Perchloridi.-Made with 5 of stronger solution, 5 of spt. rect., 10 of water; strength notaltered.

, Quinin ж.-Made with hydrochlorate, 1 gr. in $1 \mathrm{dr}$.; slightly more active.

Unguentum Hydrarg. Ammox.-Reduced from 15 to 10 percent. Plumir.-Made with glycerine of subacetate.

UngueNTA.-Many made with paraffin.

Vinum Ipecactanha. - Altered form. Dose, same.

\section{Minor Changes-Nomencluture.}

The alkaloids take the termination ina, instead of $i$-thus, atropina, morphina, quinina, strychnina, veratrina.

Salts are named after their metals-thus, potassii, sodii, etc., carbonas, ete. The present form for the oxides is, however, retained-thus, potassa causticn, potassø liquor, soda tnrtarnta, ammoniæ liquor.

Rhizoma is substituted in suitable cases for radix.

Ecbalium is to be spelt ecballium.

Assafootida as asafootida.

Arsenic is to be user only as synonym for arsenious acid, the metal boing termed arsenium. 


\section{Addenda et Corrigender.}

Acoxite.-Meningitis and epididymitis should be added to the list of inflammatory disorders for which it is advised (p. 18).

Butyi Chroral, in small and repeated doses, is serviceable in some cases of pertussis.

CAMphor.-Its value in ordinary eatarih should be referred to, but in my experience it is more effective as a vapour than internally.

Quinive.-The value of the ammoniated tincture in acute catarrh should be mentioned; also the value of the drug in anæmia generally.

Hexbane.-Its sedative effect in bladder and urethral irritation is practically similar to that of belladonna, though it is not specially named.

Giycrerine.-Its use as a nutrient should be mentioned, although distinctly less effective than cod-liver oil or maltine. The possibility of its value in diabetes should be referred to. Luchsinger proved that its subcutaneous injection in the lower animals arrested traumatic diabetes, and some observers have reported good clinical results from its internal administration. I can, in a measure, corroborate these from my own experience, which, however, is limited to a few cases.

Cociars.-A note is added on p. 1018 that panophthalmitis and toxic symptoms have sometimes followed its local use.

P. 72 , line 1, for Ischudi, rend Tschudi.

Ericatyptus Our is used as an inhalation in pertussis, croup, and diphtheria: vide also p. 300.

Saxmoxin.-Mr. Whitehead, of Manchester, recommends ten grain doses for two successive nights in amenorrhoca, especially when accompanied with chloro-anæmia.

Turpentine is used with some success in rheumatic iritis.

Certain drugs are called indifferently by Latin, English, or other names, thus: Scoparium or Broom, Thuja or Arbor Vitæ, Cimicifuga or Actra, Anacardium or Cashew, Acacia or Mucilage, Cod Oil or Cocl-liver Oil, ete. 


\section{N D E X.}

RE MEDIES.

A

Abies B.alsanea, 745 excelsa, 744

Abrin, 268

Abrus precatorius, 268

Absinthe, action of, 400

Absinthin, 399

Absinthol, 399

Acacia catechu, 261 vera, 263

Acid, abric, 268

absinthic, 400)

aconitic, 4

agaric, 825

alantic, 396

aloetic, 774

anacardic, 22j

arabic, 263

benzoic, 430

boletic, 825

cantharidic, 1006

carbolic, 830

carminic, 1004

catechu-tannic, 379

catechuic, 379

cathartic, 247

cetraric, 823

cevadic, 782

chrysophanic, 26i), 63:34

cinnamic, 429,646

cocatannic, 146

copaivic, 257
Acid, crotonic, 670

ellagie, 715

ergotinic, 804

erucic, 131

eugenic, 186

filicic, 820

gallic, 715

guaiaconic, 192

guaiaretic, 192

igasuric, 453

ipecacuanhic, 380

juglandic, 662

kinic, 346, 373

kino-tannic, 252, 347

kinovi-tannic, 347

kinovic, 346, 374 .

krameric, 144

lactic, 986

lichenostearic, 82:3

lobelic, 421

methyl-salicylic, 830

myronic, 131

myrrhic, 231

ophelic, 497

podophyllic, 34

prussic, 282

punico-tunnic, 305

pyrogallic, 718

ratanhia-tannic, 141

ricinic, 667

salicylic, 685

santonic, $\mathbf{4 0 3}$ 
Acid, sclerotic, 804

sphacelinic, 804

tannic, 715

thebolactic, 75

tiglinic, 670

tormentil-tannic, 278

toxicodendric, 226

tropic, 520

valerianic, $38 \mathrm{~s}$

Aconite, 2

physiological action, $\check{b}$

poisoning, 6

antagonists, 12

therapeutical action, 13

preparations ancl dose, 24

Aconella, 4

Aconitin, 3

Aconitum napellus, 2

Actra racemosa, 49

$\Lambda$ deps benzoatus, 996

preparatus, 995

Adonis vernalis, 33

Aëgle marmelos, 166

Æther aceticus, 927

purus, 912

sulphuricus, 912

Ethyl bromidum, 923

iodidum, 922

Agaric, white, $82 t$

fly, 826

Agaricin, 825

$\Lambda$ garicus albus, 824

muscarius, 826

Akazga, 482

Akazgia, 483

Alant-camphor, 396

Alantol, 397

Alcohol, ethylic, 877 physiological action, 883 therapeutical action, $\$ 96$

absolute, 877

amylic, 878

phenic, 830

Aldebyd, 909

Almond, 279

Aloë socotrina, 773

vulgaris, 773

Aloos, 773
Aloïn, 773

Althæa officinalis, 155

Amanita muscaria, 826

Amanitin, 826

Ammoniacum, 332

Amygdalin, 281

Amygdalus communis, 279

Amyl nitrite, 932

physiological action, 933

therapeutical action, 940

valerianate, 945

Anacardiacece, 222

Anacardium occidentale, 224

Anacyclus pyrethrum, 398

Anamirta paniculata, 66

Anamirtin, 66

Andromeda Leschenaultii, 830

Anemonin, 25

Anethol, 58, 333

Anethum graveolens, 338

Angelin, 144

Angustura, false ( $v$.nux-vomica), 201 true, 201

Angusturin, 200

Anise, 333

camphor, 58

Annelida, 1014

Anthemis nobilis, 405

Antipyrin, 948

Apis mellifica, 1003

Apocodeia, 119

Apocynacece, 447

Apomorphia, 119

physiological action, 120

therapeutical action, 122

preparation and dose, 12 ?

Aracea, 800

Arbor vite, 750

Arbutin, 426

Arctostaphylos uva-ursi, 425

Areca catechu, 799

red, 799

Argemone Mexicana, 128

Aricin, 346

Aristolochia serpentaria, 659

Aristolochincea, 659

Arnicr montana, 411

Arnicin, 411 
Arrow poison, South American, 473

Arrowroot, 758

Artanthe elongata, 682

Artemisia absinthium, 399 santonica, 402

Asafoetida, 329

Asagroea officinalis, 798

Asclepiaducere, 492

Asparagin, 156

Aspidium filix-mas, 820

Aspidosamin, 448

Aspidospermatin, 448

Aspidosperma quebracho, 447

Aspidospermin, 448

Asteracece, 395

Astragalus verus, 263

Atropa belladonna, 519

Atropia, 520

Aurantiucece, 161

Ares, 997
Bile, 987

Birthuort fumily, 659

Bistort, 641

Blood-root, 123

Boswellia, 230

Brandy, 850

Brayera anthelmintica, 285

Broom, 250

Bryonia dioica, 316

Bryonin, 317

Bryoretin, 317

Buchu, 202

Buckthorn fomily, 218

Burseracece, 230

Butter of nutmegs, 643

Butyl-chloral hydrate, 978, 1022

Buxin, $6 \pm$

Byne, 908

\section{B}

BAËL, 166

Balsamodendron myrrha, 231

Balsam of Peru, 253 of tolu, 254

Barbaloïn, T74

Bark, Winter's, 56

Barley, 818

Barosma (diosma), 202 camphor, 202

Barosmin, 202

Bean, St. Ignatius', 469

Bebeeru, 654

Beberia, 654

Belladonna, 519 active ingredients, 520 physiological action, 521

idiosyncrasy, 531

poisoning, 522

therapentical action, 534

preparations and dose, 555

Belladonnin, 520

Ben\%oate, soda, 432

Benzoin, 430

Berberin, 61
Cacao Fayily, 168

Caffein, 172 citrate, 179

Cajeputin, '296

Calabar bean, 235 active ingredients, 235 physiological action, 236 synergists-antagonists, 240 therapeutical action, 242 preparations and dose, 245

Calabarina, 235

Calendula, 419

Calendulin, 419

Calumba, 60

Culumbu family, 60

Camelliereser, 172

Camellin then, 180

Camphor, 6.t7 physiological netion, 648 thorapeutical action, 660

bromide of, 652

Camphora officinarum, 647

Canada balsam, 745

Canella alba, 186

C'anella family, 180

Cunellacedo, 186 
Canellin, 186

Cannabin, 724

Canuabis Indica, 724

physiological action, 725

therapentical action, 727

Cantharides, 1005

physiological action, 1007

therapeutical action, 1010

preparations and dose, 1014

Cantharidin, 1006

Cantharis vesicatoria, 1005

Caprifolicucer, 342

Capsaicin, 511

Capsicin, 511

Capsicol, 511

Capsicum fastigiatum, 510

physiological action, 511

therapeutical action, 513

Caraway, 337

Carbolic acid, 830

physiological action, 835

synergists, $84 j$

therapeutical action, 846

dressings, 854

preparations and dose, $86 t$

Curbon bichloride, 876

bisulphide, $8 \bar{i} 6$

tetrachloride, 876

Carbon compounds (liquid and gaseous), 875

Carbonic oxide, 875

Cardamoms, 756

Cardol, 225

Carica papayn, 752

Carmine, 1004

Carron oil, 153

Carrot, 339

Carum carui, 337

Caryophyllus aromaticus, 292

Cascara sagrada, 2200

Cascarilla, 673

Cascarillin, 673

Cashew-nut, 224

Cassia, 247

Castor, 980

Castoreum Canadense, 980

Sibiricum, 980

Castorin, 980
Castor oil, 666

Catechin, 252, 379

Catechu, pale, 378

black, 261

Cathartin, 247

Cathartocarpus fistula, 249

Celandine, 127

Celastrucea, 216

Cephaelis ipecacuanha, 380

Cera alba, 1003 flava, 1003

Cerasus lauro-cerasus, 281

Cerevisia fermentum, 907

Cetacea, 996

Cetaceum, 996

Cetraria islandica, 822

Cetyl, alcohol, 997

palmitate, 997

Chamomile, 405

Chavica Roxburghii, 680

Chelerythrin, 123

Chelidonin. 127

Chelidonium majus, 127

Cherry-laurel, 281

Climaphila umbellata, 437

Chimaphilin, 437

Chinolin, $37 \tau, 94 \bar{j}$

Chiretta, 497

Chirettin, 497

Chloral hydrate, 963

physiological action, 965

antagonists, 970

therapeutical action, 971

Chloroform, 950

physiological action, 951

therapeutical action, 956

administration, 959

preparations and dose, 962

Chrysarobin, 265

Chrysophan, 634

Cicutin, 320

Cimicifugx, radix, 49

Cimicifugin, $\overline{0} 0$

Cinchona, $3+3$

physiological action, 349

syuergists, 357

antagonists, 358

therapeutical action, 358 
Cinchona, preparations and dose, 374

Cinchonacere, 343

Cinchona-red, $3 \pm 7$

Cinchonidin, 346

physiologicalaction, 357

therapeutical action, 372

Cinchonin, 346

physiological action, 356

therapeutical action, 372

Cinnamein, 253

Cinnamomum zeylanicum, 645

Cissampelos pareira, 64

Citrullus colocynthis, 313

Citrus aurantium, 161

limonum, 163

rulgaris, 163

Clariceps purpurea, 802

Cloves, 292

Coca, 146

Cocaïn (cucaïn), 146

hydrochlorate, 151

Cocculin, 66

Cocculus Indicus, 66

physiological action, $6 \overrightarrow{7}$

therapeutical action, 70

Cocculns palmatus, 60

Coceus cacti, 1004

Cochineal, 1004

Cochlearia armoracia, $13 j$

Cocoa, 168

Codamin, 75

Codeia, if

physiological action, 84

therapeutical action, 113

Coffea Arabica, 172

Coffec, 172

physiolocrical action, 173

therapeutical action, 176

Cohosh, black, 49

Colchicin, 782

Colchicum autumnale, 781

physiological action, 782

therapeutical action, $78 j$

Coleopter", 1005

Collidin, 577

Collorlium, 160

flexile, 160

Colocynth, 313
Colocynthin, 313

Coltsfoot, 416

Columbin, 61

Compositce, $39 \mathrm{~J}$

Conia, 320

Conifere, 730

Conium maculatum, 320

physiological action, 321

poisoning, 324

therapentical action, 326

Convallamarin, 778

Convallaria majalis, 777

Convallarin, 778

Convolvulucece, 498

Convolvulin, 502

Convolvulus seammonia, 498

Conydria, 321

Copaifera multijuga, 256

Copaiva, 256

physiological action, 253

therapeutical action, 259

Copaiva resin, $25 \%$

Coriandrum sativum, 336

Cornutin, 804

Corn-poppy, scarlet, 123

Coto bark, 656

Cotoin, 657

Cotton, 157

Cotton wool, 157

iodoform, 159

salicylie, 710

Cowiteh, 264

Crocus sativus, 759

Croton-chloral hydrate, 978

Croton elenteria, 673

Croton family, 666

Croton oil, 670

Crotonol, 670

Croton tiglium, 670

Crencifere, 130

Cryptopin, 75

physiologian action, 89

Cubeha offieinalis, 680

Cubehin, 681

Cubebs, 680

Cyenmbry fumily, 308

Cucurbitacea, 308

Cumin, 33:35 
Cuminol, 335

Cuminum eyminum, 335

Cupuliferce, 713

Curare, 473

physiological action, 475

therapentical action, 479

Curarin, 474

Curcuma longa, $75 \mathrm{~s}$

Cusparia, 199

active ingredients, 200

adulterations, 201

Cusparin, 200

Cydonia vulgaris, 2is

Cymen, 629

Cymol, 297, 335

Damina, 620

Dandelion, 408

Daphne mezereum, 664

Daphnin, 664

Datura stramonium, 556

Daturin, 556

Daucus carota, 339

Delphinin, 46

Delphinium staphisagria, 46

Delphinoidin, 46

Delphisin, 46

Digitalein, 592

Digitalin, 591

Digitalis purpurea, 590

physiological action, 593

preparations and dose, 618

therapeutical action, 602

Digitoxin, 592

Dill, 338

Drimys Winteri, 56

Duboisia myoporoides, 016

Duboisin, 516

Dulcamara, 506

Dulcamarin, 506

\section{E}

EaU MFincinate, 785

Ecbalium agyeste, 308

Eccbolina, 80t
Egg, 997

Elaterin, 309

Elaterium, 308 physiological action, 310

therapeutical action, 311

Elder, 342

Elecampane, 396

Elemi, 233

Elettaria cardamomum, 756

Elm bark, $; 12$

Emetin, 380

Emodin, 219, 635

Emulsin, 28?

Ergot, 802

physiological action, $80 t$

therapentical action, $\$ 10$

preparations and dose, 817

Figotin, Bonjean's, 803

Wirgers', 803

Ergotinin, 803

Ericacere, 425

Erythroxylacee, 146

Erythroxylon coca, 146

physiological action, 146

therapeutical action, 14 ?

Eserin, $23 \bar{s}$

sulphate, 245

hydrobromate, 246

salicylate, 246

Nither, 91?

physiological action, 91:3

therapentical action, 917

preparations and dose, 9:21

administration, 9:1

Ether, acetic, 927

hrdriodic, 92.?

hydrobromic, .203

nitrous, pirits of, 929

Ethyl acetate, 927

bromide, 923

iodide, 922

Ethylate of sodium, 926

Euealyptol, 297

Eucalyptus globulus, 297

physiological action, 298

therapentical action, 29?

preparations and dose, 303

Eugenin, 293 
Enonymin, 216

Euonymite, 216

Enonrmus atro-purpureus, 216

Euphorbiucece, 666

Euryangium sumbul, 340

Exogonium purga, 501

Extract Bynes, 908

malt, 819

Pond's, 289

\section{F}

Fil movinux purificatum, 987

Fennel, 334

Ferulyl, sulphuret of, 330

Fieus carica, 721

Fig, 721

Filices, $\$ 20$

Filix-mas, 820

Flax, common, 152 purging, 154

Foniculum dulce, 334

Foxyluec family, 590

Foxglove, 590

Fuchsin, 872

Fungi, s24

G

G.IDUIX, 999

Gadus morrhua, 998

Galbanum, 332

Galipea cusparia, 199

Gallic acid, 715

Galls, 714

Grumboyle fumily, 183

Gambogria, 183

Gelsemia, 48j

Grolscmin, 486

Gelsemium sempervirens, $48 \overline{5}$ physiological action, 486

therapentical action, 489

Gentiuncueser, 494

Gentiana luten, 49.1

Gentianin, 495

Gentio-picrin, 49.5

Ginger, 7.j.)

Ginger fremily, i5j

Ginseng, 1.13
Glonoin, 144

Glucose, 1003

Gilycerine, 443

Glycocholate of soda, 987

Glycyrrhiza glabra, 261

Glycymhizin, 264

Goa powder, 26.5

Gossypium herbaceum, 157

Gourour, 170

Graminuerer, 802

Gionatuceer, 304

Granatin, 305

Grape, 187 phrsiological action, 188

Grape-cure, 188

Grepe-vine formily, 18 i

Grindelia robusta, 417

Guaiacol, 868

Guaiacum officinale, 191

Guarucusn family, 191

Guarana, 181

Guaranin, 181

Gum Arabic, 263

Gun cotton, 159

Gutta, 42i

Gutta-percha, 427

Giettiferee, 183

\section{H}

H.MMTOXYus, 256

Hatmatoxylon Campechianum, 255

HTrmomeluerere, 289

Hamamelin, 291

Hamanelis Virginica, 289

IIecuth firmily, 425

Helenin, :896;

Hellebore, black, 30

Helleborein, :30)

Hellehorin, :30

IIellehorus nigrer, 30)

Hemidesmin, 1983

IIemidesmus indicus, 492

Hemiptera, 100:3

Hemlock, :320

II emp, Indian, 724

Menlanc, 56 '2

Hespericlin, 162. 
Hirudo, 1014

Honey, 1002

Honeysuckle family, 342

Hop, 722

Hordeum vulgare, 818

Horse-radish, 135

Humulin, 722

Humulus lupulus, 722

Hydrastin, 42

Hydrastis Canadensis, 42

Hydrochinon, 872

Hygrina, 146

Hymenoptera, 1002

Hyoscin, 563

Hyoscyamin, 562

Hyoscyamus niger, 562

physiological action, $\overline{5} 6 \pm$

therapeutical action, 570

preparations and dose, 575

ICELAXD Moss, 822

Igasuria, 453

Ignatia, 469

Ilex Paraguayensis, 180

Illicium anisatum, 57

Indian liquorice, 268

Indigo, 262

Indigofera, 262

Ingluvin, 998

Insecta, 1003

Inula Helenium, 396

Inulin, 397,82 는

Ipecacuanha, 380

Iriducere, 759

Iridin, 760

Iris versicolor, 760

Isatis tinctoria, 8:30

Iso-pelletierin, 305

\section{J}

JAISORINDI, 2()-

physiological action, 2()-4

therapeutical action, 207

preparations and dose, 213
Jaborin, 204

Jalap, 50 i

Jalapin, 501

Japaconitin, 4

Jateorhiza calumba, 60

Jequirity, 268

physiological action, 269

therapeutical action, $2\lceil 1$

Jervia, 789

"Jesuit's" bark, 345

Juglundacee, 662

Juglans regia, 662

Juglone, 662

Juniper, 746

Juniperin, 746

Juniperus communis, 746

sabina, 747

K

KATRIN, 945

Kamala, 675

Kingdom, animal, 980

vegetable, 1

Kino, African, 252

Malabar, 251

Kinoïn, 252

Kinovin, 347

Kola nut, 170

Koumiss, 986

Koussin, 285

Kousso, 285

Krameria triandra, 144

Krrensol, 868

Krensote, S68

L

LA BTAT.1, 622

Lac, 986

Lactopepsin, 991

Lactose, 986

Lactucarium, 410

Lactuca sativa, $\$ 10$

virosa, 410

Lanthopin, io

Lard, benzonted, 996 prepared, 995 
Laricin, 825

Laseryl, sulphuret of, 330

Lastrea filix-mas, 820

Laudanin, 75 physiological action, 88

Laudanosin, 75 physiological action, 88

Laudanum, Sydenham's, 119

Lauracece, 615

Laurus nobilis, 655

Lavandula vera, 622

Lavender, 622

Leech, 1014

Leguminose, 234

Lemon, 163

Leptandra Virginica, 619

Leptandrin, 619

Lettuce, 410

Lichenes, 822

Lichen family, 822

Lichenin, 822

Lichen-starch, 822

Litiacece, 769

Linacee, 152

Linin, 154

Linseed, 152

Linum catharticum, 154 usitatissimum, 152

Liquor carbonis detergens, 742 pancreaticus, 994 pepticus, 991

Litmus, 824

Lobeliacece, 420

Lobelia inflata, 420

Lobelin, 421

Iogwood, 255

Lupulin, 722

Lupulite, 722

Lycoctonin, 4

Lycoperdon, $87 \bar{j}$
.Yagnolia fumily, 56 acuminata, $\check{0} 9$ glauca, $\tilde{5} 9$

Majalin, 778

Mallow fumily, 150

Malt, 908

Maltose, 818

Malcacere, 155

Mammulia, 980

Mandrake, 33

Manna, 446

Mannite, 447

Maranta arundinacea, 758

Marenturere, 758

Margarin, 985

Marsh-mallow, 155

Yastich, 222

Masticine, '223

Maté, 180

Naticin, 683

Matico, 682

Meconin, is

Mel, 1003

Melaleuca cajeputi, 295

Melanthacea, 781

Menispermucere, 60

Mentha piperita, 625 pulegium, 627 viridis, 624

ILenthol, 625

Methyl-caprinol, 190 -conia, 325 -pelletierin, 305 -theobromin, 172

Mezcreon, 66t

Milk, 986

Mimulus moschatus, 981

Mint, 624

Monkshood, "2

Morphia, 75 physiological action, 83 antagonists, (11 habit, 93 therapentical action, 110 preparations, 118

Morphia, acetate, 75 bi-meconate, ici hydrochlorate, 75
MACE, 642

Macrotin, 50

Magenta, 872

Magnoliacce, 56 
Morphia, sulphate, 76 tartrate, 76

Morphinism, 93

Morus nigra, 721

Moschus moschiferus, 982

Mucilage, 26:3

Mucuna pruriens, 264

Mulberry, 721

Muscarin, 826 nitrate, 829

Musk, 982

Mustard, 130

Mylabris, 1005

Myristica, 642

Myristicacem, 642

Myrosin, 131

Myrospermum Peruvianum, 253 toluiferum, 254

Myrrh, 231

Myrrhol, 231

Myrrhus, 231

Myrtacee, 292

MIyptte fumily, 292

N

NAPELin, 4

Narceia, $76^{\circ}$ physiological action, 86 therapentical action, 114

Narcotin, 77 physiological action, 87

Narthex asafotida, 329

Nataloin, 774

Nectandra Rodixi, 654

Nepenthe, 119

Nittle fumily, T21

Nicotiana tabacum, 576

Nicotianin, 576

Nicotin, $5 i 7$

Nightshade, deadly, 512 woody, 506

Nitre, sweet spirit of, 129

Nitroglycerine, 444

Nucin, 66?

Nucit, 662

Nutmeg, 6.t2
Nux vomica, 452

physiological action, 455

therapeutical action, 462

preparations and dose, 469

Nux romica family, 452

\section{0}

OAK, British, 713 gall, 714

Oil of almond, 279 essential, 280

anise, 333

asafœtida, 330

bays, 655

cade, 742

cajeput, 295

caraway, 337

cardamoms, 756

Carron, 153

castor, 666

chamomile, 405

cinnamon, 646

cloves, 293

cod-liver, 998

conium (volatile), 321

copaira, 257

coriander, 337

croton, 670

cubebs, 680

cumin, $33 j$

cusparia (volatile), 200

elder, 342

elemi, 233

eucalyptus, 297

fennel, 334

firwool, $7+3$

juniper, 746

lavender, 6:'3

lemon, 163

linseed, 152

mace, 643

male fern, 821

mustard, 131 volatile, 131

Neroli, 161

nutmeg, 642 
Oil of olive, 440

pennyroyal, 627

pepper, 678

peppermint, 62

pimento, $29 j$

rosemary, $62 \mathrm{~S}$

rue, $190^{\circ}$

sandal-wood (white), 272

sassafras, 653

savin, 747

spearmint, 624

tar, 740

theobroma, 169

tobacco, 577

turpentine, 731

valerian (ethereal), 388

white birch (oleum rusci), 742

Oleacea, 439

Olea Europoa, 439

Oleates, 441

Olein, 985

Olibanum, 230

Olibene, 231

Olive, 439

Otive fumily, 439

Ophelia chirata, 497

Opianin, 75

Opianyl, 77

therapentical action, 116

Opium, 73

physiological action, 78

synergists-antagonists, 90)

therapeutical action, 98

preparations and dose, 117

idiosyncrasy, 89

Opium-habit, 93

Opium denarcotisatum, 11!

Opoponax, 3:32

Opuntia cochinillifern, 100:;

Orange, bitter, 163

sweet, 161

Drange trea family, 161

Orcin, 872

Ordeal poison, West African, 482

Ornus Europoca, 446

Ovi albumen, $99 \%$ vitellus, $99 \%$

Oris aries, 985

Pulmaced, 799

Pancreatin, 992

Papain, 752

Papareracece, 73

Paparerin, $7 i$ physiological action, 87 therapentical action, 115

Papaverosin, 75

Papaver rhoas, 123 somniferum, 73

Papayacee, 752

Paracotoin, 6.๊?

Paraldehyd, 909

Pareira brava, 64

Parsley family, 319

Paullinia sorbilis, 181

Pea fumily, 234

Pelletierin, 305 sulphate, 307

tannate, 307

Pellitory, 398

Peppore fumily, 675

Pepper, black, 675 long, 680

Peppermint, 625

Pepsin, 988

Peptones, 99-1

Peptonised food, 994

Peru, balsam of, 25:3

Phaoretin, 635

Phenol, 8:30

Pliyseter macrocephalus, $99(6$

Plysosterin, 235

Physostigna venenosum, 23i)

Plysostigmin, 235

Pieraconitin, :3

Picruna cxoclsa, 214

Picropodophyllin, 34

Picrotin, (it;

Pierotoxin, $\mathbf{6 0}$

Pilocarpenc, 20.1

Pilocarpin, 20.1

hydrochlorate, 213

nitrate, 21:3 
Pilocarpus pinnatifolius, 204

Pimenta vulgaris, 294

Pimento, 294

Pimpinella anisum, 333

Pinucece, 730

Pine-tree family, 730

Pinus, 730

Piperacece, 677

Piperin, 678

Piper nigrum, 677

Pisces, 998

Pistacia lentiscus, 222 terebinthus, 223

Pitch, Burgundy, 744

Pix Burgundica, itt liquida, 740

Plum, 284

Po' de Bahia, 265

Podophyllin, 34

Podophyllotoxin, 34

Podophyllum peltatum, 33

Polychroit, 759

Polygalacere, 140

Polygala senega, 140

Polygalin, 141

Polygonacere, 633

Polygonum bistorta, 641

Pomegranate, 304

Poppy, opium, 73 prickly, 128

Poppy fumily, 73

Porphyroxin, 75, 123

Powder, Gregory's, 639

Prunus domestica, 284

Virginiana, 286

Pseudaconitin, 4

Pseudopelletierin, 305

Pterocarpus mar'supium, 251 santalinus, 262

Puccin, 123

Puftiball, 875

Pulsatilla pratensis, 25

Punica granatum, 304

Punicin, 305

Pyrethrin, 398

Pyrethrum, 398

Pyrolureee, 437

Pyrola fumily, 437
Pyrogallol, 718

Pyroxylin, 159

Q

Quassia, 214

Quussia family, 214

Quassin (quassite), 214

Quebrachin, 448

Quebracho, 447

Quercus infectoria, 714 robur, 713

Quince, 278

Quinetum, sulphate, 373

Quinidin, 346

physiological action, $35 \bar{\imath}$

therapeutical action, 372

Quinine, 345

physiological action, 350

therapeutical action, 358

Quinine, hydrochlorate, 375

sulphate, 375

and iron, citrate of, 376

Quinoidin, 345

Quinovin ( $r$ kinovin), $34 \bar{\jmath}$

R

Renunenlucece, 1

Ratanhia-red, 144

Resin of copaiva, 257

gamboge, 183

hydrastis, 42

Peru, 253

podophyllin, 34

Resorcenal, 872

Resorcin, 872

Rhamnacece, 218

Rhamnocathartin, 218

Rhamnus catharticus, 218

frangula, 219

purshiana, 220

Rhatanin, 144

Rhatany, 144

Rhatany family, 140

Rheum, 633

Rhodeoretin, 502 
Rhoadin, 75

Rhubarb family, 633

Rhubarb, 633 physiological action, 636 therapeutical action, 639

Rhus toxicodendron, 226

Ricinin, 667

Ricinus communis, 666

Roccella tinctoria, 824

Rodentic, 980

Rosacere, 276

Rosa canina, 277

centifolia, 277

gallica, 277

Rosanilin monochlorohydrate, 872

Rose, Christmas, 30

Rose family, 276

Rosein, 872

Rosemary, 628

Rosmarinus officinalis, 628

Rottlera-red, 675

Rottlera tinctoria, 675

Rottlerin, 675

Rue, 195

Rue fumily, 195

Ruminantia, 982

Rutacee, 195

Ruta graveolens, 195

Rutin, 195

Rye, ergot of, 802

SABATItLA, 798

Sabadillin, 798

Saccharum lactis, 986

Saffron, 759

Sagapenum, 332

saye fumily, 622

Salicaceer, 684

Salicin, 684

Salicylate of iron, 686

lithium, 6886

quinine, (686)

soda, 6866

Salicylicacid, (i8i)

physiological action, 688

therapentical action, 696\%
Salicylic acid, preparations and dose, 710

Salix fragilis, 684

Sambucus nigra, 342

Sandalwood family, 272

Sandalwood, white, 272 red, 262

Sanguinaria, 123

Sanguinaria canadensis, 123

Sanguisuga medicinalis, 1014 officinalis, 1014

Santalacere, 272

Santalum album, 272

Santonica, 402

Santonin, 403, 1022

Sapindacee, 181

Sapo durus, 442 mollis, 442

Saponin, 141

Sapotacere, 427

Sarothamnus scoparius, 250

Sarsaparilla, 762

Sursaparilla family, 762

Sassafras officinale, 652

Sassafrin, 653

Saviu, 747

Scammonin, 499

Scammony, 498

Scillaïn, 770

Scillin, 7 \%

Scillipierin, TTO

Scillitin, 770

Scillitoxin, 770

Scleromuein, 804

Scoparin, 250

Scoparius, 2250

Scropheluriacere, 590

Senegra, 1.10

Senegin, 141

Semna, 217

Serpentaria, $6 \overline{0} 0$

Sevim preparatum, $98 j$

Sherry, 880

Shicld-fern, common, 820 mule, 820

simurubucern, 211

Sinalbin, 131

Sinspin, 132 
Sinapis alba, 131 nigra, 130

Sinistrin, 770

Smilacere, 762

Smilacin, 763

Smilax officinalis, 762

Snake-root, 140

Soap, hard, 442 soft, 442

Socaloïn, 774

Solanacece, 505

Solanin, 506

Solanum dulcamara, 506

Spanish flies, 1005

Spartein, 250

Spearmint, 624

Spermaceti, 996

Spigelia anthelmintica, 472 Marilandica, 471

Spigelincere, 452

Spirit, proof, 879

Spiritus atheris nitrosi, 929 rectificatus, 879 tenuior, 879 vini Gallici, 880

Squill, 769

Staphisagrin, 46

Star-anise, 57

Stavesacre, 46

Steapsin, 992

Stearin, 985

Stearoptene, 623

Sterculia acuminata, 170

Sterculicucece, 168

Storax, 428

Stramonin, 556

Stramonium, 556 poisoning, 556

Strychnin, 453 poisoning, $45 \tilde{j}$

Strychnos nux-vomica, 452 Ignatia, 469

Styptic, Warren's, 736

Stypracuceer, 428

Styracin, 428

Styrax officinale, 428 benzoin, 430

Styrol, 428
Sucrose, 1003

Suet, prepared, $98 \tilde{z}$

Sugar of milk, 986

Sulpho-carbolate, copper, 865 iron, 868 soda, 865 zinc, 868

Sumach family, 22.2

Sumbul, 340

Sus scrofa, 995

T

Tamarimdes Iydica, 263

Tannin ( $x$. Acid, tannie)

Tar, 740

Taraxacerin, 409

Taraxacin, 409

Taraxacum Dens Leonis, 408

Taurocholate, soda, 98\%

Ten, 180

Paraguay, 180

Tephrosia toxicaria, 619

Terebinthina, 730

Canadensis, $7+5$

Terpene, 297

'Thebaia, 7 i

physiological action, $S 7$

therapeutical action, 116

Theobroma cacro, $16 \mathrm{~s}$

Theobromin, 169

Thorn-apple, 556

Thuja occidentalis, 750

Thujin, 750

Thymelacea, $66 t$

Thymen, 629

Thymol, 629

Thymus vulgaris, 629

Tobacco, 576

physiological action, 578

synergists-autugonists, 587

therapeutical action, 58

Tolene, 255

Tolu, 254

Tonga, 800

'Tongin, 800

Tormentilla ofticinalis, $2-8$

Tormentil-red, 278 
Tragacanth, 263

Trimethylamin, 411 action of, 412

Triolein, 985.

Tristearin, 985

Tropin, 520

Trypsin, 992

Turmeric, iss

Turpentine, 730

physiological action, 732

therapeuticalaction, 734,1022

Canadian, 745

Chian, 2023

Tussilago fartarn, 416
Veratrum viride, therapeutical action, 793

Vinum aurantii, 880

Xericum, 880

Viola canina, 139 odornta, 137

tricolor, 139

Violacere, 137

Violet family, 137

Violin, 137

Viridia, 789

Vitacea, 187

Vitis vinifera, 187
Utmacee, 71!

Ulmin, 712

Ulmus campestris, 712

Umbelliferce, 319

Umbelliferon, 340

Urginer scilla, 769

Urticacee, 721

Uva-ursi, 425
$\mathrm{U}$

V

Valeren, 388

Valeriunuced, 387

Valeriana officinalis, 387 physiologrical action, 388 therapentical action, 391

Valerianate of ammonia, 39.1 zinc, 394

Valerian-camphor, 388

Valerian family, 387

Veratria, 789

Veratroidia, 789

Veratrum album, TR8

virisle, 789

physiological action, 790
W

Walnut, 662

Wax-flowes family, 492

Wax, white, 1003 yellow, 1003

Willow, crack, 684 family, 684

Wine, orange, 880

Winter's bark, 56

Witch-hazel, 289

Wormgrass, \pm 72

Wormseed, 402

Wormwood, 399

Yeast (beer), 907

Yellow-root, 42

Yew-tree, 619

Kingiberacee, 755

Zingiber offieinale, T5o

Zyygophyllaces, 191 


\section{N D E X.}

DISEASES.

A

Abdomyal Plethora.

Grape-cure, 189

ABORTION.

Ergot, 813

Gossypium, 158

Hamamelis, 290

Opium, 109

AbsCEss.

Belladonna, 535

Carbolic acid, 849, 854, 858

Cod-liver oil, 1001

Linseed poultice, 153

Salicylic acid, 697

Sarsaparilla, 767

Stramonium, 559

Acmitr, $v$. I)rspersia, Prrosis.

ACNE.

(Belladonna)

Ergotin, 810

Glycerine, 443

Opium, 103

Soap, 443

(Purgatives)

ADENITIS.

Aconite, 18

Belladonua, 535
Ague, $v$. Iytermittent Ferer.

After-pains.
(Aconite)
Belladonna, 536
Chloral, 974
Chloroform, 95̄
Cimicifuga, 53
Dill, 339
Ergot, 813
Gelsemium, 490
Morphia, 111
Opium, 101-109

Ambuminetria.

Benzoic acid, 435

Caffein, 179

Cantharis, 1012

Chimaphila, 438

Chloral, 974

Cod-liver oil, 1000

Digitalis, 609

Elaterium, 311

Fuchsin, 872

Gallic acid, 717

Jaborandi, 209

Juniper, 747

Nitro-glycerine, $\mathbf{4 4 6}$

Opium, 109

Turpentine, 737 
Alcoholism.

Cannabis, 727

Capsicum, 515

Chloral, 971-3

Cinchona, 895

C'love, 294

Coca, 151

Coffee, 178

Gelsemium, 490

Hrdrastis, 45

Ipecacuanha, 383

Fola nut, 171

Lupulin, 723

Nux vomica, 466

Opium, 99

Quinine, 370

Strychnia, 896

Sumbul, $3+1$

AlKainoid-PoIsonitig.

Tannin, 717

Alopecia.

Cantharides, 1012

Jaborandi, 207

(Olive oil)

Rosemary, 628

Amaurosis, Amblyopia.

Arnica, 414

Santenin, 404

Strychnia, 467

Amexorrifea.

Aconite, 23

Aloes, 776

Cantharides, 1013

Cimicifuga, 5:3

Colocynth, 315

Ergot, 813

Fennel, 333

Gruaiacum, 194

(Leeches)

Myrrh, 233

Pennyroyal, 627

Pulsatilla, 29

Rosemary, 628

Rue, 198
AMENorinha-Continued.

Sanguinaria, 126

Santonin, 1022

Savin, 749

Senega, 143

Stavesacre, 48

Storax, 429

Thuja, 751

Andemia, General.

Alcohol, 904, 906

Cod-liver oil, 1002

Maltine, 819

Nux vomica, 465

Pancreatin, 992

Pepsin, 990

Quinine, 360-372

Avesthesia.

Nux vomica, 464

ANeurisir.

Amyl, 940

Chloroform (locally)

Ergot, 812

Morphia, 101-111

Veratrum viride, 797

Angina l'ectoris.

Aconite, 20

Amyl, 9t0

Cherry laurel, 283

Ether, 919

Morphia, 104, 111

Mustard, 13:3

Nitrites, 145

Nitro-glycerine, 446

Opium, 104

Quinine, 368

Stryolınia, 468

$\Lambda$ ntimonial, Porsoning.

Tannin, 717

ANus, finsure of.

Belladonna, 535

Custor oil, 663

Collodion, 160

Hydrastis, 44

Opium, 101 
Anus, Fissure of-Continued.

Rhatany, 145

Tannin, 717

(Laxatives),

Aortic Disease,

v. Heart Valve-disorder.

ApHonia.

Ben\%oin, 435

Cantharides, 1010

(Capsicum)

Ignatia, 470

Kreasote, 871

A PHTH ж.

Glycerine, 444

Mel, 1003

Quinine, 372

lihubarb, 639

Salicylic acid, 697

APoplexy.

Aconite, 24

Blistering, 1009

Cocculus, 71

Colocynth, 315

Croton oil, 672

Elaterium, 312

Mustard, 133

Appetite, Impaired.

Bibiru, 655

Calumba, 63

(and other bitters).

Appetite, Morbid.

Cod-liver oil, 1002

Ignatia, 470

Arthinitis,

v. Rieumatic Arthritis.

Ascarides, \%. Wolms.

Cajeput, 296

Jalap, 50:3

Quassia, 215

Santonin, 404
Ascites, $v$. DRopsy.

Asthenia, v. Anemia.

Cod-liver oil, 1002

Opium, 106

Asthenopia.

Eșerin, 243

Rue, 199

Tonga, 801

Asthira.

Amyl nitrite, 941

Asafotida, 332

Balsams of Tolu and Peru, 253-5

Belladonna, $5 \pm 2$

Blistering, 1011

Camphor, 650

Cannabis, 727

Chamomile, 407

Chloral, 975

Chloroform, 956

Coffee, $17 \%$

Datura, 559

Delphinin, 48

Dulcamara, 510

Ether, 918

Ethyl iodide, 922

Grindelia, $41 \mathrm{~s}$

Ipecacuanha, 385

Lobelia, 423

Mace, $6+4$

Morphia, 104

Nux vomicr, 468

Opium, 104

Pilocarpin, 210

Pulsatilla, 29

Quebracho, 450

Quinine, 371

Senega, 142

Stramonium, 559

Sumbul, 341

Tobreco, 588 
Ataxy, v. Locomotor Ataxy.

Musk, 983

Strychnia, 466

Atrophy, v. Marasmus.

B

Backache, $v$. Lumbago, Sprains.

Capsicum, 514

Coca, 151

Bedsore.
Alcohol, 897
Chloral, 971
Hydrastis, 43
Quince, 278
Tannin, 716

Belladonna-Porsoning.

Apomorphia, etc., 534

Biliousness,

v. Hepatic Congestion.

Aloes, $7 \% 6$

Angustura, 201

Bryonia, 318

Calumba, 63

Chamomile, 408

Euonymin, 217

Gentian, 496

Glycerine, 444

Hydrastis, 44

Iridin, 761

Leptandrin, 620

Nux vomica, 465

Podophyllum, 39

Rhubarb, 6399

Serpentaria, 661

Taraxacum, 409

Bites, v. S'trineis.

Bladder, Irritablit.

A conite, 24

Belladonna, i54, 551

Benroic acid, 43j

Buchu, 203

Camphor, 651
Bladder, Irritable - Continued.

Cantharides, 1011

Cocculus, 71

Cubebs, 682

Ergot, 814

Henbane, 570, 1022

Linseed, 153

Mucilage, 263

Lupulin, 723

Bradder Catarrh, v. Cystitis.

Bladder, Paralysis of.

Arnica, 414

Cantharides, 1013

Cocculus, 71

Ergot, 814

Nux vomica, 465

BlenNoRRHœA.

Hydrastis, 43

Oil of sandalwood, 273

Tannin, 717

Turpentine, 736

Blister.

Adeps, 995

Cetaceum, 997

Opium, 103

Savin, 749

BoIls.

Alcohol, 897

Belladonna, 535

Carbolic acid, $8+9$

Collodion, 160

Linseed poultices, 153

(Opium)

Bone Disease.

Cod-liver oil, 1001

Sarsaparilla, 764

Brain, v. Cenebral Congestjon.

Tratin, Concussion of.

Arnica, 114

BrantTe, Distendel.

Belladonna, 537

Henbane, 570

Stramonium, 559 
Bright's Disease, $v$. Albuminuria. Iironchiectasis.

Carbolic acid, 851

Eucalyptus, 302

Tireasote, 871

Quinine, 366

Tar, 743

Turpentine, 735

BronCHITIS-BRONCHORRHœA.

Aconite, 18

Ammonineum, 332

Aniseed, 334

Apomorphia, 122

Asafoctida, 332

BaIsams, 254, 745

Belladonna, 543

Benzoin, 434

Blister, 1010

Burgundy pitch, 744

Carbolic acid, 851

Cascarilla, 674

Cherry-laurel, 283

Chloral, 972

Cimicifuga, 53

Codeia, 113

Cod-liver oil, 1001

Colchicum, 787

Conium, 326

Copaiva, 260

Croton oil, 672

Cubebs, 682

Eucalyptus, 302

Ipecacuanha, 383

Lobelia, 423

Mustard, 133

Myrrh, 232

Narceia, $11 j$

Nux vomich, 468

Opium, 107

Paraldehyd, 911

Podophyllum, 41

Sanguinaria, 125

Senega, 14'

Squill, 772

Storax, 429

Stramonium, 560

Sumbul, 341
Bronchitis - Bronchorrhea-

Continued.

Tannin, 716

Tar, 743

Turpentine, 736

Bruises.

Arnica, 413

Capsicum, 514

Juniper, 746

Oil of bays, 656

Opium, 101

Burns aid Scalds.

Adeps, 995

Belladonna, 550

Carbolic acid, 850

Collodion, 160

Cotton wool, 157

Ether, 917

Linseed oil, 153

Salicylic acid, 697

Turpentine, 735

\section{C}

Calculi, Bilitari, $v$. Colic.

Chloroform, $95 \bar{\imath}$

Gelseminm, 490

Hydrastus, 45

Morphia, 108

Olive oil, 440

Turpentine anả ether, 737

Calculit, Rexal and Vesical.

Anssthetics, 95\%, etc.

Morphia, 108

Uva ursi, 426

Caxcer

Belladonna, 535

Calendula, 419

Carbolic acid, 851

Chloral, 971

Chloroform, 957

Codcia, 113

Conium, 326

Ethylates, 927

Eucalyptus, 300) 
Cancerr-Contimunt.

Glycerine of tamin, 71 ?

Henbane, 570

Hydrastis, 44

Opium, 101

Papain, 753

Pepsin, 990

Tiesorcin, 874

Salicylic acid, 697

Sanguinaria, 125

Thuja, 751

Thymol, 631

Turpentine, 224

Carbencle.

(Belladonna)

Carbolic acid, 849

Collodion, 160

(Opium)

Yeast, 908

Cardiac Disease, $\because$ Heart.

Cartes, $v$. Bone I iseaste.

Cat'ARACT.

Mydriatics, ฮ๊ 3, etc.

Catartir, Bitialre, $r$ Jalidice.

Catarre, (iastro-intestivat, n. I)TARRHITA.

Catarri, Gexito-urinatri, $\therefore$ Crstitis.

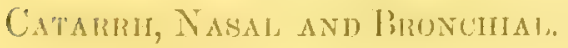

Aconite, 18

Ammoniacum, 332

Asafortida, 33.2

Belladonna, 513

liryonia, 318

('amphor, 650)

('anada balsam, iti)

Carbolic acid, 851

Cetraria, 82:3

('hamomile, $40 \%$

('herry-laurel, 28:3

('hlornl, 975

Cimicifuga, is

('od-liver' oil, 1001
Catarrh, Nasal, and Broxchials -Continued.

Copaiva, 26n

Cubebs, 68:-

Dulcamara, 509

Elecampane, 397

Eucalyptus, 30'

Grape-cure, 189

Grindclia, $41 \mathrm{~s}$

Guaiacum, 19:3

Hydrastis, $4: 3$

Ipecacuanha, :883

Jaborandi, 210

Trameria, 145

Iireasote, 871

Iinseed, 153

Liquorice, $26+4$

Mallow, 156

Mastich, 22:;

Mucilage, 26:3

Mustard oil, 13-4

Myrrh, 2:32

Nitrous ether, 931

Nux romica, $46 \mathrm{~s}$

Olibanmm, $2: 31$

Opium, 107

Pulsatilla, 2!)

Quinine, 365

Salicylates, bis

Sanguinaria, 125

Senega, 142

Squill, 7־2

Tar, 74:3

CEREIBRA, ANAMIA.

Amyl, ?t!

Chlomal, 5\%2

lither, als

(Nitro-ylyesine)

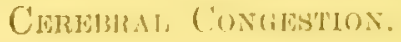

Aconite, 听

Belladomna,

lilistering, 100)!

Colocynth, 31:5

('rotom oil, (6i2)

Digitalis, 617

lirgotin, s16 
Cerebral Congestion--

Continued.

Gelsemium, 490

Jalap, 504

Ieeches, 1015

Mustard, 133

Opium, 106

Podophyllum, 39

Scammony, 500

Cererbro-spinat Mèningitis.

Aconite, 14-2t, 1022

Ergot, 817

Gelsemium, 491

Opium, 106

Quinine, $r$. Infectious Fevers.

Cerrix Uteri, Érosion of, etc.

Carbolic acid, $8 \overline{5} 0$

Hydrastis, 44

Chaycre, v. Syphilis.

Carbolic acid, 854

Chapped Hands, etc.,

v. EcZEMA.

Benzoin, 433

Collodion, 160

Glycerine, 443

Hydrastis, 43

Linseed, 153

Quince, 279

(Vaseline)

Chilblatiss.

Arnica, 413

Balsam, 254

Cajeput, 296

Cantharides, 1012

Capsicum, 513

Carbolic acid, 849

Chimbat-Poisoning.

Amyl nitrite, 944

Strychnia, etc., 970

Chloroform InHaitation.

Amyl nitrite, 942

Morphia, 112

Narceia, 115
Chloriosis,

v. ANdMIA-AMENoRRHEA.

Coceulus, 71

Esirgot, 813

Nux vomica, 465

Pepsin, 990

(Purgatives)

Cholera Asiatica.

Camphor, 651

Capsicum, 515

Carbolic acid, 862

Chloral, 977

Chloroform, 957

Ether, 918

Opium, 108

Podophyllum, 40

Strychnia, 465

Sumbul, 341

(Turpentine)

Cholera Infantum.

Camphor, 651

Carbolic acid, 862

Coto, 657

Ipecacuanha, 385

Pepsin, 990

Sulpho-carbolates, 867

Cholera Srmplex, v. Nostra.

Cajeprut, 296

Calumba, 63

Camphor, 651

Carbolic acid, 862

Chloral, 977

Ipecacuanha, $38 j$

Jaborandi, 213

Mustard, 134

Opium, 108

Salicylates, 707

Sumbul, 341

Chorder.

Aconite, 24

Belladonna, 535

Camphor, 651

Canuabis, 728

Cantharis, 1013

I.upulin, 723

Veratrum, 797 
Chorea.

Calabar bean, $24 t$

Camphor, 650

Cannabis, 727

Chloral, 975

Chloroform, 956

Cimicifuga, 51

Cocculus, 71

Cod-liver oil, 1001

Conium, 326

Curare, 482

Ergot, 816

Ether, 918

Henbane, 575

Musk, 984

Quinine, 370

Strychnia, 466

Turpentine, 739

Valerian, 392

Chororditis.

Pilocarpin, 212

\section{Chyluria.}

Benzoic acid, 430

Cirrihosis, $v$. Jaundice, I)ropsy.

Claves Hrstericus.

Ignatia, 470

Turpentine, 739

Climacteric Disorder,

v. Nervousness, Menoritiagia.

Aconite, 23

Amyl nitrite, 942

Camphor, 650

Cimicifuga, $5: 3$

Eucalyptus, 302

Nux vomica, 467

Colic, Intestinat,

v. HiATULENCE.

Anise oil, 334.

Asafixtida, 331

Belladonna, 536

Cajeput, 296:
Colic, Intestinal-Continued.

Caraway, 338

Cardamoms, 757

Castor oil, 668

Chamomile, 407

Chloroform, 957

Clores, 294

Cocculus, 71

Dill, 339

Ether, 919

Ginger, 756

Mallow, 156

Oil of bays, 656

Opium, 103

Peppermint, 626

Rue, 198

Strychnia, 465

Tobacco, 588

Turpentine, $735-7$

Colic, Lead.

Belladonna, 539

Castor oil, 668

Croton oil, 672

Opium, 103

Tobacco, 588

Colic, Renar, Hepatio,

v. Calculus.

Belladonna, 551

Chloroform, 957

Lither, 919

Opium, 10s

Turpentine, 735

CollaAs'se, $v$. Syncolde.

Opium, 106

Cома.

Blistering, 1010

Croton oil, 672

Mustard, 134

Turpentine enema, 737

Conimloma.

Carbolic neid, 851

Savin, 74!

Thuja, 751 
Congestion, Local.

Aconite, 17

Belladonna, 537

Capsicum, 513

Digitalis, 616

Ergot, 810

Leeches, 1015

Tannin, 716

Conuenctivitis.

Aconite, 17

Belladonna, 50.

Blister, 1011

Carbolic acid, 850

Castor oil, 668

Cucain, 149

Ergotin, 810

Opium, 101

Pulsatilla, 28

(Tannin)

Constipation,

Almond oil, 281

Aloes, 776

Bael, 166

Belladonna, 539

Buckthorn, 219

Carrot, 339

Cascara, 220

Castor oil, 668

Cocculus, 71

Colchicum, 788

Colocynth, 31 J

Croton oil, 67?

Elaterium, 312

(Enemata)

Euonymin, 217

Fig, 721

Gamboge, $18 j$

Glycerine, 444

Henbane, 570

(Honey)

Hydrustis, 45

Iridin, 761

Jalap, 503

Leptandrin, 620

Linseed, 154

Liquorice powder, 249
Constipation-Continued.

Lobelia, 423

Manna, 447

Muscarin, 829

Nux vomica, 465

Olive oil, 440

Opium, 103

Ox-gall, 988

Physostigma, 24j

Podophyllotoxin, 40

Podophyllum, 39

Prunes, 284

Purging flax, $15 t$

Rhubarb, 639

Scanmony, 500

Senna, 248

Strychnia, 465

Tamarinds, 263

Tar, 743

Tobacco, 588

'Turpentine, 737

Violets, 139

Convalescence, $v$. Dyspepisa.

Alcohol, 903

(Bitters)

Cascarilla, $67 \pm$

Chamomile, 407

Chiretta, 497

Coca, 150

Cod-liver oil, 1002

Gentian, 496

Grape-cure, 189

Maltine, 819

Nux vomica, 465

Pepsin, 991

Quinine, 372

Sumbul, $3+1$

Virginian prune, 287

Yeast, 908

Convulasions.

Amyl nitrite, 943

Asafectida, 331

Belladonna, 545

Blister, 1010

Chloral, 97t

Chloroform, 956 
Convulstons-Continuet.

Ergot, 816

Hemlock, 326

Henbane, 575

Ignatia, 470

Musk, 984

Mustard, 133

Opium, 109

Physostigma, 244

Rue, 198

Valerian, 391

Corneal Opactity.

Argemone, 129

Corneal Ulcer, Conical

Cornea.

Atropia, 553

Cocain, 149

Duboisin, 518

Eserin, 243

Resorcin, $87 t$

Corns.

Papain, 753

Salicylic acid, 697

Thuja, 751

Coryza, v. Catarrh, Nasal.

CoUgri.

Aconite, 19

Almond, 281

Apomorphia, 122

Asafotida, 332

Belladonna, $5+3$

Capsicum (locally)

Chamomile, 407

Chloral, 975

Chloroform, 956

Codeia, 11:3

Cod-liver oil, 1001

Coltsfoot, 417

Conium, 326;

Gelsemium, 490

(Gilycerine)

Grindclia, 418

Hyoscyamus, 5T)

Ipecacuanha, 38:3

Jaborandi, 210
Cough-Continued?

Lauro-cerasus, 283

Linseed, 153

Liquorice, $26 t$

Lobelia, 423

Mallow, 156

Morphia, 107

Mucilage, 263

Narcein, 115

Poppy, 123

Pulsatilla, 29

Sanguinaria, 125

Senega, 142

Squill, 772

Syrup of violets, 138

Tanniı, 717

Tar, $7 \pm 3$

Valerian, 392

Virginian prune, 297

Crayp, v. Colic.

Chloroform, 957

Cinnamon, 646

Croup.

Aconite, 18

Belladonna, $5 \pm 3$

Blister, 1011

Carbolic acid, $8 \check{1} 1$

Eucalyptus, 300, 1022

Ipecacuanha, 386

Lactic acid, 986, 987

Lobelia, 424

Sanguinaria, 125

Senega, 143

Squill, 772

Tamnin, 716

Cranosis.

Amyl nitrite, 912

Iocches, 101.)

(Zuebracho, tis

Cystitis.

Balsums, 254, 74.5

Belladonna, 511

Benzoin, 435

Buchu, 20:3

Cantharides, 101:3 
Crstitis-Continued.

Carbolic acid, 850

Chimaphila, 438

Copaiva, 260

Cubebs, 682

Eucalyptus, 300, 302

Hydrastis, 44

Linseed, 153

Mallow, 156

Mucilage, 263

Opium, 108

Pareira, 65

Quinine, 366

Resorcin, 874

Rhus, 229

Salicylates, 709

Sandal-wood oil, 275

Thymol, 632

Turpentine, 737

Uva ursi, 426

D

DEAFNESS.

(Astringent gargles)

(Blistering)

Cajeput, 296

Glycerine, 443

Nux vomica, 467

Oil of bays, 656

Debuity, $\%$ Convaliescence.

Delirium.

Alcohol, 903

Belladonna, 547

Camphor, 650

Cannabis, 727

Digitalis, 617

Hop, $72: 3$

Hyoscyamus, 571

Morphia, 101

Musk, 983

Opium, 99

Stramonium, 561

Sumbul, $3+1$
Delirium Tremens, $v$. Deliriok.

Actra, 54

(Calabar bean)

Capsicum, 515

Chloral, 974

Coffee, 178

Conium, 328

Lupulin, 723

Valerian, 392

Veratrum, 797

Denaue.

Belladonna, 553

DenTITION.

(Alcohol)

Belladonna, 545

Chloral, 974

Dulcamara, 509

Gelsemium, 490

(Henbane)

Diabetes Insipidus.

Atropia, 548

Cantharis, 1013

Ergot, 816

Gallic acid, 718

Jaborandi, 210

Muscarin, 829

Opium, 110

Valerian, 392

Diabetes Melitus,

$v$. Diabetes Insiphous.

Almond brend, 280

Belladonna, 548

Carbolic acid, 864

Codeia, 113

Cod-liver oil, 1001

Glycerine, 1022

Kreasote, 871

Iactic acid, 987

Quinine, 370

Salicylate of soda, 710

Thymol, 632

Uva ursi, 426 
Diartihga.

Aconite, 24

Alcohol, 905

Aloes, 776

Angustura, 201

Bael, 166

Bistort, 641

Calumba, 63

Camphor, 650

Capsicum, 515

Carbolic acid, 862

Cascarilla, 674

Castor oil, 668

Catechu, 379

Chamomile, 407

Cinnamon, 646

Coffee, 1 个 8

Coto, 657

Dulcamara, 509

Eirgot, 816

Guarana, 181

Hrmatoxylon, 256

Hydrastin, 45

Ipecacuanha, 385

Kino, 252

Kinovates, 374

Krameria, 145

Kreasote, 871

Mastich, 223

Matico, 683

Nutmeg, 643

Nux vomica, $46 \overline{5}$

Oak bark, 714

Opium, 108

Pepsin, 991

Podophyllum, 41

Pomegranate, 307

Pulsatilla, 29

Ihubarb, 640

Sevum, 985

Sumbul, 341

Tannin, 717

Tar, 743

Thymol, 6:32

Tormentil, 278

DipHTHERIA, $\because$ CROUP.

(Alcohol)
Diphtheria-Continued.

Belladonna, 538

Benzoin, 433

Carbolic acid, 850-2

Chloral, 972

Eucalyptus, 300

(Lactic acid)

Papain, 753

Pepsin, 991

Pilocarpin, 211

Quinine, 366

Resorcin, 874

Salicylate, 708

Sanguinaria, 125

Turpentine, 735

Walnut, 663

Dropsy.

Adonis, 33

Argemone, 129

Broom, 251

Bryonia, 318

Buchu, 203

Buckthorn, 219

Caffein, 178

Cajeput, 296

Cantharides, 1011

Capsicin, 516

Chimaphila, 438

Colocyuth, 315

Convallaria, 779

Copaiva, 260

1)igitalis, 607

Elaterium, 311

Gallie acid, 717

Gamboge, 185

Hellebore, 32

Horse-radish, 1:36

Hydrastis, 45

Jaboranti, 20!

Jalap, $50: 3$

Juniper, 747

Mustard oil, 1:34

Nitrous ether, 9:31

Podophyllum, 41

Seammony, 5(5)0

Senega, 14: 
Dropsx-Continued.

Squill, 771

Stavesacre, 48

(Taraxacum)

Dysentery, $v$. Diarritai.

Arnica, 415

Belladonna, 541

Benzoic acid, 436

Calumba, 63

Cetraria, 823

Cotton seeds, 158

Ergot, 816

Gamboge, 185

Glycerine, 443

Grape-cure, 189

Hamamelis, 290

Ipecacuanha, 384

Podophyllum, 41

Quinine, 385

Strychnia, 465

Dysmenorrhoca.

Aconite, 24

Actra (cimicifuga), 53

Alcohol, 907

Amyl nitrite, 94:3

Belladonna, 536

Butyl-chloral, 979

Cajeput, 296

Camphor, 6j̃

Cannabis, 728

Chloroform, 956

Cocculus, 71

Ergot, 812

Gielsemium, 489

Gossypium, 158

Gruaiacum, 194

(Leeches)

Opium, 109

Pulsatilla, 29

Rue, 198

Sumbul, 341

I) YSPEPSIA.

Alcohul, 890-901

Aloes, 756
Dyspepsia-Continumed.

Angustura, 201

Anise, 334

Bibixu, 655

Buchu, 203

Calumba, 62

Capsicum, 514

Carbolic acid, 862

Cardamoms, 757

Cascarilla, 674

Chamomile, 407

Cherry-laurel, 283

Chirata, 497

Cinchona, 372

Cloves, 294

Coccullus, 71

Damiana, 621

Drimys, 57

Elecampane, 397

Eucalyptus, 302

Gentian, 496

Ginger, 756

Hemidesmus, 493

Horse-radish, 136

Hydrastis, 45

Iceland moss, 823

Ignatia, 470

Ingluvin, 998

Ipecacuanha, 383

Lupulin, 723

Maltine, 819

Mustard, 131

Myrrh, 232

Nux vomica, $46 \bar{s}$

Oil of bays, $6 \tilde{5} 6$

Opium, 107

Orange peel, 162

Pancreatin, 994

Papuin, 754

Pepper, 679

Pepsin, 991

Pimento, 295

Podophyllin, 38

Pulsatilla, 29

Quassia, $21 j$

Quinime, 372

Rhatany, 145 
Dyspersia-Continued.

Rhubarb, 639

Salicylates, 708

Sanguinaria, 125

Serpentaria, 661

Strychnia, 465

Sumbul, 341

Tannic acid, 718

Taraxacum, 409

Virginian prune, 287

Wormwood, 401

Drsphagia.

Cajeput oil, 296

Cocain, 150

Dyspraia, $v$. Asthina, Bronchitis, Emphysema, Phthisis.

Actra (cimicifuga), 53

Amyl nitrite, 941

Blister, 1011

Convallaria, 779

Digitalis, $60 j$

Ether, 918

Iodide of ethyl, 922

Leeches, 1015

Quebracho, 449

Spigelia, 473

Valerian, 392

Virginian prune, 288

DYSURTA, $v$. STRANGURY.

Cannabis, 729

(Chimaphila)

Opium, 108

$1:$

Faracie, $v$. Dearness.

Almond oil, 281

Cajeput, 290

Carbolic acid, 819

Digitalis, 616;

Ether, 918

Leeches, 1015

Olive oil, 440
EARACHE-Contimued.

Opium, 101

Pulsatilla, 28

E.CHYyosis.

Alcohol, 896

Arnica, 413

ECTHYIA.

Cod-liver oil, 1002

Grape-cure, 189

Sulpho-carbolates, 866

Eczema.

(Anacardium)

Balsams, 254

Benzoin, 433

Blister, 1012

Carbolic acid, 849

Carron oil, 153

Celandine, 128

Chloral, 971

Cod-liver oil, 1002

Ergotin, 810

Glycerine, $\$ 44$

Grape-cure, 189

Oil of birch, 742

Oil of cade, 742

Olive oil, 440

Opium, 102

Papain, 753

Rhus, 22s

Salicylic aeid, 697, 698

Stavesacre, 48

Sulpho-carbolates, 867

'Tannin, 717

Tir, 742

líephantiasis.

Anacardium, 225

Sirsaparilia, 767

manciation, v. Marasues.

EMPIIYSEMa, D. Drapende.

Apomorphi: 122

Belladomma, jot3

C'hloral, 975

Corl-liver oil, 1001

Convallaria, 759 
Emphysema-Continued.

Ether, 919

Grindelia, 418

Iodide of ethyl, 922

Quebracho, 450

Senega, 142

Stramonium, 560

Strychnia, 468

Turpentine, 736

EMPYE Ma.

Carbolic acid, 852, 861

Salicylic acid (for washing out cavities), 697

ENDOCARDITIS.

Aconite, 17

(Blisters)

Bryony, 318

Leeches, 1015

Opium, 105

Salicylic acid, 698 , etc.

Spigelia, 473

Veratrum, 797

Endometritis.

Atropia, 536

Carbolic acid, 850

Ergot, 810

Eucalyptus, 300

(Glycerine)

Enteralgia, v. Colic:

Enteritis.

Chloral, 973

Opium, 107

Poultice, 15:3

Enuresis.

Aconite, 24

Belladonna, 541

Buchu, 203

Cantharides, 1012

Chloral, 974

Cubebs, 682

Wrot, 814

Lupulin, 72:3

Mastich, 223
ENUREsis-Continued.

Rhus, 229

Strychnia, 465

Turpentine, 738

Epididymitis.

Aconite, 1022

Belladonna, $53 \overline{5}$

Blister, 1011

Copaiba, 260

Digitalis, 616

Leeches, 1014

Opium, 108

Pulsatilla, 28

EPILEPSY.

Amyl, 943

Asafoetida, 331

Belladonna, 545

Blisters, 1010

Bryony, 317

(Calabar bean)

Chloral, 975

Chloroform, 956

Cocculus, 71

Conium, $32 \overline{7}$

Curare, 480

'

Ergot, 816

Henbane, 574

Leeches, 1014

Nitrites, 943

Nitro-glycerine, 446

Opium, 109

Picrotoxin, 72

Quinine, 363

Rue, 198

Stramonium, 560

Strychnia, 467

Sumbul, 341

Turpentine, 739

Valerian, 391

Veratrin, 797

Wormwood, 402

Epistaxis.

Aconite, 23

Arnica, 414 
EPistaxis-Continued.

Catechu, 379

Digitalis, 615

Ergot, 811

Ether, 917

Gallic acid, 717

Hamamelis, 290

Ipecacuanha, 383

Rue, 198

Turpentine, 739

Epithelioma, $v$. Ulceration.

Eucalyptus, 300

Jequirity, 271

\section{Erysipelas.}

Aconite, 15

Antipyrin, 949

Belladonna, 535, 550

Benzoin, 434

Carbolic acid, 853

Collodion, 160

Cotton-wool, 158

Digitalis, 610

Ergot, 810

Quinine, 364-369

Resorcin, 874

Rhus, 228

Salicylates, 708

Sulpho-carbolates, 867

Turpentine, 735

\section{Erythema.}

Alcohol, 897

Belladonna, 550

Quinine, 371

Rhus, 228

Exhaustion, $v$. Convalescencis.

Alcohol, 906

Arnica, 414

Castor, 981

Coca, 150

Coffee, 176

$\mathrm{Egg}, 997$

Kola nut, 171

Lupulin, 723

Maté, 180

Musk, 983
Exophthalmos.

Belladonna, 548

Digitalis, 607

Duboisia, 518

Opium, 104

Eye Operations.

Cocain, 149

Factal Palsy.

Strychnia, 462

Fiever.

Aconite, 14

Alcohol, 897-902

Ang'ustura, 201

Antipyrin, 949

Arnica, 414

Belladonna, 551

Bibiru, 654

Camphor, $6 \overline{1} 1$

Capsicum, 516

Cascarilla, 674

Cimicifuga, 52

Cinchona, 363

Cinnamon, 646

Cocculus, 71

Coffec, 177

Colehicum, 787

Digitalis, 609

Blaterium, 312

Lucalyptus, 300

Gelsemium, 491

Henbane, 570

Hydrastin, 15

Kairin, 9.46

Icmon, 16i5

Musk, 98:3

Nitrous ether, 931

Nutmeg, 644

Opium, 100, 112

Orange, $16: 2$

Pepper, 679

Podophyllum, 40 
Fever-Continued.

Pomegranate, 307

Quinine, 365

Resorcin, $87 j$

Salicin, salicylates, 705

Spigelia, 472

Sumbul, 341

Turpentine, 737

Valerian, 392

Teratrum, 796

Fibroma, $v$. Mexorritagia.

Ergot, 812

Fisisure, v. Axus.

FIS'TULA.

Capsicum, 513

Pepper, 679

Sanguinaria, 125

Flatulence, $v$. Colic, Constipation, Drspepsia.

Asafotida, 331

Caraway, 338

Cardamoms, 757

Castor, 981

Cinnamon, 646

Coriander, 337

Crocus, 760

Cumin, 336

Dill, 339

Ether, 918

Fennel, 335

Ginger, 756

Glyecrine, 443

Lavender, 623

Mallow, 156

Nutmeg, 643

Pennyroyal, 627

Pepper, 659

Peppermint, 626

Physostigma, 24j

Pimento, 295

Spearmint, 624

Valerian, :392

Findming, v. Chimagteric.
Fractures.

Benzoin, 433

Carbolic acid, 858

Gutta-percha, 427

Freckles.

Benzoin, 433

Frost-bite.

Ergotin, 810

Gall-stone, $v$. Calculi.

Gangrents.

Carbolic acid, 8551

Cinchona, 364

Eucalyptus, 300

Kreasote, 871

Opium, 102

Salicylic acid 697,709

Sanguinaria, 125

Turpentine, 735

Gastralgia, $v$. Neuralgia.

Atropia, 535

Blister, 1010

Cannabis, 727

Cherry-laurel, 283

Chloroform, $95 \mathrm{t}$

Ether, 919

Tíreasote, 871

Morphia, 107

Nux vomicn, 467

Pepsin, 990

Pulsntilla, 29

Quinine, 368

Resorcin, 875

Strychnia, 48i

Sumbul, 341

'lurpentine, 735

Gastric: Catarre, v. Dyspepsia, D) iarruma.

Catechu, 379

Ipocucuanha, 383 
Gastric Uleer, v。 Dispepsia, Gastralgia, Himatemesis.

Eucalyptus, 302

General Paralisis of Insane.

Chloral, 973

(Digitalis)

Paraldehyd, 911

Giddiness, $v$. D trspepsia,

Erilepsy, Tinnitus.

Amyl nitrite, 944

(GIAADERS.

Carbolic acid, 854

Giands, Enlarged, $v$. Ahexitis.

Ammoniacum, 332

Blister, 1011

Guaiacum, 193

Hydrastis, 44

Valerian, 392

( i LAUUCOMA.

Atropia, 554

Duboisia, 518

Eserin, 243

Pilocarpin, 207

Greet.

Balsams, 254, 7tij

Bistort, 641

Blisters, 1011

Iuchu, 203

Cantharides, 1011

Copaira, 260)

Cubebs, gri

Kino, 252

Itipulin, 72:3

Mastich, 223

(Pepper)

Sandal-wood oil, 27:3

Sulpho-carbolates, sit;

Tinnnin, 717

Turpentine, 224, 738
Globus Hrstericus.

Asafortida, 331

Ignatia, 470

Valerian, 392

Goître, $v$. Exophthalios.

Ergotin, $81 \xi$

Gonorrheea, v. Cystimis.

Bistort, 641

Cannabis, 729

Castor oil, 669

Chloral, 971

Copaiva, 260

Curbebs, 681

Digitalis, 616

Ergotin, 810

Eucalyptus, 300

Hydrastis, 44

Mallow, 156

Matico, 683

- Pulsatilla, 28

Resorcin, 874

Sandal-wood oil, 273

Sulpho-carbolates, 866

Uva ursi, 426

Gout.

Belladonna, $53 j$

Benzoic acid, 435

Buckthorn, 219

Capsicum, 516

Chimaphiln, 438

('hlor'il, 973

Cloves, 294

Codeia, 113

Colchicum, 785

Cotton-wool, 150

Duleamarn, 510

Siaterium, 312

Wither, 918

(iinger, 756

Graiacum, 193

I emon juice, 164

Iupulin, 723 
GouT-Continued.

Menthol, 626

Musk, 984

Mustard, 134

Opium, 106

Salicylates, 704

Savin, 750

(Veratria)

Wormwood, 402

Guinea-worm.

Asafoetida, 332

Carbolic acid, 848

Gums, Spongy.
(Alcohol)
Areca, 799
Bistort, 641
(Krameria)
Mastich, 223
Myrrh, 232
Pomegranate, 306
Tannin, 716

\section{H}

Homatemisis.

Bistort, 641

Ergot, 811

Gallic acid, 717

Ipecacuanha, 384

Kreasote, 870

Oak bark, 714

Tannin, 716

Turpentine, 736

Hematuria, v。 Hewatemesis.

Chimaphila, 438

Copaiva, 260

Digitalis, 614

Guaiacum, 193

Hamamelis, 290

Tireasote, 870

Matico, 683

(Pepsin)
Hemoptysis, $v$. Hematemesis.

Aconite, 23

Arnica, 414

Digitalis, 615

Gossypium, 158

Leeches, 1015

Muscarin, 829

Pyrogallic acid, 718

Thuja, 751

HemorRHaGe, Internat,

Hiemorreage, Uterine,

v. Hinmaturia.

Cannabis, 728

Cimicifuga, 53

Cinnamon, 646

Digitalis, 615

Ergot, 811

Ether, 917

Hydrastis, 45

Logwood, 256

HEMORRHOIDS.

Aloes, 776

Belladonna, 535

Capsicum, 515

Carbolic acid, 850

Castor oil, 669

Celandine, 128

Cocain, 150

Digitalis, 616

Eirgot, 810

Galls, 517

Hamamelis, 290

Henbane, 570

Hydrastis, 44

Leeches, 1015

Nux romica, $46 j$

Opium, 101

Pepper, 679

Rhubarb, 639

Senna, 248

Stramonium, 559

Tar, 743 
HAY Feyer.

Aconite, 18

Carbolic acid, 8j1

Coffee, 177

Grindelia, 418

Ipecacuanha, 383

Muscarin, 829

Quinine, 366

Salicylic acid, 698

HeADACHE.

Aconite, 22

Amyl nitrite, 94ㄴ

Argemone, 129

Balsams, 254

Belladonna, $54 \overline{7}$

Bibiru, 655

Bisulphide of carbon, 876

Bryony, 318

Butyl-chloral, 979

Caffein, 177

Cajeput, 296

(amphor, 650)

Cannabis, 727

('hamomile, 407

Cimicifuga, 52

Coca, 151

Cocculus, 71

('offee, 175

('olocynth, 315)

('roton oil, 672

Virgotin, 816

Eserin, 243

Lther, 918

Ginger, 750

Guarana, 181

Hydrastis, 4i

Ignatia, 470

Iridin, 760

I.avender, 62:3

Ieeches, 1014

Menthol, 6i26;

Nux vomica, 466

Picrotoxin, 71

Podophyllum, :3:

Pulsutilla, 2!)
Heandere-Continued.

Rosemary, 628

Tea, 180

Turpentine, 739

Valerian, 392, 393

Heartburi, $r$ Dyspepsia.

Capsicum, 514

Nux vomica, 46 T

Heart, I mgeneration of.

Belladonna, 547

Cimicifuga, 53

Ergot, 813

Ether, 918

Quebracho, 450

Strychnia, 468

Heakt, Mhatation of.

Cimicifuga, 53

Digitalis, 602

Mustard, 133

Nitrites, 941

HEART, IIYPERTROPHY OF.

Aconite, 23

Amyl nitrite, $\$ 42$

(Cumphor)

J)igitalis, 602

Frrgot, 81:3

Jeeches, 1015

Veratrum, 797

Heart', Palipitation of.

see precedini.

Adonis, 3:)

Blister, 1010

(coca, 1 i)

Convillarin, 779

Hellebore, 32

Mustard, 1333

Valerian, :3:12

Virginian prunc, 287 
Heart, Valvuiar I Mistase of. See preceding.

Amyl nitrite, 940, 942

Caffein, 178

Ergotin, 813

Morphia, 104

Opium, 104

Hectic, $v$. Fever.

Calumba, 63

Virginian prune, 287

Hemiplegia, $v$ Paralysis.

Cocculus, 71

Hepatic Congestion,

$v$. Biliousness.

Blister, 1011

Bryony, 318

Celandine, 128

Euonymin, 217

Hydrastis, $4 \bar{j}$

Ipecacuanha, 386

Lridin, 761

Leeches, 1015

Nux romica, 466

Ox-gall, 988

Podophyllum, 39

Rhubarb, 639

Sanguinaria, 125

Taraxacum, 409

Hernia.

Alcohol, 897

Chloroform, 957

Ergot, 814

Ether spray, 917

Lobelia, 423

Opium, 103

Tobaceo, 588

\section{Herpes.}

Belladonna, 5is)

Celandine, $12 \mathrm{~s}$

Ergotin, 816

(Morphia)

Quinine, 372

Rhus, 228

Veratria, 794 (unter Neuralgia)
Hiccough, $v$. Inspepsia.

Belladonna, 545

Camphor, 650

Cannabis, 727

Chloral, 974

(Chloroform)

Ether, 918

Morphia, 110

Musk, 983

Mustard, 133

Pilocarpin, 211

Hoarseness.

(apsicum, 514 (gargle)

HYDATIDS.

Turpentine, 737

HYDROCELE.

Alcohol, 897

Ergot, 811

Hydrocephalus.

Blistering, 1011

(Cod-liver oil)

Croton oil, 672

Leeches, 1015

HYDROPHOBIA.

Chloral, 976

Chloroform, 957

Curare, 480

Jahorandi, 212

(Morphia)

Pilocarpin, 212

Stramonium, 561

Hydrothorax, r. Pleeurisy.

HYPERMETROPIA.

Eserin, 243

HYPOCHONDRLASIS.

Alcohol, 906

Asafortida, 331

('imicifuga, 53

Henbane, $\bar{j} 1$ 
Hypochondriasis--Continuere.

Ignatia, 470

Lavender 623

(Leeches)

Musk, 983

Opium, 98

Ox-gall, 987

Scammony, 500

Sumbul, 341

Valerian, 392

HysteRIA.

See preceding.

Aloes, 776

Amyl nitrite, 945

Cajeput, 296

Camphor, 650

Castor, 981

Chloroform, 957

Cocculus, 71

Crocus, 760

Ether, 919

Galbanum, 332

Gentian, 496

Ignatia, 470

Lavender, 623

Lupulin, 723

Musk, 984

Orange, 162

Paraldehyd, 911

Pennyroyal, 6i27

Pulsatilla, 29

Quassia, $21 j$

Rosemary, 628

Rue, 198

Sanguinaria, 126

Strychnia, 466

Violet, 138
IMPETI(i).

Grape-cure, 189

Laurel-water, 2s3

Oil of cade, $7+2$

Salieylic acid, 698

Sanguinaria, 125

Sulpho-carbolates, su7

Tannin, 717

Tar, 742

IMPotence.

(Cannabis)

Cantharides, 1013

Cubebs, 681

Damiana, 621

Nux vomica, $463,49 j$

InCONTINENCE OF URINE, $v$. Exuresis.

INDIGESTION, $\because$ DYSPEPSIA.

INFLAMMATION.

Aconite, 17

Alcohol, 897

Antipyrin, 949

Arnica, 416

Belladonna, 5334

Blister, 1011

Bryony, 317

Chloral, 973

Colchicum, ist

(Collodion, 160)

Croton oil, 671

Digitalis, 61"

Lecches, 101.5

Mustared, 13:3

Opium, 105, 11르

Pulsatilla, 28

(Purgatives)

Quinine, 366

Salicin, Salicylates, 198

Turpentine, 73.5

Veratrum, 796

INHLEEYA, C. CATARMH.

Camphor, 150, 1022)

Garbolic acid, 8.1
Cod-liver oil, 1001

Dulcamara, 509

(Glycerine) 
Insolation, $v$. Sunstroke.

Insomana.

(Aconite)

Alcohol, 904

Butyl-chloral, 979

Camphor, 652

Cannabis, 727

Capsieum, 515

Chloral, 972

Chloroform, 957

Codeia, 11:3

Coffee, 178

Digitalis, 61$\rceil$

(Ergotin)

(Ether)

Gelsemium, 490

Henbane, 572

Hop, 723

Lactucarium, 410

Iaurel-water, 283

Lupulin, 723

Ieconin, 116

Musk, 983

Mustard, 133

Narceia, 115

Opirum, 98

Papaverin, 116

Paraldehyd, 910

Sumbul, 341

Intercostar Pain, $v$ Neuralgia.

Intermititent Fever.

Antipyrin, 949

Bibiru, 654

Bistort, 641

Capsicum, 514

Carbolic acid, 863

Cascarilla, 674

Chamomile, 408

Cinchona alkaloids, 373

Digitalis, 609

Esucalyptus, 301

Ciclsemium, 491

Gentinn, 496
Interuittent Fever-Continued.

Hydrastis, 45

Kairin, 947

Kino, 252

Magnolia, 59

Opium and derivatives, 100

Pepper, 679

Pilocarpin, 213

Podophyllin, 41

Quinine, 360

Salicin, salicylates, $70 \bar{\jmath}$

Strychnia, 468

Virginian prune, 287

Wormwood, 401

INTERTRIGo.

Carbolic acid, 849

Soap, 443

Tannin, 717

IRITIS.

Aconite, 18

Belladonna, 553

Blisters, 1011

Colchicum, 787

Copaiva, 261

Duboisia, 518

Eserin, 242

Gelsemium, 489

Leeches, 1015

(Opium)

Pilocarpin, 207

Salicylates, 705

Spigelia, 473

Tongn, 801

Turpentine, 1022

Irritant P'olsoning.

Albumen, 997

Olive oil, 440

J

Jaundice ( $v$. Prunitus,

Vomiting, etc.)

Aloes, 776

Benzoic acid, 436 
JAUNDICE-Continued.

Celandine, 128

Colchicum, 786

Dulcamara, 510

Ether, 919

Euonymin, 217

Gentian, 496

Grape-cure, 189

Hydrastis, 45

Iridin, 761

Muscarin, 829

Podophyllum, 39

Rhubarb, 639

Salicylates, 695

Sanguinaria, 125

Taraxacum, 409

Joint Disease,

v. Rheumatic Arthritis.

Ti

KeratiTIS, v. IRITIS.

L

LABOUR.

Amyl nitrite, 943

Belladonna, 536

Canuabis, 729

Carbonic oxide, 875

Chloral, 974

Chloroform and anresthetics, 957

Cimicifuga, 53

Ėrgot, 813

Gossypium, 158

Jaborandi, 213

Quinine, 355

Lactation, Deneutive.

('astor-oil leaves, 668

Gossypium, 158

Jaborandi, 213

(Mustard)
Lactation, Excessive,

v. Debility.

Belladonna, 536

Evgotin, 816

Laryngeal Irritation.

Camphor, 650

Cocaine, 150

Codeia, 113

Chloral, 972

Morphia, 107

Virginian prune, $28 \%$

Laryagisaus Strinulus.

Amyl nitrite, 941

Belladonna, 545 (under Convulsion)

Chloral, $97 t$

Chlorotorm, 956

Conium, 326

Ether, $91 \mathrm{~s}$

Ipecacuanha, $38:$

Morphia, 107

Musk, 984

Nitro-glycerine, $4+6$

Quinine, 370

Squill, 772

Tobneco, 589

LARYNGITIS.

Aconite, $1 \mathrm{ir}$

Benzoin, 43:3

Blisters, 1010

Carbolic acid, 452

Croton oil, 672

Guaincum, 19:3

Iorlide of ethyl, 9:?

1.eeches, 101:

Morphia, 10\%

Sanguinaria, 12.

Tannin, 716

Lima-pat:Y,

Eirgot, s14

Nux vomien, 4653 
IAECHIITTES,

Ether, $91 \%$

Maticn, 6x:3

LEPIROAY.

Casbew-nut, 22.5

LEUCORRHCA.

Balsams, 2.54, 745

Belladonna. 536

Cantharides, 1013

Carbolic acid, 850

Ciminifnga, 52

Cocculuss, 71

Copaiva, $2 b i$

Cubebs, 130.

Ergot, 812

Eucalyptus, 300

(Glycerine)

Hydrustis, 4,j

Kino, 252

lírameria, 145

Ireasote, 870

Logwood, 256

Myrrk, 2:32

Oak bark, 714

Pareira, $6 \bar{j}$

Pulsatilla, 29

Savin, 749

Sulpho-carbolates, 866

Sumbul, 341

Tamnin, 717

Tormentil, 278

Uva ursi, $426^{\circ}$

LivHis:

Carbolic acid, 84 ?

Chloral, a71

Iaurel-water, $28: 3$

Opium, 102

Storax, 12 ?

Tar, $7 \pm 2$

Thymol, 6:i1

\section{LitTH I A}

Jenzoic acid, 4:3.j

Buchis, 20:3

I، «mon, 16 J
Liver Disorder, v. Hepatic Congestion.

Locomotor Ataxy.

Belladonna, 545

Ergot, 817

Physostigma, 244, 24j

Pilocarpin, 212

Strychnia, 463

Lumbago.

Aconite, 22

Belladonna, $53 j$

Burgundy pitch, itt

Capsicum, 514

Chloroform, 956

Cimicifuga, 52

Ether, 919

Morphia, 111

Quinine, 36!

Rhus, 228

Salicylates, 705

Turpentine, 735

LUMBRICUS, 2 . WORMS.

Lupus.

Blister, 1012

Carbolic acid, $8 j 1$

Cod-liver oil, 1002

Ethylates, 927

Jequirity, 271

Kreasote, 871

Pyrogallic acid, $₫ 19$

Salicylic acid, 697

Soap, 443

\section{II}

Mallakiat Cachexia,

v. Ixtermitent Fever.

Bitters (gentian, etc.)

Chinolin, 37s

Eucalyptus, 301

(Purgatives)

Quinine, 358

Salicylates, 705 
MANIA.

Arnica, 414

lielladonna, j4

liryony, 318

Camphor, 650

Cannabis, 728

Chloral, 973

Colocynth, 31.5

Conium, 328

('roton oil, 672

Daturin, 561

Digitalis, 617

Elaterium, 312

Ergot, 816

Ether, 918

Gelsemium, 490

Henbane, 571

Mustard, 133

Opium, 99

Paraldehyd, 910

Physostigma, 24j

Seammony, 500)

Stramonium, 561

Veratrum, 797

MARAsMUs.

Adeps, 995

Cod-liver vil, 1000

Maltine, 819

Olive oil, $44 i$

Pancreatin, 993

Pepsin, 991

Serum, 985

MAstTTS,

1. AMFSTTS-LACTATHON.

MeAsters.

Aconite, 1.j

( ('amphor)

Ipecacuanha, :38:3

'?ulsatilla, "?

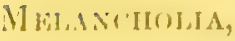

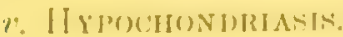

('aunabis, 728

(')lumal, 97:3
MenaxihutaA-Contimued.

Cimicifługa, jt

Ignatia, 470

Opium, 99

Valcrian, :392

MextiRti's TISEASE.

Amyl nitrite, 944

Gelsemium, 491

Pomegrauate, 30\%

Mentahtis, Cerebral, - CereBROMLIPINAT.

Acomite, 10!2:?

Belladonna, $5 \pm 7$

Blisters, 1011

Bryony, 318

Croton oil, 671

Digitalis, 617

Gelsemium, 491

H(mbane, 571

Leeches, 1015

Opium, 106

(Purgutives)

Mexurimancia.

Aconite, ㄴt

Belladomna, ji36

Cannabis, 728

('antharis, 1013

Cimicifuga, j3

Cimnamon, (346

I)igitalis, (\$14

Ergot, 81:

Gallie acid, 717

Hamamelis, 290

Pomernanate, 307

liter, 1!s

Salin, $7 \cdot t !$

Uva tusi, 46

Mentalis.

Balsams, 2j4, 74t

('arlolic acid, X4R

Col-liver oil, 100)1

Goa powder, "260

()il of cade, 742 
Mentagra-Continuer?.

Petroleum, 1019

Sulpho-carbolates, 867

Tar, 741

Turpentine, 735

Mercurialism.

Dulcamara, 510

Metritis.

Aconite, 17-23

Carbolic acid, 850

Chloral, 973

Ergotin, 810

Leeches, 1015

Opium, 105

Turpentine, 735

Migraine.

Amyl nitrite, 942

Belladonna, $5+7$

Caffein, 177

Camphor, 650

Cannabis, 727

Cimicifuga, 52

Digitalis, 618

Ergot, 816

Guarana, 181

Nitro--glycerine, 445

Quinine, 369

Sumbul, $3+2$

Miscartiage, $v$. Abortion.

Morphia-Cravingi.

Belladonna, etc., ();

Lupulin, 723

Mucous Membranes, Immtable.

Acacia, 26:3

Almond, 281

Bael, 166

Cetaceum, 997

Codeia, 113

(Gilycerine)

Hydrastis, 4:3

Linseed, 1.53
Mucous Membranes, Irritable -Continuerl.

Mallow, 156

Matico, 68:3

Myrrh, 232

Pulsatilla, 29

Storax, 429

Tragacanth, 263

Myalifa, v. Lumbago.

Aconite, 2.2

Arnica, 415

Belladonna, 535

Chlorofor'm, 956

Cimicifuga, 52

Menthol, 626

Opium, 101

Mrdriasis.

Eserin, 243

MYYeLtTIS.

Belladouna, 547

Ergot, 817

\section{$\mathrm{N}$}

NevUs.

Carbolic acid, 8.51

Chloral, 971

Ethylates, 927

Narcosis.

Amyl nitrite, 942

Apomorphia, 122

Atropia, ete., $53 \pm$

Coffee, 176

Ipecacluanha, 383 (as emetic)

I.emon-juice, 164

Musturd, 133

Nasal, Uticers, eto.

Hydrastis, 43

Pulsatilla, 29

Nausin.

Calumba, 62

Chloroform, 957 
Nausea-Continued.

Cinnamon, 646

Cloves, 294

Cocenlus, 71

Ginger, 756

Ingluvin, 998

Ipecacuanha, 384

Morphia, 108

Nux vomica, \pm 66

Pepper, 679

Peppermint, 626

Pimento, 295

Stavesacre, 48

Walnut, 663

Nephritis, Acute.

(Aconite)

Belladonna, 550

Cantharides, 1013

Digitalis, 609

Gallic acid, 717

Jaborandi, 20s

Linseed, 153

Turpentine, 737

Nervousness

("Nervols Debidty"),

$v$ Insomnia.

Aleohol, 903

Caffein, 177

Camphor, 650

Chamomile, 407

Chloral, 972

Hops, 723

Ignatia, 470

Nusk, 98:3

Pulsatilla, 28

Quinine, 368

Spigelia, 472

Strychnia, 466

Sumbul, :31

Theobromin, 170

Valcrian, 392

Nettre Rasi, v. Unticaria.
Neuralgia.

Aconite, 20

Alcohol, 907

Amyl nitrite, 942

Belladonna, 535, 538

Bibiru, 65j

Blisters, 1010

Butyl-chloral, 979

Caffein, 177

Cannabis, 727

Capsicum, 513

Carbolic acid, 854

Celandine, 128

Chamomile, 407

Chloral, 971

Chloroform, 956

Cimicifuga, 52

Cochineal, 1005́

Codeia, 113

Cod-liver oil, 1002

Convallaria, 780

Croton oil, $6{ }^{2} 2$

Digitalis, $61 \mathrm{~s}$

Ergot, 816

Ether, 918

Eucalyptus, 302

Gelsemium, 489

Ignatia, 470

Menthol, 626

Morphia, 111

Mustard, 133

Nitro-glycerine, 445

Nux vomica, 167

Opium, 101

Pulsatilla, 2!?

Pyrethrum, 3999

Quinine, 362, 368

Silicin, 705

Salieylatess, 705

Spigelia, 173

Stavesacere, 18

Stramonium, 55!)

Stryehnia, 467

Sumbul, 311

Thebaia, 116

Tomgn, 801

'T'uppentine, 738 
Neuralgia - Continum?.

Valerian, 392

Veratria, 794

Nipples, Sore.

Alcohol, 896

Arnica, 413

Balsam, $2 u t$

Benzoin, 433

Carbolic acid, 849

Collodion, 160

Glycerine, 443

Hydrastis, 44

Kreasote, 870

Quince, 279

Rhatany, $14 \bar{J}$

Tannin, 716

Nocturnal Emissions.

Belladonna, 536

Cimicifuga, 54

Digitalis, 616

Lupulin, 723

Nimphomania.

(Blisters)

Camphor, 651

Carbon bisulphide, 876

Dulcamara, 510

Lupulin, 723

Stramonium, 561

O

OBstruction, INTESTINAL, v. Constipation.

Belladonma, 539

Opium, 103

() NYOHIA.

Carbolic acid, 849

Chloral, 971

()PERATIONS.

Carbolic acid, 854

Chloroform, 957
Operations-Contimuml.

Cocain, 150)

Ether, 917, 919

Ethyl, bromide of, 924

Matico, 683

Salicrlic acid, 696

Ophthalmia, $r$ Conjunctivitis.

Spigelia, 473

Opium-Poisonisg, 2 Naruosis.

Optic Neuritis.

Pilocarpin, ㄴ12

Orchitss,

$v$. EPIDIDYMITS, I Nliamiation.

Osteitis.

Aconite, 17

Sarsaparilla, 767

Otitis, v. Eatrache.

Otorrhal.

Alcohol, 896

Calendula, 419

Carbolic acid, 849

Chloral, 971

Eucalyptus, 300 (under Mucous Discharges)

Hydrastis, 43

Pulsatilla, 2ti

Resorein, $87 t$

Salicrlates, $69 \mathrm{~s}$

(Tannin)

Oraritis, $v$. Metritis, etc.

Blister, 1011

OZ.ENA, r. ()TORLHW.

Palpitation, $\%$ M Hakt.

Cherry-laurcl, 2283

Musk, 98:3 
Paraltsis,

v. Apoplexy, ATAXY, HYSTERIA.

Arnica, 414

Calabar bean, $2 \pm 5$

Nutmeg, 643

Nux romica, 463

Oil of bars, 6.56

Pilocarpin (third nerre), 20

Rhus, 2의

Parotitis.

(Aconite)

Jaborandi, 213

Virginian prune, 287

Parterition, $v$ Labour

Pedicli.

Anise, 334

Carbolic acid, $\$ 48$

Cocculus, 70

Savadilla, 798

Stavesacre, 47

Pempuigus.

Cod-liver oil, 1002

Rhus, 22s

Sulpho-carbolates, 867

Tar, Tt2

Pericaliditis.

Aconite, 17

Alcohol, 8:7

Blister, 1011

Bryony, 317

Digitalis, 612

Leeches, 101:

Opium, 105

Quinine, 367

Spigelia, 47:3

Squill, 772

Veratrum, $7 !$

Pentostitis.

Aconite, 17

Staresacre, th
Peritonitis.

Aconite, 15, 20

Blister, 1011

Bryony, 318

Chloral, 973

Cocculus, 71

Leeches, 1015

Linseed, 153

Opium, 106

Quinine, 367

Turpentine, 735

Perspikation, Excessive.

Agaric, 825

Atropia, $\mathbf{5 4 8}$

Belladonna, 548

Calabar bean, 245

Coto, 657

Duboisia, 518

Ergot, 815

Gallic acid, 718

Hyoscin, 575

Jaborandi, 208

Logwood, 256

Muscarin, 829

(Olive oil)

Picrotoxin, 72

Pomegranate, 307

Quinine, 372

(Strychnia)

Jersmiration, Fetid.

(Carbolic acid)

Pilocarpin, 208

(Salicylic acid)

Thujal, 751

PerTUSAS.

Aeonite, 18

Asafotidn, 3:32

Belladonna, 543

Butyl-chloral, 102"

(Camnabis)

Carbolic acid, 8.52

Chamomile, 40 '

Chinolin, 378

Chomal, 974 
Pertussis-Continued.

Chloroform, 956

Cochineal, 1005

Cod-liver oil, 1001

Coffee, 177

Ergot, 817

Gelsemium, 490

Grindelia, 418

Henbane, 570

Ipecacuanha, 385

Laurel-water, 283

Lobelia, 424

Morphia, 110

Musk, 984

Myruh, 233

Narceia, 115

Pennyroyal, 627

Pulsatilla, 29

Quinine, 365, 371

Resorcin, 874

Salicylic acid, 698

Senega, 142

Squill, 772

Tannin, 716

Tar, 743

Valerian, 393

Violet, 138

Virginian prune, 287

Phagedina.

Carbolic acid, 852

Cod-liver oil, 1002

Opium, 102

Phartingitis.

Aconite, 17

Belladonna, 537

Capsicum, 514

Cimicifuga, 52

(Glycerine)

Hydrastis, 45

Ipecacuanha (spray), :3\$3

Quinine, 367

Tannin, 717

Pmimosis.

Belladonna, 535

Lupulin, 723
Phlebitis.

Hamamelis, 290

Kairin, 947

Opium, 101

Philegirasta Alba.

(Aconite)

Belladonna, 53

Blister's, 1011

Hamamelis, 290

Leeches, 1015

Opium, 101

PHOSPHATURIA.

Benzoic acid, 435

Pнотогновц.

Belladonna, 551

(Butyl-chloral)

Chloroform, 956

Cocain, 149

Conium, 326

Eserine, 24?

Phtheiriasis, $\iota$. Pedictelt.

Phthisis.

Aconite, 20

Adeps, 995

Alcohol, 907

Antipyrin, $9+9$

Atropia, $\dot{3}+9$

Benzoin, 434

Bibiru, $65 \overline{5}$

Blisters, 1011

Burgundy pitch, i 43

Calumba, 63

Carbolic acid, 852

Cetraria, 82:3

(herry-laurel, 283

(Chloral)

('hloroform, 958

Cocnin, 150

C'odein, 113

Cod-liver oil, 1001

Conium, 326

Croton oil, 672

Gelsemium, 491 
Рнтиists-Continued.

(Glycerine)

Grape-cure, 189

Henbane, 575

Iceland moss, 823

Ipecactianha, 384

Koumiss, 986

Freasote, 871

Linseed oil, 153

Logwood, 256

Maltine, 819

Myrrh, 232

Opium and derivatives, 107,113

Pepsin, 991

Picrotoxin, 72

Pilocarpin, 210

Pomegranate, $30 \%$

Quebracho, 450

Quinine, 372

Salicylates, 709

Sanguinaria, $12 \bar{\delta}$

Sarsaparilla, 767

Serum, 985

Sulpho-carbolates, 867

Sumbul, 341

Tannin, 716

Tar, $7+3$

Thymol, 631

Virginian prune, 287

Pityriasis.

Carbolic acid, 854

Chrysarobin, 266

Dulcamara, 509

(Glycerine)

\section{Puetrisy.}

Aconite, 19

Belladonna, 535

Blisters, 1011

Bryony, 318

Digitalis, 612

I.eeches, 1015

Opium, 10.5

'Trimethylamin, 116

Plelikontiata.

Belladonna, 535
Pieurodyna - Continued.

Blisters, 1011

Chloroform, 956

Cimicifuga, 52

Ether, 919

Iguatia, 470 (under Intercostal Neuralgia)

(Mustard)

Opium, 101

(Plasters)

(Poultices)

Quinine, 363, 368

Veratrum, 794

Pneumonia, $v$. Pleurisy.

Aconite, 19

Antipyrin, 949

(Apomorphia)

Belladonna, 550

Celandine, 128

Digitalis, 612

Ipecacuanha, 386

Kairin, 946

Leeches, 1015

Musk, 983

Mustard, 134

Quinine, 368

Salicylates, 709

Sanguinaria, 125

Senega, 142

Turpentine, 735

Veratrum, 794

P'OLYPUS.

Alcohol, 897

Sauguinaria, 125

'Tannin, 716

Portritio.

Cocculus, 70

Promalesus Ani.

Hydrastis, 44

Nux romica, 465

Opium, 109

Podophyllum, 40

Pomegranate, 307

'Tamin, 717 
Prolapsus Uteri.

See preceding.

Cimicifuga, 53

Galls, 717

Oak bark, 714

Prostate, Disorder of.

Blisters, 1010

Buchu, 203

Cantharides, 1011

(Conium)

Cubebs, 682

Ergot, 815

Hydrastis, 44

Rhus, 229

Turpentine, 224, 738

Prurigo-Pruritus.

Aconite, 21

Atropia, 550

Balsams, 254

Benzoin, 436

Birch oil (oleum Rusci), $7+2$

Blister, 1012

Carbolic acid, $8 \pm 9$

Chloral, 971

Chloroform, 957

Cod-liver oil, 1002

Gelsemium, 490

(Glycerine)

Jaborandi, 208

Laurel-water, 283

Liquor carbonis, it2

Oil of cade, 742

Opium, 102

Pilocarpin, 208

Stavesacre, 48

Storax, 429

Tar, 742

Tubacco, 588

Valerian and compounds, 393

\section{Psormasis.}

Blister, 1012

Carbolic acid, 819
Psoriasis-Continuert.

Chrysophanic acid, 266

Cod-liver oil, 1002

Copaiva, 261

Dulcamara, 509

Elm bark, 712

(Glycerine)

Iireasote, 871

Oil of birch, 742

Dil of cade, 747

Prrogallic acid, 719

Salicylic acid, 697

Tar, 741

Thymol, 631

Turpentine, 735

PTosis.

Ergot, 813, 814

Strychnia, 462

Ptyalisi,

Belladonna, 537

Calabar bean, 245

Tannin, $71 \%$

Puerperal Convursions.

Amyl, 943

Belladonna, $5 t j$

Chloral, 9 't

Chloroform, 957

Morphia, 109

(Mustard)

Veratrum, 797

I'verperal Fever-l'eritonitis.

Aconite, 15

Antipyrin, 949

Calumba, 63

Kairin, 946

Leeches, 1015

Opium, 105

Quinine, 36:3

Strumonium, 561

Turpentine, 735 
Puerperal Mania.

Aconite, 15

Chloral, 974

Cimicifuga, 5:?

Henbane, 571, 574

Opium, 109

Quinine, 364

Stramonium, 561

Purpura.

Ergot, 811

Gallic acid, 717

Hamamelis, 290

Lemon, 165

Nux vomica, 465

Tannin, 717

Turpentine, 736

Pyemia.

Aconite, 16

Alcohol, 905

(Antipyrin)

Digitalis, 609

Eucalyptus, 302

Pilocarpin, 210

Quinine, 364

Salicylates, 709

Turpentine, 73.5

Pyelo-NephrtTLS.

Buchu, 20:3

Cantharicles, 1013

Eucalyptus, 302

Hydrastis, 43

Salicylates, 709

PritexiA, $\because$ Fever.

Pyrosis.

Calumba, (i:?

Carbolic acid, 862

Catechu, :379

Cherry-lan'el, 283

Gallic acid, 718

Filycerine, 14:)

Nux vomica, lofi
Pyrosis-Continued.

Opium, 107

Resorcin, 875

Stryehnia, \pm 66

Q

Quinss.

Belladonna, 537

Guaiacum, 193

Salicylates, 709

Serpentaria, 661

Tannin, 716

$\mathrm{R}$

Rabies, 2 . Нydrophobia.

Rectum, IIseases of the.

Belladomna, 535

Castor oil, 669

Chloroform, 957

Copaiva, 260

Pepper, 679

Senna, 248

Stramonium, 559

Reflex Fixcitability.

Camphor, gisl

Chamomile, $40 \tau$

Chloral, 973

Cumin, 336

Valerian, 39:3

RMIAXED 'THROAT', 1. Thimon', Reinaxen.

Renittent Fever.

Aconite, 17

Salicylates, 707

Serpentaria, 661

Renat Calcelues, v. Calculus. 
Restlessness, $v$. Insomina.

Sumbul, 341

Retention of Urine.

Aconite, 24

Buchu, 203

Opium, 103, 108

Strychnia, 465

Retina, Detachment of.

Pilocarpin, 212

Rheumatic Arthritis.

Aconite, 16

Ammoniacum, 332

Arnica, 415

Blister, 1011

Cimicifuga, 52

Cod-liver oil, 1000

Colchicum, 787

Guaiacum, 193

Oleates, 441

Opium, 106

Savin, 750

Reeunatism, Acute.

Aconite, 16

Antipyrin, 949

Arnica, 415

Belladonna, 50̄3

Blister's, 1011

Bryony, 318

Carbolic acid, 853

Chloral, 973

Cimicifusn, 52

Colchicum, 787

Delphinin, 48

Digitalis, 610

Eirgot, 817

(Kairin)

Lemon juice, 164

Opium, 106

Podophyllum, 41

Quinine, 369

Salicin, Salicylates, 698

Spigelia, 473
Rheumatrsi, Acute-Contimued.

Trimethylamin, 415

Veratrum, 796

Rheumatism, Chronic.

See preceding.

Benzoic acid, 435

Buchu, 203

Buckthorn, 219

Burgundy pitch, $74 t$

Cajeput oil, 296

Canada balsam, 745

Chimaphila, 438

Codeia, 113

Cod-liver oil, 1000

Cotton-wool, 158

Cubebs, 682

Guaincum, 193

(Henbane)

Horse-radish, 136

Lupulin, 723

Magnolia, 59

Mezereon, 665

Nutmeg, 643

Pyrethrum, 399

Rhus, 228

Sarsaparilla, 767

Sassafras, 603

Sarin, 750

Senega, 143

Serpentaria, 661

Stavesacre, 48

Stramouium, 55

(Thuja)

Turpentine, $73 \cdot$

Riclets.

Atropia, 549

Cod-liver oil, 100?

Quinine, 370

Sulpho-carbolates, 868

Ringworm, $\%$. Tinea. $s$

Salifation, $v$. Ptyalisi. 
Sarcina.

Carbolic acid, 862

Kreasote, 871

Resorcin, 874

Sulpho-carbolates, 866

SCABIES.

Anise, 334

Balsams, 252

Benzoic acid, 433

Carbolic acid, 848

Cocculus, 70

Mustard oil, 133

(Petroleum)

Sabadilla, 798

Soft soap, 442

Stavesacre, 48

Storax, 429

Tar, 742

\section{Sicarlativa.}

Aconite, 14

Belladonna, 552

Carbolic acid, 863

Capsicum, 514

Digitalis, 609

Encalyptus, 300

Juniper', 747

(Mustard)

Olive oil, 4 t1

Pilocarpin, 212

Quinine, 365

Rhus, 228

Salicylates, 707

Sulpho-carbolates, 860

\section{Sictatica.}

Belladonna, 535

Blister, 1011

Burgundy pitch, 744

Butyl-chloral, 979

Canada balsam, 745

(hloroform, 9:5)

Codeia, 11:3
Solatica-Continuen.

Croton oil, 672

Digitalis, 616

Ether, 918

Guaiacum, 193

Jabor'indi, 213

Menthol, 626

Morphia, 111

(Purgatives)

Stramonium, 559

T'urpentine, 738

Veratria, 794 (under Teuralgia)

Scirritus, v. Cancer.

\section{Scrofula.}

Alcobol, 907

Chimaphila, 438

Cod-liver oil, 1001

Coltsfoot, 417

Conium, 326

Gentian, 496

Henbane, 570

Hydrastis, $4 t$

Mezereon, 665

Sanguinaria, 126

Sarsaparilla, $76 \overline{7}$

Valerian, 39.2

Walnut, 662

SOURYY.

Cancllat, 186

(Cinchona)

Citric acid, 16is

Drimys, 57

Iorse-radish, 136

Lemon juice, 164

(9range)

('Taminin)

Winter's bark, ij

SEA-SICKNIESS.

Amyl nitrite, 9.12

Capsicum, 515

(Champagne) 
Sea-sichisess-Continumet.

Chloral, 977

Chloroform, 957

Treasote, s71

Morphia, $10 \mathrm{~s}$

Nitro-glycerine, 446

(Nux romica)

Septicema, $v$ Pyima.

Aconite, 15

Hydrastin, 4j

Sexual Iebijity.

Damiana, 6리 1

Sanguinaria, 126

Sexual Desire, Excessive.

Camphor, $6 \overline{1}$

Carbon bisulphide, 876

Digitalis, 616

Dulcamara, 510

Lupulin, 723

Sntezing, Exchsive.

Belladonna, 534, 537

Camphor, 650

(Cotton-wool)

Gelsemium, 490 (under Spasm)

Sore Tirroat,

v. Toxsillitis, Quinst, ETC.

Sipasmodic Cuugh, $v$. Cough.

Asafoutida, 332

Cherry-laurel, 283

Chloral, 974

Henbane, 570

Sanguinaria, 125

Sicasmodic ITSORder,

7. Chorea, Colit.

Amyl nitrite, 941

Belladonna, $5: 35$

Camphor, 650

Castor, 981

(hloral, 97t

Chloroform, 956

Curare, 482

Ether, 91 !
Spasmiodic I)isorner-Continued.

Gelsemium, 490

Lobelia, 423

Lupulin, 723

Musk, 984

Opium, 103

Sumbul, 341

SPERMatorRHUia.

Belladonna, 536

Cantharides, 1012

Cimicifuga, $\overline{5} t$

Digitalis, 616

(Ergot)

Lupulin, 723

Nux vomica, 465

(Quinine)

Strychnia, $46 \overline{5}$

Turpentine, 736

Sphincter, Paralysis of.

Cocculus, 72

Ergot, 814

Strychnia, 463

Spina Bifida.

(Collodion)

(Cotton-wool)

(Glycerine)

Sipinal Concussron.

Arnica, 413

Leeches, 1015

Spinal Congestiox.

(Aconite)

Ergot, 817

Gelsemium, 491

Nux vomica, 463

(Turpentine)

Spinar Irritation.

Belladonna, 547

(Blisters)

Capsicum, 514

Castor oil, 671

Cimicifuga, 53

Conium, 326 (under Spasm, etc.) 
Spinal, Irritation-Continuen.

Digitalis, 616

Ergot, 817

Ignatia, 470

Leeches, 1015

Menthol, 626

Mustard, 133

Nux vomica, 463

(Opium)

(Strychnia)

Spinal Paralysis,

v. Iocomotor Ataxy.

Splenic Disorder.

Ergotin, 815

Grape-cure, 189

Hydrastis, 45

Quinine, 361

Spongy Gums, v. Scurvy.

Siprains.
(Aconite)
Arnica, 41:3
Capsicum, 514
Leeches, 1015
Oil of bays, 656
Turpentine, 734

Stertitty, $v$. Amenorrifo.

(Cantharides)

Cimicifuga, 53

D)amiana, 621

Gossypium, 158

Guaincum, 194

StTteges and Bithes.
(Aconite)
(Camphor)
('arbolic acid, 848
(Chloroform)
Tobaceo, $58 \times$

Stromatitis.

Carbolic acid, 8.50)

Ether, 917

Eucalyptus, 299

Hydrastis, 43

(Salicylates)

Sulphomcarbolates, 866 ;

('Tannin)

Strabismus,

v. Womis--JLexingitls.

Belladonna, 54j

(Purgatires)

Santonin, 404

Strangury.

Argemone, 129

Belladonna, 541

Camphor, 651

Cantharides, 1013

Gossypium, 1.58

Henbane, 570, 1022

Linseed, 15:3

Mallow, 156

(Mucilage)

Opium, 108

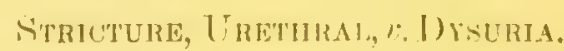

see precerting.

Belladonna, 535

- sitruma, r. ícromula.

Sithrohnia-Polsoning.

Amyl nitrite, 944

Chamomile, 407

Chloril, 976

(Jloroform, 461

Cumin, :336;

Opium, 10.5

Tobacco, ist

"STYE."

Pulsatilla, 28 
Sexstroke.

Amyl nitrite, 942

Bellidomna, $5+7$

Errot, 816

Gelsemium, 490

Honbane, ji1

(Leeches)

Nitru-glycerine, 446

Quinine, 368

Sipprissing of Mensen, ฯ. A mevorRHEA.

Aconite, 23

Gossypium, 1.58

(Jaborandi)

Mustard, $1: 33$

Opium, 109

SUPtressiox of Úrine, v. Disuria, Urfima.

Digitalis, 609

SEPPURATIOx.

Alcohol, 896

Calendula, 419

Cinchona, 364

Quinine, $36+$

Silicylates, 697

Sarsaparilla, 767

Yeast, 908

Suruical hever.

Aconite, 16

Aleohol, 90,i

(Chloral)

Pilocurpin, 2010

Quinine, 364

Sialieylates, 697

Sulpho-carbolates, 866$)$

Nirusis, 12 Menthoma.
Sympathetic Nerve, Drsolzders of, v. ExopHTHALMOS.

Belladonna, $5+7$

Pulsatilla, 29

Srncope.

Alcohol, 906

Amyl nitrite, 942

Atropia, 547

(Carnphor)

Cantharides, 1013

Chamomile, 407

Cimnamon, 646

Digitalis, 607

Ether, 918

Lavender, 623

(Musk)

Srnovitis.

Aconite, 16

Alcohol, 897

Arnien, 416

Blisters, 1011

(Bryony)

Carbolic acid, 85t

Cod-liver oil, 1001

I.eeches, 101i)

Srphin.1s.

Argemone, 129

Carbolic acid, 851

Cod-liver oil, 1001

Dulcamara, 509

Gruincum, 192

Henidesmus, 493

Jaborindi, 208

Mezereon, 665

Opium, 102

Podophyllum, 41

Salicylic acid, 710

Sanguinaria, 126

Sarsaparilla, 764

Sassafras, 653 


\section{$\mathrm{T}$}

Tabes Mesenterica.

Cod-liver oil, 1001

Olive oil, $t+0$

Tapeworm, $v$. Workms.

Tenesuus,

i. 1)YSENTERY, HaMoRRHUIDS.

Althea, 156

Tetanus.

Amyl nitrite, 944

(Arnica)

Belladonna, $5+6$

Calabar bean, 244

Cannabis Indica, 727

Chloral, 975

Chloroform, 957

Conium, 327

Curare, 479

Ether, 918

Gelsemium, 491

Henbanc, 575

Nicotin, 588

Opium, $10-1$

Paraldehyd, 911

Quinine, 370

Strvchnia, 466

Tobacco, 588

Thizont, $\because$ Tonsiletis, étc.

Trimoat, Reinxen.

Bistort, 641

Capsioum, 514

Carbolic acid, 851

Catechu, 379

Iucalyptus, 209

Ginger, 75 (5)

Horse-radish, 136

hino, 252
Throat, RElaxed-Continuert.

Kirameria, 145

Pepper, 679

Pomegranate, 307

Pyrethrum, 399

Quinine, 372

Tannin, 717

Tic Douloureux, v. Neuralgia.

Cherry-laurel, 283

Stramonium, 559

Tinea Cirunata, Tinea TonSURANS.

Carbolic acid, 818

Cocculus Indicus, 70

Cod-liver oil, 1002

Croton oil, 672

Goa powder, 266

Iireasote, 871

Menthol, 626

Pepper, 679

(Petroleum)

Salicylic acid, 6998

Sanguinaria, 125

Tar, 742

Thymol, 631

Turpentine, 73.5

(Vaselin)

Viola, 13!)

Tinea 1)ecatrans.

Cantharides, 1012

Carbolic acid, 848

Iaborandi, 208

Sapo mollis, 44:3

Tiniea favosa.

Carbolic acid, sts

()live vil, 110

(I'ctrolcum) 
Tinea Tarsi.

Stavesacre, 48

Tinnitus.

Amyl nitrite, 943

Arnica, 414

Belladonna, 547

Tongue, Inflaned, $v$. Stomatitis.

Tongte, Paralysis of.

Cinnamon, 646

Cloves, 293

Ginger, 756

Horse-radish, 136

Mezereon, 665

Nux vomica, 463

Pepper, 679

Pyrethrum, 399

Rhus, 228

TONSILIITIS.

Aconite, 18

Belladonna, 537

Benzoin, 435

Cantharides, 1011

Capsicum, 513

Carbolic acid, 851

Catechn, 379

Eucalyptus, 300

(Glycerine)

Guaiacum, 1933

Krreasote, 870

Quinine, 367

Salicylates, 709

Sanguinaria, 125

Sulpho-carbolates, 866

Tannin, 717

'Thymol, 6:31
Toothache.

Aconite, 17

Balsams, 254

Cajeput, 296

Capsicum, 513

Carbolic acid, 854

Chloral, 971

Chloroform, 956

Cloves, 293

Collodion, 160

Eucalyptus, 302

Gelsemium, 489

Ginger, 756

Henbane, 570

Kreasote, 870

Leeches, 1015

Menthol, 626

Mezereon, 66j

(Morphia)

Nitro-glycerine, $4 \mathbf{4}$

Pyrethrum, 399

$1 \quad$ Stavesacre, 48

Tonga, 801

TonTICOLLIS.

(Belladonna)

(Capsicum)

Cimicifuga, 52

(Nux romica)

Quinine, 363

Tirnohoma.

Jequirity, 271

Tremor.

Heribane, 571

Musk, 983

Nux romica, 466

Trisnus, $v$. Tetanus. 
Trmpanites, $e$ Flatulence.

Asafwetida, 331

Castor, 981

Carbolic acid, 86:2

Chamomile, 407

(Chloral)

Cocculus, 71

Ginger, 756

Nux vomica, 465

Rue, 198

Sumbul, 341

Turpertine, 737

TYPHLitis,

Belladonna, 539

Leeches, 1015

Opium, 103

Trphotid Fever.

Alcohol, 900-902

Antipyrin, 949

Arnica, 414

Belladonna, 551

Carbolic acid, 862

Chinolin, 378

Chloral, 972

Coffec, 177

Coto, 655

1)igitalis, 60(5)

lingot, 81 i

Henbanc, 571

Hydrastis, 4j

Kairin, 947

Krameria, 145

Musk, 083

Opium, 100

Quinine, 365

Ihatany, 14\%

Salicylates, 700

Serpentaria, (660)

- Sulpho-carbolates, 867

Sumbul, 341

Turpentine, 737
Typilus Fever.

See precediny.

(C'amphor)

Podophyllum, 40

U

ULCERATION.

Alcohol, 896

Argemone, 129

Balsams, 254

Belladonna, 535

Benzoin, 433

Bistort, 641

Calendula, 419

Capsicum, 513

Carbolic acid, 850

Carrot, 339

Cashew, 22.5

Catechu, 379

Chimaphila, 438

Chloral, 971

Cod-liver oil, 1002

Collodion, 160

Copaiva, 260

Ether, 917

Eucalyptus, 300

Hamamelis, 290

Henbane, 570

Hydrastis, 43

Kreasote, 870

Myrrh, 232

Opium, 101, 102

lesorein, 87.

Thatany, 145

Rhubarb, (6;3!)

Salicylio acid, 697

Simbueus, 342

Sanguinaria, 125

Suvin, 74!

Stavesacre, 48

Storax, 42 ?

Tamnin, 717

'Tur, 742

Thuiju, 7.51

Thymol, (3:31

Turmerie, 758

Turpentine, 73i 
Uramia,

Croton oil, 672

Jaborandi, 209

Urethral Fever, $r$ Surgical Fever.

URTicaria.

Belladonma, 549

Nux vomica, 468

Quinine, 371

Rhubarb, 640

Salicylates, 709

Valerian, 393

UTERINE DISORDER, v. Amenorrhaea, Menorihagia, Méritis, etc.

Livoli, $v$. Throat.

\section{V}

VAGINTSALUS.

Cocain, 150

VAGINITIS.

Alcohol, 897

Variola, Varicella.

Aconite, 14

Belladonna, 553

Carbolic acid, 849

Salicylates, 707

Sulphu-carbolates, 867

VAl11x.

Ergot, 810

Hamamelis, 291

Vomiting, $v$. SEA-SICRNIESS.

Cajeput, 296

Calendula, 419

Calumba, 63

Curluolic acid, 862

(Champagne)
Vomining, $v$. SEA-sickiness-

Continuerer.

Cherry-laurel, 28:3

Chloral, 974

Chloroform, 957

Cinnamon, $6 \pm 6$

Cloves, 294

Coceulus, 71

Ingluvin, 998

Ipecacuanba, 384

Kola nut, 171

Tioumiss, 986

líreasote, 871

Morphia, 108

Musk, 984

Nutmeg, 643

Nux vomica, 466

Pancreatin, 993

Pepper, 679

Pepsin, 991

Serpentaria, 661

Walnut, 663

WAl'IS.

Argemone, 129

Carbolic acid, 851

Cashew, 225

Celandin, 128

Papain, 753

line, 198

Savin, 749

Thuja, 751

WORMS.

(Aconite)

Aloes, 776

Areca nut, 799

Cajeput oil, 296

Carbolic acid, 864

Carrot, 339

(Castor oil)

Chamomile, 407

('od-liver' oil, 1002 
ITORus-Contimumel.

Cowiteh, ㅇt

Filix-mas, s.21

Gentian, 496

Hellebore, 32

Ignatia, 470

Jalap, 503

Kamala, $67 \overline{5}$

Konsso, 286

Olive oil, 440

Pelletierin, 307

Podophyllin, 40

Pomegranate, 306

Pulsatilla, 30

Quassia, 21j

Salicrlic acid, 710

Santonin, $40 t$

Scammony, 500

Senna, 248

Spigelia, 472, 473

Stavesacre, 49

(Tannin)

Turpentine, 7:37

Wormwood, 401
WOUADS, $v$. UTCERATION.

Arnica, 413

Balsams, $2 \tilde{4}$

Benzoin, 433

Carbolic acid, sjt

Collodion, 160

Cotton-wool, medicated, 1 cis

Ether, 917

Eucalyptus, 300

Liquor arbonis detergens, 742

Matico, 683

()pium, 101

Resorcin, 874

Salicrlic acid, $69 \%$

$\%$

"ZMinte:" Disease.

Carbolic acid, 846, 863

Sulpho-carbolates, 866 






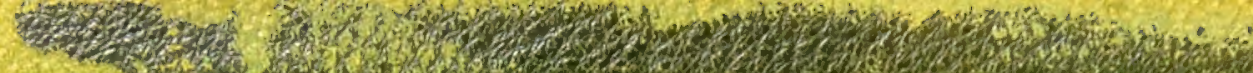

5.

34:

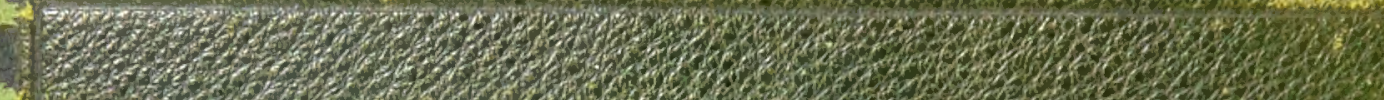

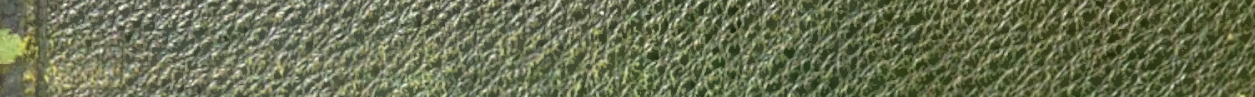

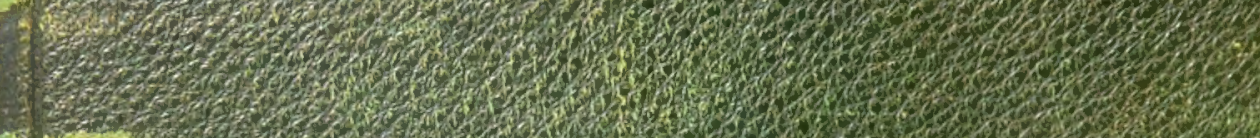

\begin{tabular}{l}
3 \\
\hline
\end{tabular}

sogs

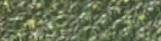

$\frac{1}{4}$

Lito 10

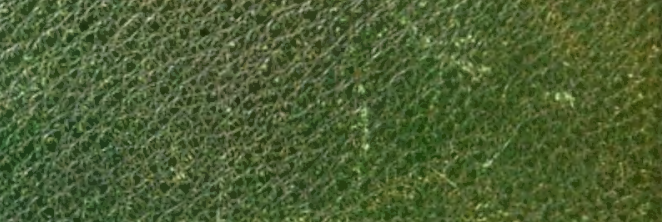

se.

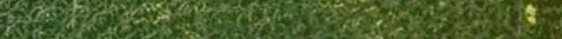

$x^{2}$

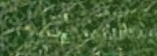

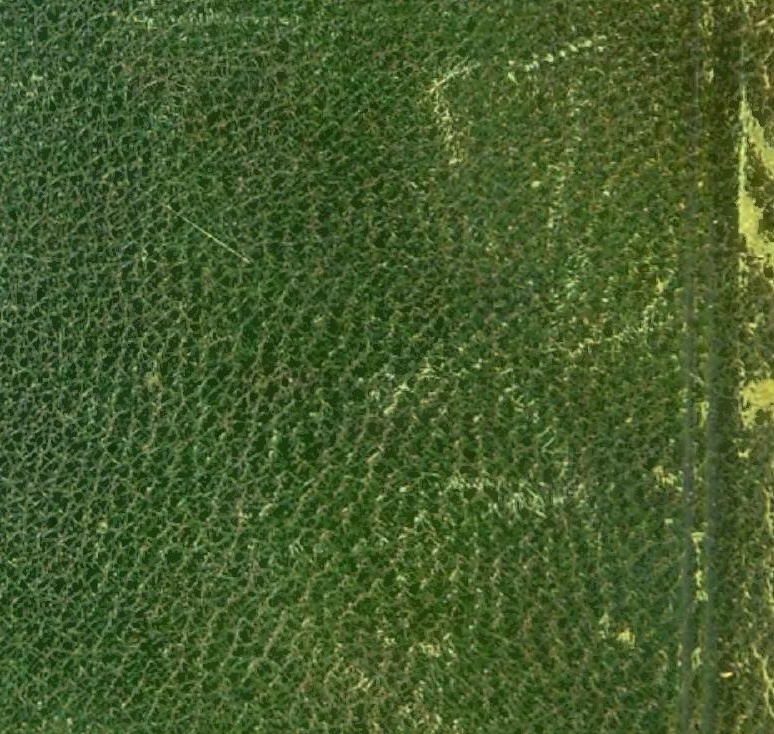

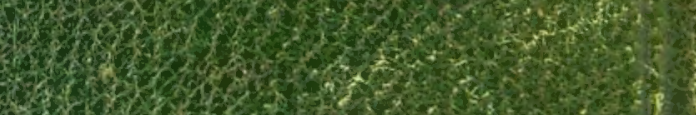
H.
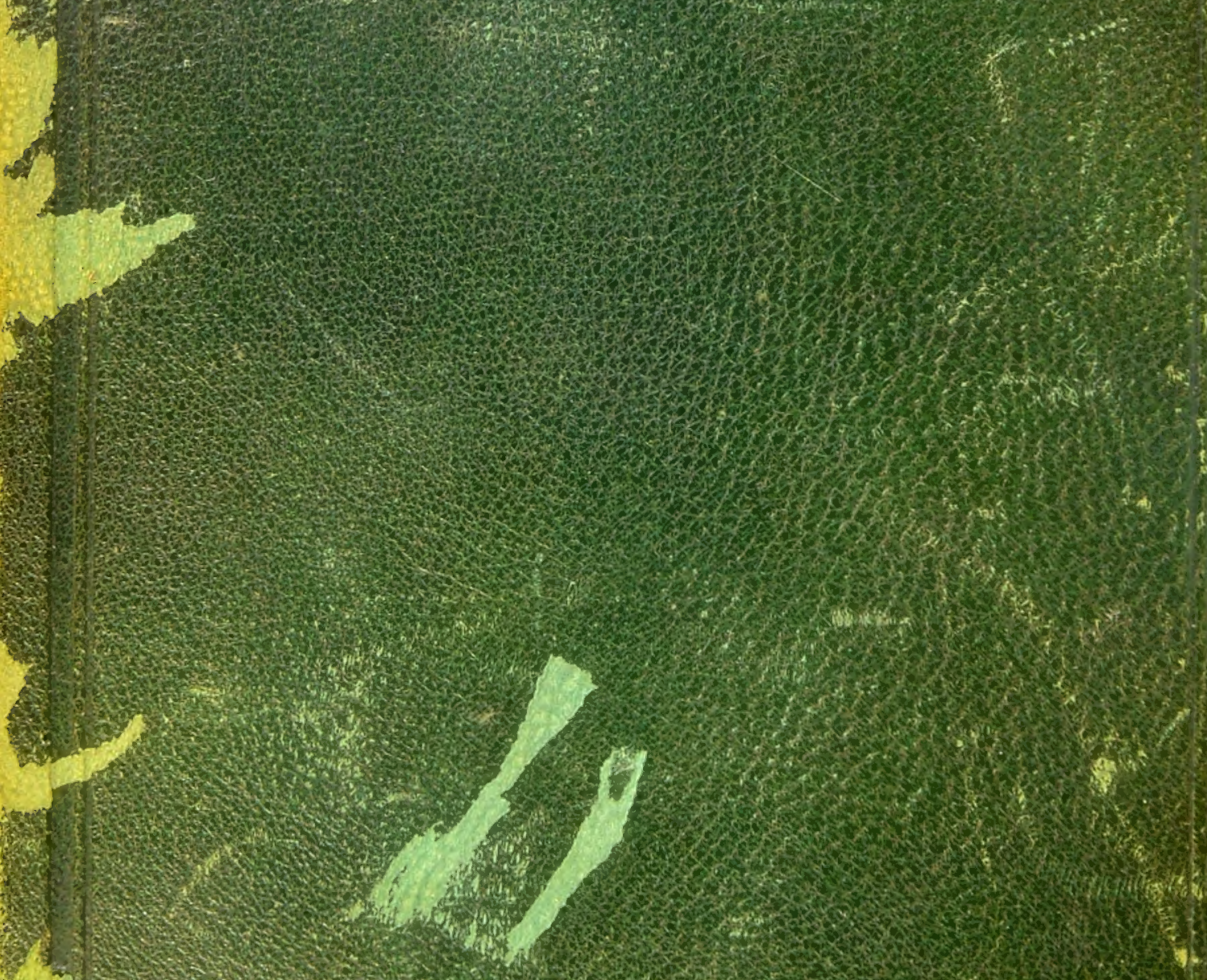



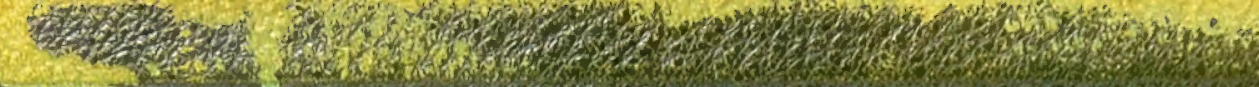

